

\section{I. 1291}
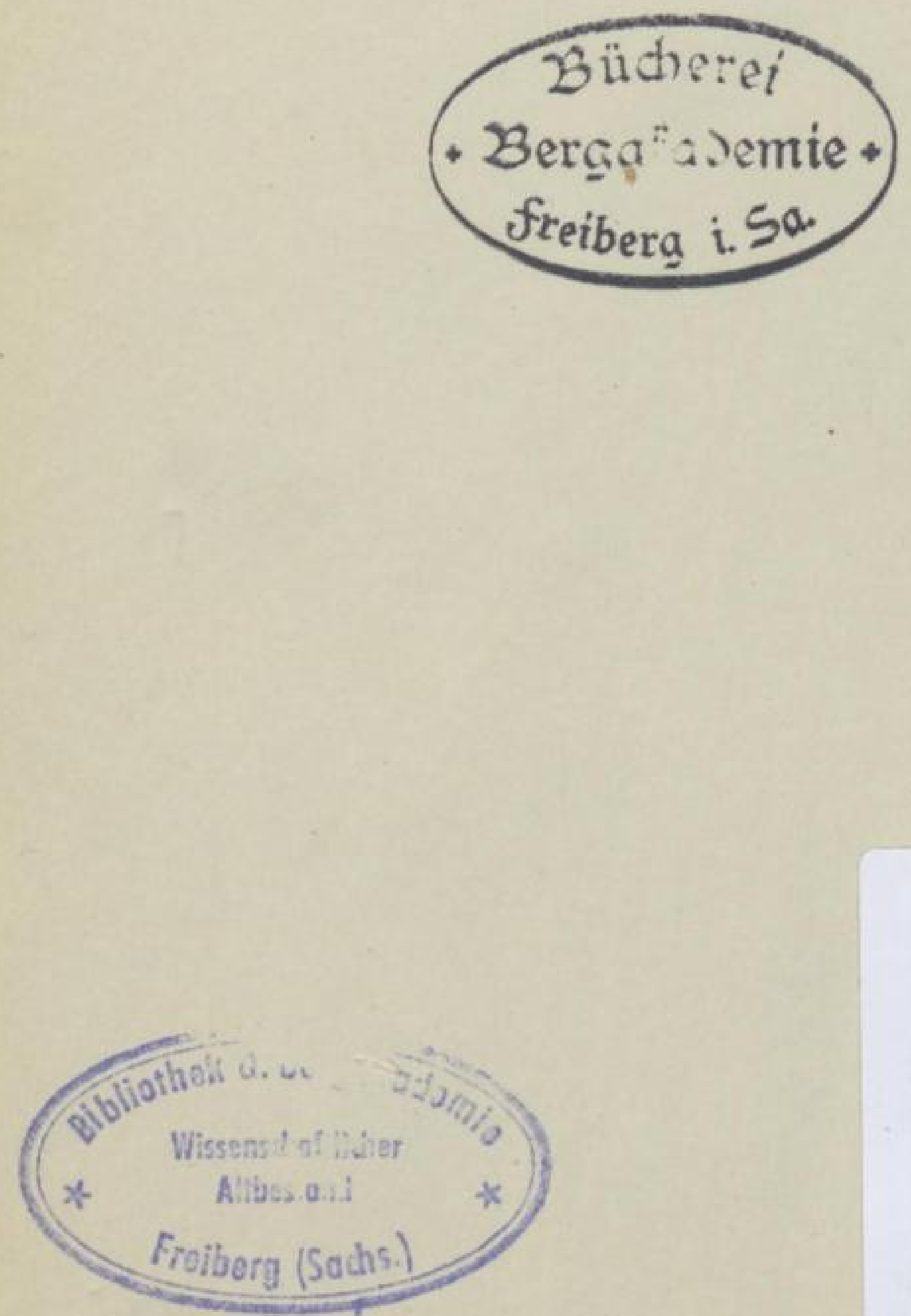

Aeseseral Wiss. Altbestand

Minerafoyic (kiveling,

\section{SLUB}

Wir führen Wissen. 


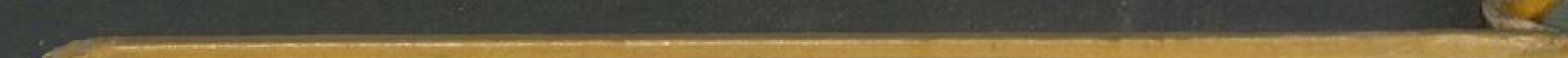

$8 x^{2}$ 
DIE

\title{
METEORITEN IN SAMIVLUNGEN
}

\section{UND IHRE+LITERATUR}

NEBST

\section{EINEM VERSUCH DEN TAUSCHWERT DER METEORITEN ZU BESTIMMEN}

$$
\text { VON }
$$

\section{Dr. E. A. WÜLFING}

A.O. PROFESSOR AN DFR UNIVERSITÄT TUUBINGEN

\author{
TÜBINGEN 1897
}

VERLAG DER H. LA UPP'SCHEN BUCHHANDLUNG. 


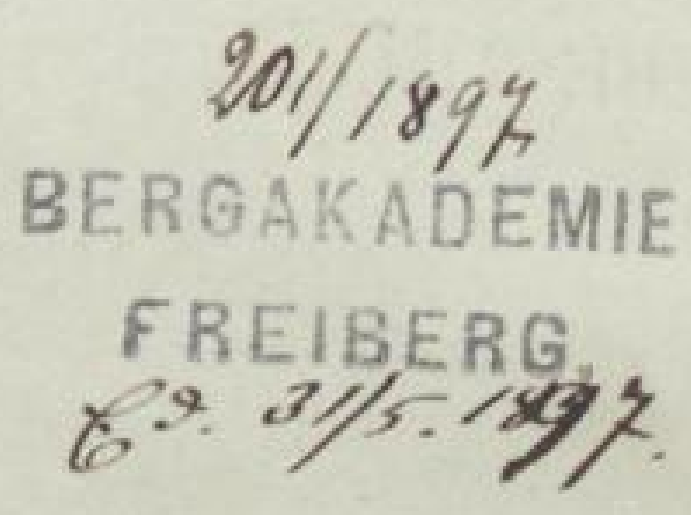

DRUCK VON H. LA UPP JR IN TUBINGEN

SLUB

Wir führen Wissen. 
Herrn Geh. Bergrat Professor Dr. H. Rosenbusch in Verehrung und Dankbarkeit gewidmet. 


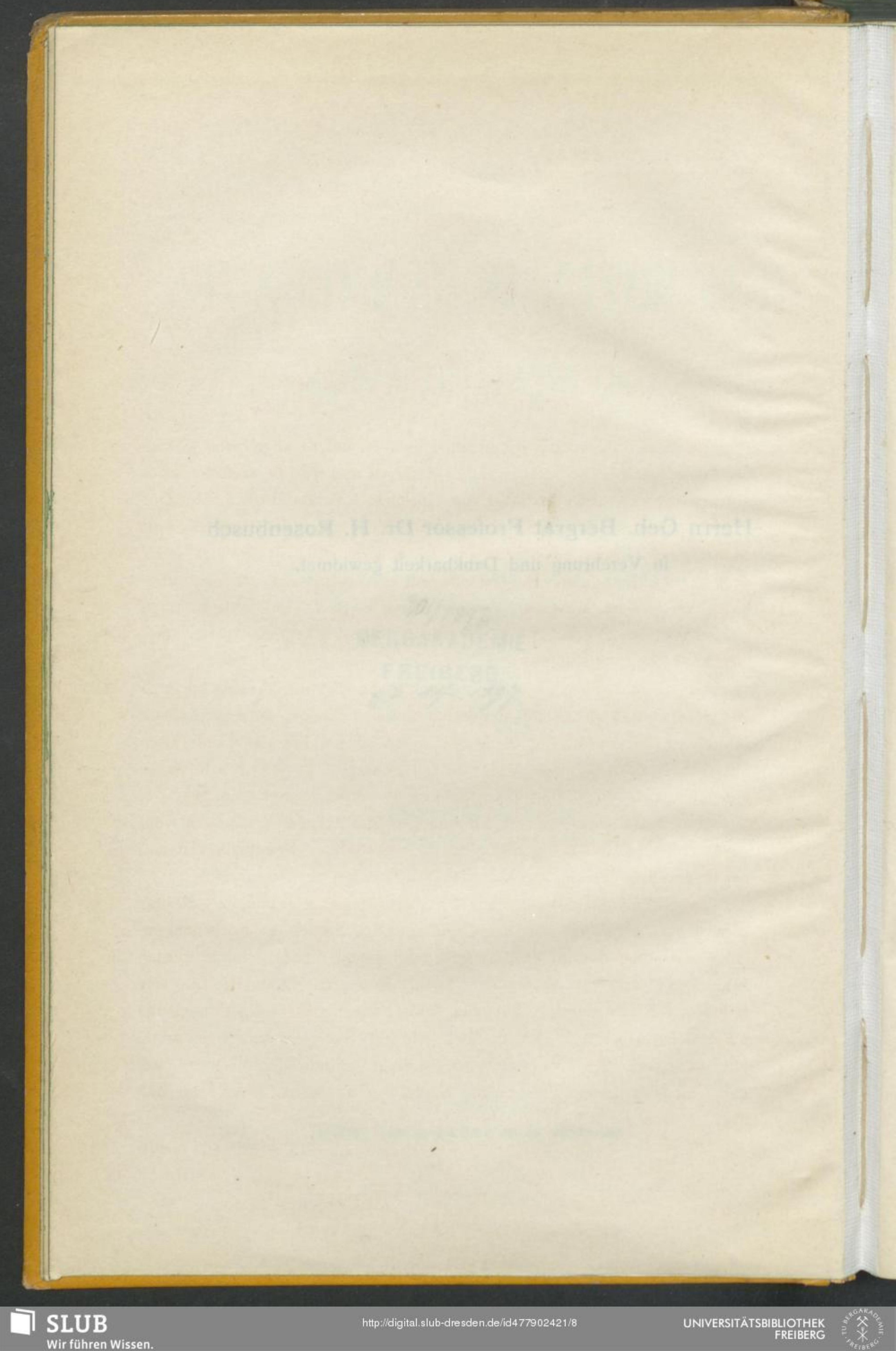




\section{Vorwort.}

Die gegenwärtige Meteoritenforschung leidet unter mangelhafter Kenntnis des in Sammlungen aufbewahrten Materials und wird demzufolge durch die Unsicherheit über den Wert der einzelnen Lokalitäten in ungünstiger Weise beeinflusst. Es dürfte daher an der Zeit sein, den Versuch, welchen $\mathrm{O}$ t to Buchner bereits im Jahre $186_{3}$ gemacht hat, in einer dem heutigen Standpunkt der Wissenschaft entsprechenden und erweiterten Form wieder aufzunehmen. Das vorliegende Buch beabsichtigt einen Ueberblick über die der Forschung heute zugänglichen Meteoriten und ihre Literatur zu gewähren.

Als ich mich vor vier Jahren dieser Aufgabe zuwandte, konnte für mich von vornherein kein Zweifel darüber bestehen, dass ich zu ihrer Lösung zahlreicher Mitarbeiter bedürfen würde. $\mathrm{Zu}$ meiner Freude ist mir diese Mitarbeit in ausgedehntem Masse zu teil geworden. Indem ich nunmehr meine Untersuchungen der Oeffentlichkeit übergebe, drängt es mich, allen Vorständen und Besitzern von Sammlungen, die auf meine zuweilen wohl etwas unbequem fallenden Bitten mit liebenswürdiger Bereitwilligkeit eingegangen sind, den Ausdruck lebhaftesten Dankes zu übermitteln.

Bei Beginn der Arbeit ahnte ich nicht die Schwierigkeiten, die mir hierbei entgegentreten würden. Erst allmählich bin ich zu der Einsicht gelangt, dass eine absolute Vollständigkeit der Angaben kaum erreicht werden kann. Das Material ist eben über die ganze Erde zerstreut und die Literatur teilweise für den einzelnen Forscher schwer zugänglich. Immerhin glaube ich annehmen zu dürfen, dass es doch gelungen ist, wenigstens vier Fünftel aller in Sammlungen aufbewahrten Meteoriten kritisch zu ordnen und über den fehlenden Bruchteil diejenigen Nachweise zu geben, welche zur Zeit überhaupt möglich sind.

Um eine weitere Verbreitung der Meteoriten anzubahnen und damit 
das Interesse an diesen wunderbaren kosmischen Massen neu zu beleben, habe ich ihren Tauschwert nach möglichst allgemeinen Gesichtspunkten zu ermitteln versucht. Ob hierbei der richtige Weg eingeschlagen worden ist, muss ich dem Urteil der Fachgenossen überlassen.

Ich darf wohl der Hoffnung Ausdruck geben, dass meine Arbeit, wenn dieselbe auch weit entfernt ist, dem mir vorschwebenden Ideale zu entsprechen, doch der Wissenschaft einige Dienste leisten wird. Ich würde glücklich sein, wenn fernere Mitteilungen über neue Meteoriten und weitere Nachrichten über ältere Fälle mich in den Stand setzten, die vorhandenen Lücken auszufüllen.

Tübingen den 25. Februar 1897 .

Ernst Anton Wülfing. 


\section{Inhalt.}

Einleitung

Zuwachs der Meteoriten XI. Rundschreiben vom Juli I 893 XII. Kurze Uebersicht der nachweisbaren Mengen aller Meteo. riten XIV. Bitte um fernere Nachrichten XVII. Gegenwärtige Verteilung der Meteoriten XVIII. Aufforderung zum Tausch XXI. Tausch durch Wertskala erleichtert XXII.

Erläuterungen zum Verzeichnis der Meteoriten in alphabetischer Reihenfolge nebst Erklärung der Abkürzungen . . . . . . . . . . . . XXIV—XLVI „Ursprüngliches Gewicht«, „Nachweisbares Gewicht XXIV. Literaturangaben und Referate XXV. Kritische Sonderung der Lokalitäten XXVI. Nomenklatur XXVI. Abkürzungen XXVIII.

Verzeichnis der Meteoriten in alphabetischer Reihenfolge

Anhang $396-407$

Meteoriten mit mangelhafter Literatur. Einige Pseudometeoriten. Drei neuere Fälle (Lesves, Madrid und Nagy-Borove).

Verzeichnis der Sammlungen

Versuch einer Bestimmung des Tauschwertes der Meteoriten

Acht Faktoren sind zu berücksichtigen: I) Nachweisbares Gewicht 430. 2) Petrographische Eigenschaften $43^{\mathrm{I}}$ (teilweise Aenderung der Gruppengewichte). 3) Zahl der Besitzer 434. 4) Das in Zukunft fallende Material 435 (Ver- 
staatlichung!). 5) Gewinnungskosten 435. 6) Erhaltungszustand 436. 7) Historisches Interesse 436. 8) Gefallen oder gefunden 436 .

Aufstellung verschiedener Formeln 437. Abrundung der Gewichte und der Zahl der Besitzer 438. Wahl unter den Formeln 439. Tabellen zur Auffindung der Werte 44I. Beispiele 445 .

Systematische Uebersicht der erhaltenen Meteoriten und ihrer Tauschwerte . . . . . . . . . 446-460

Berichtigungen . . . . . . . . . . 462 


\section{Einleitung.}

Die Erscheinung eines Meteoritenfalls hat jederzeit bei allen, die so glïcklich waren Zeugen zu sein, grosse Aufmerksamkeit und lebhaftes Interesse hervorgerufen. Allerdings haftete dieses Interesse meist nur an jenen Licht- und Schallerscheinungen, die vielfach mit überwältigender Grossartigkeit aufzutreten pflegen, und war, da die Ursache dieser Erscheinungen lange Zeit rätselhaft blieb, von keiner längeren Dauer. Erst als man darüber zu streiten begann, ob diese aus der Luft herabfallenden Massen Konkrezionen seien, welche durch irgend eine nicht näher zu definierende Wirkung der Elektrizität sich aus dem Staub der Atmosphäre bildeten oder ob sie Auswürflinge der Vulkane der Erde wären oder ob sie vom Monde, oder der Sonne und den Planeten herstammten oder ob sie schliesslich stellaren Ursprungs seien, wandte sich das Interesse diesen merkwürdigen Körpern immer mehr und mehr zu. Nach dem berühmten Steinfall von L'Aigle am 26. April r $8 \circ 3$ gelang es endlich Chladni, mit seiner Ansicht - nach welcher er sich bekanntlich im Jahre 1794 für den kosmischen Ursprung der Meteoriten ausgesprochen hatte - durchzudringen, und nun wuchs in den nächsten 30 Jahren die Zahl der Arbeiten über Meteoritenfälle ganz ungeheuer. Indessen wurden in den meisten dieser Arbeiten nur die eigentlichen Fallerscheinungen ausführlich mitgeteilt, während der Chemiker und Mineraloge dem damaligen Standpunkt seiner Wissenschaft entsprechend seine Untersuchungen mit wenig Erfolg durchführte. Die Forscher gelangten schon damals zu der Ansicht, die irrtümlicherweise auch heute noch ziemlich verbreitet zu sein scheint, dass die Meteoriten eine sehr geringe Mannig-faltigkeit in ihrer Zusammensetzung besässen, so dass das Interesse an einer genaueren mineralogischen Untersuchung allmählich erlahmte. Nur bei den Vorständen oder Besitzern grösserer Sammlungen haben die Meteoriten den Forschungsgeist dauernd zu fesseln vermocht, wie sie dies sicherlich auch in Zukunft immer thun werden. Zum Beweis brauche ich wohl nur an die Arbeiten zu erinnern, welche einerseits von den Vorständen der Sammlungen in Berlin (Rose und Rammelsberg), London (Maskelyne und Fletcher), Paris (Daubrée und Meunier) und Wien (v. Schreibers, Partsch, v. Haidinger, Tschermak und Brezina), andererseits von Chladni, v. Reichenbach, 
Shepard, Smith und Wöhler ausgeführt wurden. Dagegen hat sich die grosse Zahl der Mineralogen und Petrographen nie ernstlich mit diesen Körpern beschäftigt, wofür wohl deutlich die Thatsache spricht, dass wir bis jetzt noch kein vollständiges Handbuch oder Lehrbuch über Meteoriten in deutscher Sprache besitzen. Erst vor 3 Jahren - also gerade roo Jahre, nachdem Chladni seine berühmte Schrift „Ueber den Ursprung der von Pallas gefundenen und anderer ihr ähnlicher Eisenmassen « veröffentlichte - ist von Cohen der erste Teil einer Meteoritenkunde ${ }^{2}$ ) herausgegeben worden. Die Mehrzahl der Fachgenossen steht diesem Forschungsgebiet fremd gegenüber.

Wenn man nun aber seiner Verwunderung darüber Ausdruck geben wollte, dass dieses geringe Interesse gerade in Bezug auf jene Massen vorhanden ist, welche doch die einzigen fassbaren Belegstücke für die Beschaffenheit der extratellurischen Körper abgeben, so muss man sich erinnern, dass die Meteoriten ausserordentlich wenig verbreitet sind, und nur eine kleine Zahl von Museen grössere Sammlungen besitzt.

Bei einem genaueren Studium der Meteoriten wird man sich auch in weiteren Kreisen bald überzeugen, dass die Einförmigkeit derselben durchaus nicht so gross ist, wie man anzunehmen pflegt. Wenn man eine kleinere oder mittlere Sammlung studiert, so werden sich in einer solchen allerdings jene Meteoreisen, welche im wesentlichen aus einer EisenNickel-Legierung bestehen und jene Meteor st e in e, welche zur Gruppe der Chondrite gehören, fast ausschliesslich bemerkbar machen. Diese scheinbare Einförmigkeit schwindet aber mehr und mehr, wenn man in der Lage ist, die Gesamtmenge des vorhandenen Materials zu überblicken. Dass hierfür eine nicht ganz kleine Sammlung notwendig ist, geht schon aus der Bemerkung v. Reichenbach's hervor, welcher im Jahre 1859 [Pogg. Ann. Bd. 107 (1859), p. 157] sagt: ,Die Meteoriten zeigen sich untereinander so verschieden, manche davon stehen nach ihren Beschaffenheiten noch so isolirt, dass 200 derselben noch nicht Stoff genug gewähren, eine so weltumfassende Erscheinung nach ihrem ganzen Umfang darzustellen.

Die Untersuchung der Meteoriten pflegt heutzutage noch sehr oft nach veralteten Methoden ausgeführt zu werden. So beschränkt man sich bei der Analyse der Chondrite häufig darauf, einen in Salzsäure löslichen von einem in Salzsäure unlöslichen Teil zu trennen, wobei man den ersteren als Olivin, den letzteren als Bronzit anspricht, was doch nur im allgemeinen richtig sein dürfte. Eine genauere Trennung nach der in der Petrographie mit so grossem Erfolg angewandten Suspensionsmethode ist bei den Meteoriten bis jetzt noch nicht durchgeführt worden, obgleich sie bei vielen unzweifelhaft sehr wohl anwendbar ist. Auch pflegt man

I) Meteoritenkunde von E. Cohen, Heft I. Untersuchungsmethoden und Charakteristik der Gemengteile. Stuttgart, 1894. E. Schweizerbart. (340 Seiten und 39 Figuren). 
vergleichende Untersuchungen aller Glieder einer Gruppe bei den Meteoriten nur selten und dann auch nur in Bezug auf einzelne Eigenschaften anzustellen. Versuche, die Meteoriten hinsichtlich ihrer Bauschanalyse nach ähnlichen Gesichtspunkten zu gruppieren, wie man dies bei den Gesteinen gethan hat, sind nur in den allgemeinsten Zügen durchgeführt worden. Für eine eingehendere Betrachtung reichen die vorhandenen Analysen nicht aus; die meisten sind zudem veraltet und müssen wiederholt werden. Vor allem wäre eine vergleichende Untersuchung der interessanten Glieder der Mesosiderit-Gruppe erwünscht. Aber freilich ist heutzutage kaum eine einzige Sammlung in der Lage, solche Arbeiten anstellen zu können, weil es ihr an dem nötigen Material fehlt oder das vorhandene gar zu sorgfältig konserviert wird. Ueberhaupt muss man erst den Mut gefasst haben, mit den Meteoriten etwas weniger ängstlich umzugehen, wenn man die modernen Untersuchungsmethoden anwenden und damit unsere Kenntnis über diese Körper wesentlich fördern will. Das Material ist durchaus nicht so selten, wie man vielfach annimmt, und wird überdies, wie aus der folgenden historischen Uebersicht zu erkennen ist, in Zukunft ganz ausserordentlich wachsen. Ich habe hier die Zahl der Meteoriten angegeben, welche in den daneben aufgeführten Zeiträumen in der Literatur zuerst erwähnt werden, wobei die in diesem Buche auf p. I-395 mitgeteilten Daten zu Grunde gelegt worden sind. In der letzten Kolonne sind zum Vergleich einige der Hauptwerke über Meteoriten mit der Zahl ihrer Lokalitäten eingereiht. Die Art der Zählung geschah überall nach dem gleichen Prinzip. Diese Tabelle zeigt deutlich, dass die Aufmerksamkeit, welche man diesem Gegenstand - wenigstens was das Sammeln betrifft - gewidmet hat, in steter Zunahme begriffen ist.

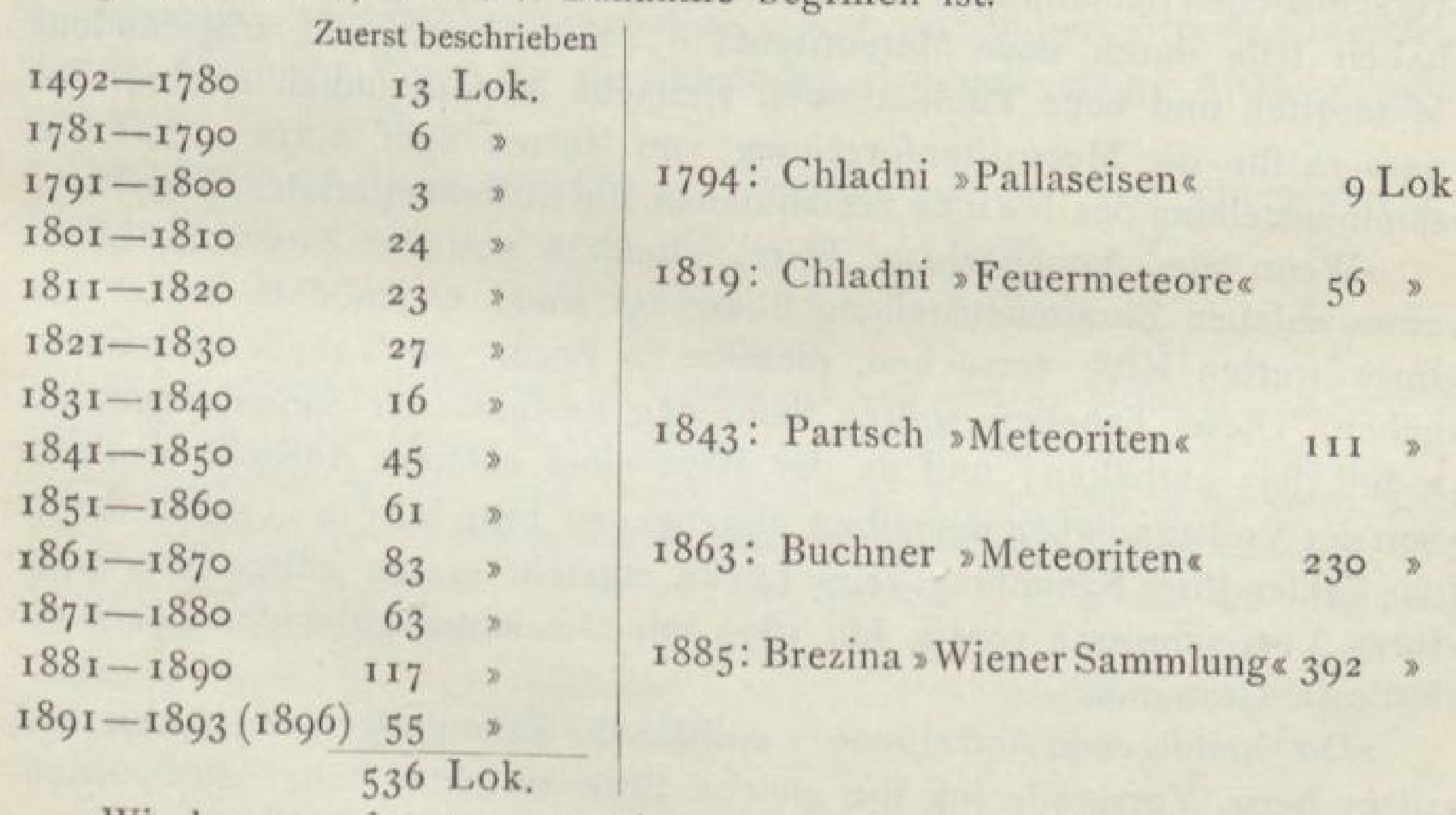

Wir kennen also gegenwärtig 536 sogenannte Lokalitäten, d. h. Meteoriten, welche gesonderten Fällen angehören. Nach anderer Zählung (Brezina, 
Wiener Sammlung 1895) erhält man etwa $55^{\circ}$ Fälle, worunter sich aber einige nicht sicher beglaubigte Meteoriten und einige unzweifelhafte Pseudometeoriten befinden; ferner mehrere Massen, welche wahrscheinlich ein und demselben Falle angehören, doppelt oder mehrfach unter verschiedener Ortsbezeichnung aufgeführt werden.

Wenn der Zuwachs in der gleichen Weise wie in den letzten 20 Jahren fortschreitet, so werden wir schon in der ersten Hälfte des folgenden Jahrhunderts mehr als 1000 Lokalitäten besitzen. v. Reichenbach hat also im Jahre 1859 [Pogg. Ann. Bd. 107 (1859) p. 157] durchaus keine zu optimistische Schätzung angestellt, wenn er vermutungsweise äussert, dass die Zahl der gesammelten Fälle nach Ablauf von 2 Jahrhunderten sich auf einige Tausend erhoben haben wird.

Ohne die Bedeutung dieses Hinweises auf das in Zukunft zu erwartende Material unterschätzen zu wollen, dürfte er doch für die Förderung des gegenwärtigen Studiums von geringem Einfluss sein. Hierfür würde es sich vielmehr vor allen Dingen darum handeln, nachzuweisen, wieviel denn eigentlich von jenen einstmals gesammelten Meteoriten erhalten blieb, und wo dieselben aufbewahrt werden. Aus diesem Grunde habe ich statistische Erhebungen über das in Sammlungen aufbewahrte Material angestellt und vor $3^{1 / 2}$ Jahren mittelst Rundschreiben um Mitteilung der Meteoritenverzeichnisse aller Sammlungen gebeten.

Der Wortlaut des Rundschreibens war folgender:

¿Vor 30 Jahren bemühte sich Dr. Otto Buchner in seinem „Werke Die Meteoriten in Sammlungens das Gewicht der einzelsnen Meteoritenfälle und die Verteilung des erhaltenen Materiales in den sverschiedenen Sammlungen zu ermitteln. Die damals gewonnenen Zahlen shaben teils durch neue Meteoritenfälle, teils durch neu aufgefundene „Meteoriten und neue Publikationen vielfache Abänderungen erfahren, so >dass es für die Meteoritenforschung von Nutzen sein dürfte, eine $\mathrm{Zu}$ »sammenstellung des $\mathrm{h}$ e u t e vorhandenen Meteoritenmateriales zu besitzen.

„Wenn Sie, hochgeehrter Herr, ebenfalls von der Zweckmässigkeit seiner solchen Zusammenstellung überzeugt sind, so möchte ich es mit shrer werten Hilfe versuchen, dieselbe in Form von Tabellen herauszuggeben. Diese Tabellen sollten dann den Bestand der Sammlungen am 》1. Juli 1893 enthalten; und da der Wert einer solchen Aufstellung allein »von der Vollständigkeit derselben abhängt, so bitte ich Sie - damit durch s das Fehlen Ihrer Sammlung keine Lücke entsteht - um gütige Mitteilung "Ihres Verzeichnisses vom I. Juli 1893 mit Gewichtsangabe der einzelnen „Fälle in Grammen.

Die beifolgende Aufzählung ${ }^{1}$ ) enthält die Sammlungen, an deren Be»sitzer bezw. Vorstände ich die gleiche Bitte gerichtet habe. Sie würden

I) Die ich hier übergehe. 
刃mich zu besonderem Danke verpflichten, wenn Sie diese Liste auf ihre $\gg$ Vollständigkeit prüfen und eventuell ergänzen wollten, soweit Ihnen dies »ohne weitere Mühe möglich ist.

»Indem ich Ihnen im voraus meinen ergebensten Dank ausspreche, sverbleibe ich u. s, w.

Tübing en, den r. Juli 1893 .

Dieses Schreiben wurde ausserdem noch in englischer und französischer Sprache verfasst. Seit jener Zeit habe ich durch Privatkorrespondenz meinen Wünschen vielfach Nachdruck verliehen, so dass es schliesslich gelungen ist, ein einigermassen übersichtliches Bild von den in Sammlungen aufbewahrten Meteoriten zu erhalten.

Leider versäumte ich, diese Aufforderung gleichzeitig auch in den verbreiteteren Zeitschriften zu publizieren, wodurch wohl mancher Fachgenosse von meiner Bitte Kenntnis erhalten hätte, die ihn vermutlich wegen ungenügender Adresse nicht erreicht hat. Bis April r894 erhielt ich 105 positive Antworten, worüber ich an anderer Stelle berichtet habe ${ }^{1}$ ). Das Ergebnis, wie es sich jetzt herausstellt, ist in der folgenden Tabelle kurz zusammengefasst. Die Charakteristik der einzelnen Gruppen ist auf p. 447 ff. gegeben.

Um einen schätzungsweisen Ueberblick darüber zu erhalten, wieviel des in Sammlungen aufbewahrten Materials hier noch nicht registriert wurde ${ }^{2}$ ), habe ich in der zweiten Reihe unter V diejenigen Gewichtsmengen aufgeführt, von denen man wohl vermutungsweise annehmen darf, dass sie noch erhalten sind. Diese hier unter $\mathrm{V}$ mitgeteilten Zahlen sind in manchen Fällen nicht so gross, wie die ursprünglichen Gruppengewichte $(\mathrm{Gu})$. So habe ich bei Gruppe $\mathbf{x}$ für das vermutungsweise erhaltene Gewicht $\mathrm{I}$ Kgr. angenommen, während ursprünglich $\mathrm{I}-2 \mathrm{Kgr}$. vorhanden gewesen sein sollen, und in ähnlicher Weise für die folgenden Gruppen einige Abänderungen getroffen. So wurde bei

$\begin{array}{clll}\text { Gruppe } 2 \text { an Stelle von } \mathrm{Gu}= & \left.108^{8}\right) & \mathrm{V}= & 100 \text { angenommen } \\ & 6 \% & 4 & \mathrm{~V}=\end{array}$

I) Verbreitung und Wert der in Sammlungen aufbewahrten Meteoriten, Jahresh. f. vaterl. Naturk. Württemberg, Bd. 51 (1895), p. 338-358 (Sep.-Abdr. von 1894).

2) Abgesehen ist von jenen Meteoritenkörpern, welche noch in der freien Natur liegen und wegen ihrer Grösse nicht in Sammlungen untergebracht werden konnten. Hier kommen besonders folgende Lokalitäten, die übrigens z. T. wieder neu aufgedeckt werden müssen, in Betracht:

\begin{tabular}{l|l|l} 
Campo del Cielo & Mejillones & Sanct Augustine's Bay \\
Great Fish River & Port Orford & Senegal \\
Huejuquilla-Gruppe & Ranchito &
\end{tabular}

3) Zur Ermittlung der ursprünglichen Gruppengewichte dienten die auf p. $447 \mathrm{ff}$. angegebenen Zahlen. 


\begin{tabular}{|c|c|c|c|c|c|c|c|c|c|}
\hline Gruppe & I0 & an & Stelle & von & $\mathrm{Gu}=$ & 156 & $\mathrm{~V}=$ & 100 & angenommen \\
\hline 3 & I3 & $\gg$ & , & $\gg$ & $\mathrm{Gu}=$ & 380 & $\mathrm{~V}=$ & 300 & x \\
\hline , & I4 & 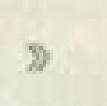 & 》 & 》 & $\mathrm{Gu}=$ & 346 & $\mathrm{~V}=$ & 300 & s \\
\hline , & I 7 & 》 & » & 》 & $\mathrm{Gu}=$ & $\left.45^{1}\right)$ & $\mathrm{V}=$ & 500 & s \\
\hline » & I 8 & " & , & , & $\mathrm{Gu}=$ & $5^{85}$ & $\mathrm{~V}=$ & 500 & s \\
\hline , & 20 & , & 》 & s & $\mathrm{Gu}=$ & $3^{8} 5$ & $\mathrm{~V}=$ & $35^{\circ}$ & " \\
\hline , & 28 & 》 & , & > & $\mathrm{Gu}=$ & $6_{3}$ & $\mathrm{~V}=$ & 50 & , \\
\hline 》 & 40 & 》 & 》 & 》 & $\mathrm{Gu}=$ & $\left.840^{2}\right)$ & $\mathrm{V}=$ & 700 & , \\
\hline , & $4 \mathrm{I}$ & 》 & $\gg$ & 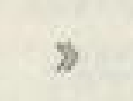 & $\mathrm{Gu}=$ & 6700 & $\mathrm{~V}=$ & 6000 & $\gg$ \\
\hline D & 42 & 》 & s & $»$ & $\mathrm{Gu}=$ & 16257 & $\mathrm{~V}=$ & 16000 & $\$$ \\
\hline 》 & 43 & 》 & $\gg$ & 》 & $\mathrm{Gu}=$ & 214 & $\mathrm{~V}=$ & 200 & $\gg$ \\
\hline , & 48 & 》 & $\Rightarrow$ & , & $\mathrm{Gu}=$ & 66 & $\mathrm{~V}=$ & 50 & , \\
\hline
\end{tabular}

Uebersicht der auf die einzelnen Gruppen entfallenden Mengen der Meteoriten in systematischer Anordnung.

16 2 vink
A. Calcium-
arm an Nic
lichen krys
I. Angrit, A
2 II. Eukrite, Eu
3 III. Shergottit, Sh
4 IV. Howardite, Ho und $\mathrm{Hob}$

$10 \&_{b} \downarrow \ell-$ B. Magnesiumreiche Steine, arm an Nickeleisen. Struktur im wesentlichen krystallinisch körnig.

$5 \quad$ I. Bustite, $\mathrm{Bu}$

6 II. Chassignit, $\mathrm{Ch}$

7 III. Chladnite, $\mathrm{Chl}$ und Chla

8 IV. Amphoterite, Am

$309-$

C. Magnesiumreiche Steine mit Nickeleisen. Struktur chondritisch.

I. Howarditische Chondrite, Cho u. Choa

\begin{tabular}{|c|c|}
\hline & $\begin{array}{l}\text { Vermut. } \\
\text { erralt. } \\
\text { Geow. } \\
\text { in KkT. }\end{array}$ \\
\hline z & \\
\hline 1 & 1 \\
\hline 4 & 100 \\
\hline 1 & 5 \\
\hline 10 & 10 \\
\hline
\end{tabular}

Nachweisb.

Grappen- Zahl gowichte Ber in $\mathrm{gr}_{\mathrm{G}}$ Bes.

397 11 $90610 \quad 180$ $4897 \quad 13$ $4707 \quad 141$

II. Weisse Chondrite

\begin{abstract}
a. Weisse Chondrite, $\mathrm{Cw}$
\end{abstract}
b. Weisse Chondrite, geadert, Cwa

c. Weisse Chondrite, breccienähnlich, Cwb

III. Intermediäre Chondrite

a. Intermediäre Chondrite, $\mathrm{Ci}$

14

b. Intermediäre Chondrite, geadert, $\mathrm{Cia}$

15

c. Intermediäre Chondrite, breccienähnlich, $\mathrm{Cib}$

1) Wegen Pultusk erhöht. - 2) Ohne Prambanan. 
IV. Graue Chondrite

a. Graue Chondrite, $\mathrm{Cg}$

b. Graue Chondrite, geadert, Cga

c. Graue Chondrite, breccienähnlich, $\mathrm{Cgb}$

V. Schwarze Chondrite, Cs

VI. Kügelchenchondrite
a. Ornansit und Ngawit, $\mathrm{Cco}_{\text {co }} \mathrm{Ccn}$
b. Kügelchenchondrite, $\mathrm{Cc}$
c. Kügelchenchondrite, geadert, Cca
d. Kügelchenchondrite, breccienähnlich, Ccb
e. Kügelchenchondrite, krystallinisch, Cck

VII. Krystallinische Chondrite

a. Krystallinische Chondrite, $\mathrm{Ck}$

b. Krystallinische Chondrite, geadert, Cka

c. Krystallinische Chondrite, breccienähnlich, Ckb

VIII. Kohlige Chondrite, $\mathrm{K}$ und $\mathrm{Kc}$

IX. Orvinit, Co

$\mathrm{X}$. Tadjerit, $\mathrm{Ct}$

XI. Ureilite, Clu

XII. Meteorsteine, welche noch keine Stellung im System gefunden haben

\section{Nickeleisen mit Silikaten.}

\section{E. Nickeleisen.}

I. Oktaëdrische Eisen

IV. Meteoreisen, welche noch keine Stellung
IV. Dichte Eisen, Db, Dn, Dp, Ds, Dt im System gefunden haben

\begin{tabular}{|c|c|c|c|}
\hline & $v$ & G & 1 \\
\hline 8 & 495 & 436114 & 146 \\
\hline 27 & 500 & 345728 & 569 \\
\hline 23 & 500 & 363575 & 408 \\
\hline & 430 & 138827 & 105 \\
\hline 3 & 13 & 8013 & 48 \\
\hline 43 & 350 & 282431 & 686 \\
\hline 8 & 63 & 48498 & 107 \\
\hline 14 & 500 & 367391 & 322 \\
\hline 7 & 32 & 24634 & 78 \\
\hline 20 & 880 & 835880 & 279 \\
\hline 2 & 14 & 7849 & 30 \\
\hline 2 & 230 & 144785 & 106 \\
\hline 8 & 50 & 29660 & 214 \\
\hline 1 & 3 & 2324 & 26 \\
\hline 1 & 9 & 8843 & 13 \\
\hline 3 & 5 & 4528 & 30 \\
\hline 21 & 114 & 12468 & 25 \\
\hline 10 & 590 & 482925 & 284 \\
\hline 1 & 1 & 970 & 11 \\
\hline 1 & 82 & 81786 & 73 \\
\hline 9 & 2000 & 1742519 & 357 \\
\hline 1 & 23 & 22939 & 13 \\
\hline 1 & 6 & 5858 & 27 \\
\hline 5 & 64 & 36054 & 95 \\
\hline 24 & 700 & 224263 & 402 \\
\hline 67 & $6000 ?$ & 4694845 & 1269 \\
\hline 21 & 16000 & 14535060 & 512 \\
\hline 4 & 200 & 107362 & 136 \\
\hline 2 & 1000 ? & 36657 & 63 \\
\hline 10 & 1950 & 1914991 & 271 \\
\hline 8 & 590 & 581409 & 74 \\
\hline 3 & 100 & 83135 & 49 \\
\hline 8 & 50 & 19402 & 85 \\
\hline 15 & 2700 ? & 1596406 & 272 \\
\hline 11 & 2245 & 2229995 & 28 \\
\hline
\end{tabular}


Für das gesamte vermutungsweise erhaltene Gewicht ergeben sich dann $39912 \mathrm{Kgr}$. und für das gesamte nachweisbare Gewicht $324 \mathrm{I} 2 \mathrm{Kgr}$., d. h. also etwas mehr als $4 / 5$ aller in Sammlungen aufbewahrten Meteoriten, Diese nachweisbare Menge verteilt sich in der Weise, dass auf die

$\begin{array}{lr}\text { Meteorsteine } & 4015 \mathrm{Kgr} . \\ \text { Mesosiderite, Pallasite etc. } & 2337 \mathrm{Kgr} . \\ \text { Meteoreisen } & 26060 \mathrm{Kgr} \text {. entfallen. }\end{array}$

Für eine Reihe von Lokalitäten zeigen sich also noch erhebliche Differenzen zwischen jenen Gewichten, welche in der Literatur angegeben werden, und jenen Mengen, welche nach meinen Erhebungen in Sammlungen vorhanden sind. Ich möchte diese Fälle in zwei Kategorien teilen und in die erste Kategorie jene Meteoriten stellen, bei denen man keine allzugrosse Hoffnung hat, einen grösseren Teil der fehlenden Menge wiederzufinden. Dahin würden z. B. zu zählen sein:

Asco
Chantonnay
Charwallas
Danville
Epinal
Lucé

Luponnas
Manegaon
Marshall County
Perth
Pittsburg
Pnompehn

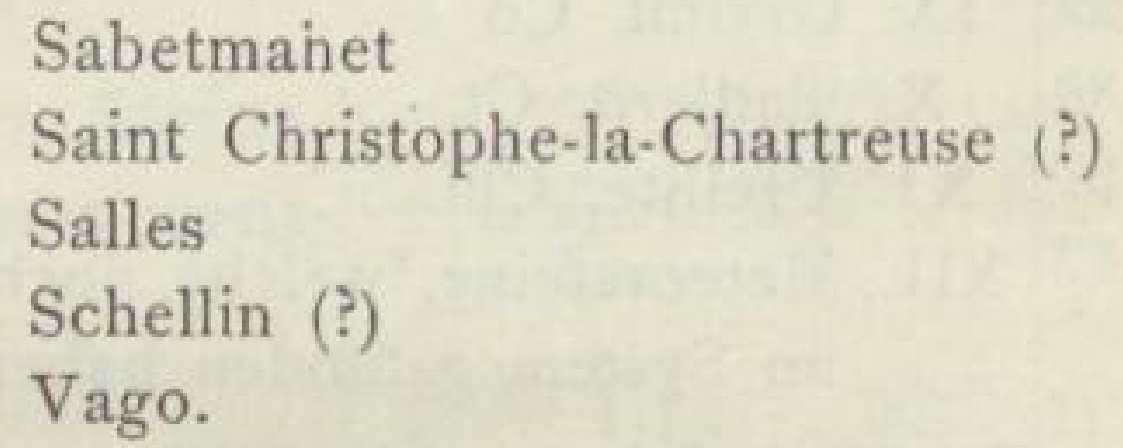

$\mathrm{Ob}$ die grosse Masse des Eisens von Jackson County verloren gegangen oder unter einem andern Namen wieder aufgetaucht ist, dürfte nicht leicht mit Sicherheit zu entscheiden sein. Wie es sich mit dem Eisen von Jenny's Creek, sowie mit so vielen andern amerikanischen Eisen verhält (Baird's Farm, Cosby's Creek), bedarf einer sorgfältigen, an Hand eines reichlichen Materials auszuführenden, kritischen Untersuchung. Vielleicht wird man bei solchen kritisch vergleichenden Arbeiten finden, dass die Eisen, welche aus benachbarten Bezirken stammen, auffallende Aehnlichkeit zeigen, wie man andererseits auf die Ueberraschung gefasst sein muss, bei manchen Eisen, welche unter dem gleichen Namen gehen, grosse Verschiedenheiten anzutreffen, was häufig auf eine Verwechslung der Etiquetten zurückzuführen sein dürfte. Solche revidierende Arbeiten können nicht bald genug vorgenommen werden, da die Verwirrung im Laufe der Zeit immer weiter um sich greifen wird.

Zur andern Kategorie rechne ich jene Meteoriten, bei denen man hoffen darf, noch weitere Massen irgendwo in Sammlungen $\mathrm{zu}$ finden. Ich habe auch für diese, bei denen also der Unterschied zwischen ursprünglichem und nachweisbarem Gewicht in auffallendem Widerspruch mit den Angaben der Literatur steht, hier eine Liste zusammengestellt. In dieser Liste sind auch manche Lokalitäten aufgeführt, bei denen nur unbedeutende Mengen fehlen; indessen waren diese kleinen Stücke unzweifelhaft 
einmal vorhanden und befanden sich in Händen von Meteoritenforschern, so dass man annehmen muss, dass sie noch irgendwo auf bewahrt werden. Z. B. hat Shepard von den Eisen von Auburn, Lion River und Orange River viel mehr besessen als sich jetzt nachweisen lässt. Auch hat er einen grösseren Teil des Eisens von Ruffs Mountain und des Steins von Searsmont in Händen gehabt. Aehnliches gilt für die Eisen von Russel Gulch und Tazewell, welche einst im Besitz von Lawrence Smith waren. Unter den aufgezählten 112 Lokalitäten befinden sich nicht weniger als 40 aus den Vereinigten Staaten von Nordamerika, nämlich 9 Steine und 3 I Eisen, d. h. etwa $1 / 3$ aller aus diesem Gebiet bekannt gewordenen Meteoriten. Die ungleichmässige Verteilung zwischen Steinen und Eisen ist auffallend, so dass man auf den Gedanken kommt, die Eisen könnten verloren gegangen und nachher unter einem andern Namen wieder aufgetaucht sein. - Ich will nicht versäumen, auf diese riz Fälle ganz besonders aufmerksam zu machen und die Bitte anschliessen, mir weitere Nachrichten darüber zukommen zu lassen. Bei einigen habe ich in () darauf hingewiesen, wo die Massen zu finden sein könnten.

\section{Meteoriten, von denen wahrscheinlich mehr erhalten ist als} sich jetzt nachweisen lässt.

\section{S t e in e.}

\begin{tabular}{l|l|l} 
Alfianello & Harrison County & Ogi (Tokio ?) \\
Angra dos Reis (Dr. Tra- & Hartford & Okniny \\
\multicolumn{1}{c}{ vassos? S. H. der Papst?) } & Inca & Orvinio (Rom?) \\
Bachmut & Indarch & Pacula (Mexico ?) \\
Baratta (Sidney ?) & Jonzac (La Rochelle?) & Petersburg \\
Bethlehem & Kalumbi & Renazzo (Bologna, Stern- \\
Bherai & Kesen (Tokio ?) & warte?) \\
Bialystock & La Charca & Sabetmahet \\
Bishunpur (Calcutta?) & Le Pressoir & Searsmont (Shepard?) \\
Bori (Calcutta?) & Limerick (Dublin?) & Sewrukof \\
Cabezzo de Mayo & Little Piney & Tjabé (Batavia?) \\
Charsonville & Maêmê (Tokio?) & Tounkin \\
Chassigny & Mainz & Tourinnes-la-Grosse \\
ColdBokkeveld(Capstadt?) & Makariwa & Veramin (Schah v.Persien ?) \\
Doroninsk & Middlesbrough (York?) & Wairarapa (Wellington ?) \\
Dundrum (Dublin?) & Milena & Warrenton \\
Favars & Monte Milone & Werchne Tschirskaja \\
Feid Chair & Mordvinovka & (Charkow ?) \\
Forsyth & Morristown (Washington?) & Zabrodje \\
Fukutomi & Nagy-Borove & \\
Gilgoin & New Concord & \\
\hline
\end{tabular}




\section{E is en.}

\begin{tabular}{l|l|l} 
Auburn (Shepard ?) & Kenton County & Russel Gulch (Smith ?) \\
Bald Eagle (Lewisburg,Pa?) & Kokomo & Saint Cristophe-la-Char- \\
Bear Creek & La Grange (Smith?) & treuse \\
Blue Tier & Lion River (Shepard ?) & Shingle Springs \\
Cachiyuyal (Santiago ?) & Losttown & Smithville \\
Cleveland (Philadelphia ?) & Madoc (Washington, Geol. & Staunton \\
Cosina (Mexico,Bergschule?) & Surv.?) & Tajgha \\
Costilla (Denver, Col.?) & Misteca (Mexico ?) & Tazewell (Smith ?) \\
Cranbourne (Melbourne?) & Moonbi (Sidney ?) & Thunda (Sidney ?) \\
Dakota & NarraburraCreek(Sidney?) & Tonganoxie (University of \\
Deep Springs (Raleigh, & Nelson County & Kansas?) \\
N. C. ?) & Oktibbeha (Philadelphia?) & Trenton \\
Dehesa (Santiago ?) & Orange River (Shepard?) & Tucson \\
Denton County (Austir ?) & Prambanan (Batavia?) & Victoria \\
Descubridora (Mexico ?) & Primitiva (Howell?) & Victoria West (Capstadt ?) \\
Eli Eluat & Putnam County & Union County \\
Grand Rapids (Ward?) & Rancho de la Pila-Ca- & Wichita (Austin, Texas?) \\
Greenbrier County & \multicolumn{1}{|c|}{ caria (Mexico ?) } & Wooster \\
Henry County & Rasgata & Zacatecas (Mexico ?) \\
Ivanpah & Ruffs Mountain (Shepard?) &
\end{tabular}

Kommen wir nunmehr auf das positive Resultat meiner Erhebungen zurück, so zeigt sich, dass von einer Seltenheit der Meteoriten nicht wohl mehr die Rede sein kann. Wenn dennoch so wenige Forscher in der Lage sind, diese Körper kennen zu lernen, so wird es nur daran liegen, dass die Massen zu wenig verbreitet sind. Diese zu geringe Verbreitung der Meteoriten kann in doppeltem Sinne verstanden werden: Einerseits giebt es überhaupt zu wenig grössere Meteoritensammlungen (kaum 20 ) und andererseits sind die einzelnen Lokalitäten grösstenteils in einer Hand. Der zweite Uebelstand lässt sich wie ich glaube beseitigen; ehe ich aber darauf näher eingehe, will ich an Hand des hier vorliegenden Materials zeigen, wieweit die 536 Lokalitäten verteilt sind.

Von dieser Verteilung gewinnt man eine Vorstellung in folgender Weise: Man dividiere das in einer Hand befindliche Maximalgewicht $M$ durch das nachweisbare Gewicht N. Für den Fall, dass ein Meteorit sich nur in einer Hand befindet, würde $\operatorname{man} \frac{\mathrm{M}}{\mathrm{N}}=\mathrm{x}$ erhalten, während in allen andern Fällen $\frac{\mathrm{M}}{\mathrm{N}}$ einen echten Bruch darstellt, der um so kleiner wird, je grösser die Verteilung ist. Teilt man hiernach die 536 Lokalitäten in ro 
Gruppen ein, von denen die erste Gruppe alle jene Meteoriten umfasst, welche gänzlich oder wenigstens zu $96 \%$ sich in einer Hand befinden, die zweite Gruppe alle jene Meteoriten enthält, welche $\mathrm{zu} 95 \%-86 \%$, im Mittel also $\mathrm{zu} \% / 10$ einem Besitzer angehören u. s. f. bis $1 / 10$, so erhält man folgenden Ueberblick:

\begin{tabular}{|c|c|c|c|}
\hline$\frac{\mathrm{M}}{\mathrm{N}}$ & In einer Hand & Zahl aller Lokalitäten & $\begin{array}{l}\text { Zahl der Lokalitä- } \\
\text { ten mit mehr als } \\
50 \text { gr. des nach- } \\
\text { weisbaren Gew. }\end{array}$ \\
\hline 1.0 & $100-96 \%$ & $\mathrm{II}_{3}$ & 85 \\
\hline 0.9 & $95-86$ & 65 & 62 \\
\hline 0.8 & $85-76$ & $5^{6}$ & 55 \\
\hline 0.7 & $75-66$ & 43 & 40 \\
\hline 0.6 & $65-56$ s & 49 & 45 \\
\hline 0.5 & $55-46$ & 60 & 55 \\
\hline 0.4 & $45-36$ 》 & 47 & 44 \\
\hline 0.3 & $35-26$, & 62 & $5^{8}$ \\
\hline 0.2 & $25-16$ & 34 & 34 \\
\hline 0.1 & $15-13 »$ & $\frac{4}{\left.533^{1}\right)}$ & $\frac{4}{482}$ \\
\hline
\end{tabular}

Berücksichtigt man die Fälle nicht, von denen sich weniger als $50 \mathrm{gr}$. nachweisen lassen und man also schon wegen des mangelnden Materials keine grössere Verteilung erwarten darf, so ist die Hälfte, nämlich $85+$ $62+55+40=24^{2}$ der übrigen Meteoriten $\mathrm{zu} \% / 10$ oder mehr in einer Hand, und nur ganz wenige haben eine solche Verteilung erfahren, dass nicht mehr als $1 / 10$ sich in einer Hand befindet. Hierzu gehören nur Coahuila, Marshall Co., Staunton und Toluca. Wenn man die nachweisbaren Gewichte hiervon im Index auf p. I-395 nachschlägt, so wird man finden, dass die Verteilung durchaus nicht proportional der vorhandenen Menge stattgefunden hat, sondern dass sie ohne Gesetzmässigkeit, rein durch zufällige Momente bedingt, vor sich gegangen ist.

Auch dem Einwand, dass die Museen in Wien, London und Paris die Hauptmassen der Meteoriten besässen und dass das Uebrige im Verhältnis zu diesen 3 Sammlungen nicht in Betracht komme, lässt sich an Hand der nachstehenden Zahlen leicht begegnen. In der folgenden Liste sind die Sammlungen ${ }^{2}$ ) nach der Zahl derjenigen Lokalitäten geordnet, von welchen sie das Maximalgewicht besitzen; jene 3 grossen Sammlungen enthalten nur von 225 Lokalitäten, also bei weitem noch nicht von der Hälfte aller Fall- und Fundorte die Hauptmassen.

1) Ohne Lesves, Madrid und Nagy-Borove.

2) Die nähere Bezeichnung dieser Sammlungen, sowie auch die Namen jener Lokalitäten finden sich auf p. $408-429$. 


\begin{tabular}{|c|c|c|c|c|c|c|}
\hline Wien, H. M. & 87 & Dorpat & & Oxford & 2 & Herzogenbusch \\
\hline London, B. M. 8 & 80 & Göttingen & & Riga & 2 & Kasan \\
\hline Paris, M. & $5^{8}$ & Howell & 5 & v. Siemaschko & 2 & Klausenburg \\
\hline Calcutta & 27 & Petersburg, B. & 5 & Berlin, G. & I & Kopenhagen \\
\hline Harvard, U. & 23 & Gregory & . & Braunau & I & Madison \\
\hline New Haven & I 8 & London, P. G. & 4 & Bremen & I & Modena \\
\hline Berlin, U. & I5 & München & 4 & Breslau & I & Möricke \\
\hline Tübingen & 15 & Odessa & 4 & Debreczin & I & Moskau \\
\hline Washingt., Sh. & I 5 & Stockholm & 4 & Detmold & I & Paris, E. \\
\hline Budapest & 13 & Belgrad & 3 & Dresden, M. & I & Parma \\
\hline Petersburg, A. & 12 & Brezina & 3 & Dublin & I & Pohl \\
\hline Ward & 12 & Kristiania & 3 & Edinburg & I & Prag, M. \\
\hline Madrid & I0 & Rom, U. & 3 & Ensisheim & I & v. Schilling \\
\hline Kunz & 8 & Turin, U. & 3 & Freiberg, i. S. & I & Sevilla \\
\hline Washington & 7 & Batavia & 2 & Gera & I & Siena \\
\hline Bailey & 6 & Bologna & 2 & Giessen & $\mathbf{I}$ & Speyer \\
\hline Kiew & 6 & Eger & & Halle & I & Würzburg \\
\hline Rio de Janeiro & 6 & Krantz & & Heidelberg & I & \\
\hline Bement & & Leiden & & Helsingfors & I & \\
\hline
\end{tabular}

Wenn ich nun eine etwas grössere Verbreitung dieser Massen in Vorschlag bringe, so möchte ich, um Missverständnissen vorzubeugen, voranschicken, dass sich eine Verteilung nur auf solche Meteoriten erstrecken soll, welche eine zu diesem Zweck notwendige Zerkleinerung ohne Schaden ertragen können. Ausgeschlossen also wären jene Meteoriten, welche noch eine vollständige Rinde tragen, eine schöne Orientierung besitzen und als einzig in ihrer Art angesehen werden; diese sind aber nur in verhältnismässig geringer Zahl vorhanden. Dagegen würden viele Meteoriten, ganz besonders die Meteoreisen in wissenschaftlicher Beziehung einen bedeutend grösseren Nutzen bringen, wenn man sie zerkleinerte. Liegen doch in vielen Sammlungen grössere Eisenblöcke schon seit Jahrzehnten immer noch mit der Rostrinde bedeckt, welche sie beim Auffinden besassen, und wodurch ihre innere interessante Struktur kaum zu erkennen ist. - Dass diese Ansicht von massgebenden Meteoritenforschern mehrfach ausgesprochen ist, möge an einigen Beispielen gezeigt werden. So sagt Partsch ${ }^{1}$ ) die Meteoreisenmassen erhalten durch Aetzen und Anlaufen eigentlich erst wissenschaftliches Interesse , und ähnlich Buchner ${ }^{2}$ ) seinen rohen Eisenklumpen kann man ob seiner Grösse und Schwere anstaunen; aber so lange er nicht polirte und geätzte Schnittflächen hat,

I) Kurze Uebersicht der im k. k. Hof-Mineralien-Kabinete zu Wien zur Schau gesteliten acht Sammlungen, 1843, p. 74-75.

2) Pogg. Ann. Bd. 116 (1862), p. 642. 
existirt er kaum für das Studium. Mit der Zubereitung steigt auch der Werth ....\&. Ferner äusserte sich Rose ${ }^{1}$ ), als er die Berliner Sammlung untersuchte $\gg$ Ich habe... sämmtliche Stein- und Eisenmeteorite anschleifen lassen, und letztere geätzt, da man nur auf diese Weise bei den ersteren einen Ueberblick über die Gemengtheile erhalten, bei den letzteren die Structur erkennen kann.... ${ }^{2}$ ).

Es entsteht nunmehr die Frage, wie man eine grössere Verbreitung der einzelnen Lokalitäten herbeiführen kann. Aus der Uebersicht auf p. XIV-XV lässt sich ersehen, welch grosse Mengen vorhanden sind; da liegt der Gedanke vielleicht nicht ganz fern, sich an die Freigebigkeit der Besitzer dieser jetzt kaum noch als selten anzusehenden Objekte $\mathrm{zu}$ wenden. Schon Haidinger ${ }^{3}$ ) hat sich in ähnlichem Sinne geäussert und eine derartige Verbreitung als dem swahren Geist der Wissenschaft * entsprechend bezeichnet, und auch die Vorstände des Calcutta-Museum's (Oldham, Atkinson und Thomson) sprechen sich in einem Gutachten ${ }^{4}$ ) dahin aus, sthat in their opinion specimens may, without injury to the Society's collections, be transmitted to the Vienna collection\&. Auch hat sich die Sammlung der Petersburger Akademie besonders hinsichtlich des Pallaseisens sehr freigebig gezeigt. Ferner möge als ein nachahmenswertes Beispiel hier erwähnt werden, dass von dem Stein von Djati Pengilon, welcher am 19. März 1884 auf der Insel Java fiel, über to Kgr. durch die niederländische Regierung an viele Sammlungen zur Verteilung gelangten. Freilich dürfte hier die unverhoffte Besitzerlangung eines Wertobjekts zu grösserer Freigebigkeit verleitet haben. Anders liegen die Verhältnisse, wenn alte Bestände, die schon seit langer Zeit in einer Sammlung aufbewahrt werden, angegriffen und verteilt werden sollen. Auch sind wohl manche grosse Sammlungen schon durch ihre Statuten an einer derartigen Freigebigkeit verhindert. So bleibt schliesslich nur noch die Möglichkeit offen, auf dem Wege des Ta uschverkehrs das Angestrebte zu erreichen. Dieser geplante Tauschverkehr setzt natürlich immer zwei Sammlungen voraus, welche sich gegenseitig ergänzen; es ist nicht schwer, aus den in diesem Buche befindlichen Angaben sehr viele derartige Sammlungen zu finden. Wie weit die Statuten eines Museums einem solchen Tauschgeschäfte hin-

1) *Meteoriten ", Abh. Berlin. Akad, 1863, p. 25 .

2) Das Zerschneiden der Meteoreisen, welches bis vor Kurzem schwierig oder mit grossem Verlust an Material verbunden war, lïsst sich gegenwärtig mit der sog. $>\mathrm{Co}$ lumbus-Kaltsäge leicht ausführen. Diese Säge schneidet bei einer Schnittbreite von nur $1 \mathrm{~mm}$. und bei äusserst geringer Kraftanwendung ein $q \mathrm{~cm}$. Eisen in etwa 3 Minuten, so dass grössere Platten in wenigen Stunden hergestellt werden können. Einzelheiten über diese Maschine sind bei Herrn Robert Zapp in Düsseldorf oder in Stuttgart, Paulinenstrasse $\mathrm{I} z \mathrm{z}$ erfahren.

3) Sitzber. Wien. Akad. Bd. 48 II (1863), p. 307-308.

4) Journ. Asiatic. Soc. Bengal Bd. 28 ( 1859 , Proc.), p. 26 I. 
derlich sind, ist hier nicht der Platz zu erörtern. Meine Aufgabe soll es nur sein, darauf hinzuweisen, dass die Forschung gewinnen kann, wenn von dieser Seite sich kein Hindernis bietet.

Als Einwand gegen diesen geplanten Tauschverkehr liesse sich vielleicht geltend machen, dass eine grössere Sammlung überhaupt nicht zu tauschen brauche, dass sie genügend Material besitze, um einen Forscher sein ganzes Leben lang zu beschäftigen. Indessen habe ich bereits oben auf p. XI bei Erwähnung der Mesosiderite gesagt, dass z. B. keine Sammlung in der Lage ist, ein vollständiges Vergleichsmaterial dieser Gruppe zu besitzen, was auch für viele andere Gruppen gilt. Aus diesem Grunde müssen die Arbeiten, welche sich mit der Beschreibung einzelner Meteoritensammlungen beschäftigen, notwendig einseitig ausfallen. Selbst die enormen Massen, welche in Wien, London und Paris vorhanden sind, vermögen einzeln kein vollständiges Bild der Erscheinungen zu geben und $\mathrm{zum}$ G 1 ü c k können auch diese grossen Sammlungen noch Desideraten-Listen aufstellen, die sie nicht anders, als durch Tauschverkehr mit andern Sammlungen zu beseitigen vermögen.

Für Sammlungen, welche überhaupt arm an Meteoriten sind, bleibt kein anderes Mittel übrig, als sich durch Kauf in den Besitz der im Handel vorkommenden, hauptsächlich neueren Lokalitäten zu setzen, um diese alsdann teilweise als Tauschmaterial zu verwenden; denn die Frage, wie Meteoriten gegen Mineralien oder Gesteine oder ähnliche Objekte eingetauscht werden könnten, lässt sich wohl kaum von allgemeinen Gesichtspunkten aus beantworten.

Höchst wahrscheinlich wäre dieser Tauschverkehr ein viel lebhafterer, falls man nur eine Vorstellung von dem Wertverhältnis der verschiedenen Meteoriten zu einander besässe. Wenn nämlich hierüber einige brauchbare Angaben sich machen liessen, so würden - wie ich glaube - die Vorstände der Sammlungen eher in der Lage sein, die Verantwortlichkeit für Tauschgeschäfte $z u$ übernehmen, für welche jetzt jede Beurteilung fehlt. Will man also in einem lebhaften Tauschverkehr Vorteile für die Meteoritenforschung erblicken, so muss man zunächst versuchen, eine Art W e rtska la aufzustellen, wie sie z. B. im botanischen Tauschverkehr in Schweden schon längere Zeit gilt und nun auch sich allgemein einzubürgern beginnt ${ }^{1}$ ). Auf diese Wertbestimmung gehe ich in dem Kapitel

I) Ich verweise besonders auf:

Points-Förteckning öfver Skandinaviens Växter. De angifna bytesvärdena äro antagna både in Lunds botaniska förening och Upsala botaniska bytesförening. I. Fancrogamer och Kärlkrytogamer. Zweite Auflage. Lund 1883 ; gedruckt bei C. W. K. Gleerup.

Von den sich an dieses Points-Förteckning anschliessenden Tausch-Katalogen liegen mir zur Zeit vor:

Katalog öfver de växter, Upsala Botaniska Bytesförening erbjuder till utbyte Vårter- 
auf p. 430-445 näher ein; hier will ich vorgreifend nur soviel bemerken, dass als Hauptmomente bei der Wertbestimmung das Gewicht der von jedem Fall erhaltenen Massen und ihre Verteilung unter den Sammlungen in Frage kommen und dass es also auch aus diesen Gründen geboten war, eine statistische Erhebung über die in Sammlungen aufbewahrten Meteoriten zu veranstalten. Das Resultat dieser Erhebungen, welches auf p. XIV-XV bereits in einer kurzen Uebersicht zusammengestellt wurde, ist in dem Verzeichnis der Meteoriten auf p. $\mathrm{I}-395$ ausführlich mitgeteilt.

minen 1892. Zusammengestellt von Herrn Konservator K. A. T. Seth. Upsala 1892; gedruckt bei Edv. Berling.

Katalog öfver de Växter, Lunds Botaniska Förening har att utbyta Höstterminen 1895. Zusammengestellt von Herman G. Simmons. Lund 1895; gedruckt bei Håkan Ohlsson.

Ferner möge verwiesen werden auf:

Jahres-Katalog pro 1894 des Wiener Botanischen Tauschvereins (Gegründet 1845). Herausgegeben von J. Dörfler, k. u. k, wissenschaftl. Beamter an der botan. Abth. des k. k. naturhistor. Hofmuseums in Wien, I. Burgring 7. Verlag des Wiener Botanischen Tauschvereins. Druck von Ch. Reisser u. M. Werthner, Wien.

Doubletten-Verzeichnis des Berliner Botanischen Tauschvereins, XXVII. Tauschjahr 1895/96. Leiter: Otto Leonhardt, Nossen i. S. Druck von E. Hensel, Nossen, i. S.

Liste générale des doubles de l'Association Pyrénéenne. Sixième année 1895/96. Druck von M. Bousrez. Poitiers 1895.

Katalog des Schweizerischen Botanischen Tauschvereins, 27stes Jahr 1896 Herausgegeben von Dr. Rob. Keller, Rektor in Winterthur u. Prof. Dr. H. Schinz, Direktor des botanischen Gartens in Zürich. 


\section{Erläuterungen zum Verzeichnis der Meteoriten nebst Erklärung der Abkürzungen.}

Im alphabetischen Verzeichnis der Meteoriten ist unter dem Stichwort „Nachweisbares Gewicht» die Gesamtmenge des Materials angegeben, über welches ich durch die eingesandten Kataloge Nachricht erhalten habe. Daran anschliessend folgt die Liste der Besitzer in alphabetischer Ordnung. Ueber die Namen der Besitzer siehe p. 409-429. - Mengen unter 0.6 gr. sind als Spl. (Splitter) bezeichnet. Mengen von 0.6 gr. -1.5 gr. sind auf 1 gr.; von 1.6 gr. -2.5 gr. auf 2 gr.; von 2.6 gr. -3.5 gr. auf 3 gr. u. s. w. abgerundet. - Ueber dieser als „Nachweisbares Gewicht bezeichneten Zahl findet sich noch eine andere Gewichtsangabe, welche „Ursprüngliches Gewich tø genannt ist. Hierunter sollen jene Mengen verstanden werden, welche, wie man mit einiger Sicherheit aus der Literatur erfahren kann, wirklich aufgesammelt wurden, und von denen man mit einiger Wahrscheinlichkeit annehmen darf, dass sie in die Hände gebildeter Personen gelangten. In vielen Fällen sind hierüber keine auch nur einigermassen sichere Angaben zu erhalten, was jedesmal eigens vermerkt worden ist. Die Originalgewichtsangaben sind, wo dies möglich war, in Kilogramme umgerechnet; doch wurden sie meistens noch in Klammern beigefügt. - Nur in wenigen Fällen erreicht das „Nachweisbare Gewicht\& die Höhe des „Ursprünglichen Gewichts ; hierauf wurde indessen nur besonders verwiesen, wenn die Angaben aus der Literatur dahin lauten, dass die grosse jetzt nicht nachweisbare Menge in einer bekannten Hand sich befand und höchst wahrscheinlich noch irgendwo aufbewahrt wird. Wenn es also z. B. bei Benares heisst: ,Ursprüngliches Gewicht: Viele Steine. Nachweisbares Gewicht: 2416 gr. «, so habe ich dies als keine auffallende Differenz angesehen, da ich geneigt bin zu glauben, dass von Benares kaum erheblich mehr als jene ${ }_{2416}$ gr. erhalten sein dürften. Dagegen habe ich beispielsweise bei Tazewell (Knoxville) die Frage sWo befindet sich die grösste Masse? « beigefügt, weil L. Smith die Hauptmasse von 55 lbs. besessen haben soll und sich doch nur $7510 \mathrm{gr}$. nachweisen lassen. 
Jene Zahlen des ursprünglichen Gewichts von neuem festzustellen, war notwendig, um zu erfahren, wie weit die statistischen Erhebungen sich der Wahrheit nähern. Hierzu war ein Durchblättern der gesamten Literatur erforderlich. Aber auch die Angaben in den mir zugesandten Verzeichnissen waren häufig recht dürftig, so dass es bei der grossen Synonymen-Verwirrung, welche bei den Meteoriten herrscht, notwendig schien, die Literatur eingehend zu studieren. Es war dies um so mehr geboten, als wir uns bis jetzt wie schon erwähnt noch keines Handbuchs der Meteoritenkunde erfreuen, welches hätte zu Rat gezogen werden können. Bei diesem Studium hielt ich es für zweckmässig, den Versuch, welchen Buchner angestellt hat, zu wiederholen, nämlich ein möglichst vollständiges Verzeichnis der Literatur, soweit sie sich auf die einzelnen Lokalitäten bezieht, zusammenzustellen.

Die Aufsätze über Meteoriten sind ganz ausserordentlich zerstreut, so dass von den Einzelwerken abgesehen die Angaben aus nicht weniger als 252 Zeitschriften zusammengetragen werden mussten. Hierbei sind die Berichte aus den Tageszeitungen so gut wie gar nicht berücksichtigt. Wohl keine Bibliothek ist in der glücklichen Lage, alle diese Zeitschriften oder auch nur die Meteoritenaufsätze daraus zu besitzen. Der Wert dieser Literaturangaben für den praktischen Gebrauch würde also sehr illusorisch sein, wenn ich nicht versucht hätte, hinter den Angaben der Originalarbeiten jedesmal die Referate anzufügen, welche in den verbreiteteren Zeitschriften erschienen sind; insbesondere wurden in Bezug auf diese Auszüge oder Referate an erster Stelle natürlich das Neue Jahrbuch für Mineralogie etc., ferner Gilbert's und Poggendorff's Annalen, Annalen der Chemie und Pharmazie, Journal für praktische Chemie, LiebigKopp's Jahresberichte, Kenngott's Uebersicht der Resultate mineralogischer Forschungen und viele andere Zeitschriften und Werke, die sich auch gegenwärtig noch einer grösseren Verbreitung erfreuen, berücksichtigt. Mit Hülfe dieser ausgedehnten Hinweise auf Referate und Auszüge ist es auch bei einer kleinen Bibliothek möglich, sich über die meisten Lokalitäten zu unterrichten. In der gleichen Weise wie Originalarbeiten sind jene mehr oder weniger referierenden Werke von v. Boguslawski, Buchner, Chladni, Flight, v. Hoff, Meunier, Rammelsberg, Shepard u. a. unter der Jahreszahl ihres Erscheinens extra zitiert worden. Auf diese Weise vermag das Literatur-Verzeichnis der Meteoriten wenigstens vorläufig ein Handbuch einigermassen $z u$ ersetzen. Umfassende vergleichende Arbeiten wurden meist, wenn sie nicht hauptsächlich theoretische Betrachtungen enthielten, bei den einzeln erwähnten Lokalitäten aufgenommen. Kleinere Aufsätze, welche sich mit der Beschreibung einer Lokalität befassen, daneben aber andere beiläufig erwähnen, sind, soweit es sich um wi chtigere Arbeiten handelt, auch vollständig ausgezogen. Aufsätze von rein theoretischem Charakter konnten nach der ganzen Anlage

W ïlfing, Meteoriten.

II 
meiner Arbeit keine Aufnahme finden. Dagegen sind einige der wichtigeren in dem nachfolgenden Verzeichnis der Abkürzungen aufgenommen. Ich habe die Arbeiten nicht alle einsehen können; doch ist dies nur dann besonders vermerkt, wenn der betreffende Aufsatz von besonderer Wichtigkeit für den einzelnen Fall sein dürfte. Eine Ergänzung zu den Literaturangaben bilden die im Druck erschienenen Kataloge, von denen die vor 1894 zuletzt erschienenen im Verzeichnis der Sammlungen erwähnt sind.

Es wurde versucht, die Angaben der eingesandten Kataloge möglichst kritisch zu ordnen, soweit dies an Hand der Literatur und ohne Besichtigung der in Frage kommenden Stücke möglich war. Es mussten hierbei notwendig eine Reihe von Fragen offen gelassen werden; so z. B. liess sich nicht entscheiden, wohin das s Abert-Eisen * und das »HumboldtEisen \& gehören, welche vorläufig als selbständige Lokalitäten aufgezählt worden sind; ebenso sind Bachmut (Alexejewka) und Mordvinovka nicht vereinigt. Andererseits aber wurden die Eisen von Jewell Hill und Duel Hill, von Lime Creek und Walker Co., von Coahuila, Sancha Estate und Fort Duncan, von Brenham und Anderson, von Stannern und Constantinopel und manche andere hintereinander aufgeführt, da es höchst wahrscheinlich ist, dass diese Meteoriten je einem einzelnen Fall angehören. Auch sind die Stücke, welche von den verschiedenen grossen Eisenschollen der Gegend von Huejuquilla herstammen sollen, vorläufig unter dem Namen „Huejuquilla-Gruppe vereinigt worden. Diese Vereinigung wurde aber in einer Weise vorgenommen, dass eine Trennung, welche infolge weiterer Untersuchungen notwendig werden könnte, leicht ausführbar ist. Ich glaube, dass wir vorläufig noch nicht in der Lage sind, mit Sicherheit $\mathrm{zu}$ entscheiden, wie weit die Differenzierung in einem Meteoritenfall gehen kann und dass wir also aus der Beschaffenheit der Stücke allein keine Trennung oder Vereinigung vornehmen dürfen. Erst die jüngsten Arbeiten Cohen's haben den Anfang zur Beantwortung derartiger Fragen gemacht. An Hand der hier mitgeteilten Listen der Besitzer einer Lokalität werden sie sich leichter verfolgen lassen. Bei mehreren Lokalitäten ist die meteorische Natur zweifelhaft; manche habe ich im Anhang aufgefüht, einige aber auch im alphabetischen Verzeichnis der Meteoriten eingeschaltet, nämlich: Grazac, Oktibbeha, Santa Catharina und Scriba.

Bei der Wahl der Bezeichnung der einzelnen Lokalitäten habe ich geschwankt, ob ich die von Brezina $188_{5}$ verwendete Nomenklatur adoptieren sollte. Ich glaube mich aber im Laufe meiner Studien überzeugt zu haben, dass hier gar zu rücksichtslos die alten historischen Namen verdrängt worden sind, um nur jenem Prinzip, wonach der dem Fallpunkt am nächsten gelegene Ort der beste zur Bezeichnung eines Meteoriten sei, zu huldigen. Ich habe also hier meine Auffassung von r894 (Jahresh. d. Ver. f. vaterl. Naturk. Württbg. Bd. 51,1895, p. 346) geändert und die alten historisch gewordenen Bezeichnungen wieder angenommen. Es 
schien mir beispielsweise nicht zweckmässig, für »Wessely $\star$ den Namen Znorow \& einzuführen und damit den Zusammenhang mit der älteren Literatur zu erschweren, wenn auch Znorow nur $1 \frac{1}{2}$ Stunde, Wessely I Stunde vom Fallort entfernt liegt ${ }^{1}$ ) und so in sehr vielen ähnlichen Fällen. Ueberhaupt habe ich mich bemüht, nach Möglichkeit keine neuen Namen einzuführen, sondern die in der Literatur am häufigsten vorkommenden zu verwenden. Diese Wiedereinführung der alten Namen kann um so unbedenklicher geschehen, als die von Brezina angewandte Bezeichnung sich noch keiner allgemeinen Zustimmung erfreut. Es ist also, um noch einige Beispiele anzuführen, wieder der Name

$\begin{array}{lcl}\text { Grosnaja anstatt } & \text { Mikenskoi } \\ \text { Krasnojarsk } & \text { Medwedewa } \\ \text { Lixna } & \text { Lasdany } \\ \text { Milena } & \text { Pusinsko Selo } \\ \text { Oesel } & \text { Kaande } \\ \text { Sikkensaare } & \text { Tennasilm } \\ \text { Tysnes } & \text { Midt-Vaage } \\ \text { Wessely } & \text { Z } & \text { Znorow }\end{array}$

gewählt worden. Wenn die ältere Bezeichnung jedoch gar zu allgemein gehalten war, wie z. B. s Gouvernement Charkow», „Gouvernement Tula«, so habe ich hierfür eine genauere Lokalitätsbezeichnung gewählt, also in diesem Fall "Jigalowka und „Netschaëvo . Auch musste der Name »Murcia « aufgegeben werden, weil wir zwei Steinfälle aus der Nähe dieser Stadt, nämlich Cabezzo de Mayo und Molina kennen; in ähnlicher Weise wurde mit einigen andern Bezeichnungen verfahren. Wenn ich noch einige Vorschläge für die Bezeichnung der Meteoriten machen darf, so möchte ich raten, Namen wie sThe Wisconsin Meteorite (Trenton), "The Iowa Meteorite (meistens Estherville), „Butcher und Couch Iron (Coahuila), sThe Lea Iron (Cleveland), "The Colorado Meteorite (Russel Gulch), "The Claywater Meteorite (Vernon County), "The Safford Meteorite" (Morristown) und dergl. nicht als Stichwörter in den Verzeichnissen zu wählen und auch die Bezeichnung nach Counties, wie sie die amerikanischen Forscher häufig anwenden, in Zukunft nach Möglichkeit zu vermeiden.

Nach dem Stichwort folgen einige nähere geographische Bezeichnungen, soweit dieselben zur Orientierung notwendig schienen. Ist neben dem von mir gewählten Namen noch ein anderer verbreitet, so wurde dieser gleich hinter meinem Stichwort angegeben. Reichlich eingeschaltete Synonyma sorgen dafür, dass jede Lokalität leicht zu finden ist. Bei

r) v. Schreibers (Baumgartner's Zeitschr. f. Phys. u. verw. Wissensch. Bd. I (18 32 ), p. 200) sagt: Die Entfernung bis zum Orte des Ereignisses beträgt von Wessely I Stunde, von der Mahlmähle des Thomas Krämer 500 Schritte, vom Walde Trny rooo Schritte, vom Dorfe Znorow $1 / 2$ Stunde. 
der Anlage meines Synonymen-Verzeichnisses waren an erster Stelle die eingesandten Kataloge massgebend.

Alsdann folgt die Bezeichnung „Stein * oder „Eisen * verbunden mit der von Tschermak eingeführten Symbolik. Hierbei habe ich mich in den allermeisten Fällen an die Wiener Bestimmungen angeschlossen, und nur in einigen Fällen bin ich davon abgewichen, wenn die Tübinger Sammlung mir für meine Auffassung genügende Stützpunkte bot Einige weitere Bemerkungen über die Systematik sind auf p. $43^{2-433}$ und im letzten Kapitel p. $446-460$ zu finden.

\section{Erklärung der Abkürzungen.}

Unter diesen Erklärungen wurden auch einige Werke erwähnt, welche in der Literatur bei den einzelnen Meteoriten nicht namentlich aufgeführt sind, da ihr Inhalt im wesentlichen mit dem anderer Arbeiten übereinstimmt, oder welche wie z. B. viele der Daubrée'schen Aufsätze die Meteoriten von allgemeineren Gesichtspunkten aus betrachten und sich weniger mit der Beschreibung der einzelnen Lokalitäten befassen. Diese Werke sind in Klammer [] gesetzt. Auch wurden nicht abgekürzte Titel einiger Aufsätze hier eingeschaltet, um bei einigen Autoren einen Ueberblick über ihre Meteoritenaufsätze zu erhalten, oder um hierbei noch auf weitere referierende Arbeiten aufmerksam zu machen.

1818- : Am. Journ. = American Journal of Science and Arts von 1818-1879; seit 1880

American Journal of Science. New Haven, Conn. U. S. A.

r886-: Ann. Hof.-Mus. = Annalen des K. K. Naturhistorischen Hofmuseums. Wien.

Bd. 1 (1886) -.

Baden-Powell s. Luminous Meteors.

1854: Balcells: Lithologia meteorica. Dr. D. Joaquin Balcells, Barcelona. Imprenta de Francisco Granell, calle de Arenas de Escudellers n ${ }^{\circ} 3$, piso 31854 (spanischer Text mit französischer und englischer Uebersetzung).

1845 : v. Baumhauer: Ursprung $=$ Ueber den mutmasslichen Ursprung der Meteorsteine, nebst einer Analyse des Meteorsteins, welcher am 2. Juni 1843 in der Provinz Utrecht gefallen ist. Von Dr. E. H. v. Baumhauer. Pogg. Ann. Bd. 66 (1845), p. 465-503. Uebersetzung der Utrechter Dissert, von 1844: Specimen meteorologico-chemicum de ortu lapidum meteoricorum, annexis duorum lapidum analysibus chemicis. - Die Uebersetzung ist zitiert. (Die auf p. 476 eingeschaltete Tafel, auf welcher Meteore und Meteoriten nach Monaten geordnet sind, wurde bei den Literaturangaben nicht berücksichtigt).

[1839: Benzenberg: Die Sternschnuppen. Hamburg 1839. Lehnt sich in Bezug auf die einzelnen Fälle im Wesentlichen an Chladni und v. Schreibers an.]

1834: Berzelius: Om Meteorstenar. Vetensk. Acad. Handl. Uebersetzt in Pogg. Ann. Bd. 33 (1834), p. I- 32, $\mathrm{Ir}_{3}-\mathrm{I}_{4} 8$; Trans. Roy. Acad. of Sc. 1834, p. $\mathrm{II}_{5}-18_{3}$; Auszüge in Philos. Magaz. Bd. $9(1836)$, p. $429-441$; Journ. Pharm. 1836 ; Am. Journ. (I) 37 (1839), p. $93-99$; N. J. 1834 , p. $530-531$ und N. J. 1836 , p. 599 
-604. S. auch v. Hoff: Bemerkungen über den Ursprung der Meteorsteine, besonders in Beziehung auf den Aufsatz von Berzelius in diesen Annalen Bd. 33, p. I u. ${ }_{13} 3$ in Pogg. Ann. Bd. 36 (1835), p. 161-186. Ref. sL'Institut « 1837, Nr. 219, p. $297-300$.

1796-1835: Bibl. Brit. = Bibliothèque Bitannique; ou Recueil Extrait des Ouvrages Anglais périodiques et autres; des Mémoires et Transactions des Sociétés et Académies de la Grande-Bretagne, d'Asie, d'Afrique et d'Amérique, en deux Séries, intitulées: Littérature et Sciences et Arts, rédigé à Genève, par une Société de Gens de Lettres (nur von $1807-1815$ in die Literaturangaben aufgenommen).

1812: Bigot de Morogues = Mémoire historique et physique sur les chutes des pierres tombées sur la surface de la terre à diverses époques. Orléans 1812. Imprimerie de Jacob ainé. $8^{\circ}$. 361 Seiten. S, auch Journ. des Mines Bd. $3^{1}$ (1812). p. $430-436$; Leonhard's Taschenbuch Bd. 8 I (I8I4), p. 268-274 u. Bd. 9 II (1815), p. $537-542$.

[1816: Björn: De indole et origine Aerolithorum. Othiniae 1816. K1. $8^{0} 88$ Seiten; bei Hempel.]

I848: Bloede : Tabelle $=$ Tabelle über die in den öffentlichen Museen zu St. Petersburg befindlichen Aërolithen und kurze Charakteristik derselben, sowie Angabe der hierüber vorhandenen Nachrichten von Bloede. Bull. de la classe physico-mathématique de l'Acad. Imp. des sciences de St. Pétersburg T. VI Nr. I (8 Seiten) $4^{0}$ (gelesen 25. Sept. 1846). (Bei Benutzung dieser Arbeit ist die Goebel'sche Kritische Uebersicht « einzusehen).

[1816: Blumhof: Nachträge zu Chladni's neuem chronologischen Verzeichnisse herabgefallener Stein- und Eisenmassen, von dem Hof kammerrath Dr. Blumhof zu Biedenkopf. Mit einem Zusatz von Dr. Chladni. Gilb. Ann. Bd. 53, p. 307-312 (diese Nachtrïge beziehen sich auf alte Meteoriten-Fälle, von denen nichts erhalten ist).]

1854: v. Boguslawski : Zehnter Nachtrag = Zehnter Nachtrag zu Chladni's Verzeichnisse der Feuermeteore und herabgefallenen Massen (Wien 1819). Pogg. Ann. Ergz-Bd. 4, p. 1-155, 353-456. (Die s Uebersichts-Tabelle der in die verschiedenen Monate des Jahres fallenden Erscheinungen von Feuermeteoren und Meteo. riten * auf P. 4I5-45I wurde bei der Literatur-Aufnahme nicht berücksichtigt). Ref. Liebig-Kopp, Jahresber. 1853 , p. 93 I.

1880: Brezina : Reichenbach'sche Lamellen = Ueber die Reichenbach'schen Lamellen in Meteoreisen; von Dr. A. Brezina, Wien. Denkschr. Wien. Akad. Bd. 43 (1880), p. 13-16 (mit 4 Tafeln). Ref. N. J. 1884 I, p. 33 .

1880: Brezina : Bericht $\mathrm{I}=$ Vorläufiger Bericht über neue oder wenig bekannte Meteoriten. Sitzber. Wien. Akad. Bd. 82 I (1880), p. $348-352$. I. Butler, Bates Co., Missouri, U. S., bekannt seit 1875. 2. Tazewell, Claiborne Co., Tennessee, U. S. 1853. 3. Casey County, Georgia, U. S. I877. 4. Whitfield County, Georgia, U. S. 1878. 5. De Calb County, Caryfort, Tennessee, U. S. 1840. 6. Kalumbi, Praesidentschaft Bombay, Ostindien, gefallen am 4. Nov. 1879. Ref. N. J. 1881 II, p. $342-$ 343 ; Liebig-Kopp, Jahresber, 1880, p. 1540.

I881 : Brezina : Bericht II $=$ Ueber die Meteoreisen von Bolson de Mapimi. Sitzber. Wien. Akad. Bd. 83 I (188r), p. $473-477$. Ref. N. J. $188_{3}$ I, p. $380-38$ r; Liebig-Kopp, Jahresber. 1882, p. 1643-1644.

1881 : Brezina :Meteoritenstudien II $=$ Meteoritenstudien II : Ueber die Orientierung der Schnittfächen an Eisenmeteoriten mittelst der Widmanstädt'schen Figuren 
(mit 4 Tafeln und II Holzschnitten). Denkschr. Wien. Akad. Bd. 44 (I88I), p. I2I -158. Ref. N. J. 1884 I, p. 33.

1881 : Brezina : Bericht III $=$ Bericht über neue oder wenig bekannte Meteoriten III. Sitzber. Wien. Akad. Bd. 84 I (1881), p. $277-283$. 8. Veramin, Teheran, Persien, gefallen April 1880. 9. Duel Hill, Madison Co., North Carolina, U. S. N. A., gefunden 1873. 10. Lick Creek, Davidson Co., North Carolina, U.S. N. A., gefunden 19. Juli 1879. II. Chulafinnee, Cleberne Co, Alabama, U. S. N. A., gefunden 1873 . 12. Natürliche Trennungsfläche an Bolson de Mapimi Ref. N. J. $188_{3}$ I, p. $3^{8} \mathrm{I}-3{ }^{8} 3$. 1882 : Brezina : Bericht IV $=$ Bericht über neue oder wenig bekannte Meteoriten IV. Sitzber. Wien. Akad, Bd. 85 I, p. 335-413. 13. Mócs, Koloscher Gespannschaft, Siebenbürgen.

1882 : Brezina : Ueber die Stellung der Mócser Meteoriten im System. Verh. k. k. geol. Reichsanst. v. 2I. Februar 1882 , p. 78 . Ref. N. J. 1883 II, p. 184 .

1885: Brezina : Wiener Sammlung $=$ Die Meteoriten-Sammlung des k. k. mineralogischen Hofkabinetes in Wien am I. Mai 1885. Mit 4 Tafeln. 126 Seiten. Jahrb. k. k. geol. Reichsanst. Bd. 35 (1885), p. 151-276. - Da die Arbeit kein Register enthält, wurde auch auf die blosse Erwähnung eines Falles hingewiesen. - Ref. N. J. 1886 I, p. 219-224; s. auch Meunier's Verteidigung gegen die Angriffe Brezina's C. R. Bd, Ior (1885), p. $728-73$; Bull. Soc. géol. (3) I4 (1886), p. $68-79$. Ref. N. J. 1887 I, p. 258 ; Liebig-Kopp, Jahresber. 1885 , p. 2322-2323.

1886: Brezina: Neue Meteoriten $I=$ Neue Meteoriten I. Ann. Hof,-Mus, Bd. I (Not.), p. 12-14. Ref. N. J. I887 II, p. 45-46; Liebig-Kopp, Jahresber. 1886, p. 2328 .

1886: Brezina : Neue Meteoriten II $=$ Neue Meteoriten II. Ann. Hof-Mus, Bd, I (Not.), p. 25-26. Ref. N. J. 1887 II, p. $45-46$.

1886/87: Brezina und Cohen : Photographien = Die Structur und Zusammensetzung der Meteoreisen, erläutert durch photographische Abbildungen geätzter Schnittflächen, herausgegeben von A. Brezina und E. Cohen. Die Aufnahmen von J. Grimm in Offenburg. Stuttgart, E. Schweizerbart'sche Verlagshandlung (E. Koch) Lieferung I (1886), Lieferung II und III (1887). Ref. N. J. 1887 I, p. 259-260 von der ersten Lieferung; von der zweiten und dritten Lieferung ist bis jetzt noch kein Referat erschienen.

1887 : Brezina : Reisebericht $=$ Reisebericht und Reisebericht aus Italien. Ann. HofMus. Bd. 2 (Not.), p. $72-74,103-112$.

1887 : Brezina: Neue Meteoriten III = Neue Meteoriten III. Ann Hof-Mus. Bd, 2

(Not.), p. II4-115. Ref. N. J. 1888 II, p. 35.

r887: Brezina : Neue Meteoriten III a $=$ Neue Meteoriten des k. k. naturhistorischen Hofmuseums. Verh. k. k. geol. Reichsanst. 1887, p. 288-289.

1889: Brezina : Cliftonit aus dem Meteoreisen von Magura, Arvaer Comitat. Ann. HofMus. Bd. 4, p. $102-106$.

[1889: Brezina: Darstellung von Meteoriten auf antiken Münzen. Monatsbl. d, numismat. Ges. Nr. 70, p. 312-314.]

1889: Brezina : Ankunft der Hidden'schen Meteoriten und Mineraliensammlung für die mineralogische Abtheilung. Ann. Hof-Mus. Bd. 4 (Not), p. $85-87$.

r8go: Brezina : Reise = Reise zur Pariser Weltausstellung. Ann. Hof-Mus, Bd. 4 (Not.), p. 116-122.

r89o: Brezina : Ueber Meteoreisen $=$ Ueber Meteoreisen, seine Unterschiede vom 
künstlichen Eisen und über das Schneiden des ersteren. Oesterr. Zeitschr. f. Bergu. Hüttenw. Bd. 38 (Nr. 31), p. $355-359$.

1890: Brezina: Untersuchungen der Herren Berthelot und Friedel in Paris über das Meteoreisen von Magura. Ann. Hof-Mus. Bd. 5 (Not.), p. 112-114.

1892: Brezina : Sternschnuppen = Sternschnuppen, Feuermeteore und Kometen. Detonierende Meteore, Meteoritenfälle, Streuungskarten. Allg. n.-ö. Volksbildungsverein, Zweig Wien und Umgebung. Volksthümliche Vorträge. Nr. 22. Sonderabdruck aus der Nummer 123 der „Volksbildungsblättere. Selbstverlag des Zweiges sWien und Umgebung «, p. 1 - 8, 8-16 (Sep.).

1893: Brezina: Ueber neuere Meteorite. Verh. d, Ges. Deutscher Naturf, u. Aerzte. Nürnberg 1893, p. $158-167$ (Sep., p. $1-10$ ).

1893: Brezina : Ankunft $=$ Die Meteoriten vor und nach ihrer Ankunft auf der Erde. Mit 15 Abb. im Text. Schriften d. Ver, z Verbreitung naturw. Kenntnisse Wien Bd. 33 , p. 503 - 542 (Sep., p. I-40).

[1894: Brezina: Die Gestaltung der Meteoriten. Mit 32 Abb. im Text. Vorträge d. Ver. z. Verbreitung naturw. Kenntnisse Wien Bd. 34, p. 249-274 (Sep., p. 1-26).]

[1894: Brezina : Bemerkungen über Meteoriten anf der Naturforscherversammlung in Wien.] [1895: Brezina: Ueber Gefüge und Zusammensetzung der Meteoriten. Mit $32 \mathrm{Abb}$. im Text. Schriften d. Ver. z. Verbreitung naturw. Kenntnisse Wien Bd. 35 , p. $1-36$ (Sep.).]

r895: Brezina: Wiener Sammlung $=$ Die Meteoritensammlung des k. k. naturhistorischen Hofmuseums am 1. Mai 1895. Mit 2 Anhängen: I. Berichte des Directors der Sternwarte Zacatecas, Prof. José A, y Bonilla, ïber den Meteoreisenfall von Mazapil. 2. Die Meteoritensammlung der Universität Tübingen. Ann. Hof-Mus. Bd, Io, p. 231-370, - Die blosse Erwähnung einer Lokalität und Einreihung in das System ohne weitere Bemerkung ist schon aus dem Grunde nicht vermerkt, weil das im Ortsverzeichnis enthaltene Register leichte Orientierung erlaubt.

1859: Buchner: Feuermeteore = Die Feuermeteore, insbesondere die Meteoriten, historisch und naturwissenschaftlich betrachtet von Dr. Otto Buchner. Giessen 1859 . J. Riker'sche Buchhandlung. $8^{\circ} 192$ Seiten.

1861 : Buchner : Versuch eines Quellenverzeichnisses zur Literatur über Meteoriten. Abh. d. Senckenb. Naturf. Ges. Bd. 3 (1859 61), p. $455-482$.

1863: Buchner: Zweites Quellenverzeichnis zur Literatur der Meteoriten. Abh. d. Senckenb. Naturf. Ges. Bd. 4 (1863), p. $1-19$ (Sep.).

1863: Buchner: Meteoriten = Die Meteoriten in Sammlungen, ihre Gewichte, mineralogische und chemische Beschaffenheit. Leipzig. Verlag von Wilhelm Engelmann. 1863. $8^{0} 202$ Seiten.

1864: Buchner: Erster Nachtrag = Die Meteoriten in Sammlungen; von Dr. Otto Buchner in Giessen. Pogg. Ann. Bd. 122 (1864), p. 317-331; s. auch Wochenschr. f. Astr, Met, u. Geogr. 1864, p. 263.

1865: Buchner: Zweiter Nachtrag $=$ Die Meteoriten in Sammlungen. Zweiter Nachtrag, Pogg. Ann. Bd. 124 (1865), p. 569-602.

1867: Buchner: Dritter Nachtrag: = Die Meteoriten in Sammlungen. Dritter Nachtrag, Pogg. Ann. Bd. 132 (1867), p. 3H-319.

1869: Buchner : Vierter Nachtrag $=$ Die Meteoriten in Sammlungen. Vierter Nachtrag, Pogg. Ann. Bd. 136 (1869), p. $437-460$ und $589-6 \mathrm{rr}$.

[1869: Buchner: Die Aetzfiguren des Meteoreisens. Ber. d. Oberhess. Ges. (1869), 
p. 99-115, mit einer Tafel. Zusammenstellung aus den Arbeiten v. Schreibers', v. Haidinger's, v. Reichenbach's u. A.]

1856: Burkart : Fundorte $I=$ Ueber die Fundorte der bis jetzt bekannten Mexikanischen Meteoreisen-Massen, nebst einigen einleitenden allgemeinen Bemerkungen über den Ursprung und die Zusammensetzung der Aerolithe von Herrn Oberbergrat Dr. H. J. Burkart N. J. 1856, p. 257-307 mit Tafel ; s. auch Verh. naturh. Ver. Bonn Bd. 13 (1856), Sitzber., p. XL-XLVIII; Kenngott, Uebersicht 1856/57, p. 15I; LiebigKopp, Jahresber. 1856 , p. 915-916.

1858: Burkart: Fundorte II $=$ Ueber die Fundorte der Mexikanischen MeteoreisenMassen, als Nachtrag zu den früheren Angaben über diesen Gegenstand unter Anschluss eines Berichtes von Friedr. G. Weidner ïher das Magneteisenstein-Vorkommen an dem Cerro del Mercado bei Durango in Mexiko. N. J. $185^{8}$, p. $769-800$ (über Meteoriten nur bis p. 780 ).

1866: Burkart : Fundorte III = Ueber einige neue Fundorte mexikanischer Meteoriten. N. J. 1866, p. 40I-408, mit Tafel. Ref. Liebig-Kopp, Jahresber. 1866, p. 1008; Verh. Naturh. Ver. Bonn Sitzber. 1865, p. 71.

I870: Burkart: Fundorte IV $=$ Ueber die Fundorte mexikanischer Meteoriten. N. J. 1870, p. $673-692$.

1889: Castillo: Catalogue $=$ Catalogue descriptif des Météorites (Fers et Pierres météoriques) du Mexique. Avec l'indication des localités dans lesquelles ces météorites sont tombées ou ont été découvertes. Paris 1889 , bei Léon Ouin. Rue du Bac 96 . $8^{\circ}$. 13 Seiten mit Situationsplan der Gegend von Huejuquilla. S. auch Note de M. Daubrée accompagnant la présentation d'un catalogue descriptif des météorites du Mexique, rédigé par M. Antonio del Castillo. C. R. Bd. 109 (1889), p. 725-727. Ref. N. J. 1891 I, p. 49.

1794: Chladni : Pallaseisen $=$ Ueber den Ursprung der von Pallas gefundenen und anderer ihr ähnlicher Eisenmassen und über einige damit in Verbindung stehende Naturerscheinungen. Riga bey Johann Friedrich Hartknoch 1794. $4^{0} .63$ Seiten.

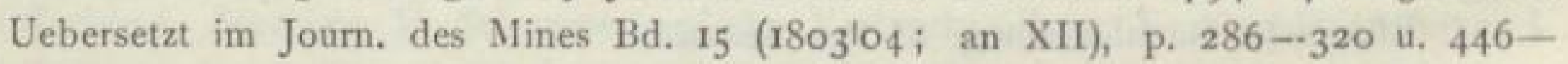
485 unter dem Titel: Réflexions sur l'origine de diverses Masses de fer natif, et notamment de celle trouvée par Pallas, en Sibérie. Traduites de l'Allemand de M. Chladni; par Eugène Coquebert. Auch besprochen in Gilb. Ann. Bd. 13 (1803), p. $350=357$ und in vielen anderen Zeitschriften und Werken, wie Bibl. Brit. $\mathrm{n}^{\circ} \mathbf{1 2 2}$, p. 73 ; Izarn: Lithologie, p. Iro-I26; Klaproth: Abh. Berlin. Akad. 1803, p. 33-34.

1803: Chladni : Chronologisches Verzeichnis $=$ Chronologisches Verzeichniss der mit einem Feuermeteor niedergefallenen Stein- und Eisenmassen, nebst einigen Bemerkungen. Gilb. Ann. Bd. 15, p. 307-328.

[r805: Chladni: Berichtigung, einen angeblichen Meteorstein betreffend. Gilb. Ann. Bd. 19, p. 243.]

[1805: Chladni: Einige kosmologisqhe Ideen, die Vermehrung oder Verminderung der Masse eines Weltkörpers betreffend. Gilb. 'Ann, Bd. 19, p. 257-281.]

r808: Chladni : Beiträge $=$ Beiträge zu den Nachrichten von Meteorsteinen. Gilb. Ann. Bd. 29, p. $375-383$.

1812: Chladni : Verzeichnis = Chronologisches Verzeichniss der herabgefallenen Steinund Eisenmassen. Schweigg. Journ, Bd, 4 Beil. I, p. 1-19.

1814: Chladni : Dr. Chladni's vergebliche Bemühungen, verschiedene ältere Meteorsteine 
aufzufinden, nebst einigen ihn selbst betreffenden Nachrichten. Gilb. Ann. Bd. 47, p. $96-104$.

1815: Chladni : Neues Verzeichnis $=$ Netues Verzeichniss der herabgefallenen Steinund Eisenmassen, in chronologischer Ordnung. Gilb. Ann. Bd. 5o, p. 225-256.

1815: Chladni: Bemerkungen $=$ Bemerkungen über Gediegen-Eisenmassen. Gilb. Ann. Bd. 50, p. 257-277.

1816: Chladni : Erste Fortsetzung = Erste Fortsetzung des Verzeichnisses der bisher bekannt gewordenen herabgefallenen Stein- und Eisenmassen, welches im 7. Stück $18 r_{5}$ oder Bd. 50, p. 225 enthalten ist; nebst neuen Beiträgen zur Geschichte der Meteorsteine, und einigen diesen Gegenstand betreffenden Bemerkungen. Gilb. Ann. Bd. 53. p. $369-392$.

1816: Chladni : Zweite Fortsetzung $=$ Zweite Fortsetzung des Verzeichnisses der vom Himmel gefallenen Massen. Gilb. Ann. Bd. 54 , p. $329-357$; s. auch Berichtigung im gleichen Band, p. 393.

1817: Chladni : Dritte Fortsetzung $=$ Dritte Fortsetzung des Verzeichnisses und der Geschichte der vom Himmel gefallenen Massen. Gilb. Ann, Bd. 56, p. 375-39o.

1819: Chladni : Vierte Fortsetzung $=$ Vierte Fortsetzung des Verzeichnisses der vom Himmel gefallenen Massen; nebst Bemerkungen über einige schon bekannte, und Beiträge zur Geschichte hierher gehörender Meteore, Gilb. Ann. Bd, 6o, p. $238-254$

1819: Chladni : Fünfte Fortsetzung $=$ Fünfte Fortsetzung des Verzeichnisses der vom Himmel gefallenen Massen; nebst weitern Nachrichten von einigen schon bekannten und von neuen Feuermeteoren (mit Zusätzen von Gilbert) Gilb. Ann. Bd. 63, p. 17 - 54 .

1819: Chladni: Feuermeteore $=$ Ueber Feuer-Meteore und über die mit denselben herabgefallenen Massen, von Ernst Florens Friedrich Chladni. Nebst zehn Steindrucktafeln und deren Erklärung von Carl von Schreibers. Wien 1819. $8^{0} .434$ Seiten, bei J. G. Heubner (die v. Schreibers'schen Tafeln erschienen 1820 ; s, unter v. Schreibers). Ref. Gilb. Ann. Bd. 68 (1821), p. 367-37o. - Die Seiten, auf denen ein Fall ausführlich besprochen wird, sind mit (!) versehen,

1821: Chladni : Erster Nachtrag $=$ Neue Beitrïge zur Kenntniss der Feuermeteore und der herabgefallenen Massen. Erste Lieferung. Gilb. Ann. Bd. 68, p. 329-370.

1822: Chladni : Zweiter Nachtrag $=$ Neue Beiträge etc. wie vorher. Zweite Lieferung. Gilb. Ann. Bd. 71, p. 359-386.

1823: Chladni : Dritter Nachtrag $=$ Neue Beiträge etc. wie vorher. Dritte Lieferung. Gilb. Ann. Bd. 75, p. 229-257.

1824: Chladni : Vierter Nachtrag $=$ Neue Beiträge etc. wie vorher. Vierte Lieferung. Pogg. Ann. Bd. 2, p. 151-168.

1826: Chladni : Fünfter Nachtrag $=$ Neue Beiträge etc. wie vorher. Fünfte Lieferung Pogg. Ann, Bd. 6, p. 2I-34, I6I-182.

1826: Chladni: Sechster Nachtrag = Neue Beiträge etc. wie vorher. Sechste Lieferung. Pogg. Ann. Bd, 8, p. 45-60; s. auch Froriep's Notizen der Natur- und Heilk. Bd. 13 (1826), p. $50-58,70-73,247-248$.

Weitere Nachträge s. unter v. Boguslawski und v. Hoff.

1852: Clark : Dissert. Gött. = On Metallic Meteorites. An Inaugural Dissertation. Göttingen 1852. Gedr. bei Kaestner. $8^{\circ} 80$ Seiten und drei Tafeln. Ref. Am. Journ. (2) 15, p. 7-22 *Notice of Professor Clark's Thesis on Metallic Meteorites «. N. J. 1853 , p. 186 ; Kenngott, Uebersicht 1852 , p. $91-92$ u. 1853 , p. 116 ; Ann. Chem. 
Pharm. Bd. 82, p. 367 ; Journ. prakt. Chem. Bd. 58 (1853), p. 55 ; Pharm. Centr. 1852 , p. 555 ; Liebig-Kopp, Jahresber. 1852 , p. $990-991$.

r889: Cohen: São Julião $=$ Chemische Untersuchung des Meteoreisens von S. Julião de Moreira, Portugal, sowie einiger anderen hexaëdrischen Eisen. N. J. 1889 I, p. $215-228$.

r89 I : Cohen u. Weinschenk: Meteoreisen-Studien. Ann. Hof-Mus, Bd. 6, p. 13I165. Ref. N. J. 1892 II, p. 244-246.

I891 : Cohen: Meteoreisen-Studien II. Ann. Hof-Mus. Bd. 7, p. 143-162. Ref. N. J. 1893 I, p. $478-479$.

1894: Cohen: Meteoritenkunde $=$ Meteoritenkunde von E. Cohen. Heft I. Untersuchungsmethoden und Charakteristik der Gemengtheile 340 Seiten mit 39 Figuren. Stuttgart. E. Schweizerbart'sche Verlagshandlung (E. Koch). 1894. Ref. N. J. 1895 I, p. 458-460. - Das Register, welches Cohen seinem Werk beigegeben hat, wurde aufgenommen, um einen Anhaltspunkt dafür zu geben, wie vollständig die einzelnen Lokalitäten besprochen werden. Nur bei Fällen, welche bei Cohen sehr oft genannt sind, wie z. B. Cosby's Creek, Magura, Toluca u. a, wurde nur erwähnt san sehr vielen Stellen .

[1847: Coulvier-Gravier et Saigey: Recherches sur les étoiles filantes. Introduction historique. Paris 1847. $8^{\circ} 192$ Seiten. Hachette et Cie.]

r835-: C. R. = Comptes Rendus Hebdomadaires des Séances de l'Académie des Sciences. Paris Bd. I (1835).

[r8II: v. Dalberg: Ueber Meteor-Cultus der Alten, vorzüglich in Bezug auf Steine, die vom Himmel gefallen. Ein Beytrag zur Alterthumskunde. Heidelberg, Mohr und Zimmer.]

[1866: Daubrée: Expériences synthétiques relatives aux Météorites. Rapprochements auxquels ces expériences conduisent, tant pour la formation de ces corps planétaires que pour celle du globe terrestre. C. R. Bd, 62 (1866), p. 200-206, 369-375, 66o-674. Ref. Bull. Soc. géol. de France (2) 23, p. $391-417$; N. J. I866, p. 738 -739; Kenngott, Uebersicht 1862/65, p. 432-433; Liebig-Kopp, Jahresber. 1866, p. 1002-1007; Journ. prakt. Chem. Bd. I05 (1868), p. 6-7.]

[1867: Daubrée: Rapport sur les progrès de la Géologie expérimentale. Paris 1867. Quatrième Partie. Météorites, p. 93-139.]

[1868: Daubrée: Expériences synthétiques relatives aux Météorites. Rapprochements auxquels ces expériences conduisent. Paris, bei Dunod. $8^{\circ} .65$ Seiten. S. auch Uebersetzung von Hauchecorne in Zeitschr. d. d. Geol. Ges. Bd. 22 (187o), p. 415--451. Ferner Uebersetzung in Annual Rep. Smithsonian Inst. 1868, p. 312-340.]

[1877: Daubrée: Recherches expérimentales, faites avec les gaz produits par l'explosion de la dynamite, sur divers caractères des météorites et des bolides qui les apportent. C. R. Bd. 85 , p. $115-122,253-259,315-319$. S. auch C. R. Bd. 84, p. 413 . Ref. Liebig-Kopp, Jahresber. 1877, p. 1390-1393.]

[1879/80: Daubrée: Etudes synthétiques de Géologie expérimentale. Paris 1879 und 1880. Deutsche Uebersetzung von Gurlt: Synthetische Studien zur ExperimentalGeologie. Braunschweig 1880. $8^{\circ} 596$ Seiten (Meteore u. Meteoriten auf p. 369-554).]

[1885: Daubrée: Les Météorites et la constitution du Globe terrestre. Revue des Deux Mondes. 15. Dez. 1885. 37 Seiten. Ref. N. J. I888 I, p. 46-47.]

[1888: Daubrée: Les Régions invisibles du Globe et des espaces célestes. Eaux souterraines, Tremblements de Terres, Météorites. Paris. Bibl. Scient. internat, 1888. $8^{\circ}$. 
202 Seiten. - IV. Les Météorites et la Constitution du Globe terrestre, p. 149-199.] 1803: de Drée: Recherches $=$ Recherches sur les masses minérales dites tombées de l'atmosphère sur notre globe. Journ. Phys. Bd. 56 (an XI), p. $3^{80-389}$ und $405-427$; s, auch Philos. Magaz. Bd. 16 (1803), p. 293; Bibl. Brit. Bd. 20.

1879: Domeyko: Mineralojia. 3. Aufl. Santiago 1879.

1890: Eastman: Met. Astron. $=$ The Progress of Meteoric Astronomy in America. Philos. Soc. of Washington Bull. Bd, I1, p. 275-358. - Die hier in Betracht kommenden Tabellen auf p. $316-323$ enthalten eine nicht sehr kritische Zusammenstellung der amerikanischen Meteoriten.

1847: Eichwald: Verzeichnis = Ein Verzeichniss von Meteorsteinfällen in Russland.

Erman's Arch. f. wissensch. Kunde Russlands Bd. 5, p. 176-184.

I808: >Encyclopédie $"=$ Encyclopédie Méthodique. Chimie et Métallurgie par M. Fourcroy. Tome Cinquième, Artikel $»$ Pierre«. Paris (1808).

1804: v. Ende: Massen und Steine $=$ Ueber Massen und Steine, die aus dem Monde auf die Erde gefallen sind. Braunschweig, 1804. $4^{0}$.

1889 : Fletcher: Atacama Meteorites $=$ On the Meteorites which have been found in the Desert of Atacama and its Neighbourhood (With a Map of the District, Plate X). Mineral, Magaz. Bd. 8, p. 223-264. Ref. N. J. 1890 II, p. 230-23I.

1890: Fletcher: Mexican Meteorites $=$ On the Mexican Meteorites, with especial regard to the supposed occurence of widespread Meteoric Showers (With Maps of Region, Plates I and II). Mineral. Magaz. Bd. 9, p. 91-178. Ref. Am. Journ. (3) 41 , p. $79-80$; N. J. 1891 II, p. $239-240$

1894: Fletcher: Introduction $=$ An Introduction to the Study of Meteorites. With a list of the Meteorites represented in the Collection (British Museum). Printed by order of the Trustees. London 1894.

1887: Flight: Meteorites = A chapter of the history of meteorites by the late Walter Flight, D. Sc. Lonid., F. R. S. With seven plates and six woodcuts. (Reprinted from the Geological Magazin with some additional notes by the Author.) London. Dulau u. Co. 37 , Soho Square, W. $1887.8^{0} 224$ Seiten. Ref. N. J. 1888 II, p. 227 Die Flight'schen Arbeiten habe ich meist nach diesem Sammelwerk zitiert. Viele dieser Arbeiten sind auch in den sLuminous Meteors $\approx$, Rep. Brit. Assoc. von 1876 an abgedruckt. Ref. über die in Bd, 2 (1879) des Geol. Magaz. erschienenen Arbeiten siehe N. J. 1876 , p. $91-93$ und 1877 , p. IOI-102.

[188x: Fouqué et Michel-Lévy: Expériences synthétiques relatives à la réproduction artificielle des météorites. C. R. Bd. 92 n 14 vom 4. April $188 \mathrm{i}$ u. Bd. 93 n 19. Ref. N. J. 1882 I, p. $366-368$ u. 1882 II, p. $64-65$.

1867: Goebe1: Kritische Uebersicht $=$ Kritische Uebersicht der im Besitze der Kaiserlichen Akademie der Wissenschaften befindlichen Aërolithen, von Ad. Goebel (Lu le 7 Juin 1866). Mit einer Tafel. Bull. Petersburg. Akad. Bd. II (1867), p. 222-282. Abgedruckt in Mélanges phys. chim tirés du Bull. Petersburg. Akad. Bd. 7, p. 255 -342 ; hiernach sind die Seitenzahlen angegeben. Referat der Arbeit findet sich in Buchner's drittem Nachtrag. - Es sind bei dieser Goebel'schen Arbeit ausnahmsweise nicht alle Seitenzahlen, auf denen das Stichwort vorkommt, angegeben, weil zum Verständnis der durch Bloede angestellten Verwirrungen doch eine vollständige Lektüre der Schrift notwendig ist.

1892: Gredilla : Meteoritos = Estudio sobre los Meteoritos. Madrid. Escuela Tipo- 
grafica del Hospicio. 1892. $8^{0}$ i2 8 Seiten. Nur der spezielle Teil (Parte Especial) p. 98-I2I über spanische Meteoriten wurde berücksichtigt.

1854: Greg : Catalogue $=$ Observations on Meteorites or Aërolites, considered Geographically, Statistically and Cosmically, accompanied by a complete Catalogue. Philos. Magaz. (4) 8, p. 329-342, 449-463. - Der $\approx$ Cataloguee ist nur ausnahmsweise erwähnt, wenn er die erste Angabe bringt; er enthält eine für die damalige Zeit sehr vollständige Zusammenstellung. Ref. > L'Institut $\approx$ I854, p. 398 ; Liebig-Kopp Jahresber. 1854, p. $909-910$.

I843: Haidinger : Uebersicht $=$ Uebersicht der Resultate mineralogischer Forschungen im Jahre I843 (Erlangen 1845 ).

[r86r : Haidinger: Ueber die Natur der Meteoriten in ihrer Zusammensetzung und Erscheinung. Sitzber. Wien. Akad. Bd. 43, p. 389-426. Ref. Kenngott, Uebersicht 1861, p. 169-170. S. auch Greg's Ansichten in Rep. Brit. Assoc. 1865. Ref. LiebigKopp, Jahresber. 1861 , p. 1117-III8; Wochenschr. f. Astr., Met. u. Geogr. I865, p. $47-48,6 \mathrm{r}-63,70-7 \mathrm{r}$; , L'Institut . Bd. 29 (186r) n० 1444, p. 303-304.]

1870: v. Haidinger: Orientierung $=$ Der Ainsa-Tucson Meteoreisenring in Washington und die Rotation der Meteoriten in ihrem Zuge. - 4. Orientierung der Bewegung. Uebersicht. Sitzber. Wien. Akad. Bd. 6I II, P, 5I2-514.

1884: Häpke: Beiträge $=$ Beiträge zur Kenntniss der Meteoriten. - I. Ein neuer Fund von Meteoreisen aus Mexiko und Bemerkungen über mexikanische Meteoriten. 2. Die Meteoriten des stältischen Museums zu Bremen. Abh. Naturw. Ver. Bremen Bd. 8 (1884), p. 517-531. Ref. N. J. 1885 I, p. 32.

1859: Harris : Dissert. Gött. = The Chemical Constitution and Chronological Arrangement of Meteorites. An Inaugural Dissertation on Promotion to the Degree of Doctor of Philosophy at the Georgia Augusta University by Elijah P. Harris. Le Roy, N. Y., U. S. A. Göttingen. Printed at the University Press by W. Fr. Kaestner 1859. Das Verzeichnis der Steinfälle geht bis Aussun, gefallen 9. Dezember $185^{8}$. Ref. Liebig-Kopp, Jahresber, 1859, p. $850,853-854$.

1830: v. Hoff: Siebenter Nachtrag $=$ Neue Beiträge zu Chladni's Verzeichnissen von Feuermeteoren und herabgefallenen Massen. Siebente Lieferung. Pogg. Ann. Bd. 18 , p. $174-197$.

1832: v. Hoff: Achter Nachtrag $=$ Neue Beiträge etc. wie vorher. Achte Lieferung Pogg. Ann. Bd. 24, p. 221-242.

1835: v. Hoff: Neunter Nachtrag $=$ Neue Beiträge etc, wie vorher. Neunte Lieferung. Pogg. Ann. Bd. 34, p. $339-370$.

1886: Huntington: Crystalline Structure $=$ On the Crystalline Structure of Iron Meteorites. Am. Journ. (3) 32, p. 284-303 (mit n Abbild. im Text); s. auch Proc. Amer. Acad. of Arts and Sc. May 12. 1886; Proc. Amer. Acad. of Arts and Sc. Bd. 23 (1887/88) Tafel I-IV und die Abhandlung Catalogue of all recorded Meteorites with a description of the specimens in the Harvard College Collection, including the cabinet of the late J. Lawrence Smithe. Zeitschr. f. Kryst. Bd. 12 (1887), p. 622.

1803: Izarn : Lithologie $=$ Des Pierres tombées du Ciel ou Lithologie Atmosphérique, Présentant la Marche et l'Etat actuel de la Science, sur le Phénomène des Pierres de foudre, Pluies de pierres, Pierres tombées du ciel, etc.; plusieurs Observations inédites, communiquées par MM. Pictet, Sage, Darcet et Vauquelin; avec un Essai de Théorie sur la formation de ces Pierres. Par Joseph Izarn, Médecin, Professeur de 
Physique etc.; Paris $\mathrm{I} 8 \mathrm{O}_{3}$ (Floréal an XI). Verlag von Delalain Fils, Libraire. $8^{0}$. 422 Seiten; s, auch Journ. Phys. Bd. 56 (1803), p. $441-456$.

1836: Kämtz: Meteorologie = Lehrbuch der Meteorologie. Dritter Band. Halle 18 36 . Der neunte Abschnitt >Problematische Erscheinungen « enthält grösstenteils kurze Angaben aus den Arbeiten Chladni's und Berzelius'.

r862: Kenngott $\mathrm{u}$. Wiser : Zürcher Sammlung = Mittheilungen über die Meteoriten der Zürcher Sammlungen. Vierteljahrschr. der Naturf. Ges. Zürich Bd. 7 (1862), p. 142-158. Ref. Kenngott, Uebersicht 186r (gedruckt 1862), p. 107, 165.

1844/65: Kenngott : Uebersicht $=$ Uebersicht der Resultate mineralogischer Forschungen in den Jahren 1844 bis 1865 (Wien 1852 bis 1855 , Leipzig 1859 bis 1868 ).

[1859/6r : Kesselmeyer: Ueber den Ursprung der Meteorsteine. Abh. d. Senckenb. Naturf. Ges. Bd. 3 (1859/6r), p. 313-454.]

r796: King: Remarks = Remarks concerning stones said to have fallen from the clouds, both in these days and in antient times. London 1796. $4^{0} \cdot 34$ Seiten; s. auch Bibl. Brit. Bd, 6 (1797), p. 51; Izarn: Lithologie, p. 99-108.

1807/15: Klaproth : Beiträge $=$ Beiträge zur chemischen Kenntniss der Mineralkörper. Bd. 4 (1807), Bd, 5 (1810), Bd. 6 (1815) (die ersten drei Bände Bd. I (1795), Bd. 2 (1797), Bd. 3 (1802) enthalten keine Arbeiten über Meteoriten). In Bd. 4 (1807): Chemische Untersuchung des Gediegen-Eisens, p. 98-106; in Bd. 5 (1810): Chemische Untersuchung einiger der jüngst gefallenen Meteorsteine, p. 245-264; in Bd. 6 (1815): Chemische Untersuchung meteorischer Stein- und Eisenmassen, p. 290-308. Uebersetzung in Mém. Acad. Roy., p. $37-66$; s. auch Gehlen's Neues allgemeines Journal der Chemie Bd. I $\left(180_{3}\right)$, p. $\mathrm{I}-77$.

1879: Klein: Göttinger Sammlung = Die Meteoritensammlung der Universität Göttingen am 2. Januar 1879. Göttingische Gelehrte Anzeigen (Nachrichten), p. 84100. Nur der Text wurde eigens unter Literatur aufgeführt; im übrigen siehe unter Gewichtslisten.

1880: Klein: Vermehrung $=$ Ueber eine Vermehrung der Meteoritensammlung der Universität. Göttingische Gelehrte Anzeigen (Nachrichten), p. 565-569. Nur der Text wurde eigens unter Literatur aufgeführt, im übrigen siehe unter Gewichtslisten.

[1825: Kratter: Versuch einer Entwicklung der Grundbegriffe über die Meteorsteine und Darstellung der vorzinglichsten Hypothesen, ihren Ursprung betreffend. Zum Gebrauch für angehende Physiker und Naturforscher. Wien 1825. $8^{0} .144$ Seiten. Enthält auf p. 134-144 eine Zusammenstellung alter Zeitschriften und Abhandlungen über den Gegenstand.]

1882 : v. Lasaulx: Vermehrung $=$ Ueber die Vermehrung der Meteoritensammlung des mineralogischen Museums. Verh. naturh. Ver. Bonn Bd. 39 (1882) (Sitzber.), p. 100-Iro. Ref. N. J. 1884 I, p. 29.

1896: Laspeyres: Ist zuweilen bei einzelnen Literaturangaben genannt. Es ist immer die Arbeit in Verh. naturh. Ver. Boun 1896 gemeint. S. Verzeichnis der Sammlungen unter Bonn.

1806: Laugier: Chromium $=$ Entdeckung des Chromiums in den Meteorsteinen. Gilb. Ann. Bd. 24 (1806), p. $377-384$; s. auch Ann. du Mus. d'hist. nat. Bd. 7 (1806), p. 392-397: >Extrait d'un Mémoire sur l'existence du chrôme dans les pierres météoriques ; ferner .Encyclopédie « Bd. 5 (1808), p. $582-5^{84}$.

[1818: Léman: Considération sur les pierres, les masses de fer, et les poussières, dites météoriques. Article extrait du Nouveau Dictionnaire d' Histoire Naturelle, volume 
26. Paris $1818.8^{\circ} .40$ Seiten. Zusammenstellung auf Chladni, Bigot de Morogue u. A. beruhend.]

r847 - : Liebig-Kopp, Jahresber. = Jahresberichte über die Fortschritte der reinen, pharmaceutischen und technischen Chemie, Physik, Mineralogie und Geologie; herausgegeben von 1847 bis 1856 von Liebig und Kopp; von 1857 bis 1864 von Kopp und Will; von 1865 bis 1867 von Will; von 1868 bis 1869 von Strecker; von 1870 bis 1874 von Naumann; von 1875 bis 1890 von Fittica. Letzter Band erschien 1895 . 1833/69: "L'Institut = L'Institut, Journal des académies et sociétés scientifiques de la France et de l'étranger. Ausgezogen Bd. I (1833) bis Bd. 37 (1869).

[189o: Lockyer: The Meteoric Hypothesis a statement of the results of a spectroscopic inquiry into the origin of cosmical systems. London. Macmillan u. Co. and New-York $1890\left(8^{\circ}\right.$. 560 Seiten mit vielen Tafeln und Abb. im Text).]

r853/80: Luminous Meteors $=$ Report on observations of Luminous Meteors. Die ersten zwölf Reports bis 1859 wurden von Baden-Powell redigiert; von 1859 an fand die Redaction durch ein Komité statt, an dessen Spitze James Glaisher stand. Diese Berichte sind abgedruckt in Rep. Brit. Assoc. Die von Flight verfassten, welche sich in seinen $>$ Meteorites e finden, wurden nicht extra aufgeführt.

[1891: Melnikow: Hat im Bergjournal für I891 ein Verzeichnis aller russischen Meteoriten herausgegeben (russisch).]

[1867: Meunier: Etude descriptive, théorique et expérimentale sur les Météorites, Paris 1867. $8^{\circ} 187$ Seiten.]

1869: Meunier : Recherches $=$ Recherches sur la composition et la structure des Météorites. Ann. Chim. Phys. (4) 17, p. $\mathrm{I}-73$; s, auch Thèse présentée à la Faculté des Sciences de Paris; ferner "La Nature * Bd. I, p. 229 (?); Chem. News Bd. I9,

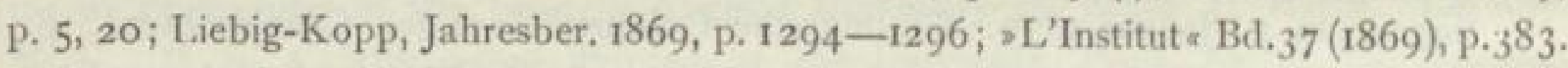

[r871: Meunier: Le Ciel Géologique, Prodrome de Géologie Comparée, Paris 1871. $8^{\circ}$. 247 Seiten.]

[1874: Meunier: Cours de Géologie Comparée, professé au Muséum d'Histoire Naturelle. Paris $1874.8^{\circ}$.]

[1880: Meunier: Imitation synthétique des fers nickelés météorifiques, Bull, Soc. Min. France Bd. 3 (1880), p. 153. Ref. N. J 1881 II, p. 184.]

[1883: Meunier: Recherches expérimentales sur le mode de formation de divers minéraux météorifiques. Mémoires présentés par divers savants à l'académie des sciences de l'Institut National de France. Bd. 27 n० 5. 28 Seiten. Ref. N. J. 1886 I, p. 405-406.]

[1883: Meunier: Essai d'application de la théorie cyclonique de M. Faye à l'histoire des météorites primitives. C. R. Bd. 96, p. 866-869. Ref. N. J. 1884 I, p. 33I.]

1884: Meunier: Meteorites = Encyclopédie Chimique, publiée sous la direction de M. Fremy. Tome II: Métalloïdes Appendice. - 2 cahier: Météorites par M. Stanislas Meunier. Paris 1884. Verlag von Dunod. Paris, Quai des Augustins 49. $8^{\circ}$. 532 Seiten mit vielen Abbildungen und Tabellen. Ref. N. J. 1885 II, P. 413-4r4.

[1885: Meunier: Sur la classification et l'origine des météorites, C. R. Bd. ror, p. 728 -730. Ref. J. 1887 I, p. 258.]

[1886: Meunier: Sur la géologie des météorites. Bull, soc. géol. de France (3) I4, p. 68 -79. Ref. J. 1887 I, p. 258.]

1893: Meunier: Revision des fers météoriques = Revision des fers météoriques de la collection du Muséum d'histoire naturelle. Bull. Soc. d'hist, nat. Autun Bd, 6 (1893) Sep., 8r Seiten. 
1895: Meunier: Revision des lithosidérites = Revision des lithosidérites de la collection du Muséum d'histoire naturelle. Bull. Soc. d'hist. nat, Autun Bd, 7 (1895) Sep, 44 Seiten.

1871/76: M. M. = Mineralogische Mittheilungen, gesammelt von Gustav Tschermak; 6 Bïnde $1871-1876$.

1875: Mohr: Entstehungsart $=$ Ueber die Natur und Entstehungsart der Meteoriten. Ann. Chem. Pharm. Bd. 179 , p. $257-282$. Ref. Liebig-Kopp, Jahresber. 1875 , P. $1308-1309$.

r878-: M. P. M. = Mineralogische und Petrographische Mittheilungen, herausgegeben von G. Tschermak Bd. I (1878) - Bd. Io (1889). Von Bd. II (1890)= Tschermak's Mineralogische und Petrographische Mittheilungen, herausgegeben von F. Becke.

[r876: Müller: Die Meteorsteine. Oeffentl. Vortr., gehalten in der Schweiz, Bd. 4 Heft 3 . 36 Seiten (Basel 1876$)$.]

1805: Münter: Ueber die vom Himmel gefallenen Steine der Alten, Bäthylien genannt, in Vergleichung mit den in neueren Zeiten herabgefallenen Steinen. Eine Verdeutschung aus dem Dänischen von J. A. Markussen. Kopenhagen u. Leipzig 1805. 80 33 Seiten.]

1888: Newton: Orbits $=$ Upon the relation which the former Orbits of those Meteorites that are in our collections, and that were seen to fall, had to the Earth's Orbit. Am. Journ. of Se. (3) 36, p. I-14.

v. Niess1: Die höchst wertvollen Arbeiten v. Niessl's, welche meist in den Verh. naturf. Ver. Brünn und in den Sitzber. Wien. Akad, erschienen sind, wurden nur insofern, als sie einen speziellen Fall behandeln, aufgenommen. In Bezug auf die Hemmungshöhe s. auch Brezina, sDie Meteoriten vor und nach ihrer Ankunft auf die Erder, p. 39; s. auch die Ref. in Wochenschr. f. Astr., Met. u. Geogr. 1887, p. $245-248,253-254,260-262,269-272,275-277$.

1890: v. Niess1 : Periheldistanzen $=$ Ueber die Periheldistanzen und andere Bahnelemente jener Meteoriten, deren Fallerscheinungen mit einiger Sicherheit beobachtet werden konnten. Verh. naturf. Ver. Brïnn Bd. 29 (189o) gedruckt 1891, p. 182-258.

1833-: N. J. = Neues Jahrbuch für Mineralogie, Geologie etc. Stuttgart. E. Senweizerbart'sche Verlagshandlung. Bd. I von 1833 .

1878: Nordenskjöld: Trenne märkeliga eldmeteorer, sedda i Sverige under îren 1876 och 1877. Geologisca Föreningens i Stockholm. Förhandl. Bd. 4 ; s, auch Uebersetzung von v. Boguslawski in Zeitschr. d. d. geol. Ges, Bd. 33 (188r). Ref. N. J. 1879, p. 77-81 ; Liebig-Kopp, Jahresber. 1879, p. 1277-1279. Die Analysentafel s. auch in v. Nordenskjöld »Studien und Forschungen «. Leipzig 1885, p. 149; hier auch Situationsplan von Hessle und allgemeine Betrachtungen unter der Ueberschrift: Ueber die geologische Bedeutung des Herabfallens kosmischer Stoffe auf die Oberfläche der Erde mit besonderer Berücksichtigung der Kant-Laplace'schen Theorie, p. $123-217$.

1884: Original Researches s. unter Smith.

1843: Partsch : Meteoriten $=$ Die Meteoriten oder vom Himmel gefallenen Steine und Eisenmassen im k. k. Hof-Mineralien-Kabinette zu Wien, Beschrieben, und durch wissenschaftliche und geschichtliche Zusätze erläutert von Paul Partsch, Custos an dem genannten Kabinette. Mit einer Abbildung. Wien 1843. Verlag von Kaulfuss Witwe, Prandel u. Comp. 80. 162 Seiten (mit 2 Tabellen).

[1803: Patrin: Bemerkungen gegen den vorhergehenden Aufsatz Howard's (aus den 
Phil. Trans, von 1802). Gilb. Ann. Bd. 13, p. 328-336. Historisch interessant;

s. auch: de Bournon in Journ. Phys. Bd. 56 (1803), p. 294-304.]

[1867: Phipson: Meteors, Aerolites and Falling Stars. With numerous illustrations. London r867. $8^{\circ}$. 240 Seiten.]

1804: Pötzsch: Kurze Darstellung $=$ Kurze Darstellung der Geschichte über das Vorkommen des gediegenen Eisens, sowohl des mineralischen als auch des problematisch-meteorischen und anderer darauf Bezug habenden Aerolithen, mit eigenen Wahrnehmungen, die auch das Daseyn der ersteren in dem Innern unsers Erdkörpers auf Lagern oder Gängen zu bestätigen scheinen. Dresden 1804. $8^{\circ}$ I19 Seiten.

Powell s Luminous Meteors,

1885: Prinz: Les Météorites tombées en Belgique et les Météorites en général. Ciel et Terre, Jahrgang 5, Sep. 39 Seiten und 1 Tafel (wurde nur in Bezug auf Saint-DenisWestrem und Tourinnes berücksichtigt). Ref. N. J. 1886 II, p. 212-213.

1843: Rammelsberg: Ueber die Bestandtheile der Meteorsteine. Pogg. Ann. Bd, 60, p. 130-139 (der wesentliche Inhalt dieser Arbeit findet sich auch in Rammelsberg's Handwörterbuch). Ref. N. J. 1844, p. 718 ; Haidinger, Uebersicht 1843, p. 108109 ; s. auch die Beiträge Rammelsberg's in Humboldt's Kosmos Bd. III (I850), p. $6 \mathrm{r} 4-6 \mathrm{r} 7$.

1860: Rammelsberg: Mineralchemie = Handbuch der Mineralchemie; von C. F. Rammelsberg, Dr. u. Professor an der Universität etc. Leipzig 1860. Verlag von W. Engelmann Anhang I, p. 9or-952 Meteoriten. - Die zweite Auflage von 1875 mit zwei Ergänzungsheften von 1886 und 1895 ist nur hinter den Arbeiten aufgeführt, auf welche die angeführte Stelle sich bezieht; diese zweite Auflage enthält nur kurze Angaben über einige meteorische Mineralien (Anorthit, Asmannit, Daubréelit, Maskelynit, Oldhamit, Troilit).

1870: Rammelsberg : Meteoriten = Die chemische Natur der Meteoriten von C. Rammelsberg. Abh. Berlin. Akad. 1870, p. 75-160. Ref. N. J. 1880 II, p. 32-34; s. auch: Ueber das Vorkommen der Augitsubstanz in den Meteoriten. Pogg. Ann. Bd. 140 (I870), p. 3II-32I. Olivin betreffend s. ferner: Autors Handbuch der Mineralchemie 2. Aufl. 1875, p. 429.

1879: Rammelsberg : Meteoriten = Die chemische Natur der Meteoriten von C. Rammelsberg. Abh. Berlin. Akad, 1879. 64 Seiten.

Rath s. vom Rath.

[1841: Reichenbach: Merkwürdiger Meteorsteinfall in Ungarn von Karl Reichenbach. Pogg. Ann. Bd. 54, p. 160.]

1850: Reichenbach: Ueber Meteoreisenanalysen. Pogg. Ann. Bd. 79, p. 478-48o.

1857: v. Reichenbach: I = Ueber den Meteoriten von Hainholz von Karl Freiherrn v. Reichenbach. Pogg. Ann. Bd, Ior, p. $3 \mathrm{II}-3 \mathrm{I} 3$.

1857: v. Reichenbach: II $=$ Zum Meteoriten von Hainholz. Pogg. Ann. Bd. 102, p. 618-621. Ref. Kenngott, Uebersicht 1856157 , p. $154-155$.

1857: v. Reichenbach: III = Ueber die Meteoriten aus dem Tolucathale in Mexiko. Pogg. Ann. Bd, 102, p. $62 \mathrm{t}-625$; s. auch Wochenschr. f. Astr., Met. u. Geogr. I858, p. $317-318$.

1858: v. Reichenbach: IV $=$ Ueber die Rinde der meteorischen Eisenmassen. Pogg. Ann. Bd. 103 , p. $637-644$. Ref. N. J. 1859, p. 444 ; Kenngott, Uebersicht $185^{8}$, p. 126-127; Liebig-Kopp, Jahresber. 1858, p. 805; Wochenschr. f. Astr., Met. u. Geogr. 1858 , p. $301-303$. 
r858: v. Reichenbach: $V=$ Ueber die Rinde der Meteorsteine. Pogg. Ann. Bd. IO4, p. 473-482. Ref. Liebig-Kopp, Jahresber. 1858 , p. $805-806$.

1858: v. Reichenbach: VI $=$ Die Meteoriten und die Kometen nach ihren gegenseitigen Beziehungen. Pogg. Ann. Bd. 105, p. 438-460. Ref. Kenngott, Uebersicht 1858, p. $166-167$, u. 1859, p. 171-172; Wochenschr. f. Astr., Met. u. Geogr. 1859, p. I16-117; Kenngott, Monatschr, d. wiss. Ver. Zürich Bd. 4, p. 346; Liebig-Kopp, Jahresber. 1858 , p. 806.

1858: v. Reichenbach: VII $=$ Ueber die Anzahl der Meteoriten und Betrachtungen über ihre Rolle im Weltgebäude. Pogg. Ann. Bd. I05, p. 557-563. Ref. Kenngott Uebersicht 1859 , p. 171-172; Kenngott, Monatschr. d. wiss. Ver. Zürich Bd. 4, p. 346; Liebig-Kopp, Jahresber. 1858 , p. $806-808$; Wochenschr. f. Astr., Met. u. Geogr. 1859 , p. $289-290$.

1859: v. Reichenbach: VIII = Die meteorischen Kügelchen des Capitain Callum. Pogg. Ann. Bd 106, p. $476-490$.

1859: v. Reichenbach: IX $=$ Anordnung und Eintheilung der Meteoriten. Pogg. Ann. Bd. 107, p. 155-182. Ref, Kenngott, Uebersicht 1859, p. 165-166.

r859: v. Reichenbach (Ohne Nummer): Notiz über den Meteoriten von Clarac. Pogg. Ann. Bd. 107, p. 191-192. Ref. N. J. 1860, p. 82; Kenngott, Uebersicht 1859, p.166. I859: v. Reichenbach: $\mathrm{X}=$ Ueber die chemische Beschaffenheit der Meteoriten. Mit einer Analysentafel. Pogg. Ann. Bd. 107, p. 353-374; s, auch Kenngott, Uebersicht 1859 , p. 166.

1859: v. Reichenbach: $\mathrm{XI}=$ Ueber das Gefüge der Steinmeteoriten, Pogg. Ann. Bd. I08, p. $29 \mathrm{I}-3$ II.

1899: v. Reichenbach : XII = Ueber die Zeitfolge und die Bildungsweise der näheren Bestandtheile der Meteoriten. Pogg. Ann. Bd. Io8, p. $452-465$.

1860: v. Reichenbach: XIII $=$ Meteoriten in Meteoriten. Pogg. Ann. Bd. III, p. 353 -386 (Mit zwei Tafeln, Abbild, von Einschlüssen). Ref. Liebig-Kopp, Jahresber. 1860, p. $844-845$.

I860: v. Reichenbach: XIV $=$ Meteoriten und Sternschnuppen. Pogg. Ann. Bd, III, p. $387-4$ or. Ref. Liebig-Kopp, Jahresber. 1860, p. 845 .

186r: v. Reichenbach: XV $=$ Ueber das innere Gefüge der näheren Bestandtheile des Meteoreisens, Pogg. Ann. Bd. I14, p. $99-$ r32. Ref. N. J. 1862, p. 598-599; Kenngott, Uebersicht I86I, p. I05-106.

r86r : v. Reichenbach: XVI = Ueber die näheren Bestandtheile des Meteoreisens. Das Bandeisen. Pogg. Ann. Bd. 1r4, p. 250-263.

1861 : v. Reichenbach: XVII = Ueber die näheren Bestandtheile des Meteoreisens. Das Fülleisen. Pogg. Ann. Bd. 114, p. 264-274.

1861: v. Reichenbach: XVIII = Ueber die nähern Bestandtheile des Meteoreisens. Die Wülste und das Glanzeisen. Pogg. Ann. Bd. 114, p. 477-491. Ref. Kenngott, Uebersicht 1861, p. 106; Liebig-Kopp, Jahresber. 186I, p. II8-III9.

1862: v. Reichenbach: XIX = Ueber die nähern Bestandtheile des Meteoreisens, Die Nadeln. Die Eisenkügelchen. Der Mohr. Pogg. Ann. Bd. 115, p. 148-156. Ref. Kenngott, Uebersicht 1861, p. 107.

1862: v. Reichenbach: XX = Ueber die nähern Bestandtheile des Meteoreisens. Ueber das Schwefeleisen. Pogg. Ann. Bd. Ir5, p. 620-636. Ref. Liebig-Kopp, Jahresber. 1862, p. $823-825$.

1862: v. Reichenbach: XXI $=$ Ueber die nähern Bestandtheile des Meteoreisens. W ï 1 fing, Meteoriten.

III 
Der Graphit und das Eisenglas. Pogg. Ann. Bd. 116, p. 576-591. Ref. s, vorige Arbeit. I863: v. Reichenbach: XXIa $=$ Ueber das chemische Verhalten des Meteoreisens gegen Säuren. Von Reinhold von Reichenbach. Pogg. Ann. Bd. Ir9, p. 172-176. Ref. Kenngott, Uebersicht $1862 / 65$, p. 265 ; Liebig-Kopp, Jahresber. 1863, p. 9II. [1863: v. Reichenbach: XXII = Ueber Erzeugung von Wärme und Licht durch Meteoriten. Von Reinhold Frhr. von Reichenbach. Pogg. Ann. Bd. 119, p. 275-287. Ref. Kenngott, Uebersicht $1862 / 65$, p. 428 ; Liebig-Kopp, Jahresber. 1863 , p. 900 -903 ; Wochenschr. f. Astr., Met. u. Geogr. 1863, p. $286-287$.]

I864: v. Reichenbach: XXIII $=$ Die Sternschnuppen in ihren Beziehungen zur Erdoberfläche. Pogg. Ann. Bd. I23, p. $368-377$.

1865: v. Reichenbach : XXIV = Geschichte der Meteoriten von Blansko, nebst Anleitung $\mathrm{zu}$ methodischer Aufsuchung frisch niedergefallener Meteoriten. Pogg. Ann. Bd. 124, p. $213-234$.

1865: v. Reichenbach: XXV $=$ Die schwarzen Linien und Ablösungen in den Meteoriten. Pogg. Ann. Bd. 125, p. 308-325, 420-44I, 600-618. Ref. Kenngott, Uebersicht $1862 / 65$, p. $43 \mathrm{I}-432$.

1863: Rose: Meteoriten $=$ Beschreibung und Eintheilung der Meteoriten auf Grund der Sammlung im mineralogischen Museum zu Berlin. Von Gustav Rose. 16r Seiten mit vier Tafeln. Abh. Berlin. Akad, 1863. Gelesen in der Akademie der Wissenschaften am 7. und 14. Aug. I862 und am II. Juni 1863. Anfang des Druckes am 4. Juli $\mathbf{r} 864$, bis wohin einige neue Zusätze hinzugefügt sind, S. auch Mon.-Ber. Berlin. Akad. 1862 , p. $551-55^{8}$, von welcher Arbeit besonders p. $555-557$ berü̈cksichtigt wurde. Auszug in Pogg. Ann. Bd. I18, p. 418-423 und Bd. I24 (1865), p. 193-213. N. J. 1862 , p. $997-998$ und 1865, p. 321-323; Kenngott, Uebersicht $186 \mathrm{I}$, p. $213,219-220$ und $1862 / 65$, p. $428-431$; Liebig-Kopp, Jahresber. 1865, p. $945-947$; Wochenschr. f. Astr., Met. u. Geogr. 1863, p. 278 und I865, p. $86-87$, II 7-II9; Zeitschr. d. d. geol. Ges. Bd, I7 (I865), p. 4-5; Vers. deutsch. Naturf. u. Aerzte Bd. 39, p. III.

[1812: Ruhland: Ueber den Ursprung der Meteorsteine. Schweigg.Journ. Bd.6, p. 14-59.]

[1843: Rumler: Die spez. Gew.-Bestimmungen Rumler's, welche so vielfach in der Literatur verbreitet sind, befinden sich in Partsch: Meteoriten 1843 abgedruckt.]

[1808: Salverte: Conjectures sur les pierres tombées de l'atmosphère par Eusèbe Salverte. sEncyclopédie Bd, 5 (1808), p. 559-56r.]

1847: Schafhäutl: Schönenberg = Ueber den bey Schönenberg, Landgerichts Burgau gefallenen Meteorstein und sein Verhältniss zu den im mineralogischen Kabinete der Akademie der Wissenschaften befindlichen Aerolithen. Gel. Anz. München. Akad, 1847 , p. $553-584$. Ref. sL'Institute Bd, 15 (1847), p. $340-341$

[r891: Schauf: Ueber Meteorsteine. Vortrag, gehalten bei dem Jahresfeste am ro. Mai 1891. Ber. Senckenberg. naturf. Ges. Frankfurt a, M. 1891, p. 3i9-335. Ref. N. J. 1892 I, p. 269.]

[1871: Schiaparelli: Entwurf einer astronomischen Theorie der Sternschnuppen. - Einzig autorisirte deutsche Ausgabe der vom Verfasser völlig umgearbeiteten, Note e Riflessioni sulla teoria astronomica delle Stelle cadenti * aus dem Italienischen übersetzt und herausgegeben von Georg v. Boguslawski. Stettin 1871, 80, 268 Seiten u. 4 Tafeln.]

r820: v. Schreibers : Beiträge $=$ Beyträge zur Geschichte und Kenntnisse meteorischer Stein- und Metall-Massen, und der Erscheinungen, welche deren Niederfall zu be- 
gleiten pflegen. $4^{\circ}$. Wien bei Heubner 1820, 97 Seiten u. ro Tafeln. S. auch Benzenberg: Sternschnuppen 1839 , p. $85-87$.

[1814: Shearman: On the origin of stones etc. De l'origine des pierres tombées de l'atmosphère. (New Monthly Magazine) Juin 1814. Bibl, Brit, ${ }^{\circ} 468$ (Juni 1815), p.18r-190.]

1829: Shepard 1): A mineralogical and chemical description of the Virginia Aerolite. Am. Journ. (I) 16, p. 19I-203 (Richmond).

1829: Shepard: Analysis of the Meteoric Iron of Louisiana, and discovery of the Stanniferous Columbite in Massachusetts. Am. Journ. (I) 16, p. 217-219.

1830: Shepard: On Crystallized Native Terrestrial Iron, Ferro-silicate of Manganese, and various other American Minerals. Am. Journ. (I) 17, p. 14I-142 (Guildford Co.). 1839: Shepard: On Meteoric Iron from Ashville, Buncombe county, N. C. Am. Journ. (I) 36 , p. $8 \mathrm{r}-84$ (Baird's Farm).

1840: Shepard: Analysis of Meteoric Stone, which fell near Little Piney, Missouri, Feb. 13. 1839. Am. Journ. (I) 39, p. $245-255$.

1841: Shepard: On Native and Meteoric Iron. Am. Journ. (1) 40, p. 366-370 (Scriba und Guilford Co.).

1842: Shepard: Analysis of Meteoric Iron from Cocke County, Tennessee, with some remarks upon Chlorine in Meteoric Iron masses. Am. Journ. (I) 43, p. 354-363.

1843: Shepard: On Phosphate of Lime (Apatite) in the Virginia Meteoric Stone. Am. Journ. (I) 45 , p. $102-103$.

1846: Shepard: Report on Meteorites. Am. Journ. (2) 2, p. 377-392. Ref. N. J. I852, p. 614-618. Uebersetzung im s'Institut * Bd. 15 (1847) Nr. 725 , p. 379-384.

1847: Shepard: Report on Meteorites. Am. Journ. (2) 4, p. 74-87. Ref. und Uebersetzung wie vorher.

1847: Shepard: Fall of Meteoric Stones in Iowa. Am. Journ. (2) 4, p. 288-289.

1848: Shepard: An Account of the Meteorite of Castine, Maine, May 20, 1848. Am. Journ. (2) 6, p. 251-253.

1848: Shepard: Observations on Rammelsberg's Analysis of the Juvenas Meteoric Stone and on the Conclusion of Fisher's Examination of the Braunau Meteoric Iron. Am. Journ. (2) 6, p. 346-349.

1848: Shepard: Report on Meteorites. Am. Journ (2) 6, p. $402-417$.

1849: Shepard: On Meteoric Iron in South Carolina. Am. Journ. (2) 7, p. 449-450 (Chesterville).

1850: Shepard: On Meteorites, Am. Journ. (2) Io, p. 127-129.

1851 : Shepard: On Meteorites. Am. Journ. (2) II, p. 36-40.

1853: Shepard: I. Notice of Meteoric Iron near Lion River, Great Namaqualand South Africa; and of the detection of Potassium in Meteoric Iron. - 2. Potassium in the

I) Das folgende Verzeichnis der Shepard'schen Arbeiten enthält jene Aufsätze, welche im Am. Journ. erschienen sind. In meinen Literaturangaben ist nicht auf jede in diesen Arbeiten beiläufig erwähnte Lokalität verwiesen worden; auch wurde der : Cataloguee von 1861 und der Aufsatz sNew Classification « von 1867 nicht aufgenommen. Da aber die „Shepard'sche Sammlung von der Sammlung des National Museum's in Washington bei mir getrennt aufgeführt ist, so gewinnt man einen Ueberblick über jene Meteoriten, über welche allenfalls noch weitere Angaben in den Shepard'schen Aufsätzen zu finden sind. 
Meteoric Iron of Ruff's Mountain, South Carolina. - 3. Figure of the Iowa Meteoric Stone, which was seen to fall Feb. 25, 1847. Am. Journ. (2) 15, p. I-7.

1853: Shepard: Notice of the Meteoric Iron found near Seneca River, Cayuga County, N. Y.7 Am. Journ. (2) 15, p. $363-366$.

1854: Shepard: Notices of three ponderous masses of Meteoric Iron at Tuczon, Sonora.

Am. Journ. (2) 18, p. $369-372$.

1856: Shepard: On a new locality of Meteoric Iron, in the Orange River County, South Africa, and a supposed new locality of the same in Mexico. Am. Journ. (2) 2I, p. 213-216 (Orange River u. Coahuila).

1857: Shepard: Notice of a Meteoric Stone, which fell at Petersburg, Lincoln county,

Tennessee, August $5^{\text {th }}$ 1855. Am. Journ. (2) 24, p. 134-137.

1860: Shepard: Notices of several American Meteorites. Am. Journ. (2) 3o, p. 204208 (Fort Pierre, Miney, Bethlehem, New Concord).

1861: Shepard: Catalogue of the Meteoric Collection of Charles Upham Shepard, deposited in the Cabinet of Amherst College, Mass. Am. Journ. (2) 31, p. 456-459 (88 Steine, 63 Eisen, darunter verschiedene Pseudo-Meteoriten, Einzelgewichte nicht angegeben. Gesamtgewicht 820 lbs.).

1866: Shepard: Brief Notices of several localities of Meteoric Iron. Am. Journ. (2) 42 , p. 249-251 (Savisavik, Botetourt, Bear Creek, „Tennessee «).

1866: Shepard: New locality of Meteoric Iron in Cohahuila, Northern Mexico. Am. Journ. (2) 42 , p. $347-35$ o.

1867: Shepard: New Classification of Meteorites, with an Enumeration of Meteoric Species. Am. Journ. (2) 43, p. 22-28. Ref. N. J. 1867, p. 719-724.

1867: Shepard: Additional Notice of the Cohahuila Meteoric Iron. Am. Journ, (2) 43. p. $384-385$.

1868: Shepard: A new locality of Meteoric Iron in Georgia. Am. Journ. (2) 46, p.257-258. 1869: Shepard: Notices of new Meteoric Irons in the United States, $1 \mathrm{~m}$. Journ. (2) 47 p. $230-234$ (Auburn, St. François, Losttown).

1871 : Shepard: Notice of the Meteoric Stone of Searsmont, Maine. Am. Journ. (3) 2, p. $133-136$.

1872: Shepard: On a Meteoric Iron lately found in El Dorado county, California, Am. Journ. (3) 3, p. $43^{8}$ (Shingle Springs).

1876: Shepard: Notice of the Meteoric Stone of Waconda, Mitchel County, Kansas. Am. Journ. (3) n, p. $473-474$.

1877: Shepard: On the Meteoric Stone of Rochester, Fulton County, Indiana. Am. Journ. (3) 13 , p. 207-2II.

1877: - Transfer of the Shepard Collections to Amherst College. Am. Journ. (3) 14, p. 167.

1879: Shepard: On the Estherville, Emmet County, Iowa, Meteorite of May $10^{\text {th }}, 1879$. Am. Journ. (3) 18, p. 186-188.

1880: Shepard: On the Ivanpah, California, Meteoric Iron. Am. Journ. (3) 19, p. $381-382$. I881 : Shepard: On the Meteoric Iron of Lexington County, South Carolina, Am. Journ.

(3) 2I, p. 117-119.

1881: Shepard: On a new Meteoric Iron of unknown locality, in the Smithsonian Museum. Am. Journ. (3) 22, p. 119.

1883: Shepard: On Meteoric Iron from near Dalton, Whitfield County, Georgia. Am. Journ. (3) 26 , p. $336-338$. 
1885: Shepard: On Meteoric Iron from Trinity County, California. Am. Journ. (3) 29, p. 469 (Glorieta).

1885: Shepard: On the Meteorite of Fomátlan, Jalisco Mexico. Am. Journ. (3) 30, p. $105-108$ (Gargantillo).

1876: Smith: Carbon compounds $=$ Researches on the Solid Carbon Compounds in Meteorites. Am. Journ. (3) 1I, p. 388-395, $433 \quad 442$; s, auch kurze Ankündigung C. R. Bd. 82 (1876), p. 1042-1043; Ann. Chem Pharm. Bd. 182 (1876), 113-127; Original Researches 1884 , p. 496-514; Liebig-Kopp, Jahresber 1876 , p. 13 II-I3I2.

1883: Smith: Concretions $=$ On the Peculiar Concretions occuring in Meteoric Iron. Am. Journ. (3) 25, p. $417-423$; s. auch Original Researches 1884 , p. 599-606. Ref. N. J. 1884 II, p. $33-34$.

1884: Smith: Original Researches $=$ Original Researches in Mineralogy and Chemistry, by J. Lawrence Smith. Membre correspondant de L'Institut de France (Académie des Sciences) etc. Printed for presentation only. Edited by J. B. Marvin, B. S. M. D. Louisville Ky. Printed by John P. Marton and Company 1884. Ref. N. J. 1885 II, p. 406.

1864: Sorby: On the Microscopical Structure of Meteorites. P oc. Roy. Soc. 1863/64, p. 333-334. Ref. Am. Journ. (2) 13 (1866), p. 136-138; Luminous Meteors: Rep. Brit. Assoc. 1865 , p. $139-140$; s. auch Note on the preceding Articles (5) and (7), by Professor Bradley im gleichen Bd., p. 140-142; Philos. Magaz. Bd. 28 (1864), p. 157-159; Liebig-Kopp, Jahresber. 1864, p. 896; "L'Institut * Bd. 33 (1865) Nr. 1623 , p. $46-47$.

1887: Sorby: On the mikroskopical structure of iron and steel. The Journal of the Iron and Steel Institut 1887 , p. $255-288$. Ref. N. J. 1891 I. p. $46-47$

[1804: Sukow: Anfangsgründe der Mineralogie nach den neuesten Entdeckungen. Leipzig 1804 Bd. 2, p. 643-649 enthält kurze Angaben über Benares, Eichstädt, Krasnojarsk und Siena, die nichts Neues bringen; auch p. 234 und 235 Krasnojarsk und Campo del Cielo erwähnt.]

1870: Tschermak: Goalpara $=$ Ueber den Meteorstein von Goalpara und über die leuchtende Spur der Meteore. Sitzber. Wien Akad Bd. 62 II (1870), p. 855-865.

1872: Tschermak: Die Meteoriten des k. k. Mineralogischen Museums am I. October 1872. M. M. Bd. 2 (1872), p. 165-172. Ref. N. J. 1872, p. $952-953$. S. auch Arbeit von 1877 .

1875: Tschermak: Vulkanismus $=$ Die Bildung der Meteoriten und der Vulkanismus. Sitzber. Wien. Akad. Bd. 71 II $(1875)$, p. $661-673$. Ref. N. J. 1875, p. $878-880$; Liebig-Kopp, Jahresber. 1875, p. 1307-1308.

1877. Tschermak: Vermehrung der Meteoritensammlung des Mineralogischen Hofmuseums bis Ende September 1877. M. M. Bd. 7 (1877), p. 309-3Ir. Ref. N. J. 1878, p. 79. Auf die beiden Verzeichnisse Tschermak's von 1872 u. 1878 ist in der Art verwiesen, dass bei der Gewichtsangabe der Sammlung des Wiener Hof-Museums, Wien, H. M. *) oder Wien, H. M.**) oder Wien, H. M. *)**) gedruckt ist.

1883: Tschermak: Beitrag = Beitrag zur Klassification der Meteoriten. Sitzber. Wien, Akad. Bd. 88 I (1883), p. 347-37I. Ref. N. J. 1884 II, p. 25-28; Liebig-Kopp, Jahresber. 1883 , p. 1951-1952.

1883/85: Tschermak: Photographien $=$ Die mikroskopische Beschaffenheit der Meteoriten erläutert durch photographische Abbildungen, 25 Tafeln mit 100 mikrophotographischen Abbildungen. Stuttgart. E. Schweizerbart'sche Verlagshandlung 
(E. Koch) 1885. Ref. N. J. 1884 I, p. 174-175; N. J. 1885 I, p. 29; N. J. 1886 I, p. $218-219$.

1808: Vauquelin: Mémoire sur les pierres dites tombées du ciel, lu à l'Institut; s, auch „Encyclopédie a Bd. 5 (I8o8), p. 564 ff.

1875: vom Rath: Meteoriten $=$ Die Meteoriten des naturhistorischen Museums der Universität Bonn. Verh. naturh. Ver. Bonn Bd, 32 (1875), p. 353-376. Ref. N.J. 1876, p. 67. Ein ausführliches Verzeichnis der vom Rath'schen Arbeiten über Meteoriten befindet sich im Sach- und Orts - Verzeichnis zu den mineralogischen und geologischen Arbeiten von Gerhard vom Rath. Im Auftrage der Frau vom Rath bearbeitet von W. Bruhns und K. Busz. Leipzig, W. Engelmann 1893.

1884: Wadsworth: Studies $=$ Lithological Studies. A description and classification of the rocks of the cordilleras. By M. E. Wadsworth. With eight Plates. Memoirs of the Museum of Comparative Zoology at Harvard College. Bd. II. Part. I. Cambridge. Printed for the Museum. October 1884. (208 Seiten Text und 33 Tafeln Analysen, die grösstenteils auch in Rammelsberg's Arbeiten enthalten sind.)

[1882: Weinland: Ueber die in Meteoriten entdeckten Tierreste. Esslingen. $4^{0}$. 12 Seiten.] - : Wien, H. M. ${ }^{*}$ ) oder Wien, H. M.**) oder Wien, H. M.*)**) s. Tschermak 1877 .

1852: Wöhler: $\gg$ Passiv $\approx$ oder $\gg$ Aktiv $c$ oder $\gg$ Intermediär = Passiver Zustand des Meteoreisens. Pogg. Ann. Bd. 85 , p. $448-449$; s, auch Ann. Chem. Pharm. Bd. 82 (1852), p. 248-249: Rammelsberg: Die chemische Natur der Meteoriten, Abhandl. Berlin. Akad. 1870, p. 80; Rammelsberg, Handwörterbuch Suppl. V, p. 14; Cohen, Meteoritenkunde (1894), p. 67; v. Boguslawski : Zehnter Nachtrag, Pogg. Ann. Ergz.-Bd. 4, p. $455-456$; Kenngott, Uebersicht 1852 , p. 9o; Liebig-Kopp, Jahresber. 1852, p. 988 ; Journ. prakt. Chem. Bd. $56\left(185^{2}\right)$, p. $244-245$.

1875: Wright: Spectroscopic Examination of Gases from Meteoric Iron. Am. Journ. (3) 9, p. 294-302, Ref. Liebig-Kopp, Jahresber. 1875, p. 1312-1314.

1876: Wright: On the Gases contained in Meteorites. Am. Journ. (3) 11, p. 253-262 und (3) 12, p. 165-176. Ref. Liebig-Kopp, Jahresber. 1876, p. 1312-1313; Wochenschr. f. Astr., Met. u. Geogr. 1876, p. $36 r-364$. 


\section{Verzeichnis der Meteoriten in alphabetischer Reihenfolge.}



Abert-Eisen, Eisen von unbekanntem Fundort; in der Sammlung von J. J. Abert gefunden und dem National-Museum in Washington durch seinen Sohn J.F. Abert geschenkt.

Eisen, Om; beschrieben 1887 .

1887: Riggs: On two new Meteoric Irons and an Iron of doubtful nature. - The Abert Iron. Bull. U. S. Geol. Surv. Nro. 42 (1887), p. 95-96 (Analyse; Abbildung einer geätzten Platte); s. auch Am. Journ. (3) 34, p. 59. Ref. N. J, 1890 I, p. 4 I.

1890: Eastman: Met. Astron., p. 320. 1894: Cohen: Meteoritenkunde, p. 54, 153 .

r895: B rezin a: WienerSammlung,p. $28_{3}$. Ursprüngliches Gewicht : $456 \mathrm{gr}$. Nachweisbares Gewicht: 324 gr. Bailey Bement II Washington 199 7 Washington, Sh. It Harvard, U. $\quad 9$ Wien, H. M. 40 London, B. M. 47

Adalia, Konia, Kleinasien. Stein, Eu; bekannt $\mathbf{1}_{88}$.

1884: Meunier: Météorites, p.295, 298. r885: Brez in a: Wiener Sammlg., p.r74. Ausser derAngabeMeunier's : sL'examen d'une lame mince m'a montré l'existence de l'anorthite très nettement caractérisé par son extinction sous des angles constamment supérieurs à $45^{\circ} \approx$ scheint in der Literatur nur noch die Bestätigung Brezina's, dass der Stein in der That ein Eukrit sei, sich zu finden.

Ursprüngliches Gewicht?

$W$ iu $1 \mathrm{fing}$, Meteoriten.
Nachweisbares Gewicht: 3 gr.

Paris, M. 3

Adare

Limerick

Adargas

Aeriotopos

Huejuquilla-Gruppe

Bear Creek

Afzelius

Sachsen, s. Anhang

Afzelius Südamerika, s. Anhang

Agen $179^{\circ}$ Barbotan

Agen 1814

Agen

Agen 1826

Galapian

Agen, Departement Lot et Garonne, Frankreich.

Stein, Cia, gefallen 5. Sept. I8r4.

1814: de Saint-Amans: Détails sur une chute d'Uranolythes, près d'Agen. Extrait d'une lettre écrite par M. de Saint-Amans à M. Thiébaut de Berneaud, sous la date d'Agen, le 17 septembre 1814. Ann. Chim. Bd. 92, p. $25-32 ;$ s, auch Bibl. Brit. Bd. 57 (Sept. 1814 No. 450 ), p. $80-87$ und p. $194-198$.

1814: De nouvelles chutes de Météorolites. Journ. Phys. Bd. 79, p. 2II-2I2 (Bericht von Lamoureux).

1814: Gilb e r t: Berichte über denSteinregen, welcher am 5. September in einigen Gemeinden des Departement des Lot und der Garonne, unweit Agen, herabgefallen ist. I) Bericht, abgestattet in der ökonom, litter. Gesellsch. zu Agen am 14. Sept. 1814 von Herrn de Saint-Amans, beständ. Sekr. d, Gesellsch. (Uebersetzung des Briefes in den Ann. Chim. s. o.). 2) Schreiben des Herrn J. Lamoureux, Ex-Pharmac. d. Armen, an den Präfekt Grafen von 
Villeneuve. 3) Notiz, aufgesetzt von Herrn Prugnieres, Gutsbesitzer. 4) Aus einem Schreiben des Herrn Prevost, Prof. d. Philos, zu Montauban, Gilb. Ann. Bd. 48, p. 395-409. Kurzes Ref. Leonhard's Mineralogisches Taschenbuch 1816 , p. $262-263$.

1815: Vauquelin: Note sur les aërolites tombées aux environs d'Agen, le 5 septembre 1814 (Qualitative Analyse). Journ. Mines Bd. 37 , p. $317-318$.

1815: Chladni: Neues Verzeichnis, Gilb. Ann. Bd. 50, p. 256.

r8r6: Chladni: Zweite Fortsetzung,

Gilb. Ann. Bd. 54 , p. $355,356$.

r8rg: Chladni: Vierte Fortsetzung, Gilb. Ann. Bd. 6o, p. 240.

r8rg: Chladni: Feuermeteore, p. 44, $58,59,66,73,91,157,289,290$, $295,305-306(1), 308,432$.

1821: Chladn i: Erster Nachtrag, Gilb. Ann. Bd. 68, p. 336.

r836: Kämtz: Meteorologie, p. 285. r843: Partsch: Meteoriten, p. 62, 63. 1854: Balcells: Lithologia meteorica, p. 23.

I854: v. B og uslaw s ki: Zehnter Nachtrag, Pogg. Ann. Ergz.-Bd. 4, p. I4. 1859: Buchner: Feuermeteore, p. 84. 1859: Harris: Dissert. Gött,, p. 72. 1863: Bu chner: Meteoriten, p.35-36. 1863: Rose: Meteoriten, p. 85, 155. r858/65: v. Reichenbach: V 475, 476,480 . VI 453 . IX 161, 171, 180. XI 294, 296, 30I. XIII 362. XX 626. XXV 317, 319, 321, 322, 324, 423, $427,43 \mathrm{I}, 432,435,601$.

1868: D a u b r é e : Note relative à l'envoi de météorites récemment fait à l'Académie par la Haute Ecole de Varsovie. C. R. Bd. 67 , p. 372 (betr. croute secondaire).

1875: Mohr: Entstehungsart, Ann. Chem. Pharm. Bd. 179, p. 259, 260, 26r. r884: Me un ier: Météorites, p. 247 , $249,479-481$.

r885: Brezina : Wiener Sammlung,

p. $155,182,232$.

I890: v. Niess 1 : Periheldistanzen,

Verh. naturf. Ver. Brünn, Bd. 29, p. 188, 195, 196, 214, 244.

r894: Cohen: Meteoritenkunde,p.223, 267.

UrsprünglichesGewicht: $30-35 \mathrm{Kgr}$. Die 2 grössten Steine je etwa $9 \mathrm{Kgr}$., davon einer gleich nach dem Auffinden grösstenteils vernichtet.

Nachweisbares Gewicht: 5274 gr.

Bailey

Berlin, U.

Böhm

Bologna

v. Braun

Budapest

Calcutta

Cleveland

Göttingen

Gregory

Greifswald

Harvard, U.

Heidelberg

Lausanne

Agra 1822

Agra 1860

Agra 1875

Agram

Agua blanca

Aigle

Ain Dep. 1753

Ain Dep. 1835

Ainsa

Akburpoor, Distrikt Saharanpoor, N. W. Provinz, Ostindien.

Stein, Cgb, gefallen 18 . April 1838 . 1854: Greg: Catalogue, p. 460 (giebt als Gewicht 4 lbs an).

1859: Haris: Dissert. Gött., p. 86. 1862: Maskel y ne: Chondritic Aërolites, Rep. Brit. Assoc. (Notices and Abstr.), p. 190. 
r863: Bu chner: Meteoriten, p. 60 (wurde mit Chandakapoor vereinigt).

1863: Ros e: Meteoriten, p. 154.

r865: v. Reichen b a ch: XXV 322 , 608 .

1877: Sorby: On the structure and origin of Meteorites, NNature $\mathrm{Bd}, \mathrm{I} 5$, p. 497 .

r884: M e uni er: Météorites, p. 268. r885: Brezina: Wiener Sammlung, p. $182,233$.

Ursprüngliches Gewicht: $1.8 \mathrm{Kgr}$. (4 lbs).

Nachweisbares Gewicht: 1663 gr.

Berlin, U.

Calcutta

9 London, P. G. $\quad 14$

Göttingen

4 New Haven

8 Paris, M.

Harvard, U.

7 Tübingen

1

2I

London, B. M.

Alabama 1832 u, 1834 Lime Creek.

Alais, Departem. Gard, Frankreich. Stein, K, gefallen 15. März 1806 .

1806: Pagès und D ho mbres-Firmas : Relation de la chute de deux aérolithes. Journ. Phys. Bd. 62 , p. 440 -442 ; s. auch $>$ Encyclopédie Bd. 5 (1808), p. $584-585$.

r806: Monge, Fourcroy, Berthollet, Vauquelin: Sitzungsprotokoll des National-Instituts vom 23. Juni 1806; s, auch Gilb. Ann. Bd. 24 (1806), p. 205. r806: Thén ard: Analyse d'un aérolithe tombé dans l'arrondissement d'A. lais, le 15 mars 1806. Ann. Chim. Juli 1806, p. 35 u. 103? ; s. auch »Encyclopédie Bd. 5 ( 1808$)$, p. $585-586$, auch p. $592-593$.

1806: Gilbert: Nachrichten über die beiden neuesten Meteorsteine, welche am 15. März 1806 im ehemaligen Languedoc (Departement du Gard) herabgefallen sind : r) Bericht von dem Herabfallen zweier Aërolithe von den HH. Pagès Doct. Med. und Dhombres-Firmas, Grundeigentümer, Mitgliedern der
Akad. des Gard-Dep. 2) Auszug aus dem Berichte des Friedensrichters des Kantons von Vezenobres, erstes Arrondissement des Gard-Departements, von einem zu Valence am 15. März $\mathbf{1 8 0 6}$ herabgefallenen Steine. 3) Analyse eines Aëroliths, welcher (zu Valence) im Arrondissement von Alais am I5. März 1806 herabgefallen ist, von Thénard in Paris. 4) Auszug aus dem Sitzungsprotokoll des National-Instituts vom 23. Junius 1806. Gilb. Ann. Bd. 24, p. $189-208 ;$ s. auch im gleichen Bd. p. $388_{3}-384$ Anm.

1808: Pictet: Description comparative etc. (s. bei Weston), Bibl. Brit. No. 296 (April 1808), p. 284-285, 287, 289, 290. I812: Ch la dn i: Verzeichnis, Schweigg. Journ. Bd. 4 Beil. I, p. 15 .

r812: Bigot de Morogues: p. 202 $-207,337-338$.

1815: Ch ladn i: Neues Verzeichnis, Gilb. Ann. Bd. 50, p. 253-254; s, auch Carlo Amoretti : Nuova scelta d'opuscoli da ... II, p. 63 u. Bibl, Brit. Bd. 32 .

r8rg: $\mathrm{C}$ b l a d n i : Feuermeteore, p. 46, $5 \mathrm{I}, 56,58,66,69,73,9 \mathrm{I}, 278-28 \mathrm{o}(!)$, 287,429 .

1834: Berzeli us: Om Meteorstenar. 4. Meteorsten från Alais. Kongl. Vetensk. Acad. Handl. (1834), p. 144158 (Analyse); Uebersetzung in Pogg. Ann. Bd. 33 (1834), p. $113-123,143$ (Beiläufig: Burkart, Fundorte I, N. J. I856, p. 267).

1836: Kämtz: Meteorologie, p. 256257 (Berzelius' Arbeit), 258, 28I.

1843: P a r ts c h: Meteoriten, p. I3-r4. 1847: Schaf häutl: S chönenberg, Sitzber. München. Akad. (1847), p. 573. r843/53 : R a m m els b e r g : Handwörterbuch, p. 435 u. Suppl. V, p. 32.

1854 : v. Bog us laws ki : Zehnter Nachtrag, Pogg. Ann. Ergz.-Bd.4, p.II-12, I4. r859: B u chner: Feuermeteore, p. 75 -76 .

1859: Harris: Dissert. Gött., p. 66-67. 
r860: R a m mels berg : Mineralchemie, p. 944.

1862: Kenngott und Wiser: Zürcher Sammlung, p. 155.

1863: B u c hner: Meteoriten, p. 19. 1863: Rose: Meteoriten, p. 25, 28, 126, 156.

1863: Roscoe: On the existence of a crystallizable carbon compound and free sulphur in the Alais Meteorite. Philos. Magaz. (4) 25, p. 319-320; s. auch Proc. of the Literary and Philos. Soc.Manchester,February 24,1863; ferner Kenngott, Uebersicht, 1862165 , p. 44I. r864: W ö hle r: Gött. Gel. Anz. r864.

Ref. $\gg$ L'Institut $\propto$ Bd. 33(1865), p.14-15. I858/65: v. Reichenbach: IV 637. VI 456 . VII $558,561,562$. IX 156 , $160,163,172,180 . X 359,362$. XI $298,299,300,304,306$. XII 458. XIII 353,376 . XIV 395,396 . XXIV 229. XXV 607.

r865: Buchner: Zweiter Nachtrag, Pogg. Ann. Bd. 124, p. $573,576 .^{\circ}$ r867: Geinitz: N. J. 1867, p. 724 . r870: R a m melsberg: Meteoriten, p. IIo-III, $138,139$.

r875: Mo hr : Entstehungsart, Ann. Chem. Pharm. Bd. 179, p. 259, 271.

r876: $\mathrm{Sm}$ it h: Carbon compounds, Am. Journ. (3) II, p. 39I, $435(!), 438$, 439. Ref. N. J. 1877 , p. 871 .

r876: Wright: On the Gases contained in Meteorites. Am. Journ. (3) 12, p. 165.

1877: Flight: Meteorites and the origin of life. The Popular Science Review. 18. Okt. 1877 , p. $7-8$ (Sep.). 1879: R a m mels be rg : Meteoriten, p. 23,24 .

r882: W ie $\mathrm{ch} \mathrm{m}$ a n n: FusionStructures in Meteorites. Ann. N. Y. Acad. Sc., Bd. 2, p. $3 \mathrm{OI}-302$.

r884: M e uni e r: Météorites, p. 79, 89, $93,95,96,97,301-302,395$. r885: Brezina: Wiener Sammlung, p. $184,233$.
1894: Co he n: Meteoritenkunde, p. 8 , $154,156,158,160,162,165,166,167$, $222,235,236,245$.

r894: F 1 e t c her: Introduction, p. 36.

Ursprüngliches Gewicht: 6 Kgr. Zwei Steine von 4 u. 2 Kgr., von denen nicht viel erhalten sein dürfte.

Nachweisbares Gewicht: $266 \mathrm{gr}$.

\begin{tabular}{lr|lr} 
Bailey & I & London, P. G. & 2 \\
Bement & I & de Mauroy & Spl. \\
Berlin, U. & 22 & Moskau & 2 \\
Bologna & I & Neumann & I \\
Budapest & I & Paris, E. & 9 \\
Calcutta & 9 & Paris, M. & I54 \\
Cambridge & Spl. & Pohl & Spl. \\
Cleveland & Spl. & v. Siemaschko & 2 \\
Dorpat & 3 & Stockholm & 4 \\
Göttingen & I & Strassburg \\
Gregory & 5 & Troyes & I \\
Harvard, U. & 6 & Tübingen & 2 \\
Klausenburg & 6 & Washington, Sh. \\
Kopenhagen & I & Wien, H. M. *) \\
Kunz & 10 & Wien, U. II & Spl. \\
London, B. M. & I3 & Zirich & I
\end{tabular}

Alastoewa Djati Pengilon Alatyr Nowo Urei Albacher Mühle Bitburg Albany $1859 \quad$ Bethlehem Albareto, bei Modena, Italien.

Stein, Cc, gefallen Mitte Juli 1766 . 1766: Troili: Della caduta di un sasso dall' aria. Modena 1766. $4^{\circ}$. r766: Troili: Ragionamento della caduta di un sasso dall' aria. Modena 1766. r794: Chladni: Pallaseisen, p. 37. r803: Chladni: Chronologisches Verzeichnis, Gilb. Ann. Bd. 15, p. 3 ro. (Hinweis auf Vassalli: Lettere fisicometeorologiche, p. I20.)

1804: v. Ende: Massen und Steine, p. $44-46$.

r 804: Pöt z s c h : Kurze Darstellung, p.64. 1804: G i l b e r $t$ : Nachtrïge zu den Aufsätzen in den Annalen über die aus 
der Luft gefallenen Steine. Gilb. Ann Bd. 18 , p. 308 .

I812: $\mathrm{C}$ hla d n i: Verzeichnis, Schweigg. Journ. Bd. 4 Beil. I, p. 13.

1812: Bigot de Morogues, p. 102 $-103$.

1814: Dr. Chladni's vergebliche Bemühungen, verschiedene ältere Meteorsteine aufzufinden, nebst einigen ihn selbst betreffenden Nachrichten. Gilb. Ann. Bd. 47 , p. 101.

r815: Chladni: Neues Verzeichnis, Gilb. Ann. Bd. 5o, p. 249.

18r9: $\mathrm{Chladni}$ : Feuermeteore, p. 66, $69,73,82,250(1)$.

1836: K i m tz: Meteorologie, p. 274.

1857: A rago: Astronomie populaire, Bd. 4, p. 193.

1859: Harris: Dissert. Gött, p. 59-6o. 1863: Hai ding er: Der Meteorit von Albareto im K. K. Hof-MineralienKabinet vom Jahr 1766, und der Troilit. Sitzber. Wien. Akad. Bd, 47 II, p. $283-298$; s. auch Kenngott, Uebersicht, 1862/65, p. 438 ; Philos. Magaz. (4) 28 (1864), p. $327-328$; Liebig-Kopp, Jahresber. 1863, p. 905 ; "L'Institute Bd. 31 (1863), p. 272. r863: B u chner: Meteoriten, p. 7. r863: R o s e: Meteoriten, p. 39, 154. 1864: Buchner: Erster Nachtrag, Pogg. Ann. Bd. 122, p. 319.

1879: Maissen: Ricerche chimica sulla meteorite di Albareto Annuario dei naturalisti XII. Modena, Ref. Liebig-Kopp, Jahresber. I88o, p. I539; N. J. 1881 I, p. 199-200; Gazetta chimica X, p. 20.

r884: M e u n i e r: Météorites, p. 61, 268. r885: Brezina : Wiener Sammlung, p. $185,233$.

1887: F light: Meteorites, p. 16y-168. 1887: Brezina: Reisebericht, Ann.

Hof-Mus. Bd. 2 (Not.), p. 72.

1894: C o h e n : Meteoritenkunde, p. 186. r895: Brezina: Wiener Sammlung, p. 255 .

Ursprüngliches Gewicht: Ein grosser Stein (von 25 Pfund ?)

Nachweisbares Gewicht: II59 gr. Bailey

Berlin, U.

9 London, B. M. 53

Bologna

1 London, P. G. Spl.

v. Braun

56 Modena

Budapest

73 Paris, M.

697

Calcutta

Göttingen

Gregory

16 Rom

3 v. Siemaschko

Spl. Washington, Sh. I

53 Wien, H. M. *) 46

\section{Albuquerque}

Glorieta

Aldsworth, Cirencester, England. Stein, Cga, gefallen 4. August 1835 .

1854: Greg: Catalogue. Philos. Magaz.

(4) 8, p. 460 (giebt als Gewicht des Steins 2 lbs, an).

1857: Powe 11: Luminous Meteors, Rep. Brit. Assoc. (1857), p. 140 (dieser Fall soll schon in einem früheren Report erwähnt worden sein).

1859: H a rris: Dissert. Gött., p. 84 . 1863: B u chner: Meteoriten, p. 58. r865: v. Reichenbach: XXV 43I. 1884: M e unier: Météorites, p. 268, 270, 335 .

r885: Brez in a: Wiener Sammlung, p. 182,233

UrsprünglichesGewicht: Nach Greg 2 lbs (Powell sagt: 9270 grs.; das wären nur 574 gr.) ${ }^{\mathrm{t}}$ ).

Nachweisbares Gewicht: 572 gr.

\begin{tabular}{lr|lr} 
Bement & $2^{\prime}$ & London, B. M. & 525 \\
Calcutta & 4 & London, P. G. & 2 \\
Gregory & 4 & Paris, M. & I I \\
Harvard, U. & 9 & Wien, H. M. *) & 15
\end{tabular}

\section{Aleppo (Haleb), Kleinasien.}

Stein, Cwb, gefallen (?) 1873 .

I) B u c h n e r sagte 1863 , dass die Hauptmasse des Steins im Museum von Cirencester sei ; nach Herrn Direktor Fletcher's gütiger Mitteilung ist das zur Zeit nicht mehr der Fall. 
1893: B r e z in a: Ueber neuere Meteoriten (Nürnberg), p. 159 .

1893; Me unier: Sur deux météorites turques récemment parvenues au $\mathrm{Mu}-$ séum d'Histoire naturelle. C. R. Bd. II7, p. 257,258 . Ref. N. J. 1895 I, p. 276. 1895 : B r e z in a: Wiener Samml, p. 246. Nach Herrn Direktor Brezina's Mitteilung brachte Herr Mineralienhändler Dr. Eger im Jahr 1893 zwei Meteorsteine mit der Fundortsangabe $>$ Haleb $*$ und "Belgradiik « aus der Türkei mit. St. Meunier erhielt 1893 zwei Steine aus der Türkei mit der Ortsangabe $>$ Tirnowa « und surbaء. Der Stein von "Tirnowa « ist identisch mit dem von sHalebs oder Aleppo; „Urba a ist identisch mit Belgradiik oder Wirba.

UrsprünglichesGewicht: Etwa ${ }_{3} \mathrm{Kgr}$. Nachweisbares Gewicht: 2225 gr.

\begin{tabular}{lr|ll} 
v. Braun & 4 & Paris, M. & 28 \\
Eger & $\left.2100^{\prime}\right)$ & Wien, H. M. & 15 \\
London, B. M. $\quad 78$ & &
\end{tabular}

Alessandria, Piemont, Italien.

Stein, Cga, gefallen 2. Febr. 1860. 186r: M issaghi: Nuovo Cimento, Bd. 13, p. 272. (Pisa 1861). Ref. Kenngott, Uebersicht 1861, p. 164-165; Liebig-Kopp, Jahresber. 1862, p. 828829 ; Wochenschr. f. Astr. Met. u.Geogr. 1864, p. $77-78$.

1863: Schrauf: Ueber den Meteorit von Alessandria. Pogg. Ann. Bd. 118, p. $36 \mathrm{r}-363$ (Auszug aus der vorigen Arbeit). Ref. Kenngott, Uebersicht, $1862 / 65$, p. 436 ; N. J. 1863 , p. 589 ; Journ. prakt. Chem. Bd. 90 (1863), p. $\mathrm{II}_{3}-\mathrm{II}_{4}$.

1863: Buchner: Meteoriten, p. 103.
1863: Rose: Meteoriten, p. 155.

1875: T s c h er ma k: Vulkanismus, Sitzber. Wien. Akad. Bd. 71 II, p. 663 .

1879: R a mmelsberg: Meteoriten, p. 26.

r884: Me unier: Météorites, p. 247, 255 .

r884: Wa d sw orth: Studies, p. 104. r885: Brezina: Wiener Sammlung, p. 182,233 .

1894: F 1 e t c her: Introduction, p. 39. 1895: Brezina: Wiener Sammlung, p. 250 .

Ursprüngliches Gewicht: Es fielen einige Steine, von denen die Fragmente 0.3 bis r.o Kgr. wogen.

Nachweisbares Gewicht: 960 gr.

\begin{tabular}{lr|lr} 
Berlin, U. & I & Neumann & 4 \\
Bologna & 12 & Paris, E. & 25 \\
Budapest & 100 & Paris, M. & 52 \\
Calcutta & 2 & Pohl & 61 \\
Cleveland & I & Rom, U. & 52 \\
Dorpat & 40 & v. Siemaschko & Spl. \\
Harvard, U. & 9 & Stockholm & 2 \\
London, B. M. & 35 & Turin, J. & $230^{2}$ ) \\
London, P. G. Spl. & Turin, U. & 256 \\
Modena & Spl. & Wien, H. M. *) & 78
\end{tabular}

Alexander Co. s. Anhang Alexejewka Bachmut Alexinac Sokobanja Alfianello bei Pontefico u. Brescia, Cremona, Italien.

Stein, Ci, gefallen 16 . Februar $188_{3}$. r883: B o m b i c c i: Sull' Aerolito caduto presso Alfianello e Verolanuova (Provincia di Brescia); sulla causa delle detonazioni che accompagnano la caduta dei bolidi; e sulla costante presenza del ferro nelle meteoriti. Reale Accade-

1) Nach dem Stand vom 1. Juli 1893. Seitdem gelangten nach Mitteilung von Herrn Dr. Eger 879 gr. an Direktor Brezina; 477 gr. an Dr. Krenner in Budapest; 306 gr. an Excellenz v. Braun; 29 gr. an St. Meunier, so dass Herr Dr. Eger Januar 1896 noch $393 \mathrm{gr}$. besass ( $16 \mathrm{gr}$. giengen bei der Zerteilung verloren). Von den $879 \mathrm{gr}$. Brezina's erhielt das H. M. in Wien $556 \mathrm{gr}$, so dass dieses gegenwärtig $571 \mathrm{gr}$. besitzt. 2) Ausserdem noch ein Steinmeteorit von $67 \mathrm{gr}$. bezeichnet *Alessandria, Agosto $1856 \mathrm{c}$. 
min dei Lincei $1882 / 83$, p. II ; s. auch Mem. di Sc. fisiche Bd. 14, p. 675 -683 .

1883: Gallia: Meteorsteinfall bei Alfianello, unweit Brescia. Verh. k. k. geol. Reichsanstalt Nro. 6 (1883), p. 92 -93. Ref.. N. J. 1883 II, p. 188.

1883: Brezin a: Weitere Nachrichten über den Meteoriten von Afianello. Verh. k. k. geol. Reichsanstalt Nro. 6 (1883), p. 93-94. Ref. N. J. $188_{3}$ II, p. 188. 1883: Denza: Chute d'une météorite à Alfianello, territoire de Brescia (Italie). C. R. Bd. 96 , p. $805-808$. Ref. Liebig-Kopp, Jahresber. $188_{3}$, p. 1952 ; s. auch "Nature Bd. 27 , p. 464,5 r. 1883: Flight: Examination of the Meteorite which fell on the r6th February 1883 at Alfianello, in the District of Verolanova, in the Province of Brescia, Italy. Proc. Ruyal Soc. Bd. 35, p. 258-260 (Analyse); s, auch Geol, Magaz. Bd, Io (1883), p. $464-466$; vgl. auch w. u. 1887 Flight.

1883: v. F oull on: Ueber die mineralogische und chemische Zusammensetzung des am 16. Februar $188_{3}$ bei Alfianello gefallenenMeteorsteines. Sitz.ber. Wien. Akad. Bd. 88 I, p. 433 -443 .

1883: Ma issen: La meteorite di Alfianello. Gazetta chimica Bd. 13, p. 369 $-374,492$; s, auch Cavazzi : Memorie del Accademia della Scienza dell' Instituto di Bologna (4) 4, p. 613; Ref N. J. 1885 II, p. 27 I. Liebig-Kopp, Jahresber. $188_{3}$, p. 1952-1953.

1883: Tschermak: Beitrag, Sitzber.

Wien Akad. Bd. 88 I, p. $355,356$.

1884: Co hen: Referat über die Arbeiten von Bombicci, Denza, Flight und v. Foullon, N. J. 1884 II, p. 30-32; s. auch Liebig-Kopp, Jahresber. $188_{3}$, p. $1952-1954$.

r884: M e unier: Météorites, p. 209, $226-227,46 \mathrm{r}, 497-498$.

1884: W a d s w or th: Studies, p. 102.

I $88_{3} / 85$ : T s ch erm a k: Photographien,

T. II, 17, p. 12, 13, 16, 18 .

1885: Brezina: Wiener Sammlung, p. 168, 169, 181, 232.

r885: S hepard: Am. Journ. (2) 30, p. 106 (Krystall von Nickeleisen).

r887: Brezina: Reisebericht, Ann. Hof-Mus. Bd, 2 (Not.), p. 108.

I887: F li g h t: Meteorites, p. 22I-223. 1888: Fried he i m: Ueber die chemische Zusammensetzung der Meteoriten von Alfianello u. Concepcion. Sitzber. Berlin.Akad.1888. Erster Halbbd.,p.345 -363 . Ref. N. J. 1889 II, p. $278-279$. r89o: v. Ni essl: Periheldistanzen, Verh. naturf. Ver. Brünn Bd. 29, p. 193.

1893: Brezin a: Ueber neuere Meteoriten (Nürnberg), p. 159.

1894: C o h e n : Meteoritenkunde, p. 57, $112,205,268,312,313$.

Ursprüngliches Gewicht : Nicht viel unter $200 \mathrm{Kgr}$. (der ganze Stein mochte $260 \mathrm{Kgr}$. gewogen haben).

Nachweisbares Gewicht: 54866 gr.

\begin{tabular}{lr|lr} 
Bailey & 205 & Frankfurt & 39 \\
Bayet & 4 & Graz & 76 \\
Bement & 1362 & Gregory & 4269 \\
Berlin, G. & 14 & Greifswald & 38 \\
Berlin, U. & 12759 & Garvard, U. & 738 \\
Blatz & 38 & Gowell & 5 \\
Böhm & 21 & Karlsruhe, M. & 34 \\
Bologna & 1057 & Kasan & 124 \\
Bonn & 22 & Klausenburg & 236 \\
v. Braun & 413 & Kopenhagen & 1540 \\
Breslau & 57 & Krantz & 600 \\
Budapest & 5014 & Kristiania & 39 \\
Calcutta & 30 & Lausanne & 9 \\
Cambridge & 48 & Lissabon & 81 \\
Catania & 393 & London, B. M. & 2515 \\
Cleveland & 40 & Lüttich & 67 \\
Darmstadt, M. & 105 & Madrid & 8 \\
Dorpat & 71 & de Mauroy & 97 \\
Dresden, M. & 74 & Meli & 2500 \\
Dublin, M. & 165 & Melion & 6 \\
Dublin, R. C. & 122 & Modena & 53 \\
Eger & 1354 & Moskau & 71 \\
& & &
\end{tabular}




\begin{tabular}{lr|lr} 
Neapel & 76 & Strassburg & 142 \\
Neumann & 49 & Strüver & 1150 \\
New Haven & 350 & Stürtz & 1317 \\
Newton & 7 & Stuttgart & 48 \\
Odessa & 117 & Troyes & 117 \\
Paris, E. & 207 & Turin, J. & 84 \\
Paris, M. & 1000 & Turin, U. & 619 \\
Parma & 134 & Upsala & 84 \\
Pech & 3080 & Utrecht & 43 \\
Petersburg, B. & 115 & Ward & 158 \\
Pohl & 29 & Washington & 61 \\
Riga & 89 & Washington, Sh. 29 \\
Rom, U. & 7620 & Wien, H, M. & $274^{1}$ ) \\
Sidney & 117 & Wien, U. I. & 140 \\
v. Siemaschko & 718 & Würzburg & 53 \\
Stockholm & 326 & &
\end{tabular}

Allahabad $18 \mathrm{r} 4$

Allahabad 1822

Alleghany Co.

Alleghany Mts.

Allen Co.

Altenburg

Amana

Amates

Ameca-Ameca

Amerika

Anderson

Angara

Angers, DepartementMaine etLoire, Frankreich.

Stein, Cwa, gefallen 3. Juni 1822 . I822: Gilbert: Nachrichten von dem am 3. Juni 1822 zu Angers herabgefallenen Meteorsteine ; frei übertragen und mit einer Nachschrift von Gilbert. - I. Aus einem Schreiben des Hrn. Desvaux, Conservateur des naturhistor. Mus. zu Angers, an d. Präs. des National-Inst, in Paris. - 2. Aus einem Brief des Herrn Boisgiraud des Aelteren, Prof. der physik. Wissensch. am königl. Colleg zu Poitiers, an Herrn

r) Gegenwärtig 652 gr.
Arago. - 3. Nachschrift von Gilbert, Gilb. Ann. Bd. 71, p. 345-353.

1822: Chladni: Zweiter Nachtrag, Gilb. Ann. Bd, 71, p. 361 .

1823: C h la d ni: Dritter Nachtrag, Gilb. Ann. Bd. 75 , p. 241.

r836: Kämtz: Meteorologie, p. 290 -291 .

1843: P a rt s ch: Meteoriten, p. I42.

1845: v. B a u m h a u er : Ursprung, Pogg. Ann. Bd. 66, p. 476 .

1854: $\mathrm{B}$ a 1 c e $11 \mathrm{~s}$ : Lithologia meteorica, p. 24 .

1854: v. Boguslawski: Zehnter Nachtrag, Pogg. Ann. Ergz.-Bd. 4, p. 22.

I859: B u c h n er: Feuermeteore, p. 88. r859: H a rris : Dissert. Gött., p. 77. r863: B u c hner: Meteoriten, p. 44. 1865: v. Reichenbach: XXV 321. 1884: Me unier: Météorites, p. 208, 214.

1885: Brezina: Wiener Sammlung, p. $177,232$.

r889: Me un ier: Sur la matière noire de la Chantonnite Bull. Soc. Franc. Min. Bd. 12, p. $76-8$ I.

r895: Brezina: Wiener Sammlung, p. $242,243$.

UrsprünglichesGewicht: Etwa r Kgr. Nachweisbares Gewicht: 149 gr.

\begin{tabular}{lr|lr} 
Bailey & I9 & v. Siemaschko & I \\
Calcutta & Spl. & Tübingen & Spl. \\
Gregory & 24 & Washington, Sh. & I \\
London, B. M. & 22 & Wien, H, M. *) & 2 \\
Paris, M. & 80 &
\end{tabular}

Angra dos Reis, Provinz Rio, Brasilien.

Stein, Angrit, gefallen 2. Hälfte Januar 1869 .

1885: Ts chermak übersendet eine Notiz über den Meteoriten von Angra dos Reis in Brasilien. Sitzber. Wien. Akad, Bd, 92 I, p. 1 o. 
1887: Ludwig und Tschermak: Der Meteorit von Angra dos Reis. M. P. M. Bd. 8 , p. $34 \mathrm{I}-355$, Ref. N. J. 1888 I, p. $47-48$, auch Ztschr. f. Kryst. Bd, 17 (1890), p. 206-208. 1888: T s c h e r m a k: Beiläufige Angabe der Fallzeit des Meteoriten von Angra, M. P. M. Bd. 9, p. 423. Ref. N. J. 1889 I, p. 239, auch Am. Journ. (3) 36 (1888), p. 157.

1893: Brezina: Ueber neuere Meteoriten (Nürnberg.), p, 158.

r894: C o h e n : Meteoritenkunde, p. 29, $49,203,259,268,295,296,297$.

1894: Fletcher: Introduction, p. 37.

1895: Brezina: Wiener Sammlung, p. 239.

UrsprünglichesGewicht: $\mathrm{I}^{1 / 2}-2 \mathrm{Kgr}$. Nachweisbares Gewicht : 397 gr.

Berlin, U.

Böhm $\quad 8 \quad 446$ gr., jetzt 344

Budapest $\quad 6$ v. Siemaschko 4

Gregory $\quad 6$ Strassburg 4

London, B. M. 6 Washington 9

Paris, M. 3 Wien, H. M. 5

Das grössere der Bruchstücke kam nach Rio, ein kleineres behielt der Schwiegervater des Dr. Travassos, Arzt in Rio. Ein Stück soll an Seine Heiligkeit den Papst nach Rom geschickt worden sein ; meine Bemïhungen hierüber Auskunft zu erhalten, waren vergeblich,

\section{Antifona}

Collescipoli

Antofogasta, Eisen, 1876

Mantos Blancos

Antofogasta, Pallasit, 1876

San Cristobal, s. Anhang

Apt

Saurette

Arizona 1851

Tucson

Arizona 1867 (Tucker's A.)

Krasnojarsk
Arizona $189 \mathrm{r}$

Arva

Arve

Asco, Insel Corsica.

Stein, Cwa, gefallen November 1805 .

r843: P a rts ch: Meteoriten, p. 64.

1854: v. Boguslawski: Zehnter

Nachtrag, Pogg. Ann. Ergz.-Bd. 4, p. II.

1859 : Harr is : Dissert. Gött., p. 66.

1859: v. Reichen ba ch: IX 162, 163, $164,169,178$.

1863: B u c hner: Meteoriten, p. 19.

1863: Rose: Meteoriten, p. 155.

r885: Brezina: Wiener Sammlung, p. $180,232$.

Ursprüngliches Gewicht: ? (Nach Partsch soll der Stein in einer Kirche aufbewahrt werden.)

Nachweisbares Gewicht: $42 \mathrm{gr}$.

Berlin, U.

v. Braun

6 Calcutta

Budapest

5 v Siemaschko $12^{\prime}$ )

Spl. Wien, H. M.*) 18

Ashville, beschr. 1839 Bairds-Farm

Ashville gefd. 1839 , beschr. 1847

Black Mountain

Ashville 1854 Jewell Hill

Asien s. Anhang

Assam, Ostindien. Nur die Provinz, kein näherer Fundort bekannt. Stein, Cgb, gefunden 1846 .

1846: Piddington: Kurze Ankündigung, dass er in the Coal Committee's Collection einen Meteoriten gefunden habe. Journ. Asiatic Soc. Bengal. Bd. 15 (Proc.), Juni 1846, p. XLVI.

1859: A $\mathrm{t} k$ inson: In einem Bericht über die Meteoritensammlung der Gesellschaft wird auch der Stein von Assam erwähnt. Journ. Asiatic. Soc. Bengal. Bd.28(Proc.), Juni 1859, p. 260.

I) Nach Herrn Direktor Brezina's Mitteilung soll dieses Stïck Siemaschko's, der dasselbe von Herrn v. Braun erhalten habe, nicht mit dem Wiener Stück übereinstimmen. 
r860: $\mathrm{H}$ ai d ing e r: Die Calcutta-Meteoriten, von Shalka, Futtehpore, Pegu, Assam und Segowlee im k. k. HofMineralien-Kabinete. Sitzber. Wien. Akad. Bd. 4I, p. 752-753. Ref. Am. Journ. (2) 32 (186I), p. 143; Luminous Meteors: Rep. Brit. Assoc. 186r (Sep.), p. 34 ; sL'Institut ๔ $\mathrm{Bd} .28$ (1860), p. 423.

r860: H a id in ge r : > Es gelang nicht, ausführlichere Nachrichten über den Meteoriten von Assam aufzufinden.* Sitzber. Wien. Akad. Bd. 42, p. 306. 1863: Buc hner: Meteoriten, p. II5 $-116$.

I865: v. Reichenbach: $\mathrm{XXV}_{422}$ $427,428,430$.

r869: Buchner : Vierter Nachtrag, Pogg. Ann. Bd. 136, p. 449.

1869: v. H a i d ing e r: Hessle, Rutlam, Assam, drei neue Meteoriten. Sitzber. Wien. Akad, Bd. 59 II, p. 229.

r884: M e un i e r : Météorites, p. 22, 260 (Abb.) 26r, 381 .

1885: Brezina: Wiener Sammlung, p. $182,233$.

r894: F let cher: Introduction, p. 10. Ursprüngliches Gewicht: In der Notiz von 1859 heisst es:

ein Bruchstück I lb. $8^{3} / 4$ oz.

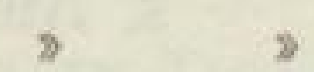

$83 / 4$ oz.

3 y

$7^{1 / 2}$ oz.

Haidinger sagt i 860: $\gg$ DreiBruch. stücke im Gewichte von I Pfund $17^{1 / 2}$ Loth, $17^{1 / 2}$ Loth u. I 5 Loth, übersetzt also einfach die eng. lischen lbs $=453.6 \mathrm{gr}$. in die österreichischenPfunde (von $560 \mathrm{gr}$ ). In der Arbeit von 1869 sagt er, dass Dr. Oldham in seinem Verzeichnis der Meteoriten in dem Museum in Calcutta für Dezember 1867 (p. 8) sAssam (history unknown) 5 Pds. 13 oz. 2.5 grs 4 angebe, was auch in der That richtig ist, aber nicht leicht mit den frïheren Angaben in Einklang gebracht werden kann. Buchner übersetzt die Gewichtsangaben Haidinger's (das Pfund zu $560 \mathrm{gr}$. gerechnet) in Gramme u. sagt: ses waren drei Bruchstücke (866g., 306 gr., 262 gr.). « Nimmt man die älteste Angabe als die der Wahrheit am nächsten kommende und das englische lb. zu 453,6 gr. = r6 Unzen, so erhält man : 702 gr., $24^{8}$ gr. u. 213 gr., in Summa: I 63 gr., was mit der nachweisbaren Menge nicht übereinstimmt. Nachweisbares Gewicht: 1247 gr.

Budapest

Calcutta

I Paris, M. 200 v. Siemaschko

Göttingen Spl. Washington, Sh. 7

London, B. M. 539 Wien, H. M.*) 188

London, P. G. 8

Assisi (Torre Assisi, auch Torre d'Andrea), Perugia, Italien.

Stein, Cc, gefallen 24. Mai 1886.

1887: B e 11 u c c i: Il meteorite di Assisi, Perugia. Tipografia di Vinçenzo Santucci. Perugia 1887. 8 Seiten.

r889: v. H a u e r: Ann. Hof-Mus. Bd, 4 (Not.), p. 64.

r89r: Terrenzi : Notizie intorno agli Aeroliti caduti nell' Umbria. Rivista Scientifico-Industriale di Firenze. (Sep.). Oktober 1891.

r89r: v. H a u e r: Ann. Hof-Mus, Bd, 6 (Not.), p. 54 .

1895: Brezina: Wiener Sammlung, p. 256.

Ursprüngliches Gewicht : I 795 gr.; ein Stein.

Nachweisbares Gewicht: ${ }^{1} 55^{1}$ gr. Bailey 3 Budapest 275

Bellucci $\quad 155$ Cleveland 5

Berlin, U. 23 Dresden, M. 40

\begin{tabular}{ll|l|l} 
v. Braun & 98 & Eger & 25
\end{tabular} 
Gregory

69 Rom, U. $\quad 126$

Harvard, U. 3 v. Siemaschko 6i

London, B. M. $\quad$ I52 Turin, U. 227 de Mauroy $\quad 2$ Washington, Sh. 29 Paris, M.

Io8 Wien, H. M. 150

Atacama Bolivia 1858 Joël Eisen

Atacama $\gg$ Daubrée's localité non indiquée "

Dehesa

Atacama Pallasit

Imilac

Atacama 1874

Cachiyuyal

Atacama Wüste, Eisen 1870 Ilimaë Atacama Wüste, Stein 1860

Lutschaunig

Atacama (ohne nähere Bez.) s. Anh.

Aubres, Bezirk Nyons, Departement

Drôme, Frankreich.

Stein, Bu, gefallen 14 . Sept. $18_{3} 6$. 1887: Gregory: Two new French Meteorites, Geol. Magaz. (3) IV, Nr. I2. Ref. N. J. 1889 I, p. 6 o.

1892: v. H a u e r: Ann. Hof-Mus. Bd. 7 (Not.), p. 73 .

1893: B r e z in a: Ueber neuere Meteoriten (Nürnberg), p. 159.

1895: Brezina: Wiener Sammlung, p. 239 .

Ursprüngliches Gewicht: $567 \mathrm{gr}$. (Der ganze Stein mochte wohl 800 gr. gewogen haben.)

Nachweisbares Gewicht: 528 gr. Gregory $\quad 23$ Paris, M.

London, B. M. 488 Wien, H. M. 8

Auburn, Macon Co.,Alabama,U.S.A. Eisen, H., gefunden 1867 .

1869: S hepard: Notices of New Meteoric Irons in the United States. 1) Meteoric Iron from Auburn, Macon county, Alabama. Am. Journ. (2) 47 , p. $230-233$ (Analyse). Ref. LiebigKopp, Jahresber. 1869, p. 1301-1302 s. auch: beiläufige Erwähnung in Smith's Arbeit über Franklin Co. (Frankfort, Eisen): On the presence of
Cobalt in Meteoric Irons. Am. Journ. (2) 49 (1870), p. 33I ; s. auch Original Researches 1884 , p. 447.

1884: M e unier: Météorites, p. 137. r885: Brezina: Wiener Sammlung, p. $218,234$.

I887: F lig h t: Meteorites, p. 164-165. 1890: E a s t m an: Met. Astron., p. 320. r89r: Cohen und Weinschenk: Meteoreisen-Studien. Ann. Hof-Mus. Bd. 6, p. 160.

r893: M e un i e r : Révision des fers météoriques, p. 75 .

r895: C o h e n : Meteoreisen-Studien IV, Ann. Hof-Mus, Bd. ro, p. 88.

Ursprüngliches Gewicht: $3^{1 / 2} \mathrm{Kgr}$. (8 lbs; die Hälfte gelangte in Shepard's Besitz.)

Nachweisbares Gewicht: 791 gr. Bailey

Bement

Berlin, U.

Bologna

v. Braun

Budapest

Cleveland I9 Minneapolis 25 22 Moskau 6 17 Newton 2 I8 Paris, M. 53 13 v. Siemaschko 7 20 Stockholm 43

Dresden, M. 9 Upsala 32 \begin{tabular}{ll|l} 
Göttingen & $\mathbf{2 7}$ & Ward
\end{tabular}

Gregory $\quad 8$ Washington 29 \begin{tabular}{ll|l} 
Kopenhagen & 28 & Washington,Sh. 228
\end{tabular} London, B. M. 37 Wien, H. M. *) 18 London, P. G. $\quad 23$

Augusta Co.

Staunton

Augustinowka, Gouvernement Ekaterinoslaw, Russland.

Eisen, gefunden 1890 im Löss, in einer Tiefe von 3 Meter ( 4 Klafter lautet eine andere briefliche Mitteilung); beschrieben 1893 .

1893: Alexejew: Ueber den Meteorit aus dem Dorfe Awgustinowka, Gouvernement Jekaterinoslaw. Verh. russ, min. Ges. Bd. 2, p. 30 und 470 (russisch) (Analyse).

r893: Me unier: Sur le fer météori- 
que d'Augustinowka (Russie). C. R. Bd. II6, p. II5I-II53, (Analyse). Ref. N. J. I894 I, p. 449.

r893: M e u ni e r : Révision des fers météoriques, p. 76.

Ursprüngliches Gewicht: $400 \mathrm{Kgr}$. (25 Pud).

Nachweisbares Gewicht: $360 \mathrm{Kgr}$., der Rest oxydierte Rinde und Schreibersit.

Petersburg, B. besitzt noch (März 1896) die ganze Masse, nur kleine Stücke der Rinde wurden nach Herrn Bergingenieur Melnikoffs gütiger Mitteilung an 3 oder 4 Sammlungen abgegeben. Paris besitzt $43 \mathrm{gr}$, Odessa $65 \mathrm{gr}$.

Anm.: Brezina vereinigt diese Lokalität mit Werchne-Dnieprowsk. Herr Professor Prendel(Odessa) hatte die Freundlichkeit mir mitzuteilen, dass Augustinowka und Werchne-Dnieprowsk zwei verschiedene Eisen und die beiden Lokalitäten sehr entfernt von einander seien. Herr Melnikoff spricht sich in gleichem Sinne aus.

\section{Aukoma}

Pillistfer

Aumale Senhadja

Aumières, Canton Massegros, Departement Lozère, Frankreich. Stein, Cwa, gefallen 3 . Juni $184^{2}$ ). 1842: de Malbos: Sur un Aérolithe tombé aux environs de Berrias (Lozère). - Extrait d'une Lettre de M. J. de Malbos à M. Elie de Beaumont. C. R. Bd. I4, p. $917-918$.

1842: de Mondesir: Sur un météore lumineux observé le 3 juin à Mende (Lozère). - Extrait d'une Lettre de M. P. de Mondesir, ingénieur des Pontset-Chaussées à M. Arago. C. R. Bd. 14, p. $918-919$.

1842; Deydier: Sur un météore lumineux observé, le 3 juin, à SaintBeauzire (Haute-Loire). Extrait d'une Lettre de M. Deydier à M. Arago. C. R. Bd. 14, p. 919-920; s. auch Pogg. Ann. Bd. 56 (1842), p. 644 ; L'Institut « Bd. Io $(1842)$, p. 214 .

1843: Petit: Sur la hauteur et la vitesse du météore lumineux du 3 juin 1842. C. R. Bd. I6, p. $485-489$.

1851 : B o is s e: Recherches sur l'histoire et la nature des Aerolithes. Rodez.

1854: v. Boguslawski: Zehnter Nachtrag, Pogg. Ann. Erg.-Bd. 4, p. 95. r863: B uc hner: Meteoriten, p. 68. 1858/65: v. Reichen b a c h: V 480. VI 456 . IX 16r, J68, r78. X $365 . \mathrm{XI}$ 295. XIII 380. XX 626. XXV 607. 1884: M e un ier: Météorites, p. 95, 97, $208,217,378,485,486$.

r885: Brezina: Wiener Sammlung, p. $180,232$.

r887: F lig h t: Meteorites, p. 110-112 (Abb.).

Ursprüngliches Gewicht : Die Hauptmasse des ganzen Falles ist in Paris. Nachweisbares Gewicht: ${ }^{1556}$ gr. Bailey Bologna $\quad 85$ Paris, M. $\left.1382^{2}\right)$ Calcutta 2 v Siemaschko 17 Harvard, U. 4 Troyes Spl. London, B. M. 43 Washington,Sh. Spl. London, P. G. I Wien, H. M. *) 5

Aussun (Clarac), Montréjeau, Depart. Haute Garonne, Frankreich. Stein,Cc, gefallen 9. Dezember 1858 . 1858: Petit: Sur l'aérolithe du 9 décembre. (Lettre de M. Petit à M. Élie de Beaumont). C. R. Bd. 47, p. 1053I055; s. auch sL'Institute B. 26 (1858),

1) de Malbos, de Mondesir u. Deydier geben den 3. Juni als den Tag an, an welchem sie das Meteor 9 h p. m. gesehen und gehört haben; es ist hiernach das allgemein verbreitete Datum des 4. Juni in den 3. Juni umzuändern.

2) Durch Vermittlung des Herrn Boisse aus dem Museum von Rodez erhalten. 
p. 425; Kenngott, Uebersicht, 1859 , p. 165; Liebig-Kopp, Jahresber. 1858,

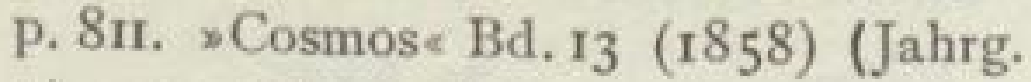
7), p. $727-728$.

r859: H a rris : Dissert. Gött., p. 4551. (Analyse) und P. I0o; s, auch Ann. Chem. Pharm. Bd. Io (1859), p. I8I, 184; Chem. Centr. 1859 , p. 565 -567 ; Kenngott, Uebersicht, 1859, p. $170-171$.

1859: Angelot donne une communication de l'extrait suivant d'une lettre qui lui a été adressée, le 26 décembre 1858 , par M. ie maire de Clarac (Haute-Garonne). Bull. Soc. Géol. Bd, 16, p. 207-208. 1859: Elie de Beaumont: (Vorzeigen einiger Stücke) C. R. Bd. 48 , p. 16, (Biot schlägt eine Kommission für die Untersuchung vor); s. auch sL'Institute Bd, 27 (1859, p. 1, 9.

1859: Fil ho 1 und Leymerie: Note sur l'aérolithe de Montrejeau. C. R. Bd. 48, p. 193- 198 (Analyse). Ref. Kenngott, Uebersicht, 1859, p. 171.

1859: $\mathrm{Chancel}$ et $\mathrm{Moit}$ ossier: Note sur la composition chimique et minéralogique de l'aérolithe de Montrejeau, tombé le 9 décembre 1858 . C. R. Bd. 48 , p. $267-269$. Ref. Kenngott, Uebersicht, 1859 , p. 166 ; Journ. prakt. Chem. Bd. 77 (1859), p. 498 -499 .

1859: Fi 1 ho 1 : Supplément à une précédente note sur l'analyse de l'aérolithe de Montrejeau. C. R. Bd. 48 , p. 348-349. (Nene Analyse); s, auch sL'Institut * Bd. 27, 1859, p. 26, 6r u. kurze Angabe in Wochenschr. f. Astr., Met. u. Geogr. 1859 , p. 56.

1859: Leymerie: Etude d'une des parties constituantes de l'aérolithe de Montrejeau. C. R. Bd. 48 , p. $446-$ 447.

1859: Cbancel et Moitessier: Deuxième Note sur la composition chimique et minéralogique de l'aérolithe de Montrejeau, C. R. Bd. 48 , p. 479
-481. (Neue Analyse).

1859: L a r oque und Bi an ch i: Note sur l'aérolithe de Montrejeau (propriétés magnétiques). C. R. Bd.48, p. $578-579$.

r859: Lar oque und Bianchi: Propriétés magnétiques de l'émail que donnent le pyroxène et le péridot par l'action de la chaleur: addition à une précédente note sur les propriétés magnétiques de l'aérolithe de Montrejeau. C. R. Bd. 48 , p. $798-799$, Fortsetzung, p. $920-921$.

r859: v. Reichen b a c h: Notiz über den Meteoriten von Clarac. Pogg. Ann. Bd. 107, p. 191-192. Ref. N. J. 1860, p. 82 .

r859: Haidinger: Notiz über den Meteorit von Aussun im k, k. Hof-Mineralien-Cabinete. Sitzber. Wien. Akad. Bd. 34, p. $265-267$.

r859: D a m o ur: Recherches chimiques et analyses sur l'aérolithe de Montrejeau. C. R. Bd. 49 , p. $3 \mathrm{I}-36$. (Neue Analyse) ; s, auch »L'Institut « Bd, 27 (I859), p. 213-2I 4 .

1859: Le y merie: Sur l'aérolithe de Montrejeau; rémarques présentées à l'occasion d'une communication récente. C. R. Bd. 49 , p. $247-248$; s. auch Act. Soc. Linn, de Bordeaux Bd, 23 (1860), p. 5I ; "L'Institut" Bd. 27 (1859), p. 266.

1859: Referate über die Arbeiten von Filhol, Leymerie, Chancel, Moitessier, Laroque, Bianchi und Damour in Liebig-Kopp, Jahresber. 1859 , p. 850 - 853 ; s. auch kurzes Ref, in der Revista minera. Madrid Bd.1o (1859), p.94. 1859: G ir a rd: zeigte ein ansehnliches Stïck eines am 9. Dez. v. J. südwestlich von Toulouse gefallenen Meteorsteins, welches derselbe für das hiesige Mineral. Museum erworben. Abh. Naturf. Ges. Halle Bd. 5 (Sitzber.), p. 5. 1859: Bu ch ner: Feuermeteore, p. $18_{3}$. 1860: $\mathrm{R}$ a m mels berg : Mineralchemie, p. $923,950$. 
1863: Buchner: Meteoriten, p. 99 - IOI.

I863: R o s e: Meteoriten, p. $85,87,98$, 99, I06, 155 .

$186_{3} / 6_{4}$ : So rb y: On the Microscopical Structure of Meteorites. Proc. Royal Soc. Bd. 13, p. 333, 334. Ref. Am. Journ. (2) 4I (1866), p. 137.

r859/65: v. Reichenbach: IX 16r, 169, 179. X 371. XI 295, 297, 298, 306. XIII $360,375,378$. XIX 152, 153. XX 623. XXIII 368. XXV 606, 607,615 .

1869: B u chner: Vierter Nachtrag, Pogg. Ann. Bd. 136, p. $446-447$.

1870: $\mathrm{R}$ a m mels berg: Meteoriten, p. 103, 105, 106, 139, 140.

r875: vom R a th: Meteoriten, Verh. naturh. Ver. Bonn. Bd. 32, p. 368 .

1876: Wright: On the Gases contained in Meteorites. Am. Journ. (3) 12, p. 171 .

1879: Rammelsberg: Meteoriten, p. 24,25 .

1883: T s c h erm a k: Beitrag, Sitzber.

Wien. Akad. Bd. 88 I, p. 355 .

I884: W a ds w ort h: Studies, p. ro4. 1884: M eunier: Météorites, p. 79,

$84,85,97,230$ (Abb.), 232-233, 349, 37o, 395, $45^{8}$ (Abb.), 522.

1883/85: Tschermak: Photographien, p. $3,18$.

r885: Brezina: Wiener Sammlung, p. $185,233$.

r885: Shepard: Am. Journ. (3) 30, p. 106 (Krystall von Nickeleisen).

r894: Coh en: Meteoritenkunde, p. 57 , $85,174,248,249,252,266,267,283$. r895: B rezina : Wiener Sammlung, p. 255 .

UrsprünglichesGewicht: $48-55 \mathrm{Kgr}$. Zwei Steine von 8-ro und 40 -
45 Kgr. Viel verschleppt.

Nachweisbares Gewicht: $95^{27}$ gr.

Bailey

Belgrad 2I Kunz

22

Bement

I4 London, B. M. 477

Berlin, U.

Böhm

Bologna

Bonn

v. Braun

Brezina

Budapest

Calcutta

Cambridge

Cleveland

Cohen

Dorpat

Dresden, M.

Edinburg

Gent

Göttingen

Gregory

Halle

Hamburg

Harvard, U.

Heidelberg

Klausenburg

Kopenhagen

I3 London, P. G. 422

546 Lüttich

4 Madrid

106 de Mauroy

13 Melion

45 Modena

20 Moskau

187 New Haven

378 Paris, E.

17 Paris, M.

2 Pohl

3 I Rom, U.

12 Sidney

27

3

40

2

17

86

I8

III

\section{3}

122

33

107 v. Siemaschko 163

I23I Stockholm 277

53 Strassburg $\quad 46 \mathrm{I}$

95 Troyes 8

262 Tühingen 493

50 Turin, U. $\quad 185$

4 Ward $\quad 5^{8}$

3I4 Washington, Sh. 20

8 Wien, H. M. *)**) 17

1038

r50 Wien, U. II. 34

Ausserdem besitzt La Faculté des Sciences in Bordeaux ein Stück dieses Falles, wie Herr Fallot mir mitteilte. Seit Juli 1893 erwarb Herr Bayet, Brüssel, n6 gr.

Austin 1856

Denton Co

Austin 1856

Australien 1825

Wichita Co

Australien

Honolulu

Authon

s. Anhang

Lancé

Avilez, bei Cuençamé, Durango, Mexico.

Stein, $\left.\mathrm{Cc}-\mathrm{Cg}^{1}\right)$, gefallen Juni $185^{2} 6^{2}$ ).

1) Der Stein steht auf der Grenze von $\mathrm{Cc} u . \mathrm{Cg}$, näher $\mathrm{Cc}$; s. auch: Bjelaja-Zerkow, Motta di Conti, Mühlau.

2) Der Fallmonat wurde Castillo entnommen; die Angaben über das Jahr des Falles schwanken zwischen 1855 u. 1856 (abgesehen von mehrfachen Druckfehlern). 
1867: W ö hl e r : Notiz über einen Meteorstein aus Mexico. Gött. Gel. Anz. (Nachr.) (1867), p. $57-58$.

r869: Buchner: Vierter Nachtrag, Pogg. Ann. Bd. 136, p. $450-451$. 1870: B urkart: Fundorte IV, N. J. 1870 , p. $683-684,689$.

r884: H ä p k e : Beiträge, Abh, naturw. Ver. Bremen. Bd. 8, p. 515-516.

1885: Brezina: Wiener Sammlung, p. $182,232$.

1887: F $1 \mathrm{ig} \mathrm{ht:} \mathrm{Meteorites,} \mathrm{p.} 92$. 1887: Brez in a: Neue Meteoriten III. Ann. Hof-Mus, Bd. 2 (Not.), p. 115. 1889: C a stillo: Catalogue, p. 13. r89o: F l e t c her: Mexican Meteorites, Mineral. Magaz. Bd. 9, p. 95.

r895: Brezin a: Wiener Sammlung. p. 249,255 .

Ursprüngliches Gewicht : ?

Nachweisbares Gewicht: 236 gr.

\begin{tabular}{ll|lr|} 
Breslau & Io & Göttingen & I42 \\
Budapest & 8r & Wien, H. M. *) & 3
\end{tabular}

Babb's Mill (Green County), Tennessee, U. S. A.

Eisen, Db, bekannt 1842 ; beschr. I845; die grosse Wiener Masse bekannt seit 1876 .

1845: Troost: Description ..... of a mass discovered in Green County, Tenn. Am. Journ. (r) 49, p. $342-344$; s. auch Am. Journ. (2) 5 p. $35^{1}$ (beiläufige Erwähnung).

1847: Shepard: Report on Meteorites. Am. Journ. (2) 4 , p. 76-77 (Abb.). Ref. Liebig-Kopp, Jahresber., $1847 / 48$, p. 1309 u. Kenngott, Uebersicht, $1850 / 5^{1}$, p. 135 .

r852: C 1 a r k: Dissert. Gött., p. 65-66. r852: Wö hler: Passiv, Pogg. Ann. Bd. 85 , p. 448,449 .

1854: v.B o g usl a ws k i : Zehnter Nachtrag, Pogg. Ann. Ergz.-Bd, 4, p. 400. r859: B u c h n e r: Feuermeteore, p.r36. 1859: H a r ris: Dissert. Gött. p. I4.
1860: $\mathrm{R}$ a m mels berg: Mineralchemie, p. $916,946$.

r859/62: v. Reichen bach: IX r76, 177, 182. X 359, 363. XI 291. XIII 354. XV 100. XVII 267, 268, 269, 273. XVIII 488. XIX 151.

1863: Bu chner: Meteoriten, p. 172. r863: Ros e: Meteoriten, p. 26, $72-$ 73, 153 .

1867: Go e b e 1: Kritische Uebersicht, Mélanges phys. chim. Bd. 7, p. 325 . 1870: R a m me 1s berg: Meteoriten, p. 79 .

1880: Brezina: Bericht I, Sitzber. Wien. Akad. Bd. 82 I, p. 35 I.

r 884: M e un i e r: Météorites S, I16, I22. r885: Brezina: Wiener Sammlung, p. 200, 20I, 203, 219, 234.

1886: Brezina: Neue Meteoriten I, Ann. Hof-Mus. Bd. 1 (Not.), p. 12, 25. r886: H u n t ing to n : Crystalline Structure, Am. Journ. (3) 32, p. $285,298$. r886: B lake: Description of a Meteorite from Green County, Tennessee. Am. Journ. (3) 31 p. 4I-46 (Abbildung). (Dieses Stück jetzt in Wien). Ref. N. J. 1887 II, p. 48 ; LiebigKopp, Jahresber. 1886, p. 2329-2330. r887 : v. Ha uer: Ann. Hof-Mus. Bd. 2 (Not.), p. $3^{8}$.

r89o: Eas tm a n : Met. Astron., p. 318, 322.

1890: Brezin a: Ueber Meteoreisen. Oesterr. Zeitschr. f. Berg- u, Hüttenw. Bd. 38, p. $35^{8}$.

r89r: Cohen und Weinschenk: Meteoreisen-Studien, Ann. Hof-Mus. Bd. 6, p. 131, 132, 142-143 (Analyse), 160.

r892: Cohen: Meteoreisen-Studien, Ann. Hof-Mus. Bd. 7 , p. $147-148$ (Analyse), 162.

1893: M e un i er: Révision des fers météoriques, p. 16, 21.

r894: Co he n: Meteoritenkunde, p. 52, $58,67,68,86,99$, 108, 109, поо, 232. r895: B r ez in a: Wiener Samml., p.297. 
1895: C o he n: Meteoreisen-Studien IV. Ann. Hof-Mus. Bd. 10, p. 83,90 , 91 .

Ursprüngliches Gewicht: 136 bis I4I Kgr.

Nachweisbares Gewicht: 133408 gr.

Berlin, U.

v. Braun

Budapest

Calcutta

Cohen

Dresden, M.

Edinburg

Göttingen

Gregory

Harvard, U.

Kopenhagen

48 London, B. M. 2164

170 London, P. G. $\quad 98$

33 Minneapolis 21

139 Moskau

I Paris, M.

3 v. Siemaschko 73

7o Stockholm 66

93 Tübingen 33

129 Washington 38

990 Washington, Sh. 21

63 Wien,H.M.*)129099 ${ }^{\text {) }}$

Bachmut (Alexejewka), bei Bachmut, Gouvernement Ekaterinos. law, Russland.

Stein, $\mathrm{Cw}$, gefallen I $_{5}$. Februar I $_{8}$ I $_{4}$. Hierher auch: „Scholakow, Stein gefallen 23. Januar 1814 \%. Vgl. auch Mordvinovka, mit welcher Lokalität diese vielleicht zu vereinigen ist, s. u. Anm. zu Kiew.

1815: Giese: Ueber die chemische Nomenklatur, und einige andre chemische und physikalische Gegenstände. Gilb. Ann. Bd. 50, p. 117, 118.

1815: Chladni: Neues Verzeichnis, Gilb. Ann. Bd. 5o, p. 256.

1819: $\mathrm{C}$ h 1 a d n i: Feuermeteore, p. 65,

$69,73,304-305$ (1).

r836: Kä m tz: Meteorologie, p. 285 .

1843: P a rts c h: Meteoriten, p.53-54.

1847: Eichwald: Verzeichnis, Erman's Archiv für Kunde Russland's, Bd. 5, p. 178 .

1847: B orrisiak: Sur l'Aérolithe tombé près de Verkhne - Tschirskaia Stanitsa. Bull. Petersburg Acad. Bd. 5, p. 198 (die Universität Charkow besitze einen Stein von Alexejewka).
1848: v. B 1 o ed e: Tabelle, Bull. Petersburg Acad. Bd. 6, p. 7 .

1854: v. Bog uslaw ski: Zehnter Nachtrag, Pogg. Ann. Ergz.-Bd. 4, p. 418. r854: B a $1 \mathrm{c}$ e $11 \mathrm{~s}$ : Lithologia meteorica, p. 23.

1859: H a rris : Dissert, Gött., p. 72. r862 : Wöhler: Ueber den Meteorstein von Bachmut. Gött. Gel. Anz. (Nachr.) Bd. 3, p. 373, 374.

1862: Greg: On the Meteorites in the British Museum. Philos. Magaz, Bd, 24, p. 538,539 .

r862 : W öh ler : Ueber die Bestand. theile des Meteorsteines von Bachmut in Russland. Sitzber. Wien. Akad. Bd. 46 , p. $302-306$; s. auch Gött. Gel. Anz. (Nachr.) 1863, p. 222. Ref. LiebigKopp, Jahresber. 1862 , p. $830-83$ r. 1862: Haidinger: Die Meteoriten von Bachmut u. von Paulowgrad, beide im Gouvernement Jekaterinoslaw. Sitz.* ber. Wien. Akad. Bd. 46 , p. $307-$ 3 Io; s, auch Wiener Zeitung vom 18. Oktober 1862, Beilage Nro. 38, p. 303 ; Kenngott, Uebersicht, $1862 / 65$, p. 437 -438 ; Liebig-Kopp, Jahresber. 1863 , p. 906-907; s'Institut \& Bd. 3I (1863), p. 57.

1863: B u c h n e r: Meteoriten, p. 34, 35. r863: Ros e: Meteoriten, p. 84,85 , 93,155 .

1863: Maskely ne u. v. Lang: Mineralogical Notes. Philos, Magaz. Bd. 25, p. $449-45$ I.

1864: B u c hn er: Erster Nachtrag, Pogg. Ann. Bd. 122, p. 319.

r859/65: v. Re i c he n b a c h: IX 16r, 162, 168, 178. X 359,363 . XI 296. XIII 372 (Fig.), $377,3^{8} 3$. XXV 438,615. r865: Buchner: Zweiter Nachtrag,

Pogg. Ann. Bd. 124, p. 577.

1865: Kuhlberg: Analyse und Beschreibung der Meteorite von Nerft, Honolulu, Lixna und eines im Gouvernement Jekatherinoslaw gefallenen

1) Ausserdem $20 \mathrm{gr}$. Babb's Mill, gefunden 1842, nach I. Juli 1893 erworben. 
Meteoriten. Arch, f. Naturk, Liv, Esth. u. Kurl. (r) 4 , p. 18-22 (Analyse). Ref. Liebig-Kopp, Jahresber. 1867, p. $1048-1049$.

1867: Go e be 1: Kritische Uebersicht,

Mélanges phys. chim. Bd.7, p.288-289.

1867: Buchner: Dritter Nachtrag,

Pogg. Ann. Bd, 132, p. 315, 319.

r869: B u chner: Vierter Nachtrag,

Pogg. Ann. Bd. 136, p. $442-444$.

1870: $\mathrm{R}$ a $\mathrm{mm}$ els ber $\mathrm{g}$ : Meteoriten,

p. 103, 105, 106, 139, 140.

r879: R a mmels ber g: Meteoriten, p. 24,25 .

1884: M e un ier: Météorites, p. 79,

$85,208,2$ II-212, 395 .

1885: Brezina: Wiener Sammlung, p. $177-180,232$.

I894: C o h e n : Meteoritenkunde, p.r36, $205,206,247,248,249,266,303$. r895: Brezina: Wiener Sammlung, p. 242 .

Ursprüngliches Gewicht: Etwa 16 Kgr. (4० Pfund).

Nachweisbares Gewicht: 2531 gr. Bailey

Berlin, U. $3 \mathrm{I}$ London, B. M. $4 \mathrm{I}$

v. Braun

99 Moskau

8 Neumann

Budapest

Calcutta

Cleveland

Göttingen

Gregory

Harvard, U.

Kasan

Kiew

I8 New Haven

I58 Paris, M.

252

3I Petersburg, B. 2I5

82 v. Siemaschko 3

17 ') Stockholm

2 Strassburg

II9 Tübingen

$57^{2}$ ) Washington, Sh

v. Leuchtenberg 8 Wien,H.M.*)**)1244

Ein grosses Stück von 20 Pfund wurde nach Charkow geschickt, 2 Stücke von
$4 \mathrm{Kgr}$. kamen an das Gymnasium zu Jekatherinoslaw (Kuhlberg); von Charkow, habe ich mich vergeblich bemüht, Nachricht zu erhalten.

\section{Bacubirito}

Ranchito

Baffinsbay

Bahia

Sowallik s. Anhang Bemdego

Baird's Farm, Asheville, Buncombe County, Nord-Carolina, U. S. A. Eisen, Om, beschr. 1839 .

Diese Lokalität ist meistens in der älteren Literatur (von Fletcher noch jetzt) als "Asheville " bezeichnet, während Asheville-Black Mountain als Black Mountain aufgeführt und Asheville-Jewell Hill als Jewell Hill bezeichnet wird.

1839: Shepard: On Meteoric Iron from Ashville, Buncombe county, N. C., Am. Journ. (I) 36, p. $8 \mathrm{I}-85$.

1843: P a r t s c h: Meteoriten, p. Ir6.

1847: S h ep a r d : Report on Meteorites, Am. Journ. (2) 4, p. 79. Ref. LiebigKopp, Jahresber. $1847 / 48$, p. $1309-$ I3IO; Kenngott, Uebersicht $1850 / 5 \mathrm{I}$, p. 135 .

1852 : C l a r k: Dissert. Gött., p. 5556 ; s. auch Ann. Chem. Pharm. Bd. $82(1852)$, p. 367 .

r854: v. Boguslawski: Zehnter Nachtrag, Pogg. Ann, Ergz.-Bd.4, p.403. 1859: H arris: Dissert. Gött., p. II2. r858/62: v. Re i c h e n b a c h: IV 638 . VII 551. IX 163, I74, I82. X 359 . XII 457. XIV 390. XV I00, กо, II 4 , 124. XVI $253,255,256,261$, XVII $264,265,266,272$. XVIII 480,484 , 487,489 . XIX 155. XX 621, 628, 629. 630. XXI 583,587 .

I) Hiervon $5 \mathrm{gr}>23$. Januar 1814 Scholakow, near Ekaterinoslaw, Russia ; auch das Brit, Mus, besitzt einen Splitter dieser Lokalität, den es von Gregory erhielt. Herr Direktor Fletcher ist ebenfalls der Ansicht, dass dieser Fall zu streichen ist, so lange nicht weitere Einzelheiten darüber bekannt werden; auch Brezina spricht sich in ähnlichem Sinne aus.

2) Mit der Bezeichnung $\$ 1814$ (Datum?) im Gouvernement Jekaterinoslaw (im Ort Pawlograd?)<.

$W$ iil fing, Meteoriten. 
1863: B u chner: Meteoriten, p. I63. r863: Ros e: Meteoriten, p. 65, I53. 1867: Go e be 1: Kritische Uebersicht, Bull. Petersburg Acad. Bd. 7, p. 325 . r884: M e u n i e r : Météorites, p. II6, I20. r885: B rez in a: Wiener Sammlung,

p. 200 (Ashville), 209, 233.

r8go: E a s tm a n: Met. Astron., p. 318. r893: Meunier: Révision des fers météoriques, p. $64-65$.

1894: C o h e n: Meteoritenkunde, p. 76, I94, 23I,

Ursprüngliches Gewicht: Eine etwa kopfgrosse Masse; Shepard erhielt 9 -roUnzen, also etwa $1 / 4 \mathrm{Kgr}$. Nachweisbares Gewicht: 837 gr.

\begin{tabular}{lr|lr} 
Bailey & 7 & Neumann & 3 \\
Berlin, U. & I3 & Paris, M. & 72 \\
Budapest & I & Pohl & 71 \\
Calcutta & 6 & Stürtz & 4 \\
Cambridge & $\left.14{ }^{1}\right)$ & Stuttgart & $5^{2}$ ) \\
Cleveland & I & Tübingen & 17 I \\
Göttingen & I & Turin, U. & $13^{3}$ ) \\
Gregory & 2 & Upsala & $\left.4^{4}\right)$ \\
Krantz & Spl. & Ward & $5^{2}$ \\
London, B. M. II5 & Washington, Sh. \\
London, P. G. & 8 & Wien, H. M.*) & 271
\end{tabular}

Baird's Plantation Baird's Farm Bald Eagle, Park Hotel, io Klm. südl. Williamsport, Pa., U. S. A. Eisen, $\mathrm{O}$,gefunden $\mathrm{x} 89 \mathrm{r}$, beschr. 1892 . 1892: O wen s: A Meteorite from Central Pennsylvania. Am. Journ. (3) 43, p. $423-424$. Ref. N. J. 1893 I, p. 479 -480 .

Ursprüngliches Gewicht: $3 \cdot 3 \mathrm{Kgr}$. Nachweisbares Gewicht ?

Das Eisen soll nach Owens an die Bucknell University, Lewisburg, $\mathrm{Pa}$, gekommen sein.
Die geographische Länge und Breite, welche Brezina angiebt, bezieht sich nach Stieler's Handatlas Blatt 85 (1880) auf die Gegend von Williamsport am Potomac, welche Stadt etwa $39^{\circ} 35^{\prime} \mathrm{n}$. $\mathrm{Br}$. und $77^{\circ} 50^{\prime}$ w. L. liegen dürfte, während das hier in Betracht kommende Williamsport am Susquehanna etwa $41^{\circ} 15^{\prime} \mathrm{n}$. Br. und $77^{\circ} 3^{\prime}$ w. L. liegt. Brezina giebt an $39^{\circ} 10^{\prime} \mathrm{n}$. Br. und $78^{\circ} 8^{\prime}$ w. L.

\section{Baldohn}

Misshof

Bambuk

Bancoorah

Senegal

Shalka

Bandong, Goemoroeh, Preanger, Insel Java.

Stein, Cwb, gefallen ro. Dez. 187 1.

1872: Everwijn: Jaarboek van het Mijnwezen in Nederlandsch Ost India. Deel 2, p. 197 .

1872: Da ubrée: Sur une météorite tombée dans l'île de Java, près Bandong, le 1o décembre 1871 , et offerte au Muséum par M. le Gouverneur général de l'Inde néerlandaise. C. R. Bd. 75 , p. $1676-1678$ (Analyse von Vlandeeren). Ref. Liebig-Kopp, Jahresber. 1872 , p. $1198-$ n99.

1873: Hirzel u, Gretsche 1: Jahrbuch der Erfindungen Bd. 9, p. 20-2r. 1879: R a m mels berg: Meteoriten, p. II, 24, 26.

r882: Brezina: Bericht IV, Sitzber. Wien. Acad. Bd. 85 I, p. 338 .

1884: M e uni er: Météorites, p. 79,256. r885: Brezina: Wiener Sammlung, p. $181,232$.

1886: Verbeeck: Jaarboek van het Mijnwezen etc. (2) I. r887: Flig ht: Meteorites, p. 50.
I) 》Ashevillea.
2) >Asheville, bekannt seit $1839 \propto$.
3) >Asheville N. Carol. « Könnte also Black Mountain (oder Jewell Hill?) sein.
4) s Asheville 1839a. 
1894: Co h e n : Meteoritenkunde, p. 249, 266.

Ursprüngliches Gewicht : II $^{1} / 4 \mathrm{Kgr}$. 6 Steine; die drei grössten $8.2 \mathrm{Kgr}$, $2.24 \mathrm{Kgr}$, u. o.68 Kgr.; die übrigen zusammen $150 \mathrm{gr}$.

Nachweisbares Gewicht: II I $10 \mathrm{gr}$.

\begin{tabular}{lr|lr} 
Bailey & 3 & Melion & I \\
Batavia & 8125 & Paris, E. & 56 \\
Bayet & 2 & Paris, M. & 2075 \\
Bement & 28 & Prag, M. & 238 \\
Berlin, U. & I & Rom, U. & 60 \\
Bologna & I & v. Siemaschko & I7 \\
Bonn & 6 & Stockholm & 2 \\
v. Braun & 12 & Strassburg & 6 \\
Budapest & 192 & Troyes & I \\
Gregory & 88 & Utrecht & 7 \\
Greifswald & 7 & Washington & b \\
London, B. M. & 14 & Washington, Sh. 5 I \\
Lüttich & 3 & Wien, H. M. **) & 112
\end{tabular}

\section{Banja}

Sokobanja

Baratta, Deniliquin, Australien.

Stein, Cs, gefallen imMai um 1845 (?) (das Falldatum ist sehr unsicher). 1871: The >Australasian $\approx$, April 22 nd. Ref. s Nature* IV (1871), p. 212.

1872: Liversidge: The Deniliquin or Baratta Meteorite. Trans. Royal Soc. New South Wales $(1872)$, p. $97-98$ u. IOI-ro3. Vergl. auch Liversidge : The Deniliquin or Baratta Meteorite. Sidney 1873 . T. Richards, government printer.

1874: Journ. of Sc. (welches?); Januar 1874 , p. 123.

1882: Liversidge: The Deniliquin or Baratta Meteorite (Second Notice) Journ. and Proc. Royal Soc, N. South Wales Bd, 16, p. $31-33$ (mit 3 Tafeln; Abbildung des Steins und eines Dünnschliffs). Ref. N. J. 1885 II, p. 270. 1884: M e unier: Météorites, p. 242. 1885: Brezina: Wiener Sammlung, p. $25 \mathrm{I}$.

1887: F lig h t: Meteorites, p. 170-I71. r888: Liversidge: The Deniliquin or Baratta Meteorite. Appendix zu: Minerals of New South Wales (London bei Trübner u. Co. 1888), p 207 -217 (Analyse; Abbildung des Steins und eines Dünnschliffs).

1889: Russell exhibited three new meteorites, Journ. and Proc. Royal Soc. of New South Wales Bd. 23, p. 46-47.

r89o: Brez in a: Reise, Ann. Hof-Mus. Bd. 4 (Not.), p. II9.

r895: Brezina : Wiener Sammlung, p. 252 .

Ursprüngliches Gewicht: Etwa I37 Kgr.; ausser den anfangs gefundenen Massen von $150+70+$ 4 lbs. wurden 1889 (nach briefl. Mitteilung von Herrn Gregory) noch zwei weitere Massen von $4^{8}+31^{1 / 2}$ lbs. aufgedeckt.

Nachweisbares Gewicht : 25 gr.

$\begin{array}{ll}\text { Gregory } & 10 \\ \text { Paris, M. } & 15\end{array}$

Die Hauptmasse war Privateigentum von Herrn Russell in Sidney und befand sich $\mathbf{1} 888$ in dessen Besitz; wo ist dieselbe gegenwärtig ?

Barbotan, Roquefort, Gascogne, Frankreich.

Stein, Cga, gefallen 24. Juli $\mathbf{7} 790$. 1790: Bertholon: Journ. des Sc. utiles Nr. 23 und 24 (1790), p. 305. (Bei Izarn wird auf Nr. 23 und 24 (1791), p. I25 verwiesen.)

I790: L a pe ir ouse: Mém. Acad. Toulouse Bd. 4, p. 189 .

1796: B a u di in: Décade philosoph., litéraire et politique Nr. 67 (29. Febr. 1796). Auszug in Gilb. Ann. Bd, 13 (1803), p. 346-349: Beschreibung eines feurigen Meteors, das am 24. Juli 1790 in Gascogne gesehen wurde.

1803: D e D r é e : Notice sur les pierres tombées près d'Ensisheim et dans les environs d'Agen (die Lokalität Barbo2* 
$\tan$ ist gemeint). Journ. Phys, Bd. 56 , p. $405,408-410,411,413,419,420$, 42I; s. auch Tilloch's Philos. Magaz. Bd. 16, p. 293 ; Bibl. Brit. Bd. $20 \mathrm{Nr}$. 154 , p. 85 (ferner Voigt's Magaz. Bd. II, p. 2).

I803: I z a r n: Lithologie, p. 80-94 (aus Bibl. Brit. Nr. 135 und 136), 108, 2I5, 255, 3II, 325, 346; s. auch Gilb. Ann. Bd.r5, p. 421 (Vauquelin) u. Gilb. Ann. Bd. 24, P. 379 Anm.; auch » Encyclopédie « I808, p. 566-568, 591 .

r8o3: Bournon: Lettre de M. le comte de Bournon, membre de la Société royale de Londres et de celle de Linnée, à M. Delamétherie en réponse à la critique de M. Patrin à l'égard des pierres tombées de l'atmosphère. Journ. Phys. Bd. 56, p. 294-3or.

r8o3: V a u quelin: Mémoire sur les pierres dites tombées du ciel. (s. bei Benares); auch $\gg$ Encyclopédie «, p. 564. 1803: Gil b e rt: Noch einiges über den Steinregen von Gascogne am 24. Juli 1790. Gilb. Ann. Bd. 15, p. 429-436 , und 310,328 .

1803: K laproth: Ueber meteorische Stein- und Metall-Massen. Abh. Berlin. Akad. (18o3), p. 34-35.

r804: Gilbert: Nachträge zu den Aufsätzen in den Annalen über die aus der Luft gefallenen Steine. Gilb. Ann. Bd. 18, p. $283-284,286$ (Analyse von Vauquelin; Zahlen nicht angeführt, dieselben seien unbedeutend verschieden von Sales).

1804: Pötzs ch: Kurze Darstellung, p. 7I-74, 1о9, по.

r804: v. Ende: Massen und Steine, p. $48-49$.

r808: L a u g i er: Chromium, Gilb. Ann. Bd. 24 (I8o6), p. $379-383$.

1808: Pictet: Description comparative etc. (s. bei Weston), Bibl. Brit. Nr. 296 (April 18o8), p. 28o-281, 286, 287, 288, 290 ; s. auch Bibl. Brit. Nr. $45^{\circ}$ (Sept. 1814), p. $88-91$ (Saint-Amans).
I812: C h la d n i: Verzeichnis, Schweigg. Journ. Bd. 4 Beil. I, p. I4.

1812: Bigot de Morogues, p. I22 $-\mathrm{I} 4 \mathrm{O}, 336$.

18r5: Chladni: Neues Verzeichnis, Gilb. Ann. Bd. 50, p. 251.

1815: $\mathrm{Chl}$ a dni: Bemerkungen, Gilb.

Ann. Bd. 5o, p. 263.

r8rg: $\mathrm{C}$ h 1 a d n i: Feuermeteore, p. 43, $48,50,51,57,66,70,71,90,132-$ 133, 236, 248, 254, 258-260 (1), 271, 295, 309, 428 .

1821: C h 1 a d n i : Erster Nachtrag, Gilb. Ann. Bd. 68, p. 339.

1823: Ch 1 ad n i: Dritter Nachtrag, Gilb.

Ann. Bd. 75, p. 23 o.

1836: K ä m tz: Meteorologie, p. 277.

r836: Gruit h u is e n: Naturgeschichte

des gestirnten Himmels, p. 407-408.

r843: P a r t s c h : Meteoriten, p. 77-78. r854: B a 1 c e 11 s : Lithologia meteorica,

p. $2 \mathrm{I}-22,40-4 \mathrm{I}$.

1857: A r a g o: Astronomie populaire,

Bd. 4 , p. 194,250 .

r859: H a rris : Dissert. Gött., p. 62.

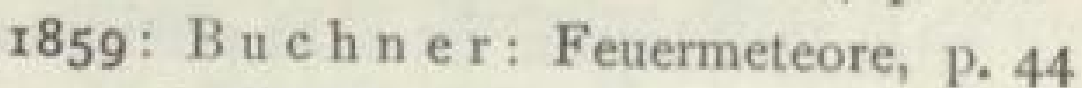
$-48,6 \mathrm{r}-62$.

1862: Kenng ot t und Wiser; Zürcher Sammlung, p. 155.

r862: Greg: On some Meteorites in the British Museum, - Note on Bluish Meteorites. Philos. Magaz. Bd. 24, p. 542 .

1863: B u c hner: Meteoriten, p. II. r863: R os e : Meteoriten, p. $87,88,92$, 154.

r858/65: v. Reichen b a ch: V 476. VI 454. IX 155, 162, 171, 18o. X 359 , 363. XI 295, 302, 309. XII $454,455$. XIII 365. XV IoI, I2I. XVIII 490. XX 623. XXV 322, 429, 432, 604, 607. 1872 : Naturee I. Febr. 1872.

1875: vom Rath: Meteoriten, Verh. naturh. Ver. Bonn Bd. 32, p. 368 369 (unter Nr, 45: New Concord). r884: M e unier: Météorites, p. 94, 95, $97,268,269,443,444,446,459$, 
$460,466-468,470$.

${ }_{188} / 85$ : T s c h e rma k : Photographien, p. 16,17 .

1885: Brezina: Wiener Sammlung, p. $182,233$.

1887: F $1 \mathrm{ight}$ : Meteorites, p. 95.

r889: F l e t c h e r : Atacama Meteorites, Mineral. Magaz. Bd. 8, p. 226 (Streufeld 6 miles).

I $690:$ v. Ni ess 1: Periheldistanzen, Verh, naturf. Ver. Brünn Bd. 29, p. 189, I95, 214, 253.

r892: Pfa hle $\mathrm{r}$ : Ueber den Meteoriten von Barbotan. 24. Juli 1790. Ueber den Meteoriten von l'Aigle. 26. April 1803. - Ueber den Meteoriten von Barbotan. Tschermak's M.P. M. Bd. I3, p. 353-362 (Analyse; Abb. von Dünnschliffen); s. auch Ann. Hof-Mus. Bd. 9 (Not.), p. 20. Ref. N. J. I895 I, p. 276 .

r894: Co hen: Meteoritenkunde, p. 59, $85,244,277$.

Ursprüngliches Gewicht: Steinschauer.

Nachweisbares Gewicht: 5911 gr. Bailey

Belgrad

Bement

Berlin, G.

Berlin, U.

Bologna

Bonn

v. Braun

Budapest

Calcutta

Cambridge

Cleveland

Darmstadt, M.

Dorpat

Frankfurt

Göttingen

Graz, J.

Gregory

\section{Halle}

4 Hamburg

14 Harvard, U.

27 Kopenhagen

300 Kunz

30 London, P. G. 185

88 de Mauroy

92 Moskau

82 Neumann

I64 New Haven

5 Paris, E.

I5 Paris, M.

I Paris, U.

2 Pohl

103 Rom, U.

657 v. Siemaschko

389 Stockholm
12 London, B. M. 858
Strassburg

Troyes

Tübingen

Upsala

Barcelona $\mathrm{I} 85^{\mathrm{I}}$

Barcelona I86I

Baré

Barea (Logroño), Provinz Logroño, Spanien.

Mesosiderit, M, gefallen 4.Juli 1842 . r854: Gr e g: Catalogue, Philos. Magaz. Bd. 8 , p. 460 .

1859: $\mathrm{H}$ a r r is : Dissert. Gött., p. 88.

r863: Buchner: Meteoriten, p. 68. 1865: Buchner: Zweiter Nachtrag,

Pogg. Ann, Bd. 124, p. 579.

1872: M e u n ier: Analyse lithologique de la météorite de la Sierra de Chaco.

Mode de formation de la logronite.

C. R. Bd. 75 , p. $1547-1548$.

I88I: B rezina: Bericht III. Sitzber.

Wien. Akad. Bd. 84 I, p. 278 .

r884: M e uni er: Météorites, p. I65, $359,36 \mathrm{r}, 362$.

r885: Brezina; Wiener Sammlung, p. 251 .

1887: Brezina: Reisebericht, Ann. Hof-Mus. Bd. 2 (Not.), p. 108.

r892: Gre dill a : Meteoritos, p.IOI-IO2. r894: C o h e n : Meteoritenkunde,p.289. 1895: M e unier: Révision des Lithosidérites, p. $33-34$.

Ursprüngliches Gewicht: Greg sagt 7 lbs., Meunier 22 I I gr. und neuerdings 2300 gr., Gredilla 2250 gr. Nachweisbares Gewicht: 2306 gr. Berlin, U. Madrid \begin{tabular}{r|l} 
Io & Paris, M. \\
2250 & Rom, U.
\end{tabular} 44

Barntrup (Krähenholz), Fürstentum Lippe, Deutschland.

Stein, Cia, gefallen 28. Mai 1886 .

I) Ausserdem besitzt Tübingen ein Stäck mit der Bezeichnung >Barbotanє, welches nach Herrn Direktor Brezina höchstwahrscheinlich zu Timochin gehört. 
r887: v. H a uer: Ann. Hof-Mus, Bd. 2 (Not.), p. 38.

1889: H äp k e: Der Meteorit von Barntrup. Abh. naturw. Ver. Bremen Bd. II p. 323-324; s. auch Weser Zeitung vom 24. August I886; "Naturforscher * 1886, p. 426-427; "Naturee Bd. 34 (1886), p. 439 ; N. J. 1891 I, p. 48. 1893: Brezina: Ueber neuere Meteoriten (Nürnberg), p. 160.

1895: Brezina: Wiener Sammlung, p. $247-248$.

Ursprüngliches Gewicht: $\mathbf{1 7 . 3}$ gr. Ein kleiner Stein.

Nachweisbares Gewicht: ${ }^{5} 5.8$ gr. (1.5 gr gingen beim Zerschneiden verloren.)

$\begin{array}{ll}\text { Detmold } & 9.5 \\ \text { Wien, H. M. } & 6.3\end{array}$

Barranca Blanca, San Francisco Pass, Cortillere Atacama, Chili, Südamerika.

Eisen, Obz, gefunden 1855 , vorläufig bestimmt 1885 , beschr. 889 . 1885: Brezina: Wiener Sammlung, p. $217-234$.

1889: F let c h e r: Atacama Meteorites, Mineral. Magaz. Bd. 8, p. 224, 262263 (Analyse).

1895: Brezina: Wiener Sammlung, p. 289 .

Ursprüngliches Gewicht: $\mathbf{1} 2.3 \mathrm{Kgr}$. Fletcher giebt II.3 Kgr. an, nach der folgenden $\mathrm{Zahl}$ dürfte ein Druckfehler vorliegen.

Nachweisbares Gewicht: 12233 gr. Bailey

Budapest

Gregory

Harvard, U. Io London, B.M. II 915 25 London, P.G. 147 28 v. Siemaschko 15 27 Wien, H. M. 66

$\begin{array}{lr}\text { Bassein } & \text { Quenggouk } \\ \text { Bates Co. } & \text { Butler }\end{array}$

\section{Batesville}

Joe Wright

Bath, South Dakota, U. S. A.

Stein, Ccb, gefallen 29. August 1892 .

r893: Foote: Preliminary Notice of

a Meteoric Stone seen to fall at Bath,

South Dakota. Am. Journ. (3) 45,

p. 64 (Abb.). Ref. N. J. 1894 I, p. 274.

r893: Brezin a: Ueber neuere Meteoriten (Nürnberg), p. 162.

1895: Brezina: Wiener Sammlung, p. 259 .

Ursprüngliches Gewicht: $21.2 \mathrm{Kgr}$. $\left(46^{3} / 4\right.$ lbs.) Der ganze Stein war nach Herrn Direktor Brezina's Mitteilung im Besitz von Herrn Bement.

Nachweisbares Gewicht: $14648 \mathrm{gr}$.

\begin{tabular}{lr|lr} 
Bailey & 57 & London, B. M. & 2119 \\
Bement & 2825 & Newton & 43 \\
Böhm & 910 & Prag, M. & 325 \\
v. Braun & 138 & Rom, U. & 92 \\
Brezina & 1457 & v. Siemaschko & 93 \\
Budapest & 246 & Ward & 1276 \\
Harvard, U. & 2265 & Wien, H. M. 2802 ')
\end{tabular}

\section{Bathurst}

\section{Battle River}

Cowra

Bear Creek, auch Bear River, Denver Co., Colorado, U. S. A.

Eisen, Of, gefunden u. beschr. 1866 . 1866: W i 1 s o n: Daily News, Denver, Colorado, 14. Mai 1866.

1866: She pard: Brief Notices of several localities of Meteoric Iron. Am. Journ. (2) 42, p. 250-251.

r866: Henry: Mass of Meteoric Iron in Colorado territory. Am. Journ. (2) 42 , p. $286-287$.

1867: S m it h : On Colorado Meteorites.

- Russel Gulch Meteoric Iron and Bear Creek Meteoric Iron. Am. Journ. (2) 43, p, 66-67 (Analyse von Schwefeleisen u. Nickeleisen); s. auch Ori-

I) Ausserdem 729 gr, nach I. Juli 1893 erworben. 
ginal Researches 1884 , p. $439-440$. r867: J a cks on: Analysis of a Meteoric Iron from Colorado. Am. Journ. (2) 43 , p. $280-281$; s, auch Boston Society 1866 , p. $71-72$.

r869: Buchner: Vierter Nachtrag,

Pogg. Ann. Bd. 136, p. 604-605.

r884: M e un i e r : Météorites, p.Ir6, I25. 1885: Brezina: Wiener Sammlung, p. $209,210,233$.

1890: Eas t m a n : Met. Astron., p. 320. r893: M eunier: Révision des fers météoriques, p. 52, 57 .

r894: Co hen: Meteoritenkunde, p. 57, 188, 191, 198.

UrsprünglichesGewicht : 500 pounds nach einer Schätzung, 436 lbs. nach Eastman.

Nachweisbares Gewicht: 2389 gr.

\begin{tabular}{|c|c|c|}
\hline Bailey & 173 & Moskau \\
\hline Bement & 125 & New Haven \\
\hline Berlin, U. & 76 & Paris, E. \\
\hline Bonn & $\mathbf{I}$ & Paris, M. \\
\hline v. Braun & 12 & Petersburg, B. \\
\hline Budapest & 80 & Pohl \\
\hline Cleveland & 21 & Stockholm \\
\hline Göttingen & 305 & Troyes \\
\hline Gregory & 8 & Upsala \\
\hline Harvard, U. & 38 & Ward \\
\hline London, B. M. & $5^{2}$ & Washington \\
\hline London, P. G. & 52 & Washington,Sh. \\
\hline Minneapolis & $4 \mathrm{I}$ & Wien, H. M.*) \\
\hline
\end{tabular}

\section{Bear River Bear Creek}

Beaufort

Orange River, Stein

Beaugency

Charsonville

Beaver Creek, Distrikt West Kootenai, British Columbia.

Stein, Cck, gefallen 26. Mai 1893 . 1893: How e 11: Beaver Creek Meteorite. "Science , July 2 Ith $^{\text {t } 893}$, p. $4 \mathrm{I}$. 1893: Brezina: Ueber neuere Meteoriten (Nürnberg), p. 162.

1894: H ow e 11: Beaver Creek Meteo-

rite. Am. Journ. (3) 47 , p. $430-43 I$ (Abb. des Steins von 22 $\frac{1 / 2}{2}$ pounds). Ref. N. J. 1896 I, p. $228-229$. r894: Hillebra nd: Chemical Discussion, Beaver Creek Meteorite. Am. Journ. (3) 47 , p. 431-435. Ref. N. J. 1896 I, p. $228-229$.

1894: M e rril1: Microscopical Discussion, Beaver Creek Meteorite. Am. Journ. (3) 47 , p. 435 . Ref. N. J. I896 I, p. 228-229.

1894: C o h e n: Meteoritenkunde, p.24I. 1895: Brezina: Wiener Sammlung, p. 260 .

Ursprüngliches Gewicht: Etwa I4 Kgr. (etwa 31 lbs.)

Nachweisbares Gewicht: I I 864 gr.

\begin{tabular}{lr|lr} 
Bailey & 155 & Paris, M. & 264 \\
v. Braun & 185 & Prag, M. & 139 \\
Cleveland & 29 & Rom, U. & 10 \\
Harvard, U. & 447 & v. Siemaschko & 99 \\
Howell & 10 206 & Washington & 330
\end{tabular}

Seit I. Juli 1893 gelangten an Wien, H.M. $885 \mathrm{gr}$, an Herrn Direktor Brezina 279gr, an Herrn Bayet, Brüssel, 17 gr.

\section{Becasse}

Bechuana Land

Belaja Zerkwa

Belgorod

Belgradjek

Bella Roca, auch La Bella Roca, Sierra de San Francisco, Santiago Papasquiaro, Durango, Mexico. Eisen,Of, gefund.r888; beschr.r 889. 1889: Whitfield: A new Meteorite from Mexico. Am. Journ. (3) 37, p. 439 -440 (Analyse u. Abb. des Eisens); s. auch Bull. United States Geol, Surv. Nr. 64 , p. 29-3o. Ref. N. J. 1891 II, p. 418 ; Liebig-Kopp Jahresber. 1889, p. 466 .

1890: E a s t m a n : Met, Astron., p. 322. 189o: F l etcher: Mexican Meteorites, Mineral. Magaz. Bd. 9, p. 155-156. 
I89r : v. H a u e r : Ann. Hof-Mus. Bd. 6 (Not.), p. $54 ;$ s. auch Bd. 7 (1892) (Not.), p. 72.

r89r: Cohen und Weinschenk: Meteoreisen-Studien. Ann. Hof-Mus. Bd. 6, p. 162 .

1892: E a s tman: The Mexican Meteorites. Bull. Philos. Soc. Washington Bd. 12, p. 45 .

1893: Brezin a: Ueber neuere Meteoriten (Nürnberg), p. $\mathbf{1 6}_{3}$.

1893: Meunier: Révision des fers météoriques, p. $44-45$ (Abb. einer geätzten Platte).

r894: C o h e n: Meteoritenkunde, p. 95, 116, 124, 125, 135, 156, 195, 196, 199.

r895: Brezina: Wiener Sammlung, p. 271 (Abb. einer geätzten Platte; auch Taf. VII, Fig. 9).

1895: Co hen : Meteoreisen-Studien IV. Ann. Hof-Mus. Bd. 10, p. 82, 90.

Ursprüngliches Gewicht: $33 \mathrm{Kgr}$. Nachweisbares Gewicht: $26{ }_{3}^{87} \mathrm{gr}$.

\begin{tabular}{lr|lr|} 
Bailey & 809 & Krantz & 613 \\
Bement & 746 & London, B. M. 3542 \\
Berlin, U. & 38 & New Haven & 315 \\
Böhm & 12 & Newton & 74 \\
v. Braun & 57 & Paris, E. & 39 \\
Brezina & 1353 & Prag, M. & 274 \\
Budapest & $15 \mathrm{I}$ & Riga & 55 \\
Calcutta & 88 & Rom, U. & 62 \\
Dorpat & 612 & v. Siemaschko & 146 \\
Gregory & 480 & Stürtz & 45 \\
Greifswald & 72 & Ward & 2003 \\
Harvard, U. & 1925 & Washington & 152 \\
Kopenhagen & 95 & Wien, H. M. 12629
\end{tabular}

\section{Belmont s. Anhang}

Bemdegó (Bahia), Monte Santo, Bahia, Brasilien.

Eisen, $O$ g, gefund. 1784 ; beschr.1816. 1816: Mornay: An account of the discovery of a mass of native iron in Brasil, Philos. Trans. (1816) p. 270280 (mit zwei Umrisszeichnungen des Eisens); s. auch Gilb. Ann. Bd.56 (1817), p. $355-368$ u. Bd. 58 , p. 169 (Abb.); Philos. Magaz. Bd. 48 (1816), p. 417 -424 .

r8r6: $\mathrm{Chl}$ a d n i: Erste Fortsetzung,

Gilb. Ann. Bd. 53 , p. $385-386$.

18r6: Wo 11 as to $\mathrm{n}$ : Observations and experiments on the mass of native iron found in Brasil. Philos. Trans. (1816) p. $28 \mathrm{r}-285$ (Analyse); s. auch Gilb. Ann. Bd. $56(1817)$, p. $369-374$; Schweigg. Journ. Bd. 23 (1818), p. 300 -308 ; $>$ Isis $\approx$ von 1818 , Heft 12 .

r8rg: Ch 1 a d n i : Feuermeteore, p. 343 -344 .

182r: Chla dni: Erster Nachtrag, Gilb. Ann. Bd. 68 , p. $343-344$.

1828: v. Spix u. v. Martius: Reise in Brasilien auf Befehl Sr. Majestät Maximilian Joseph I, Königs von Baiern, in den Jahren $1817-1820$, Teil II, p. $730-740$ u. $748-752$. München 1828. (Analyse von Fikentscher; Abb.) 1836: Kä mtz: Meteorologie, p. 277. 1843: Part s c h : Meteoriten, p.I2I- 122. 1852: C l a r k: Dissert. Gött., p. 32.

1854: v. Boguslawski: Zehnter Nachtrag, Pogg. Ann. Ergz.-Bd. 4, p. $4 \mathrm{I} 3-4 \mathrm{I} 4$.

r855: $\mathrm{H}$ a i d in g e r: Bemerkungen über die zuweilen im geschmeidigen Eisen entstandene krystallinische Structur, verglichen mit jener des Meteoreisens. Sitzber. Wien. Akad. Bd, 15 I, p. 357 (Fig. 13).

1859: $\mathrm{B} \mathrm{u} \mathrm{ch}$ n er: Feuermeteore, p. 148 -150 .

1859: H a r r is: Dissert. Gött., p. 105. 1860: Wöhler: Ueber das Meteoreisen von Bahia. Ann. Chem. Pharm. Bd. Ir5, p. 92-95 (Analyse von Martius jr. Abb. von zwei geätzten Platten); s. auch Journ. prakt. Chem. Bd, 82 (1861), p. 319-320; Liebig-Kopp, Jahresber. 1860, p. $853-854$; Chem. Centr. 1860, p. $833-834$; N. J. 1861, p. 699; Kenngott, Uebersicht r860, p. 103. 
r858/62: v. Reichen bac h: VI 448 . VII 551, 557. IX 163, 174, 181. XII 457. XIII 363 . XIV $390,393 \mathrm{XV}$ 100, IIO, 124, 128, 131. XVI 26r. XVII 266. XVIII 484,487 . XX 62I, 626, $627,628,630$. XXI 578,587 .

I863: B u c hner: Meteoriten, p. I42 -144 .

1863: (W öhle r ?): Giebt im Referat über die vorige Arbeit an, dass Dr. Fikentscher in Wunsiedel 1220 gr. von Bemdegó besitze. Gött. Gel.Anz. p. 1240. 1863: R o s e: Meteoriten, p. 59, 152. 1867: Go e be 1: Kritische Uebersicht, Mélanges phys. chem. Bd. 7, p. 312 . r869: Meunier: Recherches, Ann. Chim. Phys. (4) 17, p. 21.

r874: B jörling : Meteoriter och Kometer (cf. Hessle), p. 13.

1875 : Mo hr : Entstehungsart, Ann.Chem, Pharm. Bd. 179, p. 259, 270.

1878: P o s s n e r: Das Meteoreisen vom Bemdêgo in Brasilien. Bamberg 1878 (?), p. $\mathrm{I}-32$ (p. 33-46, Allgemeines über Meteoriten, nicht ausgezogen).

r879: K1 e in : Gött. Sammlung, Gött. Gel, Anz. 1879, p. 98 .

r884: $\mathrm{H}$ ä pke : Beiträge, Abh. naturw. Ver. Bremen Bd. 8, p. 518-519.

1884: Me un ier: Météorites, p. 23 , $35,137$.

1885: Brezina: Wiener Sammlung, p. 207, 214, 234 .

1887: de Carval ho: Bull. Soc. geograph. Bd. 3 (?) 1887 ; auch Revue Soc. geograph. 3. Juni 1887. Rapport sur le déplacement; s. auch Am. Journ. (3) 36 (1888). p. 158.

1888: D a ubrée présente à l'Academie de la part de S. M. Dom Pedro, Associé étranger, la photographie d'un fragment poli du fer météorique ou holosidère de Bendego (Brésil), C. R. Bd. 107, p. 896-897.

1888: D e r b y : Meteoritos Brasileiros. Revista do Observatorio, Rio. p. 2-3. I) Bahia?s r889: B rez in a : Cliftonit aus dem Meteoreisen von Magura, Arvaer Comitat. Ann. Hof-Mus. Bd. 4, p. 102.

r8go: E a stm a n: Met. Astron., p. 318. r89o: B r ez in a: Reise, Ann. Hof-Mus. Bd. 4 (Not.), p. II6, II8, 122.

r8gr: v. H a u er: Ann. Hof-Mus. Bd. 6 (Not.), p. 55 .

1893: Brezina: Ueber neuere Meteoriten (Nürnberg), p. 165.

1893: Meunier: Révision des fers météoriques, p. 25-26.

1894: F le t c her: Introduction, p. 14. I894: C o he n : Meteoritenkunde, p. 4I, $54,62,68,115,117,194,232$.

1895: Co he n : Meteoreisen-Studien IV. Ann. Hof-Mus, Bd. 1o, p. $82,85,90$, 91, 93 .

Ursprüngliches Gewicht : $5370 \mathrm{Kgr}$. 5361 Kgr. betrug das Gewicht bei der Ankunft in Rio; etwa 9 Kgr. waren durch Mornay, Spix und Martius abgetrennt worden. Herr Orville A. Derby teilte mir ferner mit, dass in Rio ein grosses Stück von $62 \mathrm{Kgr}$. abgesägt wurde, von welchen kleinere Schnitte durch die Herren Kunz und Ward zur Verteilung gelangten. Der grössere Teil dieser $62 \mathrm{Kgr}$. schweren Masse blieb in Rio; ausserdem erhielt das Museum dort 1868 gr. von Dr. Luiz da Rocha Dias zum Geschenk, so dass der Besitz des National Museums in Rio sich auf etwa $5335 \mathrm{Kgr}$. belaufen dürfte. NachweisbaresGewicht: $535459^{2}$ gr.

\begin{tabular}{lr|lr} 
Bailey & 98 & Brezina & 178 \\
Bement & 857 & Budapest & 585 \\
Berlin, U. & 34 & Calcutta & 60 \\
Bologna & 53 & Cambridge & $77^{\prime}$ ) \\
v. Braun & 651 & Cleveland & 17
\end{tabular}




\begin{tabular}{lr|lr} 
Cohen & 250 & Neumann & 54 \\
Dorpat & 37 & New Haven & 160 \\
Dresden, M. & 389 & Newton & 2 \\
Erlangen & 17 & Odessa & 7 \\
Göttingen & 290 & Paris, M. & 920 \\
Gregory & 65 & Pohl & 138 \\
Greifswald & 212 & Rio & 5335000 \\
Halle & 24 & v. Siemaschko & 104 \\
Harvard, U. & 14 & Stockholm & 205 \\
Howell & 260 & Strassburg & 53 \\
Kopenhagen & 15 & Troyes & 13 \\
London, B. M. & 3115 & Tübingen & 2647 \\
London, P. G. & 301 & Ward & 1987 \\
de Mauroy & 30 & Washington & 102 \\
München & 3119 & Wien, H. M.*) & 2452
\end{tabular}

Benares, Krakhut, Bengalen, Ostindien.

Stein, Cc, gefallen r9. Dezbr. 1798 . 1802: Auszüge aus Briefen u. ein Paar Zeitungsartikel: 6) Vom Himmel gefallene Steine. Gilb. Ann. Bd. Io, p. 502 (Hinweis auf die Zeitung >Moniteur Nr. 166 an $\mathrm{X}$ ).

1802: $\mathrm{H}$ o w a r d : In seiner grossen $\mathrm{Ar}$ beit: Experiments and observations u. s. w.; s, den Bericht William's: Account of the Explosion of a meteor, near Benares, in the East Indies; and of the falling of some Stones at the same time, about 14 Miles from that City. Philos. Trans, 1802, p. 175-179; s. auch Gilb. Ann. Bd. 13 (1803), p. 298 -30I u. Izarn, Lithologie, p. 176182; Fourcroy's Uebersetzung in $>$ Encyclopédie « Bd. 5 (1808), p. 547$548,548-549,550-553$.

1803: I z a r n: Lithologie, p. 171, 176 $-182,184 \quad 187,193-206,214,215$, 219, 257-274, 349 .

1803: de Drée: Recherches, Journ. Phys. Bd. 56, p. $383-384,409$, 4 II, $413,419,420,421$.

1803: Va uquelin: Ueber die aus der Atmosphäre herabgefallenen Steine. Gilb. Ann. Bd. 15, P. 422-424. Längeres Referat der Schrift Vauque- lin's aus „L'Institute vom $\mathrm{I}$. Dezember 1802 u. aus Izarn's Lithologie, p. 253274; s, auch sEncyclopédie \& Bd. 5 (1808), p. $565-567,592$.

1803: K la prot h: Ueber meteorische Stein- und Metall-Massen. Abh. Berlin. Akad. (1803), p. $3 \mathrm{I}-32$.

r803: Ch1 adn i: Chronologisches Verzeichnis, Gilb. Ann. Bd. I5, p. 311.

1804: v. Ende: Massen und Steine, p. $56-60$.

r804: Pötzsch: Kurze Darstellung, p. $85-88,92,93,118$.

1804: Gilbert: Nachträge zu den Aufsätzen in den Annalen über die aus der Luft gefallenen Steine. Gilb. Ann. Bd. 18 , p. 286 , wo auf Vauquelin's Analyse in Ann. XV, p. 424 verwiesen wird.

1806: L a u g i e r: Chromium, Gilb. Ann. Bd, 24 , p. 379.

1807: K l a p r o th: Beiträge, Bd.4, p.98. 1808: Pictet: Description comparative etc. (s. bei Weston), Bibl. Brit. Nr. 296 (April 1808), p. $282-283,288$. 1811 : Account of stones etc. Relation d'une chute de pierres tombées du ciel aux environs de Bénarés, le 20 (!) Décembre 1799; tirée des voyages de Lord Valentia, Bibl, Brit. (Januar ISII), Nr. 362, Bd, 46, p. $96-102$.

1812: $\mathrm{Ch} 1$ a d n i : Verzeichnis,Schweigg. Journ. Bd. 4 Beil. I, p. 15.

1812: Bigot de Morogues, p.17, $164-176,335-336$.

1812: Gilbert: Meteorsteine, 5) Aussagen von Hindoos über den Steinregen bei Benares den 20. (1) Dezember 1799. Gilb. Ann. Bd. 4I, p. 453-454. 1815: Chladni: Neues Verzeichnis, Gilb. Ann. Bd. 50, p. 252.

1819: $\mathrm{Chl}$ a dn i: Feuermeteore, p. 6, 10, $50,58,66,70,71,74,138,264$, $266-268$ (!), 294, 428; s, auch Bibl. Brit. Bd. 46 , p. 96.

1820: v. S c hre i bers : Beiträge, p. 62 -63 (Abb. Tafel VII). 
1836: K ä $\mathrm{mtz}$ : Meteorologie, p. 278. r836: Gruit h u is e n: Naturgeschichte des gestirnten Himmels, p. 4I2.

I843: P a r t s c h : Meteoriten, p. 43-44. r859: B u chner: Feuermeteore, p. 59 -62 .

1859: Harris: Dissert. Gött., p. 64. 1863: B u chner: Meteoriten, p.14-15. 1863: Rose: Meteoriten, p. 30, 86, 106, 154 .

1858/65: v. R e i che n b a ch: IV 637 V $475,477,479,48$ I. VI 443,444 , $449,455,456$. IX 16r, 169, 179. X $359,362,371$. XI 295, 297, 298. XIII $357,360,361,365,375$. XIX 152, 153. XX 622, 623. XXIII 368. XXIV 226. XXV 606, 615.

1865: B u chner: Zweiter Nachtrag, Pogg. Ann. Bd. 124, p. 574 .

r884: M e unier: Météorites, p. 268, $269,443,446,459,460,468-469$. 1885: Brezina: Wiener Sammlung, p. $185,233$.

1894: F le t c he r: Introduction, p. 9, $23,24$.

1894: Cohen: Meteoritenkunde, p. 7, 23,185 .

1895: Brezin a: Wiener Sammlung, p. 255 .

UrsprünglichesGewicht:VieleSteine. Nachweisbares Gewicht: 24r6 gr.

\begin{tabular}{lr|lr} 
Bailey & 60 & Moskau & 25 \\
Berlin, U. & 16 & Neumann & I \\
Bologna & I & New Haven & I \\
Budapest & I0 & Paris, M. & I4 \\
Calcutta & $77^{1}$ ) & Parma & 24 \\
Cambridge & 48 & v. Siemaschko & I \\
Göttingen & 5 & Stockholm & 8 \\
Gregory & 70 & Troyes & 6 \\
Heidelberg & I & Tübingen & 388 \\
Kopenhagen & 180 & Ward & I \\
London, B. M. & 5 III & Washington & I \\
London, P. G & 183 & Washington, Sh. & 7 \\
Madras & I5 & Wien, H. M.*) & 662
\end{tabular}

\section{Berar}

Beraun

Chandakapoor

Berdjansk

Zebrak

Berlanguillas, Burgos, Altcastilien, Spanien.

Stein, Cia, gefallen 8. Juli r $8 \mathbf{1}$. I8Ir: Comte Dorsenne: Détails sur la chute d'une pierre météorique en Espagne, Bibl. Brit. Bd. 48, p.162-I64. 1812: Gilbert: Herabfallen dreier Meteorsteine am 8. Juli 18II, unweit Burgos in Altcastilien. Gilb. Ann. Bd. 40, p. 116 ; s, auch Schweigg. Journ. Bd. 4 (1812) Beil. I, p. $20-2$ I.

1812: Bigot de Morogues: p. (52), $272-275,333$.

1812: G ilbert: Meteorsteine. 4) Noch etwas über die bei Burgos in Spanien am 8. Juli I8II herabgefallenen Meteorsteine. Gilb. Ann. Bd. 4I, p. $45^{2}$ -453 .

1812: $\mathrm{Ch}$ la dni: Verzeichnis, Schweigg. Journ. Bd. 4 Beil, I, p. 16.

1815: Chladni: Neues Verzeichnis, Gilb. Ann. Bd. 50, p. 255.

r8rg: $\mathrm{Chladni}$ : Vierte Fortsetzung, Gilb. Ann, Bd. 6o, p. 239.

r8r9: $\mathrm{C}$ h 1 a d n i: Feuermeteore, p. 57,

$66,70,74,91,273,296-297(!), 431$. r836: Käm tz: Meteorologie, p. 283 . 1843: Parts ch: Meteoriten, p. 59. 1859: Harris: Dissert. Gött., p. 70. 1863: B u chner: Meteoriten, p. 29. 1863: R os e: Meteoriten, p. 155.

1858/65: v. Reichen bach: V 476. IX 16I, 170, 179. XI 294, 301. XIII 362. XXV 318, 324.

1884: M e u n i er: Météorites, p. 197, 198. 1885: Brez ina: Wiener Sammlung, p. $182,232$.

1892: Gredilla: Meteoritos, p. 104 $-\mathrm{I05}$.

1895: Brezina: Wiener Sammlung, p. 247 .

1) 29 gr. u. 48 gr., zwei stark von einander abweichende Stücke, eins derselben wohl nicht Benares. 
Ursprüngliches Gewicht: $2-3 \mathrm{Kgr}$. Ein Stein.

Nachweisbares Gewicht: 1376 gr.

\begin{tabular}{lr|lr} 
Bement & 2 & Neumann & I \\
Berlin, U. & 38 & New Haven & I 4 \\
Calcutta & 2 & Paris, M. & I000 \\
Dublin, M. & I4 & Pohl & Spl. \\
Göttingen & 2 & v. Siemaschko & 4 \\
Gregory & 5 & Stockholm & 35 \\
Harvard, U. & 8 & Tübingen & 20 \\
London, B. M. & 26 & Wien, H. M.*) & I98 \\
Modena & 7 & &
\end{tabular}

Bethlehem, Troy, Albany Co., New York, U. S. A.

Stein, Cck, gefallen Ir. Aug. 1859 . 1859: Shepard: Meteor of August II, 1859. Am. Journ. (2) 28, p. 300-303. 1860: Bethlehem (New York) Meteoric Stone, of Aug. II, 1859. Am. Journ. (2) 30, p. 206-207 (Umrisszeichnung des etwa taubeneigrossen Steins); s. auch Liebig-Kopp, Jahresber. 186o, p. 846 (Anm.); N. J. 1862 , p. 197.

1859: $\mathrm{R}$ o ger $\mathrm{s}$ : presented the following communication on the meteor of August II, 1859, by Mr. David A. Wells. Proc. Boston Soc. of Nat. Hist. Bd. 7 (1859/61), p. 176-179.

1863: Buchner: Meteoriten, p. Io2. 1885: Brezina: Wiener Sammlung, p. 252.

1890: E a s t m a n : Met. Astron., p. 316. 1895: Brezina: Wiener Sammlung, p. 259.

Ursprüngliches Gewicht: Ein Stein von Taubeneigrösse. Wo befindet sich derselbe gegenwärtig? Nachweisbares Gewicht: 2.7 gr. $\begin{array}{lr}\text { Bailey } & \mathbf{2 . 7} \\ \text { Gregory } & \text { Spl. } \\ \text { Wien, H. M. } & \text { Spl. }\end{array}$

Beuste

Bhagur

Bhawalpur
Bueste

Dhulia

Khairpur
Bherai, Junagadh, Kathiawar, Bombay.

Stein, gefallen 28. April 1893 .

1894: F l e t c her: Introduction, p. 15. Ursprüngliches Gewicht ?

Nachweisbares Gewicht: 17 gr. London B. M. 17 gr. (Oktober 1894).

\section{Bhurtpur 1860}

Bhurtpur 1868

Kheragur stock, Russisch Polen.

Stein, Ho, gefallen 5. Okt. 1827 . 1828: Chute d'aërolithe en Russie, Ann. Chim. Phys. Bd. 39 , p. 42 I. (Abgedruckt aus > Gazette de Saint-Pétersbourg ).

1830: v. H off: Siebter Nachtrag, Pogg. Ann. Bd. 18 , p. $185-186$ (Hier ist verwiesen auf Froriep's Notizen Bd, 19 Nr. 2, p. 26 und Bd, 20 Nr. 18; p. 276). 1836: Kä m tz: Meteorologie, p. 296. 1837: Rose: Reise nach dem Ural,

Bd, I, p. 76 u. 77 (Anmerkung).

1843: Part s c h: Meteoriten, p. 27-28. 1845: v. B a u $\mathrm{m}$ h a u e r: Ursprung, Pogg.

Ann. Bd. 66, p. 476.

1847: E i chwald: Verzeichnis, Erman's Archiv $f$, wissensch.Kunde v. Russland $\mathrm{Bd} .5, \mathrm{p}, \mathrm{I} 79$.

1848: B 1 ö d e: Tabelle, Bull. Petersburg Akad, Bd. 6, p. 7 .

1854: v.Bog u sl a w s k i : Zehnter Nachtrag, Pogg. Ann. Ergz.-Bd. 4, p. 439. 1859: Harris: Dissert. Gött., p. 8t. 1863: B u c h ner: Meteoriten, p. $5 \mathrm{I}-52$. 1863: R o s e: Meteoriten, p. 26, 29, IO7, $\mathrm{HO}-1 \mathrm{I2}, \mathrm{I} 56$.

1859/65: v. Reichen b a ch: IX 160, 169, 179. X 363, 372. XIII 364, 373 . XXV 438 .

1867: Goebel: Kritische Uebersicht, Nélanges phys. chim. Bd. 7 , p. 325 -326 .

1867: Buchner: Dritter Nachtrag,

Pogg. Ann. Bd. 132, p. 317 . 
1877: Sorby: On the structure and origin of Meteorites. sNature $\mathrm{Bd}$. 15 , p. 496.

1883: T s c hermak: Beitrag, Sitzber. Wien. Akad. Bd. 88 I, p. 368.

r884: M e un i er: Météorites, p. 77, 94, $95,286,288$.

r $88_{3} / 85$ : T s c h er m a k: Photographien, p. $7-8$.

1885: Brezina: Wiener Sammlung, p. 174,232 ,

Ursprüngliches Gewicht: Mehrere Steine, von denen einer etwa 2 Kgr. wog.

Nachweisbares Gewicht: 627 gr. Berlin, U. Bonn

v. Braun Budapest

Calcutta

Cleveland

Dorpat

Göttingen

Kiew 79 Krantz

4 London, B. M.

4 London, P. G. Spl.

IOI Paris, M.

1 Petersburg, A. I12

Spl. v. Siemaschko 5

8I Stockholm 49

Spl. Wien, H. M. *) 59

120 Washington Spl.

Es ist noch keine Analyse dieses Steines ausgeführt.

\section{Bielokrynitschie Bjelokrynitschie} Bingera, New South Wales.

Eisen, Hch, gefunden 1880 (nach Gregory's Catalogue, Addenda zu 1889, p. 4 Nr. 346), beschrieben 1882 ,

1882: Liversidge: On the Bingera Meteorite, New-South-Wales (Preliminary Notice). Journ. and Proc. of the Royal Soc. New-South-Wales Bd, 16 (1882), p. 35-37 (Analyse u.Abb. des Eisens). Ref. N. J. 1885 11, p. 271 ; s. auch Liversidge: The Minerals of NewSouth-Wales 1888, p. $218-220$.

1894: C o hen: Meteoritenkunde, p. 64. 1895: Brezina: Wiener Sammlung, p. 294-295 (Abbildung einer geätzten Schnittfläche).

I) 60 Pfund 20 Zolodnik.
Ursprüngliches Gewicht: $24 \mathrm{I}$ gr.

Nachweisbares Gewicht: 86 gr.

$$
\begin{array}{lr}
\text { Gregory } & \text { I } \\
\text { Wien, H. M. } & 85
\end{array}
$$

Bischtübe, Nikolaew'scher Kreis, Turgaiski'sches Gebiet, Russland.

Eisen, $\mathrm{Og}$, gefunden $\mathbf{r} 888$, beschrieben 1890 .

r89o: K is lak owsky: Ueber den Meteoriten von Turgaisk. Bull. Soc. Imp. des Naturalistes de Moscou 1890 Nr. 2, p. 187-199 (mit Tafel). - Ref. N. J. 1892 I, p. $5 \mathrm{r}-52$; s, auch Oesterr. Zeitschr. f. Berg- und Hüttenw. Bd. 39 (I891), p. 239.

r893: M eun ier: Révision des fers météoriques, p. 25, 27.

1894: C o h en: Meteoritenkunde, p. 53 , 73, $131,134,157,267,308$.

r895: B rezina: Wiener Sammlung, p. 287.

r895: C o h e n : Meteoreisen-Studien IV. Ann. Hof-Mus. Bd. 10, p. 82, 90, 91.

Ursprüngliches Gewicht: $48^{3 / 4} \mathrm{Kgr}$. 3 Stücke von $32^{1} / 2,16$ u. $0.205 \mathrm{Kgr}$.

Pachweisbares Gewicht: $26609 \mathrm{gr}$.

\begin{tabular}{lr|lr} 
Bailey & 27 & Paris, M. \\
Cohen & III & Petersburg, B. \\
Dorpat & 72 & \multicolumn{2}{|c}{45} \\
Gregory & 45 & 656 & ') \\
Kasan & 145 & Stockholm & $\mathbf{2 2}$ \\
Krüger & 32 & Ward & 19 \\
London, B. M. & 88 & Wien, H. M. & 109
\end{tabular}

Bishopville, Sumterville, Sumter Distrikt, Süd-Carolina U. S. A.

Stein, Chla (Chladnit, geadert), gefallen 25. März 1843 .

r846: S hep ar d : Report on Meteorites. Am. Journ. (2) 2, p. $379,3^{8} 4$, 392. Auf dieser Seite sagt der Autor sdescribed by Shepard \&, während in dieser Arbeit vorher nur kurze Erwähnung einiger Mineralien geschieht, 
welche im Stein von Bishopville vorkommen. Es muss also wohl noch eine frühere Beschreibung des Steines geben, obgleich Shepard nicht darauf hinweist. Eine solche Beschreibung des Falles und des Steines findet sich indessen auch in der folgenden Arbeit Shepard's von 1848 .

1848: Shepard: Report on Meteorites. Io) Bishopville, South-Carolina. Am. Journ. (2) 6, p. 403 , p. $4 \mathrm{II}-4 \mathrm{I} 4$ (Zwei Abbildungen des Steines); s. auch Liebig-Kopp, Jahresber. $1847 / 48$, p. 1314 $-1316$.

1848: $\mathrm{H}$ a i d in g e r : Berichte der Vers. d. Freunde d. Naturw. Wien Bd, III, p. 282 .

185r: v. Waltershausen: Ueber einen Meteorstein von Bishopville in Süd-Carolina. Ann, Chem. Pharm. Bd. 79 , p. $369-374$ (Analyse). Ref. Liebig-Kopp, Jahresber. 1851 , p. 881 - 882 ; Pharm. Centr. 1852 , p. 9; Kenngott, Uebersicht $185^{\circ} / 51$, p. 179-18o.

r853: R a m mels berg : Handwörterbuch. Suppl. V, p. 33-34.

1854: v. Boguslawski: Zehnter Nachtrag, Pogg. Ann. Ergz,-Bd. 4 , p. $367-368$.

1855: Smith: Memoire on Meteorites, Am. Journ. (2) 19, p. 162, I63 Anm. (Chladnit sei ein Pyroxen).

1859: Ha r r is: Dissert. Gött., p. 89.

1860: R a mme ls berg: Mineralchemie, p. $94 \mathrm{I}$.

r86r: $\mathrm{R}$ a $\mathrm{m} \mathrm{me} \mathrm{s}$ b e $\mathrm{r} \mathrm{g}$ : Ueber einige nordamerikanische Meteoriten. I) Meteorstein von Bishopville, SüdCarolina. Monatsber. Berlin. Akad. (186r) , p. $895-899$; s. auch Journ. prakt. Chem. Bd. 85 (1862), p. 83 -87 ; Kenngott, Uebersicht 186i, p. 166-168; Chem. Centr. 1862, p. I; Zeitschr. f. d. Ges. Naturw. Bd. 19, p. 185; Liebig-Kopp, Jahresber. 186I, p. $\mathrm{II}_{3} \mathrm{O}-\mathrm{II}_{3} \mathrm{I}$, Am. Journ. (2) 38 , p. $425-426$.
1863: Buchner: Meteoriten, p 69-71. I863: Rose: Meteoriten, p. 27, 28, II7-I22, I56; s. auch Monatsber. Berlin. Akad. I862, p. 557.

1864: S mith : Chladnite of the Bishopville Meteoric . Stone proved to be a Magnesian Pyroxene. Am. Journ. (2) 38, p. 225-226; s. auch sL'Institut \& 1865 , p. 63 ; Journ. prakt. Chem. Bd. 95, p. 313; Chem. Centr. 1865 , p. 654 ; Am. Journ. (3) 5, p. 108-110 ; Original Researches 1884 , p. $429-430$, $470-472$; C. R. Bd. 76 (1873), p. 295 -297; Liebig-Kopp, Jahresber. 1864, p. 9or; Kenngott, Uebersicht $1862 / 65$, p. 450 .

r857/65: v. Reichen b a h: III 624 . IV 637 . V $476,477,480$. VI 454 , 456. VII 558. IX 160, $166,177 . \mathrm{X}$ 359,363 . XI 294, 301. XIII 353,356 , $359-360,364,375$. XIV $390,396$. $\mathrm{XV}$ iоr. $\mathrm{XX} 620,632 . \mathrm{XXV} 318,322$, $324,424,429,431$.

1865: Buchner: Zweiter Nachtrag. Pogg. Ann. Bd. 124, p. 572, 579-580. r868: Mohr: Bildung der Meteoriten.

Verh. naturh.Ver Bonn Bd.25 (Sitzber.), p. 65 .

1870: Rammels berg: Meteoriten, p. $105,121-123$; s, auch Pogg. Ann. Bd. 140 (1870), p. 315 .

1870: M a skelyne: On the Mineral Constituents of Meteorites. Philos. Trans. 1870 I, p. 194, I95; s. auch Proc. Royal Soc. $1869 / 70$ Bd. 18 , p. 149 ; Rammelsberg, Mineralchemie 2. Aufl. 1875 , p. 52 (Oldhamit).

1875: Mohr: Entstehungsart, Ann. Chem. Pharm. Bd, 179, p. 262.

1875: vom R a th: Meteoriten,Verh, naturh. Ver. Bonn Bd. 32, p. 375 , Nr. 59. 1882: Wiechmanu: Fusion Structures in Meteorites. Ann. N. Y. Acad. Bd, 2, p. 293.

1883: Wadsworth: The Bishopville and Waterville Meteorites. I) The Bishopville Meteorite. Am, Journ. (3) 
26 , p. $32-36$, auch p. 248 . Ref. N. J. 1884 II, p. 28 ; Liebig-Kopp, Jahresber. 1884, p. 2042.

1883: T s chermak: Beitrag, Sitzber.

Wien. Akad. Bd.88 I, p. $363-365,367$.

1884: Meunier: Météorites, p. 62, $73,74,80,93,94,95,96,98,278$ -280 (Abb.), 523.

r884: W a dsworth: Studies, p. 199 -201 .

1883/85: Tschermak: Photographien, T. V, p. 9-10.

r885: Brezina: Wiener Sammlung, p. $174,176,232$.

1890: Ea s tm a n n: Met. Astron., p.316.

1890: Weinschenk: Ueber zwei neue Bestandteile des Meteoriten von Sarbanovac. Ann. Hof-Mus. Bd. 4 (Not.), p nо.

1894: F 1 et ch e r: Introduction, p. 37. r894: Co he n: Meteoritenkunde, an vielen Stellen.

r895: Brezina: Wiener Sammlung, p. 239 .

Ursprüngliches Gewicht: $6 \mathrm{Kgr}$. (r3 lbs.)

Nachweisbares Gewicht: 3472 gr.

\begin{tabular}{lr|lr} 
Bailey & 5 & Frankfurt & Spl. \\
Belgrad & 2 & Frenzel & Spl. \\
Bement & 6 & Göttingen & 15 \\
Berlin, U. & 233 & Gregory & 20 \\
Bologna & 15 & Greifswald & 7 \\
Bonn & 2 & Harvard, U. & 53 \\
v. Braun & 20 & Heidelberg & 2 \\
Budapest & 20 & Klausenburg & 3 \\
Calcutta & 74 & Kopenhagen & 67 \\
Cambridge & 157 & Kristiania & 6 \\
Cleveland & 13 & Kunz & 5 \\
Dorpat & 3 & Lissabon & 5 \\
Dresden, M. & 2 & London, B. M. & 512
\end{tabular}

\begin{tabular}{lr|lr} 
London, P. G. & 166 & Roebling & 45 \\
Madrid & Spl. & Rom, U. & 9 \\
de Mauroy & 2 & v. Siemaschko & 7 \\
Melion & 3 & Stockholm & 7 \\
Minneapolis & 2 & Strasshurg & 8 \\
München & 8 & Troyes & 3 \\
Neumann & Spl. & Tübingen & 507 \\
New Haven & 213 & Upsala & 7 \\
Paris, M. & 56 & Ward & I \\
Petersburg, B. & 22 & Washingt.,Sh. & 1090 \\
Pohl & 24 & Wien, H. M *) & 45
\end{tabular}

Bishunpur, Mirzapur Distrikt, NordWest Provinz, Ostindien; hierher auch Parjabatpur.

Stein, Cs, gefallen 26. April 1895 . r895: Brezina: Wiener Sammlung, p. $253-254$.

Ursprüngliches Gewicht: $103^{8} \mathrm{gr}$. Nach gütiger Mitteilung von Herrn Direktor Holland in Calcutta fielen zwei Steine. Der eine von Bishunpur wog $94 \mathrm{r} .8 \mathrm{gr}$., der andere von Parjabatpur 96.6 gr.; von ersterem besitzt Calcutta $428.6 \mathrm{gr}$., von letzterem $6 \mathrm{r} .8 \mathrm{gr}$. Nachweisbares Gewicht: $570 \mathrm{gr}$. Es ist vermutlich noch mehr in Sammlungen enthalten, aber wegen des späten Falltermins nicht in den mir zugeschickten Listen aufgeführt.

Calcutta 490 | Wien, H. M. 8o Bissempore Shalka Bitburg, (Albacher Mühle), nördlich von Trier, Rheinpreussen. Pallasit, $\mathrm{P}^{\mathrm{t}}$ ), gefunden $\mathrm{r} 8 \mathrm{o} 2$, erwähnt I8I4.

I) Brezina trennt neuerdings von den Pallasiten eine Gruppe der breccienähnlichen Pallasite, die er als Albacher Gruppe bezeichnet und wozu er Bitburg (Albacher Mühle) und Bret.ham rechnet. Von Bitburg ist aber so wenig unverändertes Material vorhanden, dass die Bezeichnung schon aus diesem Grunde nicht sehr glücklich gewählt sein dürfte. cf. Ann. Hof-Mus. Bd. 10 (1895), p. 265. 
1812/r4: $\mathrm{G} \mathrm{ib} \mathrm{b} \mathrm{s:} \mathrm{Observations} \mathrm{on} \mathrm{the}$ Mass of Iron from Louisiana. Bruce's Am. mineralog. Journ. Bd, I (I8I4), p. $219-221$.

r8rg: $\mathrm{Ch}$ la dni: Vierte Fortsetzung, Gilb. Ann. Bd, 6o, p. 242-244.

r8rg: $\mathrm{Ch}$ l a d n i : Feuermeteore, p. 353 -354 .

1821; $\mathrm{C}$ h a d n i: Erster Nachtrag, Gilb. Ann. Bd. 68, p. 342 .

1825: Nöggerath und Bischof: Ueber die grösste europäische GediegenEisenmasse meteorischen Ursprungs. Eine historisch-physikalisch-chemische Abhandlung. Schweigg. Journ. Bd. 43, p. $\mathrm{I}-2 \mathrm{O}$.

r825: Nöggerath: Gibbs über das Gediegen-Meteoreisen von Bitburg. Schweigg. Journ. Bd. 43 , p. $20-23$. Ueber die sehr kleinen Oktaëder in der Schlacke des umgeschmolzenen $\mathrm{Me}$ teor-Eisens von Bitburg. Ebend. p. 23 -24 .

r826: Chladni: Fünfter Nachtrag, Pogg. Ann, Bd, 6, p. $33-34$.

r826: Seebeck: Ueber die magnetische Polarisation der Metalle und Erze durch Temperaturdifferenz. Pogg. Ann. Bd. 6, p. 144 .

1826: Chladni: Sechster Nachtrag, Pogg. Ann. Bd. 8, p. 51, 52.

1826: Chladni: Ueber Zusammensetzung der Meteormassen. Weitere Nachrichten von der grossen Bitburger Gediegen-Eisenmasse, gegeben von den Herren Steininger und Nöggerath und nebst einigen Bemerkungen. Schweigg. Journ. Bd. 46 , p. $385-396$.

1835: Steininger: Aufsätze über einige Gegenstände aus dem Gebiete der Physik, - III. Ueber das GediegenEisen von Bitburg. Gymnasial-Programm Trier 1835 , p. $18-21$ (Gedruckt mit Blattau'schen Schriften).

1838: Silliman: Meteoric Iron in France. Am. Journ. (r) 33, p. 257-258. 1843: P a r t s c h: Meteoriten, p. 95-98.
1852 : C 1 a r k : Dissert. Gött., p. $25-26$. 1852: W ö h le r: sAktiv «, Pogg. Ann. Bd. 85 , p. 448 .

I854: v. B ogus lawsk i: Zehnter Nachtrag, Pogg. Ann. Ergz.-Bd. 4, p. 384 -385 .

r859: B u c h n er: Feuermeteore, p. 123. 1859: H a rri s : Dissert. Gött,, p. 106.

1860: $\mathrm{R}$ a m me ls b e r g: Mineralchemie, p. 920.

1857/62: v. Reichen bac h: III 624 . IV 638. VI 442 . VII 551. IX 162 , 163, I 73, I8I. XI 296, 302, 308. XII $452-454,455$. XIII 363. XIV 399. XV 10I, 104, 107, 116. XVI 26I, 262. XVII 265. XVIII 48I. XX 622, 627. I863: B u c h ner: Meteoriten, p. 126. 1863: Rose: Meteoriten, p. 73, 77, $79,153$.

1875: vom R a th: Meteoriten, Verh. naturh. Ver. Bonn, Bd. 32, p. 376. r875: Mo hr: Entstehungsart, Ann. Chem. Pharm. Bd, 179, p. 267-268.

r879: K lein : Göttinger Sammlung, Gött. Gel. Anz. (1879), p. 98.

1879: F inkener: (Mitteilung einer Analyse). Zeitschr. d. d. geol, Ges. Bd. 31 (1879), p. 635 .

r883: Tschermak: Beitrag, Sitzber. Wien. Akad. Bd. 88 I, p. 348 . r884: Meunier: Météorites, p. 137. r884: Hä pke: Beiträge, Abh. naturw. Ver. Bremen Bd. 8, p. 518, 520. r884: Wa ds w ort h: Studies, p. 70. 1884: Wiepken: Notizen über die Meteoriten des Grossherzoglichen Museums (Oldenburg). Abh, naturw, Ver. Bremen Bd. 8, p. 528 .

1883/85: Tscherma k: Photographien, p. 23.

1885: Brezina: Wiener Sammlung, p. $206,233$.

1886: Brezina und Cohen: Photographien, T. 6.

r888: Lockyer: Researches on the Spectra of Meteorites. Proc. Royal Soc. Bd. 43 , p. 125 . 
1893: Meunier: Révision des fers météoriques, p. 70.

I894: C o h e n: Meteoritenkunde, p. 67. r895: Brezina: Wiener Sammlung, p. 265 .

1895: Cohen: Meteoreisen-Studien IV. Ann. Hof-Mus. Bd. Io, p. $8 \mathbf{2}, 85$

Ursprüngliches Gewicht : über 1600 Kgr.; grösstenteils umgeschmolzen; auch die in der nachfolgenden Tabelle aufgeführten Stücke dürften zum allergrössten Teil der umgeschmolzenen Masse entstammen. Bei wenigen kleinen Stücken ist in den Verzeichnissen ausdrücklich angegeben worden, dass sie von der $\mathrm{nicht}$ umge. schmolzenen Masse herrühren.

Nachweisbares Gewicht: 77424 gr., davon nicht umgeschmolzen $34 \mathrm{gr}$.

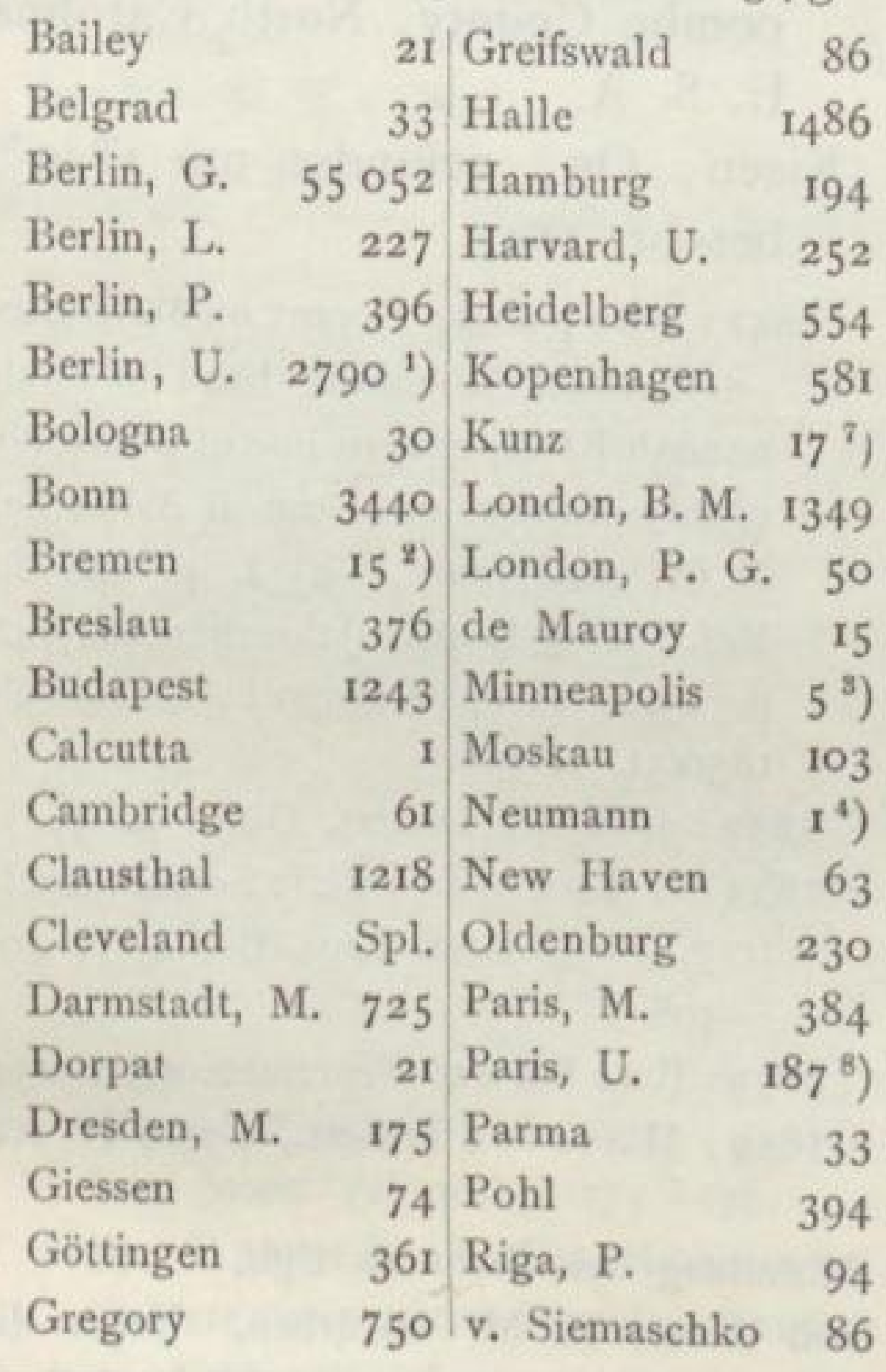

\begin{tabular}{ll|l|l|l|} 
Stockholm & $\mathbf{1 8 4}$ & Utrecht & 530
\end{tabular} Strassburg $\quad 235$ Ward $\quad 142$ Troyes $\quad 74$ Washington, M. 22 Tühingen $2792^{5}$ ) Washington, Sh. I9 Turin, U. $\quad 144$ Wien, H. M.*) $109^{6}$ ) Die Universität Basel besitzt ebenfalls ein Stück Bitburg.

Bjelaja Zerkow, Ukraine, Gouv. Kiew, Russland.

Stein, $\mathrm{Cc}-\mathrm{Cg}$, gefallen I6. Januar 1796.

1809: Stoikowitz: Nachrichten von mehreren russischen Luftsteinen, besonders von denen, die am r. Oktober 1787 im Gouvernement von Charkow herabgefallen sind. - I) Einige ältere in Russland gefallene Luftsteine. Gilb. Ann. Bd. 3I, p. 307.

I8r2: $\mathrm{Ch}$ la d ni: Verzeichnis, Schweigg. Journ. Bd, 4 Beil. I, p. 14.

1812: Bigot de Morogues, p. I6r. I8r5: Chladni: Neues Verzeichnis, Gilb. Ann. Bd, 50, p. $25^{2}$.

r8rg: Chla dni: Feuermeteore, p. 4r, $65,73,264$ (1).

1826: Chla dni: Fünfter Nachtrag, Pogg. Ann. Bd. 6, p. 29.

r836: K ä m tz: Meteorologie, p. 277.

1847: E i ch wa ld : Verzeichnis, Erman's Arch. f. wissensch. Kunde v, Russland Bd. 5, p. $176-177$.

I854: v. Bogus lawski: Zehnter Nachtrag, Pogg.Ann. Ergz.-Bd. 4, p. 416.

1857: Arago: Astronomie populaire Bd, 4, p. 195 .

1859: H a rris: Dissert. Gött., p. 63. 1863: B u c hner: Meteoriten, p. I3. 1867: Go ebel: Kritische Uebersicht, Mélanges phys. chim. Bd. 7, p. 338 . 1867: Buchner: Dritter Nachtrag, Pogg. Ann. Bd. 132, p. 314, 318.

r884: M e uni er: Météorites, p. r9r,37r.

Von der nicht umgeschmolzenen Masse: 1) Io gr., 2) 15 gr., 4) I gr., 5) $2 \frac{1}{2}$ gr., 6) $6 \mathrm{gr}-3$ ) Similar in appearance to the Ovifac iron . - 7) Mit der Bezeichnung "Trier«. - 8) Mit der Bezeichnung sTrèves? «.

W ülfing, Meteoriten. 
r885: Brezina: Wiener Sammlung, p. $182,232$.

r887: Brezina: Neue Meteoriten III. Ann. Hof-Mus. Bd. 2 (Not.), p II5. r895: Brezina: Wiener Sammlung, p. 249 .

Ursprüngliches Gewicht: „Ein ziemlich grosser Stein «.

Nachweisbares Gewicht: I826 gr.

\begin{tabular}{lr|lr|} 
Berlin, U. & 19 & Paris, M. & 80 \\
Budapest & 4 & Petersburg, A. & 30 \\
Calcutta & 4 & v. Siemaschko & I \\
Dorpat & 90 & Stockholm & 66 \\
Kiew & 1395 & Washington, M. & 10 \\
London, B. M. & 9 & Wien, H. M.*) & rr8 \\
Neumann & Spl. & &
\end{tabular}

Die meisten in der obigen Literatur verzeichneten Angaben erwähnen nur den Fall; auch Meunier sagt: Aucune analyse minéralogique de cette roche n'a été publiée jusqu' ici du moins à notre connaissance $\approx$, stellt aber trotzdem eine Gruppe der Belajite auf. Nachdem nun bereits Ioo Jahre seit dem Fall verflossen sind, wäre wohl eine Untersuchung wünschenswert; Material ist ja genügend vorhanden.

Herr Direktor Brezina teilte mir früher mit, dass der Stein auf der Grenze von $\mathrm{Cg}$ und $\mathrm{Cc}$ stehe, wie Avilez,Müihlau und Motta di Conti; neuerdings spricht er ihn als $\mathrm{Cc}$ an.

Bjelokrynitschie, Volhynien, Russland.

Stein, Cib, gefallen I. Januar 1887 .

r8gr: Agafonov: Trav. Soc. Nat. Pet. Bd, 2I, p. 20 ; s. auch Agafonov, Rev. Sc. Nat. Bd. I (1891), p. $4 \mathbf{I}$.
1893: Brezin a: Ueber neuere Meteoriten (Nürnberg), p. I60.

1895: Brezina: Wiener Sammlung, p. 249 .

UrsprünglichesGewicht: ? (Auskunft geben wahrscheinlich die russischen Aufsätze, die mir nicht zugänglich waren.)

Nachweisbares Gewicht: I662 gr.

Böhm I3 London, B. M. 54

v. Braun $\quad 77$ Odessa 35

\begin{tabular}{ll|l} 
Budapest & 49 & Paris, M.
\end{tabular}

Dorpat 3I Petersburg, A. 3I

Gregory $\quad 245$ Rom, U. 34

$\begin{array}{lll}\text { Greifswald } & 20 & \text { v. Siemaschko } 273\end{array}$

Harvard, U. $\quad 9$ Stockholm 44

Kasan II Strassburg 9')

\begin{tabular}{ll|l} 
Krüger & I2 Wien, H. M. 616
\end{tabular}

\section{Blaauw Capel}

Utrecht

Black Mountain, Asheville, Buncombe County, North Carolina, U. S. A.

Eisen, $\mathrm{Og}$, gefunden um $\left.1839^{2}\right)$, beschr. 1847 .

1847: Shepard: Report on Meteorites. - I6. Black Mountain, head of Swannanoah River, eastern line of Buncombe county (fifteen miles east of Asheville) N. C., Am. Journ. (2) 4 , p. $82-83$. Ref. Liebig-Kopp, Jahresber. $1847 / 48$, p. I3IO-I3II ; Kenngott, Uebersicht $1850 / 51$, p. 136 .

1852: Clar k: Dissert. Gött., p. 34.

r854: v. Boguslaw ski: Zehnter Nachtrag, Pogg. Ann. Ergz.-Bd. 4, p. 407 -408 .

1859: B uc h n e r: Feuermeteore, p. 134. 1859: H a rris: Dissert., Gött., p. I12.

1) Professor Bücking bezeichnet in seiner Mitteilung den Stein als Cgb.

2) Im Jahr 1839 wurde schon ein Stück von Wien, H. M. erworben. Ueber die Fundzeit finden sich in Shepard's Aufsatz folgende Angaben aus einem Brief von 1846: ${ }_{\rightarrow}$ Dr. Hardy ... gave a . . . meteoric iron, found in this county (Buncombe) to the late Col. Nicholson .... who died... six or seven years agos; ferner: she year Col. Nicholson of Charleston died, he had obtained it in Pendleton or Greenville District. \& 
186r/62: v. Reic hen b a c h: XV nо, I24. XVI 26I. XVII 265. XVIII 487. XX $622,630,63$ I. XXI $578,580,589$. r863: Buchner: Meteoriten, p. 180I8I.

I863: Rose: Meteoriten, p. 26, 65, 153. 1884: M eunier: Météorites, p. $98,138$. x885: Brezina: Wiener Sammlung, p. $207,214,234$.

r89o: Eastman: Met. Astron., p. 318.

1893: Meunier: Révision des fers météoriques, p. 72.

Ursprüngliches Gewicht: $596 \mathrm{gr}$. (2 I Unzen).

Nachweisbares Gewicht: $384 \mathrm{gr}$.

Bailey

Berlin, U.

29 London, P. G. $\quad 76$

Budapest

Calcutta

Dorpat

33 Moskau

44 New Haven

22 Paris, M.

I9 v. Siemasch

Frankfurt

Spl. Tübingen

Kopenhagen

14 Wien, H. M.*

London, B. M

$7 \mathrm{I}$

Blansko, Brünn, Mähren, Oesterreich.

Stein, Cga, gefallen 25. Nov, 1833 . 1834: v. R e i c he $\mathrm{n} \mathrm{b} \mathrm{a} \mathrm{h:} \mathrm{Auszug} \mathrm{aus}$ der $\times$ Allgem. Zeitung $\approx$, im N. J. 1834, p. 125-126; s, auch N. J. 1835 , p. 48 (Vorzeigen auf der Naturf. Vers, in Stuttgart); Jahresber. schles. Ges. 18 34 , p. 10.

1834: v. Rei c hen ba $\mathrm{ch}$ : Ueber den Meteorsteinfall zu Stannern bei Blansko in Mähren am 25. Nov, 1833 . Baumgartner's Zeitschr. f. Phys. Bd. 3, p. 73 -77 . Ref. N. J. 1836, p. $496-497$; Philos. Magaz. Bd. 6 ( 1835 ), p. I59; Am. Journ. (I) 30 , p. 175-176.

1834: Berzeli us: Om Meteorstenar. ז) Meteorsten frin Blansko. Vetensk. Acad. Handl. (1834), p. 122-141; abgedruckt in Pogg. Ann. Bd. 33 , p. 825 ; s. auch Journ. prakt. Chem. Bd. 34 (1855), p. 123 (Vergleich mit Ski).
I835: v. H of $f$ : Neunter Nachtrag, Pogg.

Ann. Bd. 34 , p. 343 .

1836: K ä m tz: Meteorologie, p. 252, 253-255 (Berzelius' Arbeit), 300.

r839: B e n z en be r g : Sternschnuppen, p. $263-264$.

r84I : R a m m e 1s b e r g: Handwörterbuch, Bd. I, p. 433-434.

I843: $\mathrm{H}$ a i.d in g e $\mathrm{r}$ : Uebersicht, p. $\mathrm{I0} 8$. r843: P a r s c h: Meteoriten, p. 65-66. r843: R a mmelsberg: Ueber die Bestandteile der Meteorsteine. Pogg. Ann. Bd. 6o, p. $137-13$ s. Ref, N. J. 1844, p. 718 .

r845/53: R a m m e ls b e r g : Handwörterbuch, Suppl. II, p.92, Suppl, V, p. I6.

r847: Schafhäut 1: Schönenberg, Gel. Anz. München.Akad, (1847), p. 573. 1849: B a d e n-P o w e 11: Luminous Meteors. Appendix Nr. 1. Fall of Meteorites at Stannern, near Blansko, Moravia, Nov. 25. 1833 . Note from W. W. Smyth. Rep. Brit. Assoc. (1849), p. 32 (Sep.).

1854: v. Boguslawski: Zehnter Nachtrag, Pogg. Ann. Ergz.-Bd. 4, p. $30-32,33$.

r859: $\mathrm{B}$ u c h ner: Feuermeteore, p. 90 -92 .

I859: H a r r i s : Dissert. Gött., p. $83-84$. 1860: R a mmels berg: Mineralchemie, p. 922 ff., 950.

r863: B u c h ner: Meteoriten, p. 56-57. 1863: Rose: Meteoriten, p. 31, 87 , IOI-103, 106, 154 .

r857/65: v. Rei c h e n b a c h: III 624 . V $477,479,480$ VI $44 \mathrm{I}, 454$. VII 56r. IX 159, 162, 171, 175, 180. X 357 , $359,366 . X I 294,297,301,302,309$. XII 454. XIII $355,356,358,362$, $364,365,370$ (Fig.), 371(Fig.). XIV 388 , 392. XV 10I, 120, 121, 131. XVI 262. XVII 269. XVIII 490. XX 623, 624, $626,634,635$. XXIV s. f. Zitat. XXV $318,321,324,428,429,433,600$, $603,607,608$.

I865: v. Reich e n b a c h: Geschichte 
des Meteoriten von Blansko nebst Anleitung zu methodischer Aufsuchung frisch niedergefallener Meteoriten. Pogg. Ann. Bd. 124, p. 213-234.

1867: W ank e 1: Ueberden Meteoritenfall im Jahre 1833 in der Nähe von Blansko. "Lotos (Prag), p. 73-77 u. 103-109 (Ein bis dahin ungedruckter Bericht Reichenbach's.).

r869: Buchner: Vierter Nachtrag, Pogg. Ann. Bd. 136, p. 446.

r870: $\mathrm{R}$ a mmelsberg: Meteoriten, p. $98,100-102,103,105,106,138$, 139, 140.

188I: v. Nordenskiöld: Zeitschr. d. d. geol. Ges. Bd. 33, p. 24 (Vergleich mit Ställdalen).

r884: M e u n i e r : Météorites, p. 35, 79, $85,256,257-258,395$.

1885: Brezina: Wiener Sammlung, p. $182,232$.

1886: M e li o n: Die Meteorsteinfälle in Mähren. Notizbl. d. mähr. schles. Ges. z. Beförd. des Ackerbau's etc. (1886) Nr. 5 und 6 , p. $4-5,7$, 10, II.

r8go: v. $\mathrm{N}$ i ess 1: Periheldistanzen, Verh. naturf. Ver. Brünn Bd, 29, p. 188, 195 , $214,242-243$.

r894: C o hen: Meteoritenkunde, p. 56, $85,208,247,248$.

Ursprüngliches Gewicht: $35^{\circ} \mathrm{gr}$. (20 Lot), 8 Steine.

Nachweisbares Gewicht: 243 gr.

\begin{tabular}{lr|lr} 
Bement & 2 & Paris, M. & 2 \\
Berlin, U. & 26 & Pohl & 20 \\
v. Braun & 3 & Stockholm & 5 \\
Budapest & 6 & Stuttgart & 19 \\
Calcutta & I & Tübingen & 88 \\
Gregory & 2 & Wien, H. M.*). & 69 \\
London, B. M. Spl. & &
\end{tabular}

Blount Co.

Summit

Blue Tier, N.O.-Küste von Tasmanien.

Eisen, gefunden 1890 , beschr. 1893 . r893: Petterd: Catalogue of Minerals of Tasmania, p. 40.

Ursprüngliches Gewicht: $\mathbf{r} \cdot 3 \mathrm{Kgr}$. Nachweisbares Gewicht: ?

Mitteilung von Herm Direktor Brezina.

Bluff, am Colorado, Lagrange, FayetteCo., Texas (s.auch TravisCo.). Stein, Ckb, gefunden um 1878 , beschrieben $\mathrm{r} 888$.

1888: Whitfield und Merrill: The Fayette County, Texas, Meteorite. Am. Journ. (3) 36, p. 113-119 (Analyse u. Abbildung). Ref. N. J. 1889 II, p. 444 -445 .

I888: Ho w e 11: „Science *; s, auch The Ward Collection of Meteorites. Rochester. N. Y. 1892 , p. $55-61$.

r888: M e un i er: Détermination lithologique de la météorite de Fayette County Texas. C. R. Bd. 107, p. 1016Ior8 (Analyse). Ref. N. J. 189o I, p. 42. r889: v. H a u e r : Ann. Hof-Mus. Bd. 4 (Not.), p. 64 und Bd. 5 (189o) Not., p. 62 .

r89o: E a s t m a n : Met. Astron., p. 320. 1893: Brezin a: Ueber neuere Meteoriten (Nürnberg), p. 162.

1893: Newton: Lines of structure in the Winnebago Co. Meteorites and in other Meteorites. Am. Journ. (3) 45, p. $152-153,355$. Ref. N. J. 1894 I, p. $273-274$.

I894: C o h e n: Meteoritenkunde, p. 6I, 202, 298, 316.

r895: Brez in a: Wiener Sammlung, p. $26 \mathrm{I}$.

Ursprüngliches Gewicht: $146 \mathrm{Kgr}$. Nachweisbares Gewicht: 74400 gr.

\begin{tabular}{lrl|lr} 
Bailey & IOor & v. Braun & 524 \\
Bayet & 10 & Brezina & 506 \\
Belgrad & 324 & Budapest & 240 \\
Bement & 1478 & Cleveland & 41 \\
Berlin, U. & 50 & Dorpat & 153 \\
Böhm & 23 & Dresden, M. & $166^{1}$ )
\end{tabular}

1) 44 gr. \$Bluff a 122 gr. \$Fayette Co. 


\begin{tabular}{lr|lr} 
Giessen & 50 & Paris, M. & 325 \\
Gregory & 1160 & Pohl & 184 \\
Greifswald & 18 & Prag, M. & 145 \\
Harvard, U. & 8040 & Roebling & 486 \\
Howell & 7680 & Rom, U. & 50 \\
Kopenhagen & 338 & v. Siemaschko & 335 \\
Kristiania & 166 & Stockholm & 108 \\
London,B.M. 12700 & Strassburg & $105^{2}$ ) \\
de Mauroy & 78 & Stürtz & 89 \\
Minneapolis & $\left.7258^{1}\right)$ & Troyes & 7 \\
München & 152 & Ward & $1233^{2}$ \\
New Haven & 780 & Washington & 247 \\
Newton & 137 & Wien,H.M. 16 738 ${ }^{3}$ ) \\
Odessa & 64 & Würzburg & 79 \\
Paris, E. & 33 & &
\end{tabular}

Bocas, Hacienda de Bocas, San Luis Potosi, Mexico.

Stein, $\mathrm{Cw}$, gefallen 24. Nov, 1804 .

I865: Burk a rt: Verh, naturh. Ver,Bonn Bd. 22 (Sitzber.), p. 71 (berichtet über Mitteilungen von Castillo über mexikanische Meteoriten).

1866: B u chner: Neue Meteoriten,

Pogg. Ann. Bd. 129, p. $35^{0}-351$.

1866: Burkart: Fundorte III, N. J.

I866, p. 4 OI.

1869: B u chner: Vierter Nachtrag,

Pogg. Ann. Bd. 136, p. $449-450$.

1870: Burkart: Fundorte IV, N. J. 1870 , p. 689.

r875: v o m R a th: Meteoriten, Verh. naturh, Ver. Bonn Bd. 32 , p. 367 Nr. 28. 1884: M e unier: Météorites, p. 209. 1885: Brezina: Wiener Sammlung,

p. $177,232$.

1889; Cas ti11 o: Météorites, p. I3. 189o: F l et c her: Mexican Meteorites,

Mineral. Magaz. Bd.9, p. 95.

Ursprüngliches Gewicht: ?

Nachweisbares Gewicht: 14 gr.
Berlin, U. $\quad 2$ London, B. M. Spl.

Bonn

I Paris, M.

\begin{tabular}{ll|l} 
Calcutta & I & Wien, H. M. *) I
\end{tabular}

Bogota Rasgata

Bohême s. Anhang

Bohumilitz, Kreis Brachin, Böhmen, Oesterreich.

Eisen, $\mathrm{Og}$, gefunden 1829.

1830: Verh. d. Ges. d, Vaterl. Museums Böhmen Bd. 3? p. 15 (oder 15. April I830 ?). (Laspeyres, Bonner Sammlung, verweist noch auf p. 17 und 29).

1830: B r ew s t e r 's Journal, Juli 1830 ; s. auch Am. Journ. (I) I9 [1831], p. $334-336$.

183r : v. Holg e r : Analyse des Meteoreisens von Bohumilitz. Baumgartner's Zeitschr. f. Phys, u. Math. Bd. 9, p. 323 -328 .

1832: Berzelius: Undersökning af en vid Bohumilitz i Böhmen funnen jernmassa. Vetensk. Acad. Handl. (1832), p. 106; Uebersetzung in Pogg. Ann. Bd. 27 (1833), p. $118-132$ : Untersuchung einer bei Bohumilitz in Böhmen gefundenen Masse; s. auch Pogg. Ann. Bd. 33 (1834), p. $137,147-148$ (v.Holger's Beryllium bestehe aus Phosphorsäure); Ann. Pharm. Bd. 8 (1833), p. 172 ; N. J. 1833 , p. $335-336$; Baumgartner's Zeitschr. f. Phys. und verw. Wissensch. Bd. I (1832), p. $289-297$. 1833: v. Holg er: Bemerkungen zur Berzelius'schen Analyse des Meteoreisen von Bohumilitz. Baumgartner's Zeitschr. f. Phys, und verw. Wissensch. Bd. 2, p. $35-37$; s. auch N. J. 1836 , p. 497 . r835: v. Hoff: Neunter Nachtrag, Pogg.

Ann. Bd. 34, p. $344-346$.

1836: K ä m tz: Meteorologie, p. 26r, 262. 184I: Rammelsberg: Handwörter-

I) $\gg 16 \mathrm{lbs} \alpha$.

2) 73 gr. $\gg$ Bluff Ck«, 32 gr. \$Fayette Co. Cg«. Auch Brezina sagt, dass der Stein auf der Grenze von Ckb u. $\mathrm{Cgb}$ stehe.

3) Ausserdem 336 gr. nach 1. Juli 1893 erworben. 
buch Bd. I, p. 424.

I843: P a r t s c h: Meteoriten, p.ri7-I20. 1848: Ha id inger: Haiding. Berichte

Bd. 3 , p. $69-71,282$ (Dieser Hinweis ist Laspeyres, Bonner Sammlung, entnommen).

1852: Wöhler: Passiv, Pogg. Ann. Bd. 85 , p. 448 .

1852: Cla rk: Dissert. Gött., p. 30-31. 1854: v. Boguslawski: Zehnter Nachtrag, Pogg. Ann. Ergz, - Bd. 4, p. $385-386$.

1855: Haidinge $r$ : Bemerkungen über die zuweilen im geschmeidigen Eisen entstandene krystallinische Structur, verglichen mit jener des Meteoreisens. Sitzber. Wien, Akad, Bd, 15 I, p. 357 (Fig. 6).

1859: B u c hner: Feuermeteore, p. 123 -124 .

1859: Harris: Dissert. Gött., p. Ho.

r860: Rammels berg: Mineralchemie, p. $903,947,948$.

1863: B uchner: Meteoriten, p. 158 -160 .

1863: Ro se: Meteoriten, p. 31, 32, 55, 56 Taf. II, 56-57, 152, 159 .

I85865: v. Reichen b a c h: IV 638. VI 449,452 . VII 55 I. VIII 488 . IX 162, 174, 175, 181, X 359. XII 457. XIII 363. XIV 390. XV I09, IIO, 124, 128. XVI 250, 255, 261. XVII 272 , XVIII $480,484,487,490$. XIX 155. XX 622, 625, 631. XXI 573, 587 . XXIV 227. XXV 437.

1869: Meunier: Recherches, Ann. Chim. Phys. (4) I7, p. 72.

1870: R a mmelsberg: Meteoriten, p. $8 \mathrm{I}, 83,84$.

1875: vom Rath: Meteoriten, Verh. naturh. Ver. Bonn Bd. 32, p. 362. I875: Mo hr: Entstehungsart, Ann. Chem.

Pharm. Bd. 179, p. 259, 268.

188I : B rezina: Bericht III, Sitzber.

Wien. Akad. Bd. 84 I, p. 282 .

r88r: S h ep a r d : Vergleich mit Lexington. Am. Journ. (3) 2I, p. $117-119$.
Ref. N. J. I881 II, p. 344 .

1884: M e unier: Météorites, p. 99, I16, 120.

1885: Brezina: Wiener Sammlung, p. $207,214,234$.

I888: Warren: Detection and estimation of Selenium in Meteoric Iron. Chem. News Bd. 57, p. 16. Ref. N. J. 1890 Il, p. 229 ; s. auch Chem. Centr. Bd.I9, p. 300 (Letzterer Hinweis ist Laspeyres, Bonner Sammlung, entnommen). 1889: C o he n: São Julião, N. J. 1889 I, p. $222,224,225$.

1890: Brezina: Ueber Meteoreisen, Oesterr. Zeitschr. f. Berg- u. Hüttenw. Bd. 38, p. 356 .

r89r: Cohen und Weinschenk: Meteoreisen-Studien. Ann, Hof-Mus. Bd. 6, p. 131, I43-I44 (Analyse), 160,164 .

1892: In diesem Jahre wurde ein $962 \mathrm{gr}$ schweres Stück Eisen auf dem Gut Skalitz (bei Smrcna?) bei Bohumilitz gefunden, welches genau mit dem im J. 1829 gefundenen Eisen übereinstimmt. Briefl. Mitteilung E. v. Lumke's an Herrn Direktor Brezina.

1893: M e unier: Révision des fers météoriques, p. 25, 26 (Abb. einer geätzten Platte).

r894: Co h e n : Meteoritenkunde, p. 22, $40,47,54,56,67,121,131,150$.

Ursprüngliches Gewicht: Ueber $57 \mathrm{Kgr}$.

Nachweisbares Gewicht : $46856 \mathrm{gr}$.

\begin{tabular}{lr|lr} 
Bailey & 5 & Gregory & $\mathbf{1 4 2}$ \\
Bement & 2 & Halle & 13 \\
Berlin, U. & I405 & Harvard, U. & 49 \\
Bonn & 44 & Leoben & 19 \\
Brezina & II2 & London, B. M. & I18 \\
Budapest $:$ & 715 & London, P. G. & 117 \\
Calcutta & 20 & Melion & 5 \\
Dresden, M. & 37 & Moskau & 10 \\
Gotha & 36 & Neumann & 57 \\
Göttingen & 31 & New Haven & 37 \\
Graz, J. & 50 & Paris, M. & 1585
\end{tabular}




\begin{tabular}{lr|l|r|} 
Pohl & 139 & Stuttgart & Io \\
Prag, B. U. & 64 & Tübingen & 293 \\
Prag, D. U. & 427 & Turin, U. & 44 \\
Prag, M. & 38282 & Washington, Sh. \\
Rom, U. & 198 & Wien, H. M.*) & 2694 \\
v. Schilling & 20 & Wrany & 65 \\
v. Siemaschko & 10 &
\end{tabular}

Bois de Fontaine Charsonville Bokkefeld Cold Bokkefeld Bolson de Mapimi Coahuila Bonanza Coahuila

Borgo San Donino (Parma), Pieve di Cusignano, Parma, Italien.

Stein, Cho, gefallen 19. April 1808 .

I808: Guidott i: Memoria fisico-chimica sulle pietre cadute dall' atmosfer? nel circondario di Borgo-San-Donino il giorno 19. April 1808. Parma bei Giuseppe Paganino kl. $8^{\circ} .43$ Seiten; s. auch »Encyclopédie Bd. 5 (1808), p. $596-602$.

1808: A moretti: Nuova Scelta d'opuscoli da C. Amoretti Bd. II, p. 275. 1808: Sur un météorolite tombé du côte de Parme. Journ. Phys. Bd, 66, p. 459. 1808: Gilbert: Einige Nachrichten von den drei neuesten Steinregen und von drei Meteorsteinen aus Russland. 2) Auszug eines Schreibens des Herrn C. Amoretti an seine Excellenz den Herrn Ritter Laudriani. Steinregen im Piacentinischen am 19. April 1808. Gilb. Ann. Bd. 29, p. 209-211.

1808: Chladni: Beiträge, Gilb. Ann. Bd. 29, p. 375 .

1809: Va uquel in: Sur l'aërolithe tombé aux environs de Parme, pour y découvrir la présence de l'alumine, annoncé par M. Sage. Ann. Chim. Bd. 69, p. $280-284$.

I809: V a u q u elin: Versuche über den von Herrn Sage angekündigten Thonerde-Gehalt eines Aërolithen, Gilb. Ann.

Bd. 33 , p. 198-201.

1812: Chladni: Verzeichnis, Schweigg.

Journ. Bd. 4 Beilage I, p. 16-17.

1812: B igot de Morogues, p. $222-$

229, $332-333$.

1815: Chladni: Neues Verzeichnis,

Gilb. Ann. Bd, 50, p. 254.

r8rg: C h 1 a d n i : Feuermeteore, p. 4I, $58,66,69,73,9 \mathrm{I}, 284-286(!), 290$, 297, 308,428 .

r836: K ä m t z: Meteorologie, p. 282.

1843: P artsch: Meteoriten, p. 30-31.

r854: Balc ells: Lithologia meteorica, p. 23 .

r859: H a rri s: Dissert. Gött., p. 68. 1863: Buchner: Meteoriten, p. 23. 1863: R os e: Meteoriten, p. 88, 154. r858/65: v. Reichenbach: V 477. VI 441 . IX $161,169,179 . X$ 359. XI 294. XIII 357,378 . XIV 389. XX $622,626 . \mathrm{XXV} 615$.

r884: M e unier: Météorites, p. 230 -231 .

1885: Brezin a: Wiener Sammlung, p. 177,232 .

1894: Cohen: Meteoritenkunde, p. 8. UrsprünglichesGewicht:VieleSteine. Nachweisbares Gewicht: I $_{5} 16 \mathrm{gr}$.

\begin{tabular}{lr|l|l|} 
Bailey & Spl. & London, P. G. & 2 \\
Bement & I & Modena & I \\
Berlin, U. & r5 & Neumann & 2 \\
Bologna & 164 & New Haven & 10 \\
v. Braun & 9 & Paris, E. & 59 \\
Budapest & 29 & Paris, M. & 428 \\
Calcutta & 4 & Parma & $477^{1}$ ) \\
Göttingen & Spl. & Rom, U. & I8 \\
Gregory & 6 & v. Siemaschko & 5 \\
Harvard, U. & Spl. & Tübingen \\
Klausenburg & II & Wien,H.M.*)**) 264 \\
London, B. M. & I0 & \\
Nach Buchner (1863) soll Graf Linati in
\end{tabular}

1) Herr Professor Vittorio Simonelli hatte die Güte, mir noch mitzuteilen : „Son poids orig. était de $893 \mathrm{gr}$. Une partie fut cédée par Guidotti à M. Chladni et des fragments se trouvent dans les collections de Bologne et de Rome*. 
Parma den grössten Stein besessen haben ; meine Bemühungen, darüber Näheres zu erfahren, waren vergeblich.

Bori, I 2 miles nordöstlich Badnur,

Betul Distrikt, Zentralprovinzen, Ostindien.

Stein, Cia, gefallen 9. Mai 1894 .

r895: Brezina: Wiener Sammlung, p. 248 .

Ursprüngliches Gewicht: 186r7 gr. Nach gütiger Mitteilung des Herrn Direktor Holland in Calcutta.

Nachweisbares Gewicht: $5_{22} 87$ gr. Es ist vermutlich noch mehr in Sammlungen aufbewahrt, aber wegen des späten Failtermines nicht in den mir zugeschickten Listen aufgeführt.

$\begin{array}{lr}\text { Brezina } & 24 \\ \text { Calcutta } & 49 \mathrm{r} 8 \text { (Dez. 1895) } \\ \text { Wien, H. M. } & 345\end{array}$

Borkut, an der schwarzen Theiss, Marmaroscher Comitat, Ungarn. Stein, Cc, gefallen $1_{3}$. Oktober 1852 .

1856 : Leydolt: Ueber den Meteoriten von Borkut. Sitzber. Wien. Akad. Bd. 20 ( $1856 \mathrm{II}$ ), p. $398-406$ (Analyse von Nurisany). Ref. N. J. I857, p. 177-179; Kenngott, Uebersicht 1855 , p. 159 u. $1856 / 57$, p. 237 ; Liebig-Kopp , Jahresber. 1856 , p. 912 ; "L'Institut \& Bd, 23 (1855), p. 459.

r859: B u c hner: Feuermeteore, p. IOI $-102,182$.

1859: Harris : Dissert. Gött., p. 95.

r860: $\mathrm{R}$ ammels berg : Mineralchemie, p. 923 ff., 950.

I863: Buchner: Meteoriten, p. $83-84$. r858/65: v. Reichenbach: V 475, 477,481 . VI $441,443,445,456$. IX I6I, I64, I7I, I80. X 359. XI 295, 306. XIII $355,357,358,36 \mathrm{I}, 369$ (Fig.), 377. XIV 399. XIX 152. XXIII 368,370 . XXV 606, 6r5.
1870: Rammels berg: Meteoriten, p. $103,105,106,138,139,140$.

1879: R a m mels berg : Meteoriten, p. 25 .

r880: $\mathrm{Hahn}$ : Die Meteorite (Chondrite) und ihre Organismen. Tübingen. T. 29 Fig. 5, T. 32 Fig. 2.

r884: Meunier: Météorites, p. 79,85 , 188, I89-I9o, 395 .

r883/85: T/ s c h erm a k: Photographien, T. 9,19, p. $3,13,16,17$.

1885: Brezina: Wiener Sammlung, p. $185,233$.

1894: Co h e n, Meteoritenkunde, p. 6o, 330.

Ursprüngliches Gewicht: $6.7 \mathrm{Kgr}$. (12 Wiener Pfund). Ein Stein.

Nachweisbares Gewicht: 4086 gr.

Bement

v. Braun

2 Neumann

Budapest

Calcutta

Gregory

6 Paris, M.

8

London,

Melion Spl. Wien, H. M.*)**) 191

22I Pohl

Spl. Siemaschko 134

41.74

41 Tübingen $343^{1}$

Borodino, Fluss Stonitza, bei Kolotscha, Moskau, Russland,

Stein, Cgb, gefallen 5. oder 6. September 1812 (neuen Stils).

r895: Brezina: Wiener Sammlung, p. 250 .

Ursprüngliches Gewicht: ?

Nachweisbares Gewicht: 325 gr.

Krüger

Paris, M.

I Petersburg, B. 320

2 Wien, H. M. 2

Der Stein wäre nach dem obigen Datum, welches Herrn Direktor Brezina von Herrn v. Siemaschko mitgeteilt wurde, kurz vor der Schlacht bei Borodino (7-8. September 1812$)$ gefallen, Herr St. Meunier teilt mir den 7. Sept. 1812 als $\mathbf{V}$ alltag mit. Die beiden Heere standen seit dem 5. September einander gegenüber. Meine Bedenken gegen die Authentizität dieses Falles hat Herr 
Direktor Brezina in seiner oben angeführten Arbeit ausgesprochen; indessen gründen dieselben sich nicht auf das eigentümliche Zusammentreffen der beiden Ereignisse, sondern vielmehr darauf, dass dieses Zusammentreffen nicht hervorgehoben wurde. Auch hatte Siemaschko als ganze Fallmasse nur 5-6 gr. angegeben. Inzwischen habe ich ganz kürzlich erfahren, dass die Petersburger Bergschule ein grösseres Bruchstück dieser Lokalität besitzt. Herr M. Melnikoff hatte die Güte, mir auf meine Anfrage mitzuteilen, dass Siemaschko eine Broschüre über diese Lokalität verfasst habe. Danach sei der Fall von einem Soldaten, der auf Wache stand, beobachtet, der Meteorit als Familienstein aufbewahrt und $\mathbf{1} 890$ von Herrn Herke für das Petersburger Berginstitut angekauft worden.

\section{Botetourt}

s. Anhang

Botschetschki, Kreis Putiwl, Gouvernement Kursk, Russland.

Stein, Cg, gefallenDezember (?) 1823 .

1843: Partsc h: Meteoriten, p. 7o.

1847: E i c hwald: Verzeichnis, Erman's Arch, f. wissensch. Kunde Russlands Bd. V, p. 180.

r848: B 1 o e d e: Tabelle, Bull. Petersburg. Akad. Bd. 6, p. 6 .

1854: H a rri s: Dissert. Gött., p. 89. 1859: v. Reichen b a c h: IX 161, 171, 180. XI 306.

1863: B u chner: Meteoriten, p. II4 $-\mathrm{II} 5$.

1867: Go e be 1: Kritische Uebersicht, Mélanges phys. chim. Bd. 7, p. 304 $-306,313$.

1867: B uchner: Dritter Nachtrag, Pogg. Ann. Bd. 132, p. 315-316.

1885: Brezina: Wiener Sammlung, p. $182,232$.
Ursprüngliches Gewicht: Wohl das von Bloede angegebene Stück von $\mathrm{I}$ Pfd. 48 Zolotnik $=6 \mathrm{I} 4 \mathrm{gr}$. Nachweisbares Gewicht: $55^{\circ} \mathrm{gr}$.

\begin{tabular}{lr|lr} 
Bailey & 3 & Krantz & Spl. ${ }^{1}$ ) \\
v. Braun & Spl. $\left.{ }^{1}\right)$ & Petersburg, A. & 544 \\
Calcutta & Spl. & Wien, H. M. *) & 3
\end{tabular}

\section{Böttcher-Insel Mauritius}

Bourbon-Vendée 1812 , Chantonnay Bourbon-Vendée 1841 , Saint Christophe la Chartreuse

\section{Bourdeaux}

Mornaus

Boyett

Cross Roads

Brahin (Rokicky), Gouv. Minsk, Russland.

Pallasit, P, bekannt 1810. In der älteren Literatur wird mehrfach r809 angegeben. Partsch sagt: $\$ 1810$ (nicht 1809)《. Herr Prof. Armaschewski (Kiew) teilt mir mit »1810 (Datum?) \&. Erwähnt 1817 .

1817: Laugier: Mém. du Mus. 1817 Bd. $6(?)$.

r8rg: $\mathrm{Ch}$ ladni: Fünfte Fortsetzung, Gilb. Ann. Bd. 63 , p. $32-33$.

1821: Chla d ni: Erster Nachtrag, Gilb. Ann. Bd. 68, p. 342 .

1823: La u g i r in Bull. des Sc. de la Soc. Philom. Juni 1823 ; Uebersetzung in Gilb. Ann. Bd. 75 (1823), p. 267 -269 unter dem Titel: Vorläufige Nachricht von der chemischen Analyse zweier in Polen gefundenen Aërolithen und zweier Meteoreisenmassen. Mit Bemerkungen von Gilbert; s. auch Ann. Chim. Phys. Bd. 25 ( 1824 ), P. $220-$ 221 und Ann. du Mus. d'hist. nat. 6. Jahrgang II. Heft ; Schweigg. Journ, Bd. 43 (1825), p. $25-26$.

r823: Ch la dn i : Dritter Nachtrag, Gilb.

I) Bezeichnet als Kursk ohne Angabe der Fallzeit, daher wohl nicht Sevrukof. 
Ann. Bd. 75, p. 230 (Anmerkung von Gilbert).

1824: Chladni: Vierter Nachtrag,

Pogg. Ann. Bd, 2, p. 16I-162.

1826: S eebeck: Ueber die magneti-

sche Polarisation der Metalle und Erze

durch Temperaturdifferenz. Pogg. Ann.

Bd. 6 , p. 144 .

184I : $\mathrm{R}$ a $\mathrm{m} \mathrm{mel} \mathrm{s}$ b e r $\mathrm{g}$ : Handwörterbuch, p. 423 .

1843: Part s ch: Meteoriten, p. 90-9r. 1847: Eichwald: Verzeichnis, Erman's Arch. f. wissensch. Kunde Russlands Bd. 5 , p. 183 .

1852: C l a r k : Dissert. Gött., p. 2I - 22. 1854: v. Boguslawski: Zehnter Nachtrag, Pogg. Ann. Ergz.-Bd. 4, p. $393-394$.

I859: B u c hn er: Feuermeteore, p. I05. 1859: Ha r ris: Dissert. Gött., p. 106-I07. I860: R a m m els b erg : Mineral chemie, p. 908-909, 949.

1859/62: v. R eich en bach: 1X 162, $163,173,181 . X 359,363,364,372$. XI 296, 302. XII 452, 454, 461. XV 104, 107, 112, 116, 126 XVI 261. XVII 265,272 . XVIII. 481,487 . XIX 155. $\mathrm{XX} 627$.

1863: B u c hner: Meteoriten, p. 129. r863: R os e : Meteoriten, p. 26, 30, 4I, $73,76,139,153$.

1867: D a u b ré e : Contribution à l'anatomie des météorites. C. R. Bd. 65 , p. 149.

r869: v. Inostranzew: Verh. der russ. K. mineralog. Ges. Petersburg (2) 4, p. 202 ; s. auch v. Kokscharow, Materialien Bd. 7 (1875), p. $216-217$ (Analyse des Olivins).

1870: Rammelsberg: Beiträge zur Kenntnis der Meteoriten. Ueber die Analyse von Meteoriten. 3) Die Analyse der Silikate. - B. Der Pallasit von Brahin. Mon.-Ber. Berlin Akad. (1870), p. $444-445$.

1870: Rammelsberg: Meteoriten, p. $79,87,88,138$; s. auch Pogg. Ann.
Bd. $140(1870)$, p. 312 .

r879: Ramme lsberg: Meteoriten, p. 6,24 .

1883: Tschermak: Beitrag, Sitzber.

Wien, Akad, Bd. 88 I, p. 348 .

r884: M e unier: Météorites, p. 79,

96, 143-144 (Abb.), 356, $35^{8}$.

r884: Wadsworth: Studies, p. 72.

r883/85: Ts c h erma k: Photographien, T. 25, p. 23,24 .

1885: Brezina: Wiener Sammlung, p. $206,233$.

r886: Brez ina und Cohen: Photographien, T. 5 .

1887: Flig ht: Meteorites, p. IOO-IOI. 1894: C o h e n : Meteoritenkunde, p. 248, $252,254,256,263$.

1895: Meunier: Révision des lithosidérites, p. 15, 17-19 (Abb. einer geätzten Platte).

1895: Brez ina: Wiener Sammlung, p. 265 .

Ursprüngliches Gewicht: Etwa 100 Kgr., es sollen zwei Massen gefunden worden sein, von denen die eine $79 \mathrm{Kgr}$. (193.5 Pfd.) wog.

Nachweisbares Gewicht: 92886 gr.

\begin{tabular}{lr|lr} 
Bailey & II & Paris, M. & 281 \\
Berlin, U. & 3 3 & Petersburg, B. & 234 \\
Budapest & 53 & Pohl & 249 \\
Calcutta & 250 & v. Siemaschko & 60 \\
Dorpat & 16 I & Stockholm & 19 \\
Göttingen & 17 & Troyes & 14 \\
Gregory & $3 \mathrm{I}$ & Tübingen & 30 \\
Harvard, U. & 35 & Washington & 14 \\
Kiew & 87 & 285 & Washington, Sh. \\
London, B. M. & 22 & Wien, H. M. *) & 3320 \\
London, P. G. & 6 & Wien, U. I. & 82 \\
Neumann & 4 & Wien, U. II. & 390
\end{tabular}

\begin{tabular}{lr} 
Brambanan & $\begin{array}{r}\text { Prambanan } \\
\text { Gramudor }\end{array}$ \\
Brandentillo \\
Brandenburg, Eisen & Seeläsgen \\
Brandera Co. Stein & Linum \\
\hline
\end{tabular}


Braunau, Hauptmannsdorf, Kreis Königgrätz, Böhmen.

Eisen, H, gefallen 14 . Juli 1847 .

1847: $\mathrm{H} \mathrm{umbol} \mathrm{d}$ : Aérolithe de Braunau, en Bohême, tombé le 14 jouillet I847 (Extrait d'une lettre de M. de H. à M. Arago). C. R. Bd. 25, p. 627 ; s. auch s'Institut $\propto$ Bd. 15 (1847), p. 354 .

r847: Göppert und v. Bogus l awski: Meteorsteinfall, Journ, prakt. Chem. Bd. 42 , p. 59-62 (Bericht von Augenzeugen); s.auch w.u.1847: Beinert.

1847: Göppert: Ueber das Braunauer u. das Seeläsgen'sche Meteoreisen. Journ. prakt. Chem. Bd. 42 , p. $428-431$; s. auch Wochenschr, f. Astr., Met. u. Geogr. Bd. 1 (1847)， p. 263,340 ; Bd. 2 (1848), p. 24.

r847: B einert: Meteoreisenfall bei Braunau in Böhmen am 14. Juli d. J., Pogg. Ann. Bd. 72, p. 170-173 (Abb.). Ref. N. J. 1848 , p. 729-731; s. auch Schles, Ges. f. vaterl. Kult, 1847 , p. 36 -38 ; Journ. prakt. Chem. Bd. 42 (1847), p. 59-62 und eine kurze Notiz im Am. Journ. (2) 5 (1848), p. 285.

1847: Duflos und $\mathrm{F}$ is cher: Analyse des. Braunauer Meteoreisens. Pogg. Ann. Bd. 72, p. 475-48o; s, auch Schles. Ges, f. vaterl. Kult, 1847 , p. 38 -46 ; Journ, prakt. Chem. Bd. 42 (1847), p. 62; Am. Journ. (2) 5 , p. $338-342$; N. J. 1847 , p. 853 u. 1848, p. 577 .

1847: F is cher: Fortsetzung der Untersuchung des Meteoreisens von Braunau. Pogg. Ann. Bd. 72, p. 575-580; s. auch Schles. Ges, f. vaterl, Kult. 20. Oktober 1847 ; N. J. 1848, p. 320-321; Am. Journ. (2) 7 (1849), p. 171-175.

1847: Haidinger: Bericht in derVersammlung am 8. Oktober. Mitteilungen d. Freunde d. Naturw. Bd. 3, p. 302 -305 . Weitere Mitteilungen nebst Abbildung einer Platte, p. $37^{8}-38$ o. Wei- tere Mitteilungen, p. 493-494; s. auch Pogg. Ann. Bd. 72 , p. $580-582$. r844/49: K en ng o t $t$ : Uebersicht, p. 222 -224 (Ref. über mehrere der obigen Arbeiten).

1847: Poggendorff legt Zeichnungen vor. Mon,-Ber. Berlin. Akad., p.243.

1847: W e iss legt ein Stïck vor. Mon,Ber. Berlin Akad,, p. 391.

1848: N e u m a n n: Mitteilung über die krystallinische Struktur des Meteoreisens von Braunau und über die krystallographische Orientierung der Linien, welche durch die Aetzung der Flächen hervortreten. Haidinger's Mitteilungen d. Freunde d. Naturw. Bd. 4, p. $86-$ 87 ; s. auch Oesterr. Bl. f. Lit. u. Kunst etc. Bd. 2 (1848) Nr. 26, p. Ioo. Ref. N. J. 1848 , p. 825 .

1848: Beinert: Der Meteorit von Braunau. Aktenmässige Darstellung, Beschreibung und Analyse nebst Ansichten über die Natur der Meteoriten (Situationsplan; 3 Tafeln mit Abb.); Breslau 1848 ; in Kommission bei Eduard Trewendt ; s. auch Haidinger's Mitteilungen d. Freunde d. Naturw. Bd. 4 ( 1848$)$, p. $349-35^{1}$ (Vorzeigen dieses Werks); v. Boguslawski im Tageblatt d, naturw. Ver. Breslau 1847 , p. $36-48$.

I848: Li ebig-K o p p : Jahresber. p. 1300 -1304 . Referate über viele der obigen Arbeiten,

1848: G lock e r: Ueber die krystallinische Struktur des Eisens. Pogg. Ann. Bd. 73 , p. $332-336$.

1848: Fis cher: Schluss der Untersuchung des Braunauer Meteoreisens. Pogg. Ann. Bd. 73 , p. $590-594$.

1848: S h ep a r d: Observation on Rammelsberg's Analysis of the Juvenas Meteoric Stone and on the Conclusion of Fischer's Examination of the Braunau Meteoric Iron. 2) Fischer's Analysis. Am. Journ. (2) 6, p. $348-349$ (Shepard's Dyslytite gleich Schreibersit). 1849: R a m m elsberg : Handwörter- 
buch, Suppl. 4, p. 15I-I52.

1849: B a d en-Powe11: Luminous Meteors; Nr. 2. Meteorite of Braunau. Note from W. W. Smyth. Rep. Brit. Assor. (1849), p. 32-34 (Sep.).

1850: Neumann: Ueber die krystallinische Struktur des Meteoreisens von Braunau. Haidinger's Naturw. Abh. Bd. 3 , p. $45-56$ (Tafel).

1852: Cl a r k : Dissert. Gött., p. 52- 55. r852: W öh 1 er : „Passiv«, Pogg. Ann. Bd. 85 , p. 448 .

1854: Preste 1: Ueber die krystallinische Structur des Meteoreisens als Kriterium desselben. Jahrb. k. k. geol. Reichsanst. 1854, p. 866 ; s. auch Zeitschr. d. d. geol, Ges. Bd. 6 (1854), p. $66_{3}-665$.

1854: v. Boguslawski: Zehnter Nachtrag, Pogg. Ann. Ergz.-Bd. 4, P. $120-122,386-388$.

1855 : $\mathrm{H}$ ai d ing e $\mathrm{r}$ : Bemerkungen über die zuweilen im geschmeidigen Eisen entstandene krystallinische Structur verglichen mit jener des Meteoreisens. Sitzber. Wien. Akad. Bd. 15, p. 354 (Abb. einer geschliffenen Platte).

r856: B u r k a r t : Fundorte I, N.J. 1856 , p. 270 .

r859: B u c hn er: Feuermeteore, p. II8 $-120$.

r859: H a rris: Dissert. Gött., p. II7. r860: R a m me ls berg : Mineralchemie, p. $904,947,948$.

I86r : R o s e legt ein Stück des Braunauer Eisens vor, vergleicht die Neumann'schen Linien mit denen am künstlichen Eisen. Zeitschr. d. d. geol. Ges. Bd. 13 (I86r), p. 356.

1862: R o s e: Ueber den Asterismus der Krystalle, insbesondere des Glimmers und des Meteoreisens. Pogg. Ann. Bd. 117 , p. $634-635$; s. auch Mon.Ber. Berlin. Akad. 1862, p. 617 .

r863: Buchner: Meteoriten, p. 176 $-\mathrm{i} 78$.

r863: Rose: Meteoriten, p. 34-35,
$37,42,43-49$ (Taf. I), $87,1_{3} 8,152$, 158.

r858/65: v. R e i c he n b a c h : IV 640 VI 448 . IX $162,176,177,182 . X_{359}$, $363,368,369$. XII 457 . XIII 354. XV 100, 116, 117, 118, 119, 122, 128, 131. XVI 255, 262. XVII 266, 273. XVIII $482,487,490$. XIX 150, 151, 155, I $56 . X X 621,622,630$. XXI 578. XXIV 226. XXV 6I2.

1865: Buchner: Zweiter Nachtrag Pogg. Ann. Bd. 124, p. 574.

1867: Daubrée: Nouveau procédé pour étudier la structure des fers météoriques. C. R. Bd. 64 , p. $685-688$. 1874: B jör ling: Meteoriter och Kometer (cfr. Hessle), p. I2.

1874: Ts c hermak: Das Krystallgefüge des Eisens, insbesondere des Meteoreisens. Sitzber. Wien. Akad, Bd. 70 I, p. $443-45^{8}$, insbesondere p. 449 $-45^{8}$ (Taf.). Ref. N. J. 1875 , p. 416 -417 ; s. auch Lehrbuch der Mineralogie, Aufl. v. 1888 , p. 582 .

1875: M o hr: Entstehungsart, Ann.Chem. Pharm. Bd. 179, p. 259, 269, 276.

1875 : S a d e b e c k : Ein neues Zwillingsgesetz im regulären System, beobachtet am gediegen Eisen. Pogg. Ann. Bd. ${ }_{5} 6$, p. $554-557$.

1875: vom Rath: Meteoriten, Verh. naturh. Ver. Bonn Bd. 32, p. 356.

r881 : D ö 11: Form, Oberfläche, Rinde, physische und chemische Zusammensetzung der Meteoriten. Mon.-Bl. d, Wiss. Club's in Wien, III. Jahrgang, Nr. 4, p. 42.

r88r: Brezina : Bericht III, Sitzber. Wien, Akad, Bd. 84 I, p. 280.

1883: K unis c h: Die Meteoriten unter besonderer Berücksichtigung der schlesischen, Vortrag gehalten am 21. Januar $\mathrm{r}_{8} 8_{3}$ im Humboldt-Verein zu Breslau, p. 3-4 (Sep.).

1883: $\mathrm{S} \mathrm{m}$ it h : Concretions, Am. Journ.

(3) 25, p. $417,422$.

r884: M eunier: Météorites, p. 35,68 
(Abb.), 94, 96, 98, 112, 113-114 (Abb.), 459. 462 .

1884: v. $\mathrm{N}$ i e s s 1: Ueber die astronomischen Verhältnisse bei dem Meteorsteinfalle von Mócs in Siebenbürgen am 3. Febr. 1882. Sitzber. Wien. Akad. Bd. 89 II, p. 29r. Ref. N. J. I886 I, p. 224.

r883/85: T s c h e r m a k : Photographien, p. 3 .

1885: Brezina: Wiener Sammlung, p. 200, 203-204, 205, 218, 234.

r886: H u n t ing t o $\mathrm{n}$ : Crystalline Structure, Am. Journ. (2) 32, p. 289, 29I, 293 (Abb.), 295, 296, 297.

r887: Flig ht: Meteorites, p.II4-II5. 1887: M eunier: Examen minéralogique du fer météorique de Fort Duncan (Texas). C. R. Bd. 104 , p. 872 -873 (Vergleich mit Braunau).

r889: C o h en: São Julião, N. J. r889 I, p. $216,217,222,224,225$.

r889: F l e t c h e r : Atacama Meteorites, Mineral-Magaz. Bd. 8, p. 226.

r889: v. H a u e r: Ann. Hof-Mus, Bd.4 (Not.), p. 64.

1890: $\mathrm{Brez}$ in a: Ueber Meteoreisen, Oesterr. Zeitschr. f. Berg- u. Hüttenw. Bd, 38, p. 358 .

1890: v. Niess 1: Periheldistanzen, Verh, naturf. Ver. Brünn Bd. 29, p. 189, I95, 2I4, 257-258.

1890: Cohen u. Weinschenk: Meteoreisen-Studien, Ann. Hof-Mus. Bd. 6 , p. 159 . r892: L in ck: Ueber die Zwillingsbildung und den orientierten Schimmer am gediegen Eisen. Zeitschr. f. Krystallogr. Bd. 20, p. 209-215. Ref. N. J. 1893 I, p. 231-232; s. auch Linck: Ueber das Krystallgefüge des Meteoreisens. Ann. Hof-Mus. Bd. 8 (1893), p.116- I17. 1893: Brezina: Ueber neuere Meteoriten (Nürnberg), p. 164 .

r893: Me unier: Révision des fers météoriques, p. 15, 16.

r894: C o h e n : Meteoreisen-Studien III, Ann. Hof-Mus. Bd. 9, p. 97, II7 (Rhabdit).

r894: Cohen: Meteoritenkunde, an vielen Stellen.

r 894 : F 1 e t $\mathrm{c}$ h er: Introduction,p.33-34. 1895: Brezin a: Wiener Sammlung, p. 290 ,

I895: C o h e n : Meteoreisen-Studien IV, Ann. Hof-Mus. Bd, Io, p. $83,88,89$.

Ursprüngliches Gewicht: 2 Massen von 23628 u. $17082 \mathrm{gr}$; die letztere noch im Besitz des Benediktinerstifts in Braunau; s. Fussnote. Nachweisbares Gewicht: $29367 \mathrm{gr}$.

\begin{tabular}{lr|lr} 
Bailey & 14 & Breslau & 1221 \\
Belgrad & 21 & Budapest & 199 \\
Bement & 23 & Calcutta & 234 \\
Berlin, U. & 1624 & Cambridge & 14 \\
Bologna & 7 & Cleveland & 1 \\
Bonn & 51 & Dorpat & 15 \\
v. Braun & 19 & Dresden, M. & 234 \\
Braunau & $\left.17082^{1}\right)$ & Frankfurt & 70
\end{tabular}

t) Im Auftrag Sr. Hochwürden des Herrn Abtes des Benediktinerstifts in Braunau teilte mir Herr Gymnasiallehrer V. Maiwald am 14. März 1896 mit, dass die kleinere der beiden gefallenen Massen von 17082 gr. noch unversehrt in der dortigen Abtei aufbewahrt werde. Von der grösseren ursprünglich $23628 \mathrm{gr}$. schweren Masse gelangten durch Verteilung seiner Zeit Stücke an: Berlin, U.; Breslau, U.; Breslau, Schles. Ges. f. vaterl. Kult.; Brünn Mähr. Schles. Ges. z. Beförd. d. Ackerb. ; Dresden, M. ; Göttingen; Graz, Johanneum; Halle; v. Haidinger; A. v. Humboldt; Leipzig; Neumann; Prag, M.; v. Reichenbach; Wien, H.M. - Als Gegenleistung kamen Stücke von 22 Fundorten an das Stift u. a. von Bohumilitz, Elbogen, Estherville, Goruckpur, Lenarto, Magura, Netschaëvo, Seeläsgen. Ueber das Gewicht dieser Stücke war zur Zeit leider keine Nachricht zu erhalten. 


\begin{tabular}{|c|c|c|}
\hline $\begin{array}{l}\text { Freiberg, i. S. } \\
\text { Giessen }\end{array}$ & & $\begin{array}{l}\text { Neumann } \\
\text { New Haven }\end{array}$ \\
\hline Giessen & I3I & $\begin{array}{l}\text { New Haven } \\
\text { New York, M. }\end{array}$ \\
\hline Göttingen & 200 & $\begin{array}{l}\text { New York, M. } \\
\text { Paris, U. }\end{array}$ \\
\hline Graz, J. & $26 \mathrm{r}$ & Paris, U. \\
\hline Gregory & 23 & Pech \\
\hline Greifswald & 41 & Pohl \\
\hline Halle & 9 & Prag, D. U. \\
\hline Hamburg & 5 & Prag, M. \\
\hline Harvard, U. & 105 & Rom, U. \\
\hline Heidelberg & 6 & v. Siemaschko \\
\hline Kopenhagen & 2 & Stockholm \\
\hline Kunz & 48 & Strassburg \\
\hline Leipzig & 29 & Stürtz \\
\hline London, B. M. & 553 & Tübingen \\
\hline London, P. G. & 89 & Turin, U. \\
\hline Marburg & 21 & Ward \\
\hline de Mauroy & 7 & Washington \\
\hline Melion & 12 & Washington, Sh. \\
\hline Moskau & & Wien,H.M. $\left.)^{* * *}\right) 24$ \\
\hline München & & Wien, U. I. \\
\hline
\end{tabular}

Brazos River 1808 (auch vielfach r814) Cross Timbers

Brazos River ${ }_{1} 8{ }_{3} 6$ (auch vielfach 1856) Wichita Co.

Breitenbach Steinbach

Bremervörde (Gnarrenburg), Prov. Hannover, Deutschland.

Stein, Ccb, gefallen r $_{3}$. Mai 1855 .

1855: Die Sammlung in Göttingen erhält einen 5 Pfund 29 Lot wiegenden Stein von Wöhler geschenkt. Gött. gel. Anz. (Nachr.) r855, p. 142.

1855: Auszug aus einem Brief des Professors Hofrat Wöhler in Göttingen, correspondierendem Mitgliede der kais. Akademie der Wissenschaften an den Vorstand des kais. Hof - MineralienCabinets P. Partsch. Sitzber. Wien Akad. Bd. 17 , p. 56-57. Auch abgedruckt in Pogg. Ann. Bd, 96 (1855), p. $626-628$. Ref. Kenngott, Uebersicht 1855 , p. 158 ; s. auch $=$ L'Institut Bd. 23 (1855), p. 233.

r856: Wöhler und Hausmann : Der Königlichen Societät wurden am
9. Juni von dem Geheimen Hofrath Hausmann die nachstehenden Bemerkungen über die im vorigen Jahre in der Gegend von Bremervörde herabgefallenen Meteorsteine, nebst der chemischen Untersuchung derselben, von dem Herm Obermedicinalrathe Wöhler vorgelegt. Gött. Gel. Anz. (Nachr.) 1856, p. 145-157 (Analyse) ; auch abgedruckt in Pogg. Ann. Bd. 98 (1856), p. 609-620. Ref. N. J. 1857, p. 332-336; Liebig-Kopp, Jahresber. 1856, p. 914; Kenngott, Uebersicht 1856/57, p. $235-236$; s L'Institut « Bd. 24 (1856), p. 289-290; Wochenschr. f. Astr. Met. u. Geogr. Bd. $11(\mathrm{t} 857)$, p. 96. r856: Wöhler: Ueber den Meteorsteinfall bei Bremervörde; Ann. Chem. Pharm. Bd. $99(1856)$, p. 244-248 (Analyse). Ref.: Journ. prakt. Chem. Bd. 69 (1856), p. 472-473; Am. Journ. (2) $2 \mathrm{I}(1856)$, p. 146 u. Am. Journ. (2) $24(\mathbf{1} 857)$, p. 295.

1856: $\mathrm{Krantz}$ zeigte zwei ganze $\mathrm{Me}-$ teorsteine von dem am 18. Mai d. J. bei Quarrenburg, Amt Bremerförde in Hannover beobachteten Falle herrührend vor und zwar einen von $4 \% / 4$ Pfund und einen von 22 Lot. Verh, naturh. Ver. Bonn (Sitzber.) Bd. 13, p. XII -XIII.

1859: B u c hn er: Feuermeteore, p. Io3. r859: Harris: Dissert. Gött., p. 97. r860: $\mathrm{R}$ a $\mathrm{mm}$ e $1 \mathrm{~s}$ ber $\mathrm{g}$ : Mineralchemie, p. 923 ff.

r863: B u c hn e r: Meteoriten,p. $88-89$. 1863: Rose: Meteoriten, p. 86,89 , $99, \mathbf{1} 55$.

r858/65: v. Reich en ba ch: VI 44 I, 443. IX 161, 171, 180. X 359, 362, 372. XI 296, 300. XIII 355,357 , 362, 366, 368 (Fig.), 370 (Fig.), 374. XIV 399. XX 623. XXIII 369. XXV 603 . I875: vom R a t h: Meteoriten, Verh. naturh. Ver. Bonn. Bd. 32 , p. 368 . 1884: H äp k e : Beitrïge, Abh. Naturw. Ver. Bremen Bd. 8, p. 523 . 
r884: M e un i er: Météorites, p. 95, 273,276 .

1885: Brezina: Wiener Sammlung, p. $182,232$.

r894: C o h e n: Meteoritenkunde, p. 68, 153.

r895: Brezina: Wiener Sammlung, p. 257 .

Ursprüngliches Gewicht: $71 / 4 \mathrm{Kgr}$. Krantz sagt $185^{6}$, dass den grössten Stein von 6 Pfund Göttingen, einen von 3 Pfund die Bergschule in Clausthal erbalten habe, dass er selbst zwei Steine von $4 / 4$ Pfund und von 22 Lot und den kleinsten Stein Wöhler besitze. Zusammen werden diese fün Steine also etwa $14^{1 / 2}$ Pfund gewogen haben.

Nachweisbares Gewicht: 6424 gr.

\begin{tabular}{lr|lr} 
Bailey & 6 & Klausenburg & 2 \\
Belgrad & 39 & Kopenhagen & 47 \\
Bement & 2 & London, B. M. 808 \\
Berlin, U. & 281 & London, P. G. & 22 \\
Bonn & 23 & Moskau & 11 \\
v. Braun & 10 & Neumann & 13 \\
Breslau & 12 & Paris, M. & 20 \\
Budapest & 5 & Pohl & 6 \\
Calcutta & 519 & Rom, U. \\
Clausthal & 1222 & v. Siemaschko \\
Cleveland & 3 & Stockholm \\
Dorpat & 32 & Troyes \\
Göttingen & 2826 & Tübingen \\
Gregory & 3 & Turin, U. \\
Greifswald & 36 & Ward & 79 \\
Harvard, U. & 3 & Washington \\
Heidelberg & 2 & Wien, H. M. *) & 348
\end{tabular}

Brenham, Brenham Township, Kiowa County, Kansas, U. S. A.

Pallasit, $\mathrm{Pb}$ (s. o. p. $3 \mathrm{I}$ Fussnote); gefunden 1885 oder 1886 ; beschr. 189o. Hierher auch vorläufig Anderson (s. Anhang am Schluss dieses Artikels). r89o: $\mathrm{K} \mathrm{unz}$ : On five new American Meteorites, I) On the group of Meteorites recently discovered in Brenham Township, Kiowa County, Kansas. Am. Journ. (3) 40, p. 312-318 (Abb. einer geschliffenen Platte. Analyse von Eakins); s. auch U. S. Geol. Surv. Nr. 78 (r89r), p. 94-95; »Science * Bd. 15 , p. 359 u. Bd. 16, p. 39 ; Proc. New-York Acad. 7. April 189o; Journ. Chem. Soc. 1891, p. 277-279; Oesterr. Zeitschr. f. Berg- u. Hüttenw. Bd. 39 (I89I), p. 228 ; N. J. 1891 I, p. $3^{85}$ -386 u. N. J. 189 I II, p. 52.

r89o: W inchell u. Dodge: The Brenham, Kiowa County, Kansas Meteorites. - Amer. Geologist Bd.5 (I89o), p. $309-3^{12}$ u. Bd. 6 (1890), p. $370-$ 377 (Analyse u. Abb. einer geätzten Platte).

r890: E a s t m a n n : Met.Astron, p.320, 322.

r89r: Huntington: The prehistoric and Kiowa County Pallasites. Proc. Amer. Acad. Arts and Sc. Bd. 26, p. $1-12$ (3 Tafeln). Ref. N. J. I892 I, p. $266-267$.

r892: H a y : The Kiowa Co. (Kansas) Meteorites. Am. Journ. (3) 43, p. So. Ref. N. J. 1892 II, p. 24 I.

I893: v. H a u e r: Ann. Hof-Mus. Bd, ro (Not.), p. 35 .

I893: M e u n i e r: Examen minéralogique et lithologique de la météorite de Kiowa, Kansas. C. R. Bd. 116 , p. $447-$ 450. Ref. N. J. 1894 I, p. $448-449$. r893: B rez in a : Ueber neuere Meteoriten (Nürnberg), p. 153.

1894: Cohen: Meteoritenkunde, an vielen Stellen.

1895: M e un i er: Révision des Lithosidérites, p. 20-24 (Abb. einer geätzten Platte).

1895: Brezin a: Wiener Sammlung, p. 265-266.

Ursprüngliches Gewicht: Ueber $900 \mathrm{Kgr}$. 
Nachweisbares Gewicht: 768836 gr.

\begin{tabular}{|c|c|c|c|}
\hline & $\left.25^{1}\right)$ & & \\
\hline Belgrad & 90 & & $n$ \\
\hline Bement & $\left.2604^{2}\right)$ & de Mauroy & \\
\hline Berlin, U. & 135 & Minneapolis 8 & $805^{1}$ \\
\hline Böhm & 2708 & München & \\
\hline 3ologna & 200 & Neapel & \\
\hline Bonn & 45 & New Haven 4 & 4500 \\
\hline Braun & 3683 & Newton & \\
\hline reslau & 12 & Paris, E. & $310^{4}$ \\
\hline Brezina & 4368 & Paris, M. & \\
\hline runner & 164 & $\mathrm{Pec}$ & \\
\hline udapest & 2810 & Pohl & \\
\hline alcutta & 60 & Prag, M. & 3 \\
\hline leveland & 367 & Prag, B. U. & 20; \\
\hline zernowitz & II5 & Prag, D. U. & \\
\hline ijon & 33 & Röbling & 125 . \\
\hline orpat & 988 & Rom, & 63 \\
\hline resden, M. & 77 & Seli & \\
\hline eiberg i. S & 120 & Sevil & \\
\hline iessen & 85 & v. Siemaschko & \\
\hline Gotha & 63 & Stockh & \\
\hline rego & 168 & Stra & \\
\hline Greifswald & 126 & Stürtz & 5 \\
\hline Harvard, U. 5 & $\left.53627^{3}\right)$ & Stuttgart & \\
\hline Heidelberg & 137 & Turin U, & \\
\hline owell & & Upsala & \\
\hline arlsruhe, M & 178 & Utrecht & \\
\hline Kopenhagen & 46 & Ward & 466 \\
\hline Krantz & 3323 & Washington & 72 \\
\hline Kristiania & 122 & Wien, H. M. & $725^{5}$ \\
\hline unz & 544000 & Wien, U. I. & 22 \\
\hline ider & $3^{I}$ & Wien, U. II. & \\
\hline oben & 76 & Würzburg & \\
\hline sadoun & 86 & Zürich & \\
\hline
\end{tabular}

Nach I. Juli 1893 erwarb Herr Bayet, Brüssel, 16 gr.

Brenham (Anderson), Little Miami
Valley, Hamilton County, Ohio, U. S. A.

Pallasit, $\mathrm{P}$, bekannt $188_{3}$, beschr. 1884 .

1884 : Kinnicut: Report on the Meteoric Iron from the Altar Mounds in the Little Miami Valley, Ohio. Rep. Peabody Museum. Amer. Archeologie and Ethnologie of Cambridge, Febr, 1884, p. $38 \mathrm{r}-384$ (Analysen und Abbildung eines Schnitts). Ref. Am. Journ. (3) 27, p. 497-498; Liebig-Kopp, Jahresber. 1884 , p. 2042 .

r884: W a d s w ort h: Studies, p. 7I.

r884: Brezina: Wiener Sammlung, p. $25 \mathrm{I}$.

r886: P u tna m: The Marriott Mound, Nr. I, and its contents. Rep. Peabody Museum etc. Cambridge 1886, p. 463 -465 (Abb. der Ohrringe).

1887: Kunz: On two new Meteorites from Carroll County, Kentucky, and Catorze, Mexico. Am. Journ. (3) 33, p. 228-232 (Abb. eines Schliffs von Turner Mound). Ref. N. J. 1888 I, p. 209-21o.

I888: v. H a u er: Ann. Hof-Mus. Bd. 3 (Not.), p. 42.

1890: K unz: s, vorige Seite.

r89o: F le t cher: Mexican Meteorites, Mineral. Magaz. Bd. 9, p. 102.

r893: Brezina: Ueber neuere Meteoriten (Nürnberg), p. 163.

1894: C o h e n: Meteoritenkunde, p.62, $252,263$.

1895: Brezin a : Wiener Sammlung, p. $263-264$.

Ursprüngliches Gewicht: 847 gr. (28 $+5^{2}+767$; ausserdem mit Me-

I) Davon 2356 gr. Haviland, Kiowa Co., Kansas (Siderite); 567 gr. Haviland Kansas (Pallasite); 305 gr. Haviland, Kansas (Pallasite-altered ?).

2) Davon 869 gr. Haviland Township, Kiowa Co. Kansas,

3) Davon $596 \mathrm{gr}$. Eigentum von O. W. Huntington.

4) Davon 77 gr. Haviland.

5) Inzwischen noch weitere 11727 gr. erworben. 
teoreisen plattierte Ohrringe, von denen einer in Wien, $H$. M.). Kinnicut giebt für das Gewicht des grössten Stücks 767.5 gr. an; dies stimmt nicht mit Huntington's Catalogue der Harvard-Sammlung überein, welcher als the greater portion of the original mass \& 186 gr. aufführt.

Nachweisbares Gewicht: 272 gr.

Gregory

I Newton

Harvard, U.

216 Washington

6) Wien, H M.

New Haven

Alfianello

Brescia

s. Anhang

Brésil

Bridgewater, Bridgewater Station, Burke Co. North Carolina. U.S. A.

Eisen, Of, gefunden und beschrieben 1890 .

1890: $\mathrm{K}$ unz: On five new American Meteorites. - 4. Meteoric iron from Bridgewater, Burke County, North Carolina. Am. Journ. (3) 40, p. $320-322$ (Abb. und Analyse, letztere von Venable). Ref, N. J. 1891 II, p. 52.

1893: Brez in a: Ueber neuere Meteoriten (Nürnberg), p. I64.

r894: Co he n: Meteoritenkunde, p. 84, 23 I.

1895: C o he n: Meteoriten-Studien IV, Ann. Hof-Mus. Bd. 10, p. 82 .

1895: Brezina, Wiener Sammlung, p. $271-272$.

Ursprüngliches Gewicht : ${ }_{1} 3.63 \mathrm{Kgr}$. Nachweisbares Gewicht : 9513 gr.

\begin{tabular}{lr|lr|} 
Bailey & 95 & Greifswald & 35 \\
Bement & I55 & Prag, M. & 174 \\
v. Braun & 152 & Rom, U. & 92 \\
Budapest & 566 & Wien, H. M. 8244 ${ }^{2}$ )
\end{tabular}

Britisch Nordamerika I 87 I

Victoria

\section{Bruce's Eisen}

Bubuowly

Bückeberg
Stein, Cgb, gefallen Mai 1859 .

1873: Daubrée: Note sur des météorites représentant deux chutes inédites qui ont eu lieu en France, l'une à Montlivault (Loir-et-Cher), le 22 juillet $188_{3} 8$, l'autre à Beuste (Basses-Pyrénées) en mai 1859 . C. R. Bd. 76, p. 3I5 -316 ; s. auch *Naturforscher \& 1873 , p. 167 ; Liebig-Kopp, Jahresber. 1873 , p. 1250 .

1884: M e unier: Météorites, p. 247, 335 .

1885: B rez in a: Wiener Sammlung, p. 253 .

r887: Flig h t: Meteorites, p. 130-r31. r889: F o u qué: Étude d'une météorite de la chute de Beuste (Basses-Pyrénées). Bull. Soc. Franc. Min. Bd, 12, p. 32-35. Ref. N. J. I89o I, p. 42 . r889: Me un i er: Sur la matière noire de la Chantonnite, Bull. Soc. Franc. Min, Bd. 12, p. 76-81.

1893: Brezin a: Ueber neuere Meteoriten (Nürnberg), p. I6o.

r895: Brezina: Wiener Sammlung, p. $25^{1}$.

Ursprüngliches Gewicht: $\mathbf{r} .82 \mathrm{Kgr}$. Zwei Bruchstücke von r.40 u. $0.42 \mathrm{Kgr}$. Das erstere gelangte an die Ecole des Mines, Paris; das andere an das Museum von Pau (Basses-Pyrénées).

Nachweisbares Gewicht: ${ }^{588} \mathrm{gr}$.

I) Ausserdem 84 gr. nach r. Juli 1893 erworben.

2) Ausserdem 172 gr. nach I. Juli 1893 erworben.

W ül fing, Meteoriten, 


\begin{tabular}{lr|lr} 
v. Braun & II & Paris, M. & 66 \\
Brezina & 38 & Pau & $\left.420^{1}\right)$ \\
Budapest & 8 & v. Siemaschko & 37 \\
Gregory & I & Stockholm & 40 \\
London, B. M. & 40 & Wien, H. M. & 66 \\
Paris, E. & 86 I & &
\end{tabular}

Bulloah

Butsura

Buncombe Co., beschr. 1839

Bairds-Farm

Buncombe Co., gefd. 1839 , beschr. I 847

Black Mountain

Bunzlau 1723

Ploschkowitz

Bunzlau 1808

Lissa

Burggraf, Der verwünschte, Elbogen

Burgos

Berlanguillas

Burke Co. 1882

Linnville

Burke Co. $1890 \quad$ Bridgewater

Burlington, Cooperstown (nicht Coopertown), Otsego County, New-York, U. S. A.

Eisen, Om, gefunden vor 1819, beschr. 1844 .

1844: Pierce: Analysis of Meteoric Iron from Burlington, Otsego Co., N. Y. Am. Journ. (I) 46, p. 40I-403 (Analyse von Rockwell).

1846: S hep a rd: Report on Meteorites. Am. Journ. (2) 2, p. 382.

r847: $\mathrm{S}$ h e p a r d: Report on Meteorites, Am. Journ. (2) 4, p. $77-78$ (Abb.); s. auch Liebig-Kopp, Jahresber. $1847 / 48$, p. 1309, 1311-1312 ; Kenngott, Uebersicht $1850 / 51$, p. 135 .

1852: C 1 a r k: Dissert. Gött., p. 61-62. r852: Wöhler: sIntermediär«, Pogg. Ann. Bd. 85 , p. 449 .

1854 : v. B o g u sl aw s k i : Zehnter Nachtrag, Pogg. Ann. Ergz.-Bd. 4, p. 402 $-403,410$.

1859: Buch n er: Feuermeteore, p. 133.
1859: Harris : Dissert. Gött., p. ro8 -. 109, $\mathrm{II}_{5}-\mathrm{II} 6$.

1860: Haid inger: Ueber das von Herrn Dr. J. Auerbach in Moskau entdeckte Meteoreisen von Tula. Sitzber. Wien. Akad. Bd. 42, p. 512 (Vergleich mit Burlington. Abb.).

I860: $\mathrm{R}$ a $\mathrm{mm}$ e l $\mathrm{s}$ b e r g: Mineralchemie, p. 914-915.

r $858 / 62$ : v. Reic h e n b a c h: IV 638 VI 448,452 . VII 551. IX 162, 174, I81. X 359,365 . XII 457. XIII 363. XV 100, 110, 114, 124, 126. XVI 255, $256,261,262$. XVII 264, 266, 272. XVIII 484,487 . XIX r50, I54, 155. XX 622, 628. XXI 589 .

1863: Buchner: Meteoriten, p. 170 -171 .

r863: R o s e: Meteoriten, p. 26,27,64,152. r869: Meunier: Recherches, Ann.

Chim. Phys. (4) 17, p. $35,72$.

r870: R a mmels berg : Meteoriten, p. 8 o.

r872: Quenstedt: Klar und Wahr, p. $3^{14}$ (Abb, einer geätzten Platte).

1884: M e un i e r : Météorites, p. 5I, 99, I33 (Abb.).

1885: Brezina: Wiener Sammlung, p. $211-212,234$.

r89o: E a s tma n: Met. Astron., p. 318. 1893: Révision des fers météoriques,

p. 49,50 (Abb. einer geätzten Platte). 1894: Flet ch er: Introduction, p. 33. r894: C o h en: Meteoritenkunde, p. 52, $62,67,76,194$ (verweist von Coopertown auf Burlington; es liegt wohl eine Verwechslung mit Cooperstown vor).

r895: B r e z in a : Wiener Samml.,p.276.

Ursprüngliches Gewicht: 100 bis $200 \mathrm{lbs}$; grösstenteils verschmiedet, etwa $6 \mathrm{Kgr}$. erhalten.

Nachweisbares Gewicht: $4867 \mathrm{gr}$.

I) Herr Soulice hatte die Freundlichkeit mir mitzuteilen, dass der Stein noch im Museum von Pau aufbewahrt werde; das Gewicht, welches leider nicht angegeben wurde, ist nach dem Aufsatz Daubrées gleich 420 gr. 


\begin{tabular}{lr|lr} 
Bailey & 25 & London, P. G. & I32 \\
Bement & 65 & New Haven & 738 \\
Berlin, U. & II & Paris, M. & 8 I \\
Budapest & 213 & Pohl & 83 \\
Calcutta & 216 & v. Siemaschko & 40 \\
Clinton & 80 & Stockholm & 57 \\
Dresden, M. & I5 & Tübingen & 850 \\
Göttingen & 62 & Ward & 32 \\
Gregory & 64 & Washington & 77 \\
Kopenhagen & 30 & Washington,Sh.r528 \\
London, B. M. & 290 & Wien, H. M. *) & 7 I
\end{tabular}

Buschhof (Scheikahr Stattan), bei Jacobstadt, Kurland, Russland. Stein, Cwa, gefallen 2. Juni 1863 . r863: Grewing k in der Rigaer Zeitung" Nr. 127 (1863).

1863: Ros e berichtet über zwei neue Meteoritenfälle nach den Mitteilungen, die ihm Hr. Prof. Grewingk in Dorpat gemacht hatte. Mon.-Ber. Berlin, Akad. (1863), p. 441 ; s, auch Pogg. Ann. Bd. 120 (1863), p. 619-620. Ref. N. J. 1864, p. 236; Kenngott, Uebersicht $1862 / 65$, p. 444 ; Liebig-Kopp, Jahresber. 1863 , p. 906.

1863: R o s e: Meteoriten, p. 155.

1864: Buchner: Erster Nachtrag, Pogg. Ann. Bd. 122, p. 323.

1864: Grewing k und $\mathrm{Schmidt}$ : Ueber die Meteoritenfälle von Pillistfer, Buschhof und Igast in Liv- und Kurland, Arch. f. Naturk. Liv. Ehst- u. Kurland's Bd. 3, Dorpat 1864, p. $452-$ $457,473-481$ (Abbildungen, Karte u. Analyse). Ref. N. J.1865, p. 99-100; Kenngott, Uebersicht $r 862 / 65$, p. 443 . 1865: He is sprach über den am 2. Juni $1864 \mathrm{zu}$ Buschhof bei Jakobstadt in Curland gefallenen Meteorstein Verh. naturh. Ver. Bonn Bd, 22, p. 60.

1870: R a mmelsberg: Meteoriten, p. 82,98 .

1875: vom R at h: Meteoriten, Verh. naturh. Ver. Bonn Bd. 32, p. 369.
I884: M e unier: Météorites, p. 209. 1885: Brezin a: Wiener Sammlung, p. $180,232$.

r893: v. H a uer: Ann. Hof-Mus, Bd. 8

(Not.), p. 49.

1894: C o h e n: Meteoritenkunde, p. II2, $205,262,303$.

1895: Brezina: Wiener Sammlung, p. 244.

Ursprüngliches Gewicht: 5 Kgr. (1 $2^{1 / 2}$ Pfund russisch).

Nachweisbares Gewicht: $35^{21}$ gr.

\begin{tabular}{lr|lr} 
Berlin, U. & 75 & Moskau & 30 \\
Bonn & 13 & New Haven & 22 \\
v. Braun & 42 & Paris, M. & 52 \\
Brezina & 134 & Pohl & 119 \\
Budapest & 126 & Prag, M. & 99 \\
Calcutta & 24 & Petersburg, B. & I2 \\
Dorpat & 1342 & Riga & 92 \\
Göttingen & 51 & Riga, Ges. & $\left.69{ }^{1}\right)$ \\
Gregory & 17 & Riga, P. & 15 \\
Heidelberg & 14 & v. Siemaschko & 2 \\
Helsingfors & 48 & Stockholm & 42 \\
Kiew & 77 & Stürtz & I \\
Krüger & 33 & Stuttgart & 10 \\
London, B. M. & 98 & Tübingen & 23 \\
London, P. G. & II & Wien, H. M.*) & 828
\end{tabular}

Bustee, Goruckpur, Nord-WestProvinz, Ostindien.

Stein, Bu, gefallen 2. Dezbr. 1852 . 1862: v. Reichenbach: XX 632 . r863: Buchner: Meteoriten, p. 84 . 1870: Maskelyne: On the Mineral Constitution of Meteorites. III. The Busti Aërolite of 1852 . IV. OldhamiteSulphit of Calcium. V. Osbornite. VI. The Augitic Constituent of the Busti Aërolite. VII. Enstatite as a Constituent of the Busti Meteorite. VIII. General Analysis of the Busti Meteorite. IX. The Action of Acids on the Enstatite and Augite. X. The Iron of the Busti Meteorite. Philos. Trans, Bd, 160,

I) Ges. = Gesellschaft für Geschichte und Altertumskunde. Riga. 
p. 193-2II (2 Tafeln mit Abbildungen des Steins und mikroskopischer Präparate); s. auch Proc. Royal Soc. Bd. I8 (1870), p. 146-156; Rammelsberg, Mineralchemie II. Aufl. (1875), p. 52 (Oldhamit); Rep. Brit. Assoc. 1862, Appendix II, p. 190 (Vorläufige Mitteilung).

1870: R a m mels berg : Meteoriten, p. 106.

r870: R a mmels berg: Die chemische Natur der Meteoriten. 5) Enstatit und Diopsid (Meteorit von Busti). Abh. Berlin. Akad. (1870), p. 123-127; s. auch Pogg. Ann. Bd. 140 (1870), p. 315 $-316,320$.

187I: Meunier: Coexistence de deux types lithologiques dans la même chute de Météorites. C. R. Bd. 73, p. 1483 -1485 .

1872: T s c hermak: Der Meteorit von Shergotty und Gopalpur. Sitzber. Wien. Akad. Bd. 65 I, p. 127.

1879: R a m mels be r g: Meteoriten, p. 25 .

r883: T s c h e rma k : Beiträge, Sitzber.

Wien. Akad. Bd. 88 I, p. $366-367$.

r884: W a d s wor th: Studies, p. 202. r884: Meunier: Météorites, p. 64, $65,85,188,192-193$.

1883/85: Tschermak: Photographien, T. 5 , p. 9.

r885: B rez in a : Wiener Sammlung, p. $155,174,232$.

r887 : F lig h t: Meteorites, p. II8-122

(Abb.); s, auch Geol. Magaz, Sept. 1875 . r889: C o h e n : São Julião, N. J. 1889 I, p. $223,224,225$.

r894: Cohen: Meteoritenkunde, an vielen Stellen.

r894: F let c h er: Introduction, p. 1o, $\mathrm{II}-\mathrm{I2}, \mathrm{I3}, 37$.

r895: Brezina: Wiener Sammlung, p. 239.

Ursprüngliches Gewicht: 1.3 Kgr. (ungefähr 3 Pfund englisch).
Nachweisbares Gewicht: II22 gr.

Budapest

Calcutta

Cambridge

I London, P. G. II

Göttingen

Gregory

I2 München

3 New Haven II

Harvard, U.

Spl. Paris, M.

4 Roebling

I 7 Stockholm

Kristiania $\quad$ I $_{3}$ Washington Spl.

London, B. M. Iooo Wien, H. M.*) 2 I

\section{Butcher Iron}

Coahuila

Butler, Bates Co., Missouri, U. S. A. Eisen, Off, gefunden vor 1874 , beschr. 1875 .

1875: B road head: On a Discovery of Meteoric Iron in Missouri. Am. Journ.

(3) 10, p. 4 oI.

1877: $\mathrm{S} \mathrm{mith}$ : Examination of the Waconda Meteoric Stone, Bates County Meteoric Iron, and Rockingbam County Meteoric Iron. - Bates County Meteoric Iron. Am. Journ. (3) I3, p. $21_{3}$ (Analyse); s. auch Original Researches 1884 , p. 525-526. Ref. Liebig-Kopp, Jahresber. 1877, p. 1396.

1880: Brezina: Vorläufiger Bericht über neue oder wenig bekannte $\mathrm{Me}$ teoriten, - I) Butler, Bates Co., Missouri, N. S., bekannt seit 1875 . Sitzber. Wien. Akad. Bd. 82, I, p. $348-35$ o, 351.

188I : B rezina: Meteoritenstudien II,

Denkschr. Wien. Akad. Bd. 44 , p. 135 -158 (Mit 4 Tafeln mit Abbildungen geätzter Platten).

1884: M e un ier: Météorites, p. 129, 131. 1884: W a d sworth: Studies, p. 6r. ${ }_{1883} / 85$ : $\mathrm{T} \mathrm{s} \mathrm{c} \mathrm{h}$ er m a k : Photographien, p. 9 , T. 5 .

1885: Brezin a: Wiener Sammlung, p. 207-208, 233.

1886: B rezin a und Cohen: Photographien, Fig. 7, 8, 9.

1886: $\mathrm{H}$ u n t in g to $\mathrm{n}$ : Crystalline Struc-

ture, Am. Journ. (3) 32, P. 290 (Abb.), 291, 295.

1887: F 1 ig h t: Meteorites, p. 187-188. 
r 889 : v. H a ue r: Ann. Hof-Mus. Bd. 4 (Not.), p. 64.

1890: E a s t m a n : Met. Astron., p. 322. r89o: Brezina: Ueber Meteoreisen, Oesterr. Zeitschr, f. Berg- u. Hüttenw. Bd. 38 , p. 356,357 (Abbildung einer geätzten Platte).

1893: M eunier: Révision des fers météoriques, p. 40-4I (Abb. einer geätzten Platte).

1893: B r e z in a : Ueber neuereMeteoriten (Nürnberg), p. 163.

r894: C o h e n : Meteoritenkunde, p. 94, 100, 105, 192, 199.

r895: Brezina: Wiener Sammlung, p. 267 (Abb. einer geätzten Platte).

1895: Co hen: Meteoreisen-Studien IV, Ann. Hof-Mus. Bd. 1o, p. $82,90$.

Ursprüngliches Gewicht: $36 \mathrm{Kgr}$. Nachweisbares Gewicht: $2755^{\circ}$ gr.

\begin{tabular}{lr|lr} 
Bailey & 480 & New Haven & 970 \\
Bement & 335 & Odessa & 126 \\
Bologna & 130 & Paris, E. & 17 \\
v. Braun & 317 & Paris, M. & 4142 \\
Brezina & 4 & Petersburg, B. & 50 \\
Budapest & 501 & Pohl & 110 \\
Cleveland & 7 & Rom, U. & 66 \\
Dorpat & 44 & v. Siemaschko & 109 \\
Gregory & 328 & Stockholm & 288 \\
Greifswald & 13 & Strassburg & 60 \\
Hamburg & 65 & Stürtz & 48 \\
Harvard, U. & 14569 & Troyes & 48 \\
Klausenburg & 66 & Ward & 76 \\
Kopenhagen & 287 & Washington & 270 \\
Kunz & 71 & Washington, Sh. 391 \\
London, B. M. & 389 & Wien, H. M. & 2959 \\
Minneapolis & 104 & Wien, U. I. \\
Moskau & 63 & 47 \\
& &
\end{tabular}

\section{Butsura, Goruckpur, Ostindien.}

Stein, Ci, gefallen 12. Mai r86r. r862: Ha iding e r : Der Meteorsteinfall im Gorukpur-Districte in Ober-Bengalen, am 12. Mai 1861. Sitzber. Wien. Akad. Bd, 45 II, p. 665-671. Ref.
Kenngott, Uebersicht $1862 / 65$, p. 433 -434; »L'Institut « Bd. 30 (I862), p. $306-307$.

1863: Maskelyne und v. Lang: Mineralogical Notes -6 . The Fall of Butsura, May 12, 1861. Philos. Magaz. Bd. 25 , p. 50-58 (Situationsplan und Abbildung der Steine); s. auch Rep. Brit.Assoc. 1862 (Notices and Abstracts), p. 190 ; Kenngott, Uebersicht $1862 / 65$; p. 440 ; Brezina, Ankunft 1893, p. 18. r863: B u c h ne r: Meteoriten, p. IO7Iog.

1863: R o s e: Meteoriten, p. 154.

r864: H a i d ing e r: Sternschnuppen, Feuerkugeln und Meteoritenschwärme im Zusammenhange betrachtet. Sitzber. Wien. Akad. Bd. 49 II, p. 6.

1867: Go e bel: Kritische Uebersicht, Mélanges phys. chim. Bd. 7, p. 338 (Goruckpoor).

1875: T s c h erm ak: Vulkanismus, Sitzber. Wien. Akad. Bd. 7I II, p. 662.

r884: W a d s worth: Studies, p. 92 -93 .

r884: M e unier: Météorites, p. 187 (Abb.), 37r.

1885: Brezina: Wiener Sammlung, p. $182,232$.

1886: Brezina: Neue Meteoriten I, Ann. Hof-Mus. Bd, I (Not.), p. I3. $^{2}$

1889: F 1 e tc her: Atacama Meteorites, Mineral, Magaz. Bd. 8, p. 226 (Streufeld 3 zu 2 miles).

r893: Brezin a: Ueber neuere Meteoriten (Nürnberg), p. 159, 164.

1894: C o h e n : Meteoritenkunde, p. 24, 208.

1894: F l et c her: Introduction, p. 10, 28-29.

1895: Brezina: Wiener Sammlung, p. 246.

Ursprüngliches Gewicht: $22 \mathrm{Kgr}$. Fünf Steine, zum Teil vollständig umrindet u. dennoch zusammenpassend. 
Nachweisbares Gewicht: 20448 gr. (ohne Calcutta).

\begin{tabular}{lr|lr|} 
Belgrad & 87 & London, P. G. & 90 \\
Bement & 3 & Moskau & 24 \\
Berlin, U. & 90 & New Haven & 92 \\
v. Braun & 9 & Paris, M. & 19 \\
Budapest & I & Petersburg, A. & 32 \\
Calcutta & 1) & v. Siemaschko & 10 \\
Debreczin & 9 & Stockholm & 46 \\
Dorpat & 99 & Ward & 2 \\
Göttingen & 48 & Washington & II \\
Gregory & 27 & Washington, Sh. 8 \\
Klausenburg & 20 & Wien, H. M.*) & 588 \\
London, B. M. 19 133 & &
\end{tabular}

\section{Cabarras Co.}

Monroe

Cabaya

Great Fish River

Cabezzo de Mayo, Murcia,Spanien.

Stein, $\mathrm{Cw}$, gefallen $\mathrm{x} 8$. August $\mathrm{I} 870$.

1870: D. Juan de Velasco: „El

Tiempo Nr. 247 vom 20. Okt. 1870. 1872: Don José Maria Solano y

Eulat e: Noticia sobre la Pietra Meteórica caïda en termino de Múrcia el dia 18 de Agosto de 1870. Anales Soc. Esp. hist. nat. Bd. I, p. $77-84$. r884: M eunier: Météorites, p. 209. 1885: Brezina: Wiener Sammlung, p. $177,180,232$.

I892: Gredilla: Meteoritos, p. I55 $-116$.

[1894: Co he n: Meteoritenkunde,p. 207, $265,28_{3}, 286$ bezieht sich nicht auf Cabezzo de Mayo wie angegeben, sondern auf Molina].

Ursprüngliches Gewicht : Etwa $25 \mathrm{Kgr}$.

Nachweisbares Gewicht: 1208 gr.

$\begin{array}{lrlr}\text { Bailey } & 4 & \text { Freiberg, i. S. } & 52 \\ \text { Bonn } & 1 & \text { Gregory } & 2 \\ \text { Budapest } & 372 & \text { London, B. M. } & 3 \\ \text { Dresden, M. } & 116 & \text { Madrid } & 520\end{array}$

Paris, M. Rom, U. v. Siemaschko

Stockholm

Strassburg

76 Stuttgart

20

4 Troyes

2 Washington, Sh,

Spl. Wien, H. M.

4

Cabin Creek, Johnson Co., Arkansas, U. S. A.

Eisen, Om, gefallen 27. März 1886. 1887: Kunz: On the Meteoric Iron, which fell near Cabin Creek, Johnson County, Arkansas, March 27th 1886. Am. Journ. (3) 33, p. 494-499 (Abb. der Masse und zweier Schnittfächen). Ref. N. J. I89i II, p. 5I-52.

1887: Whitfield: On the Johnson County, Ark., and Allen County, Ky., Meteorites. - 1) Johnson County. Ark. Am. Journ. (3) 33, p. 500 (Analyse). r887: $\mathrm{K}$ un $\mathrm{z}$ : The meteoric iron which fell in Johnson County, Ark., 3. 17 p. m. March 27, 1886. Proc. U. S. Nat. Mus. (1887) X, p. 598-655 (3 Tafeln). Ref. N. J. I89i II, p. 51-52.

I890: Eastman: Met. Astron,, p. 316. r89r: v. Hau er: Ann. Hof-Mus. Bd. 6 (Not.), p. 6, 54 .

1893: Brezin a: Ueber neuere Meteoriten (Nürnberg), p. I64.

I894: Fl et c her: Introduction, p. 34. r894: C o h e n: Meteoritenkunde, p. 57, 73, I96.

1895: Brezina: Wiener Sammlung, p. 283 .

Ursprüngliches Gewicht: $47.4 \mathrm{Kgr}$. Kunz sagt: $107^{1 / 2}$ lbs u. berechnet dieses Gewicht zu 44213 gr. anstatt $\mathrm{zu} 48.7 \mathrm{Kgr}$. Brezina teilt jüngst mit, dass von dem Stück nur wenige Plättchen im Gesamtgewicht von 29 gr. abgetrennt seien, was auch nicht ganz richtig

1) 1880 besass Calcutta nach Fedden's $>$ Popular Guides $7786 \mathrm{gr}$. (17 lbs $2 \mathrm{oz} 318 \mathrm{grs}$ ). Obgleich Herr Direktor Holland diese Zahl in dem mir gesandten Verzeichnis nicht korrigiert hat, nehme ich doch an, dass der grösste Teil nach London gelangte. 
ist, da Washington allein $34 \mathrm{gr}$. besitzt. Vielleicht bezieht sich die Abtrennung auf eine solche nach der Ankunft des Stückes in Wien.

Nachweisbares Gewicht: 47409 gr. \begin{tabular}{ll|lr} 
v. Braun & 5 & Washington & 34 \\
& &
\end{tabular} London, B. M. $\quad 5$ Wien, H. M. 47365

Cacak 1889 Jeliza Cacak i89i Guca Cacaria Rancho de la Pila Cachiyuyal, Atacama, Chili, S. A. Eisen, Om, gefunden 1874 , beschrieben $\mathrm{x} 875$.

1875: Do m e y ko: Note sur deux nouvelles météorites du désert d'Atacama, et observations sur les météorites qui ont été découvertes jusqu'ici dans cette partie de l'Amérique méridionale. C. R. Bd. 81, p. 597 (Analyse); Nachwort von Daubrée C. R. Bd. 81 , p. 600 , worin er sagt, dass dieses Eisen (wie auch Mejillones) keine Widmanstätten'schen Figuren zeige. Dagegen konstatierte Brezina an dem Pariser Stück Figuren wie bei Ruffs Mountain. Ref, Liebig-Kopp, Jahresber. 1875 , p. 1315 -1316 ; s. auch Mineralojia 3. Aufl., p. $130-131$.

r884: M e un i er: Météorites, p. n6.

1885: Brezina: Wiener Sammlung, p. $212-213,253$.

r889: F le t c h e r : Atacama Meteorites, Mineral. Magaz. Bd.8, p.224, 259-26o. r 890 : B r e z in a: Reise, Ann. Hof-Mus. Bd. 4 (Not.), p. II9 (Cachiuyal ein Glied der La Caille Gruppe, ähnlich Ruffs Mountain).

1894: Co hen: Meteoritenkunde. Verweist anf Juncal.

1895: C o h e n : Meteoreisen-Studien IV, Ann. Hof-Mus, Bd. 10, p. 82.

Ursprüngliches Gewicht: $255^{\circ} \mathrm{gr}$. Nachweisbares Gewicht: 434 gr.
London, B. M. $\quad 28$ Paris, M. Paris, E.

56

350

Fletcher sagt 1889 , dass der grösste Teil des Eisens an das Museum von Santiago gelangt sei.

Caille

La Caille

Calderilla, Caldera, Chili.

Pallasit,P (obgleich kein Olivin mehr erhalten ist), gefallen (?) $\mathrm{x} 88_{3}$, erwähnt 1890 , beschrieben 1895 .

1890: Howe 11: Description of new Meteorites. - The Calderilla Meteorite. Proc. Rochester Acad. Sc. Bd. I, p. 100.

I890: E a s t m a n: Met. Astron., p. 316. 1895: Brezin a: Wiener Sammlung, p. 264.

Ursprüngliches Gewicht: 2 I gr.

Nachweisbares Gewicht: I9 gr.

Wien, H. M. I9

Dass der Fall dieses Pallasits wirklich kürzlich stattgefunden hat, geht aus der Beschaffenheit des Wiener Stücks hervor, an welchem noch Schmelzrinde zu erkennen ist.

\section{Callac}

Kerilis

Cambria (Lockport), Niagara Co., New York, U. S. A.

Eisen, Of, gefunden 1818 ?, beschrieben 1845 .

r845: Sillima n: Notice of a mass of Meteoric Iron found at Cambria near Lockport, in the State of New York. Am. Journ. (I) 48 , p. $388-392$ (Abbildung der Masse und einer geätzten Platte). Ref, Pogg. Ann, Bd. 67 ( 1846$)$, p. 124 ; N. J. 1846 , p. $85-86$; s. auch beiläufige Erwähnung bei Burkart: Fundorte I, N. J. 1856 , p. 267 .

r846: Sill $\mathrm{im}$ an und $\mathrm{Hunt}$ : On the Meteoric Iron of Texas and Lockport. - Examination of the Lockport Iron. Am. Journ. (2) 2, p. 374-376 (Abbildung der gleichen Platte wie oben). 
Ref. Pogg. Ann. Bd. 71 (1847), p. 544 ; Pogg. Ann. Bd. 73 (1848), p. 332 ; Kenngott, Uebersicht $1844 / 49$, p. 222 ; Liebig-Kopp, Jahresber.1847/48,p. 1313 ; Journ. prakt. Chem. Bd. 42 (1847), p. $45^{8}$; >L'Institut * Bd. I4 (1846) Nr. 637 , p. 96 und Bd. 15 (1847) Nr. 725, p. 384 .

1846: S hepard: Report on Meteorites. Part IV. Summary of American Meteor-masses. Am. Journ (2) 2, p. 39I. r847: $\mathrm{R}$ a m m e ls b e r g : Handwörterbuch, Suppl. 3, p. 81 .

1847: Shepard: Report on Meteorites. - Nr. 15. Lockport (Cambria) New York. Am. Journ. (2) 4, p. 82. Ref. Liebig-Kopp, Jahresber. $1847 / 48$, p. I310 ; Kenngott, Uebersicht 1850/5I, p. 136.

1852 : C l a r k: Dissert. Gött., p. 33-34 (Abb. einer Platte s. oben).

1854: v. Boguslawski: Zehnter Nachtrag, Pogg. Ann. Ergz.-Bd. 4, p. $406-407$.

1859: H a r ri s: Dissert. Gött., p. 108.

r86o: $R$ a m me 1s b e r g : Mineralchemie, p. 914, 915 .

r858/62: v. Reiche n b a ch: IV 638. VII 552. IX 163, 174, 181. X 359, 365. XII 457. XIII 363. XV по, I24, 126. XVI 26r, 262. XVII 266, 268, 269, 272. XVIII 484,487 . XIX 150, 154. XX 622, 624, 634, 635 .

1863: B u chner: Meteoriten, p. 173 -174 .

1863: Ros e: Meteoriten, p. 27, 65, I38, 153 .

1869: Meunier: Recherches, Ann. Chim. Phys. (4) 17, p. 72.

r870: R a mmels berg: Beiträge zur Kenntnis der Meteoriten. - Lockport (Cambria), New York (Analyse mittelst Brom). Mon,-Ber. Berlin. Akad. 1870, p. 444 .

r883: Smit h: Concretions, Am. Journ.
(3) 25, p. $417,419$.

1884: M e unier: Météorites, p. wo.

r885: Brezina: Wiener Sammlung, p. 200, 210, 233.

1887: Flight: Meteorites, p. I05.

I89o: E a s t m a n: Met. Astron., p. 318. 1893: Meunier: Révision des fers météoriques, p. 47-48 (Abb, von geätzten Platten).

r894: C o h en: Meteoritenkunde, p. 40, 55, 95, I3I, 199, 208, 23 I.

r895: Brezina: Wiener Sammlung, p. $268-269$.

Ursprüngliches Gewicht: $16^{1 / 3} \mathrm{Kgr}$. (36 pounds avoirdupois).

Nachweisbares Gewicht: 12 or 8 gr. Bailey 34 London, P. G. 25

Bement 209 Moskau 19

Berlin, U. $\quad 240$ New Haven 3156

Bologna 9 Paris, M. 160

Budapest $\quad 3^{8}$ Petersburg, B. 28

Calcutta $\quad$ 150 Pohl 28

Cambridge , $\quad 5^{\mathbf{I}}$ v. Siemaschko 79

\begin{tabular}{ll|l} 
Cleveland & I Stockholm 39
\end{tabular}

Dorpat 8 Troyes Spl.

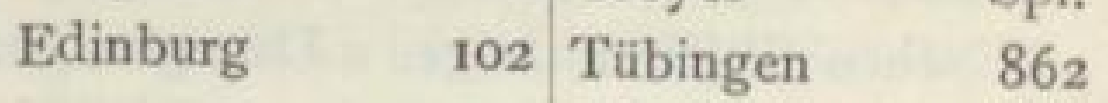

Frankfurt $\quad 39$ Turin, U. $\quad 27$

Göttingen $\quad 42$ Ward 2 10

Gregory $\quad$ IO3 Washington $\quad$ I55

Harvard, U. 692 Washington, Sh. 33

London, B. M. 5329 Wien, H. M.*) 150 ')

\section{Campbell Co.}

s. Anhang Stinking Creek

Campo del Cielo, Tucuman, Otum. pa; Gran Ghaco Gualamba, Argentinien.

Eisen, Ds, gefunden ${ }^{7} 78_{3}$, beschrieben $\quad$ 788 .

Hierher auch vorläufig ,WöhlerFisen « von unbekanntem Fundort. 1788: $\mathrm{Rubin}$ de Celis: An Account of a Mass of native Iron, found in South-America. Phil, Trans. Bd, 78 ,

I) Ausserdem noch 149 gr. nach I. Juli 1893 erworben. 
p. 37-42. Translation of Don Michael Rubin de Celis's Letter to the Royal Society, relative to a Mass of native Iron, found in South-America, p. $18_{3}$ -189 .

r794: C h 1 a d n i : Pallaseisen, p. 40-4r. 1799: Proust: Sur le fer natif du Pérou. Journ. Phys. Bd. 49 (an VII), p. 148 -149; s, auch Gilb. Ann. Bd. 24 (1806), p. 297-300: Ueber das gediegene Eisen aus Tucuman.

I802 : How a r d : Experiments and Observations on certain stony and metalline Substances, which at different Times are said to have fallen on the Earth; also on various Kinds of native Iron. - Description of various Kinds of native Iron. By the Count de Bournon. - Examination of the Iron from South-America. Phil. Trans, (1802), p. 202-203, 210.

1803: de Drée: Recherches, Journ.

Phys. Bd. 56, p. 417 .

1803: I z a rn: Lithologie, p. 218, 224, 230, 232, 343 .

1804: v. Ende: Massen und Steine, p. $70-72$.

1804: Pötzsch: Kurze Darstellung, p. $45-49,92$.

r804: S ukow: Anfangsgründe der Mineralogie. Leipzig 1804, p. 235.

1805: R e us: Lehrbuch der Mineralogie. Dritter Teil, Bd. I, p. 480 .

1807: Klap roth: Beitrigge, Bd. 4, p. IOI; s. auch Abh. Berlin. Akad, I803, p. 3r.

1812: Bigot de Morogues, p. 296 $-300,339$.

1812: $\mathrm{C}$ h 1 a d n i: Verzeichnis, Schweigg. Journ. Bd. 4 Beil. I, p. 18.

1815: Ch 1 a dn i: Bemerkungen, Gilb. Ann. Bd. 50, p. $266-268$.

1817: Chladni: Dritte Fortsetzung, Gilb. Ann. Bd. 56 , p. $3^{85}$.

r8rg: $\mathrm{Ch}$ la dni: Feuermeteore, p. 92, $318,34 \mathrm{I}-343(1), 434$; s. auch Denkschr. München. Akad. 1812, p. 106.
I82 I : C h 1 a d n i : Erster Nachtrag, Gilb. Ann. Bd. 68, p. 346.

1825: Stromeyer in Poggendorff's Zusatz zu Rose's Arbeit : Ueber die in den Meteorsteinen vorkommenden krystallisierten Mineralien. Pogg. Ann. Bd. 4, p. 195, 196 (Analyse eines Olivins aus dem Eisen von Campo del Cielo, welches dem Pallas-Eisen vollkommen ähnlich sein soll).

1826: S e e b e ck: Ueber die magnetische Polarisation der Metalle und Erze durch Temperaturdifferenz. Pogg. Ann. Bd. 6 , p. 144 .

1833: Giebel u. He intz: Zeitschr. f. ges. Naturw. Bd. I, p. 234 .

r834: Woodbine Parish: Notice as to the supposed Identity of the large Mass of Meteoric Iron now in the British Museum, with the celebrated Otumpa Iron described by Rubin de Celis in the Philosophical Transactions for 1786 . Phil. Trans. Bd. 34 , p. 53 -54 ; s. auch Buenos Ayres and the Provinzes of the Rio de La Plata, by Sir Woodbine Parish. London 1839 , p. 257,259 . Ref. N. J. 1835 , p. 94. 1836: Kä mtz: Meteorologie, p. 260 $-26 \mathrm{r}$.

1843: P a rtsch: Meteoriten, p. 128 $-130$.

1845: D a r 1 u : Nombreux aérolithes du désert d'Atacama (haut Pérou) et nombreuses masses de fer météorique des environs de Santiago del Estero (RépubliqueArgentine). C.R. Bd.20, p.1720. Ref. N. J. I846, p. III.

1852: Wöhler u. Manross: Analyse eines Meteoreisens, Ann. Chem. Pharm. Bd. 81, p. $252-255$. [Diese Analyse bezieht sich auf das sog. sWöhler Eisen \&, welches ursprünglich fast $37 \mathrm{gr}$. (4 Lot) wog und welches nach Brezina mit Campo del Cielo grosse Aehnlichkeit zeigt]; s, auch LiebigKopp, Jahresber. 1852, p. $991-992$ u. 1857, p. 734 ; Gött. Gel. Anz. (Nachr.) 
Januar 1852 ; Burkart, Fundorte I, N. J. 1856 , p. 265,268 ; Journ. prakt. Chem. Bd. 56, p. $185-186$; Pharm. Centr. 1852 , p. 555 ; s L'Institut * Bd. 20 (18 52 ), p. $159-160$.

1852: C l a r k: Dissert. Gött., p. 36-37 und $76-77$.

I854: B a l ce $11 \mathrm{~s}$ : Lithologia meteorica, p. 26.

1854: v. Boguslawski: Zehnter Nachtrag, Pogg. Ann. Ergz.-Bd. 4, p. 4I4, 453 (Wöhler-Eisen).

1855: $\mathrm{H}$ a i d ing er: Bemerkungen über die zuweilen im geschmeidigen Eisen entstandene krystallinische Structur, verglichen mit jener des Meteoreisens. Sitzber. Wien, Akad. Bd. I5 I, p. 358. 1859: $\mathrm{B} \mathrm{uchner:} \mathrm{Feuermeteore,} \mathrm{p.} 52$ $-53,127$.

1859: Harris: Dissert. Gött., p. IO3, 125-126.

r860: R a m mels berg: Mineralchemie, p. 918,949 .

r858/62: v. Rei chen b a ch: VII 551 , 557. VIII 486. IX 162, 175, 176, 182. X 359 (Wöhler-Eisen). XI 29I. XII 457. XIII 354, 364. XIV 393. XV 100. XVIII $482,483,491$. XIX $150,155$. XX 622, 627, 629, 630, 631. XXI 578 . 1863: Buchner: Meteoriten, p. ${ }_{3} 6$ -138 .

1863: Ros e: Meteoriten, p. 30, 38

(Wöhler-Eisen), 51-52, 69, I 52, 153. r869: Buchner: Vierter Nachtrag,

Pogg. Ann. Bd. 136, p. 439.

r870: Rammelsberg: Meteoriten,

p. 79 .

r874: B jörling: Meteoriter och Kometer (cfr. Hessle), p. ${ }_{3}$.

1884: M e un i e r : Météorites, p. 37, 96, 98, 112.

r885: Brezina: Wiener Sammlung, p. 200, 220, 221 (Wöhler-Eis.), 234. 1889: F l e t c h e r : Atacama Meteorites, Mineral. Magaz. Bd. 8, p. 228, 229.
1890: Eastm an: Met. Astron, p. 322. 1890: Brezina: Ueber Meteoreisen, Oesterr. Zeitschr. f. Berg- u. Hüttenw. Bd. 38 , p. $35^{8}$ (Abbildung einer geätzten Platte).

1893: Brezina: Ueber neuere Meteoriten (Nürnberg), p. 167.

1893: Meunier: Révision des fers météoriques, p. 70.

I894: F le t c h er: Introduction, p. 9, I4 (Wöhler-Eisen), 2I, 34 .

r894: C o he n : Meteoritenkunde, p. 7, $38,194,263$.

r895: Brezina: Wiener Sammlung, p. 295 .

r895: C o h e n : Meteoreisen-Studien IV, Ann. Hof-Mus. Bd. 10, p. $83,84,85$.

Ursprüngliches Gewicht : Etwa 300 Zentner (nach Rubin de Celis' Schätzung).

Nachweisbares Gewicht: $644854 \mathrm{gr}$. Belgrad Berlin, U. Bologna Budapest Calcutta Cambridge Göttingen Gregory Greifswald Harvard, U. 169 Wien, H. M. *)**) Kopenhagen $\mathbf{2 2 2 6}$

London, B. M. 20 London, P. G. 460 191 Paris, E. $\quad 982$ 10 Paris, M. 2353 67 v. Siemaschko 54 $3^{8}$ ') Stockholm 23 59 Troyes 2 5 Tübingen $\quad 354$ 177 Ward 20

8 Washington, Sh. 24 $\left.637000^{2}\right)$

\section{Campo del Pucara} $5^{\left.122^{3}\right)}$

\section{Cañada de Hierro}

Imilac

\section{Canara}

Tucson

Canellas, Villa nova bei Barcelona, Spanien.

Stein, Ci, gefallen r4. Mai r86r. r86r : Greg: On New Falls of Meteoric Stones. Philos. Magaz. Bd, 22, p. 107 -108. Ref. Kenngott, Uebersicht 186r,

Ausserdem Wöhler-Eisen : 1) 8 gr. 2) $30 \mathrm{gr} . \quad 3) 5 \mathrm{gr}$. 
p. $163-164$; N. J. 1862 , p. 497 ; Liebig-Kopp, Jahresber. 1861, p. II2I. 186r : B u c h ner: Zwei neuere Meteorsteinfälle. Briefliche Mitteilung. Pogg. Ann. Bd. I13, p. 510. Ref. Kenngott, Uebersicht 186I, p. 163 .

1863: Buchner: Meteoriten, p. 109. 1884: M e unier: Météorites, p. 26r. 1885: Brezina: Wiener Sammlung, p. $18 \mathrm{r}, 232$.

I892: Gr e dill a : Meteoritos, p.IIO-III.

Ursprüngliches Gewicht: Wohl nicht über I Kgr. Das grösste erhaltene Stück dürfte Madrid besitzen.

Nachweisbares Gewicht: 805 gr.

\begin{tabular}{ll|lr|} 
Berlin, U. & 8 & Madrid & 500 \\
Budapest & 3 & Paris, M. & 237 \\
Calcutta & I & Rom, U. & 46 \\
Gregory & I & v. Siemaschko & 7 \\
London, B. M. & I & Wien, H. M.*) & I
\end{tabular}
Der Stein scheint noch nicht analysiert worden $\mathrm{zu}$ sein.

Caney Fork

Carthago

Cangas de Onis (Elgueras), Oviedo, Spanien.

Stein, Cgb, gefallen 6. Dezbr. 1866. r873: R öm er: Geologische Reisenotizen aus der Sierra Morena. N. J. 1873, p. 257. Ref, Liebig-Kopp, Jahresber. 1873, p. $1250-1251$.

1873: M e un ier: Les pierres, qui tombent du ciel. sLa Nature Bd. 1, p. 403. 1874: Lua nco: Annales de la Sociedad de la Historia Natural (Madrid) Bd. 3 , p. 64 (Analyse s. auch Gredilla 1892 w. u.).

r884: Me unier: Météorites, p. 98 , $256,259,335,347$.

1885: Brezina: Wiener Sammlung, p. 183,233 .

1887: Flig h t: Meteorites, p. 147.

1892: Gre dilla: Meteoritos, p. 112-115.

Ursprüngliches Gewicht: Römer sagt $>6$ Pfund , was aber nach den folgenden Angaben nicht richtig sein kann.

Nachweisbares Gewicht: 6880 gr.

\begin{tabular}{lr|lr} 
Bement & 4 & de Mauroy & I \\
v. Braun & I7 & Paris, M. & 2 I55 \\
Budapest & 154 & Rom, U. & 2 \\
Cohen & 81 & Sevilla & 3627 \\
Dresden, M. & 73 & v. Siemaschko & I4 \\
Gregory & 33 & Strassburg & 8 \\
Greifswald & 66 & Tübingen & 8 \\
Harvard, U. & I3 & Turin, U. & I4 \\
London, B. M. & 96 & Wien, H. M. & II4 \\
Madrid & 400 & &
\end{tabular}

Cañon Diablo, Arizona U. S. A.

Eisen, $\mathrm{Og}$, gefunden und beschr. r $89 \mathrm{r}$.

189x: Foote: A new Locality for Meteoric Iron with a Preliminary Notice of the Discovery of Diamonds in the Iron. Am. Journ. (3) 42, p. 4I3-4I7. (Abb, einer Masse von 20I lbs und einer geätzten Platte auf Tafel 14 und 15). Ref. N. J. 1892 I, p. 513-514. r892: H u n t ing to $\mathrm{n}:$ s Science 8 . Juli I892; s. auch Amer. Acad. Arts and Sc. Bd. 22 , p. $252-253$.

1892: Mallard: Sur le fer natif de Cañon Diablo. C. R. Bd. $\mathbf{H}_{4}$, p. 812 -814 . Ref. N. J. 1894 I, p. 275.

1892: Da u brée: Quelques Observations à la suite de la Communication de M. Mallard. C. R. Bd. 114, p. 814. 1892: F riedel: Sur l'existence du diamant dans le fer météorique de Cañon

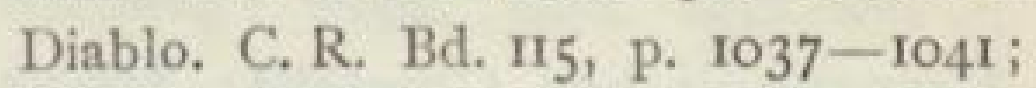
s. auch Bull. Soc. Franc. de Min. Bd. 9, p. $258-263$.

1893: Mo iss a n: Étude de la météorite de Cañon Diablo. C. R. Bd, n6, p. 288-290 (Vergrösserte Abbildung eines 0.7 auf $0.3 \mathrm{~mm}$ grossen Diamanten). Ref. N. J. 1894 I, p. 448.

1893: F riede 1: Sur le fer météorique de Cañon Diablo. C. R. Bd. ı6, p. 290 -29I. Ref. N. J. 1894 I, p. $447-448$. 
1893: Daubrée: Observation sur les conditions, qui paraissent avoir présidé à la formation des météorites. C. R. Bd. 116, p. $345-347$. Ref. N. J. 1894 I, p. 448 .

1893: Meunier: Révision des fers météoriques, p. 29, 37 .

1893: M e un i er : Remarques géologiques sur les fers météoriques diamantifères. C. R. Bd. n6, p. 409-410. Ref. N. J. 1894 I, p. 449.

r893: Kunz und $\mathrm{Hunting}$ to $\mathrm{n}$ : On the Diamond in the Cañon Diablo Meteoric Iron and on the hardness of Carborundum. Am. Journ. (3) 46, p. 470 -473 . Ref. N. J. 1895 I, p. 277.

1893: Brezin a: Ueber neuere Meteoriten (Nürnberg), p. 165.

1893: v. H a u er: Ann. Hof-Mus. Bd. 8 (Not.), p. 29.

r 894 : Hunting t o $\mathrm{n}$ : Further observations upon the occurence of diamonds in meteorites. Proc. Amer. Acad, Boston Bd. 29, p. 204-2II (Abbildung einer grossen Masse von $\mathrm{I} 87 \mathrm{lbs}$, einergeätzten Platte und eines Diamanten).

r894: C oh en: Meteoritenkunde, p. 86, IOI, I4I, I43, I44, I46, 164, 209, $212,226$.

r895: D e r b y : Constituents of the Cañon Diablo Meteorite, Am. Journ. (3) 49, p. IOI-no (Analysen).

1895: Brezina: Wiener Sammlung, p. 288.

r895: C o h e n: Meteoreisen-Studien IV, Ann. Hof-Mus. Bd, Io, p. 82 .

Ursprüngliches Gewicht: Nicht zu ermitteln.

NachweisbaresGewicht: 4 I 2 I 734 gr.

Bailey

Bement

Berkeley

Berlin, U.

Bern, M.

\begin{tabular}{r|lr}
637 & Blatz & III \\
70902 & Böhm & 62 \\
91 & Bologna & 70 \\
107 & v. Braun & 1459 \\
222 & Bremen & 22
\end{tabular}

\section{Brezina}

Budapest

Catania

Cleveland

Dresden, M.

Dublin, M.

18272 New Haven 380000 584 Newton

4I Paris, E.

880

410 Paris, M.

1982

82 Pech

169 Pierrotet

168)

397

Freiberg, i, S, 37 Prag, M.

Freiberg, iS.N. 256

Gregory 11030

Greifswald 27975 Stockholm 194000

Harvard,U.563845 $5^{1}$ ) Stuer

$\begin{array}{llll}\text { Howell } 1770400 & \text { Stürtz } & 70\end{array}$

Karlsruhe, M. 66 Troyes 3

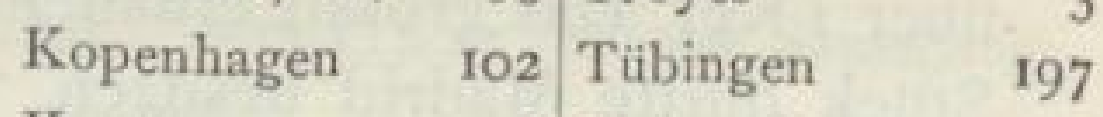

\begin{tabular}{ll|l} 
Krantz & 916 & Turin, U. \\
Kunz
\end{tabular}

Kunz II3 000 Utrecht $\quad 148$

London,B.M. 68550 Ward 708546

de Mauroy 405 Washington $\quad 1518$

Minneapolis 657 Wien, H. M. 180672

München $\quad 43$ Wien, U. I. 295

Neapel I51 Wrany $\quad$ I7

Nach Juli 1893 erwarb Herr Bayet in Brüssel 241 gr. Die Universität Basel besitzt ebenfalls ein Stück dieses Fundortes. New York M. erhielt kürzlich $2300 \mathrm{gr}$.

\section{Cany Fork}

Smithville

Caparrosa s. Anhang u. auch Toluca Cape Girardeau, Südöstliches Missouri, U. S. A.

Stein, Cc, gefallen 14. August 1846 , zuerst beschr. 1886 .

1886: D a n a und Penfiel d: On two hitherto undescribed meteoric stones, 2. Meteorite from Cape Girardeau, Missouri. Am. Journ. (3) 32, p. 229-230 (Analyse). Ref. N. J. 1887 II, p. 43 -44; Liebig-Kopp, Jahresber. 1886, p. $2331-2332$.

1890: Eas t m a n: Met. Astron., p. 316. 1894: C o h en: Meteoritenkunde, p. 249, $256,28_{3}$.

1895: B r ez in a: WienerSamml.,p. 255.

1) Hiervon $4924 \mathrm{II}$ gr. Eigentum von Herrn Huntington. 
Ursprüngliches Gewicht: 3 Stücke, von denen zwei $205^{8} \mathrm{gr}$. wogen. Nachweisbares Gewicht: $235^{8}$ gr. London, B. M. $79 \mid$ Ward New Haven 2058 Washington 4 Paris, M. $\quad 64$ Wien, H. M. 93 v. Siemaschko 43

Capeisen, Capland, Südafrika. Eisen, Hca, gefunden 1793 , erwähnt 1801 .

r8or: B a rrow : Account of travels into the interior of southern Africa, London 1801, p. 226.

1803: Klaproth: Ueber meteorische Stein- und Metallmassen. Abh. Berlin. Akad. 1803 , p. 35 .

r804 : van Marum : Naturkundige Verhandelingen van de Bataafsche Maatschapy der Wetenschapen te Haarlem. II 2, p. 257-264.

1804: P ötzsch: Kurze Darstellung, p. $49-50$.

r805: v. Da n kelmann: Voigt's Magazin d. Naturk. Bd. 1o I, p. 3-2I.

r806: Smithson Tennant: An account of an analysis, by Mr. Smithson Tennant, of a kind of native Iron found at the Cape of Good Hope, was read. Tilloch's Philos. Magaz. Bd. 25, p. 182; s. auch Ann. des Mines Bd. 6 (1821), p. 260.

r8r2: C h la d n i: Verzeichnis, Schweigg. Journ. Bd. 4 Beil. I, p. 18.

1812: B igot de Morogues, p. 3or. r815: Chladni: Bemerkungen, Gilb. Ann. Bd. 5o, p. 264-265.

18r6: Strome yer: Gött. Gel. Anz. Nr. 205; s. auch Gilb. Ann. Bd. 54 (I816), p. 393 .

1817: v. Soem merring: Ueber die Zeichnungen, welche sich bei Auflösung desMeteoreisens bilden. Schweigg. Journ. Bd. 20, p. $9 \mathrm{I}-94$.

r8r7: Stromeyer: Auffindung von Kobalt in dem Meteoreisen. Gilb. Ann. Bd. 56, p. 19I-194; Auszug aus Gött.
Gel. Anz. vom 23. Dez. 18 15 .

I8I9: Chla d n i : Feuermeteore, p. 92, $317,318,319,33^{1}-333$ (1), 433; s. auch Denkschr. München. Akad. 18I2, p. Io7. r830: v. Holger: Analyse des Cap'schen Meteoreisens. Baumgartner's Zeitschr. f. Phys, u. Math. Bd. 8, p. 279 -284 . Ref. Leonhard's Jahrb. $183 \mathrm{I}$, p. $299-300$.

1832: St r om e yer: Molybdän u. Kupfer im Meteoreisen. Pogg. Ann, Bd, 24, p. 651 .

r835: W e hrle: Analyse einiger Meteoreisenmassen. Baumgartner'sZeitschr. f. Phys, u. verw. Wiss. Bd. 3, p. 225 -226; s. auch Ann. Chem. Pharm. Bd. 14 (1835), p. 94-95.

r836: K äm t z: Meteorologie, p. 260. r84I : R a m mels ber g : Handwörterbuch Bd. I, p. 422.

I843: P a rtsch : Meteoriten, p. 13I$-\mathrm{I} 33, \mathbf{1} 45$.

r852: Wöhler: »Passiv«, Pogg. Ann. Bd. 85 , p. 448 .

r852: Cl ark: Dissert. Gött., p. $3^{8-39}$. 1854: Uricoëchea: Analyse des Meteoreisens von Toluca u. vom Cap der guten Hoffnung. - 2. Eisen vom Cap. Ann, Chem. Pharm. Bd. 9I, p. 252253; s. auch Chem. Centr. 1854, p. 816; 1856, p. 126; Journ. prakt. Chem. Bd. 63 (1854), p. $317-318$; LiebigKopp, Jahresber. 1854 , p. $917-918$; N. J. 1855, p. 455 .

1854: v. Boguslawski: Zehnter Nachtrag, Pogg. Ann. Ergz,-Bd, 4, p. $397-398$.

r855: Ha i d in g e r: Bemerkungen über die zuweilen im geschmeidigen Eisen entstandene krystallinische Structur, verglichen mit jener des Meteoreisens. Sitzber. Wien. Akad, Bd. 15 I, p. $35^{8}$. r855: B ö ck in g: Mineralanalysen, - 3 . Meteoreisen vomCap der gutenHoffnung. Ann. Chem. Pharm. Bd. 96, p. 246 ; s. auch Am. Journ. (2) 22, p. 272 ; Dissert. Gött. 1855, p. 15-18; N. J. 
I856, p. 843 ; Kenngott, Uebersicht 1856/57, p. 151 ; Liebig-Kopp, Jahresber. 1855 , p. 1029; Journ. prakt. Chem. Bd. 67 (1856), p. 208.

1859: B u c hn er: Feuermeteore, p. I28. r859: Harris: Dissert. Gött., p. 105 $-106$.

1860: $\mathrm{R}$ a m mels berg: Mineralchemie, p. $919-920,946$.

r858/62: v. R e i ch en b a ch: VI 448. VII 551, 562. IX 162, 175, I76, 182. X 359,363 . XI 29I. XII 457. XIII 354. XV 100. XVI 255, 262, XVII 266 $-267,268,269,273,274$. XVIII 488 , 490. XIX 149, 151, 156. XX 623, 629. XXI 578,590 .

r863: Buchner: Meteoriten, p. 146 $-\mathrm{I} 48$.

1863: Ros e: Meteoriten, p. 37, 7072 Taf. III, 138, 153, 160.

1867: v. Baumhauer: Sur le fer météorique du Cap de Bonne-Esperance (Neue Analyse, zwei Abb. der Masse und einer geätzten Platte. Archives Néerlandaises Bd, 2, p. $1-8$ (Sep.); s. auch Liebig-Kopp, Jahresber. 1867, p. 1050.

1869: Meunier: Recherches, Ann. Chim. Phys. (4) 17, p. 60, $71-72$. 1869: Buchner: Vierter Nachtrag, Pogg. Ann. Bd. 136, p. $60 \mathrm{I}-602$. 1870: R a mmels berg: Meteoriten, p. 79 .

1875: Mohr: Entstehungsart, Ann. Chem. Pharm, Bd. 179 , p. 259,269 -270 .

r884: M eunier: Météorites, p. 36, $39,98,112$.

r885: Brezina: Wiener Sammlung, p. 201, 203, 219, 234 .

r89o: Brezina: Ueber Meteoreisen, Oesterr. Zeitschr. f. Berg- u. Hüttenw. Bd. 38 (Nr. 3 I), p. $35^{8}$ (Abb. einer geätzten Platte).

r89r: Cohen u. Weinschenk: Meteoreisen-Studien, Ann. Hof-Mus. Bd. 6, p. 160,165 .
1893: M e unier: Révision des fers météoriques, p. 16, 20.

1894: Cohen: Meteoritenkunde, an vielen Stellen.

r895: B r ez i n a : Wiener Samml., p.293. r895: Co h e n : Meteoreisen-Studien IV, Ann. Hof-Mus. Bd. 1o, p. $83,90,91$.

Ursprüngliches Gewicht: I 70 (oder 300?) Pfund.

Nachweisbares Gewicht: 7 I 789 gr.

Bailey $\quad 82$ London, P.G. 36

\begin{tabular}{ll|l|l} 
Bement & 162 & Lüttich & 46
\end{tabular}

Berlin, U. $\quad 744$ Neumann I

Böhm 34 New Haven 12

Bologna $\quad$ I20 Paris, M. 230

\begin{tabular}{lll} 
Bonn & 86 & Pohl \\
\hline
\end{tabular}

v. Braun $\quad 144$ Rom, U. 69

Budapest 67362 v. Siemaschko 169

Calcutta $\quad 50$ Stockholm $\quad 89$

Cambridge $\quad 26$ Strassburg $\quad 49$

Cleveland 20 Troyes 18

$\begin{array}{llll}\text { Cohen } & \text { I9 } & \text { Tübingen } & 48\end{array}$

Göttingen $\quad 169$ Utrecht 4

$\begin{array}{llll}\text { Gregory } & 59 & \text { Ward } & 27\end{array}$

Greifswald $\quad$ I2 Washington 29

Harvard, U. по Washington, Sh. I82

Klausenburg $\quad$ I3 Wien,H.M.*)*** 947

Kristiania $\quad$ I3 Wien, U. I. 52

London, B. M. 329

Ausserdem besitzt Zürich: 16 gr. $\$ 1793$ vom grossen Fischfluss im Lande der Kaffern M oder $\mathrm{P}$ im Widerspruch mit Wien .

\section{Cape of good Hope Capeisen}

Caracoles Imilac

Carcoar Cowra

Carcote, Wüsten-Cordillere, Chile, Südamerika.

Stein, Ck, gefunden vor I888, beschr 1889 .

r889: v. Sandberger: Ein neuer Meteorit aus Chile. N. J. I889 II, p. 173-180. Vorläufige Mitteilung N. J. 1889 I, p. 259 (Analyse; s, auch folgende Arbeit). 
189o: Will und Pinnow: Chemische Untersuchung eines Meteoriten von Carcote (Chile). Ber. d. d. chem. Ges. Bd. 23, p. 345-353. Ref. N. J. 189i I, p. 50.

r89r: v. H a u e r: Ann. Hof-Mus, Bd, 6 (Not.), p. 55 .

r894: Cohen: Meteoritenkunde, p. 57 , $68,136,141,142,163,201,223,234$ $236,246$.

r895: Brezina: Wiener Sammlung, p. $260-26 \mathrm{I}$.

Ursprüngliches Gewicht: v. Sandberger erhielt ein Bruchstück von $79^{1 / 2}$ gr. und seine beträchtliche Quantität\& Pulver.

Nachweisbares Gewicht: 325 gr.

Dresden, M. $19 \mid$ Wien, H. M. 80

\begin{tabular}{ll|l} 
Erlangen & I5 Würzburg & 208
\end{tabular}

London, B. M. 3

\section{Carleton Tucson}

Tucson

Carlsburg

Carlton-Hamilton, Hamilton County, Texas.

Eisen, Of, gefunden 1887 , beschr. 189o. Der Name des Fundortes ist Carlton. Um jede Verwechslung mit Carleton-Tucson $=$ Tucson zu vermeiden, möge die Bezeichnung Carlton-Hamilton gewählt werden.

1890: E a s t m a n n: Met. Astron.,p.320. 189o: Howe11: Notice of two new Iron Meteorites from Hamilton Co. Texas, and Puquios, Chili, S. A. I. The Hamilton Co. Meteorite. Am. Journ. (3) 40, p. 223-224 (Analyse von Eakins, Abb.); s. auch U. S. Geol. Surv. Nr. 78 (189r), p. 95. Ref. N. J. 1891 II, p. $418 ; 1892$ II, p. 34 .

189o: How e11: Description of new Meteorites. - The Hamilton County Meteorite. Proc. Rochester Acad. of Sc. Bd. 1, p. 87-89 (Abb, einer grossen Platte in Naturselbstdruck, Analyse wie oben). Ref. Journ. Chem. Soc. 1891, p. 277-279; Oesterr. Zeitschr. f. Berg- u. Hüttenw. Bd. 39 (1891), p. 228 ; N. J. 1892 II, p. 34 .

I892: v. H a uer: Ann. Hof-Mus. Bd, 7 (Not.), p. 72.

1893: Brezin a: Ueber neuere Meteoriten (Nürnberg), p. 163.

1893: Meunier: Révision des fers météoriques, p. 65,66 (Abb. einer geätzten Platte).

I894: Co h e n: Meteoritenkunde, p. 77, $84,95,106,124,156,190,193$.

r895: Brezina: Wiener Sammlung, p. $270-27 \mathrm{I}$.

r895: C o h e n : Meteoreisen-Studien IV, Ann. Hof-Mus. Bd. 10, p. $82,90$.

Ursprüngliches Gewicht: $8 \mathrm{r}^{1} / 2 \mathrm{Kgr}$. Nachweisbares Gewicht: $6568 \mathrm{r}$ gr. Bailey $\quad 67$ Paris, E. 132 Bement $\quad 654$ Paris, M. 249 Berlin, U. $\quad 70$ Prag, M. $\quad 88$ Böhm 1055 Riga $\quad 57$ \begin{tabular}{ll|l} 
v. Braun $\quad 246$ & Rom, U. & I54
\end{tabular} Brezina IOoI Sevilla 20 Budapest $\quad 634$ v. Siemaschko II8 Dorpat $\quad 636$ Stockholm 105 Dresden, M. $\quad$ I03 Stürtz $5^{8}$ Gregory $\quad 515$ Stuttgart $\quad 65$ Greifswald $\quad 84$ Washington II5 $_{5}$ Harvard, U. 3284 Ward 41508 Howell $\quad 52$ Wien, H. M. 7576 Krantz $\quad 492$ Wien, U. I. 133 London, B. M. 6185 Wrany 52 New Haven 173

Carnawelpaita s. Anhang Ceylon Carrisalillo Vaca Muerta Carroll Co. Eagle Station Carthago (Smith County), Tennessee, U. S. A.

Eisen, Om, gefunden 1840 , beschr. 1846 .

Hiess früher auch Coney Fork. Da aber dieser Name eigentlichCaney 
Fork lautet und dadurch leicht mit Caryfort-Smithville verwechselt werden könnte, schlägt Herr Direktor Brezina vor, zum alten Namen Carthago zurückzukehren, welchemVorschlag ich gernefolge.

r846: Troost: Description of three varieties of Meteoric Iron, - I. Meteoric Iron from Carthage, Smith County, Tennessee. Am. Journ. (2) 2, p. 356 $-357$.

I847: S h e p a r d: Report on Meteorites, Am. Journ. (2) 4, p. 79.

I852 : C 1 a r k : Dissert. Gött., p. 60-6I. 1854: v. Boguslawski: Zehnter Nachtrag, Pogg. Ann. Ergz.-Bd. 4, p. 404 .

1859: Harris: Dissert. Gött., p. II5. r863: $\mathrm{Bu} \mathrm{chner:} \mathrm{Meteoriten,} \mathrm{p.} 174$ -175 .

r863: R o s e: Meteoriten, p.26,64,139,152. r858/65: v. Reichen bach : IV 638 . VI 448 . VII 551 . IX $163,174,181$. XII 457. XIV 393. XV 100, Ho, III, II4, 124, 128. XVI 250, 25I, 26I, 262. XVII 265, 266, 272. XVIII 484. XIX 154. XX 622. XXI 578. XXV 436, 600. I 866 : B o ric k y : Unter kleinerenMitteilungen, auch Angaben (u. Analyse) über das Eisen des böh mischen Museums in Prag (s. u.). N. J. 1866, p. $808-810$. Ref. Liebig-Kopp, Jahresber. 1866, p. 1008 ,

r869: Buchner: Vierter Nachtrag,

Pogg. Ann. Bd. 136, p. 602.

1872: Q u e nstedt: Klar und Wahr, p. 28I, 313 (Abb. der grossen Masse in der Tübinger Sammlung u. einer geätzten Platte).

1875: v o m R a th: Meteoriten, Verh, naturh. Ver. Bonn, Bd. 32, p. 362 .

r88r: Brezina: Bericht III, Sitzber.

Wien. Akad. Bd. 84 I, p. 282.

r884: M e un ier: Météorites, p. 99,rı6. r885: Brezina: Wiener Sammlung, p. 213,234 . r886: H un t in g t o $\mathrm{n}$ : Crystalline Structure, Am. Journ. (3) 32, p. 287.

I890: E a s tm a n n : Met.Astron., p. 318 . r893: Meunier: Révision des fers météoriques, p. 52, 55 .

r894: Co h e n : Meteoritenkunde, p. 74, $76,226,232,245,248,321$.

1895: Brez ina: Wiener Sammlung, p. 276.

Ursprüngliches Gewicht: I27 Kgr. (280 pounds).

Nachweisbares Gewicht : $123596 \mathrm{gr}$. Die Differenz zwischen dem ursprünglichen und dem nachweisbaren Gewicht ist in Hinsicht auf die grosse Verteilung auffallend gering. Ich möchte vermuten, dass unter den hier folgenden Angaben auch solche, welche zu Smithville (Caryfort) gehören, sich befinden.

\begin{tabular}{|c|c|c|}
\hline Bailey & 56 & Minneapolis \\
\hline Bement & 35 & Moskau \\
\hline Berlin, G. & $3 \mathrm{I}$ & Neumann \\
\hline Berlin, U. & 804 & New Haven \\
\hline Bologna & 64 & Paris, M. \\
\hline Bonn & 325 & Pohl \\
\hline v. Braun & 107 & Prag, M. \\
\hline Breslau & III & Rom, U. \\
\hline Budapest & 6775 & v. Siemaschko \\
\hline Calcutta & 228 & Stockholm \\
\hline Cambridge & $3^{I}$ & Strassburg \\
\hline Dorpat & 142 & Stürtz \\
\hline Dresden, M. & 140 & Stuttgart \\
\hline Dresden, P. & 95 & Troyes \\
\hline Edinburg & 104 & Tübingen \\
\hline Göttingen & 22 & Utrecht \\
\hline Gregory & $9 \mathrm{I}$ & Ward \\
\hline Harvard, U. & 18036 & Washington \\
\hline Kopenhagen & 195 & Washington, Sh \\
\hline London,B.M. & 24570 & Wien, H. M. *) \\
\hline London, P. G. & & Wien, U. I. \\
\hline
\end{tabular}

Caryfort, gefd. 1840 , beschr. 1845 Smithville 
Caryfort, gefd. 1840 , beschr. 1846 Carthago

Casale 1840

Cereseto

Casale I 868 Motta di Conti

Casas Grandes

Chihuahua

Casey County, Georgia (?) U., S. A. (Lawr. Smith und Fletcher sagen Kentucky, Flight Georgia).

Eisen, Og, gefunden und erwähnt 1877 .

1877: S mith: Two new Meteoric Irons. Am. Journ. (3) 14, p. 246 (Kurze Erwähnung).

1880: Brezi n a: Bericht I. - 3. Casey County, Georgia. U. S. 1877. Sitzber. Wien. Akad, Bd. 82 I, p. 35 I.

1884: M e unier: Météorites, p. I16, 127.

1885: Brezina: Wiener Sammlung, p. $214,234$.

1887: F 1 i g h t: Meteorites, p. 206.

1893: Meunier: Révision des fers météoriques, p. $25,27$.

r895: Brezina: Wiener Sammlung, p. 286 .

Ursprüngliches Gewicht: ?

Nachweisbares Gewicht: $73^{2}$ gr.

Bailey

Bement

Gregory 21 Minneapolis 36 134 Paris, M. 82

Harvard, U. $\quad 289$ Washington, Sh. 3

London, B. M. $\quad 45$ Wien, H. M. $\quad 65$

\section{Casignano Borgo San Donino}

Castalia, Nash County, Nord-Carolina, U. S. A.

Stein, Cgb, gefallen 14 . Mai 1874 . 1875 : Ker r: Rep. Geol. Surv, N. Carolina Bd. I. App., p. $3{ }^{1} 3$, (gedruckt zu Raleigh) Ref. N. J. 1876, p. 324.

1875: Smith : Description of the Nash County Meteorite, which fell in May 1874. Am. Journ. (3) 1o, p. $147-148$ (Analyse); s, auch Original Researches 1884 , p. 478-479; Liebig-Kopp, JahW ülfing, Metcoriten. resber. 1875 , p. 1314 ; C. R. Bd. 80 (1875 I), p. $1453-1454$.

1879: R ammelsberg: Meteoriten, p. 24,25 .

1884: Meunier: Météorites, p. 256, 260. 1885: Brezina: Wiener Sammlung, p. 183,233 .

1887: Flight: Meteorites, p. 72.

r889: B r ez in a: Ankauf der Hidden'schen Meteoriten- und Mineraliensammlung für die mineralog. Abteilung. Ann. Hof-Mus. Bd. 4 (Not.), p. 87.

1889: Flet cher: Atacama Meteorites, Mineral. Magaz. Bd. 8, p. 226 (Streufeld to auf 3 miles).

1890: v. H a u er: Ann. Hof-Mus. Bd. 5 (Not.), p. 5, 48.

r89o: Eastman: Met. Astron., p. 316. 1893: Brezina: Ueber neuere $\mathrm{Me}-$ teoriten (Nürnberg), p. 160.

1895: Brezina: Wiener Sammlung, p. 251 .

Ursprüngliches Gewicht: $7300 \mathrm{gr}$. Drei Steine von $5^{1 / 2}, \mathrm{x}$ u. $0.8 \mathrm{Kgr}$.

Nachweisbares Gewicht: 6317 gr.

Bailey

Berlin, U.

v. Braun

Budapest

Cleveland

Dorpat

Gregory

Harvard, U.

Kopenhagen

London, B. M. de Mauroy

\begin{tabular}{r|lr}
40 & Moskau & II \\
II & New Haven & 248 \\
38 & Paris, M. & 23 \\
40 & Rom, U. & I6 \\
Spl. & V. Siemaschko & 5 \\
9 & Stockholm & 16 \\
10 & Ward & 8 \\
257 & Washington & 20 \\
7 & Washington, Sh. \\
29 & I \\
8 & Wien, H. M. 5520
\end{tabular}

Castine, Hancock County, Maine, U. S. A.

Stein, Cwa, gefallen 20. Mai 1848 . 1848: Shepard: An account of the Meteorite of Castine, Maine, May 20, 1848. Am. Journ. (2) 6, p. $251-253$ (Analyse des Eisens). Ref. Liebig-Kopp, Jahresber, 1847/48, p. 1312 ; Kenngott, Uebersicht $1844 / 49$, p. 286 (hier das 
Gewicht irrtümlich zu $\mathrm{I}^{1} / 2 \mathrm{Pfd}$. anstatt $\mathbf{I}^{1} / 2$ oz. angegeben).

1854: v. Bogus lawski: Zehnter Nachtrag, Pogg.Ann. Ergz.-Bd.4, p. 16, 23, $380-38 \mathbf{r}$.

I859: B u c hn er : Feuermeteore, p. 182. 1859: H a rris: Dissert. Gött., p. 93. 1863: B u chner: Meteoriten, p 78 . 1863: R o s e: Meteoriten, p. 155. I865: v. Reichenbach: XXV 322, 607.

1884: M eunier: Météorites, p. 279. r885: Brezina: Wiener Sammlung, p. $180,232$.

1890: E a st ma n : Met. Astron., p. 316. r894: C o he n: Meteoritenkunde, p. 272.

Ursprüngliches Gewicht: 42 gr. $\left(\mathrm{I}^{1 / 2} \mathrm{Oz}\right)$. Als das Stück an Shepard gelangte, wog dasselbe $\mathrm{I} \mathrm{oz}$ 3 pwts 5 grs, was auf Troygewicht deutet und gleich 36 gr. ist. Vorher ist in dem gleichen Shepard'schen Aufsatz AvoirdupoisGewicht angegeben.

Nachweisbares Gewicht: 22 gr. Bailey Spl. London, P. G.

Berlin, U. Spl. New Haven 15 Calcutta Spl. Paris, M. I Dorpat I Stockholm Spl. Göttingen Spl. Washington,Sh.Spl. Harvard, U. Spl. Wien, H.M.*) I London, B. M. 3

\section{Catorze}

Descubridora

Cereseto, Provinz Casale, Piemont, Italien.

Stein, Ccb (früher Cgb), gefallen I7. Juli 1840 .

r840: Sismonda: Atti della secunda riunione degli scienziati Italiani tenuta in Torino nel Settembre del 1840. Torino $184 \mathrm{r}$ (cfr. N. J. 1842 , p. 844 ).

1840: de Gregory: Aérolith tombé le 17 juillet à 20 lieues à l'ouest de Milan (Extrait de la Gazetta piemontesa 25. juillet). C. R, Bd. II, p. 243
-244; s. auch Pogg.Ann. Bd.5o, p.668. I84I : L a vin i : Analisi chimica esplorativa e proporzionale di un Meteorolite caduta nel Mese di Luglio 1840 a Cereseto nelle vicinanze di Casale e Moncalvo. Mém. Acad. Torino (2) 3, p.265273. Ref. Kenngott, Uebersicht $1862 / 65$, p. $445-446$.

1843: Partsch: Meteoriten, p. 144.

1854: v. Boguslawski: Zehnter Nachtrag, Pogg. Ann. Ergz.-Bd. 4, p. 88,360 .

r859: H a rri s: Dissert. Gött., p. 87. 1863: Buchner: Meteoriten, p. 65.

1864: B uchner: Erster Nachtrag, Pogg. Ann. Bd. 122, p. 319-320 (Parnisettis Osservazioni meteorol. von 1860 entnommen).

r859/65: v. Re ic he n b a ch: IX I6I, 168, 178. XIII 362. XXV 321, 431, 432,607 .

r884: M e unier: Météorites, p. 197, 198.

1885: Brezina: Wiener Sammlung, p. $182,183,233$.

r890 : v. Ni es s 1: Periheldistanzen, Verh. naturf. Ver. Brünn Bd. 29, p. 188, 195 , 196, 214, 247.

1895: Brezin a: Wiener Sammlung, p. $250,257$.

Ursprüngliches Gewicht: $375^{\circ}$ gr.? Das ursprüngliche Gewicht wird $\mathrm{zu}$ io Pfd. 2.2 Unz. piemontesisch angegeben und hinzugefügt, dass I Pfd. $=12$ Unz, sei. Nach Sonnet's Dictionnaire des Mathématiques appliquées, Paris 1867 , ist (p. 1or2) das $>$ libbra \& für Piemont gleich 368,875 gr. ; hiernach erhielte man für die ursprüngliche Menge $375^{\circ} \mathrm{gr}$. Sollten nachträglich noch Steine gefunden worden sein, oder liegen Verwechslungen vor?

Nachweisbares Gewicht: 4361 gr. 


\begin{tabular}{lr|lr} 
Bement & I & London, P. G. & 10 \\
Bologna & 13 & Paris, M. & 3 \\
v. Braun & 156 & Rom, U. & 182 \\
Budapest & 182 & v. Siemaschko & I \\
Calcutta & Spl. & Tübingen & 2 \\
Dorpat & 39 & Turin, U. & 3372 \\
Gregory & 65 & Utrecht & 10 \\
Kopenhagen & 65 & Wien, H, M.*) & 136 \\
London, B. M. & 124 & &
\end{tabular}

\section{Cerralvo}

\section{Cerro la Bomba}

Ceylon

Chail, Allahabad, N. W. Provinz, Indien.

Stein, gefallen 5 . November 1814 . 1815: Tilloc h: Philos. Magaz. Bd. 46, p. 155 (August 1815); s. auch Proc. Royal Soc. of Edinburgh recorded as Art. XVI in Quat. Journ. of Sc. and Arts 1816 Bd. 1, p. 117 ; auch Bibl. Brit. Nr. 478 (Nov, 1815 ), p. $291-292$. 1836: Kä mtz: Meteorologie, p. 285.

Ursprüngliches Gewicht: 2I Steine sollen gesammelt sein, wenig erhalten.

Nachweisbares Gewicht: Nur das Britische Museum besitzt nach meinen Erhebungen von diesem Falle einen Splitter, welcher weniger als $\mathrm{I}$ gr. wiegt.

\section{Chañarlino}

Merceditas

Chandakapoor, Berar, Indien.

Stein, Cib (früher $\mathrm{Cgb}$ ), gefallen 6. Juni 1838 .

1854: Greg : Philos. Magaz. (4) 8, p. 460. 1859: H arris : Dissert. Gött., p. 86. 1863: Buchner: Meteoriten, p. 60. 1863: R o s e: Meteoriten, p. 155. 1858/65: v. Reichen ba ch: VI 455. IX 161. 168, 178. XI 294, 296, 300. XIII 369 Fig. XXV 32I, 324, 427, $432,607,615$.

r884: M e unier: Météorites, p. 268.
1885: Brezina: Wiener Sammlung, p. $182,233$.

1895: Brezin a: Wiener Sammlung, p. 250 .

Ursprüngliches Gewicht : ? Sowerby soll einen Stein von $4200 \mathrm{gr}$. besessen haben; Herr Dr. Traquair (Edinburg) teilt mir in seinem Verzeichnis mit: »Original mass weighed 5076 grammes .

Nachweisbares Gewicht: 10 745 gr.

Bailey

Bement

Berlin, U.

98 London, P. G. 167

Bologna

v. Braun

Budapest

Calcutta

Cambridge

Edinburg

Göttingen

Gregory

Harvard, U.

2 Moskau

2

Spl. New Haven $\quad 4 \mathrm{I}$

2 Oxford 6329

37 Paris, M. 5

49 v. Siemaschko I2

93 Stockholm 3

34 Troyes I

2651 Tübingen $204^{1}$ )

2 Upsala $\quad 36$

68 Ward 12

15 Washington 4

\begin{tabular}{lr|lr} 
Kopenhagen & II & Washington, Sh I \\
London, B. M. & $76 \mathrm{r}$ & Wien, H. M.*) & I05
\end{tabular}

Dieser Stein ist noch nicht eingehend untersucht worden.

Chandpur, bei Mainpuri, N. W. Provinz, Bengalen, Indien.

Stein, Cwa, gefallen 6. April 1885 . r885: Medlicott: Notice of the Pirthalla and Chandpur Meteorite. Records Geol. Surv. India Bd, 18, p. 148 -149 .

I886: D e w a r : Engineering Bd. 4I, p. 9I. 1886: D a u b r ée : Météorites récemment tombées dans 1 'Inde les I9 février 1884 et 6 avril 1885. C. R. Bd. 102, p. 96 -97. Ref. N, J. 1887 II, p. 45.

r894: F let c her: Introduction, p. 14. 1895: Brezina: Wiener Sammlung, p. 245.

Ursprüngliches Gewicht: I2OI gr. (etwa $1 / 12$ der ursprünglichen

1) Davon ein Stïck von $7 \mathrm{gr}$, deutlich Cia. 
Masse des Steins ist verloren). Nachweisbares Gewicht: 974 gr. Berlin, U. Calcutta 3 Paris, M.

London, B. M. 490 Wien, H. M. 4 4

Chantonnay, Bourbon-Vendée, Dep. Vendée, Frankreich.

Stein, Cgb, gefallen 5. August I $_{1} \mathrm{I}_{2}$. r8rg: Chla dni: Vierte Fortsetzung, Gilb. Ann. Bd. 6o, p. 239, 247-248. 1819: C a vo 1 e a u : Nachricht von dem bei Chantonnay in der Vendée, den 5. August 1812, herabgefallenen Meteorsteine. Gilb. Ann. Bd. 63, p. 228230 ; s. auch Journ. Phys, Bd, 88 (I8I9), p. 312 .

r8rg: Chl a dn i: Feuermeteore, p. 46, $50,56,58,66,68,73,155,301-$ $302(1), 43 \mathrm{I}$.

1821: John: Sur la Nature de grandes masses de fer métallique d'origine problématique, et sur celle du fer des aérolithes attirables par l'aimant. Ann. Chim. Phys. Bd. 18, p. 205.

r822: Chladni: Zweiter Nachtrag, Gilb. Ann. Bd. 54, p. 367.

r834: Berzeli us: Om Meteorstenar. 2) Meteorsten frin Chantonnay. K. Vetensk. Acad. Handl. (1834), p. 141I 44 (Analyse); abgedruckt in Pogg. Ann. Bd. 33 , p. $27-30$.

r836: Kämtz: Meteorologie, p. 253, 255,284 .

r84 I : R a m m els be r g: Handwörterbuch, p. 434.

1843: Haidinger: Uebersicht, p. Io8. (Die Berzelius'sche Analyse ist irrtümlich Rammelsberg zugeschrieben).

1843: Rammelsberg: Handwörterbuch, Suppl. I, p. IOI-102.

1843: Parts c h: Meteoriten, p. 38-39. 1845: R a m mels berg : Handwörterbuch, Suppl. II, p. 92-93.

1846: S hepard: Report on Meteorites. Am. Journ. (2) 2, p. 38 r.

$1847: \mathrm{Sch}$ a f hä ut 1 : Schönenberg, Gel.
Anz. München. Akad. (1847), p. 573 . r853: $R$ a m me $1 \mathrm{~s}$ ber $\mathrm{g}$ : Handwörterbuch, Suppl. V, p. 19-20.

1854: v. Bogus 1 awski: Zehnter Nachtrag, Pogg. Ann. Ergz.-Bd. 4, p. $14-15,34$.

r856: B urk a rt: Fundorte I, N. J. 1856 , p. 268 .

I859: Harris: Dissert. Gött., p. 7 I. r859: Bu chner: Feuermeteore, p. 82 $-83,92$.

r86o: R a m m els b e r g : Mineralchemie,

p. $922,930-932,950$.

1862: K e nn gott u. W i s er: Zürcher Sammlung, p. $146-147,157-158$.

1863: B u c hner: Meteoriten, p. $31-33$. r863: Ro s e: Meteoriten, p. 27, 84, 86, $92,10 \mathrm{I}-103,147,151,154,160$ (Taf. III). r858/65: v. Reichen b a ch: VI 453 , 454. IX 161, 172, 173, 180. X 359, 362 , 371. XI 294, 297, 301, 309. XIII 355, 363. XV 121, XVI 262. XX 623. XXIV 229. XXV 322, 424, 425, 426, $427,428,602,6 \mathrm{Ir}$.

1867: Go eb e 1: Kritische Uebersicht, Mélanges phys. chim. Bd. 7 , p. 336 . 1870: R a m mels berg : Meteoriten, p. $98,102,103,106,138,139,140$. 1870: R a mmelsberg: Ueber den Meteoriten von Chantonnay. Zeitschr. d. d. Geol. Ges. Bd. 22, p. $889-892$ (neue Analyse). Ref. Liebig-Kopp, Jahresber. 1870 , p. $1397-1398$.

I87I: M e un ier: Contribution au métamorphisme météorique. C R. Bd. 73, p. $1284-1285$.

1874: Tschermak: Die Trümmerstruktur der Meteoriten von Orvinio und Chantonnay. Sitzber. Wien. Akad. Bd. 7o, p. 465-470 (Abbildung von Dünnschliffen und einer grösseren Platte). Ref. N. J. 1875 , p. $418-420$.

1875: Tsch ermak: Vulkanismus, Sitzber. Wien. Akad. Bd. 71 II, p. 662 , 663,671 .

1875: vom R ath: Meteoriten, Verh. naturh. Ver. Bonn Bd. 32, p. 367 . 
1878: Ts ch ermak: Der Meteorit von Grosnaja. M. P. M. Bd. I, p. 16r. 1879: R a mme ls berg: Meteoriten, P II, 24, 25.

r882: B rez in a : Bericht IV, Sitzber. Wien. Akad. Bd. 85 I, p. $338,339$. r882: T s c her m a k : Ueber die Meteoriten von Mocs. Sitzber. Wien. Akad. Bd. 85 I, p. 204.

r882: Wiik: Mikroskopisk undersökning af naagra paa universitetets mineralkabinett befintliga meteoriter. Öfvers, af Finska Vetensk. Soc, Förh. Bd. 24. Ref. N. J. 1883 I, p. 384 .

1883: T s ch e rmak: Beitrag, Sitzber. Wien. Akad. Bd. 88 I, p. 360 .

$1883 / 85$ : T s c he rma k : Photographien, p. $3,18,19$.

1884: Me unier: Météorites, p. 79, 8I, $85,95,97,98,247,248-249,379$, 395.

1884: Wiepken: Notizen über die Meteoriten des Grossherzogl. Museums (Oldenburg). Abh, naturw. Ver. Bremen Bd, VIII, p. 525 .

r885: Brezin a: Wiener Sammlung, p. $165,182,183,233$.

1887: F $1 \mathrm{ight:}$ Meteorites, p. IOI-IO3. 1888: Newton: Orbits, Am. Journ. (3) 36, p. 4 .

1889: M eunier: Sur la matière noire de la Chantonnite. Bull. soc. franc, minéralog. Bd. 12, p. $76-8$ I. Ref. N. J. 1890 II, p. 59.

1890: v. Niess 1: Periheldistanzen, Verh. naturf. Ver. Brünn Bd. 29, p. 184.

r894: F l e t c h e r: Introduction, p. 39. r894: C o h e n, Meteoritenkunde, p. 39, $247,249,266,267,316,317$.

Ursprüngliches Gewicht : 69sPfund « nach Cavoleau's Angabe.

Nachweisbares Gewicht: 9942 gr.

\begin{tabular}{lr|lr|} 
Bailey & 19 & Bologna & 3 \\
Belgrad & 75 & Bonn & 31 \\
Bement & 5 & v. Braun & 232 \\
Berlin, U. & 290 & Breslau & 45
\end{tabular}

\begin{tabular}{|c|c|c|}
\hline Budapest & 79 & New Haven \\
\hline Cambridge & 13 & Newton \\
\hline Catania & 7 & Odessa \\
\hline Cleveland & 2 & Oldenburg \\
\hline Cohen & 3 & Oxford \\
\hline Darmstadt, M. & 77 & Paris, M. \\
\hline Debreczin & 23 & Paris, U. \\
\hline Dorpat & 36 & Pech 47 \\
\hline Dresden, M. & 14 & Petersburg, A. 167 \\
\hline Dublin, M. & II & Petersburg, B, 210 \\
\hline Frankfurt & Spl. & Pohl \\
\hline Frenzel & 10 & Rom, U. \\
\hline Göttingen & 208 & v. Siemaschko \\
\hline Gregory & 163 & Stockholm \\
\hline Greifswald & 6 & Strassburg \\
\hline Hamburg & 48 & Stuttgart \\
\hline Harvard, U. & 43 & Troyes \\
\hline Helsingfors & 62 & Tübingen \\
\hline Klausenburg & 2 & Turin, U. \\
\hline Kopenhagen & II3 & Upsala \\
\hline Kristiania & 15 & Utrecht \\
\hline London, B. M. I & 1352 & Ward \\
\hline London, P. G. & $55 \mathrm{I}$ & Washington 5 \\
\hline de Mauroy & 16 & Washington, Sh. 5 \\
\hline Melion & 2 & Wien,H.M.* $\left.{ }^{* *}\right) 2790$ \\
\hline Moskau & 52 & Zürich \\
\hline Neumann & 55 & \\
\hline
\end{tabular}

Ausserdem Lausanne: 37 gr. »Sporasidère de la Rochelle ; dies könnte auch Esnandes sein.

\section{Charca}

La Charca

Charcas, Santa Maria de los Charcas, San Louis Potosi, Mexico.

Eisen, Om, erwähnt 1804 .

[Nach Fletcher vielleicht mit Descubridora zu vereinigen].

1804: S on ne s chmid: Mineralog. Beschreibung der vorzüglichsten Bergwerks-Reviere in Mexico oder Neuspanien, p. 288. (Als Manuskript gedruckt; ein Exemplar im k. k. HofMuseum, ein anderes nach Chladni's Angabe in Freiberg i. S.)

I8Ix: v. Hu mboldt: Essai politique. Paris 1811, Bd. 2, p. $5^{82}$, 
1812: Bigot de Morogues, p. 300. r815: Chladni: Bemerkungen, Gilb.

Ann. Bd. 5o, p. 270.

1819: Chla d ni: Feuermeteore, p. 337 -338 .

1834: Burkart: Geognostische Bemerkungen auf einer Reise zwischen Ramos und Catorce. N. J. 1834 , p. $5^{89}$ -590, aus Karsten's Arch. f. Min. Bd. VI, p. 422 ff.

1843: P a r t s c h : Meteoriten, p.I45-146. 1852: Cla rk: Dissert. Gött., p. 64.

1854: v. Boguslawski: Zehnter Nachtrag, Pogg. Ann. Ergz.-Bd.4, p. 4II. 1856: Burkart: Fundorte I, N. J. 1856, p. 283, 284, 285, 286-288 (A bb. auf Taf. 4), 290, 291, 292; s. auch Burkart, Aufent halt u. Reisen in Mexico Bd. II, p. 128 (oder 127 ?), 389.

1857: Burkart: Briefliche Mitteilung, N. J. 1857 , p. 54 .

1859: Bu c h ner: Feuermeteore, p. I45. 1863: B u c hner: Meteoriten, p. I49. 1867: D a u brée: Note sur deux grosses masses de fer météorique du Muséum et particulièrement sur celle de Charcas (Mexique), récemment parvenue à Paris. C. R. Bd. 64 , p. 633 , 636-640; s, auch Weinschenk, Ann. Hof-Mus. Bd. 4 (1889), p. 100.

1867: Da ubrée: Nouveau procédé pour étudier la structure des fers météoriques. C. R. Bd. 64 , p. $685-688$. Ref. Liebig-Kopp, Jahresber. 1867, p. I044-1045 ; s'Institut \& Bd. 35 (1867), p. 106-107.

1868: S mith: A new Meteoric Iron from Mexico. Am. Journ. (2) 45, p. 77 (Analyse; nach Fletcher zu Toluca gehörig).

1869: Buchner: Vierter Nachtrag, Pogg. Ann. Bd. 136, p. 439, 606-608. r869: Meunier: Recherches, Ann. Chim. Phys. (4) 17, p. 21, 22, 23, 24, $25,27,29,33,39,43,48,52,53$, $54,55,56,60,61,66,67,68,71$. 1870: Ramme ls berg: Meteoriten, p. 8 o, 84,86 .

1870: Burkart: Fundorte IV, N. J. I870, p. 686, 6gr.

1873: Meunier: Produit d'oxydation des fers météoriques; comparaison avec les magnétites terrestres. C. R. Bd. 77, p. 645 .

r876: B a r cena: On certain Mexican Meteorites, Proc. Acad, nat. hist. Philadelphia (1876), p. I24.

r881: B rezina: Bericht III, Sitzber.

Wien. Akad. Bd. 84 1, p. 282.

1884: Meunier: Météorites, p. 21, 23, $24,26,39$ (Abb.), 40, 4I, 42, 43, 44 (Abb.), 46, 47, 6o, 6I, I16, II7-I18. 1885: Brezina: Wiener Sammlung, p. 213,234 .

I887; F lig h t: Meteorites, p. 93-94. r888: v. $\mathrm{H}$ a u e r: Ann. Hof-Mus, Bd. 3

(Not.), p. 42.

r889: C astillo: Météorites, p. 4.

r89o: Brez in a: Reise, Ann. Hof-Mus. Bd. 4, (Not.), p. 119.

189o: F l e t c her: Mexican Meteorites, Mineral. Magaz. Bd. 9 , p. $99,160-$ 162,174 .

1891: Cohen und Weinschenk: Meteoreisen-Studien, Ann. Hof-Mus. Bd. 6, p. 160,165 .

r892: C o h e n : Meteoreisen-Studien II, Ann. Hof-Mus. Bd, 7, p. 16r.

1893: M e u n i er: Révision des fers météoriques, p. 52, 54 .

1894: Cohen: Meteoritenkunde, an vielen Stellen.

1894: F le t c h e r: Introduction, p. 33. r895: Brezina: Wiener Sammlung, p. 275 .

r895: Co he n: Meteoreisen-Studien IV, Ann. Hof-Mus. Bd, Io, p. 82, 83 .

Ursprüngliches Gewicht: DieHauptmasse in Paris. Castillo sagt: $\gg \mathrm{Il}$ avait un poids de $578069 \mathrm{gr}$. lorsqu'il fut transporté à Paris, was offenbar 778069 heissen soll.

Nachweisbares Gewicht : 783649 gr. 


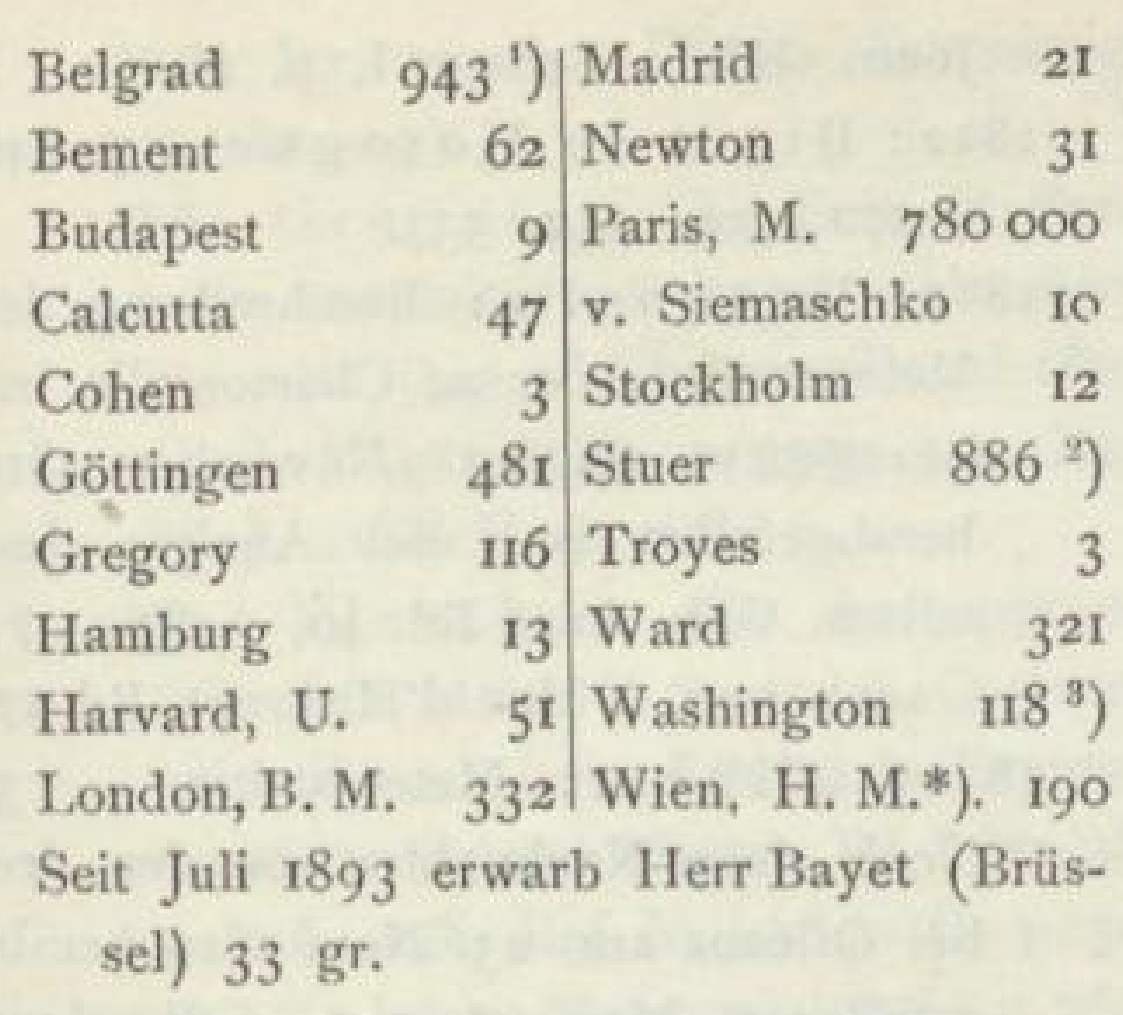

\section{Charkow}

Charleston

Jigalowka

Charlotte, Dickson Co., Nashville, Tennessee, U. S. A.

Eisen, Of, gefallen 31 . Juli oder I. August 1835 .

1845: T roost : 1 ) Description of a mass of Meteoric Iron, which fell near Charlotte, Dickson County, Tennessee, in 1835. Am. Journ. (I) 49, p. $337-340$ (Abb.); s. auch Pogg. Ann. Bd. 73 ( 1848$)$, p. 332 (Kurze Erwähnung); sL'Institut \& Bd. I4 (1846) Nr. 648, p. $194-195$.

1846: S h e p a r d : Report on Meteorites. Am. Journ. (2) 2, p. 391.

1847: $\mathrm{S}$ h e p ar d : Report on Meteorites. Am. Journ. (2) 4, p. 77.

r852: C 1 a r k : Dissert. Gött., p. 68-69. 1854: v. Boguslawski: Zehnter Nachtrag, Pogg. Ann, Ergz,-Bd. 4, p. $401-402$.

r856: B u rk a rt: Fundorte I, N. J. 1856 , p. 470 .

1859: B u c h ne r : Feuermeteore, p. 118. 1859: Harris: Dissert. Gött., p. III.

1862: Greg: On some Meteorites in

the British Museum. Philos. Magaz. Bd, 24 , p. $540-541$.
1863: B u chner: Meteoriten, p. 160. r863: R os e: Meteoriten, p. 26. 1858/65: v. Reichen bach : IV 638 , 640. VII 562. IX $163,174,182$. X 368. XII 457. XV IIO, I13, I14, 124, 128. XVI 250, 26r. XVII 266, 272. XVIII $478,484,487$. XIX 154. XX 622, 629. XXIV 226. XXV 612.

1874: B jörling : Meteoriter och Kometer (cfr. Hessle), p. 12.

1875: $\mathrm{Sm}$ it h: A Note in relation to the mass of Meteoric Iron that fell in Dickson County, Tenn., in 1835 . Am. Journ. (3) 1o, p. $349-35^{2}$ (Analyse) ; s. auch C. R. Bd. $8 \mathbf{r}(1875)$, p. 84 ; auch Chem. News Bd. 32, p. 221 ; Liebig-Kopp, Jahresber. 1875, p. 1314 -1315 .

1875: Mohr: Entstehungsart, Ann. Chem. Pharm. Bd. 179, p. 276.

r876: Wrig ht: On the Gases, contained in Meteorites. Am. Journ. (3) II, p. 257 u. (3) 12, p. 167.

1879: R a m mels berg: Meteoriten, p. 5 .

r880: Brezina: Bericht I, Sitzber.

Wien. Akad, Bd. 82 I, p. 350.

r880 : K l e i n : Vermehrung, Gött. Gel.

Anz., p. 569 .

I883: S mith: Concretions, Am. Journ.

(3) 25, p. 417 .

r884: M e un i er: Météorites, p. 18 - I9, 128 (Abb.), 129-130, 487 .

r885: Brezina: Wiener Sammlung, p. 208-209, 233 .

r886: H u n t ing to n : Crystalline Structure, Am. Journ. (3) 32, p. 295. r887: F lig ht: Meteorites, p. 107-108. r887: Brezina u. Cohen: Photographien, T. 17, 18.

1890: E a s t m a n : Met. Astron., p. 316. 1893: B r ez in a : Ueber neuere Meteoriten (Nürnberg), p. 164.

1) Mit der Jahreszahl $>1865^{\star}$.

2) Hiervon 505 gr. sSan Louis Potosi mit Daubréelite

3) Hiervon 57 gr. \San Louis Potosi. Received among the Grant relicse, 
r893: Meunier: Revision des fers météoriques, p. 4I, 42 (Abb. einer geätzten Platte).

r894: Coh en: Meteoritenkunde, p. 7o, $73,173,174,193$.

1894: F l e t c h er: Introduction, p. 34.

Ursprüngliches Gewicht : 9-10 Pfund. In Troost's Besitz gelangten 7 Pfund und $1_{3}$ Unzen (also 3539 gr.).

Nachweisbares Gewicht: 3156 gr.

\begin{tabular}{lr|lr} 
Bailey & 39 & London, B. M. & 77 \\
Bement & 4 & London, P. G. & 2 \\
Bologna & 51 & Paris, M. & 72 \\
v. Braun & 5 & v. Siemaschko & I \\
Budapest & 84 & Stockholm & 14 \\
Calcutta & 1 & Strassburg & 2 \\
Göttingen & 10 & Tühingen & 162 \\
Gregory & 5 & Ward & 58 \\
Harvard, U. & 2359 & Washington, Sh. 3 \\
Kopenhagen & 41 & Wien, H. M. *) & 166
\end{tabular}

\section{Charlottetown}

Monroe

Charsonville, Meung sur Loire, Loiret, Frankreich.

Stein, Cga, gefallen 23. Novbr. 1810. Hierher auch: Chartres, Bois de Fontaine, La Touanne.

1810: Moniteure Dez. 1810 ; Auszug in Bibl. Brit. Bd. 45 (Dezbr. I8Io Nr. 360 ), p. $397-400$; s. auch Bull. Soc. Philom. 1810 und Journ. Phys. Dezbr. 18ro (Bigot de Morogues).

181 I : Nouveaux détails sur la chute de pierres atmosphériques près d'Orléans, tirés d'une lettre particulière communiquée aux redacteurs de ce recueil par Mr. le Prof. Jurine. Bibl. Brit. Bd. 46 (18II), p. 94-96 (Januar Nr. 362); s. auch Uebersetzung Gilbert's in Gilb. Ann. Bd. 37 (1811), p. $349-351$ : Nachricht von dem Herabfallen dreier Meteorsteine am 23. November 1810, unweit Orléans.

1812: Chla dni: Verzeichnis, Schweigg.
Journ. Bd. 4 Beilage I, p. 16.

1812: Bigot de Morogues, p. 240 $-272,309,332,333$.

1812: V a uquelin: Beschreibung des Meteorsteins, der zu Charsonville bei Beaugency am 23. November r8ro herabgefallen ist, und Analyse desselben. Gilb. Ann. Bd. 40, p. 83-97; s. auch Ann. d, Mus. d'Hist. nat. Bd, 17. 1812: Gilbert: Meteorsteine. - 3 .

Noch einige Nachrichten von den drei bei Orléans am 23. Nov. 1810 herabgefallenen Meteorsteinen. Gilb. Ann. Bd. $4 \mathrm{I}$, p. $450-45^{2}$

1815: Chladni: Neues Verzeichnis, Gilb. Ann. Bd, 50, p. 255.

18r6: Chlad ni: Zweite Fortsetzung,

Gilb. Ann, Bd, 54, p. 354-355.

r8rg: $\mathrm{Ch}$ lad ni: Feuermeteore, p. 50,

$5 \mathrm{I}, 57,66,69,7 \mathrm{I}, 73,9 \mathrm{I}, 152,290$, 293-296(1), 298, 431.

r820: v. Schreibers: Beiträge, p. 65 -67 (Abb. Taf. VII).

1820: Dutrochet: De la hauteur du Météore qui a projeté des aërolithes à Charsonville, département du Loiret, le 23 novembre 18Io. Journ. Phys. (1820), p. 227-228.

I821: C h ladni : Erster Nachtrag, Gilb. Ann, Bd. 68 , p. 339.

I836: Kämtz: Meteorologie, p. 258, 283. 1843. Part s c h : Meteoriten, p. 73-74. r854: v. B o g uslaw ski: ZehnterNach-

trag, Pogg. Ann. Ergz,-Bd. 4, p. 19. 1859: Har ri s: Dissert. Gött., p. 69-70. r859: B u c hner: Feuermeteore, p. 80,81 . 1862: Kenngott u. Wiser: Zürcher Sammlung, p. 147.

r863: B u chner: Meteoriten, p. 28-29. 1863: Rose: Meteoriten, p. 155. r858/65: v. Reichenbach: V 475 , 480. IX 162, 170, 172, 179. X 359. XI 294, 297, 302, 310. XII 454. XV I01. $\mathrm{XX} 623,626$. XXIV 226. XXV $322,424,427,431,600,602$.

1884: M e u n i e r: Météorites, p. 8r, 94, $95,97,197,198,446,449,459$. 
1885: Brezina: Wiener Sammlung, p. $182,233$.

1886: Gregory: The Bois de Fontaine meteorite, and its probable identity with that of Charsonville of 18 ro. Geol. Magaz. (3) 3, p. 357-359. Ref. N. J. 1888 II, p. 226.

r889: Fletcher: On the supposed fall of a Meteoric Stone at Charsonville, Eure-et-Loir, France in September 18 ro. Mineral. Magaz. Bd. 8 Nr. 38 , p. 146-148. Ref. N. J. 1890 II, p. 59. 1894: C o h e n : Meteoritenkunde, p. 267. r895: Brezin a : Wiener Sammlung, p. $247-250$.

Ursprüngliches Gewicht: 60 Pfund. Zwei Steine, von denen der eine 40, der andere 20 Pfund wog. Im Jahre $181_{5}$ hatte Paris noch I 1 Pfund. Ein dritter Stein, dessen Fall beobachtet wurde, ist nicht gefunden worden.

Nachweisbares Gewicht: 6903 gr.

\begin{tabular}{|c|c|c|}
\hline Bailey & $\left.35^{10}\right)$ & Dresden, M. \\
\hline Bayet & 3 & Göttingen \\
\hline Belgrad & 2 & Gregory \\
\hline Bement & 24 & Harvard, U. \\
\hline Berlin, U. & $5^{1}$ & Helsingfors \\
\hline Bern, M. & 24 & Klausenburg $\quad$ I \\
\hline Bologna & 17 & London,B.M. $235^{\circ}$ ) \\
\hline Bonn & $\left.\mathbf{I}^{1}\right)$ & London, P. G. 149 \\
\hline v. Braun & 24 & de Mauroy \\
\hline Budapest & 22 & Moskau \\
\hline Calcutta & 6 & Neumann \\
\hline Cambridge & 62 & New Haven \\
\hline Cleveland & 1 & Paris, E. \\
\hline Dorpat & I & Paris, M. \\
\hline
\end{tabular}

\begin{tabular}{lr|lr} 
Paris, U. & 65 & Utrecht & $3^{11}$ ) \\
v. Siemaschko & $\left.70^{7}\right)$ & Ward & 24 \\
Stockholm & II $9^{8}$ ) & Washington & 54 \\
Troyes & IO & Washington, Sh. & 4 \\
Tübingen & 4 & Wien, H. M.*) & $694^{\circ}$ ) \\
Upsala & II & Zürich
\end{tabular}

Chartres

Charsonville

Charwallas, Hissar, Delhi, Punjab, Ostindien.

Stein, Ci, gefallen 12. Juni (oder 8. Juni ?) 1834 .

1834: Journ. Asiatic Soc. Bengal Nr. 32, August 1834 ; s. auch Philos. Magaz. Bd. 6 (1835), p. 398: Fall of a meteorite in India, on the $8^{\text {th }}(1)$ of June 1834; Am. Journ. (I) 30 (1836), p. 175. 1843: Part s ch: Meteoriten, p. 143. r85r: S he pard: On Meteorites. -2 . Charwallas, 30 Miles from Hissar, India, June $12^{\text {th }}$, 1834. Am. Journ. (2) II, p.36-37. Ref. Kenngott, Uebersicht 1850/51, p. 179; Liebig-Kopp, Jahresber. 1850 , p. $823-824$; Edinb. N. Phil. Review Bd. 53 (1852), p. 246.

1854: v. Boguslawski: Zehnter Nachtrag, Pogg. Ann. Ergz.-Bd. 4, p. $33-34$.

1859: B u c hner: Feuermeteore, p. 92. 1859: Harris: Dissert. Gött., p. 84. r $863:$ B u c hn e $\mathrm{r}:$ Meteoriten, p. 57 - 58. 1863: Ros e: Meteoriten, p. I55. 1884: Me uni er: Météorites, p. 96. r885: Brezina: Wiener Sammlung, p. $181,232$.

1894: C o h e n : Meteoritenkunde, p.237. UrsprünglichesGewicht: 7-8Pfund. Nachweisbares Gewicht: 71 gr.

1) >Meung 1825? - 2) Davon $254 \mathrm{gr}$. >Bois de Fontaine, near Meung 1825*. - 3) Davon 109 gr. Charsonville; 2227 gr. Bois de Fontaine; 20 gr. Chartres. 4) Davon $12 \mathrm{gr}$. Beaugency 1825 oder $1810<$ - 5) Davon $8 \mathrm{r}$ gr. Chartres. - 6) Davon " 5 gr. Chartres $\approx$ und $>21$ gr. Bois de Fontaine . - 7) Davon $55 \mathrm{gr}$ *Bois de Fontaine gefunden 1825^. - 8) Davon 5 gr. Bois de Fontaine. - 9) Davon 604 gr. Charsonville; 35 gr. Bois de Fontaine; $54 \mathrm{gr}$. Meung; I gr. La Touanne. - 10) Davon 20 gr. "Bois de Fontaine $\alpha$. - II) >Bois de Fontaine gefd. 1825\%. 


\begin{tabular}{lr|lr} 
Bailey & Spl. & Harvard, U. & I \\
Bement & Spl. & London, B. M. & 38 \\
Berlin, U. & Spl. & Tübingen & Spl. \\
Budapest & 9 & Wien, H. M.*) & 19 \\
Calcutta & 4 & &
\end{tabular}

v. Boguslawski sagt 1854 , dass ein Stück im Besitz des Naturhist. Museums der Universität zu Edinburg sich befinde. Der mir von Herrn Dr. Traquair mitgeteilte Katalog von Edinburg führt zwei indische Steine ohne genauere Ortsangabe von $94 \mathrm{gr}$. und $125 \mathrm{gr}$. auf und ausserdem noch einen dritten Stein von unbekanntem Fundort mit 629 gr. Buchner sagt $186_{3}$, dass London 692 gr. besässe; dies ist wohl nicht der Fall gewesen. Sollte sLondon 692 * vielleicht $\gg$ Edinburg 629 a heissen oder gehört eines der andern indischen Stücke der Edinburger Sammlung hierher?

Chassigny, Langres, Dep. Haute Marne, Frankreich.

Stein, Cha, gefallen 3 . Oktober $1_{8}$ I $_{5}$. 18r6: Pistollet: De la chute d'une pierre météorique tombée dans les environs de Langres, communiqué à $\mathrm{M}$. Virey par M. Pistollet, médecin de la même ville. Ann. Chim. Phys. Bd. I, p. $45-48$; übersetzt in Schweigg. Journ. Bd. 18 , p. $349-356$; s, auch Gilb. Ann. Bd. 58 (1818), p. $171-175$; Tilloch's Philos. Magaz. Bd. 47 (1816), p. $349-350$.

1816: V a uquelin: Analyse de l'aérolithe tombé aux environs de Langres et envoyé à l'Institut par M. Pistollet. Ann. Chim. Phys. Bd. I, p. 49-54; übersetzt in Gilb. Ann. Bd, $5^{8}$ (1818), p. $176-181$.

r8r6: C h la dn i : Erste Fortsetzung, Gilb. Ann. Bd. 53 , p. $3^{81}-3^{8} 3$.

r8r6: Calmelet und Gillet de L a u m on t: I. Note sur un aérolithe tombé aux environs de Langres, département de la Haute-Marne; par feu M. Calmelet. Ann. des Mines Bd. I, p. 489-491. - II. Note sur le même aérolithe, par M. Gillet de Laumont, p. $491-492$.

r8rg: Chla d ni: Vierte Fortsetzung, Gilb. Ann. Bd. 6o, p. 339, 34I.

r8rg: Chladni: Fünfte Fortsetzung, Gilb. Ann. Bd. 63 , p. 25 .

r8rg: $\mathrm{C}$ h 1 a d n i : Feuermeteore, p. 44, $50,58,59,66,69,72,73,91,274$, $289,307-309(1), 432$.

1820: L a u g i er: Extrait d'un Mémoire lu à l'Academie des Sciences le $\mathbf{I}^{\text {er }}$ mai 1820, par M. Laugier, et intitulé : Faits pour servir à l'histoire chimique des pierres méteoriques. Ann. Chim. Phys. Bd.13, p.440; s. auch Schweigg. Journ. Bd. 29 (1820), p. 508-510.

I82 I : C h la d n i: Erster Nachtrag, Gilb. Ann. Bd. 68 , p. 336.

1836: K ä m tz: Meteorologie, p. 258, 285 . r843: P a r s c h: Meteoriten, p. I6.

r854: v. Boguslawski: Zehnter Nachtrag, Pogg. Ann. Ergz.-Bd. 4, p. 14. r859: B u c hner: Feuermeteore, p. 84 . r860: $\mathrm{R}$ a m mel s b e r g : Mineralchemie, p. 944 .

186r: R os e legt den Stein von Chassigny vor. Zeitschr. d. d. Geol. Ges. Bd. 13 (1861), p. 526.

1862 : Kenngott u. W iser : Zürcher

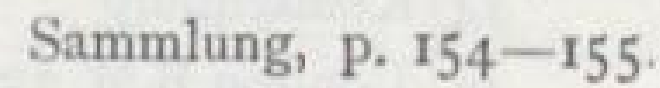

1862: Greg: On some Meteorites in the British Museum. Philos. Magaz. Bd. 24, p. 542 .

r862: D a mo ur: Note sur la pierre météorique de Chassigny. C. R. Bd. 55, p. 591-594 (neue Analyse); s. auch *L'Institute Bd. 30 (1862), p. 334, 367; Chem. Centr. 1863 , p. 240 ; Kenngott, Uebersicht $1862 / 65$, p. 447 ; N. J. 1863, p. 363 ; Liebig-Kopp, Jahresber. I862, p. $827-828$.

r863: B u c h n e r: Meteoriten, p. 36-37. r863: Rose: Meteoriten, p. $25,27, \mathrm{II}_{3}$ - I16, 140, 156; s. auch Mon,-Ber.

Berlin. Akad. 1862, p. 557.

$1858 / 65$ : v. Reichenbach: $V_{47}$. 
IX 156, 166, 177. X $359,363$. XI 294. XIII $353,365,380$. XIV 396 . XV Iол. XX 631. XXV 421, 422, 601, 607. 1870: Rammelsberg: Meteoriten, p. 118- II9, 139 .

1876: $\mathrm{S} \mathrm{mith:} \mathrm{Carbon} \mathrm{Compounds,} \mathrm{Am.}$ Journ. (3) II, p. 39 I.

1879: R a m mel sber g: Meteoriten, p. 24 .

1883: Tschermak: Beitrag, Sitzber.

Wien, Akad. Bd. $88 \mathrm{I}$, p. $36 \mathrm{r}-362$. 1884: Wa dsworth: Studies, p. 86. 1884: M e unier: Météorites, p. 70 ,

$77,79,93,94,95,96,292-293$ (Abb.), 351, 358, 373, 395, 459.

r883/85: T s c h e r m a k: Photographien, p. 3,4 , II. T. VI.

1885: Brezina: Wiener Sammlung, p. $176,232$.

r89r: v. H a u er: Ann. Hof-Mus, Bd 6 (Not.), p. 54.

r894: C o h e n: Meteoritenkunde, p. 49, $207,208,243,246,249,259,264$, $267,316$.

r895: Brezin a: Wiener Sammlung, p. 239.

UrsprünglichesGewicht : Etwa ${ }_{4} \mathrm{Kgr}$. Nachweisbares Gewicht: 854 gr.

\begin{tabular}{lr|lr|} 
Bailey & Spl. & Heidelberg & I \\
Belgrad & Spl. & Kristiania & I8 \\
Bement & Spl. & London, B. M. & 4 I \\
Berlin, U. & 13 & London, P. G. & 28 \\
Bologna & Spl. & de Mauroy & 6 \\
v. Braun & 5 & Moskau & 2 \\
Budapest & 5 & München & 12 \\
Calcutta & IO & Neumann & 4 \\
Cambridge & 14 & Paris, E. & 24 \\
Cleveland & I & Paris, M. & 415 \\
Cohen & Spl. & Paris, U. & 101 \\
Dorpat & Spl. & v. Siemaschko & 2 \\
Dublin, M. & 16 & Strassburg & Spl. \\
Frankfurt & Spl. & Tübingen & I \\
Göttingen & 5 & Wien, H. M.*) & 99 \\
Gregory & 17 & Zürich \\
Harvard, U. & 3 & II \\
\hline
\end{tabular}

Château-Renard, Montargis, Tri- guères, Dep. Loiret, Frankreich. Stein, Cia, gefallen 12. Juni I84I.

1841: D e lava ux: Note sur un aërolithe tombé le 12 juin dans les environs de Château-Renard. C. R. Bd. I2, p. II9O-IIgI; s, auch Notizen von Georges, Cordier, Dufrénoy ebenda p. 1229-1231; „L'Institute Bd. 9 (1841), p. 209, 222.

r84r: D ufrén oy : Note sur la composition de l'aërolithe de Château-Renard. C. R. Bd. 13 , p. $47-52$ (Analyse). Ref. N. J. 1843 , p. 823 ; Journ. prakt. Chem, Bd. 25 (1842), p. IOIIO3; sL'Institute Bd. 9 (1841), p. 247. 1841: Longueman: Relation de la chute de l'aërolithe du 12 juin. C. R. Bd. 13, p. 88-90; s, auch Notiz von Longueman, ebenda p. 232. Ref. Journ. prakt. Chem. Bd.25 (1842), p.IoI- 103. r84I: Meteorsteinfall, am I2. Juni 184I, unweit Château-Renard, in Frankreich. Pogg. Ann. Bd. 53, p. $4 \mathrm{II}-4 \mathrm{I} 5$ (Zusammengezogen aus den obigen Arbeiten in den C. R.); s. auch Journ.prakt. Chem. Bd. 25 (1842), p. $101-\mathrm{IO} 3$; Am. Journ. (I) 42 (1842), p. 203.

1843: H ai d ing e r : Uebersicht, p.ro8. r843: P a rts ch: Meteoriten, p. 6I. 1843: Rammels berg: Ueber die Bestandtheile der Meteorsteine. Pogg. Ann. Bd. 6o, p. 136-I37 (Berechnung der Analyse von Dufresnoy); s. auch Handwörterb. Suppl. I, p. 98-100 ; Suppl. II, p. 9I-92 ; Suppl. V, p. 23-24. Ref. N. J. 1844 , p. 718. 1854: v. Boguslawski: Zehnter Nachtrag, Pogg. Ann. Ergz.-Bd, 4, p. $362-364$.

1859: Buchner: Feuermeteore, p. 94. r859: H a r r is : Dissert. Gött., p. $87-88$. 1860: R a m mels berg: Mineralchemie, p. 922 ff., $95^{\circ}$.

1862: Gre g: On some Meteorites in the British Museum. Philos, Magaz. Bd. 24, p. 542 . 
r863: B u c h n e r : Meteoriten,p.66-67. r863: Rose: Meteoriten, p. 89,90 , $99,155$.

r858/65: v. Reichen bach: V 480. IX 16I, 168, 178. X 359. XI 292, 294, 295, 296, 297, 300. XIII 360, 365, 369 Fig., 372 Fig., $374,377,383$. $\mathrm{XX} 623$. XXV $319,320,321,324$, 438,607 .

r870: R a mmel sberg: Meteoriten, p. 105, 106, 139, 140.

1875: vom R a th: Meteoriten, Verh. naturh. Ver. Bonn Bd. 32, p. 367.

1875: Tschermak: Vulkanismus, Sitzber. Wien. Akad. Bd. 7 I II, p. 663. 1876: S m it h : Carbon compounds, Am. Journ. (3) II, p. 39 I.

I879: R a m mels berg: Meteoriten, p. 24,25 .

1882: Wie chmann: Fusion Structures in Meteorites. Ann. N. Y. Acad. Sc. Bd. 2, p. 293-295 (Taf. 20). 1883: T s c herm a k: Beitrag, Sitzber. Wien. Akad. Bd. 88 I, p. 356, 359. 1884: W a d swort h: Studies, p. 105. r884: M e un i er: Météorites, p. 79, 85 ,

95, 197, I98-201, 378, 395, 459, 487 . r884: Wiepk en: Notizen über die

Meteoriten des Grossherzogl. Museums (Oldenburg), Abh, naturw. Ver. Bremen Bd. VIII, p. $525-526$.

r883/85: Tschermak: Photographien, T. 17, 21, p. 16, 17, 18, 20.

1885: Brezina: Wiener Sammlung, p. $169,176,182,232$.

r893: B rez in a : Ueber neuere Meteoriten (Nürnberg), p. 159.

r894: F l e t c h e r : Introduction,p. 37,39. r894: C o h e n : Meteoritenkunde, p. 3I3.

Ursprüngliches Gewicht : Etwa $20 \mathrm{Kgr}$.

Nachweisbares Gewicht: I7 922 gr. Bailey

Bayet

Belgrad

37 Bement

159

3 Berlin, P.

490

1) Ein kleines Stück ohne Gewichtsangabe.

\begin{tabular}{lr|lr} 
Böhm & $4 \mathbf{I}$ & Lüttich & 46 \\
Bologna & 14 & Madrid & 7 \\
Bonn & 14 & de Mauroy & 3 \\
v. Braun & 25 & Neumann & 24 \\
Breslau & 33 & New Haven & 91 \\
Brezina & 80 & Newton & Spl. \\
Budapest & 3680 & Oldenburg & 449 \\
Calcutta & 586 & Oxford & 254 \\
Cambridge & 4 & Paris, E. & 2294 \\
Cleveland & 31 & Paris, M. & 1344 \\
Dorpat & 55 & Petersburg, B. & 440 \\
Dresden, M. & 13 & Pohl & 1 \\
Dublin, M. & 17 & Rom, U. & 5 \\
Frenzel & 22 & v. Siemaschko & 194 \\
Göttingen & 322 & Stockholm & 89 \\
Gregory & 360 & Strassburg & 74 \\
Harvard, C. & 87 & Stürtz & 27 \\
Helsingfors & 12 & Troyes & 46 \\
Klausenburg & I & Tübingen & 1043 \\
Kopenhagen & 341 & Upsala & 4 \\
v.Leuchtenbg. Spl. $\left.{ }^{1}\right)$ & Ward & 104 \\
London, B. M. 3290 & Washington, Sh. \\
London, P. G. & 367 & Wien, H. M. ${ }^{*}$ ) & 837 \\
& & &
\end{tabular}

Chatooga Co.

Cherokee Co.

Hollands Store

Losttown

Vavilovka

Cherson 1881

Grossliebenthal

Cherson 1894 Savtschénskoje

Chesterville, Chester Co.,Süd-Carolina, U. S. A.

Eisen, Hch, gefunden wenige Jahre vor 1849 , beschr. 1849 .

1849: Shepard: On Meteoric Iron in South Carolina. Am. Journ. (2) 7, p. $449-450$. Ref. Liebig-Kopp, Jahresber. 1849 , p. 828 ; Froriep's Tagsberichte über die Fortschr, d. Naturu. Heilk. Nr. 77 (1850), p. 32.

r852: Wöhler: >Activ «, Pogg. Ann. Bd. 85, p. 448 .

r852: Cla r k: Dissert. Gött., p. 66-67. r859: Harris: Dissert. Gött., p. II7-118. 1859/62: v. Reic henbach: IX 162, 175, 
176, 182. XII 457. XV 10o. XVII 273. XVIII $482,487,488$. XIX 155. XX 621, 622 .

1863: Buchner: Meteoriten, p. 182.

1863: Rose: Meteoriten, p. 69, 70, 153. r870: R a m mels berg: Metoriten, p. 80 . 1884: Me uni er: Météorites, p.94, II2, $\mathrm{II}_{3}$. r885: Brezina: Wiener Sammlung, p. $203,219,234$.

1890: Eastman: Met. Astron., p. 318. r893: Meunier: Révision des fers météoriques, p. $15,18$.

r894: Cohen, Meteoritenkunde, p. 67, 127.

1895: Brezina: Wiener Sammlung, p. 294.

Ursprüngliches Gewicht: Etwa 16 Kgr., wovon die Hälfte verschmiedet wurde.

Nachweisbares Gewicht: 6474 gr.

\begin{tabular}{lr|lr} 
Bailey & 29 & Madrid & 76 \\
Bement & 91 & Marburg & 270 \\
Berlin, U. & 395 & Moskau & 36 \\
v. Braun & 8 & New Haven & 758 \\
Budapest & 98 & Paris, M. & 88 \\
Calcutta & 178 & Pohl & 35 \\
Clinton & 79 & Rom, U. & 19 \\
Dorpat & 44 & v. Siemaschko & 64 \\
Göttingen & 115 & Stockholm & 63 \\
Gregory & 139 & Strassburg & 30 \\
Hamburg & 46 & Tübingen & 274 \\
Klausenburg & 30 & Ward & 6 \\
Kopenhagen & 79 & Washington, Sh. 115 \\
London, B. M. 2250 & Wien, H. M.*) & 884 \\
London, P. G. & 175 &
\end{tabular}

\section{Chihuahua, Mexico.}

Eisen (prähistorisch?), erwähnt 1867. 1867: T a r a y r e: Arch. de la Commission Scientifique du Mexique Bd. 3, p. 348 . Paris 1867 .

1869/70: Correjo: La Naturaleza Bd. 1, p. 256 .

1870: B urkart: Fundorte IV, N. J. 1870, p. $682-683,690$.

1873: Pi erso n: Correspondence relative to the discovery of a large me- teorite in Mexico. Annual Rep. Smithsonian Inst. (1873), p. 419-422 (schätzt das Gewicht auf 5000 lbs.).

r876: U. S. Centennial Commission. International Exhibition 1876. Reports and Awards Washington 1880 . Bd. 3, group I, p. 369 .

r876: B a r c e n a : Proc. Acad. Nat. Sc. Philadelphia 1876 , p. 122 .

1880: $\mathrm{M} \mathrm{u}$ ñ o z L u m bier: Los Aerólitos de Chihuahua. Mexico 1880 (Diese Arbeit nicht eingesehen).

1886: $\mathrm{Cl}$ a rke: The Meteorite Collection in the U. S. National Museum, Rep. Smithsonian Inst. I885/86 II. Teil, p. 257. Chihuahua, Mexico. An uncut mass to be described. Weight about $1800 \mathrm{kgr}$ \&

1890: F l e t c her: Mexican Meteorites. Mineral. Magaz. Bd. 9 , p. $98,99,102$, I19-122.

1890: E a st ma n: Met. Astron., p. 322. r892: E a s t m a n: The Mexican Meteorites. Bull. Philos. Soc. Washington Bd. 12, p. $43-44$.

Die obige Literatur bezieht sich auch auf die grosse Masse von Casas Grandes oder Casas Grandes de Malintzin, von welcher Fletcher vermutet, dass sie mit dem in Washington aufbewahrten Eisen von Chihuahua identisch sein könnte. Vergl. auch Rio Florido unter Huejuquilla-Cruppe.

Ursprüngliches Gewicht?

Nachweisbares Gewicht: Ausser der in Washington aufbewahrten Masse von $1800 \mathrm{kgr}$., welche noch nicht untersucht wurde, besitzt New Haven Chihuahua, Mexico $5^{1}$ gr.*

\section{Chili (Prof. Joy)}

Vaca Muerta

Chili, loc. inconnue

Dehesa

Chilpanzingo s. Anhang Caparrosa

\section{Chireya}

Butsura

Cholula

Misteca 
Chulafinnee, Cleberne Co., Alabama, U. S. A.

Eisen, Om, gefunden 1873 , beschrieben $\mathbf{1} 880$.

r880: Hid d e $\mathrm{n}$ : An account of the finding of a new meteorite in Cleberne County, Alabama. Am. Journ. (3) 19, p. 370-371 (Abbildung der Masse und einer geätzten Platte). Ref. N. J. I88I II, p. 180; Liebig-Kopp, Jahresber. 188o, p. $154^{2}$.

1880: Makintosh: Analysis of the Meteoric Iron from Cleberne Co., Alabama. Am. Journ. (3) 20, p. 74. I88I: Brezina: Bericht III, Sitzber. Wien. Akad. Bd. 84 I, p. 28I-282. 1885: Brezina: Wiener Sammlung, p. $155,213,214,234$.

1887: F light: Meteorites, p. 7I.

r89o: E a s tm a n : Met. Astron., p. 320.

r894: Co h e n : Meteoritenkunde, p. 74, $77,83,149$.

Ursprüngliches Gewicht: $1475^{\circ}$ gr. kamen ganz in den Besitz des k. k. Hof-Museums, nachdem etwa $3 \frac{1}{4} \mathrm{lbs}$ abgetrennt $\mathrm{u}$. verschmiedet worden waren.

Nachweisbares Gewicht: I4 020 gr. Bailey

Bement 29 Rom, U. 212

v. Braun 85 v. Siemaschko

Budapest 45 Stockholm

Harvard, U. IO3I Stürtz

London, B. M. 60 Ward de Mauroy Moskau Pohl

\section{Washington}

42 Washington, Sh. 54 188 Wien, H.M. II 975

\section{Chupaderos \\ Cincinnati \\ Cirencester \\ Claiborne 1834 \\ Claiborne 1853 \\ Clarac \\ Clarke Co.}

Huejuquilla s. Anhang Aldsworth Lime Creek Tazewell Aussun Lime Creek
Claywater Meteorite Vernon Co. Cleberne Co. Chulafinnee Cléguérec Kernouvé

Cleveland (Lea Iron of the Philadelphia Academy), Ost-Tennessee, U. S. A.

Eisen, Om, gefunden 1860, beschr. I 886.

$\mathrm{Ob}$ mit Dalton zu vereinigen? S. auch Literatur von Dalton. Hieher auch vorläufig : „Ehrenberg's Tennessee «, beschr. $186_{3}$.

r863: Ros e: Meteoriten, p. 58 (2Ehrenberg's Tennessee $\propto)$.

1866: S h e p a r d: Brief notices of several localities of Meteoric Iron. - 4. Supposed new locality in Tennessee. Am. Journ. (2) 42, p. 251 .

1886: Genth: On an undescribed meteoric iron from East-Tennessee. Proc. Acad. nat. Sc. Philad, (1886), p 366 -368 (Abbildung der Masse und geätzter Platten. Analyse). Ref. N. J. 1889 II, p. 42 ; Liebig-Kopp, Jahresber. 1886, p. $2330-2331$.

1887: v. H a u e r : Ann. Hof-Mus. Bd. 3 (Not), p. 38 .

1887: K unz: On some American Meteorites. - 3. Is the East-Tennessee Meteorite from Whitfield Co., Georgia ? Am. Journ. (3) 34, p. 473-475 (Analyse und Situationsplan). Ref. N. J. I889 I, p. 61 .

1890: E a s t m a n: Met. Astron., p. 320. 189r: v. H a u e r : Ann. Hof-Mus, Bd. 6 (Not.), p. 54.

1893: Me unier: Révision des fers météoriques, p. 52, 59-6o.

1894: Co h e n : Meteoritenkunde, p. 72, 193, 232.

Ursprüngliches Gewicht : ${ }_{1} 5_{5}^{1 / 2} \mathrm{Kgr}$. (254 lbs.) wog die von Genth beschriebene Masse, von welcher $2 \frac{1}{2} \mathrm{Kgr}$. zur Verteilung gelangten. Befindet sich der Rest noch 
in der Akademie in Philadelphia? Nachweisbares Gewicht: 2751 gr.

Bailey 87 London, B. M. 209

Bement 300 v. Siemaschko 94

Berlin, U.

Cleveland

Gregory

Harvard, U.

Howell

$39^{1}$ ) Ward

234

4 Washington 221

174 Washington, Sh. 260

$27^{2}$ ) Wien, H. M. 1055 47

Coahuila, Mexico.

Eisen, $H$, bekannt seit 1837 , beschr. 1855 .

Hierunter in Uebereinstimmung mit Fletcher folgende Fundorte vereinigt :

1) Coahuila, Bolson de Mapimi; Coahuila, Bonanza; Butcher Iron. Einige dieser Massen bekannt seit I 837 , beschr. 1855. Butcher's Expedition 1868 .

2) Santa Rosa-Saltillo. Ausser den unten aufgeführten Gewichten soll nach Eastman die National School of Engineers in Mexico $6_{3} \mathrm{Kgr}$. besitzen. Couch (Gouch) Iron, bekannt $1852 / 53$, beschr. 1855 ( 252 lbs., jetzt grösstenteils in Washington). Smithsonian Iron, Shepard, beschr. 1881 ; cf. Brezina, Ann. Hof-Mus. Bd.ro, p. 290-291.

3) Sancha Estate. Unter diesen Namen werden viele derCoahuilaEisen in den Sammlungen aufgeführt.

4) Fort Duncan,Maverick Co.,Texas, gefunden 1882 ; beschr. 1885 (44 Kgr.).

Ausserdem wären hierher wahrscheinlich auch die Massen von Cerralvo, erwähnt 1856 und von Potosi, Distr. Galeana, erwähnt
I 870 (200 lbs.) zu stellen; dieselben scheinen verloren gegangen $\mathrm{zu}$ sein.

Brezina vereinigt einerseits Bolson de Mapimi, Bonanza, ButcherIron, Santa Rosa-Saltillo zu einer Lokalität Coahuila; Sancha, Couch Iron und Fort Duncan andererseits zu einer Lokalität Fort Duncan.

1855: Smith: Memoir on Meteorites. - 3. Meteoric Iron from Coahuila, Mexico. Am. Journ. (2) 19, p. 160-16r (Couch Iron, Abbildung der Masse von 252 lbs. Dieselbe stammt aus Saltillo und befindet sich jetzt grösstenteils in Washington. Analyse); s. auch Original Researches 1884 , p. $374-376$; Journ. prakt. Chem. Bd. 66 (1855), p. $425-426$. Rep. Smithson. Inst. 1855 , p. $154-156$ (oder Rep. f. 1854 , p. 15 , 86 ?); Liebig-Kopp, Jahresber. 1855 , p. 1027 ; s. ferner Faye: C. R. Bd. $5^{8}$ (1864), p. 599-600 und hierüber Ref. Kenngott, Uebersicht $1862 / 65$, p. 269. 1856: S he pard: A supposed new locality of Meteoric Iron in Mexico. Am. Journ. (2) 21, p. 216 (Cerralvo). Ref. Kenngott, Uebersicht 1856157 , p. 153 . 1856: Burkart: Fundorte I. - 2. Meteoreisen von Coahuila. N. J. 1856 , p. $277-278$ (Couch-Meteorite. Abbildung und Analyse wie bei Smith).

1857: Schott: Report on the U. S. and Mex. Boundary Survey (von W. H. Emory) Bd. I Teil 2, p. 34.

1859: H a r r is: Dissert. Gött., p. 124. 1859: Bu chner: Feuermeteore, p. 144. r860: R a m mels berg: Mineralchemie, p. 912 .

1858/62: v. Reichen bac h: VII 552 . IX $162,174,181$. X $359,365 . \quad$ XV $100,110,114,124,128$. XVI 255, 261. XVII 266,272 . XVIII 487 . XIX 149 .

1) >Ehrenberg's Tennessee. . - 2) Eigentum von Herrn Huntington. 
1863: Wichelhaus: Analyse des Meteoreisens von der Hacienda St. Rosa in Mexico. Pogg. Ann. Bd. 118, p. 631 -633. Zusatz von Rose, p. 633-634; s, auch Kenngott, Uebersicht $1862 / 65$, p. 267; Liebig-Kopp, Jahresber. 1863 , p. 909; Journ. prakt. Chem. Bd. 90 (I863), p. $\mathrm{II}_{4}-\mathrm{II}_{5}$.

I863: B u chner: Meteoriten, p. I92. r863: R o s e: Meteoriten, p. 49, I52. 1865: Buchner: Zweiter Nachtrag, Pogg. Ann. Bd. 124, p. 598.

1866: Sh epard: A new locality of Meteoric Iron in Cohahuila, Northern Mexico. Am. Journ. (2) 42, p. 347 350 (betrifft Bonanza-Masse, Situationsplan); s. auch Journ. prakt. Chem. Bd. IOI (I867), p. 50I; \&L'Institute Bd. 35 (1867), p. 56.

1866: Veat ch (Brief an Whitney): Proc. Calif. Acad. Nat. Sc. Bd. 3 Teil 3, p. 24 r. 1867: S h e p a r d : Additional Notice of the Cohahuila Meteoric Iron. Am. Journ. (2) 43 , p. $384-385$ (Analyse). Ref. Liebig-Kopp, Jahresber. 1867, p. Io5o. r869: Buchner: Vierter Nachtrag, Pogg. Ann. Bd. 136, p. 608.

1869: S mith: The Cohahuila Meteoric Irons of 1868, Mexico. Am. Journ. (2) 47 , p. $383-385$ (Analyse; Butchers Expedition; Meteor von 1837); s, auch Original Researches 1884 , p. $44 \mathrm{I}-443$. Ref. Liebig-Kopp, Jahresber. 1869, p. 1304 .

1870: $\mathrm{B}$ urkart: Ueber die Fundorte Mexikanischer Meteoriten. N. J. 1870, p. $673-68_{3}, 690$.

1870: B u t c h e r: Circular vom 4. März 1870 (bietet das Pfund seiner 8 Eisen zu 2 Dollars bei Abnahme der ganzen Masse, zu 3 Dollars bei Abnahme einer Masse an).

187r: Burkart (briefliche Mitteilung):

N. J. 1871, p. 854 (Potosi).

1876: Barcena: On certain Mexican Meteorites. Proc. Acad, Nat, Sc. Philadelphia (1876), p. 122.
1876: $\mathrm{Sm}$ it h: Aragonite on the surface of a meteoric iron and a new mineral (Daubréelite) in the concretions of the interior of the same. Am. Journ. (3) I2, p. IO7-IIO; s, auch C. R. Bd. 82 (1876), p. 1505-1507; Rammelsberg, Mineralchemie II. Aufl, I. Ergz.-Heft (1886), p. 76 (Daubréelit); Liebig-Kopp, Jahresber. 1876, p. 13 ${ }^{1} 4-\mathrm{I}_{3} \mathrm{I}_{5}$.

r878: S mith: On the composition of the new Meteoric Mineral Daubréelite and its frequent, if not universal, occurrence in Meteoric Irons. Am. Journ. (3) 16 , p. $270-272$; s, auch Original Researches 1884, p. $540-543$; C. R. Bd. 87 (1878), p. 338-340; Ann. Chem. Pharm. Bd. I94 (1878), p. 304306 ; Z. f. Kryst. Bd. 3 (1879), p. $79-80$, 328; Liebig-Kopp, Jahresber. 1878 , p. $1315-1316$.

1879: R a m mels berg: Meteoriten, p. 23.

1879: K le in: Göttinger Sammlung, Gött. Gel. Anz, 1879, p. 98.

r880: Kle in: Vermehrung, Gött. Gel. Anz. 188o, p. 568-569.

188r: $\mathrm{Sm}$ it h: Occurrence of a nodule of Chromite in the interior of compact Meteoric Iron from Cohahuila. Am. Journ. (3) 2r, p. $46 \mathrm{r}-462$; s. auch Original Researches 1884 , p. $595-596$; C. R. Bd. 92 (1881), p. 991-992; Chem. News Bd. 43 , p. 283 ; N. J. 1882 I, p. 365 ; Z. f. Kryst. Bd. 6 (1882), p. 516 ; Liebig-Kopp, Jahresber. I88I, p. 1457. r88I: Daubrée présente à l'Académie un volumineux échantillon de météorite holosidère de Cohahuila (Mexique), dit fer de Butcher. C. R. Bd. 93 , p. 555 $-556$.

r88r: B r ez in a : Ueber die Meteoreisen von Bolson de Mapimi. Sitzber. Wien, Akad. Bd. 83 I, p. $473-477$. Ref. N. J. $\mathrm{I}_{88} \mathrm{I}$, p. $380-38 \mathrm{r}$.

r88r: $S$ hepard: On a new Meteoric Iron, of unknown locality, in the Smithsonian Museum. Am. Journ. (3) 22, 
p. II9 (Analyse). Ref. N. J. 1883 I, p. 379; Liebig-Kopp, Jahresber. 188I, p. 1458 .

r88r: B r e z in a : Bericht über neue oder wenig bekannte Meteoriten.-12) Natürliche Trennungsfläche an Bolson de Mapimi. Sitzber. Wien. Akad. Bd, 84 I, p. $282-283$. Ref. N. J. $188_{3}$ I, p. 383 . 1882: v. L a s a $1 x$ : Vermehrung, Verh. naturh. Ver. Bonn Bd. 39 (Sitzber.), p. 100.

r883: $\mathrm{S} \mathrm{m}$ it h : Concretions, Am. Journ.

(3) 25, p. $418,420,421$.

I884: vo m R a th: Verh, naturh. Ver. Bonn Bd. 4I (Sitzber.), p. 126. Ref. N. J. 1885 II, p. 27 o.

1884: W a d swort h: Studies, p. r6r. 1884: Meunier: Météorites, p. 62, 63 (Abb.), 64, 71, 87, 94, 96, 98, I12, II4, II6, I25, 440-44I.

1885: Brezina: Wiener Sammlung, p. 152, 154, 155, 209, 218, 234.

1885: Lupton: Meteoric Iron from Coahuila, Mexico. Am. Journ, (3) 29, p. 232-233 (Analyse). Ref. N. J. I886 II, p. 212 ; Liebig-Kopp, Jahresber. 1885, p. 2326.

1885: H id den: Preliminary note on an iron meteorite from Maverik Co., Texas. Trans. N. Y. Acad. Sc. Bd, 5 (1885/86), p. 231 .

1886: Brezina: Neue Meteoriten II, Ann. Hof-Mus. Bd. 2 (Not.), p. 25-26; s. auch p. 38 . Ref. N. J. 1887 II, p. $45-46$.

r886: Hidden: A new Meteoric Iron from Texas. Am. Journ. (3) 32, p. 304 -306 (Analyse von Mackintosh, Abb.). Ref. N. J. I887 II, p. 47 ; LiebigKopp, Jahresber, 1886, p. 2334.

r886: H u n ting to n: Crystalline Structure, Am. Journ. (3) 32, p. 291-292 (Abb. einer geätzten Platte), 295.

1887: B r ez in a : Neue Meteoriten IIIa,

Verh. k. k. Geol. Reichsanstalt (1887), p. 288 .

1887: Huntington: Catalogue of Wiifing, Meteoriten. all recorded Meteorites. Proc. Amer. Acad. Arts and Sc. Bd. 23, Taf. I u. II. 1887: Huntington: On the Coahuila Meteorites. Am Journ. (3) 33, p. II5- II8 (Abb. einer geätzten Platte des Butcher-Eisen's). Ref. N. J. I888 I, p. 2 II.

r887: F lig h t: Meteorites, p. 52-53. 1887: Meunier: Examen minéralogique du fer météorique de Fort Dunkan. C. R. Bd. 104 , p. $872-873$

Ref. N. J. 1888 II, p. $34-35$.

r887: v. Hauer: Ann. Hof-Mus. Bd, 2 (Not.), p. 38 ; s. auch Bd. 4 , 1889 (Not.), p. 64.

r889: Huntingt o n: The Crystalline Structure of the Coahuila Irons. Proc. Amer. Acad. Arts and Sc. Boston Bd. 24, p. $30-35$ (Mit 4 Figuren im Text); im gleichen Band auch p. $3 \mathbf{I}_{3}-\mathbf{3}_{\mathbf{1}}$.

r889: C o h e n : São Julião, N. J. 1889 I, p. 216-217, 221, 224, 225, 227, 228. r889: Cas t illo: Catalogue, p. 9-ro (Hacienda de Potosi u. Santa Rosa) r889: C 1 a rke: The Meteorite Collection etc. Report of the Smithsonian Institution 1885/86 II. Teil, p. 257 57. Locality unknown. Partially described by Shepard in 1881. Found without record in the old Smithsonian Collection. Specimen nearly entire, weighing $35 \mathrm{ro}$ grammes $\propto$.

r890: F l et c h er: Mexican Meteorites, Mineral. Magaz. Bd. 9, p. 99, 103, I04 $-\mathrm{II}_{3}, \mathrm{II}_{3}-\mathrm{Ir} 8, \mathrm{II}_{9}, \mathrm{I}_{74}-\mathrm{I}_{75}$.

r8go. Eastman: Met. Astron., p. 3r8 (Couch), 320 (Fort Dunkan), 322 (Santa Rosa, Butcher u. Coahuila).

1890: Brezina: Ueber Meteoreisen, Oesterr. Zeitschr. f. Berg- u. Hüttenw. Bd. 38 (Nr. 31 ), p. $35^{8}$.

r89r: Cohen u. Weinschenk: Meteoreisen-Studien, Ann. Hof-Mus. Bd. 6, p. 131, 159, 164 .

1892: Eastman: The Mexican Meteorites. Bull. Philos. Soc. Washington Bd. 12, P. $42-43$. 
r892: L i n ck: Ueber die Zwillingsbildung und den orientierten Schimmer am gediegen Eisen. Zeitschr. f. Krystallogr. Bd. 20 , p. 215 .

I892: Co hen: Meteoreisen-Studien, Ann. Hof-Mus. Bd. 7, p. $15^{8}(\mathrm{Cu})$.

1893: B r ez in a: Ueber neuere Meteoriten (Nürnberg), p. $\mathbf{1} 66$.

r893: Meunier: Révision des fers météoriques, p. 14, 15, 17, 19, 21-22 (Abb, einer geätzten Platte).

r894: Cohen: Meteoritenkunde, an vielen Stellen.

r894: C o h e n : Meteoreisen-Studien III, Ann. Hof-Mus. Bd. 9, p. $\mathrm{IO}_{2}-\mathrm{IO} 4, \mathrm{IO} 4$ -I07, I16, II7, Ir8 (Rhabdit).

r895: Brezina: Wiener Sammlung, p. $290-29 \mathrm{I}$.

r895: C o h e n : Meteoreisen-Studien IV, Ann. Hof-Mus, Bd. Io, p. $83,84,85$, $86,88,89,93$.

1895: v. H a u e r : Ann. Hof-Mus. Bd. ro (Not.), p. 35 .

Ursprüngliches Gewicht von I) Coahuila: Mehrere tausend Pfund, die Butcher-Eisen bestanden aus 8 Massen von $654,580,550,43^{8}$, $430,404,353,290$, zusammen 3699 lbs. $=\mathrm{I}_{67} 8 \mathrm{kgr}$.

NachweisbaresGewicht: I 615 46rgr. Bailey $\quad 157 \mid$ Cambridge $\quad 85$ Bement $\quad 4883$ Cleveland $\quad$ I55

Berlin, G. $\quad 376$ Dorpat IIO

Berlin, U. $\quad 1554$ Dresden, M. $\quad \mathbf{1 6 2}$

\begin{tabular}{ll|l} 
Bologna & 277 & Edinburg
\end{tabular}

Bonn 66o Eger 106

v. Braun $\quad 1544$ Göttingen 258I

Brunner $\quad 300$ Graz, J. 149

Budapest $\quad 13777$ Gregory $\quad 1333$

\begin{tabular}{ll|l} 
Calcutta & 26 & Greifswald \\
& 322
\end{tabular}

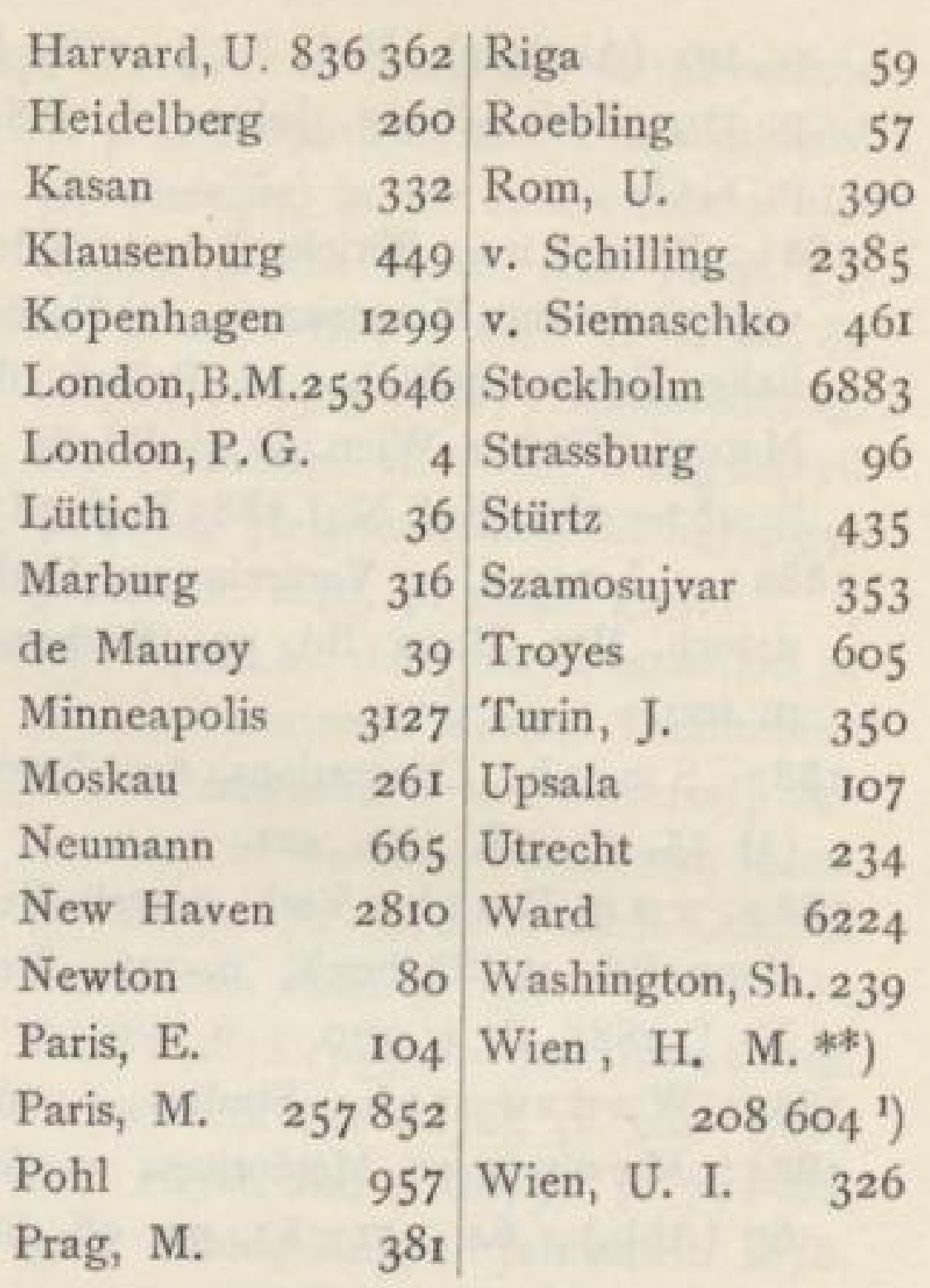

Ursprüngliches Gewicht von 2) Santa Rosa, Saltillo nicht bekannt; Couch (Gouch) Iron 252 lbs.; Smithsonian Iron Shepard - grösste Masse in Washington.

NachweisbaresGewicht: Santa Rosa, Saltillo: 1875 gr., Couch 105887 gr., Smithsonian Iron Shepard $3^{6} 3^{8} \mathrm{gr}$.

\begin{tabular}{lr|l|l} 
Bailey & $\left.6^{1}\right)$ & London, B. M. & $5^{1}$ ) \\
Berlin, U. & 22 & Paris, E. & 145 \\
Bonn & $\left.18^{1}\right)$ & Paris, M. & 22 \\
v. Braun & $4 \mathrm{r}$ & v. Siemaschko & $44^{8}$ \\
Cleveland & 37 & Stockholm & 17 \\
Cohen & 30 & Stürtz & $25^{2}$ ) \\
Dorpat & 5 & Troyes & $5^{8}$ \\
Göttingen & 74 & Tübingen & 5 \\
Gregory & I $\left.^{1}\right)$ & WashingtonIo8929 \\
Harvard, U. & $83^{3}$ & Washington,Sh. 88 \\
Krantz & 63 & Wien, H. M. 520 \\
& &
\end{tabular}

I) Ausserdem 2767 gr. nach I, Juli erworben.

I) "Smithsonian Iron Shepard. - 2) >Couch Iron. - 3) Davon $105400 \mathrm{gr}$. Couch Iron u. $3510 \mathrm{gr}$. Smithsonian Iron u. $19 \mathrm{gr}$. Santa Rosa, - 4) Davon 459 gr. Couch Iron, 6r gr. Santa Rosa Saltillo, ausserdem noch $160 \mathrm{gr}$. Couch Iron nach I. Juli 1893 erworben und $3 \mathrm{gr}$. Smithsonian Iron. 
Ursprüngliches Gewicht von 3) Sancha Estate: Nicht zu ermitteln, da Sancha Estate ein Sammelname für viele CoahuilaEisen ist.

Nachweisbares Gewicht: 4I2I gr. Bailey 2010 New Haven 380

Bement 146 Newton

v. Braun

Budapest 145 Rom, U.

51 v. Siemaschko 45

Cleveland 46 Stïrtz 167

Dresden, M.

32 Ward 70

Gregory $\quad 162$ Washington, Sh. I84

London, B. M. 573 Wien, H. M. *) $3{ }^{1}$ )

London, P. G. 19

Ursprüngliches Gewicht von 4)

Fort Duncan: $44 \mathrm{Kgr}$. $\left(97^{1 / 1 / 4} \mathrm{lbs}\right.$.).

Nachweisbares Gewicht: 20196 gr.

\begin{tabular}{|c|c|c|}
\hline Bailey & 172 & Neapel \\
\hline Belgrad & 266 & Odessa \\
\hline Bement & 299 & Paris, E. \\
\hline Berlin, G. & $5^{I}$ & Paris, M. \\
\hline Berlin, U. & 579 & Petersburg, B. \\
\hline Bologna & 71 & Prag, D. U. \\
\hline v. Braun & 3024 & Prag, M. \\
\hline Brezina & IOI3 & Rom, U. \\
\hline Budapest & 800 & Sevilla \\
\hline Cleveland & 7 & v. Siemaschko \\
\hline Dorpat & 298 & Stockholm \\
\hline Dresden, M. & 340 & Strassburg \\
\hline Graz, U. & 69 & Troyes \\
\hline Gregory & 478 & Ward \\
\hline Greifswald & 160 & Washington, Sh \\
\hline Klausenburg & IO3 & Wien, H. M. $\mathbf{I}_{3}$ \\
\hline London, B. M. & 4520 & Wien, U. I. \\
\hline de Mauroy & I9 & Wrany \\
\hline Melion & 6 & Würzburg \\
\hline München & $5^{15}$ & \\
\hline
\end{tabular}

Cobija

Cocke County

Joël Iron

Cold Bokkeveld, Tuhlbagh, Capland, Südafrika.

Stein, K, gefallen ${ }_{13}$. Okt. $\mathrm{I}_{3} 8$.
1839: M a clear und W a ter meyer (Brief an Herschel): An Account of the Fall of a Meteoric Stone in the Cold Bokkeveld, Cape of Good Hope. Phil. Trans. Royal Soc. London I839 I, p. $83-85$; s, auch: Philos. Magaz. 1839, p. 368 ; N. J. 1840 , p. $722-$ 723 ; L'Institut Bd. 7 (1839), p. 373 -374 (Analyse).

r839: F a ra d a y: Chemical account of the Cold Bokkeveld Meteoric Stone. Phil. Trans. Royal Soc. London 1839 I, p. $86-87$; s, auch London and Edinburgh Philos. Magaz. (3) 14 (1839), p. 23I, 368, 39r ; Pogg. Ann. Bd. 47 (1839), p. 384 ; Berzelius, Jahresber. Bd. 20 (I84I), p. 256 ; auch Rammelsberg: Handwörterb. Suppl. III (1847), p. 82. Ref. N. J. 1840 , p. $722-723$. 1839: African Meteorite (from the London Nautical Magazine). Am. Journ. (I) 36, p. 393 .

1839: Cold Bokkefeld Meteorites. Am. Journ. (I) 37, p. 190.

1839/40: Von älterer Literatur ist noch zu erwähnen: Mag. Nat. Hist. Bd. 3, p. 145; ferner : "L'Institut « Bd. 7 (1839) Nr. 287 , p. 224 (Uebersetzung eines Briefs von Thompson); "Athenaeum * vom 28. März 1840 und $>$ Echo du Monde Savant « vom April 1840,

1840: $\mathrm{M} \mathrm{a} \mathrm{clear}$ : Further particulars of the Fall of the Cold Bokkeveld Meteorite. Phil. Trans, Royal Soc. London (1840), p. 177-I82 (Situationsplan). r84r: Sill i man: African Meteorite of Cold Bokkeveld. Am. Journ. (I) 40, p. 199-201.

1843: P a r s c h: Meteoriten, p. 15. 1854: v. Bog uslawski: Zehnter Nachtrag, Pogg. Ann. Ergz.-Bd. 4, p. 84, $357-359$.

1859: Harris: Dissert. Gött., p. 3544 (Analyse); Ref, Kenngott, Uebersicht 1859, p. 169-170; Wochenschr. f.Astr.,

I) \$False Cañada de Hierro oder Taos, gefd, I846<. 
Met, u. Geogr. 1859, p. 199.

r859: W öhler und $\mathrm{Haidinger:}$ Ueber die Bestandtheile desMeteorsteines vom Capland. Sitzber. Wien. Akad. Bd. 35 , p. 5-12. Ref. N. J. 186o, p. 352 -353; Liebig-Kopp, Jahresber. 1859, p. 856,857 ; Journ. prakt. Chem. Bd. 77 (1859), p. $53-58$; sL'Institut e Bd. 27 (1859) Nr. 1324, p. 163.

r859: W öhler: Ueber die Bestandtheile des Meteorsteines vom Capland. Ann. Chem. Pharm. Bd. IIo, p. 369 -374 ; s. auch Chem. Centr. I859, p. $401-406$; Philos. Magaz. (4) 18 (1859), p. 213-218; Luminous Meteors, Rep. Brit. Assoc. 1865, p. 129.

1859: B u c hner: Feuermeteore, p. 93. r860: Wöhler und $\mathrm{Haidinger:}$

Neuere Untersuchungen über die Bestandtheile des Meteorsteines vom Capland.S.tzber. Wien. Akad. Bd. 4I, p. 565 -567 ; s. auch Journ.prakt.Chem.Bd.77 (1859), p. 53-58; ferner Chem. Centr. I860, p. 876-877; Luminous Meteors, Rep. Brit. Assoc. 1865 , p. 129 (Sep.); Liebig-Kopp, Jahresber. 1860 , p. 849 -850 ; s'Institut \& Bd. 28 (1860) Nr. 1396, p. 328.

1860: $\mathrm{H}$ a idinger: Einige neuere Nachrichten über Meteoriten, namentlich die von Bokkefeld, New Concord, Trenzano, die Meteoreisen von Nebraska, vomBrazos, von Oregon. Sitzber. Wien. Akad. Bd. 4I, p. 568.

r860: $\mathrm{R}$ a m m e Is berg: Mineralchemie, p. $942,949,952$.

1862: Enge l b a c h: Lithium u. Strontium im Meteorstein von Capland. Pogg. Ann. Bd. 116, p. 512. Ref. Kenngott, Uebersicht $1862 / 65$, p. 434 .

r863: Bu c hn er: Meteoriten, p. 6o-62. 1863: R os e: Meteoriten, p. 25, 27, 28, $126,156$.

r864: Wöhler: Bemerkungen über den neuesten Meteorstein-Fall. Gött, Gel. Anz. I864 (Nachr.), p. 28o-28r. Ref. *L'Institut « Bd.33(1865), p.I4 15. r858/65: v. Rei che n b a c h: V 477, 481. VI 441. VII 561. IX 156, 160, 172, 180. X 359, 362. XI 295, 298, 300, 306. XII 458. XIII 370 Fig., 377. XIV 395. XXV 426.

1865: Buchner: Zweiter Nachtrag, Pogg. Ann. Bd. 124, p. 573.

r867: Geinitz: N. J. I867, p. 724 . 1867: Go e be 1: Kritische Uebersicht, Mélanges phys. chim. Bd. 7, p. 315316,338 .

1870: $\mathrm{R}$ a m melsberg: Meteoriten, p. III-II4, ${ }_{3} 8$ (Analyse).

1875: Mo hr: Entstehungsart,Ann.Chem. Pharm. Bd. 179, p. 272.

1876: S m it h: Carbon compounds, Am. Journ. (3) II, p. $39 \mathrm{I}$.

1876: W rig ht: On the Gases contained in Meteorites. Second paper. Am. Journ. (3) 12, p. 165-176.

1877: Flight: Meteorites and the origin of life. The Popular Science Review, 18. Okt. 1877 , p. 8 (Sep.).

r879: R a m me 1s berg: Meteoriten, p. 23 .

r882: Wi e c h m a n n : Fusion-Structures in Meteorites. Ann. N. Y. Acad. Sc. Bd. 2, p. 302.

r884: W a d s w or th: Studies, p. 186. 1884: M eunier: Météorites, p. 62, $65,69,93,95,97,304-306$.

r883/85: Tschermak: Photographien, T. 20, p. $3,19,20$.

r885: Brezin a: Wiener Sammlung, p. $184,233$.

1888: N ewt on: Orbits, Am. Journ. (3) 36, p. 5 .

r889: F l e t c h er: A tacama Meteorites, Mineral. Magaz. Bd. 8, p. 227 (Streufeld $I$ auf 16 miles).

r894: F l etcher: Introduction, p. 9, 36. r894: C ohen: Meteoritenkunde, an vielen Stellen.

Ursprüngliches Gewicht: Viele Steine, zusammen mehrere Zentner; grösstenteils verschleppt. 
Nachweisbares Gewicht: 4108 gr. Bailey

Bayet

Belgrad

Bellucci

Berlin, G.

Berlin, U.

Bologna

v. Braun

Budapest

Calcutta

Cambridge

Cleveland

Cohen

Debreczin

Dorpat

Edinburg

Frankfurt

Göttingen

Gregory

Hamburg

Harvard, U.

Heidelberg

Helsingfors

Howell

Klausenburg

6 Kopenhagen

5 Kristiania

8 London, B. M. 1057

2 London, P. G. 49

38 Madras

19 Minneapolis

I München

2 Neumann

132 New Haven

I06 Oxford

4 Paris, M.

2 Petersburg, A. 244

II Pohl

Io v. Siemaschko

5 Stockholm

354 Strassburg

2 Stuttgart

30 Troyes

36 Tübingen

5 Ward

14 Washington

I Washington, Sh. 10

4 Wien, H. M.*) 666

I Wien, U. I.

2
Colfax, Colfax Township, Rutherford County, Nord-Carolina ; auch Ellenboro, Rutherford County, U. S. A.

Eisen, O, gefunden $\mathrm{r} 880$, beschr. 1890 .

1890: E a kins: Meteoric Iron from North Carolina. Am. Journ. (3) 39, p. 395-396 (Analyse). Ref. N. J. 1891 I, p. 242 ; s, auch U. S. Geol. Surv. Nr. 78 (189r), p. 93--94.

r889/90: Kunz: Meteoric Iron from Colfax Township, Rutherford County, North Carolina. Transact. New York Acad, of Sc. Bd. 9, p. 197-198 (Analyse von Cramer). Ref. N. J. 1892 I, p. 267.

1890 : E a s tma n : Met, Astron., p. 320. 1894: Co hen : Meteoritenkunde, p. 76. Ursprüngliches Gewicht: 2400 gr.
Nachweisbares Gewicht: 1954 gr. \begin{tabular}{lr|lr} 
Bailey & 16 & Washington & 315 \\
Kunz & 1623 &
\end{tabular}

\section{Collen County Mac Kinney}

Collescipoli(Antifona), Terni,Italien. Stein, Cc, gefallen 3. Februar 1890 .

r89o: Terenzi: L'aerolito di Collescipoli (Terni). Rivista di Scienze Naturali di S. Brogi, Anno X Nr. 3. Ref. Rivista di min. e crist. ital. Vol. VI 1889 (!), p. $83-84$; s. auch N. J. I89o II, p. 387 ; Oesterr. Zeitschr. f. Berg- u. Hüttenw. Bd. 39 (1891), p. 344.

1890: Trottorelli: Analisi chimica dell' aerolito caduto aCollescipoli presso Terni il 3 Febbrajo 189o. Gazetta chimica Bd. 20, p. 6II-6r5. Ref. Ber. d. d. chem. Ges. 1891 , p. 352 ; N. J. 1891 II, p. 238-239; auch eine kurze Notiz im Giornale mineral. I, p. 186.

1893: Brezin a : Ueber neuere Meteoriten (Nürnberg), p. I6r.

r894: Co he n: Meteoritenkunde, p. 22, $163,167,236$.

UrsprünglichesGewicht : DasHauptstück 3430 gr.; der ganze Stein mochte $4-5 \mathrm{Kgr}$. gewogen haben.

Nachweisbares Gewicht: I802 gr. Bement

Böhm

v. Braun

Brezina

Budapest

Dorpat

Dresden, M.

Eger

Graz, J.

Gregory

Kopenhagen $\quad$ o6

I96 Kunz 2

94 London, B. M. 105

IO3 Neumann 6

46 Paris, M. $\quad 49$

185 Rom, T. 13

15 Rom, U. 169

45 v. Siemaschko 5

192 Strüver 65

5 Turin, U. $\quad 89$

I8 Wien, H. M. 294

Professor Romolo Meli in Rom besitzt ebenfalls ein Bruchstück dieser Lokalität.

Colorado Meteorite Russel Gulch

Colorado River s. Anhang

Colorado (Wien, H. M.) Trenton 
Columbien - Copiapo.

\section{Columbien}

Concepcion, Eisen Huejuquilla Gr. Concepcion, Stein

Concord

Coney Fork

Constantinopel

Cooperstown

Coopertown, Robertson County,

Tennessee, U. S. A.

Eisen, Om, bekannt $\mathrm{x} 860$, beschr. I86r.

Cohen, Meteoritenkunde 1894 , verweist von Coopertown auf Burlington, wie auch Brezina $188_{5}$; es liegt offenbar eine Verwechslung von Cooperstown und Coopertown vor. Bei der näheren Bezeichnung von Burlington führt Brezina Cooperstown auf.

1859: Mc Donnold: Meteoric Explosion in West-Tennessee Sept. Ist $^{\text {st }}$ 1859. Am. Journ. (2) 29, p. 138 (ob auf diese Lokalität zu beziehen ?).

186r: Smith: Two new Meteorites, Am. Journ. (2) 3I, p. I5I. Ref. Kenngott, Uebersicht 186r, p. I07.

r 86r : $\mathrm{S} \mathrm{mith:} \mathrm{Description} \mathrm{of} \mathrm{three} \mathrm{new}$ Meteorites.-Robertson County(Tenn.), Meteoric Iron. Am. Journ. (2) 31, p.266 (Analyse). Ref. N. J. 186I, p. 329; s. auch $\gg$ Original Researches $\alpha$, p. 428 ; Liebig-Kopp, Jahresber. 186I, p. II27; Journ. prakt. Chem. Bd. 85 (1862), p. 515 .

1862: v. Reichenba ch: XXI 589. 1863: B uchner: Meteoriten, p. I97. 1863: Rose: Meteoriten, p. 64, 152. r884: M e un i e r : Météorites, p.r16,124. r885: Brezina: Wiener Sammlung, p. $210-2 \mathrm{II}, 233$.

r886: $\mathrm{H}$ u n t ing t o n : Crystalline Structure, Am. Journ. (3), 32, p. 294, 295 (Abb. einer geätzten Platte).

r89o: East m a n: Met. Astron., p.320. r89r: v. H a u e r: Ann. Hof-Mus. Bd. 6 (Not.), p. 54 .

1893: M e unier: Révision des fers météoriques, p. 52, 56-57.

Ursprünglich.Gewicht:Etwa $17 \mathrm{Kgr}$. Nachweisbares Gewicht: 7239 gr.

\begin{tabular}{lr|lr} 
Bailey & 73 & Minneapolis & 94 \\
Bement & 608 & Moskau & 17 \\
Berlin, U. & 172 & Neumann & 115 \\
Bologna & 23 & New Haven & 115 \\
v. Braun & 43 & Paris, M. & 296 \\
Budapest & 31 & Petersburg, B. & 73 \\
Calcutta & 210 & Pohl & 49 \\
Cleveland & 69 & Rom, U. & 19 \\
Dorpat & 30 & v. Siemaschko & 24 \\
Göttingen & 75 & Stockholm & 204 \\
Gregory & 68 & Strassburg & 31 \\
Halle & 106 & Troyes & 75 \\
Hamburg & 29 & Tübingen & 42 \\
Harvard, U. & 2065 & Upsala & 25 \\
Kopenhagen & 91 & Ward & 82 \\
v.Leuchtenberg & 149 & Washington & 633 \\
London, B. M. & 180 & Washington,Sh. 328 \\
London, P. G. & 91 & Wien,H.M.*)**) & 884
\end{tabular}

Copiapo, Sierra de Deesa, Atacama, Chile, Südamerika.

Eisen, Obc, bekannt $186_{3}$, beschr. I864.

Hierher auch vorläufig: Corrizatillo und Galleguillos (Kristiania).

1864: H a i d ing e r : Eine grosskörnige Meteoreisen-Breccie von Copiapo. Mit I Kupfertafel (Analyse der Eisenteile von v. Hauer). Sitzber. Wien. Akad. Bd. 49 II, p. $490-497$; s. auch Am. Journ. (2) 38 (1864), p. 424; Kenngott, Uebersicht $1862 / 65$, p. 268 -269 (Das sich p. 269 anschliessende Ref. bezieht sich nicht, wie Kenngott vermutet, auf Copiapo, sondern auf Vaca Muerta). S. auch Luminous Meteors, Rep. Brit. Assoc, 1865, p. 129; "L'Institut \& Bd, 32 (1864) Nr. 1613, p. $382-383$.

1865: B u chner: Zweiter Nachtrag, 
Pogg. Ann. Bd. 124, p. 591, 595-596. r868: Da ubré e: Sur trois nouveaux fers météoriques du Chili, récemment parvenus à la Collection de Géologie du Muséum, - II. Fer de la Cordillère de Deesa (irrtümlich in die Nähe von Santiago verlegt). C. R. Bd. 66 , p. 571 -572 (Analyse von Domeyko). Ref. Liebig-Kopp, Jahresber, 1868, p. 1046. r869: B u chner: Vierter Nachtrag, Pogg. Ann. Bd. 136, p. 610.

r870: Meunier, Messungen von Des Cloizeaux : Note über den krystallisierten Enstatit aus dem Meteoreisen von Deesa, daran anschliessende Bemerkungen von v. Haidinger. Sitzber. Wien. Akad. Bd. 6I II, p. 26-29; s. auch sCosmose Bd. 7 (r869), p. 188 -192; „La Nature $\mathrm{Bd}$. I (1873), p. 405 ; N. J. 1870 , p. $486-487$; Liebig-Kopp, Jahresber. 1870, p. 1396. r875: Tschermak: Vulkanismus,

Sitzber. Wien. Akad. Bd. 7I II, p. 663. 1879: R a m me 1sberg: Meteoriten, p. 7 .

1879: D o m e y ko: Mineralojia, 3 ed. 1879 , p. 132 .

I884: W a d s worth: Studies, p. 70. r884: M e unier: Météorites, p. 68, 79, 81, 99, 152 (Abb.) $=16 \mathrm{I}, 327,352$, $353,354,355,356,379,380$.

r883/85: Tschermak: Photographien, p. 3 .
1885: B rezina: Wiener Sammlung, p. $152,154,155,164,203,217-218,234$. I887: Flig ht: Meteorites, p. I47-I49. r889: F le t c her: Atacama Meteorites,

Mineral. Magaz. Bd. 8, p.224, 255-256. r889: C o h e n : São Julião, N. J. 1889

I, p. 223, 224, 225.

1894: Co he n: Meteoritenkunde (unter dem Namen Sierra de Deesa), p. 96, 133, 134, 198, 272, 275, 277, 280, 281, 286, 300. Der Name Copiapo bezieht sich bei Cohen nur auf den von Joy untersuchten Meteoriten, welcher $=$ Vaca Muerta ist.

r895: Meunier: Révision des lithosidérites, p. 9, II-I3 (Abb. einer geätzten Platte u. eines Dünnschliffs).

Ursprüngliches Gewicht: Domeyko besass etwa $7 \mathrm{Kgr}$; ; wieviel ausserdem noch gesammelt wurde, ist nicht zu ermitteln.

Nachweisbares Gewicht: 22939 gr.

\begin{tabular}{lr|lr} 
Bailey & 55 & London, B.M. & $769^{3}$ ) \\
Berlin, U. & 3443 & Neumann & $6^{3}$ ) \\
Budapest & 18 & Paris, M. & 13 990 \\
Calcutta & 22 & v. Siemaschko & 7 \\
Dresden, M. & 340 & Ward & 75 \\
Harvard, U. & $\left.333^{1}\right)$ & Wien, H. M. *) & 1 223 \\
Kristiania & $\left.268^{2}{ }^{2}\right)$ &
\end{tabular}

\section{Corrizatillo} Copiapo Cosby's Creek, Cocke County,

I) Hievon 320 gr. Iron 1860. Nineteen miles east of Copiapo 4.

2) 1330 gr. "Galleguillos n. v. Copiapo, Atacama , 1328 gr. "Corrizatillo n. v. Copiapo, Atacama ๔. Diese zwei Eisen wurden von Hrn. Director L. Sundt, der 23 Jahre lang als Bergbeamter in Chili meistens in Atacama lebte, 1884 der Sammlung in Kristiania geschenkt. Ueber die Fundzeit ist nichts bekannt geworden. Hr. Professor Brügger teilte mir ferner in Bezug auf die Beschaffenheit der beiden Eisen mit, dass dieselben stark von einander verschieden seien. In dem Stück von Corrizatillo befinden sich mehrere grosse bis $2 \frac{1}{4} \mathrm{~cm}$ im Durchschnitt messende Troilitknollen, in dem Stück von Galleguillos fehlt hingegen jede Spur von Troilit. Ob diese Stücke zur Meteoreisen-Breccie von Copiapo gehören, lässt sich ohne genaueres Studium nicht entscheiden; ich habe sie nur vorläufig hier eingereiht.

3) Sind unter Siderolithen aufgeführt; vermutlich silikatreiche Partien der Meteoreisen-Breccie von Copiapo. 
Tennessee, U. S. A., auch Sevier-Eisen.

Eisen, $\mathrm{Og}$, gefunden 1837 , erwähnt I 838 , beschr. 1840 .

1838: T roost: Briefl. Mitt. N. J. 1838 , p. 42 (kann sich wohl nur auf dieses Eisen beziehen).

1840: Troost: Description and Analysis of a Meteoric mass, found in Tennessee, composed of Metallic Iron, Graphite, Hydroxide, of Iron and Pyrites. Am. Journ. (I) 38 , p. $250-254$; s. auch Am. Journ. (2) 5 (1848), p. 351 (als älteste Eisenmasse von Tennessee aufgeführt). Ref. Journ. prakt. Chem. Bd. 25 (I842), p. 374-375; »L'Institut " Bd. 9 (184I), p. II5.

1842: S he p a r d: Analysis of Meteoric Iron from Cocke County, Tennessee, with some remarks upon Chlorine in Meteoric Iron masses. Am. Journ. (I) 43 , p. $354-359$ (Beiläufig: Burkart, Fundorte I, N. J. 1856 , p. 267).

1843: R a m mels berg : Handwörterbuch, Suppl. 2, p. 90-91.

1843: Parts ch: Meteoriten, p. II7.

1847: S hepard: Report on Meteorites. Am. Journ. (2) 4 , p. $83-85$; s. auch Liebig-Kopp, Jahresber. $1847 / 48$, p. I3II ; Kenngott, Uebersicht 1850/5I, p. 136.

1852: C 1 a r k: Dissert. Gött., p. 35.

1853: J o y: Analyse des Meteoreisens von Cosby's Creek. Ann. Chem. Pharm. Bd. 86 , p. $39-43$; s. auch Dissert. Gött. 1853, p. Io-14; ferner Journ. prakt. Chem. Bd. 60 ( 1853 ), p. 62 ; Liebig-Kopp, Jahresber. 1853 , p. 935 ; N. J. 1855 , p. $562-563$; Kenngott, Uebersicht 1853 , p. I5 $_{5}$.

1854: v. Boguslawski: Zehnter Nachtrag, Pogg. Ann. Ergz.-Bd. 4, p. $408-409$.

1857: B er g e m a n $\mathrm{n}$ : Untersuchungen von Meteoreisen. Pogg. Ann. Bd. Ioo, p. 254-255; s. auch Journ, prakt.
Chem. Bd. 7I (1857), p. 58; Chem. Centr. 1857 , p. 746; Liebig-Kopp, Jahresber. 1857 , p. $729-730,732$; Kenngott, Uebersicht $1856 / 57$, p. 153 .

1859: B u c h n e r : Feuermeteore, p. 132 -133 .

1859: Harris: Dissert. Gött., p. $\mathbf{H 2}_{2}-\mathrm{II}_{3}$. 1860: $\mathrm{R}$ a mmel $\mathrm{s}$ berg: Mineralchemie, p. $915-916,948$.

r 858162 : v. R e i c h en b a c h : IV 638. VI 448 , 449. VII 552 . IX 162, 174, 181, 182. X 359. XI 29I. XII 457 , 458,460 . XIII 363, 38r. XIV 395. XV 100, IIO, III, I12, 122, 124, 126, 127, 128, 129. XVI 253, 254, 257258 (Analyse von dem Sohne des Freiherrn v. Reichenbach; Ref. LiebigKopp, Jahresber. 186I, p. II29-II30); 261, 262, 263. XVII 265, 266, 273. XVIII $480,484,487,489$. XIX 150 , $154,155 . \mathrm{XX} \mathrm{621,} \mathrm{623,} \mathrm{625,} \mathrm{626,} \mathrm{628,}$ $629,630,634$. XXI $577,578,579$ (Fig. Graphiteinschluss), 586, 591. XXIa 172, 173, 174, 176.

I862 (?): Giebel u. Heintz: Zeitschr. f. ges. Naturw. Bd. 9, p. $5 \mathrm{ro}$; Bd, 10, p. 189.

1862: Rammels berg: Ueber das Schwefeleisen der Meteoriten. Mon.-Ber. Berlin. Akad. 1862, p. $689-691$. Ref. Liebig-Kopp, Jahresber. 1862, p. 832 -833 ; Journ. prakt. Chem. Bd. 88 (1863), p. $275-277$.

1863: B u chner: Meteoriten, p. 164 $-\mathrm{I} 66$.

r863: R o s e: Meteoriten, p. 27, 36-37,

$39,52,54,57-5^{8}, 59$, I32, I39, I52. 1864: R a m mels b e r g : Ueber das

Schwefeleisen der Meteoriten Pogg. Ann. Bd. 121, p. $366-367$; s. auch Sitzber. Berlin, Akad, 1864, p. 30 (s. w. Ref. bei Seeläsgen).

1865: Buchner: Zweiter Nachtrag,

Pogg. Ann. Bd. 124, p. 597.

1867: Go e be 1: Kritische Uebersicht,

Mélanges phys. chim. Bd. 7 , p. 325 . r869: Meunier: Recherches, Ann. 
Chim. Phys. (4) 17, p. $4^{8}$. 1870: R a m mels ber g: Meteoriten, p. $80,82,84,85$.

1875: S m it h: Troilithe; sa vraie place minéralogique et chimique. C. R. Bd. 8I, p. $976-978$.

r876: S m it h: Carbon compounds, Am. Journ. (3) II, p. $392,393,434,435$. 1877: $\mathrm{F} 1 \mathrm{ight}$ : Meteorites and the origin of life. The Popular Science Review, 18. Okt. 1877 , p. $8-9$ (Sep.). r878: S mith: On the composition of the new meteoric mineral Daubréelite etc. (cf. Coahuila). Am. Journ. (3) 16, p. 272 ; s, auch Rammelsberg, Mineralchemie 2. Aufl. 1. Erg.-Heft 1886, p. 76 (Daubréelit); Liebig-Kopp, Jahresber. 1857, p. 1309; Zeitschr. f. Krystallogr. Bd. 3 , p. 328 .

r879: $\mathrm{R}$ a m me ls be r g : Meteoriten, p. $22-23$.

1881: $\mathrm{Smith}$ : Anomalie magnétique du fer météorique de Sainte-Catherine. C. R. Bd. 92 , p. $843-844$; s. auch Original Researches 1884 , p. 597-598; Liebig-Kopp, Jahresber. 1881, p. 1458. r883: S m it h : Concretions, Am. Journ.

(3) 25, p. $417,418,419,421$.

1884: M e un ier: Météorites, p. 20, 22, $36,52,64,72,116,121,122$.

r885: Brezina: Wiener Sammlung, p. $155,200,207,214,234$.

1885: H un t ing to n: Crystalline Structure, Am. Journ. (3) 32, p. 288, 296, 302. 1887: Fletcher: Cubic Crystals of graphitic carbon. "Nature* Bd. 36, p. 305 ; s. auch Mineral. Magaz. Bd. 7 (1887), p. 130 (Arbeit über Youndegin). 1889: Brezin a : Cliftonit aus dem Meteoreisen von Magura, Arvaer Comitat. Ann. Hof-Mus, Bd, 4, p. 102.

r889: C o he n : São Julião, N. J. r889I, p. $223,224,225$.

r890: Eas tm a n : Met. Astron., p. 318. r89r: Cohen und Weinschenk: Meteoriten-Studien, Ann. Hof-Mus. Bd. 6 , p. 163,165 .

1893: Meunier: Révision des fers météoriques, p. 29, 34, 72 .

1894: Hunting ton: The Smithville Meteoric Iron. Proc. Amer. Acad. Arts and Sc. Bd. 29, p. 259 (Situationsplan von Tennessee bis W.-Virginia); s. auch Zeitschr. f. Kryst. Bd, 26 (1896), p.604.

r894: Cohen: Meteoritenkunde, an sehr vielen Stellen.

1895: C o hen: Meteoreisen-Studien IV, Ann. Hof-Mus. Bd. Io, p. $83,84,85$.

Ursprüngliches Gewicht: Etwa $95^{\circ}$ Kgr. Zwei Massen, von denen die grössere auf 2000 lbs. geschätzt wurde und die kleinere ein Gewicht von I 12 lbs. besass. Grösstenteils verschmiedet.

Nachweisbares Gewicht : 98637 gr.

Bailey

Belgrad

Bement

Berlin, U.

Bologna

Bonn

v. Braun

Breslau

Brezina

Budapest

Calcutta

Cambridge

Cleveland

Coheri

Dorpat

Giessen

Göttingen

Gotha

Gregory

Halle

Harvard,

Howell

Klausenburg

Kopenhagen

Cosina, Loma de la Cosina oder Cerro Cosina bei Dolores Hidalgo, Staat Guanajuato, Mexico. 
Stein, Ck, gefallen Januar 1844 . 1865: B u rk art: Verh, naturhist. Ver. Bonn (Sitzber.) Bd. 22, p. 7r. r866: Burkart: Fundorte III, N. J. 1866, p. $401-402$.

1866: Buchner: Neue Meteoriten, Pogg. Ann. Bd, 129, p. 350-351. 1869: Buchner: Vierter Nachtrag, Pogg. Ann. Bd. 136, p. 450.

1870: Burkart: Fundorte IV, p. 673, 689 .

1884: vo m R at h: Verh, naturh. Ver.

Bonn (Sitzber.) Bd. 41, p. 126.

Ref. N. J. 1885 II, p. 270.

1884: M e unier: Météorites, p. 188.

1885: Brezina: Wiener Sammlung, p. 19I, 233.

1889: C astill o: Météorites, p. 12. 1890: F l e tcher: Mexican Meteorites, Mineral Magaz. Bd. 9, p. 95.

Ursprüngliches Gewicht: $1.2 \mathrm{Kgr}$. Nachweisbares Gewicht: 284 gr. Berlin, U.

Bonn

Calcutta

Dorpat

London, B. M. 24 London, P. G. Spl. 2 Paris, M. I3O 9 Stockholm 18 Wien, H. M. * 2

Ein Bruchstïck befindet sich nach vom Rath in der Sammlung der Bergschule in Mexico; auch dürfte Castillo einen Teil dieses Meteoriten besitzen.

\section{Costa Rica}

Heredia

Costilla, Costilla Peak auf der Grenze von Colorado und New. Mexico.

Eisen, Om, gefunden $\mathrm{r} 88 \mathrm{I}$, beschr. I 895 .

1895: Hills: The Costilla Meteorite. Proc. Colorado Scientific Soc. Denver. Read at Meeting, January $7^{\text {th }} 1895$, 2 Seiten (Analyse v. Eakins u. Abb.).

Ursprüngliches Gewicht: $35 \mathrm{Kgr}$. (78 lbs.). Nach Hills Beschreibung im Besitz der Colorado Scientific Society in Denver; von dort erhielt Bement einen Teil. Wien H. M. besitzt gegenwärtig $1580 \mathrm{gr}$.

\section{Couch Iron}

Coahuila

Cowra, Neu Süd-Wales, Australien. Eisen, Off, gefunden vor 1888 , beschrieben 1895 .

Hierher auch Carcoar,Bathurst, Neu Süd-Wales.

1895: Brezina: Wiener Sammlung, p. 267.

Ursprüngliches Gewicht : ?

Nach brieflicher Mitteilung von Herrn Prof. Archibald Liversidge in Sidney an Herrn Direktor Brezina soll der Meteorit im Dep. of Mines in Sidney aufbewahrt werden.

Nachweisbares Gewicht: London, B. M. 192 gr., Wien, H. M. 31 gr.

Crab Orchard, Crab Orchard Mts. Powder Mill Creek, Rockwood, Tennessee.

Mesosiderit (Grahamit ?), gefunden 1887 .

1887: Whitfield: The Rockwood Meteorite. Am. Journ. (3) 34, p. 387 -390 (Analyse u. Abb. der Hauptmasse u, eines polierten Schnittes). Ref. N. J. 1889 I, p. 403 .

1887: $\mathrm{K}$ unz: On some American Meteorites. - 5. On the Powder Mill Creek Meteorite. Am. Journ. (3) 34, p. 476 -477 (Abb. eines polierten Schliffes und eines Dünnschliffes).

1887: Howe 11: Rockwood Meteorite. >Sciencer Bd. 10, p. 107.

1889: v. H a u e r : Ann. Hof-Mus. Bd. 4 (Not.), p. 64 ; s. auch Bd. 7 (1892) (Not.), p. 72.

r89o: E a s tm a n : Met. Astron,, p. 320.

1893: N e wton: Lines of structure in the Winnebago Co. Meteorites and in 
other Meteorites. Am. Journ. (3) 45, p. $152-153$ und 355 . Ref. N. J. 1894 I, p. $273-274$.

1893: Br ez in a : Ueber neuere Meteoriten (Nürnberg), p. 162.

r894: Co hen: Meteoritenkunde, p. 6I, $220,232,237,249,287,308$; p. 287 sagt Cohen: Crab Orchard (Powder Mill Creek) scheint olivinfrei zu sein - und ist daher vom Grahamit zu trennen. r895: M e unier: Révision des lithosidérites, p. 33,37 .

Ursprüngliches Gewicht: Etwa 43 $\mathrm{Kgr} .3$ Massen von ${ }^{6} 65^{8} \mathrm{gr}$., ${ }_{26} \mathrm{I}_{\mathrm{I}}$ gr. u. etwa $38 \frac{1}{2} \mathrm{Kgr}$.

Nachweisbares Gewicht: 34067 gr.

Bement $\quad 1364 \mid$ London, B. M. $\quad 148$

Berlin, U. $\quad 40$ de Mauroy $\quad 170$

Böhm 9 New Haven II4

v. Braun 221 Newton 9I

Brezina $\quad 88$ Paris, E. $\quad 34$

Budapest $\quad 130$ Prag, M. 94

Dorpat $\quad 88$ Rom, U. 165

Dresden, M. 355 v. Siemaschko 240

\begin{tabular}{ll|l} 
Gregory & 523 & Stürtz
\end{tabular}

\begin{tabular}{ll|ll} 
Greifswald & 91 & Troyes & I3
\end{tabular}

Harvard, U. 670 Ward 16112

Howell $\quad 3740$ Washington $\quad 74$

Kopenhagen $\quad 72$ Wien, H. M. $\quad 7275$

Krantz $\quad 729$ Wien, U. I. $\quad$ I60

Kunz 80

Cranberry Plains, Poplar Hill (Poplar Camp), Virginia, U. S. A. Eisen, O, gefunden $185^{2}$.

1884: Meunier: Météorites, p. $\mathbf{1 6 .}$ r885: Brez in a : Wiener Sammlung, p. 254 .

r886: H u n t in g t on : Crystalline Structure, Am. Journ. (3) 32, p. 300 (Abbildung eines Schliffs mit gekrümmten Widmannstïtten'schen Figuren).

1887: Huntington: Catalogue of all recorded Meteorites. Proc. Amer. Acad. Arts and Sc. Bd. 23, p. 70-71 (Tafel II).
1893: M eunier: Révision des fers météoriques, p. 47,48 .

Sollte Smith über dieses Eisen nichts publiziert haben, da doch nach Huntington aus seiner Sammlung $20 \mathrm{gr}$. an die Sammlung des Harvard College übergingen ?

Ursprüngliches Gewicht: ?

Nachweisbares Gewicht: 89 gr.

\begin{tabular}{lr|lr} 
Bailey & 7 & New Haven & 21 \\
Gregory & I & Paris, M. & 16 \\
Harvard, U. & 36 & Washington & 8
\end{tabular}

Cranbourne, bei Melbourne, Victoria, Australien.

Eisen, $\mathrm{Og}$, gefunden um 1854 , beschr. 186r.

Hierher auch: "Yarra-Yarra River \& I $85^{8}$ und Western Port Distrikt. r86r: v. Hochstetter: Briefliche Mitteilung, N. J. 1861, p. 316. Ref. Kenngott, Uebersicht 1861, p. 107; Liebig-Kopp, Jahresber. 1861, p. 1123 - II24. r86r: Z i m mermann: Briefliche Mitteilung, N. J. 186I, p. 557 (Auffindung der Masse durch den Mineralien-Händler Abel). Ref. Kenngott, Uebersicht 186I, p. 213-214.

r86r: Ha id ing e r : Zwei MeteoreisenMassen in der Nähe von Melbourne in Australien aufgefunden. Sitzber. Wien. Akad. Bd. 43 II, p. $583-584$. Ref. N. J. 1862 , p. 606 ; Luminous Meteors, Rep. Brit. Assoc. 1865, p. 128; Kenngott, Uebersicht 1861, p. 213; „L'Institut « Bd. 29 (1861) Nr. I445, p. $3^{\mathrm{II}}, 3^{\mathrm{I2}}$.

r86r: Haidinger: Die DandenongMeteoreisenmasse in Melbourne. Sitzber. Wien. Akad, Bd. 44 II, p. 3 I. Ref. Kenngott, Uebersicht $1862 / 65$, p. 267 ; Liebig-Kopp, Jahresber. 186I, p. 1124 ; "L'Institut \& 186r, p. 312.

r86r: Haidinger: Die zwei Cranbourne Meteoreisenblöcke in Victoria. Sitzber. Wien. Akad. Bd. 44 II, p. 378 - 
380 (Abbildung des kleineren etwa 30 Zentner schweren Blocks; Analyse von v. Hauer). Ref. Kenngott, Uebersicht 186265 , p. $266-267$; LiebigKopp, Jahresber. I86I, p. I124 ; >L'Institut \& Bd. 30 (1862), p. 45, 46.

r86r: $\mathrm{H}$ a i d ing e r: Die ersten Proben des Meteoreisens von Cranbourne in Australien. Sitzber.Wien.Akad. Bd, 44 II, p. 465-472 (Abbildung des grössten Blockes) ; s. auch Kenngott, Uebersicht 186265 , p. $266-267$; LiebigKopp, Jahresber. 186r, p. 1124.

r86I: Ha idinger: Das Meteoreisen von Cranbourne im K. K. Hofmineralienkabinet; ein Geschenk von dem königlich grossbritannischen Gouverneur von Victoria in Australien, Sir Henry Barkly. Sitzber. Wien. Akad. Bd. 45 II, p. 65-74 (mit einer Tafel, 3 Abbildungen im Text und einem Situationsplan); s, auch Am.Journ. (2) 34, p. 155 - 156; Kenngott, Uebersicht 1862165, p. 168; "L'Institut * Bd. 30 (1862) Nr. 1475, p. II9.

I863: B u c hner: Meteoriten, p. 198. 1863: R os e: Meteoriten, p. 73, 150, 152. 1866: Large mass of Meteoric Iron (Ankunft der $3750 \mathrm{kgr}$. schweren Masse im British Museum). Am. Journ. (2) 4I, p. 426 ; s. auch Wochenschr. f. Astr., Met. u. Geogr. 1866, p. 240.

1869: B u c hner: Vierter Nachtrag, Pogg. Ann. Bd. 136, p. 439 u. 6II.

1869: H a us h of er: Mineralogische Notizen. - 2) Meteorit von Cranbourne, Australien (Analyse der Kruste). Journ, prakt. Chem. Bd. 107, p. 330-331. Ref. Liebig-Kopp 1869, p. 1304-1305. 1873: B erthelot: Nouvelles contributions à l'histoire des carbones, du graphite et des météorites. Ann. Chim. Phys. Bd. 30 , p. $420-422$.

I874: B jör ling : Meteoriter och Kometer (cf. Hessle), p. 13.

1875: Ts chermak: Vulkanismus, Sitzber. Wien. Akad, Bd. 7I II, p. 664.
1877: Smith: Carbon compounds, Am. Journ. (3) It, p. 392, 394, 395, 434. 1877: Flight: Meteorites and the origin of life. The Popular Science Review, 18. Okt. 1877 , p. 9 (Sep.); s. auch unter $188_{2} / 8_{3}$.

1878: Smith: On the composition of the new meteoric mineral Daubréelite etc. (cf. Coahuila), Am. Journ. (3) I6, p. 272; s, auch Rammelsberg, Mineralchemie 2. Aufl. 1886, 2. Ergz.-Heft, p. 76 ; Zeitschr. f. Krystallogr. Bd. 3 , p. 328 .

1882/83: Flight: Report of an examination of the meteorites of Cranbourne in Australia; of Rowton in Shropshire; and of Middlesbrough in Yorkshire. Philos. Trans. Nr. I71, p. 885 -894 (Analyse, Abbildung, Situationsplan); s. auch Proc. Royal Soc. (Abstract) Bd. $33(1881 / 82)$, p. $343-345$; ferner Geol. Magaz. (2) Io (I883), p. 59 -65 ; N. J. 1884 I, p. 33-35 ; LiebigKopp, Jahresber. 1882 , p. 1644-1645; Weinschenk, Ann. Huf-Mus. Bd, 4 (1890), p. 98.

r883: S m it h: Concretions, Am. Journ. (3) 25 , p. 419, 42I, 422.

r884: $\mathrm{H}$ ä p k e: Beiträge, Abh. naturw. Ver. Bremen Bd. 8, p. 518.

r884: Meunier: Météorites, p. 20, 64,89 .

r885: Brez ina: Wiener Sammlung, p. $207,215,234$.

1887: B r e zin a: Neue Meteoriten IIIa, Verh. k. k. geol. Reichsanstalt, p. 289. r887: F $1 \mathrm{ig} \mathrm{h} \mathrm{t} \mathrm{:} \mathrm{Meteorites,} \mathrm{p.} \mathrm{135-136}$ u. $174-181$ (s. auch oben $1882 / 83$ ). 1889: C o h e n : São Julião, N. J. 1889 I, p. $222,224,225$.

1890: B r e zi n a : Reise, Ann. Hof-Mus. Bd. 4 (Not), p. n6.

r8gr: Cohen und Weins chenk: Meteoreisen-Studien, Ann. Hof-Mus. Bd. 6 , p. $144-145,162,163,164$.

r892: Cohen: Meteoreisen - Studien, Ann. Hof-Mus. Bd. 7, p. 160. 
1892: B la k e : Experiments on Meteoric Iron. Conductet by R. Smith, on portions of the largest meteorite in the British Museum and on the rust derived from it. Catalogue of the Collection of metallurgical specimens. South Kensington Museum, London, p. $307-308$.

1893: Meunier: Révision des fers météoriques, p. 74 .

1894: Co hen: Meteoritenkunde, an vielen Stellen.

r894: C o h e n : Meteoreisen-Studien III, Ann. Hof - Mus. Bd. 9, p. 98, п7 (Rhabdit).

r894: F let cher: Introduction, p. 7, 10, 37 .

Ursprüngliches Gewicht: 2 Massen, die grössere von $373^{1} \mathrm{Kgr}$. in London, die kleinere von etwa ${ }_{500} \mathrm{Kgr}$. wohl in Melbourne.

NachweisbaresGewicht: 3740970 gr.

\begin{tabular}{lrr|lr} 
Bailey & II3 & Lüttich & 8 \\
Bement & $\left.485^{1}\right)$ & de Mauroy & I4 \\
Berlin, U. & 279 & Minneapolis & II \\
Bologna & 80 & München & I7 \\
v. Braun & 67 & Paris, M. & 20 \\
Budapest & $273^{2}$ & Pohl & 4 \\
Calcutta & 59 & Roebling & 84 \\
Göttingen & 218 & v. Siemaschko & $\left.76^{8}\right)$ \\
Gregory & $\left.599^{2}\right)$ & Stockholm & 62 \\
Harvard, U. & $53^{1}$ & Troyes & 25 \\
Kopenhagen & 9 & Ward & $53^{4}$ ) \\
London, B. M. & & Washington & 113 $3^{5}$ ) \\
& 3 731 214 & Washington, Sh & $3^{2}$ \\
London, P. G. 2965 & Wien, H. M.*) & 1100
\end{tabular}

Crawford Co.

Miney

Cremona

Alfianello

Cronstadt, Orange River,Frei-Staat, S. Afrika.

Stein, Cga, gefallen 19. November 1877 . r885: Brezina: Wiener Sammlung, p. 182,233 .

r887: Flight: Meteorites, p. 205.

1894: Fletcher: Introduction, p. I4.

Ursprüngliches Gewicht: Steinschauer, wenig gesammelt.

Nachweisbares Gewicht: ${ }^{2} 285 \mathrm{gr}$.

\begin{tabular}{ll|ll} 
Kristiania & 12 & Troyes & 7
\end{tabular}

London, B. M. 1227 Washington, Sh. 12

Paris, M.

II Wien, H. M.

I6

Cross Roads, Boyett, Cross Roads Township, Wilson Co., NordCarolina, U. S. A.

Stein, $\mathrm{Cg}$, gefallen 24. Mai 1892 .

1893: How e 11: Cross Roads Meteorite. Am. Journ. (3) 46, p. 67 (Abbildung des einzigen kleinen Steins). Ref. N. J. 1895 I, p. 277.

1895: v. H a u e r : Ann. Hof-Mus. Bd.10 (Not.), p. 34.

Ursprüngliches Gewicht: I6I gr., nach brieflicher Mitteilung von Herrn Howell.

Nachweisbares Gewicht: I59 gr.

$\begin{array}{lr}\text { Howell } & 157 \\ \text { Paris, M. } & 2\end{array}$

Seit 1. Juli 1893 gelangten 26 gr. an H. M. Wien, 5 gr. an National-Mus. Washington, 9 gr. an Harvard, U.

Cross Timbers, Red River, Texas. Eisen, Om, bekannt r808, beschr. I8ro.

18ro/r4: Bruce: Mass of malleable Iron. American Mineralogical Journal Bd. I (18I4), p. 124 (diese Notiz ist I81o verfasst, cf. Silliman 1824; der I. Bd. des Bruce'schen Journals wurde 1814 beendigt); s. auch Ref. v. Patrin (Masse de fer malléable) im Journ, des Mines Bd. 32 (1812), p. $234^{-2} 35$.

Unter den obigen Gewichten sind als , Yarra-Yarra \& oder \$Yarra-Yarra River « aufgeführt: bei I) 42 gr. - 2) $142 \mathrm{gr} .-3) 17 \mathrm{gr}$ - - 4) $15 \mathrm{gr} .-5) 16 \mathrm{gr}$. 
1812: Bigot de Morogues, p. 305 $-307$.

I812/r4: Gib bs: Observations on the Mass of Iron from Louisiana. Bruce's American Mineralogical Journal Bd. I, p. $218-221$.

r8r6: Chladni: Erste Fortsetzung, Gilb. Ann. Bd. 53, p. 379.

r8rg: C h l a d n i: Feuermeteore, p. 344. 1819: Gött. Gel. Anz. (Nachr.), Stüick 47. I821 : C h la d n i : Erster Nachtrag, Gilb. Ann. Bd. 68 , p. 343 .

r824: C.H.: Notice of the Malleable Iron of Louisiana. Am. Journ. (I) 8, p. 218 -225 .

r826: Seebeck: Ueber die magnetische Polarisation der Metalle und Erze durch Temperatur-Differenz. Pogg. Ann. Bd. 6, p. 144 .

1826: $\mathrm{Ch} 1$ a d ni: Sechster Nachtrag.

Pogg. Ann. Bd. 8, p. 5I.

1829: She pard: Analysis of the Meteoric Iron of Louisiana, and discuvery of the Stanniferous Columbite in Massachusetts. Am. Journ. (1) 16, p. 217 -219 (Analyse); s. auch Schweigg. Journ. Bd. $5^{8}$ ( $\left.183^{\circ}\right)$, p. $339-343$.

I832 : v. H off : Achter Nachtrag, Pogg. Ann. Bd. 24, p. 230.

r835: Am. Journ. (r) 27, p. $3^{82}$. Unter Miscellanies : - 4. Great Mass of Meteoric Iron from Louisiana.

r838: Silliman: Meteoric Iron. - I) In Texas. Am. Journ. (I) 33 , p. 257.

r84r : $\mathrm{R}$ a m m el s ber g : Handwörterbuch, p. 423 .

r842: S hepard: Analysis of Meteoric Iron from Cocke County, Tennessee, with some remarks upon Chlorine in Meteoric Iron Masses. Am. Journ. (I) 43 , p. $35^{8}-359$.

I843: P a r t s ch: Meteoriten, p. III-II2. r846: Sill im an and $\mathrm{Hunt}$ : On the Meteoric Iron of Texas and Lockport. Am. Journ. (2) 2, p. 37o-374 (Analyse u. Abbildung einer geätzten Platte). Ref.
Pogg. Ann. Bd. 71 ( 1847$)$, p. 544 ; Journ. prakt. Chem. Bd, 42 (1847), p. $45^{8}$; Kenngott, Uebersicht $1844 / 49$, p.222 ; Liebig-Kopp, Jahresber. 1847148 , p. 1313; L'Institut \& Bd. I5 (1847) Nr. 724, p. 384 .

r847 : R a m me $1 \mathrm{~s}$ be r g : Handwörterbuch Suppl. 3, p. $80-8 \mathrm{r}$.

1852: W öhler: >Passive, Pogg. Ann. Bd. 85 , p. $44^{8}$. Ich vermute, dass hier unter Red River das Eisen von Cross Timbers und nicht jenes von Wichita gemeint ist, da letzteres in der Göttinger Sammlung nicht vorhanden ist). I852: Cl ark: Dissert. Gött., p. 59-6o (Abbildung einer geätzten Platte).

1854: v. Boguslawski: Zehnter Nachtrag, Pogg.Ann. Ergz.-Bd. 4, p.402. r858/62: v. Rei $\mathrm{ch}$ en ba ch: IV 638. VII 552. IX $163,174,18$ r. X 359 , 365. XV по. XVI 250,253 . XVII 266. XVIII 487. XIX I55. XX 623. 1859: B u c hner: Feuermeteore, p. 129. 1859: H a r ris: Dissert. Gött., p. Io6. r860: $\mathrm{R}$ a m mel $\mathrm{s}$ ber $\mathrm{g}$ : Mineralchemie, p. $917-918$.

1863: B u c hner: Meteoriten, p. 153 -154 .

1863: R os e: Meteoriten, p. 26, 57, $64, \mathrm{I} 53$.

1867: G o e be 1: Kritische Uebersicht, Mélanges phys. chim. Bd. 7 , p. 325 .

1875: vom R a th: Meteoriten, Verh. naturh. Ver. Bonn Bd. 32, p. 36r.

1875: Wright: Spectroscopic Examination of Gases from Meteoric Iron. Am. Journ. (3) 9, p. 296.

1876: Wright: On the Gases contained in Meteorites. Am. Journ. (3) II, p. 257 u. (3) I2 (1876), p. 167, I68. r884: W a dsworth: Studies, p. 6I. r884: Meunier: Météorites, p. 5I, II6, II9.

r885: Brezin a: Wiener Sammlung, p. $210,233$.

r886: H u n t ing to n: Crystalline Structure, Am. Journ. (3) 32, p. 295. 
r887: F $1 \mathrm{ight:}$ : Meteorites, p. 99-100. 1890: Eas tma n: Met. Astron., p. 318. 1893: Meunier: Révision des fers météoriques, p. 52, 54 .

r894: C o he n: Meteoritenkunde, p. 40, 63, 67 (Red River), 133, 172, 173, 232.

Ursprüngliches Gewicht : Die Hauptmasse (von Col. Gibbs) wog 1635 Pfund (nicht 3000 ) $=740^{1} / 2 \mathrm{Kgr}$., nachdem ein kleineres Stück abgeschnitten war.

Nachweisbares Gewicht: 748662 gr.

\begin{tabular}{lr|lr} 
Bailey & 169 & London, B. M. & 424 \\
Bement & 79 & London, P. G. & 139 \\
Berlin, P. & 34 & de Mauroy & 4 \\
Berlin, U. & 128 & Moskau & 34 \\
Bologna & $7^{1}$ ) & NewHaven & 740000 \\
Bonn & 2114 & Paris, M. & 25 \\
v. Braun & 25 & Petersburg, A. & 145 \\
Breslau & 1 & Rom, U. & 96 \\
Budapest & 125 & v. Siemaschko & 46 \\
Calcutta & 556 & Stockholm & 686 \\
Cambridge & $32^{2}$ ) & Stürtz & 4 \\
Cleveland & 194 & Tübingen & 116 \\
Göttingen & 8 & Upsala & 44 \\
Gregory & 36 & Ward & 55 \\
Harvard, U. & 1759 & Washington \\
Klausenburg & 8 & Washington, Sh. 64 \\
Kristiania & 197 & Wien, H. M.*) & 1297
\end{tabular}

\section{Crow Creek Silver Crown}

Cuba, Insel Cuba, Westindien.

Eisen, Fundzeit unbekannt, beschr. 1872 .

1872: Don José Maria Solano y Eulate: Noticia sobre el hierro meteórico dela Isla de Cuba. Anales Soc. Esp. hist. nat. Bd. I, p. 183 (Analyse); s. auch folgende Arbeit.

r892: Gredilla: Meteoritos, p. 99Ior (Analyse).

Ursprüngliches Gewicht: Die Madrider Masse (?).
Nachweisbares Gewicht: I329 gr. Madrid $\quad 1327$

Ausserdem führt v. Siemaschko in seinem Katalog (I89r) 2 gr. auf.

Cuernavaca

s. Anhang

Cusignano Borgo San Donino

Cynthiana, Harrison County, Kentucky, U. S. A.

Stein, $\mathrm{Cg}$, gefallen 23. Januar 1877 . r877: S m it h: Note of the recent fall of three Meteoric Stones, in India, Missouri and Kentucky. Am. Journ. (3) 13, p. 243 ; s, auch C. R. Bd. 84 Nr. 9 , p. 397 ; N. J. 1877 , p. $735-736$; C. R. Bd. 85 , p. 680 .

1877: Smith: A description of the Rochester, Warrenton, and Cynthiana Meteoric Stone which fell respectively December $2 \mathrm{I}^{\text {st }} 1876$, January $3 \mathrm{~d}$ 1877 and January $23 \mathrm{~d} 1877$, with some remarks on the previous falls of Meteorites in the same regions. Am. Journ. (3) 14, p. 224-229 (Analyse). Ref. N. J. 1878 , p. $78-79$; Zeitschr. f. Kryst. Bd. 2 (1878), p. Ino; Original Researches 1884 , p. $534-537$; Liebig-Kopp, Jahresber. 1877, p. 1394 -r395.

1877: $\mathrm{K}$ irkw ood: On eight Meteoric Fireballs seen in the United States from July 1876 , to February 1877 . - VII The Meteor of January 23, 1877. Amer. Philos. Soc., March 16 , 1877 , p. 595 -596 ; s. auch Am. Journ. (3) 14 (1877), p. 75 .

1879: R a mme lsberg: Meteoriten, p. $6 \mathrm{r}$.

r880: K le in: Vermehrung, Gött. Gel. Anz. (1880), p. 567.

1884: M e unier: Météorites, p. 273, $277-278$.

1885: Brezina: Wiener Sammlung, p. $182,232$.

r887: F 1 ig h t: Meteorites, p. 200-20I.

1) "Red River 18I4". - 2) >Red River, Texas 
1890: E a st m a n: Met. Astron., p. 316. r894: C o he n : Meteoritenkunde, p. 283 .

Ursprüngliches Gewicht: $6 \mathrm{Kgr}$.

Nachweisbares Gewicht: 4772 gr.

Bailey

Bement

v. Braun

Budapest

Göttingen

Gregory

Harvard, U.

Io London, B. M. 155

2 Minneapolis 39

8 Paris, M. 88

14 v. Siemaschko 3

142 Stockholm 108

6 Washington,Sh. 2

4093 Wien, H. M. 102

\section{Czartorya}

Zaborzika

Dacca

Shytal

Dakota, Indian Territory, U. S. A. Eisen, H, beschrieben 1863 .

1863: Haidinger: Ueber ein bisher unbekanntes Meteoreisen. Pogg. Ann. Bd. I19, p. 642-643. Ref. N. J. 1864, p. $7 \mathrm{I}$.

r863: R o s e : Meteoriten,p.149-150,152. 1863: J a ckson: Meteoric Iron from Dakota-Territory. - Description and analysis. Am. Journ. (2) 36, p.259-26r; s. auch Oesterr. Zeitschr. f. Bergu. Hüttenw. Bd. 39 (189r), p. 344 ; Kenngott, Uebersicht $1862 / 65$, p. 265 ; Bull. soc. chim. Bd. 6, p. 139; Chem. Centr. 1864, p. 334; Journ. prakt. Chem. Bd. 92, p. 240; Liebig-Kopp, Jahresber. 1863 , p. $907-908$; ,L'Institut s Bd, 23 (1864) Nr. $161_{5}$, p. 400. 1864: B uchner: Erster Nachtrag,

Pogg. Ann. Bd. 122, p. 327.

r865: Buchner: Zweiter Nachtrag, Pogg. Ann. Bd. 124, p. 599.

r88r: Brezina: Bericht III, Sitzber.

Wien. Akad. Bd. 84 1, p. 28 o.

r884: M eunier: Météorites, p. $3^{8}$, $98, \mathbf{1 1 2}, \mathrm{II}_{3}$.

1885: Brezina: Wiener Sammlung, p. 218,234 .

r889: C o h e n : São Julião, N. J. 1889

I, p. 216,217 .

1890: E a st m a n : Met. Astron., p. 320.

r89r: Cohen und Weinschenk :
Meteoreisen-Studien, Ann. Hof-Mus. Bd. 6, p. 159 .

r893: M e u n i e r: Révision des fers météoriques, p. $15,18$.

r894: C o he n : Meteoritenkunde,p. 57,96.

1895: C o he n : Meteoreisen-Studien IV, Ann. Hof-Mus. Bd. 1o, p. 83 .

Ursprüngliches Gewicht: $4800 \mathrm{gr}$. (ro pounds, ro oz).

Nachweisbares Gewicht: 790 gr.

Berlin, U. $\quad 55$ London, P. G. 26 Calcutta $\quad 95$ New Haven 4 Göttingen $\quad 5^{8}$ Paris, M. $\quad \mathrm{IO}_{3}$ Gregory $\quad 27$ v. Siemaschko 40 Harvard, U. $\quad 8$ I Stockholm 25 \begin{tabular}{ll|l|l} 
v. Leuchtenberg 45 & Wien, H. M.*) 7
\end{tabular} London, B. M. 224

Wo ist die Hauptmasse des Eisens? Dr. Jackson in Boston erhielt 1863 das obige als ursprünglich angegebene Gewicht. Die ganze Masse wurde auf 100 Pfund geschätzt.

Dalton, Whitfield County, Georgia (ob mit Cleveland zu vereinigen? vergl. auch Literatur von Cleveland).

Eisen, Om, gefunden u.erwähnt 1877 . 1877: $\mathrm{S} \mathrm{m}$ i t $\mathrm{h}$ : Two new Meteoric Irons. Am. Journ. (3) 14, p. 246.

r880: Brezina: Bericht I, Sitzber. Wien. Akad. Bd. 82 I, p. 351 .

r88I: Hidden: On the Whitfield County, Georgia, Meteoric Iron (von ungefähr $\left.1_{3} \mathrm{lbs}\right)$. Am. Journ. (3) 2I, p. $286-287$ (Abbildung einer geätzten Schliffläche). Ref. N. J. 188r II, p. 343 ; Liebig-Kopp, Jahresber. 188r, p. $145^{8}$. 1883: S hepard: On Meteoric Iron from near Dalton, Whitfield County, Georgia. Am. Journ. (3) 26, p. 336338 (Analyse; Abbildung der 117 lbsMasse und eines geätzten Schliffs). Ref. N. J. 1884 II, p. 34 ; Liebig-Kopp, Jahresber. 1884, p. 2042.

r884: M e un i er: Météorites, p. $\mathbf{n 6}$. 
1885: Brezina: Wiener Sammlung, p. 2 II, 234 .

r887: $\mathrm{K}$ unz: On some American Meteorites. - 3. Is the East Tennessee Meteorite from Whitfield Co., Georgia? Am. Journ. (3) 34, p. 473-475 (Analyse und Situationsplan). Ref. N. J. I889 I, p. 61.

1887: Flight: Meteorites, p. 206. r887: Bre z in a: Neue Meteoriten IIia, Verh. k. k. geol. Reichsanstalt (1887), p. 288 .

r889: v. H a u e r : Ann, Hof-Mus, Bd. 4 (Not.), p. 64.

r890: E as t ma n: Met. Astron., p. 320. 1893: Me unier: Révision des fers météoriques, p. 52, 58-59.

r894: C o h e n : Meteoritenkunde, p. 193, $232,242$.

r895: C o h e n: Meteoreisen-Studien IV, Ann. Hof-Mus. Bd. 1o, p. 83,84 .

Ursprüngliches Gewicht : $57^{1 / 2} \mathrm{Kgr}$. Die Masse Shepards II 7 lbs., die Masse Hiddens, von der $9 \frac{3}{4}$ lbs. erhalten blieben, soll anfangs I 3 lbs. gewogen haben.

Nachweisbares Gewicht: 55288 gr.

\begin{tabular}{lr|lr} 
Bailey & 93 & de Mauroy & 9 \\
Bement & 89 & Paris, M. & 90 \\
Berlin, U. & 30 & v. Siemaschko & 30 \\
Bologna & 9 & Strassburg & 16 \\
v. Braun & 141 & Stürtz & 40 \\
Dresden, M. & 23 & Ward & 81 \\
Gregory & 125 & Washington & 36 \\
Greifswald & II & Washington, Sh. \\
Harvard, U. & 475 & & 50917 \\
Howell & 3 & Wien, H. M. & 2924 \\
London, B. M. & 146 & &
\end{tabular}

Dandapur, Distr.Goruckpur,Indien. Stein, Cia, gefallen 5. Sept. 1878 . 1884: M e unier: Météorites, p. 209. r885: Brezina: Wiener Sammiung, p. 182,232 .

Der Stein scheint noch nicht näher untersucht worden zu sein. $W$ ül $f$ ing, Meteoriten.

Ursprüngliches Gewicht : ? Calcutta besass r 880 : $6 \mathrm{lbs} .8$ oz. 420 grs. mit der Bemerkung: „One of two pieces that formed the entire stone .

Nachweisbares Gewicht: 6047 gr. Bement 260 London, B. M. 2245

Budapest

Calcutta

Gregory

Kristiania

15 Paris, M. 295 2973 v. Siemaschko 38 16 Wien, H. M. 184 2I

Daniels Kuil, Griqua, Südafrika.

Stein, Ck, gefallen 20. März 1868. 1868: Gregory: New Meteorite from South Africa. Geol. Magaz. Bd. 5, p. 53I -532 (Analyse von Church).

1869: Church: Analysis of a Meteorite from South Africa. Journ. Chem. Soc. (2) 7, p. 22-24; s, auch Journ. prakt,Chem.Bd.106(1869),p.379-380; ferner Luminous Meteors, Rep. Brit. Assoc. 1869, p. 276 ; Liebig-Kopp, Jahresber, 1869, p. 130I.

1879: Ram melsberg: Meteoriten, p. $12,26$.

1881: Brezin a: Bericht III, Sitzber. Wien. Akad. Bd. 84 I, p. 278 .

1884: Me unier: Météorites, p. 188, 191. r885: Brezina: Wiener Sammlung, p. 19I, 233.

r885: Shep ard: Am. Journ. (3) 30, p. Ió (Krystall von Nickeleisen).

1887: F lig ht: Meteorites, p. I54- 155. Ursprüngliches Gewicht : $1048 \mathrm{gr}$. (2 lbs. 5 oz.).

Nachweisbares Gewicht: 897 gr.

Bailey

Belgrad

Bement

Bologna

Budapest

Calcutta

Cleveland

Gregory

Harvard, U.

Klausenburg
2 Kristiania 59

3 London, B. M. 449

22 London, P. G. $\quad 78$

1 Lüttich

I8 Paris, M.

50 v. Siemaschko

Spl. Stockholm 20

114 Troyes I

22 Washington, Sh. 5

11 Wien, H. M.*) I9 
Danville, Alabama, U. S. A.

Stein, Cga, gefallen 27. Nov. 1868 .

1870: Smith: Account of a fall of Meteoric Stones near Danville, Ala. with an analysis of the same. Am. Journ. (2) 49, p. $90-93$; s. auch Original Researches 1884 , p. $458-46 \mathrm{r}$; Journ. Chem. Soc. Mai 187 I.

r870: R a mmels berg: Meteoriten, p. 103, 105, 106, 139.

1876: S m it h: Carbon Compounds, Am. Journ. (3) II, P. 39I.

r879: R a mmels berg : Meteoriten, p. 10, 25 .

r884: Meunier: Météorites, p. 85 , 197, 206.

r885: Brezina: Wiener Sammlung, p. $182,233$.

1887: F lig h t: Meteorites, p. 16o-16r. 1890: E a s tm a n: Met. Astron., p. 316. r894: C o h e n : Meteoritenkunde, p. 204.

Ursprüngliches Gewicht: $2038 \mathrm{gr}$. ( $4^{1} / 2$ lbs.), von denen nur etwa 500 gr. in Smith's Besitz gelangten.

Nachweisbares Gewicht: 230 gr.

\begin{tabular}{lr|lr} 
Bailey & I & London, B. M. & 27 \\
Budapest & 2 & London, P. G & 5 \\
Cleveland & I & New Haven & II \\
Göttingen & 7 & Paris, M. & I4 \\
Gregory & 3 & Ward & 5 \\
Harvard, U. & Io5 & Wien, H. M.*)**) \\
Kopenhagen & 8 &
\end{tabular}

Darmstadt, Grossherzogtum Hessen, Deutschland.

Stein, Cga, gefunden vor 1804 .

1804: Suck ow : 》Mit diesen äusserlichen Kennzeichen (Siena und Barbo$\tan$ betreffend) kommen auch die Stücke in der Mineraliensammlung der staatswirtschaftlichen Hohen Schule zu Heidelberg uberein, welche sich in dem Darmstädtischen gefunden haben, nachdem man verschiedene Mahle heftige Knalle in der Luft gehört hatte. « An- fangsgründe der Mineralogie. Leipzig 1804. Bd. 2, p. 649.

r823: Muncke: Gilb. Ann. Bd. 73, p. 382 ; kurze Anmerkung, dass in Heidelberg einStück aufbewahrt werde. r854: v. Boguslawski: Zehnter Nachtrag, Pogg. Ann. Ergz,-Bd. 4, p. 377 .

r859: B u c h ner: Feuermeteore, p. II5. r860: $\mathrm{B} \mathrm{u} \mathrm{chner} \mathrm{:} \mathrm{UeberFeuermeteore}$ und Meteoriten. Ber. der Oberhess. Ges. Bd. 8 , p. 84 .

r859/62 : B 1 u m : Meteorstein von Darmstadt. Verh, naturh. med. Ver. Heidelberg Bd. 2 Heft 4, p. 164 (Bd. 2 erschien I862); s. auch N. Jahrb. Pharmac. Bd. 16 Heft 5 (1861), p. 297; LiebigKopp, Jahresber. 1861, p. 1127-1128. 1863: B u c hner: Meteoriten, p. II3. 1863: R o s e: Meteoriten, p. 155.

1863: Bu chner: Meteoritische Notizen aus dem Vereinsgebiet, Ber. d. Oberhess. Ges. f. Natur- und Heilk. Giessen, p. 93.

r880: K l e i n : Vermehrung, Gött. Gel. Anz. (1880), p. 567.

r885: B rezin a: Wiener Sammlung, p. 182,233 .

UrsprünglichesGewicht: Etwa roogr. Nachweisbares Gewicht: 102 gr.

Berlin, U.

Budapest

Calcutta

Göttingen

Heidelberg

\begin{tabular}{r|ll} 
I & London, B. M. & 2 \\
9 & London, P. G. & I \\
3 & Neumann & I \\
16 & Wien, H. M.*) & 5 \\
64 &
\end{tabular}

Wenn ich mich recht erinnere, hat Chladni auf die obige Suckow'sche Notiz zuerst hingewiesen; ich vermag die Stelle bei Chladni nicht mehr zu finden.

Deal, Monmouth County, NewJersey, U. S. A.

Stein, Ci, gefallen r4. August 1829 . r829: G a y - L us s a c (oder A ra g o ?): Chute d'aérolithes. Ann. Chim. Phys, Bd. 42, p. 4I9 ; s. auch Revue bibliogr. 
Ann. des Sc nat. Bd. 18 (1829), p. I28; N. J. 1833 , p. $63^{2}$.

?: Vaux u. M'Euen: Transact. Acad. Nat. Sc. Philadelph. Bd, 16, p. 181. 1832 : v. H o f $f$ : Achter Nachtrag, Pogg. Ann. Bd. 24, p. 228 (Ref. der obigen Arbeit).

1836: Kämtz: Meteorologie, p. 297. 1843: Partsch: Meteoriten, p. I43. r85I: Shepard: On the Meteoric

Stone of Deal, New-Jersey, which fell August 15 1829. Proc. Amer. Assoc. (1851), p. $188-189$.

1854: v. B oguslawski: Zehnter Nachtrag, Pogg.Ann. Ergz.-Bd. 4, p.433. 1859: Harris: Dissert. Gött., p. 82. r863: B u chner: Meteoriten, p. 53 I863: Rose: Meteoriten, p. I55.

1885: Brezina: Wiener Sammlung, p. $181,232$.

1890: E a s t m a n: Met. Astron, p. 316.

Ursprüngliches Gewicht: ? Mehrere

Steine srather more than half an ouncek.

Nachweisbares Gewicht: 10 gr.

Berlin, U. Spl. Paris, M.

5

Calcutta Spl. Rom, U.

Spl.

Gregory

I Washington, $\mathrm{Sh} .4$

London, B. M. Spl. Wien, H. M.*) Spl. London, P. G. Spl.

\section{Debreczin}

Kaba

De Calb Co., gefd. 1840 , beschr. I 845

Smithville

De Calb Co., gefd. 1840 , beschr. I 846

Carthago

Decatur Co. Prairie Dog Creek De Cewsville, Ontario Canada. Stein, Cw, gefallen 21 . Januar 1887 . 1888: Huntington führt als letate Nummer 424 seines Verzeichnisses Talbot Road, De Cewsville, Ontario, gefallen am 21, Jan. 1887, auf. Proc. Amer. Acad. Arts and Sc. Bd. 23, p. 102. r89o: Howe 11: Description of New
Meteorites. - The De Cewsville Meteorite. Proc. Rochester Acad. of Sc. Bd, I, p. 9I-93 (Abbildung des Steins; spec. Gew. $3 \cdot 5^{2}$; weiter noch nicht untersucht). Ref. N. J. 1892 II, p. 34; s. auch Ward's Collection of Meteorites, p. $64-65$.

1890: Eas tma n: Met. Astron., p. 316. r893: B re z in a : Ueber neuere Meteoriten (Nürnberg), p. I60.

r895: Brezin a: Wiener Sammlung, p. 243.

Ursprüngliches Gewicht: $340 \mathrm{gr}$.

Nachweisbares Gewicht: Der ganze Stein von $340 \mathrm{gr}$. befindet sich im Wiener Hof-Museum.

Deep Springs, Deep Springs Farm, Rockingham County, Nord-Carolina, U. S. A.

Eisen, gefunden um $\mathrm{s} 846$, beschr. I 890 .

1890: Venable: Two new Meteoric Irons, I) From Rockingham County, N. C. Am. Journ. (3) 40, p. $16 \mathrm{I}-162$ (Analyse).

I894: Co he n : Meteoritenkunde, p. 23I. Ursprüngliches Gewicht: Ir.5 Kgr. Nachweisbares Gewicht: 2259 gr. Bailey I9I6 Harvard, U. III \begin{tabular}{ll|l} 
Bement & 193 & Ward
\end{tabular} Cleveland $\quad 32$

Wo befindet sich die Hauptmasse dieses Eisens? Venable sagt, dass Herr Lindsay dasselbe dem ,State Museum * geschenkt habe. Liegt dieses $\$$ State Museum $*$ in Raleigh?

Deesa (Daubrée) Copiapo Deesa Dehesa

Dehesa, La Dehesa, bei Santiago, Chile. Daubrée's „Chili localité non indiquées. (Früher bei Brezina : Chili, Eisen, gefunden I866). Eisen, Hch, gefunden 1866 , beschr. 1868. 
1868: D a ubrée: Sur trois nouveaux fers météoriques du Chili, récemment parvenus à la Collection de Géologie du Museum. - III. Fer d'une localité non indiquée du Chili. C. R. Bd, 66 , p. 572 -573 .

1869: B uchner: Vierter Nachtrag, Pogg. Ann. Bd. 136, p. 6ro-6rr.

r869/70: O $1 \mathrm{~d}$ h a m: Rec. Geol. Surv. India Bd. 3, p. IO4 (Empfang eines Stückes).

r870: v. Haid inger: Orientierung, Sitzber. Wien. Akad. Bd. 6r II, p. 5I4. r879: Do me y ko: Mineralojia. 3. Aufl, p. $132-134$.

I884: M e uni er: Météorites, p. II2, II5. 1885: Brezina: Wiener Sammlung, p. 254 .

r889: F l e t cher: Atacama Meteorites, Mineral. Magaz. Bd. 8, p. 256.

1893: Meunier: Révision des fers météoriques, p. $16,21$.

r895: Brezina: Wiener Sammlung, p. 294 .

UrsprünglichesGewicht: Etwa $7 \mathrm{Kgr}$. Nachweisbares Gewicht: 360 gr.

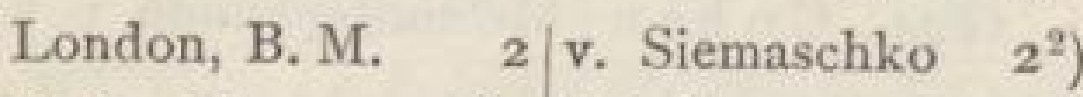
Paris, M. $\quad 282$ Washington, Sh. $5^{3}$ ) Schemnitz $67^{1}$ ) Wien, H M. 2 Wo befindet sich die grösste Masse dieses Eisens?

De Kalb Co., gefd. I 840 , beschr. I 845 Smithville

De Kalb Co., gefd. 1840 , beschr. I 846

Dellys, Algier, Nordafrika. Carthago

Eisen, Om, gefd. 1865 , beschr. 1866 . 1866: D a u bré e: Météorites tombées le 25 aout 1865 dans la tribu des Senhadja, cercle d'Aumale, province d'Alger; fer météorique signalé à Dellys. C. R. Bd. 62, p. 78 . r869: Buchner: Vierter Nachtrag, Pogg. Ann. Bd. 136, p. 602 .

r884: M e unier: Météorites, p. 133. r885: Brezina: Wiener Sammlung, p. 209, 233 .

1893: M e unier: Révision des fers météoriques, p. 50 .

r895: Brezin a: Wiener Sammlung, p. 268 .

Ursprüngliches Gewicht: „Un petit échantillon «.

Nachweisbares Gewicht: 91 gr.

Calcutta

Paris, M.

4 v. Siemaschko

76 Wien, H. M.

9

\section{Deniliquin}

Baratta

Denton Co., bei Austin, Texas, U. S. A.

Eisen, Om, bekannt 1856 ?, gelangte 1859 an einen Schmied in Mc. Kinney, beschr. 1860.

1860: Shumard: Notice of Meteoric Iron from Texas (Analyse von Riddell). Trans. St. Louis Acad. of Sc. Bd. I (185616o), p. $623-629$.

1860: $\mathrm{H}$ a idinger: Einige neuere Nachrichten über Meteoriten, namentlich die von Bokkeveld, New Concord, Trenzano, die Meteoreisen von $\mathrm{Ne}$ braska, vom Brazos, von Oregon. Sitzber. Wien. Akad. Bd. 41, p. 572 ; s. auch Liebig-Kopp, Jahresber. 1860, p. 851. r862: Madelung: Ueber das Vorkommen des gediegenen Arsens in der Natur nebst den Analysen einiger neuerer Meteoriten, - Das Meteoreisen von Denton County. Dissert. Gött. I862, p. $40-4 \mathrm{I}$.

1863: Buchner: Meteoriten, p. 192 -193 .

1863: R o s e: Meteoriten, p. $57,152$. 1884: M eunier: Météorites, p.116, 123. r885: Brezina: Wiener Sammlung, p. $213,214,234$.

I) $>$ Meteoreisen von Chili๔. - 2) sloc. inconnue, fer de Domeyko. - 3) "Chili 1866匹. Eisen. 
1890: Eastman: Met. Astron., p. 318. r893: M eunier: Révision des fers météoriques, p. 52,56 .

Ursprüngliches Gewicht: $575^{\circ} \mathrm{gr}$. (I2 Pfund I I Lot). Soviel waren noch 1860 im Staatsmuseum in Austin vorhanden; das Uebrige wurde von der anfänglich 40 Pfund schweren Masse in Mac Kinney, Collen Co., Texas verschmiedet. Nachweisbares Gewicht: 675 gr.

\begin{tabular}{lr|lr} 
Bailey & 65 & Kopenhagen & 8 \\
Bement & 2 & London, B. M. & 122 \\
Berlin, U. & II & London, P. G. & 3 \\
Budapest & 8 & New Haven & 66 \\
Calcutta & 3 & Paris, M. & 7 \\
Dorpat & 47 & Stockholm & 4 \\
Göttingen & 26 & Tübingen & 18 \\
Gregory & 3 & Ward & 13 \\
Harvard, U. & 53 & Washington, Sh. \\
Klausenburg & 5 & Wien, H. M.*) & 203 \\
Befindet sich die grösste Masse des Eisens \\
\multicolumn{1}{r}{ noch im Staatsmuseum in Austin? }
\end{tabular}

Denver Co.

Bear Creek

Deretschin s. Anhang Ruschany

Descubridora, Distr. Catorze, Staat San Luis Potosi, Mexico.

Eisen, Oml, bekannt $\mathbf{r}_{7} 80-\mathbf{r} 783$, erwähnt 1804 .

Hieher auch: Catorce, gefd. 1885, sowie die Eisen von Agua blanca, welch letztere verloren gegangẹ sind.

1804: D e $1 \mathrm{R}$ i o: Tablas Mineralogicas. Mexico 1804, p. 57.

1805: D e 1 R io: Elementos de Orictognosia, Segunda parte, p. 40.

1855: S mith: Memoir on Meteorites. - A Description of five new Meteoric Irons, with some theoretical considerations on the origin of Meteorites based on their Physical and Chemical characters. Am. Journ. (2) 19, p. 160 (Hacienda of Venagas).

1856: B u r k a r t: Fundorte I, N.J. 1856, p. $285-286,287$.

1857: Burkart: Briefliche Mitteilung,

N. J. 1857, p. $53-54$.

1858: Burkart: Fundorte II, N. J. 1858 , p. 770 .

1859: Harris: Dissert. Gött., p, I07. r869: S mith: The Cohahuila Iron of I868, Mexico. Am. Journ. (2) 47, p. $3^{8} 3$ (Venagas).

r870: Burkart: Fundorte IV, N. J. 1870, p. 691 .

1872: Zérega, Reyes, Epstein: Boletin de la Sociedad de Geografia y Estadistica de la Republica mexicana. Segunda Epoca. Bd. 4. Mexico 1872, p. 5,317 (319 ?).

1873: Comacho, Rio de 1 a Loza, Barzena, Manzano und Igles i a : sLa Naturaleza . Periodico cientifico de la Sociedad mexicana de Fistoria natural. Mexico 1873. Bd. 2, p. 277 ff. (Analyse von Murphy, p.29o); s. auch Am. Journ. (3) 7 (1874), p. 75, wo auf eine Arbeit Correjos aus dem I. Bd. der *Naturaleza \&, p. 252 verwiesen wird.

r874: B u rk a r t: Die Meteoreisenmasse von dem Berge Descubridora bei Poblazon unweit Catorze im Staate San Luis Potosi der Republik Mexico. N. J. 1874 , p. $22-28$ (Taf. III Abbildung der Masse und einer geätzten Platte; Analyse von Patricio Murphy). Ref. Liebig-Kopp, Jahresber. 1874 , p. 1349 ; Wochenschr. f. Astr. Met. u. Geogr., 1874 , p. 128 .

r876: B a r cen a : On certain Mexican Meteorites. Proc. Acad, nat. hist. Philadelphia 1876, p. 123-124.

1877 : Anales del Museo Nacional de Mexico 1877, Bd. I, p. II7 (Analyse mit $2.13 \%$ Zinn).

1881: Brez in a : Bericht II, Sitzber.

Wien, Akad. Bd. 83 I, p. 473 .

1884: Me un ier: Météorites, p. 116, 127. 1885: Brezina: Wiener Sammlung, p. 213,234 (Charcas). 
1887: B r e z in a: Neue Meteoriten IIIa. Verh. k. k. geol. Reichsanstalt (1887), p. 289 .

I887: $\mathrm{K}$ unz: On two new Meteorites from Carroll County, Kentucky, and Catorze, Mexico. Am. Journ. (3) 33, p. 233-235 (Analyse von Mackintosh; Abbildung der Masse [92 lbs.] und einer geätzten Platte). Ref. N. J. I888 I, p. 2 Io.

1887: Flight: Meteorites, p. 93 (Burkart's Aufsatz aus dem N. J.).

r888: v. H au er: Ann. Hof-Mus. Bd.3

(Not.), p. 42 ; s. auch Bd. 4 (1889) (Not.), p. 64 .

r889: C astillo: Catalogue, p. 4-5, IO, $13-15$.

r8go: F le t c her: Mexican Meteorites. Mineral. Magaz. Bd. 9, p. $66-70$, $7^{\mathrm{r}}$ $-72,99,104,156-160,174$.

r89o: Brez in a: Reise, Ann. Hof-Mus.

Bd. 4 (Not.), p. 117.

r8go: Eas t man: Met. Astron., p. 320. 1892: Eastman: The Mexican Meteorites. Bull. Philos. Soc. Washington Bd. 12 , p. 46.

r893: Meunier: Révision des fers météoriques, p. 46.

1894: Co h en: Meteoritenkunde,p. 58, 83. r895: Brezina: Wiener Sammlung, p. $269,273-274,275$.

Ursprüngliches Gewicht: Descubridora $576 \mathrm{Kgr}$., Catorze $4 \mathrm{I}^{1} / 2 \mathrm{Kgr}$. (92 lbs.)

Nachweisbares Gewicht: 42207 gr. Die grosse Masse von Descubridora befindet sich nach Castillo's Catalogue im National-Museum in Mexico.

\begin{tabular}{|c|c|c|}
\hline Bailey & 61 & Paris, M. \\
\hline Bement & 35 & Ward \\
\hline Gregory & $4 \mathrm{I}$ & Washington \\
\hline London, B. M. & 29 & Wien, H.M. \\
\hline New Haven & 195 & \\
\hline
\end{tabular}

\section{Dhenagur}

Kheragur

Dhulia, Bhagur, Khandeish, Ostind. Stein, Cwa, gefallen wahrscheinlich 27. Novbr. 1877 . Der Bericht Brezina's an die Akademie ist vom 14. Nov. 1878. Als Falljahr ist 1877 angegeben. In seinen $\mathrm{Ar}$ beiten von 1885 und 1895 ist irrtümlich das Jahr 1878 verzeichnet.

1878: B re $z$ in a überreicht einen vorläufigen Bericht über einen zu Dhulia, Hindostan, im November 1877 gefallenen Meteoriten. Anzeiger Wien, Akad. Bd. 15, p. 213-214 (Analyse, die in Bombay [von wem ?] ausgeführt wurde). 1885: Brezina: Wiener Sammlung, p. $180,232$.

1894: F let c h e r : Introduction, p. 14. Ursprüngliches Gewicht: ?

Nachweisbares Gewicht: $19 \mathrm{gr}$.

$$
\begin{array}{lr|l}
\text { Gregory } & 2 & \text { Wien, H. M. } \\
\text { London, B. M. } & \text { 10 }
\end{array}
$$

Dhurmsala, Distr. Kangra, Ostind. Stein, Ci, gefallen r4. Juli r86o. 1860 : Copy of a letter from the Deputy Commissioner, Dhurmsala, to R. H. Davies, Esquire, Secretary to Government Punjab, Nr. 927, dated the 3oth July 1860 (Sep. k. k.Hof-Mus.Wien), 4 Seiten. 1860: C o p e : Communications received - 4. From Mr. H. Cope, Umritsur, the following accounts of the aërolite which fell at Dhurmsala on Saturday the $14^{\text {th }}$ July last, accompanied by a specimen. Journ. Asiatic Soc. Bengal. (Proc.) Bd. 29 , p. $410-4 \mathrm{II}$.

r860: Sa unders: Communications received - 5. From Mr. R. F. Saunders B. C. S. Officiating Deputy Commissioner, Dhurmsala, Punjab, in reply to a letter of inquiry addressed to him by the Secretary, the following note

I) $>$ Descubridora, Mexique, gefunden 1871 4 - 2) Davon $>41530 \mathrm{gr}$. Catorze, gefunden $1885^{\circ}$. 
accompanying an account of the same meteorite. Journ. Asiatic Soc, Bengal. (Proc.) Bd. 29, p. $411-415,416-$ $418,421$.

1860: Communications received -6 .The following Extract from a letter from Dhurmsala on the same subject had been also received. Journ. Asiafic Soc. Bengal. (Proc.) Bd. 29, p. 415-416. r860: Haidinger: Die Meteorsteinfälle von Quenggouk bei Bassein in Pegu und Dhurmsala im Punjab. Sitzber. Wien. Akad. Bd. 42 , p. $305-306$; s, auch Journ. Asiatic Soc. Bengal. (Proc.) Bd. 29 , p. $416-418$; Kenngott, Uebersicht 1861, p. 169; Luminous Meteors, Rep. Brit. Assoc. 186r, p. 34 (Sep.) ; 'L'Institut « Bd. 29 (186r) Nr. 1426, p. $154-155$.

r86r: Ha id inger: Der Meteorit von Dhurmsala im k. k. Hof-Mineraliencabinet, ein Geschenk von dem kön. grossbritannischen Vicekönig und Generalgouverneur von Indien, Lord Viscount Canning. Sitzber. Wien. Akad. Bd.44 II, p. 285-288; s, auch Am. Journ. (2) 32 (186r), p. 142; Zeitschr. f. d. ges. Naturw. Bd. 19, p. 184; Liebig-Kopp, Jahresber, 186I, p. 1125 ; $>$ L'Institut Bd. 30 (1862), Nr. 1465 , p. 39 .

r861: J a c ks o n : Sur un aërolite tombé à Dhurmsalla dans l'Inde; extrait d'une Lettre de M. le Dr. Charles T. Jackson à M. Élie de Beaumont. C. R. Bd. 53, p. 1018-1019 (Analyse); s. auch Pogg. Ann. Bd. 1'5 (1862), p. 175; Kenngott, Uebersicht $1862 / 65$, p. 434 ; Proc. Boston Soc. Nat. Hist. Bd. 8, p. 233-235 (1861): Chemical Analysis of a Meteoric Stone from Dhurmsala, India ; >L'Institut * 1862, p. 39; LiebigKopp, Jahresber. 1861, p. 1125. 1862 : M a s k e 1 y n e : Chondritic Aërolites. Rep. Brit, Assoc. (1862, Notices and Abstracts), p. 190 .

r863: Bu chner: Meteoriten, p. 106. 1863: R o s e: Meteoriten, p. 98, 155.
1865: v. Reichen b a ch: XXV 427, $434,437,438,600,608$.

I866: Ha ughton: On the Chemical and Mineralogical Composition of the Dhurmsalla Meteoric Stone. Philos. Magaz. Bd. 32 (Sept. I866), p. 266269 ; s, auch Proc. Royal Soc. Dublin Nr. 85 (I866), p. 214-218; Journ. prakt. Chem. Bd. IOI, p. 499 ; LiebigKopp, Jahresber. 1866, p. Ioog. 1869: B u chner: Vierter Nachtrag,

Pogg. Ann. Bd. 136, p. $447-44^{8}$. 1870: $\mathrm{R}$ a m me $1 \mathrm{sberg}$ : Meteoriten, p. $98,103,107$.

r879: R a m mels berg : Meteoriten, p. 11,24 .

1882: Brezina: Bericht IV. Sitzber.

Wien. Akad, Bd. 85 I, p. 340.

r882: v. L a s a u $1 \mathrm{x}$ : Vermehrung, Verh. naturh. Ver. Bonn Bd. 39 (Sitzber.), p. $105-107,108$.

1883: T s c herm a k: Beitrag, Sitzber.

Wien. Akad. Bd. 88 I, p. 355.

1884: W a d s w or th: Studies, p. 88. I884: Me u n i er: Météorites, p. 79,98 , $197,203,461$.

r883/85: T s c he r m a k: Photographien, T. $8,9,14,16$, p. $12,13,14,15,16$, $17,18$.

r885: Brezina: Wiener Sammlung, p. $181,232$.

1886: Ansde11 u. Dewar: On the gaseous constituents of Meteorites. Proc. Royal Soc. Bd. 40 , p. $55^{2}$; s. auch Am. Journ. (3) 32 (r886), p. 482 ; N. J. 1887 II, p. 285 ; Liebig-Kopp, Jahresber. 1886, p. 2326.

1893: Brezina: Ueber neuere Meteoriten (Nürnberg), p. 159.

r894: Cohen: Meteoritenkunde, p. 6o,

$175,203,222,247,262,268,309$.

1894: F l et c her: Introduction, p. 10. r895: Brezina: Wiener Sammlung, p. 246 .

Ursprüngliches Gewicht: Grosse Massen, viele Steine, von denen 
Dhurmsala - Djati-Pengilon.

einer etwa $145 \mathrm{Kgr}$. wog; viel verschleppt.

Nachweisbares Gewicht: $30245 \mathrm{gr}$.

\begin{tabular}{|c|c|c|c|}
\hline Bailey & I35 & Moskau & 125 \\
\hline Bayet & I8 & München & 215 \\
\hline Bement & $5^{8} 5$ & Neumann & 146 \\
\hline Berlin, U. & 201 & New Haven & 28 \\
\hline Böhm & 9 & Newton & 94 \\
\hline Bologna & 270 & Odessa & 56 \\
\hline Bonn & 90 & Oxford & II 5 \\
\hline v. Braun & 124 & Paris, E. & $3^{8}$ \\
\hline Budapest & 1165 & Paris, M. & 231 \\
\hline Calcutta & 1317 & Pohl & 440 \\
\hline Cambridge & 444 & Prag, M. & 236 \\
\hline Cleveland & 16 & Rom, U. & 415 \\
\hline Detmold & I2 & v. Schilling & 85 \\
\hline Dorpat & 84 & Sidney & 238 \\
\hline Dresden, M. & 200 & v. Siemaschko & 67 \\
\hline Dublin, M. & 7 & Stockholm & 27 \\
\hline Edinburg & 596 & Strassburg & 36 \\
\hline Göttingen & $5^{I}$ & Stürtz & 78 \\
\hline Gregory & 1910 & Troyes & $35^{1}$ \\
\hline Greifswald & 44 & Tübingen & $\left.2^{1}\right)$ \\
\hline Harvard, U. & 546 & Turin, U. & $3^{818}$ \\
\hline Klausenburg & 79 & Utrecht & $8_{3}$ \\
\hline Kopenhagen & $24 \mathrm{I}$ & Ward & 123 \\
\hline London, B.M. I2 4 & 407 & Washington & 47 \\
\hline London, P. G. 2 & 204 & Washington, $\mathrm{Sh}$ & . 259 \\
\hline Madrid & 170 & Wien, H.M *) & $\left.1483^{2}\right)$ \\
\hline $\begin{array}{l}\text { de Mauroy } \\
\text { Melion }\end{array}$ & & Wien, U. I. & 447 \\
\hline
\end{tabular}

Diamantina Distr.

Dicson Co.

Dinagepur

Disco

Djati-Pengilon, Alastoewa, Distr.

Ngawi. Java.

Stein, Ck, gefallen 19. März 1884 .

r886: V erbeek u. Retgers: De

Meteoriet van Djati-Pengilon (Java). Met 2 af beeldingen in kleuren en $\mathbf{I}$ kaartje, benevens eene scheikundige analyse van den mijningenieur J. W. Retgers. Jaar- bock van het Mijnwezen Nederlandsch Oost-Indie Wetens. Ged. Bd. 15, p. 145 - I7 I. Amsterdam (Zwei ausgezeichnete Abbildungen des Steins, Karte von Java mit Eintragung der Fälle von I) Prambanan, 2) Tjabe, 3) Bandong, 4) Ngawi, 5) Djati-Pengilon). Ref. N. J. I888 II, p. $427-428$.

1887: Laspeyres legte der Gesellschaft ein 576 gr. schweres Stück eines Meteoriten vor, welcher am 19. März 1884 etwas nördlich vom Dorfe Djati-Pengilon innerhalb des Distrikts Gendingan, im Bezirk Ngawi in der Residenz Madioen auf der Insel Java gefallen ist. Verh. naturh. Ver. Bonn (Sitzber.) Bd. 44, p. 247-253.

1887: Brezina: Neuere Meteoriten III, Ann. Hof-Mus. Bd. 2, p. 114. Ref. N. J. 1888 II, p. 35 .

1887: D a u b r é e : Note sur la Météorite tombée le 19 mars 1884 , à Djati Pengilon (île de Java). C. R. Bd, I05, p. 203-205 (Analyse); s. auch Zeitschr. f. Krystallogr. Bd, 14 (1888), p. 604; N. J. 1888 II, p. 35 .

r887: $R$ ö $\mathrm{m}$ er legte ein 463 gr. schweres Stïck eines am 19. März 1884 bei Djati-Pengilon auf der Insel Java gefallenen Meteoriten vor. Jahresber, der Schles. Ges. f. vaterl. Cultur (1887), p. $234-236$.

I887: Ge in itz: Von Geinitz soll noch eine Notiz über Djati-Pengilon vom Jahre 1887 vorhanden sein. Wo befindet sich dieselbe?

1894: F l e t c h e r: Introduction, p. I4. r894: C o h e n : Meteoritenkunde, p. 203 , $205,246,248,266,279$.

Ursprüngliches Gewicht : $166.4 \mathrm{Kgr}$. Nachweisbares Gewicht : r6o994 gr. Bailey

Batavia

Bement

Berlin, U.
576

24 123 77

1) Einige Splitter, z. T. geadert. - 2) Ausserdem 235 gr. nach I. Juli 1893 erworben. 


\begin{tabular}{lr|lr|} 
Cleveland & Spl. & Odessa & 2 \\
Dresden, M, & 437 & Paris, M. & 486 \\
Gregory & I & Rom, U. & 37 \\
Harvard, U. & 279 & v. Siemaschko & IO \\
Leiden & 440 & Stockholm & 85 \\
Leipzig & 285 & Stürtz & 68 \\
London, B. M. & 469 & Utrecht & 139 \\
de Mauroy & 10 & Washington & 469 \\
New Haven & 250 & Wien, H. M. & 377
\end{tabular}

Doab 7. Aug. 1822

Kadonah

Doab 30. Nov. 1822 Futthepore

Dolgowoli, Kreis Luzk, Volhynien, Russland.

Stein, Cw, gefallen 26. Juni 1864 . 1864: Heis: Wochenschrift f. Astronomie 1864 , p. 328 .

1865: Buchner: Zweiter Nachtrag,

Pogg. Ann. Bd. 124, p. 591.

1867: Go e be l: Kritische Uebersicht, Mélanges phys. chim. Bd. 7, p. 338 . 1867: Buchner: Dritter Nachtrag, Pogg. Ann. Bd, 132, p. 318.

1869: Buchner: Vierter Nachtrag,

Pogg. Ann. Bd. 136, p. 455.

1884: M e uni er: Météorites, p. 209. 1885: Brezina: Wiener Sammlung,

p. $177,232$.

Ursprüngliches Gewicht: $1.6 \mathrm{Kgr}$. Nachweisbares Gewicht: 1209 gr.

\begin{tabular}{lr|lr|} 
Berlin, U. & IO & London, B. M. & I \\
Budapest & 2 & Paris, M. & IO2 \\
Calcutta & 12 & Petersburg, A. & 121 \\
Dorpat & 34 & v. Siemaschko & I \\
Göttingen & 33 & Wien, H. M.*) & 10I \\
Kiew & 792 & &
\end{tabular}

\section{Dolores Hidalgo}

Cosina

Doña Inez, Cerro de Doña Inez, Atacama, Chile, Südamerika.

Mesosiderit, M, gefunden I888, beschr. 1890 . Ist wahrscheinlich mit Inca zu vereinigen.

1890: Eas tm a n: Met. Astron., p. 320.
1890: Howe 11: Description of new Meteorites. The Doña Inez and the Llano del Inca, two new Meteorites from Atacama, Chili. Hieran anschliessend: Mineralogical Description of the Llano del Inca and the Doña Inez Meteorites vonWadsworth. Proc.Rochester Acad. of Sc. Bd. I, p. $93-98$ (Analyse von Davidson). Ref. N. J. 1892 II, p. $34-36$; noch einmal referiert: N. J. 1895 II, p. $30-3$ I.

I894 C o h e n : Meteoritenkunde, p. 24I, $259,278,294,305,307,308$.

r895: M eunier: Révision des lithosidérites, p. $3^{8-39}$ (Abb. einer geätzten Platte).

r895: Brezina: Wiener Sammlung, p. 263 .

Ursprüngliches Gewicht: $7^{1 / 4} \mathrm{Kgr}$, welche in den Besitz von Herrn Ward gelangten.

Nachweisbares Gewicht: 5686 gr.

\begin{tabular}{lr|lr} 
Bailey & 34 I & Krantz & 227 \\
Bement & I93 & London, B. M. & I0I6 \\
v. Braun & II & de Mauroy & I9 \\
Brezina & 95 & New Haven & 35 \\
Budapest & 89 & Newton & I2 \\
Cleveland & 23 & Pohl & 55 \\
Dorpat & I9 & Prag, M. & 155 \\
Gregory & 86 & Rom, U. & 60 \\
Harvard, U. & $\left.55^{\prime}\right)$ & Ward & 2317 \\
Howell & 360 & Washington & 59 \\
Kopenhagen & I35 & Wien, H.M. & 324
\end{tabular}

Dooralla

Durala

Doroninsk, Daurien, Gouv. Irkutsk, Sibirien, Russland.

Stein, Cgb, gefallen 6. April 1805 . 1808: Gilbert: Einige Nachrichten von den drei neuesten Steinregen, und von drei Meteorsteinen aus Russland. - 4. Aus den Berichten der Gelehrten, welche die neueste russische Gesandtschaft nach China begleiten soll- 
ten, an die Petersburger Akademie der Wissenschaften. Gilb. Ann. Bd. 29, p. $212-213$.

1809: Stoik owitz: Nachrichten von mehreren russischen Luftsteinen, besonders von denen, die am I. Oktober 1787 im Gouvernement von Charkow herabgefallen sind. -2 . Der Luftstein von Doroninsk im Irkutskischen Gouvernement. Gilb. Ann. Bd. 3I, p. 308 -3II (Abdruck aus der Petersburger Zeitung von 1806 No. 92, p. 1044).

I8I2: Ch1 a dni: Verzeichnis, Schweigg. Journ. Bd. 4 Beilage I, p. I5.

1812: Bigot de Morogues: Mémoire, p. 20I.

1813/r4: S c heerer : Chemische Analyse des Doroninskischen Aërolithen. Mémoires Acad, Petersburg Bd. 6 unter: Histoire de l'Acad, V. Observations, expériences et notices intéressantes, faites et communiquées à l'Academie, p. 46.

r8r5: Chladni: Neues Verzeichnis, Gilb. Ann. Bd. 50, p. 253.

r8rg: Chla d ni: Feuermeteore, p. 44, $46,53,66,69,71,73,276-277$ (1), 288,432 .

1836: K ä m t z: Meteorologie, p.258,280. I843: P a rt s c h : Meteoriten, p. 74-75. r847: Eichwa 1d: Verzeichnis, Erman's Arch. Bd, 5, p. 177.

1848: B lo e d e: Tabelle, Bull. Petersburg. Acad. Bd. 6, p. Io.

1854: B a $1 \mathrm{ce} 11 \mathrm{~s}$ : Lithologia meteorica, p. 22.

r859: H a r r i s: Dissert.Gött.,p.65-66. r863: Buchner: Meteoriten, p. 18-19. 1863: Ros e: Meteoriten, p. 154. r $858 / 65$ : v. Reichenbach: V 476 $477,479,480$. VI 453 . IX I6I, I7I,
180. X 359, 361. XI 294. XIII 362 . XXV 423, 427, 428, 433, 600, 607. 1867: Goebe 1: Kritische Uebersicht,

Mélanges phys. chim. Bd.7, p.28I-283. 1867: B u chner: D ritter Nachtrag, Pogg.

Ann. Bd. 132, p. 314.

r884: M e uni e r: Météorites,p.247,248. r885: Brezina: Wiener Sammlung, p. $182,233$.

r895: Brezina: Wiener Sammlung, p. 250 .

Ursprüngliches Gewicht: $9^{1 / 2}$ Pfund. Zwei Steine von 7 und $2^{1 / 2}$ Pfund nach der russischen Angabe.

Nachweisbares Gewicht: $57 \mathrm{I}$ gr.

\begin{tabular}{lr|lr} 
Berlin, U. & 76 & Paris, M. & I \\
Calcutta & II & v. Siemaschko & 4 \\
Dorpat & 6 & Tübingen & 215 \\
London, B. M. & 9 & W ashington & 8 \\
London, P. G. & 4 & Wien, H. M.*) & 6 I \\
Moskau & 176 & &
\end{tabular}

Der Stein wartet noch immer auf eine genauere Untersuchung. - Der Kata$\log$ der Petersburger Akademie führt unter dem Namen >Doroninsk \& $\mathbf{1 4 9 5}$ gr. auf, mit der Anmerkung: sIst in Wien 1888 fraglich geworden, Ad, Goebel . Das Stück ist vorläufig (nach Brezina) zu Simbirsk Partsch gestellt.

Drake Creek, bei Nashville, Davidson Co., Tennessee, U. S. A. Stein, (wa $^{1}$ ), gefallen 9. Mai 1827 . Hierher auch: „Sommer County, gefallen 22. Mai $1827 \%$.

1830: Silliman: Tennessee Meteorite und $\mathrm{Sey} b$ ert: Analysis of the Meteorite, which fell near Drake's Creek, eighteen miles from Nashville, Tennessee, in the year 1827 . Am. Journ.

I) v. Reichenbach sagt von dem Stück seiner Sammlung, dass es Adernbildung zeige. Pogg. Ann. Bd, I25 (1865), p. 319, 324, 428. Das in Tübingen als Nashville bezeichnete grosse Stïck lässt keine Adernbildung erkennen. Es ist nicht unwahrscheinlich, dass hier eine Verwechslung vorliegt. Ausser den oben verzeichneten 715 gr. besitzt Tübingen noch $4 \mathrm{gr}$. eines als "Nashville? * bezeichneten Chondriten, welcher Aehnlichkeit mit den oxydierten Stellen des grossen Stückes zeigt und Adernbildung aufweist. 
(I) 17 , p. $326-328$; s. auch Ann. Chim. Phys. Bd, 45 (1830 oder 1831 ), p. 416.

1830: Silliman: Tennessee Meteorite. Am. Journ. (I) I8, p. 200.

r830: Silliman: Notice of the circumstances attending the fall of the Tennessee Meteorites Mai 9, 1827 . Am. Journ. (1) 18, p. $378-379$.

r832: v. H off: Achter Nachtrag,Pogg.

Ann. Bd. 24 , p. $226-227$.

1836: K ämtz: Meteorologie, p. 296. 1843: P a r t s c h: Meteoriten, p.47-48. r845: v. Ba $\mathrm{m}$ ha uer: Ursprung, Chemische Untersuchung des am 22. Mai 1827 in Sommer County gefallenen Meteorsteins. Pogg. Ann. Bd.66, p. 465 , 498-503; s. auch Dissert. Trajecti ad Rhenum (1844), p. 45-53 u. Scheik. Onderz. Bd. I, p. 217; s. ferner Rammelsberg, Handwörterbuch Suppl. 2 (1845), p. 96-97 u. Suppl. 5 (1853), p. 27 ; Berzelius, Jahresber. Bd. 26 (1847), p. $385-387$; N.J.1849, p. 860. r848: Troost: Am. Journ: (2) 5, p. $35^{\mathrm{I}}$ (Beiläufige Erwähnung).

I854: v. Boguslawski: Zehnter Nachtrag, Pogg. Ann. Ergz,-Bd. 4, p. $26-29$.

1859: B u c h n e r: Feuermeteore, p. 90. 1859: Harri s: Dissert. Gött., p. 80.

1860: $\mathrm{R}$ a m mels berg: Mineralchemie, p. $922,924,950$.

1863: Buchner: Meteoriten, p. 50-5I. r858/65: v. Reichenbach: V 475. IX $16 \mathrm{I}, 167,168,169,178, X_{359}$, $36 \mathrm{I}, 363$. XI 294, 300. XIII 365,369 (Abb. von zwei Schliffen), 377. XX 623. XXV 319, 324, 428, 607 .

1870: Rammelsberg: Meteoriten, p. $103,107, \mathrm{I}_{3} 8$.

1879: Rammelsberg: Meteoriten, p. 24 .

1884: Wa ds worth: Studies, p. 104. r884: M e uni er: Météorites, p. 79, 95,
208, 216, 395 .

1885: S he pard: Am. Journ. (3) 30, p. 106 (Krystall von Nickeleisen). r885: Brezina : Wiener Sammlung,

p. $177,232$.

1890: Eastm a n: Met. Astron., p. 316. 1894: Co h en: Meteoritenkunde, p. 56. r895: Brezina: Wiener Sammlung. p. 242,244 .

UrsprünglichesGewicht: FünfSteine, der grösste II Pfund (amerik.). Nachweisbares Gewicht: 550r gr.

\begin{tabular}{lr|lr} 
Bailey & 21 & London, P. G. & 16 \\
v. Braun & 36 & Minneapolis & 15 \\
Budapest & 135 & Neumann & Spl. \\
Calcutta & 5 & New Haven & 422 \\
Cambridge & 2 & Newton & 3 \\
Dorpat & 4 & Paris, M. & 141 \\
Göttingen & 5 & v. Siemaschko & 2 \\
Gregory & 50 & Tübingen & $\left.715^{1}\right)$ \\
Harvard, U. & 1481 & Ward & 28 \\
Howell & 3 & Washington & 28 \\
Kopenhagen & 76 & Washington, Sh ${ }^{*}$ \\
Leiden & 2222 & Wien, H. M. *) \\
London, B. M. & 19 & \\
\end{tabular}

Duel Hill

Jewell Hill

Dundrum, Tipperary, Irland.

Stein, Ck, gefallen I 2. August I 865 . r866: Haughton: On the Meteoric Stone that fell at Dundrum in the Co. Tipperary, on the $\mathbf{2}^{\text {th }}$ of August, I865. Philos. Magaz. Bd. 32, p. 260-266; s. auch Proc. Royal Soc. Dublin 1866 (Analyse u. Abbildung des Steins); Journ. prakt, Chem. Bd. IOI, p. $498-$ 499; Liebig-Kopp, Jahresber. 1866, p. 1009 -IOIO.

r869: Buchner: Vierter Nachtrag, Pogg. Ann. Bd. 136, p. $455-456$. r870: R a mmelsberg: Meteoriten, p. $103,107,138,140,145$.

1879: R a m mels berg Meteoriten, p. $24,43,53,57$.

1) s. Anm, auf voriger Seite. 
188I : v. Nordenskiöld: Zeitschr. d. d. Geol. Ges. Bd. 33, p. 24 (Vergleich mit Ställdalen).

I884 : Me unier: Météorites, p. 79. r883/85: Ts c h e rm a k: Photographien,

T. 16,17, p. $14,16,17$.

1885: Brezina: Wiener Sammlung, p. 19I, 233.

1894: F let cher: Introduction, p. I4. Ursprüngliches Gewicht: $2225 \mathrm{gr}$. Nachweisbares Gewicht: 340 gr.

Calcutta

$$
8 \text { Newton }
$$

London, B. M. 245 Wien, H. M.*) Ist die Hauptmasse noch in Dublin?

Durala, N. W. Kurnal, Lahore, Delhi, Punjab, Ostindien.

Stein, Cia, gefallen 18. Febr. $18 \times 5$. 1820: Auszug eines Briefes von Capt. G. Bird an Major Pennington. Tilloch's Philos. Magaz. Bd. 56, p. 156-157. r82 I: C h 1 a d n i : Neue Beiträge, Gilb. Ann. Bd. 68 , p. $333-335$. I836: Kämtz: Meteorologie, p. 285. 1843: P a rts c h: Meteoriten, p. 142. 1850: In Powe 11's Luminous Meteors Mitteilung von Buist, Meteoric Stone presented to the East India Company's Museum. - Extract from a letter from Capt. G. Bird, Rep. Brit. Assoc. (1850), p. II $9-$ I2O.

I854: v. B o g uslaw ski: ZehnterNachtrag, Pogg. Ann. Ergz.-Bd. 4, p. 16. 1859: Harris: Dissert. Gött., p. 73. 1863: B u chner: Meteoriten, p. 36. 1863: Ros e: Meteoriten, p. 155. 1863: Maskelyne u. v. Lang : Mineralogical Notes. - Notices of Aërolites von Maskelyne. 12. Durala. Plate IX. Philos. Magaz. (4) 25, p. 440 -442 (Zwei Abbildungen des Steins); s, auch Rep. Brit. Assoc. (1862, Notices and Abstracts), p. I90; Kenngott, Uebersicht $1862 / 65$, p. 442 .

r865: B uchner: Zweiter Nachtrag, Pogg. Ann, Bd. 124, p. $577-578$.
1884: Meunier: Météorites, p. 208, 2II. r885: Brezina: Wiener Sammlung, p. $182,232$.

1890: v. Niess 1: Periheldistanzen, Verh. naturf. Ver. Brünn Bd, 29, p. 193. I894: F l e t c h er: Introduction, p. Io. Ursprüngliches Gewicht: „Rather more than 25 lbs. « Doch muss dies nach dem Londoner Stein wohl 29 lbs. heissen.

Nachweisbares Gewicht: I $2763_{3}$ gr. Bailey

Berlin, U.

Calcutta

Göttingen

2 London,B.M. 12589 30 London, P. G. 15 2I New Haven 3

Harvard, U. $\quad 42$ Wien, H. M.*) 42 Der Stein ist noch nicht chemisch untersucht worden.

Durango 1804 (I8II)

Humboldt - Eisen

\section{Durango (Karawinsky)}

Rancho de la Pila

Duruma, Mombas, Wanikaland, Ostafrika.

Stein, Cia, gefallen 6. März 1853 . 1862: Greg: On some Meteorites in the British Museum. Philos. Magaz. Bd. 24, p. 538. Ref. Kenngott, Uebersicht $1862 / 65$, p. 442.

1862: Buchner: Die Meteoriten in Wien u. London. Pogg. Ann. Bd. 116, p. 643 .

1863: B u chner: Meteoriten, p. $85-87$

(Enthält Originalbrief über die Herkunft des Münchener Stïcks [ $577 \mathrm{gr}$.] von Dr. Barth von Calw.)

r865: v. Reichen bach: XXV 433. 1884: Meunier: Météorites, p. 72. r885: Brezina: Wiener Sammlung, p. $182,232$.

r89o: Brezin a : Reise, Ann. Hof-Mus, Bd. 4 (Not.), p. 122.

r895: Brezina: Wiener Sammlung, p. $247,249,260$. 
Ursprüngliches Gewicht : 577 gr. Nachweisbares Gewicht: 575 gr.

\begin{tabular}{lr|lr|} 
Bailey & 3 & Krantz & I \\
Bement & I & London, B.M. & I \\
Budapest & 60 & München & 507 \\
Calcutta & Spl. & Wien, H. M.*) & I \\
Harvard, U. & I & \\
Dieser Stein & von & Duruma bedarf einer \\
\multicolumn{2}{|l}{ genaueren Untersuchung. }
\end{tabular}

Dyalpur,Sultanpur,Oude,Ostindien. Stein, $\mathrm{Cu}$, gefallen 8 . Mai 1872 .

1882: Brezina: Bericht IV, Sitzber. Wien. Akad. Bd. 85 I, p. 338,339 (Vergleich mit Mócs).

r885: Brezina: Wiener Sammlung, p. $184,233$.

1894: F l e t c h e r: Introduction, p. 27. 1895: Brezin a : Wiener Sammlung, p. $239,252,254$.

Ursprüngliches Gewicht: ?

Nachweisbares Gewicht: 304 gr. \begin{tabular}{ll|l|l|} 
Calcutta & I4 & v. Siemaschko I
\end{tabular} London, B. M. 270 Wien, H. M. 14 London, P. G. 5

Dyalpur scheint noch nicht näher untersucht worden zu sein.

Eagle Station, Carroll County, Kentucky, U. S. A.

Pallasit, P, gefunden 1880 , beschrieben $\tau 887$.

r887: Kunz: On two new Meteorites from Carroll County, Kentucky, and Catorze, Mexico. Am. Journ. (3) 33 , p. 228-232 (Abbildungen und Analyse von Mackintosh). Ref. N. J. 1888 I, p. 209-210.

1887: B r ez in a: Neue Meteoriten IIIa, Verh. k. k. geol. Reichsanstalt (1887), p. 289 .

1888: v. H a u e r: Ann. Hof-Mus. Bd. 3

(Not.), p. 42 ; s. auch Bd. 4 (1889)

(Not.), p. 64.

1889: M eunier: Sur la météorite
d'Eagle Station, nouveau spécimen de brahinite. C. R. Bd, 108, p. $762-763$;

Ref. N. J. 1890 I, p. $4 \mathrm{I}$.

1890: Kunz: On five new American Meteorites. I. On the group of $\mathrm{Me}$ teorites recently discovered in Brenham Township, Kiowa County, Kansas. Am. Journ. (3) 40, p. 317 (Little Miamy valley sei mit Brenham, nicht mit Eagle Station zu vereinigen); Ref. N. J. 1891 I, p. $3^{86 .}$

1890: E a s t m a n: Met. Astron., p. 320. 1893: B re z in a : Ueber neuere Meteoriten (Nürnberg), p. 163 .

1894: C o h en: Meteoritenkunde, p. 252, $254,256,257,263,33$ I.

r895: M eunier: Révision des lithosidérites, p. 15, 19.

1895: Brezina : Wiener Sammlung, p. 265 .

Ursprüngliches Gewicht: $36.5 \mathrm{Kgr}$. Nachweisbares Gewicht: 26 I 20 gr.

\begin{tabular}{lr|lr} 
Bailey & 78 & Paris, E. & 24 \\
Belgrad & 74 & Paris, M. & 2353 \\
Bement & 177 & Prag, M. & 133 \\
Berlin, U. & 148 & Rom, U. & $45^{8}$ \\
Bologna & 52 & v. Siemaschko & 60 \\
v. Braun & 362 & Stockholm & 104 \\
Brezina & 88 & Strassburg & 85 \\
Budapest & 840 & Stuttgart & 130 \\
Dijon & 93 & Turin, U. & 219 \\
Dresden, M. & 86 & Utrecht & 75 \\
Graz, J. & 87 & Ward & 96 \\
Gregory & 189 & Washington & 36 \\
Greifswald & 72 & Wien, H M. 18 687') \\
Kunz & 106 & Wien, U. I. & 130 \\
London, B. M. & 708 & Wien, U. II. & 63 \\
München & 94 & Wrany & 69 \\
New Haven & 70 & Würzburg & 74
\end{tabular}

East Tennessee 1837 Cosby'sCreek East Tennessee 1853 Tazewell East Tennessee 1860 Cleveland East Tennessee $\mathrm{r} 877$ Dalton East Tennessee 1887 Morristown 1) Ausserdem noch 153 gr. nach 1. Juli 1893 erworben. 
East Tennesseer 89 r Jonesborough Eau Claire

Echo

Eggenfelde Salt Lake City Mässing

Ehrenbergs Tennessee Cleveland Eichstädt, Wittmess, Mittel-Franken, Bayern, Deutschland.

Stein, Cc, gefallen 19. Febr. 1785 .

r790: Stütz: Bergbaukunde Bd. 2, p. 398,399 ; s. auch v. Moll's Ann. Berg- u. Hüttenk. Bd. 2 (I803), p. 312 -313 Anm.

I794: $\mathrm{C} \mathrm{h} \mathrm{la} \mathrm{d} \mathrm{n} \mathrm{i:} \mathrm{Pallaseisen,p.29-30.}$ I796: K in g: Remarks, p. 23-24, 25 1803: Ch 1 a d n i: Chronologisches Verzeichnis, Gilb. Ann. Bd. 15, p. 310.

1803: de Drée: Recherches, Journ. Phys. Bd. 56, p. 4I4, 416.

1803: K laprot h: Ueber meteorische Stein- und Metall-Massen. Abh. Berlin. Akad. (r803; gedruckt I806), p. 2527 (Analyse); Auszug in Gilb. Ann. Bd. 13 (1803), p. $338-339$; s. auch Klapproth's Beiträge Bd, 5 (I8Io), p. $256-257$ u. Bd. 6 (I815), p. 296 -300 ; Gilb. Ann. Bd. 24(1806), p.379.

r804: v. Ende: Massen u. Steine, p. $60-6 r$.

1804: Pötzsch: Kurze Darstellung, p. $52-54$.

r805: P i c k e l: Authentische Nachricht von einem unweit Eichstädt vom Himmel gefallenen Meteorsteine. v. Moll's Ann. Berg- u. Hüttenk. Bd. 3, p. 251 -259 .

r8r2: Bigot de Morogues, p. II7 - II9, 335 .

r812: $\mathrm{C}$ h 1 a d n i : Verzeichnis,Schweigg. Journ. Bd. 4 Beil. I, p. 14.

r8r5: Chladni: Neues Verzeichnis, Gilb. Ann. Bd. 50, p. 250.

r8rg: $\mathrm{Ch} 1$ ad ni: Feuermeteore, p. 46, $57,65,69,73,248,254,257$ (1), 260, 28I, 428 .

r820: v. S c hreiber s: Beiträge, p.r 3
-I5 (Abbildung eines Stücks; Tafel 2). 1823: Chladni: Dritter Nachtrag, Gilb. Ann. Bd. 75, p. 230.

I836: K ä m t z : Meteorologie,p.258,276. r839: B e nz e n b e r g : Sternschnuppen, p. 40.

r843: $\mathrm{P}$ a rt s c h: Meteoriten, p. 78 .

r847: S chafhä ut 1: Schönenberg,

Gel. Anz. München. Akad. (1847), p. $559-560$.

1857: A r a go: Astronomie populaire, Bd, 4, p. 194 .

1859: H a rris : Dissert. Gött., p. 6r, r862: K enng o t t u. Wis er: Zürcher Sammlung, p. 148-152 (Fallbericht). r863: B u chner: Meteoriten, p. 9-ro. I863: Rose: Meteoriten, p. 154.

r858/65: v. Reichen ba ch: V 477.

VI 441. IX 155, 161, 169, 179. X 359.

XI 294. XIV 389. XXIV 226.

r868: F r is c h m a n n : N.J. 1868 (Unter

Briefwechsel), p. $467-468$.

1869: Buchner: Vierter Nachtrag, Pogg. Ann. Bd. 136, p. 44I.

r878: $\mathrm{G}$ ï m be 1: Die in Bayern gefundenen Steinmeteoriten. - Der Meteorstein von Eichstädt. Sitzber. München.

Akad. ( 1878 ), p. $25-32$ (Sep.) (Analyse von Schwager).

1879: R a m melsberg: Meteoriten,p.II, 24,25 .

r884: Meunier: Météorites, p. 79, 208, 2IO, 460 .

r884: W a ds w or th: Studies, p. IO3. r885: Brezina; Wiener Sammlung, p. $185,233$.

189o: v. $\mathrm{N}$ i e s s 1: Periheldistanzen, Verh. naturf. Ver Brünn Bd. 29, p. 193.

r894: Cohen: Meteoritenkunde, p. 8.

Ursprüngliches Gewicht: $3185 \mathrm{gr}$.

Ein Stein von 5 Pfund 22 Lot.

Nachweisbares Gewicht: 1168 gr.

Berlin, U.

Budapest

Calcutta

Cambridge

15 Göttingen

25

84 Gregory 33

Spl. London, B. M. 14

4 München 521 
Neumann

Paris, M.

Paris, U.

v. Siemaschko

Stockholm

Eifel

Eisenberg

Ekaterinoslaw $18 \mathrm{r} 4$

7 Strassburg

Io Tübingen

7

16 Washington, Sh. 1

2 Wien, H. M.*) I28 Spl. Zïrich

293

Bitburg

Ekaterinoslaw 1826 Mordvinowka

Ekaterinoslaw 1876

Werchne Dnieprowsk

Ekaterinoslaw 1890 Augustinowka

Elbogen, der verwünschte Burggraf, Böhmen, Oesterreich.

Eisen, Om. Seit Jahrhunderten auf dem Rathaus in Elbogen aufbewahrt; erwähnt 1785 ; als meteorisch erkannt und beschrieben 1812. Als Fall- oder Fundzeit wird wohl 1400 angegeben, weil um diese Zeit kaiserliche Burggrafen in Elbogen sassen.

r785: S challer: Topographie des Königreichs Böhmen, Zweyter Theil. Ellbogner Kreis. Prag 1785, p. 6 (die Stelle ist bei Neumann, Gilb. Ann. Bd. 42 , p. 198 abgedruckt).

1812: N eumann: Der verwünschte Burggraf in Ellbogen in Böhmen, ein Meteorolith (Analyse von dem Verfasser und von Klaproth). Mit Bemerkungen von Chladni, Gilb. Ann. Bd. 42 , p. $197-209$; s. auch sHesperus « von 1812 , St. 55 , wo der Aufsatz zuerst abgedruckt wurde; ferner Schweigg. Journ. Bd. 7 (1813), p. 172 -173 , Bd. 19 (1817), p. 479, Bd. 20 (1817), p. 9I-92.

1812: K la proth: Chemische Untersuchung zweier Gediegen-Eisen-Massen. Schweigg. Journ. Bd. 5 (1812), p.I-4; s. auch Klaproth's Beiträge Bd. 6 (18r5), p. $306-308$.

1813: v.S c hreibersu.N e umann :
Briefe an den Herausgeber, Gilb. Ann. Bd. 44 , p. $103-105$ (150 Pfund gelangten nach Wien).

1815: Gillet-Laumont: Sur un Aérolithe tombé en Moravie, et sur une masse de fer nativ tombée en Bohême. Journ. Mines Bd, 38 , p. 232 -237 (Abbildungen mehrerer geätzter Platten).

I8r5: Chla d ni: Bemerkungen, Gilb.

Ann. Bd. 50, p. 265-266.

1816?: Noehden: Some account of the meteoric Stones, in the Imperial Museum at Vienna, Engl. Journ. Soc. Arts Bd. ?, p. 315 .

1817: Stromeyer: Auffindung von Kobalt in den Meteoreisen, Gilb. Ann. Bd. 56, p. 194 (findet in Elb. kein Co.). - Auszug aus den Gött. Gel. Anz. vom 23. Dez. I8r6.

r8rg: Ch lad n i : Feuermeteore, p. 316, $319,327-329$ (1), 34I, 433.

r8rg: Chladni: Fünfte Fortsetzung, Gilb. Ann. Bd. 63 , p. $28-29$.

I820: v. S c hre i bers: Beiträge, p.72 -76 (Abbildung einer geätzten Platte; T. 9); s, auch Brezina, Meteoritenstudien II. Denkschr. Wien. Akad. Bd. 44 (1881), p. 133, Anm.

r82 I: C h ladni : Erster Nachtrag, Gilb. Ann. Bd. 68, p. 346.

r82I: J o h n: Sur la Nature de grandes masses de fer métallique d'origine problématique, et sur celle du fer des aérolithes attirables par l'aimant. Ann. Chim. Phys. Bd. 18, p. 20I; s. auch Schweigg. Journ. Bd, 32, p. 258-26r. 1826: Se ebeck: Ueber die magnetische Polarisation der Metalle und Erze durch Temperatur-Differenz. Pogg. Ann. Bd. 6, p. 144.

1829: v. Holger: Neue Analyse des verwunschenen Burggrafen von Ellenbogen. Baumgartner's Zeitschr. f. Phys. u. Math. Bd. 5, p. $1-7$; s. auch N. J. I 833 , p. $192-193$.

r83o: v. Holger: Neue Analyse der 
beiden Meteoreisenmassen von Lénarto u. Agram nebst einigen Bemerkungen über den Ursprung der Meteormassen überhaupt. Baumgartner's Zeitschr. f. Phys. u. Math. Bd. 7, p. 130-134. 1834: B erzeli us: Om Meteorstenar. - 6. Meteorjern fri̊n Ellenbogen. Kongl. Vetenskaps. Acad. Handl.(I834), p. $169-172$ (Analyse); übersetzt in Pogg. Ann. Bd. 33 (1834), p. 135-137. 1834: Der verwünschte Burggraf von Elbogen. Ein Andenken an Elbogen für die T. T. Herren Carlsbader Brunnen-Gäste. Carlsbad 1834; gedruckt bei Gebr. Franieck ( 32 Seiten).

1835: Wehrle: Analyse einiger Meteoreisenmassen. Baumgartner's Zeitschr. f. Phys. u. verwandt. Wiss. Bd. 3, p. $225,226-227$; s. auch Ann Pharm. Bd. I4 (1835), p. 94.

r836: K ämtz: Meteorologie, p. 260, $261,262,263$.

r84I : $\mathrm{R}$ a m m e ls b e r g : Handwörterbuch, p. 422, 427.

r843: P a r ts c h: Meteoriten,p.Ioo-IO3. r847: S chafhäut 1: Schönenberg, Gel. Anz. München. Akad.1847, p. 578. 1852: Cla r k: Dissert. Gött., p. $44-46$ (Abbildung einer geätzten Platte).

r854 : v. B og u sl a w s k i : Zehnter Nachtrag, Pogg. Ann. Ergz.-Bd. 4, p. 385 . r855: $\mathrm{H}$ a i d ing e $\mathrm{r}$ : Bemerkungen über die zuweilen im geschmeidigen Eisen entstandene krystallinische Structur, verglichen mit jener des Meteoreisens. Sitzber. Wien. Akad. Bd. I5 I, p. 357 (Fig. II).

1859: $\mathrm{B}$ u c hn er: Feuermeteore, p. 122 -123 .

1859: H a r ris: Dissert. Gött., p. IO7. r860: R a m mels berg: Mineralchemie, p. $902-903,947,948$.

r863: B u c hn er: Meteoriten,p.r51 - I52. r863: Rose: Meteoriten, p. 30, 3I, 33, $34,53,65,153$.

r858/65: v. R e i chen b a c h: IV 638 . VI 448,452 . VII 551,562 . IX 163 ,
I74, 182. X 359. XII $457 . X V$ 100, IO9, IIO, III, II4, I24. XVI 25I, 253 , $255,261,262$. XVII $265,266,272$. XVIII 484,487 . XIX 149, r55. XX 622. XXIV 229. XXV 437.

1869: Buchner: Vierter Nachtrag, Pogg. Ann. Bd. 136, p. 439.

1872: Quenstedt: Klar und Wahr, p. $3^{\mathrm{I}} 3$ (Abb. einer geätzten Platte).

1875: vom R a th: Meteoriten, Verh. naturh. Ver. Bonn Bd. 32, p. 361.

I88I: Brezina: Bericht III, Sitzber. Wien. Akad, Bd. 84 I, p. 282 .

r884: M eunier: Météorites, p. 99, II6, п19.

1885: Brezina: Wiener Sammlung, p. 200, 209, 233.

1888: W a rren: Detection and estimation of Selenium in Meteoric Iron. Chemical News Bd. 57 , p. 16. Ref. N. J. I89o II, p. 229.

1889: Co h en : São Julião, N. J. I889 I, p. 222, 224, 225.

r89r: Cohen und Weins chenk: Meteoreisen-Studien, Ann. Hof-Mus. Bd. 6, p. 164 .

1893: M eunier: Révision des fers météoriques, p. $6_{3}, 64$ (Abb. einer geätzten Platte).

r894: C o h e n : Meteoritenkunde, p. 22, $41,56,62,12 I, 131$.

r895: B rezin a: Wiener Sammlung, p. 268 .

1895: C o h e n : Meteoreisen-Studien IV, Ann. Hof-Mus. Bd. 10, p. 83 .

Ursprüngliches Gewicht: 107 Kgr. (r9I Pfund, wahrscheinlich Wiener Gewicht).

Nachweisbares Gewicht: 103613 gr.

Bailey

Belgrad

Bement

Berlin, U.

Bologna

Bonn

v. Braun

\begin{tabular}{r|lr}
13 & Breslau & 8 \\
7 & Budapest & 454 \\
2 & Calcutta & 124 \\
225 & Debreczin & 48 \\
3 & Dorpat & 12 \\
3 & Dresden, M. & 14 \\
25 & Dublin, M. & 71
\end{tabular}




\begin{tabular}{|c|c|c|}
\hline Elbogen & $\left.60^{\prime}\right)$ & New Haven \\
\hline Frankfurt & 5 & New York, M. \\
\hline Freiberg, i. S. & 8 & Paris, M. \\
\hline Göttingen & 89 & Paris, U. \\
\hline Graz, J. & 124 & Pohl \\
\hline Gregory & 71 & Prag, D. U. \\
\hline Greifswald & 15 & Prag, M. \\
\hline Halle & 19 & Rom, U. \\
\hline Harvard, U. & 170 & v. Siemaschko \\
\hline Heidelberg & $2 \mathrm{I}$ & Stockholm \\
\hline Kopenhagen & 68 & Strassburg \\
\hline Leipzig & I & Stuttgart \\
\hline London, B. M. & 95 & Tübingen \\
\hline London, P. G. & 72 & Upsala \\
\hline Lüttich & 5 & Ward \\
\hline Madras & 3 & Washington, Sh. \\
\hline Marburg & 173 & Wien,H.M.*) 7942 \\
\hline Moskau & 18 & Wrany \\
\hline Neumann & 352 & \\
\hline
\end{tabular}

E1 Capitan Range, 40 miles von Bonito, Neu-Mexico.

Eisen, Om, gefunden 1893 , beschr. 1895 .

r895: H ow e 11: On two new Meteorites. - 2. The El Capitan Meteorite. Am. Journ. (3) 5o, p. 253-254 (Zwei Abbildungen der Masse; Analyse von Stokes).

Ursprüngliches Gewicht: $27^{1} 1 / 2 \mathrm{Kgr}$. (about 6r lbs.).

Nachweisbares Gewicht : Die Hauptmasse im Jahre 1895 noch im Besitz von Herrn Howell in Washington, ausserdem:

$$
\begin{array}{lr}
\text { Washington } & 67 \\
\text { New York, M. } \quad 455
\end{array}
$$

\section{Eldorado Co. Shingle Springs} Elgueras Cangas de Onis

Eli Eluat bei Hay, Neu Süd-Wales. Stein, gefunden 1889 .

Diese Lokalität findet sich nur in den Nachträgen, welche Gregory zu seinem
I889 gedruckten Katalog herausgegeben hat. Es heisst dort: No. 327. $\$ 1889$ Eli Eluat, near Hay, N. S. Wales, 2 gr. Small fragment, much decomposed. Auf Anfrage hatte Herr James R. Gregory die Freundlichkeit, mir mitzuteilen, dass Herr Liversidge in den Proc. Royal Soc. New South Wales I889 über diese Lokalität einen Aufsatz veröffentlicht habe. Ferner schrieb mir Herr Gregory: „The meteorite ¿Eli Eluat\& was exhibited by Liversidge at a meeting of the Royal Society of New South Wales in Sydney and was I believe the property of Mr. H. C. Russell, the Government Astronomer. I believe it was found and not seen to $\mathrm{fa} 11$ and was very much decomposed like the Gilgoin Station and Barratta stone

\section{Elisabethpol $1889 \quad$ Mighëi \\ Elisabethpol $1891 \quad$ Indarch \\ Ellenboro Colfax \\ Elmo Joe Wright \\ Elsass Ensisheim \\ Emir (Berg Emir) Krasnojarsk Emmet Co. Estherville Emmetsburg, Frederick Co., Mary- land, U. S. A. \\ Eisen, Om, gefunden 1854 . \\ 1885: Brezina: Wiener Sammlung, p. 211,234 . \\ r895: Brez in a : Wiener Sammlung, p. 277 .}

Ursprüngliches Gewicht: ?

Nachweisbares Gewicht: 177 gr.

\begin{tabular}{lr|lr} 
Bailey & 77 & London, B.M. & 7 \\
Bement & I & v. Siemaschko & 13 \\
Dresden, M. & 2 & Ward & 28 \\
Gregory & 7 & Washington, Sh. & 5 \\
Harvard, U. & ro & Wien, H. M. & 9
\end{tabular}

I) Nach gütiger Mitteilung des Herrn Bürgermeister Dörfler hatte das auf dem Ratbaus in Elbogen aufbewahrte Stück am 18. Februar 1896 das oben angegebene Gewicht.

W ü 1 fing, Meteoriten. 
Emmetsburg scheint noch nicht analysiert worden zu sein.

\section{Encantada}

Imilac

Ensisheim, Ober-Elsass, Deutschld. Stein, Ckb, gefallen I6. Nov. 1492 .

1492: Sebastian Brand (ein lateinisches Gedicht mit Uebersetzung); s. unten 1864 bei Merian.

1493: Schede 1: Liber Cronicarum, Nuremberge, A. Koberger 1493, p. 257. Augsburger Ausgabe von 1497 (Johann Schensperger), p. 289, nach der deutschen Ausgabe von 1493 , p. 257; s. auch Pogg. Ann. Bd. 121 (1863), p. 333; Kenngott, Uebersicht $1862 / 65$, p. 444. r565: Gess ner: De rerum fossilium, lapidum et gemmarum figuris. Zürich Bd. 3 , p. 66 .

1590: Trithem. Chron. Hirsau, M. S. 1590. r699: D a $\mathrm{t} t$ : Volumen rerum Germanic. UIm 1699, p. 214.

r794: C h 1 a d n i : Pallaseisen, p. 35. 1796: K ing: Remarks, p. 23.

r80o: B a r t ho $1 \mathrm{~d}$ : Analyse de la pierre de tonnerre. Journ. Phys. Bd. 50 (an 8), p. $169-176$; s. auch Klaproth, Abh. Berlin. Akad. (1803), p. 3r; ferner Izarn, p. $126-138$.

r802: $\mathrm{H}$ ow a r d : Experiments and $\mathrm{Ob}-$ servations on certain stony and metalline Substances, which at different Times are said to have fallen on the Earth; also on various Kinds of native Iron. Philos. Trans, 1802, p. 171-173; s, auch Gilb. Ann. Bd. 13 (1803), p. 295-296, 312 und die Uebersetzung Fourcroy's in sEncyclopédie * Bd. 5 (1808), p. 545,554. 1803: De Dr é e: Notice sur les pierres tombées près d'Ensisheim et dans les environs d'Agen. Journ. Phys. Bd. 56, p. 405-408; s, auch de Drée: On the Stones said to have fallen at Ensisheim, in the Neighbourhood of Agen, and at other Places. Tilloch's Philos. Magaz. Bd. 16, p. 289-293; ferner sEncyclopédies Bd. 5 (1808), p. 59r;
Gilb. Ann. 1804 etc. s. u.

1803: de Dré e: Recherches, p. 405, 406-409, 4IO, 4II, 4I3, 4I9, 420.

1803: I z a rn : Lithologie, p. 126-138, $174,325,327,328,329,333,346$. r803: K laproth: Ueber meteorische Stein- u. Metall-Massen, Abh. Berlin. Akad. 1803, p. 31 .

1803: $\mathrm{C}$ h 1 a d n i: Chronologisches Verzeichnis, Gilb. Ann. Bd. 15, P. 308, 3I3-3r4 (Anm).

1803: S a g e: Examen de la pierre météorique tombée le 7 Novembre 1492, près d'Ensisheim, village de la BasseAlsace. Journ. Phys. Bd. 57 (I803), p. $7 \mathrm{I}-73$.

r803: V a u quelin: Sur les pierres dites tombées du ciel. Ann.Chim. (an II) Bd. 45 , p. 245 ; s. auch Ann. Mus. d'hist. nat. Bd. 3 (1804), p. 108- го. I803: B u te n schön: soniteur^ (an II) Nivose 2 ; s, auch Gilb. Ann. Bd. 16, p. 74 .

1804: v. Ende: Massen und Steine, p. $29-30$.

r804: $\mathrm{Pötzs} \mathrm{ch:} \mathrm{Kurze} \mathrm{Darstellung,}$ p. $59-60, \mathrm{II}_{5}-\mathrm{II} 8$.

r804: F o u r c r oy (s, bei L'Aigle); s. auch >Encyclopédie Bd, 5 (1808), p. $563-564$.

1804: Gilbert: Nachträge zu den Aufsätzen in den Annalen über die aus der Luft gefallenen Steine. Gilb. Ann. Bd. 18 , p. $279-283$ (de Drée), 286, 291 (Vauquelin u. Fourcroy), 318-319. 1806: L a u g i e r : Extrait d'un Mémoire sur l'existence du chrôme dans les pierres météoriques. Ann. Mus, d'hist. nat. Bd. 7 , p. $392-397$; s, auch Gilb. Ann. Bd. 24 (1806), p. $378,380-384$. 1807: K l a p rot h : Beiträge, Bd.4, p.98. 1808: D e D r é e-P i c te t: Description comparative etc.; s. bei Weston, Bibl. Brit. No. 296 (April 1808), p. 279, 280, 286, 287, 289 .

1808: Gilbert: Nachrichten von den Steinen, welche zuWeston in der Provinz 
Connecticut, in den nordamerikanischen Freistaaten, am 14. Dezember I807 vom Himmel herabgefallen sind, Gilb. Ann. Bd. 29 , p. $371-372$ (der Aufsatz ist frei bearbeitet nach einem solchen in den Journ. Phys. Mai 1808); s. auch im gleichen Band, p. 97.

r809: Klaproth: Bestandteile des Smolensker Meteorsteins nach der Analyse Klaproth's. Gilb. Ann. Bd. 33, p. 2II; s. auch Ann. Chim. Bd. 70 (1809), p. 182; Gehlens Journ. Chem. Phys, u. Min. Bd. 7 (1808), p. 199.

r8ro: Klaproth: Beiträge, Bd. 5, p. $255-256$.

18ro: K la proth: Briefe, Schweitz, p. 24 .

1812: B igot de Morogues: p. 56 $-63,337$.

r8r2: $\mathrm{C}$ h 1 a d n i : Verzeichnis,Schweigg. Journ. Bd. 4 Beil. I, p. 6.

1815: Chladni: Neues Verzeichnis, Gilb. Ann. Bd. 5o, p. 235-236.

r8rg: $\mathrm{Chl}$ a d n i : Vierte Fortsetzung, Gilb. Ann. Bd. 6o, p. 239-240.

r8rg: C h la d n i: Feuermeteore, p. 40, $43,48,50,58,66,69,80,90,205$ -207 (1), 302, 427 .

r836: K ä m tz: Meteorologie, p.248,266. r839: B e n z e n b e r g : Sternschnuppen, p. $40,77-79$.

1843: P a r ts c h : Meteoriten, p.32-34. 1846: S h e p a rd: Report on Meteorites,

Am. Journ. (2) 2, p. $3^{82}$

1847: $\mathrm{S} \mathrm{ch}$ a f h ä u t 1 : Schönenberg,Gel. Anz. München.Akad.Nr.7o,p.562-563. r848: B 1 o e d e : Tabelle, Bull. Petersbourg. Acad. Bd, 6, p. 13 .

1854: B a 1 c e $11 \mathrm{~s}$ : Lithologia meteorica, p. 15 .

r859: B u c h n e r : Feuermeteore, p. 34 -36 .

1859: Harris: Dissert. Gött., p. 55 .

r862: Kenngott u. W iser: Zürcher Sammlung, p. 146.

1863: B u c hner: Meteoriten, p. 3-4. r863: R o s e: Meteoriten, p. $84,85,86$,
$90,91-92,98,147,154$. 1864: Poggendorff - Kesse1meyer: Aelteste Nachrichten über den Meteorsteinfall zu Ensisheim. Pogg. Ann. Bd, I2I, p. 333-334.

1864: Merian: Ueber den Meteorsteinfall zu Ensisheim. Pogg. Ann. Bd. 122, p. I82-186; s. auch Kenngott, Uebersicht 1862/65,p.445; Wochenschr. f. Astr., Met.u.Geogr. 1864, p. 206-207. r $858 / 65$ : v. Rei c h e n b a c h: V 480. VI 453,454 . VII 552 . IX 155, 16I, 172, 180. X 359. XI 297, 302, 309. XII 455. XIII $356,362,365$. XX 623 . XXIV 226, 227. XXV 322, 608.

1867: Go e be 1: Kritische Uebersicht, Mélanges phys. chim. Bd. 7, p. $3^{12}$. 1868: $\mathrm{Cr}$ oo $\mathrm{k}$ : On the chemical constitution of the Ensisheim, Mauerkirchen, Shergotty and Muddor Meteoric Stones. Dissert. Gött., p. $2 \mathrm{I}-26$.

1869: B u chner: Vierter Nachtrag, Pogg. Ann, Bd. 136, p. 44I.

r870: R a m mels berg: Meteoriten, p. 103, 106, 107, 139, 140.

r875: vom R at h: Meteoriten, Verh. naturh. Ver. Bonn Bd. 32, p. 367 .

1879: R a m mels berg: Meteoriten, p. 24 .

1883: T s c h erma k: Beitrag, Sitzber.

Wien. Akad. Bd. 88 I, p. 355 .

1884: M e un i er: Météorites, p. 79,

$85,93,96,179,349,350,395$.

1883/85: Tschermak: Photographien, p. 17,18 .

1885: Brezina: Wiener Sammlung, p. 19I, 233.

1890: B r e z i n a : Reise, Ann. Hof-Mus.

Bd. 4 (Not.), p. 119 .

r891: N ew to n : Document relatif à la trajectoire suivie par la météorite d'En-

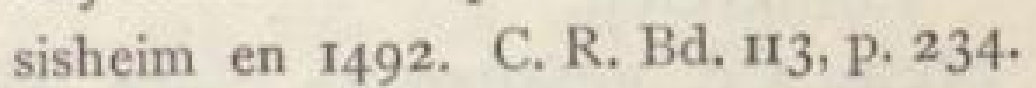
Ref. N. J. 1892 I, p. 270.

r894: F l et $\mathrm{c}$ h e r : Introduction, p. I9. 1894: Cohen: Meteoritenkunde, p. 7 , $244,246,266,267$.

$8^{*}$ 
Ursprüngliches Gewicht : Etwa $127 \mathrm{Kgr}$.

Nachweisbares Gewicht: $70385 \mathrm{gr}$.

\begin{tabular}{|c|c|c|c|}
\hline Aachen & 24 & London, P. G. & 223 \\
\hline Bailey & 7 & Lüttich & \\
\hline Bayet & I & Madras & \\
\hline Belgrad & II & Madrid & \\
\hline Bement & I 33 & de Mauroy & \\
\hline Berlin, $P$. & 15 & Melion & \\
\hline Berlin, U. & 962 & Moskau & \\
\hline Bologna & 6 & München & 224 \\
\hline Bonn & 28 & Neumann & \\
\hline v. Braun & $\left.37^{1}\right)$ & New Haven & \\
\hline Breslau & 6 & Paris, E. & \\
\hline Budapest & 962 & Paris, M. & 9074 \\
\hline Calcutta & $9^{2}$ & Paris, U. & 282 \\
\hline Cambridge & 147 & Parma & \\
\hline Cleveland & Spl. & Petersburg, B. & 12 \\
\hline Dorpat & 17 & Pohl & 54 \\
\hline Dresden, M. & 73 & Rom, U. & \\
\hline Ensisheim 548 & $\left.300^{2}\right)$ & v. Siemaschko & 25 \\
\hline Freiberg, i. S. & 27 & Stockholm & $5^{1}$ \\
\hline Gotha & 9 & Strassburg & 73 \\
\hline Göttingen & 192 & Stürtz & \\
\hline Gregory & 245 & Stuttgart & \\
\hline Greifswald & 14 & Troyes & 82 \\
\hline Hamburg & 27 & Tübingen & 368 \\
\hline Harvard, U. & 30 & Turin, J. & 32 \\
\hline Heidelberg & 4 & Upsala & 210 \\
\hline Karlsruhe, M. & 142 & Utrecht & \\
\hline Kasan & II2 & Ward & 22 \\
\hline Klausenburg & IO & Washington & \\
\hline Kopenhagen & 77 & Washington, Sh. & \\
\hline Krantz & 95 & Wien, H. M.*) & $65^{8}$ \\
\hline Leipzig & 71 & Zürich & 15 \\
\hline
\end{tabular}

Die Universität Basel besitzt ebenfalls ein Stück von Ensisheim.

Epinal, La Baffe, Dep. Vosges, Frankreich.

Stein, Cc, gefallen $\mathrm{r}_{3}$. Sept. $\mathrm{I}_{222}$. 1822: Parisot: Bericht über einen Meteorstein-Fall, der sich unweit Epinal, in der Gemeinde de la Baffe, am 13. Sept. 1822 während eines Gewitters ereignet hat. Gilb. Ann, Bd. 72, p. 323 -327 .

1822: Parisot-Gehin: Rapport à M. le Maître des Requêtes, Préfet du département des Vosges, sur l'aérolithe tombé dans la commune de la Baffe, le I3 Septembre courant, par le Régent de physique soussigné, envoyé sur les lieux pour recueillir les circonstances et les preuves de ce phénomène. Ann.Chim. (2) 21,p.17-22. 1822: Vauquelin: Examen fait, d'après l'invitation de l'Académie des Sciences d'une aérolithe tombée aux environs d'Epinal, le 13 septembre 1822 , à l'entrée de la forêt de Taunière, à trois quart de lieue de la Baffe (Vosges). Ann. Chim. Phys. (2) 21, p. $324-328$; s. auch Ann. des Mines Bd. 8 (1823), p. $332-333$.

1823: C h la dn i: Dritter Nachtrag, Gilb. Ann. Bd. 75, p. 23 I.

1836: K ämtz: Meteorologie, p. 291. 1843: Part s ch: Meteoriten, p. 42-43. 1854: B a $1 \mathrm{c}$ e $11 \mathrm{~s}$ : Lithologia meteorica, p. 24 .

1854: v. Boguslawski: Zehnter Nachtrag, Pogg. Ann. Ergz.-Bd. IV, p. 436. 1859: H a rris: Dissert. Gött., p. 77. 1859: B u c hner: Feuermeteore, p. 88; s. auch p. $94,121,184$.

1863: B u c hner: Meteoriten, p. 44. 1863: Rose: Meteoriten, p. 154. 1859/65: v. R e i c hen b a c h: IX 162, $164,169,179 . \quad$ XI 295. XIV 392. XXV 428 .

r884: M e un ie r : Météorites, p.260,26r. r885: Brez ina: Wiener Sammlung, p. 185,233 .

Ursprüngliches Gewicht: Ein Stein von der Grösse einer $\gg 6$ püundigen Kanonenkugel«.

1) Ausserdem $28 \mathrm{gr}$. fraglich. - 2) Nach Wägung vom 23. Januar 1896, welche der Herr Bürgermeister in Ensisheim die Güte hatte auf meinen Wunsch ausführen zu lassen. 
Nachweisbares Gewicht: 301 gr.

Berlin, U.

Io Paris, M. 249

v. Braun 7 v. Siemaschko

7

Calcutta

Gregory

Spl. Strassburg

3 Tübingen

London, B. M.

2 Wien, H. M. *)

Erxleben, Prov. Sachsen, Preussen, Deutschland.

Stein, Ck, gefallen 15. April 1812. I812: Ha usmann u. Vie $t h$ : Nachrichten von einem Meteorsteine, der am 15. April 1812 zu Erxleben, zwischenMagdeburg und Helmstedt, herabgefallen ist. Gilb. Ann. Bd. 40 , p. 450 -459 ; s. auch Gött. Gel. Anz. I8I2 II, p. 777-780; Gilb. Ann. Bd. 4I (1812), p. 454 .

1812: Wiedemann: Noch einige Nachrichten von dem Erxlebener Meteorstein, aus einem Brief des Herrn Dr. Wiedemann an den Prof. Gilbert. Gilb. Ann. Bd. 4I, p. 96.

r8r2: Bigot de Morogues: p.284 -285 .

1812: Strohmeyer: Analyse des zu Erxleben im Elb-Departement am 15. April 1812 herabgefallenen Meteorsteins. Gilb. Ann. Bd. 42, p.ro5-IIO. 1813: Rol off: Ueber den Aërolitben von Erxleben. Zur Bestätigung der Thatsachen aufgenommenes Protokoll. Schweigg. Journ. Bd. 7, p. 139-142. 1813: $\mathrm{B} \mathrm{u} \mathrm{chholz:}$ : Analyse des Aërolithen von Erxleben bei Magdeburg. Schweigg. Journ. Bd. 7, p. 143-172; Nachschreiben des Herausgebers, p. I72 -174, worin auch die Entdeckung Witmannstädten's, die Aetzung mit Säure betreffend, erwähnt wird.

1815: $\mathrm{Ch} 1 \mathrm{a}$ dni: Neues Verzeichnis, Gilb. Ann. Bd. 5o, p. 255.

r815: Kl a proth: Beiträge, Bd. 6, p. $303-306$.

r8rg: $\mathrm{Chladni}$ : Vierte Fortsetzung, Gilb. Ann, Bd. 6o, p. 240. r8r9: C h 1 a d n i: Feuermeteore, p. 45, 50, 58, 66, 69, 7I, 73, 9I, I54, 299 -3 or (1), 43r (Sagt, dass der Stein in Gilb. Ann. Bd. 40 , p. 458 abgebildet sei, hier findet sich aber nur die Umrisszeichnung eines kleinen Stückes). r836: $\mathrm{K}$ ä $\mathrm{m} \mathrm{tz}$ : Meteorologie, p.258,284. r84r : R a m m e $1 \mathrm{~s}$ ber $\mathrm{g}$ : Handwörterbuch, p. $427-428$.

r843: P a rt s c h: Meteoriten, p. 45-46. 1854: B a 1 c e $11 \mathrm{~s}$ : Lithologia Meteorica, p. 23.

r858/6r: v. Reichen bach: V 477, 479,48 r. IX 162, 172, 180. X 359, $362,365,371,372$. XI 294, 300, 302, 310. XII 454. XV IOI, I2I.

r 859: Buchner: Feuermeteore, p. 82. r859: Harris: Dissert. Gött., p.70-7r. r863: B u c h n e r: Meteoriten, p. $30-31$. r863: Rose: Meteoriten, p. 84,85 ,

$86,87,89,90-91,93-96$, Tafel 3, $97,100,154,159,160$.

1875: vom R a th: Meteoriten, Verh. naturh. Ver. Bonn Bd. 32, p. 367 .

1881 : v. Nordenskiö 1 d: Zeitschr. d. d. geol. Ges. Bd. 33, p. 24 (Vergleich mit Ställdalen).

1883: Tschermak: Beitrag, Sitzber.

Wien. Akad. Bd, 88 I, p. 355 .

r884: H ä p k e: Beiträge, Abh. naturw.

Ver. Bremen Bd. 8, p. 523 .

1884: Me un i e r: Météorites, p. 96, 97, 179, 180, 349 .

1883/85: Ts chermak: Photographien, T. 20 , p. $18,20$.

1885: She pard: Am. Journ. (3) 30, p. 106 (Krystall von Nickeleisen).

r885: Brez in a: Wiener Sammlung, p. $19 \mathrm{I}, 233$.

r89o: v. N i ess 1: Periheldistanzen, Verh. naturf. Ver. Brünn, Bd, 29, p.188, 194, 195, 196, 208, 214, 250-251. r894: Co h en: Meteoritenkunde,p. 8, 185.

Ursprüngliches Gewicht: $2 \frac{1 / 4}{4} \mathrm{Kgr}$. ( $4^{1 / 2}$ Pfund).

Nachweisbares Gewicht: Ir66 gr. 


\begin{tabular}{lr|lr} 
Bailey & I & Heidelberg & I6 \\
Bement & 9 & Klausenburg & 3 I \\
Berlin, U. & I30 & Kopenhagen & 30 \\
Bonn & I & London, B. M. & 3 I \\
v. Braun & I & London, P. G. & 3 \\
Breslau & 28 & Neumann & 2 \\
Budapest & 29 & New Haven & 13 \\
Calcutta & 2 I & Paris, M. & II \\
Clausthal & I8 & Petersburg, B. & 24 \\
Cleveland & I & Rom, U. & I8 \\
Dorpat & 22 & v. Siemaschko & 16 \\
Gotha & 8 & Stockholm & 46 \\
Göttingen & 297 & Stuttgart & 2 \\
Gregory & 9 & Tübingen & 48 \\
Greifswald & I80 & Washington, Sh. & 31 \\
Harvard, U. & I & Wien, H. M.*) & 88
\end{tabular}

Esnandes, Charente inférieure, Frankreich.

Stein, $\mathrm{Cg}$, gefallen August 1837 .

1837: Kurze Ankündigung im »L'Institut «

Bd. 5, Nr. 220, p. 334 .

1843: P a r s c h: Meteoriten, p. 143.

1854: v. Boguslawski: Zehnter

Nachtrag, Pogg.Ann. Ergz.-Bd. 4, p. 357.

r859: $\mathrm{H}$ a rris : Dissert. Gött., p. 85 .

1859: v. Reichenbach: IX I6I, 170, 179 .

1863: B u c hner: Meteoriten, p. 60.

r885: Brez ina: Wiener Sammlung, p. $182,232$.

I894: Fletcher: Introduction, p. 14. Ursprüngliches Gewicht: $\left.{ }^{1} 1 / 2 \mathrm{Kgr} .{ }^{1}\right)$ Nachweisbares Gewicht: $70 \mathrm{gr}$.

Calcutta

$$
3 \text { Rom, U. }
$$

22

London, B. M. $\quad 3$ Wien, H. M. *) 42

Ausserdem: Lausanne 37 gr. "Sporadosidère de la Rochelle ; könnte auch Chantonnay sein.

Estherville, Emmet County, Iowa, U. S. A.

Mesosiderit, M, gefallen 1o.Mai 1879.
I879 (?): An onymus: The Perry Meteor! The Greatest Curiosity ever placed on Exhibition in this (welcher?) City. Weighs 43 I pounds! On Exhibition at J. O. Voorhies' Building, Main Street, between Third and Fourth. (Annonce mit vielen Zeitungsberichten, ein Exemplar im k. k. Hof-Mus. Wien).

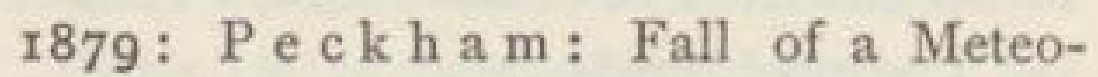
rite on the roth of May, in Iowa. Am. Journ. (3) 18, p. $77-78$.

I879: $\mathrm{S}$ he pard: On the Estherville, Emmet County, Iowa, Meteorite of May Ioth 1879 . Am. Journ. (3) I8, p. I86 -188. Ref. N. J. I880 I, p. 177-178; Liebig-Kopp, Jahresber. 1879, p. 1279 $-\mathrm{I} 28 \mathrm{o}$.

1879: Hinrichs und Daubrée: Chute de Météorites qui a eu lieu le 1o Mai 1879 dans le comté d'Emmet (Iowa) ; C. R. Bd. 88, p. 1219-1220. Ref. N. J. 1880 I, p. 47.

1880: Smith: Study of the Emmet County Meteorite, that fell near Estherville, Emmet County, Iowa, Mai 10, 1879. Am. Journ. (3) 19, p. $459-463$ (Analyse), auch p. 495 ; s. ferner Orig. Researches r884, p. 586-59r u. C. R. Bd. 90 (1880), p. 958 ; N. J. I881 I, p. 29 -3r ; Liebig - Kopp, Jahresber. 1880, p. 1541; The Eighth Annual Rep. State Geologist 1880, p. $176-180$.

1880: K l e in: Vermehrung, Gött. Gel. Anz. (1880), p. 567-568.

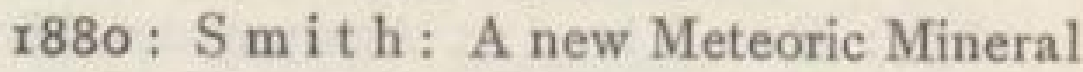
(Peckhamite) and some additional facts in connection with the fall of Meteorites in Iowa, May Ioth $1879 . \mathrm{Am}$. Journ. (3) 20, p. 136-137 (Analyse); s. auch Original Researches 1884 , p. 592 -594 ; C. R. Bd. 90 (188o), p. 1460 ; N. J. 188I I, p. 3 I.

1880: vom $\mathrm{R}$ at h: Verh. naturh. Ver.

I) Die Hauptmasse soll nach dem sL'Institute in das Naturhist. Museum nach Bordeaux gekommen sein, dort ist aber nach Herrn'Fallot's gütiger Mitteilung nichts mehr vorhanden. 
Bonn Bd. 37 (Sitzber.), p. 239-241. Ref. N. J. 1882 I, p. 175 .

1880: H of $f m$ a $n n$ : Verh, naturhist. Ver. Bonn Bd. 37 (Sitzber.), p. 285 -287 .

188r: Brezina: Bericht III, Sitzber. Wien. Akad. Bd. 84 1, p. $278,279$. I882: v. L a s a u $1 \mathrm{x}$ : Vermehrung, Verh. naturh. Ver. Bonn. Bd.39 (Sitzber.), p.ror. r882: W i e c h m a n n : Fusion-Structures in Meteorites. Ann. N. Y. Acad. Sc. Bd. 2, p. 293.

1882: M e unier: Détermination lithologique de la météorite d'Estherville, Emmet County, Iowa (Io. Mai I879). C. R. Bd. 94 (r882), p. $1659-166 r$. Ref. N. J. $188_{3}$ I, p. $3^{8} 3-384$; Liebig-Kopp, Jahresber. 1882, p. 1643 . 1883: Tschermak: Beitrag, Sitzber.

Wien. Akad. Bd. 88 I, p. 351. 1883/85: Tschermak: Photographien T. 24, p. 22, 23.

1884: M e unier: Météorites, p. 62, $87,99,165,170-174,171$ Abb., 362, $496,497$.

r 884 : W a d s w o r t h: Studies,p. $92-$ IOI. r885: Brezina: Wiener Sammlung, p. $155,168$.

r887: Flight: Meteorites, p.209-2II; s. auch eine vorläufige Notiz in Rep. Brit. Assoc. 1879 , p. 55 (Sep. der Rep. of Observ. of Luminous Meteors).

r888: New to n: Orbits, Am. Journ. (3) 36, p. 4 .

r889: F l e t c h e r : Atacama Meteorites, Mineral, Magaz. Bd. 8, p. 226.

1890: E a s t m a n: Met. Astron., p. 316. r89r: Torrey u. Barbour: The recorded Meteorites of lowa, with special mention of the last, or Winnebago Co., Meteorite (Situationsplan), Amer. Geologist. Bd. 8, p. 66 (Die grösste Masse von 500 lbs, sei an das Britische Museum gesandt und verteilt worden).
1893: B rez in a: Ueber neuere Meteoriten (Nürnberg), p. I6I, I62.

1894: C o h e n : Meteoritenkunde,p. 59,63 , 20I, 223, 24I, 258, 277, 284, 288.

r895: M eunier: Révision des lithosidérites, p. 30-32 (Abb. einer geätzten Platte u. eines Dünnschliffs).

r895: Brezina: Wiener Sammlung, p. 262 .

Ursprüngliches Gewicht: Steinschauer. Die grössten Massen wogen $437,170,92 \frac{1}{2}, 28,101 / 2$, 4 u. 2 pounds, zusammen also 744 pounds oder $337 \mathrm{Kgr}$.

Nachweisbares Gewicht: 3347 I 4 gr.

\begin{tabular}{|c|c|c|}
\hline Bailey & 516 & Howell \\
\hline Bayet & 17 & Klausenburg \\
\hline Bement & $3^{1} 30$ & Kopenhagen \\
\hline Berlin, U. & 150 & Krantz \\
\hline Böhm & 16 & Kunz \\
\hline Bologna & 400 & Lissabon \\
\hline Bonn & 103 & London,B.M. II6 \\
\hline v. Braun & 99 & London, P. G. \\
\hline Bremen & $\left.2 I^{1}\right)$ & Marburg \\
\hline Breslau & 7 & de Mauroy \\
\hline Brezina & 206 & Melion \\
\hline Budapest & 1675 & Minneapolis 60 \\
\hline Calcutta & 4 & Moskau \\
\hline Cambridge & 34 & München \\
\hline Catania & 23 & Neumann \\
\hline Clausthal & 46 & New Haven 4 \\
\hline Cleveland & 50 & Newton \\
\hline Detmold & 9 & New York, M. \\
\hline Dorpat & II & Odessa \\
\hline Dresden, M. & 44 & Paris, E. \\
\hline Dresden, P. & 9 & Paris, M. \\
\hline Dublin, M. & 33 & Pohl \\
\hline Freiberg, i. S. & N. 9 & Prag, M. \\
\hline Göttingen & 120 & Riga \\
\hline Graz, J. & 21 & Roebling \\
\hline Gregory & 596 & Rom, U. \\
\hline Greifswald & 24 & v. Schilling \\
\hline Hamburg & 18 & v. Siemaschko \\
\hline Harvard, U. & 17725 & Stockholm \\
\hline
\end{tabular}

1) Unter Meteoreisen als „Iowa aufgeführt. 


\begin{tabular}{lr|lr} 
Strassburg & 59 & Utrecht & I7 \\
Stuer & 9 & Ward & 2843 \\
Stürtz & 24 & Washington & 388 \\
Szamosujvar & 69 & Washington,Sh.3575 \\
Troyes & 55 & Wien,H.M. 23 208 ${ }^{1}$ ) \\
Tübingen & 31 & Wien, U. I. & 225 \\
Turin, U. & 26 & Wrany & 8
\end{tabular}

Die Universität Basel besitzt ebenfalls ein Stück von Estherville.

Faha

Fairweather

False Inca

Limerick

Bridgewater Inca

Farmington, Washington County, Kansas, U. S. A.

Stein, Cs, gefallen 25. Juni 1890 .

r89o: Snow: sScience«, 18. Juli 1890 Bd. 16, p. 38-39.

r89r: $\mathrm{K} \mathrm{unz}$ und W e in s c hen $\mathrm{k}: \mathrm{Me}-$ teoritenstudien. I.Washington, Washington County, Kansas. Tschermak M. P. M. Bd. 12, p. 177-182 (Analyse von Eakins). Ref. N. J. 1892 II, p.242-243. r892: Kunz und Weinschenk: Farmington, Washington Co., Kansas Aërolite. Am. Journ. (3) 43, p. 65-67. Ref. N. J. 1892 II, p. 244.

r892: Preston: Notes on the Farmington, Washington County, Kansas, Meteorite. Am. Journ. (3) 44,p.400-401 (Abbildung eines Schliffes). Ref.N.J. I893 II, p. 279.

1892 : v. H a u e r: Ann. Hof-Mus, Bd. 7 (Not.), p. 72.

1893: B rezin a: Ueber neuere Meteoriten (Nürnberg), p. 160.

r894: C o h e n : Meteoritenkunde, p.6o, 20I, 206, 26I, 268, 298, 316.

r894: F l et cher: Introduction, p. 15. r895: Brezina: Wiener Sammlung, p. 253 .

Ursprüngliches Gewicht: $84 \mathrm{Kgr}$. 2 Steine von 80 und $4 \mathrm{Kgr}$; von dem ersteren erhielt Herr Ward 61.7 Kgr.

Nachweisbares Gewicht: $53487 \mathrm{gr}$.

\begin{tabular}{lr|lr} 
Bailey & 82 & Minneapolis & 562 \\
Bement & 5369 & München & 95 \\
Berlin, U. & $5 \mathrm{I}$ & New Haven & 246 \\
Böhm & 18 & Newton & $45^{2}$ ) \\
v. Braun & 461 & Odessa & 117 \\
Brezina & $2 \mathrm{II}$ & Paris, E. & 117 \\
Budapest & 169 & Pohl & 398 \\
Cambridge & 12 & Prag, M. & 254 \\
Cleveland & 26 & Rom, U. & 147 \\
Dorpat & 45 & Sevilla & 7 \\
Frenzel & 2305 & v. Siemaschko & 7 I \\
Gregory & 274 & Stockholm & 6 I \\
Greifswald & 15 & Strassburg & 50 \\
Harvard, U. & 2792 & Stürtz & 1580 \\
Heidelberg & 44 & Troyes & 2 \\
Howell & 205 & Ward & 31 700 \\
Kunz & 3000 & Washington & 489
\end{tabular}

London, B. M. 802 Wien, H. M. ${ }_{11} 58$ de Mauroy 182 Wien, U. I. 325 Seit 1. Juli 1893 erwarb Herr Bayet, Brüssel, II4 gr.

Favars, Departement Aveyron, Frankreich.

Stein, Ci, gefallen 21. Okt. 1844.

1844: Boisse: „L'Institut * Nr. 570 Bd. 12, p. 399 ; s. auch C. R. Bd. 19 (1844), p. II8I.

1850: Filho l: Mémoires de la société des Lettres de l'Aveyron Bd. 6, p. 97. 1854: v. Boguslawski: Zehnter Nachtrag, Pogg.Ann. Ergz.-Bd,4, p.375. 1857: A rago: Astronomie populaire, Bd. 4 , p. 203.

1859: Ha r ri s: Dissert. Gött., p. 91. 1863: B u c hner: Meteoriten, p. 75. r858/65: v. Reichen ba ch: V 477. IX 161, 168, 178. XI 294, 296, 297, 300. XIII 369 Fig., 372 Fig., 383 . XXV 607.

1875: vom R a th: Meteoriten, Verh.

I) Ausserdem 659 gr. nach I. Juli 1893 erworben.

2) Als "Washington Co. unter den Meteorsteinen aufgeführt. 
naturh. Ver. Bonn Bd, 32, p. 368. r884: Me un i e r: Météorites, p. 197,20r. 1885: Brezina: Wiener Sammlung, p. $181,232$.

1894: C o h e n : Meteoritenkunde,p.267. r895: Brezina: Wiener Sammlung, p. 246.

Ursprüngliches Gewicht: 1.50 Kgr. (1050 gr. ?).

Nachweisbares Gewicht 555 gr.

\begin{tabular}{lr|lr} 
Bonn & 3 & Paris, M. & 430 \\
Calcutta & 2 & v. Siemaschko & 8 \\
Dorpat & I & Stockholm & 78 \\
Göttingen & I & Troyes & I \\
Gregory & I & Tübingen & 8 \\
London, B.M. & 6 & Wien, H. M.* & I \\
de Mauroy & 15 & &
\end{tabular}

Fayette Co.

Bluff

Fayetteville

Petersburg

Fehrbellin

Linum

Feid Chair, Prov. Constantine, Algier, Nordafrika.

Stein, Ccb, gefallen r6. August 1875 .

1877: Da ubrée: Note sur la chute d'une météorite, qui a eu lieu le 16 aon̂t I875 à Feid-Chair, dans le cercle de la Calle, province de Constantine. C. R. Bd. 84, p. $70-72$. Ref. Zeitschr. f. Kryst, Bd. I (1877), p. 91 ; LiebigKopp, Jahresber, 1877 , p. 13931394. r884: M e un ier: Météorites, p. 26I, 264, 494 .

1885: Brezina: Wiener Sammlung, p. 256.

1887: F lig h t: Meteorites, p. r9I.

r89o: B r ez in a: Reise, Ann. Hof-Mus. Bd, 4 (Not.), p. IIg.

Ursprüngliches Gewicht: $380 \mathrm{gr}$.

Nachweisbares Gewicht: 25 gr. Paris, M. 25

Wo befindet sich die Hauptmasse dieses Steins?

Fekete

Ferguson

Mezö-Madaras

s. Anhang
Fisher, Polk County, Minnesota, U. S. A.

Stein, Ci, gefallen 9. April $\mathbf{r} 894$. 1895: Brezina: Wiener Sammlung, p. 247.

Nach Direktor Brezina's Mitteilung besass Kunz 500 gr., inzwischen gelangte ein Stück an das Hof-Museum in Wien.

Fish River Great Fish River

Florac s. Anhang

Floyd Mountain, Indian Valley Township, Pulaski Co., Virginia, U. S. A.

Eisen, $\mathrm{Hb}$, gefunden 1887 , beschr. 1891 .

r89r: $\mathrm{K}$ unz u. Weinschenk: Meteoritenstudien. - 2. Floyd Mountain, Radford Furnace, Virginia. Tschermak, M. P. M. Bd. 12, p. $182-184$ (Abbildung einer geätzten Platte, Analyse von Eakins). Ref. N. J. 1892 II, p. 36, 243 ; s. auch Bull. U. S. Geol. Surv. No.9o, p. 45; Geol. Magaz. Bd.9 (I89r), p. $894-895$.

1892: Kunz u. Weinschenk: On two Meteoric Irons. I. Indian Valley Township, Floyd County, Virginia. Am. Journ. (3) 43, p. 424-425.

r894: Co hen: Meteoritenkunde, p. 77, $89,97,126,127$.

Ursprüngliches Gewicht: $14.2 \mathrm{Kgr}$. Nachweisbares Gewicht: I4 086 gr. Kunz 14000 gr. am 3r. Januar 1894.

München $\quad 7^{8} \mid$ Washington 8

Folersville

Staunton

Fomatlan

Gargantillo

Forest City, Leland, Winnebago Co., auch Kossuth Co. Iowa, U. S. A.

Stein, Ccb, gefallen 2. Mai 1890 . r89o: Torrey u. B a rbour: Fall of Meteorites in Iowa. Am. Journ. (3) 39 , P. $521-522$ (Analyse); s. auch 
Amer. Geologist. Bd. 8 (1891), p. 67 -72 (Abb. der Masse von 66 lbs., Situationsplan, Analyse).

I890: $\mathrm{Kunz}$ : On five new American Meteorites. - 2. On the Winnebago County, Iowa Meteorite. Am. Journ. (3) 40 , p. $318-320$ (Analyse von Eakins). Ref. N. J. 1891 II, p. 52; s. auch Trans. New York Akad. Sc. Bd. 9 (189o), p. 20I-203; U. S. Geol. Surv. Nr. 78 (189r), p. 95-97; Journ. Chem. Soc. 1891, p. 277-279; Oesterr. Zeitschr. f. Berg- u. Hüttenw. Bd. 39 (1891), p. 228.

1890: E a s t m a n : Met. Astron,, p. 316. r89r: v. H a u er: Ann. Hof-Mus. Bd. 6

(Not.), p. 55 u. Bd. 7 (1892, Not.), p. 72. r893: N ewto $\mathrm{n}$ : Lines of structure in the Winnebago Co. Meteorites and in other Meteorites. Am, Journ, (3) 45, p. $152-153,355$. Ref. N. J. I894 I, p. $273-274$.

1893: B r ez in a : Ueber neuere Meteoriten (Nürnberg), p. 161.

I894: C o h en: Meteoritenkunde, p. 6I, I53, 203.

1895: Brezina: Wiener Sammlung, p. 259.

Ursprüngliches Gewicht: Steinschauer,darunter 5 grössere Steine von 80,66, 10, 4, 4 Pfund u. 500 kleinere von $1 / 20-20$ Unzen.

Nachweisbares Gewicht: 122037 gr.

\begin{tabular}{lr|lr|} 
Bailey & I594 & Gregory & 1297 \\
Bayet & 18 & Greifswald & 24 \\
Bement & 110 & Harvard,U. & $650^{1}$ ) \\
Berlin, U. & 43 & Howell & 1425 \\
Böhm & 208 & Kunz & 13500 \\
v. Braun & 169 & Leipzig & 10 \\
Brezina & 244 & London, B. M. 2560 \\
Budapest & 74 & de Mauroy & 16 \\
Cambridge & 17 & Minneapolis & 32062 \\
Cleveland & 96 & München & 92 \\
Dorpat & 36 & Neapel & 28 \\
Dresden, M. & 66 & NewHaven $22000^{2}$ )
\end{tabular}

\begin{tabular}{lr|lr} 
Newton & 7494 & Stockholm & 5 \\
Odessa & 34 & Strassburg & 20 \\
Paris, E. & 36 & Stürtz & 224 \\
Paris, M. & 38 & Turin, U. & 52 \\
Pohl & 54 & Utrecht & 90 \\
Prag, M. & 85 & Ward & 726 \\
Riga & I7 & Washington & I27 \\
Rom, U. & 51 & Wien, H. M. & 580 \\
v. Siemaschko & 80 & Wrany & I5
\end{tabular}

Forsyth Mesosiderit Miney

Forsyth, Monroe County, Georgia, U. S. A.

Stein, Cwa, gefallen 8. Mai 1829. 1830: Sill i m a n : Georgia Meteor and Aerolite (Bericht von Elias Beall). Am. Journ. (I) 18, p. 388; s, auch Ann. Chim. Phys. Bd. 45 , p. 417 .

I832: v. H o ff: Achter Nachtrag, Pogg.

Ann. Bd. 24, p. 227-228.

1836: Kämtz: Meteorologie, p. 297. 1836: W a rd e n : Aërolites, C. R. Bd. 3 ,

p. 5 I.

1843: P a rts c h: Meteoriten, p. 57. r848: S h e p a r d : Report on Meteorites.

- 5. Forsyth, Georgia. Am. Journ. (2) 6, p. 406-407 (Analyse).

1854: v. Boguslawski: Zehnter Nachtrag, Pogg. Ann. Ergz.-Bd. 4, p. $29-30$.

r859: H a rr is : Dissert. Gött., p. 82 . 1863: B uchner: Meteoriten, p. 53. 1863: Rose: Meteoriten, p. 85, r55. r858/65: v. Rei chen b a c h: VI 454.

IX $161,168,178$. X $359,361,363$.

XI 295, 296, 297, 300. XIII 369 Fig.

XX 623. XXV 321, 607.

1867: Go e b el: Kritische Uebersicht, Mélanges phys. chim. Bd. 7, p. 325 . 1884: M e unier: Météorites, p. 89 , $93,230,231,381$.

1885: Brezina: Wiener Sammlung, p. $177,232$.

1890: Eastm a n : Met. Astron,, p. 316. r895: Brezina: Wiener Sammlung, p. 242,244 .

I) Davon 34654 gr. Eigentum von Herrn Huntington. - 2) 900-1000 Stück. 
Ursprüngliches Gewicht: $16 \mathrm{Kgr}$. ( 36 pounds).

Nachweisbares Gewicht: 74I gr.

\begin{tabular}{lr|lr} 
Bailey & I & London, P. G. & 67 \\
Bement & 95 & Moskau & 9 \\
Berlin, U. & I9 & New Haven & I 32 \\
Budapest & 21 & Paris, M. & 10 \\
Calcutta & 3 I & Petersburg, A. & Spl. \\
Cleveland & Spl. & Pohl & 2 \\
Dorpat & I & v. Siemaschko & I \\
Göttingen & 2 & Stockholm & IO \\
Gregory & I & Troyes & 6 \\
Harvard, U. & 70 & Tübingen & 60 \\
Kunz & 34 & Washington, Sh. \\
London, B. M. & 72 & Wien, H. M.*) & 88
\end{tabular}

London, B. M. $\quad 72$ Wien, H. M.*) 88

Befindet sich die Hauptmasse des Steins in Athens, University of Georgia?

Forsyth County, Georgia, U. S. A. Eisen, bekannt 1895 (verschieden von den früheren Forsyth P od. M. r895: Brezina: Wiener Sammlung, p. 307 (nur in der chronologischen Liste erwähnt).

Ursprüngliches Gewicht: 20943 gr., welche nach Herrn Direktor Brezina's Mitteilung sich im Besitz von Herrn Kunz befinden.

Fort Duncan

Coahuila

Fort Pierre, Nebrasca, Missouri, U. S. A.

Eisen, Om, gefunden 1856 (?), nach St. Louis geschickt 1857 , erwähnt I 858 , beschr. 1860 .

1858: Chouteau presented a mass of meteoric iron $35 \mathrm{lbs}$. weight, found in Nebraska Terr, about 20 miles from Fort Pierrea. Trans. St. Louis Acad. of Sc, Bd, I (1857/6o), p. 307 .

1860: H o $1 \mathrm{~m}$ e s: Trans. St. Luuis Acad. of Sc. Bd. I $(1856 / 60)$, p. $7 \mathrm{II}-712$ (Tafel 21. Abbildung der ganzen Masse, Analyse von Prout).

1860: Shepard: Notices of several
American Meteorites. - I. Nebrasca Iron. Am. Journ. (2) 30, p. 204-205. Ref. N. J. 1862, p. 196; Kenngott, Uebersicht 1860 , p. IOI-IO2.

1860: $\mathrm{H}$ a id inger: Einige neuere Nachrichten über Meteoriten, namentlich die von Bokkeveld, New Concord, Trenzano, die Meteoreisen von Nebrasca, von Brazos, von Oregon Sitzber. Wien. Akad, Bd. 4I, p. $57 \mathrm{I}$; s, auch Jahrb. k. k. geol. Reichsanst. I860 (Verh.), p. 104.

1860: Ha id inger: Notiz über das Meteoreisen von Nebraska. Sitzber. Wien. Akad, Bd. 42 , p. $744-746$ (Analyse von Proud. Abbildung einer geätzten Platte); s, auch Am. Journ. (2) 32, p. I46; Quarterly, Journ. Geol. Soc. Bd. 17 (186r) Translations and Notices, p. 9-10; Luminous Meteors, Rep. Brit. Assoc. 186r , p. 35 (Sep.); Kenngott, Uebersicht 186r, p. 107 ; Zeitschr. f. d. ges. Naturw. Bd. I9, p. III ; Liebig-Kopp, Jahresber. I86I, p. I126; L'Institut\& Bd. 29 (I86I) Nr. 1409, p. 8, 172-173.

1862: Madelung: Ueber das Vorkommen des gediegenen Arsens in der Natur nebst den Analysen einiger neuerer Meteoriten. - Das Meteoreisen von Nebraska. Dissert. Gött., p. $39-40$.

1863: B u chner: Meteoriten, p. 196 $-197$.

1863: Rose: Meteoriten, p. 65, 153. r $861 / 65$ : v. Re i c hen b a c h: XV nо, 124, 128. XVI 261. XVII 266, 272. XVIII 487. XIX 154. XXV 437.

r884: M e un i er: Météorites, p.rr6,123. 1885: Brezina: Wiener Sammlung, p. $213,214,234$.

r893: M e unier: Révision des fers météoriques, p. 52, 56 .

r894: C o hen: Meteoritenkunde, p. 52, $72,83,93$, 106 .

1895: Brezina: Wiener Sammlung, p. 277 . 
1895: C o he n : Meteoreisen-Studien IV, Ann. Hof-Mus. Bd. 10, p. $82,86,90$.

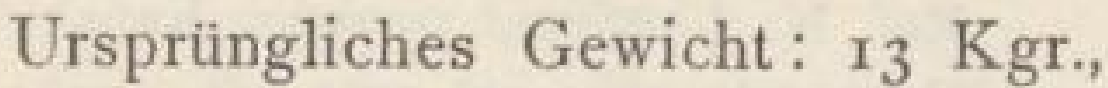
die ganze Masse wog etwa $35 \mathrm{lbs}$, wovon etwa 29 lbs. erhalten blieben.

Nachweisbares Gewicht: 7379 gr. Bement $\quad$ I732 London, B. M. $\quad 134$ Berlin, U. Brezina

Budapest 12 London, P. G. 10 Calcutta 49I New Haven 343 12 Paris, M. 4

Dorpat 30 Prag, M.

Dresden, M. II Rom, U.

Göttingen

9 Stockholm

29 Tübingen

16 Ward

Gregory

63 Wien, H. M*)

Frankfort

Francfort

Frankfort, auch Francfort, Franklin Co., Kentucky, U. S. A.

Eisen, Om, gefunden 1866, beschr. I 87 ०.

1870: Smith: Description and Analysis of the Franklin County, Meteoric Iron; with remarks on the presence of Copper and Nickel in meteoric irons; the method of analyzing the same; and the probability of the Lead in the Tarapaca iron having been originally foreign to that mass. I. The Franklin County Meteoric Iron. Am. Journ. (2) 49, p. 331 (Analyse); s, auch Origiginal Researches 1884 , p. $446-447$. I879: R a m m els b erg: Meteoriten,p.II. 1884: M e un i e r: Météorites, p. IIO, III. r885: Brezina: Wiener Sammlung, p. $21 \mathrm{I}, 2 \mathrm{I} 2,234$.

r886: H u n t in g t o n : Crystalline Structure, Am. Journ. (3) 32, p. 286. 1887: F lig ht: Meteorites, p. 147. r89o: E a s tm a n: Met. Astron., p. 320. 1893: M e unier: Révision des fers météoriques, p. 61 .

Ursprüngliches Gewicht: I I Kgr. (24 pounds).
Nachweisbares Gewicht: 8319 gr.

Bailey $\quad$ II London, B. M. 98

Bement I London, P. G. 99

$\begin{array}{llll}\text { Calcutta } & 59 & \text { New Haven } & 37\end{array}$

Göttingen $\quad 53$ Paris, M. $\quad 347$

Gregory $\quad 5$ Stockholm 53

Harvard, U. $\quad 75 \mathrm{I} 9$ 'Wien, H. M.*') 37

Frankfort, Franklin Co., Alabama, U. S. A.

Stein, Ho, gefallen 5. Dezbr. 1868 . 1869: Brush: Contributions from the Sheffield Laboratory of Yale College Nr. 2I. On the Meteoric Stone which fell Dec. $5^{\text {th }} 1868$, in Franklin Co., Alabama. Am. Journ. (2) 48 , p. 240 -244 (Abbildung des Steins; Analyse von Mixter). Ref. N. J. I87I, p. 178; Liebig-Kopp, Jahresber. 1869, p. $1302-1303$.

1870: Rammelsberg: Meteoriten, p. 140.

1879: R a m mels berg: Meteoriten, p. 26.

1883: T s cher ma k: Beitrag, Sitzber.

Wien, Akad. Bd. 88 I, p. 368.

r884: M eunier: Météorites, p. 286, 291.

r884: W a d swort h: Studies, p. 196. 1883/85: Tschermak: Photogra-

phien, p. $7-8$.

1885: Brezina: Wiener Sammlung, p. $174,232$.

1887: F light: Meteorites, p. I6r-162. r890: E a stma n: Met. Astron., p. 316. 1894: C o h e n : Meteoritenkunde,p.307.

Ursprüngliches Gewicht: 615 gr.

Nachweisbares Gewicht: 535 gr.

Bailey

Budapest 20 London, P. G. 4

Cohen

Göttingen

Gregory

Harvard, U.

Heidelberg

Kopenhagen

3 New Haven 255

Spl. Paris, M.

3 v. Siemaschko 9

2 Stockholm Spl.

I27 Strassburg 2

Spl. Ward Spl.

London, B. M. 32 Wien,H.M. * ${ }^{* *}$ ) 60 


\section{Frankfurt}

s. Anhang Nauheim

Fukutomi, Kinejima, Prov. Hizen, Japan.

Stein, Cga, gefallen 19. März r882. 1888: C 1 a r ke: New Meteorites, Am. Journ. (3) 35, p. 264. Ref. N. J. 1889 II, p. 43.

1895: Brezina: Wiener Sammlung, p. 250.

Ursprüngliches Gewicht: 7680 gr. Nachweisbares Gewicht: 34 gr.

London, B. M. $\quad 4$ Wien, H. M. 20 Washington Io

Wo befindet sich die Hauptmasse des Steins?

\section{Fulton Co.}

Rochester

\section{Fürstenberg}

Menow

Futtehpore, Allahabad, Prov. Doab, Ostindien.

Stein, Cwa, gefallen 30. Nov, 1822 . 1822? : $\mathrm{T} \mathrm{y} \mathrm{t} l$ e $\mathrm{r}$ in einer indischen Zeitschrift; s. auch Edinburgh Journ. of Sc. (1828) Nr. 15, p. 171. Uebersetzung in Sehweigg. Journ. Bd. 53 (1828), p. $47 \mathrm{I}-475$; s. ferner Monthly Magaz. Bd. 37 (I. Juli 1824), p. 553 ; Férussac, Bull. des Sc. math, Bd, II, p. 292.

1826: Chladni: Sechster Nachtrag, Pogg. Ann, Bd. 8, p. 47.

I830: v. Hoff: Siebenter Nachtrag,Pogg. Ann. Bd. 18, p. 179-181.

1832 : v. H o f : Achter Nachtrag, Pogg. Ann. Bd. 24, p. 224.

1836: K ä m tz: Meteorologie, p. 292. 1843: P a rts ch: Meteoriten, p. 142. 1851: S hepard: On Meteorites. -

1. Futtehpore Hindostan, Nov. 30, 1822. Am. Journ. (2) II, p. 36; s. auch Kenngott, Uebersicht $1850 / 51$, p. 179; Liebig-Kopp, Jahresber. 1850, p. 823. 1852: Edinburgh New. Philos. Journ. Bd. 8 (Okt, 1852), p. 245 .

1854: B a $1 \mathrm{c}$ e $11 \mathrm{~s}$ : Lithologia meteorica, p. 23 .

1854: v. Boguslawski: Zehnter
Nachtrag, Pogg. Ann. Ergz.-Bd. 4, p. $22-23$.

1857: A rago: Astronomie populaire Bd. 4 , p. 200 .

r859: A t k ins on (Verzeichnis der im Besitz der Asiatic Society befindlichen Meteoriten). Die Stelle lautet: „Nr. 2. Aërolite which fell at Allahabad, sent by Dr. Tytler, of this there are three pieces of good size, weighing 3 lbs. $8 \frac{1}{2}$ oz., 4 lbs. 3 oz., I lbs. $4 \frac{1 / 2}{2}$ oz., total 9 lbs. o oz, « Journ, Asiat. Soc. Bengal. (Proc.) Bd. 28, p. 259-260, 26I $>$ Nr. 4. Fell at Bithour and Shapur 75 (wohl Druckfehler) miles N. W. of Allahabad on the $30^{\text {th }}$ November 1822. Weight $12 \frac{1}{2} \mathrm{Oz}$, *

1859: Harr is: Dissert. Gött., p. 77. 1860: H a i inger: Die Calcutta-Meteoriten von Schalka, Futtehpore, Pegu, Assam, und Segowlee im k. k. HofMineralien-Cabinete. - I. Futtehpore, 30. November 1822. Sitzber. Wien. Akad. B. $4 \mathrm{I}$, p. $747-750$; s. auch Am. Journ. (2) 32 (1861), p. 142-143; Jahrb. k. k. geol. Reichsanst. 1860 (Verh.), p. IO4; "L'Institut « Bd. 28 (1860) Nr. 1408 , p. $422-423$.

1862: Mask el y ne: Chondritic Aërolites, Rep. Brit. Assoc. Notices and Abstracts, p. 190.

x862: v. Re i c h en b a c h: XX623,626. 1863: Ros e: Meteoriten, p. 154 .

r863: B u c h n e r : Meteoriten, P.44-45. 1884 : M e un ier: Météorites, p.208,214. 1885: Brezina: Wiener Sammlung, p. $180,232$.

1894: C o h e n : Meteoritenkunde, p.2OI. r 894 : F 1 e t ch e r: Introduction, p. ro, 28. Ursprüngliches Gewicht: Steinschauer, nicht viel erhalten.

Nachweisbares Gewicht: 3300 gr. Bailey

Bement Berlin, U. Bologna Budapest 


\begin{tabular}{lr|lr} 
Klausenburg & 20 & Paris, M. & 113 \\
Kopenhagen & 8 & v. Siemaschko & 19 \\
London, B. M. 1422 & Tübingen & 3 \\
London, P. G. & 1 56 & Wien, H. M.*) & 494 \\
Neumann & 1 & Washington, Sh. & 2 \\
New Haven & 36 & &
\end{tabular}

Galapian, bei Agen, Lot et Garonne, Frankreich.

Stein, Cwa, gefallen 25. Mai I826 (?).

(?): F ér us s a c: Bull, des Sc. naturelles Bd. II, p. 420 .

I830: v. H off. Siebenter Nachtrag,Pogg.

Ann. Bd, 18 , p. 185 .

1836: K ämtz: Meteorologie, p. 296. 1884: M eunier: Météorites, p. 208. r885: Brezina: Wiener Sammlung, p. 257.

r892: v. H a u e r : Ann. Hof-Mus, Bd, 7 (Not.), p. 73 .

r895: Brezina: Wiener Sammlung, p. 244 .

Ursprüngliches Gewicht: ?

Nachweisbares Gewicht: 56 gr.

\begin{tabular}{ll|l|l|} 
Dorpat & $9^{1}$ ) & Wien, H. M. 2 \\
Paris, M. & 45 &
\end{tabular}

\section{Galleguillos \\ Gand}

Copiapo

Gargantillo, Fomatlan (Tomatlan), Jalisco, Mexico.

Stein, Cc, gefallen August oder September $\left.1879^{2}\right)$.

r885: S he pard: On the Meteorite of

Fomatlín, Jalisco, Mexico. Am. Journ.
(3) 30, p. 105-I08. Ref. N. J. 1887 I, p. 34-35; Liebig-Kopp, Jahresber. 1885, p. $2324-2326$.

1889: C a st il1o: Catalogue, p. 13. r89o: F l e t c her: Mexican Meteorites, Mineral. Magaz. Bd. 9, p. 95.

1890: Eastman: Met. Astron., p. 316. r894: C o h en: Meteoritenkunde, p. 59. r895: Brezina: Wiener Sammlung, p. 256 .

Ursprüngliches Gewicht: Mehrere Steine, der grösste ungefähr 2 pds. Nachweisbares Gewicht: 789 gr.

\begin{tabular}{lr|lr} 
Bailey & 8 & Paris, M. & I3 \\
Bologna & 3 & v. Siemaschko & 2 \\
v. Braun & $3^{\text {I }}$ & Stockholm & 23 \\
Budapest & I6 & Washington & 4 \\
Cleveland & Spl. & Washington, Sh. & 5 II \\
Gregory & 30 & Wien, H. M. & I2 \\
London, B. M. & 136 & &
\end{tabular}

\section{Garrett Co. Lonaconing}

Garz

Schellin

Gawler Range Yardea Station

Gent Saint Denis Westrem

Gera

Politz

Ghazeepore

Mhow

Ghoordha Motecka-nugla

Gilgoin, Gilgoin Station, Neu SüdWales.

Stein, erwähnt 1889 und 1893 .

1889: Russel exhibited three new meteorites. Journ. and Proc. R. Soc. New South Wales Bd. 23. Juni r889, p. 47 .

I) in der Sammlung bezeichnet 5, IX $1814^{\star}$; also auf Agen verweisend; es wäre zu prüfen, ob der Unterschied von Cwa und Cia bei diesen beiden Meteoriten die Selbständigkeit dieses Falles unterstützen kann. Ueber das Falldatum herrscht Unsicherheit. Meunier giebt im Pariser Verzeichnis den 25. Mai 1826, v. Hoff August 1826 an. Letzterer verweist in seinem 6ten Nachtrag, Pogg. Ann. Bd. 8, p. 58, 6o, auch auf zwei Feuermeteore vom 29. Juli und 13. Sept. 1826, von denen eins vielleicht mit dem Fall von Galapian in Beziehung stehe.

2) Der Falltag scheint nicht ganz sicher bekannt zu sein. Washington und London, B. M. sagen August 1879; Paris, M. September 1879; Stockholm und Washington, Sh. 17. August 1879; Bologna, Castillo, Wien, H. M. 17. Sept. 1879. 
1893: Russe 1: On Meteorite Nr. 2 from Gilgoin Station. Proc. R. Soc. New South Wales Bd. 27, p. 36r-362.

Ursprüngliches Gewicht: $64 \mathrm{Kgr}$. ( 67 pounds und 74 pounds $5 \mathrm{oz}$.). Nachweisbares Gewicht: 8 gr.

$$
\text { Gregory }
$$$$
8
$$

Wo befindet sich die Hauptmasse des Steins?

\section{Gilpin Co.}

Russel Gulch

Girgenti, Sicilien, Italien.

Stein, Cwa, gefallen ro. Febr. 1853 .

r854: Gr e g : Catalogue, p.460 ( $>$ A large stone" ).

1859: H a rris : Dissert. Gött., p. 95.

r859: v. Reichenbach: IX 162, 168, 178. XI 308 .

? vor 1862: Gemmellaro in einem sicilischen wissenschaftlichen Journal? 1862: Greg: On some Meteorites in the British Museum. Philosoph Magaz. Bd. 24, p. 538 . Ref. Kenngott, Uebersicht $1862 / 65$, p. 442 .

r863: B u c h n er: Meteoriten, p. $84-85$. r869: vom R a t h: Mineralogische Mitteilungen. 43. Ueber den Meteoriten von Girgenti. Pogg. Ann. Bd. 138, p. $54 \mathrm{I}-545$ (Analyse). Ref. Liebig-Kopp, Jahresber. 1869, p. 1299-1300.

1873: M e un i e r : Exercices des actions mécaniques dans l'astre, aujourd'hui détruit, d'où dérivent les météorites. C. R. Bd. 76, p. 109.

I875: vom R a th: Meteoriten, Verh. naturh. Ver. Bonn Bd. 32, p. 368 .

1883: T s c hermak: Beitrag, Sitzber. Wien. Akad. Bd. 88 I, p. 355 .

1884: Me uni er: Météorites, p. 196 (Abb.), 197, 378, 525 (Abb.).

$1883 / 85$ : T's c h e r mak: Photographien, p. 18.

r885: Brezina: Wiener Sammlung, p. $181,232$.

r887: F lig h t: Meteorites, p, 123-r24. UrsprïnglichesGewicht: Mindestens
2 Steine. Greg sagt »a large stone«. Buchner sagt der Stein wog $3-4$ Kgr.

Nachweisbares Gewicht: $33^{89}$ gr.

\begin{tabular}{lr|lr} 
Bement & I & Neapel & 65 \\
Berlin, U. & 489 & Neumann & 2 \\
Bonn & 38 & Paris, M. & 474 \\
Budapest & 71 & Pohl & 208 \\
Calcutta & 23 & Rom, U. & 1104 \\
Dorpat & 51 & v. Siemaschko & 6 \\
Dresden, M. & 87 & Stockholm & 47 \\
Frankfurt & 20 & Strassburg & 19 \\
Göttingen & 28 & Tübingen & Spl. \\
Gregory & 46 & Turin, J. & 284 \\
Harvard, U. & 20 & Ward & Spl. \\
Kopenhagen & 19 & Washington, Sh. \\
London, B. M. 233 & Wien, H. M.*) & 18 \\
London, P. G. & 13 & Wien, U. I. & I8 \\
Lüttich & 4 & &
\end{tabular}

\section{Glasgow}

Glindorcha

High Possil

Indarch

Glorieta, Glorieta Mountain, Santa Fé Co., Neu-Mexico, U. S. A.

Eisen, Om, gefunden und erwähnt r 884 , beschr. I 885 .

Hieher auch: Cañon City, Trinity Co., bekannt um 1875 , beschr. I885; ferner Albuquerque, NeuMexico, gefunden um 1884 , beschrieben 1885 .

1884: Pearce exhibited a specimen of meteoric iron u. s. w., kurze Bemerkung. Proc. Colorado Scientific Soc. Denver Bd. I (1884), p. 110.

1885: Shepard: On Meteoric Iron from Trinity County, California. Am. Journ. (3) 29, p. 469 (Analyse). Ref. N. J. 1886 II, p. $35^{8}$; Liebig-Kopp, Jahresber. 1885, p. 2324.

1885: Kunz: On three Masses of Meteoric Iron from Glorieta Mountain, near Canoncito, Santa Fe County, New Mexico. Am. Journ. (3) 30, p. 235$23^{8}$ (Mit 4 Tafeln und einer Umriss- 
zeichnung. Analyse. Gewicht : $145 \mathrm{Kgr}$.); s. auch Ann. N. Y. Akad. Sc. Bd. 3 (1885), p. 329-334. Ref. N. J. 1887 I, p. 34 ; Liebig-Kopp, Jahresber. $\mathbf{1} 885$, p. $2324-2325$.

r885: Eakins: Meteoric Iron from New Mexico. Proc. Colorado Scientific Soc. Denver Bd. 2 (1885), p. $14 ;$ s. auch im gleichen Band die Bemerkung von Whitman Cross über die Arbeit von Kunz, s. o. 1885 .

1886: $\mathrm{K}$ unz: Further notes on the Meteoric Iron from Glorieta Mt., New Mexico. Am. Journ. (3) 32, p. $3 \mathrm{II}-$ $3^{1} 3$ (Mit 3 Tafeln. Analyse von Eakins. Gewicht der Massen : 1.204 Kgr., I.I26 Kgr. und I.05 Kgr.). Ref. N. J. I887 II, p. 46; Liebig-Kopp, Jahresber. 1886, p. $2334-2335$.

r886: H u n t in g t on : Crystalline Structure, p. 295, 299.

1886: Brezina: Neue Meteoriten I, Ann. Hof-Mus. Bd. I (Not.), p. I3 u. Bd.2 (Not.), p. 25, 26; s, auch Döll. Jahrb. k. k. geol. Reichsanst. Bd. 37 (1887), p. 196.

1887: B rez in a: Neue Meteoriten IIIa, Verh. k. k. geol. Reichsanstalt (1887), p. 288 .

1887: v. Hauer: Ann. Hof-Mus, Bd, 2 (Not.), p. 38 ; u. Bd. 6 (Not.) (1891), p. 54 .

I890: Ea s tm a n : Met. Astron., p. 320, 322.

r89r: Cohen und Weinschenk: Meteoreisen-Studien, Ann. Hof-Nus. Bd. 6, p. $131,132,155-158$ (Analyse), $162,164,165$.

r892: C o hen: Meteoreisen-Studien II, Ann. Hof-Mus. Bd. 7 , p. 143-145 (Analyse), $158(\mathrm{Cu}), 159,160,161$.

r893: Brez in a: Ueber neuere Meteoriten (Nürnberg), p. 164.

1893: Meunier: Révision des fers météoriques, p. 52, 59 .

I) Ausserdem 152 gr. nach I. Juli 1893 . r894: Coh en: Meteoritenkunde, an vielen Stellen.

1894: F l e t c h e r : Introduction, p. I4. r895: B rez in a : Wiener Sammlung, p. $280-282$.

r895: Co hen: Meteoreisen-Studien IV, Ann. Hof-Mus. Bd, 1o, p. $82,85,86$, 9o, 9I, 92, 93 .

Ursprüngliches Gewicht: Glorieta etwa 148 Kgr., Trinity Co. etwa $8 \%$ Kgr. (i9 lbs.), Albuquerque $2.5 \mathrm{Kgr}$.

Nachweisbares Gewicht : r45 ror gr. Bailey

Belgrad

Bement

Berlin, U.

Böhm

Bonn

v. Braun

Brezina

Budapest

Cleveland 56I Lüttich 65

Dresden, M. 213 de Mauroy 65

Dublin, M. 3790 Neapel 12 9868 New Haven 3970 27 NewYork, M, 24926 267 Paris, E. $\quad 1802$ 687 Paris, M. 21740 2530 Petersburg, B. 3915 342 Prag, M. 296 37 Rom, U. $\quad 235$ 125 v. Siemaschko 1066 2165 Strassburg $\quad \mathbf{1 6 5}$ 80 Stürtz 40

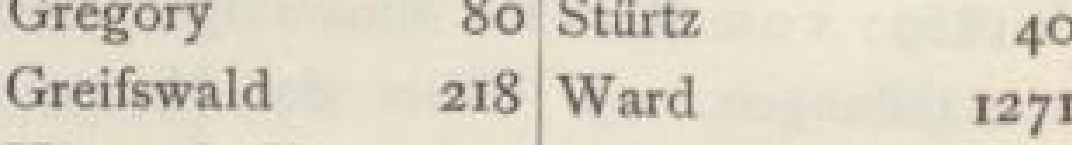
Harvard, U. $\quad 913$ Washington 380 Krantz $\quad 30$ Washington,Sh. 853 Kunz I5 Wien,H.M. 60778 ${ }^{1}$ ) London, B. M. 1588

Ausserdem Trinity County: Bailey $16 \mathrm{gr}$., Gregory Spl., Paris 3 gr. (unter »Syssidères und Oligosidèress aufgeführt), v. Siemaschko I gr., Washington 4 gr., Washington, Sh. 22 gr.; Albuquerque: Washington $63 \mathrm{gr}$.

Gnadenfrei, auch Schobergrund, preussischSchlesien,Deutschland. Stein, Cc, gefallen 17 . Mai 1879 . 1879: G a 11 e: Mitteilungen über das seltene Ereigniss des am 17. Mai stattgehabten Meteorsteinfalles bei Gna- 
denfrei. Jahresber. schles. Ges. f. vaterl. Cult. Bd. 37 , p. 166, 169. Ref. N. J. I88o I, p. 46-47; Liebig - Kopp, Jahresber. 1879 , p. 1274-1275.

1879: v. Las a u 1x: Die mineralogische Beschaffenheit der Gnadenfreier Meteorite. Jahresber. schles. Ges. f. vaterl. Cult. Bd. 37 , p. $169-170$.

1879: Ga 11 e u. v. L a s a u $1 x$ : Bericht über den Meteorsteinfall am 17. Mai 1879. Mon. Ber. Berlin. Akad., p. 750 $-77 \mathrm{r}$ (mit 2 Tafeln, Analyse). Ref. N. J. I880 II, p. $162-164$; s. auch $\times$ Naturforscher * $1879 \mathrm{Nr} .52$; Wochenschr. f. Astr., Met. u. Geogr. 188o, p. II3-II6. r880: K 1 e i n: Vermehrung, Gött. Gel. Anz., p. 567 .

1882: v. Las a $1 x$ : Vermehrung, Verb. naturh. Ver. Bd. 39, p. $\mathbf{1 0 I - 1 0 2 . ~}$

1883: Kunisch: Die Meteoriten unter besonderer Berücksichtigung der schlesischen. Vortrag, gehalten am 2I. Jan. ${ }_{188}$ im Humboldt -Verein zu Breslau (Sep.), p. 6 u. 8.

1884: Häpke: Beiträge, Abh, naturw.

Ver. Bremen Bd. 8 , p. 523 .

r884: W a d s w or th: Studies,p.9I -92. 1885: Brez in a: Wiener Sammlung, p. $156,185,233$.

r883/85: Ts chermak : Photographien, T. 13 , p. 14 .

r894: C o h e n: Meteoritenkunde, p. 7I, 206, 26I, 283 .

Ursprüngliches Gewicht: 2 Steine von I $\mathrm{Kgr}$. und $\mathrm{I} \frac{1}{2}$ Pfund.

Nachweisbares Gewicht: 1059 gr.

\begin{tabular}{lr|lr} 
Berlin, G. & 12 & Göttingen & I \\
Berlin, U. & I4 & Gregory & 6 \\
Bologna & 13 & London, B. M. & 54 \\
Bonn & 4 & Neumann & 3 \\
v. Braun & I & Paris, M. & 2 \\
Breslau & 79I & v. Siemaschko & 18 \\
Budapest & 51 & Wien, H. M. & 89
\end{tabular}

\section{Gnarrenburg Bremervörde \\ Goalpara (Assam), Ostindien.}

W ulfing, Meteoriten.
Stein, Cu, gefund.r 868 , beschr. 1869. 1869: v. Haidinger: Der Meteorit von Goalpara in Assam; nebst Bemerkungen über die Rotation der Meteoriten in ihrem Zuge. Sitzber. Wien. Akad. Bd. 59 II, p. $665-678$ (mit Tafel u. einem Holzschnitt); s. auch *L'Institut \& Bd. 37 (1869), p. 256.

1870: v. Ha i d inger: Orientierung, Sitzber. Wien. Akad. Bd. 6I II, p. $5^{\mathrm{I}} 3$. r870: $\mathrm{T}$ e $\mathrm{c} 1 \mathrm{u}$ : Chemische Untersuchung des Meteoriten von Goalpara, Assam (Indien), Sitzber. Wien. Akad. Bd. 62 II, p. $852-854$ (Analyse). Ref. N. J. I871, p. $4 \mathrm{I} 3$.

r870: Ts chermak: Ueber den Meteoriten von Goalpara und über die leuchtende Spur der Meteore. Sitzber. Wien. Akad. Bd, 62 II, p. $855-86 \mathrm{I}$ (mit Tafel, Ankündigung im gleichen Band, p. 850). Ref.N.J.1871,p.412-414. 1872: Tschermak: Die Meteoriten von Shergotty und Gopalpur. Sitzber. Wien. Akad. Bd. 65 I, p. 139.

1877: Flight: Meteorites and the origin of life. The Popular Science Review 18. Okt. 1877, p. 10 (Sep.).

1879: R a m me 1s berg: Meteoriten, p. II, 24.

r882: Brez in a : Bericht IV, p. 336 , $338,339$.

1884: W a d s w or th: Studies, p. 94. r884: M e un ier: Météorites, p. 79.

r883/85: Ts chermak: Photographien, T. 20, p. $3,19$.

1885: Brezina: Wiener Sammlung, p. $184,233$.

1887: Flight: Meteorites, p. I62-I64. r894: Co hen: Meteoritenkunde, p. 25, $59,158,163,167,260,317$.

r894: F letcher: Introduction, p. 28. r895: Brez in a: Wiener Sammlung, p. $239,252,254$.

Ursprüngliches Gewicht: ${ }_{2}^{26} 6 \mathrm{gr}$. (5 lbs. I3 oz. 2.5 grs.).

Nachweisbares Gewicht: $2166 \mathrm{gr}$. 
Bement

Calcutta

Gregory

Harvard, U.

London, B. M. $\quad$ 1187

Goldbach-Eisen Humboldt-Eisen Gopalpur, Jessore, Bengalen, Ostindien.

Stein, Cc, gefallen 23. Mai 1865 . 1865: Bábu Gour Doss Bysack (Fallbericht). Proc. Asiatic Soc. Bengal (1865), p. $94-96$.

r866: O ld ham: Katalog von Calcutta, p. 8, die Asiatic. Soc. of Bengal besitze ein Stück von 3 lbs. 9 oz. 259 grs.

r869: Buchner: Vierter Nachtrag, Pogg. Ann. Bd. 136, p. 455 .

1872: Exner: Chemische Untersuchung des Meteoriten von Gopalpur. M. M. Bd. 2, p. 4I-43. Ref. Liebig-Kopp, Jahresber. 1872, p. I196-II97.

r872: Tschermak: Die Meteoriten von Stannern, Constantinopel, Shergotty u. Gopalpur. M. M. Bd. 2, p. 95 - 100 (Analyse von Exner s. o.).

1872: Tschermak: Die Meteoriten von Shergotty u. Gopalpur. Sitzber. Wien. Akad. Bd. 65 I, p. 135-145 (mit 4 Tafeln, darunter 3 Abbildungen des Steins. Ankündigung des Aufsatzes im gleichen Band, p. II9). Ref. N. J. 1872, p. $733-734$

1879: R a m mels berg: Meteoriten, p. II, 24,25 .

1884: W a d swort h: Studies, p. 92. r884: M e unier: Météorites, p. 76, 79, 230, 234, 256.

r885: Brezina: Wiener Sammlung, p. $165,185,233$.

r887: F $1 \mathrm{ig} \mathrm{h} \mathrm{t:} \mathrm{Meteorites,} \mathrm{p.} \mathrm{I4I-142.}$ r894: C o hen: Meteoritenkunde, p. 6o, 202, 304, 310.

r894: F 1 et c h e r: Introduction, p. 13. Ursprüngliches Gewicht: ${ }^{6} 634$ gr. (3 lbs. 9 oz. $3^{1} 3$ grs.).
Nachweisbares Gewicht: ${ }_{1} 5^{82} \mathrm{gr}$.

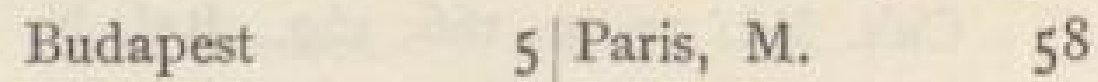
$\begin{array}{llll}\text { Calcutta } & 1214 \text { Wien, H. M.*) } & 158\end{array}$ London, B. M. 147

Goruckpur $185^{2}$

Bustee

Goruckpur 1861

Goruckpur 1865

Goruckpur 1866

Butsura

Goruckpur 1878

Supuhee

Goskowo

Pokra

\section{Gotha}

Dandapur

Gouch

Pultusk

Tabarz

\section{Gran Chaco Gualamba}

Campo del Cielo

Grand Rapids (Walker Township), Michigan, U. S. A.

Eisen, Of, gefd. $188_{3}$, beschr. 1884 . 1884: Eastman: A New Meteorite, Am. Journ. (3) 28, p. 299-300 (Analyse). Ref. N. J. 1886 I, p. 32 ; LiebigKopp, Jahresber. 1884 , p. $2042-2043$. r885: Brezina : Wiener Sammlung, p. 257 .

r885: Riggs: The Grand Rapids Meteorite. Am. Journ. (3) 30, p. 312 (Analyse). Ref. N. J. 1887 I, p. 33 ; Liebig-Kopp, Jahresber. 1885, p. 2324. r887 : v. $\mathrm{H}$ a u er: Ann. Hof-Mus. Bd, 2 (Not.), p. $3^{8}$.

1888: Riggs: On two new Meteoric Irons and an Iron of doubtful nature: The Grand Rapids Meteorite. Bull. U.S. Geol. Surv. (1888) Nr. 42, p. $94-95$ (Abb. des Eisens und einer geätzten Platte). Ref, N, J. I890 I, p. 4 I. 1890: Eas tm a n: Met. Astron., p. 320. r $89 \mathrm{r}$ : v. H a u e r : Ann. Hof-Mus. Bd. 6 (Not.), p. 54.

1893: Meunier: Révision des fers météoriques, p. 76.

r894: Co hen: Meteoritenkunde, p. 54 ,

$73,95,153$.

1895: Brez in a : Wiener Sammlung, p. 270 . 
r895: C o h en : Meteoreisen-Studien IV. Ann. Hof-Mus. Bd, 1o, p. $83,84,85,90$. Ursprüngliches Gewicht: $5^{1}{ }^{1 / 2} \mathrm{Kgr}$. (II4 lbs.).

Nachweisbares Gewicht : 16834 gr. ${ }^{1}$ )

\begin{tabular}{lr|lr} 
Bailey & 47 & de Mauroy & 159 \\
Bement & 1012 & New Haven & 638 \\
Böhm & 180 & Newton & 15 \\
Bologna & 7 & Paris, E. & 29 \\
v. Braun & 1208 & Paris, M. & 22 \\
Brezina & 481 & Pohl & 129 \\
Budapest & 209 & Prag, M. & 102 \\
Cleveland & 46 & Rom, U. & 81 \\
Gregory & 18 & Ward & 9041 \\
Greifswald & 57 & Washington & 1500 \\
Harvard, U. & 56 & Washington, Sh. 34 \\
London, B. M. I146 & Wien, H. M. & $617^{2}$ )
\end{tabular}

\section{Grasse}

La Caille

Grazac, Grazac und Montpelegry, Dep. Tarn, Frankreich.

Stein, K, gefallen ro. August 1885 . 1887: Caraven-Cachin: Sur un essaim météorique tombé le to août 1885, aux environs de Grazac et de Montpelegry (Tarn). C. R. Bd. 104, p. $1^{181} 1_{3}-1814$. Ref. N. J. 1888 II, p. $226-227$.

1887: Daubrée u. Meunier: Observations sur la météorite de Grazac ; type charbonneux nouveau qu'elle représente. C. R. Bd. 104, p. 1771-1772. Ref. N. J. 1889 I, p. 59.

1894: C o h e n : Meteoritenkunde, p. 235.

Ursprüngliches Gewicht: 20 Steine, der grösste 600 gr., wenig erhalten.

Nachweisbares Gewicht: $22 \mathrm{gr}$.

Paris, M. 22 gr. sMétéorite charbonneuse très douteuse .

\section{Great Fish River, Südafrika.}

Eisen ( $\mathrm{Hb}$ ?), gefunden 1836 , beschr. I 838 .

Hierher auch: Great Namaland z. T.; ferner Springbock River, Namaland.

1838: Sir Alexander: An Exp. of Dis. Inter. Africa (countries of the Great Namaquas, Boschmans and Hill Damaras) Bd.2 (1838), Appendix,p.272 ; s. auch Journ. Roy. Geogr. Soc London Bd. 8 ( 1838$)$, p. 24 .

r839: Herschel: Notice of a Chemical Examination of a Specimen of Native Iron, from the East Bank of the Great Fish River in South Africa. Philos. Trans. Bd. 14, p. 32-34; s, auch Pogg. Ann. Bd. 46 (1839),p.n16: Untersuchung eines gediegenen Eisens vom Ostufer des grossen Fischflusses in Südafrika; s. ferner Ann. Chem. Pharm. Bd. 31 (1839), p. 239; Bibl. univers. Avril 1839 , p. 189 .

1854: v. Bogus lawski: Zehnter Nachtrag, Pogg.Ann. Ergz.-Bd. 4, p.398. r856: Burkart: Fundorte I, N. J. 1856, p. 299.

I858/59: v. Re ic he n b a c h: VII 557. VIII 486. XVII 273. XIX I50, $_{555}$. 1859: B u c hn er: Feuermeteore, p.r28. r860: R a m mels berg: Mineralchemie, p. 920 .

r862: Kenngott u. W iser: Zürcher Sammlung, p. 144 (das Zürcher Stück sei wahrscheinlich Krasnojarsk). r863: B u chn er: Meteoriten,p.147-148. 1872: Quenstedt: Klar und Wahr, p. $3^{15}$ (Abb. einer geätzten Platte).

1885: Brezina: Wiener Sammlung, p. 257 .

1890: F l e t ch e r: Mexican Meteorites, Mineral. Magaz. Bd. 9, p 94.

1) 1889 war die Hauptmasse dieses Eisens im Gewicht von 47650 gr. im NationalMuseum, Washington, deponiert. Inzwischen wurde es, nach Mitteilung von Herrn F. W. Clarke, von dem Besitzer an Herrn Ward verkauft. Wo befindet sich gegenwïrtig die Hauptmasse? - 2) Ausserdem noch 89 gr. nach 1. Juli 1893 erworben. 
r893: Meunier: Révision des fers météoriques, p. 74 .

1894: F l e t c h e r : Introduction, p. I3.

Ursprüngliches Gewicht: Viele grosse Massen ?

Nachweisbares Gewicht: I530 gr. Bailey

Calcutta

Göttingen

Gregory

12 London, B.M. $1469^{\prime}$ )

2 v. Siemaschko Spl. ${ }^{2}$ )

I4 Tübingen

22

Great Namaland $18{ }_{3} 6$

Great Fish River

Great Namaland $185^{2}$ Lion River

Greenbrier County, 3 miles nördlichWhite SulphurSprings, Greenbrier Co., West-Virginia, U. S. A.

Eisen, $\mathrm{Og}$, gefunden um $\mathrm{r} 880$, erwähnt 1885 (oder früher in einem Londoner Katalog), beschr. 1887 . r885: Brezina: Wiener Sammlung, p. 257 .

1887: F let cher: On a Meteoric Iron (containing Crystallised Chromite) found about the year 1880 in Greenbrier County, West Virginia, U. S. A. Mineral. Magaz. Bd. 7, p. 183-186. Ref. Zeitschr. f. Kryst. Bd. 14 (1888), p. 486; N. J. 1889 II, p. $4 \mathrm{I}-42$.

1894: Co hen : Meteoritenkunde, p. 53, $58,63,68,246,248$.

1894: Huntington: The Smithville Meteoric Iron, Proc. Amer. Acad. Arts and Sc. Bd. 29, p. 259 (Situationsplan von Tennessee bis West-Virginia).

r895: Brezina: Wiener Sammlung, p. 286.

Ursprüngliches Gewicht: Etwa II Pfund.

Nachweisbares Gewicht: 2302 gr. Calcutta

Gregory 33 London, B. M. 2236 22 Washington
Green Co.

Grimma

Griqua Eisen 1855

Griqua Stein 1868

Grodno

Babb's Mill

Steinbach

Orange River

Danielskuil

s. Anhang

Grosnaja (Mikenskoi), am Fluss

Terek, Kaukasus, Russland.

Stein, Cs, gefallen 28. Juni r86r.

r862 : R o s e berichtete nach einer Mitteilung des Hrn. Staatsrat Dr. Abich in Tiflis von einem neuen Meteorsteinfall im Caucasus, der sich auf der Mekenskischen Staniza bei Grosnja an den Ufern des Terek am 16. Juni Morgens 7 Uhr des vorigen Jahres ereignet hatte. Mon. Ber. Berlin. Akad. (1862), p. 186; Ref Kenngott, Uebersicht $1862 / 65$, p. 451 ; Liebig-Kopp, Jahresber, 1862, p. 832 .

r863: B u chner: Meteoriten. - Nachträge, p. 201.

1865: Buchner: Zweiter Nachtrag, Pogg. Ann. Bd. 124, p. 572.

1867: Geinitz: N. J. 1867, p. 724. 1878: M a kows ky u. Ts chermak: Bericht über den Meteoritenfall bei Tieschitz in Mähren. Denkschr. Wien. Akad, Bd. 39, p. 196.

1878: Ts c herm a k: Der Meteorit von Grosnaja. M. P. M. Bd, I, p. 153-r64 (Analyse von Ludwig u. Tafel mit Abbildungen von Dünnschliffen). Ref. N. J. I878, p. 868-869; Liebig-Kopp, Jahresber. 1878 , p. $1320-\mathbf{r} 321$.

1879: R a m mels berg : Meteoriten, p. $12,24$.

1882: Brezina: Bericht IV, Sitzber. Wien. Akad. Bd, 85 I, p. $33^{8}, 339$. 1883: Ts c h ermak: Beitrag, Sitzber.

Wien. Akad. Bd. 88 I, p. 356.

1884: W a d s worth: Studies, p. IO2. r884: M e un i er: Météorites, p. 79, 193.

I) Hierunter: Great Fish River 20 gr., Great Namaland 1440 gr., Springbock River 9 gr.

2) Gehört nach Brezina (Ann. Hof-Mus. Bd, ro, p. 277) wahrscheinlich zu Orange River.

3) Von London, B. M. erhalten, 
r883 85 : T s c h e r m a k: Photographien, T. 20, p. $3,14,19,20$.

r885: Brezina: Wiener Sammlung, p. $155,184,233$.

r887: F $1 \mathrm{i}$ g h t: Meteorites, p. $182-185$. 1893: B r e z in a: Ueber neuere Meteoriten (Nürnberg), p. 16o.

r894: C o h e n : Meteoritenkunde, p. 49, $163,167,202,298$.

Ursprüngliches Gewicht: $3980 \mathrm{gr}$. 10 Pfund 8 Unzen 3 Drachmen $=61626$ gran Medizinalgewicht. Wurde in zwei Stücke zerschnitten, welche nach Tschermak 1970 u 1482 gr. $=345^{2}$ gr. wogen.

Nachweisbares Gewicht: 3257 gr.

Berlin, U.

Böhm

v. Braun

Brezina

Budapest

Calcutta

Dorpat

Gregory

Greifswald

Harvard, U.

Krantz

57 London, B. M. 160

9 de Mauroy II

188 Moskau 2

2 Paris, M.

44

177 Petersburg, A. 1954 Spl. Pohl

25 Rom, U.

75 v. Siemaschko

6 Stuttgart 142

7 Washington 4

Spl. Wien, H. M.*) 343

Seit 1. Juli 1893 erwarb Herr Bayet, Brüssel, 23 gr.

\section{Gross-Divina , Trentsiner Com.,} Ungarn.

Stein, $\mathrm{Cc}$ (von Tschermak als $\mathrm{Cg}$ bestimmt), gefallen 24 . Juli 1837 .

1840: $\mathrm{Z}$ ipser: Briefwechsel, N. J. 1840 , p. $89-90$.

1843: P a rts c h : Meteoriten, p.79-8o. 1854: v. Boguslawski: Zehnter Nachtrag, Pogg. Ann. Ergz.-Bd. 4, p. $356-357$.

r859: Harris : Dissert. Gött., p. 85 . 1860: Haidinger: Eine Leitform der Meteoriten, Sitzber. Wien. Akad. Bd. 40 , p. $530-533$ (4 Abbildungen des Steins auf Taf. 2). Ref. Luminous
Meteors, Rep. Brit. Assoc. 186r (Sep.), p. 33; Kenngott, Uebersicht 1859 , p. 200; 'L'Institute Bd. 28 (I860), p. 192 ; Liebig-Kopp, Jahresber. 1860, p. 844 ; s. auch Döll, Jahrb. k. k. geol. Reichsanst. Bd. 37 (1887), p. 195, 196-197.

r858/62: v. Re ic hen bach: V 475, 477. VI 456. IX 16I, 169, 179. XI 295, 297. XII 454. XX 626.

1863: B u chner: Meteoriten, p. 59. 1869: v. Haidinger: Der Meteorit von Goalpara in Assam, nebst Bemerkungen über die Rotation der Meteoriten in ihrem Zuge. - 4. Gross-Divina in berichtigter Orientierung. Sitzber. Wien. Akad. Bd. 59 II, p. $672-675$ (4 Abbildungen des Steins auf Taf. 2). r870: v. H a i d inger: Orientierung, Sitzber. Wien. Akad. Bd. 6r II, p. 513. r883: T s c h e r m a k: Beitrag, Sitzber.

Wien. Akad. Bd. $88 \mathrm{I}$, p. 355 .

1884: Meunier: Météorites, p. 95, $187,37 \mathrm{I}$.

r883/85: T s c h e rm a k: Photographien, p. 18 .

1885: Brezina: Wiener Sammlung, p. $182,232$.

r895: Brezina: Wiener Sammlung, p. 249,255 .

Ursprüngliches Gewicht: Fin Stein von etwa $101 / 2 \mathrm{Kgr}$.(19WienerPfd.). Nachweisbares Gewicht: 10 715 gr. Böhm 5 Paris, M. 260 Budapest $\quad$ I0 360 v. Siemaschko 3 \begin{tabular}{ll|ll} 
Calcutta & I & Tübingen & 4
\end{tabular} London, B. M. 3 Wien, H. M.*) 64 Neumann 15

Grossliebenthal bei Odessa, Gouv. Cherson, Russland.

Stein, Cwa, gefallen r9. Nov. r88r. 1884: Da ubrée: Météorite tombée à Grossliebenthal, près d'Odessa, le $7 / 19$ novembre 1881. C. R. Bd. 98 , p. 323 -324. Ref. N. J. 1885 I, P. 33 ; Liebig-Kopp, Jahresber. I884, p. 2039. 
1884: M eunier: Météorites, p. 209. 1885: Brezina: Wiener Sammlung, p. $180,182,232$.

I893: Melikoff u. Schwalbe: Chemische Untersuchung des Meteoriten von Grossliebenthal. Ber. d. d. chem. Ges. Bd, 26 I, p. 234-24I.

1893: Prende 1: Petrographische Untersuchung des Meteoriten Grossliebenthal. Odessa 1893. (Analyse und Abbildung des Steins. Russisch).

1894: C o h en : Meteoritenkunde, p.205, 223, 237.

Ursprüngliches Gewicht: Ein Stein von $8 \mathrm{Kgr}$. (Ein zweiter Stein, bei Sitschowska gefallen, wurde zerstückelt und verschleppt).

Nachweisbares Gewicht: $8048 \mathrm{gr}$.

Bement

Bologna

v. Braun

Budapest

Dorpat

Gregory

Kasan

Kiew

$$
\text { ro Odessa }
$$

Ior Paris, M.

7360 27 v. Siemaschko 24 Stockholm Spl. 27 Stürtz

39 Troyes

49 Washington, Sh, 8

Lond on, B. M. 62

Grüneberg, Preussisch Schlesien, Deutschland.

Stein, Cga, gefallen 22. März 1841. I84I : >Aus der Breslauer Zeitung \& : Meteorsteinfall bei Grüneberg in Schlesien. Pogg. Ann. Bd. 52, p. $495-496$.

I84I : G lo cker: Schles. Prov.-Blätter April I84I; vgl. ferner Breslauer Ztg. Nr. 174 (I840); Jahresber. Schles. Ges. 1841, p. 52 ; Berzelius, Jahresber. Bd. 22, p. 216 u. Bd. 24 , p. 329 ; Journ. prakt. Chem. Bd. 23 (184I), p. $285-287$.

r84x: Weimann: Niederfall eines Meteorsteins, Pogg. Ann. Bd. 53, p. 172-179 (hier auch Abdruck des Ber. von Glocker aus den Schles. Prov,Blättern vom April 184I). Ref. N. J. I844, p. 107; s, auch sL'Institute Bd. Io $(1842)$, p. 40
184 I : Göppert: Ueber den Meteorstein, welcher zu Schloin in Schlesien am 22. März $184 \mathrm{I}$ gefallen ist. Ref. N. J. 1844, p. 609-6ro (nach der Breslauer Zeitg. vom 9. Juni $184 \mathrm{r}$ ).

I843: P a rts c h: Meteoriten, p.68-69. 1854: v. Boguslawski: Zehnter Nachtrag, Pogg. Ann. Ergz.-Bd. 4, p. $106-107,36 \mathrm{I}-362$.

1859: B u c hner: Feuermeteore, p. 93. 1859: Harris: Dissert. Gött., p. 87 . 1863: Buchner: Meteoriten, p. 65 -66 .

1863: R os e: Meteoriten, p. 88, 92, 147, I54.

1858/65: v. Reichen bach: V 476. VI 441. IX 162, 171, 180. XI 297, 300. XII 454. XX 621, 626. XXV 422.

1883: $\mathrm{K}$ u n is c h : Die Meteoriten unter besonderer Berücksichtigung der schlesischen. Vortrag gehalten am 21. Jan. I883 im Humboldt-Verein zu Breslau (Sep.), p. 3 .

1885: Brezina: Wiener Sammlung, p. $182,233$.

1894: Cohen: Meteoritenkunde, p. 201. UrsprünglichesGewicht: Etwa $x \mathrm{Kgr}$. (2 Stücke: 1 . 30 Unzen 2 Drachmen 2 Scrupel, 2. 6 Unzen $1 \frac{1}{2}$ Drachmen).

Nachweisbares Gewicht: 97 r gr.

\begin{tabular}{lr|l|r|} 
Bailey & Spl, & Gregory & 8 \\
Bement & Spl & London, B. M. & 31 \\
Berlin, U. & 757 & London, P. G. & 2 \\
Breslau & 6 & Paris, M. & 4 \\
Budapest & 4 & v. Siemaschko & 12 \\
Calcutta & 20 & Tübingen & 108 \\
Dorpat & I & Wien, H. M.*) & 17 \\
Göttingen & I & &
\end{tabular}

\section{Guadalupe (Karawinsky)}

Rancho de la Pila

Guareña, Provinz Badajoz, Spanien. Stein, Ck, gefallen 20. Juli 1892 . r892: Calderón: Caida de un meteorito en Guareña (Badajoz). Actas 
de la Sociedad española de Hist nat. (2) I (Sep.) 2 Seiten.

1892: Gredilla: Meteorites, p. 118II9.

1893: Calderón u. Quiroga: Estudio Petrographico del Meteorito de Guareña, Badajoz. Anal. de la Soc. Esp, de Hist. Nat. Bd. 22, p. 127136 (mit 4 Tafeln, Abbildung des Steins und 6 Mikrophotographien). Ref. N. J. I895 II, p. 3 I.

1895: B rezin a: Wiener Sammlung, p. $26 \mathrm{r}$,

1896: C o h e n : Die Meteoriten von Laborel und Guareña. Ann. Hof-Mus. Bd. II, p. $36-38$.

UrsprünglichesGewicht:ZweiStücke von einem etwa $32 \mathrm{Kgr}$. schweren Steine (1. dos arrobas y quince libras, 2. 7200 gr.).

Nachweisbares Gewicht: Antonio Cánovas del Castillo besitzt das grössere Stück, La Comisión de Monumentos de Badajoz das kleinere Stück.

Ausserdem: Cohen 26 gr., Sevilla 60 gr., Wien, H. M. 9 gr.

Guěa, Serbien.

Stein, gefallen 28. September $\mathbf{r} 89 \mathbf{r}$. r89r: >Neue freie Presse * vom 14. Oktober 1891 .

Ursprüngliches Gewicht?

Nachweisbares Gewicht: 1915 gr. Belgrad (1 Stück) 1915 gr.

\section{Guernsay Co. New Concord}

Guilford Co. (auch Randolph Co.), Nord-Carolina, U. S. A.

Eisen, Om, gefunden?, beschr, 1822 . 1822: $01 \mathrm{mstedt}$ : Descriptive Catalogue of Rocks and Minerals collected in N. Carolina, and forwarded to the American Geological Society. - 59. Native Iron. Am. Journ. (I) 5, p. 262.
1830: S h e p a r d : On crystallized Native Terrestrial Iron, Ferro-Silicate of Manganese, and various other American Minerals, I. Crystallized Native Terrestrial Iron. Am. Journ. (I) 17, p. $140-142$; s. auch Liebig-Kopp, Jahresber. I847/48, p. I3II.

r84 $\mathrm{r}$ : $\mathrm{S} \mathrm{h}$ e p a r d: On Native and $\mathrm{Me}-$ teoric Iron. - Meteoric Iron from Guilford County, North Carolina. Am. Journ.

(I) 40 , p. $369-370$ (Analyse); s. auch "L'Institut $\propto$ Bd. 9 (1841), p. 452.

1843: Parts c h: Meteoriten, p. II4.

1847: Shepard: Report on Meteorites. Am. Journ. (2) 4, p. 79 ; s. auch

Kenngott, Uebersicht $1_{55}$ 이니, p. ${ }_{3} 6$. 1852: C l a r k: Dissert. Gött. Nro. 56, Randolph County, p. 74-75.

1854: v. Boguslawski: Zehnter Nachtrag, Pogg. Ann. Ergz.-Bd. 4, p. 403 - 404, 409.

1859: B u c hn er: Feuermeteore, p. r33, 136.

1859: Harris: Dissert. Gött., p. IO9. 1858/62: v. Reichen ba ch: VII 551 . IX 162, 174, 181. X 359. XV п1о, 124. XVI 26I. XVII 266. XXI 589.

1863: B u c hner: Meteoriten, p. 167. 1863: Rose: Meteoriten, p. 26, 27, 65, 153.

1867: Go e b e 1: Kritische Uebersicht. Mélanges phys. chim. Bd. 7, p. 325 . r885: Brezina: Wiener Sammlung, p. $210-211,233$.

189o: E as tman: Met. Astron., p. 318. 1894: C o h e n : Meteoritenkunde, p. 23I, 240,24 I.

Ursprüngliches Gewicht: 200 gr. (7 Unzen).

Nachweisbares Gewicht: 7I gr.

Berlin, P.

4 London, P. G. Io

Berlin, U.

Calcutta

Cambridge

Göttingen

London, B. M.
Spl. New Haven 20

I Tübingen Spl.

I Utrecht 4

8 Wien, H.M.*) 8 
Gúrdha

Motecka-nugla

Gurram Konda, zwischenPunganur

u. Kadapa, Madras, Ostindien.

Stein, gefallen 1814 .

Es scheint über diesen Fall keine ausführliche Arbeit veröffentlicht worden zu sein.

Ursprüngliches Gewicht: ?

Nachweisbares Gewicht: $10 \mathrm{gr}$. London, B. M. Io gr.

Gütersloh bei Minden, Westphalen, Deutschland.

Stein, Ccb, gefallen 17 . April 1851 . 1851: Dove: Ueber den am 17. April in Gütersloh in Westphalen herabgefallenen Meteorstein nach einer brieflichen Nachricht des Dr. Stohlmann daselbst. Mon. Ber. Berlin. Akad. (1851), p. 269-270; s. auch Pogg. Ann. Bd. 83 (1851), p. $465-467$; Zeitschr. d. d. geol. Ges. Bd. 3, p. 215; Pharm. Centr. I851, p. 594; Liebig-Kopp, Jahresber. 1851, p.879-880; Kenngott, Uebersicht $1850 / 51$, p. 180 .

185I: R o s e legt den bei Gütersloh gefallenen Meteorstein vor. Mon. Ber. Berlin. Akad. (1851), p. 287; s. auch Pogg. Ann. Bd. 83 (I851), p. $465-467$.

185I: Rose berichtet über den am 17 . April d. J. Abends 8 Uhr bei Gütersloh in Westphalen gefallenen Meteorstein. Zeitschr. d. d. geol. Ges. Bd. 3, p. 215-216.

1852: Nög gerath zeigt auf der Vers, d. naturh. Ver. f. Rheinl. etc. in Münster ein Bruchstück vor. N.J.1852, p.767. 1852: Rose berichtet über die Auffindung eines zweiten bei Gütersloh gefundenen Meteorsteins. Mon. Ber. Berlin. Akad. (1852) p. 276-278; s. auch Am. Journ. (2) 15, p. 290-29I; Pogg. Ann. Bd. 87 (1852), p. 500; Froriep's Tagsberichte über die Fortschr. d. Natur- a. Heilk., Mineralogie u. Geologie bearb. v. C. Giebel. Nro. 576 (1852), p. 318-319; Wochenschr. f.
Astr., Met. u. Geogr. 1852 , p. 380 -38 I; Pharm. Centr. I852, p. 491 ; Kenngott, Uebersicht 1852 , p. I51; sL'Institut \& Bd. 20 (1852) Nro. 975, p. 288.

1859: $\mathrm{H}$ a id ing er: Mittheilungen von Herrn J. F. Julius Schmidt über Feuermeteore. I. Ueber den Meteorfall am 17. April 1851. Sitzber. Wien. Akad. Bd. 37 , p. $804-807$; s. auch sL'Institute Bd. 27 (1859) Nro. 1353, p. $392-393$.

1859: $\mathrm{Buch}$ her: Feuermeteore, p. Ioo, 103.

1859: H a rri s: Dissert. Gött., p. 94.

1863: B u ch ner: Meteoriten, p. $80-8$ I. 1863: Ros e: Meteoriten, p. $84.85,87$, 154.

1858/65: v. Reichenbach: VI 454. IX 16I, 170, 179. XI 297. XIII 355. XXIII 226. XXV 428, 431, 608. 1884: M e uni er: Météorites, p. 260. 1884: Häp ke: Beiträge, Abh. Naturw. Ver. Bremen Bd. 8, p. 523 .

1885: Brezina: Wiener Sammlung, p. $190,233$.

1886: H äpke: Bemerkungen über Meteoriten. Abh. Naturw. Ver. Bremen Bd. 9, p. $358-360$. Ref. N. J. 1887 I, p. 258.

1890: v. Niess 1: Periheld istanzen, Verh. naturf. Ver. Brünn Bd. 29, p. 186, 189, 194, 195, 214, 255-256.

Ursprüngliches Gewicht: Etwa $I^{1 / 2}$ Kgr. ZweiSteine. Der alsbald nach dem Fall gefundene wog 937 gr. (I Pfund 261/4 Lot); der andere Stein kleiner und verwittert.

Nachweisbares Gewicht: II6I gr.

Bailey

Berlin, U.

Calcutta

Cambridge

Dorpat

Göttingen

Gregory
6 London, B. M. 109

878 London, P. G. 14

3 New Haven

Spl. Paris, M.

3 Tübingen

I Wien, H. M.*) I 


\section{Gyulatelke}

Mócs

Hacienda Concepcion Huejuquilla Hacienda Concepcion false

$\begin{array}{lr}\text { Hacienda de Bocas } & \text { Bucson } \\ \text { Hacienda de Potosi } & \text { Coahuila } \\ \text { Hainholz, Minden, Westphalen, }\end{array}$ Deutschland.

Mesosiderit (Grahamit ?), gefunden 1856, beschr. 1857 .

r857: Wöhler: Ueber einen neuen Meteoriten. Pogg. Ann. Bd, IOo, p. $342-345$. Ref. Liebig-Kopp, Jahresber. 1857 , p. $730-731$; N. J. 1858 , p. 567-586; Kenngott, Uebersicht $1856 / 57$, p. 154 .

r857: v. Rei $\mathrm{ch}$ e n b a $\mathrm{ch}$ : Ueber den Meteoriten von Hainholz. Pogg. Ann. Bd. $10 I$, p. $3 \mathrm{I}-3 \mathbf{I} 3$.

1857: R os e legte mehrere Stücke von einem merkwürdigen Meteoriten vor. Zeitschr, d. d. Geol. Ges. Bd. 9, p. r80-r81. Ref. N. J. 1859, p. 297 ; s. auch Kenngott, Uebersicht $1856 / 57$, p. 157 .

r857: v. Reichenbach: Zum Meteoriten von Hainholz. Pogg. Ann. Bd. 102 , p. $618-621$.

r859: Buc h ner: Feuermeteore, p. 106. 1859: H a rri s : Dissert. Gött., p. ror. r860: Haidinger: Ueber das von Herrn Dr. J. Auerbach in Moskau entdeckte Meteoreisen von Tula. Sitzber. Wien. Akad. Bd. 42 , p. $5^{1} 4,516-517$; s. auch Bull. Soc, Nat. Moscou Bd. 33 (1860).

1860: R a m mels ber g: Mineralchemie, p. 906, 949.

r863: B u c h n er: Meteoriten,p.130-13I. 1863: Rose: Meteoriten, p. 83,140 , I53; s, auch Mon. Ber. Berlin. Akad, 1862, p. 557 .

r857/65: v. Reic hen ba c h: III 624 . IV 638 . V $473,474,475$. VI 442 , $443,452,456$. VII 55I, 561. IX 162,
172, 174. X 363, 368. XI 294, 296, 297, 30I, 302, 305, 308, 309. XII 454, 455,458 . XIII $355,356,363,364$, $380,381,384$. XIV 390. XV IOI, 120, I2I. XVI 262. XVII 269. XVIII 484 , 490, 491. XXV 603.

1867: G o e be 1: Kritische Uebersicht, Mélanges phys. chim. Bd. 7, p. 337 -338 .

r870: $\mathrm{R}$ a m mel s berg: Ueber die $\mathrm{Zu}$ sammensetzung d. Meteorite von Shalka u. von Hainholz, - II. Der Meteorit von Hainholz. Mon.Ber. Berlin.Akad.(187o), p. 322-326 (Analyse); s. auch Pogg. Ann. Bd. 141 (I87o), p. $283-287$; Liebig-Kopp, Jahresber. I870, p. 1396 -1397 .

1870: $\mathrm{R}$ a m mels berg : Beiträge zur Kenntnis der Meteoriten, Ber.d. d. chem. Ges. Bd. 3, p. 523 .

1870: R a m mels berg: Meteoriten, p. 94-95, 105, 106, 138, 139, 140; s. auch Pogg. Ann. Bd, 140 (1870), p. 3 I2. r872: Quensted t: Klar u. Wahr, p. 292 (Abb. einer geätzten Platte).

1875: vom R a th: Meteoriten, Verh. naturh. Ver. Bonn Bd. 32, p. 366.

1875: M o hr: Entstehungsart. Ann. Chem. Pharm. Bd. 179, p. 267.

1879: K1e in: Göttinger Sammlung, Gött. Gel. Anz. (1879), p. 98.

1879: R a m me lsberg: Meteoriten, p. 24,25 .

188x: Brezina: Bericht III, Sitzber.

Wien. Akad. Bd. 84 I, p. 278.

1883: T s c hermak: Beitrag, Sitzber.

Wien. Akad. Bd, 88 I, p. 349,350 , $351,352-354$.

1884: $\mathrm{H}$ ä p k e: Beiträge, Abh. Naturw.

Ver. Bremen Bd. 8 , p. 523 .

1884: W a d sw or th: Studies, p. 74. r884: M e unier: Météorites, p. 79, 85 ,

$94,96,97,99,165,169,395$.

1883/85: Tschermak: Photographien, T. 24, p. 3,22 .

1885: Brezin a: Wiener Sammlung, p. $163,191,233$. 
I887: Flig ht: Meteorites, p. I26-127. 1890: Lipp u. Přivoznik: Mittheilungen über die im Laboratorium des k. k. General-Probieramts in Wien in den Jahren 1888 u. 1889 ausgeführten Analysen und anderweitigen Untersuchungen. - II. Meteoriten. - 2. Meteorit von Hainholz bei Burgholz, unweit Paderborn, nicht magnetischer Teil. Berg- und Hüttenm. Jahrb. Bd, 38 , p. $400-401$.

1892: Pr̃ivozniku. Schneider: Ueber die Meteorite von Knyahinya und Hainholz (Analyse des magnetischen Teils). Oesterr, Zeitschr. f. Bergu. Hüttenw. Bd 40, p. 466-468; s. auch Berg- u. Hüttenm. Jahrb. Bd. 40 (1892), p. 458 . Ref. N. J. I894 I, p. $274-275$.

1892: P ŕivoznik: Ueber die Meteorite von Knyahinya u. Hainholz, Oesterr. Zeitschr. f, Berg- u. Hüttenw. (1892). Ref. Ber. d. d. chem. Ges. Bd. 26 (1893), p. 526.

I894: C o h e n : Meteoritenkunde, p. 59, $215,232,248,258,283,309,316$.

1895: Me unier: Révision des lithosidérites, p. 33,34 .

Ursprüngliches Gewicht: $16^{1 / 2} \mathrm{Kgr}$. (33 Pfund).

Nachweisbares Gewicht: I I 344 gr.

\begin{tabular}{lr|lr|} 
Bailey & 28 & Gregory & 103 \\
Belgrad & I & Halle & 34 \\
Berlin, U. & 456 & Hamburg & 19 I \\
Bologna & 8 & Harvard, U. & 350 \\
Bonn & 63 & Helsingfors & 45 \\
v. Braun & 19 & Klausenburg & 22 \\
Breslau & 31 & Kristiania & 47 \\
Budapest & 470 & Kunz & 19 \\
Calcutta & 100 & London, B. M. 484 \\
Cleveland & 5 & London, P. G. & 107 \\
Cohen & 32 & Melion & 2 \\
Dorpat & 14 & Modena & 30 \\
Dresden, M. & 55 & Moskau & 59 \\
Frankfurt & I9 & Neumann & 47 \\
Göttingen & 198 & New Haven & 8
\end{tabular}

\begin{tabular}{lr|lr} 
Paris, M. & 49 & Troyes & 24 \\
Petersburg, A. & 105 & Tübingen & 6307 \\
Pohl & 365 & Utrecht & 2 \\
Rom, U. & 71 & Ward & 323 \\
v. Schilling & 34 & Washington & 7 \\
v. Siemaschko & 119 & Washington, Sh. 12 \\
Stockholm & 106 & Wien, H. M.*) & 760 \\
Strassburg & 13 & &
\end{tabular}

Hamblen Co.

Morristown

Hamilton Co., Pallasit,

Brenham (Anderson)

Hamilton Co., Eisen, Carlton

Hammond, Hammond Township, Saint Croix Co.,Wisconsin, U.S. A. Eisen, Oh, gefunden 1884 , beschr. 1887 .

1887: F is her: Description of an Iron Meteorite from St. Croix Co., Wisconsin. Am. Journ. (3) 34 , p. 38r383 (Abbildung des Eisens und einer geätzten Platte. Analyse). Ref. N. J. I889 I, p. 402.

1890: E a s t m a n : Met. Astron., p. 320. r89r: v. H a u e r: Ann. Hof-Mus. Bd. 6 (Not.), p. 54 .

1893: B r e z in a : Ueber neuere Meteoriten (Nürnberg), p. 166.

r894: C o h en: Meteoritenkunde, p. 57, II7, 124.

r895 : Brezina: Wiener Sammlung, p. 289 .

Ursprüngliches Gewicht: $24 \mathrm{Kgr}$. Nachweisbares Gewicht: 23910 gr. Bailey

Bement

Brezina 298 London, B. M. 62

Harvard, U. $\quad \mathbf{I}_{4} 4$ Wien, H. M. 495

Haniet-el-Beguel, Ouaregla, Algier, Nordafrika.

Eisen, Om, gefunden 1888 , beschr. 1889.

1889: D a u bré e: Météorite holosidère découverte à l'intérieur du sol en Algérie à Haniet-el-Beguel. C. R. Bd. 
108, p. 930-931. Ref. N. J. 1890 I, p. 42 .

r893: M eunier: Révision des fers météoriques, p. 52, 60 .

Urspringliches Gewicht: $200 \mathrm{I}$ gr. Nachweisbares Gewicht: 2000 gr. Paris, M. 2000

Harrison Co. 1859 Harrison Co. Harrison Co. 1877 Cynthiana Harrison Co., Indiana, U. S. A.

Stein, Cho, gefallen 28. März I 859 . r859: $\mathrm{S} \mathrm{mith:Account} \mathrm{of} \mathrm{several} \mathrm{Me-}$ teoric Stones which fell in Harrison Co., Indiana, March 28 th, 1859. Am. Journ. (2) 28, p. 409-4II (Analyse). Ref. N. J. 186I, p. $5^{84}$; s, auch Original Researches 1884 , p. 405-408; ferner Journ. prakt. Chem. Bd. 81 (1860), p. 128; Liebig-Kopp, Jahresber. 1859, p. $857-858$.

r863: B u c h ner : Meteoriten, p. IOI102.

1863: Rose: Meteoriten, p. 154.

r884: Me unier: Météorites, p. 268, $270-271,335$.

r885: Brezina: Wiener Sammlung, p. 177,232 .

1890: E a st m a n: Met. Astron., p. 316.

Ursprüngliches Gewicht: $700 \mathrm{gr}$.

Vier Steine von $538,127,25 \mathrm{u}$. $10 \mathrm{gr}$. (= I9 Oz., $4 \frac{1}{1 / 2}$ oz., 420 grs. u. 167 grs.).

Nachweisbares Gewicht: $300 \mathrm{gr}$.

\begin{tabular}{lr|lr|} 
Bailey & 4 & London, B. M. & 39 \\
Bement & 2 & London, P. G. & 6 \\
Berlin, U. & I 9 & Moskau & 8 \\
Budapest & 5 & New Haven & 17 \\
Calcutta & 28 & Paris, M. & 17 \\
Cleveland & 4 & v. Siemaschko & Spl. \\
Dorpat & I & Stockholm & 7 \\
Göttingen & 17 & Washington & 10 \\
Gregory & I & Washington, Sh & 6 \\
Harvard, U. & 85 & Wien, H. M.*)**) & I3 \\
Kopenhagen & II & \\
Wo ist die Hauptmasse der Steine?
\end{tabular}

Hartford, Linn Co., Iowa, U.S. A. Stein,Cwa, gefallen 25.Februar 1847 . 1847: Shepard: Fall of Meteoric Stones in Iowa. Am. Journ. (2) 4, p. 288 -28 . Ref. N. J. 1848 , p. 850 ; Kenngott, Uebersicht $1844 / 49$, p. 286.

1847: Iowa Meteorite (from a letter from Joshua Barney, U. S. Agent, Dubuque, to J. J. Abert, Col. Topographical Bureau, Washington). Am. Journ. (2) 4 , p. 429 .

r848: S h e p a r d : Report on Meteorites. - I. Linn Co. Iowa. Am. Journ. (2) 6, p. $403-405$ (Analyse); s. auch LiebigKopp, Jahresber. 1847/48, p. 1312 ; Pogg. Ann. Bd. 74 (1848), p. 320 ; Pharm. Centr. 1848 , p. 528 .

r85 I: Shepard: On Meteorites, 4. Description of a large Stone of the Linn Co., Iowa, fall of Feb. 25, r842. Am, Journ. (2) II, p. $3^{8-39}$ (Umrisszeichnung des im Sommer 1847 gefundenen Steins von 20 pounds; $s$. auch Kenngott, Uebersicht 1850/51, p. 179 ; auch 1853 , p. 16r; LiebigKopp, Jahresber. 1850 , p. 825 .

1853: Shepard: Notices of Meteoric Iron near Lion River, Great Namaqualand, South Africa; and of the detection of Potasium in Meteoric Iron. - 3. Figure of the Iowa Meteoric Stone, which was seen to fall Feb.25, I847 (ganzer Stein von 2 lbs. $81 / 20 z$.). Am. Journ. (2) 15, p. 6-7.

1854: v. Boguslawski: Zehnter Nachtrag, Pogg. Ann. Ergz.-Bd. 4, p. $24,378-379$.

1859: H a r r i s : Dissert,Gött., p.92-93. r863: B u c hn er: Meteoriten,p.76-77. 1863: R os e: Meteoriten, p. 84, 93, 155. 1858/65: v. Reichen b a ch: V 480.

VI 454. IX 16r, 168, 178. X 359,363 . XI 294, 300. XIII 369 (Fig.), 377. XIV 396. XX 622. XXV 321, 322, $324,607,615$.

r867: G o e be 1: Kritische Uebersicht, Mélanges phys. chim. Bd. 7, p. 336. 
1870: $\mathrm{R}$ a m m e ls berg: Beiträge zur Kenntnis der Meteoriten III. Linn Co., Iowa, Mon. Ber. Berlin. Akad. (1870), p. 457-459 (Analyse).

1870: R a mme ls berg: Meteoriten, p. $103,105,106,138,139,140$.

1875: vom Rath: Meteoriten, Verh. naturh. Ver. Bonn Bd. 32, p. 368.

1879: Ra mmelsberg: Meteoriten, p. 24,25 .

I882: W i e c h m a n n : Fusion-Structures in Meteorites. Ann. N. Y. Acad, Sc. Bd. 2, p. 293.

1884: Wa d sw orth: Studies, p. 104. 1885: M e un i er: Météorites, p. 79, 8o, $98,208,218$.

1885: Brezina: Wiener Sammlung, p. $176,180,232$.

1887: Flig h t: Meteorites, p. I3- II4. 1890: E a s t m a n : Met. Astron., p. 316. r89r: Torrey u. B arbour: The recorded Meteorites of Iowa, with special mention of the last of Winnebago Co. Meteorite (Analyse, Situationsplan). Amer. Geologist Bd 8, p. 65.

r894: Cohen: Meteoritenkunde, p. 237, 272.

1895: B rezina: Wiener Sammlung, p. 244 .

Ursprüngliches Gewicht: 2 I Kgr. Gleich nach dem Fall gefunden I Stein, vollständig umrindet, von 2 lbs. $8 \frac{1}{2} \mathrm{Oz}$, gelangte an Shepard. Ein Stein von 42 Pfund, von welchem ein Stück von 2 lbs. $u$. I I Oz. an Col. Abert, Washington, gelangte. Im Frühjahr 1847 gefunden ein Stein von 46 (oder 40?) Pfund, von welchem Shepard 2 I lbs. 7 oz. erhielt. Im Sommer 1847 gefunden ein Stein von 20 Pfund, welchen Shepard erhielt. Es wird dann noch von einer Masse von etwa 50 Pfund ge-

1) "Linn Co.s sprochen, die aber wohl mit einer der obigen Steine identisch ist. Erhalten sollten also mindestens sein $2 \mathrm{r}$ Kr. (46 Pfund $101 / 2$ Unzen). Nachweisbares Gewicht: 9177 gr.

Bailey

Bayet

Bement

Berlin, U.

Bologna

Bonn

v. Braun

Budapest

Calcutta

Cleveland

Dorpat

Dresden, M. 418 Madrid

$\left.\mathbf{I}^{1}\right)$ de Mauroy

$$
132 \text { Minneapolis }
$$

59

21

348 Modena 5

14 Moskau 8

74 Neumann 3

16 New Haven 1046

155 Paris, M. 103

643 Petersburg, A. 75

262 Petersburg, B. 95

$5^{8}$ Pohl

83 Rom, U.

40

Edinburg

Göttingen

Gregory

Hamburg

Harvard, U.

Howell

Kopenhagen

445 v. Siemaschko $8_{3}$

84 Stockholm $\quad 189$

92 Strassburg $\quad 54$

6 Stürtz 17

374 Troyes $\quad 6$

Kristiania $\quad 74$ Ward

Leiden $\quad 102$ Washington 24

London, B. M. 942 Washington,Sh.1602

London, P. G. 333 Wien, H. M.*) 241

Lüttich

3

Wo befindet sich der Rest der Steine, welche Shepard besass?

Hassi Jekna, Valleé de l'Oued Meguiden, Algier, Nordafrika.

Eisen, Of, gefunden um 1890 (Fall beobachtet?).

1892: Meunier: Fer météorique récemment tombé à Hassi Jekna, en Algérie. C. R. Bd. II5, p. $53 \mathrm{I}-533$ (Analyse), Ref. N. J. I894 I, p. 275.

1893: M eunier: Révision des fers météoriques, p. 40, $4 \mathrm{I}$.

Ursprüngliches Gewicht: I250 gr. Nachweisbares Gewicht: 1250 gr. Paris, M. 1250 
Hauptmannsdorf

Haviland Township

Hawaii

Haywood Co.

Haywood Co. s. Anhang Ferguson

Hebriden

Heidelberg

Heinrichsau

Hemalga

s. Anhang Tiree

s. Anhang

Grüneberg

Tarapaca

Henry Co., Virginia, U. S. A.

Eisen, gefunden 1889 , beschr. 1890 .

r89o: Venable: Two new Meteoric Irons. - 2. From Henry County, Va. Am.Journ.(3) 40, p.162-163 (Analyse). 1894: C o h e n : Meteoritenkunde, p.23I. Ursprüngliches Gewicht: $1.7 \mathrm{Kgr}$. und $0.22 \mathrm{Kgr}$. Rindenabfälle.

Nachweisbares Gewicht: $\mathbf{1}^{2} \mathbf{2}$ gr.

Bailey

v. Braun

21 Krantz

Cleveland

Gregory

\begin{tabular}{l|l}
3 & Kunz \\
9 & Washington \\
7 &
\end{tabular} 3

Wo befindet sich die Hauptmasse des Eisens? Ob hierher Locust Grove? s. Anhang.

Henry Co. 1857 s.Anh.LocustGrove Heredia, San José, Costa Rica. Centralamerika.

Stein, Ccb, gefallen I. April 1857 . r859: H a r r is: Dissert. Gött., p. 99 -100 (als Costa Rica 1. August 1858 aufgeführt).

r859: D o m e y k o: Annales de la Univ. de Chile (1859), p. 325.

1863: B u c h n e r: Meteoriten, p.93-94. r858/65: v. R e i c he n b a c h: VI 455.

IX 162, 171, 180. XI 294, 301. XII 454. XXIV 228. XXV 422, 427, 431, 608. r884: M eunier: Météorites, p. 26o, 262.

r885: Brezina: Wiener Sammlung, p. $190,233$.
1890: E a stma n : Met. Astron., p. 316. r895: Brezina: Wiener Sammlung, p. 257 .

Ursprüngliches Gewicht: Mehrere Steine,von denen einer etwa $\mathrm{I}$ Kgr.?

Nachweisbares Gewicht: 707 gr.

Bement 2 London, P. G. 2

Berlin, U.

2 Paris, E. 79

\section{Calcutta}

I Paris, M.

Göttingen 422 Tübingen

London, B. M. $\quad 54$ Wien, H. M.*)

Hessen

s. Anhang Nauheim

Hessle, Mälar See, Schweden.

Stein, Cc, gefallen x. Januar 1869 . r869: Fahne hjelm: Meteorfallet i Fittja socken af Upsala län d. I: sta Januari 1869. Oefversigt af Vetensk.Akad, Förhandl (1869) Nro. I, p. 59-60; s. auchWochenschr. f. Astr., Met. u.Geogr. 1869, p. 136.

r869: Lindström: Kemisk Undersökning af meteorstenar ne från Hessle. Oefversigt af Kongl. Vetensk,-Akad. Förhandl (1869) Nro. 8, p. 715-724 (Analyse).

1869: D a u b rée présente des météorites provenant de la chute qui a eu lieu le I er janvier r869 aux environs d'Upsal. C. R. Bd. 68 , p. 363 ; s. auch sL'Institute Bd. 37 (1869), p. 49. r869: Nordenskiö 1 d: Ny. Illustrererd Tidenske 1869; s. auch Gött. Gel. Anz. (1869); „L'Institut « Bd, 37 (1866), p. 351 .

r869: Fredholm: Om Meteorstens fallet vid Hessle den I : sta Januari $\mathbf{1} 869$. Akademisk Afhandling. Upsala (W. Schultz 1869), 43 Seiten mit Karte. $8^{\circ}$. 1869: Bu chner: Vierter Nachtrag, Pogg. Ann. Bd. 136, p. 596.

r869: v. Haid ing er: Hessle, Rutlam, Assam, 3 neue Meteoriten. I. Hessle. Sitzber. Wien. Akad. Bd, 59 II, p. 226-227.

I) Als $>$ Siderolite aufgeführt. Vielleicht oxydierte Rinde? 
1870: Tschermak: Goalpara, Sitzber. Wien. Akad. Bd. 62 II, p. 864 (den Kohlenstoffgehalt betreffend).

r870: Nordenskiö1d: Meteorstens falled vid Hessle den I: sta Januari 1869. Kongl. Vetensk. Akad. Handl. Bd, 8 Nro. 9 (mit Tafel u. Karte; Analyse von Lindström), I4 Seiten; s. auch Pogg. Ann. Bd, 14I (1870), p. 205-224; auch 1893: Brezina, Ankunft, p. 21 ; LiebigKopp, Jahresber. 1870 , p. $1398-1399$; *L'Institut « Bd. 37 (1869), p. 35I.

1870: R a mme ls berg: Meteoriten, p. $158-159$.

1874: B jör ling: Meteoriter och Kometer. Populära Föredrag. Med 4 Plancher och 18 Figurer i Texten. Stockholm (F. u. G. Beijers Förlag I874), 95 Seiten $8^{\circ}$, p. 9.

I875: vom R at h: Meteoriten, Verh. naturh. Ver. Bonn Bd. 32, p. 374 .

1877: F light: Meteorites and the origin of life. The Popular Science Review. 18. Okt. 1877 , p. 10-n (Sep.). 1878: Nordenskjöl d: Trenne märkeliga eldmeteorer, sedda i Swerige under i̊ren 1876 och 1877 . Geologiska Förennigens i Stockholm. Förhandl. Bd. 4, p. 6r.

r879: R a m mels berg : Meteoriten, p. 24 .

r88x: v. Nordenskiöld: Zeitschr. d. d. geol. Ges. Bd. 33, p. 24 (Vergleich mit Ställdalen).

r882: W i ik: Mikroskopisk undersökning af naagra paa universitetets mineralkabinett befindliga meteoriter. Ofvers. af Finska Vetensk. Soc. Förh. Helsingfors Bd. 24. Ref. N. J. $1888_{3}$ I, p. 384 .

I884: W a d s w ort h: Studies, p. 105. r884: Meunier: Météorites, p. 79, 98, 230, 234-236, 421, 425 (Sit.-Plan), 491.

1884: Lin dström: Förteckning öfver Riksmusei Meteoritsamling. Oefver-

I) Ein ganz umrindeter Stein von $0.3 \mathrm{gr}$. sigt af Kongl. Vetensk. Akad. Förhandl. (1884) Nro. 9. Ref. N. J. I886 II, p. 39 .

r885: Brezina: Wiener Sammlung, p. $164,185,233$.

1886: R e us c h: Ueber den Tysnesmeteorit u. drei andere in Skandinavien niedergefallene Meteorsteine, - II. Die Hesslemeteorite (r. Januar 1869 ungefähr $12 \frac{1}{2}$ Uhr Nachmittags). N. J. B. Bd. IV, p. 487-490 (mikroskopische Abbildungen); s. auch Nyt Magazin for Naturvidenskaberne B.1. 29, p. 320 -323 .

1887: F 1 ig ht: Meteorites, p. 2-4. r889: Fl et cher: Atacama Meteorites, Mineral. Magaz. Bd. 8, p. 226 (Streufeld to auf 3 miles).

r89o: v. N i ess l: Periheldistanzen, Verh. naturf. Ver. Brünn Bd. 29, p. 188, 189, 194, 214, 247.

r892: B rez i n a : Sternschnuppen, p. 15. 1893: N e w t o n: Lines of structure in the Winnebago Co. Meteorites and in other Meteorites. Am. Journ. (3) 45, p. $152-153,355$. Ref. N. J. 1894 I, p. $273-274$.

1894: Co he n: Meteoritenkunde, p. 6r, $156,164,165,166,167,168,207,237$, $304,310,330$.

1894: F letcher: Introduction, p. 26. 1895: Brezina: Wiener Sammlung, p. 255 .

Ursprüngliches Gewicht: Steinschauer. Die Grösse der einzelnen ganz umrindeten Steine von 0.07 gr. bis I Kgr.

Nachweisbares Gewicht: 22895 gr.

Bailey

Belgrad

Bellucci

Bement

Berlin, U.

Bologna

Bonn 


\begin{tabular}{|c|c|c|c|}
\hline Freiberg, i. S. & 47 & New Haven & \\
\hline Göttingen & 173 & Newton & \\
\hline Gregory & 137 & Paris, M. & \\
\hline Harvard, U. & 549 & Pech & \\
\hline Helsingfors & 128 & Pohl & \\
\hline Klausenburg & 66 & Prag, M. & \\
\hline Kopenhagen & 137 & Rom, U. & \\
\hline Krantz & 34 & v. Siemaschko & \\
\hline Kristiania & 193 & Stockholm & \\
\hline Kunz & 5 & Strassburg & \\
\hline Leipzig & 59 & Troyes & \\
\hline London, B. M. & 910 & Turin, J. & \\
\hline London, P. G. & 237 & Upsala & \\
\hline Lund, G. & 278 & Utrecht & \\
\hline Lund, M. & 62 & Ward & \\
\hline Madrid & 89 & Washington & \\
\hline de Mauroy & 33 & Washington,Sh. & \\
\hline Moskau & Io & Wien, H. M.*) & \\
\hline München & $2 \mathrm{II}$ & Würzburg & \\
\hline Neumann & 12 & & \\
\hline
\end{tabular}

Hex River, Capland, Südafrika.

Eisen, H, gefunden 1882 , erwähnt 1885 .

1885: Brezina: Wiener Sammlung, p. $155-156,218-219,234$ Taf. III. 1887: B r e z in a : Neue Meteoriten III. Verh. k. k. geol. Reichsanstalt (1887), p. 289 .

1888 : v. H a u e r : Ann. Hof-Mus. Bd, 3 (Not.), p. 42 u. Bd. 4 (1889) (Not.), p. 64 .

1890: Brezina: Ueber Meteoreisen. Oesterr. Zeitschr. f. Berg- n. Hüttenw. Bd. 38 , p. $357-35^{8}$ (Abbildung einer geätzten Platte).

r89r: Cohen u. Weinschenk: Meteoreisen-Studien. Ann. Hof-Mus. Bd. 6, p. 143 (Analyse), 159 .

r893: Brezin a: Ueber neuere Meteoriten (Nürnberg), p. 166.

r893 : M e u n i er: Révision des fers météoriques, p. 15, 17 .

1894: Co h e n: Meteoreisen-Studien III. Ann. Hof-Mus. Bd. 9, p. 108-III, II6, II7, II8 (Rhabdit).
1894: C o h en : Meteoritenkunde, p. 96, $98,126,127,128,132,157,191,194$, 209, 212, 216, 248, 298, 320, 321.

1895: Brezina: Wiener Sammlung, p. $291-292$.

1895: C o h e n: Meteoreisen-Studien IV. Ann. Hof-Mus. Bd. Io, p. $82,85,88$, 89, 93.

Ursprüngliches Gewicht: ?

Nachweisbares Gewicht : 45644 gr.

\begin{tabular}{lr|lr} 
Bailey & 96 & Krantz & 90 \\
Bement & 2657 & Paris, E. & 45 \\
Böhm & 79 & Paris, M. & 288 \\
v. Braun & 108 & Prag, M. & $\mathbf{1 8 2}$ \\
Breslau & 90 & Rom, U. & 66 \\
Budapest & 2500 & Stockholm & 175 \\
Dorpat & $33^{8}$ & Strassburg & 12I \\
Dresden, M. & 25 & Ward & 693 \\
Gregory & 332 & Wien, H.M. 37 704 ${ }^{1}$ ) \\
Greifswald & 55 & &
\end{tabular}

Hidalgo

Pacula

High Possil (Glasgow) bei Glasgow, Schottland.

Stein, Cw, gefallen 5. April 1804 . 1806: $\mathrm{T} i 11 \mathrm{och}$ : Nachrichten von einem Steine, der am 5. April 1805 unweit Glasgow aus einer Wolke herabgefallen ist. Gilb. Ann. Bd. 24, p. 369 -376 (Uebersetzung aus Tilloch's Philos. Magnz. Mai $\mathrm{I}^{804}$ und Bibl. Brit. Bd. 26, p. 203).

1812: Ch la dni: Verzeichnis,Schweigg. Journ. Bd. 4 Beil. I, p. 15 .

1812: Bigot de Morogue, p. 2or. r812: Sowerby hat 1812 eine Tafel anfertigen lassen mit Abbildungen der Steine von Wold-Cottage, Mooresford und High Possil (Exemplar im k. k. Hofmus, Wien).

1815: Chladni: Neues Verzeichnis, Gilb. Ann. Bd. 5o, p. 253.

1819: $\mathrm{Chl}$ a dni: Feuermeteore, p. $57,66,69,73,275(1), 293,429$; (auch auf v. Moll's Ann. der Berg- u. Hüttenkunde Bd, 4, p. 92 verwiesen).

1) Ausserdem 182 gr. nach I. Juli 1893 erworben. 
I836: K ä mtz: Meteorologie, p. 280. 1843: P a r ts c h: Meteoriten, p. 58.

1854: B a l c e $11 \mathrm{~s}$ : Lithologia meteorica, p. 22.

1854: v. Boguslawski: Zehnter Nachtrag, Pogg. Ann. Ergz.-Bd. 4, p. 423.

1857: Arago: Astronomie Populaire Bd. 4 , p. 196.

I859: Harris: Dissert. Gött., p. 65.

1859: v. Reic hen b a c h: IX I6I, 168, 178. XI 296.

1863: B u c hner: Meteoriten, p. 18. 1863: Rose: Meteoriten, p. 155. 1884: M e un i e r: Météorites, p 208,210. r885: Brezina: Wiener Sammlung, p. $177,232$.

r889: F l e t c h er : Atacama Meteorites, Mineral. Magaz. Bd. 8, p. 232.

r894: F le t c her : Introduction, p. 27.

UrsprünglichesGewicht: ZweiBruchstücke eines Steins, das eine 2 Zoll, das andere 6 auf 4 Zoll gross, von dem letzteren aber nur ein Teil erhalten.

Nachweisbares Gewicht: 152 gr.
Bailey

Bement

Berlin, U.

Budapest

Calcutta

Cambridge

Göttingen

\section{Gregory}

I London, B. M. 91

Spl. London, P. G:

Spl. Paris, M.

24 Pohl

I5 Tübingen

I Wien, H. M.*
2

I

I

Spl. Spl.

15
Hill, Stein v. Prof. Hill Travis Co.

Hiokomo s. Anhang Yokahima Hislugari

Maêmê

Hizen Ogi

Hollands Store, Chattooga Co., Georgia, U. S. A.

Eisen, $\mathrm{Hb}$, gefunden u. beschr. $\mathrm{r} 887$. 1887: $\mathrm{K}$ unz: On some American Meteorites. - 2. The Chattooga County, Georgia Meteorite. Am. Journ. (3) 34, p. $47 \mathrm{I}-472$ (Abbildung, Analyse von Whitfield); s. auch Huntington: Proc. Amer. Acad. Arts and Sc. Bd, 24 (1889), p. 34; Am. Journ. (3) 43, p. 425 ; N. J. I889 I, p. 6r.

r89o: Eastman : Met. Astron.,p.320. r89o: Flet cher: Mexican Meteorites,

Mineral. Magaz. Bd. 9, p. 106.

r89r: Cohen u. Weinschenk: Meteoreisen - Studien. Ann. Hof-Mus, Bd. 6 , p. 160 .

r89r : v. H a u e r : Ann. Hof-Mus. Bd. 6 (Not.), p. 54 .

1893: B r e z in a: Ueber neuere Meteoriten (Nürnberg), p. $\mathbf{I} 66$.

1893: Meunier: Révision des fers météoriques, p. 76 .

r894: C o he n : Meteoritenkunde, p. 62, $77,89,95,97,127,232$.

r895: Brezina: Wiener Sammlung, p. $292-293$.

r895: C o he n : Meteoreisen-Studien IV. Ann. Hof-Mus. Bd. 1o, p. 88.

Ursprüngliches Gewicht : I 2.5 Kgr., wovon aber nur $5 \mathrm{Kgr} .\left(9+\mathrm{I}^{1} / 2\right.$ $+1 / 2$ lbs.) erhalten blieben und an Herrn Kunz gelangten.

Nachweisbares Gewicht: 3402 gr.

Bailey

Bement

I7 Paris, M.

30

v. Braun 214 Rom, U.

27

Budapest 944 v. Siemaschko 90

London, B. M. 204 Washington I9

Homestead (West Liberty), Iowa, U. S. A.

Stein, $\mathrm{Cgb}$, gefallen 12 .Februar 1875 . 1875: Fall of a Meteor in Iowa (3) 9, p. 407 .

1875: T s c her ma k: Meteorit von Iowa. M. M. (I875), p. 209.

1875: Hinrichs: Sur une chute de météorites tombées dans l'Etat d'Iowa (mit Anerkennung Daubrées). C. R.

I) Ausserdem 419 gr. nach I. Juli 1893 erworben. 
Bd. 8o, p. II75; s. auch Popular Science New York, September 1875. The great Iowa Meteor (Situationsplan mit Abbildung von 9 Steinen); $>$ Der Naturforscher * 1875 , p. 258 ; Am. Chemist. Bd. 7, p. 19I ; Liebig-Kopp, Jahresber. 1876, p. I318.

1875: Smith: Rapport sur la chute de deux pierres météoriques dans les Etats - Unis, - Météorite du Comté d'lowa. C. R. Bd. 8 o, p. $1455^{-}-1453$ (Analyse); s. auch "Der Naturforscher * 1875, p. 294 .

1875: Wright: Preliminary Note on an Examination of Gases from the Meteorite of February 12, 1875. Am. Journ. (3) 9 , p. $459-460$.

1875: W right: Examination of Gases from the Meteorite of Feb. 12, 1875 . Am. Journ. (3) 10, p. $44-49,206-$ 207 ; s. auch Pogg. Ann. Ergz.-Bd, 7 (1876), p. 336 ; >Der Naturforscher 1875, p.357-359; Wochenschr.f. Astr. Met, u. Geogr. 1875 , p. $364-368$.

I875: L e o n a r d : Iowa County Meteor and its Meteorites. Am. Journ. (3) Io, p. $357-363$ (Analyse von Smith. Kleine Karte mit der Verteilung der Steine u. der Bahn am Himmel). Ref. LiebigKopp, Jahresber. 1875 , p. $1311-1312$. r875: Ir is h: An account of the detonating Meteor of February 12, 1875 . Gedruckt Iowa City, Iowa, Daily Press Iob Printing office $1875.4^{\circ}$, 16 Seiten (mit Karte; Analyse von Hinrichs ; Abdruck zahlreicher brieflicher Mitteilungen an den Verfasser).

1875 : Ueber die Beschaffenheit der Steinmeteoriten vom Fall am 12. Februar 1875 in der Grafschaft Iowa N.-A. Sitzber. München. Akad. Bd. 5, p. 313330 (Analyse u. Abbildung einesSchliffs). Ref. N. J. 1876 , p. 440 .

r 876 : W r i g h t : On the Gases contained in Meteorites. Am. Journ. (3) II, p. 259, 260.

r 876: W r i g h t : On the Gases contained W ül fing, Meteoriten. in Meteorites. Am. Journ. (3) 12, p. 167, 169, 170 .

1879: Ra m me 1sberg: Meteoriten, p. $12,24,25$.

1879: N e w to n: Relation of Meteorites to Comets. »Nature \& Bd.19, p.315-317, $340-342$. A lecture delivered in the Mechanics Course of the Sheffield Scientific School of Yale College. U.S. by H. A. Newton.

r882: W i e c h m a n n: Fusion-Structures in Meteorites. Ann. N. Y. Akad. Sc. Bd. 2, p. 293, 295 (Taf. 20).

1882: Brezina: Bericht IV, Sitzber.

Wien, Akad. Bd. 85 I, p. 343 .

1882: Las a u $1 x$ : Vermehrung. Verh. naturh. Verein Bonn (Sitzber.) Bd. 39, p. $102-105,108$.

r884: W a dsworth: Studies, p. 86. 1884: v. $\mathrm{N}$ i ess 1: Ueber die astronomischen Verhältnisse bei dem Meteoritenfalle von Mócs in Siebenbürgen vom 3. Februar 1882. Sitzber. Wien. Akad. Bd. 89 II, p. 29I. Ref. N. J. 1886 I, p. 224.

I884: M eu nier: Météorites, p.268,272. r883/85: Tschermak: Photographien,

T. 7,10, p. $12,13,14$.

1885: Brezina: Wiener Sammlung, p. $155,165,168,182,183-184,233$. 1887: F lig h t: Meteorites, p. 76-8o. r888: N e wto n: Orbits, Am. Journ. (3) 36, p. 4 .

r889: F l etc h er: Atacama Meteorites, Mineral. Magaz. Bd. 8, p. 226.

r890: Eas tma n: Met, Astron., p. 316. r89o: v. Ni ess 1: Periheldistanzen, Verh. naturf. Ver. Brünn Bd. 29, p. 186, 188, 194, 195, 214-250.

r89r: Torrey u. Barbour: The recorded Meteorites of Iowa, with special mention of the last or Winnebago Co. Meteorite (Analyse; Situationsplan). Amer. Geologist Bd. 8, p. 66.

1892: Eastman: The Mexican Meteorites. Bull. Philos. Soc. Washington Bd. 12 , p. 50.

10 
1893: B re $z$ in a: Ueber neuere Meteoriten (Nürnberg), p. 160, I61.

r894: C o h e n : Meteoritenkunde, p. 6r, 172, 173, 174, 179, 180, 206, 262, 298, 319.

1894: Fl et cher: Introduction, p. I3. r895: Brezina: Wiener Sammlung, p. $251-252$.

Ursprüngliches Gewicht: Steinschauer. Etwa 700 lbs. gesammelt; der grösste Stein soll I20 lbs. gewogen haben.

Nachweisbares Gewicht: 124492 gr.

\begin{tabular}{lr|lr|} 
Bailey & 339 & Heidelberg & 12 \\
Bayet & I 44 & Karlsruhe, M. & 27 \\
Belgrad & 72 & Klausenburg & 21 \\
Bellucci & 10 & Kopenhagen & 3060 \\
Bement & 10224 & Krantz & 68 \\
Berlin, P. & $\left.580^{1}\right)$ & Kristiania & 2248 \\
Berlin, U. & 2314 & Lausanne & 886 \\
Böhm & 30 & London, B. M. & 3780 \\
Bologna & 63 & London, P. G. 704 \\
Bonn & 56 & Lüttich & 21 \\
v. Braun & 1486 & Madrid & 87 \\
Brezina & 168 & de Mauroy & $31^{2}$ ) \\
Budapest & 3586 & Minneapolis & 307 \\
Catania & 2 & Moskau & 482 \\
Cleveland & 64 & München & ${ }^{2} 541$ \\
Cohen & 20 & Neumann & 60 \\
Dorpat & 86 & New Haven & 35719 \\
Dresden, M. & 994 & Newton & 360 \\
Göttingen & 367 & New York, M. & 583 \\
Graz, J. & 50 & Odessa & 2 \\
Gregory & 577 & Paris, E. & 18 \\
Harvard, U. & 17389 & Paris, M. & 7468
\end{tabular}

Petersburg, A. 3262 Szamosujvar 19

Petersburg, B. 120 Troyes 26

Pohl II9 Upsala 215

Prag, M. $\quad 155$ Utrecht $\quad 32$

Rom, U. $\quad$ I39 Ward $\quad$ I2 545

v. Siemaschko 76 Washington $40 \mathrm{I}$

Stockholm 2460 Washington,Sh. 3186

Strassburg $\quad 18$ Wien,H,M.**) $5115^{2}$ )

Stürtz $\quad 64$ Wien, U. I. $\quad{ }_{54}$

Die Military Academy in West Point, N. Y. besitzt ebenfalls einStück dieserLokalität.

\section{Hommoney Creek s. Anhang}

Honolulu, Owahu, Sandwich Inseln, Stiller Ozean.

Stein, Cwa, gefallen 27. September ${ }^{4}$ ) (15. September alten Stils) I825. 1823/26: Kotzebue: Reise um die Welt in den Jahren $1823,24,25,26$ Teil II (Weimar 1830 bei W. Hoffmann), p. 139. Für die Bestimmung des Datums s. auch Teil I, p. 3 u. Teil II, p. 138 ; s. w. u. bei v. Hoff 1832 u. bei Kuhlberg 1865 .

r829: Hof $\mathrm{m}$ a n n: Geognostische Beobachtungen angestellt auf einer Reise um die Welt u. s. w. Berlin 1829 bei G. Reimer, p. 69 (hier wird der 15. Sept. als Falltag angegeben).

1830: Aérolithe dans les îles Sandwich. Ann. Chim. Phys. Bd. 39, p. 42 I. r830: v. H off: SiebenterNachtrag, Pogg. Ann. Bd, 18, p. 184

r832 : v. H off: Achter Nachtrag, Pogg. Ann. Bd. 24, p. 225-226 (hier ist der Bericht Kotzebue's wörtlich abgedruckt).

1) Nur sIowa Wisconsin * bezeichnet. Die verhältnismässig grosse Menge lässt vermuten, dass der Stein zu Homestead gehört. - 2) >5 gr. pierre verte; 306 gr. pierre grise . - 3) Ausserdem I4I gr. nach I. Juli 1893.

4) Ueber das Datum des Falles herrscht einige Verwirrung. In den Ann. Chim. Phys. von 1830 stebt $\$ \mathbf{I} 4$. September $;$; daraus schöpft v. Hoff in seinen Beiträgen von 1830 . Das gleiche Datum geben an: Gregory (1854), Tschermak (1872), Brezina (1885). Den $\rightarrow$ r5. September geben an: Rose (1837), Partsch (1843), Harris (1859) und Fletcher (1893). Den 18. Sept. giebt Haidinger (Sitzber. Wien. Akad. Bd. 54 II, p. 205), den 27. Sept. Bingham (1845) an. Kotzebue nennt den 15. Sept. als Falltag und sagt ausdrücklich, dass die Daten nach altem Stil gerechnet seien. 
1836: K ä m t z: Meteorologie, p. 294. r837: Ro s e: Reise nach dem Ural, p. $32-33$ (sieht den Stein in der Dorpater Sammlung u. erhält ein kleines Stück für Berlin).

1843: P a r t s c h: Meteoriten, p. 51-52. r845: B ing ha m: Particulars of the fall of Meteorites in the Sandwich Islands Am. Journ. (1) 49, p. 407-408; s. auch Pogg. Ann. Ergz.-Bd. 2 (1848), p. 367-368; Kenngott, Uebersicht I $844 / 49$, p. 286 .

I854: v. Bog uslaw ski: Zehnter Nachtrag, Pogg. Ann. Ergz.-Bd. 4, p. 19.

r857: A ra g o: Astronomie populaire,p.2or. 1859: Harris: Dissert. Gött., p. 79.

r863: B u c h n e r : Meteoriten, p. $49-50$. r863: Ros e: Meteoriten, p. $\mathbf{r} 55$.

I859/65: v. Re i c h e n b a c h: IX 16r, 168, 172, 178. XIII 373. XX 626. $\mathrm{XXV} 318,321,322,324,422,427$.

r865: K u h 1 b er $\mathrm{g}$ : Analyse u. Beschreibung der Meteorite von Nerft, Honolulu, Lixna u. eines im Gouvernement Jekatherinoslaw gefallenen Meteoriten. Arch. Naturk. Liv. Ehst. Kurl. (I) 4, p. 14-18 (Analyse u. Abbildung). Ref. Liebig-Kopp, Jahresber. 1867 , p. 1048 . r867: Go e be 1: Kritische Uebersicht, Mélanges phys. chim. Bd. 7, p. 302-303,338 .

r869: B u chner: Vierter Nachtrag, Pogg. Ann. Bd. 136, p. 445-446.

r870: $\mathrm{R}$ a m mels berg : Meteoriten, p. 82,98 .

1884: Meunier: Météorites, p. 208, $215-216$.

r885: Brezina: Wiener Sammlung, p. $180,232$.

1893: v. H a u e r: Ann. Hof-Mus. Bd. 8 (Not.), p. 49.

1893: Brezin a: Ueber neuere Meteoriten (Nürnberg), p. 159.

r894: C o hen: Meteoritenkunde, p. 53, 57, 112, 136, 206, 249.

r895: Brezina: Wiener Sammlung, p. 243,246 .
Ursprüngliches Gewicht: Zwei Steine; sjeder der herabgefallenen Steine mochte vor der Zerbröckelung ${ }_{15}$ Pfund schwer gewesen sein «. Nach Kuhlberg's Beschreibung wog das Dorpater Stück ${ }_{1142}$ gr. und scheint nach der Abbildung ein Monolith gewesen zu sein. Hofmann sagt: „Ausser zahlreichen kleineren Stücken sah ich nur zwei, die gegen 3 Pfund schwer waren «.

Nachweisbares Gewicht: 2395 gr.

\begin{tabular}{lr|lr} 
Bayet & 7 & London, P. G. & I0 \\
Bement & 91 & Madrid & 7 \\
Berlin, U. & 64 & Moskau & 17 \\
v. Braun & 38 & New Haven & 577 \\
Brezina & 74 & Paris, E. & 2 \\
Budapest & 76 & Paris, M. & 16 \\
Calcutta & 13 & Petersburg, A. & 438 \\
Dorpat & 549 & Pohl & Io \\
Göttingen & 3 & v. Siemaschko & Spl. \\
Gregory & 13 & Troyes & 3 \\
Harvard, U. & 37 & Tübingen \\
Klausenburg & 4 & Washington, Sh. \\
London, B. M. & 81 & Wien, H. M.*) & 26r
\end{tabular}

\section{Horzowic}

Zebrak

Howard Co.

Kokomo

Hraschina, Agram, Croatien, Oesterreich.

Eisen, Om, gefallen 26. Mai $175 \mathrm{I}$.

1785: Güssmann: Lythophylaceum Mitisianum, Dissertatione praeuia et observationibus perpetuis physico mineralogicis explicatum a Francisco Güssmann. Viennae typis Josephi Nobilis de Kurzbeck 1785 Bd. 2 (nicht ${ } \mathrm{Bd}$. $\mathbf{I} \approx$, wie Haidinger sagt), p. 127-r31.

r789: Gron a u: Einige Demerkungen über die Gewitter. Schriften der Ges. naturf. Freunde, Berlin Bd. 9 (Bd, 3 der Beobacht. u. Entdeck.), p. 47 (Entstehung durch Blitzschlag).

r79o: Stü tz: sBergbaukundeđ Bd. II, 
p.398-407. Diese »Bergbaukunde $₫$ war die Zeitschrift der 1786 zu Schemnitz gestifteten 》 Societät der Bergbaukunde « . Die hier in Frage kommende Stelle ist bei v. Ende u. bei Haidinger s, u. abgedruckt.

1794: C h la dn i: Pallaseisen, p.31-33. r796: K ing: Remarks, p. 23-25.

1803: I z a rn: Lithologie, p. 120-12I, 141.

1803: de Dré e: Recherches, Journ. Phys. Bd. 56, p. 4I3-4I4.

r803: C h l a d n i: Chronologisches Verzeichnis, Gilb. Ann. Bd. 15, p. 309.

1803: K 1 a p r ot h: Ueber meteorische Steine und Metallmassen. Abh. Berlin. Akad, 1803 , p. $21-41$; s. auch Klaproth, Beiträge Bd. 4 (1807), p. 99Ior ; Gilb. Ann. Bd. 13 (1803), p. 339 u. Gilb. Ann. Bd. 24 (1806), p. 379.

r804: P öt z s h: Kurze Darstellung, p. $54-56$, 100

1804: v. End e: Massen und Steine, p. $36-41$ (Urkunde von 1751).

1804: G i 1 b e r t: Nachträge zu den Aufsätzen in den Annalen über die aus der Luft gefallenen Steine, Gilb. Ann. Bd. 18, p. 287.

1812: $\mathrm{Ch}$ la d n i: Verzeichnis, Schweigg. Journ. Bd. 4 Beil. I, p. $12-\mathbf{I} 3, \mathbf{I} 8$.

r812: Bigot de Morogues, p. 96 -98 .

1812: $\mathrm{N}$ e u ma n n: $>$ Hesperuse Nr. 55. Ref. in Leopold's Jahrbuch 1814 , p. 258 (beobachtet die nachWidmanstätten genannten Aetzfiguren); s. auch Cohen, Meteoritenkunde 1894 , p. 330 ; ferner Schweigg. Journ, Bd, 7 (1813), p. 173 . 1815: Chladni: Neues Verzeichnis, Gilb. Ann. Bd. 50, p. 248.

r8r5: Chla dni: Bemerkungen, Gilb. Ann. Bd. 5o, p. $257,263-264$.

1817: Chladni: Dritte Fortsetzung, Gilb. Ann. Bd. 56, p. $379-380$.

1817: v. Soemmering: Ueber die Zeichnungen, welche sich bei Auflösung des Meteoreisens bilden. Schweigg.
Journ. Bd. 20, p. 9I-94.

r8I9: $\mathrm{Ch}$ la dni: Fünfte Fortsetzung,

Gilb. Ann. Bd. 63 , p. 28 .

1819: Chlad n i: Feuermeteore, p. 27, $37,4 \mathrm{I}, 59,66,69,7 \mathrm{I}, 74,92,115-$ I16, 245-246 (I), 319, 327, 333, 433; s. auch Denkschr. München. Akad, 1812, p. $104-105$.

1820: v. Schreibers: Beiträge, p. I-9 (Abbildung in wirklicher Grösse); s. auch Brezina, Meteoritenstudien II (1881), p. 133 Anm.

1826: S e e beck: Ueber die magnetische Polarisation der Metalle und Erze durch Temperatur-Differenz. Pogg. Ann. Bd. 6, p. 144 .

1830: v. H o lg e r : NeueAnalyse der beiden Meteoreisenmassen von Lénarto in Agram, nebst einigen Bemerkungen über den Ursprung der Meteormassen überhaupt. Baumgartner's Zeitschr. f. Phys. u. Math. Bd. 7, p. 129-139; s. auch N. J. 1833, p. 192-193.

r832: v. H off: Achter Nachtrag, Pogg. Ann. Bd. 24, p. 230.

1835: We hrle: Amalyse einiger Meteoreisenmassen. Baumgartners Zeitschr. f. Phys. u. verw. Wiss. Bd. 3, p. 224 $-225,226$; s. auch Ann. Pharm. Bd. 14 (1835), p. 94.

1836: Kä m tz: Meteorologie, p. 260, $262,273$.

1836: Gruit huis e n : Naturgesch, d. gestirnten Himmels. München $188_{3} 6$ bei Fleischmann, p. 404-405.

r839: B e n z e n b e r g: Sternschnuppen, p. 40.

184r: R a m mels ber g : Handwörterbuch Bd. I, p. 422.

1843: Partsch: Meteoriten, p. $\mathrm{IO}_{3}-$ I08.

1847: St ed ler: Ueber die in Ungarn herabgefallenen Meteorsteine. Oesterr. Bl. für Lit. 1847 Nro. 86, p. 343 . Ref. N. J. 1848 , p. 65 .

1852: W ö h l e r : >Intermediär «, Pogg. Ann. Bd. 85 , p. 449. 
1852 : C l'a r k : Dissert. Gött., p. 42-44 (Abbildung einer geätzten Platte auf Tafel III).

I854: B a 1 c e $11 \mathrm{~s}$ : Lithologia meteorica, p. 20 .

1854: v. Boguslawski: Zehnter Nachtrag, Pogg. Ann. Ergz.Bd. 4, p. 392.

r855: $\mathrm{H}$ a i d in g e r : Bemerkungen über die zuweilen im geschmeidigen Eisen entstandene krystallinische Struktur verglichen mit jener des Meteoreisens. Sitzber. Wien. Akad. Bd. 15 I, p. 357 (Fig. 7).

1856: Burkart: Fundorte I, N. J. 1856, p. 270.

1858: B urk art: Fundorte II, N. J. 1858 , p. 774 .

1859: $\mathrm{B} \mathrm{u} \mathrm{c} \mathrm{h} \mathrm{n} \mathrm{e} \mathrm{r} \mathrm{:} \mathrm{Feuermeteore,} \mathrm{p.} 40$ -41 .

1859: Harris : Dissert. Gött., p. IO3. 1859: Ha id inger : Der Meteoreisenfall von Hraschina bei Agram am 26. Mai 1751. Sitzber. Wien. Akad. Bd. 35, p. $361-388$ (mit einer chromolithographischen Tafel und zwei Figuren im Text. Zwei Urkunden von 1751). Ref. Luminous Meteors, Rep.Brit.Assoc. r86r (Sep.), p. 32-33; s. auch sL'Institut Bd. 27 (1859) Nr. 1329, p. 203. r860: $\mathrm{R}$ a m mel s ber g : Mineralchemie, p. 906-907.

1860: Haidinger: Eine dritte Urkunde über den Meteoreisenfall von Hraschina bei Agram. Sitzber. Wien. Akad. Bd. 39 , p. $519-525$; s. auch s'Institut \& Bd. 28 (1860) Nr. 1377, p. 174 .

r862: Kenng ott u. Wiser: Zürcher Sammlung, p. 144-I45.

r863: B u chner : Meteoriten, p. 135136.

1863: R os e: Meteoriten, p. 30, 42, 153.

1858/65: v. Re i chen ba c h: IV 640. VI 452 . VII 557 . IX $155,163,174$, 182. X 359, 368, 369. XV 109, по,
124, 126. XVI 250, 255, 256, 261, 262, $265,266,272$. XVII 264. XVIII 484,487 . XIX 150, 155. XXIV 226. XXV 612, 613, 6r4, 618.

1869: Bu chner: Vierter Nachtrag, Pogg. Ann. Bd, 136, p. 439.

r870: v. H a i d in g er: Orientierung, Sitzber. Wien. Akad. Bd. 6r II, p. 512 .

1870: $\mathrm{R}$ a m mels ber $\mathrm{g}$ : Meteoriten, p. 80 .

r874: B jörling: Meteoriter och Kometer (cfr. Hessle), p. I2.

1875: Mohr: Entstehungsart, Ann. Chem. Pharm, Bd. 179, p. 259, 269, 276.

1875: Tschermak: Vulkanismus, Sitzber. Wien. Akad. Bd. 71 II, p. 662. r884: M e uni er: Météorites, p. 94, 98, II5, II6-II7, 459.

r884: v. $\mathrm{Ni}$ iess 1: Ueber die astronomischen Verhältnisse bei dem Meteoritenfalle von Mócs in Siebenbïrgen am 3. Februar 1882. Sitzber. Wien. Akad. Bd. 89 II, p. 29I. Ref. N. J. $1886 \mathrm{I}$, p. 224.

1883/85: Tschermak: Photographien, p. 3 .

1885: Brezina: Wiener Sammlung, p. $209,233$.

1888: N e w to n: Orbits, Am. Journ. (3) 36, p. 4 .

1889: F l e t c h e r : Atacama Meteorites, Mineral. Magaz. Bd. 8, p. 225.

1890: v. Niess 1: Periheldistanzen, Verh. naturf. Ver. Brünn Bd. 29, p. 186, 188, 194, 214, 239-241.

r89r: Cohen u. Weinschenk: Meteoreisen-Studien. Ann. Hof-Mus.Bd. 6, p. 131, 148-149 (Analyse), 164 .

r892: B r e z in a: Sternschnuppen, p.II, 16.

1893: B r e zin a : Ueber neuere Meteoriten (Nürnberg), p. 164 .

1893: M e u nier: Révision des fers météoriques, p. $63-64$.

r894: C o h en: Meteoritenkunde,p. 38,40 , $54,56,76,131,189,330$. 
1894: F le t c h e r: Introduction, p. 33 34 .

1895: B rez in a : Wiener Sammlung, p. 268 .

Ursprüngliches Gewicht: $48^{3 / 4} \mathrm{Kgr}$. Zwei Massen; die eine 7I, die andere 16 Wiener Pfund. Die letztere scheint verloren gegangen zu sein.

Nachweisbares Gewicht: 3967 I gr.

Berlin, U.

Budapest

27|Neumann

Calcutta

Göttingen

Harvard, U.

2 New Haven

I Paris, M.

20 Stockholm

6 Tübingen

London, B. M. $\quad 282$ Washington, Sh.

London, P. G. $\quad 9$ Wien,H.M.*) 39245

Marburg

Moskau

I Würzburg

Spl. Zürich

Huejuquilla-Gruppe(Jimenez), Chihuahua, Mexico.

Unter diesem Namen sind vorläufig vereinigt :

I. Chupaderos, Rancho de Chupaderos, Eisen, Of.

II. Concepcion (Adargas) bei $\mathrm{Za}$ pote, Valle de San Bartolomé (Valle de Allende), Eisen, Oml.

III. San Gregorio (Morito), Valle de Allende, Eisen, Oml.

IV. Rio Florido (verloren gegangen, nach Brezina die aus Chihuahua in Washington liegende Masse).

V. Sierra Blanca (z. T. von Brezina zu Toluca gestellt); hierher auch Villa Nueva.

VI. Tule.

I. Chupaderos, bekannt seit Jahrhunderten, wieder aufgefunden 1852 .

1854: B artlett: Personal Narrative of Explor. in Texas, New Mexico, California, Sonora and Chihuahua.
New York 1854 Bd. 2, p. 453,458 . 187I: S m it h: The precise Geographical position of the large masses of meteoric iron in North Mexico, with the description of a new mass. - The SanGregorio Meteorite. Am. Journ. (3) 2, p. 337 , wo nur gesagt wird: $\gg$ The locality of a huge meteorite lately discovered, of which no specimen has yet been detached, and is said to be larger than any one yet found in that locality «; s. auch Original Researches 1884, p. 467.

187 I : Burkart: Briefl. Mitt. N. J. I 871 , p. $85 \mathrm{I}-852$ (könnte sich auch allenfalls auf Ranchito beziehen).

1876: $\mathrm{S} \mathrm{mith:} \mathrm{Aragonite} \mathrm{on} \mathrm{the} \mathrm{sur-}$ face of a meteoric Iron and a new Mineral (Daubréelite) in the concretions of the interior of the same. Am. Journ. (3) 12, p. 107, wo es heisst: We have some account of one even larger than the last (nämlich der San-Gregorio Masse von $3500 \mathrm{Kgr}$.), located in the very center of the desert.e; s, auch C. R. Bd. 83 (1876), p. 74 ; Original Researches 1884, p. 514 .

1876: B a r cen a : On certain Mexican Meteorites. Proc. Acad. nat. hist. Philadelphia (1876), p. 122 .

1879: Rammelsberg : Meteoriten, p. 32 .

r880: $\mathrm{M}$ u ñ oz L u m bi e r: Los Aerólitos de Chihuahua. Mexico 188o (nicht eingesehen).

1884: $\mathrm{H}$ ä $\mathrm{p} \mathrm{ke} \mathrm{:} \mathrm{Beiträge,} \mathrm{Abh}$. naturw, Ver. Bremen Bd, 8, p. 516-519.

r889: Flet c her: Mexican Meteorites, Mineral. Magaz. Bd. 9, p. 99, 103, 104 , 122-15I, 175. Ref. N. J. 1891 II, p. 240.

1889: C a s tillo: Météorites, p. 6-8. r89o: B r e z in a : Reise, Ann. Hof-Mus.

Bd. 4 (Not.), p. 1 7.

189o: Brezina: Ueber Meteoreisen, Oesterr. Zeitschr. f. Berg- u. Hüttenw. Bd. 38 (Nr. 31$)$, p. 355 . 
r89r: Cohen u. Weinschenk: Meteoreisen-Studien I. Ann. Hof-Mus. Bd. 6, p. 131, 132, 147-148 (Analyse), 160,164 .

r892: E a st $m$ a n : The Mexican Meteorites. Bull. Philos. Soc. Washington Bd. 12, p. 44 .

r892: C o hen: Meteoreisen-Studien II. Ann. Hof-Mus. Bd. 7, p. 150-151 (Analyse), ${ }_{15} 8(\mathrm{Cu}), 159,160,161$.

1893: Meunier: Révision des fers météoriques, p. 52, 53 .

1894: C o h e n : Meteoritenkunde, p. 53, 10I, 102, 104, 196.

r895: Brezina: Wiener Sammlung, p. 269 .

UrsprünglichesGewicht: Etwa 24890 Kgr. Zwei sehr grosse Schollen,

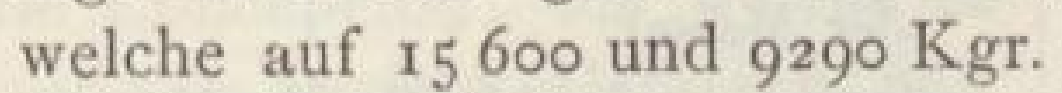
geschätzt werden.

Nachweisbares Gewicht: I47I gr. Paris, M. $\quad 787 \mid$ Wien, H. M. $\quad 65^{8}$ v. Siemaschko 26

II. Concepcion (Adargas), bekannt seit Jahrhunderten, wieder aufgefunden 1780 oder 1784 (?) (auch Hacienda Concepcion und Sierra de las Adargas).

[r625: Purchas his Pilgrines in five bookes etc. London 1625 . Fourth part. The Eighth booke. Chap. III, p. ${ }^{56} 65$ -r567.] (Nicht eingesehen)

1854: B a rt l e t $t$ : Personal Narrative of Explor. in Texas, New Mexico, California, Sonora and Chihuahua. New York 1854 Bd. 2, p. 457.

1855: S mith: Memoir on Meteorites: A Description of five new Meteoric Irons, with some theoretical considerations on the origin of Meteorites based on their Physical and Chemical characters. Am. Journ. (2) 19, p. 160 (Weidner's Parral-Masse), 163 (zwei Abbildungen der Masse von 3853 lbs.); s. auch Original Researches 1884, p.378-379; Kenngott, Uebersicht 1855 , p. 97; Journ. prakt. Chem.Bd.66 ( 1855$)$, p. $427-428$.

r856: B urkart: Fundorte I, N.J. 1856, p. 280-281 (Abbildung der Smith'schen Masse auf Taf. 4).

1857: Pietro Gar cia Conte: Boletin de la Sociedad Mexicana de Geog. y Estad. Prima época. México 1857, Bd. 5 , p. 251 .

1858: Burkart: Fundorte II, N. J. 1858 , p. 770,772 .

1865: C on o 11 y: Smithsonian Report for 1865 , p. 124 .

1866: B u rk a rt: Fundorte III, N. J. I866, p. 408.

1867: S i m s o n : Smithsonian Report for 1867 , p. 472.

r870: B urkart: Fundorte IV, N. J. I870, p. 69r.

1871: Burkart: Briefl. Mitt. N. J. 187I, p. $852-854$.

187 I: Urquidi: Boletin de la Sociedad Mexicana de Geog. y Estad. Segunda época. México I871 Bd. 3, p. 275. 1872: Urgin di: On the Meteorites of the Hacienda $\$ \mathrm{La}$ Concepcion and San Gregorio (Extract from a letter to Prof. Henry, Secretary of the Smithsonian Institution, to whom we are indebted for the communication). Am. Journ. (3) 3, p. 207-208. Gewicht: $154^{192 / 1000}$ ×arrobas ( $($ arrobas zu 25 lbs.), also etwa $r_{700} \mathrm{Kgr}$. Fletcher sagt nach Castillo $3^{1} 30 \mathrm{Kgr}$. Die Volumbest. Urgindi's ergiebt 39299 Kubikzoll oder etwa $2 / 8$ Kubikmeter oder ca. 5000 Kgr. Ref. Liebig-Kopp, Jahresber. I872, p. 1200 .

1876: B a r c en a : Proc. Acad, nat, hist. Philadelphia 1876 , p. 122.

1879: R a mmelsberg: Meteoriten, p. $3 \mathbf{I}$.

1880: Muños Lumbier: Los Aerólitos de Chihuahua. Mexico 188o, p. 16,17 .

1884: Meunier: Météorites, p. 44r. 1884: vom R a th: Meteoriten. Verh. 
naturh. Ver. Bonn (Sitzber.) Bd. 4I, p. 126.

1885: B rezin a: Wiener Sammlung, p. 22I, 234 .

1889: Cast i 11 o: Catalogue, p. 6-7. r89o: F l et c her: Mexican Meteorites, Mineral. Magaz. Bd. 9, p. 122, 123, $124,127-128,131,139,140-144,150$, $151-152$.

r89o: B r e z in a: Reise, Ann. Hof-Mus. Bd. 4 (Not.), p. 117 .

1892: Eastman: The Mexican Meteorites, Bull. Philos. Soc. Washington Bd. 12, p. 44.

r894: Co he n : Meteoritenkunde, p. 62, 95, 196.

Ursprüngliches Gewicht: EineMasse von etwa 3853 Pfund spanisch oder von $3^{1} 30 \mathrm{Kgr}$., wie Castillo angiebt.

Nachweisbares Gewicht: 734 gr.

\begin{tabular}{lr|lr} 
Bailey & $\left.\mathbf{I}^{1}\right)$ & Göttingen & Spl. \\
Bement & $\left.32^{2}\right)$ & Gregory & $\mathbf{I I}^{6}$ ) \\
v. Braun & $\left.\mathbf{I}^{3}\right)$ & Harvard, U. & $3^{7}$ ) \\
Cleveland & $\left.\mathbf{I}^{4}\right)$ & Wien, H. M. $\left.*^{*}\right)$ & $574^{8}$ )
\end{tabular}

III. San Gregorio (Morito oder El Morito), bekannt $\mathrm{r} 600$, erwähnt I6I9.

(Eisen mit der Inschrift: "Solo dios con un poder « etc.).

r6rg: Luis Cabrera de Cordob a: Historia de Felipe Segundo Rey de Espagña, Madrid 16I9 Lib. 13, p. 1163; auch Edicion publicada de Real orden, Madrid 1876-1877 Bd, 2, p. 677 .

1829: $\mathrm{Hardy}$ : Travels in the interior of Mexico in $1825-1828$. London 1829 , p. $48 \mathrm{I}$.

1854: B a r $\mathrm{t} 1$ e $\mathrm{t} t$ : Personal Narrative of Explor. in Texas, New Mexico, Cali- fornia, Sonora and Chihuahua. NewYork I854 Bd. 2, p. $45^{8}$.

1858: B urkart: Fundorte II, N. J. I858, p. $770,771-772$.

r866: B u r k a rt : Fundorte III, N. J. I866, p. 408.

r870: Burkart: Fundorte IV, N. J. 1870, p. 690.

1871 : Burkart: Briefl. Mitt. N. J. I871, p. $852-853$. Ref. Liebig-Kopp, Jahresber. 1871, p. 1244 .

r87 I: $\mathrm{S} \mathrm{mith}$ : The precise Geographical position of the large masses of meteoric iron in North Mexico, with the description of a new mass u. s, w.; s. bei Chupaderos. Am. Journ. (3) 2, p. 336 ; s. auch Original Researches 1885 , p. 465-466 (Analyse u. Abbildung der Masse).

1872: U r g in d i: On the Meteorites of the Hacienda "La Concepcion" and San Gregorio (Extract from a letter to Prof. Henry etc. s, bei Concepcion). Am. Journ. (3) 3, p. 207-208.

1879: R a m mels berg: Meteoriten,p.31. 1880: M u ñ o z L u m b i e r: Los Aerólitos de Chihuahua. Mexico 188o, p.16,17. 1889: H untingt on: The Crystalline Structure of the Coahuila Irons. Proc. Amer. Acad. of Arts and Sc. Boston Bd. 24 (1889), p. 35.

1889: C a st ill o: Catalogue, p. 7.

r89o: E a s t m a n : Met. Astron., p. 322.

1890: Brez in a: Reise, Ann. Hof-Mus. Bd. 4 (Not.), p. 117 .

r8go: F let c her: Mexican Meteorites, Mineral, Magaz. Bd. 9, p. 122, 123, I24-I25, 13I-I32, 137-I40, 150, 151. 1892 : Eas t m a n: The Mexican Meteorites. Bull. Philos. Soc. Washington Bd. 12, p. 44 .

1893: M e un i e r : Révision des fers météoriques, p. 52, 53.

1) sHacienda Conception, Chihuahua Mex. 1784<. - 2) >Adargas

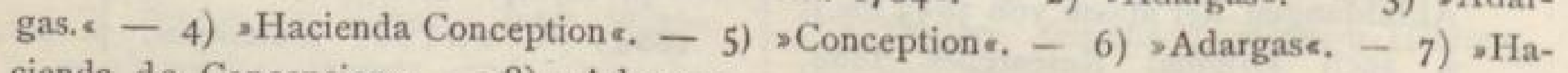
cienda de Concepcion $\alpha .-8$ ) . Adargas $\propto$. 
I894: C o hen: Meteoritenkunde, p. 196. r895: Brezin a: Wiener Sammlung, p. $269,272-273$.

r895: Co hen: Meteoreisen-Studien IV. Ann. Hof-Mus. Bd. X, p. 88.

UrsprünglichesGewicht : I $1000 \mathrm{Kgr}$. Nachweisbares Gewicht: II 2 gr. Harvard, U. $\quad 36$ v. Siemaschko 9 London, P. G. I Wien, H. M. $\quad$ I $8^{1}$ ) Paris, M. $\quad 48$

IV. Rio Florido, bekannt $185^{8}$, soll verloren gegangen sein. Brezina vermutet, dass die in Washington aufbewahrte Masse von Chihuahua (s. dort) hiermit identisch ist. Fletcher sagt: etwa halb so gross, wie Concepcion, für welche er $3130 \mathrm{Kgr}$. angiebt. Uebrigens hält Fletcher es nicht für unmöglich, dass Rio Florido u. Concepcion identisch sind.

1858: B urkart: Fundorte II, N. J. $185^{8}$, p. $77^{2}$.

1858: Weidner: Bol. Soc. Mex, de Geog. y Estad. Primera época Bd. 6 (1858), p. 6r.

1866: Burkart: Fundorte III, N. J. I866, p. 408 .

1866: Wh itney: Proc. Calif. Acad. Nat. Sc. Bd. 3, p. 24 I.

r867: W o o d w or th : Smithsonian Report for 1867 , p. 472 .

187r: Burkart: Briefl. Mitt. N. J. 1871 , p. 852,854 .

1889: C a st il1 o: Catalogue, p. 6, 9. r89o: F l e t c h er: Mexican Meteorites, Mineral. Magaz. Bd. 9, p. 122, 123, $144,146-148$.

1892: Eastman: The Mexican Meteorites. Bull. Philos. Soc. Washington Bd. 12, p. 44 .

V. Sierra Blanca, bekannt ${ }^{7} 784$. Das unter diesem Namen von
Rose aus der Berliner Sammlung beschriebene Stück wird für Toluca gehalten. Da aber Brezina neuerdings eine Aehnlichkeit von Toluca u. Chupaderos erwähnt (Wiener Sammlung 1895 , p. 269 u. 274), so wäre es nicht unmöglich, dass das Berliner „Sierra Blanca* zur Huejuquilla-Gruppe gehört. Hierher auch vorläufigVillaNueva. 1784: Gazeta de Mexico, año de 1784 et 1785 Bd. I, p. $146,200$.

1817: Chladni: Dritte Fortsetzung, Gilb. Ann. Bd. 56, p. $3^{8} 3-3^{84}$.

1819: C h la d n i : Feuermeteore, p. 338 -339 .

1834: K a y s e r: Beschreibung der Min.

Samml. des Herrn Bergemann. Berlin 1834, p. 465.

1843: Parts ch: Meteoriten, p. 145. 1852 : C l a r k: Dissert. Gött., p. 64. r854: v. B o g us la w ski: ZehnterNachtrag, Pogg. Ann. Ergz.-Bd. 4, p. $4^{12}$. 1856: B urk art: Fundorte I, N. J. 1856, p. $278-28$ o.

r858: Burkart: Fundorte II, N. J. 1858 , p. 770 .

r863: Rose: Meteoriten, p. 63 .

1870: B urk art: Fundorte IV, N. J. 1870 , p. 690 .

1890: F l et c h e r: Mexican Meteorites, Mineral. Magaz. Bd. 9, p. 122-123, 149-150.

1892: Eastman: The Mexican Meteorites. Bull. Philos. Soc. Washington Bd. 12, p. 44 .

1895: B rezina : Wiener Sammlung, p. 274 .

Ursprüngliches Gewicht: ?

Nachweisbares Gewicht: $39^{2}$ gr.

Berlin, U. $\quad 147$ London, B.M. 15 Dorpat 230

Auch die Ecole des Mines in Mexico besitzt nach Castillo ein Stück dieses Eisens.

I) Aus der Dr. H. Schulze'schen Sammlung, Santiago, stammend. 
VI. Tule. Von Castillo zuerst 1889 erwähnt.

1889: Castillo: Catalogue, p. 7. r89o: F let cher: Mexican Meteorites, Mineral. Magaz. Bd. 9, p. 123, 150.

1892: Eastman: The Mexican Meteorites. Bull. Philos, Soc. Washington Bd. 12, p. 44.

r895: Brezina: Wiener Sammlung, p. 269.

Ein kleines Stück befand sich 1889 in der Ingenieur-Schule in Mexico.

\section{Huesca}

Roda

Humboldt-Eisen, auch vielfach ? Mexico 1804 von Humboldt mitgebracht oder "Durango 1811 \& bezeichnet, da Humboldt's Werk, in welchem er dieses Eisen erwähnt, I 81 I erschien. Wahrscheinlich zur Huejuquilla-Gruppe gehörig.

Eisen, die Tübinger Stücke Om.

r804: Del R i o: Tablas Mineralogicas, Mexico 1804 , p. 57 ; s, auch Elementos de Orictognosia. Mexico $1805 \mathrm{Bd} .2$, p. 41 .

1807: K la proth: Beiträge, Bd. 4, p. $\mathrm{IOI}-\mathrm{IO} 2$.

I8II: v. $\mathrm{Humboldt}$ : Essai politique sur le royaume de la nouvelle Espagne. Paris I8II Bd. I, p. 293 u. Bd. 2, p. 582 .

I8r2: $\mathrm{Ch} 1$ a d n i: Verzeichnis, Schweigg. Journ. Bd. 4 Beil. I, p. 17; s, auch Denkschr. München. Akad. 1812, p. 107. I812: Bigot de M orogues, p. 300. r8r5: Chla dni: Bemerkungen, Gilb. Ann. Bd. 50, p. $268-27 \mathrm{I}$.

1819: Chla d ni: Feuermeteore, p. 337 -338 .

r820: v. Schreibers: Beiträge, p. $78-79$ (Tafel VIII * Mexico $\%$ ). Vielleicht gehört dieses Eisen der Wiener Sanmlung hierher. Die Umrisse und die gebogenen Lamellen sind nicht unähnlich einem Stücke "Xiquipilco der Göttinger Sammlung (s. Clark 1852, T. III, so dass möglicherweise beide Schliffe benachbarten Teilen ein und desselben Stücks angehörten; sicher nicht Zacatecas, wie Laspeyres, p. 206 annimmt.

I82I: J o hn: Chemische Untersuchung der gediegenen Eisen- u. Meteormassen. - 4. Chemische Zerlegung der mexikanischen Eisenmasse. Schweigg. Journ. Bd. 32 , p. 263-264; s, auch Ann. Chim. Phys. Bd. 18 (1821), p. 263-264. r843: R a mirez: El Museo Mexicano ó Miscelanea Pintoresca de Amenidades Curiosas e Instructivas, México I843/45 Bd. I, p. 29.

1843: Partsch: Meteoriten, p. 112 (nicht P. $\mathrm{II}_{3}$ u. II4, welche sich auf $\mathrm{Ka}$ rawinsky's Guadalup gleich Rancho de la Pila beziehen).

I848: $\mathrm{R}$ a m m e $1 \mathrm{~s}$ be $\mathrm{r}$ : : Handwörterbuch, p. 422 .

r852: W öhler : >Aktiv«, Pogg. Ann. Bd. 85 , p. 448 (als > Mexico aufgeführt; ich vermute, dass darunter das *Humboldt-Eisen * gemeint ist, da hiervon Göttingen ein Stück besitzt).

r852 : C 1 a r k : Dissert. Gött., p. $56-$ 57 u. Abbildung einer geätzten Platte Tafel III, Fig. 4.

1854: v. Boguslawski: Zehnter Nachtrag, Pogg. Ann. Ergz.-Bd. 4, p. $4 \mathrm{II}$.

1855: Haidinger: Bemerkungen über die zuweilen im geschmeidigen Eisen entstandene krystallinische Struktur, verglichen mit jener des Meteoreisens. Sitzber. IVien. Akad. Bd, I5 I, p. 357 (Abbildung einer geätzten Platte; Tafel I, Fig. 8, die gleiche Abbildung wie bei Clark).

1856: Burkart: Fundorte I, N. J. 1856 , p. $281-285,290,291,292$. 1857: Burkart: Briefl. Mitt. N. J. 1857 , p. $53-54$. 
1857: Harris: Dissert. Gött., p. 107. r858: We idner: Bol. Soc. Mex. de Geog, y Est. Primera época, Mexico $185^{8}$ Bd. 6, p. 6r.

r858: Burkart: Fundorte II, N. J. I858, p. 77I, 773-775, 779 .

r859: B u c h n e r: Feuermeteore, p. 143. r860: $\mathrm{R}$ a m mels berg: Mineralchemie, p. 909 .

r86r: F or c h h a m m e r: Oversigt K. Dansk. V. S. Forh. 1861, p. 226.

I858/62: v. Re i che n b a c h: VI 448 .

VII 551,557 . IX 163, 174, 181. X 559 .

XII 457. XV 100, $110, \mathrm{II}_{4}, \mathrm{I24}, \mathrm{I2} 8$. XVI 25I, 254, 255, 261, 262. XVII 266, 272. XVIII $480,484,487,490$. XIX 149, $152,153,155 . X X 621,622 . X X I_{586}$, $588,589$.

1862: Kenngott u, Wiser: Zür-

cher Sammlung, p. 144 .

1863: B u c hner: Meteoriten, p. 149I50.

r863: R o s e : Meteoriten, p. 23, 64-65, 153 .

1864: Bu chner: Erster Nachtrag, Pogg. Ann. Bd. I22, p. 32 I.

1866: B u rk a rt: Fundorte III, N. J. 1866, p. 408.

1867: T a r a yre: Archives de la Commission Scientifique du Mexique. Bd. 3 , p. 270.

1869: B u chner: Vierter Nachtrag, Pogg. Ann. Bd, 136, p. 605.

1870: Burkart: Fundorte IV, N. J. 1870 , p. 684,691 .

1871: Burkart: Briefl. Mitt. N. J. 1871 , p. $851-852$.

r875: Mohr: Entstehungsart, Ann.

Chem. Pharm. Bd. 179, p. 259, 270.

1876: B a r c e n a : Proc. Acad, nat. hist.

Philadelphia 1876, p. 123.
1884: M e uni e r: Météorites, p. 96, 116, 118 .

1884: Häpke: Beiträge, Abh, naturw. Ver. Bremen Bd. 8 (1884), p. 516. 1890: E a s t m a n: Met. Astron, p. 318. I890: Flet cher: Mexican Meteorites, Mineral. Magaz. Bd, 9, p. 104, 122, 123, $132-138,150,151$.

1894: C o h e n : Meteoritenkunde, p. 54. r895: Brezina: Wiener Sammlung, p. $268,274,276$.

Ursprüngliches Gewicht: $30000-$ 40000 Pfund (I900 myriagrammes). Nach dieser Gewichtsangabe sollte man an eine der grossen Schollen der HuejuquillaGruppe in dem nördlich von dem Staate Durango liegenden Staate Chihuahua oder an die grosse Masse von Ranchito (Bacubirito) im Staate Sinaloa, westlich von Durango denken. Im Staat Durango selbst scheint bis jetzt noch keine so grosse Masse gefunden zu sein; in früherer Zeit dehnte sich dieser Staat weiter nach Norden aus, so dass damals die grossen Schollen von Huejuquilla in Durango lagen (Fletcher). Zunächst wäre es wohl erwünscht, die nachfolgenden Massen auf ihre Einheitlichkeit zu prüfen.

Nachweisbares Gewicht: $284^{2}$ gr.

Berlin, U. $\left.\quad 782^{1}\right)$ Gregory $\quad 74^{5}$ )

Calcutta $\quad 7^{2}$ ) Kopenhagen $219^{6}$ )

Cambridge $\quad 8^{3}$ ) London, B. M. 440 ${ }^{7}$ ) Göttingen $\quad 59^{4}$ )

1) Bei Klein $\$ 1804$ Rancho de la Pila, Durango, Mexico $\approx$, bei Rose $\$ 1804$ Durango, Mexico « bezeichnet. - 2) "Durango $18 \mathrm{r} \times$. - 3) "Durango is - 4) "Durango I8r $\approx$. 5) $>$ Found $\mathbf{r} 804$. Durango, Mexico. Etched slice, fine Widmanstätten figures $\propto$. - 6) $>1805$ Durango, Mexiko . - 7) In dem neueren Londoner Kataloge nicht aufgeführt, da nach gütiger Mitteilung des Herrn L. Fletcher über den Ursprung des Stückes Unsicherheit herrscht: st was said by Mr. Heuland (in 1846) to be Zacatecas, but I do not know on 


\begin{tabular}{lr|lr} 
Minneapolis & $\left.10^{1}\right)$ & Tübingen & $197^{6}$ ) \\
Moskau & $\left.9^{2}\right)$ & Washington & $45^{7}$ ) \\
Neumann & $\left.1 \mathbf{1}^{3}\right)$ & Wien, H. M. & $\left.826^{8}\right)$ \\
Paris, E. & $\left.91^{4}\right)$ & Zürich & $\left.7^{9}\right)$ \\
Stockholm & $\left.57^{5}\right)$ & &
\end{tabular}

Hungen, Hessen, Deutschland.

Stein, Cga, gefallen 17 . Mai 1877 .

1877: B u c hner: Der Meteorstein von Hungen. M. M (1877), p. 3'3-315. Ref. N. J. 1878, p. $411-412$; s, auch Z. f. Kryst. Bd, 2 (1878), p. 630 ; LiebigKopp, Jahresber. 1877 , p. 1393.

1877: Ts chermak: Anhang zu der vorstehenden Mitteil. M. M. (1877), p. $315-316$.

1878: $\mathrm{B}$ u chner: Ueber den Meteorstein von Hungen und über Meteoriten im Allgemeinen. Programm der Grossherzoglichen Realschule zu Giessen 1878 , Nr.522. Giessen bei W.Keller. 1884: W a d s w or th: Studies, p. IO2. 1884: M e un i er: Météorites, p. 209. 1885: Brezina: Wiener Sammlung, p. $155,182,233$.

1887: F $1 \mathrm{ig}$ h t: Meteorites, p.2O2 - 203. 1894: C o h en : Meteoritenkunde, p.298.

Ursprüngliches Gewicht: II 2 gr.

Zwei Steine von 86 und 26 gr.

Nachweisbares Gewicht: $1 \mathrm{II}^{1 / 2} \mathrm{gr}$.

v. Braun

Budapest
$56^{1 / 2}$

$1^{1 / 2}$
London, B. M. $\quad 5^{1 / 2} /$ v. Siemaschko $\quad 61 / 2$ Paris, M. 2 Wien, H. M. 26

Ibbenbühren, Westphalen,Preussen, Deutschland.

Stein, Chl, gefallen 17. Juni 1870. 187 1 : vom $\mathrm{R}$ a t h legte ein Modell des am 17 . Juni 1870 in der Gegend von Ibbenbühren in Westphalen gefallenen Meteorsteins vor, welchen er der Güte des Herrn Prof. Heis in Münster verdankte. Verh. naturh, Ver. Bonn Bd. 28 , p. $127-128$; s. auch Wochenschr. f. Astr., Met. u. Geogr. 1871, p. 291. 1871: vom $\mathrm{R}$ at h hielt einen Vortrag über die mineralogische und chemische Constitution des am 17. Juni 1870 in der Gegend von Ibbenbühren niedergefallenen Meteorsteins. Verh. naturh. Ver. Bonn Bd, 28, p. 142-145.

1872: vom Rath: Ueber den Meteoriten von Ibbenbühren (Westphalen), gefallen am 17. Juni r870 (zwei Abbildungen des Steins). Mon. Ber. Berlin. Akad. (1872), p. 27-36; s, auch Pogg. Ann. Bd. $146(1872)$, p. $463-474$; Am. Journ. (3) 4 , p. 78 ; N. J. 1872 , p. 648; Liebig-Kopp, Jahresber. 1872, p. II93-II94; Wochenschr. f. Astr., Met. u. Geogr. 1873, p. 225-229. 1875: vom Rath : Meteoriten, Verh. naturh. Ver. Bonn Bd, 32, p.374-375.

what authority. The name Durango was suggested by one of my predecessors. I myself think it may be Toluca (?)<. - 1) Von Shepard eingetauscht: "Durango Mex. 1804 (?)«. -

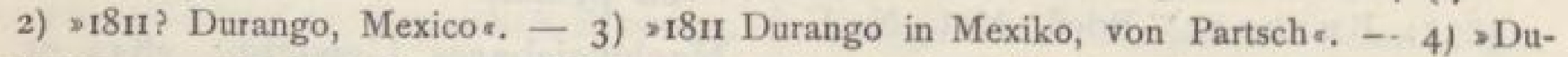
rango. - 5) $>\mathrm{I} 805$ Durango, Mexico. 4 smärre stycken från Berzelii samling etiketterade "Fer de Mexique. Humboldt«". - 6) $\gg$ I8n Durango, Mexico , oktaëdrisches Eisen mit mittlerer Breite der Lamellen. - 7) „Durango, Mexico. 1804*. - 8) Davon 36 gr. I804, Mexico von Humboldt mitgebracht. Goldbach's Eisenc. Of, welche Brezina zu Teposcolula stellt und $790 \mathrm{gr}$. $* 1804$ (Pila) Durango, Mexico $\star$ Om. Ausserdem noch ein als Toluca e bezeichnetes Stück, welches von v. Humboldt an Bergmann in Berlin gelangte u. I810 von Wien erworben wurde. "Goldbach's Eisens ist dunkler Herkunft, da über die salte Sammlung * nichts Näheres zu erfahren war. Wenn Humboldt es eigenhändig von einem Block, der einem Schmied als Ambos diente, abgeschlagen hat (Bre-

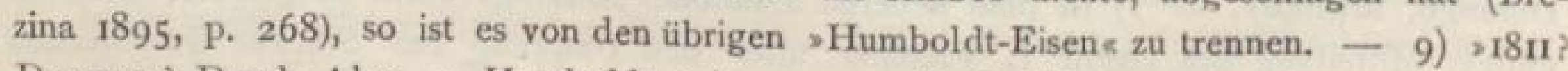
Durango? Durch Alex, v. Humboldt gebr. u. von Prof. Chladni gekauft. 
r879: R a m m e ls b e r g : Meteoriten,p.I4 $-15,25$.

1883: T s chermak: Beitrag, Sitzber. Wien. Akad. Bd. 88 I, p. $365-366$. r884: W a d s w or th: Studies, p. 202. 1884: Häpke: Beiträge, Abh. naturw. Ver. Bremen Bd. 8, p. 523 .

r883/85: T s c h e r mak: Photographien, T. VI, p. 3, 10.

r885: Brezina: Wiener Sammlung, p. $174,232$.

r887: Flight: Meteorites, p. 17-18. 1894: Co he n: Meteoritenkunde, p.207,

$243,247,277,281,287,319$.

Ursprüngliches Gewicht: 2064 gr. Ein Stein, von welchem 2034 gr. gleich nach dem Fall und $30 \mathrm{gr}$. später gefunden wurden.

Nachweisbares Gewicht: 2019 gr.

Aachen

Berlin, U.

Bonn

v. Braun

Dorpat

Göttingen

\begin{tabular}{r|lr}
3 & Klausenburg & 5 \\
1944 & Krantz & 5 \\
23 & London, B. M. & 3 \\
3 & Riga & 2 \\
I & v. Siemaschko & I \\
13 & Wien, H, M.*) & 16
\end{tabular}

\section{Igast}

s. Anhang

Iglau Stannern

Iharaota (Lalitpur), zwischen Iharaota und Nyagong, Pargana Marwara, Lalitpur Distrikt, NordwestProvinz, Indien.

Stein, Choa, gefallen 7. April 1887. 1887: M a 11 et: Note on the "Lalitpur \& Meteorite. Rec. Geol. Surv. Bd. 20, p. $153-154$.

r894: F l e t c h e r : Introduction, p. 14. r895: Brezin a : Wiener Sammlung, p. 241 .

Ursprüngliches Gewicht: $37^{2} \mathrm{gr}$. Nachweisbares Gewicht: 3r4 gr.

\begin{tabular}{lr|ll} 
Calcutta & 144 & Paris, M. & 22 \\
Gregory & 37 & Wien, H. M. & 29 \\
London, B. M & 82 & &
\end{tabular}

\section{Ihung}

Jhung
Ilimaë, Wüste Atacama, Chile, Südamerika.

Eisen, Om, gefunden?, bekannt 1870, beschr. I871. Tschermak's ¿Wüste Atacama 1870* (Brezina vereinigt dieses Eisen mit Juncal).

1871: T s chermak: Das Meteoreisen aus der Wüste Atacama. Denkschr. Wien. Akad. Bd. 3r, p. 187-I96 (Analyse von Ludwig, 4 Tafeln, 3 Holzschnitte) Ref. N. J.1872, p. 429-430; Liebig-Kopp, Jahresber. 18 71, p. 1243 -1244; Wochenschr. f. Astr., Met. u. Geogr. 1870 , p. 280.

1871: Ludwig: Chemische Analyse des Meteoreisens aus der Wüste Atacama. Sitzber. Wien. Akad, Bd, 63 II, p. $323-324$.

r875: Tschermak: Vulkanismus, Sitzber. Wien. Akad. Bd. 71 II, p. 662. 1880: B r e $z$ in a : Reichenbach'sche Lamellen, Denkschr. Wien. Akad. Bd. 43 , p. 14 .

r88x: Brez in a: Bericht III, Sitzber. Wien. Akad. Bd. 84 I, p. 283 .

r882: W i e $\mathrm{c} \mathrm{h}$ m a n n : Fusion-Structures in Meteorites. Ann. N. Y. Acad. Sc. Bd. 2, p. 293,294 (Das hier von Ludwig untersuchte Mejillones ist offenbar Ilimaë).

1885: B rezina: Wiener Sammlung, p. 152,154 .

1887: F $1 \mathrm{ight}$ : Meteorites, p. 19-23. 1889: Flet cher: Atacama Meteorites, Mineral. Magaz. Bd.8, p. 224, 260-26r. 1894: C o h e n: Meteoritenkunde, p. 45, $51,83,92,93,98$, 101, 103, 107, 125, 193, 196.

Ursprüngliches Gewicht: $5^{\mathrm{I}} \cdot 7_{\mathrm{Kgr}}$. Nachweisbares Gewicht: $5^{1} 19^{8} \mathrm{gr}$. London, B. M. 39 (Wien,H.M.*) 51159

Imilac, Atacama, Bolivia.

Pallasit, $\mathrm{P}$, bekannt etwa 1822 ( 800 ?), beschr. 1828 , sehr häufig wird sgef. 1827 * angegeben. 
Hierher auch : Atacama Pallasit(San Pedro de Atacama), Potosi,Campo del Pucara, Caracoles, La Encantada.

r828: A 11 a n: On a Mass of Native Iron from the Desert of Atacama in Peru. Edinburgh. Phil. Trans. Bd. II, p. $223-226$.

1828: Turner: Examination of the Specimen presented to the Society by Mr. Allan in the name of Mr. Woodbine Parish, his Majesty's Consul-General at Buenos Ayres. Edinburgh Phil. Trans. Bd. II, p. 226-228 (Analyse des Eisens); s. auch Pogg. Ann. Bd. I4 (1828), p. 469; Ann. Chim. Phys. Bd. 39 (1828), p. $423-424$.

1830: v. H off: Siebenter Nachtrag,Pogg. Ann. Bd. 18 , p. $188-189$.

r834 : P e n t 1 a n d: Vorzeigen eines grösseren Stücks. «L'Institut « Bd. 2 Nr. 78 , p. 378 ; s. auch Pentland: Ueber das schlackenförmige Eisen von Atacama. N. J. 1835, p. 197.

r839: Woodbine Paris h: Buenos Ayres and the Provinces of the Rio de la Plata. London 1839 , p. 260.

1839: Morren (u. Juben): Chronique scientifique 1 . année 24. Févr. 1839 (angeblich Beilage des ,L'Institut ); s. auch London, Edinb. Philos. Magaz. Bd. 14 (1839), p. 394; Pogg. Ann. Bd. 47 (I839), p. 470 »Meteoreisen von Potosi (von Herrn Juben mitgebracht, von Herrn Morren analysiert) ; Am. Journ. (I) 37 (1839), p. I90-r9r; N. J. 1840, p. 229.

r840: R u m ler: Entdeckung der arsenigen Säure in dem olivenähnlichen Mineral aus dem Meteoreisen von Atacama in Bolivia und von Krasnojarsk in Sibirien. Pogg. Ann. Bd. 49. p. 59I595 ; s. auch Berzelius, Jahresber. Bd. 21 ( 1842$),$ p. 233.

r84r: R a m mels ber g : Handwörterbuch Bd. I, p. 423 . r843: Parts c h: Meteoriten, p.85-86.

1845: D a r 1 u : Nombreux aérolithes du désert d'Atacama (haut Pérou) et nombreuses masses de fer météorique des environs de Santiago del Estero (République Argentine). C. R. Bd. 20, p. I720; s. auch Kenngott, Uebersicht I $844 / 49$, p. 283 ; N. J. I846, p. III.

r848: $\mathrm{S}$ h e p a rd: Report on Meteorites, Am. Journ. (2) 6, p. 403.

185x: Ried (Fletcher sagt R e id) : Chamber's Edinburgh Journ. März I851 ; s. auch N. J. 1855 , p. 8.

185 $\mathrm{I}$ : B o 11 a e r $\mathrm{t}$ : Observations of Southern Peru, including a Survey of the province of Tarapaca, and route to Chili by the coast of the desert of Atacama; read before the Royal Geogr. Soc. of London 28. (oder 2I. ?) April 1851, p. 128; s, auch Rep. Brit. Assoc. 1851 , p. 84 (Notices and abstracts); Liebig-Kopp, Jahresber. 1852, p. 993; Kenngott, Uebersicht 1852 , p. 93.

r85I: S c h mid: Chemisch-mineralogische Mitteilungen. -3 . Olivin aus dem Meteoreisen von Atakama. Pogg. Ann. Bd. 84 , p. $50 \mathrm{O}-504$; s, auch Zeitschr. d. d. Geol. Ges. Bd. 3 (1851), p. $37 \mathrm{I}$; Liebig-Kopp, Jahresber. 1851, p. 775 ; N. J. 1852, p. 53,864 .

1851: v. Kobell: Korr. Bl, zool. mineral. Ver. Regensburg 5. Jahrg. Nr. 7, p. II2.

1852: C 1 a r k: Dissert. Gött., p. 17-19. r852: W ö hl e r : sIntermediärs, Pogg. Ann. Bd. 85 , p. 449 .

1852: Scherer: Verh. phys. med. Ges. Würzburg Bd. 2 (40-42) [nach Laspeyres].

1854: v. Boguslawski: Zehnter Nachtrag, Pogg. Ann. Ergz.-Bd. 4, p. $4 \mathrm{I}_{2}-4 \mathrm{I}_{3}$.

1855: Rö mer legt eine Masse von 9 Pfund 3 Lot vor. Verh. naturh. Ver. Bonn Bd. 12 (Sitzber.), p. 26 u. 300 (Nöggerath). Ref. N. J. 1856, p. 441. r855: Philippi: Ueber das Vorkom- 
men des Meteoreisens in der Wüste Atacama. N. J. 1855 , p. $\mathrm{I}-8$; auch N. J. 1854 , p. 568 ; s, auch Petermann's Mittheil. 1856 , p. 64 ; LiebigKopp, Jahresber. 1854, p. 914 ; Kenngott, Uebersicht 1855 , p. 94-95; ferner Anales de la Universidad de Chile, for June 1854 (Meteoric Iron of Atacama) u. Naval Astron. Exped. to the Southern Hemisphere 1855 Bd. 2, p. 287. 1856: F i e l d: Quart. Journ. Chem. Soc. Bd. 9, p. 143; s. auch Liebig-Kopp, Jahresber. 1856 , p. 918 ; N. J. 1858 , p. 216. Ref. Kenngott, Uebersicht $1856 / 57$, p. 152,$156 ;$ s, auch Faye in C. R. Bd. $5^{8}$ (1864), p. $598-599$; Liebig-Kopp, Jahresber. 1864, p. 904 ; Journ. prakt. Chem. Bd. 69 (1856), p. $250-251$.

1857: S a e m a n n: Briefl. Mitt. N. J. 1857 , p. 415 ; s. auch Journ. prakt. Chem. Bd. 69 (1856), p. $250-251$. •Analyse eines Meteorsteins aus der Wüste Atakama ; N. J. 1857, p. I66 -167 ; Kenngott, Uebersicht $1856 / 57$, p. 156 .

1857: B unsen u. Bronn: Ueber die chemische Zusammensetzung des Meteoreisens von Atacama. N. J. 1857 , p. $257-265$ (Tafel; Analyse von Frapolli); s. auch Liebig-Kopp, Jahresber. 1857, p. 735 ; Kenngott, Uebersicht $1856 / 57$, p. $155-156$.

r859: B u c h n er: Feuermeteore, p. 105. r859: Ha rris : Dissert. Gött., p. IOI, I2I -122 .

r859: S a e m a n n : Briefl. Mitt. N. J. 1859 , p. 178 .

r86o: R a m m els berg: Mineralchemie, p. $438,913,949$.

r862: Kenng ott u. W iser: Zürcher Sammlung, p. 143-144, 157 .

1863: B u c hner: Meteoriten, p. I27 -129 .

r863: R os e: Meteoriten, p. 26, 4I, 43, $76,77, \mathrm{I} 53$.

r864: D o m e y ko: Rapport sur deux
Mémoires de M. J. Domeyko, relatifs, l'un à de grandes masses d'aérolithes trouvées dans le desert d'Atacama, près de Taltal, l'autre à plusieurs espèces minérales nouvelles du Chili. C. R. Bd. 58 , p. $55^{1}-55^{2}$. Nach Meunier auch Annales des Mines (6) 5 (1864), p. 431 .

r864: H a i d ing e r: Eine grosskörnige Meteoreisen-Breccie von Copiapo (Brief von Tschudi über das \$Meteoreisen von Atacama \&). Sitzber. Wien. Akad. Bd. 49 II, p. $494-495$.

1865: Buchner: Zweiter Nachtrag, Pogg. Ann. Bd. 124, p. 59 I.

r857/65: v. Reichen b a c h : III 624 . VI $442,443,454,456$. VII 55 I. IX $162,163,173,181 . X_{371}, 372$. XI 296, 298, 302, 308, 309. XII 452-454, 455. XIII 363. XV 104, 107, I08, 112, II5, 126. XVI 255, 261, 262. XVII 265, 27I, 272. XVIII 48I, 487. XIX 155 . XX 623, 627. XXIV 226. XXV 422, $429,431,600,608$.

1867: D a u bré e: Contribution à l'anatomie des météorites. C. R. Bd. 65 , p. $148-151$.

1867: Go e b e 1: Kritische Uebersicht, Mélanges phys. chim. Bd. 7, p. 336. 1868: M o hr: Bildung der Meteoriten. Verh. naturh. Ver. Bonn Bd. 25 (Sitzber.), p. 66,67 .

r869: Me unier: Recherches, Ann. Chim. Phys. (4) 17 , p. $52,53,56$.

1870: R a m mels berg: Meteoriten, p. $79,87-89,138$; s, auch Pogg. Ann. Bd. 140 (1870), p. 312 .

r872: M e un i er: Analyse lithologique du fer météorique d'Atacama, premier exemple de filons concrétionnés parmi les Météorites. C. R. Bd. 75 , p. $5^{88}$ - 590; s. auch Liebig-Kopp, Jahresber. 1872 , p. II 93 .

1875: D o me yko cfr. Vaca Muerta. r875: vom R a th: Meteoriten, Verh. naturh. Ver. Bonn Bd. 32, p. 365 . r879: $\mathrm{R}$ a m mel s ber g : Meteoriten,p.24. 
1879: Do me y k o: Mineralojia 3. Aufl, p. $127-129$. que de la roche empâtée dans la syssidère d'Atacama. C. R. Bd. 95, p. 1384 -1386. Ref. N. J. 1884 I, p. 32 ; Liebig-Kopp, Jahresber. 1882 , p. 1644. 1883: Tschermak: Beitrag, Sitzber. Wien. Akad. Bd. 88 I, p. 348 . r884: W a d sw ort h: Studies, p.70,72. 1884: Hä p k e: Beiträge, Abh. Naturw.

Ver. Bremen Bd, 8, p. 521 .

1884: Me un i er: Météorites, p. 70, 77, 79, 93, 94, 96, 97, 99, 148 (Abb.) $-152,356,357,358,377,380,499$. (Meunier verweist auch auf Viage al Desierto de Atacama, porel Doctor R. A. Philippe. Halle en Saponia I86o, p. 121).

1883/85: Tschermak: Photographien, p. 23, 24.

1885: $\mathrm{Brez}$ in a: Wiener Sammlung, p. $200,206,233$.

r886: Brezina u. Cohen: Photographien, T. 3 .

1886: $\mathrm{H}$ äp k e: Bemerkungen über Meteoriten. Abh. Naturw. Ver. Bremen Bd. 9, p. 359. Ref, N. J. 1887 I, p. 258. r887: Co h e n : Der Pallasit von Campo de Pucará in der Argentinischen Republik. N. J. 1887 II, p. 45-52.

r887: F lig ht: Meteorites, p. 23 (Anmerkung).

1888: W a r r e n : Detection and estimation of Selenium in Meteoric Iron. Chem. News Bd. 57, p. 16. Ref, N. J. 1890 II, p. 229.

r889: F le t c her: Atacama Meteorites,
I882: M e unier: Analyse minéralogi-

Mineral. Magaz. Bd. 8, p. 224, 225 , 229-230, 243-255.

1893: M e unier: Révision des fers météoriques, p. 15, 20.

r894: C o hen: Meteoritenkunde, p. 22, $62,64,248,252,256,257,263,265$.

r894: F l e t c her: Introduction, p. 14. 1895: M eun ier: Révision des lithosidérites, p. 26, 27-30 (Abb. einer geätzten Platte und eines Dünnschliffs).

1895: Brez ina: Wiener Sammlung, p. 265 .

Ursprüngliches Gewicht: Mehrere Zentner.

Nachweisbares Gewicht: $282317 \mathrm{gr}$.

\begin{tabular}{|c|c|c|}
\hline Bailey & $\left.157^{1}\right)$ & Dijon \\
\hline Bayet & $\left.9^{2}\right)$ & Dorpat \\
\hline Belgrad & 7 & Dresden, M. 13 \\
\hline Bement & 80 & Dublin, M. $\quad \mathbf{I}^{\mathbf{I}^{7}}$ ) \\
\hline Berkeley & $\left.129^{3}\right)$ & Edinburg \\
\hline Berlin, G. & 51 & Eger \\
\hline Berlin, U. & $\left.3793^{4}\right)$ & Frankfurt $\quad 75$ \\
\hline Bern, M. & 49 & Freiberg, i. S. 109 \\
\hline Bologna & $\left.53^{5}\right)$ & Freiberg, i. S. N. $5^{8}$ \\
\hline Bonn & 561 & Göttingen $\quad 2224$ \\
\hline v. Braun & 199 & Graz, J. \\
\hline Bremen & 8 & Gregory \\
\hline Breslau & 97 & Greifswald \\
\hline Brezina & 628 & Halle \\
\hline Budapest & $38 \mathrm{ro}$ & Hamburg \\
\hline Calcutta & 588 & Harvard, U. \\
\hline Cambridge & 48 & Heidelberg \\
\hline Catania & 56 & Howell \\
\hline Clausthal & 183 & Klausenburg \\
\hline Cohen & $\left.134^{6}\right)$ & Kopenhagen \\
\hline Debreczin & 37 & Krantz \\
\hline Detmold & 265 & Kristiania \\
\hline
\end{tabular}

I) 9 gr. $\gg 1877$, Caracoles Atacama Bolivia $;$; 42 gr. $>1800$, Imilac Atacama Bolivia ; 5 gr. "1879, Campo del Pucara, Argentina, S. A. - 2) A Atacama ع. - 3) $\rightarrow 18$ small iron meteorits from Atacama Chili. - 4) Davon 1. 5 gr. "Campo del Pucara «, - 5) *Atacama (Bolivie) $1827 \alpha_{.}$- 6) Davon $30 \mathrm{gr}$. >Campo de Pucara «. - 7) >Atacama Syssiderite, - 8) > Copiapo. It more nearly resembles the Eagle Station than the Sierra de Chaco $\alpha$. Also Pallasit, daher vorläufig hier untergebracht. - 9) $1427 \mathrm{gr}$. "Caracoles, $\mathrm{Pe} ; 178 \mathrm{gr}$. "La Encantada, ö. v. Corrizalillo $\mathrm{P}_{\alpha} ; 60 \mathrm{gr}$. $\diamond$ Imilac P . Herr Professor Brögger teilte mir noch folgendes mit: Nach dem Aussehen allein zu urteilen, wäre es wohl immer- 


\begin{tabular}{|c|c|}
\hline Leiden & Petersburg, A. 423 \\
\hline Leipzig & Petersburg, B. 4 \\
\hline v. Leuchtenberg & Pierrotet \\
\hline London,B.M.227328 & Pohl \\
\hline London, P. G. ${ }^{1251}$ & Rom, U. \\
\hline Madrid $\quad 102$ & Sidney \\
\hline Manchester & v. Siemaschko \\
\hline Marburg & Stockholm \\
\hline de Mauroy & Strassburg \\
\hline Melion & Stuer \\
\hline Modena & Stürtz \\
\hline Moskau & Stuttgart \\
\hline München & Troyes \\
\hline Münster & Tübingen \\
\hline Neapel & Turin \\
\hline Neumann & Upsala \\
\hline New Haven & Utrecht \\
\hline Newton & Ward \\
\hline New York, M. 4889 & Washington $\quad 197$ \\
\hline Odessa $\quad 9$ & Washington,Sh. 37 \\
\hline $\left.10500^{2}\right)$ & Wien, H. M. ") **) \\
\hline Paris, E. & $\left(3692^{6}\right)$ \\
\hline Paris, M. & Wien, U. I. $\quad$ I8o \\
\hline Paris, U. & Würzburg \\
\hline Pech & Zürich \\
\hline
\end{tabular}

Die Universität Basel, die Military Academie in West Point, das Museu National in Lissabon, sowie die Herren Dr. Ulex, Dr. Plagemann, F. Worlée und F. Cappel, alle in Hamburg, besitzen ebenfalls je ein Stück von Imilac.

Inca (Llano del Inca),Atacama,Chile. Mesosiderit, M, gefunden 1888 , beschr. 189o. Ist wahrscheinlich mit Doña Inez zu vereinigen.

r8go: How e11: Description of New Meteorites. The Doña Inez and the Llano del Inca, two New Meteorites from Atacama Chili. Daran anschliessend Mineralogical Description of the Llano del Inca and the Doña Inez Meteorites von Wadsworth. Proc. Rochester Acad. Sc. Bd. I (I89o), p. 93 -98 (Analyse von Eakins); s. auch U. S. Geol, Surv. Nr. 78 (189r), p. 97. Ref. N. J. 1892 II, p. $34-36$; noch einmal referiert N. J. 1895 II, p. 30-3I. r894: Co he n: Meteoritenkunde, p.24I, $259,283,293,294,305,307,308$. r895: M e unier : Révision des lithosidérites, p. 38,39 .

1895: Brezina: Wiener Sammlung, p. 263 .

Ursprüngliches Gewicht: $121 / 4 \mathrm{Kgr}$. Das grösste Stück (nur I $27^{1 / 2}$ gr. schwer) gelangte in Herrn Prof. Ward's Besitz.

Nachweisbares Gewicht: 3r45 gr.

\begin{tabular}{lr|lr} 
Bailey & 99 & London, B. M. & 376 \\
Bement & $16 \mathrm{r}$ & de Mauroy & 15 \\
v. Braun & 60 & New Haven & 45 \\
Brezina & 78 & Newton & 39 \\
Budapest & 17 & Paris, M. & 15 \\
Cleveland & 18 & Rom, U. & 34 \\
Dorpat & 17 & v. Siemaschko & 71 \\
Gregory & 79 & Ward & 240 \\
Howell & 1440 & Washington & 66 \\
Krantz & 83 & Wien, H. M. & $\left.192^{7}\right)$
\end{tabular}

hin möglich, dass die 2 Lokalitäten Caracoles u. Imilac vereinigt werden könnten. Dagegen hat das Stïck mit der Etiquette: La Encantada ö. v. Corrizatillo, Atacama ein abweichendes Aussehen, indem die Olivinkörner mehrmals grösser, als in dem hiesigen Stück von Caracoles sind und zum Teil $2-2^{1 / 2} \mathrm{~cm}$ im Durchschnitt messen. * Hiernach dürfte das Stück kein Mesosiderit sein und nicht zu Vaca Muerta gehören (cfr. Brezina : Ann. Hof-Mus. (1895) Bd. 10, p. 265). - 1) 22 gr. $>$ Ferro meteorico, Imilac, prov. di Atacama ; 103 gr. s Meteorite (pallasite), Atacama Chilie. - 2) 10 oder II Kgr. »Atacama๔. - 3) 408 gr. >Atacama ; 97 gr. >Atacama ; 250 gr. „Caracoles «. - 4) Da-

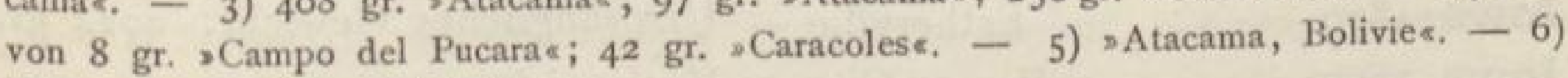
Davon 5 gr. >Campo del Pucarae. -

7) Ausserdem $\gg$ False Inca, Om\& to gr.

$W$ it Ifing, Meteoriten. 
Indarch, Schuscha, Elisabethpol, Kaukasien, Russland; auch: Indarh-Choucha, Gindorcha und Glindorcha, Elissawetpol.

Stein, Kc, gefallen 7. April r89r. r89r: v. Sie mas ch ko: Catalogue de la Collection des Météorites de Julien de Siemaschko. St. Petersbourg 189I, "p. 55-56 (russisch).

1892: Tarassow: Ueber den im Kirchdorfe Gindorcha des Schuschinskischen Kreises gefallenen Aerolithen (russisch). Verh. russ, min. Ges. (2) 29 , p. $185-186$.

r893: B re z in a: Ueber neuere Meteoriten (Nürnberg), p. I6r.

r895: Brezina: Wiener Sammlung, p. 254,255 .

Ursprüngliches Gewicht: Etwa 27 Kgr. (r Pud $25 \% \frac{1}{4}$ Pfund, falls ich die russ. Angabe richtig erraten habe).

Nachweisbares Gewicht 949 gr.

Berlin, U. 14 London, B. M.

v. Braun

Brezina

Budapest

Gregory

Krantz

I38 Petersburg, B.

I Stockholm

326 Upsala

5 Wien, H. M.

Wo befindet sich dieHauptmasse desSteins ?

Independence

Independence Co.

Kenton Co.

Indian Valley Township

Indien

Inka

Floyd Mountain

Invercargill

Iowa $\mathrm{r} 847$

Iowa 1875

Iowa 1879

Iowa 1890
Homestead

Estherville

Forest City
Iquique, Prov. Tarapaca, Peru, S. A. Eisen, Hca, gefunden u.beschr. 1871 . 187I: R a im on di: (Rose verweist auf eine Beschreibung u. Analyse des Herrn Raimondi vom 21. Sept. 187r; s. f. Arbeit).

1873: Rose: Ueber das Meteoreisen von Iquique in Peru. Festschr. d. Ges, nat.-forsch. Freunde. Berlin (1873) (7 Seiten mit 2 Tafeln; Analyse von Rammelsberg); s. auch Am. Journ. (3) 8 (1874), p. $398-399$.

1885: Brezina: Wiener Sammlung, p. 2OI, 203, 219, 234 .

1887: F lig ht: Meteorites, p. 24-26. I894: C o h e n : Meteoritenkunde, p. Io8, 109, 110.

Ursprüngliches Gewicht: $12^{1 / 2} \mathrm{Kgr}$. (25 Pfund).

Nachweisbares Gewicht: 10691 gr. Berlin, U. 10 650 Wien, H. M. 41

Irapuato

Irkutsk 1805

Irkutsk 1824

La Charca

Iron Creek

Doroninsk

Ironhannock Creek

Tounkin

Victoria

Tomhannock Creek

Irwin Ainsa

Tucson

Isle de France

Mauritius

Itapicuru-mirim, Prov. Maranhão, Brasilien.

Stein, Cc, gefallen März 1879 .

1888: D e r by: Meteoritos Brasileiros.

Extrahito da Revista do Observatorio.

Rio de Janeiro (bei Lombaerts u. Comp.) r888, p. 8-9; s. auch Am. Journ. (3) 36 (1888), p. 147.

1895: Brezina: Wiener Sammlung, p. 256 .

Ursprüngliches Gewicht: 2024 gr. Nachweisbares Gewicht: 2024 gr.

I) Nach I. Juli 1893 erworben, 
London, B. M. $\quad 6$ v. Siemaschko 5 Paris, M. $\quad 2$ Washington 10 \begin{tabular}{ll|l} 
Rio & $\left.1995^{1}\right)$ & Wien, H. M. 6
\end{tabular}

Ivanpah, San Bernardino Co., Californien, U. S. A.

Eisen, Om, gefunden u. beschr. 1880 . r880: Shepard: On the Ivanpah, California, Meteoric Iron. Am. Journ. (3) 19, p. $381-382$ (Analyse). Ref. Liebig-Kopp, Jahresber. 188o, p. 1542. r883/84 : Ge hring: Mineral. Resources of the United States. Washington $1883 / 84$, p. 290.

1884: M eunier: Météorites, p. 137. r885: Brezina : Wiener Sammlung, p. $25^{8}$.

I887: F lig ht: Meteorites, p. 213.

189o: Eastma n : Met. Astron., p. 320. r89r: W h itfie $1 \mathrm{~d}$ : Bull. U. S. Geol.

Surv. Nr. 78 , p. 97 .

r89r: Cohen u. Weins chenk: Meteoreisen-Studien, Ann. Hof-Mus,

Bd, 6, p. 131, 145 (Analyse), 160, 165. r892: Co hen: Meteoreisen-Studien II.

Ann. Hof-Mus, Bd. 7, p. 149-150, 158 (Cu).

1893: M e un i e r: Révision des fers météoriques, p. 76.

r894: Cohen: Meteoritenkunde, p. 53,

$54,76,146,147,153,216,28_{3}, 321$. r894: F let c her: Introduction, p. 14. r895: Brezina: Wiener Sammlung, p. $279-28$ o.

Ursprüngliches Gewicht: Wird auf I 20 lbs. geschätzt.

Nachweisbares Gewicht: $34^{8}$ gr.

\begin{tabular}{lr|lr|} 
Bailey & 6 & Dresden, M. & 4 \\
Bement & 4 & Gregory & I \\
Bonn & 3 & London, B. M. & 33 \\
Budapest & 9 & de Mauroy & 2 \\
Cleveland & 12 & Minneapolis & 4
\end{tabular}

\begin{tabular}{|c|c|c|}
\hline Moskau & I & Ward \\
\hline New Haven & 4 & Washington \\
\hline Paris, M. & 2 & Washington, Sh. \\
\hline v. Siemaschko & 7 & Wien, H. M. \\
\hline Turin, U. & 47 & \\
\hline
\end{tabular}

Berkeley bei S. Francisco, U. S. A. besitzt ein Stück eines Meteoreisens *Locality not given on label, marked $>$ California «? 230 gr * Könnte auch Shingle Springs sein. Wo ist die Hauptmasse dieses Eisens?

Iwate $185^{\circ}$

Kesen

Iwate $\mathrm{I} 880$

Ixtlahuaca

Toke-uchi-mura

Toluca

Jacala

Pacula

Jackson Co., Tennessee, U. S. A.

Eisen, $\mathrm{Om}$, gefunden ?, beschr. 1846 . (Ist dieses Eisen vielleicht mit Cosby's Creek zu vereinigen?)

1846: Troost: Description of three varieties of Meteoric Iron. - 2. Meteoric Iron from Jackson County, Tennessee. Am. Journ. (2) 2, p. 357 ; s. auch Am. Journ. (2) 5, p. $35^{1}$ (beiläufige Erwähnung).

r846: S h e pard: Report on Meteorites. Am. Journ. (2) 2, p. 39 I.

r852: C 1 a r k : Dissert, Gött., p. 64-65. r854: v. Boguslawski: Zehnter Nachtrag, Pogg.Ann. Ergz,-Bd. 4, p.404. r859: Harri s: Dissert. Gött., p. In6. 1863: B u c hner: Meteoriten, p. 175. 1885: Brezina: Wiener Sammlung, p. 211,234 .

r890: Eas t m a n : Met. Astron., p. 3 I8.

Ursprüngliches Gewicht: Grosse Masse, welche geheim gehalten wurde und von welcher Troost nur $425 \mathrm{gr}$. ( $15 \mathrm{oz}$.) und $88 \mathrm{gr}$. (31/s oz.) Rinde erhielt.

I) Im Katalog von 1888 ist das ursprüngliche Gewicht von 2024 gr. angegeben. Eine Randbemerkung Derby's vom 10. Juli 1894 sagt: "Some $3-4$ grams were cut away and distributed $\propto$. Statt $>3-4 \mathrm{gr}$. $\ll$ muss es offenbar heissen $30-40 \mathrm{gr}$. 
Nachweisbares Gewicht: 209 gr.

Bailey

Bement

Gregory

Jakobshavn

Jalisco

Jamaica 46 London, B. M. 9 I3 Ward 2 Wien, H. M. *)

Jamestown, Stutsman Co., NordDacota, U. S. A.

Eisen, Of, gefd. 1885 , beschr. 1890 .

1890: Huntington: A new meteoric iron from Stutsman County, North Dacota. Proc. Amer. Acad. Arts and Sc. (2) 17, p. 229-232 (Analyse, Abbildung der Masse und einer geätzten Platte). Ref. N. J. 1892 I, p. 266.

1893: B re z in a : Ueber neuere Meteoriten (Nürnberg), p. I03.

r894: C o he n : Meteoritenkunde, p. 63 , $73,181,230$.

r895: Brezina: Wiener Sammlung, p. 270.

Ursprüngliches Gewicht: $4015 \mathrm{gr}$. Nachweisbares Gewicht: 3578 gr.

\begin{tabular}{lr|l|l|} 
Bailey & 74 & London, B. M. & 1627 \\
Bement & 7 & v. Siemaschko & 30 \\
Gregory & 98 & Washington & 74 \\
Harvard, U. & $\left.1570^{1}\right)$ & Wien, H. M. & 98
\end{tabular}

Jamkheir, Ahmednuggur, Bombay, Indien.

Stein, gefallen 5. Oktober 1866.

r869: Buchner: Vierter Nachtrag, Pogg. Ann. Bd. 136, p. 460.

r885: Brezina: Wiener Sammlung, p. 259 .

1894: F letcher: Introduction, p. I3. Ursprüngliches Gewicht : ? In Fedden's »Popular Guide « der Samm- lung von Calcutta ist diese Lokalität nicht genannt.

Nachweisbares Gewicht: 24 gr.

London, B. M. I9/London, P. G. 5

Eine ausführliche Arbeit ist über diesen Fall noch nicht veröffentlicht.

Jamyschewa (Pawlodar), Semipalatinsk, Russland.

Pallasit, P, gefunden 1885 .

Hierher auch: Samyschewa.

1893: Brezin a: Ueber neuere Meteoriten (Nürnberg), p. 163.

r894: C o h e n: Meteoritenkunde, p. 256, 257.

1895: Me unier: Révision des lithosidérites, p. $15,19-20$.

1895: Brez in a : Wiener Sammlung, p. $264-265$.

Ursprüngliches Gewicht: $6 \mathrm{Kgr}$.? Nachweisbares Gewicht: 4036 gr.

Berlin, U.

Böhm

v. Braun

Brezina

Budapest

Dorpat

Gregory

Kasan

Krantz

Krüger

27 Kunz

15 London, B. M.

3I3 Paris, M. 93

19 Petersburg, A. 622

174 Riga

52 Rom, U. $\quad 34$

9 v.Siemaschko $2198^{2}$ )

I88 Strassburg $\quad 58$

25 Wien, H. M, $48^{3}$ ) 42

Bei Herrn Staatsrat v. Braun sah ich eine Platte, welche die halbe Grösse dieses Blattes haben mochte. Sehr viel Olivin (so schien es) in grossen Krystallen, wie Brenham, und wenig Eisen. Silikat wahrscheinlich die Menge des Eisens bedeutend überwiegend. In der russischen Notiz von v. Siemaschko wird gesagt, dass der grössere Teil ungefähr Io Pfund, also $4 \mathrm{Kgr}$. gewogen habe.

I) Eigentum von Herrn Huntington.

2) In v. Siemaschko's satalogue de la Collection de Météorites S. Petersburg 189i ", p. 23 findet sich noch eine kurze Notiz (russisch) über diese Lokalität.

3) Ausserdem $3 \mathrm{gr}$. nach I. Juli 1893 erworben. 


$\begin{array}{lr}\text { Janacera-Pass } & \text { Vaca Muerta } \\ \text { Jarquera } & \text { Vaca Muerta } \\ \text { Jasly } & \text { Bialystock } \\ \text { Jefferson City } & \text { Little Piney } \\ \text { Jefferson Co. s. Anhang Long Creek } \\ \text { Jekaterinoslaw } & \text { Ekaterinoslaw } \\ \text { Jeliza-Gebirge, Serbien. }\end{array}$

Stein, Amphoterit, Am, gefallen I. Dez. 1889 .

r89o: Dö 11: Der Meteorfall im JelizaGebirge in Serbien am I. December 1889. Verh. k. k. geol. Reichsanst. 1890 , p. $70-77$. Ref. N.J. 189 I I, p. 48 . r89o: M e u n i er: Examen lithologique et géologique de la météorite de Jelica (Serbie). C. R. Bd. I1o, p. 871-873. Ref. N. J. 189r II, p. 5 I.

1890: v. N i e s s 1 berichtet über den am I. Dezember 1889 um $2 \frac{1}{2}$ Uhr Nachmittags bei Cac̉ak, am Abhange des Jeliza-Gebirges in Serbien stattgehabten Meteoritenfall. Verh. naturh. Ver. Brünn Bd. 29, p. 36 .

1890: v. N i e s s 1: Ueber die Bahn der am 1. December 1889 bei Cačak am Jeliza-Gebirge in Serbien gefallenen Meteoriten. Verh. naturh. Ver. Brünn Bd, 29, p. 166-178 (mit zwei Fig. im Text).

1890: v. Niess 1: Periheldistanzen, Verh. naturf. Ver. Brünn Bd. 29, p. 188, I95, 214, 248.

r89x: v. Hauer: Ann. Hof-Mus. Bd, 6 (Not.), p. 54, 55 .

r892: Los anitsch: Analyse des Meteoriten von Jelica. Ber. d, d, chem. Ges. Bd. 25, p. 876-88o.

1893: B rez in a : Ueber neuere Meteoriten (Nürnberg), p. 159.

1893: Me unier: Ein Aufsatz in den Ann, géol. de la Peninsula Balkanique (Belgrade) Bd. 4, p. 3-21 (serbisch ?). 1894: C o h e n: Meteoritenkunde, p. II2, $204,223,236,248$.

I) 25 Stück.
1895: B rezina: Wiener Sammlung, p. $238,239-240$.

Ursprüngliches Gewicht : Nach Döll wurden 26 Steine im Gewicht von 33830 gr. gesammelt; später scheinen noch mehr Steine gefunden worden zu sein.

Nachweisbares Gewicht: 37634 gr.

\begin{tabular}{lr|lr} 
Bailey & 5 & Kopenhagen & IIO \\
Bayet & 5 & London, B. M. 1879 \\
Belgrad & $\left.30830^{1}\right)$ & München & 75 \\
Bement & $15^{8}$ & Paris, M. & 1050 \\
Berlin, U. & 112 & Pech & 46 \\
Böhm & 61 & Prag, M. & 20 \\
v. Braun & 468 & Rom, U. & 122 \\
Brezina & 362 & v. Siemaschko & 82 \\
Budapest & 154 & Stockholm & 114 \\
Cleveland & 1 & Strassburg & 144 \\
Dresden, M. & 37 & Stürtz & 7 \\
Gregory & 69 & Utrecht & 31 \\
Greifswald & 23 & Wien, H. M. & 1507 \\
Harvard, U. & 35 & Wien, U. I. & 127
\end{tabular}

Jenny's Creek (OldFork of Jenny's Creek), Wayne County, WestVirginia, U. S. A.

Eisen, Ogb, gefd. $188_{3}$, beschr.1885. 1885: Kunz: Proc. Amer. Assoc. Bd. 34 (1885), p. 246; s. auch Am. Journ. (3) $30(1885)$, p. 326 u. Am. Journ. (3) $3^{1}$ (1886), p. 145-148: Meteoric Iron from Jenny's Creek, Wayne County, West-Virginia (Analyse von Mackintosh, Abbildung der kleinen erhaltenen Masse u. einer geätzten Seite); auch *Science Bd. 6 (1885), p. 222 u. Bd. 7 (1886), p. II-12. Ref. N. J. 1887 I, p. 3334 ; Liebig-Kopp, Jahresber. I886, p. $2333-2334$.

1886: Brezina: Neue Meteoriten I, Ann. Hof-Mus, Bd. I (Not.), p. 14. r887: Huntington: On the Coahuila Meteorites. Am. Journ. (3) 33, p. 118 . 
1887: K unz: On some American Meteorites. Am. Journ. (3) 34, p. 475.

189o: E a s tman : Met.Astron., p. 320. r893: M e u n i er: Révision des fers météoriques, p. 76 .

1894: H un ting ton: The Smithville Meteoric Iron. Proc. Amer. Acad, Arts and Sc. Bd. 29, p. 259 (Situationsplan von Tennessee bis West-Virginia). r894: F l e t c her: Introduction, p. r4. r895: Brezina: Wiener Sammlung, p. 286.

Ursprüngliches Gewicht: Drei Massen von 23 lbs., $2-3$ lbs. u. 535 gr.; letztere 1885 aufgedeckt. Die ersten beiden Massen dïrften grösstenteils verloren gegangen sein.

Nachweisbares Gewicht: r rog gr.

Bailey

Berlin, U. 229 London, B. M. 78

v. Braun

Budapest

$$
5 \text { de Mauroy }
$$

I7 Newton

67 v. Siemaschko

Io Stockholm

Cleveland

Gregory

Harvard, U.

I2 Troyes

Krantz

Kunz

$\left.3^{1}\right)$ Washington

Iо $^{2}$ ) Wien, H. M.

I6

Jewell Hill, Madison Co., NordCarolina, U. S. A.

Eisen, Of, gefd. 1854 , beschr. 1860. Hierher auch vorläufig „Duel Hill « (s. Anhang dieses Artikels). Brezina hebt die grosse Verschiedenheit der beiden Wiener Eisen von Jewell Hill und „Duel Hills hervor. Da wir aber über das Mass der Verschiedenheit der Eisen einer Lokalität noch wenig unterrichtet sind, und da ausserdem in den Sammlungen die Vereinigung grösstenteils unter dem Namen Jewell Hill durchgeführt ist, so möge vorläufig sDuel Hill « als Anhang zu dem älteren und verbreiteteren Namen Jewell Hill gestellt werden. Ein weitererVergleich der von Jewell Hill und „Duel Hill« vorhandenen Massen ist sehr erwünscht.

r860: Smith: Description of three new Meteoric Irons, from Nelson County, Ky., Marshall County, Ky., and Madison County, North Carolina. Am. Journ. (2) 30 , p. 240 ; s. auch Original Researches 1884 , p. 409-410; LiebigKopp, Jahresber. 1860 , p. 853 ; Journ. prakt. Chem. Bd. 84 (186r), p. 59-6o. r862: v. Reichen b a ch: XX 622 . 1863: B u chner: Meteoriten, p. 194. 1863: Rose: Meteoriten, p. 65, 153. r869: Me unier: Recherches, Ann.

Chim. Phys. (4) 17, p. 29, 34, 71, 72. 1870: R a m m e ls berg: Meteoriten, p. $8 \mathrm{I}$.

r872: Quenstedt: Klar u. Wahr, p. $3^{1} 3$ (Abb. einer geätzten Platte).

r872: Ts chermak: Ein Meteoreisen aus der Wüste Atacama, Denkschr. Wien. Akad. Bd. 3I, p. 195.

1875: vom R a th: Meteoriten, Verh. naturh. Ver. Bonn Bd. 32, p. 364.

I880: Brez in a: Reichenbach'sche Lamellen, Denkschr. Wien. Akad. Bd. 43, p. I4.

1880: Brezina: Bericht I, Sitzber.

Wien. Akad. Bd, 82 I, p. 350.

1884: Meunier: Météorites, p. 47, 5I, 129, 130 .

1885: Brezina: Wiener Sammlung, p. 209, 233.

r886: Hunting ton: Crystalline Structure, Am. Journ. (3) 32, p. 295.

r887: Brezina u. Cohen: Photographien, T. 23.

1887: Flig h t: Meteorites, p. 127.

1890: Eastman: Met. Astron., p. 318.

1) Ausserdem noch 31 gr. Kanwahoe Co. West Virginia 1882,

2) sKanahiva von S. C. H. Bailey herstammende. 
1893: Meunier: Révision des fers météoriques, p. $37,3^{8}$ (Abbildung einer geïtzten Platte).

1894: Co h e n: Meteoritenkunde, p. 48, $73,84.193,232$.

r894: Fletcher: Introduction, p. 33 . r895: Brezina: Wiener Sammlung, p. $233,269$.

r895: C o h e n: Meteoreisen-Studien IV. Ann. Hof-Mus. Bd. 10, p. 82 .

Ursprüngliches Gewicht: $4 \mathrm{Kgr}$. (8 lbs. ${ }_{3} 3$ oz.).

Nachweisbares Gewicht: 9607 gr. Ein Teil der Bradley'schen Masse ist offenbar in diesem letzteren Gewicht enthalten; s. Fussnote I).

Bailey

Bement

Berlin, U.

Bonn

Calcutta

Cleveland

Cohen

Dorpat

Göttingen

Gregory

Hamburg

Harvard, U.

London, B. M. $\quad 142$

Jewell Hill-Duel Hill, Madison

Co. Nord-Carolina, U. S. A.

Eisen, Og, gefd. 1873 , beschr. 1876 .

r876: Burton: Notice of a Meteorite, from Madison Co., N. C. Am. Journ.

(3) 12, p. 439 (Analyse). Ref. LiebigKopp, Jahresber. 1876, p. 1317.

1881: Brezina: Bericht III, Sitzber.

Wien. Akad. Bd. 84 I, p. $279-28$ o. 1885: Brezina: Wiener Sammlung, p. $215,216,234$. r885: Ge nth u. Kerr: The Minerals and Mineral Localities of North Carolina, p. 14 (gedruckt in Raleigh). r887: F light: Meteorites, p. 68. r89r: Cohen u. Weinschenk: Meteoreisen-Studien. Ann. Hof-Mus. Bd. 6, p. 161 .

1894: C ohen: Meteoritenkunde, p. 115, 194 .

1895: Brezina : Wiener Sammlung, p. $233,277,286$.

Ursprüngliches Gewicht : $9 \frac{1}{2} \mathrm{~K}$ gr. (2 I Pfund erhielt Bradley, nachdem einige Pfund vorher abgeschlagen waren).

Nachweisbares Gewicht: I510 gr. Bailey $\quad 8$ London, B. M. ${ }^{12}{ }^{2}$ ) Budapest $\quad 29$ v. Siemaschko $\quad$ I2 $\begin{array}{lll}\text { Gregory } & \text { I Wien, H. M. } & \text { I2O2 }^{3} \text { ) }\end{array}$ Harvard, U. 246

Jhung, Punjab, Ostindien.

Stein, Cc, gefallen Juni 1873 .

1884: Meunier: Météorites, p. 23I.

1885: Brezina: Wiener Sammlung, p. 185,233 .

1894: F le t c her: Introduction, p. 14. Ursprüngliches Gewicht: ?

Nachweisbares Gewicht: 34I4 gr. Bailey $\quad$ I8 London, P. G. I8 Bement 26 New Haven 16 Budapest 6 Paris, M. 102 Calcutta 1122 v. Siemaschko 5 Gregory $\quad 7$ Ward 6 Harvard, U. 53 Washington I Kristiania $\quad 25$ Wien, H, M.*) 25 London, B. M. 1984

Der Stein scheint noch nicht näher untersucht worden zu sein.

Jigalowka (Charkow), Gouv Charkow, Russland.

Stein, Cwa, gefallen 13 . Okt. 1787 .

1) $>1856$ u. 1873 Jewell Hille; also offenbar auch "Duel Hille.

2) Unter Jewell Hill aufgeführt: >Described as from Duel Hill in 1876 by Burton $\propto$.

3) Nach 1. Juli 1893 erworben. 
Hierher auch: Goebel's tuffartiger Chondrit von ungewisserHerkunft. 1808: Einige Nachrichten von den drei neuesten Steinregen und von drei Meteorsteinen aus Russland. - 5. In den Russischen Miscellen, Jahrgang 1804, findet sich folgende Anzeige des berühmten Chemikers Staatsrath Low it z (dass die Steine von Charkow u. von L'Aigle sich ähnlich sind). Gilb. Ann. Bd, 29, p. 213 .

I809: S to ik owit z: Nachrichten von mehreren russischen Luftsteinen, besonders von denen, die am I. Oktober I787 im Gouvernement von Charkow herabgefallen sind. -3 . Von den in dem Slobodsko-Ukrainer Gouvernement herabgefallenen Luftsteinen. A. Die Geschichte dieses Ereignisses. B. Aeussere Kennzeichen, beschrieben von Emanuel Krüger, Adjunkt der kaiserlichen Charkower Universität. C. Analyse der im Charkower Gouvernement gefallenen Meteorsteine, von den Herren Professoren der Chemie Schnaubert und Giese. Gilb. Ann. Bd. 3I, p. $3 \mathrm{II}-322$.

I8r2: $\mathrm{Ch}$ l a d n i: Verzeichnis,Schweigg. Journ. Bd. 4, Beil. I, p. I4.

1813/r4: Scheerer: Chemische Analyse des Charkov'schen Meteorsteins. Mémoires Petersburg. Akad. Bd. 6: Histoire de l'Acad. V. Observations, expériences et notices intéressantes faites et communiquées à l'Academie, p. 47.

18I5: Chladni: Neues Verzeichnis, Pogg. Ann. Bd. 50, p. 250.

r8r5/r6: S c heerer présenta l'Analyse faite par Mr. le Professeur Giese de la pierre météorique du poids de 50 livres, tombée de l'Atmosphère aux environs de Kharkoff au mois de Mars 1814. Mémoires Petersburg. Acad. Bd. 7. Observations, expériences et notices intéressantes faites et communiquées à l'Academie, p. 3I, 32.

r8rg: C h l a d n i: Feuermeteore, p. 45, $57,66,69,73,90,253,257-258(1), 271$. r836: $\mathrm{K}$ ä $\mathrm{m} \mathrm{tz}$ : Meteorologie, p. 276. 1843: P a r s c h: Meteoriten, p. 52- 53. 1847: Eichwald: Verzeichnis, Ermann's Arch. f. wissensch. Kunde v. Russland Bd. 5, p. 176 (der Charkower Stein sei teils nach Petersburg, teils nach Charkow gekommen).

1848: B l o e de: Tabelle, Bull. Petersburg. Acad. Bd, 6, p. 10.

1859: $\mathrm{H}$ a r r is : Dissert.Gött., p.6r-62. r863: Buchner: Meteoriten, p. Io. 1863: Rose: Meteoriten, p. 155.

I859 65: v. Re i ch en b a c h: IX I6I, $168,178 . \mathrm{X}_{359}, 361,362,363$. XXV $429,432,434,436,607$.

r867: Bu chner: Dritter Nachtrag, Pogg. Ann. Bd. 132, p. 314.

1867: Go e bel: Kritische Uebersicht, Mélanges phys. chim. Bd. 7, p. 278281, 330-33I, 342 .

1884: M e uni er: Météorites, p. 197. r885: Brezina: Wiener Sammlung, p. $177,232$.

1894: Cohen: Meteoritenkunde, p. 8. r895: Brezina: Wiener Sammlung, p. 242,243 .

Ursprüngliches Gewicht: Mehrere Steine(?), von denen einer erhalten blieb. In der Literatur ist keine Gewichtsangabe vorhanden. Den grössten Teil soll die Universität Charkow besitzen; ich habe leider keine Nachricht darüber erhalten können.

Nachweisbares Gewicht: ${ }_{15} 55^{2}$ gr.

Berlin, U.

Calcutta

Debreczin

Dorpat

Göttingen

Gregory

Greifswald

Hamburg

Kiew

4 London, B. M. 437

2 London, P. G. 33

7 Paris, M.

34 Petersburg, A. 932

32 v. Siemaschko 7

Io Tübingen 12

Spl. Washington 26

1 Wien, H. M.*) 2 12

Jimenez
Huejuquilla-Gruppe 
Jodzie

Yodzé

Joël Iron, Wüste Atacama, S. A. Eisen, Om, gefunden $185^{8}$, beschr. 1885 (in Verzeichnissen schon früher erwähnt).

Hierher auch: »Atacama, Bolivia, $\mathrm{Oml} 1858 \ll$.

r885: Brezina: Wiener Sammlung, p. $155,213,214,234$.

1889: F l et c her: Atacama Meteorites, Mineral. Magaz. Bd. 8, p. 224, 263264 (Analyse).

r893: M eun ier: Révision des fers météoriques, p. 74 .

1895: Brezina: Wiener Sammlung, p. 277 .

Ursprüngliches Gewicht: $1300 \mathrm{gr}$. Nachweisbares Gewicht: 1296 gr.

Calcutta $\left.20^{1}\right)$ v. Schilling $\quad 42^{4}$ )

Harvard, U. $\quad 8^{2}$ ) v. Siemaschko 9

London, B. M. 1144 Wien, H. M. $\quad 5^{5}$ )

London, P. G. $\quad 39$ Wrany $24^{6}$ )

Paris, M. $\left.\quad 5^{3}\right)$

Joe Wright Mountain, 7 miles östlich von Batesville Independence Co., Arkansas, U. S. A.

Eisen, Om, gefd. 1884 , beschr. 1886. Hierher auch: Elmo.

1886: $\mathrm{Hid}$ d e $\mathrm{n}$ : On two masses of Meteoric Iron of unusual interest. -1 . The Independence County, Arkansas Meteorite (Abbildung, Analyse von Mackintosh). Am. Journ. (3) 3I, p. 460--463. Ref. N. J. 1888 I, p. 47 ; Liebig-Kopp, Jahresber. 1886, p. 2332-2333.

1886: Brezina: Neue Meteoriten I,

Ann. Hof-Mus. Bd, I (Not.), p. 13-14. 1886: Brezina: Neue Meteoriten II, Ann. Hof-Mus. Bd. I (Not.), p. 25.
I887: v. H a u e r : Ann, Hof-Mus, Bd. 2 (Not.), p. $3^{8}$.

r887: B r e z in a : Neue Meteoriten IIIa, Verh. k. k. geol. Reichsanst. (1887), p. 288 .

r89o: E a s t m a n : Met. Astron., p. 320. r89r: Cohen u. Weinschenk: Meteoreisen-Studien. Ann. Hof-Mus. Bd. 6, p. 131, 158-159 (Analyse), 162, 164,165 .

1893: Br ez in a: Ueber neuere Meteoriten (Nürnberg), p. 164 .

r894: C o h e n : Meteoritenkunde, p. I24, $125,193,195,197,199$.

r895: Brezina: Wiener Sammlung, p. 280 .

r895: C o he n : Meteoreisen-Studien IV. Ann. Hof-Mus. Bd. Io, p. 90.

Ursprüngliches Gewicht: $42^{1 / 2} \mathrm{Kgr}$. (94 pounds).

Nachweisbares Gewicht: 37497 gr. Bailey $\quad 60$ Paris, E. 27

Belgrad $\quad 154$ Prag, M. 137

Bement $\quad 276$ Rom, U. $\quad 375$

Berlin, U. $\quad 28$ v. Siemaschko 265

Bologna 240 Stockholm $\quad 163$

v. Braun 401 Strassburg 26

\begin{tabular}{ll|l} 
Brezina & 12 & Stürtz \\
I7I
\end{tabular}

Budapest $\quad 225$ Stuttgart 10

$\begin{array}{llll}\text { Calcutta } & \text { 2ro } & \text { Troyes } & 14\end{array}$

Dresden, M, 39 Utrecht 19

Gregory $\quad 398$ Ward 6

Howell $\quad 114$ Washington 309

Klausenburg $\quad 44$ Washington, Sh. 20

Kunz $\quad 33$ Wien, H.M. 33126

London, B. M. 372 Würzburg 120

New Haven 103

Johanngeorgenstadt Steinbach Johnson Co. Cabin Creek Jonesboro, Ost-Tennessee, U. S. A. Eisen, Of, gefd. 1891 , erwähnt r 892 .

1) Atacama desert (? 20 leagues north of Cobija) $\%$ - 2) ^Atacama Bolivia $1858 \%$. - 3) sAtacama Bolivie $1858 \%$ - 4) >Atacama Bolivia (ob hierher gehörig ?). - 5) -Atacama Bolivia 1858\%. - 6) >Atacama Bolivia 1858\%,

7) Ausserdem 149 gr. nach I. Juli 1893 erworben. 
I892: W a r d : Illustrated descriptive Catalogue (Rochester N. Y. bei R. W. Lace) 1892 , p. 15.

1895: Brezina: Wiener Sammlung, p. 272 .

Ursprüngliches Gewicht: $\gg 30 \mathrm{gr}$. ( $\mathbf{~ o z .}$ * giebt Ward an.

Nachweisbares Gewicht: $28 \mathrm{gr}$, Wien, H. M. 28

Jonzac (Saintonge), Dep. Charente inférieure, Frankreich.

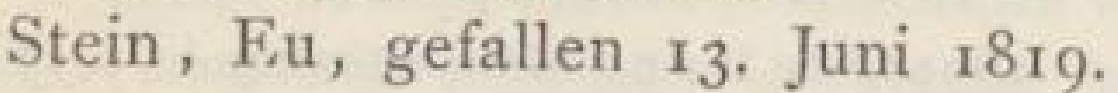
1819: Chladni: Fünfte Fortsetzung (mit Zusätzen von Gilbert), Gilb. Ann. Bd, 63 , p. 24 .

1820: La ug i er: Extrait d'un Mémoire lu à l'Académie des Sciences, le I er mai 1820, intitulé: Faits pour servir à l'histoire chimique des pierres météoriques. Ann. Chim. Phys. Bd. 13, p. 440 -442; s. auch Mém. du Muséum Bd. 6, p. 234-238; Schweigg. Journ. Bd. 29 (1820), p. 508-510; Bibl, Univ. Bd. 15 (1820), p. 3II; Tilloch's Philos. Magaz. Bd. 56 (1820), p. $157-158$; Ann. des Mines Bd. 6 (1821), p. 258. 1820: S o w e r b y: Thoms. Ann. Phil. Sept. 1820, p. 234.

182I: Chladni: Erster Nachtrag, Gilb. Ann. Bd. 68, p. $335-336$.

I82I: Fleurian de Bellevue : Mémoires sur les pierres météoriques, et notamment sur celles tombées près de Jonzac, au mois de Juin r8rg. Journ. Phys. Bd. 92 , p. $159-182$; s, auch Am. Journ. (I) 5, p. 170-171. In dieser Arbeit Fleurian's sind bereits einige der 39 Jahre später von Haidinger geäusserten Ideen über Leitform der Meteoriten (Sitzber, Wien, Akad. Bd. 40 II, I860, p. $525-536$ ) ausgesprochen; s. auch Döll, Jahrb.k. k. geol. Reichsanst. Bd. 37 (1887), p. 194. 1822: La ugier: Analyse de la Pierre météorique de Juvénas. Ann. Chim. Phys. Bd, 19, p. $264-265,270-271$; s. auch Gilb. Ann. Bd. 7r (1822), p. 209. r822: Chladni: Zweiter Nachtrag,

Gilb. Ann. Bd, 71, p. 360, 36r.

1824: Chla dni: Vierter Nachtrag,

Pogg. Ann. Bd. 2, p. 154

1834: B erzel i us: Om Meteorstenar. Kongl. Vetensk. Acad. Handl. (1834), p. $179-180$.

I836: K ä m tz: Meteorologie,p.258,287. 1843: P a r t s c h: Meteoriten, p. 26-27. 1854: v. Boguslawski: Zehnter Nachtrag, Pogg. Ann. Ergz.-Bd. 4, p. I4, 19, 20.

1859: B u c h n e r: Feuermeteore, p. 84 -85 .

1859: Ha rris: Dissert. Gött., p. 75. 1860: R a m mels berg: Mineralche-

mie, p. $940,950,951,952$.

I863: B u c h n e r : Meteoriten, p 39-40. 1863: Rose: Meteoriten, p. 26, 27, 126,

I35, 156.

r858/65: v. Reichen b a ch: $\mathrm{V}_{476}$ 478 . VI 441 . IX 160, 166, 177. X 359.

XI 296. XIII 353,356, 378. XV IOI. XX 631. XXV 6o7.

1872: Tschermak: Die Meteoriten von Shergotty u. Gopalpur. Sitzber.

Wien. Akad. Bd. 65 I, p. 127. I883: Tschermak: Beitrag, Sitzber.

Wien. Akad. Bd. 88 I, p. 368, 369 . 1884: Me un i er: Météorites, p.95,295, 296.

I884: W a d s w or th: Studies, p. 196. I883/85: Ts chermak: Photographien, p. 6 .

r885: Brezina: Wiener Sammlung, p. $173,232$.

r894: C o he n: Meteoritenkunde, p.304. Ursprüngliches Gewicht: Steinschauer; die grössten Steine 2 und $3 \mathrm{Kgr}$. schwer; nicht viel erhalten.

Nachweisbares Gewicht: ${ }^{5} 55^{2} \mathrm{gr}$.

Bailey

Berlin, U.

Budapest

Calcutta
7 Cleveland I

2 Göttingen Spl.

Spl. Gregory Spl. 
London, B. M. $\quad 9 \mid$ v. Siemaschko 3 London, P. G. Spl. Stockholm J Neumann I Strassburg Spl. New Haven Oxford 2 Tübingen I 2 Washington, Sh.Spl. Paris, E. 53 Wien, H. M.*) 1157 Paris, M. 298 Wien, U. I.

$\mathrm{I}_{3}$

Ein Stein von 4 Pfund, der bei Fleurian de Bellevue abgebildet ist, zeigt ausgezeichnete Orientierung; er gelangte an das Cabinet d'histoire naturelle de la Rochelle. Befindet sich derselbe noch dort?

\section{Juchnov}

Timoschin

Judesegeri, Distr. Tumkur, Mysore, Ostindien.

Stein, Cc, gefallen 16 . Febr. 1876 . 1876: M e d li c o t $t$ : The Secretary exhibited some specimens of Meteorites recently fallen in India and read some remarks upon them. - Record of the Judesegeri Meteorite of 16 th February, 1876. - Judesegeri stone. Report of the Deputy Commissioner of Túmkúr. Journ. Asiat. Soc. Bengal (Proc.), p. 22I. 1884: Meunier: Météorites, p. 257. (stellt diesen Stein zu seiner Gruppe der Mesminite; weiter keine Angaben). 1883/85: T s c h e r ma k: Photographien, p. 6,7 .

1885: Brezina: Wiener Sammlung, p. $185,233$.

1890: v. Niess 1: Periheldistanzen, Verh, naturf. Ver. Brünn Bd, 29, p. 193. 1894: F let c her: Introduction, p. 36.

Ursprüngliches Gewicht: $716 \mathrm{gr}$. (1 lb., 9 oz., 136 grs.).

Nachweisbares Gewicht: 531 gr.

Calcutta 355 Paris, M. 25

London, B. M. 135 Wien, H. M.

Juncal, Atacama, Chile,Südamerika. Eisen, Om, gefd. 1866 , beschr. 1868 (s. auch Ilimaë).
1868: D a u bré e: Sur trois nouveaux fers météoriques du Chili, récemment parvenus à la Collection de Géologie du Muséum, - I. Fer trouvé entre le Rio-Juncal et Pedernal, dans la HauteCordillère d'Atacama. C. R. Bd. 66, p. 568-57I (Analyse von Damour). Ref. Liebig-Kopp, Jahresber. I868, p. 1045-1046.

1869: Buchner: Vierter Nachtrag, Pogg. Ann. Bd, 136, p. 439, 609-6ro. r875: D o m e y k o: Note sur deux nouvelles météorites du désert d'Atacama et observations sur les météorites qui ont été découvertes jusqu'ici dans cette partie de l'Amérique méridionale. C. R. Bd. 81, p. 599. Ref. Liebig-Kopp, Jahresber, 1875 , p. 1316 .

r879: Do m e y k o: Mineralojia, 3. Aufl. (1879), p. 129-130.

1880: B rez in a: Reichenbach'sche Lamellen, Denkschr. Wien. Akad. Bd. 43 (2. Abt.), p. I6 (zwei Abbildungen von geätzten Platten).

1884: M e unier: Météorites, p.116,125. 1885: Brezin a: Wiener Sammlung, p. $152,154,204-205,211-213,234$, Taf, III.

1889: F le t cher: Atacama Meteorites, Mineral. Magaz. Bd. 8, p. 224, 259, $261-262$.

r89o: B r e z i n a : Reise, Ann. Hof-Mus. Bd. 4 (Not.), p. II9.

I892: v. H a u e r: Ann. Hof-Mus. Bd. 7 (Not.), p. 72.

1893: M eunier: Révision des fers météoriques, p. 52,57 .

1894: Cohen: Meteoritenkunde, p. 72, 133, 193, 196.

1895: Brezina: Wiener Sammlung, p. $278-279$.

Ursprüngliches Gewicht: Etwas über $104 \mathrm{Kgr}^{1}$ )

Nachweisbares Gewicht: 105604 gr.

1) Herr St. Meunier hatte die Freundlichkeit mir mitzuteilen, dass stout ce qui existe de Juncal vient du Muséum et a été prelevé sur l'échantillon de 104 Kgr. ₹ 
Bement

Böhm

v. Braun

Budapest

Cohen

Gregory

Krantz

165 London, B. M. 72

8 Paris, M. 104000

30 Rom, U. 30

200 v. Siemaschko 50

3 Stockholm 8

50 Ward 60

57 Wien, H. M.*) 87 I

Juvinas, Département de l'Ardêche, Languedoc, Frankreich.

Stein, Eu, gefallen 15. Juni 1821 . 182I: Sur la pierre météorique qui est tombée dans le département de l'Ardèche, le 15 juin 1821 . - Verny: Procès-verbal du Maire d'Aubenas. - Delaigue: Extrait du procès-verbal du Maire de la commune de Juvenas (Stein von $92 \mathrm{Kgr}$., davon $45 \mathrm{Kgr}$. an Herrn Alleysson). - Extrait d'une lettre de M. Jules de Malbos, cet extrait a été communiqué à l'Académie des Sciences. Ann. Chim. Phys. Bd. 17, p. 434-439; s. auch Schweigg. Journ. Bd, 33 (1821), p. 230 .

I82I: Gilb ert: Herabfallen eines sehr grossen Meteorsteins am 15. Juni 1821, zu Juvenas im ehemaligen Languedoc. Ausgezogen aus mehreren Berichten.I. Bericht des Herrn Flaugergues in Viviers. - 2. Aus einem Schreiben des Herrn Jules de Malbos, Gutsbesitzer zu Bariat. - 3. Auszug aus dem Protokoll des Maire der Gemeinde Juvenas. 4. Aus dem Sitzungsprotokoll der $\mathrm{Pa}$ riser Akademie der Wissenschaften. Gilb. Ann. Bd. 69 , p. $407-416$.

r82I: Vauquelin: Aérolithe tombé à Juvenas, près d'Aubenas, département de l'Ardéche, le 15 juin 1821. Ann. Chim. Phys, Bd. 18, p. $421-423$ (Analyse); s, auch Gilb. Ann. Bd. 7I (1822), p. 201-202; Schweigg. Journ. Bd. 35 (1822), p. 80.

r82r: Flaugergues: Sur P'Aérolithe tombé à Juvinas le 15 juin dernier. Journ. Phys. Bd. 92 (1821), p. $463-$ 466 ; s. auch Bd. 93 (1821), p. $71-73$.
1822: Ha üy: Traité de minéralogie. Paris (1822) Bd. III, p. 537.

r822: La ug i e r: Analyse de la Pierre météorique de Juvénas, Ann. Chim. Phys. Bd. I9, p. $264-273$; s, auch Ann. des Mines Bd. 8 (1823), p. 330 -332; Gilb. Ann. Bd. 7r (1822), p. 203-2I2; Schweigg. Journ. Bd. 35 (1822), p. $414-421$

1822: Chladni: Zweiter Nachtrag, Gilb. Ann. Bd. 71, p. 360, 362, 372. 1824: Chladni: Vierter Nachtrag, Pogg. Ann. Bd. 2, p. 154 .

1825: R o s e: Ueber die in den Meteorsteinen vorkommenden krystallisirten Mineralien. Pogg. Ann. Bd. 4, p. 173 -185 ; s. auch Burkart, Fundorte I, N. J. 1856, p. $267,268,269$.

1834: Berzeli us: Om Meteorstenar. Kongl. Vetensk. Acad. Handl. (1834), p. $179-180 ;$ s. auch Pogg. Ann. Bd 33 (1834), p. $144-145$.

1836: K ä m t z : Meteorologie, p.251, 258, 289.

1840: F o r bes: Edinb. New. Phil, Journ. Bd. 28, p. 385-387. Ref. N. J. 1840, p. 593 .

r84r: $\mathrm{R}$ a m m els b e r g : Handwörterbuch Bd. I, p. $429-430$.

r842: de Malbos: Sur un aërolite tombé aux environs de Berrias (Lozère). Extrait d'une lettre de M. J. de Malbos à M. Elie de Beaumont. C. R. Bd. 14, p. 917-918; s. auch Pogg. Ann. Bd. 56, p. 644 .

1843: Rammeis berg: Ueber die Bestandteile der Meteorsteine, Pogg. Ann. Bd. 60 , p. 130, 131, 132.

1843: P a rts c h: Meteoriten, p. 16-r7. I843: H a i d in g e r: Uebersicht,p.ro8. r848: S h e p a r d : Report on Meteorites, Am. Journ. (2) 2, p. $3^{88}$.

1848 : R a m me $1 \mathrm{~s}$ b e r g: Ueber die $\mathrm{Zu}$ sammensetzung des Meteorsteins von Juvenas, und seinen Gehalt an Phosphorsäure und Titansäure. Pogg. Ann. Bd. 73 , p. $585-590$; s. auch Ram- 
melsberg, Handwörterbuch Suppl. IV, p. I54-155 u. Suppl. V, p. 30-3I; Zeitschr. d. d. geol. Ges. Bd. I (1849), p. 244 (Vergleich mit der TjorsaLava des Hekla); Liebig-Kopp, Jahresber. $1847 / 48$, p. $1298-1299$; v. Humboldt, Kosmos Bd. 3 (1850), p. 6r6; Kenngott, Uebersicht $1844 / 49$, p. 285 . N. J. 1850, p. $63,455-456$.

r848: Shepard: Observations on Rammelsberg's Analysis of the Juvenas Meteoric Stone, and on the Conclusion of Fisher's Examination of the Braunau Meteoric Iron. Am. Journ. (2) 6, p. $346-348$.

I854: B a $1 \mathrm{c}$ e $11 \mathrm{~s}$ : Lithologia meteorica, p. 24 .

1854: v. Boguslawski: Zehnter Nachtrag, Pogg. Ann. Ergz.-Bd. 4, p. $14,19-22$.

r859: B u chner: Feuermeteore, p. 85 -88 .

1859: Harris: Dissert, Gött., p. 76. r860: Rammels berg: Mineralchemie, p. 937-940, 950, 951, 952.

r86x : W ö h 1 er veranlasst Bunsen, die Meteoriten spektralanalytisch zu untersuchen; er findet in Juvenas und Parnallee Lithion. Ann. Chem. Pharm. Bd. 120, p. 253-254; s. auch Gött. Gel, Anz. (Nachr.) 1863, p. $222-223$; Liebig-Kopp, Jahresber. I861, p. II32 ; Kenngott, Uebersicht 186265 , p. 440. 1862: Greg: On some Meteorites in the British Museum. Philos, Magaz. Bd, 24, p. 542 .

r863: B u c h n e r: Meteoriten,p. $42-43$. 1863: Rose: Meteoriten, p. 26, 27, $30,126-133,134,135,139$, I 41 , I51, 156. r $857 / 65$ : v. R e i chen b a c h: III 624 . V $475,476,478,48$ r. VI 452 . VII 558. VIII 476,477 . IX 159, 160, 167, 177. X 359, 362, 371, 372. XI 295. 297. XIII $356,364,367$ (Fig.), 368, 377. XIV 396. XX 626, 631, 633. XXV 615 .

1865: Buchner: Zweiter Nachtrag,
Pogg. Ann. Bd. 124, p. 573-574.

1867: v. L a n g: Messung des Anorthits aus dem Meteorstein von Juvenas.Sitzber. Wien. Akad. Bd. 56 I, p. $839-840$; s. auch Pogg. Ann. Bd. I33 (1868), p. $188-189$; 'L'Institut $\propto$ Bd. 36 (1868), p. 136; Liebig-Kopp, Jahresber. 1868, p. 1044 .

r869: B u c hner : Vierter Nachtrag, Pogg. Ann. Bd, 136, p. $439,445$.

r870: $\mathrm{R}$ a $\mathrm{mmel} \mathrm{s}$ berg : Meteoriten, p. $127-130$; s. auch Rammelsberg, Mineralchemie II. Aufl, 1875, p. 557 (Anorthit); Pogg. Ann. Bd, I40 (1870), p. 321 .

1870: T s c hermak: Goalpara, Sitzber. Wien. Akad. Bd, 62 II, p. 862 . I872: Tschermak: Die Meteoriten von Shergotty und Gopalpur. Sitzber. Wien. Akad. Bd. 65 I, p. 127.

I874: T s cherm a k: Der Meteoritenfund bei Ovifak in Grönland, - Meteorischer Eukrit. M. M. (I874), p. 168 $-17 \mathrm{I}$.

1879: R a mmels berg: Meteoriten, p. 25 .

r88x: Fouqué et Michel-Lévy: Reproduction artificielle des diabases, dolérites et météorites à structure ophitique, C. R. Bd. 92, p. 89 I.

1883: T s cherma k: Beitrag, Sitzber. Wien. Akad. Bd. 88 I, p. 349, 368-37o. 1884: Me unier: Météorites, p. 62, $74,75,76,89,93,95,295$ (Abb.), 296 $-297,373,459,48 \mathrm{I}-485$.

1883/85: Tscherma k: Photographien,

T. $1,2,3,21$, p. $3,4,6,7$.

1884: Wa d swort h: Studies, p. 197. 1885: Brezina: Wiener Sammlung,

p. $173,232$.

r89o: B re z in a: Reise, Ann. Hof-Mus.

Bd. 4 (Not.), p. II9.

r894: C o hen: Meteoritenkunde, an vielen Stellen.

r894: F l e t c h e r: Introduction,p.37,39.

Ursprüngliches Gewicht : Ein Stein 
von über 9r Kgr., der aber nur zur Hälfte erhalten blieb. Es sollen noch mehrere Steine in der Umgegend gefallen sein.

Nachweisbares Gewicht: 5064r gr.

Bailey

Bayet

Belgrad

Bement

Berlin, U.

Bologna

Bonn

v. Braun

Breslau

Budapest

Calcutta

Cambridge

Cleveland

37 Lüttich

1 Madras

317

I2 Madrid

8 de Mauroy

IO12 Melion

5 Minneapolis

20 Modena

23 Moskau

6 München

220 Neapel

305 Neumann

44 Paris, E.

14 Paris, M.

Darmstadt, M.

45 Paris, U.

Debreczin

Dorpat

Edinburg

Göttingen

Gregory

Greifswald

Hamburg

Harvard, U.

Heidelberg

Karlsruhe, M.

Kasan

Kiew

Klausenburg

Lausanne

Leipzig

55 Parma

5 Petersburg, B.

72 Pierotet

I50 Pohl

II 2 v. Siemaschko

6 Stockholm

7 Strassburg

72 Stürtz

55 Stuttgart

136 Troyes

108 Tübingen

55 Turin, J.

3 Ward

100 Washington

146 Washington,Sh. 39

London, B. M. 940 Wien, H. M.*) 682

London, P. G. $\quad 389$ Wien, U. I.

43

Kaaba , Mecca

s. Anhang

Kaande

Ösel

Kaba bei Debreczin, Nordbibarer Com., Ungarn.

Stein, K, gefallen ${ }_{5} 5$. April 1857 . 1858: v. Törö k: Ueber den KabaDebreczin-Meteorit. Pogg. Ann. Bd. 105, p. 329-334 (drei Abbildungen); s. auch Am. Journ. (2) 27, p. 424 ; Kenngott, Uebersicht $185^{8}$, p. 165. 1858: Hörnes: Ueber den Meteorsteinfall bei Kaba, südwestlich von Debreczin am 15. April 1857. Sitzber. Wien. Akad. Bd. $3 \mathrm{I}$, p. $347-35^{\circ}$ (2 Abbildungen des Steins); s, auch Liebig-Kopp, Jahresber. 1858, p. 810 -811 ; Chem. Centr. 1858, p. 820 -822 . Ref. N.J. 1859, p. 192 ; Wochenschr. f. Astr., Met. u. Geogr. 1858 , p. 360; sL'Institute Bd. 26 (1858), Nr. 1292, p. 33 .

1858: W ö h 1 e r: Ueber die Bestandteile des Meteorsteines von Kaba in Ungarn. Sitzber. Wien. Akad. Bd. 33, p. 205 - 209 (Analyse); s, auch Ann. Chem. Pharm. Bd. 109, p. $344-348$. Ref. N. J. 1860, p. 77 ; Journ. prakt. Chem. Bd. 77 (1859), p. 44-48; >L'Institute Bd. 27 (1859), Nr. 1312, p. 63, 67.

r859: Wöhler: Die organische Sub$\operatorname{stanz}$ im Meteorsteine von Kaba. Sitzber. Wien. Akad. Bd. 34 , p. $7-8$; s. auch N. J. I86o, p. $352-353$ (Cold Bokkefeld); Journ. prakt. Chem. Bd. 77 (1859), p. 49-50; Ann. Chem. Pharm. Bd. 109, p. $349-350$; Kenngott, Uebersicht 1859 , p. 167-168; Chem. Centr. Bl, 1859, p. 403 ; Philos. Magaz. (4) 18, p. 215; kurze Bemerkung in Revista minera Bd. 10 (1859), p. 199; Luminous Meteors, Rep. Brit. Assoc. 1865, p. 129; Liebig-Kopp, Jahresher. 1859 , p. $855-856$; $>$ L'Instituts Bd. 27 (1859), Nr. 1317, p. I05. 1859: Wöhler: Composition d'une pierre mctéorique tombée en avril 1857 . C. R. Bd. 48 , p. $403-404$.

1859: B u c hn er: Feuermeteore, p. 183. 1859: H a r r i s: Dissert. Gött., p. 98-99. r860: $\mathrm{R}$ a mmels berg: Mineralchemie, p. $942-944,952$.

1863: B u chner: Meteoriten, p. 94 -96 .

1863: R o se: Meteoriten, p. 126, 156.

1) Davon 46 gr. Aërolito 1821 in Francia *. 
1864: Wöhler: Bemerkungen über den neuesten Meteorstein-Fall. Gött.Gel. Anz. 1864 (Nachr), p. 279-28o. Ref. sL'Institut \& Bd. 33 (1865), p. 14. I859/65: v. Reich en b a c h: IX I6I, 172, 180. X 359. XI 295, 306. XII 458. XIII $355-356,361,362,373$ (Fig.), 380, 383. XIV 395, 399. XIX 152. XXIII 368. XXV 607.

r865: B u chner: Zweiter Nachtrag, Pogg. Ann. Bd. 124, p. 573.

r867: Geinitz: N. J. (1867), p. 724. r870: Rammelsberg: Meteoriten, p. ${ }^{11} 5-\mathrm{II}_{7}, \mathrm{I}_{3} 8$.

1870: Ts chermak: Goalpara, Sitzber. Wien, Akad. Bd. 62 II, p. 860 . 1875: Mohr: Entstehungsart, Ann. Chem. Pharm. Bd. 179, p. 271-272. r876: $\mathrm{S} \mathrm{mit} \mathrm{h:} \mathrm{Carbon} \mathrm{compounds,} \mathrm{Am.}$ Journ. (3) II, p. 39I, 435.

1877: Flight: Meteorites and the origin of life. The Popular Science Review 12. Okt. 1877, p. 9 (Sep.).

r879: R a m mels berg: Meteoriten, p. 23.

r882: W i e chmann: Fusion-Structures in Meteorites. Ann. N. Y. Acad. Sc. Bd. 2, p. 302 .

r884: M e uni er: Météorites, p. 95, 96, 97, 304, 306 .

r885: Brezin a: Wiener Sammlung, p. $184,233$.

1894: C o hen : Meteoritenkunde,p.r54, $155,156,161,162,205,223$.

Ursprüngliches Gewicht: Ein Stein von 7 Pfund, von dem aber nur etwa $5 \frac{1}{4}$ Pfund erhalten blieben ${ }^{1}$ ). Nachweisbares Gewicht: 2316 gr.

\begin{tabular}{lr|lr} 
Bailey & I & Berlin, U. & Spl. \\
Bement & I & Böhm & 6
\end{tabular}

\begin{tabular}{lr|lr} 
Budapest & 27 & Neumann & 2 \\
Calcutta & IO & New Haven & Spl. \\
Debreczin & 2IO2 & Paris, M. & I \\
Göttingen & 2 & Pohl & 4 \\
Greifswald & I & v. Siemaschko & I \\
Harvard, U. & I & Szamosujvar & I \\
Klausenburg & 6 & Tübingen & 7 \\
London, B. M. & IO4 & Washington,Sh. Spl. \\
London, P. G. & Spl. & Wien, H. M.*) & 39
\end{tabular}

\section{Kadapa}

Gurram Konda

Kadonah, Agra, Provinz Doab, Ostindien.

Stein, Cga, gefallen 7. August 1822 .

1834: M a 1 t e B r u n: Nouvelles Annales des Voyages, de la géographie et de l'histoire - par Malte Brun - Series 3 (vol. I-24. 1834-39) Bd, 2, p. 415. Paris. (Nach Herrn Direktor Fletcher's gütiger Mitteilung).

1850: P owe 11: Luminous Meteors, No. 23: Meteoric Stone which fell near Agra on the $7^{\text {th }}$ August 1822. Rep. Brit. Assoc. (1850), p. 120.

1854: v. Boguslaws ki: Zehnter Nachtrag, Pogg. Ann. Ergz.-Bd. 4, p.33. 1859: H a r r is: Dissert, Gött., p. 77. 1862: M a skel y ne: Chondritic Aërolites. Rep. Brit.Assoc. (Not. and A bstr.), p. 190.

r863: Buchner: Meteoriten, p. 44. r865: v. Reichen ba ch: XXV 321 , $427,428,432$.

1884: M e un i e r: Météorites, p. 97, 208. 1885: Brezina: Wiener Sammlung, p. $182,233$.

Ursprüngliches Gewicht: ?

Nachweisbares Gewicht: 89 gr.

1) Herr Professor Johann Kovaćs bestätigte mir auch die in der Literatur vorhandene Angabe, dass der Stein ursprïnglich 7 Pfund gewogen haben mochte, dass aber nur $5 \frac{1 / 4}{4}$ Pfund an das Debrecziner Collegium gelangten. Dieses liess 5 Stücke abbrechen und verteilte dieselben an Wöhler und an die Museen von Budapest, Klausenburg, London und Wien. 
Budapest

Calcutta

Gregory

London, B. M.

4 London, P. G. 2 I

4 Paris, M.

13 v. Siemaschko

39 Wien, H. M.*)

Kaee, Hardoi Distr.,Oude,Ostindien.

Stein, $\mathrm{Cc}$ (früher als $\mathrm{Cg}$ angesprochen), gefallen 29. Januar 1838 . 1864: B u c h n e r : Erster Nachtrag,Pogg. Ann. Bd. 122, p. 317.

1864: Maskelyne u. v. Lang : Mineralogical Notes. - Notices of Aërolithes von Maskelyne. - Kaee, Oude. Philos. Magaz. Bd. 28, p. 149-150; s.auch Kenngott, Uebersicht 1868,p.443. 1869: Buchner: Vierter Nachtrag, Pogg. Ann. Bd. 136, p. 450.

1884: M e uni er: Météorites, p. 268. 1885: Brezina: Wiener Sammlung, p. $182,232$.

r894: F let c her: Introduction, p. Io. r895: Brez ina: Wiener Sammlung, p. 249,255 .

Ursprüngliches Gewicht : » 17 tollahs and 6 massahs . 7 o\%. 160 grs. erhielt Maskelyne.

Nachweisbares Gewicht: 2 I4 gr.

Calcutta Spl. Paris, M.

London, B. M. 209 Wien, H. M.*) 4

Kahangarai - Kakangarai

Kakangarai, auch Kahangarai Tirupathar Talug, Salem Distrikt, Madras, Ostindien.

Stein, gefallen 4. Juni 1890.

Herr Dr. Warth, vom Government Central Museum Madras, teilt mir am 12. August 1893 mit: A larger portion of this fall was sent to the Indian Museum, Calcutta and weighed $307 \mathrm{gr}$. (dies soll wohl $317 \mathrm{gr}$. heissen?).

r894: F l e t c her: Introduction, p. 14.

Ursprüngliches Gewicht: 347 gr., nach Mitteilung von Herrn Holland, Calcutta.

Nachweisbares Gewicht: 342 gr.

\section{Calcutta $\quad$ I9I $\mid$ Madras}

London, B. M. 122

Kakowa, Temeser Banat, Ungarn. Stein, Cga, gefailen 19. Mai $185^{8}$. r859: H a r r i s : Dissert. Gött., p. 22-34 (Analyse), Ref, Kenngott, Uebersicht 1859 , p. 168.

r859: Wöhler u. Haidinger : Ueber die Bestandteile des Meteorsteins von Kakova im Temeser Banate. Sitzber. Wien. Akad. Bd. 34 , p. 8-II (Harris' Analyse) ; s, auch Liebig-Kopp, Jahresber. 1859 , p. $854-855$.

1859: Ha id ing e r: Der Meteorit von Kakova bei Oravitza. Sitzber. Wien. Akad. Bd. 34 , p. II-15 (mit 2 Abbildungen). Ueber diese Arbeiten von Wöhler, Harris u. Haidinger vgl, auch Ann. Chem. Pharm. Bd. 110 (1859), p. I2I-125; Jahrbuch geol. Reichsanst. Bd. 9, p. I; Journ. prakt. Chem. Bd. 77 (1859), p. $50-53$; N. J. 1859, p. 292 u. 1860 , p. $82-84$; Liebig-Kopp, Jahresber. 1859, p. $854-857$; Chem. Centr. 1859 , p. 325 ; Kenngott, Uebersicht 1859 , p. $166-167$; sL'Institut a Bd. 27 (1859), Nr. 1317, p. 105-106. 1860: $\mathrm{R}$ a $\mathrm{mme}$ l $\mathrm{s}$ be r g: Mineralchemie, p. 923 ff., 950 .

r863: B u c h n e r: Meteoriten, p.98-99. r863: Ros e: Meteoriten, p. IOI, IO3, 105,155 .

1859/65: v. Re ichen b a ch: IX 161, 168, 178. XX 623. XXV 321, 322, $433,603,607$.

r870: R a m me ls berg : Meteoriten, p. 103, 106, 139, 140.

1879: R a m mels ber g : Meteoriten, p. 24 .

1884: M e unier: Météorites, p. 79, 209, 220-221, 395 .

r885: B rez in a : Wiener Sammlung, p. 182,233 .

r894: C o h e n : Meteoritenkunde,p.r53, $247,248,266$.

r895: B rez in a : Wiener Sammlung, p. 250 . 

1893: B re z in a : Ueber neuere Meteoriten (Nürmberg), p. 166.

r894: C o he n: Meteoritenkunde, p. 89, 131.

1895: Brezina: Wiener Sammlung, p. 292.

I895: C o h e n: Meteoreisen-Studien IV. Ann. Hof-Mus. Bd. 10, p. 83 .

Ursprüngliches Gewicht : $20^{5} / 6 \mathrm{Kgr}$. Nachweisbares Gewicht: I4666 gr.

\begin{tabular}{lr|lr} 
Bailey & 28 & München & I12 \\
Bement & 368 & Paris, E. & 60 \\
Böhm & I2 & Paris, M. & 270 \\
v. Braun & 747 & Prag, M. & 345 \\
Budapest & 582 & Rom, U. & 294 \\
Dorpat & 198 & v. Siemaschko & 20 \\
Dresden, M. & IIO & Stockholm & 46 \\
Giessen & 42 & Strassburg & 6 I \\
Gregory & 168 & Utrecht & 85 \\
Greifswald & 49 & Ward & 118 \\
Howell & 45 & Wien, H.M. Io I53 $\left.{ }^{1}\right)$ \\
Krantz & 92 & Wrany & 32 \\
London, B. M. & 556 & Würzburg & 73
\end{tabular}

Kenton Co., 8 miles nördlich von Independence, Kentucky, U.S. A. Eisen, Om, gefunden 1889 (ob gefallen 7 . Juli 1873 ?), beschr. 1892 .

Der Name Independence wurde schon wegen einer möglichenVerwechslung mit Joe Wright, Independence Co. nicht als Stichwort gewählt.

r892: Preston: Preliminary Note of a new Meteorite from Kenton County, Kentucky. Am. Journ. (3) 44, p. 163 -164 (Abbildung und Analyse von Davison). Ref. N. J. 1893 II, p. 279; Proc. Rochester Acad. Sc. (1892), p. 151-153 (?).

r893: Meunier: Revision des fers météoriques, p. 52, 60 .

1893: Brezin a: Ueber neuere Meteoriten (Nürnberg), p. 164.
1894: Cohen: Meteoritenkunde, p. I56, 199.

r895: Brezina: Wiener Sammlung, p. 284 .

1895: Co he n: Meteoreisen-Studien IV. Ann. Hof-Mus. Bd. 1o, p. 82.

Ursprüngliches Gewicht: $16_{3} \mathrm{Kgr}$. Nachweisbares Gewicht: 39724 gr.

\begin{tabular}{lr|lr} 
Bailey & 93 & de Mauroy & 1 56 \\
Bement & 9000 & New Haven & 5700 \\
v. Braun & 59 & Paris, E. & 1 37 \\
Brezina & 2619 & Paris, M. & 657 \\
Budapest & 1293 & Prag, M. & 317 \\
Harvard, U. & 3855 & Ward & 9921 \\
London, B. M. & 2520 & Wien, H. M. & 3397 \\
Wo befindet sich die grösste Masse des \\
\multicolumn{2}{l}{ Eisens? }
\end{tabular}

Kerilis, Dep. Côtes du Nord, Frankreich.

Stein, Cga, gefallen 26. Nov. 1874 . r880: Da ubrée: Sur une météorite tombée le 26 novembre 1874 à Kerilis, commune de Maël-Pestivien, canton de Callac (Côtes-du-Nord). C. R. Bd, 9I, p. $28-30$. Ref. N. J. I88I II, p. $182-18_{3}$; Liebig-Kopp, Jahresber.188o, p. $1539-1540$.

r884: M e un ier: Météorites, p. 26I, $263-264,493-494$.

1885: B rezina: Wiener Sammlung, p. 182,233 .

r887: Flig ht: Meteorites, p. 74.

r894: C o h e n : Meteoritenkunde, p. 246.

Ursprüngliches Gewicht: 5 Kgr., von welchen $4200 \mathrm{gr}$. nach Paris kamen.

Nachweisbares Gewicht: 4409 gr.

\begin{tabular}{lr|lr} 
Bologna & 2 & Paris, E. & 46 \\
Calcutta & 77 & Paris, M. & 4168 \\
Gregory & 4 & v. Siemaschko & 6 \\
London, B. M. & 75 & Washington, Sh. & 3 \\
Newton & 3 & Wien, H. M. & 25
\end{tabular}

I) Ausserdem 327 gr. nach 1. Juli 1893 erworben. 
Kernouvé (Cléguérec), Morbihan, Bretagne, Frankreich.

Stein, Ck, gefallen 22. Mai 1869 . r869: de Li mur: Bolide tombé le 22 mai 1869 dans la commune de Cléguérec, arrondissement de Napoléonville (Morbihan). C. R. Bd. 68 , p. 1338 $\rightarrow$ I339.

1869: P is a n i : Analyse de la météorite de Kernouvé, près Cléguérec, arrondissement de Napoléonville (Morbihan), tombée le 22 mai 1869 . C. R. Bd. 68 , p. I489-I49I. Ref. N. J. 187o, p. 106 -Io7; Luminous Meteors, Rep. Brit. Assoc. 1869, p. 278; Liebig-Kopp, Jahresber. 1869, p. 1299 .

r869/70: O 1 d h a m: Rec. Geol. Surv. India Bd. 3, p. 104 (Empfang von zwei Stücken).

1879: R a mmelsberg: Meteoriten, p. 12,25 .

1880 : K l e i n : Vermehrung, Gött. Gel. Anz. (1880), p. 567.

1884: Me unier: Météorites, p. 98, 179, 180-182 (Abb.), 349, 350, 491 $-492,52 \mathrm{~T}$.

1883/85: Tschermak: Photographien, p. 20.

1885: Brezina: Wiener Sammlung. p. 19I, 233.

1887: F1 ight: Meteorites, p. 8-9. r89r: v. H a uer: Ann. Hof-Mus. Bd. 6 (Not.), p. 54.

r894: Co hen: Meteoritenkunde, p. 60. 1895: $\mathrm{R}$ in n e: Ueber rhombischen Augit als Kontaktprodukt, chondrenartige Bildung aus künstlichen Schmelzen und über Konkretionen in Basalten. N. J. 1895 II, p. 246 (Abbildung eines Dünnschliffs).

Ursprüngliches Gewicht： Ein Stein von $80 \mathrm{Kgr}$. , von welchem etwa $40 \mathrm{Kgr}$. erhalten.

Nachweisbares Gewicht: 3 I 142 gr. Bailey Belgrad

\begin{tabular}{|c|c|c|c|}
\hline Böhm & & Marburg & $2 \mathrm{I}$ \\
\hline Bologna & 200 & de Mauroy & \\
\hline v. Braun & 95 & Moskau & \\
\hline Breslau & 22 & Neumann & \\
\hline Budapest & 183 & New Haven & \\
\hline Calcutta & 273 & Newton & \\
\hline Cleveland & I & Paris, M. & 1500 \\
\hline Dorpat & 2 & Pohl & \\
\hline Dresden, M. & II & Rom, U. & \\
\hline Frenzel & 3 & v. Siemaschko & \\
\hline Gent & 76 & Stockholm & 123 \\
\hline Göttingen & 37 & Strassburg & \\
\hline Gregory & 192 & Stürtz & \\
\hline Harvard, U. & 406 & Troyes & \\
\hline Kopenhagen & $2 \mathrm{I}$ & Upsala & 324 \\
\hline v. Leuchtenberg & 21 & Utrecht & \\
\hline London, B. M. 9 & 9347 & Ward & \\
\hline London, P. G. & 507 & Washington, $\mathrm{S}$ & \\
\hline Lüttich & 2 & Wien,H.M.*) & \\
\hline Madrid & 3 & & \\
\hline
\end{tabular}

Kesen, Iwate, Japan.

Stein, Ccb, gefallen $\mathrm{I}_{3}$. Juni 1850 . r893: W a rd: Preliminary Note of a new Meteorite from Japan. Am Journ. (3) 45, p. 153-155 (Abbildung eines Stückes, welches Ward erhielt und das $6 \frac{1}{2}$ auf 5 Zoll gross war; das Gewicht dieses Stücks wird auf $61 / 16$ Unzen angegeben, welcher Angabe wohl ein Druckfehler zu Grunde liegt); s. auch Proc. Rochester Acad. Sc. Bd. 2 (1892), p. 171-173. Ref. N. J. 1894 I, p. 273.

1893: Brez in a: Ueber neuere Meteoriten (Nürnberg), p. I6I.

1895: Brezina: Wiener Sammlung, p. 257 .

UrsprünglichesGiewicht: Der grösste Stein etwa $\gg I^{1 / 2}$ Kubikfuss $\propto$, ausserdem noch io Stücke oder mehr. Nachweisbares Gewicht: 7088 gr.
Bailey

Bayet

Bement

Böhm

v. Braun
34 Brezina 8

4 Budapest 245

$185 \mathrm{x}$ Cleveland 6

222 Harvard, U. 698

23 London, B. M. 1281 $12 *$ 
New Haven $\quad 424$ Stockholm 60

Prag, M. 2I Utrecht 26

Rom, U. $\quad$ I16 Ward $\quad \mathbf{1 2 8 8}$

v. Siemaschko $\quad 32$ Wien, H. M. $\quad 749$

Khairpur, Bhawalpur, Mooltan, Ostindien.

Stein, Ck, gefallen 23. Sept. 1873 .

1873: The Pioneer. Sept. (1873).

r874: Medlicott: Record of the

Khairpur Meteorite of $23^{\text {rd }}$ Sept. 1873 .

- Note by the Rev. G. Yeates, Church

Missionary Society Multan received through Major Minchin. Journ. Asiat. Soc. Bengal Bd. 43 II, p. $33-38$; s. auch $\gg$ Der Naturforscher $\ll 1875$, p. I35.

I874: Luminous Meteors, Rep. Brit.Assoc. (1874), p. 300

1885: Brez in a: Wiener Sammlung, p. I9I, 233.

1887: F light: Meteorites, p. 69-71;

s. auch Rep. Brit. Assoc. (1875), p.237.

r887: Brezina: Neue Meteoriten III,

Ann. Hof-Mus. Bd. 2 (Not,), p. II4.

1888: N e w to n: Orbits, Am. Journ. (3)

36 , p. 4 .

1889: F l e t c h e r : Atacama Meteorites,

Mineral. Magaz. Bd. 8, p. 226 (Streu-

feld. 16 auf 3 miles).

I890: v. Niess 1: Periheldistanzen,

Verh. naturf. Ver. Brünn Bd. 29, p. 188, $195,214,247-248$.

r894: F let c her: Introduction, p. I4.

Ursprüngliches Gewicht: Viele

Steine. Flight giebt für die indischen Museen etwa ${ }_{3} 3 \mathrm{Kgr}$. an. Nachweisbares Gewicht: 9809 gr.

Bement

Budapest

Calcutta

Gregory

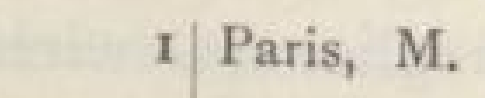

I Pohl

6386 v. Siemaschko

I05 Stockholm

Harvard, U.

69 Troyes

Klausenburg

52 Utrecht

London, B. M. 299I Washington, Sh

London, P. G. I22 Wien, H.M.**)

New Haven

\section{Khandeish 1843 \\ Khandeish 1877 \\ Kharkov}

Manegaon

Dhulia

Jigalowka

Kheragur (Khiragurh), Agra, Staat

Bhurtpur, Dhenagur, Ostindien.

Stein, Cc, gefallen 28. März 1860 .

1860: Presentations were received, I. From Major Bouverie, GovernorGeneral's Agent at Bhurtpore, a meteorite which fell at a village about fifteen miles south of Bhurtpore. Proc. Asiat. Soc. Bengal, Bd. 29, p. 212.

1860: Maskelyne u. v. Lang: Mineralogical Notes. - Notices of Aërolites von Maskelyne. - 14. Dhenagur. Philos. Magaz. Bd. 25, p. 446 -447 u. Bd. 26 , p. 134. Ref. Kenngott, Uebersicht $1862 / 65$, p. 442 ; Rep. Brit.Assoc. 1862 (Not.and Abstr.), p.rgo. 1860: $01 \mathrm{~d} \mathrm{~h}$ a m: Schlussbemerkung, über einen neuen Meteoriten von Bhurtpore, zu dem Aufsatze Haidinger's: Die Meteoritenfälle von Quenggouk bei Bassein in Pegu u. Dhurmsala im Punjab. Sitzber. Wien. Akad, Bd. 42,p.306. 1863: $\mathrm{Bu} \mathrm{chner:} \mathrm{Meteoriten,} \mathrm{p.} \mathrm{IO4.}$ 1863: R os e: Meteoriten, p. 155.

1863: M askel y ne u. v. Lang: Mineralogical Notes. - Notices of Aërolithes von Maskelyne. - 20. Khiragurh. Philos. Magaz. Bd. 26, p. 134 .

Ref.Kenngott,Uebersicht 1862/65,p.442. 1865: Buchner: Zweiter Nachtrag,

Pogg. Ann. Bd. 124, p. 582.

1885: Brezina: Wiener Sammlung, p. $18 \mathrm{I}, 232$.

I894: F1 et cher: Introduction, p.ro, 36. r895: Brezina: Wiener Sammlung, p. 246 .

Ursprüngliches Gewicht: ?

Nachweisbares Gewicht: 545 gr.

Berlin, U. $\quad 4$ London, P. G. 19

\begin{tabular}{ll|l} 
Calcutta & 146 & Wien,H.M. $\left.{ }^{*}\right) \quad 23$
\end{tabular}

London, B. M. 353 
Khetree (Saonlod), Staat Shaikhanati, Rajpotanah, Ostindien.

Stein, Cgb, gefallen r9. Jan. 1867 . 1867: $\mathrm{O} 1 \mathrm{~d} \mathrm{~h} \mathrm{a} \mathrm{m:} \mathrm{Catalogue} \mathrm{von} \mathrm{Cal-}$ cutta, p. 8 (Asiatic Soc. of Bengal besitze $2 \mathrm{oz} .300$ grs.).

1869: B u chner: Vierter Nachtrag, Pogg. Ann. Bd. 136, p. 460.

1869: W a 1 d i e: Analysis of the Kettree Meteorite with an account of its fall. Journ, Asiat. Soc. Bengal Bd. 38 , p. 252-258; s, auch Chemical News v. 19. Juni 1870 (Bd. 21, p. 278 ?).

r869/70: O 1d h a m: Rec. Geol. Surv. India Bd. II, p. Ior u. Bd. III, p. 10. (Nur zwei Stücke seien erhalten, das eine in der Asiatic Soc.e, das andere in der sGeol. Surv.«).

1879: R a mmel sber g : Meteoriten,p.24, 25 .

1884: M eunier: Météorites, p. 79, 26I, 263 .

1885: Brezina: Wiener Sammlung, p. 183,233 .

1887: F light: Meteorites, p. 149-15I. x 894 : F 1 e t c her: Introduction, p. 13 .

UrsprünglichesGewicht: VieleSteine (gegen 40); wenig erhalten.

Nachweisbares Gewicht: $7^{8}$ gr.

Bement

Calcutta

6|London, P. G. 5

44 Paris, M.

London, B. M.

13 Wien, H. M.*)

6

Khiragurh

Kiew ${ }^{7} 79^{6}$

Kiew $187 \mathrm{I}$

Kheragur

Bjelaja Zerkow

Oczeretna

Kikino, Wjasemsk, Gouv. Smolensk, Russland.

Stein, Cwa, gefallen r8og.

1847: Eichwa1d: Verzeichnis, Erman's Archiv f, wissensch. Kunde Russl. Bd. 5 , p. 177 .

1859: Harris: Dissert. Gött., p. 69.

1859: v. Reichenbach: IX 16r, 168,178 .
1885: Brezin a: Wiener Sammlung, p. $180,232$.

Ursprüngliches Gewicht: ?

Nachweisbares Gewicht: 193 gr.

v. Braun

Budapest

Gregory

I Paris, M. 5

67 Rom, U.

6i v. Siemaschko

London, B. M. $\quad 25$ Wien, H. M.*)

9

4

21

Killeter, Tyrone Co., Irland.

Stein, Cwa, gefallen 29. April 1844 .

r854: Greg: Catalogue, Philos. Magaz.

(4) 8 , p. 460.

r859: Ha r r is: Dissert. Gött., p. $9 \mathrm{I}$.

r858/65: v. Reichenbach: V 476, 480. VI 453. IX 161, 168, 178. XI $294,295,296 . X X 626 . \quad X X V{ }_{318}$ $321,322,324$.

186r: Buchner: Notiz über den Meteorsteinfall zu Killeter in Irland, Pogg. Ann. Bd. 113 , p. 508-509 (Analyse von Haughton); s, auch Trans. Irish Acad, r86r; Zeitschr. f. d. ges. Naturw. Bd. 18, p. 168; Liebig-Kopp, Jahresber, I86I, p. II28-II29.

r862: Haughton: On the Shower of Aëroliths that fell at Killeter, Co. Tyrone, on the $29^{\text {th }}$ of April, 1844 . Philos. Magaz. Januar 1862, 6 Seiten (Analyse). Ref. Kenngott, Uebersicht 1861, p. 163 ; N. J. I862, p. 882.

r863: B u c hner: Meteoriten, p. 74. 1884: M eunier: Météorites, p. 8o, 208,218 .

1885: Brez in a: Wiener Sammlung, p. $180,232$.

1895: Brezina: Wiener Sammlung, p. 244 .

Ursprüngliches Gewicht: Steinschauer, aber sehr wenig gesammelt und erhalten.

Nachweisbares Gewicht: 140 gr.

\begin{tabular}{ll|l} 
Calcutta & 2 & London, P. G. Spl.
\end{tabular}

Göttingen Spl. Paris, M. Spl.

\begin{tabular}{ll|l} 
Gregory & 2 & Tübingen $\quad 30$
\end{tabular}

London, B, M. 105 Wien, H. M *) I 
Kinejima

Fukutomi

Kiowa Co. Brenham (Anderson)

Kirgisen Steppe

Kiusiu 1730

Kiusiu 1886

\section{Klausenburg}

Klein-Menow

Klein-Wenden , Thüringen,

Deutschland.

Stein, Ck, gefallen 16 . Sept. 1843 .

r843: Ein mutmasslicher und ein thatsächlicher Meteorsteinfall. Pogg. Ann. Bd, 60 , p. $157-158$.

1844 : v. $\mathrm{H} \mathrm{u} \mathrm{mboldt}$ : Mon.-Ber. Berlin. Akad. 25. Januar 1844 ; s. auch \&L'Institut * Bd. I2 (I844), p. I54-I55.

1844: R a m mels ber g: Chemische Untersuchung des Meteorsteins von Klein-Wenden. Mon,-Ber. Berlin. Akad. 1844, p. 245 ; s, auch Pogg. Ann. Bd. 62 , p. $449-464$; Journ. prakt. Chem. Bd. 33 (1844), p. $229-232 ;$ N. J. 1844, p. 721 u. N. J. 1846 , p. $75-76$; Berzelius, Jahresber. Bd. 25 (1846), p. 396; Rammelsberg, Suppl. II (I845), p. $93-95$ u. Suppl. V (1853), p. 20 -23 ; Kenngott, Uebersicht $1844 / 49$, p. $282-283$; »L'Institut \& Bd.12 (1844), p. $377-378$ u. sL'Institute Bd. 14 $(1846)$, p. 308.

1847: v. Humboldt: Aérolithe de Braunau, en Bohême, tombé le 14 juillet 1847 (Extrait d'une lettre de M. de H. à M. Arago). C. R. Bd. 25, p. 627 .

1854: v. B o g us law s ki: ZehnterNachtrag, Pogg. Ann. Ergz.-Bd. 4, p. 371 -374 .

1859: H a r r i s : Dissert.Gött., p. 90-91. 1859: Buchner: Feuermeteore, p. 95 -97 .

1860: R a mmels berg: Mineralchemie, p. 923 ff., 950.

1863: B u c hner: Meteoriten, p. 73. 1863: R os e: Meteoriten, p. $86,87,89$,
91, 93, 96 (Taf. III), IOI-103, 154, I60. 1859/65: v. Rei che n b a ch: IX 159, $162,172,180 . \times 359,362,371,372$. XIII 365. XV 101, 131. XXV 615. 1870: Rammels berg: Meteoriten,

p. 103, 105, 106, 138, 139, 140.

r879: $\mathrm{R}$ a $\mathrm{m} \mathrm{m}$ e ls b e r g: Meteoriten,p. 25. 1884: M e un i er: Météorites, p. 35, 79,85 .

1884: $\mathrm{Häp} \mathrm{k} \mathrm{e} \mathrm{:} \mathrm{Beiträge,} \mathrm{Abh.} \mathrm{naturw.}$

Ver. Bremen Bd. 8, p. 523 .

r883/85: T s c h e r mak: Photographien, p. $16,20$.

1885: Brezina: Wiener Sammlung, p. I9I, 233.

r887: Brezin a: Neue Meteoriten III, Ann. Hof-Mus. Bd, 2 (Not.), p. II4.

1894: C o he n : Meteoritenkunde, p. 56, $246,249,265$.

Ursprüngliches Gewicht: $3^{1 / 4} \mathrm{Kgr}$. ( $6 \frac{1}{2}$ Pfund).

Nachweisbares Gewicht: 2719 gr.

Bement

Berlin, U.

Calcutta

Cleveland

Dorpat

Göttingen

$$
\begin{array}{r|lr}
18 & \text { Greifswald } & 2 \\
2508 & \text { London, B. M. } & 5 \\
1 & \text { London, P. G. } & 1 \\
\text { Spl. } & \text { Stockholm } & \text { Spl. } \\
5 & \text { Wien, H. M.*) } & 174 \\
5 & &
\end{array}
$$

\section{Knasta}

Knoxville

Bialystock

Tazewell

Knyahinya, Unghvarer Com., Ung.

Stein, $\mathrm{Cg}$, gefallen 9. Juni 1866 .

r866: $\mathrm{H}$ a i d ing e r : Der Meteorsteinfall am 9. Juni 1866 bei Knyahinya nächst Nagy-Berezna im Ungher Comitat. Sitzber. Wien. Akad. Bd. 54 II, p. 200 -205 (Situationsplan). Ref. Luminous Meteors, Rep. Brit. Assoc. I868, p. $387-388$; N. J. 1866 , p. $826-827$; >L'Institute Bd. 34 (1866), p. 360, 400. r866 : v. $\mathrm{H}$ a u e r : Der Meteorsteinfall von Knyahinya. Verh. k. k. geol. Reichsanst. Bd. 16, p. I05 (Geschenk eines 249 gr. [14 1/4 Lot] schweren Steins 
durch Hern Franz Kistler an die k. k. Reichsanst.).

r866: S z a b ó: (Kurze Mitteilung, dass Steine bei Knyahinya und O-Sztusica niederfielen). Verh. k. k. geol. Reichsanst. Bd, 16, p. 115.

1866: Haidinger: Der Meteorsteinfall am 9. Juni $\mathbf{1 8 6 6}$ bei Knyahinya. Zweiter Bericht mit 3 Tafeln und 12 Fig. im Text (zwei Abbildungen des grossen Steins, Erscheinungen am Himmel). Vorwort. Quellen: I. Die Erscheinungen im Falle nahe am Fallorte. II. Beobachtungen aus grösseren Entfernungen. Sitzber. Wien. Akad. Bd. 54 II, p. $475-522$; s. auch Am. Journ. (2) 44 (1867), p. 131; 1893: Brezina, Ankunft, p. 9. Ref. N. J. 1867, p. 371 -372 ; :L'Institute Bd. 35 (1867), p. $55-56$.

I866: $\mathrm{S} z \mathrm{ab}$ b : Meteorite of Hungary of June 1866. Am. Journ. (2) 42, p. 432. r867: Goe be 1: Kritische Uebersicht, Mélanges phys. chim. Bd. 7 , p. $33^{8}$. 1867: Ros e legte den Meteorstein von Knyahinya vor, den die Ungarische Akademie der Wissenschaften dem mineralogischen Museum der Berliner Universität geschenkt hat. Mon.-Ber. Berlin. Akad. 1867, p. $203-205$.

1869: Buchner: Vierter Nachtrag, Pogg. Ann. Bd. 136, p. 439, 459-46o. r869: Kenngott: Ein Dünnschliff einer Meteorsteinprobe von Knyahinya. Sitzber. Wien. Akad, Bd. 59 II, p. 873-880 (mit r Tafel). Ref. N. J. 1869, p. 866 ; Liebig-Kopp, Jahresber. 1869 , p. 1298-1299; Philos. Magaz. Bd. 37 (1869), p. $424-428$ : Microscopical investigation of thin polished laminae of the Knyahinya Meteorite (mit Tafel); "L'Institut \& Bd. 37 (1869), p. 270. I870: v. H a i d i n g er: Orientierung, Sitzber. Wien. Akad. Bd. 61 II, p. 513. r87 1 : $\mathrm{S}$ c hi a p a re 11 i: Entwurf einer astronomischen Theorie der Sternschnuppen. Achte Note. Beweis, dass die Me- teoriten von Knyahinya u. von Pultusk nicht aus derselben Gegend des Himmelsraumes haben herkommen können. Deutsche Ausg. $8^{\circ}$. Stettin 1871 (Th. von der Nahmer), p. $267-268$.

r872: v. B a u $\mathrm{m}$ h a u e r: Sur la météorite de Knyahinya dans le Comitat d’Unghvar. Arch. Néerl. Bd. 7, p. 146 - I53 (Analyse).

1875: T s chermak: Vulkanismus, Sitzber. Wien. Akad. Bd. 71 II, p. 662, 664,671 .

1875: vom Rath: Meteoriten, Verh. naturh. Ver. Bonn Bd. 32, p. 369-370. 1880: H a h n: Die Meteorite(Chondrite) und ihre Organismen. Tübingen. (Zahlreiche $\mathrm{Abb}$, von Dünnschliffen).

1882: v. L a s a u $1 \mathrm{x}$ : Vermehrung, Verh. naturh. Ver. Bonn (Sitzber.) Bd. 39, p. 104 .

1883: Ts chermak: Beitrag, Sitzber. Wien. Akad, Bd. 88 I, p. $355,356,359$. r884: W a d s w orth: Studies, p. 8891.

I884: v. $\mathrm{Niess} \mathrm{1:} \mathrm{Ueber} \mathrm{die} \mathrm{astrono-}$ mischen Verhältnisse bei dem Meteorsteinfalle von Mócs in Siebenbürgen am 3. Februar 1882. Sitzber. Wien. Akad. Bd. 89 II, p. 29I. Ref. N. J. I886 I, p. 224.

1884: M eunier: Météorites, p. 98,268 (Abb.), 271, 321, 42I, 444, 445 (Abbildung der Fallerscheinung, Situationsplan), 446, 448, 459, 460 (Abb.). r883/85: Tschermak: Photographien, p. $13,14,15,16,17,18,20$. T. X, XI, XII, XIII, XIV.

r885: Brezina: Wiener Sammlung, p. $165,166,168,182,232$.

1887: F $1 \mathrm{ig} \mathrm{h} \mathrm{t:} \mathrm{Meteorites,} \mathrm{p.} \mathrm{145-I46.}$ r889: F let c her: Atacama Meteorites, Mineral Magaz. Bd. 8, p. 226 (Streufeld 9 auf 3 miles).

1890: v. Niess 1: Periheldistanzen, Verh. naturf. Ver. Brünn Bd, 29, p. 186, 189, 194, 214, 256.

1892: P ́́ivoznik: Ueber die Meteo- 
rite von Knyahinya u. Hainholz. Oesterr. Zeitschr. f. Berg- u. Hüttenw. Bd. 40, p. $465-466$; s. auch Berg- u. Hüttenm. Jahrb. Bd. 40 (1892), p. $457-458$. Ref. N. J. 1894 I, p. $274-275$.

I892 : Brezina: Sternschnuppen, p. 8 -II, 16.

1893: B r e z in a : Ueber neuere Meteoriten (Nürnberg), p. 160.

r894: C o he n : Meteoritenkunde, p. 205 , 249, 262, 268, 276, 286, 298.

r894: F l et c h e r : Introduction, p. I3, $28,36,3^{8}, 39,53$.

Ursprüngliches Gewicht: Etwa 500 Kgr. Ueber tausend Steine. Es wurden gesammelt:

I Stück 550 Pfund
Je $\mathrm{I}$ Stück von $73^{1 / 2}, 30,17$
$14,6=140^{1 / 2}$ Pfund
20 Stücke zwischen 4 und
2 Pfund = 60 Pfund
Kleinere Steine zusammen
roo Pfund

Nachweisbares Gewicht: 423 I 20 gr.

\begin{tabular}{lr|lr} 
Bailey & 174 & Calcutta & 1395 \\
Bayet & 63 & Cambridge & 85 \\
Belgrad & 148 & Clausthal & 31 \\
Bement & 1262 & Cleveland & 219 \\
Berkeley & $\left.119^{1}\right)$ & Darmstadt, M. & 88 \\
Berlin, G. & 57 & Debreczin & 484 \\
Berlin, L. & 15 & Dorpat & 151 \\
Berlin, P. & 133 & Dresden, M. & 60 \\
Berlin, U. & 1817 & Eger & 440 \\
Bern, M. & 100 & Frankfurt & 185 \\
Böhm & 635 & Frenzel & 48 \\
Bologna & 229 & Freiberg, i. S. & 19 \\
Bonn & 9417 & Gent & 89 \\
v. Braun & 940 & Giessen & 6 \\
Breslau & 217 & Göttingen & 391 \\
Brezina & 64 & Graz, J. & 103 \\
Budapest & 47423 & Gregory & 827
\end{tabular}

\begin{tabular}{|c|c|c|}
\hline Greifswald & 26 & Paris, U. \\
\hline Halle & 236 & Petersburg, A. II3 \\
\hline Hamburg & 94 & Pohl $\quad 4814$ \\
\hline Harvard, U. & 885 & Prag, M. \\
\hline Heidelberg & 84 & Prag, D. U. \\
\hline Karlsruhe, P. & 120 & Riga \\
\hline Kasan & 1203 & Rom, U. \\
\hline Klausenburg & 1473 & Schemnitz $\quad 26$ \\
\hline Kopenhagen & 421 & v. Siemaschko 283 \\
\hline Krantz & 234 & Seligmann \\
\hline Kristiania & 157 & Stockholm \\
\hline Kunz & 82 & Strassburg \\
\hline Leipzig & 36 & Stuer \\
\hline v. Leuchtenberg & g 122 & Stürtz \\
\hline London, B.M. I3 & 13053 & Stuttgart \\
\hline London, P. G. & 1703 & Szamosujvar \\
\hline Madrid & 10 & Tharand \\
\hline Marburg & $4^{19}$ & Troyes \\
\hline de Mauroy & 79 & Tübingen \\
\hline Melion & 14 & Turin, J. \\
\hline Minneapolis & 20 & Turin, U. \\
\hline Modena & Io & Upsala \\
\hline Moskau & 227 & Ward \\
\hline München & 54 & Washington 28 \\
\hline Neapel & 269 & Washington, Sh. 33 \\
\hline Neumann & 208 & Wien, H. M. *) **) \\
\hline New Haven & 65 & 299216 \\
\hline Newton & 46 & Wien, U. I. \\
\hline New York, M. & 35 & Wien, U. II. \\
\hline Odessa & 12 & Wrany \\
\hline Paris, E. & 43 & Würzburg \\
\hline Paris, M. & 8515 & Zürich \\
\hline
\end{tabular}

Die Universität Basel besitzt ebenfalls ein Stück Knyahinya. Hierher gehört vielleicht auch ein 75 gr. schwerer Stein von »Ungarn \&, von welchem Herr Hovey mir mitteilt: $>$ The surface is indented with closely-set small pits «, was mit der charakteristischen Oberfläche der Steine von Knyahinya übereinstimmen könnte.

Kokomo, Howard Co., Indiana, U. S. A.

1) $>$ Stony meteorite, Hungary\& $103+16$ gr.; ist wohl Knyahinya,

2) Davon ein Stein von $129 \mathrm{gr}$., früher als Siena bezeichnet, von Herrn Direktor Brezina als Knyahiuya erkannt. 
Eisen, Hca, gefd. 1862 , beschr. 1873 . 1873: Cox: On a new Meteorite found in Indiana. Am. Journ. (3) 5, p. 155 -156 .

1874: S m it h: On a Mass of Meteoric Iron of Howard Co. Ind., with some remarks on the molecular structure of meteoric iron, and a notice concerning the presence of solid protochloride of iron in Meteorites. Am. Journ. (3) 7, p. 391-392 (Analyse); s. auch C. R. Bd. 77 (1873), p. I193-1197; Original Researches (1884), p. 473-474. Ref. Liebig-Kopp, Jahresber, 1873 , p. 1251 u. 1874, p. $1342-1343$.

1884: M e unier: Météorites, p. Ior (Abb.).

1885: Brezina: Wiener Sammlung, p. 200, 201, 203, 219, 234 .

1887: F lig ht: Meteorites, p. 19, 138. 1890 : Eastman: Met. Astron., p. 320. 1893: Meunier: Revision des fers météoriques, p. 6 .

1894: Co he n: Meteoritenkunde, p. 70, 108, no.

Ursprüngliches Gewicht: $5^{8} 5^{\circ} \mathrm{gr}$. Cox's Eisen: 1850 gr. (4 pounds $I^{1} / 2$ ounces); Smith's Eisen : $4 \mathrm{Kgr}$. Nachweisbares Gewicht: 655 gr.

Bailey

Budapest

Gregory

Harvard, U,

Kopenhagen

35 London, B. M. $\quad 38$

35 London, P. G. 7

7 Paris, M.

418 v. Siemaschko

34 Wien, H. M.**) 15

Wo ist die grösste Masse dieses Eisens?

Kokstad, Griqualand, Ost-Südafrika. Eisen, Om, bekannt 1887 .

1887: B rez in a: Neue Meteoriten IIIa, Verh. k. k. geol. Reichsanst. (1887), p. 289 .

1888: v. H a u e r: Ann. Hof-Mus. Bd. 3 (Not.), p. 42 u. Bd. 4 (1889) (Not.), p. 64 .

r89r: Cohen u. Weinschenk: Meteoreisen-Studien. Ann. Hof-Mus.Bd.
6 , p. $131,159,165$.

1894: C o h e n : Meteoritenkunde,p. 216,

$287,298,317,319,321$.

1895: Brezina: Wiener Sammlung, p. 284 .

Ursprüngliches Gewicht: $42.6 \mathrm{Kgr}$. Nachweisbares Gewicht: 4081 2 gr. Wien,H.M. 40812

\section{Kolotscha}

Borodino

Konia

Adalia

Kossuth Co.

Forest City

Köstritz

Politz

Krähenberg, Bairische Pfalz, Deutschland.

Stein, Cho, gefallen 5. Mai 1869. r869: Keller: Der Meteorstein von Krähenberg (Analyse) Palatina, Beibl.z. Pfälzer Zeitung Nr. 79. Speyer, 3. Juli 1869, p. 318 . Gedruckt in der Jägerschen Druckerei in Speyer.

1869: $\mathrm{Kr}$ antz: Den Meteorstein, der am 5. d. M. bei Krähenberg halbwegs zwischen Landstuhl und Zweibrücken gefallen ist, haben schon die Zeitungen erwähnt; etwas Näheres erlaube ich mir noch in der Kürze zu berichten.Verh. naturh. Ver. Bonn (Corresp.) Bd. 26, p. 40-4r. Ref. Wochenschr. f. Astr., Met. u. Geogr. 1869, p. 168, 264.

r869: N e u m a y er: Bericht über das Niederfallen eines Meteorsteines bei Krähenberg, Kanton Homburg, Pfalz. Sitzber.Wien. Akad. Bd, 60 II, p. 229 24I (Situationsplan u. 4 [nicht gute] Abbildungen des Steins) ; s. auch *L'Institute Bd. 37 (1869), p. 328.

r869: $\mathrm{Bu} \mathrm{c} \mathrm{hn} \mathrm{e} \mathrm{r:} \mathrm{Der} \mathrm{Meteorstein} \mathrm{von}$ Krähenberg. Pogg. Ann. Bd, 137, p. I76. 1869: vom R a th: Ueber den Meteoriten von Krähenberg, gefallen am 5. Mai 1869. Pogg. Ann. Bd, 137, p. 328-336 (Analyse). Ref. N. J. 1870, p. 23I-232; Journ. prakt. Chem. Bd. Io8 (1869), p. $163-172$; Wochenschr. f. Astr., Met. u. Geogr. 1869, p. 4 I2 -4 I5 
1869: W e is s: Ueber den Meteorstein von Krähenberg bei Zweibrücken. Pogg. Ann. Bd, 137, p, 617-624.

r869: Weiss: Briefl. Mitteilung mit einer Skizze des Steins. N. J. 1869, p. $727-729$.

I869: Referat über die Arbeiten von Buchner, vom Rath, Weiss u. Neumayer. Liebig-Kopp, Jahresber. I869, p. $1296-1297$.

1869/70: O l d h a m: Rec. Geol. Surv. India Bd. 2, p. IOI (Empfang eines Stückes).

r870: R a mmels berg: Meteoriten, p. 98 .

1870: N e um a y er: Der Meteorit von Krähenberg. Neustadt $8^{\circ}$. Mit Tafel, zwei Abbildungen des Steins, Analyse von Keller. 28 Seiten.

1870: v. Haidinger: Der AinsaTucson-Meteoreisenring in Washington und die Rotation der Meteoriten in ihrem Zuge. - I. Die Rotation, Krähenberg. Sitzber. Wien. Akad. Bd. 6I II, p. 499-50I (Abbildung des Steins). Ref. Luminous Meteors, Rep.Brit. Assoc. I870, p. $92-93$.

r872: Reinsch: Suite Mikroskopischer Praeparate über die Structurverhältnisse und die mineralogischen Bestandtheile des im Jahre 1869 gefallenen KrähenbergerMeteorsteines.Zweibrücken 1872 ; s. auch Tagebl. Vers. d. d. Naturf. Leipzig 1872 , p. $132-134$; Brezina, Bericht IV, Sitzber. Wien. Akad. Bd. 85 I (1882), p. 343 ; Hirzel u. Gretschel's Jahrb. d. Erfind. Bd. 9, p. 22-23. Auch erschien eine Abhandlung von Reinsch: "Die Meteorsteine , welche am Schluss den Fall von Krähenberg bespricht (Sep, im k, k. Hof-Museum Wien).

I875: vo m R a th: Meteoriten, Verh. naturh. Ver. Bonn Bd. 32, p. 374.

I878: G üm be I: Die in Bayern gefundenen Steinmeteoriten, - Der Meteor- stein von Krähenberg bei Zweibrücken in der Rheinpfalz. Sitzber. München. Akad. Bd. 8, p. 47-72 (Sep.; zwei Abbildungen von Dünnschliffen. Analyse von Schwager).

1879: R a mmelsberg: Meteoriten,p.r2, $24,26$.

r882: Brezina: Bericht IV, Sitzber.

Wien. Akad. Bd. 85 I, p. 343 .

r884: W a d sw ort h: Studies, p. 103. I884: M e un ier: Météorites, p. 79, 268, 271-272, 459.

1885: Brezina: Wiener Sammlung, p. $176,177,233$.

I887: F lig h t: Meteorites, p. 5-7.

1888: v. $\mathrm{N}$ i es s1: Bestimmungen der Bahnverhältnisse einiger Meteore. Ueber die Bahnen der Meteoriten von Pillistfer am 8. August 1863 u. Krähenberg am 5. Mai 1869. - B. Meteoritenfall bei Krähenberg i, d. Pfalz. Verh. naturf. Ver. Brünn Bd. 27, p. 270-273. 1888: Newt o n: Orbits, Am. Journ. (3) 36, p. 4 .

1890: Weinschenk: Ueber zwei neue Bestandteile des Meteoriten von Sarbanovac. Ann. Hof-Mus. Bd. 4 (Not.), p. IIo.

1890: v. Niess 1: Periheldistanzen, Verh. naturf. Ver. Brünn Bd. 29, p. 186, 188, 194, 209, 210, 21I, 2I4, 248. r894: C o hen: Meteoritenkunde, p.203, 319.

r894: F l e tc her: Introduction, p. 13. Ursprüngliches Gewicht : Ein Stein von etwa $16^{1 / 2} \mathrm{Kgr}$.

Nachweisbares Gewicht: 15 ogr gr. Berlin, U.

Bonn

v. Braun

Budapest

Calcutta

Göttingen

Krantz

London, B. M.

London, P. G.
5 de Mauroy 1

I7 München 4

Spl. New Haven 2

Spl. Paris, M. 3

I Pohl I

3 Speyer $14950^{1}$ )

5 Stockholm Spl.

3 Wien, H. M. *) 93

3

I) Städtisches Mus, naturh. Abt. Gewicht 1893 durch den Herrn Konservator neu bestimmt. 
Eine neue Analyse dieses Steins wäre sehr erwïnscht.

\section{Krähenholz \\ Krakhut}

Krasnojarsk Eisen

Krasnojarsk (Medwedewa), Gouv. Jeniseisk, Sibirien, Russland.

Pallasit, P, gefunden um 1749 , von Pallas wieder aufgedeckt 177I, von Chladni als meteorisch erkannt 1794 .

Hierher auch vorläufig: „Tuckers Arizona s s.Brezina, Wiener Sammlung (1895), p. 264 .

Um 1774: Vorläufige Nachrichten in Petersburger $u$. andern Zeitungen.

Um 1774: Engeström in einer Rede vom 4. Mai 1774 , gehalten an der schwedischen Akademie.

1776: P a ll a s: Reise durch verschiedene Provinzen des russischen Reichs 1721-1773. Petersburg 1776 Bd. 3, p. 411 ff.; abgedruckt in v. Ende, Massen u. Steine 1804 s, u. Ein ausführlicher Auszug der Reisebeschreibung in 3 Bänden erschien in Frankfurt und Leipzig bei Fleischer. Bd. 3 von 1778 , p. 315-324. Franz. Uebersetz. von Gauthie de la Teyronie avec notes de Lamarck (an II).

1777: Meier: Versuche mit der von dem Herrn Prof. Pallas in Sibirien gefundenen Eisenstufe, nebst einigen allgemeinen Erfahrungen vom Eisen. Beschäftigungen der Berlinischen Gesellsch. naturf. Freunde Bd. 3 , p. $3^{85}$ -394 ; s. auch Klaproth, Abh. Berlin. Akad. 1803 , p. $3 \mathrm{I}$.

1783: R omé de l'I s le : Cristallographie. 2. Aufl. Bd, 3, p. 167-168.

1789: Gro n a u : Einige Bemerkungen über die Gewitter. Schriften der Gesellsch. naturf. Freunde. Berlin Bd. 9 (Bd. 3 der Beobacht, u. Entdeck.), p.
47 (Vermutung, das Eisen möchte durch den Blitz entstanden sein).

r794: Chla dn i: Pallaseisen, p. 39$40 \mathrm{u}$. an vielen anderen Stellen.

1796: K ing : Remarks, p. 23-24, 26. r8or (?): P atrin: Bibl.Brit. Nr. 122, p. 73 u. Nr. 2, Bd. 18; s. auch Izarn, p. 150.

1801/02: Deluc: Sur la masse de fer de Sibérie. Journ. des Mines Bd. II (an X), p. 213-220.

1802: Ho w a r : Experiments and Observations on certain stony and metalline Substances which at different Times are said to have fallen on the Earth; also on various Kinds of native Iron. - Description of various Kinds of native Iron. By the Count de Bournon. Philos. Trans. (1802), p. 204-210, 212 ; s. auch Gilb. Ann. Bd. I3 (1803), p. 319-325, 327; Fourcroy's Uebersetzung in «ncyclopédie « Bd. 5 (1808), p. $55^{8}$. 1802/03: Delu c: Sur la Masse de fer de Sibérie, et sur les Pierres supposées tombées de l'atmosphère. Journ. des Mines Bd. $\mathrm{I}_{3}$ (an XI), p. 82-107.

1803: de Dré e: Recherches, Journ. Phys. Bd. 56 (an XI), p. 4I4, 4I 7 .

I803: C h 1 a d n i: ChronologischesVerzeichnis, Gilb. Ann. Bd. 15, p. $3^{18}$ -319 .

1803: I z a r n: Lithologie, p. IIo-126 (Chladni's Werk von 1794, welches aus einer Bearbeitung in den Philos. Magaz. in die Bibl. Brit. gelangte), 138-149 (De Luc aus Bibl. Brit. I8or Bd. 17, Nr. 3, p. 809 ), 150-169 (Patrin aus Bibl. Brit, 18or Bd. 18, Nr. 2), $218-$ 229 (Howard u. Bournon), 230, 232, $333-342,347$.

1803: K la proth: Ueber meteorische Stein- u, Metallmassen. Abh. Berlin. Akad. (1803), p. 29-30; s, auch Klaproth, Beiträge Bd. 6 (1815), p. 300303; Gilb. Ann. Bd, 18 (1804), p. 289; Gehlen's Neues allgem. Journ, d. Chem. Bd. I, p. 3 . 
1804: $\mathrm{P}$ öt z s c h: Kurze Darstellung, p. $36-45,93,98$.

1804: v. Ende: Massen u. Steine, p. $6 \mathrm{I}-70$ (enthält den Bericht von Pallas abgedruckt).

1807: K laproth: Beiträge Bd. 4, p. 99.

r812: Bigot de Morogues, p. 288 $-295,338$

I8I2: $\mathrm{C} \mathrm{hl}$ a d n i : Ueber Gediegeneisen und besonders über eine noch unbekannte im Mayländischen gefundene Gediegeneisenmasse. Denkschr. München. Akad. (1812), p. 105; s. auch Schweigg. Journ. Bd. 4 (1812), p. 117 (17 ?).

r8r5: Chladni: Neues Verzeichnis, Gilb. Ann. Bd. 50, p. 256.

r815: Chla dni: Bemerkungen, Gilb. Ann. Bd. 5o, p. $25^{8}-259,263$.

I8I5: Gillet-Laumont: Sur un Aérolithe tombé en Moravie, et sur une Masse de fer natif tombée en Bohême. Journ. des Mines $18 r_{5}$, p. 234 -237 (Abbildung von kleinen geätzten Stücken),

I8r6: J o h n : »Eine höchst merkwürdige Entdeckung habe ich rücksichtlich der Mischung des Siberischen Meteoreisens gemacht. Dieses enthält ausser $96 \mathrm{p}$. C. Eisen 3 Th. Nickel und $1 \mathrm{Th}$. Kobalt, « Leonhard's Taschenb. f. Mineralogie Jahrg. Io (1816), p. 604-605; s. auch Gilb. Ann. Bd, 57 (1817), p. $119-120$. 1817: La ugier: Expériences propres à confirmer l'opinion èmise par des naturalistes sur l'identité d'origine entre le fer de Sibérie et les Pierres Météoriques ou Aérolithes. Ann. Chim. Phys, Bd. 4, p. 363-366; s. auch Mém. Mus. Hist. Nat. Bd. 3 ( 1817 ), p. $34 \mathrm{r}$ -352 ; ferner Gilbert's freieBearbeitung: Neue Analyse des Pallas'schen sibirischen Eisens, zur Bestätigung eines gemeinschaftlichen Ursprungs desselben mit den Meteorsteinen. Gilb. Ann, Bd, 58 (1818), p. $182-186$.
1817: v. Soemmering: Ueber die Zeichnungen, welche sich bei Auflösung des Meteoreisens bilden. Schweigg. Journ. Bd. 20, p. 9I-94.

r8r7: Stromeyer: Auffindung von Kobalt in dem Meteoreisen. Gilb. Ann. Bd. 56, p. 194 (findet bei Krasnojarsk kein Co). Auszug aus Gött. Gel. Anz. vom 23. Dezember I816.

r8rg: $\mathrm{Ch}$ l a d ni: Feuermeteore, p. 9, $46,92,300,309,319,320-323$ (!), $325,340,341,421,432$.

1820: B iot: Sur la structure de la substance verte, qui se trouve dans les cavités de la masse de fer natif découverte en Sibérie par Pallas (weist nach, dass der Olivin optisch zweiaxig sei). Bull. Soc. Philomatique ( 1820 oder I8I9?), p. 89 .

r820: v. Schreibers: Beiträge, p. 84-86 (Abbildung auf Taf. 8).

I82I : John: Sur la nature de grandes masses de fer métallique d'origine problématique, et sur celle du fer des aérolithes attirables par l'aimant. Ann. Chim. Phys. Bd. 18, p. 201; s, auch Schweigg. Journ. Bd. 32 (182I), p. 256 -257 .

r82I: Chladni: Erster Nachtrag, Gilb. Ann. Bd. 68 , p. 346 .

1822: Chladni: Zweiter Nachtrag, Gilb. Ann. Bd. 7I, p. 369 .

I824: W a $1 \mathrm{mste} d \mathrm{t}$ : Försökatt bestämma Peridotens sammensättning. Kongl. Vetensk. Acad. Handl, r824, p. $36_{3}-364$.

1824: Strom e y e r: De Olivini, Chrysolithi et fossilis, quod celulas et cavernulas ferri meteorici Pallasii explet, analysi chemica, Gött. Gel. Anz, 27. Dezember 1824, p. 1957, 2073-2083 (Analyse).

1825: Ros e : Ueber die in den Meteorsteinen vorkommenden krystallisirten Mineralien. Pogg. Ann. Bd. 4, p. 186 -192 (Taf. 2), 193-20r.

1826: Seebeck: Ueber die magneti- 
sche Polarisation der Metalle und Erze durch Temperatur-Differenz. Pogg. Ann. Bd. 6, p. 144 .

1834: B erzeli us: Om Meteorstenar. - 5. Pallasjernet och Pallasolivin. Kongl. Vetensk. Acad. Handl. (1834), p. $158-169$ (Analyse), auch abgedruckt in Pogg. Ann. Bd. 33 (1834), p. 123 -135 ; s. auch Auszug in Berzelius, Jahresber. Bd. I5 (1836), p. 23I, 234. I835: H es s : Vermischte Notizen. Pogg. Ann. Bd. 36 , p. 560 (Gewicht der Pallas'schenEisenmasse I270 russ.Pfund). 1836: Kämtz: Meteorologie, p. 260, 26I, 262.

1837: R o s e: Reise nach dem Ural. Bd. I, p. $43-44$.

r839: B e n z e n b e r g : Sternschnuppen, p. 40.

1840: $\mathrm{R}$ u $\mathrm{m} 1$ e r: Entdeckung der arsenigen Säure in dem olivinähnlichen Mineral aus dem Meteoreisen von Atacama in Bolivia u. von Krasnojarsk in Sibirien. Pogg. Ann. Bd. 49, p. 591 -595 . Ref. Berzelius, Jahresber. Bd. 21 (1842), p. 233.

r84r : $\mathrm{R}$ a m m e ls ber g : Handwörterbuch Bd, I, p. $426-427$.

r843: P a rts c h: Meteoriten, p. $87-90$. r847 : E i chwald: Verzeichnis, Erman's Arch. f. wissensch. Kunde Russlands Bd. 5, p. 183 .

r847: S c hafhä ut 1: Schönenberg, Gel. Anz. München. Akad. 1847, p. $574-578$.

r848: B 1 o e d e. Tabelle, Bull. Petersburg. Akad. Bd. 6, p. 9-10.

r852: W ö hler: > Passiv๔, Pogg. Ann. Bd. 85 , p. 448 .

1852: Cl a rk: Dissert. Gött., p. 15-17. 1854: v. Boguslawski: Zehnter Nachtrag, Pogg. Ann. Ergz.-Bd. 4, p. $394-396$.

r859: B u c h ner: Feuermeteore, p. 48 -50 .

r859: H a r r is : Dissert, Gött., p. IOI. 1860: R a mmels berg: Mineralche- mie, p. 909, 947, 948, 949.

1862: Kenng ott u. W is er: Zürcher Sammlung, p. $143,157$.

r863: Buchner: Meteoriten, p. I2I124.

I863: R os e: Meteoriten, p. 23, 26, 28, 30, 3I, 32, 39, 40, 4I, 73 (Taf. IV) $-76,77,79,80$, 153, 159, 161 ; s. auch Mon.-Ber. Berlin. Akad, 1862, p. 556 .

r857/65: v. Reiche $\mathrm{n}$ b a c h: III 624 . $\mathrm{V} 473,475$. VI $442,443,452$. VII 551, 56r. IX 162, 163, 173, 181. X 359, $363,371,372$. XI 296, 302, 305, 309. XII $452-454,455,461$. XIV 393 . $\mathrm{XV}$ 104, 107, 108, 112, 116, 126. XVI 26I, 262. XVII 265, 271. XVIII 48I, 487. XIX 155. XX 627. XXI 578 . XXV $422,429,436,6 \mathrm{rr}$.

1866: v.K o k s c h a r o w : Materialien zur Mineralogie Russlands Bd. 5, p. I7 $-2 \mathrm{O}$.

1867: Helmersen: Bericht an die physikalisch-mathematische Classe über die Durchschneidung der Pallas'schen Eisenmasse (Auszug). ( $\mathrm{Lu}$ le 8 mars 1866) Bull. Petersburg. Akad. Bd. Io, p. 296-305; s. auch Mélanges phys. chim. Bd. 6, p. $7 \mathrm{II}-724$.

r867: Go e b e l: Ueber die Pallasmasse, Bull. Petersburg. Akad. Bd. Io, p. 305 -324 ; s. auch Mélanges phys. chim. Bd, 6, p. $724-75$ I.

1867: G o e b e 1: Kritische Uebersicht, Mélanges phys, chim. Bd, 7 , p. 274278.

1867: Buchner: Dritter Nachtrag, Pogg. Ann. Bd. 132, p. 312-314, 319. 1869: Meunier: Recherches, Ann. Chim Phys. (4) 17, p. 52, 55.

r870: v. Kok s charow: Ueber den Olivin aus dem Pallaseisen. Materialien zur Mineralogie Russlands Bd. 6, p. I -60, T. $75-77$ (neue Analyse von Seiner Kaiserlichen Hoheit Herzog Nicolai Maximilianowitsch von Leuchtenberg). Ref.N.J.187o, p.778-779; Mem. 
Akad. Sc. Petersburg. Bd, I5, Nr. 6 (40 Seiten mit 4 Tafeln); Bull. Petersburg. Akad. Bd. 20 (1870), Nr. 3.

1870: R a m mels berg: Meteoriten, p. $79,83,87-88,138$; s. auch Pogg. Ann. Bd. 140 (1870), p. 312.

I87r: v. Ba u $\mathrm{mhauer}$ : Sur l'olivine du fer météorique de Pallas. Arch. Néerland. Bd. 6, p. 162-167.

1872: de Chancourtois: Observations (vergleicht das Pallaseisen mit dem von Grönland). Bull. Soc. Géol. France Bd. 29, p. 175-177, 210, 214. 1872: Q uensted t: Klar u. Wahr, p. 291 (Abb. einer geätzten Platte).

1873: v. H el mers e n: Briefliche Mitteilung an Herrn G. Rose, die Fundstätte des Pallaseisens betreffend. Zeitschr. d. d. geol. Ges. Bd. 25 , p. $347-$ 349 .

1874: B jörling : Meteoriter och Kometer (cfr. Hessle), p. I2.

1875: M o br: Entstehungsart, Ann. Chem. Pharm. Bd. 179 , p. 258.

1875: vom R a th: Meteoriten, Verh. naturh. Ver. Bonn Bd. 32, p. 364-365.

1875: Go e bel: Ueber die neuerdings gegen den kosmischen Ursprung des Pallas-Eisens erhobenen Zweifel, nebst einer Widerlegung derselben. Bull. Petersburg. Akad. Bd. 20, p. 100-I29 (auf p. $\mathrm{IO}_{4}$ wird der Kosake Medwedjew aus Medwedjewo als einer der ersten Finder genannt, daher der jetzt ziemlich verbreitete Name Medwedewa $\propto$ ); auch abgedruckt in Mélanges phys. chim, Bd. IX. Die Arbeit ist von $9 / 21$ April 1874.

1879: R a mmels berg: Meteoriten, p. 6,24 .

r882: M e uni er: Contribution à 1 'histoire géologique du fer de Pallas. C. R. Bd. 95 , p. $938-94$ I. Ref. N. J. I884 I, p. $3 \mathrm{I}-32$.

r883: Ts c herma k: Beitrag, Sitzber. Wien. Akad. Bd. 88 I, p. 348 .
1884: W a d s worth: Studies, p. 68, $71-72$,

1884: Wi ep ken: Notizen über die Meteoriten des Grossberzoglichen Museums. - II. Meteoreisen. Abh. naturw. Ver. Bremen Bd. 8 , p. $526-527$. r884: $\mathrm{Hä}$ p ke: Beiträge, Abh. Naturw.

Ver. Bremen Bd. 8, p. 520.

r884: Meunier: Météorites, p. 35, 77, 79, 93, 94, 96, 97, 139-142 (Abb.), $356,360,361,373,380,477,498-499$. r883/85: Ts chermak: Photographien, T. 25, p. 3,23 .

r885: Brezina: Wiener Sammlung, p. 200, 203, 206, 233.

r886: Brezina u. Cohen: Photographien, T. 4 .

I887: F lig ht: Meteorites, p. 85-90. r888: C o h e n: São Julião, N. J. 1889 I, p. 221, 224, 225.

r888: W a r r e n : Detection and estimation of Selenium in Meteoric Iron. Chem. News Bd. 57 , p. 16 . Ref. N. J. 1890 II, p. 229.

I89r: v. H a u er: Ann. Hof-Mus, Bd, 6 (Not.), p. 55 (Tucker's Arizona).

1893: Brezina: Ueber neuere Meteoriten (Nürmberg), p. 163.

r894: Cohen: Meteoritenkunde, an vielen Stellen.

I894: F letcher: Introduction, p. 8, 20-21, 32 .

r895: Brezina: Wiener Sammlung, p. 264 (Tucker's Arizona).

r895: M eunier: Revision des lithosidérites, p. 14, 15, 17 (Abb. einer geätzten Platte und eines Dünnschliffs).

r895: C o he n: Meteoreisen-Studien IV. Ann. Hof-Mus. Bd. 10, p. 82 .

Ursprüngliches Gewicht: $687 \mathrm{Kgr}$. (1680 russ. Pfund) nach Pallas' Schätzung. Nach der Wägung von Hess im Jahre $18_{35}$ waren in der Petersburger Akademie noch $520 \mathrm{Kgr}$. (1270 russ. Pfund). 
Dasselbe Gewicht giebt Bloede i. J. 1848 an.

Nachweisbares Gewicht : 567995 gr.

\begin{tabular}{|c|c|c|}
\hline Aachen & Göttingen & 802 \\
\hline Bailey & Graz, J. & 1938 \\
\hline Bayet & Gregory & 298 \\
\hline Belgrad & Greifswald & $4^{8}$ \\
\hline Bement & Halle & 156 \\
\hline Berlin, G. & Hamburg & 270 \\
\hline Berlin, U. & Harvard, U. & 235 \\
\hline Bern, M. & Heidelberg & 17 \\
\hline Bologna & Helsingfors & 77 \\
\hline Bonn & Karlsruhe, M. & 106 \\
\hline v. Braun & Karlsruhe, P. & 66 \\
\hline Bremen & Kasan & 315 \\
\hline Breslau & Kiew & 442 \\
\hline Brezina & Klausenburg & 85 \\
\hline Brunner & Kopen] & 004 \\
\hline Budapest & Krantz & 18 \\
\hline Calcutta & Kristiania & 1074 \\
\hline Cambridge & Krüger & 57 \\
\hline Catania & Kunz & 76 \\
\hline Clausthal & Leiden & $49 \mathrm{I}$ \\
\hline Cleveland & Leipzig & 78 \\
\hline Danzig & v. Leuchtenberg & g 409 \\
\hline Darmstadt, M. I50 & Lissabon & $\left.501^{2}\right)$ \\
\hline Debreczin $\quad 45$ & London, B. M. & 3736 \\
\hline Dorpat & London, P. G. & II 28 \\
\hline Dresden, M. & Lund, M. & 106 \\
\hline Dublin, M. & Madras & 30 \\
\hline Dublin, R. C. & Madrid & 493 \\
\hline Edinburg & Marburg & 270 \\
\hline Frankfurt & de Mauroy & 7 \\
\hline Freiberg, i. S. $49 \mathrm{I}$ & Melion & 2 \\
\hline Freiberg, i. S. N. 32 & Minneapolis & I 44 \\
\hline Frenzel & Modena & 12 \\
\hline Gent & Moskau & 162 \\
\hline Giessen & München & 353 \\
\hline Gotha & Neapel & II5 \\
\hline
\end{tabular}

\begin{tabular}{lr|lr} 
Neumann & 52 & Seligmann & 37 \\
New Haven & $\left.1780^{9}\right)$ & v. Siemaschko & 220 \\
Newton & 16 & Stockholm & 710 \\
NewYork, M. & $\left.109^{1}\right)$ & Strassburg & 283 \\
Odessa : & 96 & Stuer & 12 \\
Oldenburg & $5^{8}$ & Stürtz & 150 \\
Oxford & 1714 & Stuttgart & 140 \\
Paris, E. & 505 & Troyes & 57 \\
Paris, M. & 1884 & Tübingen & 2012 \\
Paris, U. & 200 & Upsala & 1959 \\
Parma & 63 & Utrecht & 9 \\
Pech & 329 & Ward & 951 \\
Petersburg, A. & & Washington & 7 \\
& 520000 & Washington,Sh. 327 \\
Petersburg, B. & 794 & Wien, H.M.*) & $3644^{5}$ ) \\
Pohl & 173 & Wien, U. I. & $15^{2}$ \\
Prag, D. U. & 296 & Wien, U. II. & 224 \\
Riga & 32 & Wrany & 3 \\
Rom, U. & 48 & Zürich & 284 \\
\hline
\end{tabular}

Die Universität Basel besitzt ebenfalls ein Stäck von Krasnojarsk.

Krasnoj-Ugol, Gouv. Räsan, Russl. Stein, Cc, gefallen 9. Sept. 1829 . 1830: Pog gendorff unter Notizen. - 3. Meteorstein in Russland. Pogg. Ann. Bd. 17 , p. $379-380$; s. auch Leonhard's Jahrb. 1830 , p. 492.

1832: v. H off: Achter Nachtrag, Pogg. Ann. Bd. 24, p. 228--229; s. auch Preuss, Staatszeit. 1830 No. 97, p. 720. 1836: K ä m tz: Meteorologie, p. 297. 1837: Rose: Reise nach dem Ural. Bd. I, p. 76- 77 (erhält von Kupffer ein Stück für die Berliner Sammlung); s, auch Pogg. Ann. Bd, 54 (184r), p. 29 r. r843: Parts c h: Meteoriten, p. 45.

r847: Eichwald: Verzeichnis, Erman's Arch. f. wissensch. Kunde v. Russland Bd. 5, p. 179.

I) s, auch Leonhard's Jahrb. 1832 , p. 268 . - 2) Davon $168 \mathrm{gr}$, fraglich. - 3) Ausserdem $37 \mathrm{gr}$. >Tucker's Arizonaء. - 4) Hiervon $78 \mathrm{gr}$. Berg Emir, Siberia und $3^{1} \mathrm{gr}$. $\rightarrow$ Mount Kemis Siberias. Herr E. O. Hovey teilte mir noch brieflich mit: $>$ One specimen from, Mt. Kemis, Siberia " has exactly the characters of the well known Pallas iron and it is barely possible that the one labeled $\$$ Berg Emir, Siberiae is from the same falle. Auch ein kleines Bruchstück von $4 \frac{1}{2}$ gr. mit der Bezeichnung > Ensisheim, Elbogen, Bohemia dürfte hierher gehören. - 5) Ausserdem 5 gr. >Tucker's Arizona, « 
1848: B l o e d e: Tabelle,-Bull. Petersburg. Akad. Bd. 6, p. 10.

1854: v. Boguslawski: Zehnter Nachtrag, Pogg. Ann. Ergz.-Bd.4,P.436.

1859: v. Reichenbach: IX 16r, I7I, 180 .

1859: Harris: Dissert. Gött., p. 82.

I863: B u chner: Meteoriten, p. 53- 54 .

r863: Rose: Meteoriten, p. $85,86,87$,

$88,92,97$, Taf. IV, 98, I54, 16r.

1867: G o e b e 1: Kritische Uebersicht,

Mélanges phys. chim. Bd. 7 , p. 327 -329 .

1867: Buchner: Dritter Nachtrag,

Pogg. Ann. Bd. 132, p. 317.

1883/85: Tschermak: Photographien, p. 14.

1885: Brezina: Wiener Sammlung, p. $165,185,233$.

Ursprüngliches Gewicht: I oder 2 Steine.

Nachweisbares Gewicht: 2530 gr.

Berlin, U.

62 Tübingen

Calcutta

Spl. Washington

Petersburg, A, 2450 Wien, H, M.*)

\section{$\mathrm{Kr}$ asnoslobodsk}

Krawin

Nowo Urei

Tabor

Kuleschowka, Gouvernement Poltawa, Russland.

Stein, Cwa, gefallen r2. März r8r 1.')

I8rI: Gil bert: Ein neuer in Russ-

land am 13. März 18II herabgefallener

Meteorstein. Gilb. Ann. Bd. 38, p. 120 (Zeitungsberichte)

I8Ir: Scheerer: Darbringung eines Aërolithen von über 15 Pfund. Mém. Petersburg. Akad. Bd. 4 (hist.), p. 26. (Gedruckt $\mathrm{I}_{1} \mathrm{I}_{3}$ ).

1812: $\mathrm{C} \mathrm{h} 1$ a d n i: Verzeichnis,Schweigg. Journ. Bd. 4 Beil. I, p. 16 .

18r2: Bigot de Morogues, p.272. 1815: Chladni: Neues Verzeichnis, Gilb. Ann. Bd. 5o, p. 255 .

1) Um Mitternacht vom 12. zum 13. März.

2) Ausserdem 12 gr. *Gouv. Poltawa. r8rg: C h 1 a d n i: Feuermeteore, p. 66, $68,73,296(1)$.

1836: Kä m tz: Meteorologie, p. 283.

1843: Partsch: Meteoriten, p. 44, $54-55$.

1847: E i c hwa 1 d: Verzeichnis, Erman's Arch. f. wissensch. Kunde Russl. Bd. 5, p. $177-178,180$.

1848: B l o e d e: Tabelle, Bull. Petersburg. Akad., p. 2.

1854: v. Bog uslaw ski: Zehnter Nachtrag, Pogg. Ann. Ergz.-Bd. 4, p. 23.

1857: A rago: Astronomie populaire Bd. IV, p. 197.

1859: H a r r i s: Dissert. Gött., p. 70.

r860: v. Reichenbach: XIII 360 $-36 \mathrm{r}$.

1863: B u c hner: Meteoriten, p. 29. 1863: Rose: Meteoriten, p. 155.

r867: Buchner: Dritter Nachtrag,

Pogg. Ann. Bd. 132, p. 315.

1867: Go e bel: Kritische Uebersicht, Mélanges phys. chim. Bd. 7, p. 285 , 288,313 .

r884: M e uni e r: Météorites, p.208,211. r885: Brezina: Wiener Sammlung, p. $180,232$.

r895: Brezina: Wiener Sammlung, p. 243 .

Ursprüngliches Gewicht: Ueber $6 \mathrm{Kgr}$.

Nachweisbares Gewicht: 5219 gr.

Berlin, U.

Bologna

3 London, P. G. $\quad 75$

v. Braun

Budapest

Calcutta

Cambridge

Dorpat

Göttingen

Gregory

Harvard, U.

Kiew

Io Moskau

Spl. Neumann

39 Paris, M.

10 .

8o Petersburg, A. 4196

69 Petersburg, B. 2

139 v. Siemaschko 33

I Tübingen 32

95 Utrecht 2

5 Washington 5

$63^{2}$ ) Washington, Sh. 6

London, B. M. $\quad 5^{8}$ Wien, H. M.*) 194 


\section{Kurla \\ Kursk 1823 \\ Kursk 1874 \\ Pillistfer \\ Botschetschki \\ Sevrukof \\ Kusiali, Distr. Gurlwhal, Ostindien. Stein, $\mathrm{Cw}$, gefallen 16 . Juni 1860. \\ 1864: Buchner: Erster Nachtrag, Pogg. Ann. Bd. 122, p. 317. \\ 1864: Maskel yne u. v. Lang: Mi- neralogical Notes, - Notices of Aëro- lites von Maskelyne. - Kusiali,Kumaon. Philos. Magaz. Bd. 28, p. 148-149. Ref. Kenngott, Uebersicht $1862 / 65$, p. $442-443$. \\ 1869: B u c hner: Vierter Nachtrag, Pogg. Ann. Bd, 136, p. 454. \\ 1885: B r ez ina: Wiener Sammlung, p. $177,232$. \\ 1894: F let cher: Introduction, p. 10.}

Ursprüngliches Gewicht : ?

Nachweisbares Gewicht: $4 \mathrm{gr}$.

Calcutta Spl. (Wien, H. M.*) Spl.

London, B. M. $\quad 4$

\section{La Baffe}

Epinal

La Bécasse, Dep. Indre,Frankreich.

Stein, Cw, gefallen 31 . Januar 1879 .

1879: D a ubrée: Sur une météorite sporadosidère tombée le $3 \mathrm{I}$ Janvier 1879 à la Bécasse, commune de Dunle-Poëlier (Indre). C. R. Bd. $89 \mathrm{Nr}$. I4, p. 597. Ref. N. J. 188 o I, p. 178.

1884: Meunier: Météorites, p. 209, 225, 495-496.

1885: B rezina : Wiener Sammlung, p. $177,180,232$.

1890: B r e z i n a : Reise, Ann, Hof-Mus. Bd. 4 (Not.), p. II9.

Ursprüngliches Gewicht: Ein Stein von $2.8 \mathrm{Kgr}$.

Nachweisbares. Gewicht: 2785 gr.
v. Braun
5I Paris, M. 2580
Calcutta
IOI v. Siemaschko I
Gregory
London, B. M,
II Washington, Sh. 5
19 Wien, H. M.
17

La Bella Roca Bella Roca

Laborel, Dep. Drôme, Isère, Frankreich.

Stein, Ci (nach Cohen, Ccb nach Brezina), gefallen 14 . Juni ${ }^{1}$ ) 187 r.

r895: Brezina: Wiener Sammlung, p. 249 .

r896: C o h e n : Die Meteoriten von Laborel und Guareña. Ann. Hof-Mus. Bd. II, p. $3 \mathrm{r}-36$.

Ursprüngliches Gewicht: 2257 gr. Zwei Steine von 2166 u, 9I gr. Nachweisbares Gewicht: Nach den Mitteilungen des Herrn Prof. Kilian kamen der kleine Stein, sowie 102I gr. (Herr Dr. Krantz sagt: r160) von dem grösseren durch Kauf an Herrn Mineralienhändler Krantz. 840 gr. befinden sich in der Sammlung der Faculté des Sciences in Grenoble, 200 gr. giengen beim Durchsägen verloren, den Rest erhielt Prof. Cohen. Krantz besitzt zur Zeit (März × 896 ) noch das kleine, ganz umrindete Stück von 9r gr. u. 449 gr. von der grösseren Masse, von welcher Bruchstücke an: v. Braun, Berlin, M., London, B. M., de Mauroy, Paris, M., Wien, H. M. (an letzteres 2 gr.) gelangten.

La Caille, Dep. Var, (Alpes maritimes), Frankreich.

Eisen, Om, gefunden s vor Jahrhunderten, auch wird zuweilen

I) Nicht Juli, wie Brezina angiebt, sondern Juni (Abends $8 \mathrm{Uhr}$ ), wie Herr Professor Kilian auf meine Anfrage nochmals feststellte. 
sum 1600\& angegeben, erwähnt I 828 .

1828: B r a r d : s Minéralogies unter dem Artikel $\approx$ Fer $\propto$.

r829: Acad, Sc. Bordeaux (1829), p. 39. 1830: v. Hoff: Siebenter Nachtrag, Pogg. Ann. Bd. 18, p. 187-188.

r843: Part s c h: Meteoriten, p. Ir5.

1844: D uc de Luynes: Analyse du fer météorique de Grasse. Ann. des Mines (4) 5, p. 16I-164 ; s. auch Kenngott, Uebersicht $1844 / 49$, p. 226 ; Berzelius, Jahresber. Bd. 25 (1846), p. 399. Ref. N. J. 1845 , p. 324 ; »L'Institute Bd. I2 (1844), Nr. 566 , p. 360 .

I845: R a m mels ber g : Handwörterbuch Suppl. II, p. 90-9I.

1852: C 1 a r k: Dissert. Gött., p. 56.

1854: R i vot: Analyse du fer météorique du département du Var. Ann. des Mines (5) 6, p. $554-558$; s. auch Kenngott, Uebersicht 1855 , p. 94; Liebig-Kopp, Jahresber. 1855, p. IO24.

1854: v. Boguslawski: Zehnter Nachtrag, Pogg. Ann, Ergz,-Bd.4, p.39o. 1859: B u c hner: Feuermeteore, p.128. r859: H a rri s : Dissert. Gött., p. Iog. r860: $\mathrm{R}$ a m mels ber g : Mineralchemie, p. 908.

1863: Buchner: Meteoriten, p. 158. r863: Rose: Meteoriten, p. 65, r53; s. auch Mon.-Ber, Berlin, Akad, 1862, p. 555 .

I 858165 : v. Reiche n b a ch: IV 638. VII 55t. IX 162, 174, 175, 176, 182. XII 457. XV 100, Iо, III, I4, 124 . XVI 253, 254, 26r. XVII 266. XIX 155. $\mathrm{XX} \mathrm{621,} \mathrm{628,} \mathrm{629,} 630$.

1867: D a u br é e: Notesur deux grosses masses de fer météorique du Muséum et particulièrement sur celle de Charcas (Mexique), récemment parvenue à Paris. C. R. Bd. 64, p. $633-636,685-688$. Ref. Liebig-Kopp, Jahresber. 1879, p. Io44-IO45; Weinschenk, Ann. HofMus. Bd. 4 (1889), p. 100.

r869: F i z e a u: Tableau des dilatations par la chaleur de divers corps simples métalliques ou non métalliques, et de quelques composés hydrogénés du carbone. C. R. Bd. 68, p. 1130 .

r869: Me unier: Recherches, Ann. Chim. Phys. (4) I7, p. 24, 27, 29, 3I, $32,33,37,48,52,53,54,56,5^{8}$, $60,63,68,70,71,72$.

r869: B u chner: Vierter Nachtrag, Pogg. Ann. Bd, 136, p. 439, 598.

r870: R a m mels berg: Meteoriten, p. 81,84 .

r872: B o ussing a $1 \mathrm{t}$ : Recherche et dosage du carbone combiné dans le fer météorique, C. R. Bd. 74 , p. 1287 -1288 ; s. auch Ann. Chim. Phys. (4) 28 (1873), p. 124-126; Chem. News Nr. 688 , p. 59 ; Liebig-Kopp, Jahresber. 1872 , p. II95.

r880: Brez in a: Reichenbach'sche Lamellen, Denkschr. Wien. Akad. Bd. 43, p. 14 .

r88x: Brezina: Bericht III, Sitzber.

Wien. Akad. Bd. 84 I, p. 282.

r884: M eunier: Météorites, p. 21, 25, 33 (Abb.), 38, 39, 4I, 43 (Abb.), 44, $45,46,47,48,49,50,53,54,57$ (Abb.), 93, 94, 96, 98, п15, п16, п19 -I20, 352, 353, 356, 361, 373 .

r885: Brezina: Wiener Sammlung, p. 200, 20I, 213, 234 .

r886: Hunting t on : Crystalline Structure, Am. Journ. (3) 32, p. 285, 293, 294 (Abbildung einer geätzten Platte). 1887: F 1 ig ht: Meteorites, p. 105-106. r89o: Brezin a : Reise, Ann. Hof-Mus. Bd. 4 (Not.), p. ng.

r89r: Cohen u. Weinschenk: Meteoreisen-Studien. Ann. Hof-Mus. Bd. 6, p. $160,163,165$.

1892: Co h e n: Meteoreisen-Studien II. Ann. Hof-Mus, Bd, 7, p. 16 r.

r893: M e un i er : Revision des fers météoriques, p. 51, 52, 54-55 (Abb, einer geätzten Platte).

1894: C o hen: Meteoritenkunde, an vielen Stellen. 
Ursprüngliches Gewicht : Etwas mehr als $625 \mathrm{Kgr}$.

Nachweisbares Gewicht: 626896 gr.

\begin{tabular}{lr|lr} 
Bailey & 32 & London, P. G. & I4 \\
Berlin, U. & rO2 & Moskau & 53 \\
Bologna & Spl. & Paris, E. & 107 \\
v. Braun & 8 & Paris, M. & 625000 \\
Budapest & 73 & Pohl & 5 \\
Calcutta & 165 & v. Siemaschko & 66 \\
Cleveland & II & Stockholm & 75 \\
Dorpat & 51 & Troyes & 30 \\
Göttingen & 46 & Tübingen & 5 \\
Gregory & 33 & Washington, Sh. & I \\
Harvard, U. & 304 & Wien, H. M.*) & 340 \\
London, B. M. & 375 & \multicolumn{2}{|l}{}
\end{tabular}

La Charca bei Irapuato, Mexico. Stein, C, gefallen $x$. Juni $x 878$.

1884 : vo m R a t h: Verh, naturh. Ver. Bonn (Sitzber.) Bd. 41 (1884), p. 126.

Ref. N. J. 1885 II, p. 270.

r885: Brezin a : Wiener Sammlung, p. 260 .

1889: C a st i 11 o: $^{\circ}$ Catalogue, p. 13. 1890: Flet cher: Mexican Meteorites, Mineral. Magaz. Bd. 9, p. 95.

Ursprüngliches Gewicht: 399 gr. Nachweisbares Gewicht: 1/2 gr.

Kunz Spl, (1/2 gr.)

Den ganzen Stein besitzt nach vom Rath das Collegio zu Guanajuato in Mexico; nach Castillo soll Prof. S. Navia in Guanajuato eine Beschreibung dieses Steins veröffentlicht baben.

\section{La Encantada}

La Grange 1860

La Grange 1878

Imilac

La Grange, Oldham Co., Kentucky, U. S. A.

Eisen, Of, gefd, r860 (nach Rose I856), beschr, 186r.

186x: Smith: Two new Meteorites. Am. Journ. (2) 31, p. 151. Ref. Kenngott, Uebersicht 186t, p. 106. 186r: Smith: Description of three new Meteorites. - Lincoln County, Meteoric Stone which fell in August, 1855. - Oldham County (Ky.) Meteoric Iron. - Robertson County (Tenn.) Meteoric Iron. Am. Journ. (2) 3I, p. 265-266 (Analyse); Journ. prakt. Chem. Bd. 85 (1862), p. 515. Ref. N. J. 1861, p. 329 ; Original Researches 1884 , p. 427-428; Liebig-Kopp, Jahresber. 186I, p. II27.

I862: v. Reichenbach: XX 622. XXI 588 .

1863: Bu c hner: Meteoriten, p. 197.

r863: Ros e: Meteoriten, p. $65,153$. r869: Me unier: Recherches, Ann. Chim. Phys. (4) 17, p. 29, 35.

r870: $\mathrm{R}$ a m mel sberg: Meteoriten, p. $8 \mathrm{I}$.

1875: vom R a th: Meteoriten, Verh. naturh. Ver. Bonn Bd. 32 , p. 364 .

1884: M e un i er: Météorites, p. 47, 51, 129, 130 .

r885: B rezina: Wiener Sammlung, p. $208-209,233$.

r886: H unt ing t o n: Crystalline Structure, Am. Journ. (3) 32, p. 295.

r887: Brezina u. Cohen: Photographien, T. $20,2 \mathrm{I}$.

I890: E a s t m a n: Met. Astron., p. 320. r893: M e u n i er: Revision des fers météoriques, p. 37,38 .

1894: Cohen: Meteoritenkunde, p. 48, 191, 212.

r894: F let c her: Introduction, p. 33.

Ursprüngliches Gewicht: $5^{\mathrm{I}} \mathrm{Kgr}$. (I 12 lbs.).

Nachweisbares Gewicht: 6544 gr.

Bailey

Bement

Berlin, U. 46| Gregory

173

Bonn

Budapest

Calcutta

Cleveland

3 I Harvard, U. $\quad$ I96

Göttingen

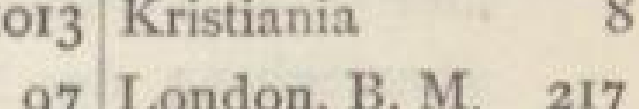

49 London, P. G. r9o

536 Madrid 108

40 Manchester $\quad 52$ 382 Moskau $\quad 7$ 


\section{New Haven \\ Paris, M. \\ 46 Tübingen \\ Rom, U. \\ v. Siemaschko \\ 370 Ward \\ Stockholm \\ 24 Washington \\ 8 Washington, Sh. \\ Strassburg 136 \\ 52 Wien, H. M. \\ 2002 \\ Wo ist die grösste Masse dieses Eisens, welches einst in L. Smith's Besitz war ? \\ L'Aigle, Normandie, Dep. de l'Orne, Frankreich.}

Stein, Cib, gefallen 26. April 1803 . Hierher auch: Waldau, Oberpfalz.

1803: B i o t: Relation d'un voyage fait dans le Département de l'Orne, pour constater la réalité d'un météore observé à l'Aigle le 6 floréal an Ir. Mém. de l'Institut Bd. 7, p. 224 (Situationsplan; 47 Seiten); s, auch Gilb. Ann. Bd, 16 , p. 44-71; „Encyclopédie * Bd. 5 (1808), p. $568-580,592$.

1803 : B i o $t$ : Vorläufige Nachricht von dem Steinregen zu l'Aigle am 26. April 1803. Gilb. Ann. Bd, 15, p. $74-76$; aus dem Bull. Sc. Soc. Philom. Nr. 77, p. 129; s, auch Tilloch's Philos. Magaz. I8O3, p. 224-228.

1803: Ch la d n i: ChronologischesVerzeichnis, Gilb. Ann. Bd. 15, p. 3 II.

r8o3: de Dré e: On the Stones said to have fallen at Ensisheim, in the neighbourhood of Agen, and at other Places. Tilloch's Philos. Magaz. Bd. 16, p. 296-297; s. auch Journ. Phys. Bd. 56 ( 1803$),$ p. 428 Sur les pierres tombées à Aigle, département de l'Orne «. 1803: Klaproth: Nachtrag zur Geschichte der Meteorsteine. Abh. Berlin. Akad. (1803), p. $42-43$.

1803: R itter: Einiges über Nordlichter und deren Periode, und über den $\mathrm{Zu}$ sammenhang des Nordlichts mit dem Magnetismus und des Magnetismus mit den Feuerkugeln, dem Blitze und der Elektrizität. Gilb. Ann. Bd. 15, p. 221. 1803: L a m bot in (auch Brief von Ma- rais): Sur les pierres tombées de l'atmosphère le 6 Floréal à Aigle, département de l'Orne. Journ. Phys. Bd. 56 (Juni 1803), p. 458; s. auch Gilb. Ann. Bd. 18 , p. $310-314$.

1803: S a ge: Examen comparé de la Pierre météorique d'Aigle et de celle de Villefranche. Journ. Phys. Bd. 57, p. 70 (Juli 1803 ); s. auch Gilb. Ann. Bd, 18, p. 314-316.

1804: Thénard: Mém. de l'Institut Bd. 7, p. 224; s, auch Gilb. Ann. Bd. 16, p. $44-71$ (7o!).

1804: Pötzsch: Kurze Darstellung, p. $88-92$.

r804: F o u r cr oy (Vauquelin): Sur les Pierres tombées de l'atmosphère et spécialement sur celles tombées auprès de l'Aigle le 6 floréal an II; lu à l'Institut le 28 fructidor an II. Ann. Mus. d'hist, nat. Bd, 3, p. $10 \mathrm{I}-107$, 110II2 ; s. auch Gilb. Ann. Bd. 18, p. 316 -32r; Journ. de Pharm. Bd. 56 (1803), p. 458 u. Bd. $57(1803)$, p. 70 ; $» \mathrm{En}-$ cyclopédiec Bd. 5 (1808), p. $561-563$. 1806: La u g i e r: Extrait d'un Mémoire sur l'Existence du chrôme dans les pierres météoriques, Ann. Mus, d'hist. nat. Bd. 7, p. 392-397; s. auch Gilb. Ann. Bd. 24, p. 380,383 .

1807: K laproth: Beiträge Bd, 4, p. 98 .

1808: de Dré e-Pictet: Description comparative etc. s. bei Weston. Bibl. Brit. Nr. 296 (April 1808), p. 283$284,287,288$.

r808: S a g e: Du procédé que j’ai employé pour déterminer l'existence de l'alumine dans les pierres météoriques. Journ. Phys. Bd. 66 (1808), p. 460 -462 .

1812: Ch 1 a d n i: Verzeichnis, Schweigg. Journ. Bd. 4 Beil. I, p. 15.

1812: Bigot de Morogues, p. 18 $-19,176-197,333$.

1815: Chladni: Neues Verzeichnis, Gilb. Ann. Bd. 50 , p. 252 ; s. auch 
Gilb. Ann. Bd. 47 , p. 96. r8rg: Chla dni: Vierte Fortsetzung, Gilb. Ann. Bd. 60, p. 239, 240. r819: $\mathrm{Ch}$ la dni: Fünfte Fortsetzung, Gilb. Ann. Bd. 63, p. 23.

r8I9: $\mathrm{Ch} 1$ a d n i : Feuermeteore, p. VII, $6,12,38,43,48,50,51,57,66,69$, 71, 73, 9I, 14I, 253, 254, 258, 260, $266,268,269-272(!), 273,275,293$, $302,305,428$.

1820: v. S c hreibers: Beiträge, p. 12 -I3 (mit Abbildung von einem der grössten Steine).

r82 I : John: Mémoire sur la Nature de grandes masses de fer métallique d'origine problématique, et sur celle du fer des aérolithes attirables par l'aimant. Ann. Chim. Phys, Bd, 18, p. 205. r822: $\mathrm{Chladni:}$ Zweiter Nachtrag, Gilb. Ann. Bd. 71, p. 367 .

1836: K ä m tz: Meteorologie, p.248, 257, 279-280.

1836: Gru it h u is e n: Naturgeschichte des gestirnten Himmels, p. 4I5-4I7. 1843: P a rt s ch: Meteoriten, p. 34-36. r847: Sch a fhäut 1: Schönenberg,

Gel. Anz. München. Akad, Bd. 24, Nr. 7I, p. 574 .

r848: B l o e d e: Tabelle, Bull. Petersburg. Akad. Bd. 6, p. II.

1854: B a 1 c e $11 \mathrm{~s}$ : Lithologia meteorica, p. 22 .

1859: H a r r is : Dissert. Gött., p. 65 . 1859: Kenngott: Mineralogische Mitteilungen. - II. Ueber Rutil, Granat und einen Meteorstein. Vierteljahrsschr. Naturforsch. Ges, Zürich Bd. 4, p. 303 -308 (Aufdeckung eines Betrugs, einen untergeschobenen $>$ Meteorstein vonWaldau, Oberpfalz« betreffend); s, auch Kenngott, Uebersicht $185^{8}$, p. 214 .

1859: B u c hner: Feuermeteore, p. 64 -67 .

1860: $\mathrm{H}$ a i d ing e $\mathrm{r}$ : Eine Leitform der Meteoriten. Sitzber. Wien. Akad. Bd. 40 , p. 532,533 .

r862: Kenngott u. Wiser: Zür- cher Sammlung, p. 152-153, I57. 1863: B u c hner: Meteoriten, p 15-17. r863: Ros e: Meteoriten, p. $90,93,98$, IOO, 155 .

1864: D a u bré e: Météorite offerte au Muséum par M. le Cte Charles de Saporta et paraissant être la principale masse tombée à l'Aigle (Orne), le 26 avril 1803 . Catalogue de Météorites du Muséum au 14 décembre 1864. C. R. Bd, 59, p. $1065-1067$; s. auch $>$ L'Institute Bd. 32 (1864), Nr. 1617, p. 409 u. Bd. 33 (1865), Nr. 1618, p. 4. 1864: Sorby: On the Microscopical Structure of Meteorites. Proc. Royal Soc. Bd. '3, p. 333-334. Ref. Am. Journ. (2) 4I (1866), p. 138.

r858/65: v. Reichen b a ch: V 479. VI $445,453,454$. VII 552,56 I. IX 155, 161, 170, 171, 179. X $359,363$. XI 294, 297, 302. XIII 362, 365. XV 101, 121, 131. XVI 262. XVII 269. XVIII 490. XXIV 226, 227. XXV 317 , $324,427,429,432,435,600,607,608$. r865: Buchner: Zweiter Nachtrag, Pogg. Ann. Bd. 124, p. $575-576$.

1867: Buchner: Dritter Nachtrag, Pogg. Ann. Bd. 132, p. 314.

r867: Go e be 1: Kritische Uebersicht, Mélanges phys. chim. Bd, 7, p. 28I283,330 .

r872: v. B a u $\mathrm{m}$ h a u er: Sur la météorite de l'Aigle. Arch. Néerl. Bd. 7, p. 154-160 (neue Analyse).

r875: M o hr: Entstehungsart, Ann. Chem. Pharm. Bd. 175, p. 259, 260. 1875: vom Rat h: Meteoriten, Verh. naturh. Ver. Bonn Bd. 32 , p. 367 .

1877: $\mathrm{G}$ ü m b e 1: Ueber die Beschaffenheit des Steinmeteoriten vom Fall am 12. Februar 1875 in der Grafschaft Iowa, N. A. Sitzber, München. Akad. Bd. 5 , p. 322 .

1878: G ü m b e 1: Ueber die in Bayern gefundenen Steinmeteoriten, Sitzber. München.Akad.(1878), p.39-40 (Sep.). 1882: Wie chmann: Fusion-Struc- 
tures in Meteorites. Ann. N. Y. Acad. Sc., p. 293, 295 (Taf. 20), 299-300. 1883: T sehermak: Beitrag, Sitzber. Wien. Akad. Bd. 88 I, p. 355 .

1884: Me unier: Météorites, p. 35, 93, $95,97,98,268,269,421-423$ (Sit.Plan), 446-449, 457 (Abb.), 459, 460, 46r, 469, 475 (Biot's Bericht). 1884: W a dsworth: Studies, p. 105. 1884: Wiepken: Notizen über die

Meteoriten des Grossherzoglichen Museums. Abh. naturw. Ver. Bremen Bd. 8 , p. $524-525$.

1883/85: Tschermak: Photographien, T. 9, p. 18 .

1885: Brezin a: Wiener Sammlung, p. $182,232$.

1887: Flight: Meteorites, p. 96-97. I888: N e w t o n: Orbits, Am. Journ. (3) 36 , p. 5 .

r889: F l et c h e r: Atacama Meteorites, Mineral. Magaz. Bd. 8, p. 226 (Streufeld 6 auf $2 \frac{1}{2}$ miles).

r89o: v. Niess 1: Periheldistanzen, Verh. naturf. Ver. Brünn Bd. 29, p. 188, 194, 214, 216, 248-250.

r89r: v. Ha ue r: Ann. Hof-Mus. Bd, 6 (Not.), p. 54.

r892: $\mathrm{P}$ f a h l e r : Ueber den Meteoriten von Barbotan 24. Juli 179o. Ueber den Meteoriten von l'Aigle 26. April 1803 . - Ueber den Meteoriten von l'Aigle. M. P. M. Bd. 13 , p. $362-372$ (Analyse u. Abbildungen von Dünnschliffen); s, auch Ann. Hof-Mus.Bd. 9 (Not.), p. 20. Ref. N. J. 1895 I, p. 276.

1894: Co h en : Meteoritenkunde,p.39,6r, $223,244,246,267,304$.

r894: F let c her: Introduction, p.8,25. Ursprüngliches Gewicht: Ein Steinschauer von 2000-3000 Steinen. Der grösste etwa $9 \mathrm{Kgr}$, wovon $6 \mathrm{Kgr}$. erhalten in Paris. Der nächstgrösste von $x^{1} / 2 \mathrm{Kgr}$.inWien.
Nachweisbares Gewicht: 36843 gr.

\begin{tabular}{lr|lr} 
Bailey & 195 & London, B. M. 2242 \\
Bayet & 7 & London, P. G. 898 \\
Belgrad & 28 & Lüttich & 127 \\
Bement & 94 & Madrid & 3 \\
Berlin, U. & 1920 & Marburg & 61 \\
Bern, M. & 103 & de Mauroy & 20 \\
Blatz & 10 & Melion & 2 \\
Bologna & 328 & Modena & $\left.73^{1}\right)$ \\
Bonn & 84 & Moskau & 89 \\
v. Braun & 425 & München & 129 \\
Breslau & 60 & Neapel & 62 \\
Budapest & 3479 & Neumann & 89 \\
Calcutta & 513 & NewHaven & 902 \\
Cambridge & 849 & Newton & 2 \\
Cleveland & 5 & Odessa & 42 \\
Debreczin & 207 & Oldenburg & 10 \\
Dorpat & 73 & Oxford & 117 \\
Dresden, M. & 235 & Paris, E. & 943 \\
Dublin, M. & 218 & Paris, M. & 8549 \\
Frankfurt & 110 & Paris, U. & $130^{2}$ ) \\
Freiberg, & 776 & Para & 44
\end{tabular}

- Freiberg, i. S. 776 Parma 44

- Freiberg, i.S. N. 73 Pech 51

Frenzel $\quad$ I04 4 Petersburg, A. I9

Gotha $\quad 44$ Petersburg, B. 400

Göttingen 241 Pierrotet 10

Graz, J. $\quad 676$ Pohl 305

Gregory $\quad 1364$ Prag, M. 160

$\begin{array}{llll}\text { Greifswald } & 3 & \text { Riga, P. } & 49\end{array}$

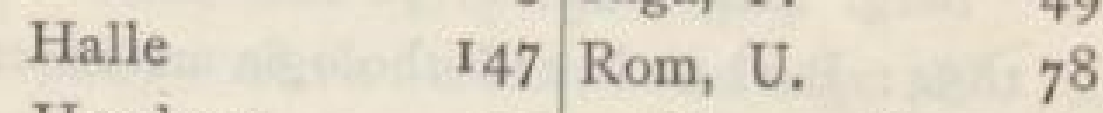

Hamburg $\quad 70$ v. Siemaschko $46 \mathrm{I}$

Harvard, U. $\quad 166$ Stockholm 639

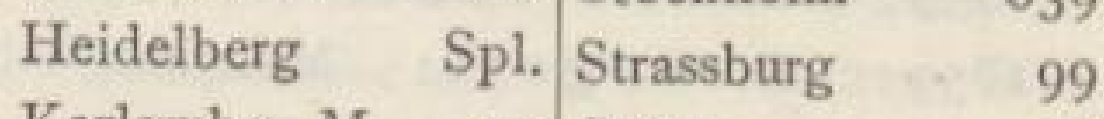

Karlsruhe, M. 44 Stürtz 38

Kasan $\quad 566$ Stuttgart $\quad 335$

\begin{tabular}{lr|lr} 
Kiew & 25 & Troyes & II4
\end{tabular}

\begin{tabular}{ll|l} 
Klausenburg $\quad 46$ & Tübingen $\quad 476$
\end{tabular}

Kopenhagen $\quad 256$ Turin, U. $\quad 17$

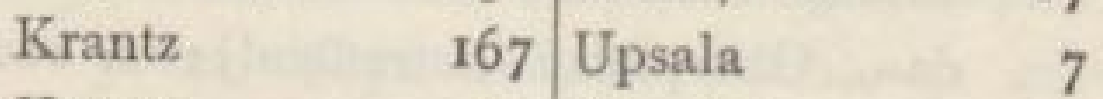

\begin{tabular}{ll|ll} 
Krüger & $7 \mathrm{I}$ & Utrecht & 89
\end{tabular}

\begin{tabular}{ll|ll} 
Kunz & 60 & Ward & 129
\end{tabular}

Lausanne $\quad 30$ Washington $\quad 56$

Leipzig $\quad \mathrm{II}_{3}$ Washington,Sh, II

\footnotetext{
1) >Fe meteorico $\alpha .-2$ ) Davon 32 gr. $>$ Waldau, Ht Palatinat, tombé dans la
} semaine avant Pentecôte année I809ء. 
Wien, H. M.*) 4054 Zürich

Wien, U. II. 124

Die Universität Basel besitzt ebenfalls ein Stück von L'Aigle.

\section{Laissac}

Favars

\section{Lake Superior}

s. Anhang Thunder Bay

\section{Lalitpur}

Iharaota

Lancé, Dep. Loir et Cher, Frankreich.

Stein, Cc, gefallen 23. Juli 1872 . Wird auch neuerdings mit Indarch zu einer Gruppe der kohligen Kügelchenchondrite $\mathrm{Kc}$ vereinigt.

1872: de Tastes: Chute d'un aérolithe dans la commune de Lancé, canton de Saint-Amand (Loir-et-Cher). C. R. Bd. 75, p. 273-276 (Situationsplan).

r872: D a ubré e: Note sur la découverte d'une seconde météorite tombée le 23 juillet 1872 , dans le canton de Saint-Amand (Loir-et-Cher). C. R, Bd. 75 , p. $308-309$.

1872: Da ubré e: Examen des météorites tombées le 23 juillet 1872 à Lance et à Authon (Loire-et-Cher). C. R. Bd. 75 . p. $465-468$ (Analyse). Ref. Liebig-Kopp, Jahresber.1872, p.II95-II96. 1872: J o 11 y: Manifestation, dans le département de la Vienne, du bolide qui a apporté, le 23 juillet 1872 , de météorites dans le canton de Saint-Amand (Loir-et-Cher). Extrait d'une lettre communiquée à M. Daubrée, par M. Jolly. C. R. Bd. 75, p. 505-506 nebst $\mathrm{Zu-}$ satz von Daubrée, p. 506.

1872: de Fleury: Note sur les Météores d'origine cosmique à propos de l'Aérolithe du 23 juillet 1872 , Blois bei P. Dufresne 1872 .

1873: Noue 1: Notices sur le bolide du 23 Juillet 1872 , qui a projeté des météorites dans le canton de SaintAmand, arrondissement de Vendôme, département de Loir-et-Cher. Bull. Soc. A rchéologique, Scientifique et Littéraire du Vendômois. Vendôme 1873 . Typographie Lemercier et fils. 56 Seiten. Abbildung.

r874: D a ubrée: Note additionelle sur la chute de météorites, qui a eu lieu le 23 Juillet 1872 , dans le canton de Saint-Amand (Loir-et-Cher). C. R. Bd. 79, p. 277. Ref. Liebig-Kopp, Jahresber. 1874 , p. 1344; "L'Institut * v. 5. Aug. 1874 ; La Nature Bd. II, p. 159 .

1875: v. D r a s c h e: Ueber den Meteoriten von Lancé. M. M. (I875), p. I-8 (zwei Abbildungen des Steins u. Abbildungen von Dünnschliffen). Ref. N. J. I875, p. 652 ; Liebig-Kopp, Jahresber. 1875, p. 1310; Am. Journ. (3) 1o (1875), p. 74 .

1875: Ts chermak: Vulkanismus, Sitzber. Wien. Akad. Bd. 7I II, p. 662. 1879: R a m mels ber g : Meteoriten, p. $12,24,26$.

1884: M e u nier: Météorites, p. 79, 89, $18_{3}, 185-187$ (Abb.), 52r.

1884: W a d s worth: Studies, p. 93. $-83 / 85:$ Ts c h erm a k: Photographien, T. $7,8,18$, p. $12,14,16$.

1885: Brezina: Wiener Sammlung, p. $185,233$.

1887: Flight: Meteorites, p. 54-58. r890: v. Niess 1: Periheldistanzen, Verh. naturf. Ver. Brünn Bd. 29, p. 188 , I95, 196, 214, 245-246.

1894: C o h e n: Meteoritenkunde, p.223, $236,237,261$.

1894: F let cher: Introduction, p. 3I. r895: Brezina : Wiener Sammlung, p. 254,255 .

Ursprüngliches Gewicht : $5^{\mathrm{I}^{3} / 4} \mathrm{Kgr}$. 6 Steine von $47 ; 3 ; 0.62 ; 0.60$; 0.30 u. $0.25 \mathrm{Kgr}$.

Nachweisbares Gewicht: $48883 \mathrm{gr}$.

\begin{tabular}{lr|lr} 
Budapest & 6 & Gregory & 4 \\
Calcutta & 58 & London, B. M. & 333
\end{tabular}


Lancé - Lenarto.

London, P. G. I2 Pohl de Mauroy

New Haven

Paris, E.

Paris, M.

2 v. Siemaschko

27 Stockholm

I466 Wien,H.M.**) 46915

Landes Dep.

Langres

La Primitiva

Laramie Co.

La Rochelle 1812

La Rochelle 1837

Lasdany

La Touanne

Barbotan

Chassigny

Primitiva

Silver Crown

Chantonnay

Esnandes

Lixna

Laurens Co., Süd-Carolina, U.S.A.

Auch Laurens Court House.

Eisen, Of, gefd. 1857 , beschr. 1886 .

r886: Hid d e n : On two masses of Me-

teoric Iron of unusual interest. - 2. The

Cuboidal Mass of Meteoric Iron, from

Laurens County, South Carolina. Am.

Journ. (3) $3 I$, p. $463-465$ (Ana-

lyse von Mackintosh, Abbildung der

Masse und einer geätzten Platte). Ref.

N. J. I888 I, p. 47 ; Liebig-Kopp, Jahresber. 1886, p. 2333.

1886: Brezina: Neue Meteoriten I Ann. Hof-Mus. Bd. I (Not.), p. I4.

1886: Brezina: Neue Meteoriten II Ann. Hof-Mus. Bd. I (Not.), p. 25. 1887: B r e z in a: Neue Meteoriten IIIa, Verh. k. k. geol.Reichsanst,(1887),p.288. 1887 : Brezina u. Cohen: Photographien, T. 24 .

r887: v. Hauer: Ann. Hof-Mus. Bd, 3 (Not.), p. 38 .

1890: E a s t m a n: Met. Astron., p. 318.

1893: B re zin a: Ueber neuere Meteoriten (Nürnberg), p. 163.

r894: C ohe n: Meteoritenkunde, p. 73, 106, 125, 229.

1895: Brezina: Wiener Sammlung, p. 269 .

I) Ausserdem 53 gr. nach I. Juli 1893 erworben.

2) Kürzlich gelangten noch weitere $96 \mathrm{gr}$. an Wien, H, M.
Ursprüngliches Gewicht: $2220 \mathrm{gr}$. (4 lbs. II oz.).

Nachweisbares Gewicht: I750 gr.

\begin{tabular}{lr|lr} 
Bement & 40 & Paris, M. & $3^{8}$ \\
v. Braun & 85 & v. Siemaschkor & 40 \\
London, B. M. & 63 & Wien, H. M. $\left.1484^{1}\right)$
\end{tabular}

La Vivionnère, Le Teilleul, Dep. Manche, Frankreich.

Stein, Ho, gefallen 14 . Juli 1845 .

1879: Da ubrée: Sur une météorite appartenant au groupe des eukrites, tombée le 14 juillet 1845 , dans la commune du Teilleul (Manche). C. R. Bd. 88 , p. $544-547$ (Analyse vonSorel). Ref. N. J. 1879, p. 905-906; LiebigKopp, Jahresber. 1879, p. 1277; Wochenschr. f. Astr., Met. u. Geogr. 1879, p. $16 \mathrm{I}-\mathrm{I} 62$.

1883: T s cherma k: Beitrag, Sitzber.

Wien. Akad. Bd. 88 I, p. 368.

1884: M e unier: Météorites, p. 286

(Abb.), 289-291 (Abb.), 524.

I884: W a d s wort h: Studies, p. 198. r883/85: Tscherma k: Photographien, p. 7,8 .

1885: Brezina: Wiener Sammlung, p. $174,232$.

1893: B r ez in a : Ueber neuere Meteoriten (Nürnberg), p. 159.

1894: C o h e n : Meteoritenkunde, p. 294, 306.

UrsprünglichesGewicht: Etwa 78ogr. Nachweisbares Gewicht: 779 gr.

Budapest

Gregory

I5 Paris, E.

287

London, B. M.

I Paris, M.

467

Lea Iron

2 Wien, H. M.

Leavenworth Co. Tonganoxi

Lee Co. s. Anhang Pilot Grove

Leland

Forest City

Lenarto, Saroser Com., Ungarn. 40

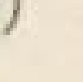


Eisen, Om, gefd. 1814 , beschr. 1815 . 1815: T e he 1: Auffinden einer neuen Masse Meteor-Eisen auf den Karpathen (Auszug eines Briefes Tehel's an Tihavsky, mitgeteilt von v. Jacquin). Gilb. Ann. Bd. 49 , p. $181-182$; s. auch $\gg$ Hesperus $\approx$ 6. Heft (18I5).

1815: $\mathrm{Schol} z$ : Ueber eine in Ungarn gefundene Gediegen-Eisenmasse, über Jodine und Platinaverbindungen (aus einem Schreiben des Herrn Scholz an Gehlen). Schweigg. Journ. Bd. 12, p. $347-348$.

1815: S e n nowitz: (Unter vermischte Nachrichten u. Anzeigen). - VI. Geognostische: v. Moll's Neue Jahrbücher der Berg- u. Hüttenk. Bd. 3 , p. 465 -468 .

1815: $\mathrm{Ch} 1 \mathrm{adn}$ i: Bemerkungen, Gilb. Ann. Bd. 5o, p. $272-273$.

1819: $\mathrm{Ch}$ ladni: Feuermeteore, p. 92, $318,319,329-33^{1}(!), 340,432$.

1820: v. S chreibers: Beiträge, p. 70,77 , T. 8 (Abb. einer geätzten Platte); s. auch Brezina, Meteoritenstudien II, Denkschr. Wien. Akad, Bd. 44 (I881), p. 133.

1822: Chladni: Zweiter Nachtrag, Gilb. Ann. Bd. 7I, p. 368-369.

1825: Chladni: Sechster Nachtrag, Pogg. Ann. Bd. 8, p. 52.

1830: v. Holger: Neue Analyse der beiden Meteoreisenmassen von Lénarto u. Agram, nebst einigen Bemerkungen über den Ursprung der Meteormassen überhaupt. Baumgartner's Zeitschr. f. Phys. u. Math. Bd. 7, p. 129-139. Ref. N. J. 1833, p. 192-193.

1832: Chladni: Achter Nachtrag, Pogg. Ann. Bd. 24, p. 230.

1835: We hrle: Analyse einiger Meteoreisenmassen. Baumgartner's Zeitschr. f. Phys, u. verw. Wissensch. Bd. 3, p. 226-227; s. auch Ann. Chem. Pharm. Bd. 14 (1835), p. 95.

r $836: \mathrm{Ka} \mathrm{a} \mathrm{tz} \mathrm{z:} \mathrm{Meteorologie,} \mathrm{p.} 260$. 1841: $R$ a m mels berg : Handwörter- buch Bd. I, p. 423 .

I843: P a r t s c h : Meteoriten, p.Io8-IIO. 1847: Sted ler: Ueber die in Ungarn herabgefallenen Meteorsteine. Oesterr. Bl. Lit, 1847 Nr. 86, p. 343 . Ref. N.J. 1848, p. 65 .

1852: Wöhler: "Aktiv«, Pogg. Ann.

Bd. 85, p. 448,449 .

1852: C l a r k: Dissert. Gött., p. 39-42

(Analyse); s. auch Ann. Chem. Pharm. Bd. 82 (1858), p. 368 ; Journ. prakt. Chem. Bd. $5^{8}$ (1853), p. 55 ; Kenngott, Uebersicht 1852, p. 91. Ref. N. J. 1853, p. 186.

1854: v. Boguslawski: Zehnter Nachtrag, Pogg. Ann. Ergz.-Bd. 4, p. 39I-392, 455 .

r855: $\mathrm{H}$ a i d ing e r: Bemerkungen über die zuweilen im geschmeidigen Eisen entstandene krystallinische Structur, verglichen mit jener des Meteoreisens. Sitzber. Wien. Akad. Bd. 15 I, p. 357 (Fig. 12).

1859: $\mathrm{B} \mathrm{u} \mathrm{c} \mathrm{h} \mathrm{n} \mathrm{er:} \mathrm{Feuermeteore,} \mathrm{p.} 123$. 1860: R a m mels berg: Mineralchemie, p. 907.

r86r: Boussing a ult: Sur la présence de l'azote dans un fer météorique. C. R. Bd. 53 , p. $77-79$; s. auch "L'Instituta 1861, p. 24I; Dingl. pol. J. Bd. I6I, p. 396; Ann. Chim. Phys. (3) 63 (186r), p. 336-343; Chem. Centr. 1861, p. 768; Pogg. Ann. Bd. 114, p. 336. Ref. N. J. 1862, p. 600 ; Kenngott, Uebersicht 1861, p. 106; Liebig-Kopp, Jahresber, 1861, p. II32 ; Journ. prakt. Chem. Bd. 85 (1862), p. $461-462$.

1858/62: v. Reichen bach: IV 638 . VI $448,449,452$. VII 55 I. IX 163 , 174, 182 X 359 . XI 29I. XII 456 . XIII 363. XV 100, nо, III, II4, 124. XVI 261, 262. XVII 266, 271. XVIII $480,484,485,487,489,490$. XIX 155. XX 621, 629, 630. XXI 578. 1863: B u chner: Meteoriten, p. 152 -153 . 
I863: Rose: Meteoriten, p. 54, 64, 138, 153 .

1867: Go e bel: Kritische Uebersicht, Mélanges phys. Bd. 7, p. 312.

r867: Grah a m: On the Occlusion of Hydrogen Gas by Meteoric Iron. Proc. Royal Soc. Bd. 15, p. 502-503; s. auch: Sur l'occlusion du gaz hydrogène par le fer météorique. C. R. Bd. 64 (1867), p. 1067-1069; Pogg. Ann. Bd. 131 (1867), p. 151-153; Am. Journ. (2) 44 (1867), p. 109; Chen. News Bd, 15, p. 273 (May 3I, 1867); Bull. Soc. chim. (2) 8, p. I64; Journ. prakt. Chem. Bd. 102, p.191-I92; Chem. Centr. 1867, p. 973 ; Liebig-Kopp, Jahresber. 1867, p. I045-IO46; »L'Institut « Bd. 36 (1868), p. $46-47$.

r869: Buchner: Vierter Nachtrag, Pogg. Ann. Bd, 136, p. 598.

r87o: R a m mels berg: Meteoriten, p. 8 o, 84 .

1871/72: M a $11 \mathrm{et}$ : Examination of the Gases occluded in Meteoric Iron from Augusta Co., Virginia. Proc. Royal Soc. Bd. 20, p. 368,369 (Vergleich mit Lenarto); s. auch Am. Journ. (3) ro, p. 206; Philos. Magaz. Bd. 44 (1872), p. 314, 315; Pogg. Ann. Bd. I47 ( 1872$)$, p. $138,139$.

1872: Salet: sDie im Meteoreisen von Lenarto eingeschlossenen Gase bestehen aus $\mathrm{H}$ u. $\mathrm{CO}, \mathrm{Hr}$. Salet hat keinen Stickstoff darin gefunden, « Ber. d. d. chem. Ges. Bd. 5, p. 222; s, auch Revue Scientifique vom 9. März 1872 ; The Academy\& Bd. 3, p. 113.

1872: B oussing a $1 \mathrm{t}$ : Recherche et dosage du carbone combiné dans le fer météorique. C. R. Bd. 74, p. 1288 -1289 (Analyse); s. auch Ann. Chim. Phys, (4) 28 (1873), p. 126-127; Chem. News Nr. 688 , p. 59 ; Liebig-Kopp, Jahresber. 1872 , p. 1195 .

1875: vom R a th: Meteoriten, Verh. naturh. Ver. Bonn Bd. 32, p. 36r. 1876: Wright: On the Gases con- tained in Meteorites. Am, Journ. (3) I2, p. 167 (Graham).

r879: W ö h l e r: Briefl. Mitt. N. J. 1879. p. 370 .

r879: Kl e in : Göttinger Sammlung, Gött. Gel. Anz., p. 99-I00 (Polen). 1880: B r ez in a: Reichenbach'sche Lamellen, Denkschr. Wien. Akad. Bd. 43 , p. $13,14$.

1880: K l e in : Vermehrung, Gött. Gel. Anz., p. 568.

1884: M eunier: Météorites, p. II, I2 $-13,17,18,25,55,96,136,322$.

1885: Brezina: Wiener Sammlung, p. 200, 210-2II, 233.

1885: Willi a ms: Note on the occlusion of hydrogen by zinc dust and the meteoric iron of Lenarto. Chem. News Bd. 51, p. $146-147$ u Bd. 52, p. 205 -207 .

1887: F lig ht: Meteorites, p. 104-I05. r8gr: Cohen u. Weinschenk:

Meteoreisen-Studien. Ann. Hof-Mus. Bd. 6, p. $13 \mathrm{I}$.

1893: Me unier: Revision des fers météoriques, p. $62-63$ (Abb. einer geätzten Platte).

I894: C o h e n: Meteoritenkunde, p. 4I, $54,56,62,67,149,170,171,189,193$. I895: Co he n: Meteoreisen-Studien IV. Ann. Hof-Mus. Bd. 10, p. $83,84,85$.

UrsprünglichesGewicht: EineMasse von ro8.6 Kgr. (r94Wiener Pfund).

Nachweisbares Gewicht: 9I 595 gr.

\begin{tabular}{lr|lr} 
Bailey & 54 & Calcutta & 377 \\
Bayet & 37 & Cambridge & 120 \\
Belgrad & 40 & Debreczin & 166 \\
Bement & 175 & Dorpat & 28 \\
Berlin, G. & 363 & Dresden, M. & 35 \\
Berlin, U. & 532 & Eger & 26 \\
Bern, M. & 13 & Gotha & 81 \\
Bologna & 96 & Göttingen & 102 \\
Bonn & 81 & Graz, J. & 57 \\
v. Braun & 61 & Gregory & 116 \\
Breslau & 60 & Greifswald & $5 p 1$. \\
Budapest & 76649 & Halle & 63 \\
& & &
\end{tabular}




\begin{tabular}{|c|c|c|}
\hline Hamburg & $7 \mathrm{I}$ & v. Schilling \\
\hline Harvard, U. & II9 & v. Siemaschko \\
\hline Klausenburg & 223 & Stockholm \\
\hline Kopenhagen & 16 & Strassburg \\
\hline London, B. M. & 2028 & Stürtz \\
\hline London, P. G. & 569 & Troyes \\
\hline Madras & 35 & Tübingen \\
\hline Marburg & 553 & Turin, U. \\
\hline de Mauroy & 10 & Upsala \\
\hline Moskau & 83 & Utrecht \\
\hline Neumann & Io & Ward \\
\hline New Haven & 120 & Washington, Sh. I7 \\
\hline Oxford & 205 & Wien, H. M.*) 324 \\
\hline Paris, E. & 186 & Wien, U. I. 8 \\
\hline Paris, M. & $18_{3}$ & Wien, U. II. \\
\hline Pohl & 445 & Zürich \\
\hline Rom, U. & 77 & \\
\hline
\end{tabular}

Le Pressoir, Louans, Dep. Indre et Loir, Frankreich.

Stein, Cc, gefallen 25 . Januar 1845 . 1881: D a u brée: Météorite tombée à Louans (Indre-et-Loir) le 25 janvier 1845 et dont la chute est restée inédite. C. R. Bd. 92 , p. $984-985$. Ref. N. J. 1882 II, p. 30 ; Liebig-Kopp, Jahresber. $188 \mathrm{I}$, p. 1457.

r884: M eunier: Météorites, p. 230, 487 .

r885: Brezina: Wiener Sammlung, p. 185,232 .

r887 : v. Ha uer: Ann. Hof-Mus, Bd. 2 (Not.), p. 39.

1895: Brez ina: Wiener Sammlung, p. 247,255 .

Ursprüngliches Gewicht: Von einem etwa ${ }_{3} \mathrm{Kgr}$. schweren Stein dürften I133 gr. erhalten sein.

Nachweisbares Gewicht: 376 gr. Bailey

Bement

Böhm

Bologna

Budapest

Cleveland

Harvard, U.

de Mauroy
32 New Haven 10

4 Newton

6 Paris, M.

58 Rom, U.

76 v. Siemaschko

51 Washington,Sh.

3 Wien, H. M. 15
Wo ist der Rest des Stïckes, welches Daubrée der Akademie 188 I vorzeigte?

Les Ormes, Dep.Yonne,Frankreich. Stein, $\mathrm{Cw}$, gefallen $\mathrm{I}$. Oktober 1857 . 1857: S éguier: "L'Institute Bd. 25 (1857), p. 363. Ref. Kopp u. Will, Jahresber. 1857, p. 731; Kenngott, Uebersicht 1858 , p. 167 ; Wochenschr. f. Astr., Met. u. Geogr. 1857, p. 387 -388 .

1862: B u chner: Die Meteoriten in Wien u. London, Pogg. Ann. Bd. 116 , p. 643 .

1863: B u c hner: Meteoriten, p. 96.

1864: Buchner: Erster Nachtrag,

Pogg. Ann. Bd. 122, p. 317, 321.

1884: M e u ni e r: Météorites,p.209,220. 1885: Brezina: Wiener Sammlung, p. 261.

1886: v. H a u e r: Ann. Hof-Mus. Bd. 2

(Not.), p. 39.

r892: M e uni e r: Examen minéralogique de deux Météorites Bourguignonnes. Luponnas, Ain, 8 Sept. 1753 , et Les Ormes, Yonne, 4 Octobre 1857. Bull. Soc. Hist. Nat. Autun Bd. 5 (1892), (30 Seiten, 2 Tafeln).

1895: Brezina: Wiener Sammlung, p. 242.

Ursprüngliches Gewicht: Ein Stein, von welchem $125 \mathrm{gr}$. an die franz. Akademie gelangten.

Nachweisbares Gewicht: Ir3 gr.

Budapest

2 v. Siemaschko 2

Calcutta Spl. Washington, Sh. I

London, B. M. I2 Wien, H. M. 2 Paris, M. 94

\section{Le Teilleul La Vivionnère}

Lexington Co. 1844 Ruffs Mountain Lexington Co. $188 \circ$ Lexington Co. Lexington Co., Süd-Carolina,U.S.A. Eisen, $\mathrm{Og}$, gefunden u. erwähnt 1880, beschr. 1881.

r880 : K l e in : Vermehrung, Gött. Gel. Anz., p. 569 . 
1881: S hep a r d : On the Meteoric Iron of Lexington County, South Carolina. Am. Journ. (3) 2I, p. II7-II9 (Analyse). Ref. N. J. 188I II, p. $343-344$; Zeitschr. f. Kryst. Bd. 5 (188I), p. 516; Liebig-Kopp, Jahresber. I88I, p. I457.

1885: Brezina: Wiener Sammlung, p. $26 \mathrm{r}$.

1887: F 1 ig h t: Meteorites, p.215-2I6. r89o: Eastm a n : Met. Astron, p. 318, 320.

1893: M e u n i er : Revision des fers météoriques, p. $25,27$.

1894: C o he n: Meteoritenkunde, p. 57, $68,199,24 \mathrm{I}, 242$.

1895: Brez ina: Wiener Sammlung, p. 286.

Ursprüngliches Gewicht: $4 \frac{3}{4}$ Kgr. (10 $1 / 2$ lbs.).

Nachweisbares Gewicht: 5053 gr. $\left.{ }^{1}\right)$

Bailey

Bement

v. Braun

Budapest

Cleveland

Göttingen

Gregory

London, B. M.

19 Minneapolis 17

206 New Haven 49

I Paris, M.

17 v. Siemaschko 20

9 Ward 226

20 Washington 65

76 Washington,Sh.3992

Liberty 1875

Liboschitz

Lick Creek, Davidson Cor,

Carolina, U. S. A.

Eisen, H, gefd. 1879 , beschr. 1880 . r880: $\mathrm{H}$ i d d e n : A New Meteoric Iron from North Carolina. Am. Journ. (3) 20, p. 324-326 (Abbildung des ganzen Eisens u. der Rinde. Analyse von Mackintosh u. Smith); s. auch > Illustrated Scientific News. . NewYork, 15. März I880 Bd. III, Nr. 6, p. 62-66. Ref. N. J. I88I II, p. I80; LiebigKopp, Jahresber, 188o, p. I542. r88I : Brezina: Bericht III, Sitzber.
Wien. Akad. Bd. 84 I, p. $280-28$ r. 1884: M e un i er: Météorites, p. II2.

1885: Brezina: Wiener Sammlung, p. $155,218,234$.

I887: F lig h t: Meteorites, p. 2II-2I2. r889: C o h e n : São Julião, N. J. r889

I, p. 216,217 .

r89o: E a s t m a n: Met. Astron., p. 320. r89r: Cohen u. Weinschenk: Meteoreisen-Studien. Ann. Hof-Mus. Bd. 6, p. 159.

r893: M e un i e r : Revision des fers météoriques, p. $15,17$.

r894: C o he n : Meteoritenkunde, p. 58, 70, 96, 126, 191, 212, 242.

r895: Brezina: Wiener Sammlung, p. 291.

r895: Cohe n: Meteoreisen-Studien IV. Ann. Hof-Mus. Bd. Io, p. $83,85,88,89$. Ursprüngliches Gewicht: $1.24 \mathrm{Kgr}$. Nachweisbares Gewicht: I230 gr. Bailey

Bement

Budapest

Greifswald

Harvard, U.

London, B. M. $3^{8}$ Paris, M. 15 v. Siemaschko 25 40 Stürtz I2 28 Washington, Sh. Io 6 Wien, H. M. 995

Lime Creek, Claiborne, Clarke Co., Alabama, U. S. A.

Eisen, H, gefd. $\mathrm{I}_{34}$ (nach Harris I833), beschr. $18{ }_{3} 8$.

Hierher auch vorläufig Walker Co., s. Anhang dieses Artikels.

1838: Jack son: Chemical Analysis of Meteoric Iron, from Claiborne, Clarke Co., Alabama. Am. Journ. (I) 34 , p. $332-337$; s. auch Philos. Magaz. Bd. 13 (1838), p. 350-353; Rep. Brit. Assoc. 1839 (Abstracts etc.), p. 54; Am. Journ. (I) 43 (1842), p. 169, 359; Pogg. Ann. Ergz.-Bd. I (1842), p. 371 -372; Journ. prakt. Chem. Bd, 16 (1839), p. 239-243; Berzelius, Jahres-

1) Sollte unter diesem nachweisbaren Gewichte vielleicht Ruffs Mountain Lexington 
ber. Bd. 20 (r84r), p. $255-256$ u. Bd. 23 (1844), p. 296; N. J. 1839 , p. 187 ; wiederholt N. J. $184 \mathrm{I}$, p. 464 ; *L'Institut \& Bd. 7, Nr. 268 (1839), p.48.

r84r : $\mathrm{R}$ a m mels berg: Handwörterbuch Bd. I, p. 423 .

r843: P a rt s c h: Meteoriten,p.r33-134. 1843: $\mathrm{R}$ a m mel s b erg : Handwörterbuch Suppl. 3, p. 8r.

r845: Jackson: I. Remarks on the Alabama Meteoric Iron, with a Chemical Analysis of the drops of Green Liquid which exude from it. 2. Letter from Mr. A. A. Hayes on the same subject, with remarks on the origin of the Chlorine found in the Alabama Iron, and a description of new methods employed in the Analysis of Meteoric Irons. Am. Journ. (I) 48, p. 145-156 (Analyse von Hayes); s. auch sL'Institut « Nr. 606, p. 290; Berzelius, Jahresber. Bd, 26 (1847), p. $3^{8} 7-3^{88}$; Kenngott, Uebersicht $1844 \mid 49$, p. 224. r847: $\mathrm{S}$ h e p a rd: Report on Meteorites. Am. Journ, (2) 4, p. 77.

r852 : C 1 a r k : Dissert. Gött., p. 57-58. 1854: v. Boguslawski: Zehnter Nachtrag, Pogg. Ann. Ergz.-Bd. 4, p. $400-401$,

1859: Harris: Dissert. Gött., p. III. 1860: $\mathrm{R}$ a m mels berg: Mineralchemie, p. $917-918$.

r85862: v. Rei $\mathrm{ch}$ e $\mathrm{n} \mathrm{b} \mathrm{ach}:$ IV 638. VI 448 . VII 551. VIII 488. IX 162, 175, 176, 182. X 359, 363. XII 457, 458. XIV 390. XV 100, II9, 122, 128, 13I. XVI 262. XVIII 479, 48I, $483,489,490$. XIX 150, 151, 156. XX 621, 622, 629, 630. XXI $578,5^{84}, 588,589,590$. r863: B u c h n e r: Meteoriten,p.16r-162. 1863: R os e: Meteoriten, p. 49, 152.

1880: Brez ina: Reichenbach'sche Lamellen, Denkschr. Wien. Akad. Bd. 43 , p. 14 (bezieht sich wohl auf Walker Co.).

1884: Me unier: Météorites, p. 35, 94,134 .
1885: Brezina: Wiener Sammlung, p. 218,234 .

r89o: Eas t m a n : Met. Astron., p. 318. r89r: Cohen u. We inschenk: Meteoreisen-Studien. Ann. Hof-Mus. Bd. 6, p. 160 .

1893: M e unier: Revision des fers météoriques, p. I5, 19.

r894: C o h en: Meteoreisen-Studien III. Ann. Hof-Mus. Bd. 9, p. I14-116, II7, II8 (Rhabdit).

1894: C o hen: Meteoritenkunde, an sehr vielen Stellen.

r894: C o h en: Meteoreisen-Studien IV. Ann. Hof-Mus. Bd, 10, p. 82, 87, 88, 89 .

Ursprüngliches Gewicht: Das von Jackson beschriebene Eisen stammte von einem ro Zoll langen und 5-6 Zoll dicken Stück. Der Finder (Hubard) berichtet aber, sthat there are many other similar masses near the spot where this was founds. Es scheint, da die unter diesem Namen nachweisbaren Eisen die erste Masse von Jackson weit übertreffen, dass von jenen Hubard'schen Stücken noch nachträglich gesammelt worden ist. Reichenbach's Angabe, Pogg. Ann. Bd. 114, p. I I9 spricht dafür,dass jenes vonLondon Walker County « genannte Eisen und das von Lime Creek dem gleichen Fundort angehören. Reichenbach sagt: „Die eine Hälfte davon (nämlich von Claiborne) befindet sich im britischen Museum, die andere, etwa ein Zentner schwer, fast in Halbkugelgestalt ist in meiner Sammlung «. Das Claiborne Reichenbachs ist aber Lime Creek, denn er führt in seiner Sammlung nur ein Claiborne und bezieht auf dies die Hayes'sche 
Analyse, die zu Lime Creek gehört. Nun besitzt London 65 gr. Claiborne u. 22295 gr. Walker Co.; beide sind nach Brezina hexaedrische Eisen (früher wurde Walker Co. als dichtes Eisen aufgefasst).

Nachweisbares Gewicht: 40888 gr.

Bailey

Berlin, U.

Calcutta

Cleveland

Cohen

Göttingen

Gregory

7 Neumann 20

157 New Haven 64

4 Paris, M. I3

4 Pohl

I6 Stockholm

2 Strassburg

I I Tübingen

62

London, B. M.

London, P. G.

35

Lime Creek - Walker Co., Alabama, U. S. A.

Eisen, H, gefd. 1832 , beschr. 1845 .

Hierher auch: Morgan Co., Alabama, U.S.A., bekannt 1849 (welches wohl pseudometeorisch ist).

r845: Troost : Description.... - 4 . Of a mass discovered in Walker County, Alabama. Am. Journ. (I) 49, p. 344 -346 u. Am. Journ. (2) 5, p. $35^{1}$ (beiläufige Erwähnung).

I846: S h e p a r d: Report on Meteorites. Am. Journ. (2) 2, p. 382, 391.

1847: S h e p a r d: Report on Meteorites.

- Walker county, Alabama. Am. Journ.

(2) 4, p. 74-75 (Abbildung und Analyse); s. auch Liebig-Kopp, Jahresber. $1847 / 48$, p. 1308 ; Kenngott, Uebersicht $1850 / 51$, p. 135.

1852 : C l a r k: Dissert. Gött., p. 74.

1854: v.B o g u s l a w ski: ZehnterNach-

trag, Pogg. Ann. Ergz.-Bd. 4, p. 399.

1859: $\mathrm{B}$ u c h n e r: Feuermeteore, P. I32.

r859: H a rris : Dissert.Gött.,p.Ho-III.

I86I: v. Reichenbach: XV II9.

1863: B u chner: Meteoriten, p. 172 $-173,181$.

I) $>$ Walker County, or Morgan County $1832 \mathrm{e}$.
1863: R o s e: Meteoriten, p: 26.

r880: Brez in a: Reichenbach'sche Lamellen, Denkschr. Wien. Akad. Bd. 43 , p. 14; s, auch das Zitat bei Lime Creek. r884: M e un i er: Météorites, p.rr6,I2O. 1885: Brezina: Wiener Sammlung, p. $218,221,234$.

1886: H un t ing t o n : Crystalline Structure, Am. Journ. (3) 32, p. 295.

1890: Eas tman: Met. Astron., p. 318. r8gr: Cohen u. Weinschenk: Meteoreisen-Studien. Ann. Hof-Mus. Bd. 6, p. 160 (Morgan Co.).

1893: B rez in a: Ueber neuere Meteoriten (Nürnberg), p. 164.

1893: M e un i er: Revision des fers météoriques, p. 72.

1894: C o h e n : Meteoritenkunde, p. 52, 231, 232.

r895: Brezina: Wiener Sammlung, p. 290.

Ursprüngliches Gewicht: $74^{1 / 2} \mathrm{Kgr}$. (16 6 lbs.).

Nachweisbares Gewicht: $2573^{8}$ gr.

Bailey

Bement

4I London, B.M. 22295

Berlin, U.

v. Braun

6 London, P. G. 27

Budapest

Calcutta

Cambridge

Cleveland

Gregory

Harvard, U.

Heidelberg

I 46 New Haven 357

23 Newton 21

33 Paris, M. 62

12 $\left.{ }^{1}\right)$ Pohl $\quad 36$

$\left.6^{2}\right)$ v. Siemaschko 26

30 Stockholm 80

29 Ward 147

Ausserdem Morgan Co: Baitey 7 gr.; Tübingen 5 gr.; Wien, H. M. 65 gr. Die beiden letzteren sind wahrscheinlich Pseudometeoriten.

Limerick, Adare, Irland, Grossbritannien.

Stein, Cga (Bestimmung nach dem Tübinger Stück), gefallen 10. Sept. $\mathbf{r}^{8} \mathrm{r}_{3}$.

2) >Alabama probably Walker Co.\& 
I813: Gentleman's Magazine (1813), p. 390. 1814: T e nnant: Journ. Pharm. Sept. I8I4, p. 211 ; s. auch Journ. Phys. Bd. 79 (1814), p. 2II; Gilb. Ann. Bd. 49 (1815), p. 180.

1815: Chladni: Neues Verzeichnis, Gilb. Ann. Bd. 50, p. 255.

18r6: $\mathrm{Ch} 1 \mathrm{adni}$ : Zweite Fortsetzung, Gilb. Ann. Bd. 54, p. 355 .

1818: Higgins: Account of a Shower of Meteoric Stones which fell in the County of Limerick. Philos. Magaz. Bd. 5I, p. $355-356$ (Brief Maxwell's); s, auch Uebersetzung in Gilb. Ann. Bd. 6o, p. $233-237$.

Die Bibliothek des k. k. Hof-Museum's Wien enthält einen Ausschnitt mit Angaben Maxwell's iber Limerick: Analecta August 1818 Bd. 3, Nr. 4, p. 313. Aus welcher Zeitschrift ist dieser Ausschnitt?

18r9: $\mathrm{Chl}$ a d ni: Vierte Fortsetzung, Gilb. Ann, Bd. 6o, p. 248-249.

1819: $\mathrm{Ch} 1$ a d n i: Fünfte Fortsetzung, Gilb. Ann. Bd. 63, p. 23.

r8rg: $\mathrm{C}$ h 1 a d n i : Feuermeteore, p. 66, $69,70,72,73,293,303-304(1), 431$. r836: K ä m tz: Meteorologie, p. 285.

1837: A pjohn: Analysis of a Meteoric Stone which fell near Adair, in the County of Limerick on September ro, 1813. Trans. Irish Acad. Bd, 18 (1837), p. $17-30 ;$ s. auch Philos. Magaz. Bd. 23 (1862), p. $49-50$. Ref. Kenngott, Uebersicht $1862 / 65$, p. $441-442$; Liebig-Kopp, Jahresber. 1862, p. 828 . r843: Partsch: Meteoriten, p.67-68. 1859: Harris: Dissert. Gött., p. 71. r863: B u c hner: Meteoriten, p. 33. 1863: R o s e : Meteoriten, p. 154. r858/65: v. Reichen ba c b: V 480. VI 454. IX 161, 170, 179. XI 294, 302. XXIV $227,228 . X X V_{318}, 324$ $427,429,433,600,607,608$.

1869: B u chner: Vierter Nachtrag, Pogg. Ann. Bd. 136, p. 442.

1874: Apjohn: On the Analysis of a Meteoric Stone and the Detection of Vanadium in it. Journ. Chem. Soc. (2) 12, p. 104-106 (Analyse). Ref. Liebig-Kopp, Jahresber. 1874, p. I340, 1345 .

1879: Rammels berg: Meteoriten, p. Io, 26.

1884: M e un i er: Météorites, p. 227, 228-229.

1885: $\mathrm{Brez}$ in a : Wiener Sammlung, p. $182,233$.

r887: F lig ht: Meteorites, p.103-IO4. r889: F letch er: Atacama Meteorites,

Mineral Magaz. Bd. 8, p. 226 (Streufeld 3 miles).

1894: C o hen: Meteoritenkunde, p. 22, 285,320 .

r894: F l e t c h e r : Introduction, p. 9.

r895: Brezina: Wiener Sammlung, p. 250 .

Ursprüngliches Gewicht: ro6 lbs. Es sollen drei Steine von 17 , 24 u. 65 lbs. gesammelt worden sein, die aber wohl nur zum Teil erhalten blieben.

Nachweisbares Gewicht: 10 878 gr.

\begin{tabular}{ll|l|l} 
Bement & 2 & London, P. G. $\quad 126$
\end{tabular}

Berlin, U. $\quad 3$ Neumann 8

Budapest $\quad 23$ Newton I

Calcutta $\quad 43$ Oxford 8505

Cambridge $\quad 83$ Paris, M. $\quad$ I85

Dublin, M. $\quad 135$ Rom, U. 27

Göttingen $\quad 109$ v. Siemaschko 3

Gregory $\quad 74$ Stockholm $\quad$ I6

Greifswald $\quad 8$ Strassburg 8

Harvard, U. 50 Tübingen 1156

Kopenhagen 6 Ward 30

London, B. M. $\quad{ }_{4} 4$ Wien, H. M. *) $\quad 163$

Linn Co.

Hartford

Linnville, Linnville Mountain,Burke Co., Nord-Carolina, U. S. A.

Eisen, Hch, gefunden um 1882 , beschr. 1888 .

1888: $\mathrm{Kunz}$ : On two new masses of meteoric iron. - I. Meteoric Iron 
from Linnville Mountain, Burke Co., North Carolina. Am. Journ. (3) 36, p. 275-276 (Analyse von Whitfield). Ref. N. J. 1889 II, p. 446.

I89o: E a s tm a n: Met. Astron., p. 320. r $89 \mathrm{r}$ : v. H a uer: Ann. Hof-Mus, Bd. 6 (Not.), p. 54.

1894: C o hen: Meteoritenkunde, p. 77, $94,232$.

1895: Brezina: Wiener Sammlung, p. 295 .

Ursprüngliches Gewicht : 442 gr., davon I4 gr. für Analyse verwendet.

Nachweisbares Gewicht: 394 gr.

Bailey

Bement

Gregory

\begin{tabular}{l|lr}
24 & London, B. M. & 2I \\
69 & Ward & 38 \\
28 & Wien, H. M. & 214
\end{tabular}

Linum bei Fehrbellin, Prov, Brandenburg, Preussen, Deutschland. Stein, gefallen 5 . Sept. 1854 .

1854: R ose legte den kürzlich bei Linum gefallenen Meteorstein vor. Berichte Berlin. Akad. (1854), p. $525-$ 527 ; s. auch Journ. prakt. Chem. Bd. 63 (1854), p. $356-358$; Pogg. Ann. Bd, 94 (1855), p. $169-171$; L'Institut * (1855) Bd. 23 Nr. III9, p. 206 ; Pharm. Centralblatt 1854 , p. 923 ; Kenngott, Uebersicht 1854 , p. 167 ; Liebig-Kopp,Jahresber.1854,p.910-9II. I859: B u c hn er: Feuermeteore,p.ro2. 1859: Harris: Dissert. Gött., p. 95. r86o/6r: v. Reichenbach : XIII 365. XV ror.

1863: Buchner: Meteoriten, p. 87. 1863: B o 11: Meteorstein bei Meno in - Mecklenburg gefallen. Archiv der Freunde der Naturgeschichte. Neubrandenburg Bd. 17 (1863), p. 285.

1863: Ros e: Meteoriten, p. 93, 155. r885: Brezina: Wiener Sammlung, p. 261,
Ursprüngliches Gewicht ${ }^{1}$ ) : I 862 gr. (3 Pfd. $2 x^{3} / 4$ Lot).

Nachweisbares Gewicht: I730 gr.

Berlin, U. $1730 \mid$ Calcutta Spl.

Der Stein scheint noch nicht näher untersucht worden zu sein.

Lion River, Great Namaland, Südafrika.

Eisen, Of, bekannt?, nach London gebracht 1852 , beschr. 1853 .

1853: Shepard: Notice of Meteoric lron near Lion River, Great Namaqualand, South Africa; and of the detection of Potassium in Meteoric Iron. Am. Journ. (2) 15, p. I-4 (Analyse und Abbildung einer Masse von 178 lbs. $=80^{1 / 2} \mathrm{Kgr}$.). Ref. N. J. $\mathbf{1 8 5 3}$, p. 473 u. N. J. 1855 , p. 562 ; Journ. prakt. Chem. Bd. $5^{8}$ (1853), p. $3^{25}$ -327 ; Kenngott,Uebersicht 1853.p.II5 ; Liebig-Kopp, Jahresber. 1853 , p. 935. 1859: Buc h ner: Feuermeteore, p. 128 -129 .

1859: H a r r is: Dissert, Gött., p. I22.

1860: Rammels berg: Mineralchemie, p. 920 .

r858/62: v. Reic hen bach: VII 55 I, IX $163,174,182 . X V$ nо, $1_{3}, \mathrm{II}_{4}, \mathrm{I2}_{4}$. XVI 261, 262. XVII 266, 272. XVIII $478,484,487$. XIX 155. XX $622,627$. r863: B u c h ner: Meteoriten,p.r86-187. 1863: Rose: Meteoriten, p. 65, I53. r869: Meunier: Recherches, Ann.

Chim. Phys. (4) 17, p. 72.

1872: Quenstedt: Klar und Wahr,

p. 313 (Abb. einer geätzten Platte). 1880: Brezina: Bericht I, Sitzber.

Wien. Akad. Bd. 82 I, p. 350.

I884: M e un i e r: Météorites, p.r29,130. 1885: B rezin a : Wiener Sammlung, p. 200, 208-209, 233.

1887: Brez ina u. Cohen: Photographien, T. 19.

I) Buchner giebt als ursprüngliches Gewicht dasjenige des Steins in Berlin an. 
r893: Meunier: Revision des fers météoriques, p. 42.

r894: Cohen: Meteoritenkunde, p. 56, 62,68 .

r895: Brezina: Wiener Sammlung, p. 269 .

Ursprüngliches Gewicht: Die von Shepard beschriebene Masse wog $80^{1 / 2} \mathrm{Kgr}$. ( $7_{7} 8 \mathrm{lbs}$.).

Nachweisbares Gewicht: 1913 gr.

\begin{tabular}{lr|lr} 
Bailey & 30 & Marburg & I27 \\
Bement & I19 & Moskau & 24 \\
Berlin, U. & 60 & New Haven & 40 \\
Calcutta & 178 & Paris, M. & 38 \\
Dorpat & 30 & v. Siemaschko & 44 \\
Göttingen & I4 & Stockholm & 15 \\
Gregory & 24 & Tübingen & 129 \\
Kunz & 49 & Ward & 106 \\
Leiden & 74 & Washington & 35
\end{tabular}

London, B. M. 390 Washington, Sh. 2 I London, P. G. $\quad 86$ Wien, H. M.*) 280 Wo befindet sich die von Shepard beschriebene grosse Masse?

\section{Lipowitz}

Lippe

Oczeretna

Liptoer Com. s. Anh. Nagy Borove

Lissa, Bunzlau,Böhmen,Oesterreich.

Stein, Cwa (teils Cwa, teils Cwb nach Brezina), gefall. 3 . Sept. 1808.

1808: v. S chreibers: Nachrichtvon einem neuen Steinregen, der am 3. Sept. 1808 einige Meilen von Prag herabgefallen ist. Gilb. Ann. Bd. 30 , p. $35^{8}$ $-3^{6} \mathrm{r} ;$ s. auch Bibl. Brit. Nr. $3^{12}$ (Dez. 1808), p. 369-371.

1809: Reuss u. K laproth: Ueber die bei Lissa gefallenen Aërolithe. 1. Nachrichten von dem Steinregen, der sich am 3. September 1808 bei Lissa in Böhmen ereignete. - 2. Chemische Untersuchung des Meteorsteins von Lissa. Gehlen's Journ. für Chemie Bd. 8 , p. $43^{8}, 467$; s. auch Reuss, Mémoire sur les Aérolithes tombés près W it 1 fing, Meteoriten. de Lissa en Bohême, le 3 Septembre 1808. Ann. Chim. Phys. Bd. 74 (1810), p. $84-95$; v. Dalberg, Ueber Meteorkultus der Alten. Heidelberg $\mathbf{1} 8 \mathrm{II}$, bei Mohr u. Zimmer, p. $4^{8}-49$.

r809: v. Schreibers: Auszug aus einem Schreiben des Herrn v. Schreibers an den Professor Gilbert. Gilb. Ann. Bd. 32, p. 125-126.

18ro: K laproth: Beiträge, Bd. 5, p. $246-253$ (Analyse).

r812: Bigot de Morogues, p. 233 -238 .

r812: $\mathrm{C}$ h 1 a d n i: Verzeichnis,Schweigg. Journ. Bd, 4 Beil. I, p. 16.

r8r5: Chladni: Neues Verzeichnis, p. 254 .

18r6: $\mathrm{Ch} 1$ a dni: Zweite Fortsetzung, Gilb. Ann. Bd. 54, p. 354, 355 .

r8x9: Chla d n i: Feuermeteore, p. 4I, 50, 5I, 57, 66, 69, 73, 9I, 286, 289 -290 (I), 295, 296, 308, 430.

r820: v. Schreibers: Beiträge, p. 17-19 (Abbildung des grossen Wiener Steins auf Taf. III).

r836: K ä m tz: Meteorologie,p.258,282. 1836: Ro s e: Reise nach dem Ural, Bd.

I, p. 77 (Vergleich mit Honolulu).

1843: P a r s c h: Meteoriten, p. 50-51. r854: B a 1 c e $11 \mathrm{~s}$ : Lithologia meteorica, p. 23 .

r854: v. B o g us law ski: ZehnterNachtrag, Pogg. Ann. Ergz.-Bd, 4, p. 436. 1859: Ha rris: Dissert.Gött., p.68-69. 1859: B u ch ner: Feuermeteore, p. 81 . 1863: B u c hn er: Meteoriten, p.26-27. 1863: R os e: Meteoriten, p.90, 93, 155. r858/65: v. Reichen bach: V 477 , 479,480 . VI 455 . IX 16I, 168, 178. X 359,363 . XI 294. XIII 369 (Fig.). XX 626. XXIV 226. XXV 322, 324, 423,427 .

r883: Tscherma k: Beitrag, Sitzber.

Wien. Akad. Bd 88 I, p. 355 .

r884: M e un i er: Météorites,p.208,2II. 1883/85: T s c h e r ma k: Photographien, p. 18 .

14 

Kopenhagen $\quad{ }_{5}$ Stockholm Spl.

London, B. M. IO4 Troyes

London, P. G. $\quad 6$ Tübingen

Minneapolis

New Haven

I Washington

24 Washington, Sh. 75

Paris, M.

v. Siemaschko

14 Wien, H. M.*) 62

I

Livingstone Co.

Smithland

Lixna (Lasdany),Dünaburg,Witebsk, Russland.

Stein, Cga, gefallen 12. Juli 1820. r820: M ein e cke: Meteorfall an der

Düna. Notizen, zusammengezogen aus öffentlichen Blättern. Schweigg. Journ. Bd. 29 , p. $5 \mathrm{II}-5 \mathrm{I} 3$.

r82r: v. Grotthuss: Untersuchung eines in Kurland im Dünaburg'schen Kreise, am 30. Juni (12. Juli) 1820 herabgefallenen Meteorsteins. - I. Geschichtliches. - 2. Aphorismen, den Steinregen im Allgemeinen betreffend. - 3. Aeussere Beschaffenheit u. physikalische Merkmale des Dünaburg'schen Meteorsteins, - 4. Chemische Prüfung der vom Magnet ausgezogenen Metalltheile. - 5. Analyse des Dünaburgschen Meteorsteins. Gilb. Ann. Bd, 67, p. $337-367$ (Analyse u. Abbildung eines Bruchstücks); s.auch Froriep'sNotizen d. Natur- u. Heilk. 1827.

1821: Chladni: Erster Nachtrag, Gilb. Ann. Bd. 68 , p. $337-338$. .

1823: La ug ier: Bull. Soc. Philom. Juni 1823 (Analyse); s. auch Ann. du Mus, d'hist. nat. 6. Jahrg. II, Heft. Uebersetzung in Gilb. Ann. Bd. 75 (1823), p. $265-266$ unter dem Titel: Vorläufige Nachricht von der chemischen Analyse zweier in Polen gefundenen Aërolithen u. zweier Meteor-Eisen mit Bemerkungen von Gilbert; Ann. Chim. Phys. Bd. 25 (1824), p. 219220; Schweigg. Journ. Bd. 43 (1825), p. 26 .

r836: K ä m tz: Meteorologie, p. 258, 288 .
1842: v. D a l wit z: Ueber die imJahre 1820 in Weiss-Russland zur Erde gefallenen Aërolithen. Schriften der russ. k. Ges. f. d. gesamte Min. Bd. I. I. (1842), p. LXX-LXXII (Analyse von v. Grotthuss).

1843: Partsch: Meteoriten, p. 70 -71 .

1847: Eichwald: Verzeichnis, Erman's Arch. f. Kunde Russl. Bd. 5, p. 179 ; s. auch Pharm. Centr. 1848 , p. 289 .

r848: B loe d e: Tabelle, Bull. Petersburg. Akad. Bd. VI, p. Io.

1852: Eichwald: Der Meteorstein von Lixna. Pogg. Ann. Bd. 85, p. 574 -579 (Analyse von Grotthaus); s. auch Journ. prakt. Chem. Bd. 56, p. 315316; Liebig-Kopp, Jahresber. 1852, p. 989 ; Kenngott, Uebersicht 1852 , p. I50 -151; Am. Journ. (2) 16, p. 148; N. J. 1854, p. 184 .

r853 : R a m m e $1 \mathrm{~s}$ berg: Handwörterbuch Suppl. V, p. 28-29.

1854: v. Boguslaw ski: ZehnterNachtrag, Pogg. Ann. Ergz.-Bd. 4, p. 16-19. 1859: B u c hner: Feuermeteore, p. 85. 1859: Harris: Dissert. Gött., p. 75.

r860: Ram mels berg: Mineralchemie, p. 922 ff.

r863: B u c h n e r: Meteoriten, p.4I -42. I863: R os e: Meteoriten, p. $89,90, \mathbf{r} 54$. r $858 / 65$ : v. Reichen ba ch: VI 454 . IX 162, 171, 180. X 359. XI 294. XXV $433,602,603,607,608$.

r865: K u h 1 berg: Analyse und Beschreibung der Meteorite von Nerft, Honolulu, Lixna und eines im Gouvernement Jekatherinoslaw gefallenen Meteoriten. Arch. Naturk. Liv, Ehst. Kurl. (1) 4 , p. 23-29 (Analyse). Ref. LiebigKopp, Jahresber. 1867, p. 1049.

I865: K u h 1 berg: Der Meteorit von Lixna. Ergänzung zu p. 23 nach einem Manuskript des Grafen Plater-Sieberg. Arch. Naturk. Liv. Ehst. Kurl. (1) 4 , p. $33-34$ (Sit.-Plan).

$14 *$ 
1867: Goe be 1: Kritische Uebersicht, Mélanges phys. chim. Bd, 7, p. 300 $-302,338$.

1867: Buchner: Dritter Nachtrag, Pogg. Ann. Bd. 132, p. 315, 318.

1869: Buchner: Vierter Nachtrag,

Pogg. Ann, Bd, 136, p. $444-445$.

I875: vo m R a th: Meteoriten, Verh. naturh. Ver. Bonn Bd. 32, p. 367 .

r88I: v. Nordenskiöld: Zeitschr. d. d. geol. Ges. Bd. 33 , p. 24 (Vergleich mit Ställdalen).

I884: M e unier: Météorites, p. 73, $75,208,213-214$.

1883/85: Tschermak: Photographien, p. 3 .

1885: Brezina: Wiener Sammlung, p. $182,233$.

1894: C o he n : Meteoritenkunde, p. 57, 136, 206, 248.

r895: Brezina: Wiener Sammlung, p. 250 .

Ursprüngliches Gewicht: Ein Stein von $20 \mathrm{Kgr}$, von dem viel verschleppt wurde.

Nachweisbares Gewicht: 5213 gr.

\section{Bement}

Berlin, U,

Bonn

v. Braun

Budapest

Calcutta

Dorpat

Dresden, M.

Freiberg, i. S.

Göttingen

Gregory

Greifswald

Harvard, U.

Heidelberg

Kiew

\section{Krüger}

65 London, B. M.

20 London, P. G.

19 Madrid

64 Moskau

65 Neumann

399 Paris, M.

312 Petersburg, A. 284

I7 Pohl 284

200 Riga

12 v. Siemaschko

I9 Stockholm

5 Tübingen

36 Washington, Sh. 2770 Wien, H. M.*) 268
Lundsgård

Inca

Cambria

s. Anhang
Lodran, Mooltan, Punjab, Ostindien. Lodranit, Lo, gefallen r. Okt. 1868. r869/70: T. O. (O $1 \mathrm{~d}$ h a m): Rec. Geol. Surv. India Bd. 2 I, p. 20, 34 (giebt an beiden Stellen den 17. Oktober als Falltag an).

1870: R a m mels berg: Meteoriten, p. $92-93,106,138,139$.

1870: Ts c her ma k: Der Meteorit von Lodran. Sitzber. Wien. Akad, Bd. 6r II, p. 465-475 (Analyse; Tafel; Abbildung mikroskopischer Präparate); s. auch Pogg. Ann. Bd, 140 (1870), p. 321-324 (Auszug vom Verfasser); Am. Journ. (2) 50 (1870), p. 293; „L'Institut\& v. 20. Juli 1870, p. 20; N. J. 1870 , p. 483 ; Cohen, N. J. I887 II, p. 49, vgl. die dendritenartigen Gebilde von Erz mit solchen des Pallasiten von Imilac (Campo de Pucará).

1870: Tschermak: Goalpara, Sitzber. Wien. Akad. Bd. 62 II, p. 860. r879: R a mmels berg : Meteoriten, p. 25 .

r882: Meunier: Histoire géologique de la syssidère de Lodran, C. R. Bd. 95, p. $1176-$ II79. Ref. N. J. 1884, p. 32 .

1883: T s c hermak: Beitrag, Sitzber. Wien. Akad. Bd, 88 I, p. 354.

r884: Me unier: Météorites, p. 78,79 , $84,85, \mathbf{1 4 4}$ (Abb.) -147, 356, 363, $395,520-521$.

1884: W a d s w or th: Studies,p.74-75. r883/85: Ts chermak : Photographien, p. 22.

r885: Brezina: Wiener Sammlung, p. 175, 192-199, Taf. IV, 233.

r887: F l ig h t: Meteorites, p. 158-160. 1887: Brezin a : Neue Meteoriten III, Ann. Hof-Mus. Bd. 2 (Not.), p. 114, 115. r894: F l e t c h er: Introduction, p. 13. 1894: Cohen: Meteoritenkunde, an vielen Stellen.

r895: Meunier: Revision des lithosidérites, p. 40-42 (Abb. einer geätzten Platte u. eines Dünnschliffs). 
r895: B r e z in a : Wiener Samml., p.263. Ursprüngliches Gewicht: ?

Nachweisbares Gewicht: 970 gr.

Bailey

v. Braun

Budapest

Calcutta

Gregory

Greifswald

\section{Logroño}

\section{Loma de la Cosina}

Barea

Lonaconing, Garrett Co., Maryland, U. S. A.

Eisen, $\mathrm{Og}$, gefd. 1888 , beschr. 1892. 1892: Foote: A new Meteoric Iron from Garrett Co., Maryland. Am. Journ. (3) 43, p. 64. (Nach König über Ir $\%$ Nickel u. Cobalt; Abbildung der Masse und einer geätzten Seite). Ref. N. J. 1892 II, p. 244.

I894: Co he n: Meteoritenkunde, p. 73. 1895: B r e z in a: Wiener Samml., p. 287.

Ursprüngliches Gewicht: 45 oz., aber durch Analyse, Schneiden und Polieren etc. auf $36^{1 / 2} \mathrm{oz}$. $=1026$ gr. reduziert, was mit dem nachweisbarenGewicht nicht übereinstimmt.

Nachweisbares Gewicht: Ir34 gr.

\begin{tabular}{ll|lr} 
Bailey & 36 & Paris, E. & 819 \\
Bement & 99 & Ward & 24 \\
v. Braun & 23 & Wien, H. M. & 133
\end{tabular}

\section{Long Creek}

s. Anhang

Long Island (Phillips Co.), Kansas, U. S. A.

Chondrit, gefd. $189 \mathrm{r}$, beschr. 1895 . r895: We in s c he n k: Meteoritenstudien II. - 4. Long Island, Phillips Co, Kansas. M.P. M. Bd.14, P. $471-473$.

Ursprüngliches Gewicht: $55^{8} \mathrm{Kgr}$., nach Mitteilung von HerrnGeorge F. Kunz, der die ganze Masse besass. Weinschenk giebt 1184 lbs., also etwa $936 \mathrm{Kgr}$., als ursprüngliches Gewicht an. Das Field Columbian Museum in Chicago besitzt, nach dem Verzeichnis vom August $\mathrm{x} 895,534467 \mathrm{gr}$.

\section{Lontolax}

Luotolaks

Lorana

s. Anhang

Los Angeles Shingle Springs

Losttown, Cherokee Co., Georgia, U. S. A.

Eisen, Om, gefd. 1867 , beschr. 1868. Auch: "The Cherokee Meteorite«, gefunden 1894, vorläufig hierher gestellt.

1868: S h ep a rd: A new locality of Meteoric Iron in Georgia. Am. Journ.

(2) 46 , p. $257-258$. Ref. N. J. 1869, p. 580 .

r869: S h e p a rd: Notices of New Meteoric Irons in the United States. 3. Composition of Meteoric Iron from Losttown, Cherokee Co., Georgia. Am. Journ. (2) 47, p. 234 (Analyse). Ref. Liebig-Kopp, Jahresber. 1869, p. 1302. 1885: Brezina: Wiener Sammlung, p. $26 \mathrm{r}$.

r887: F lig ht: Meteorites, p. r55-r56. 1890: Eas tma n: Met. Astron., p. 320. r893: Meunier: Revision des fers météoriques, p. 47,48 .

1895: Brezina: Wiener Sammlung, p. 279 .

1895: How e11: On two new Meteorites. - 1. The Cherokee Meteorite. Am. Journ. (3) 5o, p. 252-253 (Tafel 4, Abb. einer geätzten Platte; Analyse von Stokes; Aufdecken einer Masse von $8 \frac{1}{2}$ lbs.).

Ursprüngliches Gewicht: 3000 gr. (6 lbs ro oz.), ohne die neuerdings aufgedeckte Masse von $8^{1 / 2}$ lbs.

Nachweisbares Gewicht: 235 gr. 


\begin{tabular}{lr|lr} 
Bailey & 48 & London, P. G. & 5 \\
Bement & 3 & Paris, M. & I0 \\
v. Braun & 2 & v. Siemaschko & 17 \\
Budapest & 22 & Washington & 7 \\
Gregory & 6 & Washington, Sh. & 75 \\
London, B. M. & 6 & Wien, H. M. & 34 \\
\hline
\end{tabular}

Wo ist die Hauptmasse des Eisens?

$\begin{array}{lr}\text { Louans r845 } & \text { Le Pressoir } \\ \text { Louisa Co. } & \text { Staunton } \\ \text { Louvain } & \text { Tourinnes-la-Grosse } \\ \text { Löwenfluss } & \text { Lion River } \\ \text { Lozère } & \text { Aumières } \\ \text { Lucé, Dep. Sarthe, Frankreich. }\end{array}$

Stein, Cwa, gefallen ${ }_{3}$. Sept. ${ }_{7} 68$.

I769: B a chela y: Hist. de l'Acad. Royale 1769, gedr. 1772 , p. $20-21$ (legt der Akademie einen Stein von Lucé vor).

1772: Fougeroux, Cadet et Lavo is i e r: Rapport fait à l'Académie Royale des Sciences, par M. M. Fougeroux, Cadet et Lavoisier, d'une observation communiquée par M. l'Abbé Bachelay, sur une pierre qu'on prétend être tombée du Ciel pendant un orage. Journ. Phys. Bd. 2 (1772), p. 251-255; s. auch sEncyclopédie $\mathrm{Bd}$. 5 (1808), p. 59r; Mém. de l'Acad. Royal. Paris 1769, p. 21-34; Laugier, Chromium, p. 377 ; Izarn (s. u.), p. 59-71; Klaproth, Abh. Berlin. Akad. 1803, p. 30.

r796: K in g: Remarks, p. 33-34.

1802: How ard: Experiments and Observations on certain stony and metalline Substances, which at different times are said to have fallen on the Earth; also on various Kinds of native Iron. Phil. Trans. (1802), p. 170 -17r; s. auch Gilb. Ann. Bd, 13 (1803), p. 293-295; Fourcroy's Uebersetzung in sEncyclopédie Bd. 5 (1808), p. 545. r803: I z a rn: Lithologie, p. 59-71, I92, 30I.

1803: de Drée: Recherches, Journ.
Phys. Bd. 56, p. $415-416$.

r803: $\mathrm{Ch} 1$ a d n i: ChronologischesVerzeichnis, Gilb. Ann. Bd. 15, p. 31 o.

I804: v. En d e : Massen u. Steine, p. 46 -48 .

1808: De Dré e-Pict e t: Description comparative etc. (s. bei Weston), Bibl. Brit. Nr. 296 (April 1808), p. 28o, 288. 1812: Bigot de Morogues, p. 16 $-\mathrm{I} 7, \quad \mathrm{IO}_{5}-\mathrm{II}_{3}, 287$ (nicht Salles, s. Chladni I8I9, p. 252), 335.

1812: Ch la d n i: Verzeichnis,Schweigg. Journ. Bd. 4 Beil. I, p. 13 .

r8r5: Chladni: Neues Verzeichnis, Gilb. Ann. Bd, 50, p. 249.

r8r6: Chla dni: Zweite Fortsetzung,

Gilb. Ann. Bd. 54, p. $348-349$.

1818: Nouv. Dict, de l'hist. nat, (1818).

Art. Pierres météoriques Bd, 26.

I8r9: $\mathrm{Ch}$ l ad ni: Feuermeteore, p. 57,

$90,25 \mathrm{I}-252$ (1), 293.

1829: Pes che: Dictionnaire topographique et statistique de la Sarthe tome II, p. 669 .

1836: Kämtz: Meteorologie, p. 275.

1843: P a r ts c h: Meteoriten, p. $48-50$.

I854: B a $1 \mathrm{c}$ e $11 \mathrm{~s}$ : Lithologia meteorica, p. 2 I.

I859: Bu chner: Feuermeteore, p. 42 -44 .

1859: Harri s: Dissert. Gött., p. 60. r862: Kenngott u. Wiser: Zürcher Sammlung, p. 148.

1863: Buchner: Meteoriten, p. 7-8. I863: R os e: Meteoriten, p. $87,154$. 1858/65: v. Re i che n b a c h: VII 552. IX 16r, 169, 179. XI 297. XXIV 226. XXV 322, 324, 433 .

r881: Guilli er : Notes sur les météorites et spécialement sur celles tombées au Grand-Lucé le 13 septembre 1768. Bull. Soc. d'Agriculture, Sciences et Arts de la Sarthe. Gedruckt bei Ed. Monnoyer in Le Mans. Déc. 1881, 7 Seiten. r884: M e un ier: Météorites, p. 95. 207 (Abb.), 208, 209, 459, 464-466, 522. 
1883/85: Ts chermak: Photographien, p. 16.

r885: Brezina: Wiener Sammlung, p. 180 .

1889: M e u n i e r : Sur la matière noire de la Chantonnite. Bull. Soc. Franc. Min. Bd. 12, p. 76-81.

I894: Cohen: Meteoritenkunde, p. 7. r894: F l e t c h e r: Introduction, p. 20. r895: Brezina: Wiener Sammlung, p. 243.

Ursprüngliches Gewicht : $71 / 2$ Pfund. Nachweisbares Gewicht: $26_{3}$ gr.

Berlin, U.

Budapest

Calcutta

Freiberg, i. S. 23 Paris, M.

Gregory

Hamburg

London, B. M. I2 Zürich v. Siemaschko 3 5 Stockholm Spl. Io Tübingen 3 3
Spl. $\mathbf{I} 66$

Lucky Hill, St. Elizabeth, Jamaica. Eisen, Om, gefunden 1885 .

Ich habe hierher auch die Literatur über einen älteren Fall vom Jahre 1700 , erwähnt 1719 , welcher auf Jamaica stattfand, gestellt, obgleich die unten aufgeführten Massen vielleicht in keinem $\mathrm{Zu}$ sammenhang damit stehen.

1720: B a r h a m: Philos. Trans. Bd. 30

Nr. 157, p. 837 oder Nr. 357 , p. 148.

Bd. 30 ist der Jahrgang $1717-1719$ (gedruckt 1720).

r794: C h 1 a d n i: Pallaseisen,p.28-29. r815: Chladni : Neues Verzeichnis,

Gilb. Ann. Bd. 50, p. 247.

1819: $\mathrm{C}$ h 1 a d n i: Feuermeteore, p. 32, 105-106 (1).

1821: $\mathrm{Ch} 1$ a d n i: Neue Beitrïge, Gilb.

Ann. Bd. 68 , p. $338-339$.

1836: Kä m tz: Meteorologie, p. 269. 1859: H a r r i s: Dissert. Gött., p. 58.

r886: v. H a u e r: Ann. Hof-Mus. Bd. 2

(Not.), p. 39. r89r: v. Siem as chko: Catalogue de la Collection de Météorites de Julien de Siemaschko. St. Petersburg 189I, p. Io (kurze Anmerkung, russisch).

1893: Meunier: Revision des fers météoriques, p. 76 .

r894: F le t c her: Introduction, p. I4. r895: Brez in a: Wiener Sammlung, p. 282 .

Ursprüngliches Gewicht: ?

Nachweisbares Gewicht : 3406 gr.

Gregory $\quad 45 \mid$ Paris, M. 30

London, B. M. Spl. v. Siemaschko 30

London, P. G. 3280 Wien, H. M. 2 I

Lujan, Villa Lujan, Argentinische Republik.

Mesosiderit, M, gefunden ?, erwähnt 1892 .

1892: W a r d: Villa Lujan, Argentine Republic, S. A.: Dark-brown fragment of an old Aerolite found by Prof. Florentine Ameghino, a league and a half southwest of the city of Villa Lujan, in an undisturbed Quarternary formation, at a depth of nearly 6 metres, and about 5 metres below a megatherium rib. Weight 9 grammes. The Ward Collection of Meteorites, p. 37 Nr. 147. Rochester 1892 (Lace, Printer). r895: Brezina: Wiener Sammlung, p. 261.

Ursprüngliches Gewicht: 9 gr. Nachweisbares Gewicht: $8 \mathrm{gr}$.

$$
\text { Wien, H. M. } \quad 8^{1} \text { ) }
$$

Lumpkin, Stewart Co., Georgia, U. S. A.

Stein, Cck, gefallen 6. Okt. 1869. r870: $\mathrm{Sm}$ it h: Fall of a Meteorite in Stewart Co., Georgia. Am. Journ. (2) 50, p. 293.

1870: Willet: Account of the fall of a Meteoric Stone in Stewart County, Georgia. Am. Journ. (2) 5o,p.335-338.

1) Juni 1893 war das Stück noch im Besitz von Herrn Ward. 
1870: $\mathrm{Sm}$ it h: Description and Analysis of a Meteoric Stone that fall in Stewart County, Ga. (Stewart Couraty Meteorite), on the $6^{\text {th }}$ of October 1869 . Am. Journ. (2) 5o, p. 339-34I (Analyse); s, auch Original Researches I884, p. $442-457$.

I879: Rammelsberg: Meteoriten, p. 24,25 .

r885: Brezina: Wiener Sammlung, p. I9I, 233.

1887: F1 ig h t: Meteorites, p. IO-II.

1890: Eastman: Met. Astron., p. 316. I894: Cohen: Meteoritenkunde, p. 285.

r895: Brezina: Wiener Sammlung, p. 260 .

Ursprüngliches Gewicht: $357 \mathrm{gr}$. ( $12 \%$ Oz.).

Nachweisbares Gewicht: 128 gr.

Bailey

Cleveland

Göttingen

Gregory

Harvard, U,

Kopenhagen

I London, B. M. I I7

Spl. London, P. G. 4

8 v. Siemaschko Spl.

4 Ward

6I Washington

4 Wien, H. M.*)**) 25

Lundsgård, Kirchspiel Ljungby, Schonen, Schweden.

Stein, $\mathrm{Cw}$, gefallen 3. April $\mathrm{r} 889$.

r889: Svedmark: Om en i Stine den 3 sistlidne April fallen meteorsten. Geol, Fören. i Stockolm Förh. (1889) Bd. XI, p. 245-246. Ref. N. J. 189oI, p. 4I4; Nature Bd. 40 (1889), p. 179, 229.

189o: v. Nordenskiöld, A. E. : Ueber einen neuen Meteorsteinfall in Schweden. Geol. Fören, i Stockholm Förh. Bd. XII, p. II-I2. Ref. N. J. I891 I, p. 49.

189r : N or dens k iöld, O.: Kemisk undersökning af meteoriten från Oestra Ljungby i Skåne. Geol, Fören.i Stockholm Förh. Bd. VIII p. $470-475$; s. auch N. J. 1892 I, p. $138-140$ (Chemische Untersuchung des Ljungby
Meteoriten von Otto Nordenskiöld) u. Ref. im gleichen Bd., p. 268.

1894: Cohen: Meteoritenkunde, p. 156, 236.

I895: Brezin a: Wiener Sammlung, p. 243 .

Ursprüngliches Gewicht: $10^{1 / 2}$ I $\mathrm{Kgr}$.

Nachweisbares Gewicht: 10523 gr. Böhm

v. Braun

Budapest

London, B. M. 214 24 Paris, M. $\quad 104$ 86 Stockholm 9643 $45^{2}$ Upsala Spl.

Ausserdem Wien, H. M. $5^{8}$ gr. nach I. Juli 1893 erworben.

Luotolaks (Felsenbucht), Gouv. Wiborg, Finnland, Russland.

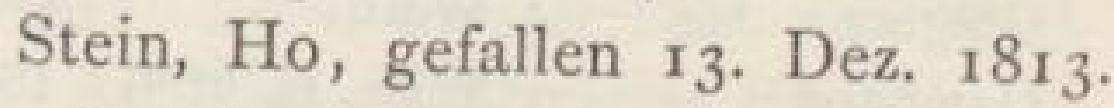
1815/r6: Scherer teilt den Fall der Petersburger Akademie mit. Bull. Petersburg. Akad. Bd. 7. Observations, Experiences et notices intéressantes faites et communiquées à l'Académie, p. 3 I. r8r8: Scherer: Allg. Nord, Ann. Chem. Bd. I, p. 407 oder 474 oder 174 (ist ausserdem noch etwas in Nord. Blätter Bd. 4, p. 107 erschienen? Chladni sagt: Nord. Blätter für die Chemie B. I H. 4, p. 407).

r8rg: $\mathrm{Chl}$ a d n i: Feuermeteore, p.304 -305 .

r82I : Gilbert: Zusatz, von dem Kurländischen meteorischen Papiere und einem Finnländischen Meteorstein. Gilb. Ann. Bd. 67 , p. 370.

I82r : N ordenskiöld, Nils: Beschreibung des in dem Finnländischen Gouvernement Wiborg gefallenen Meteorsteins. Schweigg. Journ. Bd. 3I, p. $160-162$; s, auch Bidrag till närmare kännedom af Finlands mineralier och geognosie Bd. 1, p. 99 (1820); Neues Journ. f. Chem. u. Phys. Bd, I Heft 2, p. 160.

1821: Chladni: Erster Nachtrag, Gilb. Ann. Bd. 68, p. 339-340. 
1821: L a ug i e r: Analyse de la Pierre météorique de Juvénas. Ann. Chim. Phys. Bd. 19, p. 270, 271, 272; s, auch Schweigg. Journ. Bd. 35 (1822), p. 419 -420 ; Gilb. Ann. Bd. 71 (I822), p. 209-2II.

r824: Chla dni: Vierter Nachtrag, Pogg. Ann. Bd. 2, p. 154.

r824: Nordenskiöld: Description minéralogique des aérolithes qui tombèrent près de Wiborg, en Finlande, le 13 décembre 1822 (muss $181_{3}$ heissen). Ann. Chim. Phys. Bd. 25, p. 78-80. 1825: Rose: Ueber die in den Meteorsteinen vorkommenden krystallisirten Mineralien. Pogg. Ann. Bd. 4, p. 185 . 1834: Berzelius: Om Meteorstenar. - 3. Meteorsten från Lautolax, Kongl. Vetensk. Acad Handl. (1834), p. 144 -147 (Analyse); s. auch Burkart, Fundorte I, N. J. 1856 , p. 267.

r836: R o s e : Reise nach dem Ural,p.77. 1836: $\mathrm{K}$ ä $\mathrm{mtz}$ : Meteorologie, p. 253, $255-256,285$.

r84r: R a m mels berg : Handwörterbuch, p. $429-430$.

r843: Rammels berg: Ueber die Bestandtheile der Meteorsteine. Pogg. Ann. Bd. 6o, p. 130, 132.

1843: P a rts c h: Meteoriten, p. 28.

1847: S chafhäut1: Schönenberg, Gel. Anz. München. Akad, 1847, p. 573 .

1847: E i c hwa 1d: Verzeichnis, Erman's Arch. f. wissensch. Kunde Russl. Bd. 5 , p. $\mathbf{r} 78$.

r848: B 1 o e d e: Tabelle, Bull. Petersburg. Akad. Bd. 6, p. 14 .

1853: R ammelsberg: Handwörterbuch Suppl. V, p. 30.

1854: v. Boguslawski: Zehnter Nachtrag, Pogg. Ann, Ergz.-Bd. 4, p.r5. 1859: H a r r i s : Dissert. Gött., p.71-72. r859: $\mathrm{B}$ u c h n e r : Feuermeteore, p. 83 $-84,92$.

r860: R a m mels berg: Mineralchemie, p. 940--94I, 949, 950, 952.
1862: Greg: On some Meteorites in the British Museum etc. - 5. Lontalax. Philos. Magaz. Bd. 24, p. 539. Ref.Kenngott,Uebersicht 1862/65,p.442. 1862: Buchner: Die Meteoriten in Wien u, London. Pogg. Ann. Bd, n6, p. 643 .

1863: B uchner: Meteoriten, p. 34 . 1863: Maskelyne u. v. Lang:

Mineralogical Notes. Notices of Aërolites von Maskelyne. - I9. Wiborg. Philos. Magaz. Bd. 25, p. $452-453$. Ref.Kenngott,Uebersicht 1862/65,p. 442 . 1863: Rose: Meteoriten, p. 26, 29, I07, I08-IIO, II2, 156; s. auch Mon.Ber. Berlin. Akad. 1862, p. 557 ; Pogg. Ann. Bd. 124 (1865), p. 207-208.

I858/65: v. Reichen b a ch: V 476. VI 441,455 . IX 159, 160, 167, 169, 179. X 363,373 . XIII $353,355,356$, $364,376,380$. XXIII 369 . XXIV 229. $\mathrm{XXV} 43^{8}$.

1865: Buchner: Zweiter Nachtrag, Pogg. Ann. Bd, 124, p. 574, 577.

1867: B u chner: Dritter Nachtrag, Pogg. Ann. Bd. 132, p. 319.

1867: Arppe: Om Meteorstenar fr. Luotolaks. Acta soc. scient. Fennicae Bd. 8, p. 37-100 (Analyse u. Abb.). Diese Angabe ist Friedländer's Kata$\log \mathrm{Nr} .400$ entnommen.

r869: Buchner: Vierter Nachtrag, Pogg. Ann. Bd, 136, p. 442.

r870: R a mmels berg: Meteoriten, p. $13 \mathbf{I}-136,139,140$.

r879: R a mmel sber g: Meteoriten,p.24. r882: W i i k: Mikroskopisk undersökning af naagra paa universitetets mineralkabinett befindliga meteoriter. Öfversigt af Finska Vetensk. Soc. Förh. Helsingfors Bd. 24 , p. $63-64$. Ref. N. J. I $88_{3}$ I, p. 384 .

1883: T s c hermak: Beitrag, Sitzber. Wien. Akad, Bd. 88 I, p. $349,368$. r884: Wa ds w or th: Studies, p. 190. r884: Me unier: Météorites, p. 78,79, $85,94,286-288,395$. 
r883/85: T s c he r m a k: Photographien, T. 4, p. $3,7,8$.

1885: Brezina: Wiener Sammlung, p. $174,232$.

I894: C o he n: Meteoritenkunde,p.240, 249, 293, 294, 296.

Ursprüngliches Gewicht: Mehrere Steine, von denen wenig gesammelt wurde.

Nachweisbares Gewicht: 894 gr. Bailey Berlin, U. Bonn v. Braun Budapest Calcutta Dorpat

Heidelberg Helsingfors Krantz I London, B. M. 2I 5 London, P. G. Spl. Spl. Paris, M. 12 Spl. Petersburg, B. 24 Io v. Siemaschko 2 Spl. Stockholm 90 39 Strassburg I Tübingen 654 Upsala I Wien,H.M.*)

Luponnas, Dep. de l'Ain,Frankreich. Stein, Cib, gefallen 7. Sept. 1753. r756: J er ôm e de 1 a L a nde: Etrennes historiques de la province de Bresse. 1756, p. 32 .

1802: J e rôme de la Lande: Lettre de Jerôme de la Lande à C. Delamétherie sur les Pierres de foudre. Journ. Phys. Bd. 55 , p. $45 \mathrm{I}-453$; s. auch Jerôme de la Lande: Nachricht von Steinen, die in Bresse aus Bd. I3 (1803), p. $343-345$, wo die Stelle aus den Etrennes historiques aus dem Jahre 1756 abgedruckt ist.

r803: Chla dn i: ChronologischesVerzeichnis, Gilb. Ann. Bd, 15, p. 309.

1803: de Drée: Recherches, Journ. Phys. Bd. 56, p. 416.

1803: Izarn: Lithologie, p. $55-59$ (aus den »Etrennes historiques * vom Jahr 1756), 299-300.

I) Ausserdem war noch ein Stück von r804: Pötzsch: Kurze Darstellung, p. $108-109$.

I812: Bigot de Morogues, p. 100, IOI, 334 .

I812: $\mathrm{Ch} 1$ a d n i: Verzeichnis,Schweigg. Journ. Bd, 4 Beil. I, p. I3.

I8r5: Chladni: Neues Verzeichnis, Gilb. Ann. Bd. 50, p. 248.

I8I9: C h la d n i: Feuermeteore, p. 248

(1), 432; verweist auch auf Richard, Hist. natur. de l'air et des météores Bd. 8, p. 434 .

I836: K ä m tz: Meteorologie, p. 273. I843: P a rt s c h: Meteoriten,p. 36-37. r854: B a 1 c e 11 s : Lithologia meteorica, p. 40.

r859: B u chner: Feuermeteore, p. 42. 1859: Ha rris: Dissert. Gött., p. 59. I863: B u chner: Meteoriten, p. 6-7. 1858/65: v. Reichen ba ch: V 476.

VII 552. IX 16I, 170, I7I, 179. XI 294. XIII 362. XX 622. XXV 427, 428. 1884: M e unier: Météorites, p.95,247. 1885: Brezina: Wiener Sammlung, p. $182,232$.

1892: M e unier: Examen minéralogique de deux Météorites Bourguignonnes. Luponnas, Ain, 8 Sept. 1753, et Les Ormes, Yonne, 4 Octobre 1857. Bull. Soc. Hist. Nat. Autun Bd, 5 (1892, 30 Seiten u. 2 Tafeln).

1894: Co hen: Meteoritenkunde,p.223

Ursprüngliches Gewicht: $31_{1}^{1} / 2$ Pfund. Zwei Steine von 20 u. I I $1 / 2$ Pfund; der erstere wurde alsbald zerkleinert und verteilt; der zweite gelangte nach Dijon; wenig erhal. ten. Von einem $3 \mathrm{Kgr}$. schweren Stein, welchen 1812 de Drée besass, vermutet Bigot de Morogues, dass er zum Fall von Luponnas gehöre.

Nachweisbares Gewicht: $277 \mathrm{gr}$.

ist nach (1) Direktor Brezina's Bestimmung Stannern. 
Berlin, U.

Calcutta

Gregory

I Paris, M.

Spl. v. Siemaschko 2

London, B. M. $\quad 8$ Wien, H. M.*) 84

Paris, E. 47

Lusignan d'Asso

Siena

Lutschaunig, Wüste Atacama, Südamerika.

Stein, $\mathrm{Cg}$, gefd. $\mathrm{I} 860$, erwähnt $\mathrm{I} 885$.

Fletcher giebt bei dem Stück, welches nach Wien kam, als Fundzeit 1860 an (Mitteilung von Herrn Direktor Brezina).

1885: Brezina: Wiener Sammlung, p. 251 .

1889: F letc he r : Atacama Meteorites, Mineral. Magaz. Bd. 8, p. 224, 225, 234. Ref. N. J. 189o II, p. 230.

1892 : v. H a u e r : Ann. Hof-Mus. Bd. 7 (Not.), p. 73.

r894: F le t c her: Introduction, p. 13. r895: Brezina: Wiener Sammlung, p. 249.

Ursprüngliches Gewicht: ?

Nachweisbares Gewicht: ${ }_{5} 6 \mathrm{gr}$.

London, B. M. 105 Wien, H. M.*) 2 London, P. G. 49

Macao, Prov. Rio Grande do Norte, Brasilien.

Stein, Cia (früher als Ci aufgefasst), gefallen 11 . November $188_{3} 6$.

r837: B e r th o u : Chute de pierres observée au Brésil; extrait d'une lettre de M. F. Berthou, transmise par M. d'Abbadie et datée d'Olinda. C. R. Bd. 5, p. 2II; s. auch Pogg. Ann. Bd. 42 (1837), p. 592 ; "L'Institute Bd, 5 (1837), p. 287.

I837 : Athenäum «, London, 16. Dezbr. 1837, p. 915 ; s, auch Am. Journ. (1) 34 $\left(183^{8}\right)$, p. $209-210$.

1839: Benzen ber g: Sternschnuppen, p. $269-270$.

1) $2048 \mathrm{gr}$. war das ursprünglich von Rio
1843: P a rts c h: Meteoriten, p. $8 \mathrm{I}-84$ (ausführliche Beschreibung des Falles). I847 : Oesterr. Blätter f. Litt, 1847 .

I854: v. Boguslawski: Zehnter Nachtrag, Pogg. Ann. Ergz.-Bd, 4, p.82, $355-356$.

r859: B u c hn er: Feuermeteore, p. 93. r859: H a r r is: Dissert. Gött., p. 85. 1863: Bu chner: Meteoriten,p. $58-59$. r863: Ros e: Meteoriten, p. $87,154$. r858/65: v. Rei chen b a c h: VI 445 . IX 162, 170, 179. XI 294, 296, 303 (Fig.), 304, 308, 310. XIII 365. XV Ior. XXIV 226. XXV 433.

1867: G o e b e 1: Kritische Uebersicht, Mélanges phys. chim. Bd. 7, p. 312. I884: M eu nier: Météorites, p.I9I, I92, 371.

r885: Brezina : Wiener Sammlung, p. $18 \mathrm{I}, 232$.

r888: D e r by: Meteoritos Brasilieiros. Revista do Observatorio. Rio de Janeiro, p. $7-8$.

r889: Fle t c her: Atacama Meteorites, Mineral. Magaz. Bd. 8, p. 227 (Streufeld 14 miles).

r893: Meunier: Revision des fers météoriques, p. 39-40 (Abb. einer geätzten Platte).

r894: C o hen : Meteoritenkunde, p.85.

Ursprüngliches Gewicht: Steinschauer. Der französische Bericht spricht von Steinen bis 80 Pfund; dagegen hat Partsch ermittelt, dass die Steine klein, meist von der Grösse eines Taubeneis, gewesen sind; wenig erhalten.

Nachweisbares Gewicht: 2902 gr.

\begin{tabular}{lr|lr} 
Bailey & I & Harvard, U., & 17 \\
Berlin, U. & 37 & London, B. M. & 6 \\
Budapest & 3 & Paris, M. & 9 \\
Calcutta & 6 & Petersburg, A. & 17 \\
Göttingen & 10 & Rio $2048^{1}$ ) \\
Gregory & 6 & v. Siemaschko & 12
\end{tabular}

Gesessene Gewicht, worter ein Stein 
Macao - Maêmê.

Stockholm

Tübingen

Utrecht

Macerata

Mac Kinney, U. S. A.

Stein, Cs, gefallen 1870 (?).

1895: v. H a uer: Ann. Hof-Mus, Bd, Io (Not.), p. 34 .

r895: Brezina: Wiener Sammlung p. $252-253$.

Ursprüngliches Gewicht: Zwei Massen, die grössere etwa roo Kgr. Nachweisbares Gewicht: 79613 gr. Brezina $\quad 32639$ Wien, H. M. 46667 Prag, M. $\quad 307$

Ein Bericht über den Fall ist meines Wissens bis jetzt noch nicht erschienen.

Macon Co.

Macquaire River

Madison 1854

Madisonville Brenham (Anderson)

Madoc, Madoc Township, Ober-

Canada, British-Amerika.

Eisen, Of, gefd. 1854 , beschr. 1855 .

1855: Hunt: Extract from a letter $T$.

S. Hunt to J. D. Dana, dated Mont

Real, Canada, March 12, 1855 . - On

a newly discovered Meteoric Iron.

Am. Journ. (2) 19, p. 417. Ref. Journ. prakt. Chem. Bd. 66 (1855), p. 431;

Kenngott, Uebersicht 1855 , p. 98 ; Liebig-Kopp, Jahresber. 1855, p. 1024.

1859: B u c hner: Feuermeteore,p.r33.

r859: H a r r is : Dissert. Gött,, p. I2I

r $858 / 62: v$. R e ic hen b a c h: IV 638.

VI 448 . VII 552,561 . IX 163,174 ,

I8i. XII 457. XV 110, 114, 124, 126.

XVI 250, 261, 262. XVII 266, 272 .
XVIII 480,487 . XIX 150. XX 622. XXI 589 .

1863: B u chner: Meteoriten, p. I9I. 1863: Ros e: Meteoriten, p. 65, 153. r884: M e un i er: Météorites, p. Ir6. r885: Brezina: Wiener Sammlung, p. 200, 210, 233.

1890: E a s t m a n: Met. Astron., p. 318. r894: Cohen: Meteoritenkunde, p. 72. r895: Brezina: Wiener Sammlung, p. $246,247$.

Ursprüngliches Gewicht : $167^{1} / 2 \mathrm{Kgr}$. (370 lbs.).

Nachweisbares Gewicht: 1734 gr.

\begin{tabular}{|c|c|c|c|}
\hline Bailey & 20 & Moskau & 20 \\
\hline Bement & I7 & New Haven & 26 \\
\hline Berlin, U. & 29 & Paris, E. & 190 \\
\hline Budapest & 12 & Paris, M. & II2 \\
\hline Calcutta & $4 \mathrm{I}$ & v. Schilling & 50 \\
\hline Dorpat & 28 & v. Siemaschko & \\
\hline Dresden, M. & 43 & Stockholm & \\
\hline Göttingen & I9 & Strassburg & 08 \\
\hline Gregory & Spl. & Tübingen & 170 \\
\hline Harvard, U. & 86 & Turin, J. & \\
\hline Kopenhagen & 4 & Washington, Sh. & 2 \\
\hline London, B. M. & 216 & Wien, H. M.*) & $2 \mathrm{IC}$ \\
\hline London, P. G. & 301 & & \\
\hline $\begin{array}{l}\text { Befindet sich d } \\
\text { noch im Besi } \\
\text { in Washingtc }\end{array}$ & $t z d$ & $\begin{array}{l}\text { sste Masse des Ei } \\
\text { Geological Sur }\end{array}$ & \\
\hline
\end{tabular}

\section{Madrid}

Maël Pestivien

s. Anhang Kerilis

Maêmê, Hislugari, Prov. Satsuma, Japan.

Stein, Cwa, gefallen Io. Nov. 1886.

Hierher auch vorläufig gestellt: "Oshima (London) u. ferner: ,Oynchi mura gefallen 29. Okt. I886 ع.

von 800 gr.; einige kleinere Steine wurden inzwischen abgegeben; diese sind in dem obigen nachweisbaren Gewicht vielleicht doppelt gezählt.

I) Ausserdem $15 \mathrm{gr}$. als Macao bezeichnet gezählt.

angesehen werden und in der That ein 
1888: $\mathrm{C}$ la rk e: New Meteorites. Am. Journ. (3) 35 , p. 264 . Ref. N. J. 1889 II, p. 43 .

r895: Brezin a : Wiener Sammlung, p. 245 .

Ursprüngliches Gewicht: 328 gr. (?) Nachweisbares Gewicht: $27 \mathrm{gr}$.

London, B. M. $\quad 6 \mid$ Wien, H. M. II Washington Io

Nach Clarke's Mitteilung scheint der Stein im Educational Museum in Tokio zu sein.

\section{Magdeburg}

Erxleben

Magura (Arva), Szlanicza, Arvaer Com., Ungarn.

Eisen, $\mathrm{Og}$, gefd. 1840 , beschr. 1844 . 1844: $\mathrm{H}$ a i d ing er: Wiener Zeitung 17. April 1844 und Allg. Preuss, Zeitung $1844 \mathrm{Nr}$. I15: Meteoreisen aus Ungarn; s. auch Pogg. Ann. Bd, 6I, p. $675-676$; N. J. 1844 , p. 598 .

r845: Weiss: Wiener Zeitung vom 30. März 1845 .

r846: H a i d ing e r: Graphit, pseudomorph nach Schwefelkies. Pogg. Ann. Bd. 67 , p. $437-439$; s. auch Berzelius, Jahresber. Bd, 27 (1848), p. 237 ; Fletcher in sNature * Bd. 36 (1887), p. 304 .

1847: P a t e r a legte die Resultate der chem. Analyse des Arva'er Meteoreisens vor. Haidinger's Ber. über die Mitth, v. Freunden der Naturw. Bd. 3, p. 62, $69-71$; s. auch Oesterr. Bl. f. Litt. 1847 Nr. 169, p. 670 u. Nr. 175, p. 694 ; Journ. prakt. Chem. Bd. 46 (1849), p. 183 u. Bd. $7 \mathrm{r}(1857)$, p. $59-6 \mathrm{r}$; Liebig-Kopp, Jahresber. 1847/48, p. 1307; Kenngott, Uebersicht 1844149 , p.224 - 225, 226; Rammelsberg, Handwörterbuch Suppl. IV (I849), p. I54 ; Am. Journ. (2) 8 (1849), p. 439-440; Cailletet in sL'Institut^, Nouv. Série Bd. III, p. 44. Ref. N. J. 1848 , p. 698 u. N. J. 1849 , p. 199.
1847: Haidinger macht eine Mitteilung "Schreibersit « betreffend. Haidinger's Ber. über die Mitth. v. Freunden der Naturw. Bd. 3, p. 282.

1852 : C1 a r k: Dissert. Gött., p.48-49. 1852: Wöhler u. Partsch: Analyse des Meteoreisens von Rasgatà in Neugranada, von Prof. Wöhler in Göttingen, mit Notizen über das Vorkommen u, die physikalischen Eigenschaften desselben. Sitzber. Wien. Akad. Bd.8, p. 499. Ref. Kenngott, Uebersicht 1852 , p. 92 .

1852: W ö h l e r : >Intermediär «, Pogg. Ann. Bd. 85 , p. 449.

1854: v. Boguslawski: Zehnter Nachtrag, Pogg. Ann. Ergz.-Bd.4, p.392 -393 .

1857: B e r g e m a n n : Untersuchungen von Meteoreisen, -- Meteoreisen von Arva. Pogg. Ann. Bd. 100, p. $256-$ $25^{8}$ (Analyse); s, auch Chem. Centr. 1857, p. 746-747; Journ. prakt. Chem. Bd. 71 (1857), p. 59-6r; Liebig-Kopp, Jahresber. 1857 , p. 730, 731; Kenngott, Uebersicht $1856 / 57$, p. 153 .

1859: B u c hner: Feuermeteore, p.II7, 126-127.

r859: H a r r is : Dissert. Gött., p. II4 -II5.

1860: R a mmels berg: Mineralchemie, p. 907-908, 947, 948 .

1862: Ha id inger: Sitzber. Wien. Akad. Bd. 46 II, p. 293 (Stereotypdruck einer Platte zum Vergleich mit Sarepta).

r862: K enngott u. $W$ is er: Zürcher Sammlung, p. 157.

1863: B u chner: Meteoriten, p. 168 -170 .

1863: R o s e: Meteoriten, p. $33,34,52$ $-54,55,57,138,152$.

r $858 / 65$ : v. Rei chen b a c h: IV 638. VI 448. VII 551. IX 163, I75, I76, I82. X 359, 362. XI 29I. XII 457. XIII 363. XIV 393. XV 100, I12, 128. XVI 26r. XVII 273. XVIII 487,488 , 
490. XIX 156. XX 622, 629, 634 . XXI $578,579,580,582,586,5^{87}$, 589. XXIa 172, 173, 175. XXV 436 r865: Buchner: Zweiter Nachtrag,

Pogg. Ann. Bd. 124, p. 597.

1867: Go ebe 1: Kritische Uebersicht, Mélanges phys. chim. Bd. 7 , p. 336 . r 870: R a m mels berg: Meteoriten,p.80, 82,84 .

r875: vom Rat h: Meteoriten, Verh. naturh. Ver. Bonn Bd. 32, p. 362 .

1875: Wright: Spectroscopic Examination of Gases from Meteoric Iron. Am. Journ. (3) 9, p. 298.

1875: Mohr: . Entstehungsart, Ann. Chem. Pharm. Bd. I79, p. 259, 268 -269 .

r876: Wright: On the Gases contained in Meteorites. Am. Journ. (3) II, p. 256,257 u. (3) 12, p. $167,168$. r88r: Brezina: Bericht III, Sitzber. Wien. Akad. Bd. 84 I, p. 279.

r884: M e un i er: Météorites, p.94, 96, 98, 99, 112.

r884: Wi epken: Notizen über die Meteoriten des Grossherzoglichen Museums [Oldenburg]. - 7. Arva, Abh. naturw. Ver. Bremen Bd. 8 , p. 258 -259 .

1884: Häp k e: Beiträge, Abh. naturw. Ver. Bremen Bd. 8, p. 519-520.

r885: Brezina: Wiener Sammlung, p. $200-201,207,215,216,234$.

I887: F $1 \mathrm{ight}$ : Meteorites, p. I09-no. r887: Sorby: On the microscopical structure of iron and steel. Journ. Iron and Steel Inst. 1887 , p. $255-288$. Ref. N. J. 1891 I, p. 47.

I888: K unz: >Science* Bd. II, p. II9 (Laspeyres entnommen).

1889: B r e z in a : Cliftonit aus dem Meteoreisen von Magura, Arvaer Comitat. Ann. Hof-Mus. Bd. 4, p. $102-106$. Ref. N. J. 1890 II, p. 59 ; Zeitschr f. Kryst. Bd. 20 (I892), p. 292.

1889: Weins chenk: Ueber einige Bestandtheile des Meteoreisens von Ma- gura, Arva, Ungarn. Ann. Hof-Mus, Bd. 4, p. 93-Ior. Ref. Zeitschr. f. Kryst. Bd. 20 (1892), p. $29 \mathrm{I}-292$; N. J. I89o II, p. $57-59$.

r889: C o h e n : São Julião, N. J. 1889 I, p. 223, 224, 225.

r89o: Li p p u. Pr̃ivoznik: Mittheilungen über die im Laboratorium des k. k. General-Probieramtes in Wien in den Jahren $\mathbf{1} 888$ u. $\mathbf{1} 889$ ausgeführten Analysen u. anderweitigen Untersuchungen. - II. Meteoriten, I. Meteoreisen aus Slanitza am Fusse der Magura im Arvaer Comitat in Ungarn. Bergu. Hüttenm. Jahrb. Bd. 38, p.399-400. r89o: Berthelot u. Friedel: Sur le fer météorique de Magura Arva (Hongrie). C. R. Bd, III, p. 296-300. Ref. Zeitschr. f. Kryst. Bd. 20 (1802), p. 292 ; Ber. d. d. chem. Ges. I89o, 24. November, p. 679 ; N. J. 1891 I, p. $3^{84}-3^{85}$

189o: Brezina: Untersuchungen der Herren Berthelot und Friedel in Paris über das Meteoreisen von Magura. Ann. Hof-Mus. Bd. 5 (Not), p. II2-II4. Ref. N. J. r891 I, p. $384-385$.

I89I : v. H a uer: Ann. Hof-Mus, Bd. 6 (Not.), p. 29.

r89r: Cohen u. Weinschenk: Meteoreisen-Studien. Ann. Hof-Mus.Bd. 6, p. 131, 149-152 (Analyse), 161, 162, 163,$164 ; 165$.

1892: C o he n: Meteoreisen-Studien. Ann. Hof-Mus. Bd. 7, p. $155-156$ (Analyse), $158(\mathrm{Cu}), 159,160,161$.

r893: M e unier: Remarques géologiques sur les fers météoriques diamantifères. C. R. Bd. 116, p. 409-410. Ref. N. J. 1894 I, p. 449.

r893: M e unier: Revision des fers météoriques, p. 29, 30-31.

1893: B r e z in a : Ueber neuere Meteoriten (Nürnberg), p. 165.

r894: Cohen: Meteoritenkunde, an sehr vielen Stellen.

1895: Co he n: Meteoreisen-Studien IV. 
Ann. Hof-Mus. Bd. 10, p. 82,86 , 9r. r895: Brezin a : Wiener Sammlung, p. 286 .

Ursprüngliches Gewicht: Wohl an 30 Zentner, von welchen aber der grösste Teil eingeschmolzen wurde, $\mathrm{u}$, snur etwa 2 Zentner erhalten blieben.

Nachweisbares Gewicht : $1_{3}^{8} 3^{8} 7 \mathrm{gr}$.

\section{Bailey}

Belgrad 253 Klausenburg 91

Bement

Berlin, G. 214 Kopenhagen 346 Krantz 390 Berlin, P. Berlin, U. 100 London, B. M. 9oI 1107 London, P. G. 537 Bologna Bonn v. Braun Bremen Breslau Brezina Budapest Calcutta Cambridge

Clausthal

Cleveland

Czernowitz

Debreczin

Dorpat

Dresden, M, 10 I06 Lüttich 216 Marburg 441 de Mauroy 238 Melion

II Minneapolis 708 Modena 46 Moskau 19050 München 1193 Neumann

86 New Haven

19 Newton

236 New York, M. ${ }_{115}$ Odessa 629 Oldenburg 171 Oxford

Dresden, P. Dublin, R. C. 236 Paris, M.

5 Petersburg, B.

Edinburg

Erlangen

- Freiberg, i. S. Göttirgen

Graz

Graz, J.

Gregory Greifswald

Hamburg

Harvard, U.

Heidelberg

Helsingfors

Karlsruhe, P.

Kasan
Turin, J.

I6I Wien, H. M. *) **)

Turin, U.

Upsala

Utrecht

Ward

Washington

399

30452

498 Wien, U. I. 270

59 Wien, U. II. 20

309 Wrany 157

Mainpuri Chandpur

Mainz, Grossherzogtum Hessen, Deutschland.

Stein, $\mathrm{Ck}$ (von Brezina neuerdings zu Cia gestellt, während Segowlee, mit welchem Mainz ausserordentlich grosse Aehnlichkeit hat, noch als $\mathrm{Ck}$ aufgeführt wird), gefunden I850 (1852?), beschr. 1857 .

1857: S e e $1 \mathrm{~h}$ e i $\mathrm{m}$ : Untersuchung eines bei Mainz gefundenen Meteorsteins. Jahrb. d. Ver. f. Naturk. Grossherz. Nassau (1857) Heft 12, p. 405 (Analyse); auch abgedruckt im Journ. prakt. Chem. Bd. 73 ( 1858 ), p. $235-240$; s. auch Kenngott, Uebersicht $1856 / 57$, p. 239-240; Chem. Centr. 1858, p. 487 ; Liebig-Kopp, Jahresber. 1858 , p. 808-809; Zool. Min. Ver. Regensburg Bd. 13 (1859), p. I19; Am. Journ. (2) $26\left(185^{8}\right)$, p. 436 . Ref. N. J. 1859 , p. $194-195$.

1859: B u c hner: Feuermeteore,p.ro4. 1860: Haidinger: Die Calcutta-Meteoriten, von Shalka, Futtehpore, Pegu, Assam und Seegowlee im k. k. HofMineralien - Kabinete. Sitzber. Wien. Akad, Bd. 4I, p. 755 (Vergleich mit Segowlee).

1860: R ammels berg : Mineralchemic, p. $932 \mathrm{ff}$.

I863: B u chner: Meteoriten, p. n6. r863: B u c h n e r : Meteoritische Notizen aus dem Vereinsgebiet. Ber. d. Oberhess. Ges, f. Natur- u. Heilk. Giessen, p. $93-94$.

1863: Ros e: Meteoriten, p. 155.

r858/65: v. Reichenbach: V 480 . 
VI 454. VII 551. IX 16r, 172, I73, I80. $\mathrm{X} 359,362,368,371 . \mathrm{XI} 302$, 309. XIII $355,363,365$. XV гог.

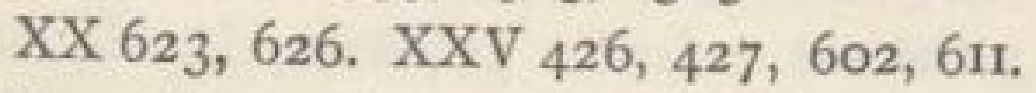
1884: M e unier: Météorites, p. 96, $247,249-250$.

I885: Brezina: Wiener Sammlung, p. 19I, 233.

r895: Brezina : Wiener Sammlung, p. $247,260$.

Ursprüngliches Gewicht: $I^{3 / 4} \mathrm{Kgr}$. ( $3^{1 / 2}$ oder $2^{1 / 2}$ (?) Pfund).

Nachweisbares Gewicht: 685 gr. Bailey

Berlin, U.

Budapest

Calcutta

Cleveland

Göttingen

Gregory

Harvard, U.

I2 Moskau

2 Neumann

25 Paris, M.

201 Pohl

2 v. Siemaschko

40 Stockholm

I3 Tübingen

Io Ward

London, B. M.

London, P. G.

34 Wien, H. M.*)

3

Wo befindet sich der grösste Teil des Steins ?

Makariwa bei Invercargill, NeuSeeland.

Stein, C, gefd. 1879, beschr. 1893.

1893: Ulrich: On a Meteoric Stone found at Makariwa near Invercargill, New Zealand. Proc. Royal. Soc. Bd. 53 , p. 5464 (Abbild. von Dünnschliffen).

1894: F l et c h er: Chemical Analysis of the Meteoric Stone found at Makariwa, near Invercargill, New Zealand, in the year 1879. Mineral. Magaz. Bd, Io, p. $287-326$ (sehr ausführliche chemische Untersuchung).

1894: C o hen : Meteoritenkunde,p.24I, 26I, 330, 33 I.

1894: F l e t c her: Introduction, p. 15.

Ursprüngliches Gewicht : $4-5$ lbs.

Nachweisbares Gewicht: 63 gr.

Am I. Juli 1893 war der Stein offenbar noch in keiner Sammlung vorhanden, wenigstens sind mir keine diesbezüglichen Angaben mitgeteilt worden. London besass 1894: $63 \mathrm{gr}$.

Manbhoom, Bengalen, Ostindien.

Stein, Amphoterit, Am, gefallen 22. Dezember 1863 .

1864: Ha iding er: Der Meteorstein von Manbhoom in Bengalen im k, k. Hof-Mineralien-Kabinete aus dem Fall am 22. Dezember 1863. Sitzber.Wien. Akad. Bd. 50 II, p. 24I-246; s, auch Am Journ. (2) 40 (1865), p. 134; Kenn-

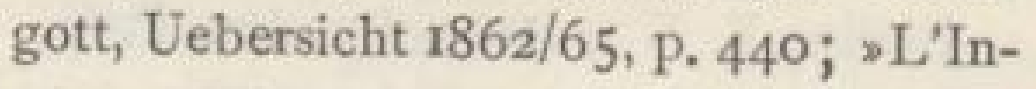
stituta Bd. 32 (1864) Nr.1617, p. 415. 1865: Buchner: Zweiter Nachtrag, Pogg. Ann. Bd, 124, p. 587 .

1883: Ts c her m a k: Beitrag, Sitzber. Wien. Akad. Bd. 88 I, p. $362-363$. 1884: M e unier: Météorites, p. 195 (Abb.), 350, 522.

r884: W a d s worth: Studies, p. 167. 1883/85: Tschermak: Photographien, T. VI, p. Io.

1885: Brezina: Wiener Sammlung, p. $175,232$.

1888: v. F o u 11 o $\mathrm{n}$ : Untersuchung der Meteorsteine von Shalka u. Manbhoom.

- 2. Der Meteorit von Manbhoom. Ann. Hof-Mus. Bd. 3, p. $202-208$. Ref. N. J. 1889 II, p. 279-281.

r894: C o hen: Meteoritenkunde, p. In2, $248,249,282,3$ Io.

r894: F letcher: Introduction, p. 13. r895: Brezina: Wiener Sammlung, p. $238,239$.

Ursprüngliches Gewicht: Mehrere Steine, der grösste $\mathrm{I}^{1 / 2} \mathrm{Kgr}$.

Nachweisbares Gewicht: 2136 gr.

Budapest

Calcutta

Göttingen

Gregory

I London, P. G. $3 \mathbf{I}$

1654 München 8

3 Paris, M. 94

Harvard, U. 3 v. Siemaschko 6

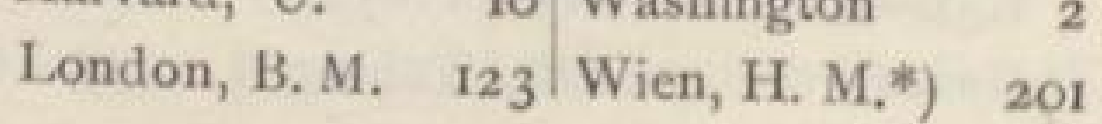


Manegaon, Khandeish, Dekan, Ostindien.

Stein, Chl, gefallen 29. Juni 1843 .

1844: A b b o t $t$ : Fall of a Meteoric Stone in Khaundes (einschliesslich verschiedener Briefe). Journ. Asiat, Soc. Bengal. Bd. 13, p. $880-886$.

1850 : Powe 11: Luminous Meteors (Mitteilungen von Abbott und Piddington), Rep. Brit. Assoc. (185o), p. 122 $-126$.

1854: v. Boguslawski: Zehnter Nachtrag, Pogg. Ann. Ergz.-Bd. 4, p. $370-37 \mathrm{x}$

r859: H a rri s: Dissert. Gött., p. 90. 1859: A t kinson: Verzeichnis derim Besitz der Asiatic Society befindlichen Meteoriten. Die Stelle lautet: Nr. 6. Fall at Manegaon in Kandeish, July 1843, procured from Captain J. Abbott, Bengal Artillery, and J. Bell, Esq. Bombay, Civil Service, Collector of Kandeish. Weight $2 \frac{1}{2}$ oz. (70 gr.). Journ. Asiat. Soc. Bengal. Proc. Bd. 28 , p. 26 o.

1863: Maskelyne u. v. Lang: Mineralogical Notes, - Notices of Aërolites von Maskelyne. 21. Manegaum. Philos. Magaz. Bd 26, p. 135 -r39; s. auch Rep. Brit. Assoc. (Not. and Abstr.) 1862, p. 191; Kenngott, Uebersicht $1862 / 65$, p. 442.

1863: B u c h n er: Meteoriten, p. 72. r863: Ros e: Meteoriten, p.ro7, I13, 156. 1865: Buchner: Zweiter Nachtrag, Pogg. Ann. Bd. 124, p. $580-5^{81}$.

r862/65: v. Reichen bach: XX631. XXV 607, 6r5.

1870: Maskely ne: On the Mineral Constituents of Meteorites, - XI. On the Manegaum Meteorite of 1843 . Philos. Trans. Bd. 160 (1870), p. $2 \mathrm{II}-2 \mathrm{I} 3$ (Analyse); s. auch grösseren Auszug in Proc. Royal Soc. Bd. 18 (1870), p. 156-157; Philos. Trans, Bd, 161 (1871), p. 367 .

W ül fing, Meteoriten.
1870: R a m m e ls berg: Meteoriten, p. 106, 120, 139; s, auch Pogg. Ann. Bd. 140 (1870), p. 319-320.

r875: vom Rath: Meteoriten, Verh. naturh. Ver. Bonn Bd. 32, p. 374.

1879: R a mme ls berg: Meteoriten, p. 25 .

1883: T s che r mak: Beitrag, Sitzber.

Wien. Akad. Bd. 88 I, p. $365-366$. 1884: Meunier: Météorites, p. 79, 85,96 .

1884: W a d s w or th: Studies, p. 201 $-2 \mathrm{O} 2$.

1883/85: Tschermak: Photographien, p. Io.

r885: Brezina: Wiener Sammlung, p. $174,232$.

r887: F 1 i g h t: Meteorites, p. II2-II3. r894: C o h e n : Meteoritenkunde, p. 29, $275,281,286,287$.

r894: F l e t c h e r: Introduction, p. Io. Ursprüngliches Gewicht: Ein grosser Stein, von welchem aber nur einige Unzen erhalten blieben.

Nachweisbares Gewicht: 44 gr.

Berlin, U. Spl, Gregory I Bonn Spl. London, B. M. II

Calcutta $\quad 31$ Wien, H. M.*) I

Mañi

Toluca

Mantos Blancos (Mount Hicks), Wüste Atacama, Chile, S. A.

Eisen, Of, gefunden um $1876, x 879$ an das British Museum geschenkt, beschr. 1889 (in Verzeichnissen schon früher erwähnt).

1885: Brezina: Wiener Sammlung, p. 262 .

r889: F l e t c h e r: Atacama Meteorites, Mineral. Magaz. Bd. 8, p. 224, 230, $257-258$ (Analyse).

1892: v. H a u e r: Ann. Hof-Mus, Bd, 7 (Not.), p. 73.

r894: F 1 e t c h er: Introduction, p. 14. r895: Brezina : Wiener Sammlung, p. 270 .

15 
Ursprüngliches Gewicht: $10.3 \mathrm{Kgr}$. Nachweisbares Gewicht: 9436 gr. Bailey I3 v. Siemaschko 9 London, B. M. gor5 Wien, H. M. 399

\section{Manzanares}

s. Anhang

Marmande,Montignacb.Marmande,

Dep. Lot et Garonne, Frankreich.

Stein, Cc, gefallen 4. Juli 1848 .

Hierher auch: Montignac, Stein,

Cc, gefunden 1848 .

1862: Greg: On some Meteorites in

the British Museum. Philos. Magaz.

Bd. 24, p. 540.

1863: B u c hner: Meteoriten, p. $7^{8}$.

1885: Brezina: Wiener Sammlung, p. 262 .

r895: Brezina: Wiener Sammlung, p. 255 .

Ursprüngliches Gewicht: Ein Stein, von welchem nur ein kleines Stück erhalten blieb.

Nachweisbares Gewicht: $63 \mathrm{gr}$.

Gregory 2 Stockholm

London, B. M.

5 Troyes

de Mauroy 25 Wien, H. M.

Marmaroscher Com.

Borkut Maros Mezö-Madaras

Marshall Co., Indiana 1893 Plymouth

Marshall Co., beschrieben 1860 Marshall Co.

Marshall Co., Kentucky, U. S. A. Eisen, Om, gefunden?, beschr. I860. r860: $\mathrm{S} \mathrm{m}$ it h: Description of three New Meteoric Irons, from Nelson County, Ky., Marshall County, Ky, and Madison County, North Carolina. Am. Journ. (2) 30, p. 240 (Analyse); s. auch Original Researches 1884, p. 409 ; Liebig-Kopp, Jahresber, 1860, p. 853 ; Journ. prakt. Chem. Bd. 84 (186r), p. $59-60$.

I863: B u c h n e r : Meteoriten, p. 194. I863: R o s e: Meteoriten, p. 64, I52. r862/65: v. Re i c hen b a ch: XXI 589 . XXV 437.

1884 : M e un ier: Météorites, p.I16,123. ז885: Brezina: Wiener Sammlung, p. $213,214,234$.

I890: E a s t m a n: Met. Astron., p. 318. r893: Meunier: Revision des fers météoriques, p. 52,56 .

1894: Co he n: Meteoritenkunde, p. 93. r895: C o h e n: Meteoreisen-StudienIV. Ann. Hof-Mus. Bd. Io, p. 82,86 .

Ursprüngliches Gewicht: $6.8 \mathrm{Kgr}$. (I 5 lbs.).

Nachweisbares Gewicht: ${ }_{13} 88 \mathrm{gr}$.

\begin{tabular}{ll|l} 
Bailey & 20 Kopenhagen & I35
\end{tabular}

Berlin, U. $\quad 72$ London, B. M. $\quad 80$

Calcutta $\quad$ I 82 London, P. G. 38

Cleveland $\quad$ 19 Moskau $\quad 80$

Cohen 6. Paris, M. $17 \mathrm{r}$

\begin{tabular}{ll|l|l|l|l|l|l} 
Göttingen & $\mathbf{1 4 2}$ & Tübingen & 45
\end{tabular}

\begin{tabular}{ll|l} 
Gregory & 17 & Washington, Sh. 68
\end{tabular}

Hamburg $\quad 5^{1}$ Wien, H. M.*) 73

Harvard, U. $\quad$ I 89

Wo befindet sich die grösste Masse des Eisens, von welcher Buchner vermutungsweise äussert, dass Smith den Hauptteil besessen habe? Smith sagt 1860, dass er die ganze Masse, deren Gewicht zu 15 lbs. angegeben werde, nicht gesehen habe.

Maryland 1825

Maryland 1854

Maryland 1888

Mascombes, Corrèze, Frankreich. Stein, $\mathrm{Cw}$, gefallen 3 r. Januar 1835 . 1864: D a u bré e: Note sur deux aérolithes, l'un tombé à Vouillé (Vienne), le 13 mai 1831 , et offert au Muséum d'Histoire naturelle par la ville de Poitiers; l'autre tombé à Mascombes, département de la Corrèze, le $3 \mathbf{1}$ janvier 1836 , et dont la chute était restée sans publicitée, C. R. Bd. 58 , p. 229-230. 
1864: Buchner: Erster Nachtrag. Pogg. Ann. Bd. 122, p. 322, 329. 1865: B u chner: Zweiter Nachtrag, Pogg. Ann, Bd. 124, p. 579. I884: M e unier: Météorites, p. 208. 1885: Brezina: Wiener Sammlung, p. $177,232$.

Ursprüngliches Gewicht: Ein Stein von etwa I Kgr.

Nachweisbares Gewicht: 566 gr.

\begin{tabular}{lr|l|r|} 
Calcutta & I & Paris, M. & 522 \\
Gregory & 7 & v. Siemaschko & 8 \\
London, B. M. & 5 & Wien, H. M.*) & I \\
Newton & 22 & &
\end{tabular}

Mässing(SanktNicolas)bei Altötting, Kreis Niederbayern, Deutschland; in der älteren Literatur auch Eggenfelde genannt.

Stein, Ho, gefallen $x_{3}$. Dez. $\mathrm{I}_{80}$. 1804: B l u m e n b a c h: Voigt's Magaz. f. Naturk. Bd. 7, p. 233 (oder 247 ?). 1804: Gil ber t: Nachträge zu den Aufsätzen in den Annalen über die aus der Luft gefallenen Steine (Bericht Imhof's aus dem kurpfalzbaierischen Wochenblatt von 1804 , p. 3 ff.), Gilb. Ann. Bd. 18, p. $330-33$ I.

r804: Pötzsch: Kurze Darstellung, p. $\mathrm{II}_{3}-\mathrm{II}_{5}$.

1808: Ch la d n i: Beitrïge, Gilb. Ann. Bd. 29 , p. $3^{82}$.

1812: Ch 1 a d n i: Verzeichnis, Schweigg. Journ. Bd. 4 Beil. I, p. 15.

1812: Bigot de Morogues, p.200 -201 .

r8r5: Chladni: Neues Verzeichnis, Gilb. Ann. Bd. 50, p. 253.

1819: $\mathrm{Ch} 1$ a d n i : Feuermeteore, p. 50, $58,59,69,73,9 \mathrm{I}, 271,273-275(1)$, $308,309,432$.

1830: $\mathrm{K} \mathrm{ä} \mathrm{m} \mathrm{tz}$ : Meteorologie, p. 280. 1843: P a r ts ch: Meteoriten, p. 29-30. r847: S chafhiiut 1: Schönenberg, Gel. Anz. München. Akad. Bd. 24, p. 558-559 (Analyse; München be- sass damals von diesem Stein nur $\mathrm{I}^{1 / 2}$ Lot). r854: v. Boguslawski: Zehnter Nachtrag, Pogg. Ann. Ergz.-Bd.4, p. 16. r859: $\mathrm{B} \mathrm{u} \mathrm{c} \mathrm{hner:} \mathrm{Feuermeteore,} \mathrm{p.} 74$ -75 .

r859: H a rris: Dissert. Gött., p. 65 . 1860: Rammels berg: Mineralchemie, p. 945 .

r862: Kenngott u. W is er: Zürcher Sammlung, p. I54.

r863: B u c hner: Meteoriten, p.r7-r8. 1863: Ros e: Meteoriten, p. 26, 28, 107 , $\mathrm{II}_{2}-\mathrm{II}_{3},{ }^{1} 56$.

r858/65: v. Reichen bach: V 475. IX $159,160,169,179 . \times 359,362$, 372. XI 294, 295. XIII $355,357$. XXIII 369 . XXIV 226. XXV $438,6 \mathrm{r}_{5}$. r865: Buchner: Zweiter Nachtrag, Pogg. Ann. Bd. 124, p. 574.

r878: Gü m b e 1: Ueber die in Baiern gefundenen Steinmeteoriten. - Der Meteorstein von Mässing. Sitzber. München. Akad. (1878) (Sep.), p. 32-40 (Analyse von Schwager; Abbildung eines Dünnschliffs).

1879: Ram melsberg: Meteoriten, p. $20-22$.

r883: T s cherma k: Beitrag, Sitzber. Wien, Akad. Bd. 88 I, p. 368.

1884: Me unier: Météorites, p. 94, 208, 210 .

1884: W a d s wort h: Studies, p. 197. ${ }^{888} / 85: T s \mathrm{ch}$ er m a k: Photographien,

T. 4 , p. 7,8 .

1885: Brezina: Wiener Sammlung, p. $174,232$.

r894: C o h e n: Meteoritenkunde, p.275. Ursprüngliches Gewicht: $1.6 \mathrm{Kgr}$. $\left(3^{1 / 4}\right.$ Pfund). Schafhäutl sagt 1847 : „Von diesem Stein, der anfangs $3 \frac{1}{4}$ Pfund wog, sind nur noch $I^{1 / 2}$ Loth vorhanden.\&

Nachweisbares Gewicht: $74 \mathrm{gr}$.

Bailey I Cleveland

Berlin, U. 22 Cohen 2

Calcutta ILondon Spl. $15^{*}$ 
München

Neumann

Paris, M.

v. Siemaschko

16 Strassburg

Spl.

Spl. Tübingen

Spl.

22 Wien, H. M.*) 2

I Zürich

3

Mauerkirchen, Oberösterr. (früher Oberbayern), Innviertel.

Stein, Cw, gefallen 20. Nov. 1768 .

r769: ?: Nachricht u. Abhandlung von einem in Bayern unfern Maurkirchen d. 20. Nov. 1768 aus der Luft gefallenen Steine (Straubingen 1769) $8^{\circ}$. I769: Bericht von dem Wunderstein, welcher unweit Maurkirchen in Bayern aus den Wolken herabgefallen sein soll. München 1769 .

r803: C h l a d n i: Chronologisches Verzeichnis, Gilb. Ann. Bd. 15, p. 310, $316-317$.

r804: B l u m en b a c h: Vogt's Magaz. Bd. 7 , p. 244 .

1804: Gilbert: Nachträge zu den Aufsätzen in den Annalen äber die aus der Luft gefallenen Steine (Bericht Imhof's aus dem kurpfalzbaierischen Wochenblatt von 1804, p. 3 ff.). Gilb. Ann. Bd. 18, p. 328-330.

r804: Pötz sch: Kurze Darstellung, p. $69-70$, II8.

r812: $\mathrm{C}$ h 1 a d n i: Verzeichnis,Schweigg. Journ. Bd. 4 Beil. I, p. 13.

r8r2: Bigot de Morogues, p.rI3, 334.

1815: Chladni: Neues Verzeichnis, Gilb. Ann. Bd. 50, p. 249 (hier auch Imhof's Analyse, die wahrscheinlich im kurpfalzbaierischen Wochenblatte I8O4, p. 3 ff, zuerst erschien).

r8rg: Chladni: Feuermeteore, p. 5I, $57,66,69,73,90,252-253(1), 266$, 271,428 .

1836: Kämtz: Meteorologie, p. 275.

1837: Rose: Reise nach dem Ural

Bd. I, p. 77 .

1843: Partsch: Meteoriten, p. 47.

1847: Schafhäut 1: Schönenberg,

Gel. Anz. München. Akad. Nr. 7o
(1847), p. $563-564$.

r859: B u c hner: Feuermeteore, p. 44. r859: H a r r i s: Dissert. Gött., p. 60. r862 : Kenngott u. Wiser: Zürcher Sammlung, p. 155 .

r863: B u c hner: Meteoriten, p. 8-9. r863: R os e: Meteoriten, p. 84,86 , $92-93,98,154$.

1863: Anonymus (wahrscheinlichWöhler) giebt im Referat über Buchner's s Meteoriten « an, dass Göttingen 1927 gr. besitze. Gött, Gel. Anz. (1863), p. 1240. I $858 / 65$ : v. Reichen b a $\mathrm{c}$ : IV 637 V 475. VI 456. VII 552 . IX 155,161 , 167, 168, 178. X $359,363,372$, XI $294,297,300,306$. XIII 360,373 (Fig.), 383. XX 622, 626. XXIV 226. XXV $438,607,609,615$.

1868: Crook: On the Chemical Constitution of the Ensisheim, Mauerkirchen, Shergotty and Muddoor Meteoric Stones.Dissert.Gött.,p.26-3o(Analyse).

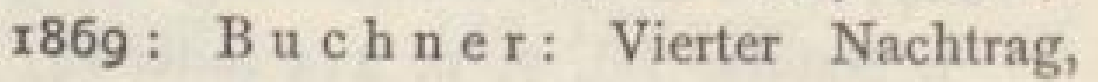
Pogg. Ann. Bd. 136, p. 44I.

1870: Rammelsberg: Meteoriten, p. $103,107,139,140$.

1878: G ü m b e 1: Die in Bayern gefundenen Steinmeteoriten, - Der Meteorstein von Mauerkirchen. Sitzber. München. Akad. (1878), p. 16-24 (Analyse von Schwager; Abbildung von einem Dünnschliff).

1879: Rammelsberg: Meteoriten, p. $12-13,24$.

1883: Tschermak: Beitrag, Sitzber.

Wien. Akad. Bd. 88 I, p. 355 .

1884: W a d s w or th: Studies, p. Io3. r884: M eunier: Météorites, p. 79, 208, 209.

r883/85: Tscherma k: Photographien, p. 18.

r885: Brezina: Wiener Sammlung, p. $177,232$.

r89o: B r e z in a : Reise, Ann, Hof-Mus, Bd. 4 (Not.), p. 122.

Ursprüngliches Gewicht: I9 $_{9} \mathrm{Kgr}$. (38 Pfund). 
Nachweisbares Gewicht: I2 $55^{8}$ gr.

\begin{tabular}{lr|lr} 
Aachen & 52 & Madrid & I \\
Belgrad & 7 & Moskau & 12 \\
Bement & 84 & München & 77 II \\
Berlin, U. & 221 & Neumann & 55 \\
Bologna & 1 & New Haven & 12 \\
v. Braun & 5 & Paris, M. & 212 \\
Budapest & 133 & Petersburg, B. & 33 \\
Calcutta & 7 & v. Siemaschko & 5 \\
Cambridge & 169 & Stockholm & 20 \\
Cohen & Spl. & Strassburg & 22 \\
Darmstadt, M. & 80 & Stürtz & 45 \\
Dorpat & 17 & Stuttgart & 6 \\
Gotha & 44 & Troyes & 64 \\
Göttingen & I920 & Tübingen & 149 \\
Gregory & 75 & Utrecht & 6 \\
Harvard, U. & 9 & Ward & I8 \\
Kopenhagen & 114 & Washington,Sh. Spl. \\
Kunz & II0 & Wien, H. M.*) & 588 \\
London, B. M. & 302 & Würzburg & I56 \\
London, P. G. & 92 & Zürich & I
\end{tabular}

Mauritius, Isle de France, Indischer Ozean (Isle aux Tonneliers im Nordwesten der Insel Mauritius). Stein, Cho, gefallen zwischen 22. Dez. 1801 u. 20. Januar 18021 ). r804: B ory de Saint-Vincent: Voyage dans les quatre principales îles des mers d'Afrique fait par ordre du gouvernement pendant les années neuf et dix de la République (1801 u. 1802) Bd. 3 an XIII (1804), p. 254-262. Paris bei Buisson.

1806: L u c a s: Tableau méthodique des espèces minérales Bd. 2, p. 367. Paris 1806 bei d'Hautel.

1819: $\mathrm{Ch}$ la dni: Vierte Fortsetzung, Gilb. Ann. Bd. 6o, p. 246-247.

r8rg: $\mathrm{Ch} 1$ a d n i : Feuermeteore, p. 7r, $74,140,268$ (!).

r836: Kä m tz: Meteorologie, p. 278 .
1854: B a 1 c e 11 s: Lithologia meteorica, p. 22.

1857: Arago: Astronomie populaire Bd. 4 , p. 195 .

1859: Harris: führen den Fall 1863: Buchner: nicht auf. r885: Brezina: Wiener Sammlung, p. 177,232 .

1895: Brezina: Wiener Sammlung, p. $24 \mathrm{I}$.

UrsprünglichesGewicht:DreiSteine; „einer war von der Grösse einer Melone, und zwey wie Orangen $\%$. Nachweisbares Gewicht: 243 gr.

\begin{tabular}{lr|ll} 
Bailey & 33 & Harvard, U. & I \\
Bement & 2 & Wien, H. M. & 3 \\
Edinburg & 204 & &
\end{tabular}

Mavrick Co. Coahuila (FortDuncan) Mazapil, Staat Zacatecas, Mexico. Eisen, Om, gefallen 27 . Nov, 1885 . r887: Hid den: On the Mazapil Meteoric-iron, which fell November $27^{\text {th }}$ I885. Am. Journ. (3) 33, p. 221-226 (Analyse von Mackintosh; Abbildung des Eisens und einer geätzten Platte; Karte mit Fallort); s, auch Ann. of the N. Y. Acad. of Sc. Bd. 4, p. 45 -65 . Ref. N. J. 1888 I, p. 210-2II; Liebig-Kopp, Jahresber, 1888, p. 574. 1889: Brezina: Ankunft der Hidden'schen Meteoriten- und Mineraliensammlung für die mineralogische Abteilung. Ann. Hof-Mus. Bd, 4 (Not.), p. 86. r89o: v. H a u er: Ann. Hof-Mus. Bd. 5 (Not.), p. $5,48$.

1890: F let c her: Mexican Meteorites, Mineral. Magaz. Bd. 9, p. 95-99.

1890: E a stm a n : Met. Astron, p. 316. 1890: Brez in a: Ueber Meteoreisen, Oesterr. Zeitschr. f. Berg- u. Hüttenw. Bd. 38, p. 355 .

1892: P f la u m: Der Meteorit von Ma-

I) An X. Nivôse. Nach der Zeittafel von Major Ed. Jäger, Stuttgart (Meyer's Konvers.-Lex. 4. Aufl. Bd. 9, p. $3^{85}$ ) reicht der Monat Nivôse des zehnten Jahres vom 22. Dezember 1801 bis 20. Januar 1802 . 
zapil - ein Bruchstïck des Biela'schen Kometen. Korresp. des Naturf,-Ver. Riga Bd. 35 , p. 8-16 u. Sitzber. im gleichen Heft, p. $37-38$; s. auch Bibl. géol. de la Russie Bd. 8, p. 126, I27. Ref. N. J. 1895 I, p. 33.

1892: B r e z in a: Sternsehnuppen, p. 8. 1893: B r e z in a : Ueber neuere Meteoriten (Nürnberg), p. 164.

r894: Co h e n: Meteoritenkunde, p. 73, $83,149,232$.

r894: F 1 e t c h e r : Introduction, p. 34,47. r895: Brezina: Wiener Sammlung, p. $282-28_{3}, 308-327$. Berichte des Directors der Sternwarte Zacatecas, Professor José A. y Bonilla, an Herrn William E. Hidden in Newark über den Sternschnuppenfall vom 27. Nov. I885 und über den Fall des Meteoreisens von Mazapil.

Ursprüngliches Gewicht: $3950 \mathrm{gr}$. (Bonilla giebt das Gewicht zu 4090 gr. an).

Nachweisbares Gewicht: 3780 gr.

Bailey

Bement

Gregory

\section{Mecca}

Mecherburg

Medwedewa

Mejillones Eisen

5 I London, B. M. I4

93 Ward

I9 Wien, H. M. 3546

\section{Mejillones Mesosiderit (Grahamit)}

Vaca Muerta

Mejillones, Wüste Atacama, Bolivia, S. A.

Eisen, Hb, gefd. ?, beschr. 1875 .

Hierher auch vorläufig das»PolankoEisen Domeyko's gestellt.

1875: D o m e y k o : Note sur deux nonvelles météorites du désert d'Atacama, et observations sur les météorites qui ont été découvertes jusqu'ici dans cette partie de l'Amérique méridionale. II. Fer météorique des environs de Mejil- lones C. R. Bd. 81, p. $597-598$ (Analyse). Mit Anmerkung Daubrée's im gleichen Band, p. 60o. Ref. Licbig-Kopp, Jahresber. 1875, p. 13151316. S. auch Domeyko's Mineralojia 3. Aufl. (1879), p. $131-132,137$.

1884: Me unier: Météorites, p. 133. 1884: Wadsworth: Studies, p. 74. r885: Brezina: Wiener Sammlung, p. 262 .

1889: F let c her: Atacama Meteorites, Mineral. Magaz. Bd, 8, p. 224, 257.

1893: M e un i er: Revision des fers météoriques, p. $75-76$.

1894: C o he n: Meteoritenkunde,p.r34. r895: Brezina: Wiener Sammlung, p. 292.

Ursprüngliches Gewicht: Grosse Masse, die nur mittelst Wagen fortzuschaffen wäre.

Nachweisbares Gewicht: I 185 gr.

Bailey

Bement

v. Braun

Cleveland

Dresden, M.

8 Harvard, U. 703

37 Paris, M. $\quad 164$

59 v. Siemaschko 2

I Washington, Sh. 30

5 Ward $\quad 176$

\section{Melbourne}

Cranbourne

Menow, Mecklenburg, Deutschland. Stein, Cck (früher als Ck aufgefasst), gefallen 7 . Oktober 1862 .

1862: Meteorsteinfall bei Menow in Mecklenburg-Strelitz. Mitteilung zusammengestellt aus Zeitungsberichten. Pogg. Ann. Bd. I17, p. $637-638$; s. auch Kenngott, Uebersicht $1862 / 65$, p. 435 . Ref. N. J. 1863, p. I00-101; LiebigKopp, Jahresber. 1862 , p. 825 .

r862: Gre g: On some Meteorites in the British Museum. Philos. Magaz. Bd. 24, p. 541 .

r863: B u c hn er: Meteoriten,p.ro9-Iro. 1863: Ros e: Meteoriten, p. 154.

1863: B o 11: Meteorstein bei Menow in Mecklenburg-Strelitz gefallen. Arch, d. Freunde d. Naturgesch. Neubranden- 
burg (1863) Bd. 17, p. $282-284$. r865: v. Reichen b a c h: XXV 606, $6 \mathrm{I}_{5}$.

1868/70: Rec. Geol. Surv. India Bd. I, p. 72 u. Bd. 2, p. 34 (das Museum der Geol. Surv, erhält von Nevill ein Stück).

r87r(?): Haidinger: Der Meteorstein von Meno. In einem Ausschnitt einer Zeitschrift im Besitz des k. k. Hof-Mus. Wien (welche Zeitschrift vorlag, liess sich nicht erkennen) giebt Haidinger an, dass v. Reichenbach den Stein für $100 \mathrm{Pfd}$. Sterl. an Nevill verkauft habe. Nach einer Angabe Buchner's hatte v. Reichenbach den Stein für 400 Thlr, erworben.

1875: vom Rath: Meteoriten, Verh. naturh. Ver. Bonn Bd. 32, p. 369 .

1876: S m ith: An account of a New Meteoric Stone that fell on the $25^{\text {th }}$ March 1865, in Wisconsin, identical with the Meno-Meteorite. Am. Journ.

(3) 12, p. 207-209 (Analyse); s, auch

C. R. Bd. $83(1876)$, p. $161-166$; Original Researches 1884 , p. 519-522. Ref. N. J. 1877 , p. $4 \mathrm{II}$; Cohen, N. J. 1887 II, p. 52 .

r879: R a m mels ber g : Meteoriten, p. 13.

1884: M e unier: Météorites, p. $97,18_{3}$ -184 .

1885: Brezina: Wiener Sammlung, p. $191,233$.

r889: F l e t c h er: Atacama Meteorites, Mineral. Magaz. Bd. 8, p. 252.

1895: Brezina: Wiener Sammlung, p. $259,260$.

Ursprüngliches Gewicht: 101/2 Kgr. (21 Pfund).

Nachweisbares Gewicht: $6325 \mathrm{gr}$.

\begin{tabular}{lr|lr|} 
Bailey & 4 & Budapest & II3 \\
Belgrad & 8 & Calcutta & 2621 \\
Berlin, U. & 498 & Cambridge & 22 \\
Bonn & 23 & Dorpat & 2 \\
v. Braun & 22 & Göttingen & 36
\end{tabular}

\begin{tabular}{lr|lr} 
Gregory & 80 & Rom, U. & 5 \\
Harvard, U. & 146 & v. Siemaschko & 35 \\
Kopenhagen & 12 & Stockholm & 290 \\
London, B. M. & 1132 & Strassburg & 3 \\
London, P. G. 918 & Tübingen & 6 \\
Neumann & 28 & Ward & 2 \\
Paris, M. & 113 & Washington & 2 \\
Pohl & 44 & Wien, H. M.*) & 160
\end{tabular}

Merceditas, auch El Chañaralino Meteorit genannt, Valparaiso, Chile, S. A.

Eisen, Om, gefunden vor 1884, beschr. 1890 .

r89o: E a st m a n : Met. Astron., p. 322. I890: H o we11: Description of New Meteorites. - The El Chañaralino Meteorite. Proc. Rochester Acad, Bd. I, p. 99-100 (mit Abbildung); auch abgedruckt in The Ward Collection of Meteorites, Rochester 1892 (bei Lace). Ref. N. J. 1892 II, p. 36.

1892: v. H a u e $r$ : Ann. Hof-Mus. Bd. 7 (Not.), p. 72.

1893: B rez in a: Ueber neuere Meteoriten (Nürnberg), p. I64.

1893: M e un i er: Revision des fers météoriques, p. 52,59 .

r894: Co h e n : Meteoritenkunde, p. 58, $59,73,193,195,199$.

1895: Brezina: Wiener Sammlung, p. 280 .

1895: C o h e n: Meteoreisen-Studien IV. Ann. Hof-Mus. Bd. Io, p. 82, 90.

Ursprüngliches Gewicht: $43.4 \mathrm{Kgr}$. Nachweisbares Gewicht: $30749 \mathrm{gr}$.

\begin{tabular}{lr|lr} 
Bailey & 336 & Krantz & 90 \\
Bement & 2554 & London, B. M. & 1917 \\
Böhm & 530 & Paris, E. & 126 \\
v. Braun & 518 & Paris, M. & 220 \\
Brezina & 2410 & Prag, B. U. & 171 \\
Budapest & 2124 & Prag, M. & 141 \\
Calcutta & 180 & Riga & 60 \\
Dorpat & 882 & Rom, U. & 237 \\
Gregory & 255 & Sevilla & 20 \\
Greifswald & 102 & v. Siemaschko & 172
\end{tabular}


Stockholm

Strassburg

Ward

$$
\begin{array}{r|l}
108 & \text { Wien, H. M } \\
51 & \text { Wrany } \\
10149 &
\end{array}
$$

Mern bei Prastö, Dänemark.

Stein, C, gefallen 29. August 1878 .

1878: T rom hold: Meteorsteinfall in

Dänemark. Wochenschr. f. Astr., Met.

u. Geogr. Jahrg. 1878 (Halle 1879), p. $391-392$.

Ursprüngliches Gewicht: Ein Stein von $4^{1 / 8} \mathrm{Kgr}$. ( $8^{1 / 4} \mathrm{Pfd}$. dänisch). Nachweisbares Gewicht: 3790 gr.

Kopenhagen 3790

\section{Mesquital}

San Francisco del Mesquital Meung sur Loire Charsonville Mexico Eisen Humboldt-Eisen Mexico Stein Mexico

Mexico s. auch Anhang

Mexico, Dorf Mexico, Prov. Pampanga, Philippinen.

Stein, Cgb, gefallen 4. April $1859^{1}$ ). 1868: D a ubrée: Météorite tombée (an $1859^{\text {? }}$ ) aux îles Philippines, C, R. Bd. 66 , p. $637-639$. Ref, LiebigKopp, Jahresber. I868, p. 1045.

r869: B uchner: Vierter Nachtrag,

Pogg. Ann. Bd, 136, p. 452-453.

r884: M e uni e r: Météorites,p.8I(Abb.), 246 (Abb.), 247, 255, 379.

r885: B rezina: Wiener Sammlung, p. $183,233$.

1894: C ohen: Meteoritenkunde, p.3I7. 1895: Brezina: Wiener Sammlung, p. 251 .

\begin{tabular}{|c|c|c|}
\hline Calcutta & I & Paris, M. \\
\hline Gent & 27 & v. Siemaschko \\
\hline Condon, B. M. & 2 & Wien, H. M. \\
\hline
\end{tabular}

Ursprüngliches Gewicht : ?

Nachweisbares Gewicht: 169 gr.
Mezö-Madaras, Maros, Siebenbürgen, Oesterreich-Ungarn, auch vielfach Fekete (auf deutsch: Weiler) bezeichnet.

Stein, $\mathrm{Cgb}$ (früher als Cga aufgefasst), gefallen 4. Sept. $185_{2}$.

I852: K n ö p fl e r: Bericht über den am 4. September d. J. bei Mezö-Madaras stattgefundenen Aerolithenfall. Verh, d. Siebenbürg. Ver. Bd. 3, p. 153--154i s. auch Zipser, Ein Aerolithenfall. Corr. Bl. d. Regensburg. Ver. Bd. 6 (1852), p. 128 .

r853: Kn öpfler: Bericht über den am 4. September 1852 bei Mezö-Madaras in Siebenbürgen statt gehabten Meteoriten-Fall nach ämtlichen Berichten. Verh. d. Siebenbürg. Ver. Bd. 4, p. $19-24$; s. auch Corr, Bl. d. Regensburg. Ver. Bd. 7 (1853), p. I6. Ref. N. J. 1853 , p. 725 u. 1856 , p. 694 ; Liebig-Kopp, Jahresber. 1852, p. 988 ; Kenngott, Uebersicht 1852 , p. $151-152$ u. 1853 , p. $159-16 \mathrm{I}$; Wochenschr. f. Astr., Met. u. Geogr. 1853 , p. $73-74$.

1853: Parts ch u. Kn öp fler: Ueber den Meteorstein-Niederfall unweit MezöMadaras in Siebenbürgen am 4. September 1852. - Auszug aus den ämtlichen Berichten über den am 4. Sept, 1852 bei Mezö-Madaras in Siebenbürgen stattgehabten Meteoriten-Fall (verfasst von Knöpfler). Sitzber. Wien. Akad. Bd. II, p. 674-68I ; s, auch LiebigKopp, Jahresber. 1853, p. $934-935$; Pharm. Centr. 1854, p. 203 ; Pogg. Ann. Bd. 91 (1854), p. $627-628$.

r854: $\mathrm{Kr}$ an tz legte einenschönen Me-

1) Das Datum nach Herrn Direktor Brezina, der mich darauf aufmerksam machte, dass eine Arbeit von Llanos publiziert sei: Obs. y diseno de los aerol. caido en Pampagna 4. V1. 1859. Das Musée Royale d'histoire naturelle in Brüssel besitzt nach Brezina ebenfalls ein Stüick von dieser Lokalität. 
teorstein, zwei Pfund schwer, von dem Meteorstein-Niederfall unweit Mezö-Madaras in Siebenbürgen am 4. September 1852 vor. Verh. naturh. Ver. Bonn Bd. II (Sitzber.), p. XVIII-XIX.

r855: K nöpfler: Verh. d. Siebenbürg. Ver. Bd. 6, p. 87 (nicht eingesehen). Ref. Kenngott, Uebersicht 1855 , p. 158.

r855: Wöhler (und Atkinson): Analyse der Meteorsteine von MezöMadaras in Siebenbürgen. Sitzber. Wien. Akad. Bd. 17, p. 284-287; auch abgedruckt in Ann. Chem. Pharm. Bd, 96 (1855), p. 251-255; Philos. Magaz. (4) II (1856), p. 14I; Journ. prakt. Chem. Bd. 68 (1856), p. 357-359; Pharm. Centr. 1855 , p. 777 ; $\gg$ L'Institute Bd. 23 (1855) Nr. 1477 , p. 459 u. Bd. 24 (1856) Nr. 1168, p. 187-188; Am. Journ. (2) 22 (1856), p. 272; Verh. u. Mitt. d. Siebenb. Ver. Bd. 9 (1858), p.166-167; Kenngott, Uebersicht 1855 , p. 158-159; Liebig-Kopp, Jahresber. 1855, p. $1022-1023$.

1856: Hausmann u. Wöhler: (Vergleich mit dem Stein von Gnarrenburg). Gött. Gel. Anz. (Nachrichten), p. 150 ; s, auch Pogg. Ann. Bd. 98 (I856), p. 6r4.

?: Giebel u. Heintz: Zeitschr, f. d. gesamte Naturw. Bd. 7, p. 77.

1859: H a r r i s : Dissert.Gött.,p.94-95. r859: Buch ner: Feuermeteore, p. 100 $-\mathrm{IOI}, \mathrm{IO} 3$.

1860: $\mathrm{R}$ a m mels berg: Mineralchemie, p. 923 ff., 95 I.

1863: B u c h n er : Meteoriten,p. $82-83$. r863: R o s e : Meteoriten, p. $85,86,87$, 92,154 .

r864: S o r b y : On the Microscopical Structure of Meteorites. Proc. Royal Soc. Bd, 13 , p. $333-334$. Ref. Am. Journ. (2) 41 (1866), p. 138 .

r859/65: v. Re ichen b a ch: IX r6r, 171, 180. X 359, 362, 372. XI 296, 300. XIII $355,362,366,376,378$.
XIV 399. XX 623. XXI 577. XXIII 369. XXV 428, 433, 603, 607 .

r870: R a m me lsberg : Meteoriten, p. $103,107$.

r870: Ts chermak: Goalpara, Sitzber.Wien. Akad. Bd. 62 II, p. 860 .

I87I : v. B a u $\mathrm{m}$ h a uer: (Vergleich mit Tjabé). Arch. Néerl. Bd. 6 Nr. 4 ( $187 \mathrm{r})$.

187r: Ra mmelsberg: Ueber den Meteorstein von Mezö-Madaras. Ber. d. d. geol. Ges. Bd. 23 , p. $734-737$ (neuc Analyse). Ref. Liebig-Kopp, Jahresber. 1871, p. 1237-1238.

1875: Ts chermak: Vulkanismus, Sitzber. Wien. Akad. Bd. 71 II, p. 671. 1875: vom R a th: Meteoriten, Verh. naturh. Ver. Bonn Bd. 32, p. 368 .

1875: $\mathrm{M}$ ohr: Entstehungsart, Ann. Chem. Pharm. Bd. 179, p. 259-260. 1877: Sorby: On the structure and

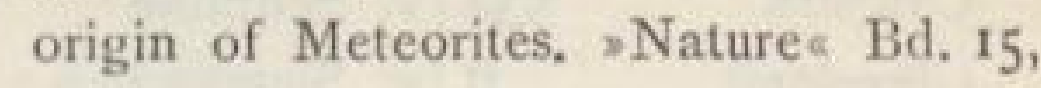
p. 496 .

1879: R a m mels berg: Meteoriten, p. $13,24,25$.

1882: W i e c hman n: Fusion-Structures in Meteorites. Ann. N. Y. Acad. of Sc. Bd. 2, p. 293, 295 (Taf. 20). 1883: T s c herm a k: Beitrag, Sitzber. Wien. Akad. Bd. 88 I, p. 355,356 , $357,35^{8}$.

r884: W a d s w or th: Studies, p. 104. 1884: Meunier: Météorites, p. 79 , $95,273,275-276,335,348$.

1884: $\mathrm{H}$ äpke: Beiträge, Abh, naturw. Ver. Bremen Bd. 8, p. 521 .

1883/85: Tschermak: Photographien,

T. $7,10,11,13,14,15,18,19$, p. 12, $13,14,15,16,17,18$.

r885: Brezina: Wiener Sammlung, p. $182,233$.

1887: Flig ht: Meteorites, p. Io, II7. 1890: v. Niess 1: Periheldistanzen, Verh. naturf. Ver. Brünn Bd. 29, p. 188, 195, 196, 214, 244-245.

r894: Cohen: Meteoritenkunde, an vielen Stellen, 
r894: F l e t c her: Introduction,p, 27,39. 1895: Brezina: Wiener Sammlung, p. 250 .

Ursprüngliches Gewicht : Steinschauer; der grösste Stein ro Kgr. (I8 Wiener Pfund). Aufgefunden wurden nach Knöpfler I 855 (s. o.) grösstenteils Bruchstücke im Gesamtgewicht von $40 \mathrm{Pfd}$. I9 Lot $=22.7 \mathrm{Kgr}$.

Nachweisbares Gewicht: 24393 gr.

\begin{tabular}{|c|c|c|}
\hline Dalley & 12 & Marburg \\
\hline Bement & 79 & Melion \\
\hline Berlin, U. & 2977 & Neumann \\
\hline Bologna & I & New Haven \\
\hline Bonn & 333 & Paris, M. \\
\hline Braun & II5 & Pohl \\
\hline remen & & Prag, M. \\
\hline est & 1409 & Rom, U. \\
\hline Cal & & v. Schilling \\
\hline Clev & II & v. Siemaschko \\
\hline Jorpat & 48 & Stockholm \\
\hline resden, M. & 12 & Strüver \\
\hline ien & 19 & Stürtz \\
\hline Göttingen & $5^{2}$ & Szamosujvar \\
\hline iraz, J. & 160 & Troyes \\
\hline Gregory & 25 & Tübingen \\
\hline Halle & 267 & Turin, U. \\
\hline ard, U. & 73 & Upsala \\
\hline Heid & 51 & \\
\hline Klausenburg & 225 & Ward \\
\hline Kopenhagen & 129 & Washington,Sh. \\
\hline London, B. M. & 734 & Wien, H. M.*)* \\
\hline ondon, P. & & \\
\hline - & & 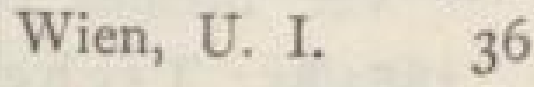 \\
\hline
\end{tabular}

Mhow, Distrikt Azim Gur, N.W.Provinz, Ostindien.

Stein, Ci, gefallen 16. Febr. 1827 . 1828: Edinburgh Journ. of Sc. Juli 1828, p. 172 .

I) Davon 15 gr. als Macao bezeichnet, s. Anm. p. 220.

2) Buchner's Angaben, dass Calcutta 354, London 153 und Wien 25 gr. besessen hätten, sind wohl Katalogen entnommen, welche aus verschiedenen Zeiten stammen.

I 828 : ? F e r us s a c : Bull, des Sc. math. Bd. II, p. 292.

I832: v. H off: Achter Nachtrag, Pogg. Ann. Bd. 24, p. 226.

r836: K ä m t z : Meteorologie, p. 296.

1843: P a rts ch: Meteoriten,p.142-143. 1854: v. Boguslawski: Zehnter Nachtrag, Pogg. Ann. Ergz.-Bd. 4, p. 418 .

1859: Harris: Dissert. Gött., p. 8o. 1859: A tkinson: Verzeichnis der im Besitz der Asiatic Society befindlichen Meteoriten, Die Stelle lautet: $>$ Nr. 5 . Fell at Mow, Ghazepore, Februar 1827, procured from R. Borlow, Esq. Weight $12 \frac{1}{2}$ oz. $\approx$ Journ. Asiat. Soc, Bengal (Proc.) Bd. 28, p. 260.

1863: Maskel yne u. v. Lang: Mineralogical Notes, - Notices of Aërolithes von Maskelyne.-15. Mhow. Philos. Magaz. (4) 25, p. $447-449\left(5^{1 / 4}\right.$ oz. von der Asiatic Soc. an das Brit. Museum); s, auch Rep. Brit. Assoc. 1862 (Not. and Abstr.), p. 190; Kenngott, Uebersicht $1862 / 65$, p. 442 .

r863: B u c hner: Meteoriten, p. 50. 1863: Rose: Meteoriten, p. I55.

r865: Buchner: Zweiter Nachtrag,

Pogg. Ann, Bd. 124, p. 578 .

I884: M e un i e r : Météorites,p.208,216. r885: Brezina: Wiener Sammlung, p. $18 \mathrm{I}, 232$.

1894: F let c her: Introduction, p. ro. Ursprüngliches Gewicht: Erhalten blieb wohl nur das 1859 in Calcutta vorhandene Stiuck von 354 gr. $\left.\left(\begin{array}{lll}12 & 1 / 2 & \text { Oz. }\end{array}\right)^{2}\right)$.

Nachweisbares Gewicht: 333 gr.

Berlin, U. I London, P. G. II

\begin{tabular}{ll|l} 
Calcutta & 127 & Paris, M.
\end{tabular}

London, B. M. 163 Wien, H. M.*). 24

Middlesbrough (Pennyman's Si- 
ding), zwischen Middlesbrough u. Ormesby, Yorkshire, England. Stein, Cw, gefallen r4. März 188 r. 188r: Hers che 1: Notice of the fall of an aërolite near Middlesbrough, Yorkshire on the $14^{\text {th }}$ of March 188I and brief account of some of the particulars of the occurrence. York. Printed by Benjohnson and Co. Micklegate; s. auch Monthly Notices of the Astron. Soc. Bd.4I, p.444; $\gg$ DerNaturforscher $\propto$ (188I) Bd. I4, p. $46 \mathrm{I}$; N. J. 1883 I, p. 380 ; Newcastle Daily Chronicle $*$, 30. März 1881 u. April 1 st. 1881 (Additional note of the sound of the explosion heard at Northalleston etc.). Newcastle-onTyne.

1882/83: F lig ht: Report of an examination of the Meteorites of Cranbourne, in Australia ; of Rowton in Shropshire; and of Middlesbrough, in Yorkshire. Philos. Trans, of the Royal Soc, 1882 III, p. 896-899. London 1883 (Analyse u. Abb.). Ref. N. J. 1884 II, p. 29-30. Auszug in Proc. Royal Soc. Bd. $33(188 \mathrm{I} / 82)$, p. $346-347$; Journ. Chem. Soc. 1884 , p. 977 ; Liebig-Kopp, Jahresber. 1882 , p. 1642 .

r884: M e u ni er: Météorites,p.209,225 -226 .

1885: Brezina: Wiener Sammlung, p. $156,177,180,232$.

1887: F l ig h t: Meteorites, p. 218-219;

s. auch oben $188_{2} / 188_{3}$.

1894: Cohen: Meteoritenkunde, p. 53, II2, 303 .

r894: Flet c h er: Introduction, p.14,26.

Ursprüngliches Gewicht: Ein Stein von 1594 gr. (3 lbs. $81 / 4$ oz.).

Nachweisbares Gewicht: $28 \mathrm{gr}$.

\begin{tabular}{l|l|l|} 
Gregory & I & Paris, M. I
\end{tabular}

London, B. M. 26 Wien, H. M. Spl.

Nach Flight's Angaben soll der Stein im Museum in York sein. Wenn derselbe nicht hoch orientiert ist, so wïre es wünschenswert, noch einige Stücke zur Verteilung an wissenschaftliche Sammlungen gelangen zu lassen.

\section{Midt-Vaage}

Tysnes

Mighëi, Transkaukasien, Russland. Stein, K, gefallen 18 . Juni 1889 .

1890: v. Siemaschko: (Ref. einer Mitteilung v. Siemaschko's aus $>$ L'A stronomie a). s Nature Bd. 4I, p. 272.

1890: M e un ie r: Analyse dela météorite de Migheï (Russie); présence d'une combinaison non signalée jusqu'ici dans les météorites. C. R. Bd. Io9, p. 976 -978 (Analyse). Ref. N. J. 189o II, p. $229-230$; Ber. d. d. chem. Ges. Bd. 23 (189o), p. 141.

r893: B rez in a : Ueber neuere Meteoriten (Nürnberg), p. r6 1 .

r894: Cohen: Meteoritenkunde, p. $156,162,167,236,283$.

1895: Brezina: Wiener Sammlung, p. 254 .

Ursprüngliches Gewicht: ?

Nachweisbares Gewicht: 7948 gr.

\begin{tabular}{lr|lr} 
Bailey & 29 & Odessa & 2765 \\
Belgrad & 6 & Paris, M. & 67 \\
Berlin, U. & 20 & Petersburg, B. & 215 \\
Böhm & 40 & Riga & 48 \\
v. Braun & 58 & Rom, U. & 13 \\
Budapest & 168 & v. Siemaschko & 4 III \\
Gregory & 27 & Stockholm & 18 \\
Kasan & 35 & Strassburg & 12 \\
Klausenburg & 6 & Turin, J. & 6 \\
Krantz & 4 & Utrecht & 4 \\
Krüger & 7 & Ward & 10 \\
Kunz & 44 & Wien, H. M. & 127 \\
London, B. M. & 87 & Wien, U. II. & 3 \\
München & 18 & &
\end{tabular}

\section{Mikenskoi Grosnaja}

Milena (Pusinsko Selo), Warasdiner Com., Croatien, Oesterreich. Stein, Cw, gefallen 26. April 1842 . 1842: K o c e var: Meteorsteinfall bei Milena in Croatien, Pogg. Ann.Bd. 56, p. 349-350. Auszug aus dem Bericht von Dr, Kocevar in der Wiener Zei- 
tung vom 22. u. 23. Mai 1842 ; s, auch sL'Institut \& Bd, 10 (1842), p. 320, 460. r843: v. Rosthorn: Briefl. Mitt. N.

J. 1843 , p. $79-8$ o.

1843: P a rts c h: Meteoriten, p.56-57. I854: v.B o g u s l a w sk i: ZehnterNach-

trag, Pogg. Ann. Ergz.-Bd. 4, p. 366. 1859: Harris: Dissert. Gött., p. 88. 1863: B u c hn er: Meteoriten, p.67-68. 1863: R o s e : Meteoriten, p. I55.

r858/65: v. Reichen b c h: V 477. VI 441. IX 16I, 167, 168, 178. X 372 . XI 294, 297. XIII 360,369 (Fig.), 378. XX 623, 626. XXV 607, 615. 1883: Tschermak: Beitrag, Sitzber. Wien. Akad. Bd. 88 I, p. $355,359$. r884: M e unier : Météorites,p.208,217. r883/85: Tschermak: Photographien,

T. 16, p. $12,16,18$.

r885: B rezina: Wiener Sammlung, p. $176,177,180,232$.

1894: Cohen: Meteoritenkunde,p.313.

Ursprüngliches Gewicht: Etwa 5$6 \mathrm{Kgr}$. (ro-I I Pfund). Im Ganzen zwei, nach Anderen drei Steine; Kocevar erhielt ein Stück von $2 \frac{1}{8}$ Pfund und sah ein anderes von 4 Lot.

Nachweisbares Gewicht: 1946 gr.

Bailey

Berlin, U.

I London, P. G. I7

Bonn

v. Braun

Budapest

Calcutta

Cleveland

Göttingen

Graz, J.

Gregory

Harvard, U.

Klausenburg

9 Minneapolis

3 Neumann

2 New Haven

260 Paris, M.

13 Pohl

3 Rom, U.

Io v. Siemaschko

45 Tübingen

Io Turin, U.

83 Ward

Io Washing

London, B. M. Bien, H. M.*) 192 Befindet sich die Hauptmasse des Steins noch im Museum in Agram? Zirkulare wurden vergeblich an die Franz-JosephsUniversität und an das National-Museum daselbst gesandt.

\section{Miljana}

Millers Run

Milwaukee

Minas Geraës Eisen

Santa Catharina

Minas Geraës Stein Minas Geraës

Minas Geraës (?), Brasilien.

Stein, Cwa, Fallzeit unbekannt, beschr. I 888 .

I888: Der b y: Meteoritos Brasileiros; Notas sobre Meteoritos Brasileiros. Revista do Observatorio Rio de Janeiro (1888), p. 12 (Sep.); s. auch Am. Journ. (3) 36 (1888), p. 157.

r895: Brezina: Wiener Sammlung, p. 245 .

Ursprüngliches Gewicht: I 224 gr. Nachweisbares Gewicht: 1212 gr. London, B. M. 4| Washington II Rio I9O $^{1}$ ) Wien, H. M. 3 v. Siemaschko 4

Miney, Taney Co., U. S. A.

Mesosiderit, M,gefunden sum 1856 。 (ob gefallen $1857 / 58$ ?) zuerst beschrieben 1860 .

Hierher auch: Forsyth, Taney Co., beschr. 1887; Newton Co., Arkansas, beschr. 1865 ; Crawford Co., beschr. 1860, Buchner sagt 4. Juli 1859 gefallen.

1860: Shepard: Notices of several American Meteorites. - 2. Forsyth (Taney County, Missouri) Iron. Am. Journ. (2) 30, p. 205-206 (2 grosse Massen geheim gehalten; Shepard erhielt nur ein kleines Stück). Ref. N. J. 1862, p. 196; Kenngott, Uebersicht 1860, p. 102. I) Herr O. Derby teilte mir mit, dass von dem 1224 gr. schweren Stein $30-40 \mathrm{gr}$.
zur Verteilung gelangten. 
1860: C o x : Second Report Geol. Reconnoissance of Arkansas (Philadelphia I860), p. 408 (oder 308 ?).

1863: B u chner: Meteoriten, p. 102, II7, I3I.

1865: S m it h: A new Meteorite from Arkansas. Am. Journ. (2) 39, p. 372. 1865: Sm it h: A new Meteorite from Newton county, Arkansas, containing on its surface Carbonate of Lime. Am. Journ.(2) 40, p.213-216 (Analyse ; Gew. $2 \mathrm{O}^{1 / 2}$ ounces); s. auch Original Researches 1884 , p. 434-437; Liebig-Kopp, Jahresber. 1865 , p. 947; Kenngott, Uebersicht 1862/65, p. 45I; Chem. Centr. 1865 , p. ${ }_{13} 6$.

r869: B u chner: Vierter Nachtrag, Pogg. Ann. Bd. 136, p. 453-454.

1875: vom Rath: Meteoriten, Verh. naturh. Ver. Bonn Bd. 32 , p. 366 .

1881: Brezina: Bericht III, Sitzber. Wien. Akad. Bd. 84 I, p. $278,279$. r882: W i e chmann: Fusion-Structures in Meteorites. Ann. N. Y. Acad. Sc. Bd. 2, p. 293.

r883: S m it h: Concretions, Am. Journ. (3) 25, p. $42 \mathrm{I}$.

r884: M e un i er: Météorites, p. 87,165 , 169-170.

1884: W a d s w or th: Studies, p. 71,74. 1885: Brezina: Wiener Sammlung, p. 19I, 233, 256, 264 .

r887: $\mathrm{K}$ unz: On some American Meteorites. - I, The Taney Co., Missouri Meteorite. Am. Journ. (3) 34, p. $467-471$ (Abbildung des Steins u. eines Schliffs; Analyse von Whitfield; Gewicht 89796 gr.). Ref. N. J. 1889 I, p. 60-6r.

r89r: v. H a u e r: Ann, Hof-Mus. Bd. 6 (Not.), p. 8, 54.

1890: E a s t m a n : Met. Astron., p. 318, $320,322$.

1893: B re zi n a : Ueber neuere Meteoriten (Nürnberg), p. 162.
I894: Fl e t c her: Introduction, p. 69. 1894: Co hen: Meteoritenkunde, p. 6o, 206, 246, 258.

r895: Brez in a : Wiener Sammlung, p. 262 .

1895: M e uni er: Revision des lithosidérites, p. 32, 33, 34-35 (Abb. einer geätzten Platte).

Ursprüngliches Gewicht: $9 \circ \mathrm{Kgr}$.

Nachweisbares Gewicht: $57022 \mathrm{gr}$.

\begin{tabular}{|c|c|c|c|}
\hline Bailey & 89 & Moskau & \\
\hline Bement & 747 & München & \\
\hline Bonn & 30 & New Haven & \\
\hline v. Braun & 2540 & Paris, E. & \\
\hline Breslau & 59 & Paris, M. & \\
\hline Budapest & 4230 & Pohl & \\
\hline Calcutta & 3 & Prag, M. & \\
\hline Cleveland & 23 & Rom, U. & \\
\hline Dorpat & 2756 & v. Siemaschko & \\
\hline Dresden, M. & 102 & Stockholm & \\
\hline Göttingen & 23 & Strassburg & \\
\hline Gregory & 3 & Utrecht & \\
\hline Greifswald & 71 & Ward & 24 \\
\hline Harvard, U. & II8 & Washington & \\
\hline Howell & II8 & Wien, H. M.*) & \\
\hline Kopenhagen & 6 & & \\
\hline $\begin{array}{l}\text { London, B. M } \\
\text { London, P. G. }\end{array}$ & 2404 & Wien, U. I. & \\
\hline
\end{tabular}

Minsk $1810 \quad$ Brahin

Minsk $1856 \quad$ Zmenj

Misshof, Ritterg.Misshof in Kurland. Stein, Cc, gefallen ro. April 1890. ז89o: Bericht in einer lettischen Zeitung ,Baltyas Wehstnesis * vom 17. April 1890 (neuen Stils); übersetzt in der folgenden Arbeit von Bruno Doss.

r891: Doss: Der Meteorit von Misshof in Kurland, Arbeiten d. Naturf. Ver. Riga N. F. Heft 7 (189r). Situationsplan und 3 Tafeln, p. $1-68$; s, auch Geinitz, \Isis^ (I89I, Juli-Dezember), p. $28-29$.

r89r: Johan s o n: Chemische Unter-

I) Hiervon 39077 gr. nach 1. Juli 1893 erworben, ausserdem 36 gr. false Newton Co. Arkansas, U. S. A. 
suchung des Meteoriten von Misshof. Arbeiten d. Naturf. Ver. Riga. N. F. Heft 7 , p. 69-9o.

r892: S chweder: Meteoritensammlung des Naturforscher-Vereins zu Riga. - Verteilung des Meteoriten von Misshof. Korr. Bl. d. Naturf. Ver. Riga Bd. 35 , p. $19-20$ (Abbildungen).

I892: D o s s: Ueber den Meteoriten von Misshof in Kurland und die Ursachen der Schallphänomene bei Meteoritenfällen im Allgemeinen. N. J. I892 I, p. 7I- $\mathrm{II}_{3}$ (Analyse von Johanson; Abbildung des Steins; 8 Figuren im Text; Photographie einer Kanonenkugel im Fluge zur Demonstration der Knallwelle); s. auch v. Niessl in Naturf. Ver. Brünn Bd. 3I (1892), p. $3 \mathrm{I}-32$.

1893: B r ez i n a : Ueber neuere Meteoriten (Nürnberg), p. I6I.

r894: Cohen: Meteoritenkunde, an vielen Stellen.

1895: Brezina: Wiener Sammlung, p. 25 .

Ursprüngliches Gewicht : Etwa 5800 gr., wovon 5636 nach Riga gelangten. Nach dem Zerschneiden u. nach der Untersuchung 499r gr.

Nachweisbares Gewicht: 4I82 gr.

\begin{tabular}{lr|lr} 
Berlin, U. & I8 & Krantz & 65 \\
Bremen & 78 & Krüger & 36 \\
Brezina & II2 & London, B. M. & 134 \\
Budapest & 79 & Paris, M. & 37 \\
Dorpat & 97 & Petersburg, B. & 273 \\
Doss & 33 & Riga & 2303 \\
Dresden, M. & 165 & Riga, P. & 219 \\
Frankfurt & III & v. Siemaschko & 176 \\
Gregory & 145 & Wien, H. M. & 10I
\end{tabular}

Zur Ergänzung und zum Vergleich seien auch hier die Angaben über die Verteilung des Meteoriten von Misshof, wie sie im Korr.-B1. d. Naturf. Ver. Riga Bd. 35, p. 19-20 mitgeteilt sind, aufgeführt:

\begin{tabular}{|c|c|c|}
\hline Berlin, U. $\quad 37$ & Riga & 2303 \\
\hline Bremen & Riga, P. & 184.2 \\
\hline Dorpat & v. Siemaschko & o 464.8 \\
\hline Dresden, M. $\quad 165.4$ & 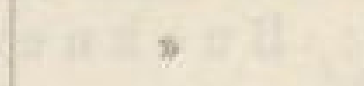 & $\mathbf{1 7} 6.8$ \\
\hline Frankfurt & s & $134 \cdot 5$ \\
\hline Krüger & * & II $7 \cdot 5$ \\
\hline II & Wien, H. M. & $484 \cdot 3$ \\
\hline Mitau, Ges, f.Lit. & 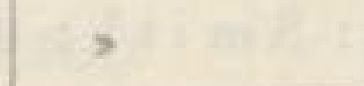 & II 7.0 \\
\hline $\begin{array}{l}\text { u. Kunst } 34^{8.2} \\
\text { St. Petersburg, }\end{array}$ & Zusammen & 4991.4 \\
\hline
\end{tabular}

Missouri s. auch Anhang

Missouri, südöstl., SaintFrançoisCo.

Misteca (Yanhuitlan), Staat Oaxaca, Mexico.

Eisen, Om, gefunden?. erwähnt I 804 , beschr. 1843 .

Hierher auch vorläufig: Cholula (Teposcolula), Chalco. Brezina stellt hierher auch: >Mexico von Humboldt mitgebrachte $=$ Goldbach's Eisen.

1804: D e 1 R i o: Tablas Mineralogicas, p. 57 .

1840: El Mosaico Mexicano Bd. 3, p. 219 (die bezïgliche Stelle ist bei Fletcher übersetzt).

1843: P a rts c h: Meteoriten,p.134-135. 1852: Cla r k: Dissert. Gött., p. 71.

1856: Burkart: Fundorte I, N. J. 1856, p. $305-307$; s, auch Journ.prakt. Chem. Bd. 71 (1857), p. 57 .

1857: B e r g e $\mathrm{m}$ a $\mathrm{n} \mathrm{n}$ : Untersuchungen von Meteoreisen. - Meteoreisen aus der Misteca im Staate von Oojaca. Pogg. Ann. Bd. Ioo, p. 246-249 (Analyse); s. auch Chem. Centr. (1857), p. 745; Kenngott, Uebersicht $1856 / 57$, p. 153 ; Liebig-Kopp, Jahresber. 1857 , p. 729 $-730,733$.

r859: B u c h ner: Feuermeteore, p. I46 -147 .

1859: H a r r is: Dissert, Gött., p. I22. 1860: R a m mels berg: Mineralchemie, p. $912,948$. 
?: Giebel u. H e int z: Zeitschr. f. d. gesamte Naturw. Bd. 9, p. $5^{10}$ u. Bd. 1o, p. 189 .

1858/62: v. Reichen ba $\mathrm{ch}$ : VII 55 I. IX 162, 174, 181. X $359 . X V$ 114, 124. XVI 261, 262, XVII 266, 272, XVIII 484,487 . XIX 150, 155, 156. XX 621,622 .

1863: Buchner: Meteoriten, p. 148 -149 .

I863: R os e: Meteoriten, p. 55, 62, I38, 152.

r865: Buchner: Zweiter Nachtrag, Pogg. Ann. Bd. 124, p. 599 (Cholula). r865: Castillo u. Rio de-la Loz a: Bull. Soc. Mex. de Geogr. y Estad. Bd. 10, p. 66I (Analyse von Rio de la Loza); s. auch Burkart, Verh. naturh. Ver. Bonn. (Sitzber.) Bd. 22 (1865), p. 7x.

r866: Burkart: Fundorte III (Abbildung der grossen Masse von Misteca, damals noch $42 \mathrm{I}$ kgr. schwer). N. J. 1866, p. 402-408 (Auszug aus der Arbeit Castillo u. Rio de la Loza's; letztere erschien $\mathbf{1 8 6 5}$ in Mexico unter dem Titel sDescripcion del Aerolito de Yanhuitlan por D. Leopoldo Rio de la Loza. 8 Seiten mit gleichen Abbildungen wie bei Burkart).

r869: Buchner: Vierter Nachtrag, Pogg. Ann. Bd, 136, p. 605-606.

r869: R a m mels berg: Ueber zwei Meteoreisen aus Mexico, Zeitschr. d. d. geol. Ges. Bd. 21 , p. 83 (Analyse von Misteca alta u. Yanhuitlan). Ref. Liebig-Kopp, Jahresber. 1869, p. 1304. 1870: Burkart: Fundorte IV, N. J. I870, p. $688-689,692$.

1875: M o hr: Entstehungsart, Ann. Chem. Pharm. Bd. 179, p. 259, 269. r876: B a rcen a : On certain Mexican Meteorites. Proc. Acad, nat. hist. Philadelphia 1876 , p. $125-126$.

r880: M u ñ oz L u mbier: LosAerólitos de Chihuahua Mexico. 188o, p. 6. r88x : Brezina: Bericht III, Sitzber.
Wien. Akad. Bd. 84 I, p. 282.

r884: Hä p k e: Beiträge, $A b h$, naturw.

Ver. Bremen Bd. 8, p. 518 .

r884: M e u n ier: Météorites,p. 38 (Abb.), 116, 121.

I884: $\mathrm{v}$ o m R a th: Meteoriten, Verh. naturh. Ver. Bonn (Sitzber.) Bd. 4I, p. 126. Ref. N. J. 1885 II, p. 270.

r885: Brezina: Wiener Sammlung, p. 213,234 .

1887: v. Hauer: Ann. Hof-Mus. Bd, 2

(Not.), p. $39, \mathrm{Bd} .3$ (Not.) (I888), p. 42 u. Bd. 4 (1889) (Not.), p. 64.

r889: Castillo: Catalogue, p. 2-3. r889: C o h e n : São Julião, N. J. 1889 I, p. 222, 224, 225.

r89o: F l et ch er: Mexican Meteorites, Mineral. Magaz. Bd. 9, p. 96, 99, 102, 104, $171-173$.

r8go: Brez in a : Reise, Ann. Hof-Mus. Bd. 4 (Not.), p. II7.

r89o: East man: Met. Astron., p. 322. r89r: Cohen u. Weinschenk: Meteoreisen-Studien. Ann. Hof-Mus. Bd. 6, p. I3I, 165.

r892: Cohen: Meteoreisen-Studien.

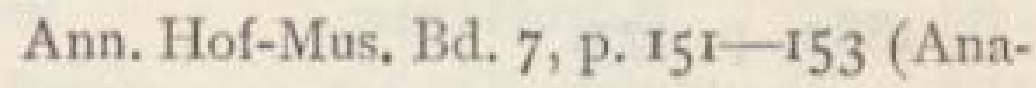
lyse), $158(\mathrm{Cu}), 159,160,16 \mathrm{r}$.

r892: Eastman: The Mexican Meteorites. Bull. Philos. Soc. Washington Bd. 12, p. 47.

r893: B r ez in a: Ueber neuere Meteoriten (Nürnberg), p. 164.

1893: M e un ier: Revision des fers météoriques, p. $52-55$.

r894: Cohen: Meteoritenkunde, an vielen Stellen.

1895: Brezina: Wiener Sammlung, p. $268,275-276$.

r895: C o hen: Meteoreisen-Studien IV. Ann. Hof-Mus. Bd, 1o, p. 82,85 .

Ursprüngliches Gewicht : Die grosse Masse von Yanhuitlan von $42 \mathrm{I}$ Kgr. befindet sich nach Castillo im Museum in Mexico.

Nachweisbares Gewicht: 6263 gr. 


\begin{tabular}{lrr|lr} 
Bailey & 87 & Melion & II6 \\
Bement & 7 I & Paris, E. & 46 \\
Berlin, U. & I23I & Paris, M. & 87 \\
v. Braun & 43 I & Pohl & I48 \\
Budapest & 317 & Prag, M. & I4I \\
Calcutta & 16 & Rom, U. & 345 \\
Cohen & I6 & v. Siemaschko & 287 \\
Detmold & 44 & Stockholm & 104 \\
Dorpat & 2 & Strassburg & 2 \\
Dresden, M. & 66 & Stürtz & 334 \\
Göttingen & 3 & Troyes & I2 \\
Gregory & 98 & Ward & 86 \\
London, B. M. & 316 & Washington, Sh. \\
London, P. G. & I28 & Wien, H. M.*) & 17 II ${ }^{1}$ )
\end{tabular}

\section{Mittel-Stuhre}

Misshof

Mócs bei Klausenburg,Siebenbürgen.

Stein, Cwa, gefallen 3. Febr. 1882.

I882 : v. $\mathrm{H}$ a u e r : Meteorsteinfall bei Klausenburg. Verb. k. k. geol. Reichsanst. 21. Februar 1882 , p. $77-78$.

1882: Brezin a: Ueber die Stellung der Mócser Meteoriten im Systeme. Verh. k. k. geol. Reichsanst. 21. Februar I882, p. 78 ; s, auch > Der Naturforscher $\approx$ 1882, p. 175 .

r882: $\mathrm{K}$ o c h : Bericht über den am 3 . Februar 1. J. stattgefundenen Meteorsteinfall von Mócs in Siebenbürgen. Sitzber. Wien. Akad. Bd. 85 I, p. II6 -132 (Situationsplan); s. auch "Der Naturforscher \& 1882 , p. $425-429$.

r882: Tschermak: Anz. Ak. d, Wiss. Wien (1882), p. $52-53,83-84$. 1882: Ts chermak: Ueber den Meteoriten von Mócs. Sitzber.Wien. Akad. Bd. 85 I, p. $195-208$ (mit 2 Tafeln). 1882: Brezina: Bericht IV, Sitzber.

Wien. Akad. Bd. 85 I, p. 335-344; s. auch Anz. Ak. d. Wiss, I882, p. 104 -105; Liebig-Kopp, Jahresber, 1882, p. $164 \mathrm{I}$.

1882 : D ö 11: Ueber die Form u. Oberfläche der Meteorsteine von Mócs und

I) Ausserdem 234 gr. nach 1. Juli 1893 erworben und 15 gr. „Teposcolula a, welches Brezina als eigenen Fundort aufführt. eine merkwürdige Fallzone, in welche dieser Fall gehört. Verh. k. k. geol. Reichsanst. 31. Mai 1882, p. 159-I6o. I882: Dö11: Die Meteorsteine von Mócs. Bemerkungen über die rundlichen Vertiefungen, die Gestalt und die Rotation der Meteoriten und eine Fallzone derselben. Jahrb. k. k. geol. Reichsanst. Bd. 32 , p. $42 \mathrm{I}-434$ und Tafel 5-8 (mit Abbildung vieler Steine); s, auch Liebig-Kopp, Jahresber. 1882 , p. $1639-1640$; ,Der Naturforscher 1882 , p. 428 .

1882 : v. L a s a u $1 \mathrm{x}$ : Vermehrung,Verh. naturh.Ver. Bonn Bd. 39 (Sitzber.), p. 107-108; Corresp. B1., p. 8o-8I.

1883: Cohen: Referat über die Arbeiten von v. Hauer, Brezina, Koch, Tschermak und Döll. N. J. 1883 II, p. 184 $-187$.

1882/83: Ko c h: ChemischeZusammensetzung des bei Mócs am 3. Februar 1882 gefallenen Meteorsteines. Mathem. u. naturw. Ber. aus Ungarn Bd, r, p. $345-346$.

1883: D ö 11: Der Meteorsteinfall von Mócs in Siebenbürgen. Monatsblätter d. wissensch. Clubs Nr. 5 vom 15 . Febr. $188_{3}, 3$ Seiten.

1883: $\mathrm{K} \circ \mathrm{c} \mathrm{h}$ : Ergänzender Bericht über den Meteoritenfall bei Mócs in Siebenbürgen am 3. Februar 1882. M. P. M. Bd. 5, p, 234-244 (Analyse von Franz Koch). Ref. N. J. 1883 II, p. 188 ; Liebig-Kopp, Jahresber, $188_{3}$, p. 1952. r883: Ts c hermak: Beitrag, Sitzber.

Wien. Akad. Bd. 88 I, p. 354,355 , $356,359,360$.

1884: W a d s worth: Studies, p. 96. 1884: v. Niess 1: Ueber die astronomischen Verhältnisse bei dem Meteoritenfall von Mócs in Siebenbürgen am 3. Februar 1882. Sitzber. Wien, Akad, Bd. 89 II, p. 283-293. Ref. N. J. 1886 I, p. 224. 
r884: M e un i e r: Météorites, p.98, 209, 459 .

1883/85: Ts chermak: Photographien, T. 12, 16, 21, 22, p. 4, 14, 16, $18,19,20$.

r885: Brez in a : Wiener Sammlung, p. $155,172,176,180,232$ u. Taf. IV. I886: v. $\mathrm{H}$ a u e r: Ann. Hof-Mus. Bd. I, p. 26. Durch Kauf erworben : „Ein über acht Kilo schwerer, beim Auffallen in zwei Stücke zerborstener Meteorstein von Mócs, mit blasiger Rinde auf der Rückseite ; s, auch Bd. 5 (189o) (Not.), p. 62 u. Bd. 6 (189r) (Not.), p. 54, 55 . r886: A nsde11 u. Dewar: On the gaseous constituents of Meteorites. Am. Journ. (3) 32, p. 482 . Ref. N. J. 1887 II, p. 285 ; Liebig-Kopp, Jahresber. 1886, p. 2326.

1887: D ö 11: Zwei neue Kriterien für die Orientierung der Meteoriten. III. Beschreibung zweier Meteoriten von Mócs. Jahrb. k. k. geol. Reichsanst. Bd. 37 , p. 204-206 (mit 4 Tafeln). Ref. N. J. 1889 I, p. 59-60.

r887: F light: Meteorites, p.220-22I. r889: F l et c her: Atacama Meteorites, Mineral. Magaz. Bd, 8, p. 226 (Streufeld 3 auf 0.6 miles).

1890: v. Niess I: Periheldistanzen, Verh. naturf. Ver. Brünn Bd. 29, p. 186, 188, 194, 214, 250.

r892: Brezina : Sternschnuppen, p. $15,16$.

1893: B rez in a : Ueber neuere Meteoriten (Nürnberg), p. 159, 160.

r894: F l e t c her: Introduction, p. 27. r894: C ohen: Meteoritenkunde, an vielen Stellen.

r895: Brezina: Wiener Sammlung, p. $244,245,259$.

Ursprüngliches Gewicht: Steinschauer. Koch stellt in seinem ergänzenden Bericht (s. oben) eine Liste von 912 Steinen im
Gesamtgewicht von I74 I I3 gr. auf. Er schätzt die Zahl der herabgefallenen Steine auf 3000 Stück (Brezina auf mehr als tooooo St.) u. deren Gewicht auf $300 \mathrm{Kgr}$. Flight erwähnt noch ein Stück von $70 \mathrm{Kgr}$., welches später gefunden sei. Nach den in Sammlungen aufbewahrten Massen scheint die Angabe auf einem Irrtum zu beruhen, da dieser Monolith doch wohl erhalten wäre.

Nachweisbares Gewicht: ${ }_{5} 55632$ gr.

\begin{tabular}{lr|lr} 
Aachen & 31 & Frenzel & 72 \\
Bayet & 6 & Giessen & 84 \\
Bailey & 331 & Goldschmidt & 39 \\
Belgrad & 49 & Graz, J. & 115 \\
Bellucci & 4 & Graz, U. & 3 \\
Bement & 1374 & Gregory & 1687 \\
Berlin, U. & 1384 & Greifswald & 326 \\
Bern, M. & 115 & Hamburg & 62 \\
Blatz & 93 & Harvard, U. & 1350 \\
Böhm & 457 & Heidelberg & 21 \\
Bologna & 864 & Helsingfors & 60 \\
Bonn & 240 & Howell & 68 \\
v. Braun & 1433 & Karlsruhe, M. & 88 \\
Breslau & 52 & Kasan & 239 \\
Brezina & 206 & Klausenburg 42816 $)$ \\
Budapest & 20930 & Kopenhagen & 1596 \\
Cambridge & 106 & Krantz & 415 \\
Catania & 271 & Kristiania & 123 \\
Cleveland & 37 & Krüger & 22 \\
Czernowitz & 41 & Kunz & 86 \\
Darmstadt, M. & 107 & Leiden & 153 \\
Debreczin & 646 & Leipzig & 19 \\
Dijon & 22 & London, B, M, 14 511 \\
Dorpat & 171 & Lüttich & 13 \\
Dresden, M. & 200 & Madrid & 145 \\
Dresden, P. & 40 & Marburg & 38 \\
Dublin, M. & 310 & de Mauroy & 325 \\
Dublin, R. C. & 42 & Melion & 314 \\
Edinburg & 671 & Modena & 171 \\
Eger & 1816 & München & 371 \\
Frankfurt & 102 & Münster & 137
\end{tabular}

r) Der grösste Stein 35700 gr.

W ü If $\mathrm{fing}$, Meteoritew.

16 
Mócs - Molina.

\begin{tabular}{lr|lr} 
Neapel & 59 & Stockholm & 954 \\
Neumann & 442 & Strassburg & 589 \\
New Haven & 154 & Stuer & 15 \\
Newton & 42 & Stürtz & 954 \\
New York, M. & 201 & Stuttgart & 1311 \\
Odessa & 28 & Szamosujvar & 1470 \\
Paris, E. & 227 & Troyes & 195 \\
Paris, M. & 3361 & Tübingen & 182 \\
Pech & 279 & Turin, J. & 91 \\
Petersburg, B. & 180 & Turin, U. & 269 \\
Pohl & 904 & Upsala & 425 \\
Prag, B. U. & 141 & Utrecht & 56 \\
Prag, D. U. & 63 & Ward & 763 \\
Prag, M. & 370 & Washington & 95 \\
Riga, P. & 212 & Washington, Sh. & 18 \\
Roebling & 160 & Wien, H.M. 36 & $\left.570^{1}\right)$ \\
Rom, U. & 598 & Wien, U. I. & 2137 \\
v. Schilling & 417 & Wien, U. II. & 107 \\
Sevilla & 8 & Wrany & 45 \\
Sidney & 611 & Zürich & 109 \\
v. Siemaschko & 3200 & &
\end{tabular}

Die Universität Basel und Herr Dr. Plagemann in Hamburg besitzen ebenfalls ein Stück Mócs; ausserdem befinden sich noch eine grosse Zahl (1300 ?) von Steinen dieser Lokalität im Besitz von Frau Wtwe. Eggerd in Wien.

Zur Ergänzung und zum Vergleich möge die Tabelle von Koch, M. P. M. Bd. 5 (1883), p. 237 hier aufgeführt werden: Besitzer Stücke Gewicht

Azbey, Kaufmann I 2130

v. Bánffy in Bonczhida $10 \quad 500$

Báré, verschied. Einw. das. 5 1010

Benke, F., Kaufmann in Klausenburg 294

44525

Bethlen, Gräfin in V.Kamarás $3 \quad \mathbf{I 5 0 0}$

Bogdán, L., Kaufmann, verkauft durch denselben 56

v. Braun

3029

Budapest

1240

1600

Budapest, min. Inst. 52 2135

Duret, Prof. Jos., Klausenb. $2 \quad 500$

Elekes, Grundbesitzer I 40

Gaal, Jul. > in M. Kalyan I 400

I) Am I. Juli 1893, jetzt 23553 gr.

\section{Besitzer}

Stïcke Gewicht

Gáspár, ref. Pastor, Visa I 500

Genersich, Prof.A., Klausenbg. I 75

Kanitz, Prof. Aug., $\quad$ I 145

Klausenburg, M. $\quad 404 \quad 78513$

Klausenburg, ref. Coll. $\quad 9 \quad 3095$

Klausenburg. unitar. Coll. 22428

Kol. Monostor, Landw.Anst. $2 \quad 105$

London, B. M. 2 I3100

Marosvásárhely, ref. Coll. $2 \quad 135$

Mártonfi, Dr. L. $\quad 73 \quad 5233$

Nagy-Enyed, ref. Coll. $13 \quad 6902$

Paal, Fr.,Preparandie-Direkt. I 500

Szab6, Al., Apahidaer Notar $8 \quad 573$

Wien, H. M. I 5600

Winkler, Dr. Fr., Kreisarzt

in Mócs

$$
\begin{array}{rr}
1 & 600 \\
\hline 912 & 174113
\end{array}
$$

\section{Modena}

Albareto

\section{Mohilew}

s. Anhang

Molina, Murcia, Spanien.

Stein, Cgb, gefallen 24. Dez. 1858 .

1868: Da ubrée u. Meunier: Météorite tombée à Murcie, Espagne, le 24 décembre 1858. C. R. Bd. 66, p. 639-642(Analyse von Meunier); s.auch Ann. Chim. Phys. (4) 17 (1869), p. 9 --12; Pogg. Ann. Bd, 133 (1868), p. 683 - 684 ; Liebig-Kopp, Jahresber. I868, p. 1045.

1869: Buchner: Vierter Nachtrag, Pogg. Ann. Bd, 136, p. $451-45^{2}$.

1870: R a mmelsberg: Meteoriten, p. $98,103,105,106,138$.

1882: Ts chermak: Ueber die Meteorite von Mocs. Sitzber. Wien. Akad. Bd. 85 I, p. 203.

1883: Ts c hermak: Beitrag, Sitzber.

Wien. Akad. Bd. 88 I, p. 355 (Murcia). 1884: Meunier: Météorites, p. 79, $247,250-252$.

r883/85: T s c hermak: Photographien,

T. 16, p. $18,19$. 
1885: Brezina: Wiener Sammlung, p. $18_{3}, 233$.

1887: F 1 i g h t: Meteorites, p.128-I29. r892: Gredilla: Meteoritos, p. Io8 -IIO.

1894: Co he n: Meteoritenkunde,p.207, $265,283,286$ (nicht Cabezzo de Mayo).

Ursprüngliches Gewicht: Ein Stein von $114 \mathrm{Kgr}$. (Gredilla sagt,p.108: $144 \mathrm{Kgr}$; ; ist aber vielleicht ein Druckfehler).

Nachweisbares Gewicht : II 4298 gr. Bailey

Bement 3 Madrid II4 OOO

Berlin, U. 9 de Mauroy 3

v. Braun

Budapest

Calcutta

Gregory

London, B. M.

70 Paris, M.

3 Rom, U.

39

13r. Siemaschko

2 Washington, $\mathrm{Sh}$

I Wien, H. M.

Monroe, Cabarras Co., Nord-Carolina, U. S. A.

Stein, Cga, gefallen 3 r. Okt. 1849 . 1850: Gibbon: Meteorite in North Carolina, Am. Journ. (2) 9, p. I43146 ; s. auch $>$ L'Institute 1850 , p. 183 ; Philos. Magaz. (3) 36, p. 240 ; LiebigKopp, Jahresber. 1850 , p. 824 ; Kenngott, Uebersicht $1850 / 51, p, 180$.

1850: Shepard: Described the recently fallen stone of Cabarras County N. C. (Oct. 31, 1849). Am. Journ. (2) Io, p. 127-I28 (Analyse). Ref. N. J. 1852, p. 617 ; Liebig-Kopp, Jahresber. 1850 , p. 824 ; Proc. Amer. Assoc. (1850), p. 148-151; Gött. Gel. Anz. 1852, p. 313 .

1853: $\mathrm{R}$ a m mels ber g : Handwörterbuch Suppl. 5, p. 34 .

1854: v. Boguslawski: Zehnter Nachtrag, Pogg. Ann, Ergz.-Bd. 4, p. 34, $3^{81}-3^{82}$.

1859: B u c hn er: Feuermeteore, p. 99. r859: Harri s: Dissert.Gött., p.93-94. r860: $\mathrm{R}$ a m me $1 \mathrm{~s}$ berg: Mineralchemie, p. 934 .
1862: Kenngott u. Wiser: Zürcher Sammlung, p. 156.

I863: B u c hner: Meteoriten, p. 79-80. I863: Rose: Meteoriten, p. $85,92,{ }_{54}$. 1859/65: v. Re ich en ba ch: IX I62, I71, 180. X 359, 363. XIII 358. XX $623 . X^{2} 432,607$.

I875: vom $\mathrm{R}$ a th: Meteoriten, Verh. naturh. Ver. Bonn Bd. 32, p. 368 .

1880: Hahn: Die Meteorite (Chondrite) und ihre Organismen. Tübingen. T. 13, Fig. 6; T. 24, Fig. 6.

1882: Wi e chmann: Fusion-Structures in Meteorites. Ann. N. Y. Acad. Sc. Bd, 2, p. 293, 295 (Taf. 19), 306. r884: W a d swort h: Studies, p. Io3 $-104,187$.

r884: M e uni e r: Météorites,p.r79,18o. 1883/85: Ts chermak: Photographien, T. 19, p. $16,17$.

r885: Brez in a : Wiener Sammlung, p. $182,233$.

I890: E a s t m a n : Met. Astron., p. 316. Ursprüngliches Gewicht: $8.8 \mathrm{Kgr}$. ( $19^{1 / 2}$ lbs.).

Nachweisbares Gewicht: $479^{8}$ gr. Bailey $\quad \mathbf{I}_{4} \mid$ London, P. G. 70 Bement $\quad 86$ Madrid 2000 Berlin, U. $\quad 132$ de Mauroy 27 Bologna $\quad 3$ Melion I Bonn I Minneapolis 3 v. Braun $\quad 36$ Moskau 15 Budapest $\quad 63$ New Haven 231 \begin{tabular}{ll|l} 
Calcutta & 146 & Paris, M. \\
\hline
\end{tabular}

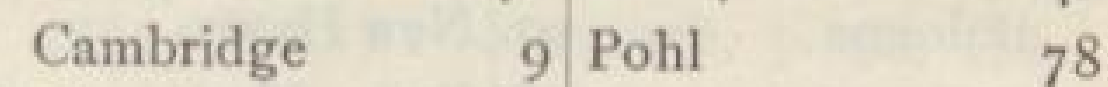
Cleveland 84 Rom, U. II Dorpat $\quad 29$ v. Siemaschko 25 Dresden, M. 8 Stockholm 53

-Freiberg, i. S. 27 Strassburg 10 Göttingen $\quad 33$ Stuttgart 39 \begin{tabular}{ll|l} 
Gregory & 152 & Troyes \\
\hline
\end{tabular} $\begin{array}{llll}\text { Hamburg } & \text { I9 } & \text { Tübingen } & 8\end{array}$ Harvard, U. $\quad 176$ Turin, J. 12 \begin{tabular}{ll|ll} 
Klausenburg & I & Upsala & I5
\end{tabular} Kopenhagen $\quad 99$ Washington, Sh. 344

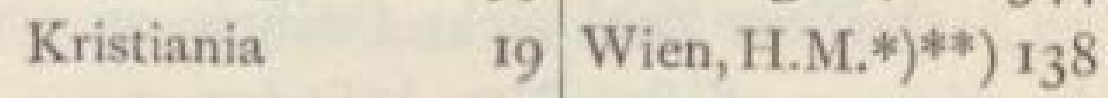
London, B. M. $3^{8}{ }_{5}$ Zürich 2 $16 *$ 
Monroe Co.

Montauban

Monte Alto

Monte Milone (Macerata) Marc Ancona, Italien (ehemals Kirchenstaat).

Stein, Cwb (früher als $\mathrm{Cw}$ aufgefasst), gefallen 8. Mai 1846 .

I846: S p a d a : Raccolta scientif. Bd, 2, Nr. II; s. auch >L'Institut \& Bd. I4 (1846), p. 340; Am. Journ. (2) 3 (1847), p. $141-142$.

r854: v. Boguslawski: Zehnter Nachtrag, Pogg. Ann. Ergz.-Bd. 4, p. $375-376$.

r859: H a rri s: Dissert. Gött., p. 9r.

r863: B u c hner: Meteoriten, p. 75 .

I859/65: v. Reich en b a ch: IX I6r, 168, 178. XI 298, 307. XIII 354, 360, 380. XXV 32I, $427,428,607$.

1884: M e unier: Météorites, p. 197.

1885: Brez in a: Wiener Sammlung, p. 177,232 .

1887: Brezina: Reisebericht, Ann. Hof-Mus. Bd. 2 (Not.), p. 73, 108.

r895: Brezina: Wiener Sammlung, p. 242,246 .

Ursprüngliches Gewicht : Etwa 5 Kgr. (Es werden mehrere Steine angeführt von II Unzen, I, 3 u. 6 Pfund).

Nachweisbares Gewicht: 2489 gr.

Bologna

v. Braun 97 New Haven 3

Budapest Io8 Paris, M.

I6r

Calcutta

39 Rom, U. 2044

Gregory 12 v. Siemaschko 4

London, B. M. 8 Washington, Sh. I London, P. G. Spl. Wien, H. M.*) 4

Der Stein scheint noch nicht näher untersucht worden zu sein.

\section{Montezuma}

Chihuahua

\section{Montignac}

Marmande

Montlivault, Dep. Loir et Cher, Frankreich.

Stein, $\mathrm{Cw}$, gefallen 22. Juli $183^{8} 8$. 1873: D a u b r é e: Note sur des météorites représentant deux chutes inédites qui ont eu lieu en France, l'une à Montlivault (Loir-et-Cher), le 22 juillet 1838, l'autre à Beuste (Basses-Pyrénées) en mai 1859. C. R. Bd. 76, p. 314315 ; s. auch $\gg$ Der Naturforscher 1873 , p. 167; Liebig-Kopp, Jahresber. 1873, p. 1250.

r884: M e un ier: Météorites, p. 208. 1885: Brezina: Wiener Sammlung, p. $177,180,232$.

r887: F 1 ig ht: Meteorites, p. Io8.

Ursprüngliches Gewicht： Ein Stein von 510 gr. $\left.{ }^{1}\right)$.

Nachweisbares Gewicht: 539 gr.

\begin{tabular}{lr|lr} 
Budapest & I & Paris, M. & 515 \\
Gregory & I & v. Siemaschko & 3 \\
London, B. M. & II & Wien, H. M. & 8
\end{tabular}

London, B. M. II Wien, H. M. 8

Montpelegry

Grazac

Montréjeau

Aussun

Mooltan 1868

Lodran

Mooltan 1873

Khairpur

Moonbi bei Tamworth, Neu-SüdWales, Australien.

Eisen, Of, gefd. 1892 , beschr. 1893 . 1893: Ming a ge: Notes and analysis of a Meteorite from Moonbi, near Tamworth, N. S. Wales. Journ, and Proc. Royal Soc. N. S. Wales Bd. 27 , p. 82-83 (zwei Abbildungen und Analyse). Ref. N. J. 1896 I, p. 229.

1895: Brezina: Wiener Sammlung, p. 272 .

Ursprüngliches Gewicht: $13 \mathrm{Kgr}$. (29 lbs.).

1) Diese Zahl giebt Daubrée an, während nach dem Pariser Katalog sich 515 gr. im Museum d'Hist. Nat, befinden. 
Nachweisbares Gewicht: 2 gr. Wien, H. M. 2 gr.

Wo befindet sich die grosse Masse?

\section{Mooradabad, Delhi, N. W. Provinz, Ostindien.}

Stein, Cw, gefallen 1808 .

Hierher auch: »Panganoor, gefallen 23. November 1811«.

(?): Ferrussac: Bull. Soc, Math. Bd. II, p. 292.

1828: Edinburgh Journ, of Sc. (Juli 1828), p. 172.

1832: v. H of f: Achter Nachtrag, Pogg. Ann. Bd. 24, p. 223, 226.

1844 : (Beiläufige Erwähnung) Journ. Asiat.

Soc. Bengal Bd. 13 , p. 884,885 .

1859: H a rri s: Dissert. Gött., p. 67.

1859: Atkinson: Verzeichnis der im Besitz der Asiatic Society befindlichen Meteoriten. Die bezügliche Stelle lautet : -Nr, I. Fell at Moradabad in 1808 , procured from Captain Herring, 3 fragments. The total weight of these fragments is $2^{1 / 2}$ ounces.e Journ. Asiat. Soc. Bengal (Proc.) Bd. 28, p. 259. 1863: B u chn er: Meteoriten, p.23, 30. 1863: Maskel yne u, v. Lang: Mineralogical Notes, - Notices of Aërolites von Maskelyne. - 16. Morarlabad. Philos. Magaz. Bd. 25, p. 449 ; s. auch Rep. Brit. Assoc. 1862 (Not, and Abstr.), p. 19o; Kenngott, Uebersicht 1862/65, p. 442.

1863: R o se: Meteoriten, p. 155. 1865: Buchner: Zweiter Nachtrag, Pogg. Ann. Bd. 124, p. 576-577. 1885: Brezina: Wiener Sammlung, p. 177,232

1894: F l e tc her: Introduction, p. ro. Ursprüngliches Gewicht : Es scheint nur das 1859 in Calcutta aufbewahrte Stück von $70 \mathrm{gr}$. $\left(2^{1 / 2} \mathrm{Oz}\right.$. $)$ erhalten $\mathrm{zu}$ sein.

Nachweisbares Gewicht: 49 gr.
Berlin, U.

Budapest

Calcutta

London, B. M.

Mooresfort (Tipperary), Irland.

Stein, Cga (von Brezina neuerdings zu Ccb gestellt), gefallenAug.r 8 ro. 1811: H ig g in s: Description and Analysis of the Meteoric Stone, which fell in the County of Tipperary in Ireland in the Month of August 1810. Philos. Magaz. Bd. $3^{8}$ (I8II). p. 262-268.

1812: Sowerby hat (I812) eine Tafel anfertigen lassen mit Abbildungen der Steine von Wold Cottage, Mooresfort u. High Possil (Exemplar im k, k. Hof-Mus. Wien).

1819: Maxwe11: Bericht von dem Steinregen, welcher sich am Io. September ${ }_{18} 81_{3}$ in der Grafschaft Limerick in Irland ereignet hat (nachträgliche Bemerkung). Gilb. Ann. Bd. 6o, p. 236 -237 .

1819: Chladni: Fünfte Fortsetzung, Gilb. Ann. Bd. 63, p. 22-23.

1819: $\mathrm{Ch}$ la d n i : Feuermeteore, p. 57, $58,66,69,73,75,91,292-293$ (l), 304, 431 .

1836: K ä m tz: Meteorologie, p. 258, 283 .

1843: P a rt s c h: Meteoriten, p.69-7o. 1859: H a rri s : Dissert. Gött., p. 69. 1863: B u c h n er: Meteoriten, p. 27-28. 1863: R o s e: Meteoriten, p. 92, 154. r858/65: v. Reichenbach: V 480. VI 454. IX 161, 170, 179. X $359,363$. XI 294, 300, 302. XXIV 227. XXV $427,429,431,607,608$.

1884: M e u nier: Météorites,p.268,27o. 1885: Brezina: Wiener Sammlung, p. $182,233$.

1894: F let c her: Introduction, p. 9. 1895: Brezina : Wiener Sammlung, p. $250,257$.

Ursprüngliches Gewicht: $3^{1 / 2} \mathrm{Kgr}$. $\left(7^{3 / 4}\right.$ lbs. $)$. Buchner giebt 3326 gr.an. 
Nachweisbares Gewicht: 2974 gr.

Bailey

Bement

Berlin, U.

v. Braun

Budapest

Calcutta

Cambridge

Dresden, M.

I London, B. M. 243

Dublin, M.

Göttingen

Gregory

Greifswald

Harvard, U.

Kopenhagen

Kunz

\section{Moradabad}

Morbihan

I London, P. G. $\quad 89$

$3^{8}$ Modena

47 Moskau

74 Neumann

25 Paris, M.

II2 ${ }^{1}$ ) Pohl

6 Rom, U.

1255 v. Siemaschko

I8 Stockholm

II7 Stürtz

I4 Troyes

7 Tübingen

32 Washington, Sh.

7 Wien, H. M.*) 278

Mordvinovka, Pawlograd, Gouv. Ekaterinoslaw, Russland.

Stein, Cw, gefallen 19. Mai 1826 . Ist vielleicht mit Bachmut (Alexejewka) zu vereinigen. Ein $57 \mathrm{gr}$. schweres Stück der Universität Kiew hat folgende Bezeichnung: ग814 (Datum?) im Gouvernement Jekaterinoslaw (im Ort Paw. lograd?)\&; das Jahr deutet auf Bachmut, die Ortsbezeichnung auf Mordvinovka; ich habe das Stück vorläufig $\mathrm{zu}$ Bachmut gestellt. Ueber Berdjansk, Tourisches Gouvernement, s.Anm. w. u.

1826: Arch. des découvertes (1826), p. 186. 1830: v. Hoff: Siebenter Nachtrag, Pogg. Ann. Bd. 18 , p. 185 (Ref. aus d. obigen Archiv, dass auf den Feldern einer Frau v. Sorbinoff ein Meteorstein von 80 Pfund niedergefallen sei, eine Nachricht, die wohl der Bestätigung bedürfe).

1836: K ä $\mathrm{m} \mathrm{tz}$ : Meteorologie, p. 295. 1847: E i c hw a 1 d: Ein Verzeichnis der

1) Ausserdem $591 \mathrm{gr}$. fraglich.
Meteorfälle in Russland. Erman's Archiv f. wiss. Kunde Russlands Bd. 5, p. 178 Anmerk.

1854: v. Boguslawski: Zehnter Nachtrag, Pogg.Ann. Ergz.-Bd.4, p.425. 1859: Ha r ris : Dissert. Gött., p. 79 (Stein von $86 \mathrm{lbs}$.).

1862: Gre g: On some Meteorites in the British Museum etc. - 4. Ekatherinoslaw. Philos, Magaz, Bd. 24, p. 538 539. Es wird hier schon die Vermutung Haidinger's u. Hörnes' ausgesprochen, dass dieser Meteorsteinfall vielleicht mit Alexejewka zu vereinigen sei. Das Stück, auf welches sich Greg bezieht, stammt aus der Sammlung des Herrn Allan von einem 85 lbs. schweren Stein, $>$ which fell in the government of Ekatherinoslaw 1825 , - the principal mass being in the (?) Museum of Odessa. Odessa besitzt nichts von diesemFundorte.

1862: Haidinger: Die Meteoriten von Bachmut und von Paulowgrad, beide im Gouvernement Jekaterinoslaw. Sitzber. Wien. Akad. Bd, 46 II, p. 307-310; s. auch Kenngott, Uebersicht $1862 / 65$, p. $43^{8}$.

1863: B u chner: Meteoriten, p. 35. 1863: Maskelyne u. v. Lang: Mineralogical Notes, - Notices on Aërolites von Maskelyne. - r7. Paulowgrad. Philos, Magaz. Bd. 25, p. $449-451$. 1862/65: v. Reichen b a c h: XX 626. XXV 322.

1865: Buchner: Zweiter Nachtrag, Pogg. Ann. Bd. 124, p. 577.

r865: K uh1 berg : Analyse und Beschreibung der Meteorite von Nerft, Honolulu, Lixna und eines im Gouvernement Jekatherinoslaw gefallenen Meteoriten, Arch. f. Naturk, Liv. Ehst. u. Kurl. (r) 4, p. 18-22.

1879: Kle in: Göttinger Sammlung, Gött. Gel. Anz. (1879), p. 92.

1883: Tschermak: Beitrag, Sitzber.

Wien. Akad. Bd. 88 I, p. 355. 
r884: M e uni er: Météorites, p.98,208. r885: Brezina: Wiener Sammlung, p. $177-180,232$.

r894: C o he n : Meteoritenkunde, p. 53, 57 , II2.

r895: Brezin a: Wiener Sammlung, p. 242 ,

Ursprüngliches Gewicht: $80-86$ Pfund (russisch?).

Nachweisbares Gewicht: 348r gr.

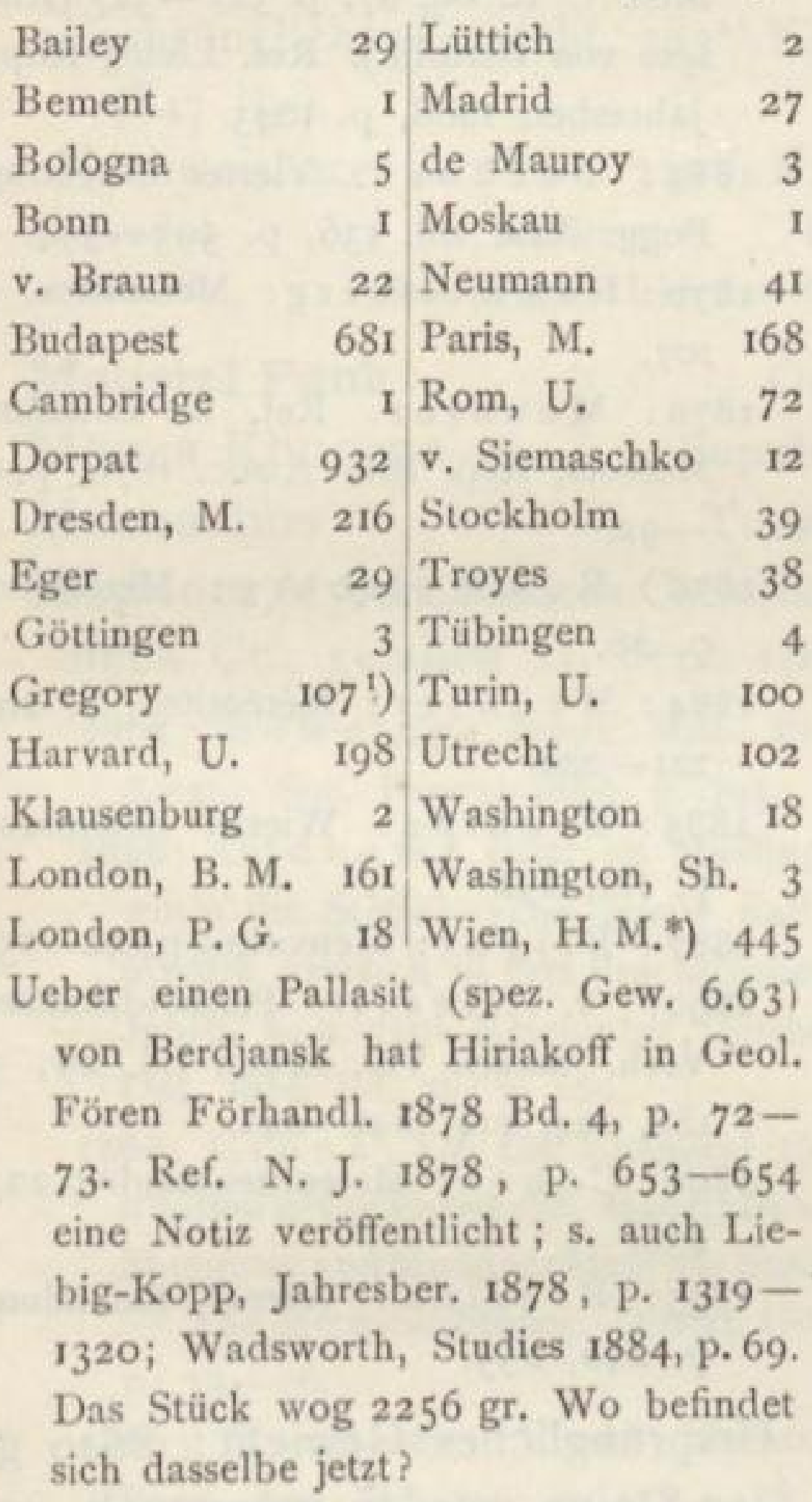

\section{Morelos}

s. Anhang Amates

Morelos s. Anhang Cuernavaca MorganCo. Lime Creek(WalkerCo.) Morito Huejuquilla-Gruppe Mornans, Bezirk Bourdeaux, Dep. Drôme, Frankreich.
Stein, Cga, gefallen Sept. 1875 . 1887: Gregory: Two new French Meteorites. Geol. Magaz. (3) 4 Nr. 12. Ref. N. J. 1889 I, p. 60.

1892: v. H a u e r: Ann. Hof-Mus. Bd. 7 (Not.), p. 73 .

r895: Brez in a: Wiener Sammlung, p. 250 .

Ursprüngliches Gewicht: I300 gr. ${ }^{2}$ ) Nachweisbares Gewicht: 1187 gr. \begin{tabular}{ll|l|l} 
Gregory & 22 & Paris, M. & 40
\end{tabular} London, B. M. 1 Io8 Wien, H. M. 17

Moro do Riccio Santa Catharina Morradal bei Grjotlien, Kirchspiel Skiaker, Norwegen.

Eisen, $\mathrm{Db}$, gefunden 1892 .

Herrn Professor W. C. Brögger verdanke ich die Nachricht von diesem Eisen, welches der Sammlung in Kristiania angehört und $275^{\circ} \mathrm{gr}$. schwer ist.

1895: Brezina: Wiener Sammlung, p. 297.

Wien, H. M. erhielt inzwischen 21 gr.

Morristown, Hamblen Co., Tennessee, U. S. A.

Stein, gefunden 1887 , beschr. 1893 . r893: E a kins: A New Meteorite from Hamblen County, Tennessee. Am. Journ. (3) 46, p. $283-285$ (Analyse), 482 (Berichtigung), s. auch Bull. U. S. Geol. Surv. Nr. 113, p. 6r. Ref. N. J. 1895 I, p. 276-277.

Ursprüngliches Gewicht: 16.3 Kgr. (36 lbs., zwei Bruchstücke von II und 13 lbs.).

Nachweisbares Gewicht: I820 gr. Washington $1820 \mathrm{gr}$.

Motecka-nugla, Staat Bhurtpur, Rajputana, Ostindien.

I) Davon $20 \mathrm{gr}$. snear Ekaterinoslaw, fell $1825 \%$.

2) Gregory giebt das Gewicht des Steins, an welchem er seine Beobachtungen anstellte, zu $\mathrm{m} 70 \mathrm{gr}$. an. 
Stein, Ck, gefallen 22. Dezbr. 1868 . I880: Im Popular Guide to the Geological Collections in the Indian Museum Calcutta ; unter Nr. 3: Meteorites, p. 26 giebt Fedden an: Moti-ka-nagla, hamlet of Gúrdha, Biáva district, Bhurtpore State, Rájputána, India 1868 Dec. 22. Two portions A. S. B. 35 (aus der Sammlung der Asiatic Soc. of Bengal) the larger 3 lbs. 4 oz. I54 grs. See model Nr. $\mathrm{I}_{3}(\mathrm{~A})$ and $(\mathrm{C})$. \& $1 \mathrm{~m}$ ganzen 3 lbs. 9 oz. I4I. 5 g rs.

1884: M e unier: Météorites, p. 179.

1885: Brezina: Wiener Sammlung, p. I9I, 232 .

1887: F lig ht: Meteorites, p. 162 (sagt nur, dass das Brit. Mus. ein Stück von $14 \mathrm{oz}$. erhalten habe).

1887: Brez in a: Neue Meteoriten III, Ann. Hof-Mus. Bd. 2 (Not.), p. 114. I894: F let $\mathrm{ch}$ er: Introduction, p. 13. Ursprüngliches Gewicht: ?

Nachweisbares Gewicht: 2295 gr. Bement 71 London, B, M. 408 v. Braun $\quad 8$ London, P. G. $\quad{ }_{5}$ Budapest Io Paris, M. 4 Calcutta $\quad 1624$ v. Siemaschko 7 Gregory $\quad 63$ Washington 3 Harvard, U. $\quad 7$ Wien, H. M. $\quad 75$ Sind über diesen Stein weitere Angaben in indischen Zeitschriften vorhanden?

Motta di Conti, Villanova, Casale, Piemont, Italien.

Stein, $\mathrm{Cc}$ (früher als $\mathrm{Ci}$ angesprochen), gefallen 29. Februar 1868. 1868: Karlsruher Zeitung vom п. März 1868. Ref. N. J. 1868 , p. $36 \mathrm{I}$; >L'Institute Bd. 36 (1868), p. 112 .

r868: Goiran, Bertolio, Zannetti u. Musso: Sopra gli Aeroliti caduti il giorno 29 febbraio $1868 \mathrm{nel}$ territorio di Villanova e Motta dei Conti, Piemonte, circondario di Casale. Con Introduzione del patre Denza. I868, Torino; s. auch Bulletino meteorolo- gico dell' Oserv. del R. Coll. Carlo Alberti in Montialieri, 31. März, 30. April u. 3o. Juni 1868 Bd. III, p. 2I (Analyse von Bertolio). Ref. Luminous Meteors, Rep. Brit. Assoc. 1868, p. 390. 1868: D e nz a : Sur les météorites tombées le 29 février 1868 dans le territoire de Villeneuve et de Motta dei Conti, arrondissement de Casale, Montferrat (Piémont) mit Nachtrag von Daubrée. C. R. Bd. 67 , p. $322-327$ (Analyse von Bertolio). Ref. Liebig-Kopp, Jahresber. I868, p. 1043.

r869: Buchner: Vierter Nachtrag, Pogg. Ann. Bd. 136, p. 593-594.

r870: Rammelsberg: Meteoriten, p. 107.

r870: Meunier: Ref, in Luminous Meteors, Rep. Brit Assoc. I870, p. 9I -92 .

1879: R a m melsber g : Meteoriten, p. 26 .

r884: M e un ier: Météorites, p. 209, $221-222$.

1885: Brezina: Wiener Sammlung, p. $181,232$.

1887: Flig ht: Meteorites, p.152-154. r89o: v. Niess 1: Periheldistanzen, Verh. naturf. Ver. Brünn Bd. 29, p. 188, 194, 214, 24I-242.

r894: C o he n : Meteoritenkunde,p.223, 236.

1895: Brezin a : Wiener Sammlung, p. 246,255 .

Ursprüngliches Gewicht: $8620 \mathrm{gr}$. 2 Steine wurden gesammelt, von 1920 und $6700 \mathrm{gr}$.

Nachweisbares Gewicht: 8135 gr.

\begin{tabular}{lr|l|r|} 
Bologna & 15 & Turin, J. & 138 \\
Budapest & 5 & Turin, U. & 6309 \\
Paris, M. & 19 & Washington, Sh. & 2 \\
Strüver & 1645 & Wien, H. M.*) & 2
\end{tabular}

Mount Hicks Mantos Blancos Mount Joy, Mount Joy Township 
(8 Klm. von Gettysburg), Adams Co., Penn., U. S. A.

Eisen, Hb, gefd. 1887 , beschr. $189^{2}{ }^{\prime}$ ). 1892: Howe11: Description of the Mt.

Joy Meteorite. Am. Journ. (3) 44, p. 4I5-4I6 (2 Abbildungen; Analyse von Eakins). Ref. N. J. 1893 II, p. 279. r894: Co h e n: Meteoritenkunde, p. 64. 1895: Brezina: Wiener Sammlung, p. 293 .

Ursprüngliches Gewicht : $3^{8} 3^{1 / 2} \mathrm{Kgr}$. (847 lbs).

Nachweisbares Gewicht: $3_{8}^{8} 3^{1 / 2} \mathrm{Kgr}$. Brezina 2 Washington 135 Howell 6800 Wien, H.M. den Rest

Moustel Pank

Ösel

\section{Mouza Khoorna}

\section{Muchachos}

Supuhee

Tucson

Muddoor,Mysore,Madras,Ostindien. Stein, Cc, gefallen 21. Sept. 1865 . 1865: Bowring: Briefl, Mitt. Proc. Asiatic Soc. Bengal 1865, p. 195.

1866: $01 \mathrm{~d} \mathrm{~h} \mathrm{a} \mathrm{m}$ : Brief an Haidinger, worin die Stelle: >Der zweite Fall in Mysore geschah in drei Bruchstücken. Jahrb. k. k. geol. Reichsanst. (Sitzber.) Dez. 1866 Bd, 16, p. 199.

1868: $\mathrm{Crook}$ : On the Chemical Constitution of the Ensisheim, Mauerkirchen, Shergotty and Muddoor Meteoric Stones.

- The Meteoric Stones. Dissert. Gött., p. $33-36$.

1869: B u chner: Vierter Nachtrag, Pogg. Ann. Bd. 136, p. $457-45^{8}$.

1870: R a mmelsberg: Meteoriten, p. $103,138,140$.

1879: R a mmelsberg: Meteoriten, p. 24,25 .

1880: Im Popular Guide to the Geological Collections in the Indian Museum
Calcutta unter Nr. 3: Meteorites, p. 26 giebt Fedden an: »Maddur taluk (near Annay Doddi), Mysore, Madras, India. 1865, Sep. 21. 4 lbs. 2 oz. 196 grs. Two specimens the larger 3 lbs. 8 oz. 60 grs. $\propto$

r884: M e uni e r: Météorites, p.79, 230, 233 (Abb.).

1885: Brezina: Wiener Sammlung, p. $185,233,263$.

r894: F l e t c h er: Introduction,p.r3,36.

UrsprünglichesGewicht:ZweiSteine, der eine kam unzerbrochen, der andere in Stücken nach Calcutta. Nachweisbares Gewicht: 2475 gr.

\begin{tabular}{lr|lr} 
Calcutta & 1883 & Paris, M. & 67 \\
Göttingen & 4 & Pohl & 10 \\
Gregory & I & v. Siemaschko & 6 \\
London, B. M. & 407 & Wien, H. M.*) & 5I \\
London, P. G. & 46 & &
\end{tabular}

Mühlau, zwischenWeiherburg (nicht Mecherburg) u. Mühlau, bei Innsbruck, Oesterreich.

Stein, $\mathrm{Cc}$ bis $\mathrm{Cg}$, gefunden um $1877^{2}$ ), beschr. 1887. Der Stein steht auf der Grenze von Cc, wie z. B. auch Avilez und Bjelaja Zerkow, auch Motta di Conti; cfr. Brezina, 1895 , p. 255 .

1887: B rezina: Neue Meteoriten III, Ann. Hof-Mus. Bd. 2, p. II5. Ref. N.J. I888 II, p. 35.

1888: v. Ha u e r: Ann. Hof-Mus Bd. 3 (Not.), p. 43 .

1893: B rezin a : Ueber neuere Meteoriten (Nürnberg), p. 161.

1895: Brezina: Wiener Sammlung, p. 255 .

Ursprüngliches Gewicht: $5 \mathrm{gr}$.

1) Nach Herrn Direktor Brezina's gütiger Mitteilung hat Howell in einem Flugblatt von 1891 das Eisen schon erwähnt.

2) Nach brieflichen Angaben des Herrn Prof. Pichler v. Rautenkar an Herrn Direktor Brezina. 
Nachweisbares Gewicht: 5 gr. Wien, H. M. 5 gr.

Murcia $185^{8}$

Molina

Murcia 1870

Cabezzo de Mayo

Murfreesboro bei Nashville, Rutherford Co., Tennessee, U. S. A. Eisen, Om, gefunden ?, beschr. 1848 .

1848: Troost: Description of a mass of Meteoric Iron, discovered near Murfreesboro', Rutherford county, Tenn. Am. Journ. (2) 5 , p. $35^{\mathrm{I}-35^{2}}$ (Analyse); s. auch Liebig-Kopp, Jahresber. I847/48, p. 1314; Pharm. Centr. 1848 , p. 844 ; Kenngott, Uebersicht 1850 / $5 \mathrm{I}$, p. $136-137$.

1852 : C l a r k : Dissert. Gött., p.67-68. r854: v. Boguslawski: Zehnter Nachtrag, Pogg.Ann.Ergz-Bd. 4, p.409. r859: B u c hn er: Feuermeteore,p.r3o. 1859: H a rris: Dissert. Gött., p. 123. 1862: v. Reichenbach: XX 622. 1863: Buchner: Meteoriten, p. I8I. 188I: Brez in a: Bericht III, Sitzber.

Wien. Akad. Bd. 84 I, p. 282.

1884: Me unier: Météorites, p. 97, II6, 122 .

1885: Brezina: Wiener Sammlung, p. $210,233$.

I890: Eastman: Met. Astron., p. 3r8. 1893: M e unier: Revision des fers météoriques, p. 52,55 .

1895: Brezina: Wiener Sammlung, p. 276 .

Ursprüngliches Gewicht: $8^{1 / 2} \mathrm{Kgr}$. (19 lbs., wovon Troost io lbs. I4 oz. besass).

Nachweisbares Gewicht: 6848 gr.

Bailey

Budapest

Calcutta

Cleveland

\begin{tabular}{r|lr}
38 & Gregory & 46 \\
63 & Harvard, U. & 2428 \\
5 & London, B. M. & 2794 \\
2 & London, P. G. & 32
\end{tabular}

\begin{tabular}{lr|lr} 
Minneapolis & 66 & Upsala & Spl. \\
Moskau & 3 & Ward & 95 \\
Paris, M. & 202 & Washington & 57 \\
v. Siemaschko & 3 & Washington, Sh. & 6 \\
Stockholm & 50 & Wien, H. M.*) & 949 \\
Tübingen & 9 & &
\end{tabular}

Muroshna

Mysore 1865

s. Anhang Angara

Muddoor

Mysore 1876

Judesegeri

Nagaya bei Concepcion, Prov. Entre Rios, Argentina, S. A.

Stein, K, gefallen $\mathbf{r}$. Juli $1879^{1}$ ). I882: W e bsky: Ueber einen v. Hrn.

Burmeister der Akademie übersandten Meteoriten. Sitzber. Berlin. Akad. 1882 I, p. 395-396. Ref. N. J. 1884 II, p. $32-33$.

1883: D a u bré e: Météorite charbonneuse tombée le 30 juin 1880 dans la république Argentine, non loin de Nogoga (province d'Entre-rios). C. R. Bd. 96, p. $1764-1766$. Ref. N. J. 1884 II, p.32-33; Liebig. Kopp, Jahresber.1883, p. 1954 .

r884: M eunier: Météorites, p. 304 (Abb.), 306-307.

r885: Brezina: Wiener Sammlung, p. $184-185,233$.

1887: F1 ig h t: Meteorites, p. 2rr.

r888: Fried heim: Ueber die chemische Zusammensetzung der Meteoriten von Alfianello und Concepcion. - 2. Der Meteorit von Concepcion. Sitzber. Berlin. Akad. 1888 I, p. $363-367$. Ref. N. J. 1889 II, p. 279.

r894: C o he n: Meteoritenkunde, p.r56, $163,167,214,222,223,232,235,236$. r895: Brezina: Wiener Sammlung, p. 254 .

Ursprüngliches Gewicht: Websky erhielt 2213 gr. (2 Stücke 1239 u. 974 gr.) u. 32 Splitter.

I) Ueber die Fallzeit herrscht Ungewissheit; es wird angegeben: Winter r880; 1. Juli 1879; r. August 1879. Das obige Datum erhielt Brezina von Herrn W. Seekamp in Concepcion del Uruguay. 
Nachweisbares Gewicht: 2528 gr.

\begin{tabular}{lr|lr} 
Bailey & 2 & Klausenburg & 4 \\
Bement & 9 & London, B. M. & 7 \\
Berlin, U. & 1797 & de Mauroy & 1 \\
v. Braun & 98 & München & 32 \\
Budapest & 13 & Paris, M. & 210 \\
Cleveland & Sp1. & v. Siemaschko & 2 \\
Cohen & 21 & Strassburg & 3 \\
Dresden, M. & 1 & Tübingen & 3 \\
Gregory & 24 & Ward & 15 \\
Greifswald & 61 & Washington \\
Howell & I & Wien, H. M. & 221
\end{tabular}

Nageria, Distr. Agra, N.W. Provinz, Ostindien.

Stein, gefallen 24. April 1875 .

1876: Med $1 \mathrm{icot} t$ : The Secretary exbibited some specimens of Meteorites recently fallen in India and read some remarks upon them. Record of the Nageriá Meteorite, of 22 nd April $1876 .-$ Nageria stone. - Tahsíldár's Report. Journ. Asiatic Soc. (Proc.), p. 222-223. 1880: Im Popular Guide to the Geological Collections in the Indian Museum Calcutta, unter Nr. 3: Meteorites, p. 16 giebt Fedden an: "Nageria, Fatehabad pargana, Agra distr., N.W. P., India. 1875, April 24. 230.5 grains (etwa $13 \frac{1}{2}$ gr.). Two specimens; the heavier 'A. S. B. (Asiatic Soc. Bengal) $5^{\prime}$ ' gravelly fragments with some black vitreous crust, weights 156 grains. The date here given is the correct one, though differing from that recorded in Proceedings, Asiatic Society, December 1876 , p. 222. «

1885: Brezina: Wiener Sammlung, p. 263 .

1894: F 1etcher: Introduction, p. I4.

Ursprüngliches Gewicht: $18 \mathrm{gr}$. (26 lbs., wovon aber nur 300 grs. erhalten blieben).

Nachweisbares Gewicht: $14 \mathrm{gr}$.

Gregory I I London, B.M. 13

Von Calcutta wurde mir noch mitgeteilt, dass es ${ }_{3} 3$ gr. dieses Falls besitze, ich möchte aber vermuten, dass diese $\mathbf{1} 3$ gr. inzwischen nach London gelangten.

Nagy-Borove s. Anhang Nagy-Divina Gross Divina Nagy-Vazsony, Veszprimer Comitat, Ungarn.

Eisen, Om, gefunden 1890 , erwähnt I89r, beschr. 1895 .

I89r : v. H a u e r: Ann. Hof-Mus. Bd. 6 (Not.), p. 54 (zeigt die Erwerbung eines Stückes von $1.9 \mathrm{Kgr}$. an).

1895: B rezina: Wiener Sammlung, p. 284 .

Ursprüngliches Gewicht: $1980 \mathrm{gr}$. Nachweisbares Gewicht: I750 gr.

\begin{tabular}{lr|l|l} 
Bement & 218 & London, B. M. & 70 \\
v. Braun & 36 & Ward & 37 \\
Gregory & 36 & Wien, H. M. & 1353
\end{tabular}

Namaland ${ }_{1} 8{ }_{3} 6$ Great Fish River Namaland $185_{2} \quad$ Lion River Nammianthal, Prov. Madras, Ostindien.

Stein, Cc, gefallen 27. Januar 1886 . 1886: M e d li c ott: Notice of the Nammianthal aerolite. - Rec. Geol, Surv. India Bd. 19, p. 268.

r886: D a u b r é e: Météorite tombée le 27 janvier 1886 , dans l'Inde, à Nammianthal, province de Madras. C. R. Bd. 103 , p. 726-727. Ref. N. J. 1887 II, p. 285,287 .

r894: F l e t ch e r: Introduction, p. 14. 1895: Brezina: Wiener Sammlung, p. 257.

Ursprüngliches Gewicht: 45 I9 gr. Nachweisbares Gewicht: 4244 gr.

Berlin, U. 13 London, B. M. 1623 Budapest Spl. Madras $\quad 356$ Calcutta $\quad 1267$ Paris, M. $\quad 885$ Gregory I Wien, H. M. 99

Herr Dr. H. Warth in Madras teilte mir noch mit: $>$ a larger portion of this fall 
is elsewhere, probably most of it in Calcutta. There must be $\mathbf{1 2 6 7}$ grammes more in addition to the above 356 grammes $\approx$, hiernach scheinen mehrere Steine gefallen zu sein.

Nanjemoy, Maryland, U. S. A. Stein, Cc (früher als $\mathrm{Cg}$ aufgefasst), gefallen 10 . Februar 1825 .

1825: Carver: Notice of a Meteoric Stone which fell at Nanjemoy, Maryland, February io th, 1825. Extracted from two letters to the Editor, dated Nanjemoy, Md. March to th, 1825 and April $29^{\text {th }}, 1825$. - Statement of W. D. Harrison, Esq. Am. Journ. (I) 9, p. $35 \mathrm{I}-353$; s. auch Ann. Chim. Phys. Bd. 30 (1825), p. 422 ; Boston Journal, Aug. 1825 , p. 604 ; Ferrussac, Bull. universelle, November 1825 , p. 212.

1826: Chilt on: Analysis of the Maryland Aerolite. Am. Journ. (I) ro, p. I3I-I35. - Additional notice of the physical characters of the Maryland Aerolite (von Silliman) im gleichen Band, p. 135-137.

1826: $\mathrm{C} \mathrm{h} 1$ a d $\mathrm{n}$ i: Ueber Zusammensetzg. der Meteormassen. - 2. Meteorsteinfall zu Nanjemoy in Maryland. Schweigg. Journ. Bd. 46 , p. $396-402$; s. auch Froriep's Notizen aus dem Gebiete der Natur- u. Heilkunde Bd. 13 (1826), p. 184 .

1826: Chla dni: Fünfter Nachtrag, Pogg. Ann. Bd. 6, p. 33.

r826: Chladni: Sechster Nachtrag, Pogg. Ann. Bd. 8, p. 47-49.

I830: v. Hoff: Siebenter Nachtrag, Pogg. Ann. Bd. 18 , p. 184 .

r836: K ä m tz: Meteorologie, p. 294. 1843: P a r ts c h: Meteoriten, p.63-64. I848: S hep ard: Report on Meteorites. Am. Journ. (2) 6, p. 406.

1854 : v.B o g u s l a w s ki: ZehnterNachtrag, Pogg. Ann. Ergz.-Bd. 4, p. 24. 1857: Arago: Astronomie populaire Bd. 4 , p. 201 . r859: H a r r i s: Dissert. Gött., p.78, 79. 1863: B u c h n er: Meteoriten,p. $4^{8}-49$. 1863: Rose: Meteoriten, p. 154. r858/65: v. Reic he n b a c h: IV 637. V 575. VI 441. IX 162, 164, 169, 179. XI 294, 296, 297. XIX 153. XXIII 369. XXV 6r5.

1865: Buchner: Zweiter Nachtrag, Pogg. Ann. Bd, 124, p. 574.

1867: Go e b e 1: Kritische Uebersicht, Mélanges phys. chim. Bd. 7, p. 325 . I882: W i e $\mathrm{ch}$ m a n $\mathrm{n}$ : Fusion-Structures in Meteorites. Ann. N. Y. Acad. Sc. Bd. 2, p. 293,295 (T. 19).

I884: Meunier: Météorites, p. 8o, 208, 215 .

1884: W a d s w or th: Studies, p. 104. 1885: Brezina: Wiener Sammlung, p. $182,232$.

r89o: Eastman: Met. Astron., p. 316. r895: Brezina: Wiener Sammlung, p. 249,255 .

Ursprüngliches Gewicht: Der Stein soll 16 lbs. $7 \mathrm{oz}$. gewogen haben. 4 lbs. 5 oz. (1950 gr.) gelangten an Silliman. Nicht viel mehr als dieses Bruchstück dürfte erhalten sein, wie auch die nachfolgendenZahlen vermuten lassen.

Nachweisbares Gewicht: 2525 gr.

\begin{tabular}{lr|lr} 
Bailey & 45 & London, P. G. & 237 \\
Bement & 30 & Neumann & Spl. \\
Berlin, U. & 33 & New Haven & 897 \\
v. Braun & 5 & Paris, M. & 4 \\
Budapest & 93 & Petersburg, A. & 6 \\
Calcutta & 70 & v. Siemaschko & 2 \\
Göttingen & 9 & Strassburg & 2 \\
Gregory & 13 & Tübingen & 183 \\
Harvard, U. & 123 & Ward & Spl. \\
Kopenhagen & 66 & Washington, Sh. 31 \\
London, B. M. & 325 & Wien,H.M.*) & 351
\end{tabular}

\section{Napoléonsville Kernouvé}

Narraburra Creek, I2 miles östlich Temora, Australien. 
Eisen, Ogg, gefd. 1854 , beschr. 1890. 1890: Russe11: The Narraburra Meteor. Nature\& $(1890)$, p. $526-527$ (Abb.); s. auch Royal Soc. Sidney 1890. r895: Brezina: Wiener Sammlung, p. 288.

UrsprünglichesGewicht: $70 \mathrm{lbs} .40 \mathrm{oz}$. Nachweisbares Gewicht: $5^{2}$ gr.

(Die Hauptmasse soll im Besitz d. Observatoriums in Sidney sein). Wien, H. M. 52 gr.

Das Eisen ist noch nicht analysiert worden.

Nash Co.

\section{Nauheim}

Nebraska

s. Anhang

Nedagolla, Mirangi, Distr. Vizagapatam, Madras, Ostindien.

Eisen, Dn (früher als Df bezeichnet), gefallen 23. Januar 1870 .

1870: S a x t o n : Briefl. Mitt. Proc. Asiat. Soc. Bengal (1870), p. $64-65$.

1870: Bericht aus den Indischen Zeitungen: sHomeward Mail« 1870, 14. März und aus dem »Madras Athenaeum\&, Luminous Meteors, Rep.Brit,Assoc. (1870), p. 93.

1884: M e uni er: Météorites, p. 133. r885: Brezina: Wiener Sammlung, p. $204-205,220,234$ u. Taf. II.

1887: Flig ht: Meteorites, p. 16; s. auch Geol. Magaz. (2) 2 (1875), p. 71. 1888: Newton: Orbits, Am, Journ. (3) 36, p. 10.

1893: Bre z in a : Ueber neuere Meteoriten (Nürnberg), p. 164, 167.

r893: M eunier: Revision des fers météoriques, p. 75 .

r894: Co hen: Meteoritenkunde, p. 58, 64,73 .

r 894 : F l e t c h e r: Introduction,p. 28,34 .

Ursprüngliches Gewicht: Etwa $4^{1} / 2$ $\mathrm{Kgr}$. (507 tolas or about ro lbs.).
Nachweisbares Gewicht: 470r gr.

\begin{tabular}{ll|ll} 
Calcutta & 214 & Paris, M. & 6
\end{tabular}

Gregory $\quad 4$ v. Siemaschko 5

Harvard, U. 20 Stockholm 8

London, B. M. 4380 Wien, H. M. 39

London, P. G. $\quad 25$

Nejed, Wadee Banee Khaled, Zentral-Arabien.

Eisen, Om, gefallen $186_{3}$ (Fletcher), beschrieben 1887 . Nach Brezina trägt das Eisen eine limonitische Verwitterungsrinde, so dass er die Beobachtung des Falls in Zweifel zieht.

r887: F le t c her: On a Meteoric Iron seen to fall in the District of Nejed, Central Arabia, in the year 1863. Mineral. Magaz. Bd. 7, p. 179-I82 (Analyse). Ref. Zeitschr. f. Kryst, Bd. I4 (1888), p. 397 ; N. J. 1889 II, p. 41. 1888: Lockyer: Researches on the Spectra of Meteorites. Proc. Royal Soc. Bd. 43 , p. 125.

r89o: Fle t c her: Mexican Meteorites, Mineral. Magaz. Bd. 9, p. 92.

I892 : v. H a u er: Ann. Hof-Mus, Bd, 7 (Not.), p. 73.

1893: B r ez in a: Ueber neuere Meteoriten (Nürnberg), p. 164, 165 .

r893: Meunier: Revision des fers météoriques, p. $25,26$.

r894: L o c k y e r : On the photographic arc spectrum of iron meteorites. Proc. Royal Soc. Bd. 55, p. 139-140.

1894: C o hen: Meteoritenkunde, p. 22, $68,72,329$.

r895: Brezina : Wiener Sammlung, p. 278 .

Ursprüngliches Gewicht: London besitzt die Hauptmasse, deren Gewicht von Fletcher zu 59.4 $\mathrm{Kgr}$. angegeben wird.

Nachweisbares Gewicht: 59800 gr. 


\begin{tabular}{|c|c|c|}
\hline Calcutta & 47 & v. Siemaschko \\
\hline Cambridge & 7 & Stockholm \\
\hline Gregory & 22 & Troyes \\
\hline London, B.M & 420 & Washington, Sh. 37 \\
\hline New Haven & 30 & Wien, H. M. \\
\hline Paris, M. & 80 & \\
\hline
\end{tabular}

\section{Nellore}

Yatoor

Nelson Co., Kentucky, U. S. A.

Eisen, Ogg, gefunden 1860 (Shepard u. Buchner sagen $185^{6}$ ), beschr. 1860 .

1860: Smith: Description of three New Meteoric Irons, from NelsonCounty, Ky., Marshall County Ky., and Madison County, North Carolina. - Nelson County (Ky.) Meteorite. Am. Journ. (2) 30, p. 240 (Analyse); s. auch Original Researches 1884 , p. 409; Liebig-Kopp, Jahresber. 1860, p. 853 ; Kenngott, Uebersicht 1860, p. IO2 ; Journ. prakt. Chem. Bd. 84 (1861), p. $59-60$.

r86r: S h e pard: Am. Journ. (2) 3r, p. 459 (sagt: gefunden 1856 ).

r86r: v. Reichenbach: XV 100, I28. XVII 273.

1863: Buchner: Meteoriten, p. 193. 1863: Ros e: Meteoriten, p. 5I, 152.

r869: Meunier: Recherches, Ann. Chim. Phys. (4) 17, p. 30, 72.

r884: M e un i er: Météorites, p. 48 , 94, 98, 109 (Abb.), IIо, III.

r885: Brezina: Wiener Sammlung, p. 217,234 .

I886: H u n ting t o n : Crystalline Structure, Am. Journ. (3) 32, p. 285, 294. 1887: B r e z in a : Neue Meteoriten IIIa, Verh. k. k. geol. Reichsanst. (1887), p. 289 .

r 889 : v. Ha uer: Ann. Hof-Mus. Bd. 4 (Not.), p. 64 .

r89o: E a s t m a n : Met. Astron, p. 318 . r89r: v. H a u e r: Ann. Hof-Mus. Bd, 6 (Not.), p. 54.
I89r: Co h e n, Meteoreisen-Studien II. Ann. Hof-Mus. Bd. 7, p. 153-154 (Analyse), $15^{8}(\mathrm{Cu})$.

r893: M eunier: Revision des fers météoriques, p. 22, 23-24 (zwei Abb. von geätzten Platten).

r894: C o he n: Meteoritenkunde, p 53, 83,92 , 101, 19I, 212.

r894: F le t c her: Introduction, p. 33. r895: Brezina: Wiener Sammlung. p. $288-289$.

r895: C o h e n: Meteoreisen-Studien IV. Ann. Hof-Mus. Bd. Io, p. 83,84 .

Ursprüngliches Gewicht: $73 \mathrm{Kgr}$. (r6 I lbs.).

Nachweisbares Gewicht: $3^{8} 703$ gr.

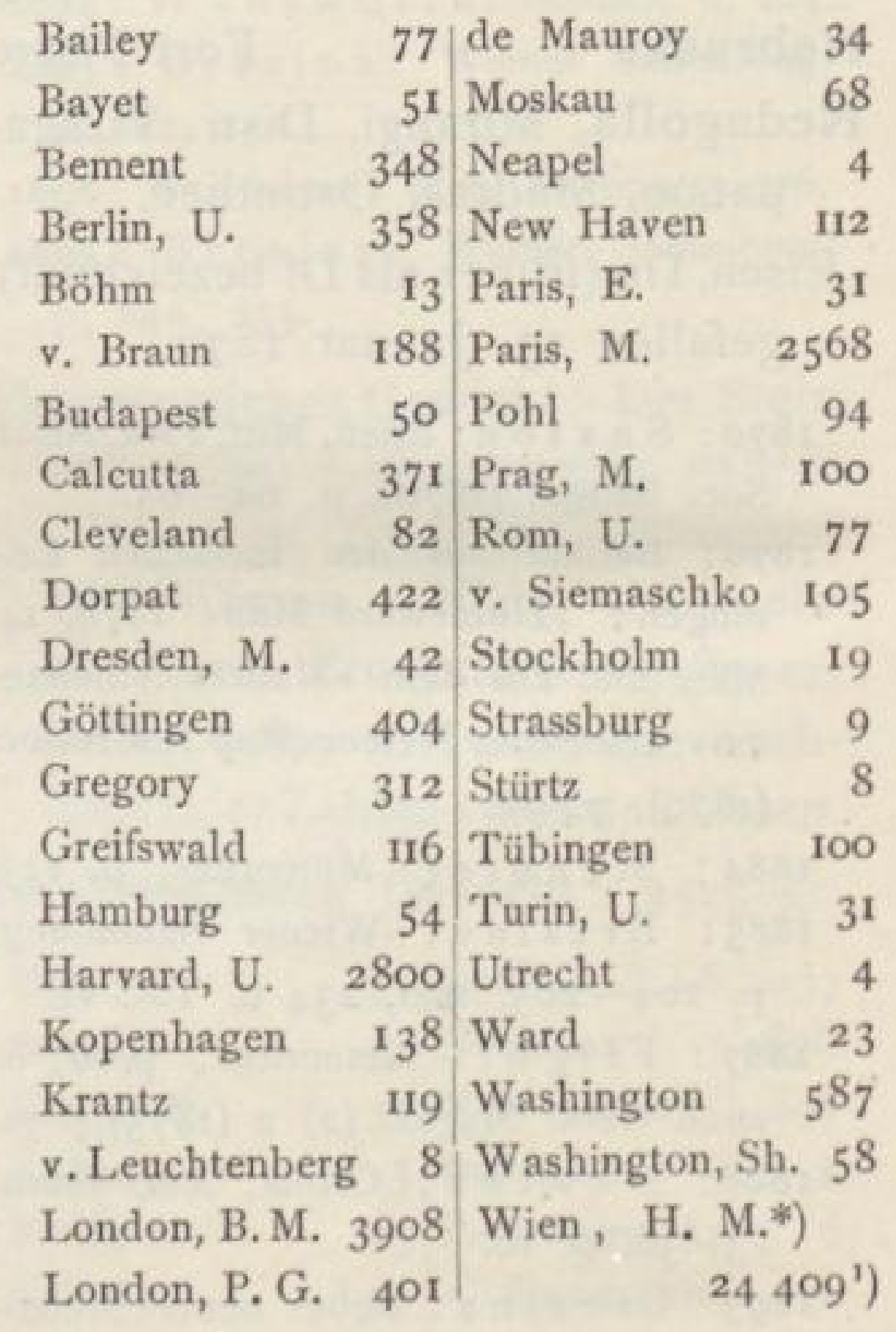

Nenntmannsdorf bei Pirna, Sachsen, Deutschland.

Eisen, H, gefunden und erwähnt 1 872 , beschr. 1873 .

1872: Geinitz im Dresdener Journal vom 31. Dezember 1872 (Nr. 303);

1) Ausserdem 05 gr. nach 1. Juli 1893 erworben. 
s. auch N. J. 1873, p. 221; Am. Journ. (3) 6 (1873), p. 237; Liebig-Kopp, Jahresber. 1872, p. II94; Wochenschr. f. Astr., Met. u. Geogr. 1873, p. 256. 1873: Lichtenberger: Sitzber. sIsis๘. Dresden 1873 , p. 4 (Analyse); s. auch N. J. 1873 , p. 221 .

r876: Ge in itz: Das Nenntmannsdorfer Meteoreisen im Dresdener Museum. N. J. 1876 , p. $608-612$ (neue Analyse); s. auch Rammelsberg, Mineralchemie 2. Aufl. I. Ergz.-Heft (1886), p. ${ }_{23} 8$ (Troilit); Liebig-Kopp, Jahresber. 1876 , p. 1315 .

1879: $\mathrm{R}$ a m m e ls berg: Meteoriten, p. 5 .

r885: Brezina: Wiener Sammlung, p. 218,234 .

r887 : v. H a uer: Ann. Hof-Mus. Bd, 2 (Not.), p. 38 .

1887: F1 ig ht: Meteorites, p. 64, I86 -187 .

r889: C o h e n: São Julião, N. J. 1889 I, p. $216,217$.

r89r: Cohen u. Weinschenk: Meteoreisen-Studien. Ann. Hof-Mus. Bd. 6, p. 159.

1893: M e u n i er: Revision des fers météoriques, p. 15, I6.

1894: C o h e n : Meteoritenkunde, p. 52, $97,98,190,197,225,232$.

1894: Fl et ch er: Introduction, p. 14. 1895: Brezina: Wiener Sammlung, p. 291.

1895: Co he n : Meteoreisen-Studien IV. Ann. Hof-Mus. Bd. 10, p. $82,88,89$.

Ursprüngliches Gewicht: 12500 gr. Nachweisbares Gewicht: 12002 gr.

\begin{tabular}{lr|lr} 
Bailey & 16 & New Haven & 47 \\
Bologna & 40 & Paris, M. & 9 \\
Budapest & 48 & v. Siemaschko & 34 \\
Dresden, M. 11636 & Stockholm & 48 \\
Freiberg, i. S. & 2 & Washington, Sh. & 15 \\
Gregory & 22 & Wien, H. M.**) & 69 \\
London, B. M. & 16 &
\end{tabular}

Nerft, Kurland, Russland.

Stein, Cia, gefallen 12. April 1864 . Auch: Pohgel-Meteorit und Swajahn-Meteorit.

1864: Grewingk und Schmidt: Ueber die Meteoritenfälle von Pillistfer, Buschhof und Igast in Liv. u. Kurland. Arch. f. Naturk. Liv. Ehst. u. Kurl. (1) 3, p. 554. - Meteoritenfall von Nerft in Kurland (aus der Riga'schen Zeitung vom 16/4 April 1864 Nr.9).Ref.Kenngott, Uebersicht 1862/65, p. 444 .

1865: B u chner: Zweiter Nachtrag, Pogg. Ann. Bd. 124, p. $587,588$.

r865: $\mathrm{Kuhlberg}$ : Analyse und Beschreibung der Meteorite von Nerft, Honolulu, Lixna und eines im Gouvernement Jekatherinoslaw gefallenen Meteoriten. Arch. f. Naturk. Liv. Ehst. u. Kurl, (I) 4, P. 2-14 (Analyse, Abbildung u. Situationsplan). Ref. LiebigKopp, Jahresber. 1867, p. 1047-1048. 1869: Buchner: Vierter Nachtrag, Pogg. Ann. Bd, 136, p. $448-449$. 1870: R a m mels berg: Meteoriten,p. 98 . r875: vom R a th: Meteoriten, Verh. naturh. Ver. Bonn Bd. 32, p. 369 .

1884: M e un ier: Météorites, p. 197, 201-202.

1885: Brezina: Wiener Sammlung, p. $182,232$.

r89r: v. S i e m a s c h ko: Kurze Notiz in seinem Katalog, p. $4^{\mathrm{I}}$ (russisch).

1893: Brezina: Ueber neuere Meteoriten (Nürnberg), p. 159.

1893: v. H a u er: Ann. Hof-Mus. Bd. 8 (Not.), p. 29.

r894: Cohen: Meteoritenkunde, p. 53, $57,112,136,206,248$.

1894: F le t c h er: Introduction, p. 13. r895: Brezin a: Wiener Sammlung, p. 247 .

Ursprüngliches Gewicht: I0 349 gr. Zwei Steine von 5645 gr.u,4704 gr. Nachweisbares Gewicht: 9972 gr. 


\begin{tabular}{lr|lr} 
Bailey & I & London, P. G. & I5 \\
Berlin, U. & $5 \mathbf{I}$ & Moskau & 68 \\
Bonn & I & New Haven & 48 \\
v. Braun & 58 & Odessa & 15 \\
Brezina & 407 & Paris, E. & 36 \\
Budapest & 93 & Paris, M. & 45 \\
Calcutta & 2 & Prag, M. & I 20 \\
Dorpat & 7376 & Stockholm & 7 \\
Göttingen & 3 I & Stuttgart & 10 \\
Helsingfors & 19 & Tübingen \\
Kiew & I8 & Wien, H. M.*) 1417 ${ }^{1}$ ) \\
London, B. M. & 69 &
\end{tabular}

Netschaëvo, Gouvernement Tula, Russland.

Eisen, Omn, stellenweise sehr reich an Silikaten, so dass es einem Mesosiderit ähnlich wird; gefunden 1846 , erwähnt $185^{8}$, beschr. I 860 .

1858: A u e r b a ch: (Kurze Mitteilung über den neuen Fund) Bull, de la Soc. impér. des Naturalistes Moskou. Bd. 3I (1858), p. $331-332$.

r860: $\mathrm{H}$ a idinger: Ueber das von Herrn Dr. J. Auerbach in Moskau entdeckte Meteoreisen von Tula, Sitzber. Wien. Akad, Bd. 42 , p. $507-518$ (Abbildungen von drei geätzten Schliffen); s. auch Bull. de la Soc. impér. des Naturalistes Moskou Bd. 33 (1860), p. 362-376; Liebig-Kopp, Jahresber. 186I, p. I129; Kenngott, Uebersicht 186I, p. 108; »L'Institut « Bd. 29 (186I) Nr. 1419, p. 98-100; Am. Journ. (2) 32 (1861), p. 144-146; Luminous Meteors, Rep. Brit. Assoc, 1861, p. 35 (Sep.); N. J. 1862, p. 109-III.

r862: Nöggerath zeigt ein prachtvolles Stück Meteoreisen etc., Verh. naturh. Ver. Bonn Bd. 19 (Sitzber.), p. 159.

r862: A uerba c h : Chemische Zusammensetzung des Meteoriten von Tula. Bull. de la Soc. impér. des Natura- listes Moskou Bd. 35 (1862), p. 628 -633 . Ref. N. J. 1863 , p. $362-363$; auch abgedruckt in Pogg. Ann. Bd. I18 (1863), p. $363-367$; Liebig-Kopp, Jahresber. 1862, p. $831-832$; Kenngott, Uebersicht $1862 / 65$, p. 446 ; Journ. prakt. Chem. Bd. 90 (1863), p. III-II2. r863: B u chner: Meteoriten, p. 195 -196 .

1863: Rose: Meteoriten, p.63-64,152. r864: $\mathrm{H}$ a i d in g e $\mathrm{r}$ : Eine grosskörnige Meteoreisen-Breccie von Copiapo. Sitzber. Wien. Akad. Bd. 49 II, p. 496. r86r/65: v. Re i c hen b a c h: XVino, 124, 128. XVI 250, 251, 261. XVII 266, 272. XVIII 484, 489. XIX 150. $\mathrm{XX}$ 629. XXV 603 .

I865: Buchner: Zweiter Nachtrag, Pogg. Ann. Bd. 124, p. 572.

1867: Buchner: Dritter Nachtrag, Pogg. Ann. Bd. 132, p. 319.

1869: $\mathrm{B} \mathrm{u} \mathrm{c} \mathrm{hner:} \mathrm{Die} \mathrm{Aetzfiguren} \mathrm{des}$ Meteoreisens. - Tula, Ber. Oberhess. Ver. f. Natur- u. Heilk. Giessen (1869), p. $109-111$.

1870: Ra mmels ber g: Beiträge zur Kenntnis der Meteoriten. - A, Meteoreisen. - III. Tula (Netschaëvo). Mon,Ber. Berlin. Akad. (1870), p. 444 (neue Analyse).

1870: $\mathrm{H}$ a i d ing er: Orientierung, Sitzber. Wien. Akad, Bd. 6I II, p. 512.

r870: R a mmels berg : Meteoriten, p. 82,108 .

r875: Ts chermak: Vulkanismus, Sitzber. Wien. Akad. Bd. 7I II, p. 663 . r875: v om R at h: Meteoriten, Verh. naturh. Ver. Bonn Bd. 32 , p. 362 .

r879: R a m mels berg: Meteoriten, p. 26 .

r884: Meunier: Météorites, p. 96, 177 (Abb.) -178 .

1883/85: T s c h e r m a k: Photographien, p. 3 .

1885: Brezina: Wiener Sammlung, p. $163,203,214,234$.

1) Ausserdem 167 gr. Nerft (Pohgel) nach 1 . Juli 1893 erworben. 
r887: F1 ig ht: Meteorites, p. II3.

1890: Brezina: Ueber Meteoreisen,

Oesterr. Zeitschr. f. Berg- u. Hüttenw. Bd. 38 , p. 356 (Abb. einer geätzten Platte).

r894: Co he n: Meteoritenkunde, p. 57. r894: F l e t c h e r: Introduction, p. ro. r895: Brezina: Wiener Sammlung, p. 289.

I895: Meunier: Revision des lithosidérites, p. 8-9 (Abb. einer geätzten Platte).

1895: Las pe yres u. K a is e r : Mitteilungen aus dem mineralogischen $\mathrm{Mu}$ seum der Universität Bonn. VI Teil Nr. 38. Die Silikate im Meteoreisen von Netschaëvo in Russland. Zeitschr. f. Kryst. Bd. 24, p. $495-496$.

Ursprüngliches Gewicht: $250 \mathrm{Kgr}$. (mehr als ${ }_{5}$ Pud), wovon ein grosser Teil verschmiedet wurde.

Nachweisbares Gewicht: $5^{8} 5^{8} \mathrm{gr}$.

Bailey 20 London, B. M. 1077

Berlin, P. $\quad 139$ London, P. G. 93

Berlin, U. $\quad 562$ Madrid 2

Bonn $\quad 153$ Moskau 607

Breslau II Neumann II

Budapest $\quad 33$ New Haven 3 I

Calcutta $\quad 324$ Paris, M. $\quad 106$

Cleveland $\quad 19$ Petersburg, B. 205

Dorpat $\quad 258$ Pohl II6

- Freiberg, i. S. $\quad 25$ v. Siemaschko 150

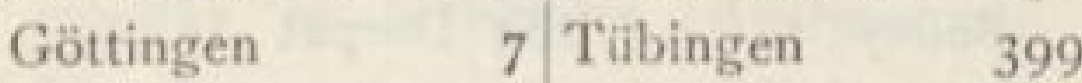
Gregory $\quad 36$ Washington, Sh. 62 Halle 206 Wien, H. M.*) H92 $^{2}$ Kopenhagen

14

Neu Mexico (Genth 1854) Tucson Neu-Seeland 1864 Wairarapa Neu-Seeland 1878 Makariwa Nevada

s. Anhang

Newberry Ruffs Mountain

New Concord, Guernsay Co., Ohio, U. S. A.

Stein, Cia, gefallen r. Mai r 860. Wü $1 \mathrm{fing}$, Meteoriten.
1860: Andrews, Evans, Johnson u. S mith: An account of the fall of Meteoric Stones at New Concord, Ohio, May I st. I86o; by Prof. E. B. Andrews of Marietta College. With (2.) Computations respecting the Meteor; by Prof. E. W. Evans, of the same Institution. To which added further notices of the same by D. W. Johnson, Esq. and Dr. J. Lawrence Smith. Am. Journ. (2) 30, p. IO3-III (Analyse von Johnson. Abbildung des Steins von Io $_{3} \mathrm{lbs}$. [Marietta College]).

r860: Shepard: Notices of several American Meteorites. - 4. Remarks upon the Ohio stones of May I, 1860. Am. Journ. (2) 30, p. 207-208; s. auch Am. Journ. (3) 30 (1885), p. 106 (Krystall von Nickeleisen).

1860: Evans: Further Notice of the New Concord (Ohio) Meteor, of May I, 1860. Am. Journ. (2) 30, p. 296.

1860: Haidinger: Einige neuere Nachrichten über Meteoriten, namentlich die von Bokkeveld, New Concord, Trenzano, die Meteoreisen von Nebraska, vom Brazos, von Oregon. Sitzber.Wien. Akad. Bd. 4I, p. 569, 572.

1860: L i e b ig-K o p p : Jahresber. 1860, p. $851-853$ (Ref. über Andrews, Shepard, Evans, Smith u. A.).

186r: S m it h: The Guernsey County (Ohio) Meteorites, a complete account of the phenomena attending their fall with a chemical analysis of them. Am. Journ. (2) 3I, p. 87-98 (neue Analyse, Karte und Anführung der Gewichte von 24 Steinen von IO 3 lbs. bis $1 / 2$ Jb.); s. auch Original Researches 1884 , p. $4 \mathrm{II}-425$. Ref. Kenngott, Uebersicht 1861, p. 168-169; Journ, prakt. Chem. Bd. 85 (I862), p. 184 $-186$.

r86r: Evans: On the Path and Velocity of the Guernsey County (Ohio) Meteor of May Ist, 1860. Am. Journ. (2) 32 , p. $30-38$ (Karte). 
186r : Ein Brief Silliman's an Haidinger wird von Poggendorf mitgeteilt.- Meteorsteinfall zu New Concord Muskingum County, im Staat Ohio, Pogg. Ann. Bd. II2, p. 493-494. Ref. Kenngott, Uebersicht I861, p. 163 .

r862 : K enngott u. W iser: Zürcher Sammlung, p. 155-156.

1862: Madelung: Ueber das Vorkommen des gediegenen Arsens in der Natur nebst den Analysen einiger neuerer Meteoriten. - Der Meteorstein von New Concord. Dissert. Gött., p. 4I -47. r863: B u chner: Meteoriten, p. 104 -105 .

I863: Ros e: Meteoriten, p. 25, 85, 93 , 98,155 .

r864: Sor by: On the Microscopical Structure of Meteorites. Proc. Royal Soc. London Bd. 13, p. 333. Ref. Am. Journ, (2) 4I (I866), p. 138.

r865: v. Reichenbach: XXV 32I, $431,607,615$.

1865: Buchner: Zweiter Nachtrag, Pogg. Ann. Bd. I24, p. 572.

r869: Bu chner: Vierter Nachtrag, Pogg. Ann. Bd, 136, p. $43^{8}$.

1870: R a m me ls berg: Meteoriten, p. $105,106,139$.

r87r: Hen r y: Fall of Meteoric Stones at Concord, Ohio. Additional Notice of the same fall, from the Zanesville Courier of May 5, 1860. Am. Journ. (3) I, p. $308-309$.

r872: Meunier: Application du métamorphisme météorique à la crôte noire des météorites grises. C. R, Bd. 75 , p. 502.

1875: vom R ath: Meteoriten, Verh. naturh. Ver. Bonn Bd. 32, p. 368-369.

1876: Wright: On the Gases contained in Meteorites. Am. Journ. (3) II, p. $258,259,260,26 \mathrm{r}$ u. (3) 12, p. 167. 1876: $\mathrm{S} \mathrm{m}$ it h, L.: Carbon compounds, Am. Journ. (3) II, p. 39I.

1879: R a m mels berg: Meteoriten, p. 25 . r882: W i e c h m a n n : Fusion-Structures in Meteorites. Ann. N. Y. Acad. Sc. Bd. 2, p. 293.

1883: T s c her m a k: Beitrag, Sitzber.

Wien. Akad. Bd. 88 I, p. 355

$\mathrm{r} 884$ : W a d s w or $\mathrm{t} \mathrm{h}$ : Studies,p. 95-96. 1884: M e unie r : Météorites, p. 85, 95 ,

$97,197,202,395,460$.

1883/85: T schermak: Photographien, p. 18 .

1885: Brezina: Wiener Sammlung, p. $181,232$.

1887: F light: Meteorites, p. 131.

1889: F l e t c her: Atacama Meteorites,

Mineral, Magaz. Bd. 8, p. 226 (Streufeld 3 auf 2 miles).

1890: v. Niess 1: Periheldistanzen, Verh. naturf. Ver. Brünn Bd. 29, p. 189, $194,214,256-257$.

1890: Ea s tma n: Met. Astron., p. 316. 1894: Cohen: Meteoritenkunde, p. 173 , I74, 267.

1895: Brezina: Wiener Sammlung, p. 246,247 .

Ursprüngliches Gewicht: Ueber 30 Steine ; 24 , worunter die grössten, wogen nach der Liste von Smith $209 \mathrm{Kgr}$. (460\%/4 lbs.). Nach andern Angaben soll das Gesamtgewicht etwa $350 \mathrm{Kgr}$. betragen haben.

Nachweisbares Gewicht: 978I I gr. Bailey

Bayet

Belgrad

Bement

Berlin, U.

Bern, M.

Böhm

Bologna

Bonn

v. Braun

Budapest

Calcutta

Cleveland

Cohen 284 Dorpat

2 Dresden, M. $4 \mathrm{I}$

4I Giessen 3

2361 Göttingen $\quad 846$

13845 Gregory $\quad 257$

I8 Halle 98

I6 Hamburg 90

2171 Harvard, U. 2936I

156 Heidelberg 20

61 Howell 4200

483 Klausenburg $\quad 18$

4606 Kopenhagen II9

54 London, B.M. 195 I9

3 London, P. G. 414 


\begin{tabular}{lr|lr} 
Lüttich & 179 & Strassburg & 35 \\
Madrid & 80 & Stürtz & 33 \\
de Mauroy & II & Stuttgart & 113 \\
Melion & 2 & Troyes & 125 \\
Moskau & 21 & Tübingen & 370 \\
München & 113 & Turin, U. & 100 \\
Münster & 23 & Utrecht & 127 \\
New Haven & 6718 & Ward & 1roo \\
Newton & 283 & Washington & 1917 \\
New York, M. & 44 & Washington,Sh.3312 \\
Odessa & 2 & Wien, H. M. *)**) \\
Paris, M. & 1630 & & 1341 ${ }^{1}$ ) \\
Pohl & 152 & Wien, U. II. & $4 \mathrm{I}$ \\
v. Siemaschko & 23 & Zürich \\
Stockholm & 810 & 6 \\
Wen &
\end{tabular}

Wenn die vier grössten Steine von Io3, $_{3}$ $56,5^{2}$ u. 50 lbs. (zus. $118 \mathrm{Kgr}$.) erhalten blieben, so befinden sich dieselben nicht in dieser Liste. An welche Sammlungen sind sie gekommen? 1866 besass das Marietta College, Marietta, Ohio, 103 Pfund; s. Haidinger, Sitzber. Wien. Akad. Bd. 54 II (1866), p.504.

\section{New Granada New Jersey \\ Rasgata \\ Deal \\ Newstead, Roxburghshire, Schottl.}

Eisen, Dn (früher als Df bezeichnet), gefunden 1827 , beschr. 1862 .

r862: $\mathrm{S} \mathrm{m}$ it h: Notice of a Mass of Meteoric Iron found in the village of Newstead, Roxburghshire with some general remarks on Meteorites (Analyse von Thomson u. Abbildung); Edinborough New Philos. Journ. Bd. 16 (July 1862), 24 Seiten (Sep.); s. auch Am. Journ. (2) 36 (1863), p. 149-r5o. Ref. N. J. 1863, p. 203; Kenngott, Uebersicht 186r, p. I08, ro und $1862 / 65$, p. 266. 1863: B u chner: Meteoriten, p. 199 -200 .

1863: R o s e: Meteoriten, p. 24.

1864: Buchner: Erster Nachtrag,

Pogg. Ann. Bd. 122, p. 321.

1879: K l e in: Göttinger Sammlung,
Gött. Gel. Anz. (1879), p. 98.

1884: Meunier: Météorites, p. 97, 131, 132 .

1885: Brezina: Wiener Sammlung, p. 220,234 .

1893: M eunier: Revision des fers météoriques, p. $7 \mathrm{r}-72$.

Ursprüngliches Gewicht: I $4_{4} 8_{3} \circ \mathrm{gr}$. ( 32 lbs. 1 I $0 z$. I $1 / 2$ drachms avoirdupois).

Nachweisbares Gewicht: I I $45^{8} \mathrm{gr}$.

Bailey

Belgrad

Berlin, U.

Budapest

Calcutta

Debreczin

Edinburg

Göttingen

Gregory

8 London, P. G. 203 212 New Haven 96

I1 Odessa I2

21 Paris, M. 236

184 Prag, M. 153

I90 v. Siemaschko 33

I321 Stockholm III

II Troyes 14

London, B. M. 8129 Wien, H. M.*) 429

Newton Co.

Miney

Ngawi, Karang-Modjo, Gentoeng, Residentschaft Madioen, Java.

Stein, Ccn, gefallen 3. Okt. $188_{3}$. r884: v. B a u $\mathrm{m}$ h a u e r: Sur la météorite de Ngawi, tombée le 3 octobre $188_{3}$, dans la partie centrale de l'ile de Java. Arch. Néerl. des Sciences exactes et naturelles Bd, 19 II, p. 175 -185 (Analyse, 2 Tafeln). Ref. N. J. 1885 II, P. 30-31; Liebig-Kopp, Jahresber. 1884, p. 2041.

1884: v. B a u $\mathrm{mb}$ a u e r spreekt over de op 3 October $188_{3}$, des namiddags am 5 uren, in Midden-Java gevallen meteorieten. Verl, en Meded. Afd. Natuurk, 3 de Reeks Deel. I, p. 5-6 (Sep.).

x884: v. B a u h ha uer: Over den op 3 October $188_{3}$ te Ngawi in MiddenJava gevallen meteoriet. Versl. en Meded. Afd. Natuurk. $3^{\text {de }}$ Reeks Deel. I Haarlem (1884), p. 8-I8 (Sep.) (Ana-

1) Ausserdem $220 \mathrm{gr}$, nach 1. Juli 1893 erworben. 
lyse; Abbild. der Steine und von Schliffen; 2 Tafeln); s. auch Jaarb. Neerl. Bd. 19, p. 177.

1885: Brezina: Wiener Sammlung, p. 264 .

1887: Boss cha, jr.: Ueber den $\mathrm{Me}-$ teorit von Karang-Modjo oder Magetan auf Java. N. J. B.-Bd. V, p. 126-I44 (3 Tafeln; Abbildung des grösseren Steins u. Phototypien von Schliffen); s. auch Arch. Néerl. Bd. 2I (1887).

1893: Brezina: Ueber neuere Meteoriten (Nürnberg), p. 162.

r894: C o hen : Meteoritenkunde, p. 6o, 206, 262,

r895: Brezin a: Wiener Sammlung, p. 259.

Ursprüngliches Gewicht: 1393 gr. (Zwei Steine von II9I u. 202 gr). Nachweisbares Gewicht: I340 gr. v. Braun $36 \mid$ Leiden $\quad$ 1166 ${ }^{1}$ )

Budapest

Greifswald

Gregory

Krantz 105 Paris, M. Spl. I v. Siemaschko I 9 Wien, H. M. 16 6

\section{Niakornak Nikolaew}

Niro

\section{s. Anhang Ovifak} Bischtübe

Nobleborough, Augusta, Lincoln Co., Maine, U. S. A.

Stein, Ho, gefallen 7 . August 1823 . 1824: Cleaveland: Notice of the late Meteor in Maine. Brunswick, Oct. II th, 1823. Am. Journ. (I) 7, p. 170 -171 .

1824: We bster: Chemical Examination of a Fragment of a Meteor which fell in Maine, August 1823. Philos. Magaz. Bd. 63 (1824), p. $16-19$ (Analyse); auch tibersetzt in Schweigg. Journ. Bd. 42 (1824), p. 104-109: Chemische Untersuchung eines Meteorsteins von Webster; s. auch Am. Journ. (I) 9, p. 400;
Ann. of Philosophy (2) 7 (1824), p. 236-237.

1824: B rayley, jr.: An Account of the principal Phaenomena of Igneous Meteors which were observed in the year 1823; forming part of a Review of the Progress of Meteorological Science during that Period: with remarks on the Characters of certain Meteorites. Ann. of Philosophy (2) 7 (1824), p. 466.

r824: Chladni: Vierter Nachtrag, Pogg. Ann. Bd. 2, p. $153-\mathbf{I} 55$.

I836: K ä m tz: Meteorologie, p. 292.

r843: P a rts c h: Meteoriten, p. 29.

1848: She pard: Report on Meteorites, - 6. Nobleboro, Maine. Am. Journ. (2) 6, p. 407.

r854: v. Boguslawski: Zehnter Nachtrag, Pogg. Ann. Ergz.-Bd. 4, p. $16,23$.

r859: H a r r is : Dissert.Gött.,p. 77-78. r860: $\mathrm{R}$ a $\mathrm{mm}$ e $\mathrm{l} \mathrm{s}$ b e r g : Mineralchemie, p. $945,949$.

1863: B u chner: Meteoriten, p. 46. 1863: M askel yne u, v. Lang: Mineralogical Notes, - Notices of Aerolites von Maskelyne. - 2I. Manegaum.

Philos. Magaz. Bd, 26, p. 137.

1863: Ros e: Meteoriten, p. 26, 27, 107, $\mathrm{II}_{3}, \mathrm{I} 37, \mathrm{I56}$.

$1859 / 64$ : v. Re i c hen b a c h: IX 160, 169, 179. X 359, 361, 362. XIII 373 . XXIII 369 .

1865: B u chner: Zweiter Nachtrag, Pogg. Ann. Bd. 124, p. 578 .

r870: R a m mels berg: Meteoriten, p. 136 .

1883: T s c h er ma k: Beitrag, Sitzber.

Wien. Akad. Bd. 88 I, p. 368 .

I884: W a ds w orth: Studies, p. 105. r884: M e un ie r: Météorites, p. 73, 95. 1883/85: Tschermak: Photographien, p. 7,8 .

r885: Brezina: Wiener Sammlung, p. 174,232 .

I) Ngawi 202 gr., Karang-Modjo 964 gr. 
1890: Eas t m a n: Met. Astron., p. 316. r894: Cohen: Meteoritenkunde, p. 317, 3 I8.

Ursprüngliches Gewicht : 4-6 lbs. ; sehr wenig erhalten.

Nachweisbares Gewicht: 78 gr.

\begin{tabular}{lr|lr} 
Bailey & Spl. & Harvard, U. & 3 \\
Berlin, U. & Spl. & London, B. M. & Spl. \\
Calcutta & Spl. & New Haven & 9 \\
Cleveland & Spl. & Tübingen & Spl. \\
Gregory & Spl. & Wien, H. M.*) & 6 \\
Halle & 60 & &
\end{tabular}

Eine neue Analyse wäre wegen des howarditischen Charakters dieses Meteoriten sehr erwünscht.

Nogoya

Nordamerika

Nord-Brabant
Nagaya

s. Anhang

Uden

Nowo-Urei, Krasnoslobodsk, Gouv. Pensa, Russland ${ }^{1}$ ).

Stein, Cu, gefallen 22. Sept. 1886. r887: Vorläufige Notiz von v. Jerofeieff u. v. Latschinoff, über welche ein Ref. in Nature $\mathrm{Bd}$, 37, p. no-III.

I888: v. H a u er: Ann. Hof-Mus. Bd, 3 (Not.), p. 42 ; s. auch Bd. 4 (I889) Not., p. 64 u. Bd. 7 (1892) Not., p. 72. 1888: v. Jer ofeieff und v. Lat$\mathrm{schinoff:} \mathrm{Der} \mathrm{Meteorit} \mathrm{von} \mathrm{Nowo}$ Urei. Verh, d. Russ, Kais. Mineralog. Ges. zu St. Petersburg (2) 24, p. 263 -297 (mit 3 Tafeln, Analysen); s. auch den ausführlichen Auszug (Analyse und Abbildungen des Steins und von Dünnschliffen) in v. Kokscharow's Mineralien Bd. Io (1888), p. 82-103. Ref. N. J. 1889 I, p. 227-229; Ber. d. d. chem. Ges. 1888 (Ref.), p. 887 ; Zeitschr, f. Kryst. Bd, 15 (1889), p. $550-552$; Weinschenk, Ann.Hof-Mus. Bd.4 (1889), p. 99; C. R. Bd. 106 (1888), p. 1679 -168I ; Liebig-Kopp, Jahresber. I888, p. 532 .

1888: D a u b ré e : Météorite diamantifère tombée le $1 \% / 22$ septembre $\mathbf{1} 886$, en Russie, à Nowo-Urei, gouvernement de Penza. C. R. Bd. 106, p. I68II682, Ref. N. J. I89I I, p. 45.

r888: $\mathrm{Kunz}$ : Diamonds in Meteorites. science Bd. II, p. II8-II9. Ref. N. J. 1891 I, p. 45.

r89r: Schweder sprach noch über die Meteoriten von Ochansk und NowoUreisk. Korr. Bl. d. Naturf. Ver. Riga Bd. 34 , p. $33-35$.

1893: Brezin a : Ueber neuere Meteoriten (Nürnberg), p. I6r.

r894: C o h en: Meteoritenkunde, p.r4o, I4I, 156, 259, 265, 293, 294, 296.

r895: B rezina: Wiener Sammlung, p. 239,254 .

Ursprüngliches Gewicht: Von zwei aufgefundenen Steinen wurde das Gewicht des einen auf etwa 1.9 Kgr. geschätzt; 1762 gr. dieses Steins gelangten nach Petersburg. Nachweisbares Gewicht: $205^{8} \mathrm{gr}$.

\begin{tabular}{lr|lr} 
Bailey & I & Paris, M. & 38 \\
Berlin, U. & 4 & Petersburg, B. & 1738 \\
Bonn & 7 & Rom, U. & 6 \\
v. Braun & 7 & v. Siemaschko & 53 \\
Budapest & 64 & Stockholm & 6 \\
Greifswald & 2 & Strassburg & 13 \\
Gregory & 83 & Washington & 4 \\
London, B. M. & 22 & Wien, H. M. & IO $^{2}$ )
\end{tabular}

\section{Nuevo Leon Coahuila}

Nulles, Vilabella, Tarragona, Catalonien, Spanien.

Stein, Cgb, gefallen 5. Nov. 1851 . r852: Luis de 1 a Escosura: Aná-

1) v. Siemaschko sagt in seinem Katalog 1891: Nr. 359: 1886, 4. Sept. Alatyr, Karamzinka (Ardatow), Petrowka (Loukojanow), Gouv. Nijni Nowgorod. False: Nowy Urej, Krasnoslobodsk, Penza๙.

2) Ausserdem 48 gr. nach 1 . Juli 1893 erworben. 
lisis del aerolito que cayó en las inmediaciones del pueblo de Nulles de la provincia de Tarragona en 5 de noviembre de 1851. Revista Minera Bd. 3 , p. $246-247,407-4$ Io. Ref. Kenngott, Uebersicht 186265 , p. $450-45^{1}$.

r854: B a $1 \mathrm{c}$ e $11 \mathrm{~s}$ : Lithologia meteorica, p. $4-9,28-39$. Barcelona 1854 (Analyse von Balcells und Escosura); s. auch Gredilla 1892 .

1862: Greg: On some Meteorites in the British Museum. - 1. Nulles. Philos. Magaz. Bd. 24 , p. $536-538$ (Auszug der Arbeit Balcells'). Ref. Kenngott, Uebersicht $1862 / 65$, p. 442 ; LiebigKopp, Jahresber. 1862 , p. $829-830$. r863: B u chner: Meteoriten, p. 81 -82 .

I865: v. Reichen bach: XXV 428 , 607.

1879: R a m me ls berg: Meteoriten, p. II, $24,25,26$.

1884: M e un i e r: Météorites,p 247,249. 1885: Brezina: Wiener Sammlung, p. 183,233 .

r892: Gre dilla: Meteoritos, p. I05 -106.

1895: B rezin a : Wiener Sammlung, p. 250 .

Ursprüngliches Gewicht: Viel€ Steine, verhältnismässig wenig erhalten. Balcells giebt I9 libras 8 onzas bei Nulles an, ausserdem sehr viele Stücke von I bis $5 \mathrm{Pfd}$. bei Vilabella und Brafim.

Nachweisbares Gewicht: $8116 \mathrm{gr}$.

\begin{tabular}{lr|l|r|} 
Budapest & 26 & Rom, U. & 2 \\
Calcutta & 8 & v. Siemaschko & I \\
Gregory & I & Tübingen & I \\
London, B. M. & 4 & Utrecht & I \\
Madrid & 7862 & Wien, H. M.*) & 27 \\
Paris, M. & I66 & &
\end{tabular}

$\begin{array}{lr}\text { Nurrah } & \text { Sitathali } \\ \text { Nyons } & \text { Aubres } \\ \text { Oaxaca } & \text { Misteca }\end{array}$

Obernkirchen (Bückeberg), Oldenburg, Deutschland.

Eisen, Of, gefunden u. beschr. 1863 .

1863: Wöhler u. W i cke: Ueber ein neu aufgefundenes Meteoreisen. Gött. Gel. Anz. (Nachr.) 1863, p. 364 -367 (Analyse u. Abbildungen); s. auch Pogg. Ann. Bd. 120, p. 509-510; Ann. Chem. Pharm. Bd. 129, p. 123; Chem. Centr. 1864, p. 224; Bull. Soc. chim. Bd, 6, p. 139; Liebig-Kopp, Jahresber.1863, p.904-905; Kenngott, Uebersicht $1862 / 65$, p. 265 -266; Wochenschr. f. Astr., Met, u. Geogr. 1864, p. 55 .

1864: Buchner: Erster Nachtrag, Pogg. Ann. Bd. I22, p. 326-327.

1884: $\mathrm{H}$ ä p k e : Beiträge, Abh, naturw.

Ver. Bremen Bd. 8, p. 523 .

1884: Wiepken: Notizen über die Meteoriten des Grossherzoglichen Museums (Oldenburg). Abh. naturw. Ver. Bremen Bd. 8, p. 530-53I. Ref. N. J. 1885 I, p. 33 .

1884: M e un i er: Météorites, p. 116.

1885: Brezina: Wiener Sammlung, p. 209,233 .

1886: H un ting t o n : Crystalline Structure, Am. Journ. (3) 32, p. 295.

1893: M e unier: Revision des fers météoriques, p. 37,38 .

r894: L o c k y er: On the photographic arc spectrum of iron meteorites Proc. Royal Soc. Bd. 55, p. $139-140$.

1894: Cohen: Meteoritenkunde, p. 68, 230, 329-330.

UrspringlichesGewicht: Fast $41 \mathrm{Kgr}$. Nachweisbares Gewicht : 37674 gr. Bailey Belgrad Budapest Calcutta Dresden, M. Göttingen Gregory Harvard, U 21 Klausenburg 130 134 Kristiania 218 184 London,B.M. 35366 Spl. London, P. G. 209 26 Marburg 139 New Haven 89 7 I Odessa 30 408 Oldenburg $\quad 92$ 
Paris, M.

Pohl

v. Siemaschko

Stockholm

Ober-Pfalz

Ocatitlan

no Ward

7 Washington $\quad 152$

127 Washington, Sh. 23 9o Wien, H.M.*) 19

L'Aigle

Toluca

Ochansk (Taborg), Gouv. Perm, Stein, Ccb, gefallen 30 .August 1887 . 1887: D a u brée: Météorite tombée le 18/30 aout 1887 en Russie, à Taborg, dans le gouvernement de Perm, C. R. Bd. 105 , p. $987-988$; s. auch $\circ \mathrm{Na}$ ture s Bd. 37 (1888), p. 120. Ref, N. J 1889 II, p. $40 \quad 41$.

1888: $\mathrm{P}$ a v 1 off: Ueber eine Mitteilung an die Soc. des Naturalistes Moscou siehe Ref. in s Nature Bd. 37 (1888), p. 544 (der Stein soll noo Pfund gewogen haben). Auch Tschernischew soll in den Verh. russ. min. Ges. und Kuotow in den Trav. de la Soc. d. nat. de Kazan Mitteilungen veröffentlicht haben.

1888: Tichomirow u. Petrow: Chemische Untersuchung einiger Proben des Meteorits von Ochansk. Journ, d. russ phys.-chem. Ges, I888 I, p. 5 I 3 -518 . Ref. Ber. d. d. chem. Ges, Bd. 21 (1888), p. 604.

1889: Eine russische Abhandlung von Pavlow. Moskau 1889. 29 Seiten (Abbildung des Meteors).

189o: D ö 11: Ueber den Meteoriten von Ochansk. Verh. k. k. geol. Reichsanst. (1890), p. Io9-IIo.

r89o: M e un i er: sa Nature (1890), p. $12-14, \mathrm{Nr} .914$.

r89o: v. Si e m a s ch ko: Einige Beobachtungen an dem Meteorsteine von Ochansk, M. P. M. Bd. II, p. $87-90$ (Analyse). Ref. N. J. 1891 I, p. 47-48; Zeitschr. f. Kryst. Bd. 20 (1892), p. $312-313$.

r89r: v. H a uer: Ann. Hof-Mus. Bd. 6 (Not.), p. 54. r89r: Me unier: "La Nature Bd. 43 , (1891), p. 228.

r89I: S chweder sprach noch über die Meteoriten von Ochansk und NowoUreisk. Korr. Bl. Naturf. Ver. Riga Bd. 34 (1891), p. $32-33,35$.

1893: B r ezin a: Ueber neuere Meteoriten (Nürnberg), p. I6I.

1894: C o he n: Meteoritenkunde, p. 57, $86,208$.

r895: B r ez in a : Wiener Sammlung, p. $258-259$.

Ursprüngliches Gewicht: Da aus der Literatur (abgesehen von der russischen) hierüber keine sicheren Daten zu ermitteln sind, mögen die Angaben des Herrn Prof. Stuckenberg über die in Kasan aufbewahrten Stücke ausführlich eingeschaltet werden:

Die im Ochanskischen Kreise des Permischen Gouvernements den 30. August 1887 gefallenen Meteoriten :

A. Die Hauptmasse des in der Nähe des Kirchendorfs Tabor (sic) gefallenen Meteoriten 100300 gr. (245 russ. Pfd.).

B. Fünf Bruchstücke desselben Meteoriten:

Nr. I 7975 gr. (19 $1 / 2$ russ. Pfd.)

Nr. 25575 gr. $\left(1_{3}^{3 / 5}\right.$, 》)

Nr. 32089 gr. $\left(5^{1 / 10}\right.$ 》 》)

Nr. 43280 gr. $(8$, 》)

Nr. 52355 gr. $\left(5^{3 / 4}\right.$ » 》)

C. Gefallen in Polom (Ochansk) 1724 gr. (4 $4^{1 / 5}$ russ. Pfd.).

D. Gefallen in Ochansk 1549 gr. $\left(3^{4} / 5\right.$ russ. $\mathrm{Pfd}$.).

E. Gefallen S.S.W. von Ochansk 640 gr. ( $1^{1 / 2}$ russ. Pfd.).

Nachweisbares Gewicht : 169203 gr. 


\begin{tabular}{|c|c|c|}
\hline Bailey & 14 & Neumann \\
\hline Bayet & 13 & New Haven \\
\hline Bement & 207 & Odessa \\
\hline Berlin, U. & 55 & Paris, E. \\
\hline v. Braun & $9 \mathbf{I}$ & Paris, M. \\
\hline Budapest & 240 & Pech $\quad 340$ \\
\hline Calcutta & II & Petersburg, A. $4^{8} 78$ \\
\hline Cleveland & 3 & Petersburg,B. $\mathbf{I}_{4}$ 120 \\
\hline Dorpat & 5 & Prag, M. $\quad 230$ \\
\hline Dresden, M. & 17 & Riga \\
\hline Gregory & 175 & Rom, U. $\quad 442$ \\
\hline Greifswald & 37 & v. Siemaschko 12289 \\
\hline Kasan & 5487 & Stockholm \\
\hline Krantz & 357 & Strassburg \\
\hline Krüger & 16 & Stürtz \\
\hline London, B. M. I & 1222 & Troyes \\
\hline de Mauroy & 60 & Utrecht \\
\hline Melion & 6 & Ward \\
\hline Moskau & 808 & Washington $\quad \mathbf{I} 74$ \\
\hline München & 814 & Wien, H. M. $3736^{1}$ ) \\
\hline
\end{tabular}

Oczeretna, Lipowitz, Gouv. Kiew, Russland.

Stein, Cga, gefunden 1871 ,

r885: Brezina: Wiener Sammlung, p. $182,233$.

Ursprüngliches Gewicht: ?

Nachweisbares Gewicht: 124 gr.

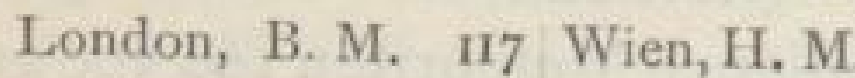

v. Siemaschko 3

Der Stein scheint noch nicht näher untersucht worden zu sein.

\section{Odessa}

Grossliebenthal

Ogi, Hizen, Kiu-Siu, Japan.

Stein, Cw, gefallen um 1730 , beschr. 1882 .

1882: Divers: On two Japanese Meteorites. Transact. Asiat. Soc. of Japan Tokiyo (Febr. 1882) Bd, Io II, p. 199; s. auch Chemical News Bd. 45 (1882), p. 316 (Analyse von Shimidzu); Nordenskiöld, Studien und Forschungen
Leipzig 1885 , p. 149; Liebig-Kopp, Jahresber. 1882 , p. $1642-1643$.

r885: Brez ina: Wiener Sammlung, p. 264 .

1887: F lig ht: Meteorites, p.166-167. r892: v. H a u er: Ann. Hof-Mus. Bd. 7 (Not.), p. 73 .

1894: F let c her: Introduction, p. 14. 1895: Brezina: Wiener Sammlung, p. 242 .

Ursprüngliches Gewicht: $10,2 \mathrm{Kgr}$, Zwei Steine von 5.6 u. $4.6 \mathrm{Kgr}$. Nachweisbares Gewicht: $4283 \mathrm{gr}$. v. Braun Calcutta 2I Stockholm 14 I Paris, M 40 London, B. M. 4185 Wien, H. M. 22

Ohaba (Veresegyhaza), Carlsburg, Blasendorfer Bez., Siebenbürgen. Stein, Cga, gefallen 11. Okt. $\left.{ }^{2}\right)$ I 857. 1857: N e u g e b o r e n: Meteorstein-Fall in der Nacht zwischen dem 10. und II. October d. J. bei Ohaba im Blasendorfer Bezirke nach ämtlich eingegangenem Berichte, Verh. u. Mitt. d. Siebenb. Ver. Bd. 8, p. $229-230$.

r858: H örnes: Ueber den Meteorsteinfall bei Ohaba im Blasendorfer Bezirke in Siebenbürgen, in der Nacht zwischen dem 10. und II. October 1857 . Sitzber. Wien. Akad. Bd, 3I, p. $79-84$ (Analyse von Bukeisen). Auszug in Pogg. Ann. Bd. 105 (1858), p. $334-336$. Ref. N. J. I859, p. 79-80; Am. Journ. (2) 27, p. 424 ; Liebig-Kopp, Jabresber. 1859, p. 809-810; Journ. prakt. Chem. Bd. 76 (1859), p. 127-128; Pharm. Centr. (1858), p. 785 (820 ) ; Verh. u. Mitt. d. Siebenb. Ver. Bd. $9\left(185^{8}\right)$, p. 167-168; Corr. d. zool. min. Ver. Regensburg Bd. I3 (1859), p. I17; Kenngott, Uebersicht 1856157 , p. 239 und 1858 , p. $165-166,167$; $>$ L'Insti-

1) Ausserdem 402 gr. nach 1. Juli 1893 erworben.

2) In der Nacht vom IO. zum n. Oktober setwa bald nach Mitternacht « s. Hörnes $\left(18_{53}\right)$, p. 8 . 
tute Bd. 26 (1858) Nr. 1292, p. 330. r859: Harris: Dissert. Gött., p. 99. 1859: $\mathrm{Buch}$ ner: Feuermeteore, p.103. 1860: $\mathrm{R}$ a m mels berg: Mineralchemie, p. $923 \mathrm{ff}$.

1863: B u c hn e r: Meteoriten,p.96-97. 1863: R o s e: Meteoriten, p. 154. 1859/65: v. Reichen bach: IX 160, 171, 180. X 359. XI 306. XIII 355 , 361, 373. XXV 317, 324, 429, 431, 433. r881: Nordenskiöld: (Vergleich mit Ställdalen). Zeitschr. d. d, geol. Ges. Bd. 33 , p. 24 .

r884: M eunier: Météorites, p. 227, $229,379,381$.

r885: Brezina: Wiener Sammlung, p. 182,233

1894: C o h e n : Meteoritenkunde,p.ro,68. 1895: Brezina: Wiener Sammlung, p. 250 .

Ursprüngliches Gewicht: $16 \% \frac{1}{4} \mathrm{Kgr}$. (29 Pfund). Nachdem Hörnes zur Untersuchung Stücke abgetrennt hatte, blieben noch $16080 \mathrm{gr}$. (28 Pfd. 20 Lot).

Nachweisbares Gewicht: 16 022 gr.

Bement

Berlin, U.

Calcutta

Göttingen

Greifswald

I London, B. M. 40

Harvard, U. 30 Wien, H.M.*) 15764 Klausenburg Spl.

Okniny, Kreis Kremenetz, Gouv. Volhynien, Russland.

Stein, Cgb (früher als Cga aufgefasst), gefallen 8. Januar 1834 .

1842: W tors chetzk it: Ueber einen am 27. December 1833, Morgens zwischen 9 und to Uhr im Dorfe Okaninach im Volhynschen Gouv. und Kremenetzkischen Kreise niedergefallenen Meteorstein. Schriften der russ, k. Ges, f. d. ges. Min. Bd, I I (1842), p. LXXII -LXXIII. r 847 u. 1848 : Der Stein wird weder in Eichwald's Verzeichnis (Erman's Archiv f. d. Kunde Russlands Bd. 5 ( 1847 ), p.176 184 , noch in Bloede's Verzeichnis (Bull. Petersburg. Akad. Bd. 6, 1848, p. $1-16$ ) erwähnt. Aus dem Gouvernement Volhynien wird bei Bloede nur der Stein von Zaborzika, Cw, gefallen 10. April I8I8, aufgeführt.

1854: Greg: Catalogue, p. $459 \gg 1833$ Dec. 28. Okaninak, Volhynia, Russia, Iron or stone $30 \mathrm{lbs}$. (27 or 29 Dec.).e Das Datum bezieht sich auf alten Stil. 1859: Harris: Dissert. Gött., p. 84. 1863: Buchner: Meteoriten, p. 57. r863: Ros e: Meteoriten, p. 85, r55. 1859/65: v. Re ic ke n b a ch: IX I6I, 170, 171, 179. XI 296. XX 626. XXV $322,427,433,607$.

1885: Brezina: Wiener Sammlung, p. $182,233$.

1895: Brezina: Wiener Sammlung, p. 250 .

Ursprüngliches Gewicht: 12 Kgr. (gegen 30 Pfund)?

Nachweisbares Gewicht: 309 gr.

\begin{tabular}{lr|lr} 
Bement & 3 & London, B. M. & 7 \\
Berlin, U. & 65 & v. Siemaschko & I \\
Calcutta & Spl. & Tübingen & 24 \\
Dorpat & 2 & Wien, H. M.*) & 110 \\
Kiew & 97 & &
\end{tabular}

Oktibbeha, Mississippi, U. S. A.

Eisen, $\mathrm{Db}(62 \% \mathrm{Ni} !)$, prähistorisch, beschr. 1857 .

(Die meteorische Natur dieses Eisens ist wohl zweifelhaft).

1857: T a y 1 o r : Examination of a Nickel Meteorite, from Oktibbeha County, Mississippi. Proc. Acad. Nat. Sc. Philadelphia. April 1857 (2 Seiten); s. auch Am. Journ. (2) 24, p. 293-295: Examination of a Nickel Meteorite, from Oktibbeha County, Mississippi (Analyse); Liebig-Kopp, Jahresber, 1857 , p. $732-733$. Ref. N. J. $185^{8}$, p. 823 ; 
Kenngott, Uebersicht $1856 / 57$, p. 157 -158 .

1859: $\mathrm{B}$ u c hner: Feuermeteore, p.r3o. I863: B u c hner: Meteoriten, p. 194 $-\mathbf{1 9 5}$.

1869: M e unier: Recherches, Ann. Chim. Phys. (4) 17, p. 21, 35, 72.

r870: R a mmelsberg: Meteoriten, p. 82 .

1884: M e unier: Météorites, p. 23, 5I, 97, 101, 102.

1885: Brezina: Wiener Sammlung, p. 200, 201, 203, 219, 234.

I890: F 1 et $\mathrm{ch}$ er: Mexican Meteorites,

Mineral. Magaz. Bd. 9, p. 102.

I89o: E a s t m a n : Met. Astron., p. 318 .

1892: Cohen: Meteoreisen-Studien.

Ann.Hof-Mus.Bd.7, p.146 (Analyse),162.

I893: M e uni er: Revision des fers metéoriques, p. 5-6.

I894: Co he n: Meteoritenkunde, p. 48, 51, 65, 66, 68, 108, н10,

1894: F l etcher: Introduction, p. 33 . 1895: Brezina: Wiener Sammlung, p. 293 .

Ursprüngliches Gewicht: I55 gr. $\left(5 \frac{1}{2} \mathrm{oz}.\right)$, wovon die Hälfte verschmiedet wurde.

Nachweisbares Gewicht: I5 gr.

Calcutta

Göttingen

Harvard, U.

I New Haven I

I Paris, M. I

4 Washington Sh. 2

London, B. M. Spl. Wien, H. M.*) 3 London, P. G. $\quad 2$

Befindet sich die Hauptmasse noch in der Akademie in Philadelphia?

\section{Old Fork of Jenny's Creek}

Oldham Co. Jenny's Creek riep, Südafrika.

Eisen, Om, bekannt 1855 , beschr. 1856 .

1856: Shepard: On a new locality of Meteoric Iron, in the Orange River
County, South Africa, and a supposed new locality of the same in Mexico. Am. Journ (2) 2I, p. 213-216 (Abbildung und Analyse). Ref. LiebigKopp, Jahresber. 1856 , p. 915 ; Kenngott, Uebersicht $1856 / 57$, p. 153 .

1859: Harris: Dissert. Gött., p. I24.

1860: R a m m els berg: Mineralchemie, p. 920.

r86r/62: v. Reichen ba ch: XV 124, 128. XVI 261, 262. XVII 266, 272. $\mathrm{XVIII}_{4} 87$. XIX 150, 153. XX 622,630. 1863: B u chner: Meteoriten, p. 193. 1863: R o s e: Meteoriten, p. 64, 152. r869: Me unier: Recherches, Ann.

Chim. Phys. (4) 17, p. 35

1884: M e un i e r: Météorites, p.r16,123. 1885: Brezina: Wiener Sammlung,

p. 211, 234.

1893: M e un i e r : Revision des fers météoriques, p. 52, 56 .

1894: C o he n: Meteoritenkunde, p. 48. r895: Brezina: Wiener Sammlung, p. 277.

Ursprüngliches Gewicht : $1488^{1} / 2 \mathrm{Kgr}$. ( 328 lbs.).

Nachweisbares Gewicht: $1063 \mathrm{gr}$. Bailey

Bement

Berlin, U.

27 London, P. G. 52

Calcutta 90 New Haven 27 28 Paris, M. 21

Cleveland

Göttingen 60 Tübingen 2I4 7 Ward 214
95 Harvard, U. $\quad 32$ Washington, Sh. 21 Kunz II4 Wien, H. M.*)

London, B. M. $\quad 98$

Wo wird die grosse Masse aufhewahrt, welche Shepard besass ? Hierher gehört wahrscheinlich auch ein Spl. *Spring. bock River der v. Siemaschko'schen Sammlung; s. 1895. Wiener Sammlung, p. 277 .

\section{Orange River, Südafrika.}

Stein, Cia, gefallen ca. 8 Sept. 1887 . Hierher vielleicht auch Beauford, 
Capland, Südafrika. Stein, bek. 1895. cfr. Brezina 1895 , Synonymen-Verzeichnis.

1895: Brezina: Wiener Sammlung, p. 248 .

Ursprüngliches Gewicht: ?

Nachweisbares Gewicht: 8 gr

Wien, H. M. 8 gr.

\section{Oregon}

Port Orford

Orgueil, Montauban, Dep. Tarn et Garonne, Frankreich.

Stein, K, gefallen 14. Mai 1864 .

1863: Ros e: Meteoriten, p. 126, 156 (mit dem Druck wurde 4. Juli 1864 begonnen).

1864: P a que ré e: Météore lumineux observé à Castillon (Gironde). C. R. Bd. 58 , p. 9 ro.

r 864: B o urri è r e s: Observation d'un météore lumineux à Agen (Lot-et-Garonne). C. R. Bd. 58 , p. 9IO-9II.

1864: Brogniart, D a ubrée, Le Verrier: Météore lumineux et chute de pierres météoriques du 14 mai. Communication de M. Brogniart. Communication de M. Daubrée d'après sa correspondance et celle de M. Le Verrier. C. R. Bd. 58 , p. $932-937$. Ref. Wochenschr. f. Astr., Met, u. Geogr. 1864, p. 230-232, 233.

1864: D a u b ré e : Note sur les météorites tombées le 14 mai aux environs d'Orgueil (Tarne-et-Garonne). C. R. Bd. 58 , p. $984-986$. Ref. Kenngott, Uebersicht $1862 / 65$, p. 440 ; LiebigKopp, Jahresber. 1864, p. $898-899$; Journ. prakt. Chem. Bd. 95 (1865), p. $360--365$.

1864: C 1 o ëz: Note sur la composition chimique de la pierre météorique d'Orgueil. C. R. Bd. 58, p. 986-988. Ref. Kenngott, Uebersicht $1862 / 65$, p. 440. 1864: L e y m e rie: Sur l'aërolithe d'Orgueil (Tarn-et-Garonne), tombé le $\mathbf{1}_{4}$ mai, à 8 heures du soir. C. R. Bd. 58 , p. $988-990$.

1864: D a u bré e: Nouveaux renseignements sur le bolide du mai $\mathbf{1 8 6 4}$. C. R. Bd. 58 , p. $1065-1072$ (mit brieflichen Mitteilungen von Lespiault, Baget, Lajous, Pauliet, Jacquot, Paructeau-Léon, Saint-Amans, Laurentie, Cruzel, extrait d'un journal de Périgueux, Madame la marquise Puylaroque, Mgr l'Evêque de Montauban, Triger, Hende u. Leymerie).

1864: La ussedat: Sur la méthode employée pour déterminer la trajectoire du bolide du 14 mai. C. R. Bd. $5^{8}$, p. 1100 Ho5 (grosser Situationsplan). Ref Wochenschr. f. Astr., Met.u. Geogr. 1864, p. 232.

1864: Laroque et Bianchi adressent une Note sur l'aërolithe du 14 mai. C. R. Bd. 58 , p. 1164 ; s. auch Mém. de l'Acad. des Sc. Toulouse 1864, p. $373-378$ : Note sur l'aérolithe charbonneux du 14 mai 1864 .

1864: Lespia u 1t: Note à l'occasion du bolide du 14 mai. C. R. Bd. $5^{8}$, p. $1212-1213$.

r864: Kesse 1 meyer: Der Meteorsteinfall zu Orgueil und Nohic bei Montauban in Südfrankreich am 14. Mai 1864. Pogg. Ann. Bd. 122, p. $654-$ 658. Zusammenstellung der meisten obigen Berichte u. derjenigen aus l'Etoile Belge Nr. 140, 142, 144, 166 vom 19., 21., 24. Mai und 14. Juni 1864 u. aus dem Journ. d'Agen vom 15. Mai 1864 und dem Courier du Tarn et Garonne. Ref.Kenngott,Uebersicht $1862 / 65$, p.447. 1864: Filhol et Melliés: Note sur la composition chimique de l'aérolithe du 14 mai 1864. Mém. de l'Acad. des Sc. Toulouse (1864), p. $379-382$. 1864: $\mathrm{Cl}$ o ëz: Analyse chimique de la pierre météorique d'Orgueil, C. R. Bd, 59 , p. $37-40$. Ref, Kenngott, Uebersicht $1862 / 65$, p. $440-44$ I.

1864: La us sedat: Sur la trajectoire 
du bolide du 14 mai. Note, en réponse à celle de M. Lespiault. C. R. Bd. 59, p. $74-76$.

1864: $\mathrm{P}$ is a n i: Étude chimique et analyse de l'aërolithe d'Orgueil. C. R. Bd. 59, p. 132-135. Ref. Liebig-Kopp, Jahresber. 1864, p. 899 ; Kenngott, Uebersicht 1862/65, p 44I ; L'Instituts 1864, p. 251; Bull. Soc Chim. (2) 2, p. 197 .

1864: Des C loizea ux: Sur la présence d'un carbonate de magnésie et fer cristallisé dans la météorite d'Orgueil. C. R. Bd. 59 , p. $829-830$.

1864: D a ubrée: Observation sur la présence de la breunérite dans la météorite d'Orgueil. C. R. Bd. 59 , p. 830 . r864: W öhler: Bemerkungen über den neuesten Meteorsteinfall. Gött. Gel. Anz. I864 (Nachr.), p. 281-282. Ref. "L'Institut « Bd. 33 (1865), p. 14-15. 1864: C lo ëz: Dosage de l'acide carbonique contenu dans la météorite d'Orgueil. C. R. Bd. 59, p. $830-83$ I. Ref. Luminous Meteors, Rep. Brit. Assoc. 1865, p. 129.

1864: Referate über die obigen Arbeiten : $\rightarrow L^{\prime}$ 'nstitut $\ll \mathrm{Bd}, 32$ (1864), p. 16I, 169, $181-182, \quad 251-252, \quad 272, \quad 361-362$, $371-372$.

1864/65: Des C1o izea ux: Carbonate de magnésie et fer dans la météorite d'Orgueil. Bull. soc. géol. de France Bd. 22, p. 24-25; s. auch Pogg. Ann. Bd. 124, p. 191-192: Ueber die Gegenwart eines krystallisierten Magnesia-Eisen-Carbonat's im Meteorit von Orgueil. Ref. Kenngott, Uebersicht 1862165 , p. 444, 447; Liebig-Kopp, Jahresber. 1864, p. $899-$ 900 ; N. J. 1865 , p. 479 ; s. auch C. R. Bd. 59 (1864), p. 829 .

I865: Buchner: Zweiter Nachtrag, Pogg. Ann. Bd. 124, p. 572, 573, 588 -591 .

1865: Referate über die französischen Arbeiten: Am. Journ. (2) 39, p. 230-231;
Journ. prakt. Chem. Bd 105 (1865), p. $360-365$.

I866: D a u b r é e présente à l'Académie une météorite au nom de M. le Maréchal Vaillant. C. R. Bd. 62 , p. 283 -284 ; s. auch >L'Institute Bd. 34 (1866), p. $43,53$.

1867: Geinitz: N. J. 1867, p. 724. 1867: D a u bré e : Complément d'observations sur la chute de Météorites qui a eu lieu le 14 Mai I864 aux environs d'Orgueil (Tarne-et-Garonne). Nouvelles Archives du Muséum d'hist. nat. Paris. Bd. 3, p. I-I9 (I Tafel mit Abbildungen von Steinen, darunter sehr schön orientierte; Karte)

1870: R a m mels ber g: Meteoriten, p. $116-117,138$.

1870: v. $\mathrm{Ha}$ id inger: Orientierung, Sitzber.Wien. Akad. Bd. 61 II, p. $5^{13}$. 1870: $\mathrm{B}$ erthelot: Recherches sur les états du carbone. - IV. Les états actuels du carbone. - A. Variétés usuelles et naturelles Ann. Chim. Phys. (4) 17 , p. 417: $: 13$. La matière charbonneuse de la météorite d'Orgueil, purifiée autant que possible par les dissolvants, s'est ensuite oxydée entièrement«; s. auch Journ. prakt. Chem. Bd. 106 (1869), p. 254 ; C. R. Bd. 67 , p. 849 .

1870: Berthelot: Méthode universelle pour réduire et saturer d'hydrogène les composés organiques, - Cinquième et dernière partie. Matières charbonneuses. - 5. Matière charbonneuse des météorites. Ann. Chim. Phys. (4) 20 , p. $53 \mathrm{I}-532$.

I875: vom R a th: Meteoriten, Verh. naturh. Ver. Bonn Bd. 32, p. 375-376. 1876: Smith: Carbon Compounds, Am. Journ. (3) II, p. 39I, 392, 434, $435-438(1), 439,440$.

1877: F $1 \mathrm{ight}$ : Meteorites and the origin of life. The Popular Science Review. 18. Okt. 1877 , p. 9-10 (Sep.). 1879: v. $\mathrm{Ni}$ ess 1 : Untersuchungen über 
die Bahnverhältnisse des Meteoriten von Orgueil in Frankreich am 14. Mai 1864. Verh. naturf. Ver. Brïnn (Abh.) Bd. 18 , p. $143-163$.

r879: R a m mels berg: Meteoriten, p. 23 .

I882: W i e c h ma n n: Fusion-Structures in Meteorites. Ann. N. Y. Acad. Sc. Bd. 2, p. 300-30I.

r884: v. $\mathrm{N}$ i es s l: Ueber die astronomischen Verhältnisse bei dem Meteoritenfalle von Mócs in Siebenbürgen am 3. Februar 1882. Sitzber. Wien. Akad. Bd. 89 II, p. 29r (Hemmungshöhe 23 klm.). Ref. N. J. 1886 I, p. 224.

r884: Meunier: Météorites, p. 62, $88,97,301,302-303,373,421,443$, $446,447,448,459,46 r$.

r883/85: Ts chermak: Photographien, p. 24.

1885: Brezina: Wiener Sammlung, p $166,184,233$.

I886: Ansdell u. Dewar: On the Gaseous Constituents of Meteorites. Proc. Royal Soc. Bd. 40 , p. $557-55^{8}$; s. auch Am. Journ. (3) 32 , p. 483 . Ref. N. J. 1887 II, p. 285 ; LiebigKopp, Jahresber. 1886, p. 2327.

1888: N ew t o n: Orbits, Am. Journ. (3) 36, p. 4.

1890: Brezin a: Reise, Ann. Hof-Mus. Bd. 4 (Not.), p. 119 .

r89o: v. Niess 1: Periheldistanzen, Verh. naturf, Ver. Brünn Bd. 29, p. 186, $187,188,194,210,214,231,232,247$. 1894: Cohen: Meteoritenkunde, an vielen Stellen.

1894: F l e t c h e r: Introduction,p. 27,29.

Ursprüngliches Gewicht: Ueber 20 Steine von Kopf-bisFaust-Grösse.

Nachweisbares Gewicht: 11523 gr.

\begin{tabular}{lr|lr} 
Bailey & 2 & Bonn & 36 \\
Bayet & I & v. Braun & 22 \\
Belgrad & 2 & Breslau & 54 \\
Berlin, U. & 150 & Budapest & 73 \\
Bologna & 5 & Calcutta & 40
\end{tabular}

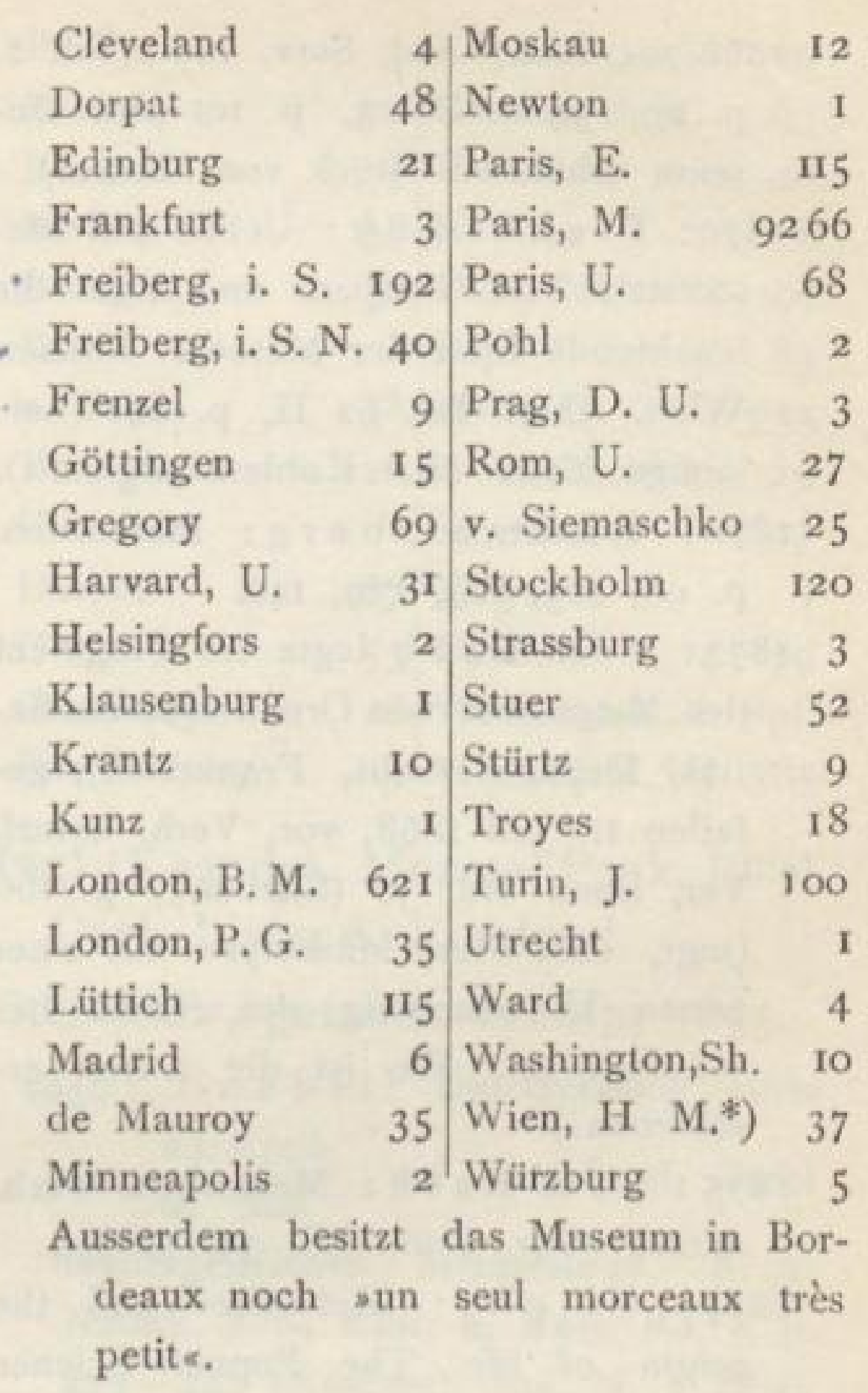

Orléans 18 ro

Orléans 1872

Ormes

Charsonville

Lancé

Les Ormes

Ornans, Salins, Doubs, Frankreich. Stein, Cco, gefallen II. Juli 1868. 1868: $\mathrm{P}$ is a n i: Analyse d'une météorite tombée le II juillet $\mathbf{I} 868$ à Ornans (Doubs). C. R. Bd. 67 , p. $663-665$ (Analyse). Ref. N. J. 1869, p. $371-$ 372 ; Luminous Meteors, Rep. Brit. Assoc. 1869, p. 277; Liebig-Kopp, Jahresber. 1868, p. 1044; $\rightarrow$ L'Institute Bd. 36 (1868), p. 330-33I.

1868169: Marc o u: Notes sur une météorite tombée le II juillet 1868 , à Lavaux, près Ornans (Doubs). Bull, Soc. géol. France Bd. 26, p. 92-94.

1868/69: D a u b r é e: Observations sur la météorite d'Ornans et sur l'imitation artificielle de la structure globulaire ou chondritique. Bull. Soc. géol. France Bd. 26, p. 95-100; s. auch s L'Institute Bd. 37 (1869), p. 249. 
1868/70: Rec. Geol. Surv. India Bd. 2, p. 20,34 u. Bd. 3 , p. Ior (das Museum erhält ein Stück von Marcou).

I870: Tschermak: Ueber den Meteorstein von Goalpara und über die leuchtende Spur der Meteore. Sitzber. Wien. Akad. Bd. 62 II, p. $86 \mathrm{I}$ (beiläufige Notiz über Kohlenstoffgehalt). 1870: R a m me 1s berg: Meteoriten, p. $98,103,107,139,140$.

1873: vom R a th legte ein Fragment des Meteoriten von Ornans (unfern Salins, Depart. Doubs, Frankreich), gefallen II. Juli I868, vor. Verh, naturf. Ver. Bonn Bd. 30 (Sitzber.), p. 166 (sagt, dass Story-Maskelyne mit einer neuen Untersuchung des Steins beschäftigt sei. Wo ist die Arbeit erschienen ?)

1875: vom R a th: Meteoriten, Verh. naturh. Ver. Bonn Bd. 32, p. 374.

1877: Flight: Meteorites and the origin of life. The Popular Science Review 18. Okt. 1877 , p. Io (Sep.).

1879: R a m mel sberg: Meteoriten, p. 26.

1884: M e unier: Météorites, p. 280, $28 \mathrm{r}-284$ (Abb.), 336, 337 .

r883/85: T schermak: Meteoriten, p.r7. 1885: Brezin a: Wiener Sammlung, p. 190-19I, 233.

r887: F1 ig h t: Meteorites, P.r56-r57. r894: Cohen: Meteoritenkunde, p. $163,205$.

Ursprüngliches Gewicht: Ein Stein von $6050 \mathrm{gr}$.

Nachweisbares Gewicht: $5059 \mathrm{gr}$.

Bailey

Bement

Berlin, U.

Bonn

Budapest

Calcutta

Gregory

Greifswald

Harvard, U.
21 London, B. M. 1018

4 London, P. G. 19

Spl. Newton 102

5 Paris, M. 3707

II v. Siemaschko I3

89 Stockholm 2I

2 Washington, 6

3 Wien, H. M.*) 26
12 Troyes Spl.

\section{Oroville}

s. Anhang

Orvinio, Umbrien, Italien.

Stein, Co, gefallen 31 . August 1872 .

1872: F errari: Richerche fisico-astronomiche intorno all' Uranolito caduto nell' agro Romano il $3 \mathbf{I}$ di Agosto 1872. Roma. Tip. Belle Arti 1873. $4^{\circ}$. 46 Seiten u. Karte. Ref. Wochenschr. f. Astr., Met. u. Geogr. 1873, p. 230 $-232,239-240 ; 1874$, p. 408 .

1872: Secchi: Sur les diverses circonstances de l'apparition d'un bolide aux environs de Rome et sur les spectres stellaires. C. R. Bd. 75 , p. 656 $-658$

1873: de Rossi u. Bellucci: Atti dell' Acc. pontif. di nuovi Lincei (1873). 1873: > Les Mondes vom 25. Dezember 1873 .

1873: K e 11 er: Der Meteorsteinfall von Orvinio im August 1872. Pogg. Ann. Bd. 150 , p. $171-176$. Ref. LiebigKopp, Jahresber, 1874, p. 1343-1344. 1874: Keller: Nachträgliches über den Meteorsteinfall von Orvinio. M. M. (1874), p. $258-260$.

1874: vom $R$ ath legte ein kleines Fragment des Meteoriten von Orvinio (früher Canemorto), Provinz Umbrien, $45 \mathrm{klm}$. nordöstlich von Rom, vor. Verh, naturh. Ver. Bonn Bd, 31 (Sitzber.), p. II8-II9.

1875: Tschermak: Die Trímmerstruktur der Meteoriten von Orvinio u. Chantonnay. - Orvinio. Sitzber.Wien. Akad. Bd. 70 I, p. $459-465,469-$ 470 (Analyse von Sipöcz, Abbildung eines Steins, Ansicht eines Schnitts u. Abb. von Dünnschliffen); s, auch M. M. 1874, p. 244 246. Ref. N. J. 1875 , p. $418-420$.

r875: Ts cherm a k: Vulkanismus, Sitzber. Wien. Akad. Bd. 71 II, p. 662, 663,671 .

1875: vom R a th: Meteoriten, Verh. naturh. Ver. Bonn Bd. 32, p. 374. 
1878: Ts cherma k: Der Meteoritvon Grosnaja. M. P. M. Bd. I, p. I6ı.

1878: M a k ow s y u. T s chermak:

Bericht über den Meteoritenfall bei

Tieschitz in Mähren. Denkschr. Wien. Akad. Bd. 39, p. 196.

1879: Rammelsberg: Meteoriten, p. 13 .

r88r: v. Nor denskiöld: Zeitschr. d. d. geol. Ges, Bd. 33, p. 24 (Vergleich mit Ställdalen).

r882: Brez ina: Bericht IV, Sitzber. Wien. Akad. Bd. 85 I, p. 338,339 . 1882: Ts chermak: Ueber die Meteorite von Mocs. Sitzber.Wien. Akad. Bd. 85 I, p. 204.

r883: Tschermak: Beitrag, Sitzber. Wien. Akad. Bd. 88 I, p. $360,36 \mathrm{r}$.

1884: Meunier: Météorites, p. 241 (Abb.), 242, 245-246, 450, 492-493. r884: W a d swort h: Studies, p. ro5 -106 .

r883/85: Tscherma k: Photographien, p. 3,19 .

r885: Brezina: Wiener Sammlung. p. $165,184.233$.

r887: Flight: Meteorites, p. 58-62. 1889: M e un i er: Sur la matière noire de la Chantonnite. Bull. Soc. Min. France Bd. 12, p. $76-8 \mathrm{I}$.

r89o: v. Ni e s s 1 : Periheldistanzen, Verh. naturf. Ver. Brünn Bd. 29, p. 184 , r86, 188, 191, 195, 214, 245 .

r894: C o he n: Meteoritenkunde, p. 59, $316,317$.

1894: F l e t c h er: Introduction, p. 39. r895: Brezina: Wiener Sammlung, p. 252 .

Ursprüngliches Gewicht: $3396 \mathrm{gr}$. 6 s Fragmente (1 $242 \frac{1}{2}, 1003$, $622,43^{2}, 92,4^{3 / 4}$ gr.).

Nachweisbares Gewicht: 2324 gr. ').

\begin{tabular}{lr|lr} 
Bailey & 3 & Kopenhagen & 10 \\
Bellucci & 20 & London, B. M. & 63 \\
Bement & 3 & Neumann & 21 \\
Berlin, U. & 38 & Newton & I \\
Bologna & 32 & Paris, M. & 109 \\
Bonn & 2 & Pohl & 83 \\
v. Braun & 58 & Rom, U. & 735 \\
Budapest & 226 & v. Siemaschko & 16 \\
Cleveland & I & Stockholm & 97 \\
Dorpat & 67 & Troyes & I \\
Gregory & 53 & Turin, U. \\
Greifswald & 18 & Washington,Sh. Spl. \\
Klausenburg & 3 & Wien, H. M.**) & 585
\end{tabular}

Ösel (Kaande), Moustel Pank, Insel Ösel, Livland, Russland.

Stein, $\mathrm{Cw}$, gefallen 11 . Mai 1855 .

1856: Go e be 1: Untersuchung eines am $\frac{\text { 29. April }}{\text { II. Mai auf der Insel Oesel }}$ niedergefallenen Meteorsteins. Arch. Naturk. Liv., Ehst. u. Kurl. Bd. I, p. 477-482 (Analyse). Ref. N. J. 1856, p. $690-692$ u. N. J. 1858 , p. 320 ; Pogg. Ann. Bd. 99 (1856), p. $642-$ 644 ; Ann. Chem. Pharm. Bd. 98 (1856), p. $387-388$; Journ. prakt. Chem. Bd. 69 (1856), p. 307-308; Am. Journ. (2) 24 (1857), p. 295; Kenngott, Uebersicht $1856 / 57$, p. $236-237$; Chem. Centr. 1856 , p. $603-604$; LiebigKopp , Jahresber. 1856, p. 913-914. 1857: >L'Institut « Bd. 25 (1857), p. 136. Ref. N. J. 1857 , p. $842-843$; Wochenschr. f. Astr., Met, u. Geogr. 1857 , p. $173-174$.

1859: B u c h ner: Feuermeteore, p.IO2, 182 .

r859: H a r r is : Dissert,Gött., p.96-97. r860: R a m mels berg: Mineralchemie, p. 923 ff., 950 .

r863: B u c h ner: Meteoriten,p. $87-88$. r863: Ros e: Meteoriten, p. 155.

1) Herr Keller, der sich um das Sammeln der Steine grosse Verdienste erwarb, schenkte dem Mineralienkabinet der römischen Universität und dem von S. Maria sopra Minerva Stücke dieses Meteoriten, dem ersteren ein solches von $735 \mathrm{gr}$. 
1864: Grewingk u. Schmidt: Ueber die Meteoritenfälle von Pillistfer, Buschhof u. Igast in Liv- u. Kurland. Arch f. Naturk. Liv., Ehst. u. Kurl. Bd. 3 , p. $41-45,66-69$ (betrifft den Pseudometeoriten Igast).

r859/65: v. Reichen ba ch: IX I6r, $168,178, X_{359}, 363,365,372$. XI 294, 295. XIII 360, 362, 369 (Fig.). XX 626. XXV 607, 615.

1865: Buchner: Zweiter Nachtrag, Pogg. Ann. Bd. 124, p. $583-585$.

1867: Buchner: Dritter Nachtrag, Pogg. Ann. Bd. 132, p. 318

1867: Go e b e 1: Kritische Uebersicht, Mélanges phys. chim. Bd, 7, p. 337 . 1870: Rammelsberg: Meteoriten, p. 103, 105, 106, 138, 139, 140.

1875: vom Rath: Meteoriten, Verh. naturh. Ver. Bonn Bd. 32, p. 368.

1879: R a mmels berg: Meteoriten, p. 24 .

r882: v. L a s a u $1 \mathrm{x}$ : Vermehrung, Verh. naturh. Ver. Bonn Bd. 39 (Sitzber.), p. I08-IIo (Igast als Pseudometeorit erkannt).

r884: M e un ier: Météorites, p. 79, 85 , $87,209,219-220(293-294,352$, 524 Igast).

r885: Brezina: Wiener Sammlung, p. $177,232$.

1894: Cohen: Meteoritenkunde, p. 68, 206, 248, 303, 32I.

Ursprüngliches Gewicht: Mehrere Steine; nur Bruchstücke von einem grossen Stein im Gesamtgewicht von höchstens $6 \mathrm{Kgr}$. erhalten.

Nachweisbares Gewicht: $1500 \mathrm{gr}$.

\begin{tabular}{lr|lr} 
Bailey & 20 & Calcutta & 9 \\
Bement & 3 & Dorpat & 465 \\
Berlin, U. & 21 & Göttingen & 12 \\
Bologna & 3 & Gregory & 22 \\
Bonn & 9 & Helsingfors & 20 \\
v. Braun & 4 & Kiew & 133 \\
Budapest & 232 & Krüger & 27
\end{tabular}

\begin{tabular}{lr|lr} 
v. Leuchtenberg & 27 & v. Siemaschko & 22 \\
London, B. M. & 18 & Stockholm & 50 \\
London, P. G. & I & Stürtz & 3 \\
Moskau & 214 & Tübingen & 21 \\
New Haven & 6 & Utrecht & 29 \\
Paris, M. & 4 & Ward & 3 \\
Petersburg, A. & 47 & Washington & 13 \\
Riga, N. & 1 & Washington, Sh. & 4 \\
Riga, P. & 4 & Wien, H. M.*) & 21 \\
Rom, U. & 32 & &
\end{tabular}

\section{Oshima}

Ostrolenka

Maêmê

Oswego Co.

Otsego Co.

Otumpa

Ouaregla

Oude 1838

Oude 1872

Oviedo 1856

Oviedo 1866

Pultusk

Scriba

Burlington

Campo del Cielo

Haniet-el-Beguel

Kaee

Dyalpur

Oviedo

Cangas de Onis

Oviedo, Asturien, Spanien.

Stein, Cw, gefallen 5. August 1856 . r865: Bu chner: Zweiter Nachtrag, Pogg. Ann. Bd. 124, p. 591.

r867: L u a n c o: Revista de los Progressos de las Ciencias Exactas, Fisicas y Naturales. Madrid 1867 (Analyse; s. auch bei Gredilla 1892).

r884: M e un ier: Météorites, p.209,220. r885: Brezina: Wiener Sammlung, p. 265 .

r8go: Brezin a : Reise, Ann. Hof-Mus. Bd. 4 (Not.), p. $\mathbf{\text { I9. }}$

1892: Gredilla: Meteoritos, p. 106 -108 .

Ursprüngliches Gewicht: ?

Nachweisbares Gewicht: $30 \mathrm{gr}$.

Madrid

I6 Paris, M.

Ovifak

Oynchi-mura

s. Anhang

Maêmê

Pacula, Tacal, Hidalgo, Mexico.

Stein, Cwb, gefallen r8. Juni 188 1. 1889: C a st i 11 o: Catalogue, p.12-I5. 
189o: F let cher: Mexican Meteorites, Miner 1. Magaz. Bd. 9, p. 95.

1895: Brezina: Wiener Sammlung, p. 246 .

Ursprüngliches Gewicht: $3361 \mathrm{gr}$. Drei Stücke; das grösste im Gewicht von 2 II 5 gr. u. das kleinste, dessen Gewicht bei Castillo nicht angegeben wird, besass 1889 Castillo.

Nachweisbares Gewicht: I I91 gr. Bailey

Bement

v. Braun

Cleveland

Paderborn

Pallas-Eisen

Pampanga

Panganur

Papas quiaro

Parjabatpur

Park Hotel

Parma

33 London, B. M. 28

797 Paris, M. 64

I Wien, H. M. 266 2

Hainholz
Krasnojarsk
Mexico (Stein)
Mooradabad
Bella Roca
Bishunpur
Bald Eagle
San Donino

Parnallee, Madura, Ostindien.

Stein, Cga (früher als Cg aufgefasst), gefallen 28. Februar 1857 .

1857: Taylor: Trans. Geogr. Soc. Bombay (1857). Eine Notiz soll auch im "Atheneum * (wahrscheinlich Madras-Athenaeum) enthalten sein; ferner im: Catalogue of Minerals, Ores and Roches with a note on Meteorites, of which the fall in southern India has been recorded. Government Central Museum, Madras 1890, p. 102-104; s. auch Am. Journ. (2) 3I, p. 302. 186x: Haidinger: Der Meteorsteinfall von Parnallee bei Madura in Hindustan. Sitzber.Wien. Akad. Bd. 43 II, p. 307-309; Kenngott, Uebersicht 1861, p. 169; s L'Institut * Bd. 29 (1861), p. 296 .

186r: Casse 1s: Notice of a Meteorite W il I fing, Meteoriten. which fell in Hindostan in 1857. Am. Journ. (2) 32, p. $40 \mathrm{O}-403$ (Abbildung des kleineren Steins); s. auch Kenngott, Uebersicht 186r, p. 169 u. $1862 / 65$, p. 436 ; sL'Institut « Bd. 30 (1862), p. 276.

r86r: Hai dinger: Der Meteorit von Parnallee bei Madura im k. k. HofMineralien-Kabinete Sitzber.Wien.Akad. Bd. 44 II, p. II7-120. Ref. N. J. 1862, p. 490; Kenngott, Uebersicht $1862 / 65$, p. $436-437$; Liebig-Kopp, Jahresber. 1861, p. I122-II23; Am. Journ. (2) 34 (1862), p. I53-154; Luminous Meteors, Rep. Brit. Assoc. 186r, p. $35-36$ (Sep.).

r86r: W ö h 1 e r veranlasst Bunsen, die Meteoriten spektralanalytisch zu untersuchen; er findet in Juvenas u. Parnallee Lithion. Ann. Chem. Pharm. Bd. 120, p. 253-254; s. auch Gött. Gel. Anz. (Nachr.) 1863, p. 222-223; Kenngott, Uebersicht $1862 / 65$, p. 440. r862: M a s k e 1 y n e: Chondritic Aërolites, Rep. Brit. Assoc. (Not, and Abstr.), p. 190. Ref. Kenngott, Uebersicht 186265 , p. 442 .

1863: H a i d ing e r: Parnallee. Dritter Bericht. Sitzber. Wien. Akad. Bd, 47 II, P. 420-425 (Abbildung des grösseren Steins); s. auch »L'Institut « 1863, p. 295 ; Chem. Centr. 1864 , p. 398 ; Liebig-Kopp, Jahresber. 1863 , p. 910 -9 II.

1863: P fe iffer: Prozentische Zusammensetzung des Meteorsteines von Parnallee bei Madura in Ostindien. Sitzber. Wien. Akad.Bd.47 II, p.46o-463 (Analyse); s. auch Kenngott, Uebersicht 1862/65, p. $438-439$; Liebig-Kopp, Jahresber. 1863, p. 910 ; sL'Institut * Bd. 31 (1863), Nr. 1550, p. 295-296.

r863: B u c hner: Meteoriten, p. $91-92$. 1863: Rose: Meteoriten, p. 155. 1863: Maskelyne u. v. Lang : Mineralogical Notes. - Notices of Aërolites von Maskelyne. - п. Parnallee. 
Philos, Magaz. Bd. 25 , p. $43^{8}-440$ (zwei Abbildungen des grossen Steins, Taf. VIII).

1864: S or b y: On the Microscopical Structure of Meteorites. Proc. Royal Soc. Bd. 13, p. $333-334$; s. auch Am. Journ. (2) 4I (1866), p. 137, 138. 1864: Buchner: Erster Nachtrag, Pogg. Ann. Bd. 122, p. 320-321. 1864/65: v. Reichenbach: XXIII 369. XXV 322, 421, 422 .

1865: Buchner: Zweiter Nachtrag, Pogg. Ann. Bd, I24, p. $5^{82}$. 1869: Buchner: Vierter Nachtrag, Pogg. Ann. Bd. 136, p. 439.

1870: Rammels berg: Meteoriten, p. $98,103,105,106,139$.

r87x: Me unier: Coexistence de deux types lithologiques dans la même chute de météorites. C.R. Bd.73,p.I483-1485. r87r: Meunier: Étude lithologique de la météorite de Parnallee. C, R. Bd. 73 , p. $346-350$.

1875: M o hr: Entstehungsart, Ann. Chem. Pharm. Bd, 179, p. 259.

r876: W right: On the Gases contained in Meteorites. Am. Journ. (3) II, p. 258,260 u. (3) $12(1876)$, p. 167 , 171 (Sorby).

1877: $\mathrm{S} \mathrm{m}$ it h: (vergleicht Parnallee mit Cynthiana). C. R. Bd. 85. p. 680.

1879: Rammelsberg: Meteoriten, p. 24,25 .

1880: Hahn: Die Meteorite (Chondrite) und ihre Organismen. Tübingen. T. II, Fig. 5; T. 13, Fig. 1.

r884: M e uni er: Météorites, p. 79, 95, 98,273 (Abb.), 276-277, 347, 348, 380,395 .

1885: Brezina: Wiener Sammlung, p. $182,232$.

1887: F l i g h t: Meteorites, p. 127-128.

1894: Cohen: Meteoritenkunde, an vielen Stellen,

r894: F let c her: Introduction, p. Io.

1895: Brezina: Wiener Sammlung, p. $249,250$.
Ursprüngliches Gewicht: $74 \mathrm{Kgr}$. Zwei Steine von zusammen 74 Kgr. (1 27 u. 37 lbs.).

Nachweisbares Gewicht: 72237 gr.

\begin{tabular}{lrl|l} 
Bailey & I 565 & London, B. M. 6r 36I \\
Belgrad & I & London, P. G. & 187 \\
Bement & 352 & Madrid & 84 \\
Berlin, U. & 423 & Minneapolis & 8 \\
Bologna & 45 & Modena & I \\
v. Braun & 12 & Moskau & 12 \\
Budapest & 47 & München & 7 \\
Calcutta & 150 & Neumann & 24 \\
Cleveland & 2153 & New Haven & 2370 \\
Dorpat & 60 & Newton & I \\
Dresden, M. & 67 & Paris, M. & 482 \\
Giessen & 2 & Pohl & 115 \\
Göttingen & 87 & v. Siemaschko & 23 \\
Gregory & 104 & Stockholm & 162 \\
Greifswald & 5 & Strassburg & 58 \\
Hamburg & 92 & Stürtz & 4 \\
Harvard, U. & 421 & Troyes & 27 \\
Heidelberg & I & Tübingen & 135 \\
Howell & 31 & Ward & 356 \\
Klausenburg & 8 & Washington & 87 \\
Kopenhagen & 15 & Washington, Sh. 331 \\
Kristiania & 7 & Wien, H. M.*) & 739
\end{tabular}

Das Western Reserve College in Hudson, Ohio, welches den Stein von 37 lbs. erhielt und verteilte, dürfte auch noch ein Stück von diesem Meteoriten besitzen.

Pau

Bueste

Pawlodar

Pawlograd

Jamyschewa Mordvinovka

Pawlowka, Bez, Balaschew, Gouv. Sarátow, Russland.

Stein, Ho, gefallen 2. August 1882. r883: Tschernyschow: Ueber einen im Gouvernement Sarátow am 2I. Juli 1882 gefallenen Meteoriten. Zeitschr. d. d. Geol. Ges. Bd. 35, p. 190-192, Aus den Verh, d. kaiserl. russ. mineral. Ges, zu St. Petersburg (2) 18 (1883), p. 205-211 auszugsweise mitgeteilt durch A. Arzruni. Ref. 
N. J. 1884 I, p. 33 ; Liebig-Kopp, Jahresber. $1888_{3}$, p. 1954 .

r884: W a d s w o r th: Studies, p. 198. r884: M e unier: Météorites, p. 286. 1885: Brezina: Wiener Sammlung, p. $174,232$.

r894: C o he n: Meteoritenkunde,p.246, 293.

1895: Brezina: Wiener Sammlung, p. 241 .

Ursprüngliches Gewicht: $2 \mathrm{Kgr}$. (5 Pfund russisch?).

Nachweisbares Gewicht: I123 gr.

\begin{tabular}{lr|lr} 
Bement & 4 & Paris, M. & I22 \\
Bologna & 20 & Petersburg, B. & 15 \\
v. Braun & 8 & Sevilla & Spl. \\
Breslau & 8 & v. Siemaschko & 170 \\
Budapest & 285 & Stockholm & 150 \\
Cohen & 3 & Strassburg & 4 \\
Gregory & 119 & Utrecht & 12 \\
Klausenburg & 3 & Washington, Sh. & 6 \\
London, B. M. & 78 & Wien, H. M. & 89 \\
Madrid & 27 & &
\end{tabular}

Eine quantitative Analyse scheint noch nicht ausgeführt zu sein; dieselbe wäre um so wünschenswerter, als der Stein der seltenen Gruppe der Howardite angehören soll und von ihm genügend Material vorhanden ist.

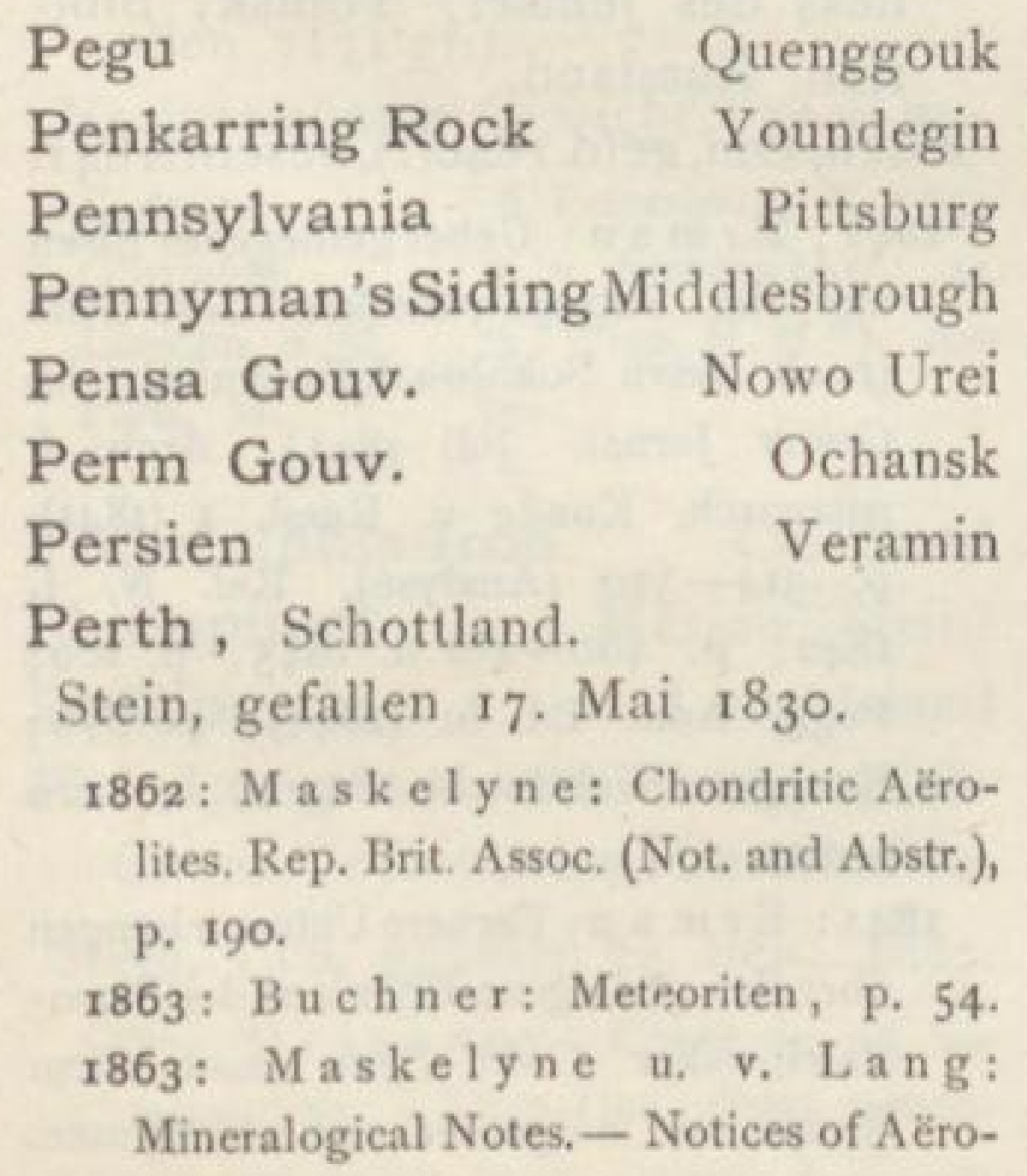

lites von Maskelyne. - ro. Perth. Philos. Magaz. Bd. 25, p. 437. Ref. Kenngott, Uebersicht 1862165 , p. 442. r865: Buchner: Zweiter Nachtrag,

Pogg. Ann. Bd. 124, p. $578-579$. 1868/70: Rec. Geol. Surv. India Bd, I, p. $72-73$ u. Bd. 2, p. 34 (das Mus. der Geol. Surv. erhält von Nevill ein Stück von 6.5 grs.).

1885: Brezina: Wiener Sammlung, p. 265 .

Ursprüngliches Gewicht: Ein Stein von 7 Zoll Durchmesser, von welchem wie es scheint nur Nevill ein Stück besass, soll gefallen sein. Das Nevill'sche Stück gieng teils nach London, teils nach Calcutta. Weitere Nachforschungen wären wohl von Glasgow oder Edinburg aus anzustellen. London besitzt $\mathrm{I}^{1} / 2$ gr., Calcutta Spl.

$\begin{array}{lr}\text { Peru } 1840 & \text { Tarapaca } \\ \text { Peru } 1871 & \text { Iquique } \\ \text { Perugia } & \text { Assisi }\end{array}$

Petersburg, Lincoln Co., Tennessee, U. S. A.

Stein, Ho, gefallen 5 . August 1855 . 1856: Smith: (In Saffords report of the geology of Tennessee for 1855). Geol,Reconnoissance Surv. Tenn. Nashville 1856 .

1857: Shepard: Notice of a Meteoric Stone which fell at Petersburg, Lincoln county, Tennessee, August $5^{\text {th }}$, 1855. Am. Journ. (2) 24, p. I34-137 (Analyse von J. L. Smith); s, auch Pogg. Ann. Bd. 103 (1858), p. 434; sL'Institut \& Bd. 26 (1858), Nr. 1263, p. 98; Liebig-Kopp, Jahresber. 1857 , p. 732. Ref. N. J. $185_{5}^{8}$, p. 686 ; Kenngott, Uebersicht 1856157, p. 238 u. 1858 , p. 164 u. 1859 , p. $164-165$; Wochenschr. f. Astr., Met. u. Geogr. 1858 , p. 208. 
1858: Giebel u. Heintz: Zeitschr. f. d. ges. Naturw. Bd. II, p. 563 (nach Buchner's Quellenverzeichnis).

1859: $\mathrm{B}$ u c h n e r : Feuermeteore, p.Io3, I83 (führt die Analyse von Snith als von Irmisch stammend auf).

r859: Jla rris: Dissert. Gött., p. 97 -98 .

r86r: $\mathrm{S} \mathrm{m} \mathrm{ith:} \mathrm{Description} \mathrm{of} \mathrm{three}$ new Meteorites. - Lincoln County Meteoric Stone which fell in August, 1855 . - Oldham County (Ky) Meteoric Iron. - Robertson County (Tenn.) Meteoric Iron. - Lincoln County Meteorites. Am. Journ. (2) 3I, p. 264-265 (inhaltlich gleich der obigen Arbeit von Shepard, in welcher Smith auch genannt ist); s, auch Original Researches (1884), p. 426-427. Ref. N. J. 1861, p. 328; Kenngott, Uebersicht 186I, p. 164; Zeitschr. f. d. ges. Naturw. Bd. 17, p. 474; LiebigKopp, Jahresber. I861, p. II26-II27 ; Journ. prakt. Chem. Bd. 85 (1862), p. $5^{15}$.

r 863: Bu c hn er: Meteoriten, p. $89-9$ o. I863: Ros e: Meteoriten, p. 30, 126, I35-137, 156 .

1859/65: v. Reichenbach: VIII 476,477 . X 372 . XIII $353,356,384$. $\mathrm{XV}$ 101. XX 620,623,631,632. $\mathrm{XXV}_{422}$. 1865: Buchner: Zweiter Nachtrag, Pogg. Ann. Bd. 124, p. 574 .

1870: R a mmels berg: Meteoriten, p. 127, 129-131; s, auch Pogg. Ann. Bd, 140 (1870), p. 321 .

1874: Ts chermak: Der Meteoritenfund bei Ovifak in Grönland. - Meteorischer Eukrit, M. M. (1874), p. 17 o.

1877: Sor by: On the structure and origin of Meteorites. $>$ Naturee Bd, 15 , p. 496.

1883: T s c hermak: Beitrag, Sitzber. Wien. Akad. Bd. 88 I, p. 368 .

1884: Meunier: Metéorites, p. 75, 77, $85,96,286,288$.

r884: W a d s w or th: Studies, p. I96. r883/85: T s c hermak: Photographien, p. 6,7 .

1885: Brezina: Wiener Sammlung, p. $174,232$.

r890: Eastman: Met. Astron., p. 316. r894: C ohen: Meteoritenkunde, p. 159, 296, 307, 319.

r894: F l et ch e r: Introduction, p. 37 . Ursprüngliches Gewicht: Ein Stein von $1764 \mathrm{gr}$. (3 lb. $14 \frac{1}{2}$ oz.). Nachweisbares Gewicht: 399 gr.

\begin{tabular}{lrr|r} 
Bailey & 4 & Moskau & 6 \\
Bement & I & New Haven & 15 \\
Berlin, U. & 73 & Paris, M. & 14 \\
Bonn & I & Petersburg, B. & I \\
v. Braun & I & Pohl & 12 \\
Budapest & I & Riga & Spl. \\
Calcutta & 41 & Rom, U. \\
Cleveland & 5 & v. Siemaschko & 5 \\
Dorpat & 23 & Stockholm & 20 \\
Göttingen & 14 & Troyes & Spl. \\
Gregory & 2 & Tübingen \\
Harvard, U. & 14 & Upsala \\
London, B M. & 53 & Washington, Sh. \\
London, P. G. & 16 & Wien, H. M.*) & 24 \\
Madrid & Spl. &
\end{tabular}

Wo befindet sich dieHauptmasse desSteins?

Petropawlowsk am Mrass, Nebenfluss des Jenisei, Tomsk, Sibirien, Russland.

Eisen, Om, gefd.r840(?), beschr.r841.

184I : E rm a n: Ueber gediegenes Eisen aus der Petropawlowsker Gold-Seife (nach Herrn Sokolowskji's Aufsatz im Gorny Jurnal, Juli 1841). Arch. f. wissensch, Kunde v. Russl. I (184I), p. 314-320 (Analyse). Ref. N. J. 1842 , p. $460-46 \mathrm{I}$ u. 1845 , p. 106 ; Pogg. Ann. Bd. 61 (1844), p. 676; Kenngott, Uebersicht $1844 / 49$, p. 226 -227 .

184r: Erm a n: Fernere Untersuchungen über das gediegene Eisen aus der PetroPawlowsker Gold-Seife nach dem Russischen mitgeteilt von A. Erman. 
Arch. f. wissensch. Kunde v. Russl. Bd. 1 (1841), p. $723-725 ;$ s. auch "L'Institut « Bd. 14 (1846), p. 96. 1843: Partsch: Meteoriten, p. I44 $-\mathrm{I} 45$.

1847: Eichwald: Verzeichnis, Erman's Arch. f. wissensch. Kunde v. Russl. Bd. 5 (1847), p. 184.

1848: B l o e d e: Tabelle, Bull. Petersburg. Akad. Bd. 6, p. II.

1852: C 1 a r k: Dissert. Gött., p. 72.

1854: v. B o g us la w s k i: ZehnterNachtrag, Pogg. Ann. Ergz.-Bd. 4, p. 396. r859: B u c h ner: Feuermeteore, p. 116 $-\mathrm{II7}$.

r859: Ha rris: Dissert. Gött., p. II3. r859/6r: v. Reich en ba ch: IX 163 ,

174,182 . XV 110. XVI 261.

r863: B u c h n e r : Meteoriten, p.163,164. 1867: Buchner: Dritter Nachtrag,

Pogg. Ann. Bd. 132, p. 319.

1885 : Brezina: Wiener Sammlung,

p. $211-212,234$.

r893: M eunier: Revision des fers météoriques, p. 29, 34 .

r894: C o hen : Meteoritenkunde, p. 95.

Ursprüngliches Gewicht: Etwas über $7 \mathrm{Kgr}$. ( $17 \frac{1}{2}$ Pfund russisch, welche angegeben werden, entsprechen $7171 \mathrm{gr}$.).

Nachweisbares Gewicht: 7200 gr.

\begin{tabular}{lr|lr|} 
Calcutta & 6 & Petersburg, B. 7050 \\
Göttingen & 6 & v. Siemaschko & 14 \\
London, B. M. & 12 & Wien, H. M.*) & 100 \\
Paris, M. & 12 & &
\end{tabular}

\section{Philadelphia-Iron}

Cleveland Philippinen Mexico (Stein) Phillips Co. Long Island

Phû Long oder Phu-Hong, BinhChanh,Cochinchina,Hinterindien. Stein, Cca, gefallen 22, Sept. 1887 . r887: De 1 a uney: Chute le 25 octobre 1887 , à Than-Duc, d'une météorite qui parait avoir disparu à la suite d'un ricochet. C. R. Bd. 105, p. I294, wo am Schluss Phu-Long erwähnt wird. 1888: D a u bré e: Météorite tombée le 22 septembre 1887 , à Pha Long, BinhChanh (Cochinchine). C. R. Bd. I06, p. $3^{8}$. Ref. N. J. 1889 I, p. 227.

r889: Meunier: Analyse de la météorite de Phu-Hong; remarques sur le type limerickite. C. R. Bd. Io9, p. $875-878$ (Analyse). Ref. N. J. I891 I, p. $45-46$.

r89o: B r e z in a: Reise, Ann. Hof-Mus. Bd. 4 (Not.), p. 119.

r894: Coh en: Meteoritenkunde,p.265. Ursprüngliches Gewicht: ?

Nachweisbares Gewicht: 365 gr.

$$
\text { Paris, M. } \quad 365
$$

Piacenza Borgo San Donino

Piemont $1840 \quad$ Cereseto

Piemont $1860 \quad$ Alessandria

Piemont r868 Motta di Conti

Pila Rancho de la Pila

Pila (Brezina) Humboldt-Eisen

Pillistfer, Aukoma, Wahhe, Kurla, Kreis Fellin, Livland, Russland. Stein, Ck, gefallen 8. August 1863 . I863: R o s e berichtete über zwei netue Meteoritenfälle nach den Mitteilungen, die ihm Hr. Prof. Grewingk in Dorpat gemacht hatte. Mon.-Ber. Berlin. Akad. (1863), p. $44 \mathrm{I}-443$. Ref. N. J. 1864, p. 237; Pogg. Ann. Bd. I2O (1863), p. 620-623; Kenngott, Uebersicht 1862165 , p. 444 ; Liebig-Kopp, Jahresber. 1363 , p. 906 .

1863: R os e: Meteoriten, p. 9I, 154. 1864: Buchner: Erster Nachtrag, Pogg. Ann, Bd. 122, p. 323-325.

1864: Grewingk u. S chmidt: Ueber die Meteoritenfälle von Pillistfer, Buschhof, und Igast in Liv- und Kurland. Arch, Nat, Kunde Liv. Ehst. u. Kurl. Bd, 3, p. $425-452,469-472$ (Abbildungen u. Analyse). Ref. N. J. 
1865, p. 99; Kenngott, Uebersicht 186265 , p. 443 ; Liebig-Kopp, Jahresber. 1864 , p. $900-901$.

1875: v om R a th: Meteoriten, Verh. naturh. Ver. Bonn Bd. 32, p. 369 .

188I: v. Nordenskiöld: Zeitschr. d. d. geol. Ges. Bd. 33, p. 24 (Vergleich mit Ställdalen).

r884: Meunier: Météorites, p. 179. I883/85: T s c h e r m a k: Photographien, p. 20 .

1885: Brezina: Wiener Sammlung, p. I9I, 233.

1887: B rezin a: Neue Meteoriten III, Ann. Hof-Mus. Bd. 2 (Not.), p. II4. r888: v. Niess 1: Bestimmungen der Bahnverhältnisse einiger Meteore, Ueber die Bahnen der Meteoriten von Pillistfer am 8. August 1863 u. Krähenberg am 5. Mai 1869. - A. Meteoritenfall von Pillistfer in Livland, Verh. naturf. Ver. Brünn Bd, 27, p.265-270. 1889: F l e t c h er: Atacama Meteorites, Mineral. Magaz. Bd. 8, p. 227 (Streufeld 8 auf $2^{1 / 4}$ miles).

1890: v. Niess 1: Periheldistanzen, Verh. naturf.Ver. Brünn Bd, 29, p. 186, 188, 195, 210, 211, 214, 246-247.

r893: B r e z in a : Ueber neuere Meteoriten (Nürnberg), p. 159, 162.

I893: v. H a u er : Ann. Hof-Mus. Bd. 8 (Not.), p. 49.

I894: Co he n: Meteoritenkunde, p.205, 303.

r895: B rez in a: Wiener Sammlung, p. 260 .

Ursprüngliches Gewicht: In der Rose'schen Mitteilung wird das ursprüngliche Gewicht von 3 Steinen zu etwa 5 I Pfund angegeben. Die Hauptmasse ist in Dorpat (s. Anm. zu Dorpat.).

Nachweisbares Gewicht: $23561 \mathrm{gr}$.

\begin{tabular}{lr|lr} 
Bailey & 2 & London, B. M. & I57 \\
Bement & I & Melion & 2 \\
Berlin, U. & I8 & Moskau & 13 \\
Bonn & 83 & New Haven & I4 \\
v. Braun & 74 & Paris, E. & 34 \\
Brezina & 544 & Paris, M. & II \\
Budapest & 67 & Pohl & 60 \\
Calcutta & 25 & Prag, M. & 123 \\
Dorpat & $20250^{1}$ ) & Riga & 2 \\
Frankfurt & 41 & Rom, U. & 13 \\
Göttingen & 44 & Stockholm & 52 \\
Gregory & 16 & Stuttgart & 7 \\
Heidelberg & 3 & Tübingen & 43 \\
Helsingfors & 22 & Washington, Sh. & 3 \\
Kiew & 128 & Wien, H. M.*) & 1709
\end{tabular}

Seit Juli 1893 erwarb Herr Bayet, Brïssel, 94 gr.

\section{Pilot Grove}

s. Anhang Pine Bluff Little Piney

Pipe Creek, San Antonio, Brandera Co., Texas.

Stein, Cka, gefd. 1887 , beschr. 1888 . 1888/89: Led o ux: The Pipe Creek Meteorite. Trans. New-York Acad. Sc. Bd. 8, p. 186-187 (Analyse). Ref. N. J. 1891 I, p. 50; Oesterr, Zeitschr. f. Berg- u.Hüttenw. Bd. 39 (I89r), p. 344 . 1893: B r ez in a: Ueber neuere Meteoriten (Nürnberg), p. 162.

1895: Brezin a: Wiener Sanmlung, p. 261 .

Ursprüngliches Gewicht: Etwa $13 \frac{1}{2} \mathrm{Kgr}$.

Nachweisbares Gewicht: 7414 gr. Bailey $\quad 333$ London, B. M. 821 Bement $\quad 342$ Minneapolis 25 Budapest $\quad 106$ Paris, M. Cleveland $\quad 22$ Rom, U. 44 Gregory $\quad 3965$ v. Siemaschko 25 Harvard, U. 295 Strassburg 41 Howell $\quad 35$ Ward 603 Krantz $\quad 37$ Wien, H. M. $\quad 428$ Kunz $\quad$ roo Wien, U.I. 77

1) Aukoma, ganzer Stein 11781 ; Kurla, halber Stein $683_{2}$; Wahhe, Bruchstück 1479; Sawiauk, Bruchstïck 158. 


\section{Piprassi}

Butsura

Piquetberg, Capkolonie, Südafrika.

Stein, Cca (Grundmasse ähnlich Ck), gefunden 1881 .

1887: B rez in a: Neue Meteoriten III, Ann. Hof-Mus. Bd. 2, p. $\mathrm{I}_{4}-\mathrm{II}_{5}$.

r889: v. Ha u e r: Ann. Hof-Mus. Bd. 4 (Not.), p. 64.

r895: B r e zi n a : Wiener Sammlung, p. 257 .

Ursprüngliches Gewicht: ?

Nachweisbares Gewicht: ${ }_{3}^{8}$ gr.

Wien, H. M. $\quad 38$

Ueber den Fall scheint noch keine ausführliche Arbeit publiziert worden zu sein.

Pirgunje, Dinagepur, Ostindien.

Stein, Cwa, gefallen 29. Aug. 1882. 1892: v. H a u e r: Ann. Hof-Mus, Bd, 7 (Not.), p. 73 .

r895: Brezin a: Wiener Sammlung, p. 245 .

Ursprüngliches Gewicht: ?

Nachweisbares Gewicht: 743 gr.

London, B. M. 734 | Wien, H. M. 9

Ueber diesen Fall scheint noch keine ausführliche Arbeit publiziert worden zu sein,

\section{Pirna}

Nenntmannsdorf

Pirthalla, Distr. Hissar, Prov. Pandschab, Ostindien.

Stein, Ccb, gefallen 9. Febr. 1884 . 1885: Medlicott: Notice of the Pirthalla and Chandpur Meteorites. Rec. Geol. Surv. India Bd. 18 , p. 148 ; s. auch $>$ Science e Bd. 6, p. 333 ; Engineering $\mathrm{Bd}$. 4I (I886), p. 9I.

$1886: D$ a u b r é e : Météorites récemment tombées dans l'Inde les 19 février 1884 et 6 (muss heissen 9. Febr.) avril 1885 . C. R. Bd. 102, p. $96-97$. Ref, N. J. I887 II, p. 45.

r894: F l e t c h er: Introduction, p. I4. r895: Brezina : Wiener Sammlung, p. 258.
Ursprüngliches Gewicht : $1160 \mathrm{gr}$. (510, 426 u. 224 gr.); $1 / 8$ des ursprünglichen Gewichtes mag verloren gegangen sein.

Nachweisbares Gewicht: 1041 gr.

Calcutta $\quad 480$ Paris, M. 104

London, B. M. 427 Wien, H. M. 30

Pittsburg (Millers Run), Alleghany Co., Pennsylvania, U. S. A.

Eisen, $\mathrm{H}$, gefunden um $185^{\circ}$, beschr. 1850 .

r850: Silliman jr.: Notice of two Meteoric Irons Proc. Amer. Assoc. Fourth meeting held at New-Haven (1850), p. 37 .

r851: Shepard: On Meteorites. 6. Specific gravities of two meteoric irons: "Meteoric iron of Pittsburg, $\mathrm{Pa}$. 7.380\%. Am. Journ. (2) II, p. 40. Ref. Liebig-Kopp, Jahresber. 1850, p. 826 ; Kenngott, Uebersicht $1850 / 51$, p. 137.

1852 : C l a r k: Dissert. Gött., p.75-76. 1859: H a rris: Dissert. Gött., p. 119. r86r: v. Reichenbach: XVI 26r. XVIII 487 .

r863: B u c h ner:Meteoriten,p.r8 5-186. 1875: Genth: On the Pittsburg Meteoric Iron, Rep. Geol. Surv. Penn. I875 (Bd. 6, p. 7 ?) (Analyse); s. auch Am. Journ. (3) $12(1876)$, p. $72-73$. 1883: S m it h: Concretions, Am. Journ.

(3) 25 , p. 419.

1885: Brezina: Wiener Sammlung, p. 218,234 .

1887: F1ig ht: Meteorites, p.172.

1890: Eas tm a n: Met. Astron., p. 3r8. r89r: Cohen u. Weinschenk: Meteoreisen-Studien. Ann. Hof-Mus. Bd. 6, p. 160.

r894: C o h e n: Meteoritenkunde, p. 57. r895: C o h en: Meteoreisen-Studien IV. Ann. Hof-Mus. Bd, Io, p. 88.

Ursprüngliches Gewicht: $132 \mathrm{Kgr}$., grösstenteils verschmiedet. 
Nachweisbares Gewicht: 592 gr.

Bailey

Berlin, U.

Budapest

Calcutta

Göttingen

Gregory

Harvard, U.

3 London, B. M. 208

1 London, P. G. 2

5 New Haven $2 I_{3}$

7 Pohl

99 Tübingen

2 Wien, H. M.*)

I 5 34

Plescowitz

Ploschkowitz

Ploschkowitz, Liboschitz, Bunzlauer Kreis, Böhmen, Oesterreich.

Stein, Ccb (früher als Cgb aufgefasst), gefallen 22 . Juni 1723 .

1725: Rost: Von Steinen, so aus der Lufft gefallen, und noch etwas von dem im Suppl. I, Art. 5 angeführten Diamant-Regen. Sammlung von Natur- u. Medicin - etc, Geschichten (Breslauer Sammlungen), 3I. Versuch, WinterQuartal 1725 , p. $44-47$ (besass einen Stein von $5^{1} / 2$ Pfund)

1754: Stepling: De pluvia lapidea Anni 1753 ad Strkow et ejus Causis meditatio. Typis Francisci Ignatii Kirchner, Prag 1754, p. 33; s. auch Troili, Ragionamento della Caduta di un Sasso dall' Aria. Modena 1766.

I794: C h l a d n i: Pallaseisen, p. 36, 37. 1796: K ing: Remarks, p. 29.

1803: C h 1 a d n i : Chronologisches Verzeichnis, Gilb. Ann. Bd. 15, p. 309.

1803: de Dré e: Recherches, Journ. Phys. Bd. 56, p. $4 \mathrm{I} 5$.

1804: v. End e: Massen u. Steine, p. $35-36$.

1804: G i 1 b e r t : Nachträge zu den Aufsätzen in den Annalen über die aus der Luft gefallenen Steine. Gilb. Ann. Bd. 18, p. 28 r.

1804: Pötzsch: Kurze Darstellung, p. 64 .

r805: M a y er: Beitrag zur Geschichte der meteorischen Steine in Böhmen, Dresden 1805 (Waltherische Hof buchhandlung), p. 8.
I812: $\mathrm{Ch}$ la d n i: Verzeichnis, Schweigg. Journ. Bd. 4 Beil. 1, p. 12.

1812: Bigot de Morogues, p. 86 -87 .

1815: Chla dni: Neues Verzeichnis,

Gilb. Ann. Bd. 5o, p. 247.

r8rg: $\mathrm{Chl}$ a d n i: Feuermeteore, p. 69,

$73,240-241(1)$.

r836: Kä $\mathrm{mtz}$ : Meteorologie, p. 270.

1857: A rago: Astronomie populaire

Bd. 4, p. 192, 193 .

1859: B u c hn er: Feuermeteore, p. 39. r859: H a rris: Dissert. Gött., p. 58. 1862: $\mathrm{Buchner:} \mathrm{Die} \mathrm{Meteoriten} \mathrm{in}$

Wien u. London. Pogg. Ann. Bd. 116, p. $640-641$

1862: Greg: On some Meteorites in the British Museum. - Stones. - 6 . Plescowitz. Philos. Magaz. Bd. 24, p. 540 .

1863: Buchner: Meteoriten, p. 5.

1863: Maskelyne u. v. Lang: Mineralogical Notes. - Notices of Aërolites, von Maskelyne. - 18. Pleskowitz Aërolite. Mineral, Magaz. Bd. 25 , p. $451-452$. Ref. Kenngott, Uebersicht $1862 / 65$, p. 442 .

1865: Buchner: Zweiter Nachtrag, Pogg. Ann. Bd. 124, p. $574-575$.

1885: Brez ina: Wiener Sammlung, p. $182,232$.

r895: B rez in a : Wiener Sammlung, p. 249,257 .

Ursprüngliches Gewicht: Von 33 Steinen wird berichtet; wenig erhalten.

Nachweisbares Gewicht: 37 gr.

London, B. M. $\quad 26$ Pohl

London, P. G. $\quad 7$ Wien, H. M.**) 3

Plymouth, Marshall Co., Indiana, U. S. A.

Eisen, Om, gefunden 1893 ( $188_{3}$ ?), beschr. 1895 .

1895: Ward: Preliminary Notice of the Plymouth Meteorite, Am, Journ. 
(3) 49 , p. $53-55$ (Abbildung der Masse und einer geätzten Platte, die aber wenig erkennen lässt; Analyse von Davison). Ref. N. J. 1896 II, p. 265. r895: Brezina : Wiener Sammlung. p. 285 .

1895: C o he n: Meteoreisen-Studien IV, Ann. Hof-Mus. Bd. 10, p. 82, 90.

Ursprüngliches Gewicht: HerrWard giebt kein Gewicht an; er sagt nur, dass das Stück $12 \frac{1}{2}$ Zoll lang u. $7^{3} / 8$ Zoll dick gewesen sei. Nachweisbares Gewicht: $3676 \mathrm{gr}$.

Bement 3640 Prag, M.

Seit Juli 1893 gelangten an Herrn Direktor Brezina $44 \mathrm{I}$ gr.; an Herrn Bayet, Brüssel $96 \mathrm{gr}$. und an Wien,H.M. 946 gr.

Pnompehn, Cambodga, Cochinchina, Hinterindien.

Stein, $\mathrm{Cw}$, gefallen zwischen 20. u. 30. Juni 1868 .

I868: sLes Mondes « vom 20. Nov, 1868. Ref.Luminons Meteors, Rep.Brit Assoc. 1869, p. 276-277.

1884: M e un i er: Météorites, p. 23 o. r885: Brezina: Wiener Sammlung, p. 265 .

1892 : v. Ha uer: Ann. Hof-Mus. Bd. 7 (Not.), p. 73 .

1895: Brezina: Wiener Sammlung, p. 243 .

Ursprüngliches Gewicht: I Kgr.? [The meteorite separated into three pieces, of which one fell at the door of the King's palace, the other two pieces were picked up at a considerable distance. The first piece, forwarded to France by M. Latou, Naval Surgeon, residing at Pnompehu, is pyramidal, weighing about $2 \mathrm{lbs}$.]

Nachweisbares Gewicht: $4 \mathrm{I}$ gr.

Paris, M. 41 Wien, H. M. Spl.
Poblazon

Descubridora

Pohgel

Poinsett Iron

Nerft

Toluca

Vouillé

Poitiers

Pokra bei Bustee, Goruckpur, N. W. Provinz, Ostindien.

Stein, Ck, gefallen 27. Mai 1866 (dieAngaben schwanken zwischen 27. u. 28. Mai; Calcutta u. London geben den 27. Mai an).

r866: O1d ha m: $=$ Ein Fall fand statt im verflossenen Mai bei Bustee, nahe wo der Fall im Jahre 1852 sich ereignete, nur ein Stück etwa 12 Unzen schwer u. nahe vollständig $\propto$. Verh. k. k. geol. Reichsanst. (Sitzber.), 18. Dez. 1866 Bd. 16, p. 199.

r869: Buchner: Vierter Nachtrag, Pogg. Ann. Bd, 136, p 458.

1885: Brezina: Wiener Sammlung, p. 19I, 233.

1887: Brezina: Neue Meteoriten III, Ann. Hof-Mus. Bd. 2 (Not.), p. 114. 1894: Fletcher: Introduction, p. 13. Ursprüngliches Gewicht: Ein Stein von etwa $340 \mathrm{gr}$. (etwa 12 Unzen '. Nachweisbares Gewicht: 342 gr.

Calcutta $\quad 257 \mid$ Paris, M. $\quad 1_{3}$ London, B. M. $\quad 46$ Wien, H. M.*) 26

\section{Polanko}

Polen (Berzelius)

Mejillonès

Politz, awischen Politz und Langenberg, Köstritz, Gera, Reuss, Deutschland.

Stein, Cwa, gefallen 13 . Okt. 18r9. 1819: B raun: Nachrichten von einem Meteorsteine, der am 13. October 18 I9 unweit Köstritz im Reussischen herabgefallen ist. Gilb. Ann. Bd. 63 , p. 217 -2.28 .

r8rg: Strome y er: Chemische Zerlegung des Köstritzer Meteorsteins, Gilb. Ann. Bd. 63 , p. $451-452$ (Analyse); 
S. auch Schweigg. Journ. Bd. 26 (18I9), p. 251-252; Thomson's Ann. of Philosoph. Okt. 1820, p. 380 ; Ann. Mines Bd, 6 (1821), p. 259.

1814: Lindig jr. : Ueber den am 13. Oct. $18 \mathrm{r} 9$ in der Gegend von Gera gefallenen Meteorstein. (Auf Anordnung des Ober-Berghauptmanns Gerland wird der Bergscirreiber Lindig veranlasst, nähere Erkundigungen über den Meteorstein einzuziehen. Bericht ist abgedruckt). Schweigg. Journ, Bd, 26, p. $243-250$.

1820: Osterländische Blätter: s. u. I860 unter Liebich.

182I: Chladni: Erster Nachtrag, Gilb. Ann. Bd. 68, p. 336.

1843: Partsch: Meteoriten, p. 54.

I854: v.B o g usla w ski: ZehnterNachtrag, Pogg. Ann. Ergz.-Bd. 4, p. 439.

1856: $\mathrm{Kr}$ ant $\mathrm{z}$ slegte zwei Fragmente von 20 und ${ }_{14}$ Loth Gewicht von dem seltenen, am 13. October 1819 zu Kösteritz bei Gera gefallenen, 7 Pfd. schweren Meteorstein vor, welche er kürzlich angekauft hatte. Verh, naturh.Ver. Bonn Bd. I3 (Sitzber.), p. XIII.

1859: H a rris: Dissert. Gött, p. 75.

1860: Liebich: Das Thatsächliche über den Meteorsteinfall von Pohlitz. Nach mehrseitigen Wünschen unserer Mitglieder mitgetheilt in Auszügen aus den gleichzeitigen Beobachtungen. Jahresber. d. Ges. v. Freunden d. Naturw. Gera Bd. 3 (1860), p. 15-24. I. Nachricht von einem in der Herrschaft Gera vom Himmel gefallenen Stein (Osterländische Blätter, herausgegeben von den Sekretären der Naturf. Ges. des Osterlandes I820 Nr. 2. - II. Zusatz zu jener Nachricht Osterländ. Blätter Nr. 3. - III. Aussagen über das Luftgetöse, mit welchem der Aërolith am 13. Oktober 1819 Morgens zwischen 7 und 8 Uhr unfern Köstritz und Pohlitz herabfiel. Genau nach der eigentümlichen Mundart der Zeugen niedergeschrieben und mitgeteilt erhalten von Herrn Hofrat Dr. Schottin in Köstritz. - IV. Noch zur Geschichte des Meteorsteins gehörig. Auszug aus einem Schreiben des Hrn. Hofr. Dr. Schottin in Köstritz. Osterländ. Blätter Nr. 9. - V. Abdruck aus Moll's N. Jahrbüchern 1824, p. 147-I54.

1863: Buchner: Meteoriten, p. 40 $-4 \mathrm{I}$.

1863: R o s e: Meteoriten, p. 90, 93, 155. r858/65: v. Reichen b a ch: V 480.

VI 455. IX 161, 168, 178, X 359,363 ,

372. XI 294, 297, 300. XIII 356 ,

37I (Fig.), 374, 377. XX 626. XXV $321,433,607,615$.

r884: M e u n i e r: Météorites, p.208,213. 1885: Brezina: Wiener Sammlung, p. $180,232$.

r895: Brez in a : Wiener Sammlung, p. 243 .

Ursprüngliches Gewicht: Ein Stein von 7 Pfd. I Lot. Das grösste Stück desselben, welches 1819 beschrieben wurde, wog $25 \mathrm{I} 8 \mathrm{gr}$. (5 Pfund I Lot I Quentchen). Dass mehr als $2518 \mathrm{gr}$. von diesem Stein erhalten blieben, folgt schon aus den Mitteilungen Buchner's vom Jahre 1863 . Er verzeichnet als Besitzer ohne nähere Gewichtsangabe: Altenburg, Auerbach, Freiberg, Göttingen, Heidelberg, Jena, Lobkowitz (jetzt Budapest), London, Nevill (jetzt Calcutta), v. Reichenbach (jetzt Tübingen), Shepard (jetzt Washington Sh.) und ferner mit Gewichtsangabe: Berlin,Ferber(jetzt Pohl) Gotha, Greg, Wien mit zusammen 1768 gr. Ergänzt man in der ersteren Reihe die Gewichte nach meinen Erhebungen, so erhält man in Summa $2784 \mathrm{gr}$. 
Nachweisbares Gewicht: 2757 gr.

Berlin, U. $\quad 713$ Harvard, U. Budapest Io Heidelberg 3

Calcutta II London, B. M. 87

Cambridge I London, P. G. 2

Cleveland Spl. Moskau 29

Dorpat II Paris, M. 9

Freiberg, i. S. 12 Pohl 422

Gera $\left.73^{\prime}\right)$ v. Siemaschko 2

Gotha $\quad 136$ Tübingen $\quad 152$

Göttingen 5 Ward Spl.

Gregory $\quad 5$ Washington,Sh. Spl.

$\begin{array}{llll}\text { Greifswald } & 2 & \text { Wien, H. M.*) } & 404\end{array}$

Halle

Polk Co.

Fisher

Poltawa i8I I Kuleschowka

Poltawa (Bloede) z.T.Kuleschowka

Poltawa (Partsch) Slobodka 1818

Ponta Grossa s. Anhang

Ponte de Lima São Julião

Poplar Camp Cranberry Plains

Poplar Hill Cranberry Plains

Port Orford, Rogue River Mountains, Oregon, U. S. A.

Pallasit, P, gefd. r859, beschr. r860.

1860: J a c ks on: (Kurze Erwåhnung).

Proc. of Boston Soc. Nat, Hist. Bd. 7

(1859/6r), p. 16r, 174, 175-176, 279,

289 ; s. auch C. R. Bd. 50, p. 105; sL'Institut e Bd. 28 (1860), p. 72 ; Liebig-Kopp, Jahresber. 1860, p. 850 ; Kenngott, Uebersicht 186I, p. I07; Revue de géol. par Delesse 1860, p. 55. 1860: Haidinger: Einige neuere Nachrichten über Meteoriten, namentlich die von Bokkeveld, New-Concord, Trenzano, die Meteoreisen von Nebraska, vom Brazos, von Oregon. Sitzber. Wien. Akad. Bd. 4I, p. 572 ; s. auch Liebig-Kopp, Jahresber. 1860, p. $845,850$.

186r: Haidinger: Meteoreisen von Rogue River Mountain in Oregon und von Taos in Mexico, gesandt von Hrn. Dr. Charles T. Jackson. Sitzber.Wien. Akad. Bd. 44 II, p. 29-30. Ref. N. J, 1862, p. 597; *L'Institut « Bd. 29 (186r), p. 3 I2 ; Zeitschr. f. d. ges. Naturw. Bd. I9, p. III.

1863: Buchner: Meteoriten, p. I3I.

1863: Ros e: Meteoriten, p. 15I, 153. 1885: Brezina: Wiener Sammlung, p. 206,233 .

1886: Brezina u. Cohen: Photographien, T. 6.

1890: E a s tma n: Met. Astron,, p. 320. r895: Brez in a: Wiener Sammlung, p. 264 .

UrsprünglichesGewicht: Sehr grosse Masse, welche von Evans auf $10000 \mathrm{Kgr}$. geschätzt, nach dessen Tode aber nicht wieder aufgefunden wurde.

Nachweisbares Gewicht: 4 gr.

Berlin, U. Spl, London, P. G. Spl. Calcutta Spl. Wien, H. M.*) 4

\section{Port Oxford}

Potosi Eisen

Port Orford

Potosi Pallasit

Coahuila

Powder Mill Creek Crab Orchard Prachin Bohumilitz

Prairie Dog Creek, Decatur Co., Kansas, U. S. A.

Stein, Cck, bekannt 1893 , beschr. 1895 .

r895: W ein s c hen k : Meteoritenstudien II. - 5. Prairie Dog Creek, Decatur Cy, Kansas. M. P. M. Bd. I4, p. $473-475$. Ref. N. J. 1896 II, p. 265. 1895: Brez in a : Wiener Sammlung, p. 260 .

Ursprüngliches Gewicht: Nach Hrn. Direktor Brezina's Mitteilung wog der Stein, welcher in Herrn Kunz' Besitz war, 2.9 Kgr.

1) Hiervon erhielt Dr. Eger, Wien, ein Stück von 332 gr. im Januar 1896. 
Nachweisbares Gewicht: 44I (bez. 2900) gr.

Brezina

I40 Wien, H. M. 30I

Prambanan, auch Brambanan, Soeracarta, Java.

Eisen, Of, bekannt seit 1797 , beschr. 1866.

Es wurden zwei Massen von Prambanan transportiert, die eine 1784 die andere 1797 ; die erstere soll verloren gegangen sein, von der letzteren stammen die in den Sammlungen befindlichen Stücke. 1866: v. Baumhauer: Sur le fer météorique de Prambanan dans le district de Soerakarta (Île de Java). Arch. Neerl. Haarlem Bd. I, p. 465-467 (Analyse von van der Boon Mesch; Abbildungen von geätzten Platten); $\mathrm{s}$, auch Meteoorijzer te Soerakarta: Natuurkundige Tijdschr. Nederlandsch Indië Bd. 29 (1867), p. $268-270$ (Analyse). Ref. Liebig-Kopp, Jahresber. 1867, p. IO49-I050; Wochenschr. f. Astr., Met. u. Geogr. 1867, p. 40.

1866: Buchner: Neue Meteoriten. Pogg. Ann. Bd. 129, p. 350.

1869: B u chner: Vierter Nachtrag, Pogg. Ann. Bd. 136, p. 600-601. 1884: M e un ier: Météorites, p.127,I28. 1885: Brezina: Wiener Sammlung, p. $204-205,208,233$ u. Taf. III.

1887: Brezina u. Cohen: Photographien, T. $15,16$.

1893: Meunier: Revision des fers météoriques, p. $47,48$.

r894: Co h en:Meteoritenkunde,p.72,193. 1895: Brezina: Wiener Sammlung, p. 268.

1895: C o h e n : Meteoreisen-Studien IV. Ann. Hof-Mus. Bd. 1o, p. 82 .

UrsprünglichesGewicht: Eine grosse Masse von etwa $8000 \mathrm{Kgr}$.

Nachweisbares Gewicht: 252 gr.

v. Braun

Budapest

Greifswald

London, B. M.

Paris, M.

Praskoles

Prastö

Zebrak

Primitiva, Salitra, Tarapaca, ${ }_{4} \mathrm{Km}$.

(40 miles) östl, von Iquique, Chile.

Eisen, Dp, gefd. 1888 , erwähnt 1890.

1890: Howe11: Description of new Meteorites. Proc. Rochester Acad, of Sc. Bd. 1, p. 100 .

1895: Brez in a : Wiener Sammlung, p. 297.

UrsprünglichesGewicht: 6 oder 8lbs. Nachweisbares Gewicht: ${ }^{1} 34^{8}$ gr. Brezina II73 | Wien, H. M.

175

\section{Princetown}

\section{Pseudo-Mejillones}

Pulaski Co. 1839

Pulsora, Rutlam, Indore, Malwa,

Centralindien, Ostindien.

Stein, Cib, gefallen 16. März 1863 . r867: $01 \mathrm{dham}$ : Catalogue of the specimens of Meteoric Stones and Meteoric Irons. Calcutta, Dezember 1867 , p. 8 (die Asiatic Soc. of Bengal besitze 1 lb. 7 oz. 165 grs.).

1869: Bu chner : Vierter Nachtrag,

Pogg. Ann. Bd. 136, p. 454-455.

r869: v. H a id ing er: Hessle, Rutlam, Assam, drei neue Meteoriten. Vorläufiger Bericht. Sitzber. Wien. Akad. Bd. 59 II, p. 228 - 229. Ref. LiebigKopp, Jahresber. 1869 , p. I30I.

r872: Ts chermak: Meteoriten, M. M. $(1872)$, p. $167,170$.

r884: M eunier: Météorites, p. 195 -196 (Abb.).

1883/85: Tschermak: Photographien, p. 3 . 
r885: Brezina: Wiener Sammlung, p. $164,182,232$.

r887: F $1 \mathrm{ight:}$ : Meteorites, p. 139.

r894: F let cher: Introduction, p. I3.

UrsprünglichesGewicht: DreiSteine, von denen einer etwa ein Pfund wog.

Nachweisbares Gewicht: 755 gr.

v. Braun I Paris, M. I43

Calcutta 5 Io $\quad$ v. Siemaschko 4

London, B. M. $\quad 48$ Wien, H. M.*) 49

Pultusk, zwischen Pultusk u. Ostrolenka am Narew, Polen, Russland.

Stein, Cga (jetzt von Brezina auch als $\mathrm{Cg}$ u. $\mathrm{Cgb}$ aufgefasst), gefallen 30. Januar 1868 .

1868: Sz y man ski : Briefl. Mitt. N.J. 1868, p. 326.

1868: He is: Wochenschr. f. Astr., Met. u. Geogr. Bd. II, p. $68-70,93-96$, 113- $118,228-230$.

1868: Wa wnikiewicz: Notice sur la météorite tombée le 30 Janv. I868 aux environs de la ville Pultusk. Publiée par la haute École de Varsovie (Analyse und Situationsplan). Varsovie. Imprimerie de Jean Jaworski. 1868. 16 Seiten; s. auch 1893: Brezina, Ankunft, p. 23 ; Zeitschr. d, d. geol. Ges. Bd. 20 (1868), p. 744.

r868: W e r ther: Analyse des Meteorits von Pultusk. Journ, prakt. Chem. Bd, 105, p. $\mathrm{I}-6$. Ref. Liebig-Kopp, Jahresber. 1868, p. 1042; s. auch Schriften d. phys. ökon. Ges. Königsberg Bd. 9, p. $34-40$.

1868: vom Rath: Ueber die Meteoriten von Pultusk. Ber. d. d. chem. Ges. Bd. 1, p. 124-125. Ref, LiebigKopp, Jahresber. 1868, p. $1042-\mathrm{IO} 43$. 1868: Prof. vom Rath zeigte einen der am 30. Januar d. J. nahe dem Dorfe Sielc, District Maków, Gouvernement Lomze (Königr. Polen), gefallenen Meteoriten, im Besitze des Hrn.
Dr. A. Krantz, vor (Gewicht $32^{7 / 10}$ Lot). Verh. naturh. Ver. Bonn Bd. 25 (Sitzber.), p. $47-48$; s. auch Döll, Jahrb. k. k. geol. Reichsanst. Bd. 37 (1887), p. 195, 197, 198, 199 .

1868: Mo hr: Bildung der Meteoriten. Verh. naturh. Ver. Bonn Bd. 25 (Sitzber.), p. 66,67 .

1868: Szymanski im Briefwechsel des N. J. 1868 , p. 326.

1868: H a i d in g e r: Der Meteorsteinfall vom 30. Jänner 1868 unweit Warschau. Sitzber. Wien. Akad. Bd. 57 II, p. 405-4II (Situationsplan). Ref. Rec. Geol. Surv. India Bd. I (1868), p. 39 $-40,70$; N. J. 1868, p. 499; *L'Institute Bd. 36 (1868), p. $279,280$.

1868: vom Rath: Ueber die Meteoriten von Pultusk im Königreich Polen, gefallen am 23. Januar 1868 (mit Tafel der Abbildung von vielen Steinen). Festschrift der niederrh. Ges, f. Nat.u. Heilkunde z. 50 jähr. Jubiläum der Universität Bonn, 27 Seiten (Analyse) [Sep.]. Ref. N. J. 1869 , p. $80-82$.

r868: v. F rit s c h: Bemerkungen über die Gemengtheile eines der am 30. Jänner 1868 bei Pultusk in Polen gefallenen Aërolithen. Verh. geol. Reichsanst. p. 92-94. Ref. N. J. 1868, p. $498-499$.

1868: Ga 11 e: Ueber die Bahn des am 30. Januar r 868 beobachteten u. bei Pultusk im Königreiche Polen als Steinregen niedergefallenen Meteors durch die Atmosphäre. Abh. schles. Ges. f. vaterl. Kultur 1868, p. 79-I2I (eine der besten Arbeiten über die Bestimmung der Bahn eines Meteoriten). Ref. Luminous Meteors, Rep. Brit. Assoc. I868, p. $388-389$.

r868: T is chler: Vortrag über den Meteoriten vom 30. Januar d. J., gestützt auf eine kleine Schrift von Galle, in welcher die Bahn desselben genau bestimmt wird. Sitzber. d. phys. öcon. Ges. Königsberg (1868), p. 38-40. 
r868: D a u bré e: Note relative à l'envoi de météorites récemment fait à l'Académie par la Haute-École de Varsovie. C. R. Bd. 67 , p. $369-373$.

I868: Z e us ch ner: N. J. I868, p. 499 (unter I20 Stücken zwei Stücke von gediegenem Eisen von der Grösse einer welschen Nuss).

r868: Rose legte einen der am 30. Januar gefallenen Meteoriten von Warschau vor. Zeitschr. d. d. geol. Ges. Bd. 20 , p. 744 .

1869: Buchner: Vierter Nachtrag, Pogg. Ann. Bd. 136, p. 590-593.

r869: N a u ck: Briefl. Mitt. Zeitschr. d. d. geol. Ges. Bd. 21 (1869), p. 472 -474 .

1870: R a m mels ber g: Beiträge zur Kenntnis der Meteoriten. - 3. Die Analyse der Silikate. - C. Die Chondrite von Pultusk, Richmond u. Iowa. - I. Pultusk. Mon.-Ber. Berlin. Akad. 1870, p. $448-452$.

r870: $\mathrm{R}$ a m mels berg : Meteoriten, p. 103, 105, 106, 138, 139, 140.

187x: v. B a u m h a u e r: Sur la météorite de Tjabé dans l'Inde Néerlandaise. Arch. Néerl. Bd. 6, p. 310 (Vergleich mit Pultusk).

r872: M eunier: Application du métamorphisme météorique à l'étude de la crotate noire des météorites grises. C. R. Bd. 75 , p. $499-503$; s. auch Hirzel u. Gretschel, Jahrb. d, Erf, Bd. 9 (1873), p. $24-26$.

r875: Ts chermak: Vulkanismus, Sitzber. Wien. Akad. Bd. 71 II, p. 662, 663 .

1875: vom R a th: Meteoriten, Verh. naturh.Ver. Bonn Bd. 32, p. $370-374$.

1875: Mohr: Entstehungsart, Ann. Chem. Pharm. Bd, 179, p. 259, 260, 26r.

1876: Wright: On the Gases contained in Meteorites. Am. Journ. (3) $\mathrm{II}_{\text {, }}$ p. 258,260 u. (3) 12 (1876), p. 167.

1877: G ä m b e 1: Ueber die Beschaffenheit der Steinmeteoriten vom Fall am
12. Februar 1875 in der Grafschaft Iowa, N. A. Sitzber. München. Akad. (1877), p. 314.

r879: R a mmels berg: Meteoriten, p. 24,25 .

r882: Brezina: Bericht IV, Sitzber.

Wien. Akad. Bd. 85 I, p. 344 .

r882: v. L a s a u 1 x: Vermehrung, Verh. naturh. Ver. Bonn Bd. 39 (Sitzber.), p. $\mathrm{IO}_{3}$.

I882 : W i i k: Mikroskopisk undersökning of naagra paa universitetets mineralkabinett befindliga meteoriter. Ofers. Finska Vetensk. Soc. Förh. Bd. 24. Ref. N. J. 1883 I, p. 384 .

1883: Tschermak: Beitrag, Sitzber. Wien. Akad. Bd. 88 I, p. 355 .

r884: W a d s w or th: Studies, p. 94 -95 .

1884: M e unier: Météorites, p. 79, 81, $84,85,98,247,252 \quad 255,395,421$, 423 (Sit.-Plan). 424, 450, 455, 456, $459,489-49$ r.

1884: H äpke: Beiträge, Abh. naturw. Ver. Bremen Bd. 8, p. 521.

1884: v. $\mathrm{Niess} 1$ : Ueber die astronomischen Verhältnisse bei dem Meteoritenfalle von Mócs in Siebenbürgen am 3. Februar 1882. Sitzber. Wien. Akad. Bd. 89 II, p. 29I, Ref. N. J. I886 I, p. 224.

r883/85: Tschermak: Photographien, T. 12, p. 4, 14, 17, 18, 19.

r885: Brez ina : Wiener Sammlung, p. $155,164,168,182,233$.

1886: A nsdell u. Dewar: On the

Gaseous Constituents of Meteorites. Proc. Royal Soc, London Bd. 40, p. $55^{2}, 553$; s. auch Am. Journ. (3) 32, p. 482 . Ref. N. J. 1887 II, p. 285 ; Liebig-Kopp, Jahresber. 1886, p. 2326. 1888: N ew to n: Orbits, Am. Journ.

(3) 36 , p. 4 .

r889: F 1 e t c her: Atacama Meteorites,

Mineral. Magaz. Bd. 8, p. 226 (Streufeld ro auf 3 miles).

1889: $\mathrm{M}$ e unier: Sur la matière noire 
de la Chantonnite. Bull. Soc. Française Min. Bd. 12, p. 76-81. Ref. N. J. 1890 II, p. 59.

r89o: Brezin a: Reise, Ann. Hof-Mus, Bd. 4 (Not.), p. II9.

r89o: v. Niess 1: Periheldistanzen, Verh, naturf. Ver. Brünn Bd. 29, p. 186, 187, 189, 194, 214, 231, 232, 256. r892: B r ez in a : Sternschnuppen, p. 13 $-14,15$.

1893: New to n: Lines of structure in the Winnebago Co. Meteorites and in other Meteorites. Am. Journ. (3) 45, p. I52, 355. Ref. N. J. 1894 I, p. 273 -274 .

1893: B r e z in a: Ueber neuere Meteoriten (Nürnberg), p. 160.

1894: Cohen: Meteoritenkunde, an vielen Stellen.

r894: F 1 e t c h e r : Introduction, p.27,39. r895: Brezina: Wiener Sammlung, p. $251,259$.

Ursprüngliches Gewicht: Ein Steinschauer von gegen roo ooo Steinen, wie gewöhnlich schätzungsweise angegeben wird. Der grösste Stein wog $7 \mathrm{Kgr}$., einige $4 \mathrm{Kgr}$.; die meisten klein bis auf $\mathbf{I}$ gr. Krantz zählte 2 I 2 Steine auf 1 Kgr.

Nachweisbares Gewicht : 200932 gr.

\begin{tabular}{lr|lr} 
Aachen & 7 & Bonn & 18 904 \\
Bailey & 480 & v. Braun & 691 \\
Bayet & I0 & Bremen & 122 \\
Belgrad & 27 & Breslau & 2574 \\
Bellucci & 94 & Brezina & 25 \\
Bement & 997 & Budapest & 5469 \\
Berkeley & 163 & Calcutta & 105 \\
Berlin, G. & 1077 & Cambridge & 30 \\
Berlin, L. & 127 & Cleveland & 175 \\
Berlin, P. & 161 & Clinton & 71 \\
Berlin, U. & 10649 & Cohen & 520 \\
Bern, M. & 830 & Czernowitz & 73 \\
Blatz & 25 & Danzig & 99 \\
Böhm & 107 & Darmstadt, M. & 200 \\
Bologna & 375 & Debreczin & $16 \mathrm{I}$
\end{tabular}

Dorpat $\quad 2097$ de Mauroy $\quad 765$

Dresden, M. $5 \mathrm{II}$ Melion ca, I000

Dresden, P. $\quad$ I22 Minneapolis 118

Dublin, M. 25 Modena $\quad 5$

Dublin, R. C. 43 Moskau 531

\begin{tabular}{ll|l} 
Edinburg & 339 & München $\quad 234$
\end{tabular}

\begin{tabular}{ll|ll} 
Eger & 250 & Münster & 467
\end{tabular}

$\begin{array}{llll}\text { Erlangen } & 53 & \text { Neapel } & 20\end{array}$

$\begin{array}{lll}\text { Frankfurt } & 38 & \text { Neumann }\end{array}$

、 Freiberg, i. S. $\quad 57$ New Haven 364

- Frenzel $\quad 37$ Newton 224

Gent $\quad 138$ New York, M, 5I

$\begin{array}{llll}\text { Giessen } & 83 & \text { Odessa } & 33\end{array}$

Göttingen $\quad 324$ Oxford $\quad$ II6

Graz, U. $\quad 6$ Paris, E. $\quad 304$

Graz, J. $\quad 49$ Paris, M. 29787

Gregory $\quad 383$ Pech $\quad 1559$

Greifswald $\quad$ I43 Petersburg, A. 8490

Halle $\quad$ II5 Petersburg, B. 908

Hamburg $\quad 525$ Pohl 2394

Harvard, U. 1461 Prag, D. U. 442

Heidelberg $\quad 3^{16}$ Prag, M. $\quad 346$

Helsingfors $\quad 469$ Riga $\quad 54$

Howell $\quad 450$ Riga, P. 127

Karlsruhe, M. $\quad$ III Roebling 38

Karlsruhe, P. $\quad 32$ Rom, U. 1368

Kasan 292 Rom, S. Ap. ${ }_{1052}$

Kiew 593 Schemnitz So

Klausenburg $\quad 433$ v. Schilling $\quad \mathbf{I}_{3}$

Königsberg, M. 33 Seligmann 125

Kopenhagen $57 \mathrm{I}$ Sevilla 90

Krantz $\quad 34000$ v. Siemaschko 8530

Kristiania $\quad 473$ Stockholm 4913

\begin{tabular}{ll|l|l} 
Krüger & 217 & Strassburg & 615
\end{tabular}

Kunz 2 Stuer $\quad 223$

\begin{tabular}{ll|l|l} 
Leipzig & 88 & Stürtz & 606
\end{tabular}

$\begin{array}{llll}\text { Lissabon } \quad 277 & \text { Stuttgart } \quad 748\end{array}$

London,B.M. 17905 Szamosujvar 45

London, P. G. 1305 Troyes $\quad 225$

Lund, G. $\quad$ n6 Tübingen $\quad 795$

Lund, M. $\quad 340$ Turin, J. $\quad 184$

Lüttich $\quad 500$ Turin, U. $\quad 137$

\begin{tabular}{ll|l} 
Madrid & 509 & Upsala \\
\hline
\end{tabular}

\begin{tabular}{ll|l} 
Manchester & 29 & Utrecht \\
\hline
\end{tabular}

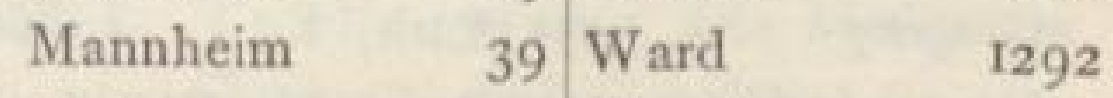

\begin{tabular}{ll|l} 
Marburg & 271 & Washington \\
& ${ }_{15} 8$
\end{tabular} 
Washington,Sh. 305 Wien, U. II III Wien, H. M.*)**) Wrany 67 I $5564^{1}$ ) Würzburg $\quad 52$

Wien, U. I. I25I Zürich 1049

Die Universität Basel besitzt ebenfalls ein Stück Pultusk.

Puquios, Chile, Südamerika.

Eisen, Om, gefunden um 1885 , beschr. 1890 .

r8go: Howe 11: Notice of two new Iron Meteorites from Hamilton Co., Texas and Puquios, Chili, S. A. 2. The Puquios, Chili, Meteorite. (Analyse von Eakins; Abbildung des Eisens u. einer geätzten Platte). Am. Journ. (3) 40 , p. $224-226$. Ref. N. J. $189 \mathrm{I}$ II, p. 418 u. N. J. 1892 II, p. 34 ; Proc. Rochester Acad of Sc. Bd. I (1890), p. 89-9I; Journ. Chem. Soc. I891, p. 277-279; Oesterr. Zeitschr. f. Berg- u. Hüttenw. Bd. 39 (189r), p. 228; U. S. Geol. Surv. Nr. 78 (189i), p. 95.

r 890 : E a s t m a n : Met. Astron,, p. 320. 1893: Brezina: Ueber neuere Meteoriten (Nürnberg), p. 3 .

1893: Meunier: Revision des fers météoriques, p. 52,59 .

r894: C o h e n : Meteoritenkunde, p. 84, $95,156$.

r892: v. Ha u er: Ann. Hof-Mus. Bd. 7 (Not.), p. 72 .

1895: Brezina : Wiener Sammlung, p. 282 .

Ursprüngliches Gewicht: $6^{1} / 2 \mathrm{Kgr}$.

Nachweisbares Gewicht: 5339 gr.

Bailey

5oi Howell

Bement 479 Krantz

I4

Böhm

v. Braun

I7 London, B. M.

Brezina

Budapest

I52 de Mauroy

I35 München

23

Dorpat

Gregory

29 New Haven

219 Newton

171 Paris, E.

Harvard, U. $\quad 254$ Paris, M.

I) Ausserdem 1045 gr, nach I. Juli 1893 erworben.

\begin{tabular}{lr|lr} 
Rom, U & 29 & Ward & 1620 \\
v. Siemaschko & 71 & Washington & 28 \\
Strassburg & 27 & Wien, H. M. & 1363 \\
Stürtz & 28 & &
\end{tabular}

\section{Pusinsko Selo}

Putiwl

Putnam

Milena

Botschetschki

s. Anhang

Putnam Co., Georgia, U. S. A.

Eisen, Of, gefd. 1839 , beschr. 1854 .

1854: Wille t: Description of Meteoric Iron from Putnam County, Georgia. Am. Journ. (2) 17 , p. 331-332 (Analyse von Shepard); s. auch Journ. prakt. Chem. Bd. 62 (1854), p. 348 -349 ; Kenngott, Uebersicht 1854 , p. I2O-I2I. Ref. Liebig-Kopp, Jahresber. 1854, p. 917.

1859: $\mathrm{B}$ u c h n e r: Feuermeteore, p. I3I. 1859: Harris: Disșert, Gött., p. II2.

1860: R a m mels berg: Mineralchemie, p. $917,918$.

r858/62: v. Re i chen ba c h: IV 638. VI 448 . VII 552 . IX $162,174,175$,

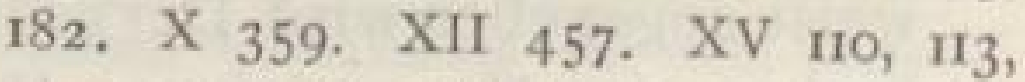
$\mathrm{II}_{4}, \mathrm{I24}, 128$. XVI $250,26 \mathrm{t}, 262$. XVII 266,272 . XVIII 478,484 . XX 622. XXI $585,5^{8} 9$.

r863: B u c hner: Meteoriten, p. 162. 1863: Rose: Meteoriten, p. 65, I53. 1869: Meunier: Recherches, Ann.

Chim. Phys. (4) 17, p. 35.

1870: R a mmels berg : Meteoriten, p. 143 .

1875: vom R a th: Meteoriten, Verh. naturh. Ver. Bonn Bd. 32, p. 363 . r884: M e un i e r: Météorites, p. 5I, 99, 129, 130.

1885: Brez ina: Wiener Sammlung, p. $208-209,233$.

r886: H unting t o n : Crystalline Structure, Am. Journ. (3) 32, p. 294, 295 (Abbildung einer geiitzten Platte).

1890: East m a n : Met. Astron, p. 318. s 3 
r893: M e un i er: Revision des fers météoriques, p. $42-43$.

1894: C o h en: Meteoritenkunde, p. 48, $56,62,191,212$.

1895: Brezina: Wiener Sammlung, p. 269 .

I895: C o he n : Meteoreisen-Studien IV. Ann. Hof-Mus. Bd. 1o, p. 82,85 .

(r896): Brezina u. Co hen: Photographien, T. 22. Im Buchhandel noch nicht erschienen. (Es war nach der Ankündigung in Lief. III beabsichtigt, Abbildungen von Muddor herzustellen; es werden aber nach den im k. k. HofMus. befindlichen Exemplaren auf T, 22 Abbildungen von Putnam Co. erscheinen und der IV. Lief. beigelegt werden).

Ursprüngliches Gewicht : $32^{1 / 2} \mathrm{Kgr}$. ( 72 lbs.).

Nachweisbares Gewicht: 4ror gr.

\begin{tabular}{|c|c|c|c|}
\hline Bailey & 30 & London, P. G. & 50 \\
\hline Bement & 26 & Madrid & 55 \\
\hline Berlin, U. & 24 & de Mauroy & \\
\hline Bologna & 5 & Minneapolis & \\
\hline Bonn & 15 & Moskau & \\
\hline v. Braun , Kry & talle $\alpha$ & München & \\
\hline Budapest & 15 & New Haven & \\
\hline Calcutta & IOI & Paris, M. & \\
\hline Cleveland & $5^{2}$ & Petersburg, B. & \\
\hline Cohen & 4 & v. Siemaschko & \\
\hline Dorpat & 29 & Stockholm & \\
\hline Dresden, M. & 27 & Stürtz & \\
\hline Göttingen & 61 & Tübingen & \\
\hline Gregory & 26 & Upsala & \\
\hline Hamburg & $\left.46^{1}\right)$ & Washington & \\
\hline Harvard, U. & $23 \mathrm{II}$ & Washington, Sh. & \\
\hline Kunz & 4 & Wien,H.M.*) & \\
\hline London, B. M & II2 & & \\
\hline
\end{tabular}

Wo befindet sich die grösste Masse dieses Eisens?

Queensland Thunda

Quenggouk bei Bassein, Pegu, Hinterindien.

Stein, Cc, gefallen 27 . Dezbr. 1857 . r860: Ha i ding er: Die Calcutta-Meteoriten, von Shalka, Futtehpore, Pegu, Assam und Segowlee im k. k. HofMineralien-Cabinete. - 2. Pegu. Aufgefunden 1854. Sitzber. Wien. Akad. Bd. 4I, p. 750-75I; s. auch Jahrb. k. k. geol. Reichsanst. 1860 (Verhandl.), p. I04; Journ. Asiat. Soc. Bengal. (Proc.) Bd. 29, p. 416-418; Quarterly Journ. Geol. Soc. Bd. I7 (186r) (Translations and Notices), p. 8-9; Am. Journ. (2) 32 (186r), p. I43; Luminous Meteors, Rep. Brit. Assoc. 186I, p. 34 (Sep.); L'Institut Bd. 28 (1860), Nr. 1408, p. 423 .

1860: Haidinger: Die Meteoritenfälle von Quenggouk bei Bassein in Pegu und Dhurmsala im Punjab. Sitzber. Wien. Akad. Bd. 42 , p. $30 \mathrm{I}-305$ (Sit.-Plan). Ref. Kenngott, Uebersicht 186r, p. 169; sL'Institut s Bd. 29 (186r), Nr. 1426, p. $153-154$.

r86r: Haidinger: Das Meteor von Quenggouk in Pegu, und die Ergebnisse des Falles daselbst am 27. Dezember 1857. Sitzber.Wien. Akad. Bd. 44 II, p. 637-642 (mit Tafel, die glänzende Lichterscheinung darstellend, beobachtet von Lieutenant Aylesbury); s. auch Kenngott, Uebersicht $1862 / 65$, p. 437 ; Am. Journ. (2) 32 (186r), p. I42 ; s'Institut \& Bd. 30 (1862), Nr. 1474, p. III.

1863: B u c hner: Meteoriten,p.97-98. 1863: R o se: Meteoriten, p. 154.

r 86 r $/ 64$ : v. Re ic he n b a c h: XV Ior. XIX 152. XXIII 369.

1864: H a i d ing er: Sternschnuppen, Feuerkugeln und Meteoritenschwärme im Zusammenhange betrachtet, Sitzber. Wien, Akad, Bd. 49 II, p. 6.

1870: v.H a i d in g e r: Orientierung, Sitzber. Wien. Akad. Bd. 6I II, p. 512, 5I3. 1872: Tschermak: Die Meteoriten von Shergotty und Gopalpur. Sitzber. Wien. Akad. Bd. 65 I, p. 139.

I) Nach Bestimmung von Cohen u. Brezina; von Bryce Wright als Zacatecas verkauft. W ül fing, Meteoriten. 
r884: M e unier: Météorites, p. 97, 230, 231, 232, 349 .

1885: Brezina: Wiener Sammlung, p. 185,233 .

r89o: v. Niess 1: Periheldistanzen, Verh. naturf. Ver. Brünn Bd. 29, p. 188, 195, 196, 214, 252.

r892: B r ez in a : Sternschnuppen, p. II. r894: Co he n: Meteoritenkunde,p. 201. 1894: F let c h er : Introduction,p.Io,36.

Ursprüngliches Gewicht: 6044 gr. ${ }_{3}$ Stücke von einem Stein (229r; I909 u. I844 gr.).

Nachweisbares Gewicht: 554I gr.

\begin{tabular}{lr|lr} 
Bailey & 8 & Klausenburg & Spl. \\
Berlin, U. & I7 & London, B. M. & 654 \\
Bologna & 7 & London, P. G. & 49 \\
Budapest & 4 & Paris, M. & 74 \\
Calcutta & 4036 & v. Siemaschko & 7 \\
Cleveland & II & Stockholm & 4 \\
Dorpat & 2 & Tübingen & 70 \\
Giessen & I7 & Upsala & Spl. \\
Göttingen & 20 & Washington, Sh. 20 \\
Gregory & 34 & Wien, H. M.*) & 506 \\
Harvard, U. & I &
\end{tabular}

Quinçay, Dep. Vienne, Frankreich. Stein, Cgb, gefallen Sommer $185 \mathrm{I}$. 1884: M eunier: Météorites, p. 24I. r885: Brezina: Wiener Sammlung, p. $182,233$.

Ursprüngliches Gewicht: ?

Nachweisbares Gewicht: 3r gr.

London, B. M. Io v. Siemaschko 8

Paris, M.

Io Wien, H. M.

Ueber diesen Fall scheint keine ausführliche Arbeit publiziert worden zu sein.

\section{Qutahar Bazar}

Butsura

Rakowka, Gouv. Tula, Russland. Stein, Ci, gefallen 20 . Nov, 1878 . 1879: Trautschold: Briefl. Mitt. N. J. 1879 , p. 144-145. Ref. LiebigKopp, Jahresber. 1879, p. 1279.

1880: Gregoriew: Der Meteorit von Rakowska im Gouvernement Tula in
Russland, Zeitschr. d. d. geol. Ges. Bd. 32, p. 417-420 (Analyse). Ref. N. J. 188I II, p. 181; Liebig-Kopp, Jahresber. I880, p. 1540.

r884: Meunier: Météorites, p. 209. 1885: Brezina: Wiener Sammlung, p. $181,232$.

r894: Cohen: Meteoritenkunde, p. $57,202,249$.

UrsprünglichesGewicht:Kopfgrösse. Nachweisbares Gewicht: 2642 gr.

\begin{tabular}{lr|lr} 
Budapest & I2 & New Haven & Spl. \\
Dorpat & 277 & Paris, M. & I03 \\
Gregory & I & v. Siemaschko & I5 \\
London, B. M. & 375 & Ward & 358 \\
Madrid & 46 & Washington, Sh. 23 \\
Moskau & 896 & Wien, H. M. & 536
\end{tabular}

Ranchito, Bacubirito Sinaloa, Mex. Eisen, Off, gefunden 1871 , erwähnt 1876 .

r876: B a r c e n a : On certain Mexican Meteorites. Proc. Acad. Nat. Sc. Philadelphia 1876 , p. 122 .

1884: $\mathrm{H}$ ä $\mathrm{p} \mathrm{k}$ e: Beiträge, $\mathrm{Abh}$. naturw. Ver. Bremen Bd. 8, p. 517-518.

r889: C a stillo: Catalogue, p. 5. 1890: F let c h e r : Mexican Meteorites, Mineral, Magaz. Bd. 9, p. 151, 174.

r892: Eastman: The Mexican Meteorites. Bull. Philos. Soc. Washington Bd. I2, p. 45.

1893: Brezin a: Ueber neuere Meteoriten (Nürnberg), p. 163.

1893: M e un ie r: Revision des fers météoriques, p. 75 .

1894: C o hen: Meteoritenkunde, p. 49. r895: Brezina: Wiener Sammlung, p. 267.

UrsprünglichesGewicht:Etwa 50000 Kgr. Das Eisen, welches $3^{1 / 2} \mathrm{~m}$. lang, $2 \mathrm{~m}$. breit und $\mathrm{x}^{1 / 2} \mathrm{~m}$. hoch sein soll, würde jedes andere Meteoreisen an Grösse übertreffen. Nachweisbares Gewicht: 306 gr. 


\begin{tabular}{lr|lr} 
Bailey & 26 & Stockholm & 34 \\
Bement & II & Washington & 14 \\
Harvard, U. & $9^{1}$ ) & Washington, Sh. 171 \\
Paris, E. & 4 & Wien, H. M. & 20 \\
Paris, M. & I7 & &
\end{tabular}

Rancho de la Pila (Pila), Durango, Mexico.

Eisen, Om, gefd. 1882 , beschr. 1884 .

Hierher auch vorläufig: Guadalupe (Karawinsky), bekannt $\mathrm{I} 834$; Cacaria, Hacienda de Cacaria, 50 $\mathrm{Km}$. nördlich v. Durango, Mexico. Eisen, Oh, gefunden?, erwähnt $\left.1867^{2}\right)$.

r843: P a r s c h: Meteoriten, p. Ir3II4 (Karawinsky's Masse).

1856: Burk art: Fundorte I, N. J. $\mathbf{r} 856, \mathrm{p} .282-283$ (Karawinsky's Masse).

1867: T a r a y re: Archives de la Commission Scientifique du Mexique. Paris 1867 Bd. 3, p. 270.

r876: B a r c en a : On certain Mexican Meteorites. Proc. Acad. Nat. Sc. Philadelphia 1876 , p. 123 (Cacaria).

r884: v o m R a th: Verh. naturh. Ver. Bonn (Sitzber.) Bd, 4I, p. 126. Ref. N. J. 1885 II, p. 270 .

1884: Hä p ke: Beiträge, Abh, naturw. Ver. Bremen Bd. 8, p. $513-515,517$ (Analyse von Janke). [Die Arbeit wurde 1883 geschrieben; der Band erschien 1884].

1885: Brez in a: Wiener Sammlung, p. $155,213,234$.

I888: v. Ha uer: Ann. Hof-Mus, Bd, 3 (Not.), p. 42.

1889: Cas ti11 o: Catalogue, p. 5 (Cacaria).

r89o: F le t c he r: Mexican Meteorites, Mineral. Magaz. Bd. 9, p. 104, 152154, 156.

1) Eigentum von Herrn Huntington.

2) Nach Fletcher ist es wahrscheinlich, dass dieses Eisen mit Rancho de la Pila dem gleichen Falle angehört.
1892: E astman: The Meexican Meteorites. Bull. Philos, Soc,Washington Bd. 12, p. 45 .

1893: M e un i er: Revision des fers météoriques, p. 52, 53 .

r894: Cohen: Meteoritenkunde, p. 58,83 .

1895: Brezina: Wiener Sammlung, p. 276 .

Ursprüngliches Gewicht: von Rancho de la Pila: $46.4 \mathrm{Kgr}$. (I02.3 Pfd. englisch nach Häpke, was nach der Londoner Angabe nicht ganz richtig sein kann). Von $\mathrm{Ca}$ caria befindet sich nach Castillo eine Masse von 41 $422 \mathrm{gr}$. im Museum in Mexico.

Nachweisbares Gewicht: $475^{\mathrm{I}} 3 \mathrm{gr}$. Berlin, U. $\quad 782$ London,B.M. 465 I2 Bremen $\quad 137$ London, P. G. 2 Häpke $\quad 7$ v. Siemaschko 20 Harvard, U. $\quad 34$ Wien, H. M. $\quad$ I9

Ursprüngliches Gewicht von Cacaria: 4I 442 gr.

Nachweisbares Gewicht: Ausser der Hauptmasse im Museum inMexico besitzen noch:

Paris, M. $\quad 5 \mid$ Wien, H. M. I5

\section{Rancho de los Amates}

\section{Randolph Co. Guilford Co.}

Rasgata, zwischen Bogota u. Pamplona bei Zipaquira, Columbien, Südamerika, gefunden 1810, beschr. 1824 .

Hierher auch: Santa Rosa, Tocavita, Columbien, (Boussingault). Santa Rosa, Tocavita, Tunja,
Eisen, Ds (früher als Dr bezeichnet), 
Columbien, feinkörniges Eisen (Rose). Ferner wurden verschiedeneStücke oktaëdrischen Eisens, welche unter der Bezeichnung Santa Rosa gehen und deren Ursprung noch nicht sicher ermittelt ist, hier eingereiht.

1824: Mariano de Riverou. Boussingault: Mémoire sur différentes masses de fer qui ont été trouvées sur la Cordillère orientale des Andes. Ann, Chim. Phys. Bd. 25, p. 438-443. Traduit de l'espagnol. Le Mémoire original a été imprimé à SantaFé de Bogota. S. auch Schweigg. Journ. Bd. $5^{8}$ (1830), p. 343-345. Die Masse vom Hügel Tocavita wird auf $750 \mathrm{kgr}$. geschätzt. Das Eisen ist zellig (caverneux), Struktur körnig, spezifisches Gewicht 7.3, Analyse: Eisen 9I.4I, Nickel 8.59. Von einer andern bei Santa Rosa, I8Io, gefundenen Masse von 681 gr. wird angegeben: spezifisches Gewicht 7.6 ; Eisen 91.23; Nickel 8.21; unlöslicher Rückstand 0.28 . Von einer dritten bei Santa Rosa, 1810, gefundenen Masse von $56 \mathrm{r}$ gr. von zelliger Struktur: Eisen 91.76; Nickel 6.39. Von einer vierten Masse von Rasgata, bei Zapaquirá von 4 r kgr.: spezifisches Gewicht 7.6; Eisen 90.76; Nickel 7.87. Von einer fünften Masse, ebenfalls von Rasgata von 22 kgr. mit vielen Höhlungen (vacuoles) : $7-8 \%$ Nickel; s. auch Viajes Scientificos a los Andes ecuatoriales etc. por M. Boussingault et por el Dr. Roulin traducidos con aruencia de los autores por J. Agosta. Paris Laserre, editor, 1849, p. 6r.

1824: Chladni: Vierter Nachtrag, Pogg. Ann. Bd. 2, p. 159-16r.

1826: Se e beck: Ueber die magnetische Polarisation der Metalle und Erze durch Temperatur-Differenz. Pogg. Ann. Bd. 6, p. 144 .

I832: v. Hoff: Achter Nachtrag,Pogg.
Ann. Bd. 24, p. 232.

-4I: R a m mels ber g : Handwörterbuch Bd. 1, p. 423 .

r843: Parts c h: Meteoriten, p. 125128. Es ist nur die Rede von den Massen bei Rasgata, nicht von dem grossen, $750 \mathrm{kgr}$. schweren Eisen vom Hügel Tocavita bei Santa Rosa ; s. auch p. I45 (hier wird „Santa Rosae oder sTocavita\& des Berliner Museums erwähnt und für ganz ähnlich mit Rasgata befunden).

1852: Wöhler u. Partsch: Analyse des Meteoreisens von Rasgatá in Neugranada von Prof. Wöhler in Göttingen, mit Notizen über das Vorkommen u, die physikalischen Eigenschaften desselben von Direktor Partsch. Sitzber. Wien. Akad. Bd. 8, p. $496-504$ (Abbildung von geätzten Platten. Die Analyse wurde an einem Stück Rasgata des Wiener Hofmuseums angestellt und ergab $6.7 \%$ Nickel); s. auch Ann. Chem. Pharm, Bd. 82 , p. $243-247$; Pharm. Centr. 1853, p. 157; >L'Institut * Bd. 20 (1852), Nr. 960 , p. 171 ; Weinschenk, Ann. Hof-Mus. Bd. 4 (1889), p. 100; Clark, Dissert. Gött. 1852, p. 27-28; Liebig-Kopp, Jahresber. 1852, p. $989-990$; Kenngott, Uebersicht 1852 , p. 91 ; N. J. 1853 , p. 54 $-5^{8}$; beiläufig auch : Burkart,Fundorte I, N. J. 1856 , p. 268.

r852: W ö hler : sAktiv «, Pogg. Ann. Bd. 85 , p. 448,449 .

r852 : C l a r k: Dissert.Gött., p.28 (Analyse der grossen Masse von Tocavita ergiebt $8.21 \%$ Nickel, - also ganz ähnlich dem Eisen von Rasgata).

1854: v. Boguslawski: Zehnter Nachtrag, Pogg. Ann. Ergz.-Bd. 4, p. 419, 454-455.

1854: Uricoe chea: Ann. Chem. Pharm. Bd. 9r, p. 249. Ref. Journ. prakt. Chem. Bd. 63 (1854), p. $3^{17}$ -318 .

1855: Ha id ing er: Bemerkungen über 
die zuweilen im geschmeidigen Eisen entstandene krystallinische Struktur, verglichen mit jener des Meteoreisens. Sitzber. Wien. Akad. Bd. 15 I, p. 357. 1857: Arag o: Astronomie populaire Bd. 4, p. $196-197,228-229$ (Abbildung. Es handelt sich um die $750 \mathrm{kgr}$. schwere Masse von »Santa Rosa Tocavita «, von welcher behauptet wird, dass sie in der Nacht vom 20. auf $2 \mathbf{I}$. April r8ro gefallen sei).

r859: B u c h n er: Feuermeteore, p.II7, I30, 184 .

1859: H a r r i s: Dissert. Gött., p. 107. r860: R a m mels berg: Mineralchemie, p. 914.

1862: Ros e: SystematischesVerzeichnis der Meteoriten in dem mineralogischen Museum der Universität von Berlin Nr. 36: St. Rosa (Tocavita) bei Tunja, Columbien u. Nr. 37: Rasgatà, Zipaquira bei Bogota, Columbien. Abh. Berlin. Akad,, p. 552, 555-556; beide unter der Abteilung: sEisenmeteoriten aus feinkörnigen Individuen bestehend a aufgeführt. Ersterer Fundort wurde durch Humboldt, letzterer durch Partsch erhalten. Humboldt's Etikette sagt: „Fer natif de St. Rosa, près Thunga . Rose vermutet, dass diese Eisen von den einzelnen Stücken herstammen, die um den Hügel von Tocavita herumgelegen haben und von denen Boussingault selbst einige gesammelt hat, Ausserdem beschreibt Rose noch ein sehr kleines Stückchen Eisen von etwa $1 / 2 \mathrm{gr}$. Gewicht, welches Carsten mitbrachte und welches von dem $750 \mathrm{kgr}$. schweren Eisen stammen soll, Dieses Stück zeigt merkwürdiger WeiseWidmannstätten'sche Figuren, was von den andern Eisen dieses Fundortes ausdrücklich verneint wird. In dem neueren Katalog von Klein von 1889 (Sitzber. Berlin, Akad, Bd, 41 (1889), p. $855,857)$ ist aber : ${ }_{18} 810$ St. Rosa, Tunja, Columbia 973.5 gr. $*$ unter den oktae- drischen Meteoreisen und 1810, Rasgata, Tocavita, Columbia I 30 gr.\& unter den dichten Meteoreisen aufgeführt.

1863: B u chner: Meteoriten, p. 155 -157 .

1863: R os e: Meteoriten, p. 38, 4I, 64, $67-69,152,153$.

1858/65: v. Rei c hen b a c h: VII 552 . IX $162,175,176,182$. X 359,363 , 364. XI 29r. XII 457. XIII 354, 364. XV 10o. XVI 255. XVII 268, 269, 273. XVIII 488, 489 . XIX 149, 15I. $\mathrm{XX} 621,628,630 . \mathrm{XXV} 437$

1867: G o e b e 1: Kritische Uebersicht, Mélanges phys. chim, Bd, 7, p. 3I2.

1869: Meunier: Recherches, Ann.

Chim. Phys. (4) I7, p. 68.

r870: R a mmelsberg: Meteoriten, p. 79,8 o.

1884: L a s a u $1 \mathrm{x}$ : Ueber das Meteoreisen von Santa Rosa, Columbien, I8ro (neue Analyse von dem auf $750 \mathrm{kgr}$. geschätzten Block Boussingault's; $8.2 \%$ Nickel, $0.32 \%$ Silikate. Abbildung nach einer Skizze Stübel's). Sitzber. naturh. Ver. Bonn Bd, 4I, p. $150-$ 154. Ref. N. J. 1885 II, p. $269-$ 270; Zeitschr. f. Kryst. Bd. II (I886), p. 174-175; Liebig-Kopp, Jahresber. 1884 , p. $2045-2046$.

1884 : Me unier: Météorites,p.96,98, no. $188_{3} / 85$ : T s c h e r m a k: Photographien, p. 3 .

1885: Brezin a : Wiener Sammlung, p. $155,220,234,267$.

r889: C a st i 11 o: Catalogue, giebt p. 9 ein Santa Rosa mitWidmannstättenschen Figuren an.

r89o: E a s tma n: Met. Astron., p. 318, 322.

r8gr: Cohen u. Weinschenk: Meteoreisen-Studien, Ann. Hof-Mus. Bd. 6, p. 165.

1892: C o hen: Meteoreisen-Studien II. Ann. Hof-Mus. Bd. 7, p. ${ }^{158}(\mathrm{Cu})$.

1893: Me un i er: Revision des fers météoriques, p. $70-71$. 
1894: Cohen: Meteoritenkunde, p. 44, $53,56,57,157,194,216,248,298$, $319,320,321$.

1894: C o h e n: Meteoreisen-Studien III. Ann. Hof-Mus. Bd. 9, p. $\mathrm{II}-\mathrm{II}_{3}, \mathrm{II}_{7}$, II8 (Rhabdit).

1895: C o h e n: Meteoreisen-Studien IV. Ann. Hof-Mus. Bd. Io, p. 83 , 90, 91.

Ursprüngliches Gewicht: Die Masse vom Hügel Tocavita wurde auf $750 \mathrm{Kgr}$. geschätzt, ausserdem sind mehrere kleine Massen ( 56 I gr., 68r gr.) in jener Gegend gefunden worden. Die beiden Hauptmassen von Rasgata bei Zapaquira wogen 22 und $4 \mathrm{r} \mathrm{Kgr}$.

Nachweisbares Gewicht: 9443 gr.

\begin{tabular}{|c|c|c|}
\hline Bailey & $\left.17^{1}\right)$ & Krantz \\
\hline Belgrad & 27 & London, B. M. $159^{7}$ ) \\
\hline Bement & $\left.2^{2}\right)$ & London, P. G. $156^{8}$ ) \\
\hline Berlin, U. & $\left.1103^{3}\right)$ & München I \\
\hline v. Braun & 2 & Neumann \\
\hline Bonn & 8 & New Haven \\
\hline Budapest & $\left.163^{t}\right)$ & Newton \\
\hline Calcutta & 68 & Paris, M. $\quad 565$ \\
\hline Cohen & 2 & Petersburg, A. 67 \\
\hline Dresden, M. & Spl. $\left.{ }^{5}\right)$ & Pohl 3 \\
\hline Göttingen & 17 & v. Siemaschko \\
\hline Gregory & $\left.102^{6}\right)$ & Tübingen $\left.5655^{12}\right)$ \\
\hline Greifswald & 2 & Washington $\quad I^{18}$ ) \\
\hline Hamburg & 17 & Washington, Sh. I5 \\
\hline Harvard, U. & 4 & Wien, H. M.*) 1266 \\
\hline Hügel & & $\mathrm{Ma}$ \\
\hline
\end{tabular}

Rasoumowski s. Anh. Kamschatka Red River I808(I814) CrossTimbers Red River $1836 \quad$ Wichita Co. Reichstadt Ploschkowitz

Renazzo bei Cento, Prov. Ferrara, Italien.

Stein, Cs, gefallen I5. Jan. I824. r824: Orioli: Nuova Collezione di opusculi scientifici di Bologna, Heft 3 , p. 151 ; s. auch Froriep's Notizen aus dem Gebiete der Natur- und Heilkunde Bd. I2 (1826), p. $151-152$; Pogg. Ann. Bd. 5, p. 122; Ann. Chim. Phys. Bd. 30 (1825), p. $42 \mathrm{I}$.

1824: Chla dni: Vierter Nachtrag, Pogg. Ann. Bd. 2, p. I55 (Auszug aus verschiedenen Zeitungen).

1826: Chla dn i: Fünfter Nachtrag, Pogg. Ann. Bd. 6, p. 27-28.

1827: Cordier: Rapport fait à l'Académie des Sciences, sur une Pierre météorique tombée près de Ferrare en 1824. Ann. Chim, Phys, Bd, 34, p. 132-139 (Analyse von Laugier).

1827: La ugier: Note sur la composition chimique de la pierre météorique de Ferrare. Ann. Chim. Phys. Bd. 34, p. 139-142. Uebersetzt nebst Cordier's Bericht in Schweigg. Journ. Bd. 49 (1827), p. $402-411$.

1830: v. Hoff: Siebenter Nachtrag, Pogg. Ann. Bd. 18 , p. $18 x-183,184$. 1836: K äm tz: Meteorologie, p. 293. 1843: Partsch: Meteoriten, p. 39. 1854: v. Boguslawski: Zehnter

1) Davon 10 gr. Santa Rosa Tunga. - 2) Santa Rosa, New Granada. - 3) Davon 973 gr. Santa Rosa, Tunja, Columbia unter oktaëdrischen Meteoreisen aufgeführt. - 4) Davon 4 gr. »1810 Santa Rosa, New Granada, Columbia « bezeichnet und unter hexaëdrischen Eisen aufgeführt; sollte dies nicht ein dichtes Eisen sein? - 5) Tocavita, Santa Rosa, Boyaca Fluss, Neu Granada. - 6) Davon 6 gr. Tocavita. - 7) Davon IOI gr. Santa Rosa, Tocavita. - 8) Davon $3^{8}$ gr. Santa Rosa, Neu Granada. - 9) Von Partsch erhalten, - Io) Santa Rosa, Tocavita 1824, ausserdem noch ein Stück Eisen Columbia S. A.? 57 gr.a - II) Nur als Santa Rosa bezeichnet, daher nicht sicher einzuordnen. - I2) Ausserdem 44 gr, oktaëdrisches Eisen Santa Rosa, Rasgata. - 13) 1810 Tocavita. 
Nachtrag, Pogg. Ann. Ergz.-Bd. 4, p. $23-24$.

r 859 : B u c hner: Feuermeteore, p. 88 -89 .

r859: H a r r i s: Dissert. Gött., p. 78.

r863: B u c h n e r: Meteoriten,p.76-77. r863: Rose : Meteoriten, p. 27, 154;

s. auch Mon.-Ber. Berlin. Akad, 1862, p. 557 .

1858/64: v, Re i c hen ba ch: V 476. VI 44I. IX 16r, 172, 180. X 359, 362. XI 293, 306. XIII $353,355,356$, $362,365,370$ (Fig.), 374. XIV 395. XXIII 369 .

1870: Tsch e r mak: Goalpara, Sitzber.

Wien. Akad. Bd. 62 II, p. 860.

1882: Brezina: Bericht IV, Sitzber.

Wien. Akad. Bd. 851 , p. $33^{8}-339$. 1883: Ts chermak: Beitrag, Sitzber.

Wien. Akad. Bd. 88 I, p. 357.

1884: M e u n ier: Météorites, p. 96,97, 193-195 (Abb.), 521-522.

r883/85: Tschermak: Photographien,

T. 15,18, p. $3,15,16,19$.

1885: Brezina: Wiener Sammlung, p. 184,233 .

1886: Brezina: Neue Meteoriten II, Ann. Hof-Mus. Bd. I (Not.), p. 73.

r894: C o he n : Meteoritenkunde, p. 6o, $163,298$.

UrsprünglichesGewicht: DreiSteine, der grösste etwa 12 Pfund.

Nachweisbares Gewicht: ${ }_{108} \mathrm{gr}$.

\begin{tabular}{lr|lr} 
Bailey & Spl. & Neumann & 7 \\
Berlin, U. & 2 & Paris, M. & I13 \\
Bologna & 441 & Parma & 13 \\
v. Braun & 4 & Pohl & 8 \\
Budapest & 99 & Rom, U. & 40 \\
Calcutta & 2 & v. Siemaschko & 5 \\
Dorpat & 6 & Stockholm & 40 \\
Graz, J. & 38 & Troyes & I \\
Gregory & 4 & Tübingen & 7 \\
Greifswald & I & Ward \\
Harvard, U. & 75 & Washington, Sh. \\
London, B. M. & 15 & 7 \\
Modena & 17 & Wien, H. M.*) & I13 \\
& &
\end{tabular}

Wo ist die grösste Masse der Steine? Wird der grösste Stein noch auf der Sternwarte in Bologna aufbewahrt?

Rensselaer Co. Tomhannock Creek Richmond, Virginia, U. S. A.

Stein, Cck, gefallen 4. Juni 1828 . 1829: C o cke: Virginia Aerolite. To the Editor. Bremo Fluvanna County,

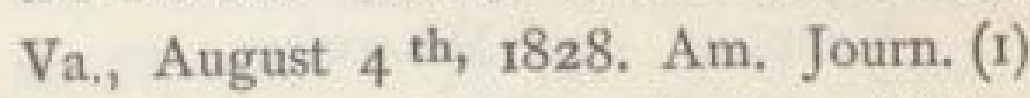
15, p. 195-196; s. auch Ann. Chim. Phys. Bd. 39 (1828), p. $421-422$; Schweigg. Journ. Bd. 57 (I829), p. 47 -50 .

r829: S h e p a r d : A mineralogical and chemical description of the Virginia Aerolite. Am. Journ. (1) 16, p. 191203 (Abbildung eines Trölit-Krystalls); s. auch Pogg. Ann. Bd. 17 (1829), p. 380 ; Berzelius, Jahresber. Bd. Io ( $18_{30}$ ), p. 179-180; Schweigg. Journ. Bd. 57 (1829), p. 50-69; Leonhard's Jahrb. I830, p. $305-306$.

1830: v. Hoff: Siebenter Nachtrag, Pogg. Ann. Bd. 18, p. 186-187.

1836: K ä m tz: Meteorologie, p. 297. 1843: Shepard: On Phosphate of Lime (Apatite), in the Virginia Meteoric Stone. Am. Journ. (I) 45, p. $102-103$; s. auch Berzelius' Bedenken in Berzelius, Jahresber. Bd. 25 (1846), p. 399 -400 ; Haidinger, Uebersicht 1843, p. I09; Pogg. Ann. Bd. 54 (184r), p. 28I ; beiläufig auch bei Burkart, Fundorte I, N. J. 1856, p. 268 .

r843: P a r s c h: Meteoriten, p. 40. 1848: Shepard: Report on Meteorites. Am. Journ. (2) 6, p. $4 \mathrm{II}$; s. auch (3) 30 (1885), p. 106 (Krystall von Nickeleisen).

I854: v. Bog uslaw s k i: Zehnter Nachtrag, Pogg. Ann. Ergz.-Bd. 4, p. 29. r859: B u c hner: Feuermeteore, p. 90. 1859: H a rris : Dissert. Gött., p. 8I. r863: Buchner: Meteoriten, p. 52. r863: R os e: Meteoriten, p. 27, 85, 86, $88,89,139,147, \mathbf{1 5 4}$. 
r858/65: v. Reichen bach: V 48r. VI 452. IX 159, 161, 169, 179. XI 295. XII 454. XIII 36r. XX 626. XXIII 369. XXV 606.

r865: Buchner: Zweiter Nachtrag, Pogg. Ann. Bd. 124, p. 574.

1867: Go e b e 1: Kritische Uebersicht, Mélanges phys. chim, Bd. 7, p. 325 . r870: $\mathrm{R}$ a $\mathrm{mme}$ m s berg: Beiträge zur Kenntnis der Meteoriten. - 3. Die Analyse der Silikate. - C. Die Chondrite von Pultusk, Richmond u. Iowa. - II. Richmond. Mon.-Ber. Berlin. Akad. (1870), p. 453-457 (Analyse). r870: R a m mels berg: Meteoriten, p. 103, 105, 106, 139, I40.

r879: R a m me l s berg : Meteoriten, p. 24,25 .

r884: M e unier: Météorites, p. 74, 79,

$85,93,95,97,238-239$ (Abb.), 395. r883/85: Tschermak: Photographien, p. $4,19$.

1885: Brezina: Wiener Sammlung, p. 191, 233.

1887: Flig ht: Meteorites, p. I07.

r890: Eas tma n: Met. Astron., p. 316.

1894: Cohen: Meteoritenkunde, p. $200,283,317$.

1895: Brezin a: Wiener Sammlung, p. 260 .

Ursprüngliches Gewicht: Ein Stein von etwa 4 Pfund. Cocke erhielt I444 gr. (3 lbs. 3 oz.).

Nachweisbares Gewicht: 849 gr.

Bement

Berlin, U.

Budapest

Calcutta

Cambridge

Dorpat

Göttingen

Gregory

Harvard, U.

Klausenburg

Kunz

\section{Rincon de Caparrosa}

s. Anhang Caparrosa

Rio de Janeiro r 869 Angra dos Reis Rio de Janeiro 1873 Santa Barbara Rio de Janeiro I 888 Minas Geraës Rio Florido Huejuquilla-Gruppe Rio Grande do Sul Santa Barbara Rio San Francisco do Sul

Santa Catharina

Rittersgrün

Robertson Co. Steinbach

Roche Servières

Saint Christophe-la-Chartreuse

Rochester, Fulton Co., Indiana, U. S. A.

Stein, Cc, gefallen 21. Dez, 1876 . 1877: Newton: Meteor of Dec. 21 st 1876. Am. Journ. (3) I3, p. 166-167. 1877: Shepard: On the Meteoric Stone of Rochester, Fulton County, Indiana. Am. Journ. (3) 13, p. 207-2II u. (3) 30 (1885), p. 106 (Krystall von Nickeleisen).

1877: $\mathrm{Sm}$ it h: Note of the recent fall of three Meteoric Stones, in Indiana, Missouri, and Kentucky. Am. Journ. (3) I3 $_{3}$ p. 243 ; s auch C. R. Bd, 84 (1877), p. 399 ; Ref. N. J. 1877, p. 735. 1877: Kirkwood: MeteoricFireballs. Am. Journ. (3) I4, p. 75 (verschiedene Berichte von ihm s, oben bei Shepard); s. auch Amer. Philosoph. Soc. March 16, 1877: On eight Meteoric Fireballs seen in the United States from July I876, to February I877. - IV. The Meteor of December 21, 1876, p. 592-595.

1877: Smith: A description of the Rochester, Warrenton, and Cynthiana Meteoric Stones, which fell respectively December 21 st, 1876, January 3 d, 1877, and January 23 d, 1877 , with some remarks on the previous falls of Meteorites in the same regions, - 1 . Rochester (Indiana) Meteorite. Am. Journ. 
(3) I4, p. 219222 (Analyse); s. auch Original Researches 1884 , p. 528-532; Auszug in C. R. Bd. 85 ( 1877$)$, p. 678-679 (mit Anmerkung von Daubrée). Ref. N. J. I878, p. $78-79$; Zeitschr. f. Kryst. Bd. 2 (1878), p. Iо; Liebig-Kopp, Jahresber. 1877, p. 1394. 1879: R a m mels b e rg: Meteoriten, p. $6 \mathrm{I}$.

r880: K l e in : Vermehrung, Gött, Gel. Anz. p. 567 .

1884: Meunier: Météorites, p. 23I, $237-238,494-495$.

r885: Brezina: Wiener Sammlung, p. $185,233$.

r887: F 1 ig h t : Meteorites, p.197-198. 1888: Newt on: Orbits, Am. Journ. (3) 36, p. 4 .

1890: v. Niess 1: Periheldistanzen, Verh. naturf. Ver. Brünn Bd. 29, p. 189 , 195, 196, 214, 253-254.

1890: F l et ch e r: Mexican Meteorites, Mineral. Magaz. Bd. 9, p. 94.

r89o: E a s tm a n : Met. Astron., p. 316. r894: C o hen: Meteoritenkunde, p. 272, $28_{3}$.

Ursprüngliches Gewicht: Ein Stein von $340 \mathrm{gr}$. ( $\% / 4$ lbs.).

Nachweisbares Gewicht: 195 gr.

\begin{tabular}{lr|lr|} 
Bailey & I & London, B. M. & 8 \\
Bologna & Spl. & New Haven & 15 \\
Bonn & 3 & Paris, M. & 4 \\
v. Braun & 4 & Roebling & 12 \\
Budapest & I & v. Siemaschko & 1 \\
Cleveland & Spl. & Stockholm & 5 \\
Göttingen & 2 & Washington, & 2 \\
Gregory & 2 & Washington, Sh. 48 \\
Harvard, U. & 75 & Wien, H. M. & 12
\end{tabular}

Rockingham Co. 1846 Deep Springs Farm

Rockingham Co. 1863

Smith's Mountain

\section{Rockwood}

Crab Orchard

Roda, Huesca, Aragonien, Spanien. Stein, welcher infolge eines Feldspat- gehaltes eine besondere Gruppe, Rodit, bilden soll. Der Feldspatgehalt ist aber noch nicht sicher nachgewiesen. Vielleicht gehört der Stein zu den Amphoteriten. Gefallen im Frühjahr 1871 .

r874: $\mathrm{P}$ is a n i: Analyse d'une météorite tombée dans la province de Huesca, en Espagne. C. R. Bd. 79, p. 15071509; s. auch Bolletino Commiss. geol. Espagna Bd. 3, p. 277. Ref. LiebigKopp, Jahresber. 1874 , p. $1344-\mathbf{I} 345$. 1874: Da u bré e : Observations relatives à la météorite de Roda. C. R. Bd. 79, p. $1509-15 \mathrm{II}$.

r876: $\mathrm{Sm}$ it h : Carbon compounds, Am. Journ. (3) II, p. 392 (Anm.).

1879: R a mmelsberg: Meteoriten, p. $15-16,26$.

r884: M eun ier: Météorites, p. 299, $300-301$.

r885: Brezina : Wiener Sammlung, p. $175-176,232$

I887: F $1 \mathrm{ig} \mathrm{h} \mathrm{t:} \mathrm{Meteorites,} \mathrm{p.} \mathrm{49-50.}$ 1892: Gredilla: Meteoritos, p. 116 -II7.

1894: C o he n : Meteoritenkunde, p. 49, $277,279,283$.

1894: F l et c her: Introduction, p. 37. r895: Brez in a : Wiener Sammlung, p. 240.

Ursprüngliches Gewicht : Ein Bruchstück von $200 \mathrm{gr}$. besass Pisani. Der ganze Stein mochte etwa 400 gr. gewogen haben.

Nachweisbares Gewicht: 179 gr.

\begin{tabular}{lr|lr} 
Bologna & I & London, B. M. & 8 \\
v. Braun & I & Paris, M. & 125 \\
Budapest & I & v. Siemaschko & 30 \\
Gregory & 2 & Wien, H. M. & 1I
\end{tabular}

\section{Rogue River Mountains}

\section{Rokicky}

Port Orford

Rom

Brahin

Orvinio 


\section{Roqueford}

Rowton, Wellington, Shropshire, England.

Eisen, Om, gefallen 20. April 1876 . 1882/83: F light: Report of an examination of the Meteorites of Cranbourne, in Australia; of Rowton, in Shropshire ; and of Middlesbrough, in Yorkshire. Philos. Trans. Royal Soc. Bd. 3 (1882), p. $894-896$ (Analyse u. Abbildung). Ref. N. J. 1884 II, p. 29; Auszug in Proc. Royal Soc. Bd. 33 (I88I/82), p. 345-346; Liebig-Kopp, Jahresber. 1882 , p. $1641-1642$.

1885: Brezina: Wiener Sammlung, p. 204-205, 2II, 213, 234, Taf. II. 1887: Flight: Meteorites, p. 194-195 (Abbildung des Eisens); s. auch oben 1882/83.

1893: $\mathrm{Brez}$ in a : Ueber neuere Meteoriten (Nürnberg), p. 164.

1893: Meunier: Revision des fers météoriques, p. 16, 21.

1894: C o h en: Meteoritenkunde, p. 73, I74, I9I, I97.

r894: F 1 et cher: Introduction, p. $\mathrm{I}_{3}$, 33,34 .

Ursprüngliches Gewicht: $3.5 \mathrm{Kgr}$. $\left(7^{3 / 4}\right.$ lbs.).

Nachweisbares Gewicht: 3228 gr. Gregory $\quad I_{3} \mid$ v. Siemaschko 2 Harvard, U. $\quad 17$ Stockholm Io London, B. M. 3109 Washington 19 London, P. G. $\quad 16$ Wien, H. M. 39 Paris, M. 3

\section{Roxburghshire}

Newstead

Ruffs Mountain, Newberry, Lexing. ton Co., Süd-Carolina, U. S. A. Eisen, Om, gefd. 1844 , beschr. 1850 . 1850: S hepard: On Meteorites, Am. Journ. (2) 10, p. 128 (Analyse); s, auch Proc. Amer. Assoc. 1850 , p. 152-153; Gött. Gel. Anz. 1852, 26. Febr.; Kenngott, Uebersicht $18_{50} / 51$, p. 138 ; Lie-
big-Kopp, Jahresber, 1850, p. 825826 ; N. J. 1853 , p. 617 .

185x: Shepard: On the probable date of the fall of the Ruff's Mountain Meteoric Iron. Proc. Amer. Assoc. New Haven 185I, p. 189-19I.

1852: C 1 a r k: Dissert. Gött., p. 47.

1853: S hepard: Notice of Meteoric Iron near Lion River, Great Namaqualand, South Africa; and of the detection of Potassium in Meteoric Iron. 2. Potassium in the Meteoric Iron of Ruff's Mountain, South Carolina. Am. Journ. (2) 15, p. 5-6 (Abbildung des Eisens von 55 lbs.). Ref. N. J. 1853 , p. 474 u. N. J. 1854 , p. 72 ; Kenngott, Uebersicht 1853, p. I15; Liebig-Kopp, Jahresber. 1853, p. 934-935; Journ. prakt. Chem. Bd.58 (1853), p.326-327. 1854: v.B o g u s 1 a w s ki: ZehnterNachtrag, Pogg. Ann. Ergz.-Bd. 4, p. 405. 1855 : B ö c k i n g: Dissert. Gött., p. Io -I5 (Analyse). Ref. N. J. 1856, p. 51; Kenngott, Uebersicht $1856 / 57$, p. 151 . 1859: Harris: Dissert.Gött., p.113-r14. r858/62: v. Reichen bach: IV 638. VI 448,452 . VII 552 . IX I62, 174 , 181. X 359, 364. XII 457. XV II 4 , 124, 126. XVI 255, 261, 262, XVII 266, 272. XVIII 484, 487. XIX I50, 155, 156. XX 622.

1863: B u chner: Meteoriten, p. 184 -185 .

1863: R os e: Meteoriten, p.35, 60, 152. 1870: $\mathrm{R}$ a m mels berg: Beiträge zur

Kenntnis der Meteoriten. A. Meteoreisen.

- I. Ruff's Mountains, Newberry (oder Lexington County), Südcarolina. Mon.Ber. Berlin. Akad. (1870), p. 444 (neue Analyse).

1872: Quenstedt: Klar und Wahr, p. 314 (Abb. einer geätzten Platte). 1875: vo m R a th: Meteoriten, Verh. naturh. Ver. Bonn Bd. 32 , p. 363 . 1880: Brezina: Reichenbach'scheLamellen, Denkschr.Wien, Akad. Bd. 43, p.16 (Abbildungen von geätzten Platten). 
r88r: Brezina: Bericht II, Sitzber.

Wien. Akad. Bd. 83 I, p. 476 .

r88r: Brezina: Bericht III, Sitzber.

Wien. Akad. Bd. 84 I, p. 282 .

r884: M e uni er: Météorites, p.r16, 122. r885: Brezina : Wiener Sammlung,

p. $200,2 \mathrm{I}_{3}-2 \mathrm{I} 4,234$.

r887: F light: Meteorites, p. I6-II7. 1887: Sorby: On the microscopical structure of Iron and steel. Journ. of the Iron and Steel Intitute (1887), p. 255-288. Ref. N. J. 1891 I, p. 47.

r8go: B r e z in a : Reise, Ann. Hof-Mus.

Bd. 4 (Not.), p. II9.

r89r: v. H a uer: Ann. Hof-Mus, Bd. 6 (Not.), p. 54 .

1893: M e un i er: Revision des fers météoriques, p. 52,55 .

r894: Cohen: Meteoritenkunde, p. 74, 93, 115, 193, 319, 320.

r895: B rezin a : Wiener Sammlung, p. 277,278 .

1895: Co hen: Meteoreisen-Studien IV. Ann. Hof-Mus, Bd. 1o, p. 83,85 .

Ursprüngliches Gewicht: $53 \mathrm{Kgr}$. (II7 lbs.).

Nachweisbares Gewicht: I I 300 gr.

\begin{tabular}{lr|lr} 
Bailey & 34 & Hamburg & 24 \\
Belgrad & 32 & Harvard, U. & 575 \\
Bement & 125 & Heidelberg & 10 \\
Berlin, U. & 275 & Klausenburg & 71 \\
Bologna & 95 & Kopenhagen & 84 \\
Bonn & 155 & London, B. M. & 499 \\
v. Braun & 73 & London, P. G. & 463 \\
Budapest & 289 & Moskau & 140 \\
Calcutta & 227 & Neumann & 14 \\
Cambridge & 14 & New Haven & 536 \\
Cleveland & 34 & Paris, M. & 190 \\
Cohen & 2 & Petersburg, B. & 105 \\
Dorpat & 36 & Pohl & 85 \\
Dresden, M. & 62 & Prag, M. & 57 \\
Göttingen & 102 & Sevilla & 20 \\
Gregory & r18 & v. Siemaschko & 11 ${ }^{1}$ ) \\
Halle & 59 & Stockholm & 144
\end{tabular}

Troyes

Tübingen

Turin, J.

Upsala

Wo befindet sich die grösste Masse dieses Eisens?
Rügen
s. Anhang
Ruschany
s. Anhang

Russel Gulch, Gilpin Co., Colorado, U. S. A, auch s The Colorado meteorites.

Eisen, Of, gefd. $186_{3}$, beschr. 1866 . 1866: Smith: A new Meteoric Iron, sthe Colorado meteorite\&, from Russel Gulch, near Central City, Colorado Territory. Am. Journ. (2) 42, p. 218 219 (Analyse); s. auch Journ. prakt. Chem. Bd. ror, p. 499-50r. Ref. N. J. 1867, p. 365 ; Liebig-Kopp, Jahresber. I866, p. IOIO.

1867: $\mathrm{S} \mathrm{m}$ it h: On Colorado Meteorites. - Russel Gulch Meteoric Iron, and Bear Creek Meteoric Iron. Am. Journ. (2) 43 , p. $66-67$; s. auch Original Researches 1884 , p. $438-439$. Ref. Liebig-Kopp, Jahresber. 1867, p. 1050. r869: Buchner: Vierter Nachtrag, Pogg. Ann. Bd. 136, p. 604.

r882: W i e ch mann: Fusion-Structures in Meteorites. Ann. N. Y. Acad. Sc. Bd. 2, p. 293.

r884: M e unier: Météorites, p.r16,124. r885: Brezina: Wiener Sammlung, p. 208-209, 233.

r887: Brezina u. Cohen: Photographien, T. 2 I.

r89o: E a s $t \mathrm{~m}$ a n : Met. Astron., p. 320. 1893: M e uni er: Revision des fers météoriques, p. 52, 57 .

Ursprüngliches Gewicht: I3 Kgr. (29 lbs.).

Nachweisbares Gewicht: 4278 gr.

1) Ausserdem sollen nach Brezina (Wiener Sammlung, p. 277) noch 16 gr. >SenekaFallsø wahrscheinlich zu Ruffs Mountain gehören, 


\begin{tabular}{lr|l|r|} 
Berlin, G. & 17 & London, B. M. & 245 \\
Berlin, U. & 502 & London, P. G. & 35 \\
v. Braun & 23 & München & 33 \\
Budapest & 10 & New Haven & 126 \\
Calcutta & 131 & Paris, M. & 184 \\
Dorpat & 86 & v. Siemaschko & 6 \\
Dresden, M. & 18 & Strassburg & 34 \\
Göttingen & 442 & Ward & 303 \\
Gregory & 13 & Washington & 76 \\
Harvard, U. & 1771 & Wien, H. M.*) & 105 \\
Kopenhagen & 118 & &
\end{tabular}

Rutherford Co. 1848 Murfreesboro Rutherford Co. $1880 \quad$ Colfax Rutlam

Pulsora

Sabetmahet, Mathruraghat, Gonda Distr., Oudh, Ostindien.

Stein, C, gefallen 16. August 1885 . 1885: Medlicott: Notice of the Sabetmahet Meteorite. Rec. geol. Surv. India Bd, 18, p. 237-238.

Ursprüngliches Gewicht: 1297 gr. Der Stein war $\mathrm{I}_{88}$ im Besitz der Eingeborenen, welche ihn nicht herausgeben wollten.

Nachweisbares Gewicht: 3 gr. Calcutta 3

\section{Sachsen (Afzelius) \\ Sachsen-Altenburg \\ s. Anhang}

s. Anhang Eisenberg

Saharanpur

Akburpoor

Saint Apre s. Anhang Tocane Saint Augustines Bay

Sanct Augustine's Bay

Saint Caprais, Saint Caprais de Quinsac, Gironde, Frankreich. Stein, Ci, gefallen 28. Januar 1883 . 1883: Lespiault et L. Forquignon: Sur une météorite ferrifère, tombée le 28 janvier $188_{3}$ à Saint-Caprais-de-Quinsac (Gironde). C. R, Bd. 97, p. 1022-1023. Ref, N. J. 1884 II, p. 32 ; Liebig-Kopp, Jahresber. $188_{3}$, p. 1954 .
1884: M e u n ier: Météorites,p.268,272. 1885: Brezina: Wiener Sammlung, p. 181,232 .

Ursprüngliches Gewicht: 282 gr. Nachweisbares Gewicht: 202 gr.

London, B. M. $\quad 9$ Wien, H. M. 33 Paris, M. $\quad 160$

Saint Christophe-la-Chartreuse, Roche-Servières, Vendée, Frankreich.

Stein, gefallen 6. September $184 \mathrm{I}$. Hierher auch: "Bourbon Vendée, Roche-Servières, 5. November $\mathrm{I} 84 \mathrm{I}$ * (dies letztere Datum ist vielleicht richtig, da es schon bei v. Boguslawski 1854 genannt wird. Die älteste Literatur aus „Echo du monde sav." habe ich nicht einsehen können).

1842: Echo du monde sav. (1842) Nr. 683 vom 24 . November.

1843: Part s c h: Meteoriten, p. 144. r854: v.B o g u s la w s ki: ZehnterNachtrag, Pogg. Ann. Ergz.-Bd. 4, p.92, 366. 1859: H a r r i s: Dissert. Gött., p. 88. 1863: Buchner: Meteoriten, p. 67. 1880: D a ubrée: Sur une météorite tombée le 6 septembre $184 \mathrm{I}$ dans les vignes de Saint-Christophe-la-Chartreuse, commune de Roche-Servières (Vendée). C. R. Bd. 9I, p. 30-3I. Ref. N. J. 188I II, p. 344 ; LiebigKopp, Jahresber. 188o, p. 1540.

1887: Flig ht: Meteorites, p. r7o.

Ursprüngliches Gewicht: $5^{1 / 2} \mathrm{Kgr}$. Nachweisbares Gewicht:

Dieser von Daubrée unter dem obigen Namen aufgeführte Meteorit ist offenbar der gleiche, von welchem Buchner sagt, dass um denselben ein Prozess zwischen Finder und Besitzer geführt worden ist, der zu Gunsten des Finders entschieden wurde. Im Jahre 1880 erlaubte der damalige Besitzer keine Untersuchung des Steins. 
Saint Croix Co.

Hammond

Saint-Denis-Westrem bei Gent, Ost-Flandern, Belgien.

Stein, Cca (früher als Cc aufgefasst), gefallen 7 . Juni 1855 .

1855: D uprez: Note sur l'aërolithe tombé à St. Denis-Westrem. Bull. Acad. Belgique Bd. 22 II, p. $54-5^{8}$; s. auch Pogg. Ann. Bd. 99 (1856), p. 63-64; Giebel u. Heintz, Zeitschr. f. ges. Naturw. Bd. 8, p. 355; Am. Journ. (2) 24 (1857), p. 296; >L'Institut \& 1855, p. 380 . Ref. N. J. 1859 , p. 743 ; Kenngott, Uebersicht 1855 , p. 159 u. 1859 , p. I71; Liebig-Kopp, Jahresber, 1855 , p. 1024 .

1859: $\mathrm{B} \mathrm{u} \mathrm{c} \mathrm{hn} \mathrm{er:} \mathrm{Feuermeteore,} \mathrm{p.102.}$ 1860: $\mathrm{H}$ a i ding e r: Der Meteorit von St. Denis-Westrem im k. k. Hof-Mineralien-Cabinete. Sitzber. Wien. Akad. Bd. 42 , p. $9-18$ (zwei Abbildungen des Steins). Ref. N. J. 186I, p. 612-613; Kenngott, Uebersicht 1861, p.169; Am. Journ. (2) 32 (186r), p. 140; Luminous Meteors, Rep. Brit. Assoc. 186r, p. 33-34 (Sep.); sL'Institute Bd. 29 (1861), Nr. 1410, p. 14.

r863: B u chner: Meteoriten, p. 89. 1862/65: v. Re i c hen ba ch: XX623, 626. XXV $317,322,324,607$.

r870: Duprez: Bull. Acad. Belgique (2) 29, p. I6r.

r87o: M e un i er: Bull. Acad. Belgique (2) 29, p. 210.

1872: Dewa 1 que: Acad. royale de Belgique. Centième Anniversaire de Fondation (1772-1872) Bd. II: Rapport séculaire (Sciences minérales), p. 23. 1884: M e uni er: Météorites,p.209,220. r885: Brezina: Wiener Sammlung, p. $176,190,23 \hat{3}$.

1885: Prinz: Les Météorites tombées en Belgique et les Météorites en général. Ciel et Terre V. Brüssel 1885. 39 Seiten (Tafel. Analyse von Rénard, über SaintDenis s. p. 17-26 (Sep.). Ref. N. J.
I886 II, p. $212-213$.

r886: K l ement: Notice sur la composition chimique de la Météorite de Saint-Denis-Westrem(Flandre orientale). Bull. Musée royale d'hist, nat. Belgique Bd. 4 , p. $273-282$ (neue Analyse). Ref. N. J. 1888 I, p. 45.

r887: F lig ht: Meteorites, p.125-126. r887: Brezina: Neue Meteoriten III,

Ann. Hof-Mus. Bd. 2 (Not.), p. ${ }^{1} 5$.

Ursprüngliches Gewicht: Ein Stein von $700 \mathrm{gr}$.

Nachweisbares Gewicht: 575 gr.

\begin{tabular}{lr|lr} 
Bayet & 3 & Lüttich & 3 \\
Calcutta & Spl. & Paris, M. & I \\
Gent & 189 & Pohl & 3 \\
Göttingen & 43 & Tübingen & 5 \\
London, B. M. & I & Wien, H. M.*) & 326 \\
London, P. G. & I &
\end{tabular}

\section{Saint Elizabeth Lucky Hill}

Saint François Co., Südöstliches Missouri, U. S. A.

Eisen, Og. Mit diesem Eisen von Saint François Co. wurde auch das früher als „Südöstliches Missouri bezeichnete, r 863 gefundene und 1869 beschriebeneEisen vereinigt (Cohen, Meteoritenkunde, p. 115 u. Brezina, 1895, p. 286). 1869: Shepard: Notices of New Meteoric Irons in the United States. 2. Meteoric Iron from Southeastern Missouri (Analyse). Am. Journ. (2) 47, p. 233-234. Ref. Liebig-Kopp, Jahresber. $\mathbf{1 8 6 9}$, p. $\mathbf{1} 302$.

1881: Brezina: Bericht III, Sitzber. Wien. Akad. Bd. 84 I, p. 279,28 o. 1884: M e un i e r : Météorites, p. $112,115$. r885: Brezina: Wiener Sammlung,

p. $207,215,216,234$.

I887: F 1 ig h t : Meteorites, p. 139-I40. I890: Eastman: Met. Astron., p. 320. r89r: v. H a uer: Ann. Hof-Mus. Bd, 6 (Not.), p. 54. 
r89r: Cohen u. Weinschenk: Meteoreisen-Studien. Ann. Hof-Mus.Bd, 6 , p. 160 .

r893: M eunier: Revision des fers météoriques, p. 29, 32 .

r894: C o h e n: Meteoritenkunde, p. II5. 1895: Brez in a : Wiener Sammlung, p. 286 .

Ursprüngliches Gewicht: Das als „S. E. Missouri« bezeichnete Eisen der St. Louis Academy of Sc. war nur 3.3 Zoll lang, 1.5 Zoll breit und r.r Zoll dick und wog höchstens $35^{\circ} \mathrm{gr}$. Das ursprüngliche Gewicht von »Saint François Co.» war nicht $\mathrm{zu}$ ermitteln.

Nachweisbares Gewicht: 2418 gr.

\begin{tabular}{lr|lr} 
Bement & $\left.75^{1}\right)$ & Paris, M. & $\mathrm{II}^{2}$ ) \\
Brezina & $\left.\mathrm{I} \mathrm{I}^{1}\right)$ & Prag, M. & $35^{1}$ ) \\
Budapest & $4^{2}$ ) & v. Siemaschko $4^{2}$ ) \\
Harvard, U. & $48^{2}$ ) & Ward & $64 \mathrm{I}^{1}$ ) \\
London, B. M. $\mathrm{IO}^{2}$ ) & Washington, Sh. $6^{2}$ ) \\
London, P. G. $4^{2}$ ) & Wien, H. M. $\left.{ }^{*}\right)^{* *}$ ) \\
New Haven & $68^{2}$ ) & $\left.6 \mathrm{I}^{1}\right) \mathrm{u}^{2}$ )
\end{tabular}

Saint-Mesmin bei Troyes, Dep. Aube, Frankreich.

Stein, Cib (früher als Cgb aufgefasst), gefallen 30. Mai 1866.

r866: R a y: Notice sur les Mét. de Saint-Mesmin le 30 Mai 1866. Mém. Soc. Académique de l'Aube Bd. 30 Troyes 1866. I8 Seiten (Analyse von Pisani).

1866: D a u bré e: Météorites tombées le 30 mai 1866 sur le territoire de Saint-Mesmin, département de l'Aube. C. R. Bd. 62 , P. 1305-1310; s. auch Haidinger in der Nachschrift zu Knyahinya. Sitzber. Wien. Akad. Bd. 54 II, p. 205; $>$ L'Institute Bd. 34 (1866), p. $211-212$.

1866: $\mathrm{P}$ is a n i: Analyse de l'aérolithe de Saint-Mesmin, canton de Méry-surSeine, département de l'Aube, tombé le 30 mai 1866 . C. R. Bd. 62 , p. 1326. Ref. N. J. 1866, p. $827,83 \mathrm{I}-832$; Liebig-Kopp, Jahresber. 1866, p. 1008 -1009; sL'Institute 1866, p. 212 ; Bull. soc. chim. (2) 6. p. 457 ; Chem. Centr. 1867 , p. 286.

r869: B u chner: Vierter Nachtrag, Pogg. Ann. Bd. 136, p. $45^{8}-459$.

r870: Rammelsberg: Meteoriten, p. 103, 106, 107, 138, 139, 140.

1879: R a mmels berg: Meteoriten, p. 24 .

1884: M e un ier : Météorites, p. 79, 85 , 256,257 (Abb.), 258-259, 347, 373, $380,395,443,448,449$.

1883/85: Tschermak: Photographien, p. 3 .

1885: Brezina: Wiener Sammlung, p. 183,233 .

1894: Cohen: Meteoritenkunde,p.249. r895: Brezin a: Wiener Sammlung, p. 250.

Ursprüngliches Gewicht: $8720 \mathrm{gr}$. Nach den Angaben Daubrée's wurden 3 Steine gefunden ( 4200 , 22 10 u. 1860 gr.). Die nachweisbare Menge deutet darauf hin, dass die Masse schwerer war, oder dass nachher noch mehr gefunden wurde.

Nachweisbares Gewicht: 9601 gr.

\begin{tabular}{lr|lr} 
Bailey & I & London, P. G. & 3 \\
Belgrad & 35 & Newton & 2 \\
Bologna & I & Paris, M. & $530^{3}$ ) \\
v. Braun & 26 & v. Siemaschko & 6 \\
Calcutta & 120 & Stockholm & 2 \\
Göttingen & Spl. & Troyes & 30 II $\left.^{1}\right)$ \\
Gregory & $43^{8}$ & Upsala & I6 \\
Harvard, U. & 7 & Washington, Sh. \\
London, B. M. & ro & Wien, H. M. \\
& & 491
\end{tabular}

I) Saint François Co. - 2) Südöstliches Missouri.

3) Der grösste Stein 4200 gr. - 4) 2905 + 106 gr. 


\section{Saintonge \\ Salem}

Salem Distr.

Salins

Salitra

\section{Jonzac \\ Smithland \\ Kakangorai \\ Ornans \\ Primitiva}

Salles, Villefranche bei Lyon, Dep. Rhône, Frankreich.

Stein, Cia, gefallen 8/12 März 1 798 . Höchst wahrscheinlich ist der 8 . März als Falltag anzunehmen; s. Prevost 1802 .

1802: de Dr é e: Sur les masses minérales dites tombées de l'atmosphère sur notre globe. Journ. Phys. Bd. 56, p. $383-389$; s. auch Gilb. Ann. Bd. 16 (1804), p. 75 (Anmerkung).

1802: P r e vos t: Remarques sur l'époque de la chute d'une pierre. Journ. Phys. Bd. 56, p. $465-469$.

r802: Sage: Sur une pierre qu'on dit tombée du ciel dans les environs de Villefranche, département du Rhône. Journ. Phys. Bd. 56, p. 314-315 ; s. auch Bd. 57 (1803), p. $70-73$.

r803: I z a r n : Lithologie, p. 108-109 (Mitteilung v. Sage; Lelievre's Bericht). 1803: C h la d n i: ChronologischesVerzeichnis, Gilb. Ann. Bd. 15, p. 310, $319-320$ (Anmerkung von Gilbert; Lelievre's Bericht).

1803: de Dré e : Account and Description of a Stone which fell from the Clouds in the Commune of Sales, near Ville-Franche, in the Department of the Rhone. Tilloch's Philos. Magaz. Bd, (?), p. 217--224; s, auch BibI. Brit. Bd. 22, p. 371 ; Bd. 23, p. 61 , ${ }_{11} 3,213$.

1803: de Drée: Recherches, Journ. Phys. Bd. 56 (an XI), p. $383-389$, $408,409,410,411,413,419,420,421$. r804: Gil bert: Nachträge zu den Aufsätzen in den Annalen über die aus der Luft gefallenen Steine. Gilb. Ann. Bd. 18, p. 270-279 (Analyse von Vauquelin).
1804: Pötzsch: Kurze Darstellung, p. IIo.

1808: Sa g e: Du procédé que j’ai employé pour déterminer l'existence del'Alumine dans les pierres météoriques. Journ. Phys. Bd. 66 (1808), p. $460-462$.

1808: d e Dré e-P i c te t: Description comparative etc. (s. bei Weston) Bibl. Brit. Nr. 296 (April r808), p. 28r282, 286, 287, 288.

r809: V a uquelin: Expériences sur l'aërolithe tombé aux environs de Parme, pour y découvrir la présence de l'alumine, annoncée par M. Sage. Ann. Chim. Phys. Bd. 69, p. $280-284$. Uebersetzung in Gilb. Ann. Bd, 33, p. $198-201$.

1812: Bigot de Morogues, p. 154 $-16 \mathrm{I}, 336$.

1812: C h l a d ni: Verzeichnis,Schweigg. Journ. Bd. 4 Beil. I, p. 15 .

1815: Chladni: Neues Verzeichnis, Gilb. Ann. Bd. 50, p. 252.

1816: $\mathrm{Ch} 1$ a d n i: Zweite Fortsetzung, Gilb. Ann. Bd. 54, p. $35^{2}$.

r8rg: $\mathrm{Ch}$ la d n i : Feuermeteore, p. 50 , $57,65,70,72,73,91,136,137,253$, $265-266(1), 271,273,297,428$.

r820: v. Schreibers: Beiträge, p. 68-69 (Abb.).

1836: Gruith u is en: Naturgeschichte

des gestirnten Himmels, p. $4 \mathrm{II}-412$. 1836: K äm tz: Meteorologie, p. 278. r843: P a rts c h: Meteoriten, p. 6r-62. 1854: B a $1 \mathrm{c}$ e $11 \mathrm{~s}$ : Lithologia meteorica, p. $4 \mathrm{I}-43$.

r859: Harris: Dissert.Gött., p.63-64. r859: Buchner: Feuermeteore, p. 57 -58 .

1862: Kenngott u. W iser: Zürcher Sammlung, p. 155 .

r863: B u c hn e r: Meteoriten, p. 13-r4. r863: Ros e: Meteoriten, p. 155.

1864: B u chner: Erster Nachtrag, Pogg. Ann. Bd. 122, p. 3 I9.

1858/65: v. Reichenbach: V 475. IX 161, 169, 179. X 359. XI 295, 300. 
XIII 365. XX 622, 626. XXIV 226. XXV 321, 322, 423, 427, 433, 434. 1875: Mohr: Entstehungsart, Ann.

Chem. Pharm. Bd. 179, p. 259, 260, 26I. 1884: M e un i er: Météorites, p.95, 247, $378,459,468$.

1885: Brezina: Wiener Sammlung, p. $182,232$.

r894: C o h en : Meteoritenkunde,p.8,223. 1895: Brezina: Wiener Sammlung, p. 247 .

Ursprüngliches Gewicht: $10 \mathrm{Kgr}$. (20 Pfund).

Nachweisbares Gewicht: 2900 gr.

\begin{tabular}{lr|lr} 
Berlin, U. & 16 & Paris, E. & 53 \\
Bologna & I & Paris, M. & 1746 \\
Budapest & I & Paris, U. & I18 \\
Calcutta & I8 & Parma & 6 \\
Cambridge & 95 & v. Siemaschko & 4 \\
Göttingen & I & Stuttgart & 4 \\
Gregory & 125 & Troyes & 53 \\
Harvard, U. & 6 & Tübingen & 12 \\
London, B. M. & 165 & Washington,Sh. \\
London, P. G. & 135 & Wien, H. M.*) & 333 \\
Newton & I & Zürich \\
\end{tabular}

\section{Saltillo}

Coahuila

Salt Lake City (Echo), zwischen Echo u. Salt Lake City, Utah, U. S. A.

Stein, Cgb, gefd. 1869 , beschr. 1886 . 1886: D ana u, Penfield: On two hitherto undescribed Meteoric Stones.1. Meteorite from Utah. Am. Journ. (3) 32, p. 226-229 (Analyse von Penfield); s. auch Liebig-Kopp, Jahresber. 1886, p. 233I-2332. Ref. N. J. I887 II, p. $42-43$.

I890: E as t m a n : Met. Astron., p. 320. r894: C o hen: Meteoritenkunde,p.248, $283,309$. r895: B rezin a: Wiener Sammlung, p. 251 .

Ursprüngliches Gewicht: $875 \mathrm{gr}$. Nachweisbares Gewicht: 875 gr. London, B. M. $\quad 5$ v. Siemaschko $\quad 7$ New Haven $834^{1}$ ) Washington 3 Paris, M. $\quad 14$ Wien, H. M. 12

Salt River, Kentucky, U. S. A.

Eisen, Hch, beschr. 1850 .

1850: Silliman jr.: Notice of two American Meteoric Irons. Proc. Amer.

Assoc. (Fourth meeting held at New Haven, $185^{\circ}$ ), p. $36-37$ (Analyse). r851: Shepard: Am. Journ. (2) II (185I), p. 40; s. auch Liebig-Kopp, Jahresber. 1850 , p. 826 .

1852: C 1 a r k: Dissert. Gött., p. 76. 1859: Harris: Dissert. Gött., p. 123. 1859/62: v. Reichen b a ch: IX I62, 175, 176, 182. XI 291. XV 100. XVII 268,269 . XVIII 488. XXI $578,588$. r863: B u c hner: Meteoriten, p. 185. 1863: Rose: Meteoriten, p. 70, 153. r884: Me uni er: Météorites, p. 99, r31 (Abb.), 132.

r885: Brezina: Wiener Sammlung, p. 203, 219, 234.

r89o: Eastman: Met. Astron., p. 318. r893: Meunier: Revision des fers météoriques, p. 15, 18.

1894: F le t c h er: Introduction, p. 34. UrsprünglichesGewicht: Etwa $4 \mathrm{Kgr}$. Nachweisbares Gewicht: 2281 gr.

\begin{tabular}{lr|lr} 
Berlin, U. & I9 & London, B. M. & 524 \\
Budapest & 5 & London, P. G. & 2 I \\
Calcutta & 78 & New Haven & 985 \\
Cleveland & 3 & Paris, M. & 34 \\
Dorpat & 81 & Stockholm & 27 \\
Dresden, M. & I9 & Tübingen & 61 \\
Göttingen & 13 & Washington, Sh. & 51 \\
Gregory & II & Wien, H. M.*) & 45 \\
Harvard, U. & 304 & &
\end{tabular}

I) In dem mir 1893 mitgeteilten Katalog von New Haven (der gedruckte Teil von 1886) ist das Gewicht des Steins noch mit $875 \mathrm{gr}$. angegeben; wahrscheinlich ist dieses Gewicht um die an andere Sammlungen abgegebenen Mengen zu verkleinern. 
Samyschewa

San Antonio Eisen

Jamyschewa

San Antonio Stein

Sanarka

Kendall Co.

Pipe Creek

s. Anhang

San BartoloméHuejuquilla-Gruppe

San Bernardino Co. I880 Ivanpah

San Bernardino Co. 1887

San Emigdio Range

\section{Sancha}

Coahuila

Sanchez Estate

Coahuila

San Cristobal

s. Anhang

Sanct Augustine's Bay, Madagascar, Ostafrika.

Eisen, Dn (früher als Df bezeichnet), gefunden ?, erwähnt $\left.1843(1845 \text { ? })^{\prime}\right)$.

1845: Proc. Amer. Assoc. of the Geologists New Haven, April 1845, p. 40 ; s. auch Am. Journ. (2) 15, p. 22.

r853: Silli m a n's Anmerkung zum Referat über Clark's Dissertation. Am. Journ. (2) 15, p. 22.

r859: H a r r is: Dissert, Gött., p. II4. 1863: B u chner: Meteoriten, p. 171. 1885: Brezina: Wiener Sammlung, p. 220, 234 .

r894: Co hen: Meteoritenkunde, p. 62.

Ursprüngliches Gewicht: Es sollen grosse Massen vorhanden sein.

Nachweisbares Gewicht: 20 gr.

\begin{tabular}{lr|lr} 
Calcutta & 2 & New Haven & 7 \\
London, B. M. & 6 & Wien, H. M.*) & $1^{2}$ ) \\
London, P. G. & 4 & &
\end{tabular}

\section{Sandwich-Inseln Honolulu}

San Emigdio Range, San Bernardino Co., Californien.

Stein,Cc, gefd. vor 1887 , beschr.1 888 . 1888: Merril1: On the San Emigdio Meteorite. Proc. of N.S. National Museum (1888), p.161 -167 (mit Tafel; Ana- lyse von Whitfield); s. auch Am. Journ. (3) 35 , p. $490-49 \mathrm{r}$ : $>$ On a New Meteorite from the San Emigdio Range, San Bernardino County, California «. Ref. N. J. 1889 II, p. 281 u. 1891 II, p. 417 .

r889: M e un i er: Détermination lithologique de la météorite de San Emigdio Range, Californie. C. R. Bd. 109, p. 206-207 (neue Analyse). Ref. N. J. I89o I, p. 413.

I890: Ea stman: Met. Astron., p.322. r89I : v. H a ue r: Ann. Hof.-Mus. Bd. 6 (Not.), p. 55.

r894: C o h e n: Meteoritenkunde,p.298. r895: Brezina: Wiener Sammlung, p. 256 .

Ursprüngliches Gewicht: Ein Stein, der in einer Stampfmühle vollständig zermalmt wurde und von dem nur wenig erhalten ist.

Nachweisbares Gewicht: 180 gr.

\begin{tabular}{lr|lr} 
Bailey & I9 & Howell & 18 \\
Bement & 4 & Paris, M. & 5 \\
v. Braun & I & v. Siemaschko & 3 \\
Cleveland & I & Washington & II9 \\
Gregory & 4 & Wien, H. M. & 6
\end{tabular}

SanFranciscoCo. SaintFrançoisCo. San Francisco del Mezquital (Mezquital), Durango, Mexico.

Eisen, Hch, bekannt kurz vor 1868 , beschr. 1868 .

1868: D a u b r é e : Fermétéorique trouvé à San Francisco del Mezquital, Durango, Mexique. C. R. Bd. 66, p. 573 -574 (Analyse von Damour). Ref. Liebig-Kopp, Jahresber, 1868, p. 1046. r869: Meunier: Recherches, Ann. Chim. Phys. (4) 17, p. 68.

r869: B uchner: Vierter Nachtrag, Pogg. Ann. Bd. 136, p. 609.

1) Brezina sagt bekannt 1843. Die Arbeit in den Proc. Amer. Assoc., in welcher eine Angabe darüber enthalten sein dürfte, habe ich nicht einsehen können.

2) Ausserdem eine Pfeilspitze aus diesem Eisen.

W ï 1 fing, Meteoriten. 
1870: Burkart: Fundorte IV, N. J. 1870, p. 685,691 .

I876: B a r c en a : On certain Mexican Meteorites. Proc. Acad. nat. hist. Philadelphia (1876), p. 123.

r884: M e un i e r: Météorites, p.44, II6, 125.

r885: Brezina: Wiener Sammlung, p. $210,233$.

1889: M eunier: Altération remarquable du fer météorique de San Francisco del Mezquital. C. R. Bd, 108, p. $1028-1029$. Ref. N. J. 1890 II, p. 60. r89o: $\mathrm{Fl}$ e t $\mathrm{c} \mathrm{h}$ er: Mexican Meteorites, Mineral. Magaz. Bd. 9, p.154-155, 156. 1892: Eastman: The Mexican Meteorites. Bull. Philos. Soc. Washington Bd. 12, p. 45 .

I892: v. H a u e r: Ann. Hof-Mus. Bd. 7 (Not.), p. 73 .

1893: M e unier: Revision des fers météoriques, p. 74 .

1894: C o h e n: Meteoritenkunde, p. 72, 196, 237, 242 .

r895: $\mathrm{Brez}$ in a: Wiener Sammlung, p. 294.

Ursprüngliches Gewicht: 7513 gr. wog die Hauptmasse.

Nachweisbares Gewicht: 7507 gr.

\begin{tabular}{lr|lr} 
Budapest & I4 & London, P. G. & 46 \\
Gregory & I2 & Paris, M. & 175 \\
Harvard, U. & 52 & Utrecht & 15 \\
London, B. M. 7120 & Wien, H. M.*) & 73
\end{tabular}

San Francisco do Sul

Santa Catharina

SanFranciscoPass BarrancaBlanca San Giovann d'Asso Siena

San Gregorio Huejuquilla-Gruppe San Jago del Estero

San José

San Juliano

Sankt Nicolas
Campo del Cielo

Heredia

São Julião

Mässing
San Luis de la Paz

s. Anhang Manzanares

San Luis Potosi Eisen 1780

Descubridora

San Luis Potosi Eisen 1804 Charcas

San Luis Potosi Stein 1804 Bocas

San Pedro deAtacama,Mesosiderit

Vaca Muerta

San Pedro de Atacama, Pallasit Imilac

San Pedro Springs, San Antonio, Texas, U. S. A.

Stein, gefunden 1887 . Herr Bement führt diesen Meteoriten in seinem Katalog vom Juni 1894 mit einem Gewicht von $72 \mathrm{gr}$. auf. Nach Brezina (Wiener Sammlung 1895 , p. 306) gehört derselbe $\mathrm{zu}$ einem besondern Fall.

Santa Barbara, Rio Grande do Sul, Brasilien.

Stein, Cho, gefallen 26. Sept. 1873 . 1888: Derby: Meteoritos Brasileiros. Notas sobre Meteoritos Brasileiros. Revista do Observatorio Rio de Janeiro, p. 9-12. Ref. Am. Journ. (3) 36 , p. 157 ; N. J. 1889 II, p. 281 u. 1891 I, p. 243.

I89r: v. H a u e r: Ann. Hof-Mus Bd. 6 (Not.), p. 55.

1895: Brezina: Wiener Sammlung, p. $24 \mathrm{I}$.

Ursprüngliches Gewicht: Die nach Rio gelangten Stücke dürften etwa $93 \mathrm{Kgr}$. gewogen haben.

Nachweisbares Gewicht: $85167 \mathrm{gr}$.

London, B.M. $\quad 2$ Rio $\quad 85161$

Paris, M. 2 Wien, H. M. 2

Santa Catharina, auch Morro do Ricio, Rio San Francisco do Sul, Brasilien.

Eisen, Dn (früher als Df bezeich- 
net), bekannt $1873^{1}$ ), beschr. 1876 . Die meteorische Natur ist fraglich.

1876: Guignet u. Ozorio de A 1meida: Sur un fer météorique trèsriche en nickel, trouvé dans la province de Santa-Catharina (Brésil). Mit Nachtrag von Daubrée. C. R. Bd. 83, p. 917-919 (Analyse). Ref. LiebigKopp, Jahresber, 1876, p. 1319.

1877 : D a mour: Sur un fer métallique trouvé à Santa-Catarina (Brésil). Mit Nachtrag von Boussingault. C. R. Bd, 84 , p. $478-482$ (neue Analyse von Boussingault). Ref. Zeitschr. f. Kryst. Bd. I (1877), p. 407.

1877: D a u brée : Observations sur le fer natif de Sainte-Catherine, sur la pyrrhotine et la magnétite qui lui sont associées. C. R. Bd. 84 , p. $482-485$. Ref. Zeitschr. f. Kryst. Bd. I (1877), p. 407 .

r877: Guignet: Note sur les travaux de Chimie à l'Ecole Polytechnique de Rio-de-Janeiro. C. R. Bd. 84 , p. 1085 . r877: Guignet: Sur le fer nickelé de Sainte-Catherine au Brésil. Extrait d'une lettre de M. Guignet à M. Daubrée. Mit einigen Bemerkungen Daubrée's. C. R. Bd. 84, p. 1507-1509.

1877: Lunay: Sur le fer nickelé de Sainte-Catherine. Extrait d'une lettre adressée à M. Daubrée. C. R. Bd. 85, p. $84-85$ (neue Analyse).

1877: D a u b r é e : Constitution et structure bréchiforme du fer météorique de Sainte-Catherine (Brésil); déductions à tirer de ses charaktères, en ce qui concerne l'histoire des roches météoriques et notamment l'association habituelle du carbone au sulfure de fer. C. R. Bd. 85 , p. $1255-1260$. Ref. Zeitschr. f. Kryst. Bd, 2 (1878), p. 516-517 ; Liebig-Kopp, Jahresber. 1885 , p. 2326 ; s. auch C. R. Bd. 86 (1878), p. 1433 . Ref. Liebig-Kopp, Jahresber. 1878 , p. 1322 auch 1877, p. $1398-1400$; an welcher Stelle sich Referate über die Arbeiten von Boussingault, Damour, Daubree, Guignet u. Lunay befinden. 1877: Eine den 9. März 1877 datierte spanische Uebersetzung einer Notiz über Santa Catharina aus dem sEngineering * besitzt die Bibliothek des k. k. HofMuseums in Wien.

1879: D o m e y k o: Mineralojia. Dritte Aufl., p. 138 - 139 .

1879: Oesterr, Zeitschr. f. Berg- und Hüttenw. Bd. 27, p. 96: Kurzes Referat aus Le Technologiste , dass man $25000 \mathrm{Kgr}$. gewonnen habe.

r880: Kle in : Vermehrung, Gött. Gel. Anz. (1880), p. 569 .

r88I : B e c quere 1: Sur les propriétés magnétiques du fer nickelé de SainteCatherine. C. R. Bd. 93, p. 794-797. Ref. Liebig-Kopp, Jahresber. I882, p. 1644 .

r88I: $\mathrm{S} \mathrm{m}$ it h: Anomalie magnétiqne du fer météorique de Sainte Catherine. C. R. Bd. 92 , p. $843-844$; s, auch Original Researches (1884), p. $597-598$. Ref. 'N. J. 1882 I, p. 365 ; LiebigKopp, Jahresber, $188 \mathrm{I}$, p. $145^{8}$ u, 1885 , p. 2327.

r884: M e uni e r: Météorites, p.26, 5 I, $52,64,70,99,102-108$ (Abb.), 320, $364-367,501-502$.

1885: B rezina: Wiener Sammlung, p. 154, 199-200, 221, 234 .

1885: Der by: The Santa Catharine Meteorites. Am. Journ. (3) 29, p. 33 -35 . Ref. N. J. 1886 II, p. 357 ; Liebig-Kopp, Jahresber. 1885 , p. $2326-$ 2327.

1886: H unt ing to n: Crystalline Struc-

ture, Am. Journ. (3) 32 , p. 285. 1888: D erby: Meteoritos Brasileiros.

1) Wien erhielt im Jahre 1873 ein Stück von dieser Lokalität. Viele Autoren geben 1875 als Fundjahr an. 
- Notas sobre Meteoritos Brasileiros. - Nota sobre a localidade do Ferro Nativo de Santa Catharina por Luiz F. Gonzaga de Campos. Revista do Observatorio. Rio de Janeiro 1888 , p. 3 $-6,23-29$ (mit zwei Karten). Ref. Am. Journ. (3) 36 , p. 157 ; N. J. 1889 II, p. 28 I u. 189 I I, p. 243.

I89o: E a s t m a n : Met. Astron., p. 320. r89o: Brez in a: Reise, Ann. Hof-Mus. Bd. 4 (Not.), p. IIg.

r89r: v. H a u e r: Ann. Hof-Mus. Bd. 6 (Not.), p. 54 .

1892: Ca log eras: Le fer nickelé de Sainte-Catherine. Revue scientifique Bd. 50, p. $59 \mathrm{I}-594$. Ref. N. J. 1893 I, p. 480 .

1893: Brezin a: Ueber neuere Meteoriten (Nürnberg), p. 167.

1893: Meunier: Revision des fers météoriques, p. 7-14.

1894: Co he n: Meteoritenkunde, p. 34, $5 \mathrm{I}, 65,70$, ro 8, III, 138 .

r894: F l et c h e r: Introduction, p.r4,33. 1895: Brezina: Wiener Sammlung, p. 296.

1895: C o h en: Meteoreisen-Studien IV. Ann. Hof-Mus. Bd. 1o, p. 83 .

Ursprüngliches Gewicht: Grosse Massen, von denen $25000 \mathrm{Kgr}$. nach England gebracht und auf Nickel verarbeitet worden sein sollen.

Nachweisbares Gewicht: I37 453 gr. Bailey

Belgrad

Bement

Bologna

v. Braun

Breslau

Brezina

Budapest

Cambridge

Cleveland

Dorpat

Madrid
de Mauroy
Minneapolis
Moskau
München
New Haven
Newton
Odessa
Paris, E.

Paris, M.

Petersburg, B. 691 Washingt., Sh. 7454

Pierrotet . Spl. Wien,H.M. $45234^{2}$ )

\section{Santa Fé Co.}

Santa Giulietta

Santa Rita

Santa Rosa 1810

Santa Rosa 1837

$7 \mathrm{I} \mid$ Pohl $\quad 46$

I42 Prag, M.

25 Rio

182

260 v. Siemaschko 378

IOI5 Stockholm 177

13 Stuer

60 Troyes

776 Ward

30

25

8093

82

São Julião de Moreira bei Ponte de Lima, Minho, Portugal.

Eisen, $\mathrm{Hb}$, gefunden?, nach Lissabon gesandt $188_{3}$, beschr. 1888 .

1888: B e n-S a ude: Note, sur une météorite ferrique trouvée à S. Julião de Moreira près de Ponte de Lima (Portugal). Communicaçòes da Commissão dos Trabalhos Geologicos de Portugal Bd. II (1888/89), p. $14-16$ (mit 2 Tafeln; Analyse von Bonhorst). Ref. N. J. 1888 II, p. $371-372$.

r889: Cohen: Chemische Untersuchung des Meteoreisens von S. Julião de Moreira, Portugal, sowie einiger anderen hexaëdrischen Eisen. N. J. 1889 I, p. 215-228.

r89r: Cohen u. W einschenk: Meteoreisen-Studien. Ann. Hof.-Mus. Bd. 6, p. 159,164 .

r894: C o hen : Meteoritenkunde, p. 68, $89,96,123,129,131,225,230,321$. 1895: Brezina: Wiener Sammlung, p. $292,293$.

1895: C o h e n: Meteoreisen-Studien IV. Ann. Hof-Mus. Bd. 10, p. 83,88 .

1) $2-3$ Kgr. - 2) Ausserdem 347 gr. nach 1. Juli 1893 erworben. 
Ursprüngliches Gewicht: Etwa I $62 \mathrm{Kgr}$.

Nachweisbares Gewicht: 162569 gr. Berlin, U. $\quad$ I7 $\mathrm{Krantz} \quad 2 \mathrm{I}$ v. Braun II Lissabon 184 Brezina $\quad 162000$ London, B.M. 9 Cohen 310 Wien, H.M. 17

Herr Prof. Cohen gab eine kleine Menge an Herrn v. Siemaschko ab, was in dessen Katalog von 1891 noch nicht verzeichnet sein konnte.

\section{Saonlod}

Saratow Eisen 1854

Saratow Stein 1882

Sarbanovac

Sarepta, Kalmücken-Steppe, Gouvernement Saratow, Russland.

Eisen, Og, gefd. u. erwähnt 1854 . 1854: A u e rb a ch présente de la part de Mr. Glitsch de Sarepta une masse de fer météorique pesant 32 livres et 58 zolotniks u. s, w. (kurze Ankündigung). Bull. Soc. Imp. des Naturalistes de Moscou (1854) Nr. 4, p. 504. 1862: Haidinger: Das Meteoreisen von Sarepta (mit 2 Tafeln; drei Abbildungen der Masse und Stereotypdrucke von geätzten Platten). Sitzber. Wien. Akad. Bd, 46 II, p. 286-297 (genauere Angabe der damaligen Besitzer); s. auch Am. Journ. (2) 36 (1863), p. $150-152$; >L'Institute Bd. 30 (1862), p. 354-355.

1863: B u chner: Meteoriten, p. 190. 1863: Ros e: Meteoriten, p. 34, 54, 58 $-59,138,152$.

1864: $\mathrm{H}$ a i d in g e r : Einegrosskörnige Meteoreisen-Breccie von Copiapo (am Schluss Mitteilung Auerbach's über Sarepta nebst Analyse). Sitzber. Wien. Akad, Bd. 49 II, p. 496-497; s. auch sL'Institut* Bd. 32 (1864), p. 398399 ; Am. Journ. (2) 38 (1864), p. 424 ; Liebig-Kopp, Jahresber. 1864, p. 90I;
Jahrb. k. k. geol. Reichsanst. Bd. 37 (1887), p. 197, 20 I.

r86r/65: v. Reichen b a ch:XVioo, I26. XVI 250. XVII 273. XVIII 487. XIX 150, 155. XXV 437.

1865: B u chner: Zweiter Nachtrag,

Pogg. Ann. Bd. 124, p. 598.

1867: Buchner: Dritter Nachtrag,

Pogg. Ann. Bd, 132, p. 319.

1867: Go e b e 1: Kritische Uebersicht, Mélanges phys. chim. Bd. 7 , p. 338 . r869: M eunier: Recherches, Ann.

Chim. Phys. (4) 17, p. 68.

1875: vom R a th: Meteoriten, Verh. naturh. Ver. Bonn Bd. 32, p. 363 .

1881: B rezina: Bericht III, Sitzber.

Wien. Akad. Bd. 84 I, p. 279.

r884: M e un i e r: Météorites, p. 44, 94, 96, 98, 112, I14.

1885: Brezin a: Wiener Sammlung, p. 20I, 207, 234 .

1888: v. Ha uer: Ann. Hof-Mus, Bd. 3

(Not.), p. 42.

189r: Cohen u. Weinschenk: Meteoreisen-Studien. Ann. Hof-Mus. Bd, 6, p. 161 .

1893: Meunier: Revision des fers météoriques, p. 29-32.

1894: C o h e n: Meteoritenkunde, p. 57, II5, 136.

Ursprüngliches Gewicht: Das Gewicht wird meist zu $14325 \mathrm{gr}$. angegeben; 32 livres 56 zolotniks (s. o.), die doch vermutlich russ. Gew. sein sollen, sind aber nur gleich $1335^{2} \mathrm{gr}$.

Nachweisbares Gewicht: 6544 gr.

\begin{tabular}{lr|lr} 
Bailey & 5 & Dresden, M. & 90 \\
Bement & 8 & Erlangen & 104 \\
Berlin, U. & 1962 & Göttingen & 20 \\
Bonn & 3 & Gregory & 27 \\
v. Braun & 23 & Harvard, U. & 446 \\
Budapest & 254 & Klausenburg & 2 \\
Calcutta & 177 & London, B. M. & 296 \\
Cleveland & 4 & London, P. G. & 36 \\
Dorpat & $4 \mathbf{I}$ & Melion & 4
\end{tabular}




\begin{tabular}{lr|lr} 
Minneapolis & 2 & Stockholm & 80 \\
Moskau & 72 & Stuttgart & 632 \\
Neumann & 4 & Tübingen & 247 \\
Paris, M. & 329 & Utrecht & II \\
Petersburg, A. & 252 & Washington, Sh. & 3 \\
Petersburg, B. & 44 & Wien, H. M.*) & 75 I \\
Pohl & 120 & Würzburg & 488 \\
v. Siemaschko & 7 &
\end{tabular}

\section{Saroser Comitat}

Sarte

Lenarto

\section{Saskatchewan River}

Lucé

Satsuma

Victoria

Maêmê

Sauguis, Saint Etienne, Basses-Pyrenées, Frankreich.

Stein, Cwa, gefallen 7. Sept. r868. r868: D a u bré e: Note sur une chute de météorites, qui a eu lieu le 7 septembre 1868 à Sauguis-Saint-Etienne, canton de Tardets, arrondissement de Mauléon (Basses-Pyrénées). C.R. Bd.67, p. $873-877$ (Analyse von Meunier). Ref. Liebig-Kopp, Jahresber. 1868, p. 1043-1044; Ann Chim. Phys. (4) 17 (1869), p. 17-19; Luminous Meteors, Rep. Brit. Assoc. 1869 , p. 277 ; sL'Institute Bd. 36 (1868), p. 353.

r869: Buchner: Vierter Nachtrag, Pogg. Ann. Bd. 136, p. 595-596.

r870: Rammelsberg: Meteoriten, p. 103 .

r884: Me unier: Météorites, p. 209, $222-224$

1885: Brezina: Wiener Sammlung, p. $180,232$.

r887: F li g h t: Meteorites, p. I57-I58.

1894: Co h e n: Meteoritenkunde,p.20r.

Ursprüngliches Gewicht : Ein Stein, welcher in einen Bach fiel und in kleine Stücke zerbrach. Das Gesamtgewicht dieser Stücke wird auf $2-4$ Kgr. angegeben.

Nachweisbares Gewicht: 208 gr.

Bement

Bologna

I Gregory

1 Harvard, U.
Kunz

I2 Paris, M.

150

London, B. M. $\quad 16$ v. Siemaschko 5

de Mauroy $\quad 3$ Wien, H. M.*) I3

Saurette (Apt), Dep. Vaucluse, Frankreich.

Stein, Cga, gefallen 8. Okt. 1803 .

1803: Urkunde über den Fall im \$Moniteur a vom 24. November 1803 .

r803: B o urdon: Account of a Shower of Stones in a Letter from the Prefect of the Department of Vaucleure to the French Minister of the Interior, dated November 10, 1803. Philos. Magaz. Bd. I7, p. 27I--274.

1804: L a ugi e r: Analyse d'une pierre tombée de l'atmosphère. Ann. Mus. d'hist. nat. Bd. 4, p. $249-257$; s. auch Gilb. Ann. Bd. 16, p. 72-74; sEncyclopédie * Bd. 5 (1808), p. $5^{80}-5^{82}$. 1804: Gi lb e r t : Nachträge zu den Aufsätzen in den Annalen über die aus der Luft gefallenen Steine. Gilb. Ann. Bd. 18, p. $321-326$ (Laugier's Analyse). 1804: $\mathrm{P}$ ötzsch: Kurze Darstellung, p. $\mathrm{III}-\mathrm{II}_{3}$.

1806: L a u g i e r : Extrait d'un Mémoire sur l'existence du chrôme dans les pierres météoriques. Ann. Mus. d'hist. nat. Bd. 7, p. $392-397$; s, auch Gilb. Ann. Bd. 24 , p. $380-3^{8} 3$; s Encyclopédie « Bd. 5 (1808), p. $582-584$, 592 ; Voigt's Magaz. Bd. 8, p. 434.

r812: $\mathrm{Ch} 1 \mathrm{ad}$ n i: Verzeichnis,Schweigg. Journ. Bd. 4 Beil. I, p. 15.

1812: Bigot de Morogues, p. 198 -200 .

I8I5: $\mathrm{Ch} 1 \mathrm{adn}$ i: Neues Verzeichnis, Gilb. Ann. Bd. 50, p. 253.

1819: Chladni: Vierte Fortsetzung, Gilb. Ann. Bd. 6o, p. 239.

1819: $\mathrm{Ch}$ la d ni: Feuermeteore, p. 5I, $57,66,69,73,91,265,271,273$ (!), $275,290,295,296,297,429$.

1836: Käm tz: Meteorologie, p. 280. 1843: Parts ch: Meteoriten, p. 59-60. 1859: Harris : Dissert. Gött., p. 64 
1863: B u chner: Meteoriten, p. 17. 1863: Rose: Meteoriten, p. 93, 155. r 858/65: v. Reichen ba ch: V 476. IX 16I, 168, 178, X 359. XI 294, 30I. XIII 365. XXIV 226. XXV 321, 322. 1884: M eunier: Météorites, p. 95, 197, 198, 459.

r885: Brezin a: Wiener Sammlung, p. $182,233$.

r894: Co he n: Meteoritenkunde, p.244. Ursprüngliches Gewicht: Etwa 3 Kgr. ( $7^{1 / 2}$ lbs.).

Nachweisbares Gewicht: I560 gr.

\begin{tabular}{|c|c|c|c|}
\hline Bailey & Spl. & Harvard, U. & 127 \\
\hline Bement & I & London, B. M. & 37 \\
\hline Berlin, U. & 16 & Paris, M. & 1000 \\
\hline Bologna & 24 & Paris, U. & 33 \\
\hline Budapest & 4 & v. Siemaschko & 5 \\
\hline Calcutta & Spl. & Tübingen & 2 \\
\hline Gotha & 8 & Wien, H. M.*) & 297 \\
\hline Gregory & 6 & & \\
\hline
\end{tabular}

Savtschénskoje, Gouv. Cherson, Russland.

Stein, Cck, gefallen 27. Juli 1894 . r895: Herr Prof. Prendel teilte mir in dem russischen Katalog der Meteoritensammlung in Odessa vom Februar 1895 mit, dass dieser Meteorit am 27. Juli I894 gefallen sei u. dass das in Odessa vorhandene Gewicht 2565 gr. betrage. Kürzlich ist eine Arbeit Prendel's : sNotiz über den Meteoriten von Savtschenskoje mit Abbild. erschienen (russisch).

r895: Brez in a: Wiener Sammlung, p. 260 .

Inzwischen erhielt Wien, H. M. 33 gr.

\section{Sawiauk}

Pillistfer

Scheikahr Stattan Buschhof

Schellin, Garz, Stargard, Pommern, Preussen, Deutschland.

Stein, Cia, gefallen 11 . April 1715 , beschr. 1822 .

1822: Gil bert: Nachricht von einem noch unbekannten Meteorsteinfall unweit Stargard in Pommern am II. April I715. Gilb. Ann. Bd. 71, p. 213-223 (Urkunde des Predigers Granzin) u. Bd. 72 (1822), p. 328.

1823: Chladni: Dritter Nachtrag,

Gilb. Ann. Bd. 75, p. 229-230.

1836: Kä mtz: Meteorologie, p. 27o. 1843: P a rts ch: Meteoriten, p. I4I. 1854: v. Boguslawski: Zehnter Nachtrag, Pogg. Ann. Ergz.-Bd.4, p.422. 1857: A rago: Astronomie populaire. Bd. 4, p. 192.

1859: H a rris: Dissert. Gött., p. $5^{8}$. r863: Buchner: Meteoriten, p. 5 .

1863: Ros e: Meteoriten, p. 155.

1863: Bo 11: Meteorstein bei Meno in Mecklenburg. Arch, der Freunde d. Naturgesch, in Mecklenburg (Neubrandenburg 1863) Bd. 17, p. 285.

r859/65: v. Reichen b a c h: IX I6I, I68, 178. XXV 433.

1866: B o 11: Meteorsteinfall bei Schellin in Pommern. Arch. der Freunde d. Naturgesch. in Mecklenburg Bd. 20, p. 76 ; s, auch Th. Schmidt: Ein Ausflug nach dem Saziger Kreise. Balt. Studien. Stud. XXI, p. 218 .

r885: Brezina: Wiener Sammlung, p. $181,232$.

r895: Brezin a : Wiener Sammlung, p. 247 .

Ursprüngliches Gewicht: Zwei Steine. Der grössere $1_{5}$ Pfund, der kleinere »wie ein Gänseei«; wenig erhalten. Im Jahr 1822 besass Freiherr v. Bredow auf Wagenitz ein Stück von $\mathrm{II}^{3} / 4$ Lot, von welchem Gilbert $4 \frac{1}{2}$ Lot erhielt; ausserdem müssen noch kleinere Stücke vorhanden gewesen sein, da nach gütiger Mitteilung von Freifrau v. Bredow auf Wagenitz bei Paulinenau, Provinz Brandenburg, das Gesamtgewicht des ge- 
genwärtig (Juli $\mathrm{r} 896$ ) in der Familie aufbewahrten Materials 34I gr. beträgt.

Nachweisbares Gewicht: $34^{8} \mathrm{gr}$.

Berlin, U.

v. Bredow

5 Tübingen Spl.

London, B. M. Spl.

Eine neue Untersuchung dieses Steins wäre erwünscht.

Schie

\section{Schobergrund}

Scholakoff

Schonen

Schönenberg, Pfaffenhausen, Burgau, Prov. Schwaben, Baiern, Deutschland.

Stein, Cwa, gefallen 25. Dez. 1846 . 1847 : Augsburger Allg. Zeitung vom I. Januar 1847 ; s, auch Pogg. Ann. Bd. 70 (1847), p. 334-335; Kenngott, Uebersicht $1844 / 49$, p. 283 ; sL'Institute Bd. I5 (1847) Nr. 686, p. 7 I.

r $847: \mathrm{S} \mathrm{c} \mathrm{h} \mathrm{a} \mathrm{f} \mathrm{h} \mathrm{ä} \mathrm{u} \mathrm{t} 1$ : Schönenberg,Gel. Anz. München. Akad. Bd. 24, p. 553 $-5^{8} 8_{3}$, insbesondere $565-570$ u. 580 , $5^{81}$; s. auch Liebig-Kopp, Jahresber. $1847 / 48$, p. 1308 .

1847: Landbeck: Bericht über das Niederfallen eines Meteorsteines. Jahreshefte d. Ver. f. vaterl, Naturk, Württemberg Bd. II, p. 383-386 (Abbildung des Steins, aber - nach den Gewichtsangaben zu urteilen - nicht in natürlicher Grösse, wie der Verfasser sagt, sondern höchstens in $1 / 2$ bis $1 / 3$ natürlicher Grösse); s, auch Plieninger, Jahresh. d. Ver. f. vaterl. Naturk. Württemberg Bd. II (1855), p. $454-455$.

1854: v.B o g u s l a w sk i: ZehnterNachtrag, Pogg. Ann. Ergz.-Bd. 4, p. 377 -378 .

I859: B u c h ne r: Feuermeteore, p. 98. 1859: H a rris : Dissert. Gött., p. 92. r863: Bu c h ner: Meteoriten, p. 75-76. 1860/65: v. Reichenbach: XIII $365 . X X V_{436}, 600,601,602,609,612$. 1878 : G ü m b e 1: Die in Bayern gefundenen Steinmeteoriten. - Der Meteorit von Schönenberg. Sitzber. München. Akad. 1878 I (Sep.), p. 40-46 (Analyse).

1879: Rammelsberg : Meteoriten, p. $13,25,26$.

1883: T s c hermak: Beitrag, Sitzber.

Wien. Akad. Bd. 88 I, p. 355 .

I884: W a d swor $\mathrm{t} \mathrm{h}$ : Studies, p. Io3. r885: Brezina: Wiener Sammlung,

p. $180,232$.

r89o: Br e z in a: Reise, Ann, Hof-Mus, Bd. 4 (Not.), p. 122.

r895: Brezina: Wiener Sammlung, p. 244 .

Ursprüngliches Gewicht: Die älteren Gewichtsangaben lauten 17 Pfund 5 Lot württembergisch oder 14 Pfund 17 Lot bayrisch. Gümbel sagt: 8015 gr.

Nachweisbares Gewicht: 7846 gr.

\begin{tabular}{lr|lr} 
v. Braun & 4 & Paris, M. & 41 \\
Brezina & 7 & v. Siemaschko & I \\
Eger & 79 & Stockholm & Spl, \\
Kunz & 17 & Stuttgart & 4 \\
London, B. M. & 42 & Wien, H. M.*) & I $\left.^{1}\right)$ \\
München & 7650 & &
\end{tabular}

Schuscha

Indarch

Schwetz a. d. Weichsel, Preussen, Deutschland.

Eisen, Om, gefd. 1850 , beschr. 1851 . r85I : R o se berichtet über eine neuerdings bei Schwetz aufgefundene Meteoritenmasse. Mon.-Ber. Berlin. Akad. (1851), p. 104-106, 369; s, auch Pogg. Ann. Bd. 83, p. 594-596; Zeitschr. d. d. geol. Ges. Bd. 3 (185r), p. 214215 ; sL'Institute 1851 , p. 208; Am.Journ. (2) $\mathbf{I}_{3}(1852)$, p. $142 ;$ Philos. Magaz. (4) I (18 51$)$, p. $517-518$; Froriep's Be-

I) Ausserdem 22 gr. nach 1 . Juli 1893 erworben. 
richte über d. Fortschr. d. Natur- u. Heilk, 1851, Nr. 321; Arch. ph, nat. Bd. 18 , p. 6r; Kenngott, Uebersicht $1850 / 51$, p. 137 ; Liebig-Kopp, Jahresber. 1851, p. 881 ; N. J. 1852 , p. $847-848$.

r85 I: Ram mels berg: Ueber das Meteoreisen von Schwetz an der Weichsel. Pogg. Ann. Bd. 84, p. $153-154$ (Analyse); s. auch Journ, prakt. Chem. Bd. 55 (1852), p. 60; Pharm. Centr. 1851, p. 947 ; Kenngott, Uebersicht 185ol 51, p. $137-{ }_{3} 8$; N. J. 1852 , p. 863 . r852: W ö h l e r : >Passiv«, Pogg. Ann. Bd. 85 , p. 448 .

1852 : C l a r k: Dissert. Gött., p.58-59. 1853: K a rsten: Ueber Feuer-Meteore und über einen merkwürdigen Meteormassen-Fall, der sich früher bei Thorn ereignet hat. Abh. Berlin. Akad, 1853 , p. 9 (sei von Schwetz verschieden).

1854: v. Boguslawski: Zehnter Nachtrag, Pogg. Ann, Ergz.-Bd, 4, p. 390,454 .

1859: B u c hn er: Feuermeteore, p. 127 -128 .

1859: H arri s: Dissert. Gött., p.rI8-II9. r860: $\mathrm{R}$ am mels berg: Mineralchemie, p. 905-906, 947,948 .

1863: Bu chner: Meteoriten, p. 182 $-188_{3}$.

r863: Rose: Meteoriten, p. 55, 59-6o, 139,152 .

1858.65 : v. Reichen b a c h: VI 448 , 452. VII 551. IX 163, 174, 181. X 359 , 365. XI 29I. XIl 457. XV nо, 114, 124, 128. XVI 250, 261, 262. XVII 266, 272. XVIII 484, 487. XIX 150, 152, 153. XX 621. XXI 587. XXV 437. r869: M e unier: Recherches, Ann. Chim. Phys. (4) 17, p. 72.

I875: vom R a th: Meteoriten, Verh. naturh.Ver. Bonn Bd 32, p. $362-363$. 1875: Mohr: Entstehungsart, Ann, Chem. Pharm. Bd, 179, p. 259, 270. 1879: Kle in: Göttinger Sammlung, Gött. Gel. Anz. (1879), p. 98. r884: M e un i er: Météorites, p. 96, $127,128$.

1885: Brezina: Wiener Sammlung, p. $200,2 \mathrm{II}, 234$.

1889: C o h e n: São Julião, N, J. 1889 I, p. 221, 224, 225.

r89r: Cohen u. Weinschenk: Meteoreisen-Studien. Ann. Hof-Mus. Bd.6, p. I46-147 (Analyse), I6o, I64165.

r892: Cohen: Meteoreisen-Studien. Ann. Hof-Mus. Bd. 7 , p. $148-149$ (Analyse), 159.

1893: Meunier: Revision des fers météoriques, p. $45,46-47$ (Abbildung einer geätzten Platte).

r894: C o h e n : Meteoreisen-Studien III. Ann. Hof-Mus. Bd. 9, p. III, II7, 118 (Rhabdit).

r894: Cohen: Meteoritenkunde, an vielen Stellen.

Ursprüngliches Gewicht: 21637 gr. (43 Pfund $8^{1 / 4}$ Lot).

Nachweisbares Gewicht: I 5923 gr.

Berlin, G. $\quad 107 \mid$ Madrid $\quad 152$

Berlin, P. $\quad$ II3 Marburg 93

Berlin, U. Io 178 Moskau 1 6

Bonn $\quad 54$ Münster 205

Budapest $\quad 656$ Neapel 82

Calcutta $\quad 157$ New Haven 256

Danzig $\quad 63$ Paris, M. 6I

Dorpat $\quad 162$ Rom, U. 119

Göttingen $\quad 303$ v. Siemaschko 23

Gregory $\quad 9 \mathrm{r}$ Stockholm 19

Harvard, U. $\quad 3 \mathrm{I}$ Strassburg 24

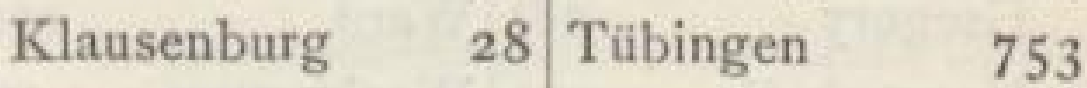

London, B. M. 1062 Washington Sh, Io

London, P. G. $\quad 162$ Wien, H. M.*) 843

\section{Schwiebus Seeläsgen}

Scottsville, Allen Co., Kentucky, U. S. A.

Eisen, H, gefd. 1867 , beschr. 1887 . 1887: Whitfield: On the Johnson County, Ark., and Allen County, Ky., 
Meteorites. Am. Journ. (3) 33, p. 500 - 501 (Analyse).

r889: v. H a u e r: Ann. Hof-Mus. Bd. 4 (Not.), p. 64 .

r889: C o h e n: São Julião, N. J. r889 I, p. $217,227$.

I889: $\mathrm{H}$ unt ingt o $\mathrm{n}$ : The Crystalline Structure of the Coahuila Irons. Proc. Amer. Acad. Arts and Sc. Bd. 24, p. 33. 1890: Eastman: Met. Astron., p. 320. 1890: F l et c her: Mexican Meteorites, Mineral. Maga7. Bd. 9, p. 106.

r89r: Cohen u. Weinschenk: Meteoreisen - Studien. Ann. Hof-Mus. Bd. 6 , p. 159.

r893: B r ez in a: Ueber neuere Meteoriten (Nürnberg), p. I66.

1893: Meunier: Revision des fers météoriques, p. 15, 19-20.

r894: Cohen: Meteoritenkunde, p. 96, 98, 156, 191, 199, 209.

r895: Brez in a: Wiener Sammlung, p. 291.

1895: $\mathrm{C}$ o h e $\mathrm{n}:$ Meteoreisen-Studien IV. Ann. Hof-Mus. Bd. 1o, p.82,85,88,89, 93 .

UrsprünglichesGewicht: Etwas über ro $\mathrm{Kgr}$.

Nachweisbares Gewicht: 677I gr.

\begin{tabular}{lr|lr} 
Bailey & I43 & London, B. M. & 410 \\
Bement & 532 & de Mauroy & 4 \\
Berlin, U. & 72 & New Haven & 76 \\
v. Braun & II2 & Newton & 10 \\
Brezina & 57 & Paris, M. & 50 \\
Budapest & 222 & Rom, U. & 39 \\
Greifswald & 52 & v. Siemaschko & 67 \\
Gregory & 281 & Ward & 1516 \\
Harvard, U. & 555 & Washington & 100 \\
Kopenhagen & 78 & Washington, Sh. 713 \\
Krantz & Iro & Wien, H. M. & 1572
\end{tabular}

Scriba, Oswego Co., New York, U. S. A.

Eisen, Dn (früher als Df bezeichnet), gefunden um 1835 , beschr, 1841. Die meteorischeNatur dieses Eisens ist fraglich.

r84r: S hepard: On Native and Meteoric Iron. - Native Iron from near Oswego, N. Y. Am. Journ. (I) 40, p. 366-369 (Analyse, welche neben sehr kleinen Mengen $\mathrm{SiO}_{2}, \mathrm{CaO}$ und $\mathrm{Al}_{2} \mathrm{O}_{3} \quad 99.68 \% \quad \mathrm{Fe}$ ergab; Abbildung). Ref. N. J. 184I, p. $698-699$; sL'Institut * Bd. 9 (184I), p. 45I-452. 1846: She pard: Report on Meteorites. Am. Journ. (2) 2, p. 382 .

1847: S h e p a r d : Report on Meteorites. 2. Scriba (Oswego), N. Y. Am. Journ. (2) 4 , p. 75 ; s, auch Liebig-Kopp, Jahresber. $1847 / 48$, p. 1308; Kenngott, Uebersicht $1850 / 51$, p. 135 .

1852: C l a r k: Dissert. Gött., p. 73. 1854: v. Boguslawski: Zehnter Nachtrag, Pogg. Ann. Ergz. - Bd. 4, p. $399-400$.

1859: B u c h ne r : Feuermeteore, p. r3I. 1859: H a rris: Dissert. Gött., p. ror. 1862: Greg: On some Meteorites in the British Museum. Philos. Magaz. Bd. 24, p. 541 (Qualitative Analyse von Heddle, welche unter anderen Elementen auch Nickel nachweist). Ref. Kenngott, Uebersicht 1862165, p. 269. I863: B u c h ner: Meteoriten,p.166-167. 1863: R o s e: Meteoriten, p. 24, 26.

r884: M e unier: Météorites, p. $3^{8}$, $93,97,131-132$.

r885: $\mathrm{Br}$ ezin a: Wiener Sammlung, p. 220,234 .

r89o: E a st m a n : Met. Astron, p. 318 . 1893: Meunier: Revision des fers météoriques, p. 72.

1894: Co hen: Meteoritenkunde, p. 52.

Ursprüngliches Gewicht: $3^{1 / 2} \mathrm{Kgr}$. (ungefähr 8 lbs.).

Nachweisbares Gewicht: I685 gr.

Bailey

Berlin, U.

Budapest

Calcutta

Cleveland

Dorpat
24 Göttingen $\quad 17$

42 Gregory 49

I6 Halle 3 I

117 Harvard, U. 486

27 London, B. M. I32

28 London, P. G. 13 
Moskau

New Haven

Paris, M.

Pohl

v. Siemaschko

Stockholm

28 Strassburg

I73 Tübingen

36

27 Washington

32

I64 Washingt

7 Wien, H. M.*) $8_{3}$ 83

Searsmont,Waido Co.,Maine,U.S.A. Stein, Cc, gefallen 21. Mai I87 I. 1871: Shepard: Notice of the Meteoric Stone of Searsmont, Maine. Am. Journ. (3) 2, p. 133-136. Ref. LiebigKopp, Jahresber. 1871, p. 1241-1242. r87r: $\mathrm{S} \mathrm{mith:} \mathrm{Mineralogical} \mathrm{and} \mathrm{Che-}$ mical Composition of the Meteoric Stone that fell near Searsmont, Maine, May 21, 1871. Am. Journ. (3) 2, p. 200 -2OI (Analyse); s. auch Original Researches 1884, p. $462-463$. Ref. LiebigKopp, Jahresber. 1871, p. 1242.

1876: S m i t h : Carbon compounds, Am. Journ. (3) II, p. $39 \mathrm{r}$.

r879: R a mmels berg: Meteoriten,p. 25. 1884: M e unier: Météorites, p. 230, $236-237,349$.

1885: Brezina: Wiener Sammlung, p. $185,233$.

r887: Flig h t: Meteorites, p. 47-48. r89o: E a stma n: Met. Astron., p. 3 I6. r894: Cohen: Meteoritenkunde, p. 153, 303 .

1894: F le t c h er: Introduction, p. 13. Ursprüngliches Gewicht: Ein 12 lbs. schwerer Stein, von welchem Shepard 900 gr. (2 lbs.) besass. Wahrscheinlich ist nur dies letztere Stück erhalten.

Nachweisbares Gewicht: 345 gr.

\begin{tabular}{lr|lr|} 
Bailey & 2 & Kopenhagen & 2 \\
Bernent & Spl. & Kunz & 3 \\
Budapest & 22 & London, B. M. & 5 I \\
Calcutta & 5 & London, P. G. & 8 \\
Cleveland & 2 & Madrid & 9 \\
Göttingen & II & Minneapolis & I \\
Gregory & 4 & New Haven & Io \\
Harvard, U. & 30 & Newton & I
\end{tabular}

New York, M. $\quad 4$ Upsala I

Paris, M. $\quad 36$ Ward Spl.

Petersburg, B. II Washington I6

Pohl 6 Washington, Sh. 62

v. Siemaschko II Wien, H. M.*)**) 30 Strassburg

s. Anhang

Seassport 7

Seeläsgen, Kreis Schwiebus, $\mathrm{Re}$ gierungsbezirk Frankfurt a. O., Brandenburg, Preussen, Deutschland.

Eisen, Ogg, gefunden 1847 .

1847: G ö p p e rt teilt der Akademie das Auffinden des 2 Ztr. schweren Meteoreisens mit. Verh. Berlin. Akad. (1847), p. 488 .

1847: Duflos: Meteormasse zu Seeläsgen in der Mark Brandenburg. Tageblatt d. Naturw. Vers. Breslau (1847), p. $49-50$; s. auch sL'Institut Bd. 17 (1849), Nr. 784, p. 15.

r847: G ö p p e r t: Ueber das Braunauer u. das Seeläsgen'sche Meteoreisen. Nach den Mittheilungen des Herrn Prof. Göppert über die Verhandlungen der naturwissenschaftl. Section der schlesischen vaterländischen Gesellschaft. Journ. prakt. Chem. Bd. 42 , p. $428-$ $43 \mathrm{I} ;$ s. auch Haidinger's Berichte d. Vers, d. Freunde d. Naturw. Wien, Bd. III (1847), p. 471, 472 , wo die Berichte aus der Breslauer Zeitung $\mathrm{Nr}$. 287 u. 288 vom 8. und 9. Dezember 1847 abgedruckt sind.

1848: P o g g e n d or $f f$ : Auffindung einer Meteoreisen-Masse in der Mark Brandenburg. - I. Aus einem Schreiben des Herrn Prof. Glocker-Breslau, 7. December 1847. - 2. Aus den Berichten der schlesischen Gesellschaft für vaterländische Kultur. Naturwissenschaftliche Section, 8. Dec. 1847 (Göppert's Bericht). - Zusätzliche Bemerkung vom Herausgeber. Pogg. Ann. Bd. 73, p. $329-332$; s, auch Am. Journ. (2) 6 (I848), p. 426 . 
1848: G lo c k er: Ueber die krystallinische Struktur des Eisens. Pogg. Ann. Bd. 73 , p. $332-336$ (s. auch Anmerkung von Fischer), p. 593.

r848: P a r s c h berichtet über das bei Seeläsgen, unweit Frankfurt an der Oder, gefundene Meteoreisen. Sitzber. Wien. Akad. Bd. I I, p. 153-156.

1848: S c hne id er: Ueber das Meteoreisen von Seeläsgen bei Schwiebus. Pogg. Ann. Bd. 74 , p. $57-6 \mathrm{r}$ (Abbildung). Ref. N. J. 1849, p. 86.

r848. Duf 1 os: Chemische Zerlegung der Meteoreisenmasse von Seeläsgen. Pogg. Ann. Bd. 74, p. 6r-65 (Analyse). Ref. N. J. 1848 , p. $808-809$; s. auch Ann. Chem. Pharm. Bd. 68 (1848), p. $259-261$.

1848: R a mmels berg: Ueber die chemische Zusammensetzung des Meteoreisens von Seeläsgen. Pogg. Ann. Bd. 74 , p. $443-448$ (Analyse des Schwefeleisens und des Phosphornickeleisens); s, auch Ann. Chem. Pharm. Bd. 68 (1848), p. 260 ; Burkart, Fundorte I, N. J. 1856 , p. 267 ; N. J. $185 \mathrm{I}$, p. $696-697$ u. 1852 , p. $2 \mathrm{II}-2 \mathrm{I} 4$; Kosmos Bd. III (1850), p. 6I4.

I847/48: Li e b ig-K o p p : Jahresber. p. 1304-1307 (Referate über die obigen Arbeiten); s. auch Pharm. Centr. 1848 , p. 143,428 .

1844/49: Kenngott: Uebersicht, p. 225-226 (Referate über die obigen Arbeiten).

r849: R a m m el s berg: Handwörterbuch, Suppl. 4, p. $152-154$.

1852 : C l a r k: Dissert. Gött., p. $49-52$.

I854: v. B o g us law ski: ZehnterNachtrag, Pogg. Ann. Ergz.-Bd. 4, p. 388 -389 .

1859: B u c hn e r : Feuermeteore, p. I24 $-126$.

1859: Harris: Dissert, Gött., p. Ir8. 1860: R a m m els berg: Mineralchemie, p. $904,947,948$ u, 2. Aufl. (1875), p. 53 . r862: R a mme ls berg: Ueber das Schwefeleisen der Meteoriten. Mon.Ber. Berlin. Akad. (1862), p. $689-69 \mathrm{r}$; s. auch Journ. prakt. Chem. Bd. 88 (1863), p. $275-277$.

1862: R o s e: Ueber den Asterismus der Krystalle, insbesondere des Glimmers u. des Meteoreisens. Pogg. Ann. Bd.In7, p. $634-635$; s. auch Mon.-Ber. Berlin. Akad. (1862), p. 617.

r 863 : B u c hn er: Meteoriten,p. $178-180$. r863: Ros e : Meteoriten, p. 39, 49$5^{1}$ (Taf. II), 56, 59, 138, 139, 152, 159 . r864: R a mmels berg: Ueber das Schwefeleisen der Meteoriten. Pogg. Ann. Bd. 121, p. $367-368$; s, auch Sitzber. Berlin. Akad, 1864, p.29; Journ. prakt. Chem. Bd. 91, p. 396; Chem. Centr. 1865 , p. 17; Kenngott, Uebersicht $1862 / 65$, p. 266 ; Liebig-Kopp, Jahresber. 1864, p. 904.

I $858 / 65$ : v. Re i c h e $\mathrm{n} \mathrm{b}$ a c h: IV 638 . VI 452 . VII 55 I. IX $162,174,175,18$ I. X 359. XII 456, 457. XIII 363. XV $100,110,112,122,124,125,128$. XVI 261. XVII $265,266,273$. XVIII 480 , $484,487,490$. XIX 150, 153. XX 62I, $625,627,628,629,631,633,634$. XXI $578,579,580,582,586,589$. XXV 437 .

1869: Meunier: Recherches, Ann. Chim. Phys. (4) 17, p. 68, 69.

r869: Buchner: Vierter Nachtrag, Pogg. Ann. Bd. 136, p. $43^{8}$.

r870: Ra m mels ber g : Ueber dasSchwefeleisen des Meteoreisens. Zeitschr. d. d. geol. Ges. Bd. 22 , p. $893-896$. Ref. Liebig - Kopp, Jahresber. 1870, p. 1395 .

1870: R a m mels berg : Meteoriten, p. $79,84,85$.

1872: Quenstedt: Klar u. Wahr. p. 294 (Abb. einer geätzten Platte). I875: v o m R a th: Meteoriten, Verh. naturh. Ver. Bonn Bd. 32, p. 357-358. 1875: Mohr: Entstehungsart, Ann. Chem. Pharm. Bd.179, p.259,266-267. 
1883: S m it h: Concretions, Am. Journ.

(3) 25, p. 417.

r884: M e un i er: Météorites, p. 44, 45, 94, 98, 99, rio-rir.

r885: Brezina: Wiener Sammlung, p. $207,216-217,234$.

1886: H u n ting t o n : Crystalline Structure, Am. Journ. (3) 32, p. 302.

1889: C o h e n : São Julião, N. J. 1889 I, p. $223,224,225$.

r892: Co h e n: Meteoreisen - Studien. Ann. Hof-Mus, Bd. 7, p. $158(\mathrm{Cu})$.

r893: Me unier: Revision des fers météoriques, p. $25,26-27$.

1894: C o h e n : Meteoreisen-Studien III. Ann. Hof-Mus. Bd. 9, p. 97, 99-102, 116, 117,118 (Rhabdit).

1894: Cohen: Meteoritenkunde, an zahlreichen Stellen.

r894: F l et c h e r: Introduction, p. 33. r895: Co he n: Meteoreisen-Studien IV. Ann. Hof-Mus. Bd, ro, p. $83,84,85$.

Ursprüngliches Gewicht: $\mathrm{IO}_{2} \mathrm{Kgr}$. (Schneider sagt $218^{\prime}$ Pfund).

Nachweisbares Gewicht: $67896 \mathrm{gr}$.

\begin{tabular}{lr|lr|} 
Bailey & 183 & Göttingen & 37 \\
Belgrad & 17 & Gregory & 197 \\
Bement & 761 & Greifswald & 32 \\
Berlin, G. & 1 & Halle & 257 \\
Berlin, P. & 51 & Hamburg & 182 \\
Berlin, U. & 4385 & Harvard, U. & 954 \\
Böhm & 32 & Howell & 68 \\
Bonn & 3544 & Karlsruhe, P. & 92 \\
v. Braun & 244 & Klausenburg & 163 \\
Breslau & 2010 & Kopenhagen & 341 \\
Brezina & 92 & Krantz & 880 \\
Budapest & 900 & Leipzig & 4796 \\
Calcutta & 2259 & Leoben & 15 \\
Cleveland & 174 & London,B.M. & 9846 \\
Dorpat & 128 & London, P. G. & 632 \\
Dresden, M. & 75 & Lüttich & 57 \\
Dresden, P. & 17 & Marburg & 145 \\
Edinburg & 87 & de Mauroy & 15 \\
Freiberg, i. S. & 3359 & Melion & 13 \\
Frenzel & 10 & Moskau & 58 \\
Giessen & 42 & München & 98
\end{tabular}

\begin{tabular}{lr|lr} 
Neapel & 202 & Stockholm & 201 \\
Neumann & 257 & Strassburg & 210 \\
New Haven & 174 & Stürtz & 133 \\
Newton & 3 & Stuttgart & 88 \\
New York, M. & 18 & Troyes & 74 \\
Paris, M. & 127 & Tübingen & 14919 \\
Pech & 123 & Turin, U. & 929 \\
Petersburg, A. & 1083 & Upsala & 231 \\
Petersburg, B. & 22 & Utrecht & 14 \\
Pohl & 3626 & Ward & 53 \\
Prag, B. U. & 121 & Washington & I04 \\
Prag, M. & 732 & Washington, Sh. 112 \\
Rom, U. & 126 & Wien, H. M.*) 6580 \\
v. Schilling & 29 & Wrany & 29 \\
v. Siemaschko & 302 & Zürich & 25
\end{tabular}

Herr Dr. Ulex in Hamburg besitzt ebenfalls ein Stïck dieses Fundortes.

Segowlee, Distr. Chumparun, Bengalen, Ostindien.

Stein, Ck, gefallen 6. März 1853 . 1854: S herwil1: (Kurzer Fallbericht an die Asiatic Society. Uebersendung eines kleinen Meteoriten). Journ. Asiatic Soc. Bengal (Proc.) Bd. 23, p. 746 -747 .

1855: G lover schenkt der Asiatic Society einen Meteoriten. Journ. Asiatic Soc. Bengal (Proc.) Bd. 24 , p. 247. r856: Grote übersendet einen Stein von I4 lbs. an die Asiatic Society, nebst kurzem Fallbericht von Dr. Evan Mc Donell. Journ. Asiatic Soc. Bengal (Proc.) Bd. 25, p. 169-170.

r859: At kinson: Verzeichnis der im Besitz der Asiatic Society befindlichen Meteoriten. Es werden unter Nr. 9, 10 u. II von Segowlee aufgeführt $7^{1 / 2} \mathrm{oz}$., I lb. $2 \frac{1 / 2}{2}$ oz. u. 14 lbs. Journ. Asiatic Soc. Bengal (Proc.) Bd. 28, p. 261.

r860: H a id ing er: Die Calcutta-Meteoriten, von Shalka, Futtehpore, Pegu, Assam und Segowlee im k. k. HofMineralien - Cabinete. - 4. Segowlee, 6. März 1853 . Sitzber. Wien. Akad. Bd. 4I, p. $754-758$ (Abbildung); s. auch Am. Journ. (2) 32, p. 143-144; Hai- 
dinger, Quarterly Journal of Geol. Soc. Bd. 17 (186r), (Miscellaneous), p. 8. Ref. Luminous Meteors, Rep. Brit. Assoc. 186r (Sep.), p. 34; >L'Institut " Bd. 29 (186r) Nr. 1409, p. 7.

r863: B u chner: Meteoriten, p. 85 . I863: Ros e: Meteoriten, p. I54. r865: v. Reichen bac h: XXV 427. r884: Me u n i er : Météorites, p. 96, I9I, I92.

1887: B rezina: Neue Meteoriten III, Sitzber. Wien. Akad, Bd. 84 I, p. 114 . r894: Flet ch er: Introduction, p. ro. 1895: Brezina: Wiener Sammlung, p. $260-26 \mathrm{r}$.

Ursprüngliches Gewicht: Etwa 30 Steine wurden gesammelt von $1 / 2$ lb. bis 14 lbs.

Nachweisbares Gewicht: 7099 gr.

\begin{tabular}{lr|lr} 
Bailey & I & London, P. G. & 20 \\
Berlin, U. & 6 & de Mauroy & 14 \\
Bologna & I & New Haven & 46 \\
v. Braun & I & Paris, M. & 9 \\
Budapest & 3 & v. Siemaschko & 10 \\
Calcutta & 4596 & Stockholm & 23 \\
Göttingen & 6 & Tübingen & 5 \\
Gregory & 10 & Ward & 6 \\
Harvard, U. & 125 & Wien, H. M.*) & 996 \\
London, B. M. 1206 & &
\end{tabular}

Semipalatinsk Jamyschewa

Sena (Sigena), Bezirk Sigena, Aragonien, Spanien.

Stein, Cgb, gefallen 17 . Nov. 1773 .

1774: Carta filosofica sobre un fenómeno etc. Zaragoza 1774 (Mitteilung von Herrn Direktor Brezina).

1803: Proust: Sur une pierre météorique tombée aux environs de Sigena, en Aragon, dans l'année 1773. Journ. Phys. Bd. 60 (an XIII, 1803), p. 185-205; s. auch Ann. Chim. Bd. 49 (an XIII, 1803), p. 186. - Freie Bearbeitung Gilbert's in Gilb. Ann. Bd. 24 (1806), p. 26r-292 unter dem Titel: Ueber einen Meteorstein, der im
J. 1773 unweit Sigena in Aragonien herabgefallen ist, und über die Meteorsteine überhaupt (Analyse von Proust). 1804: Proust: Análisis de una pietra meteórica caida en las immediaciones de Sigena (Aragón). Madrid I804.

1804: Gi 1 b e r t: Nachträge zu den Aufsätzen in den Annalen über die aus der Luft gefallenen Steine. Gilb. Ann. Bd. 18 , p. 290.

r812: Bigot de Morogues, p.II3 -117 .

1815: $\mathrm{Ch} 1$ a dni: Neues Verzeichnis, Gilb. Ann. Bd. 50, p. 250.

r8rg: C h 1 a d n i : Feuermeteore, p. 57, $66,69,74,253-254$ (1), 260, 432.

1836: K ä m tz: Meteorologie,p.258,275. 1843: P a rts c h: Meteoriten, p. 76. I854: B a $1 \mathrm{c}$ e $11 \mathrm{~s}$ : Lithologia meteorica, p. 2 I.

1857: A r a g o: Astronomie populaire. Bd. IV, p. 194 .

r859: H arris: Dissert. Gött., p. 60. 1863: B u c hner: Meteoriten, p. 9. 1865: Buchner: Zweiter Nachtrag, Pogg. Ann. Bd. 124, p. 575.

r858/65: v. Reichenbach: V 477. VII 552. IX 155, 162, 170, 179. X 359,363 . XXIV 226,228. XXV 322,430. r87r: Me unier: Coexistence de deux types lithologiques dans la même chute de météorites. C.R. Bd.73,p.1483-1485. r884: M eunier: Météorites, p. 95 , 188, 189 (Abb.), 273-274. 381, 466,521. 1885: Brezina: Wiener Sammlung, p. $155,182,183,233$.

1892: Gredilla: Meteoritos, p. Io3 -IO4.

1894: Cohen: Meteoritenkunde, p. 24, 185.

Ursprüngliches Gewicht: Ein Stein von 9 Pfund $x$ Unze (nach Gilbert's Auszug); 1900 gr. nach einer Pariser Etiquette (Meunier p. 275).

Nachweisbares Gewicht: 2045 gr. 
Bailey

Berlin, U.

Budapest

Dorpat

Madrid

Seneca Falls (Seneca River) bei Waterioo, Seneca Co., New-York, U. S. A.

Eisen, Om, gefd. $185^{\circ}$, beschr. $185^{2}$. [r85r: Shepard: On Meteorites. 5. Meteoric Stone of Waterloo, Seneca Co., N.Y.; fell in the summer of 1826 or 1827. Am. Journ. (2) II, P.39-40; s.auch Rammelsberg, Mon.-Ber. Berlin. Akad. 1861, p.899-900; v.Boguslawski, Zehnter Nachtrag, Pogg. Ann. Ergz.-Bd, 4 (1854), p. 25-26; Liebig-Kopp, Jahresber. $186 \mathrm{r}$, p. 1132 u. 1850 , p. 825 . Bezieht sich auf einenPseudometeoriten.] 1852: Root: On a mass of Meteoric Iron from near Seneca River. Am. Journ. (2) 14, p. 439-440; Ref. Liebig-Kopp, Jahresber. 1852 , p. 992 ; Kenngott, Uebersicht 1852 , p. 92 ; Edinburg Phil. Journ. Bd. 53, p. 245; "L'Institut $\propto$ Bd, 2 I (1853) Nr. 102I, p. 248. 1853: Shepard: Notice of the Meteoric Iron found near Seneca River, Cayuga Co., N. Y. Am. Journ. (2) 15 , p. $363-366$ (Analyse und Abbildung einer geätzten Platte); s. auch Pogg. Ann. Bd. 88 (1853), p. 176; Journ. prakt. Chem. Bd. 59 (1853), p. 406410 ; Liebig-Kopp, Jahresber. 1852, p. 992-993 ; N. J. 1853 , p. 694 ; Kenngott, Uebersicht 1852 , p. $92-93$.

1854: v. Boguslawski: Zehnter Nachtrag, Pogg. Ann. Ergz.-Bd. 4, p. $453-454$.

r859: Bu chner: Feuermeteore, p.r35. 1859: Harris: Dissert. Gött., p. 122. r859/62: v. Reichen b a c h: IX 162, 174, 181. XIV 390. XV 124, 126. XVI 261, 262. XVII 266, 272. XVIII 487 .
XIX $149,155,156 . X X 622,625,634$. XXI $588,5^{89}$.

1863: B u chner: Meteoriten, p. I86. I863: R o s e: Meteoriten, p. 6o, I52.

1884: M eunier: Météorites, p. 7o, I16, 122 .

1885: Brezina: Wiener Sammlung, p. 213,234 .

r89o: E a s t m a n : Met. Astron., p. 318. r89r: v. Ha uer: Ann. Hof-Mus. Bd. 6 (Not.), p. 54 .

r893: Meunier: Revision des fers. météoriques, p. 52, 55 .

1894: Coh en: Meteoritenkunde, p. 56, $58,76,91,122,245,246,248$.

r894: F let c her: Introduction, p. 33. r895: Brezina: Wiener Sammlung, p. 277 .

Ursprüngliches Gewicht: $4 \mathrm{Kgr}$. (9 lbs.).

Nachweisbares Gewicht: 2067 gr.

Berlin, U. $\quad 17 \mid$ London, P. G. 22

Budapest $\quad 88$ New Haven 30

Calcutta 126 Paris, M. 45

Clinton $\quad 450$ v. Siemaschko 16')

Dorpat $50 \mid$ Stockholm I2

Göttingen $\quad$ 120 Tübingen $\quad 59$

Gregory $\quad 54$ Upsala $\quad$ I4

Harvard, U. Io Washington, Sh. 8o

London, B. M. $\quad 54$ Wien, H. M.*) 820 Ausserdem befindet sich in dem Field Columbian Museum in Chicago ein Herrn Murray Guion gehöriges Stück Seneca Falls von $300 \mathrm{gr}$, welches unter den obigen Gewichten noch nicht aufgeführt sein dürfte.

\section{Seneca River}

Seneca Falls

Senegal (Siratik), Bambuk und Siratik, Westafrika.

Eisen, Ds. Fletcher stellt dieses Eisen unter die Siderolithe; schon Bigot de Morogues vergleicht es mit dem Pallaseisen. Nach Fletcher's

1) Gehört nach Brezina (Ann. Hof-Mus, Bd, Io, p. 277) wahrscheinlich zu Ruffs Mountain. 
Beschreibung überwiegt das Eisen aber in sehr hohem Masse und die Olivine sind fast mikroskopisch ausgebildet. Sollten mehrere Glieder dieser Art gefunden werden, so würde man wohl eine eigene Gruppe bilden müssen (cfr. Tucson). Bekannt I 7 16 (nach Fletcher), erwähnt $\mathbf{I} 748$.

1748: C om pagnon in Schwabe's: Allgemeine Historie der Reisen zu Wasser und Lande. Leipzig 1748. Bd. 2 Buch 5 Kap. I3 $_{3}$ p. 5 Io (nach Fletcher). Clark sagt: Compagnon: Allgemeine Reisen zuWasser und zu Lande, von Ehrmann in Frankfurt a. M. I79I Bd. 2, p. 5 IO.

I748: W a 11 e rius: Systema Mineralogicum Bd. 2, p. 233.

r78r/83: Forster u. Sprengel: Kurze Nachrichten über den Zustand von Senegal u. s. w. aus dem mündlichen Berichte des Herrn Doktor Schott. Beyträge zur Völker- u. Länderkunde. I. T., p. 61-62 (Leipzig $1781)$ u. 3. T. $\left(1_{7} 8_{3}\right)$, p. $138-139$.

1783: Romé de l'Is le: Cristallographie. 2. Aufl. Bd. 3, p. $165-167$.

1802: Golberry: Fragments d'un voyage en Afrique (Paris 1802 ) Bd. I Kap. 7, p. 291 (die Stelle ist abgedruckt bei Chladni s. u.).

1802: $\mathrm{H}$ ow a $\mathrm{rd}$ : Experiments and $\mathrm{Ob}-$ servations on certain stony and metalline Substances, which at different Times are said to have fallen on the Earth; also on various Kinds of native Iron. - Description of various Kinds of native Iron. By the Count de Bournon. Examination of Iron from Senegal, brought by General O'Hara, and given to me by Mr. Hatchett. Philos. Trans. (1802), p. 9II-9I2 (Analyse); s, auch Fourcroy's Uebersetzung in Encyclopédie « Bd. 5 (1808), p. 558, 559.

1803: de Dré e: Recherches, Journ.
Phys. Bd. 56 (an XI), p. $4 \mathrm{I} 7$.

1803: I z a r n : Lithologie, p. 224,231,232. r8o3: K la proth: Ueber meteorische Stein- und Metall-Massen. Abh. Berlin. Akad. (1803), p. $35-36$.

1804: v. Ende: Massen und Steine, p. $72-73$.

r804: Pötzsch: Kurze Darstellung, p. $34-36,93$.

I8I2: Bigot de Morog ues, p. 301 $-302,339-340$.

1812: Chla dn i: Verzeichnis, Schweigg. Journ. Bd. 4, Beil. I, p. 17.

r8r5: Ch la dni: Bemerkungen, Gilb. Ann. Bd. 50, p. $271-272$.

1819: $\mathrm{Ch}$ la dni: Vierte Fortsetzung, Gilb. Ann. Bd. 60 , p. $24 \mathrm{I}-242$.

r8rg: $\mathrm{Ch} 1$ a d ni: Feuermeteore, p. 318, 333-336; s. auch Denkschr. München. Akad. 1812, p. 106.

r836: K ämtz: Meteorologie, p. 260. r843: P a r t s c h: Meteoriten, p.r3O-r3I. 1852: C 1 a r k : Dissert. Gött., p. 37-38. r852: W öhler: sAktiv «, Pogg. Ann. Bd. 85, p. 448 .

1854: v. B og us lawski: Zehnter Nachtrag, Pogg. Ann. Ergz-Bd. 4, p. 398. 1855: v. $\mathrm{H}$ a id inger: Bemerkungen über die zuweilen im geschmeidigen Eisen entstandene krystallinische Structur, verglichen mit jener des Meteoreisens. Sitzber. Wien. Akad. Bd. 15 I, p. $35^{8}$.

r859: B u c h n e r : Feuermeteore, p. 127. 1859: Harris: Dissert. Gött., p. IO2. 1858/62: v. Rei c hen ba c h: VI 447 , 448 . VII 552,557 . VIII 486 . IX 162 , $175,176,182$. XI 291. XII 457. XIII 354,364 . XIV $390,393 . \quad X V$ roo. XVIII 483, 490, 49I. XIX 150, 155. XXI 578,587 .

r863: Bu chner: Meteoriten, p. I36. 1863: Rose: Meteoriten, p. 30, 69$70,153$.

I 870: $\mathrm{R}$ ammels berg: Meteoriten, $\mathrm{p}$. 79,8 o.

1884: M e un i e r: Météorites, p. 37, 137. 
1885: Brezin a : Wiener Sammlung, p. $220,234$.

r893: Meunier: Revision des fers météoriques, p. $69-70$.

1894: C o he n: Meteoritenkunde, p. 7, 62,67 .

r895: Brezina: Wiener Sammlung, p. 295 .

Ursprüngliches Gewicht: Grosse Massen. Forster u. Sprengel sagen in ihren »Beyträgen «, dass Herr D. Schott ein Stück von 30 Pfund besessen habe, welches aber nicht nach Europa gelangt sei.

Nachweisbares Gewicht: $1710 \mathrm{gr}$.

\begin{tabular}{lr|lr} 
Bailey & 68 & London, B. M. & 396 \\
Bement & 197 & München & 43 \\
Berlin, U. & 73 & Neumann & 2 \\
Budapest & I & Paris, M. & 4 I \\
Calcutta & $\left.20^{1}\right)$ & v. Siemaschko & 10 \\
Göttingen & I & Tübingen & 24 I \\
Gregory & 103 & Wien, H. M.*) & 49 I \\
Harvard, U. & 23 & &
\end{tabular}

Senhadja, Aumale, Constantine, Algier, Nordafrika.

Stein, Cwa, gefallen 25. August 1865 . 1866: D a u bré e: Météorites tombées le 25 aotat 1865 dans la tribu des Senhadja, cercle d'Aumale, province d'Alger ; fer météorique signalé à Dellys. C. R. Bd. 62 , p. $72-78$; s. auch Am. Journ. (2) 4r, p. 426 ; LiebigKopp, Jahresber. 1866, p. 1007 ; „L'Institut * Bd. 34 (1866), Nro, 1671, p. 9. 1869: Buchner: Vierter Nachtrag, Pogg. Ann. Bd, 136, p. $456-457$.

1877: G ü $\mathrm{m} \mathrm{bel}$ : Ueber die Beschaffenheit des Steinmeteoriten vom Fall am 12. Februar 1875 in der Grafschaft Jowa N. A. Sitzber. München. Akad. Bd. 5 , p. 314 .
1884: M e unier: Météorites, p. 197, 204-206, 460 .

r85: Brezina: Wiener Sammlung, p. 177.232.

1890: B r ez in a : Reise, Ann. Hof-Mus. Bd, 4 (Not.), p. II9.

r894: C o h e n: Meteoritenkunde, p. 208, $237,245,246,267$.

r895: Brezina: Wiener Sammlung, p. 244 .

UrsprünglichesGewicht:ZweiSteine von etwa $50 \mathrm{Kgr}$., jeder etwa 25 Kgr. schwer, welche z. T. zu Amuletten verarbeitet wurden.

Nachweisbares Gewicht: Io 334 gr.

Bailey

Bement

Göttingen

Gregory

Harvard, U.

I Paris, M. 992 I

I Rom, U. 42

2 v. Siemaschko 8

25 Stockholm II9

London, B. M.

London, P. G.

20 Upsala

9 Ward 18

Madrid

I Washington

27 Wien, H. M.*) 23

Seres, Macedonien, Türkei.

Stein, $\mathrm{Cg}$, gefallen Juni (?) $18 \mathrm{r} 8$.

(?) r8rg: Chla d n i: Feuermeteore, p. 167 (wenn das hier angegebene Meteor mit dem Stein von Seres in Zusammenhang steht, so müsste der Fall am 3 I. Oktober 1818 stattgefunden haben); s. auch Stedler: Ueber die in Ungarn herabgefallenen Meteorsteine. Oesterr. B1. f. Lit. 1847 Nr. 86, p. 343 . Ref. N. J. 1848 , p. 65 .

1828: Berzelius: Undersökning af en Meteorsten. K. Vetensk. Acad.Handl. $(1828)$, p. $156-163$ (Analyse); übersetzt in Pogg. Ann. Bd. 16 (1829), p. $6 \mathrm{II}-6 \mathrm{r} 8$. Ref. Leonhard's Jahrb. 1830 , p. $309-3$ Io.

I830: v. H o ff: Siebenter Nachtrag, Pogg. Ann. Bd, 18, p. 190-191 (Ref. der Arbeit von Berzelius); s. auch

I) Davon 19 gr. s? Senegal, Africa. Formerly in W. G. Lettsom's collection, said to have been brought from Senegal«.

W ü I fing, Meteoriten. 
Berzelius: Ueber Meteorsteine. Pogg. Ann. Bd. 33 (1834), p. 25-26.

1832: v. S cherer: Abbildung des 15

Pfund schweren Meteorsteines von Seres in Macedonien, gefallen im Juni 1818 (5 Tafeln). Wien, Druck von Anton Schweiger.

I835: v. H off: Neunter Nachtrag, Pogg. Ann. Bd. 34 , p. $340-34 \mathrm{I}$ (Hinweis auf Chladni, s. o.); s, auch: >Isis von Oken 1833 , Heft $4-6$, p. 479.

1836: Kämtz: Meteorologie, p. 252, 256, 287 .

r843: P a r s c h: Meteoriten, p.75-76 (giebt als Fallzeit „Juni $1818 \propto$ an, wahrscheinlich den v. Scherer'schen Tafeln entnommen, auf welchen >VI I8I8* geschrieben ist).

r847: S ch a fhä ut 1: Schönenberg,

Gel.Anz. München. Akad. Bd.24, p.573. 1853: Rammels berg: Suppl. V, p. $27-28$.

1854: v. Boguslawski: Zehnter

Nachtrag, Pogg. Ann. Ergz.-Bd. 4, p. 16. 1859: B u c hn er: Feuermeteore, p. 84. 1859: H a r r is: Dissert. Gött., p. 74.

1860: $\mathrm{R}$ a m mel s ber $\mathrm{g}$ : Mineralchemie, p. 922,926 ff., 950.

r863: B u c h n e r : Meteoriten,p.38-39. 1863: R os e: Meteoriten, p. 87, 92, 154. r857/65: v. Re i chen bach: III 624 . V 476, 48r. VI 44I, 454. IX I62, 17I, 180. X 359. XI 294, 295, 297, 301, 302. XII 454. XIII 355,362 . XIV 399. XV 121. XVI 262. XXIV 229. XXV 429,607 .

1867: Goe be 1: Kritische Uebersicht, Mélanges phys. chim. Bd, 7, p. $33^{8}$.

r870: R a mmels berg : Meteoriten, p. $98,105,106,139,140$.

1875: Ts chermak: Vulkanismus, Sitzber. Wien. Akad. Bd, 71 II, p. 662. 1879: R a mmels berg: Meteoriten, p. 25,102 .

r884: M e unier: Météorites, p. 85, 273, $274-275$.

1883/85: Ts chermak: Photogra- phien, T. 8,9, II, p. 12, 13, 14, 16. 1885: B rez in a : Wiener Sammlung, p. $182,232$.

r894: F let cher: Introduction, p. 36. Ursprüngliches Gewicht: $8.4 \mathrm{Kgr}$.

(I5 Pfund).

Nachweisbares Gewicht: 7287 gr.

Bailey

Belgrad

Berlin, U.

Budapest

Calcutta

Cohen

Gent

Göttingen

Gregory

Harvard, U.

I London, B. M. 400

2 London, P. G. 77

48 Moskau $\quad 46$

Io Paris, M. 2

Io Petersburg, A. 5

Spl. Pohl 2

50 Stockholm 4

64 Tübingen 61

1 Wien, H. M.*) 6485

Serrania de Varas

Varas

Setif

Sevier Eisen

Tadjera

Sevilla, Andalusien, Spanien.

Stein, Cho, gefallen r. Okt. 1862 .

1865: Buchner: Zweiter Nachtrag, Pogg. Ann. Bd. 124, p. 591.

1884: Meunier: Météorites, p. 230 (stellt den Stein zu seiner Gruppe der Montréjite).

1885: Brezina: Wiener Sammlung, p. 268 .

r89o: B r e z in a : Reise, Ann. Hof-Mus.

Bd. 4 (Not.), p. II9.

r892: v. H a u e r: Ann, Hof-Mus, Bd, 7 (Not.), p. 73.

1892: Gr e dill a: Meteoritos,p.II-II2. r895: Brezina: Wiener Sammlung, p. $24 \mathrm{I}$.

Ursprüngliches Gewicht: ?

Nachweisbares Gewicht: 95 gr.

Madrid $\quad 92$ v. Siemaschko Spl.

Paris, M. $\quad 3$ Wien, H. M. Spl.

Der Stein scheint noch nicht analysiert worden $z u$ sein.

Sewrukof, Bezirk Belgorod,Gouvernement Kursk, Russland. 
Stein, Cs, gefallen 1 I. Mai 1874 . In der Nacht vom 29. April (I I. Mai neuen Stils) auf den 30 . April, ungefähr um Mitternacht.

1874: Moskauer Nachrichten (Wedomosti 31. Mai 1874 Nr. 135).

1875: D a u bré e: Chute d'une météorite survenue le I2 mai ( 30 avril, vieux style) 1874 , à Sevrukow, district de Belgorod, gouvernement de Koursk. C. R. Bd. 81 , p. $661-663$; s, auch Liebig-Kopp, Jahresber. 1875, p. 13II; Wochenschr. f. Astr., Met. u. Geogr. 1876, p.30-31; N Naturforscher $(1875)$, p. 486 .

1882: Brezina: Bericht IV, Sitzber. Wien. Akad. Bd. 85 I, p. 338 (Vergleich mit Mócs, s. d.).

1882: E berhard: Der Meteorit von Sewrjukowo, gefallen am 12. Mai (30. April) 1874. Arch. f. d. Naturk. Liv. Ehst, u. Kurl. Bd. 9 (Sep.), 27 Seiten (Abbildung u. Analyse). Ref. N. J. I884 I, p. 30-3I; Liebig-Kopp, Jahresber. 1884, p. 2040.

1884: M e u n i e r: Météorites,p.242,246. 1885: Brezina: Wiener Sammlung, p. $184,233$.

1890: B r z z i na : Reise, Ann. Hof-Mus. Bd. 4 (Not.), p. rig.

189r: $\mathrm{K}$ unz u. Weinschenk: Meteoritenstudien. M. P. M. Bd. 12, p. 8 (Vergleichung mit Farmington).

1894: Co hen: Meteoritenkunde,p. 246. 1895: Brezina: Wiener Sammlung, p. 253.

Ursprüngliches Gewicht: Ein Stein von $98 \mathrm{Kgr}$.

Nachweisbares Gewicht: $79^{2}$ gr.

Bailey

Budapest

Dorpat

Gregory

London, B. M.

Moskau

Odessa

\begin{tabular}{r|lr|}
5 & Paris, M. & 365 \\
63 & Petersburg, B. & 13 \\
96 & v. Siemaschko & 12 \\
3 & Stockholm & 36 \\
20 & Ward & 33 \\
8 & Wien, H. M.**) & 30 \\
8 & &
\end{tabular}

Befindet sich die grösste Masse des Steins noch im Museum in Charkow?

Shalka bei Bissempore in Bancoorah, Bengalen, Ostindien.

Stein, Chl, gefallen 30 . Nov. 1850 .

185r: $\mathrm{P}$ i d d ing t on : Examination and Analysis of the Shalka Meteorite (Zillah West Burdwan). Ausführlicher Fallbericht von Herrn Mactier. Journ. Asiat. Soc. Bengal. Bd. 20, p. 299-307. II. Mineralogical and Chemical Examination, p. $308-3_{13}$ (Analyse). Appendix, in welchem der Fragebogen Piddington's abgedruckt ist, p. 3133 I 4 .

1852: B a den-Pow e 11: Luminous Meteors, Rep. Brit. Assoc. (1852), p. I, 47. Ref. Liebig-Kopp, Jahresber. 1852, p. $987-988$; Kenngott, Uebersicht 1852 , p. 151 .

r854: v.B og u s l a w s k i: ZehnterNachtrag, Pogg. Ann. Ergz-Bd. 4, p. 382 .

1859: Harris: Dissert. Gött., p. 94.

1859: A $\mathrm{t} \mathrm{k}$ ins o $\mathrm{n}$ : In einem Verzeichnis der im Besitz der Asiatic Society befindlichen Meteoriten werden mehrere grosse u. kleine Stücke im Gesamtgewicht von 9 lbs, erwähnt. Journ. Asiat. Soc. Bengal. (Proc.) Bd. 28 , p. 260. 1859: B u c hn er: Feuermeteore, p.roo. r $60:$ Ha iding er: Der Meteorit von Shalka in Bancoorah und der Piddingtonit. Sitzber. Wien. Akad, Bd, 4I, p. 251 -26o, 745 (Analyse von v. Hauer); s. auch Am. Journ. (2) 32 (I86r), p. I4I-I42; Jahrb. k. k. geol. Reichsanst. 1860 (Verh.), p. 104; Luminous Meteors, Rep. Brit. Assoc. I86r (Sep.), p. 34; Liebig-Kopp, Jahresber. 1860, p. 848-849; Chem. Centr. I860, p. $835-838$; s L'Institute Bd. 28 (I860), Nr. 1391, p. $286-287$.

1862: $\mathrm{M}$ a s k el y ne: Chondritic Aërolites. Rep. Brit. Assoc. (Not. and Abstr.) (1862), p. 190.

r863: Bu chner: Meteoriten, p. 80. $2 \mathrm{I} *$ 
1863: Rose: Meteoriten, p. 28, 122 $-125,156$.

r86r/65: v. Reichen b a ch: XV ror. XX 63т. XXV 427, 607, 615.

1867: Go e b e 1: Kritische Uebersicht, Mélanges phys. chim. Bd. 7, p. $33^{8}$. 1870: R a mmelsberg: Ueber die Zusammensetzung der Meteorite von Shalka und von Hainholz. - I. Der Meteorit von Shalka. Mon.-Ber. Berlin. Akad. (187o), p. 316-322, 325-326; s. auch Pogg. Ann. Bd. I4I (1870), p. $277-283$; Chemical News $\approx$ Bd. 22 (1870), p. 95.

1870: Rammelsberg: Meteoriten, p. 106, II9-120, 139; s, auch Pogg. Ann. Bd. 140 (187o), p. 312 ; Ber. d. d. chem. Ges. Bd. 3 (1870), p. 522 .

r87 I: $\mathrm{M}$ as kelyne: On the Mineral Constituents of Meteorites. - XVI. The Shalka Aërolite. Philos. Trans. Bd. 16r, p. $366-367$ (Analyse); s. auch Proc. Royal Soc. Bd. 19, 1870/71, p. 266-268; Liebig-Kopp, Jahresber. 1871 , p. 1238.

1872: Tschermak: Die Meteoriten von Shergotty und Gopalpur. Sitzber. Wien. Akad. Bd. 65 I, p. I3I.

r879: $\mathrm{R}$ a m mels berg: Meteoriten, p. $15,24,25$.

1883: T s c hermak: Beitrag, Sitzber.

Wien. Akad. Bd. 88 I, p. $362-363,365$. r884: Meunier: Météorites, p. 72, 79,

8o, 85, 93, 95, 96, 298 (Abb.), 299, 300,395 .

1884: W a d s w or th: Studies, p. 202. 1883/85: T s c h e r m a k: Photographien, T. 6, p. $3,5,10$.

r885: Brezina: Wiener Sammlung, p. $174,232$.

1887: Flight: Meteorites, p. II5-II6. r888: v. F o u 11 o $\mathrm{n}$ : Untersuchung der Meteorsteine von Shalka und Manbhoom. - Der Meteorit von Shalka. Ann. Hof-Mus. Bd. 3, p. 195-201 (Neue Analyse). Ref. N. J. 1889 II, p. $279-281$.
1894: C o h e n : Meteoritenkunde, p. 49, $245,246,249,272,281,283,287$. 1894: F l e t c h e r: Introduction, p. ro. r895: Brezina: Wiener Sammlung, p. 239 .

UrsprünglichesGewicht: Ein grosser Stein, welcher 3 Fuss (?) lang gewesen sein soll. Im Verhältnis hierzu wenig erhalten.

Nachweisbares Gewicht: 3626 gr.

\begin{tabular}{lrr|r} 
Bement & I & London, P. G. & 53 \\
Berlin, U. & 79 & Moskau & 5 \\
Bologna & 2 & München & 24 \\
Budapest & 4 & Neumann & 3 \\
Calcutta & I922 & New Haven & I \\
Cambridge & 2 & Paris, M. & 8 \\
Cleveland & I & Petersburg, A. & 16 \\
Dorpat & 2 & Pohl & II \\
Göttingen & I & Roebling & 6 \\
Gregory & 53 & v. Siemaschko & 9 \\
Harvard, U. & 3 & Stockholm & 33 \\
Heidelberg & 2 & Troyes & Spl. \\
Klausenburg & I & Tübingen & 10 \\
Kristiania & 43 & Wien, H. M.*) & 199 \\
London, B. M. II32 & &
\end{tabular}

Shergotty (Umjhiawar), Behar, Bengalen, Ostindien.

Stein, She, gefallen 25. August 1865 . 1865: B a y ley u. Cost ley: Briefl. Mitt. Proc. Asiat. Soc. Bengal. (1865), p. 193-195.

r866: $\mathrm{O} 1 \mathrm{~d}$ h a m sagt im Catalogue etc. von Calcutta, p. 8, dass die Asiatic Soc. einen Stein von 1o lbs. $6 \mathrm{oz}$. I2I grs, besitze.

1868: Crook: Dissert. Gött.: On the Chemical Constitution of the Ensisheim, Mauerkirchen, Shergotty and Muddoor Meteoric Stones. - The Shergotty Stone, p. 30-33 (Analyse). [Ist nicht Shergotty, s. u. 1871 Tschermak]. r869: Buchner: Vierter Nachtrag, Pogg. Ann. Bd. 136, p. 457. r870: R a m me ls berg : Meteoriten, p. 103, 106, II9-120, 138, 139, 140. 
1871: L u m p e: Analysen aus dem Laboratorium des Herrn Prof. E. Ludwig. M. M. $(187 \mathrm{I})$, p. $55-56$. Ref. LiebigKopp, Jahresber, 1871, p. 1238.

187 I : T s c hermak: Der Meteoritvon Shergotty. M. M. (1871), p. 56 ; s, auch Rammelsberg, Mineralchemie 2. Aufl. (1875), p. 698-699.

r87 I : L u d w ig : Der Meteorit von Shergotty. Jahrb. geol. Reichsanst. Bd, 2 I (Not.), p. $56-57$.

1872: Tschermak: Die Meteoriten von Shergotty und Gopalpur. - Shergotty. Sitzber. Wien. Akad. Bd, 65 I, p. 122--135 (Mit Tafel; Abbildung mikroskopischer Präparate). Ref. N. J. 1872, p. 733-734; Am. Journ. (3) 4 (1872), p. 78 .

1872: Ts chermak: Die Meteoriten von Stannern, Constantinopel, Shergotty u. Gopalpur. - Shergotty. M. M. (1872), p. 87-95 (Analyse von Ludwig s. o.). Ref. Liebig-Kopp, Jahresber. 1872 , p. II97- II98.

r879: R a m mels berg : Meteoriten, p. $16-20,24,25$.

1883: T s c h e r m a k: Beitrag, Sitzber.

Wien. Akad. Bd. 88 I, p. $355,370$. r884: M e un ier: Météorites, p. 69, 74, $85,295,297-298,395$.

r884: W a d s w ort h: Studies, p. 197 -198 .

1883/85: Tschermak: Photographien, T. 3 , p. $3,4,7$.

1885: Brezina: Wiener Sammlung, p. $165,174,232$.

1887: F l ig h t: Meteorites, p. 142-144. r894: Cohen: Meteoritenkunde, an vielen Stellen.

r894: F let c h er: Introduction, p. I3, 37.

Ursprüngliches Gewicht: Ein Stein von $4 \frac{1}{2} \mathrm{Kgr}$. (ro lbs. 6 oz. $)^{1}$ ). Nachweisbares Gewicht: 4897 gr.
Bailey

Budapest

Calcutta

Cambridge

Cleveland

Göttingen

London, B. M.

7 London, P. G. 26

7 München 5

4436 Paris, M. 91

1 Roebling 7

7 v. Siemaschko I

7 Wien, H. M.*) $\quad 183$

Shingle Springs, El Dorado Co., Californien.

Eisen, Hch, gefunden $1869 / 70$, beschr. 1872 .

Hierher auch: Los Angeles, Californ.

1872: S hepard: On a Meteoric Iron lately found in El Dorado county, California. Am. Journ. (3) 3, p. 438 (Analyse). Ref, Liebig-Kopp, Jahresber. I872, p. 1199 .

1872: Jackson: Analysis of the Meteoric Iron of Los Angeles, California. Am. Journ. (3) 4, P. 495-496 (Analyse). 1873: Silliman: On the Meteoric Iron found near Shingle Springs, Eldorado county, California. Am. Journ. (3) 6, p. 18-22 (Analyse und Abbildung). Ref. Liebig-Kopp, Jahresber. 1873, p. 1251 .

r876: Wright: On the Gases contained in Meteorites. Am. Journ. (I) II, p. 256,257 u. (3) $12(1876)$, p. 167. 1885: Brezina: Wiener Sammlung, p. 201.

I887: F l ig h t: Meteorites, p.12-13,63. I888: v. Hauer: Ann. Hof-Mus. Bd, 3

(Not.), p. 43.

r89o: Eas tma n: Met. Astron., p. 320, 322.

1893: Brezin a: Ueber neuere Meteoriten (Nürnberg), p. $\mathbf{1 6 6 .}$

r893: M.e u n i er: Revision des fers météoriques, p. 75 .

1894: Co hen: Meteoritenkunde, p 57, 109, $110,127,156,173,174$.

1894: F l et c her: Introduction, p. 13.

I) Nach anderen Angaben (Proc. Asiatic Soc. 1865) II lbs, 2 oz. 368 grs. 
1895: Brezina: Wiener Sammlung, p. 294 .

Ursprüngliches Gewicht: $3^{8 / 2} \mathrm{Kgr}$. (85 lbs.).

Nachweisbares Gewicht: 1650 gr.

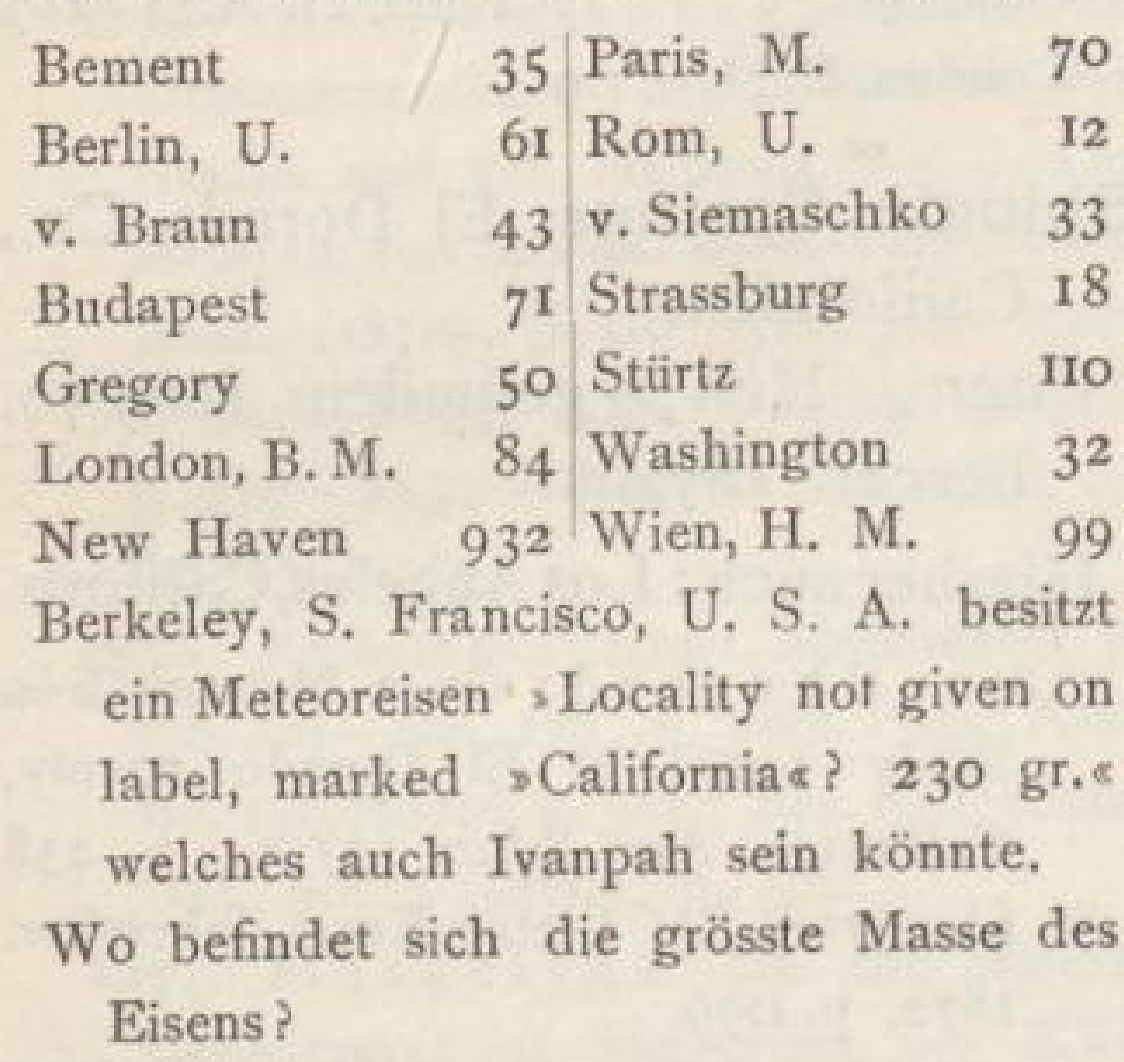

Shropshire

Rowton

Shytal, Dacca, Bengalen, Ostindien.

Stein, Cib, gefallen I1. August 1863 .

r863: $\mathrm{H}$ a i dinger: Der Fall eines Meteoriten bei Dacca in Bengalen am II. August I863. Sitzber. Wien. Akad. Bd. 48 II, p. 595-600 (Fallbericht nach dem Calcutta-Zeitungsblatt „Phönix « vom Donnerstag den 24. September 1863. Zwei Abbildungen des Steins); s. auch Pogg. Ann. Bd. I2O (1863), p. 659-66o; Liebig-Kopp, Jahresber. I863, p. 909; Kenngott, Uebersicht 1862/65, p. 440, 444; >L'Institut « Bd. 32 (1864), Nro. 1574 , p. 70 ; N. J. 1864, p. 237. Ref. Luminous Meteors, Rep. Brit. Assoc. 1864 (Sep.), p. 97. In Oldham's Catalog von Calcutta 1867, p. 8 ist das damals noch im Besitz der Asiat. Soc. Bengal befindliche Stück mit 5 lbs, I oz. 3 r 3 grs, aufgeführt. 1864: Buchner: Erster Nachtrag, Pogg. Ann. Bd. 122, p. 326.

1865: Buchner: Zweiter Nachtrag, Pogg. Ann. Bd. 124, p. $5^{85}$.

1865: Pıoc. Asiat. Soc. Bengal (1865), p. 39 (Vorzeigen eines Modells).
1866: He in: Analyse eines Meteoriten von Dacca in Bengalen. Sitzber. Wien. Akad. Bd. 54 II, p. 558-561; s. auch Chem. Centr. 1867 , p. 35I ; s'Institut " Bd. 35 (1867), p. 62 ; Liebig-Kopp, Jahresber. 1866, p. 1010.

r869: Buchner: Vierter Nachtrag, Pogg. Ann. Bd. 136, p. 448.

1884: Meunier: Météorites, p. 247. r883/85: Tsch e r mak: Photographien, p. 3 .

1885: Brezina: Wiener Sammlung, p. $182,232$.

r894: F le t c her: Introduction, p. I3. Ursprüngliches Gewicht: Calcutta und London dürften die Hauptmasse besitzen.

Nachweisbares Gewicht: 3188 gr.

\begin{tabular}{lr|lr} 
Calcutta & 2473 & London, P. G. & 6 \\
Göttingen & 3 & Paris, M. & 4 \\
Gregory & 8 & v. Siemaschko & 4 \\
Harvard, U. & 2 & Washington, Sh. & I \\
London, B. M. & 463 & Wien, H. M.*) & 224
\end{tabular}

Sibirien (Krantz) Werchne Udinsk

Siena, Cosona, Pienza, San Giovann d'Asso, Toscana, Italien.

Stein, Cho, gefallen 16. Juni 1794.

r794: Domen ico Tata: Memoria sulla pioggia di pietre avvenuta nella Campagna Sanese, il di 16. di Giugno di questo corrente anno (74 Seiten), Napoli 1794 bei Aniello Nobile e Comp.; s. auch Antologia Romana Bd, 21, p. 94 (oder 97 ?); Auszug von Leopold v. Buch in Gilb. Ann. Bd. 6 (180o), p. 156-169: Ueber den Steinregen zu Siena am 16. Juni 1794, von Abbé Domenico Tata zu Neapel; s. auch im gleichen Bd., p. 46 .

1795: Hamilton: Bericht des Earl of Bristol, Bishop of Derry. Abgedruckt in: Phil. Trans, Bd. 85 (1795), p. $1_{3}-$ 105: An Account of the late Eruption of Mount Vesuvius. s, auch Gilb. Ann. Bd. 6 (180o), p. $43-46$; Bibl. Brit. 
Nr. 3 von 1796; Izarn (s. u.), p. 94 -98 .

1796: Ambrogio Soldani: Dissertazione sobra una pioggetta di sassi accaduta nella sera di 16. Giugno I794 in Lusignan d'Asso nel Sanese, del P. D. Ambrogio Soldani Abb. Camaldolense, e. P. Prof. di Mathematiche nell' Universitá di Siena; s. auch Opuscoli Scelti sulle scienze e sulle arti Bd. 18, p. $33-45,136,185,285$ (Milano 1796).

1796: K ing : Remarks, p. $3-9$, 16, $32-33$.

r796?: S p a l a nz a n i in den Atti dell' Accademia di Siena Bd. 9.

1797: Lichten berg : Steinregen zu Siena. Taschenbuch zum Nutzen und Vergnügen für's Jahr 1797. Göttingen, p. $16 \mathbf{I}-169$; s. auch Voigt's Magazin Bd. I, p. 17.

1802: T a rgi o n i: Giorn. Pisano 3. r802: $\mathrm{H}$ o w a rd: Experiments and $\mathrm{Ob}-$ servations on certain stony and metalline Substances, which at different Times are said to have fallen on the Farth; also on various kinds of native Iron. Phil. Trans. (1802), p. 173-174. - Mineralogical Description of the various Stones said to have fallen upon the Earth. By the Count de Bournon. - Stone from Italy, p. 184-185. - Examination of the Stone from Sienna, p. 195-197 (Analyse); s. auch Gilb. Ann. Bd. I3 (1803), p. $296-297,305-306,312$; Fourcroy's Uebersetzung in $\gg$ Encyclopédie « Bd. 5 (1808), p. 546, 549$550,553-554,555$.

1803: v. No 11: Steinregen, Feuerkugeln in Ann. Berg- u. Hüttenk. Bd, 2, p. $306-328$.

1803: O 1 b e r s: Ueber die vom Himmel gefallenen Steine. Gilb. Ann. Bd. 14, p. $3^{8}$.

1803: de Drée: Recherches, Ann. Chim. Phys. (4) 17, p. 412, 413, 419, 420,423 .
1803: C h 1 a d n i : ChronologischesVerzeichnis, Gilb. Ann. Bd. 15, p. 310.

1803: $\mathrm{K} 1$ a prot h: Ueber meteorische Stein- und Metallmassen. Erster Abschnitt. - I. Meteorsteine von Siena. Abh. Berlin. Akad. (1803), p. 22-25 (Analyse); s. auch Gilb. Ann. Bd. 13 (1803), p. $337-338$; Klaproth's Beiträge Bd. 6 (I815), p. $290-296$.

1803: I z a r n : Lithologie, p.94- 98,171 , $174,175,183,188-189,206-209$, 231, 232, 325, 343, 344 .

1804: v. Ende: Massen und Steine, p. 49-54 (Abdruck mehrerer Berichte und Mitteilungen aus Olbers' Vorlesung). 1804: Pötzsch: Kurze Darstellung, p. $74-78,92$.

1804: G i 1 b e r $t$ : Nachtrïge zu den Aufsätzen in den Annalen über die aus der Luft gefallenen Steine, Gilb. Ann. Bd. 18, p. $285-286$.

r806: L a u g i e r: Chromium, Gilb.Ann. Bd, 24, p. 379 .

1806: S a n ti: Viaggio terzo etc. seguito del Viaggo al Monte Amiata. Pisa 1806, p. 353 .

1807: K la proth: Beiträge, Bd. 4, p. 98 .

1808: de Dré e-Pictet: Description comparative etc. (s. bei Weston). Bibl. Brit., Nr. 296 (April t808), p. $28 \mathrm{r}, 287,288$.

r8xo: Kl a proth: Beiträge, Bd. 5 . p. 245 .

18ro: Bull, Soc. Philom. Mai r8ro.

1812: C h 1 a d n i: Verzeichnis,Schweigg. Journ. Bd. 4 Beil. I, p. I4.

1812: Bigot de Morogues, p. $(85)$, 141-I50, 334 .

1815: Chladni: Neues Verzeichnis, Gilb. Ann. Bd. 50, p. 251; s, auch Gilb. Ann. Bd. 47, p. 96.

1816: $\mathrm{Ch} 1$ a d n i: Zweite Fortsetzung, Gilb. Ann. Bd. 54 , p. $349-35$ o.

r8rg: $\mathrm{Ch}$ la dn i: Feuermeteore, p. 6, 10, $50,66,70,72,73,90,135-136$, $261-262(1), 309,420,421,428$. 
I820: v. S c hreibers: Beiträge, p. 15 $-16,61-62$ (Abb.).

I82I: John: Sur la Nature de grandes masses de fer métallique d'origine problématique, et sur celle du fer des aérolithes attirables par l'aimant. Ann. Chim. Phys. (182I), p. 205.

1822: $\mathrm{Chl}$ adni: Zweiter Nachtrag, Gilb. Ann. Bd, 71, p. 367 .

1827: In Edinburgh Journ. of Sc. Bd. 7, p. 15 werden die Steine wieder auf einen Ausbruch des Vesuv's zurückgeführt. 1832: v. H o ff: Achter Nachtrag, Pogg. Ann. Bd. 24, p. 222223.

I834: B e rz e li u s: Ueber Meteorsteine, Pogg. Ann. Bd. 33, p. 3 .

1836: K ä mtz: Meteorologie, p. 258, 277, 312.

1839: B e n z e n b e r g: Sternschnuppen, p. $4 \mathrm{I}, 43-44$.

184I : R a m m els ber g : Handwörterbuch, p. 428 (Analyse von Klaproth). r843: P a rts c h: Meteoriten, p. 31-32. 1854: B a 1 c e 11 s: Lithologia meteorica, p. 22.

1859: B u c h n e r : Feuermeteore, p. 55 $-56$.

1859: H arris: Dissert.Gött., p. 62.

r862: Kenngott u. Wiser: Zürcher Sammlung, p. 154 .

r863: B u c h n er: Meteoriten, p. II-I2. 1863: R o s e: Meteoriten, p. 84, 147, 155, 159, Taf. II.

1858/65: v. Re ichen ba ch: V 477 . VI $440,443,449$. VII 562. IX 16I, I69, 179. X 359, 362. XI 294, 297, 302, 309. XIII $355,356,364,371$ (Fig.), 375-377, 380. XIV 399. XV I2I. XVIII 490. XX 622, 623, 626. XXIII 369. XXIV 226.
1875: vom R a th: Meteoriten, Verh. naturh. Ver. Bonn Bd, 32, p. $367,370$. r880: Ha h n : Die Meteorite (Chondrite) und ihre Organismen. Tübingen. T. 4 Fig. 2; T. 6 Fig. I, 3; T. 13 Fig. 3 ; T. 24 Fig. 1 ; T. 25 Fig. 5 .

1883: Tschermak: Beitrag, Sitzber. Wien. Akad. Bd. 88 I, p. 355.

r884: M e un i er: Météorites, p. 4, 68, $95,227-228,459$.

1883/85: Tschermak: Photographien, p. $18,20$.

r885: Brezina: Wiener Sammlung, p. $177,232$.

1887: Brezina: Reisebericht, Ann, Hof-Mus. Bd. 2 (Not.), p. 73, 109, 1 1о. r887: Meunier: La giovanite, nouvelle roche cosmique. C. $\mathrm{R}$. Bd. 104, p. 193-I94. Ref. N. J. 1888 I, p. 2 II. 1893: Newton: Lines of structure in the Winnebago Co. Meteorites and in other Meteorites. Am. Journ. (3) 45, p. $152-153,355$. Ref. N. J. 1894 I, p. $273-274$.

1894: Cohen: Meteoritenkunde, p. $7,8,23,39,6 \mathrm{r}, 185$.

r894: F l e t c h e r : Introduction, p. 22, 23,24 .

1895: Brezina: Wiener Sammlung, p. $24 \mathrm{I}$.

Ursprüngliches Gewicht: Steinschauer; der grösste Stein 7 Pfund $7^{1 / 2}$ Unzen. Mehrere von einigen Pfund und viele kleine Steine.

Nachweisbares Gewicht: 4163 gr.

\begin{tabular}{lr|llr} 
Bailey & I & v. Braun & 4 \\
Berlin, U. & 60 & Budapest & 26 I \\
Bologna & 985 & Calcutta & 205 \\
Bonn & $\left.32 \mathrm{I}^{1}\right)$ & Cambridge & I (?)
\end{tabular}

I) Hiervon betrachtete vom Rath $317 \mathrm{gr}$. als zweifelhaft. Laspeyres kann dem nicht beipflichten (vergl. s die Meteoritensammlung der Universität Bonn «. Verh. naturh. Ver. Bonn Bd. 51, p. 127-128). Bei der ausserordentlich charakteristischen Beschaffenheit der Rinde. welche die Sieneser Steine zeigen, ist es auffallend, dass vom Rath Zweifel erheben konnte; Laspeyres betont ausdrücklich, dass die Rinde eines $3^{1 / 2} \mathrm{gr}$. schweren Stïckes, dessen Herkunft von Siena nicht bezweifelt wird, mit der Rinde des grossen Stückes übereinstimme. 
Siena - Simbirsk.

\begin{tabular}{|c|c|c|}
\hline Clausthal & 56 & Newton \\
\hline Cleveland & Spl. & Paris, M. \\
\hline Dresden, M. & 55 & Parma \\
\hline Gotha & 12 & Pohl \\
\hline Göttingen & 17 & Rom, U. \\
\hline Gregory & $5^{1}$ & v. Siemaschko \\
\hline Harvard, U. & 5 & Siena \\
\hline Klausenburg & 3 & Stockholm \\
\hline Kopenhagen & 7 & Troyes \\
\hline London, B.M. & 129 & Tübingen \\
\hline London, P. G. & 105 & Turin, U. \\
\hline Marburg & 2 & Upsala \\
\hline Modena & 43 & Ward \\
\hline Moskau & 1 & Washington, Sh. \\
\hline Neumann & 29 & Wien, H. M.*) \\
\hline New Haven & $\mathbf{I}$ & Zürich \\
\hline
\end{tabular}

Die Universität Basel besitzt ebenfalls ein Stück von Siena.

Sierra Blanca Huejuquilla-Gruppe Sierra de Chaco Vaca Muerta Sierra de Deesa 1863 Obc Copiapo Sierra de Deesa I 866 Hch Dehesa Sigena

Signet Iron Sena Sikkensaare Tucson Tennasilm

Silver Crown, Crow Creek, Laramie Co., Wyoming Territory, U. S. A.

Eisen, $\mathrm{Og}$, gefd. 1887 , beschr. 1888 . r888: $\mathrm{Kunz}$ : On two new masses of Meteoric Iron, - 2 Meteoric Iron from Laramie County, Wyoming. Am Journ. (3) 36 , p. 276-277 (Analyse von Mc. Ilvain). Ref. N. J. 1889 II, p. 446. 1890: E a s t m a n: Met. Astron., p. 320. r891: v. Ha u er: Ann. Hof-Mus, Bd, 6 (Not.), p. 54.
I893: B r e z i n a : Ueber neuere Meteoriten (Nürnberg), p. 165.

1894: Co he n : Meteoritenkunde, p. 72, 124, 125.

r895: Brezina: Wiener Sammlung, p. 287 .

1895: C o h e n: Meteoreisen-Studien IV. Ann. Hof-Mus, Bd, I0, p. 82, 87, 90. Ursprüngliches Gewicht: i $616 \mathrm{gr}$. Nachweisbares Gewicht: 8592 gr. Bailey $\quad 88$ Prag, M, $\quad 156$ Bement $\quad 136$ Rom, U. 100 v. Braun $\quad 98$ Stockholm 74 Gregory $\quad 172 \mid$ Strassburg $\quad 72$ Greifswald $\quad 41$ Ward 71 Krantz III Wien, H. M. 689o London, B. M. $\quad 5^{8} 3$

Simbirsk (Bloede) Slobodka I8 18 Simbirsk (Bloede) Krasnoi Ugol(?) Simbirsk (Partsch), Russland.

Stein, Ck, Fallzeit unbekannt; bekannt vor 1838 ; erwähnt 1843 . Unter diesem Stichwort sind vereinigt: Simbirsk (Kupffer $\mathrm{r}_{3} 8$ ); „Simbirsk Partșch 1843 ; Poltawa (Bloede 1846); Meteorit von ungewisserHerkunft(Goebelı867).

r843: Parts ch: Meteoriten, p. 46 (die Wiener Sammlung erhielt $18{ }_{3} 8$ ein Stück aus Petersburg).

r847: Eichwald: Verzeichnis, Erman's Arch. f. wissensch. Kunde v. Russl. Bd. 5 , p. 18 o.

I848: B 1 o e d e: Tabelle, Bull. Petersburg. Akad. Bd. 6, p. 5-6.

1859: H a rris : Dissert. Gött., p. 89. r863: B u chner: Meteoriten, p. II5

1) sLibbre 3 , dramme 5 e denari 12. Libbra fiorentina $=339.520 \mathrm{gr}$.; Dramma $=3.516$ gr.; Denaro $=\mathbf{1 . 1 7} 8 \mathrm{gr}$. Im Besitz der Accademia dei Fisiocritici di Siena, nach Mitteilung von Herrn Prof. Gucci,

2) Unter der Bezeichnung ${ }^{\text {Siena }}$ befinden sich in Tübingen noch 3 Steine. Herr Direktor Brezina bestimmte einen davon (129 gr.) als Knyahinya, die beiden anderen sind vorläufig nicht $\mathrm{zu}$ identifizieren.

3) Ausserdem 180 gr. nach I. Juli 1893 erworben. 
(Dürfte sich sowohl auf Bloede's als auch auf Partsch's Simbirsk beziehen.) 1863: R os e: Meteoriten, p. 86, I54

("Gouv. Pultawa ; in Klein's Katalog als „Simbirsk Partsch * bezeichnet).

1867: Buchner: Dritter Nachtrag,

Pogg. Ann. Bd. 132 , p. 315.

1867: G o e be 1: Kritische Uebersicht,

Mélanges phys. chim. Bd. 7 , p. 339 (sei vielleicht Doroninsk oder Tounkin).

(Brezina hebt die Aehnlichkeit mit dem Wiener "Simbirsk Partsch * hervor). 1885: Brezina: Wiener Sammlung, p. I9I, 233.

r895: Brezina : Wiener Sammlung, p. 260 .

Ursprüngliches Gewicht: Der früher in der Sammlung der Petersburger Akademie unter dem obigen Namen geführte Stein wird jetzt als Doroninsk aufgeführt, von welchem es heisst: sist 1888 in Wien fraglich geworden «; s. auch unter Doroninsk p. Io6.

Nachweisbares Gewicht: ${ }_{15}^{17}$ gr. Kiew $12^{1}$ ) Wien, H. $\mathrm{M}^{*}$ ) 10 Petersburg, A. 1495

$\begin{array}{lr}\text { Simonod } & \text { s. Anhang Belmont } \\ \text { Sinaloa } & \text { Ranchito } \\ \text { Siratik } & \text { Senegal }\end{array}$

Sitathali bei Nurrah, Raipur, Rajpootanah, Central-Prov., Ostind. Stein, Cho, gefallen 4. März I875. 1876: Medlicott exhibited a Meteorite from Raipur, Central Provinces, and read the following note regarding it - Record of the Sitathali Meteorite of $4^{\text {th }}$ March, 1875 . Proc. Asiatic Soc. Bengal. (1876), p. I15-116.

1884: M e unier: Météorites, p. 23I.

1885: Brezina: Wiener Sammlung, p. $177,232$.

Ursprüngliches Gewicht: I 4I 3 gr. Zwei Stücke, $3 / 4$ miles von einander gefunden, zusammenpassend (2 lbs. o oz, 430 grs. u. I lb. $10 \mathrm{oz}$. 160 grs.).

Nachweisbares Gewicht: I 788 gr. $^{2}$ )

Bement

Budapest

Calcutta

Gregory

Harvard, U.

London, B. M. 600

Ski, Amt Akershuss, Norwegen.

Stein, Cwa, gefallen 27. Dez, 1848 .

1854: Ditte n: Analyse eines Meteorsteins. Chemisches Laboratorium der Universität Christiania und die darin ausgeführten chemischen Untersuchungen. Herausgegeben von A. Strecker. Universitätsprogramm für das zweite Halbjahr 1854. Christiania 1854, p. 82 ; s. auch Journ. prakt. Chem. Bd. 64 (1855), p. 121-123; Pogg. Ann. Bd. 96 (1855), p. 341-344; Liebig-Kopp, Jahresber. 1854 , p. $912-913$; Kenngott, Uebersicht 1854,p.167-168; Giebel u.Heintz, Zeitschr. f. ges. Naturw. Bd. 4, p. 395 u. Bd. 6 , p. 414 ; N. J. 1856 , p. 435 u. N. J. 1857, p. $83 \mathrm{I}-832$.

1859: B u c hner: Feuermeteere, p. 102. r859: H a r r i s : Dissert.Gött., p. 95-96. 1860: Rammels berg: Mineralchemie, p. $923 \mathrm{ff}$., 950 .

1863: B u c hn e r : Meteoriten,p. 78-79. 186o/65: v.Rei chen b a c h: XIV 392. XX $623,626$. XXV 324 .

1870: R a m mels berg: Meteoriten, p. $103,105,106,139,140$.

I) „Gouv. Poltawa ".

2) Der Widerspruch zwischen ursprünglichem und nachweisbarem Gewicht ist wahrscheinlich auf die Weise zu erklären, dass der Fedden'sche Katalog über die Sammlung von Calcutta nicht mehr in allen Teilen Gültigkeit hat. 
1879: R a m melsberg: Meteoriten, p. 24,25 .

r884: M eunier: Météorites, p. 79, $85,209,219$.

r886: Reu s c h: Ueber den Tysnesmeteorit u. drei andere in Skandinavien niedergefallene Meteorsteine. - IV. Der Skimeteorit (27.December 1848 Abends). N. J. B. B. IV, p. 493-495 (Tafel VIII : 2 Abbildungen des Steins).

1894: F let c her: Introduction, p. 13. 1895: Brezina: Wiener Sammlung, p. 244 .

Ursprüngliches Gewicht: Ein Stein von $85^{\circ} \mathrm{gr}$.

Nachweisbares Gewicht: 750 gr.

\begin{tabular}{lr|lr|} 
Bailey & Spl. & Paris, M. & I \\
Budapest & Spl. & Stockholm & 4 I \\
Calcutta & Spl. & Tübingen & 32 \\
Dorpat & I & Washington & Spl. \\
Kristiania & 636 & Washington,Sh. & I \\
London, B. M. & 6 & Wien, H. M.*) & 30 \\
London, P. G. & 2 & &
\end{tabular}

Slavetic zwischen Agram und Jaska, Croatien, Oesterreich.

Stein, Cgb (früher als Cga aufgefasst), gefallen 22. Mai 1868 .

1868: v, Haidinger: Der Meteorsteinfall in Croatien am 22. Mai 1868. Vorläufiger Bericht Sitzber.Wien.Akad. Bd. 58 II, p. 162-168; s, auch $>$ L'Institut< Bd. 36 (1868), p. 392.

r868: v. Haidinger: Der Meteorsteinfall am 22. Mai I 868 bei Slavetič. Zweiter Bericht (mit Tafel, 3 Abbildungen des Steins und fünf Holzschnitten, Situationsplan, Schnittflächen). Sitzber. Wien, Akad, Bd. 58 II, p. 943 -954 ; s. auch >L'Institut \& Bd. 37 (1869), p. 96.

1869: Buchner: Vierter Nachtrag, Pogg. Ann. Bd, 136, p. 594-595. r869/70: O l d h a m: Rec. Geol. Surv. India Bd.2,p.ror (Empfang eines Stücks). 1884: M e unier: Météorites, p. 268,
27I, 448 (Abb. der Fallerscheinung). 1885: Brezina: Wiener Sammlung, p. $164,182,233$.

I890: v. Niess 1: Periheldistanzen, Verh. naturf.Ver. Brünn Bd. 29, p. 189, 194, 195, 214, 258.

Ursprüngliches Gewicht: I708 gr. Zwei Steine von ${ }_{1} 5^{8} 3$ und $I_{2} 5$ gr. Nachweisbares Gewicht: 1420 gr. Budapest $\quad 6 \mid$ Paris, M. 32 Calcutta 2I v. Siemaschko I Göttingen $\quad 7$ Stockholm I London, B. M. $\quad 21$ Wien, H. M.*) 1331 Besitzt das Museum in Agram noch den kleineren Stein?

Slobodka, Kreis Juchnow, Gouv. Smolensk, Russland.

Stein, Cc, gefallen ro. August 1818. Bloede'sSimbirsk. Partsch'sPoltawa.

r8rg: $\mathrm{Much}$ in: Ein kleines in russ. Sprache geschriebenes Buch. - Die in Betracht kommende Stelle ist bei Goebel (s. u. 1867) übersetzt.

r8rg: $\mathrm{Chl}$ adni: Vierte Fortsetzung, Gilb.Ann Bd.6o, p.254 (Fall eines Steins von 7 Pfund mit dem Falldatum Ir. Juli, 29. Juni alten Stils). Nachtrag von Gilbert $>$ Noch stehe hier folgende Nachricht aus den neuesten Zeitungen $\mathrm{Ham}$ burg. Corresp. 1818, 158; Schwäb. Merkur Nr. 243.

1819: $\mathrm{Chlad}$ n i: Fünfte Fortsetzung, Gilb. Ann. Bd. 63 , p. 24 .

1819: $\mathrm{Chl}$ a d n i: Feuermeteore, p. 66, 73. 310 (!) (Falldatum 10. August, 29. Juli alten Stils).

1823: Chladni: Dritter Nachtrag, Gilb. Ann. Bd. 75, p. 266. Anmerkung von Gilbert (Erwähnung des Steins mit dem Falldatum Chladni's).

1836: K ä m tz: Meteorologie, p. 287. 1837: Ros e : Reise nach dem Ural Bd. I, p. $44,75-76$ (der Stein sehe aus wie einer von Stannern; Rose erhielt ein 
Slobodka - Smithland.

Stück, welches aber in seinem Katalog von I $\$ 62$ unter den Chondriten aufgeführt wird).

1843: P a r s ch: Meteoriten, p. 44. r845: B a 1 c e 11 s : Lithologia meteo rica, p. 23-24.

I847: Eichwa 1d: Verzeichnis, Erman's Archiv f. wissensch. Kunde Russlands Bd. 5, p. $178-179$.

r848: B 1 o e d e: Tabelle, Bull. Petersburg. Akad. Bd. 6, p. to (unter jenen russischen Meteoritenfällen aufgeführt, von welchen Stücke in der Petersburger Sammlung fehlen).

1857: Arago: Astronomie populaire. Bd. 4 , p. 199.

I859: Ha r ris : Dissert.Gött.,p.74-75,89. r863: Bu chner: Meteoriten, p. 39. 1863: R o se: Meteoriten, p. 93, 155. r859/64: v. Reichen ba ch: IX 162, 172, 180. X 372 . XIII $362,363,373$, 380. XXIII 368 .

1867: B u chner: Dritter Nachtrag, Pogg. Ann. Bd. 132, p. 315 .

1867: Go e bel: Kritische Uebersicht, Mélanges phys. chim. Bd. 7, p. 29r $-300,3^{1} 3$ (Abb. des Steins).

r884: M eunier: Météorites, p. 19I, 192, 371 .

r85: Brezina: Wiener Sammlung, p. $185,233$.

r895: Brezina: Wiener Sammlung, p. 255 .

Ursprüngliches Gewicht: Ein Stein von $2^{3 / 4} \mathrm{Kgr}$. (7 Pfund russisch). Nachweisbares Gewicht: 2696 gr. Berlin, U. Calcutta

\begin{tabular}{r|r}
$127^{\text {I }}$ & Harvard, U. \\
3 London, B. M. & 27
\end{tabular}

London, P. G. Spl. Petersburg, A. 236r

\begin{tabular}{lr|lr} 
Neumann & I & Tübingen & $28^{2}$ ) \\
New Haven & 2 & Washington & 4 \\
Paris, M. & 49 & Wien, H. M.*) & 90
\end{tabular}

\section{Slobodka-Partsch.}

Stein, Cwa (auch das Tübinger Stück ist Cwa und hat den gleichen Ursprung, wie ein Teil der Wiener Bruchstücke), bekannt vor $18{ }_{3} 8$, beschr. 1843 von Partsch als "Slobodka, Gouv. Smolensk, gefallen 10. August 1818\%.

I843: P a r s c h : Meteoriten, p.55-56. 1847: E i chwa 1d: Verzeichnis, Erman's Arch f.wissensch.Kunde Russlands Bd. 5 , p. 180 .

1863: Ros e: Meteoriten, p. 93, 155. r858/65: v. Re ichen b a c h: V 480. VI 44I, 443. IX 16I, 168, 178. XI 294, 300. XIII 378. XV 101. XX 626. XXV $319,322,324$.

1885: Brezina: Wiener Sammlung. p. $177,232$.

1895: Brezina: Wiener Sammlung, p. $242,244,255$.

Ursprüngliches Gewicht: ?

Nachweisbares Gewicht: $940 \mathrm{gr}$. Calcutta Spl. ${ }^{3}$ ) Wien, H. M.*) 148 Tübingen 792

\section{Slonin s. Anhang Ruschany Smith Co. Carthago}

Smithland, Salem, Livịngston Co., Kentucky, U. S. A.

Eisen, Db (früher als Hca aufgefasst), gefunden um $1839-1840$, beschr. 1846 .

I) Ausserdem 490 gr., welche aus der Erzherzog Stephan-Rumpffschen Sammlung stammen, als "Grüneberg \& u. >Stannern * bezeichnet waren und nach Klein mit Slobodka oder Timoschin grosse Aehnlichkeit aufweisen. Das nachweisbare Gewicht von diesem Slobodka (Cc) deutet darauf hin, dass die Stücke nicht von dem gleichen Fall sein können.

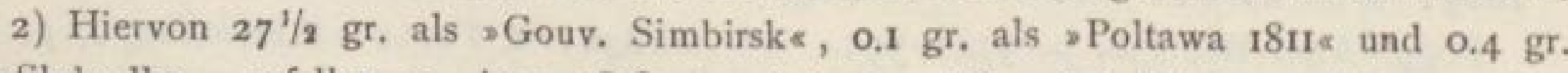
als $>$ Slobodka, gefallen 10. Aug. 1818 \& bezeichnet. Alle drei Stücke gehören zu den ausgesprochenen Kügelchenchondriten und sind nach Brezina Slobodka.

3) „Poltawa Gouvernment, Little Russia $18{ }_{3} 8$ (ante) 6 grains, * 
1846: Troost: Description of three varieties of Meteoric Iron. - 3. Meteoric Iron from Livingston Co., Kentucky. Am. Journ. (2) 2, p. 357-358; s. auch Am. Journ. (2) 5 (1848), p. 351 (beiläufige Erwähnung).

1847: S hepard: Report on Meteorites. - 5. Livingston county, Kentucky. Am. Journ. (2) 4, p. 77.

1852: C 1 a r k: Dissert. Gött., p.69-7o.

1854: v. Boguslawski: Zehnter Nachtrag, Pogg. Ann. Ergz.-Bd.4, p. 40I. r859: H a rr is: Dissert. Gött,, p. r17. 1859/62: v. Reichen b a h: 1 X 162. 176, 177, 182. XI 291. XIII 354. XV I00. XVII 268, 273. XX 630.

r862: Greg: On some Meteorites in the British Museum. - Irons 8. Livingston County, Kentucky. Philos. Magaz. Bd. 24, p. 540 .

1863: B u chner: Meteoriten, p. 174. 1863: Ros e: Meteoriten, p. 24.

1884: M e un i e r: Météorites, p. 116, I2I. r885: Brezina: Wiener Sammlung, p. $203,219,234$.

1890: Eastman: Met. Astron., p. 318. r893: M e unier: Revision des fers météoriques, p. 16, 20.

Ursprüngliches Gewicht: Grössere Masse, die zum Teil verschmiedet wurde; 8-ro lbs. wurden gerettet, wovon Troost 2.1 Kgr. ( $4^{3} / 4$ lbs.) erhielt.

Nachweisbares Gewicht: 4969 gr.

Berlin, U. $\quad 14 \mid$ Paris, M. $\quad 75$

$\begin{array}{llll}\text { Calcutta } & 70 & \text { Pohl } & 34\end{array}$

Göttingen $\quad 8$ Stockholm 45

Harvard, U. 1877 Ward 49

London, B. M. 2556 Washington, Sh. I3

London, P. G. no Wien, H. M. 118

In Brezina's 'Synonymen-Verzeichnis ist bei Smithland auch $\mathrm{Sa} 1 \mathrm{e} \mathrm{m}$ angeführt. Um Verwechslungen vorzubeugen, möge hier erwähnt werden, dass Hayes, Am. Journ. (2) 25, p. $135>$ On the Supposed Meteorite from Marbleheade einige
Angaben veröffentlicht hat, die dessen Pseudonatur nachweisen. Dieser vermeintliche Meteorit sei in einer Zeitung von Salem beschrieben worden. M a rbl e h e a d erwähnt auch Kesselmeyer, p. 473 und verweist auf Jackson in Proc. Boston Soc. Nat, Hist, Bd, 7, p. 294.

Smith's Mountain, Rockingham Co., Nord Carolina, U. S. A.

Eisen, Of, gefunden um 1863 , erwähnt 1872 .

1872: T s c her mak: Meteoriten, M.M. Bd. 2 , p. 172 .

r874: S m ith: On a Mass of Meteoric Iron of Howard Co., Ind.; with some remarks on the molecular structure of meteoric iron, and a notice concerning the presence of solid protochloride of iron in Meteorites. Am. Journ. (3) 7, p. 395 (ausser in Tazewell in diesem Eisen entdeckt); s, auch Original Researches 1884 , p. 477 ; Liebig-Kopp, Jahresber. 1874, p. 134I.

1875: K e rr: Rep. Geol. Surv, N. Carolina (gedruckt zu Raleigh), Bd. I, app. C, p. 313 (oder 56?). Ref, N. J. 1876, p. 324 ; s, auch Min. and Min. Soc. of N. Carolina 1885 , p. 15 (gedruckt zu Raleigh).

1877: Smith: Examination of the Waconda Meteoric Stone, Bates County Meteoric Iron and Rockingham County Meteoric Iron. Am. Journ. (3) 13, p. 213 -2I4 (Analyse); s, auch Original Researches 1884 , p. $526-527$; LiebigKopp, Jahresber. 1877 , p. 1396.

1883: S m it h : Concretions, Am. Journ.

(3) 25, p. 420.

1885: Brezina: Wiener Sammlung, p. $210,233$.

1887: F lig h t: Meteorites, p. 54.

1890: Eastman: Met. Astron., p. 322.

1894: C o he n: Meteoritenkunde, p. 133, 229. 
1895: Brezin a : Wiener Sammlung, p. 269 .

Ursprüngliches Gewicht: 5 Kgr. (I I lbs.).

Nachweisbares Gewicht: 2271 gr.

\begin{tabular}{lr|lr} 
Bailey & 737 & London, B. M. & 77 \\
Bement & 208 & London, P. G. & 40 \\
Cleveland & 32 & v. Siemaschko & 33 \\
Göttingen & 54 & Stockholm & 12 \\
Gregory & 8 & Ward & 13 \\
Harvard, U. & 821 & Washington & 59 \\
Kopenhagen & 53 & Wien,H.M.*)**) & 124
\end{tabular}

\section{Smithsonian Iron}

Coahuila

Smithville (Caryfort), Decalb Co., Tennessee, U. S. A.

Eisen, Og, gefunden $\mathrm{I} 840$, beschr. 1845 (neuer Fund 1893).

Hierher auch: Caryfort und Cany Fork. Ist vielleicht mit Cosby's Creek zu vereinigen, $\mathrm{cf}$. Cohen, Meteoritenkunde, p. 146.

1845: Troost: Description of a mass of Meteoric Iron discovered in De Kalb County, Tenn.; Am. Journ. (1) 49, p. 34I -342 u. (2) 5 , p. 35 I (beiläufige Erwähnung).

r846: Shepard: Report on Meteorites. Am. Journ. (2) 2, p. 390, 39I. 1852: C l a r k: Dissert. Gött,, p. 68.

r854: v.B o g u s l a w s k i: ZehnterNachtrag, Pogg. Ann. Ergz.-Bd. 4, p. 403. 1859: H a rris: Dissert. Gött., p. II6. r858/62: v. Re i c he n b a c h: IV 638. VI 452. VII 551. VIII 488. IX 162 , $175,176,182$. XII 457. XIII $363,364$. XIV 390. XV 100, III, 128. XVI 26I, 262. XVII 273 XVIII $484,487,489$. XX 621, 625, 629, 631, 634. XXI $578,579,580,586$.

1863: Bu chner: Meteoriten, p. r7i, 1863: Rose: Meteoriten, p. 26, 73, r53. r876: S m it h : Carbon Compounds, Am. Journ. (3) II, p. 392, 394, 434. r880: Brezina: Bericht I, Sitzber.
Wien. Akad. Bd. 82 I, p. $351-352$. 188I: Brezina: Bericht III, Sitzber. Wien. Akad. Bd. 84 I, p. 279,28 o. 1884: Me unier: Météorites,p.20,116,122. 1885: Brezina: Wiener Sammlung, p. $215,216,234$.

1886: H u n t in g t o n: Crystalline Structure, Am. Journ. (3) 32, p. 287,288 (Abbildung einer geätzten Platte).

1887: Fligh t: Meteorites, p. 170.

I887: v. H a ue r: Ann. Hof-Mus, Bd. 3 (Not.), p. 38 .

1890: Brezina: Ueber Meteoreisen, Oesterr. Zeitschr. f. Berg- u. Hüttenw. Bd. 38 , p. 357 (Abbildung einer geätzten Platte).

1890: E as t m a n : Met. Astron., p. 318. 1893: M e unier: Remarques géologiques sur les fers météoriques diamantifères. C. R. Bd, n6, p. 4ro. Ref. N. J. 1884 I, p. 449.

1893: M e un ie r: Revision des fers météoriques, p. 29, 31-32.

1894: Huntington: The Smithville meteoric iron. Proc. Amer. Acad. Arts and Sc. Boston Bd. 29, p. $251-260$ (Analyse. Abbildung der 1893 gefundenen Masse von 65 lbs. Abb. einer geätzten Platte. Uebersichtsplan von Tennessee bis West-Virginia); s. auch Zeitschr. f. Kryst. Bd. 26 (1896), p. 604. r894: Co hen: Meteoritenkunde, p. 76, $142-143,146,149,150,165,194$.

r895: Brezina: Wiener Sammlung, p. $285-286$.

Ursprüngliches Gewicht: 36 lbs. Neuerdings (1893) wurden noch drei Massen von 65,15 u. 7 lbs. gefunden.

Nachweisbares Gewicht: 13923 gr.

\begin{tabular}{lr|lr} 
Bailey & 224 & Cambridge & Spl. \\
Bement & 47 & Gregory & 15 \\
Berlin, U. & 2 & Harvard, U. & 3315 \\
Brezina & 329 & Kopenhagen & 124 \\
Budapest & 138 & London, B. M. & 4 \\
Calcutta & 12 & London, P. G. & 35
\end{tabular}




\begin{tabular}{lrr|lr} 
New Haven & 6I & Tübingen & 8059 \\
Paris, M. & II5 & Ward & 55 \\
Prag, M. & $\left.6 \mathbf{I}^{1}\right)$ & Wien, H. M.*) & I2I2 \\
Stockholm & II5 & &
\end{tabular}

Sokobanja (Sarbanovac), auch Banja bei Alexinac, Serbien.

Stein, Cc, gefallen 13. Okt. 1877 . 1876 (?): P a n è i č : (Der erste Meteorit in Serbien, Belgrad 1876 (?); 30 Seiten (serbisch); s, auch I885: Brezina, Wiener Sammlung, p. 186-188. 1877: D ö 11: Der Meteorsteinfall von Soko-Banja nordöstlich vonAleksinaćam 13. Oktober 1877. Verh. k. k. geol. Reichsanst. (1877), Nr. 16, p. $283-287$ (mit zwei Situationsplänen). Ref. Liebig-Kopp, Jahresber. 1878 , p. $1318-$ 1319.

1878: Los a itsch: Das Meteor von Sokol-Banja in Serbien. Ber. d. d. chem. Ges. 1878 I, Bd. II, p. $96-98$ (Analyse). Ref.Liebig-Kopp, Jahresber. 1878 , p. 1318 .

1879: Klein: Göttinger Sammlung, Gött. Gel. Anz. (1879), p. $92-98$.

r88I: M e un i er: Examen lithologique et géologique de la météorite, tombée le 13 octobre 1872 (doch wohl 1877 !) aux environs de Soko-Banja, en Serbie. C. R. Bd, 92, p. 331-332. Ref. N. J. I88I II, p. $188_{3}-184$.

188x : Brezina: Bericht II, Sitzber. Wien. Akad, Bd. 83 I, p. 475 .

1883: T s c hermak: Beitrag, Sitzber. Wien. Akad. Bd. 88 I, p. 355 .

r884: M e u n i e r: Météorites, p. 98, 264 -267 (Abb.), 349, 350, 351, 380 .

1883/85: Tschermak: Photographien,

T. 12, p. $14,17,18,20$.

1885: $\mathrm{Brez}$ in a $(\mathrm{P}$ an $\breve{c} \mathrm{i} \tilde{\mathrm{c}})$ : Wiener

Sammlung, p. 155, 185, 186-188 (Taf.

IV, Situationsplan), 233.

r887; F lig h t: Meteorites, p. 203-205. 1889: Wein schenk: Ueber zwei neue Bestandtheile des Meteoriten von Sarbanovac. Ann. Hof-Mus, Bd, 4 (Not.), p. IO9-IIO.

1893: B rez in a: Ueber neuere Meteoriten (Nürnberg), p. I6r.

I894: C o h e n : Meteoritenkunde, p. Ir2, $169,204,248,284,300,304,317$. 1895: Brez in a : Wiener Sammlung, p. 255,256 .

Ursprüngliches Gewicht: Etwa 48 (80 ?) Kgr. Mehrere Steine, der grösste $38 \mathrm{Kgr}$. Döll sagt: r Stein von ${ }_{23} \mathrm{Oka}$, I Stein von 15 Oka, 2 Stücke von je 2 Oka; ausserdem noch mehrere Steine u.Stücke. Ein Oka soll $=1260-1280 \mathrm{gr}$. sein.

Nachweisbares Gewicht: $40329 \mathrm{gr}$.

\begin{tabular}{lr|lr} 
Bailey & 40 & Minneapolis & 2 \\
Belgrad & $26263^{2}$ ) & Modena & 14 \\
Bement & 185 & Moskau & 1275 \\
Berlin, U. & 70 & München & 29 \\
Böhm & 43 & Neumann & 30 \\
Bologna & 76 & New Haven & 2 \\
Bonn & 2 & Newton & 20 \\
v. Braun & 64 & Paris, E. & 40 \\
Budapest & 639 & Paris, M. & 1850 \\
Cohen & 2 & Pohl & 208 \\
Czernowitz & 4 & Prag, B. U. & 22 \\
Dorpat & 156 & Prag, D. U. & 28 \\
Dresden, M. & 230 & Prag M. & 83 \\
Frankfurt & 273 & Rom, U. & 83 \\
Göttingen & 1023 & Sidney & 170 \\
Graz & 52 & v. Siemaschko & 167 \\
Gregory & 219 & Stockholm & 885 \\
Harvard, U. & 256 & Strassburg & 42 \\
Heidelberg & I3 & Stürtz & 43 \\
Klausenburg & 215 & Stuttgart & 77 \\
London, B. M. 1975 & Szamosujvar & II \\
London, P. G. & 225 & Troyes & 42 \\
Lüttich & 3 & Tübingen & 43 \\
Madrid & n18 & Turin, U. & 72 \\
de Mauroy & 4 & Utrecht & 4 \\
Melion & 27 & Ward & 39 \\
& & \\
& &
\end{tabular}

1) \Smithville, Dekalb Co, $1892 \varepsilon$.

2) Drei Steine 16285 , 9695 u. 254 gr.; im Katalog steht als Summe 26263 gr. 
Sokobanja - Ställdalen.

\begin{tabular}{ll|l} 
Washington & 2 & Wien, U. I. 300
\end{tabular}

Washington,Sh. 16 Wrany

Wien, H. M. $255^{\circ}$

Sommer Co.

Drake Creek

Sonora

Sörakarta

Tucson

South East Missouri

Saint François Co.

$\begin{array}{ll}\text { Swallik } & \text { s. Anhang } \\ \text { Sparta } & \text { s. Anhang }\end{array}$

Springbock River Great Fish River

Ssyromolotow, Angara, Gouv. Jenisey, Sibirien, Russland.

Eisen, Om, gefd. 1873 , beschr. 1874 .

1874: Goebe 1: Bericht über einen neuen Eisenmeteoriten vom Ufer der Angara aus dem Gouv. Jenisseisk. Bull. Petersburg. Akad. Bd. 19, p. 544-554 (Analyse. Abbildung der Masse); abgedruckt in den Mélanges phys, chim. Tome IX (1874), p. 95-109. Ref. Liebig-Kopp, Jahresber. 1874 , p. 1346. r885: Brezina: Wiener Sammlung, p. 269 .

1887: F lig ht: Meteorites, p. 67-68. 1892 : v. $\mathrm{H}$ a u e r : Ann. Hof-Mus. Bd, 7 (Not.), p. 73 .

1893: Meunier: Revision des fers météoriques, p. 75 .

1894: Co he n: Meteoritenkunde, p. 68. 1895: B rez in a : Wiener Sammlung, p. 279.

Ursprüngliches Gewicht: I2 Pud, welche Gewichtsangabe Goebel in $196568 \mathrm{gr}$. umrechnet. Diese Angabe von 12 Pud war aber wohl nur eine rohe Bestimmung, welche auf keiner genaueren Wägung beruhte und Goebel mitgeteilt wurde; daher offenbar der Widerspruch mit der gegenwärtig in Petersburg befindlichen Menge.
Nachweisbares Gewicht : 2 I 6766 gr.

Gregory

London, B. M. 4 v. Siemaschko 24

Paris, M.

I Wien, H. M. 6

Staartje

Stade

Uden

Ställdalen bei Kopparberget, Dalekarlien, Schweden.

Stein, Cgb, gefallen 28. Juni 1876 .

1877: v. Nordenskiöld: Föredrag i Mineralogi vid $A$ kademiens ärshögtid den 3 April, Stockholm 1877 ; s, auch „Nature « vom 19. Juli 1877 .

1877: Lindström: Analys af de vid Ställdalen den 28 Juni 1876 nedfallna meteorstenar. Öfversigt af Kongl. Vetenskaps-Akad. Förhandl., No. 4 (1877), p. $35-40$ (Analyse).

1878: v. Nordenskiöld: Trenne märkeliga eldmeteorer, sedda i Sverige under ăren 1876 och 1877 (härtill tafl. 2, 3 , p. 6-II). Geologiska Föreningens i Stockholm Förhandl. Bd. 4 Nr. 2 ; s. auch Uebersetzung von v. Boguslawski in Zeitschr. d. d. geol. Ges. Bd. 33 (1881), p. 14-25: Ueber drei grosse Feuermeteore, beobachtet in Schweden in den Jahren 1876 u, 1877 (mit 2 Karten. Analyse von Lindström). 1879: R a m mels berg: Meteoriten, p. $24-25$.

1884: M e uni e r: Météorites, p. 79, 247. r884: v. $\mathrm{N}$ iess 1: Ueber,die astronomischen Verhältnisse bei dem Meteorsteinfall von Mócs in Siebenbürgen am 3. Februar 1882. Sitzber. Wien. Akad, Bd. 89 II , p. 291 (Hemmungshöhe). Ref. N. J. I886 I, p. 224.

r884: L i n d s t r ö m : Förteckning öfver Ricksmusei Meteoritsamling. Öfversigt af Kongl, Vetenskaps-A kademiens Förh. Stockholm (1884), Nr. 9. Ref. N. J. 1886 II, p. $38-39$.

1883/85: Tschermak: Photographien, p. 19. 
1885: Brezina: Wiener Sammlung, p. $183,184,233$.

1886': R e u s c h: Ueber den Tysnesmeteorit und drei andere in Skandinavien niedergefallene Meteorsteine. - III. Der Ställdalmeteorit (28. Juni 1876 II Uhr 32 Min. Vormittags, mittlere Zeit des Ortes). N. J. B. B. IV, p. 490 -493 (mit Abbildung von Dünnschliffen); s, auch Nyt Magazin for Naturvidenskaberne Bd. 29 , p. 323-328. 1887: F lig h t: Meteorites, p. 196-197. r89o: v. Niess l: Periheldistanzen, Verh. naturf. Ver. Brünn Bd. 29, p. 184, $185,186,188,189,194,214,251-252$. r892: B r ez i n a : Sternschnuppen, p. 14. r894: Cohen: Meteoritenkunde, p. $237,283,316$.

Ursprüngliches Gewicht: $34 \mathrm{Kgr}$. (Elf Steine von 2 I gr. bis $12400 \mathrm{gr}$.) Nachweisbares Gewicht: 31 926 gr.

\begin{tabular}{|c|c|c|}
\hline Bayet & 16 & Lund, M. \\
\hline Belgrad & 12 & Madrid \\
\hline Berlin, U. & 41 & de Mauroy \\
\hline Bologna & 8 & Paris, M. \\
\hline v. Braun & 224 & Pech \\
\hline Budapest & 342 & Rom, U. \\
\hline Cohen & 7 & v. Siemaschko $\quad 126$ \\
\hline Dorpat & 7 & Stockholm $\quad 23689$ \\
\hline Dresden, M. & II4 & Strassburg \\
\hline Freiberg, i. S. & $5^{2}$ & Turin, U. \\
\hline Gregory & 17 & Upsala $\quad 354^{8}$ \\
\hline Klausenburg & 70 & Washington, Sh. ${ }^{152}$ \\
\hline Kristiania & 221 & Wien, H. M. $\quad 3$ \\
\hline Kunz & ${ }^{15}$ & Wien, U. I. \\
\hline
\end{tabular}

Stannern, Iglau, Mähren, Oesterr.

Stein, Eu, gefallen 22. Mai 1808 . Hierher auch: Constantinopel, s. Anhang zu diesem Artikel.

1808: v. Ja cquin: Ein neuer Steinregen am 22. Mai 1808 , in Mähren (aus einem Briefe des Herrn Freiherrn von Jacquin in Wien an den Prof. W ül fing, Meteoriten.
Gilbert in Halle). Gilb. Ann. Bd. 28, p. 491 .

1808: Gil bert: Einige Nachrichten von den drei neuesten Steinregen, und von drei Meteorsteinen aus Russland. - 1. Steinregen bei Stannern in Mähren (aus einem Schreiben des Herrn Commissionsraths Busse in Freiberg an den Prof. Gilbert. Wien den 8. Junius 1808). Gilb. Ann. Bd. 29, p. 207-209.

1808: v. Schreibers: Nachrichten von dem Steinregen, der sich am 22sten Mai 1808 in und um Stannern in Mähren ereignet hat; gesammelt auf einer Reise nach Stannern, in Gesellschaft des Directors von Widmannstätten. Gilb. Ann. Bd. 29, p. $225-250$, 375; s. auch De Carro: Lettre du Dr. De Carro aux Editeurs de la Bibl. Brit. sur un Aérolithe tombé en Moravie. Vienne ce 4 juin 1808. - Bibl. Brit. Nr. 300 (Juni 1808), p. 193-195; Vaterl. Blätter f. d. Oesterr. Kaiserstaat Nr. I3, 2I. Juni 1808; Uebersetzung von De Carro in Bibl. Brit. Nr. 304 (August 1808), p. $392-402$ : Rapport plus détaillé de la chute d'Aérolithes, qui a eu lieu à Stannern en Moravie et dans ses environs. r808: M os e r: Darstellung der physischchemischen Eigenschaften der Steine, welche am 22. Mai 1808 bei und in Stannern in Mähren aus der Luft gefallen sind. Gilb. Ann. Bd. 29, p. 309 -327 (Analyse); s. auch Bibl. Brit. Nr. 306 (September 1808), p. 7I-90. r8og: Scherer: Bemerkungen über die mährischen Meteorsteine, vorzüglich in Hinsicht auf ihre Inkrustirung. Gilb. Ann. Bd. 3I, p. $1-22$ (mit 3 Tafeln). r8og: v. Schreibers: Beschreibung der mährischen Meteorsteine nach ihrem Aeussern, vorziiglich der Rinde, und nach ihrer Masse, und einige Folgerungen, auf welche diese Beschreibung führt. Gilb. Ann. Bd. 31, p. 23-77 (bezieht sich auf die gleichen Figuren, wie Scherer). 
r809: V a u que lin: Analyse del'aérolite tombé à Stannern en Moravie, le 22 mai 1808. Ann. Chim. Bd. 70, p. $32 \mathrm{I}-330$; s. auch Uebersetzung in Gilb. Ann, Bd. 33, p. 202-210.

1810: Kl a proth: Beiträge, Bd. 5, p. 257-264; s. auch Ann. Chim. Bd. 74 (1810), p. 94-95 (am Schluss der Arbeit über Lissa).

I8II: v. Da 1 berg: Meteorkultus der Alten. Heidelberg $\mathbf{I} 8 \mathbf{I}$, p. $\mathbf{I} 86$.

I81 2: $\mathrm{C} \mathrm{h} 1$ a d ni: Verzeichnis, Schweigg. Journ. Bd, 4 Beil. I, p, 16.

1812: Bigot de Morogues, p.229 -233 .

1815: Gillet-Laumont: Sur un Aérolithe tombé en Moravie, et sur une Masse de fer natif tombée en Bohême. Journ. des Mines Bd. $3^{8}$, p. 232233.

1815: Chladni: Neues Verzeichnis, Gilb. Ann, Bd. 50, p. 254.

1816: $\mathrm{Ch} 1$ adn i: Erste Fortsetzung, Gilb. Ann. Bd. 53 , p. 383 .

18rg: $\mathrm{Chl}$ a d n i: Feuermeteore, p.VII, $44,45,46,49,50,51,53,55,56$, $57,58,59,66,68,72,73,91,150$, $257,271,277,286-289$ (1), 308, 423 . 1820: La ug i er: Extrait d'un Mémoire lu à l'Académie des Sciences le $I_{\mathrm{er}}$ mai 1820, et intitulé: Faits pour servir à l'histoire chimique des pierres météoriques. Ann. Chim. Phys. Bd. 13, p. $440--442$; s, auch Gilb. Ann. Bd. 68 (1821), p. 428.

1820: v. S c hreibers: Beiträge,p.20 $-39,40,4 \mathrm{I}, 59-60,69,87-92$ (mit Tafel IV-VII und Situationsplan); s. auch Döll's Jahrb. k. k. Geol. Reichsanst. Bd. 37 (1887), p. 193-194.

1822: Ha tï y: Traité de Minéralogie. II. Auflage. Paris 1822 Bd. 3 , p. 536 . 1824: Chladni: Vierter Nachtrag, Pogg. Ann. Bd. 2, p. 154.

1824: M o h s: Grundriss der Mineralogie. Dresden 1824 Bd. II, p. 313 ; s. auch Mohs-Zippe, Naturgeschichte des Mineralreichs 2. Aufl. Bd. 2 (1839), p. 309 .

1825: R o s e: Ueber die in den Meteorsteinen vorkommenden krystallisirten Mineralien. Pogg. Ann. Bd. 4, p. 185 (beiläufig: Burkart, Fundorte I, N. J. 1856, p. 269).

1833: v. Holger: Analyse des Meteorsteins von Stannern. Baumgartner's Zeitschr. f. Phys, u. verw. Wissensch. Bd, 2, p. 293-307; s. auch N. J. 1836 , p. 497 . 1834: Berzeli us: Om Meteorstenar. Kongl. Vetensk. Acad. Handl. (1834), p. $178-181$; s. auch Pogg. Ann. Bd. 33 (1834), p. $144-146$.

1836: K ä m t z: Meteorologie,p.258,282. r84I : R a m me $1 \mathrm{~s}$ ber $\mathrm{g}$ : Handwörterbuch, Bd. I, p. $427-428$.

1843: Parts ch: Meteoriten, p. 17-26. 1846: Shepard: Report on Meteorites. Am. Journ. (2) 2, p. 379, 38r. 1847: S chafhäut l: Schönenberg,

Gel. Anz. München. Akad. Bd. 24, p. $556-55^{8}, 571,580$.

I848: B lo ed e: Tabelle, Bull. Petersburg. Akad. Bd. 6, p. 13-14.

r85r: R a mmelsberg: Ueber die Zusammensetzung des Meteorsteins von Stannern. Pogg. Ann. Bd. 83 , p. $591-$ 593 (neue Analyse). Ref. N. J. 1853 , p. 699-700; Am. Journ. (2) 14 (1852), p. 279; Liebig-Kopp, Jahresber. 1851, p. 880 ; Pharm. Centr. 1851, p. 857 ; Kenngott, Uebersicht $1850 / 51$, p. 179. 1853: R a mme ls berg: Suppl, V, p. $3 \mathrm{I}-32$.

1854: v. Boguslawski: Zehnter Nachtrag, Pogg. Ann. Ergz.-Bd. 4, p. 13-14, 19.

1857: A rago: Astronomie populaire. Bd. 4, p. 196.

1859: H a rri s: Dissert. Gött., p. 68 -69 .

1859: B u chner: Feuermeteore, p. 78 -80 .

1860: H a i d ing er: Eine Leitform der Meteoriten. Sitzber. Wien. Akad. Bd. 
40, p. 526-528 (mit Tafel); s auch "L'Institut « Bd. 28 (1860) Nr. 1379, p. 192 ; Liebig-Kopp, Jahresber. 1860, p. 844 ; Luminous Meteors, Rep. Brit. Assoc. 186I (Sep.), p. 33; Kenngott, Uebersicht 1859, p. 200.

1860: R a m mels berg: Mineralchemie, p. 92I, $936-937,950,951,952$. r862: Kenng ott u. W is er: Zürcher Sammlung, p. $\mathbf{I}_{4} 8, \mathbf{I}_{5} 8$.

1862: Greg: On some Meteorites in the British Museum etc. - Note on Bluish Meteorites. Philos. Magaz. Bd. 24 , p. 542 .

1862: Haidinger: Stannern. Ein zweiter Meteorstein, durch seine Rinde genau in seiner kosmischen Bahn orientiert. Sitzber. Wien. Akad. Bd. 45 II, p. 790-795 (mit Tafel); s auch sL'Institut a Bd. 30 (1862) Nr, 1500, p. 323. 1863: B u c hner: Meteoriten,p.23-26. 1863: R o s e: Meteoriten, p. 26, 27, 126, $133-134,135,147,151,156$.

1864: Sor by: On the Microscopical Structure of Meteorites. Proc. Royal Soc. Bd, 13, p. 333-334. Ref. Am. Journ. (2) 41 (1866), p. 138.

r864: H a id ing e r: Sternschnuppen, Feuerkugeln und Meteoritenschwärme im Zusammenhange betrachtet. Sitzber. Wien. Akad. Bd, 49 II, p. 6.

1857/65: v. Reichen b a h: III624. IV 637 . V $475,476,477,478,479$. VI 445 . VII $552,558,56 \mathrm{I}, 562$. VIII 476,477 . IX $155,159,160,167,177$. $\mathrm{X} 359,362,365,371,372$. XI 295, $296,297,308$. XIII $353,356,364$, 368 (Fig.), 37 I (Fig.), 372, 377. XIV $389,390,396$. XV ror. XVIII 490. $\mathrm{XX} 621,623,626,631$. XXIV 226. XXV $315,421,422,428,438,600$, 601,607 .

1865: R o s e: Systematische Einteilung der Meteoriten. Pogg. Ann. Bd, I24, p. $210-2 \mathrm{II}$.

1867: Go e b e 1: Kritische Uebersicht, Mélanges phys, chim. Bd.7,p.284-285. r868: M o h r: Bildung der Meteoriten. Verh. naturh. Ver. Bonn Bd. 25 (Sitzber.), p. 65 .

1870: v.H a i d ing e r: Orientierung, Sitzber. Wien. Akad. Bd. 6I II, p. 512.

1870: Tschermak: Goalpara, Sitzber. Wien. Akad, Bd, 62 II, p. 862 . r870: Rammels berg: Meteoriten, p. 127-129; s, auch Pogg. Ann. Bd. 140 (1870), p. $32 \mathrm{I}$.

1872: Tschermak: Die Meteoriten von Stannern, Constantinopel, Shergotty und Gopalpur. - Stannern. M. M. (1872), p. 83-87. Ref. Liebig-Kopp, Jahresber. 1872 , p. $1194-1195$.

x872: Tschermak: Die Meteoriten von Shergotty und Gopalpur. Sitzber. Wien. Akad. Bd. 65 I, p. 127.

1874: $\mathrm{T} \mathrm{s} \mathrm{ch} \mathrm{erma} \mathrm{k:} \mathrm{Der} \mathrm{Meteoriten-}$ fall bei Ovifac in Grönland. M. M. (1874), p. 168.

1875: Tschermak: Vulkanismus, Sitzber. Wien. Akad. Bd, 7I II, p. 662.

1875: Mohr: Entstehungsart, Ann. Chem. Pharm. Bd, 179, p. 259, 260, 261, 262 .

1875: v o m Rath: Meteoriten, Verh. naturh. Ver. Bonn Bd. 32, p. 375 .

1877: Sorby: On the structure and origin of Meteorites. Nature Bd. 15 , p. 495 .

1879: R a m me ls berg: Meteoriten, p. 25 .

1882: Brezina: Bericht IV, Sitzber. Wien. Akad. Bd. 85 I, p. $336,34 \mathrm{I}, 342$. r882: W i e c hmann: Fusion-Structures in Meteorites. Ann. N. Y. Acad. Sc. Bd. 2, p. 293, 295 (Taf, 20).

r883: Ts c herm a k : Beitrag, Sitzber. Wien. Akad. Bd. 88 I, p. 349,368 -370 .

1884: Wa d s w or th: Studies, p. 195. r884: Meunier: Météorites, p. 75, 93, 95, 96, 98, 295, 296 (Abb.), 351,524 .

1883/85: Ts chermak: Photographien, T. 1,3 , p. $3,6,7,8$.

$22 *$ 
1885: Brezina: Wiener Sammlung, p. $173-174,176,232$.

r886: Meli o n: Die Meteorsteinfälle in Mähren. Notiz-Bl. d. mähr. schles. Ges. zur Beförd. d. Ackerbau's etc. (1886) Nr. 5 u. 6 , p. I-4, 7, 9, I0, II. 1887: F1 ig ht: Meteorites, p. 98-99. 1888: N ew to n: Orbits, Am. Journ. (3) 36, p. 5 .

r889: v. H a u e r : Ann. Hof-Mus, Bd. 4 (Not.), p. 64.

1889: F l e t c h er: Atacama Meteorites, Mineral. Magaz. Bd. 8, p. 226 (Streufeld 8 auf 3 miles).

1890: v. $\mathrm{N}$ i e s s 1 : Periheldistanzen, Verh. naturf. Ver. Brünn, Bd. 29 , p. 188 , I94, 105, 214, 243.

1892: B r e z in a : Sternschnuppen, p. 12 -13 .

1894: Co hen: Meteoritenkunde, an vielen Stellen.

r894: F le t c h er: Introduction, p. 9, I3, 27, 37 .

Ursprüngliches Gewicht: Von 200 -300 Steinen, welche (nach Partsch) gefallen sein mögen, wurden 66 mit einem Gesamtgewicht von etwa $52 \mathrm{Kgr}$. (93 Pfund) gesammelt.

Nachweisbares Gewicht: $3_{8}^{8} 408$ gr.

\begin{tabular}{lr|lr} 
Aachen & 83 & Breslau & I 27 \\
Bailey & 73 & Brezina & 170 \\
Bayet & I & Brüssel & 13 \\
Belgrad & 63 & Budapest & 2223 \\
Bement & 58 & Calcutta & 592 \\
Berlin, G. & 71 & Cambridge & 104 \\
Berlin, P. & 4 & Cleveland & 28 \\
Berlin, U. & 1496 & Cohen & I \\
Böhm & 82 & Czernowitz & 108 \\
Bologna & 525 & Darmstadt, M. & 40 \\
Bonn & 13 & Debreczin & I14 \\
v. Braun & 334 & Dorpat & 27
\end{tabular}

\begin{tabular}{|c|c|c|}
\hline Dresden, M. & 400 & Odessa \\
\hline Dublin, M. & 265 & Paris, E. \\
\hline Frankfurt & 17 & Paris, M. \\
\hline Freiberg, i. S. & 168 & Paris, U. \\
\hline Frenzel & 20 & Parma \\
\hline Gent & 32 & Petersburg, A. \\
\hline Gotha & $33^{8}$ & Petersburg, B. \\
\hline Göttingen & 312 & Pohl \\
\hline Graz, J. & 390 & Prag, B. U. \\
\hline Gregory & 161 & Prag, D. U. \\
\hline Greifswald & 36 & Prag, M. \\
\hline Hamburg & 14 & Riga \\
\hline Harvard, U. & 225 & Riga, P. \\
\hline Helsingfors & 63 & Rom, U: \\
\hline Karlsruhe, P. & $3^{8}$ & v. Schilling \\
\hline Kasan & 16 & v. Siemaschko \\
\hline Klausenburg & 104 & Stockholm \\
\hline Kopenhagen & 310 & Strassburg \\
\hline Krantz & 132 & Stürtz \\
\hline Kristiania & 29 & Stuttgart \\
\hline Leipzig & II9 & Szamosujvar \\
\hline London,B.M. & I 584 & Troyes \\
\hline London, P. G. & 508 & Tübingen \\
\hline Lüttich & I & Turin, U. \\
\hline Madras & IOI & Upsala \\
\hline Madrid & 8 & Utrecht \\
\hline Mannheim & $\left.9^{\prime}\right)$ & Ward \\
\hline Marburg & 87 & Washington $\quad 47$ \\
\hline de Mauroy & 5 & Washington, Sh. 26 \\
\hline Melion & 98 & Wien, H. M.*)**) \\
\hline Minneapolis & I & $\quad 15588$ \\
\hline Modena & 379 & Wien, U. I. \\
\hline Moskau & 22 & Wien, U. II. \\
\hline München & 442 & Wrany \\
\hline Neumann & 805 & Würzburg \\
\hline New Haven & 107 & Zürich \\
\hline
\end{tabular}

St annern-Constantinopel, Türkei. Stein , Eu, soll gefallen sein Juni I 805 , erwähnt 1807 . Es ist sehr wahrscheinlich, dass die unter dem Namen Constantinopel in den Sammlungen aufbewahrten

1) >Vom Steinfall in Mähren 1807 herrührend «.

2) Davon 6 gr. als sLuotolaks \& bezeichnet, von Brezina als Stannern bestimmt, dem es in der That vollkommen gleicht. 
Steine von Stannern herrühren. 1807: Haïr-Kougas-Ing is i an (Ingig i a n) hat nach Bigot de Morogues u. a. über den Fall geschrieben u. zwar in einem armenischen Werk : Egang (Eghang) - Busankian (Buzankian), welches 1807 im armenischen Kloster in Venedig gedruckt sein soll; s, auch Anmerkung im Journ. des Mines $\mathrm{Bd} 23$ (1808), p. 140.

1812: Bigot de Morogues, p. 20 I -202 .

1812: C h la d n i: Verzeichnis,Schweigg. Journ. Bd. 4 Beil. I, p. 15 .

r8r5: Chladni: Neues Verzeichnis, Gilb. Ann. Bd. 50, p. 253.

r8rg: $\mathrm{Ch}$ l a d n i: Feuermeteore, p. 4I, $66,74,278$ (1).

1822: Chlad n i: Zweiter Nachtrag, Gilb. Ann. Bd. 71, p. $36 \mathrm{I}-362$.

1836: Käm tz: Meteorologie, p. 280. r843: Parts c h: Meteoriten, p. 26. I854: B a $1 \mathrm{c}$ e $11 \mathrm{~s}$ : Lithologia meteorica, p. 22.

1854: v. Boguslawski: Zehnter Nachtrag, Pogg. Ann. Ergz.-Bd.4, p.427. 1859: H arris: Dissert. Gött., p. 66. r863: B u c hn er: Meteoriten, p. r9. 1863: Ros e: Meteoriten, p. 26, 27, 126.

r858/65: v. Rei chen ba ch: V 476, 478 . IX $160,167,177 . X_{362,365}$ 372. XI 294. XIII 368 (Fig.), 370, 372 (Fig.). XIV 396. XV ror, XX 631 . XXV 32I, 607.

1872: Ts ch ermak: Die Meteoriten von Stannern, Constantinopel, Shergotty und Gopalpur. M. M. 1872 , p. 85 -87 (Analyse von Ludwig).

1883: T s chermak: Beitrag, Sitzber. Wien. Akad. Bd. 88 I, p. 368.

1884: W a d s w or th: Studies, p. 195. 1883/85: Ts chermak: Photographien, p. 6 .

1885: Brezina: Wiener Sammlung, p. $173-174,232$.

1887: Flight: Meteorites, p. 97, 98.
Ursprüngliches Gewicht: Nicht bekannt.

Nachweisbares Gewicht: 6 gr.

Bailey Spl. v. Siemaschko Spl.

Calcutta Spl. Tübingen Spl.

Pohl Spl. Wien, H. M.*) 6

Staunton, Augusta Co., Virginia, U. S. A.

Eisen, Om, gefunden $185^{8}$ oder 1859 , beschr. 1871 . Brezina trennt die $185^{8}$ gefundene und 1878 beschriebene vierte Masse, Staunton IV, von den übrigen 4 Massen: 1869 Staunton I u. II; 187 I Staunton III; 1887 Staunton V. Hierher auch vorläufig: Louisa Co., Virginia(s.Anm.zu diesemArtikel).

1871: M a 11 et: On three Masses of Meteoric Iron, from Augusta Co., Virginia. Am. Journ. (3) 2, p. 10-I5 (Analyse; Abbildung der drei ersten Massen und geätzter Platten); s. auch Rep. Brit. Assoc. 1872 (Brighton), p. 77; Ber. d. d. chem. Ges. Bd. 5 ( 1872 ), p. 591 $-592,813$; Liebig-Kopp, Jahresber. I871, p. 1242-1243.

1872: Mallet: Examination of the Gases occluded in Meteoric Iron from Augusta Co., Virginia. Proc. Royal Soc. Bd. 20 (1872), p. $365-370$; s. auch Philos. Magaz. Bd. 44 (1872), p. $3 \mathrm{II}-3 \mathrm{I} 5$; Am. Journ. (3) Io (I875), p. 206 u. (3) II (I876), p. 257-258; Chemical News Bd. 25 , p. 292 ; „L'Institute 1872, p. 365; Pogg. Ann. Bd. 147 (1872), p. 134-140; LiebigKopp, Jahresber. 1872 , p. III-II92. 1875: Mallet: Note on the Gases accompanying Meteorites. Am. Journ. (3) Io, p. 206.

r876: Wright. On the Gases contained in Meteorites. Am. Journ. (3) 12, p. 167 (Mallet).

1878: Mallet: On a fourth mass of Meteoric Iron from Augusia County. 
Virginia. Am. Journ. (3) 15, p. 337$33^{8}$ (Analyse von Santos; Abbildung der vierten Masse). Ref. Liebig-Kopp, Jahresber. 1878 , p. I321.

1879: R a m me ls berg : Meteoriten, p. $4-5$.

r880: Bre z in a: Reichenbach'scheLamellen, Denkschr.Wien. Akad. Bd. 43, p. 14-15 (Abbildungen von geätzten Platten).

I882: v. L a s a u $1 x$ : Vermehrung, Verh. naturh. Ver. Bonn Bd. 39 (Sitzber.), p. 100 .

1883: S m it h : Concretions, Am. Journ.

(3) 25, p. 418

1884: Meunier: Météorites, p. 14-r8, I9, 99, I16, 126-I27, 502-503.

r884: Wad sw or th: Studies, p. 60. 1885: Brezina: Wiener Sammlung,

p. $155,207,2 \mathrm{II}, 234$.

I887: F lig ht: Meteorites, p. I3-I5, $\mathrm{I} 8 \mathrm{I}-\mathrm{I} 82$.

I887: K unz: A fifth mass of Meteoric Iron from Augusta Co., Va. Am. Journ.

(3) 33 , p. 58-59 (Analyse von Mallet; Abbildung der fünften Masse). Ref. N. J. 1888 II, p. 34 .

I890: E a s tm a n: Met. Astron., p. 320, 322.

I89r: v. H a u er: Ann. Hof-Mus. Bd. 6. (Not.), p. 54 .

r8gr: How e 11 : Proc. Rochester Acad. Bd. I, p. $173-174$.

r89r: Cohen u. Weinschenk: Meteoreisen-Studien, Ann. Hof-Mus. Bd. 6, p. I3I, I45-I46 (Analyse), I63, $164,165$.

1892: Co he n: Meteoreisen-Studien II. Ann. Hof-Mus. Bd. 7, p. $156-157$ (Analyse), ${ }_{5} 8$ (Cu), 160.

r893: Brez in a: Ueber neuere Meteoriten (Nürnberg), p. 165, I66.

r893: M eunier: Revision des fers météoriques, p. 52, 57-58 (Abb. einer geätzten Platte).
1894: Cohen: Meteoritenkunde, an vielen Stellen.

r895: Brez ina: Wiener Sammlung, p. 278,279 .

I895: C o h e n : Meteoreisen-Studien IV. Ann. Hof-Mus, Bd, 10, p. $82,90$.

1896: R a m s a y: On Argon and $\mathrm{He}-$ lium. Chem. News Bd. 71 (1895), p. 259 (Nr. 14). Ref. N. J. 1896 II, p. 232. Ursprüngliches Gewicht : I I $3964 \mathrm{gr}$. 5 Massen: I) 25429 gr. ; 2$)$ I6 44 I gr. ; 3) r644 gr.; 4) 68950 gr.; 5) etwa $I_{500}$ gr. nach Abbild. geschätzt.

Nachweisbares Gewicht: 51 627 gr.

\begin{tabular}{|c|c|c|}
\hline Bailey & $\left.219^{1}\right)$ & Moskau \\
\hline Belgrad & 22 & München \\
\hline Bement & 1143 & Münster \\
\hline Berlin, U. & 1475 & Neumann \\
\hline Böhm & 120 & New Haven \\
\hline Bologna & 96 & Newton \\
\hline Bonn & 354 & New York, M. 217 \\
\hline v. Braun & 103 & Paris, M. $\quad 2780$ \\
\hline Budapest & 6785 & Petersburg, B. \\
\hline Calcutta & 1180 & Pohl \\
\hline Cleveland & 73 & Prag, M. \\
\hline Dorpat & 74 & Roebling \\
\hline Dresden, M. & 128 & Sidney $\quad 1346$ \\
\hline Edinburg & I064 & v. Siemaschko 1263 \\
\hline Göttingen & 318 & Stockholm \\
\hline Graz, J. & 227 & Strassburg \\
\hline Gregory & 220 & Strüver \\
\hline Greifswald & 160 & Stürtz \\
\hline Harvard, U. & 4595 & Stuttgart \\
\hline Howell & 220 & Troyes \\
\hline Kopenhagen & $7^{8}$ & Turin, U. \\
\hline Leiden & $5^{8}$ & Upsala \\
\hline Leoben & 60 & Utrecht \\
\hline London, B. M. & 2797 & Ward \\
\hline London, P. G. & $3^{\mathrm{II}}$ & Washington $\quad$ I55 \\
\hline Lüttich & $5^{2}$ & Washingt.,Sh.1662 ${ }^{2}$ ) \\
\hline de Mauroy & 36 & Wien, H.M.*) $6001^{3}$ ) \\
\hline $\begin{array}{l}\text { Melion } \\
\text { Minneapolis }\end{array}$ & $\begin{array}{r}4 \\
90\end{array}$ & Wien, U. I. $\quad 1750$ \\
\hline
\end{tabular}

1) Davon 152 gr. von der 1858 gefundenen Masse, - 2) Vom Jahre 1869 . 3) 4828 gr. Staunton IV. Ausserdem 453 gr, von Staunton V nach I. Juli 1893 erworben. 
Ueber Louisa Co, , wovon Howell und Ward je I gr. besitzen, teilte mir ersterer mit: ? There were only a few grains found of the Louisa Co., Va., iron. It was much decomposed. It closely resembles the Augusta Co. iron but was found a large distance from where any of the pieces of that iron were found ๙. Ferner teilte mir Herr Howell mit, dass er die Lokalität im Am. Journ. erwähnt habe. Die Stelle konnte ich leider nicht finden.

Wo befinden sich die grössten Massen dieses Eisens?

Stawropol, Kaukasus, Russland.

Stein, Ck, gefallen 24. März 1857 . 1860: A b i ch: Ueber einen bei Stawropol gefallenen Meteorstein. Bull. Petersburg. Akad. Bd. 2 , p. $404-422$, 433-440 (Analyse). Ref. N. J. 1862, p. 108-109; Liebig-Kopp, Jahresber. 186o, p. $847-848$; Kenngott, Uebersicht $186 \mathrm{I}$, p. $165-166$.

1862: Greg: On some Meteorites in the British Museum etc. Philos. Magaz. Bd. 24 , p. $54 \mathrm{I}$.

1863: B u c hner: Meteoriten, p.92-93. 1863: Rose: Meteoriten, p. 85,89 , 91, 93, 96-98(Taf. IV), 105, 154, 16r. 1867: Go be 1: Kritische Uebersicht, Mélanges phys. chim. Bd, 7, p. 337 . r867: Buchner: Dritter Nachtrag, Pogg. Ann. Bd, 132, p. 318.

r870: Rammels berg: Meteoriten, p. 102, 103, 105, 106, 138, 139, 140. 1879: R a m mels berg : Meteoriten, p. 24,25 .

1884: Meunier: Météorites, p. 74, 77, $79,85,96,255-256$ (Abb.), 369, $370,371,395$.

r883/85: Ts ch ermak: Photographien, T. 18 , p. 20.

1885: Brezina: Wiener Sammlung, p. 191,233 .

Ursprüngliches Gewicht : $163^{2}$ gr. (nach Buchner's Referat).
Nachweisbares Gewicht: 1527 gr.

\begin{tabular}{lr|lr} 
Berlin, U. & 93 & Paris, M. & I9 \\
Budapest & 6 & Petersburg, A. I278 \\
Calcutta & I & v. Siemaschko & 5 \\
Dorpat & 3 & Tübingen & 9 \\
Gregory & I & Washington, & 52 \\
London, B. M. & 23 & Wien, H. M.*) & 22 \\
London, P. G. & I5 & &
\end{tabular}

Steinbach, Sachsen, Deutschland. Siderophyr, S, gefunden?, beschr. I 751 bez. 1565 .

Hiermit sind vereinigt:

I) Grimma, Sachsen; vielleicht gefallen um $1540-1550$, beschrieben ${ }_{15} 65$, in Sammlungen nachweisbar um 1724, jetzt nur in Gotha. Stromeyer (1824) sagt: ... Eisenmasse, welche in dem Herzogl. Naturalien-Cabinete zu Gotha verwahrt wird, und in der vor etwa roo Jahren für das Cabinet angekauften MineralienSammlung des ehemaligen Sächsischen Oberberghauptmanns von Schönberg enthalten war.

2) Rittersgrün, bei Schwarzenberg, Sachsen; gefunden 1847 (Weisbach sagt 1833), beschr. 186r.

3) Breitenbach, Bezirk Platten,Kreis Elbogen,Böhmen; gefunden 186I, beschr. 1862 .

1565: Conrad Gessner: De omni rerum fossilium etc. Tiguri 1565. III Teil, p. 26 unter: De metallicis rebus ac nominib. Observationes variae ex Schedis Georgij Fabricij (s. Chladni 1819).

1590: Petrus A l bin us: Meissnische Bergchronik, p. 135 (enthält die deutsche Uebersetzung der obigen Angabe von Fabricius).

1751: Lehman n, J. G.: Kurze Einleitung in einige Theile der Bergwerkswissenschaft, Berlin 1751, p. 79-80. 
I769: Spicilegium quarundam rerum naturalium subterranearum Lipsiae collectarum. Leipzig 1769. Taf. II; s. auch Chladni in Gilb. Ann. Bd. 50, p. 259 -262 (Anmerkung von Gilbert).

1776: Verzeichniss der Fossilien in dem zur allgemeinen Oekonomie gewidmeten Gebäude der kaiserlichen Theresianischen Akademie. Wien 1776.

1802: $\mathrm{H}$ ow a $\mathrm{rd}$ : Experiments and $\mathrm{Ob}-$ servations etc.; Philos. Trans. (1802), p. 209-2Io; s, auch Gilb. Ann. Bd. $I_{3}(\mathrm{I} 803)$, p. 325 .

1803: K la proth: Ueber meteorische Stein- und Metallmassen. Abh. Berlin. Akad. (1803), p. 39; s. auch Gilb. Ann. Bd. 13 ( 1803$)$, p. 340.

1807: K lap r oth: Beiträge, Bd, 4, p. 99.

1808: Ch la d n i: Beiträge, Gilb. Ann. Bd. 29, p. 379 (Abdruck der Angabe von Fabricius; anstatt: sin siluis Neuhorianis\& schreibt Chladni sin silvis Neuhofianise).

1812: Bigot de Morogues, p. 67. 1815: Chladni: Neues Verzeichnis, Gilb. Ann. Bd. 50, p. 237.

1815: Chla dni: Bemerkungen, Gilb. Ann. Bd. 5o, p. 259-262.

18r6: Chladni: Zweite Fortsetzung, Gilb. Ann, Bd. 54 , p. 344 .

r817: v. Soemmering: Ueber die Zeichnungen, welche sich bei Auflösung des Meteoreisens bilden. Schweigg. Journ. Bd. 20 , p. 92 .

r8rg: Chla d n i: Feuermeteore, p. 92, 212-213, 326 (Gothaer Stück), 324 (1), 433.

1824: Strome yer: (Referat einesVortrags über Olivin). Gött. Gel. Anz. $(1824)$, p. $2082-2083$ (Analyse, welche Stromeyer auf ein Trisilikat deutet).

r825: Ros e: Ueber die in den Meteorsteinen vorkommenden krystallisirten Mineralien. Pogg. Ann. Bd. 4, p. 194 - I96 (Stromeyer'sAnalyse des Minerals von dem Siderolith in Gotha).
1826: See beck: Ueber die magnetische Polarisation der Metalle und Erze durch Temperaturdifferenz. Pogg. Ann. Bd. 6 , p. 144 .

1843: P a r t s c h : Meteoriten, p. 91 -95 (das erwähnte Stück aus der Schlotheim'schen Sammlung, welches wie das Gotha'sche von Grimma kommen sollte, ist in Wien nicht mehr vorhanden).

1852: C 1 a r k: Dissert. Gött., p. 20-2I, 1854: v. Boguslawski: Zehnter Nachtrag, Pogg. Ann. Ergz, - Bd. 4, p. 384 .

r859: B u c hner: Feuermeteore, p. 37. 1859: Harris: Dissert. Gött., p. IO2. r860: R a mmels berg: Mineralchemie, p. 503 (Stromeyer's Analyse des sog. Trisilikates); s. auch Liebig-Kopp, Jahresber. I89I, p. II3I.

I86r: B reit ha u $\mathrm{t}$ : Auf einer Reise im Inlande während unserer letzten Hauptferien habe ich glücklicher Weise eine bereits vor 14 Jahren gefundene und ${ }_{173}$ Pfund schwere Eisenmasse ausgekundschaftet, und zwar bei dem Finder, einem Bauer zu Rittersgrün bei Schwarzenberg «. Zeitschr. d. d. geol. Ges. Bd. 13, p. 148. Ref. N. J. 1862, p. 490-49I; Liebig-Kopp, Jahresber. I86r, p. 1128 .

1862: Breithaupt macht das Ergebniss der von Herrn Dr. Rube ausgeführten Analyse des Rittersgrüner Meteoreisens bekannt. Berg-u.Hüttenm. Zeitung, Jahrg. 21, p. 72. Ref. Kenngott, Uebersicht 1862165 , p. 266,267 ; Liebig-Kopp, Jahresber. 186I, p. 1128. 1862: B r e it h a up t: Vorläufige Nachricht über den Eisen-Meteorit von Rittersgrïn. Berg- u. Hüttenm. Zeitung, Jahrg. 21, p. $321-322$; s, auch Wochenschr. f. Astr., Met. u. Geogr. 1864, p. $13-14$.

I858/62: v. Reichen ba ch: VI 442. IX 162, 163, I73, 181. XI 302. XII 452-454. XV 10I, 107, 108, 109, 112, 
II3, 116, 122, 131. XVI 261. XVII 265, 272. XVIII 48r. XX 627.

1863: B u chner: Meteoriten, p. 124 -126 .

1863: R o s e: Meteoriten, p. 4I, 73, 77, $78-79$ (Grimma), 80, 153; s. auch Mon.-Ber. Berlin. Akad. I862, p. 556 -557 .

1864: Kenng o $t$ : Notiz über ein Meteoreisen in der Universitäts-Sammlung in Zürich. Sitzber. Wien. Akad. Bd. 49 II, p. 467-469. Ref. Kenngott, Uebersicht $1862 / 65$, p. 266 .

r864: $\mathrm{H}$ a i d ing er: Bemerkungen über das von Fierrn Professor Kenngott in der Züricher Universitätssammlung aufgefundene Meteoreisen. Sitzber. Wien. Akad. Bd. 49 II, p. $469-470$.

1864: R o s e legte als neue Erwerbung des mineralogischen Museums 2 neue Meteoriten vor, ein Meteoreisen (Werchne Udinsk) und einen andern Meteoriten, der wahrscheinlich ein Mesosiderit ist. - Der zweite Meteorit wurde bei Breitenbach in Böhmen von Herrn Osius in Freiberg gefunden. Zeitschr, d. d. geol. Ges. Bd. r6, p. 355-356.

1864: B u chner: Erster Nachtrag, Pogg. Ann. Bd. 122, p. 318.

1867: D a u b r é e : Contribution à l'anatomie des météorites. C. R. Bd. 65 , p. $148-151$.

r869: Meunier: Recherches, Ann. Chim. Phys. (4) 17, p. 52.

r869: B u c h n er: Die Aetzfiguren des Meteoreisens,- Rittersgrün bei Schwarzenberg in Sachsen. Ber. Oberhess,Ver. f. Natur- u. Heilk. Giessen (1869), p. III - II2.

1869: v. L a n g : Ueber den Enstatit im Meteoreisen von Breitenbach. Sitzber, Wien. Akad. Bd. 59 II, p. $848-856$ (Tafel); s. auch Pogg. Ann. Bd. 139 (1870), p. $315-318 ;$ s. ferner Meunier's Arbeit über den Enstatit von Copiapo (Deesa) in Sitzber.Wien. Akad, Bd. 6r II (1870), p. $26-28$.
1869: M a k el y ne: Preliminary Notice on the Mineral Constituents of the Breitenbach Meteorite. Proc. Royal Soc. Bd. I7 (Nr. III), p. $370-372$ (Analyse von Enstatit und Tridymit). Ref. Verh. naturh. Ver. Bonn (Sitzber.) Bd, 27 (1870), p. 159-160; Zeitschr. f. Kryst. Bd. 2 (1878), p. $273-274$; Proc. Royal Soc. Bd. 19 (187r), p. 266 267 ; Liebig-Kopp, Jahresber. I869, p. $1297-1298$ u. 1871 , p. 1237.

r869: B u chner: Vierter Nachtrag, Pogg. Ann. Bd. 136, p. 598-599.

r870: R a mmelsberg: Meteoriten, p. $79,89-91,139$; s. auch Pogg.Ann. Bd. 140 (1870), p. $316-319$.

r87r: Maskelyne: On the Mineral Constituents of Meteorites. - XII. The Breitenbach Meteorite. XIII. Bronzite of the Breitenbach Siderolite (Analyse). XIV. Silica crystallized in the Rhombic System, as a Constituent of the Breitenbach Siderolite (Analyse). XV. Iron of the Breitenbach Siderolite (Analyse). Philos. Trans. Bd. 16r, p. $359-365$; s. auch Proc. Royal Soc. Bd. 19 (1871), p. 266-267; Philos. Trans. 1870, p. 212 ; Verh. naturh.Ver. Bonn Bd. 30 (1873), p. 107-108; Bergu. Hüttenm. Zeitung Bd. 32 (1873), p. 245; Zeitschr. d. d. geol. Ges. Bd. 25 (1873), p. IO7; Pogg. Ann. Ergz.-Bd. 6 (1874), p. $3^{82}-3^{84}$; Rammelsberg, Mineralchemie 2. Aufl. 1875 , p. 164 . r872: Q uenstedt: Klar und Wahr, p. 29I-292 (Abbildung einer geätzten Platte).

1873: vom R a th: Pogg. Ann. Ergz.Bd. 6, p. 382 (Analyse des Asmanit). Ref. Liebig-Kopp, Jahresber. 1873, p. 1250.

1875: G oe be 1: Bull.Petersburg.Akad. Bd. 20 , p. 129-130 (Vergleich mit Krasnojarsk).

r875: vom R at h: Meteoriten, Verh. naturh. Ver. Bonn Bd. 32 , p. $365-$. 366. 
1876: We is b a ch: Der Eisenmeteorit von Rittersgrün im sächsischen Erzgebirge. Freiberg 1876 ( 3 Seiten. Abbildung in Farbendruck). Verlag d. k. Bergakademie, gedruckt bei J. C. F. Eichler, Freiberg; s. auch Verh, naturh. Ver. Bonn Bd. 33 (1876), p. 92 ; N. J. 1876, p. 934-935 ; Liebig-Kopp, Jahresber. 1876 , p. 1316.

1877: W e bs ky legt ein Stück des Meteoreisens von Rittersgrün vor. Zeitschr. d. d. geol. Ges. Bd. 29 (1877), p. 418 . 1878: Winkler: Die Untersuchung des Eisenmeteorits von Rittersgrün. Nova Acta K. Leop. Karol, Akad. Bd. 40 Nr. 8, p. $333-382$ (Analyse). Ref. N. J. 1879, p. 902-905; LiebigKopp, Jahresber. 1879, p. 1275-1276. 1879: R a m mels berg : Meteoriten, p. $7-10,24,25$.

I88I : Fouqué et Michel Lévy : Expériences synthétiques relatives à la reproduction artificielle des météorites. C. R. Bd. 93, p. 675 .

I882: W e is b a c h: Mineralogische Notizen II. N. J. 1882 II, p. 253 (BroncitMessung). Ref. Liebig-Kopp, Jahresber. I882, p. 1557 .

1883: Ts chermak: Beitrag, Sitzber.

Wien. Akad. Bd. 88 I, p. 348 .

r884: M e un i er: Météorites, p. 35, 7I, $73,79,80,84,85,93,97,99$, 16r (Abb.) $-164,395,499-500$.

r884: Wa ds worth: Studies, p. 72 -73 .

1883/85: Ts chermak: Photographien, T. 25, p. $4,5,18,22$.

1885: Brez in a : Wiener Sammlung,

p. 152, 154, 155, 203, 206, 233.

1886/87: B rezina u. Cohen: Photographien, T. $1,2$.

r887: F lig h t: Meteorites, p. 85, r3I136, $168-170$.

r889: C o h e n: São Julião, N. J. 1889 I, p. $222-223,224,225$.
1890: Brezina: Ueber Meteoreisen, Oesterr. Zeitschr. f. Berg- u. Hüttenw. Bd. 38 (Nr. 3r), p. 355 .

1893: B rez in a: Ueber neuere Meteoriten (Nürnberg), p. 162 ,

1894: Cohen: Meteoritenkunde, Steinbach: p. 220; Breitenbach: p. 23, 217, $219,220,245,246,248,273,274$, $276,280,286$; Rittersgrün: p. Io, 55, $62,68,114,115,133,138,191,198$, $219,220,248,274,276,280,300$.

1894: F l et c her: Introduction, p. 12, $31,32$.

r895: Meunier: Revision des lithosidérites, p. 23-26 (Abb. einer geätzten Platte u, eines Dünnschliffs).

1895: Brezina: Wiener Sammlung, p. 263 .

Ursprüngliches Gewicht:

Grimma: Grosse Masse (oder wohl Massen ?), von der nur das inGotha befindliche Stück erhalten ist. Steinbach: ?

Rittersgrün: $86^{1} / 2 \mathrm{Kgr}$. (173 Pfund); Hauptstück in Freiberg i, S.

Breitenbach: etwa ro $1 / 2 \mathrm{Kgr}$.; Hauptstück in London.

Nachweisbares Gewicht: 81 786 gr.

\begin{tabular}{lr|lr} 
Bailey & 148 & Cleveland & 37 \\
Belgrad & 86 & Darmstadt, M. & $3 \mathbf{1}$ \\
Bement & 58 & Debreczin & 96 \\
Berlin, G. & 407 & Dorpat & 86 \\
Berlin, P. & 24 & Dresden, M. & 580 \\
Berlin, U. & 4447 & Dublin, M. & 35 \\
Bern, M. & 28 & Erlangen & 17 \\
Bologna & 62 & Freiberg, i. S. 55561. \\
Bonn & 90 & Freiberg, i. S. N. $30^{*}$ \\
v. Braun & 78 & Frenzel & 105 \\
Breslau & 114 & Gotha & $\left.917^{1}\right)$ \\
Brezina & 12 & Göttingen & 189 \\
Brunner & 63 & Graz, J. & $\mathbf{1 4 0}$ \\
Budapest & 483 & Gregory & 119 \\
Calcutta & 563 & Greifswald & $\mathbf{1 2 4}$ \\
Cambridge & Spl. & Halle & 54
\end{tabular}

I) Bei Grimma in Sachsen, aus der Sammlung des Berghauptmanns v. Schönberg ๔. 


\begin{tabular}{|c|c|c|}
\hline Hamburg & 70 & Pohl \\
\hline Harvard, U. & 419 & Prag, M. \\
\hline Helsingfors & $9 \mathrm{I}$ & Riga \\
\hline Klausenburg & 93 & Roebling \\
\hline Kopenhagen & 60 & Rom, U. \\
\hline Kristiania & 53 & Seligmann \\
\hline Leipzig & 148 & v. Siemaschko \\
\hline Lissabon & $5^{8}$ & Stockholm \\
\hline London, B. M. & 7057 & Strassburg \\
\hline London, P. G. & 408 & Troyes \\
\hline Madrid & 6 & Tübingen \\
\hline de Mauroy & 4 & Turin, $\mathrm{J}$. \\
\hline Melion & 5 & Turin, U. \\
\hline Moskau & 415 & Ward \\
\hline Neumann & 136 & Washington \\
\hline New Haven & 25 & Washington, Sh. \\
\hline New York, M. & $\mathrm{II}_{7}$ & Wien, H. M. $\left.{ }^{*}\right)^{*}$ \\
\hline Odessa & 16 & \\
\hline Paris, E. & 240 & Wien, U. I. \\
\hline Paris, M. & $23^{8}$ & Wien, U. II. \\
\hline Petersburg, B. & 580 & Zürich \\
\hline
\end{tabular}

Stein Prof. Hill

Steward Co.

Travis Co.

Stinking Creek

Stockholm

Stonitza

Stutsman Co.

Lumpkin

s. Anhang

Hessle

Borodino

Jamestown

Südamerika (Afzelius) s. Anhang

Südöstliches Missouri 1863

Saint François Co.

Sultanpur

Dyalpur

Summit, Blount Co.,Alabama,U.S.A. Eisen, $\mathrm{Hb}$, gefunden?, beschr. 1890 .

r89o: K unz: On five new American Meteorites. - 5. Meteoric Iron from Summit, Blount County, Alabama. Am. Journ. (3) 40, p. 322-323 (Analyse von Venable). Ref. N. J. $189 \mathrm{I}$ II, p. 53 . r894: Co he n : Meteoritenkunde, p. 89 , $97,193,232$.

1895: Brezina: Wiener Sammlung,

p. 293.
1895: Co h e n: Meteoreisen-Studien IV. Ann. Hof-Mus. Bd. 1o, p. 88.

Ursprüngliches Gewicht: I Kgr.

Nachweisbares Gewicht: 47 I gr.

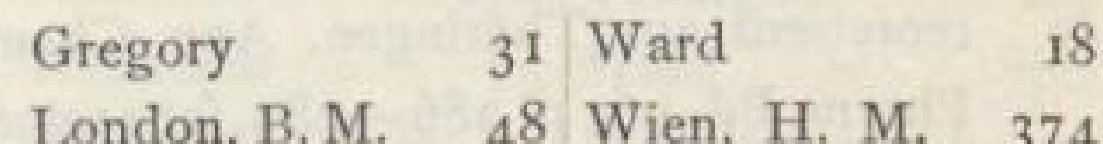

Sumterville Bishopville

Supuhee (Mouza Khoorna), Distr. Goruckpur, Ostindien.

Stein, Cgb, gefallen r 9 . Januar 1865 . 1869: Buchner: Vierter Nachtrag, Pogg. Ann. Bd. 136, p. 455 (es wird nur mitgeteilt, dass London $357^{8}$ gr. besitze).

1880: Im Popular Guide to the Geological Collections in the Indian Museum Calcutta unter Nr. 3 - Meteorites, p. 19 giebt Fedden an: >Supuhi $\left(1^{1} / 2\right.$ and 4 miles to northward), I4 miles southsouth-east of Padrauna, Sidhua-Jobna pargana, Gorakhpur district, NorthWestern Provinces, India. 1865, Jan. 19, I oz. , 1.3 grs. 'A. S. B. $4 \mathbf{r}^{\prime}$ (Asiatic Soc. Bengal) crust on natural surface. See model Nr. 7 .

1884: M e u n i e r: Météorites,p. $247,256$. r885: Brezin a: Wiener Sammlung, p. $155,183$.

r894: F l e t c h e r: Introduction, p.13,28.

Ursprüngliches Gewicht: ?

Nachweisbares Gewicht: 4496 gr. Belgrad 20 London, B. M. 425 I Bement I London, P. G. 84 Budapest $\quad 4$ Paris, M. 20 Calcutta $\quad 28$ v, Siemaschko 4 Gregory $\quad 15$ Washington, Sh. 4 Harvard, U. $\quad 36$ (Wien, H. M.*) 29

\section{Susquehanna \\ Bald Eagle \\ Swajahn \\ Syromolotow \\ Szlanicza}

1) Ausserdem ein Stück von 16 gr. svom grossen Fischfluss im Lande der Kaffern «, welches nach Herrn Prof, Grubenmann sehr grosse Aehnlichkeit mit Steinbach zeigt. 
Tabarz, Sachsen-Gotha, Thüringen, Deutschland.

Eisen, Og, gefd. 1854 , beschr. 1855 . 1855: E b e r h a rd: Analyse eines Meteoreisens aus Thüringen. Ann. Chem. Pharm. Bd, 96, p. 286-289 (aus seiner Dissert. Gött. 1855: Analysen einiger Thüringer Mineralien); s. auch Am. Journ. (2) 22 (1856), p. 271-272; Journ. prakt. Chem. Bd. 67 (I856), p. $3^{82}-3_{83}$; Chem. Centr. 1856, p.213; Kenngott, Uebersicht $1856 / 57$, p. 152 ; Liebig-Kopp, Jahresber. I855, p. 1022. 1859: B u c hner: Feuermeteore, p. I2I, I24.

I859: H a r r i s : Dissert.Gött.,p. $120-121$. r860: $\mathrm{R}$ a mmels berg: Mineralchemie, p 906.

1862: v. Reichen bach: XXI 588 . 1863: B u chner: Meteoriten,p.I89-19o. 1885: Brezina: Wiener Sammlung, p. $200,207,214,234$.

1887: Hidden führt in einer Anmerkung Tabarz unter jenen Meteoreisen auf, deren Fall beobachtet sei. Am. Journ. (3) 33, p. 221.

r893: Brezin a: Ueber neuere Meteoriten (Nürnberg), p. 164.

Ursprüngliches Gewicht: 50 gr. Fast 3 Lot wog das von Eberhard untersuchte Stück; mehr scheint nicht erhalten zu sein.

Nachweisbares Gewicht: $50 \mathrm{gr}$.

Calcutta

Göttingen 5 London, B. M. 9 20 Wien, H. M.*) 16

Tabor (Krawin), Böhmen, Oesterr. Stein, Ccb (früher als Cgb aufgefasst), gefallen 3. Juni 1753 .

1754: St ep ling: De pluvia lapidea Anni 1753 ad Strkow et ejus Causis meditatio. Typis Francisci Ignatii Kirchner. Prag 1754. 33 Seiten; s. insbesondere, p. $3-6$.

1772 : Lythophylactum Bornianum. Prag 1772 Bd. I, Nr. 125.
1790: St ü t z: Bergbaukunde II (s. bei Hraschina); s, auch Gilb. Ann. Bd. 6 (18or), p. r6r; v. Moll's Ann. Berg- u. Hüttenk. Bd, $2(1803)$, p. $313-318$, (Anmerkung).

r794: Chla dni: Pallaseisen, p. 3I. 1796: K in g: Remarks, p. 23.

1802: How ard: Experiments and Observations on certain stony and metalline Substances, which at different Times are said to have fallen on the Earth; also on various Kinds of native Iron. Philos. Trans. (1802), p. 179I80, I85-I86, I97- I98 (Analyse von de Bournon); s, auch Gilb. Ann. Bd. I3 (1803), p. 30I-302, 306, 311-312, 325 ; Ann. Chim. an X (180r); Fourcroy's Uebersetzung in „Encyclopédie * Bd. 5 (1808), p. $548,550,554,556$ $-558,559$; Voigt's Magaz.Bd.ro, p.220. I803: I z a r n : Lithologie, p. $182-183$, $190-193,210,219,221,223,231,232,344$. 1803: de Drée: Recherches, Journ. Phys. Bd. 56, p. 412, 413, 417, 419, 420,421 .

1803: C h 1 a d n i : Chronologisches Verzeichnis, Gilb. Ann. Bd. 15, p. 309.

1804: v. Ende: Massen und Steine, p. $4 \mathrm{I}-44$.

1804: Pötzs ch: Kurze Darstellung, p. $63-68,92$.

1805: M a y e r: Beytrag zur Geschichte der meteorischen Steine in Böhmen. Dresden bei Walther $1805.8^{\circ}$, p. 5-10, 14-15 (Abdruck der Protokolle); s. auch den Auszug in Voigt's Magazin Bd. Io, p. 220.

1806: L a u g i e r: Chromium, Gilb. Ann. Bd. 24, p. 379 .

1807: K la prot h : Beiträge, Bd.4,p.99. I8r2: Bigot de Morogues, p. 98 -100 .

I8r2: Chla dni: Verzeichnis, Schweigg. Journ. Bd. 4, Beil. I, p. 13. 1815: $\mathrm{Ch}$ ladni: Neues Verzeichnis, Gilb. Ann. Bd. 5o, p. 248.

r8rg: Chla dni: Feuermeteore, p. 50, 
$56,57,66,70,73,90,236,246-$ $248(1), 254,260,271,324,427$.

1820: v. Schreibers: Beiträge, p. 10-12 (Abb.).

1836: K ä m tz: Meteorologie, p. 273. 1837: R o s e : Reise nach dem Ural, Bd,I, p. $76-77$ (Anmerkung).

1843: P a rts ch: Meteoriten, p. 7 $1-73$. 1854 : B a 1 c e 11 s: Lithologia meteorica, p. 20.

1859: B u chner: Feuermeteore, p. 4I -42 .

r859: H a r ri s : Dissert. Gött., p. 59. 1863: B u chner: Meteoriten, p. 6. 1863: Ros e: Meteoriten, p. 89, 92, 154. r858/65: v. Reichen bach: V 477 , 481. VI 44I, 443, 454. VII 552. IX $162,171,180 . X_{359}, 362,363$. XI $294,295,297,301,304$. XII 454. $\mathrm{XIII}_{365,377 .} \mathrm{XXIV}_{22} 8, \mathrm{XXV}_{324,607 .}$ 1865: Buchner: Zweiter Nachtrag, Pogg. Ann, Bd. 124, p. 574-575.

1875: Tschermak: Vulkanismus, Sitzber. Wien. Akad, Bd. 71 II, p. 662. 1880: $\mathrm{H}$ a h n : Die Meteorite (Chondrite) und ihre Organismen. Tübingen. T.29, Fig. 3 .

1884: M e un i e r: Météorites, p.95,227. 1885: Brezina: Wiener Sammlung, p. $18_{2}, 183,233$.

r894: Cohen: Meteoritenkunde, p. 7 , 23,185 .

1894: Flet cher: Introduction, p. 8, $23,24$.

1895: Brezina: Wiener Sammlung, p. 250 .

Ursprüngliches Gewicht: Mehrere Steine; der grösste soll etwa I $3_{3}$ Pfund gewogen haben.

Nachweisbares Gewicht: 6606 gr.
Bailey

Bement

Berlin, U.

v. Braun

Budapest

\begin{tabular}{r|l} 
I & Calcutta \\
43 & Cambridge \\
70 & Dorpat \\
45 & Dresden, M. \\
762 & Gregory
\end{tabular}

762 Gregory $\left.39^{1}\right)$

3

29
Harvard, U. $\quad{ }_{4}$ Prag, M. 128

London, B. M. I 5 I Rom, U. $\quad 58$

London, P. G. $\quad$ I 4 v. Siemaschko 54

$\begin{array}{llll}\text { Moskau } & 2 & \text { Stockholm } & \text { I4 }\end{array}$

München $\quad 2$ I 4 Tübingen $\quad$ II3

Neumann $\quad 75$ Ward Spl.

Paris, M. $\quad 7$ Washington, Sh. $\quad 2$

Pohl 6o Wien, H. M.*) 4063

Prag, D. U. $\quad 640$

Eine chemische Untersuchung ist seit I802 nicht mehr ausgeführt worden.

\section{Taborg Ochansk \\ Tabory Ochansk}

Tadjera, Setif, Constantine, Algier, Nordafrika.

Stein, Chondrit-Tadjerit, $\mathrm{Ct}$, gefallen 9 . Juni 1867 .

1867: A ug e ra u d: Chute d'aérolithes dans la plaine de Tadjera (Amer Guebala) à 15 kilomètres sud-est de Sétif le 9 juin 1867 , vers to h $30 \mathrm{~m}$ du soir. C. R. Bd. 65 , p. $240-242$; s. auch "L'Institut * Bd. 35 (1867), p. 249.

1868: D a u bré e: Météorite tombée le 9 juin 1867, en Algérie, à Tadjera, près Sétif, province de Constantine. C. R. Bd. 66 , p. 513-519 (Analyse von Meunier). Ref. Liebig-Kopp, Jahresber. 1868, p. I046; "L'Institut * Bd. 36 (I868), p. 99- IOI.

1868: "Cosmos « 21. März 1868, p. 25 u. 28. März 1868.

r869: Buchner: Vierter Nachtrag, Pogg. Ann. Bd. 136, p. 589-59o.

r869: Meunier: Recherches, Ann. Chim. Phys. (4) 17, p. 13-16. Ref. Liebig-Kopp, Jahresber. 1869, p. 1300 -130 .

r870: R a m me ls berg : Meteoriten, p. 103, 105, 106, 138, 139, 140.

1871: Me un i e r: Étude chimique de la matière colorante noire de la Tadjérite. C. R. Bd. 72 , p. $339-343$. Ref. Liebig-Kopp, Jahresber. 1871, p. 1237. 
1871: M e unier: Contribution au métamorphisme météorique. C. R. Bd. 73, p. $1284-1285$.

1872: Me unier: Application du métamorphisme météorique à l'étude de la croûte noire des météorites grises. C. R. Bd. 75 , p. 500 ; s. auch Hirzel u. Gretschel: Jahrb. d. Erfindungen Bd. 9 (1873), p. 25 .

1875: Gümbe 1: Ueber die Beschaffenheit der Steinmeteoriten vom Fall am 12. Februar 1875 in der Grafschaft Iowa, N. A. Sitzber. München. Akad. Bd. 5 , p. 330 .

r875: T s c h e r m a k: Vulkanismus, Sitzber. Wien. Akad. Bd. 71 II, p. 670. I879: R a mmels berg: Meteoriten, p. 24,25 .

1882: Brezina: Bericht IV, Sitzber. Wien. Akad. Bd. 85 I, p. 338,339 (Vergleich mit Mócs).

1884: M e unier: Météorites, p. 6r, $62,79,81,83,85,242-245$ (Abb.), $354,355,356,367-369,371,395$, $450,487,489,523$.

1883/85: Ts cher mak: Photographien, p. $3,19$.

1885: Brezina: Wiener Sammlung, p. $172,184,233$.

r887: Flight: Meteorites, p. I5I.

1889: M e un ie $r$ : Sur la matière noire de la Chantonnite. Bull. Soc. Franc. Min. Bd. 12, p. 76-8I. Ref. N. J. 1890 II, p. 59.

r89o: B r e zin a : Reise, Ann. Hof-Mus. Bd. 4 (Not.), p. 119.

I890: v. $\mathrm{N}$ i e s s 1 : Periheldistanzen,Verh. naturf. Ver. Brünn Bd. 29, p. 184.

1894: Cohen: Meteoritenkunde,p. 214, 284,317 .

1894: F l et ch er: Introduction, p. 39.

r895: Brezina: Wiener Sammlung, p. 252 .

Ursprüngliches Gewicht: 8843 gr. Zwei Steine von 5760 u. 1700 gr. Im ganzen giebt Daubrée 7659 gr. an, worin abgestossene Teile des grösseren Meteoriten, der nach dem PariserKatalog 5900 gr.wiegt, mitinbegriffen zu sein scheinen. Das Pariser Gewicht zeigt, dass nachträglich noch weitere Stücke gesammelt worden sind.

Nachweisbares Gewicht: 8843 gr.

\begin{tabular}{lr|lr} 
Belgrad & I7 & London, B. M. & 40 \\
Bement & 2 & New Haven & 43 \\
Bologna & 7 & Paris, M. & 8515 \\
Cohen & Spl. & v. Siemaschko & 5 \\
Dorpat & I & Upsala & I4 \\
Gregory & I & Wien, H. M.*) & 166 \\
Harvard, U. & 32 & &
\end{tabular}

Taïga

Tajgha

Tajgha, Krasnojarsk, Sibirien.

Eisen, Om, gefunden r891.

Hierher auch vorläufig: „Krasnojarsk, Eisen, 1894«. Dieses Eisen, welches noch nicht beschrieben worden ist, wurde nach Herrn Melnikoff's Mitteilung 1894 in der Nähe der Stadt Krasnojarsk in Sibirien gefunden. Die Masse von $21960 \mathrm{gr}$. (I Pud $13 \%$ Pfund) befindet sich in Petersburg, B. Ein kleines Stück gelangte an Petersburg, A.

1894: Cohen: Meteoritenkunde, p. 93 (Taïga).

1895: Brezina : Wiener Sammlung, p. 284 .

Ursprüngliches Gewicht: $300-400$ gr. (nach Mitteilung des Herrn Direktor Brezina).

Nachweisbares Gewicht: 2 I gr.

London, B. M. Io /Wien, H. M. II

v. Siemaschko dürfte auch von dem Eisen von Tajgha besessen haben; in seinem Katalog von 1891 ist es noch nicht erwähnt. 


\section{Tajima}

Talbot Road

Taltal r 86r

Taltal r 888

\section{Tamentit}

Taney Co.

Taos

Toke uchi mura

De Cewsville

Vaca Muerta

Inca

s. Anhang

Miney

Tucson

Tarapaca, Tarapaca-Wüste, Hemalja, Chile, Südamerika.

Eisen, Db, gefd. 1840 , beschr. 1855 .

Hierher auch vorläufig ein oktaëdr. Eisen von Tarapaca (s, u. in der Gewichtsliste bei Brezina).

1855: Greg: Description of a new Meteoric Iron from Chili, containing a Native Lead. Philos. Magaz. (4) ro, p. 12-I4 (Analyse von Darlington), aus dem Liverpool Liberary and Phil. Soc. Journ.; Ref. Am. Journ. (2) 23 (1857), p. 118-Ir9; Liebig-Kopp, Jahresber. 1855 , p. $1027-1028$; Pogg. Ann. Bd. 96 (1855), p. 176; Journ. prakt. Chem. Bd. 66 (1855), p. 430 $-43 \mathrm{I}$; C. R. Bd. $4 \mathrm{I}$ (1859), p. 490 ; N. J. 1856 , p. 553 u. N. J. 1857 , p. 68; "L'Institute Bd. 23 (1855), N. 1145 , p. 435 .

1855: Giebel u. Heintz: Zeitschr. f. d. ges, Naturw. Bd. 6, p. 327 ; s. auch Kenngott, Uebersicht 1855, p. 97-98. 1859: B u c hner: Feuermeteore, p. 150. 1859: H arri s: Dissert. Gött., p. ${ }_{13}$. 1859/60: v. Reichenbach: IX 162, $176,177,182 . X_{358}, 359$. XIII 364 . 1860: R a mmels berg : Mineralchemie, p. 914 .

1863: B u chner: Meteoriten, p. 190 -191.

1863: R o s e: Meteoriten, p. 24.

1870: $\mathrm{S} \mathrm{m}$ it h : Description and Analysis of the Franklin County Meteoric Iron; with remarks on the presence of Copper and Nickel in meteoric irons; the me- thod of analyzing the same; and the probability of the Lead in the Tarapaca iron having been originaly foreign to that mass. Am. Journ. (2) 49 , p. 334-335; s, auch Original Researches 1884 , p. $450-451$; Liebig-Kopp, Jahresber. 1870, p. 1395 .

1884: M e u n i e r : Météorites, p.153,352. 1884: W a d s worth: Studies, p. 69. 1885: Brezina: Wiener Sammlung, p. $221,234$.

r886: H u n tin g t o n : Crystalline Structure, Am. Journ. (3) 32, p. 303 (die meteorische Natur des unter diesem Namen im Harvard-Kabinet befindlichen Eisens wird angezweifelt).

1887: F 1 ig h t: Meteorites, p. Io.

1890: E a s t m a n : Met. Astron, p. 318. 1894: Co hen: Meteoritenkunde, p. 34 . r895: Meunier: Revision des lithosidérites, p. 9-II.

Ursprüngliches Gewicht: $7^{1 / 2} \mathrm{Kgr}$. (beinahe 17 lbs.).

Nachweisbares Gewicht: 5368 gr.

\begin{tabular}{lr|lr} 
Bailey & 63 & London, B. M. & 1656 \\
Bement & 7 & London, P. G. & 69 \\
Berlin, U. & 119 & New Haven & 17 \\
Brezina & $\left.462^{1}\right)$ & Paris, E. & 127 \\
Budapest & 252 & Paris, M. & 156 \\
Calcutta & 121 & Pohl & 34 \\
Cleveland & 7 & v. Siemaschko & 80 \\
Edinburg & 293 & Stockholm & 10 \\
Gregory & 36 & Troyes & I \\
Harvard, U. & 189 & Tübingen & 76 \\
Klausenburg & 10 & Washington, Sh. 84 \\
Kopenhagen & 17 & Wien, H. M.*) & 329 \\
Leipzig & 1153 & &
\end{tabular}

\section{Tarne}

Grazac

Tarragona

Nulles

Tasmania

Blue Tier

Tazewell (Knoxville), Claiborne

Co., Tennessee, U. S. A.

Eisen, Off, gefd. 1853 , beschr, 1854 . 
1854: $\mathrm{Sm}$ it h: A new Meteorite from

Tennessee. Am. Journ. (2) 17, p. 131; s. auch Journ. prakt. Chem. Bd. 6I (1854), p. 255; Kenngott, Uebersicht I854, p. IIg.

1854: Shepard: New Localities of Meteoric Iron. Am. Journ. (2) 17,p. 325 -327 ; s. auch Journ. prakt, Chem. Bd. 62 (1854), p. $345-346$; Kenngott, Uebersicht 1854, p. 120; LiebigKopp, Jahresber. 1854, p. 914-915 ; s. ferner Am. Journ. (2) 18, p. $380-381$; Journ. prakt. Chem. Bd. 63 (1854), p. $46 \mathrm{r}-462$.

1855: $\mathrm{Sm}$ ith: Memoir on Meteorites. - A Description of five New Meteoric Irons, with some theoretical considerations on the origin of Meteorites based on their Physical and Chemical charakters. - I) Meteoric Iron from Tazewell County, East Tennessee. Am. Journ. (2) 19, p. 153-159 (Analyse u. Abbildung); s. auch Original Researches 1884, p. 367-373; Journ. prakt. Chem. Bd. 66 (I 855 ), p. $42 \mathrm{I}$ -425; Liebig-Kopp, Jahresber. 1855 , p. 1024-IO26; Rammelsberg, Mineralchemie, 2. Aufl. 1875 , p. 53 (Troilit); Report, Smithsonian Inst. 1855 , p. 151 , 154 (Abb.).

1859: $\mathrm{B} \mathrm{u} \mathrm{c} \mathrm{hner:} \mathrm{Feuermeteore,} \mathrm{p.} 137$ -138 .

1859: H a rris: Dissert. Gött., p. II9. r860: $\mathrm{R}$ ammels berg: Mineralchemie, p. $916-917,946,948$.

I862: R a m mels berg: Ueber dasSchwefeleisen der Meteoriten. Mon.-Ber.Berlin. Akad. (1862), p. 689-691; s. auch Journ. prakt. Chem. Bd. 88 (1863), p. $275-277$.

1863: Buchner: Meteoriten, p. 187 -189 .

1863: Rose: Meteoriten, p. 66, 153. 1858.65: v. Reichen bach: VII 55I. IX 162, 174, 182. XII 458. XV no, I13, 124. XVI 26r. XVIII $478,484$. XX 621, 634. XXV 436. r869: M eunier: Recherches, Ann. Chim. Phys. (4) I7, P. 4I, 44-46, 53.7I. 1870: R a mmels berg: Meteoriten, p. 84,85 .

1872: Quenstedt: Klar u. Wahr, p. 3I3 (Abb. einer geätzten Platte). 1875: Wright: Spectroscopic Examination of Gases from Meteoric Iron. Am. Journ. (3) 9, p. 297-298.

r876: Wright: On the Gases contained in Meteorites. Am. Journ. (3) II, p. 256,257 u. (3) 12, p. $167,168,169$. r880: Brez ina: Bericht I, Sitzber.

Wien. Akad. Bd, 82 I, p. $350-351$. I88I: Brezina: Bericht II, Sitzber.

Wien. Akad. Bd. 83 I, p. 476 .

r88r : B rezin a : Bericht III, Sitzber.

Wien. Akad. Bd. 84 I, p. 280 .

r883: S m it h : Concretions, Am. Journ.

(3) 25, p. $417,419$.

r884: M e un i er: Météorites, p. 58, 59, $66,89,93,96,99,108-109$ (Abb.). r885: Brezina: Wiener Sammlung, p. $207,208,233$.

r886: H un ting to $n$ : CrystallineStructure, Am. Journ. (3) 32, p. 289 (Abbildungen geätzter Platten), 293, 295. 1887: Brezina u. Cohen: Photographien, T, IO, II.

r887: Flight: Meteorites, p. 122,174. r887: Sorby: (On the microscopical structure of iron and Steel. Journ. Iron and Steel Inst. 1887, p. $255-288$ ) nach Ref. N. J. 189 I I, p. 47.

r889: C q h e n : São Jutlião, N. J. 1889 I, p. 22I, 222, 224, 225.

r890: E a s t m a n : Met. Astron., p. 318. r89r: Cohen u. Weinschenk : Meteoreisen-Studien. Ann. Hof.-Mus. Bd. 6 , p. 164 .

1893: Meunier: Revision des fers météoriques, p. $43-44$.

1894: Huntington: The Smithville Meteoric Iron. Proc. Amer. Acad. Arts and Sc.Bd. 29, p.259 (Situationsplan von Tennessee bis West-Virginia). 
1894: Cohen: Meteoritenkunde, an sehr vielen Stellen.

1895: Brez in a: Wiener Sammlung, p. 267.

r895: C o h e n : Meteoreisen-Studien IV. Ann. Hof-Mus. Bd, Io, p. 82 .

Ursprüngliches Gewicht: Ueber 60 lbs. Smith besass die Hauptmasse von 55 lbs.

Nachweisbares Gewicht: $7510 \mathrm{gr}$.

\begin{tabular}{lr|lr} 
Bailey & 53 & Madrid & 75 \\
Belgrad & 17 & Moskau & 35 \\
Bement & 17 & New Haven & 418 \\
Berlin, U. & 722 & New York, M. & 52 \\
v. Braun & 34 & Paris, M. & 223 \\
Brezina & 34 & Petersburg, B. & 161 \\
Budapest & 380 & Pohl & 18 \\
Calcutta & 376 & Rom, U. & 43 \\
Cleveland & 5 & v. Siemaschko & 20 \\
Dorpat & 56 & Stockholm & 39 \\
Dresden, M, & 56 & Strassburg & 58 \\
Göttingen & 270 & Stuttgart & 78 \\
Gregory & 89 & Troyes & 19 \\
Greifswald & 16 & Tübingen & 294 \\
Hamburg & 71 & Upsala \\
Harvard, C. & 754 & Ward \\
Klausenburg & 74 & Washington \\
Kopenhagen & 58 & Washington,Sh.1943 \\
London, B. M. & 336 & Wien, H. M.*) & 165 \\
London, P. G. & 124 & \\
Wo befing &
\end{tabular}

Wo befindet sich die grösste Masse dieses Eisens?

Teheran

Teilleul

Tejupilco

Temora

Tennasilm (Sikkensaare), Esthland, Russland.

Stein, Cca, gefallen 28. Juni 1872 . 1873: v.S c h i $11 \mathrm{ing}$ : Mittheilungen über den am 16. 28 . Juni 1872 Mittags, beim Dorfe Tennasilm, im Kirchspiel Turgel, des Kreises Jerwew in Ehstland nieder-

1) $9734 \mathrm{gr}$., $1595 \mathrm{gr}$. und $720 \mathrm{gr}$.

W it $1 \mathrm{fing}$, Meteoriten. gefallenen Steinmeteoriten. Arch. f. Naturk. Liv. Ehst. u. Kurl. Bd, 8, p. I-20 (Abbildung des Steins u. Situationsplan).

r884: M e unier: Météorites, p. 227.

r885: Brezina: Wiener Sammlung, p. $155,190,233$.

1887: Brezina: Neue Meteoriten III, Ann. Hof-Mus. Bd. 2 (Not.), p. II5. 1894: C o he n: Meteoritenkunde, p.203, 303, 310.

Ursprüngliches Gewicht: $28^{1} / 2 \mathrm{Kgr}$. (69-70 Pfund russisch). EinStein, von welchem Freiherr v. Schilling $25 \mathrm{Kgr}$. rettete. (Gewicht der einzelnen Stücke $24 ; 9 \frac{3 / 4}{8} ; 8$; $5 ; 4 ; 3$ Pfund).

Nachweisbares Gewicht: 22 714 gr. Berlin, U.
v. Braun Budapest Dorpat Gregory London, B. M. 30 Paris, M. 7 8 Reval, P. M. 3742 86 v. Schilling $12049^{\prime}$ ) 3015 v. Siemaschko 21 3 Utrecht 2 16 Washington 48 de Mauroy I Washington, Sh. I Melion

1 Wien, H.M.**) 3684

Tennessee (Ehrenberg) Cleveland Tennessee (Lea Iron) Cleveland Teposcolula

Terek

Misteca Grosnaja

Ternera, Sierra de la Ternera, Atacama, Chile.

Eisen, Hch, gefunden?, beschr. $189 \mathrm{I}$. r89r: $\mathrm{K}$ unz u. Weins chen k: Meteoritenstudien, - 3. Sierra de la Ternera, Provinz Atacama, Chile. M. P. M. Bd, 12, p. $184-185$ (Abb, und Analyse von Weinschenk). Ref. N. J. 1892 II, p. 243-244.

1892: Kunz u. Weinschenk: On two Meteoric Irons. - 2. Sierra de la Ternera, Province of Atacama, Chile. 
Am. Journ. (3) 43 , p. $425-426$ (Analyse von Weinschenk).

I894: C o h e n : Meteoritenkunde, p. IO9, IIO, 135 .

Ursprüngliches Gewicht: $65^{\circ} \mathrm{gr}$. Nachweisbares Gewicht: 650 gr., welche sich im Besitz von Herrn Dr. Wilhelm Moericke in Stuttgart befinden (August 1896).

\section{Terni}

Collescipoli

Texas 1808 ( 18 r 4 ) Cross Timbers

Thunda, Windorah, Distr Diamantina, Queensland, Australien.

Eisen, Om, gefd. 1886, beschr. 1887. r886: Liversidge: Metallic Meteorite, Queensland. Journ. and Proc. Royal Soc. of New South Wales Bd. 20, p. 73,285 . Ref. N. J. 189r I, p. 47 ; s. auch Liversidge: Metallic meteorite, Queensland. Minerals of New South Wales 1888, p.221 (s Professor Liversidge exhibited a polished section through the Thunda Meteorite from Queensland, and a model from the complete Meteorite before cutting $\alpha$ ); s. ferner Journ, and Proc. Royal Soc. N. S. Wales Bd. 23 (1889), p. 158.

r89r: v. H a u er: Ann. Hof-Mus. Bd, 6 (Not.), p. 55.

1893: Brezina: Ueber neuere Meteoriten (Nürnberg), p. 164.

1893: Meunier: Revision des fers météoriques, p. 61-62 (Abbildung einer geätzten Platte).

r894: C o he n : Meteoritenkunde, p. 72, $83,193$.

I 894: F l et c he r: Introduction, p. 15. 1895: Brezina: Wiener Sammlung, p. 283 .

Ursprüngliches Gewicht: „I Zentner oder mehr».

Nachweisbares Gewicht: $9287 \mathrm{gr}$.

Bailey II 4 Böhm

Bement 508 v. Braun
Brezina

Budapest

Cleveland

Gregory

Harvard, U.

London, B. M.

Paris, E.

Paris, M.

Wo befindet sich die Hauptmasse dieses Eisens?

\section{Thunder Bay \\ s. Anhang \\ Thüringen \\ s. Anhang}

Tieschitz, Tischtin, Bez. Prerau, Mähren, Oesterreich.

Stein, Cc, gefallen $\mathbf{5}$. Juli 1878 .

I878: T s c he r m a k: Der Meteorit von

Tieschitz. M. P. M. Bd. I, p. 289.

1878: Ma k o w sk y legt den am 15. Juli

d. J. bei Tieschitz in Mähren gefallenen Meteoriten von $27^{1 / 2} \mathrm{Kgr}$. Gewicht vor und berichtet über die $\mathrm{Re}$ sultate der diesfälligen Untersuchungen. Verh, naturf. Ver. Brünn Bd.17(Sitzber.), p. $37-39$.

1878: Ts chermak: Der Metenritenfall bei Tieschitz in Mähren (Erster Bericht). Sitzber. Wien. Akad. Bd. 78 I, p. $440-443,580-582$. Ref. Liebig-Kopp, Jahresber.1878, p.1316-1317. 1879: M a k ow k y u. T s chermak: Bericht über den Meteoritenfall bei Tieschitz in Mähren. Denkschr. Wien. Akad. Bd. 39, p..187-202 (Analyse von Habermann ; 5 Tafeln; Situationsplan u. Holzschnitte); s. auch Anzeig. Wien. Akad. Bd. $15(1878)$, p. 215. 1882: T's chermak: Ueber die Meteoriten von Mócs. Sitzber. Wien. Akad. Bd. 851, p. 205.

I882: v. L a s a u $1 \mathrm{x}$ : Vermehrung, Verh. naturh. Ver. Bonn (Sitzber.) Bd. 39, p. 102.

r883: Ts c hermak: Beitrag, Sitzber.

Wien. Akad. Bd. 88 I, p. $356,357$. r884: M e unier: Météorites, p. 239 241 (Abb.), 523. 
1884: W a d s worth: Studies, p. Ior102.

1883/85: Tschermak: Photographien, T. 8,10, p. $12,13,14,15,17$.

1885: Brezina: Wiener Sammlung, p. I55, I56, 165, 188-I90, 233, Taf. IV (Situationsplan).

1886: Meli o n: Die Meteorsteinfälle in Mähren. Notiz-Bl. d. mähr. schles. Ges, zur Beförd, d. Ackerbaus etc. 1886, Nr. 5 u. 6 , p. $5-8$, 10, Ir.

1887: F lig h t: Meteorites, p. 207-209. 1890: v. Niess 1: Periheldistanzen, Verh. naturf.Ver. Brünn Bd, 29, p. 188, 189, 19I, 195, 214, 216, 238.

r892: Brez i n a : Sternschnuppen,p.r7. r894: Co hen: Meteoritenkunde, p. 59, $60,202,26 \mathrm{I}, 279,286,29 \mathrm{I}, 298$.

r895: Brezina: Wiener Sammlung, p. 256.

Ursprüngliches Gewicht: „Etwa $27^{1 / 2}$ Kgr.

Nachweisbares Gewicht: 28233 gr.

\begin{tabular}{lr|lr} 
Berlin, U. & 5 & Melion & 2 \\
Bonn & 3 & Moskau & 3 \\
v. Braun & 50 & Paris, M. & 34 \\
Budapest & 83 & Pohl & 42 \\
Cohen & 6 & Rom, U. & 18 \\
Dorpat & 8 & v. Siemaschko & 41 \\
Gregory & 2 & Strassburg \\
Harvard, U. & 18 & Wien, H. M. 27 847 \\
London, B. M. & 17 & Washington,Sh. 27 \\
de Mauroy & 23 & \multicolumn{2}{|l}{}
\end{tabular}

Das Franzens-Museum in Brünn besass 1886 ein Bruchstück von 6I gr.

\section{Till Porter Mound}

Brenham (Anderson)

Timoschin, Kreis Juchnow, Gouv. Smolensk, Russland.

Stein, Cc, gefallen 25. März 1807 . 1807: Gil b e r : Zeitungsnachricht von einem Meteorsteine, der in Russland herabgefallen ist. Aus einem Schreiben, Petersburg den 18. Julius, im Hamburger nnparteiischen Corresp. 1807. Nr. 126.
Gilb. Ann. Bd. 26, p. $238-239$. 1807: Chute d'un aërolithe. Bibl. Brit. Nr. 280 (August 1807 ), p. $362-363$; s. auch Journ. Phys, vom 15. Mai 1807. 1808: Gilbert: Einige Nachrichten von den drei neuesten Steinregen und von drei Meteorsteinen aus Russland. 6. Aus öffentlichen Blättern im Mai 1808. Gilb. Ann. Bd. 29, p. 213-214 (Analyse von Scherer).

r808: Klaproth: Analyse des Steins aus dem Kreise Juchnow. Gehlens Journ. Chem. Phys. Mineralog. Bd. 7, p. 198-199; s, auch Tassaert: Extrait d'une lettre de M. Klaproth à M. Gehlen. Ann. Chim. Bd. 70 (1809), p. 18I -184 (Analyse); ferner Gilb. Ann. Bd. 33 (1809), p. $210-211$; Klaproth, Beiträge Bd. 5 (I8Io), p. $254,257$. 1812: Bigot de Morogues, p. 207 $-210$.

1812: $\mathrm{C}$ h 1 a d ni: Verzeichnis,Schweigg. Journ. Bd, 4 Beil. I, p. 16.

r815: Chladni: Neues Verzeichnis, Gilb. Ann. Bd. 50, p. 254.

1816: Chla dni: Zweite Fortsetzung, Gilb. Ann. Bd. 54, p. 353.

r8rg: $\mathrm{Ch}$ la d n i: Feuermeteore, p. 66, $69,73,75,9 \mathrm{I}, 28 \mathrm{O}-28 \mathrm{I}(1), 429$.

1820: v. S chreibers : Beiträge, p. 63 -65 ; Tafel VII.

1836: $\mathrm{K}$ ä m tz: Meteorologie, p.257,281. r837: K o s e: Reise nach dem Ural Bd. I, p. $75-76$ (Anmerkung).

1843: P a rts c h: Meteoriten, p. 8o-81. 1847: Borissiak: Sur l'Aérolithe tombé près de Verkhne Tchirskaia Stanitsa, Bull. Petersburg. Akad. Bd. 5, p. 198 (die Universität Charkow besitze einen Stein von Timoschin).

1847: Eichwald: Verzeichnis, Erman's Arch, f. wissensch. Kunde v. Russl. Bd. 5, p. 177.

1848: B 1 o e d e: Tabelle, Bull. Petersburg. Akad. Bd. 6, p. I-4.

1854: B a 1 c e 11 s: Lithologia meteorica, p. 22 .

$23^{*}$ 
r859: $\mathrm{Buch}$ uer: Feuermeteore, p. 77. 1859: Harris: Dissert.Gött., p. 67.

r862: Kenngott u. W iser: Zürcher Sammlung, p. 153-I54.

r863: B u chner: Meteoriten, p. 21. I863: Rose: Meteoriten, p. 86, 93, $98,154,16 \mathrm{r}$, Taf. IV.

1857/65: v. Re i c hen b a ch: III 624 . V 475. VI 44I. IX 16r, 169, 179. X 359. XI 294, 295, 296, 298, 30I, 302. XII 454. XV I2I. XVIII 490. XX 626. XXV 606,615 .

1867: G o e be 1: Kritische Uebersicht, Mélanges phys. chim. Bd. 7 , p. $28_{3}$ $-284,313$.

1867: B u chner: Dritter Nachtrag, Pogg. Ann. Bd. 132, p. 314, 319. 1875: vom R at h: Meteoriten, Verh. naturh. Ver. Bonn Bd. 32, p. 367 . 1884: M e uni e r: Météorites, p. 191, 192. 1885: B rez in a: Wiener Sammlung, p. 185,233 .

I894: Co he n: Meteoritenkunde, p. 8. r895: Brezina: Wiener Sammlung, p. 255 .

Ursprüngliches Gewicht : $65^{1} / 2 \mathrm{Kgr}$. (160 russ. Pfund).

Nachweisbares Gewicht : 50099 gr. Berlin, U. $\quad 433$ Neapel 6 Bonn $\quad 87$ Paris, E. $\quad 56$

Budapest $\quad 117$ Paris, M. 50

Calcutta I4 Petersburg,A. 48500

Cambridge $\quad 4^{\prime}$ ) Petersburg, B. 125

Darmstadt, M. 9 Pohl 52

Dorpat $\quad 45$ v. Siemaschko 37

Göttingen Io Strassburg 17

Gregory $\quad 69$ Tübingen $\quad 30^{\frac{9}{}}$ )

$\begin{array}{llll}\text { Greifswald } & \text { 2I Ward }\end{array}$

Harvard, U. $\quad 7$ Washington $\quad 7$

Kasan $\quad 43$ Washington, Sh. 15

\begin{tabular}{ll|l} 
Klausenburg & 2 & Wien, H. M.*) I41
\end{tabular}

London, B. M. $\quad{ }_{3} 8$ Zürich $\quad 7$

Moskau
Tipperary 1810

Tipperary 1865

Tiree

Tirlemont

Tirnowa

Tjabé, Bodgo-Negoro, Padang, Res.

Rembang, Java.

Stein, Ck, gefallen 19. Sept. 1869.

r87r: v. Baumhauer: Sur la météorite de Tjabé dans l'Inde néerlandaise. Arch. Néerl. Bd. 6 (Nr. 4), p. 305-325 (Analyse); s, auch v. Baumhauer: Over den Meteoriet van Tjabé in Nederlandsch Indië. Versl, en Mededeel. Amsterdam. Akad. Bd. 6 (1872), p. 54-73 (Analyse); ferner Naturkundig Tidschrift Nederlandsch Ind. Bd. 32 , p. $242-250$.

1872: Da ubrée: Sur une météorite tombée dans lîle de Java, près Bandong, le io décembre 1871 , et offerte au Muséum par M. le Gouverneur général de 1'Inde néerlandaise. C. R. Bd. 75, p. $1676-1678$.

1873: Hirzel u. Grets ch e 1: Jahrb. d. Erfind. Bd. 9, p. 20.

1879: R a mmelsberg: Meteoriten, p. $14,24,25$.

1880: K 1 e in: Vermehrung, Gött. Gel. Anz. (188o), p. 567 .

1884: Meunier: Météorites, p. 79, 179, 182.

1885: Brezina: Wiener Sammlung, p. 191, 233.

1887: Flig h t: Meteorites, p. 9-ro. 1887: Brezina: Neue Meteoriten III, Ann. Hof-Mus. Bd, 2 (Not.), p. 114.

Ursprüngliches Gewicht: Ein Stein von $20 \mathrm{Kgr}$.

Nachweisbares Gewicht: III2 gr.

1) \$Smolensk, Timoschin ? - 2) Ausserdem $90 \mathrm{gr}$., welche als Barbotan bezeichnet sind, vielleicht aber zu Timoschin gehören, s. o. p. 2I, Anmerkung. 


\begin{tabular}{lr|lr} 
Bement & I & Neumann & 3 \\
Berlin, U. & Spl. & Newton & I \\
Bologna & 7 & Paris, E. & 70 \\
Bonn & I & Paris, M. & II2 \\
v. Braun & 4 & Pohl & II \\
Budapest & 500 & Rom, U. & 24 \\
Göttingen & 3 & v. Siemaschko & 47 \\
Gregory & 22 & Stockholm & 66 \\
Greifswald & 3 & Troyes & 2 \\
Klausenburg & 33 & Utrecht & I \\
London, B. M. & 134 & Washington, Sh. & 29 \\
Melion & I & Wien, H. M.**) & 37 \\
Wo befindet sich die Hauptmasse? &
\end{tabular}

\section{Tocane}

s. Anhang

Tocavita

Rasgata

Toke-uchi-mura, Tajima, Yofugori,

Tamba, Japan.

Stein, Ck, gefallen 18 . Febr. 1880 .

r88r: Korschelt: Der Meteorit von

Tajima Februar 1880. Mitteilungen der deutsch. Ges. f. Natur- u. Völkerk. Ostasiens. Yokohama Heft 25 (188r), p. 204. r885: Brezina: Wiener Sammlung, p. 270.

r887: Flight: Meteorites, p. 167, $212-213$.

r887: Brezina: Reisebericht, Ann. Hof-Mus. Bd. 2 (Not.), p. 108.

Ursprüngliches Gewicht: Etwa $65 \mathrm{gr}$. (etwa 100 grs. Troy).

Nachweisbares Gewicht: 66 gr.

Berlin, U. $\quad 6 \mathrm{I}$ Rom, U.

Cohen Spl.

Toluca, Xiquipilco, Ixtlahuaca, Tolucathal, Mexico.

Eisen, Om, bekannt vor 1776 (nach Fletcher), erwähnt 1784 .

1784: Gazetas de Mexico. Mittwoch den 15. Dez. 1784 Nr. 25, p. 201 (Clark sagt: Bd. I, p. 146, 200 u, Bd, 5, p. 59).

1804: D e $1 \mathrm{R}$ i o: Tablas mineralogicas (1804), p. 57.

I $8 \mathrm{x} \mathrm{x}: \mathrm{v} . \mathrm{H} \mathrm{u} \mathrm{m} \mathrm{b} \mathrm{o} 1 \mathrm{~d} \mathrm{t}$ : Essai politique sur le royaume de la Nouvelle Espagne. Bd, 2, p. 582 .

1812: Bigot de Morogues, p. 300. 1812: Chla d n i: Verzeichnis,Schweigg. Journ. Bd. 4 Beil. I, p. 18.

1817: Chladni: Dritte Fortsetzung, Gilb. Ann. Bd. 56, p. $3^{84}$.

r8r9: $\mathrm{Chladni}$ : Fünfte Fortsetzung, Gilb. Ann. Bd. 63 , p. 25-26.

r8I9: C h la d n i: Feuermeteore, p. 66, 319, $339(1), 434$.

1826: Chladni: Sechster Nachtrag, Pogg. Ann. Bd, 8, p. 51-52.

1826: Nögger ath: Ueber Meteoreisen aus Mexico; ein Schreiben an Herrn Dr. Chladni. Schweigg. Journ. Bd. 47 , p. $74-76$.

1827: B erthier: Analyse du fer météorique de Toluca au Mexique. Ann. d. Mines (2) I, p. $337-33^{8}$.

r831: R a m ir e z: Gazetas de Literatura de Mexico. Puebla 183i. Bd. 2, p. $3^{8}$ I. 1843: P a r t s c h: Meteoriten,p.99- roo. 1852: C 1 a rk: Dissert. Gött., p. 63.

r852: W ö hle r : »Passiv ^, Pogg. Ann. Bd. 85 , p. 448 .

r853: Nög g e r a th: Meteoreisenmassen mit Widmannstädt'schen Figuren. N. J. 1853 , p. 174 (soll aus Verh, der niederrh. Ges. Bonn vom 12. Dez. 1852 sein; ist aber dort nicht zu finden); s. auch Kenngott, Uebersicht 1853 , p. 116.

1854: v.B o g u s l a w s ki: ZehnterNachtrag, Pogg. Ann. Ergz.-Bd. 4, p. 4II $-4 \mathrm{I} 2$.

1854: Urico e c h ea: Analyse derMeteoreisen von Toluca und vom Cap der guten Hoffnung. - I. Eisen von Toluca, Ann. Chem. Pharm. Bd, 91, p. 249-252; s, auch Pharm. Centr, 1854, p. 816 ; Journ. prakt. Chem. Bd. 63 (1854), p. $317-318 ;$ N. J. 1855 , p. 572 -573; Kenngott, Uebersicht 1854 , p. 122 ; Liebig-Kopp, Jahresber. 1854, p. 917; Weinschenk, Ann. Hof-Mus. Bd. 4 (1889), p. 100. 
1855: H a i d ing er: Bemerkungen über die zuweilen im geschmeidigen Eisen entstandene krystallinische Structur, verglichen mit jener des Meteoreisens. Sitzber. Wien. Akad. Bd. 15 I, p. 357.

1855: Krantz legte ein sehr schönes durchgeschnittenes u. geätztes Stück mexicanischen Meteoreisens vor, und berichtete darüber. Verh. naturh. Ver. Bonn Bd, 12 (Sitzber.), p. XLVIIXLVIII; s. auch im gleichen Band: Nöggerath, p. 300. Ref. N. J. 1855, p. 446; Kenngott, Uebersicht 1855 , p. 98 u. $185^{8}$, p. 126. Ref. über Cotta's Beob. der Widmanstätten'schen Fig. aus Berg- u. Hüttem. Zeit. Bd, 17, p. 304 .

r856: $\mathrm{Pugh}$ : Analyse von Meteoreisen aus Mexico. Ann. Chem. Pharm. Bd. 98 , p. $383-386$; s. auch Journ. prakt. Chem. Bd. 69 (1859), p. 309-310; Chem. Centr. 1856, p. 601-603; Am. Journ. (2) 24, p. 295; Liebig-Kopp, Jahresber. 1856, p.916-917 ; Kenngott, Uebersicht $1856 / 57$, p. 152-153; Weinschenk, Ann. Hof-Mus. Bd. 4, (1889), p. 100.

1856: T a y 1 or: Examination of the Meteoric Iron from Xiquipilco, Mexico. Am. Journ. (2) 22, p. $374-376$ (Analysen). Aus Proc. Acad. Nat. Sc. Philadelphia Bd. 8 (1856) Nr. 3, p. 128 -130; s. auch Journ, prakt. Chem. Bd. 70 (1857), p. $189-190$; N. J. 1857 , p. 578-579; Liebig-Kopp, Jahresber. 1856, p. 918; Kenngott, Uebersicht $1856 / 57$, p. 155 .

1856: Jordan: Ueber ein mexicanisches Meteoreisen. Ann. Chem. Pharm. Bd. IOI, p. $356-35^{8}$ (Analyse von Nason); Journ, prakt. Chem. Bd. 71 (1857), p. 122-123; Chem. Centr. 1857, p. 399 ; Liebig-Kopp, Jahresber. 1857 , p. 733-734; Kenngott, Uebersicht 1856157 , p. 155 .

1856: Burkart: Fundorte I, N. J. $(1856)$, p. $268,282,284,285,290$,
297-305 (Xiquipilco, Istlahuaca, Tejupilco; neue Analysen von Boecking); s, auch Journ. prakt. Chem. Bd. 71 (1857), p. $57-58$; N. J. $185^{8}$, p. 770 ; Liebig-Kopp, Jahresber. 1856 , p. 916 -918; Verh. naturh. Ver. Bonn Bd, I3 (1856), p. 40.

1856: Wöhler: Ueber das Meteoreisen von Toluca in Mexico. Sitzber. Wien. Akad. Bd. 20, p. 217-224. Ref. Liebig-Kopp, Jahresber. 1856 , p. 916.

1856: v. B a b o : Analyse eines Meteorsteins. Verh. Freiburg. Naturf. Ges. Bd. I Heft 2 (1856), p. 256-257 (Analyse).

1855/57: G i e b e 1 u. H e in tz: Zeitschr. f. d. ges. Naturw. Bd. 6, p. 99 u. Bd. 9 , p. 510 u. Bd. Io, p. 189,525 .

1857: B ergem a n n: Untersuchungen von Meteoreisen. Pogg. Ann. Bd, Ioo, p. $245-246,252-254$; s. auch Liebig-Kopp, Jahresber. 1857 , p. $733-$ 734; Kenngott, Uebersicht $1856 / 57$, p. 153; Chem. Centr. 1857, p. 745; Journ. prakt. Chem. Bd. 71 (1857), p. $57-5^{8}$.

1857: $\mathrm{Kr}$ a n tz: Ueber Meteoreisen vom Toluccathal in Mexico. Pogg. Ann. Bd, IoI, p. 152-153. Ref. N. J. 1857 , p. 830 ; Liebig-Kopp, Jahresber. 1857 , p. 734; Kenngott, Uebersicht 1856/57, p. 154 .

I857: v. Reich en b a ch: Ueber die Meteoriten aus dem Tolucathale in Mexico. Pogg. Ann. Bd. IO2, p. 62I625 ; s. auch Wochenschr. f. Astr., Met u. Geogr. 1858 , p. $317-318$.

1859: B u c hner: Feuermeteore, p. 106 $-107,138-143,147$.

1859: Ha rris: Dissert. Gött., p. 103 $-104,124-125$.

r860: $\mathrm{R}$ a m mel $\mathrm{s}$ b er $\mathrm{g}$ : Mineralchemie, p. 910-912, 948, 1000.

1860: W öhler: Analyse eines mexicanischen Meteoreisens. Ann. Chem. Pharm, Bd. 115, p. 95-96 (neue Ana- 
lyse von Martius); s, auch Journ. prakt. Chem. Bd. 82 (186r), p. 320 ; LiebigKopp, Jahresber. 1860 , p. $854-855$; Chem. Centr. 1860, p. $834-835$. 1861: Ros e: Ueber das Vorkommen von krystallisirtem Quarz in dem Meteoreisen von Xiquipilco in Mexico. Mon.-Ber. Berlin. Akad. (1861), p. 406 -409 ; s. auch Pogg. Ann. Bd. II3 (186r), p. 184-188; Verh. naturh. Ver. Bonn (Sitzber.) Bd. 30 (1873), p. 108; Chem. Centr. 186r, p. 494 ; sL'Institute Bd. 29 (186r) Nr, 1456, p. $400-401$; Zeitschr. f. d. ges. Naturw. Bd. 18, p. 60 ; N. J. 1862 , p. $82-83$; Liebig-Kopp, Jahresber. 186I, p. I131 ; Kenngott, Uebersicht 186I, p. 104-105. r858/62: v, Reichen bach: IV 643 .

VI 450,452 . VII 551, 557. VIII 487 . IX $162,164,173,174,181 . \quad X 359$, 364. XII $454,457,458$. XIII 355 , 363,384 . XIV 390 . XV Iro, III, II4, 124, 126, 128. XVI 250, 252, 26I, 262. XVII 266, 272. XVIII 480 (Fig. Magnetkies), $484,487,489,490$. XIX 153. XX 621, 622, 625, 626, 629, 630,634 XXI $578,579,582,5^{84}$, $585,586,5^{87}, 590$.

1862: R a mmelsberg: Ueber das Schwefeleisen der Meteoriten. Mon.Ber. Berlin. Akad. (1862), p. 689-69r; s. auch Journ. prakt. Chem. Bd. 88 (1863), p. 275-277.

1862: Kenngott u. W is er: Zürcher Sammlung, p. 156-157.

r863: B u chner: Meteoriten, p. ${ }^{1} 3^{8}$ -142 .

1863: R o s e: Meteoriten, p. 25, 42, 43, $60-62,64,138,139,152 ;$ s. auch Pogg. Ann. Bd. 124, p. 198, 201. 1866: B u rk a r t: Fundorte III, N. J. 1866, p. 402 (Caparrosa).

1867: M o h r: (Graphit auf dem Meteoreisen von Toluca). Verh, naturh. Ver. Bonn Bd. 25 (Sitzber.), p. 66.

1868: $\mathrm{S} \mathrm{mith:} \mathrm{A} \mathrm{new} \mathrm{Meteoric} \mathrm{Iron}$ from Mexico. Am, Journ. (2) 45, p.
77 (Analyse) (nach Fletcher Mex. Met., p. I7o zu Toluca gehörig). Ref. LiebigKopp, Jahresber. 1868, p. I045 ; Journ. prakt. Chem. Bd. I05 (1868), p. 8 (Poinsett-Eisen).

r869: $\mathrm{B} \mathrm{u} \mathrm{chner:} \mathrm{Die} \mathrm{Aetzfiguren} \mathrm{des}$ Meteoreisens, - Toluca. Ber. Oberhess. Ges. f. Natur- u. Heilk. Giessen 1869, p. $106-108$

r869: Meunier: Recherches, Ann. Chim. Phys. (4) 17, p. 39, 42, 44, 45, $46,49,50,51,54,56,57$.

1870: Burkart: Fundorte IV, N. J. 1870, p. 692 (Caparrosa).

r870: R a m mels berg : Meteoriten, p. $83,84,85,86$.

r872: Quenstedt: Klar und Wahr, p. 295 (Abb. einer geätzten Platte).

1875: vom R a th: Meteoriten, Verh. naturh. Ver. Bonn Bd. $3^{2}$, p. $35^{8}-36 r$. 1875: Mohr: Entstehungsart, Ann. Chem. Pharm. Bd. 179, p. 259, 264, 265-266.

1876: $\mathrm{S} \mathrm{m}$ it h : Carbon Compounds, Am. Journ. (3) II, p. 392.

r876: B a r cen a: On certain Mexican Meteorites. Proc. Acad. nat. hist. Philadelphia (1876), p. $124-125$.

$1878: \mathrm{Sm}$ ith: On the composition of the new meteoric Mineral, Daubréelite etc. (s, bei Coahuila). Am Journ. (3) 16, p. 272; s, auch Zeitschr. f. Kryst. Bd. 3, p. 328 ; Rammelsberg, Mineralchemie, 2. Aufl,, 1. Ergz.-Heft 1886, p. 76 .

1879: B e c k-K r a f t: Archiv f,Anthropologie Bd. 12, p. 307-3II.

r88r: Brezina : Bericht II, Sitzber.

Wien. Akad. Bd. 83 I, p, 476 .

r88r: Brezina: Bericht III, Sitzber.

Wien. Akad. Bd. 84 I, p. 282 .

r883: S m it h: Concretions, Am. Journ.

(3) 25 , p. $417,418,419$.

1884: M e un i e r: Météorites, p. $5^{8}, 60$, $64,67,69,70,72,93,96,98,116,117$. r884: $\mathrm{H}$ ä p k e : Beitrïge, Abh, naturh.

Ver. Bremen Bd. 8, p. 520 . 
r884: vom $R$ ath: Verh, naturh. Ver, Bonn (Sitzber.), p. 126. Ref. N. J. I885 II, p. 270.

1884: Wa d sworth: Studies, p. 6r. 1884: W i epken: Notizen über die Meteoriten des Grossherzoglichen Museums. - 5. Xiquipilco im Thale von Toluca, Mexico. Abh, naturw.Ver. Bremen Bd. 8, p. $527-528$.

r883/85: T s c h e r m a k: Photographien, p. 3 .

r885: Brezina: Wiener Sammlung, p. 210, 211, 268 (Sieron Blanca), 233. I886: Ansde11 u. Dewar: On the Gaseous Constituents of Meteorites. Proc Royal Soc. Bd. 40, p. 554 ; s. auch Am. Journ. (3) 32, p. 483 . Ref. Liebig-Kopp, Jahresber. I886, p. 2326. 1887: Flig h t: Meteorites, p. 93-95. 1887 : v. H a u e r: Ann. Hof-Mus. Bd. 2 (Not.), p. 39.

1887: Sorby: On the microscopical structure of iron and steel. Journ. Iron and Steel Inst. 1887, p. $283-288$. Ref. N. J. 1891 I, p. 47.

r889: F let c her: Atacama Meteorites, Mincral Magaz. Bd. 8, p. 226.

r889: v. Hauer: Ann. Hof-Mus. Bd. 4 (Not.), p. 64.

i889: C a s ti11o: Catalogue, p. 3-4. r889: C o h e n: São Julião, N. J. 1889

I, p. 221, 222, 223, 224, 225.

1890: E a s t m a n : Met. Astron., p. 322. r890: F let c her: Mexican Meteorites, Mineral. Magaz. Bd. 9, p. 99, IO3, 164-I7I, 174 .

1890: Brezina: Ueber Meteoreisen.

Oesterr. Zeitschr. f. Berg- u. Hüttenw. Bd. 38 , p. 356 .

r89r: Cohen u. Weins chenk: Meteoreisen-Studien. Ann, Hof-Mus. Bd. 6, p. 13I, 135-142 (Analyse), 162, 164,165 .

r8gr : v. Ha u er: Ann. Hof-Mus. Bd. 6 (Not.), p. 54.

1892: Co he n: Meteoreisen - Studien. Ann. Hof-Mus. Bd. 7 , p. $157-158$
(Analyse), $\mathbf{I}_{58}(\mathrm{Cu}), \mathbf{1 5 9}, \mathbf{1 6 0}, \mathbf{1 6}$.

r892: Eastman: The Mexican Meteorites. Bull. Philos. Soc. Washington Bd. 12, p. $46-47$.

1893: L i n c k: Ueber das Krystallgefüge des Meteoreisens. Ann. Hof-Mus. Bd. 8, p. II5-II6.

r893: Meunier: Revision des fers météoriques, p. 52, 53 .

r894: Cohen: Meteoritenkunde, an sehr vielen Stellen.

1894: F le t c her: Introduction, p. 33. 1895: L a s p y res u. Ka is er: Mittheilungen aus $d$. mineralogischen Museum der Universität Bonn. - 36 . Quarz- und Zirkonkrystalle im Meteoreisen von Toluca in Mexico. Zeitschr. f. Kryst. Bd, 24 , p. $485-493$.

1895: Brezina: Wiener Sammlung, p. $274-275$.

1895: Cohen: Meteoreisen-Studien IV. Ann. Hof-Mus. Bd. Io, p. $82-86,90$, 91, 92.

Ursprüngliches Gewicht: Zahlreiche grosse bis zu 220 (300?) Pfd. schwere und viele kleine Massen. Nachweisbares Gewicht: 972407 gr.

\begin{tabular}{lr|lr} 
Bailey & 406 & Calcutta & 7067 \\
Bayet & 4 & Cambridge & 289 \\
Belgrad & 421 & Catania & 84 \\
Bement & 7310 & Clausthal & 340 \\
Berkeley & 2906 & Cleveland & 692 \\
Berlin, G. & 1332 & Cohen & 89 \\
Berlin, P. & 73 & Czernowitz & 421 \\
Berlin, U. & 54861 & Darmstadt, M. & 2317 \\
Bern, M. & 523 & Dijon & 174 \\
Blatz & 391 & Dorpat & 406 \\
Böhm & 3055 & Dresden, M. & 504 \\
Bologna & 395 & Dresden, P. & 117 \\
Bonn & 62761 & Dublin, M. & 803 \\
v. Braun & 14630 & Edinburg & 175 \\
Bremen & 87 & Eger & 327 \\
Breslau & 1246 & Erlangen & 17 \\
Brezina & 154 & Frankfurt & 639 \\
Brunner & 338 & Freiberg, i. S. & 5485. \\
Budapest & 37254 & Freiberg, N. & 735 .
\end{tabular}




\begin{tabular}{|c|c|}
\hline Frenzel & Moskau \\
\hline Gent & München \\
\hline Giessen & Münster \\
\hline Goldschmidt & Neapel \\
\hline Göttingen & Neumann \\
\hline Graz, J. & New Haven \\
\hline Graz, U. & Newton $\quad 1690$ \\
\hline Gregory & New York, M. 1968 \\
\hline Greifswald $\quad \mathbf{1 1 7 6}$ & Odessa $\quad 49$ \\
\hline Greifswald, $\mathrm{Ph} .63$ & Oldenburg \\
\hline Halle $\quad 596$ & Paris, E. \\
\hline Hamburg & Paris, M. \\
\hline Harvard, U. $\quad 3423 \mathrm{I}$ & Paris, U. \\
\hline Heidelberg $\quad 55^{8}$ & Parma \\
\hline Helsingfors $\quad 418$ & Pech \\
\hline Karlsruhe, M. II40 & Pierrotet \\
\hline Karlsruhe, P. 200 & Pohl \\
\hline Kasan & Prag, B. U. \\
\hline Klausenburg & Prag. D. U. \\
\hline Kopenhagen $\quad 1560$ & Prag M. \\
\hline Krantz $\quad 25000$ & Riga \\
\hline Kristiania & Rom, U. \\
\hline Krüger & Schemnitz \\
\hline Kunz & Seligmann \\
\hline Lausanne & Sidney $\quad 2631$ \\
\hline Leiden & v. Siemaschko 5209 \\
\hline Leipzig $\quad 360$ & Stockholm $\quad 43278$ \\
\hline v. Leuchtenberg 160 & Strassburg \\
\hline Leoben $\quad 294$ & Stuer \\
\hline Lissabon $\quad 63$ & Stürtz \\
\hline London,B.M.106548 & Stuttgart \\
\hline London,P.G. 21813 & Szamosujvar \\
\hline Lund, M. & Troyes \\
\hline Madison & Tübingen \\
\hline Madras & Turin, J. \\
\hline Madrid & Turin, U. \\
\hline Manchester & Upsala \\
\hline Marburg & Utrecht \\
\hline de Mauroy & Ward $\quad 145474$ \\
\hline Melion & Washington $\quad 32148$ \\
\hline Minneapolis & Washington, Sh. \\
\hline Modena & 18488 \\
\hline
\end{tabular}

Wien, H. M.*)**) Wrany 53

I20 $472^{4}$ ) Würzburg $\quad 239$

Wien, U. I. II42 Zürich 1002 Wien, U. II. $\quad 374$

Das Museo Nacional in Mexico besitzt nach vom Rath eine kubikfussgrosse Masse. Die Universität Basel und die Herren Dr. Plagemann u. F. Cappel in Hamburg besitzen ebenfalls je ein Stück von Toluca.

Tomatlan

Gargantillo

Tomhannock Creek (Ironhannock Creek), Rensselaer Co., N. Y., U. S. A.

Stein, Cgb, gefunden um 1863 , erwähnt 1885 , beschr. 1887 .

Hierher vorläufig auch: Yorktown, New York, U. S. A. Stein sgefallen September I $869 \%$.

r885: B rez in a : Wiener Sammlung, p. $183,233$.

1887: Baile y: On an Aerolite from Rensselaer County, New York. Am. Journ. (3) 34, p. $60-62$ (Analyse von F. A. Wilber u. Abb. des Steins). 1890: Eastman: Met. Astron,, p. 320. 1893: N e w t o n: Lines of structure in the Winnebago Co. Meteorites and in other Meteorites. Am. Journ. (3) 45, p. 153,355 . Ref. N. J. 1894 I, p. 273 -274 .

r894: C o he n: Meteoritenkunde, p.6r. 1895: Brezina: Wiener Sammlung, p. 251 .

1895: Meunier: Revision des lithosidérites, p. $33,36-37$.

Ursprüngliches Gewicht: Etwa $1^{1} / 2 \mathrm{Kgr}$.

Nachweisbares Gewicht: 1496 gr.

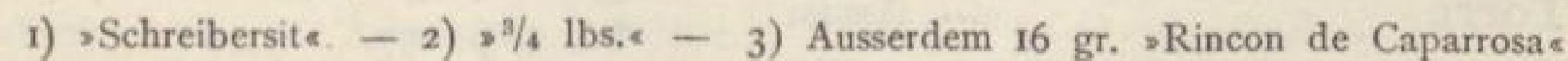
(s. Anhang Caparrosa). - 4) Ausserdem 20 gr. "Rincon de Caparrosa, 1858\& (s. Anhang Caparrosa); Toluca Mañi 42 gr., Xiquipilco 74 gr., letztere beide nach I. Juli 1893 erworben. 


\begin{tabular}{lr|l|r|} 
Bailey & I345 & de Mauroy & I \\
Bement & I6 & New Haven & 28 \\
v. Braun & I & Newton & 7 \\
Budapest & 5 & Paris, M. & 5 \\
Cleveland & 5 & v. Siemaschko & 2 \\
Dresden, M. & 6 & Ward & 8 \\
Gregory & 3 & Washington \\
Harvard, U. & 9 & \multicolumn{2}{|c}{ Dünnschliff } \\
Krantz & 7 & Washington, Sh. & 9 \\
London, B. M. & I & Wien, H. M. & 22 \\
Ausserdem unter & dem Namen Yorktown : \\
Bailey & 177 & London, B. M. \\
Bement & 6 & Paris, M. \\
Gregory & 27 & v. Siemaschko & I
\end{tabular}

\section{Tomsk Petropawlowsk}

Tonganoxie, Leavenworth Co., Kansas, U, S. A.

Eisen, Om, gefd. 1886 , beschr. 1891 . r8gr: Snow : Vorläufige Notiz in „Science (1891) 2. Januar.

r8gr: B ailey: The Tonganoxie Meteorite. Am. Journ. (3) 42, p. 385387 (Analyse; Abb. des Eisens und einer geätzten Fläche, Tafel XIII). Ref. N. J. 1892 II, p. 36.

1894: Co hen: Meteoritenkunde, p. 64, 232.

Ursprüngliches Gewicht : I $1 \frac{1}{2} \mathrm{Kgr}$. (26 lbs.).

Nachweisbares Gewicht: Bailey sagt 1891, dass die Hauptmasse dieses Eisens, welche in das Museum der Universität von Kansas gelangte, ro.55 Kgr, wiege. Berkeley führt 2 gr. eines Eisenmeteoriten von Kansas auf, welcher vielleicht hierher gehört.

\section{Tonnelier}

Torre

Mauritius

Touanne

Assisi

Toulouse, Dep. Haute-Garonne, Frankreich.
Stein, Cia (früher als Cga aufgefasst), gefallen ro. April 1812 .

1812: soniteur $;$; 6. Mai 1812, Nr. 127. 1812: Gi 1 b e rt : Meteorsteine. $-\mathrm{I}$. Neuster Steinregen in der Gegend von Toulouse am 10 April 1812. - A. Schreiben eines Ingenieurs des Brücken- und Wegebau's an den Präfekten des Departement der Dordogne; Perigueux, den 16. April 1812. - - B. Aus einem Schreiben des Hrn. Puymaurin, Mitglied des Gesetzgeb-Corps, an den Senator Hrn Chaptal. Gilb. Ann. Bd 4I, p. 445-449. Aus Bibl. Brit. Bd. 50, Nr. 394 u. 396, p. $62-67,159-171$. r812 : Journ. des Mines (Nr. 186), p. 419. 1812: G i 1 b e rt: Bericht über den Steinregen bei Toulouse am 10. April 1812 . Von einer Commission aus Mitgliedern der Akad. d. Wiss, zu Toulouse, Gilb. Ann. Bd. 42 , p. III-II5 (aus Journ. Phys. Bd. 74 (1812), p. $467-469$ ).

1812: Gi l bert: Einige Nachträge $z u$ dem Berichte über den Steinregen bei Toulouse, am 10. April 1812. Gilb. Ann. Bd. 42, p. 343-346 (wohl aus Mém. Acad. Toulouse (5) 3).

1812: Bigot de Morogues, p.275 -284 .

1815: Chladni: Neues Verzeichnis, Gilb. Ann. Bd, 5o, p. 255.

1819: Ch 1 a d n i: Feuermeteore, p. 50, 57 , $70,72,73,75,9 \mathrm{I}, 153-154,297-$ 299 (!), 431.

1819: Chladni: Vierte Fortsetzung, Gilb. Ann. Bd. 6o, p. 239.

1836: Kämtz: Meteorologie, p. 283 -284 .

I837: R o s e: Reise nach dem Ural. Bd.I,

p. 77 (Vergleich von Krasnoj-Ugol mit Toulouse und Krawin).

1843: Partsch: Meteoriten, p. 65. 1857: A rago: Astronomie populaire.

Bd. 4, p. 197.

1859: Harris: Dissert. Gött, p. 70. 1863: Bu chner: Meteoriten, p. 30 . 1863: R o se: Meteoriten, p. 92, 154. 
r858/65: v. Reichen ba ch: V 477 , 480 . IX 162, 168, 178. XI 295, 300. XX 626. XXV 431 .

r884: M e un i er: Météorites, p. 268, 270 (Abb.), 459, 523.

1885: Brezina: Wiener Sammlung, p. $182,233$.

r89o: v. Niess 1: Periheldistanzen, Verh. naturf. Ver. Brünn Bd. 29, p. 188, 194, 196, 214, 238-239.

r895: Brezina: Wiener Sammlung, p. 247,250 .

Ursprüngliches Gewicht: Viele Steine. Einer von I Kgr., ein anderer von $1 / 4$ Kgr., 6 weitere Steine von 4 Unzen und weniger gelangten an die UntersuchungsKommission.

Nachweisbares Gewicht: $420 \mathrm{gr}$.

\begin{tabular}{lr|lr} 
Bailey & 4 & de Mauroy & Spl. \\
Berlin, U. & 29 & Neumann & 2 \\
Budapest & Spl. & Paris, M. & 208 \\
Calcutta & I & Paris, U. & II \\
Gregory & 3 & v. Siemaschko & 12 \\
Halle & I & Stockholm & 4 \\
Klausenburg & Spl. & Troyes & 9 \\
London, B. M. & 32 & Tübingen & I2 \\
London, P. G. & 76 & Wien, H. M.*) & I6
\end{tabular}

Tounkin, Tunga, Irkutsk, Sibirien, Russland.

Stein, $\mathrm{Cg}$, gefallen 18 . Februar 1824 . 1824: Chladni: Vierter Nachtrag, Pogg. Ann. Bd. 2, p. 155-156. r828: F é r u s s a c : Bull. des Sc.Mathém. Bd. 9. p. 325 .

1832 : v. H off: Achter Nachtrag, Pogg. Ann. Bd. 24, p. 224-225. 1836: K äm tz: Meteorologie, p. 293. 1843: Parts ch: Meteoriten, p. I42. 1854: v. Boguslawski: Zehnter Nachtrag, Pogg. Ann. Ergz.-Bd.4, p.418. 1857: A rago: Astronomie populaire. Bd. 4, p. 200.

1858'59: v. Reichenbach: V 476. IX $162,171,180$.
I859: B u c hne r: Feuermeteore, p. 89. r859: H a r r is: Dissert, Gött, p. 78.

r863: B u chner: Meteoriten, p. 47 . 1867: Goebel: Kritische Uebersicht, Mélanges phys. chim. Bd. 7, p. 329 -330 .

1867: Buchner: Dritter Nachtrag, Pogg. Ann. Bd. 132, p. 317-318.

r885: Brezina: Wiener Sammlung, p. $182,233$.

I890: v. N i e s 81 : Periheldistanzen, Verh. naturf. Ver. Brünn Bd. 29, p. 193.

Ursprüngliches Gewicht: 2 Kgr., (5 Pfund russisch?).

Nachweisbares Gewicht: Ein Splitter, welcher aus der Tübinger Sammlung an das k. k. Hof-Museum in Wien gelangte. Der Stein soll in den 3 oer Jahren an die Petersburger Akademie geschickt worden sein; schonGoebel'sNachforschungen waren vergeblich.

Tourinnes-la-Grosse, Tirlemont, Beauvecchin, Belgien.

Stein, Cw, gefallen 7. Dezbr. 1863 . 1863: V a n Beneden: Bull. Acad. Roy. Belgique Bd. 16 (1863), p. 621 ; s. auch *L'Institut \& Bd. 32 (1864), Nr. 1573 , p. 62 .

r863: Desrumea ux : sLes Mondese (1863), p. 607.

1863: R o s e : Meteoriten, p. 155.

1863: Quetelet: Sur un aérolithe aperçu en Belgique le 7 décembre 1863. Bull. Acad. Roy. Belgique Bd, 16, Nr. 12, p. 9-10 (Sep.).

1864: Quetelet: Sur un aérolithe tombé dans les environs de Louvain, le 7 décembre 1863. Bull. Acad, Roy Belgique (2) 17, Nr. I, p. 12 - I6 (Sep.). I864: $\mathrm{T}$ h i e 1 e $\mathrm{n}$ s: Quelques mots à propos des Aërolithes, tombés en Brabant, le 7 Décembre 1863 . Brïssel $1864 ; 8^{n}$ (1o Seiten, gedruckt bei ?). 
1864: S a e m a n n: Note sur la météorite de Tourinnes-la-Grosse (Belgique), tombée le 7 décembre 1863 présentée par Daubrée. C. R. Bd. 58 , p. $74-$ 77 ; s. auch "L'Institut 1864 , p. 3.

1864: $\mathrm{P}$ is a n i: Analyse de l'aérolithe de Tourinnes-la-Grosse, près Louvain (Belgique), tombé le 7 décembre 1863. C. R. Bd. 58 , p. 169-171. Ref. N. J. I864, p. 476 ; Liebig-Kopp, Jahresber. r864, p. 897-898; Journ. prakt. Chem. Bd. 91 (1864), p. 255, 256; Kenngott, Uebersicht $1862 / 65$, p. 448 (wo die Analyse irrtümlich Daubrée zugeschrieben ist); sL'Institut \& 1864 Bd. 32, p. 19; Bull. Soc. Chim. (2) 2, p. 43; Chem. Centr. 1864, p. 311 ; Chem. News Bd. 9, p. 57 .

1864: $\mathrm{F}$ a $v$ a $\mathrm{rt}$ donne quelques renseignements sur l'état du ciel et les indications des instruments météorologiques au moment de la chute, qui eut lieu à $\mathrm{II}^{\mathrm{h}} \mathrm{I}_{5} \mathrm{~m}$ du matin. C. R. Bd. $5^{8}$, p. $517-518$.

r864: K ess e $1 \mathrm{~m}$ e yer: Meteorsteinfall bei Tirlemont in Belgien, am 7. Dec. 1863 und über den angeblichen Meteorsteinfall bei Brest, am Io. Jan, I864. Pogg. Ann. Bd. 122, p. 187-189. Ref. Kenngott, Uebersicht $1862 / 65$, p. 445 . 1864: Buchner: Erster Nachtrag, Pogg. Ann. Bd. 122, p. $322-323,330$ -331 .

1864: H a idinger: Der Meteorsteinfall von Tourinnes-la-Grosse bei Tirlemont im k. k. Hof-Mineralien-Cabinete. Sitzber. Wien. Akad. Bd. 49 II, p. 123 -127, 158-I59 (Mitteilungen von Dr. A. Thielens). Ref. Luminous Meteors, Rep. Brit. Assoc. 1864 (Sep.), p. 97 -98; Kenngott, Uebersicht 1862165, p. $447-448$; Wochenschr. f. Astr., Met. u. Geogr. 1864, p. 184 ; >L'Institut Bd. 32 (1864) Nr. I579, p. II2. 1865: Buchner: Zweiter Nachtrag, Pogg. Ann. Bd. 124, p. 585-586.

?: Renard: Sur la structure microscopique et la composition minéralogique de la météorite de Tourinnes (Brabant). Mém. Soc. belge de microscopie Bd. 5 , p. $43-50$.

r870: R a mmels berg: Meteoriten, p. 103, 105, 106, 138, 139, I40.

I875: v o m R a th: Meteoriten, Verh, naturh. Ver. Bonn Bd. 32, p. 369 .

r879: R a m mels berg : Meteoriten, p. 24,25 .

r883: T s c h er mak: Beitrag, Sitzber.

Wien. Akad. Bd. 88 I, p. 355 .

r884: M e un i er: Météorites, p. 79, 85, 197, 203-204, 395, 454 (Abb.).

r884: W a d swort h: Studies, p. 93. r883/85: T schermak: Photographien, p. 18 .

1885: Brezina: Wiener Sammlung, p. 169, 177, 232.

1885: P rinz: Les Météorites tombées en Belgique et les Météorites en général. "Ciel et Terre $\mathrm{Bd}$. 5. Brüssel (1885), p. $4,26-28$ (Sep.). Ref. N. J. 1886 II, p. 213.

r8gr: v. H a u e $r$ : Ann. Hof-Mus. Bd. 6 (Not.), p. 55.

Ursprüngliches Gewicht: $14^{1 / 2} \mathrm{Kgr}$.

Zwei Steine von 7 und $7 \frac{1}{2} \mathrm{Kgr}$. Nachweisbares Gewicht: $3440 \mathrm{gr}$.

Bailey

Bayet

Bement

Berlin, U.

Bonn

v. Braun

Brüssel

Budapest

Calcutta

Cleveland

Cohen

Dorpat

Frankfurt

Gent

Göttingen

Gregory
I Harvard, U. 17

135 Kopenhagen $\quad 35$

I London, B. M. 203

493 London, P. G. 4

5 Lüttich 129

I5I New Haven 4

72 Oilessa

I08 Paris, E. 95

22 Paris, M. 1300

I Rom, U. II6

Spl. v. Siemaschko 19

18 Stockholm 4

89 Stürtz 2

34 Ward 16

48 Washington, Sh. 9

42 Wien, H. M.*) 266 
Ist der grösste Teil der beiden Steine verschleppt oder befindet sich derselbe in Belgischen Museen? Der kleinere Stein wurde von der Landbevölkerung zerkleinert und verteilt, der grössere zerbrach beim Fall.

\section{Transsylvania}

s. Anhang

Travis Co., Texas, U S. A.

Stein, gefunden?, beschr. I89o.

Gehört dieser Stein vielleicht zum Fall Bluff?

1890: E a k in s: A new Stone Meteorite. Am. Journ. (3) 39, p. 59-6r (Analyse). Ref. N. J. 1891 I, p. 46; s, auch Bull. U. S. Geol. Surv. 1891, Nr. 78, p. 91 -93. Ref. N. J. I892 II, p. 242.

Ursprüngliches Gewicht: Ein Stein von setwa $2^{1 / 1} / 2 \mathrm{Kgr}$.

Nachweisbares Gewicht: 2657 gr. Bement 7 | Washington 2650

Trenton, 30 miles N. W. Milwaukee, Washington Co., Wisconsin, U. S. Eisen, Om, gefunden 1858 , beschr. 1869 (oder 1868 wie Klein 1879 sagt). Weitere Massen wurden 1869 und $187 \mathrm{I}$ gefunden.

Hierher auch: sThe Wisconsin Meteorites «.

Im J. 1868 wurde der Wisconsin Society of Nat, Hist, ein Bericht über dieses Meteoreisen vorgelegt. Ist dieser Bericht im Drucke erschienen?

1869: Brenndecke: On Meteorites. - Extract from a discourse, February 7 , 1869 , before the Society of natural history of Wisconsin. Rep. Smithsonian Institution for 1869 , p. 417-419 (Analyse von Bode)

1869: $\mathrm{S} \mathrm{m}$ it h : A New Meteoric Iron,sThe Wisconsin Meteoritese with some remarks onWidmannstätten Figures. Am. Journ. (2) 47, p. $271-272$ (Analyse); s. auch Original Researches 1884 , p. $444-445$; C. R. Bd. 68 (1869), p. $620-621$; N. J. 1871, p. 178-179; Liebig-Kopp, Jahresber. 1869, p. 1303; s. ferner Smith's Mineralogy and Chemistry, p. 349 ; Brezina, Meteoreisenstudien II, Denkschr. Wien. Akad. Bd. 44 (I88I), p. 133 (Anmerkung).

1872: L a pham: The Wisconsin Meteorite. Am. Journ. (3) 3, p. 69.

1879: Kle in: Göttinger Sammlung, Gött. Gel. Anz. (1879), p. 99.

r880: B rez in a : Reichenbach'sche Lamellen, Denkschr. Wien. Akad. Bd. 43, p. 15 (Abbildungen geätzter Platten). 1884: M eunier: Météorites, p. 116. 1884: Wiepke n: Notizen über die Meteoriten des Grossherzoglichen Museums. - 8. Washington-County Wisconsin. Abh. naturw. Ver. Bremen Bd. 8 , p. $529-530$.

r885: Brezina: Wiener Sammlung, p. 152, 2II, 212 (Colorado vielleicht Trenton), 234.

r887: Flig h t: Meteorites, p. 15, 130. r89o: Ea s tm a n : Met.Astron.,p.318,320. 1892: F o o t e: (Vergleich mit dem Eisen von Lonaconing). Am. Journ. (3) 43.p.64. 1893: Meunier: Revision des fers météoriques, p. 52,58 .

1894: C o h e n: Meteoritenkunde, p. 44, 107, 193.

r895: C o h e n: Meteoreisen-Studien IV. Ann. Hof-Mus, Bd. 1o, p. 82 .

Ursprüngliches Gewicht: $65 \mathrm{Kgr}$. 6 Massen: $60 ; 16 ; 10 ; 8 ; 16 \frac{1}{4}$ und $33 \mathrm{lbs}$; d die beiden letzten I 869 u. I 87 I gefunden.

Nachweisbares Gewicht: 24615 gr.

\begin{tabular}{lr|lr} 
Bailey & 67 & Budapest & 677 \\
Bement & 639 & Göttingen & 25 \\
Berkeley & 73 & Gregory & 64 \\
Berlin, U. & 1420 & Greifswald & 5 \\
Bonn & 126 & Harvard, U. & 6249 \\
v. Braun & 111 & Howell & 254 \\
Brüssel & 9 & Kopenhagen & 163
\end{tabular}


London, B. M. 223 Rom, U.

London, P. G. 25 v. Siemaschko

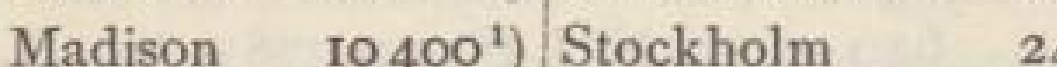

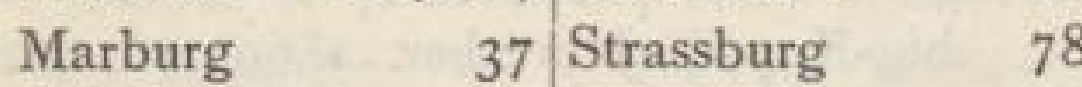

de Mauroy $\quad 42$ Stïrtz I30

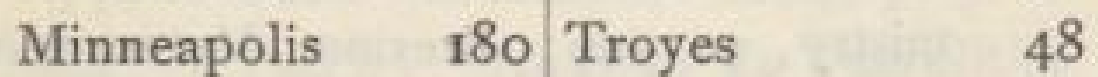

Moskau

Neumann

152 Upsala

67 Utrecht

7

New Haven

65 Ward

I8

Newton

Oldenburg

19 Washington 327

Paris, M.

Pohl

216 Washington, Sh. 91

961 Wien,H.M.*)**)пog I 99

Wo befindet sich der grösste Teil der Eisenmassen?

Unter der Bezeichnung $\gg$ Colorado, Om, bek. 1868 \& besitzt Wien, H. M. ein Eisen von $32 \mathrm{gr}$., von welchem Brezina vermutet, dass es vielleicht hierher gehört; cfr. 1885 . Brezina, Wiener Sammlung, p. 211, 212, 234; 1895: Brezina, Wiener Sammlung, p. 272, 276.

Trenzano, Brescia, Lombardei, Italien.

Stein, Cca (früher als Cc aufgefasst), gefallen 12. November 1856 .

1860: Curioni: Intorno al Bolide caduto nel Territorio di Trenzano il 12 Novembre 1856. Atti R. Instit. Lomb. di Scienze, Lettere et Arti. Milano 1860 Bd. I, p. $357-364$ (Analyse).

1860: Haidinger: Einige neuere Nachrichten über Meteoriten, namentlich die von Bokkeveld, New Concord, Trenzano, die Meteoreisen von Nebraska, vom Brazos, von Oregon. Sitzber. Wien. Akad. Bd. $4 \mathrm{I}$, p. $569-570$; s. auch $*$ L'Institute Bd. 29 (186r), Nr. 1409, p. 8.

1862: v. Reichenbach: XX 63 t.

r863: B u c hn e r: Meteoriten, p. 90-9r.

1863: R os e: Meteoriten, p. 154.

187x: M e unier: Coexistence de deux types lithologiques dans la même chute 1) $\$ 23 \mathrm{lbs}, \mathrm{a}$ de météorites. C. R. Bd. 73 , p. 1483 -1485 .

1873: Jervis: I Tesori Sotterranei dell' Italia Bd I, p. 277. Turin bei Loescher 1873 .

1884: M e un i er: Météorites, p 97, 188, I90-I9I, 273, 276, $3^{8}$ I.

r885: Brezina: Wiener Sammlung, p. $155,185,233$.

1887: Flig ht: Meteorites, p. 126.

1887: Brezina: Reisebericht, Ann. Hof-Mus. Bd. 2 (Not.), p. 73.

1893: B rez in a: Ueber neuere Meteoriten (Nürnberg), p. 16r.

r895: Brezina: Wiener Sammlung, p. $255,256-257$.

Ursprüngliches Gewicht: Zwei Steine, der eine 17 Pfund schwer.

Nachweisbares Gewicht: 105'5 gr.

\begin{tabular}{lr|lr} 
Bailey & 33 & New Haven & 43 \\
Bayet & 9 & Paris, E. & 62 \\
Berlin, U. & 6 & Paris, M. & 38 \\
Böhm & 991 & Pech & 4 \\
Bologna & 5505 & Riga & 47 \\
v. Braun & 81 & Roebling & 17 \\
Brezina & 471 & Rom, U. & 69 \\
Budapest & 121 & Sevilla & 15 \\
Calcutta & 25 & v. Siemaschko & 10 \\
Cleveland & 5 & Stockholm & 2 \\
Dorpat & 262 & Strassburg & 18 \\
Göttingen & 2 & Stürtz & 58 \\
Graz, J. & 16 & Troyes & 6 \\
Gregory & 24 & Turin, U. & 155 \\
Klausenburg & 10 & Utrecht \\
Kunz & 26 & Ward \\
London, B. M. & 158 & Washington, Sh. \\
London, P. G. & 24 & Wien, H. M.*) & 2131 \\
de Mauroy & 4 &
\end{tabular}

Trier

Trinity Co.

Troizk

Troy

Bitburg

Glorieta

s. Anhang Sanarka

Tuat

Bethlehem

s. Anhang Tamentit 
Tuckers Arizona Krasnojarsk

Tucson (Muchachos), Arizona, Mex.

Eisen, Dt, bekannt seit Jahrhunderten, wieder aufgefunden $185 \mathrm{I}$ ( 1846 ?), zuerst erwähnt 1852 . Dieses Eisen wird auch vielfach als Siderolith angesprochen (vgl. Senegal). Die Einsprenglinge von steinigem Material sind an den Wiener und Tübinger Stücken sehr klein; über das Londoner Stück teilte mir Herr Direktor Fletcher mit: scontains so much stony matter, that it seems unsatisfactory to keep away from other siderolithes .

Hierher auch: Cañada de Hierro (Thal des Eisens) 1846, auch Tucson 1846; Tucson Ainsa oder Tucson Sonora 1869; Carleton Tucson (1850); "The Tucson Irwin, Ainsa or Signet Meteorite * auch »The Ring Meteorite «; Muchachos(1660); Santa Rita; Taos.

1852: Le Conte: Notice of Meteoric Iron in the Mexican Province of Sonora. Am. Journ. (2) I3, p. 289-29o (erwähnt werden zwei grosse Massen; s, auch Proc. Amer, Assoc. advancement Sc. 6 th meeting, p. 188; Journ prakt. Chem. Bd. 64 (1855), p. Ir $8-120$; Kenngott, Uebersicht 1852 , p. $90-91$. 1854: Genth: On a New Meteorite from New Mexico. Am. Journ. (2) 17, p. 239-240 (Analyse). Es ist nicht sicher, ob dieses Eisen hierher gehört. Die Angabe einer deutlich oktaëdrischen s Spaltbarkeite spricht dagegen; s. auch Philos. Magaz (4) 7 (1854), p. 378-379; Journ. prakt Chem. Bd. 62 (1854), p. $188-189$, ferner Bd. 64 (1855), p. $118-120$ u. Bd. 66 (1855), p. $429-430$; N. J. 1857 , p. 166 ; Kenngott, Uebersicht 1855 , p. 97 ;
Liebig-Kopp, Jahresber. 1854, p. 913 -914; sL'Institut $\mathrm{Bd} .22$ (1854), Nr. $108_{3}$, p. 348 .

1854 : $S$ h e $p$ ar $d$ : Notice of three (1) ponderous masses of Meteoric Iron at Tuczon, Sonora. Am. Journ. (2) 18, p. 369 -372 ; s. auch Journ. prakt. Chem. Bd. 64 (1855), p. I18-12I; Kenngott, Uebersicht 1854 , p. 122 ; auch beiläufige Erwähnung bei Burkart, Fundorte I, N. J. 1856, p. 269.

1854: B a rt lett: Personal Narrative of Explorations in Texas, New Mexico, California,Sonora, and Chihuahua Bd.2, p. 297 (298?); Ref. Liebig-Kopp, Jahresber. 1855 , p. 1027.

1855: S mith: Memoir on Meteorites. - A description of five new Meteoric Irons, with some theoretical considerations on the origin of Meteorites based on their Physical and Chemical characters. - 4. Meteoric Iron from Tucson, Mexico. Am. Journ. (2) 19, p. 161-163 (Analyse u. Abbildung); s. auch Original Researches 1884 , p. 376-378; Journ. prakt. Chem. Bd. 66 (1855), p. $426-427$; Kenngott, Uebersicht 1855 , p. 96.

1855: Gen th: Analyses of the Meteoric Iron from Tucson, Province of Sonora, Mexico. Am. Journ. (2) 20, p. 119 -120 ; s. auch Proc. Acad. Nat. Sc. Philad. (1855) Bd. 7, p. $317-318$; Journ. prakt. Chem. Bd. 66 (1855), p. $429-430$; N. J. 1857 , p. 166 ; Liebig-Kopp, Jahresber. 1855 , p.1027. 1856: B u r k a r t: Fundorte I, N. J. 1856,

p. $273-277$ (Abb. Taf. IV).

1854/57: G ie bel u. He in tz: Zeitschr.

f. d. ges. Naturw. Bd. 3, p. 489 , Bd. 5 ,

p. 472 u. Bd. 9 , p. 330.

1857: Michler: Rep. of the U. S.

and Mex. Bound. Surv. Bd. I, Teil I, p. 118 .

1859: B u chner: Feuermeteore, p. 127, $147-148$.

1859 : H a r r i s : Dissert.Gött., p. 123. 
1860: R a mmelsberg: Mineralchemie, p. 913 .

r86r: Haidinger u. Jackson: Meteoreisen von Rogue River Mountain in Oregon u. von Taos in Mexico. Sitzber. Wien. Akad, Bd. 44 II, p. 29-30 (dieses Taos sollte Tucson sein, ist aber ein oktaëdrisches Eisen und daher verwechselt worden); s. auch .L'Institut $\alpha$ Bd. 29 (186I), Nr. 1446, p. 312.

r863: Brus h: Meteoric Iron from Tucson, Arizona. Am. Journ. (2) 36, p. I52 -154 (Analyse); s. auch Proc. California Acad. Nat. Sc. Bd. (I) 3, p. 33 , 48 ; Am. Journ. (2) 36, p. 301 ; LiebigKopp, Jahresber. 1863, p. 908 ; Kenngott, Uebersicht $1862 / 65$, p. $269-270$. r863: B u chner: Meteoriten, p. 183 -184 .

1863: Ros e: Meteoriten, p. 73, 150, 152. 1863: $\mathrm{H}$ a i d in g e $\mathrm{r}$ : Das Carleton-Tucson-Meteoreisen im k.k.Hof-MineralienCabinete. Sitzber. Wien. Akad. Bd. 48 II, P. $30 \mathrm{I}-308$ (Tafel). Ref. Kenngott, Uebersicht $1862 / 65$, p.268 ; sL'Institute Bd. 32 (1864), Nr. 1572, p. 55 -56 .

1863: Ir w in and A in s a : Smithsonian Rep. for 1863 , p. 85,86 ; s. auch unter Henry im gleichen Band, p. 55. r863: W hitney: Proc. Calif. Acad. Nat. Sc. Bd. 3 , Teil I, p. $33,48$. 1865: B u chner: Zweiter Nachtrag, Pogg. Ann. Bd, 124, p. 597-598.

1866: Wh it ney: Proc. Calif. Acad. Nat. Sc. Bd. 3, Teil III, p. 24 I.

1869: M e unier: Recherches, Ann. Chim. Phys. (4) 17, p. 52, 53, 68.

r870: v. Haidinger: Der Ainsa-Tucson-Meteoreisenring in Washington und die Rotation der Meteoriten in ihrem Zuge. - 3. Die Meteoreisenmassen Carleton-Tucson und Ainsa-Tucson. Sitzber. Wien. Akad. Bd. 6r II, p. 506 $-511,514$ (Abbildung).

r870: $\mathrm{R}$ a $\mathrm{mmel} \mathrm{sb}$ erg: Meteoriten, p. 84 .
187 $\mathrm{I}: \mathrm{S} \mathrm{m}$ it h: The precise geographical position of the large masses of meteoric iron in North-Mexico, with the description of a new mass - the San-Gregorio Meteorite: Am. Journ. (3) 2, p. $337-338$.

1873: $\mathrm{M}$ eu $\mathrm{n}$ i er: Détermination minéralogique des Holosidères du Muséum. C. R. Bd. 76, p. 1281 .

1883/84: W i 11 i a m s : Mineral Resources of theUnitedStates for $188_{3} / 84, \mathrm{p} .290$.

r884: Me unier: Météorites, p. 44, 97, I34-I35, 44I, 459 .

r884: W a dsworth: Studies, p. 69. 1885: Brezin a: Wiener Sammlung, p. 218, 220, 221, 234 .

r885/86: Clarke: The Meteorite Collection in The U.S. National Museum. Rep. Smithsonian Inst., p. 257, T. I (Abbildung).

1886: Brezina: Neue Meteoriten I, Ann. Hof-Mus. Bd, I (Not.), p. 13. 1887: B r e z in a: Neue Meteoriten IIIa, Verh. k.k.geol. Reichsanst. (1887), p.289. r887: v. Ha u er: Ann. Hof-Mus. Bd, 2 (Not.), p. $3^{8}$.

1887: F $1 \mathrm{ig} \mathrm{ht:} \mathrm{Meteorites,} \mathrm{p.124-125.}$ (Abbildung).

r8go: Flet cher: The Meteoric Iron of Tucson. Mineral. Magaz Bd. 9, p. $16-36$ (Zusammenfassung aller früheren Arbeiten; s, auch im gleichen Band die Arbeit über die mexikanischen Meteoriten, p. 104). Ref. N. J. 1891 I, p. 242.

1890: Eastm a n : Met.Astron.,p.318,320. r89r: Cohen u. Weinschenk: Meteoreisen-Studien, Ann. Hof-Mus. Bd. 6, p. $160,165$.

1893: Meunier: Revision des fers météoriques, p. 36 .

1894: B rez in a: Die Gestaltung der Meteoriten. Vortr, z. Ver. Verbr. naturw. Kenntnisse. Wien. Jahrgang 34, Heft 9, p. $20-2 \mathrm{I}$.

r894: Co he n : Meteoritenkunde, p. 52, $197,256,267$. 
r894: F let ch er: Introduction, p. II. r895: Brezina: Wiener Sammlung, p. $273,295-296$.

r895: C o h e n : Meteoreisen-Studien IV. Ann. Hof-Mus. Bd. 10, p. 83,90 .

Ursprüngliches Gewicht : Etwa ı 000 Kgr. Zwei (oder auch drei) grosse Massen, die eine 1400 , die andere rooo lbs. schwer.

Nachweisbares Gewicht: 637224 gr.

\begin{tabular}{|c|c|c|}
\hline Bailey & 1003 & London, B. M. 443 \\
\hline Bement & 88 & London, P. G. 20 \\
\hline Berlin, U. & 27 & Madrid \\
\hline Bonn & 40 & New Haven \\
\hline v. Braun & 117 & Paris, M. \\
\hline Budapest & II & Rom, U. \\
\hline Calcutta & 99 & v. Siemaschko \\
\hline Cleveland & 8 & Stockholm \\
\hline Dorpat & 40 & Strassburg \\
\hline Dresden, M. & 82 & Stürtz \\
\hline Göttingen & 16 & Tübingen \\
\hline Gregory & 177 & Ward \\
\hline Greifswald & 24 & Washington 630000 \\
\hline Harvard, U. & 253 & Washington, Sh. I2I \\
\hline Howell & 40 & WienH.,M.". $\left.\left.{ }^{* *}\right) 622^{1}\right)$ \\
\hline
\end{tabular}

\section{Tucuman}

Tula 1846

Tula $\quad$ 874

Tula 1878

Tule

Tulisca

Turanaki

Turgaisk
Campo del Cielo Netschaëvo Sewrukof Rakowka Huejuquilla-Gruppe Gargantillo Wairarapa Bischtübe

TurnerMound Brenham(Anderson) Turon River

Tyree

s. Anhang

Tysnes (Midt-Vaage), Gehöft MidtVaage auf der Tysnes-Insel südlich von Bergen, Norwegen.

Stein, Cgb, gefallen 20. Mai r 884 .
I886: R e u s ch: Ueber den Tysnesmeteorit und drei andere in Skandinavien niedergefallene Meteorsteine. - I. Der Tysnesmeteorit (20. Mai 1884 Abends zwischen 8 und 9 Uhr). N. J. B. B. IV, p. $473-486$ (mit 4 Tafeln, Abbildung des Steins und mikroskopischer Präparate); s, auch Nyt Magazin for Naturvidenskaberne Bd. 29, p. 309320: Om Tysnesmeteoriten og tre andre i Scandinavien nedfaldne meteorstene (Tafel); Liebig-Kopp, Jahresber. 1886, p. 2328.

1886: $\mathrm{R}$ e u s c h : Om Meteorstene. $>\mathrm{Na}-$ turen $\approx$ (1886), Nr. 9-io. Ref. N. J. 1889 II, p. 43; übersetzt in American Naturalist Bd. 22 (Febr. 1888), Nr. 254, p. 97.

r887: $\mathrm{H}$ i or d a h 1: Analyse af TysnaesMeteoriten. Nyt Magazin for Naturvidenskaberne Bd. 30 , Heft 3 .

r887: v. H a uer: Ann. Hof-Mus, Bd. 2 (Not.), p. 39.

r89o: v. $\mathrm{N}$ i e s s 1 : Periheldistanzen, Verh. naturf. Ver. Brïnn Bd, 29 , p. $183-I 84$, 189, 191, 194, 195, 214, 254-255.

1894: C o h e n : Meteoritenkunde, p.26r, 262.

r895: B r e z i n a: Wiener Samml., p.252. Ursprüngliches Gewicht: 21.7 Kgr. Der grosse Stein wog 18.95 Kgr., ein grosses Bruchstück von einem andern Stein 0.9r Kgr., ausserdem kleinere Bruchstücke.

Nachweisbares Gewicht: 20894 gr.

\begin{tabular}{lr|lr} 
Bement & IO & Paris, M. & 73 \\
Berlin, U. & 30 & Rom, U. & 28 \\
v. Braun & 35 & v. Siemaschko & 5 \\
Budapest & 82 & Stockholm & 98 \\
Gregory & II & Strassburg & II \\
Kristiania & 19 359 & Stürtz & 62 \\
London, B. M. 896 & Utrecht & 7 \\
de Mauroy & 12 & Washington & 29 \\
München & 47 & Wien, H.M. & 93 \\
Odessa & 6 & &
\end{tabular}

I) Davon I gr. "Muchachos, False Hacienda Concepcion, Chihuahua, Mexico \&. W ül fing, Meteoriten.

24 
Uden (Staartje), Gemeinde Uden, Herzogenbusch, Nordbrabant, Holland.

Stein, Cwb, gefallen 12. Juni 1840 .

1843: v. R ees: Zwei Meteorsteinfälle in Holland, ein neuer und ein älterer. Pogg. Ann. Bd. 59, p. $349-350$; s. auch Haidinger, Uebersicht 1843 , p.rog. 1845: v. Ba $\mathrm{mhauer}$ : Ueher den mutmasslichen Ursprung der Meteorsteine, nebst einer Analyse des Meteorsteins, welcher am 2. Juni 1843 in der Provinz Utrecht gefallen ist. Pogg. Ann. Bd. 66 , p. 467 ; s. auch bei Utrecht. 1854: v. Bog us law s ki: Zehnter Nachtrag, Pogg. Ann. Ergz.-Bd. 4, p. 360. r859: B u c hn er: Feuermeteore, p. I8r -182 .

r859: H a rris: Dissert. Gött., p. 87. 1862: v. B a um ha er: Scheikundig onderzoek van den Meteorsteen van Uden. Verslagen en Mededeelingen Amsterdam. Akad. Bd. 14, p. I-8 (Analyse in Gemeinschaft mit Seelheim; Abbildung); s. auch Pogg. Ann. Bd. II6 (1862), p. 184-188: :Chemische Untersuchung des Meteorsteins von Uden«. Ref. Kenngott, Uebersicht 1862165, p. 434; Liebig-Kopp, Jahresber. 1862, p. $826-827$; Chem. Centr. 1862, p. 864 .

I863: B u c hner: Meteoriten, p. 64 . 1860/65: v. Reichen bach: XIV 392. XV ior XX 623. XXV 607.

r870: Rammels berg: Meteoriten, p. 106, 139, 140.

1884: Meunier: Météorites, p. 85, 95, 208, 216, 395 .

r885: Brezin a : Wiener Sammlung, p. $181,232$.

r894: C o he n : Meteoritenkunde, p. 248, 303.

Ursprüngliches Gewicht: 720 gr.
Nachweisbares Gewicht: 708 gr.

Bailey I London, B. M. 5

Budapest $\quad 3$ Paris, M. Spl.

Calcutta Spl. Tübingen 9

Göttingen Spl. Wien, H. M.*) Spl. Herzogenbusch690 ${ }^{1}$ )

\section{Uderei s. Anhang Angara}

Udipi, Süd-Canara, Malabar-Küste, Ostindien.

Stein, Cga, gefallen April I866.

1884: Meunier: Météorites, p. 209

(nur aufgeführt unter seiner grossen Gruppe "Lucéite «).

1885: Brezina: Wiener Sammlung, p. $182,233$.

1894: F l et cher: Introduction, p. I3 $_{3}$

Ursprüngliches Gewicht: ?

Nachweisbares Gewicht: 3673 gr.

\begin{tabular}{ll|l} 
Bement & I London, B. M. 3306
\end{tabular}

Budapest $\quad 6$ London, P. G. 82

Calcutta $\quad 53$ Paris, M. 51

Gregory $\quad$ I5 v. Siemaschko 8

Harvard, U. $\quad 62$ Wien, H.M.*) 89

Der Stein scheint noch nicht näher untersucht worden zu sein; auch habe ich über eine Beschreibung des Falls nichts erfahren können.

Umbala, Delhi, Ostindien.

Stein, Cga, gefallen 1822 oder 1823 . 1859: A $t k$ inson sagt in seinem Verzeichnis der im Besitz der Asiatic Society befindlichen Meteorite̊n : >Nr. 3 : Aerolite which fell about 40 miles to the west of Umbala between the Jumna and Punjab in $1832-3$, obtained by Captain Murray; given by Mr. J. Bird to Mr. Cracroft. Weight $3^{1} / 2 \mathrm{Oz}$. Journ. Asiat. Soc. Bengal. (Proc.) Bd. 28, p. 260 .

1862: M a s k el y ne: Chondritic Aërolites, Rep. Brit. Assoc. 1862 (Not. and Abstr.), p. 190.

r) Museum der Provincial Genootschap van Kunsten en Wetenschappen in NoordBrabant, nach Mitteilung des Freiherrn v. Ryckevorsel vom 3. April 1896. 
r863: Bu chner: Meteoriten, p. 56. r869: Buchner: Vierter Nachtrag, Pogg. Ann. Bd, 136, p. 446.

1885: Brezina: Wiener Sammlung, p. $182,233$.

1894: F let c h er: Introduction, p. Io. Ursprüngliches Gewicht: $100 \mathrm{gr}$. $\left(3^{1 / 2}\right.$ oz. $)$.

Nachweisbares Gewicht: 68 gr. Bailey

Calcutta Göttingen 2 London, P. G. I 34 New Haven I 2 v. Siemaschko 4 London, B. M. 2I Wien, H. M.*) 3

\section{Umjhiawar}

Shergotty

Unbekannte Fundorte von Eisen s. Anhang

Unbekannte Fundorte von Steinen s. Anhang

Union Co., Georgia, Nordamerika. Eisen, Ogg (früher als Obn aufgefasst), gefunden 1853 , beschr. 1854 .

r854: Shepard: New Localities of Meteoric Iron. - 3. Union county, Georgia. Am. Journ. (2) 17 , p. 328 (analytisch nur $3.32 \% \mathrm{Ni}$ nachgewiesen); s. auch Journ. prakt. Chem. Bd. 62 (1854), p. 347 ; Kenngott, Uebersicht 1854, p. 120; Liebig-Kopp, Jahresber. 1854 , p. $915-916$.

1859: B u c h ne r : Feuermeteore, p. 135. 1859: H arris : Dissert. Gött., p. 119 -120 .

r859/62: v. Reich en ba ch: IX 162, 174, 181. XV no. XVII 266. XX 621, 622. XXI 586,589 .

1863: Buchner: Meteoriten, p. 187.

1863: Ros e: Meteoriten, p. 5I, 152.

r869: Meunier: Recherches, Ann.

Chim. Phys. (4) 17, p. 68.

r884: M e un i e r: Météorites, p. 44, 73, 96, iII.

r885: Brezina: Wiener Sammlung, p. 217,234 .
I890: Eastma n: Met. Astron., p. 318 . 1893: Meunier: Revision des fers météoriques, p. $23,24-25$.

r894: Co h e n : Meteoritenkunde, p. 194. r895: Brezina: Wiener Sammlung, p. 288.

Ursprüngliches Gewicht: $6.8 \mathrm{Kgr}$. (I 5 lbs.). Shepard erhielt $502 \mathrm{gr}$. (I lb. $I^{3} / 4$ oz.).

Nachweisbares Gewicht: 7 II gr.

Bailey

Bement

I8 London, P. G. $\quad$ I13

Berlin, U.

Budapest

Calcutta

Cleveland

Dorpat

Göttingen

Gregory

Harvard, U.

59 de Mauroy 2

54 Minneapolis I

4 New Haven 15

32 Paris, M. 73

5 v. Siemaschko 2

7 Strassburg $\quad 7$

I4 Tübingen $\quad 67$

7 Ward

35 Washington, Sh. 124

London, B. M. $\quad 55$ Wien, H. M.*) 16

Urba

Urei

Wirba

Utah

Nowo-Urei

Utrecht (Blaauw-Kapel), Holland. Salt Lake City Stein, Cca (früher als Cc aufgefasst), gefallen 2. Juni 1843 .

1843: Quetelet: Sur deux aérolithes tombés le 2 juin, près d'Utrecht, C. R. Bd. 16 , p. $1311-I_{3} 12$; s. auch $\gg$ L'Institut \& Bd. II (1843), p. 197.

1843: v. Rees: Zwei Meteorsteinfälle in Holland, ein neuer und ein älterer. Pogg. Ann. Bd, 59 , p. $348-349$; s, auch sL'Institut * Bd. 11 (1843), p. 297; Haidinger, Uebersicht 1843, p. Iog.

1845: v. B a u hauer: Ueber den mutmasslichen Ursprung der Meteorsteine, nebst einer Analyse des Meteorsteins, welcher am 2. Juni 1843 in der Provinz Utrecht gefallen ist. Pogg. Ann. Bd. 66 , p. $465-467,485-498$ (Analyse). Ref. Kenngott, Uebersicht $1844 / 49$, p. $283-284$; s. auch v. Baumhauer's Dissert. De ortu lapidum $24^{*}$ 
meteoricorum annaxis duorum lapidum analysibus chemicis. Trajecti ad Rhenum 1844. Sectio altera. Analysis lapidis qui cecidit prope Rheno-Traiectum, p. $54-8$ o. Ref. N. J. 1847 , p. 730 ; s. auch Rammelsberg, Suppl. II (18 +5), p. $97-99$ u. Suppl.V (1853), p. 25-27; Berzelius, Jahresber. Bd. 26 (1847), p. $385-387$.

I854: v. Bog us law s ki: Zehnter Nachtrag, Pogg. Ann. Ergz.-Bd. 4, p. 368 -370 .

r859: B u c hner: Feuermeteore, p. 94 -95 .

1859: H a rris: Dissert. Gött., p. 89 $-90$.

1860: R a mmels berg: Mineralchemie, p. $923,924,950$.

r863: B u c h n e r: Meteoriten, p. $7 \mathrm{I}-72$. 1859/65: v. Reichen b a h: IX 16I, 169, 179. X 359, 362, 371. XI 295, 298. XIII 361. XIV 399. XIX 152. $\mathrm{XX}$ 623. XXV 607.

1870: Rammelsberg: Meteoriten, p. 103, 105, 106, 138, 139, 140.

r872: Tschermak: Die Meteoriten von Shergotty und Gopalpur. Sitzber. Wien. Akad. Bd. 65 I, p. 139.

1879: R a m me ls berg: Meteoriten, p. 24,25 .

r882: K re cke: De Meteorsteene,p. 32 (nach Meunier).

1884: M e unier: Météorites, p. 79, $85,208,217-218,395$.

r885: Brezina: Wiener Sammlung, p. $185,233$.

r894: C o h e n : Meteoritenkunde, p. 56, 303.

1894: F l e t c her: Introduction, p. 36. 1895: Brezina: Wiener Sammlung, p. $255,256$.

Ursprüngliches Gewicht : Etwa 9.7 Kgr. Zwei Steine von 7 u. 2.7 Kgr. Nachweisbares Gewicht: $9^{8} 7^{2}$ gr. ${ }^{1}$ )

\begin{tabular}{lr|lr} 
Bailey & I & Melion & 2 \\
Bayet & I & Neumann & I \\
Belgrad & 4 & New Haven & 4 \\
Bologna & 4 & Paris, M. & 24 \\
Bonn & 20 & Pohl & 38 \\
v. Braun & 8 & Rom, U. & 23 \\
Budapest & 6598 & v. Siemaschko & 50 \\
Calcutta & Spl. & Stockholm & 200 \\
Frankfurt & 18 & Strassburg & 4 \\
Göttingen & I & Stürtz & 15 \\
Gregory & 25 & Tübingen \\
Harvard, U. & 9 & Utrecht \\
Klausenburg & 4 & Washington \\
London, B. M. & 70 & Washington, Sh. \\
de Mauroy & 4 & Wien,H.M.*)**) & 373
\end{tabular}

Vaca Muerta (Sierra de Chaco), Quebrada di Vaca Muerta, San Pedro de Atacama, Janacerapass, Taltal, Südamerika.

Mesosiderit (Grahamit), bekannt 1861, beschr. 1862.

Hierher auch: Vegas i Carrisalillo und »Mejillones« (Fletcher).

r862: Domeyko: Mémoire sur les grandes masses d'aérolithes trouvées au désert d'Atacama dans le voisinage de la Sierra de Chaco. C. R. Bd. 55, p. $873-874$.

1863: B u chner: Meteoriten, p. 131 -132 .

r863: Ros e: Mittheilung über den Meteorit von der Sierra de Chaco in Chile. Mon.-Ber. Berlin. Akad. (1863), p. 30-34 (schlägt den Namen Mesosiderit vor; Analyse des metallischen Teils von Domeyko); s. auch $>$ L'Institute Bd. 31 (1863), Nr. 1548, p. 278 -279 ; N. J. 1863 , p. $361-362$; Liebig-Kopp, Jahresber. 1863, p. 909 ; Kenngott, Uebersicht $1862 / 65$, p. 435 -436 .

1863: Rose: Meteoriten, p. $81-83$, I4I, 148-149, 153 .

r) Hiernach scheint der Stein von >7 Kgr. etwas mehr gewogen zu haben. 
I864: D o m e y k o : Meteorologia. Sobre las grandes masas de aerolitas halladas en el Desierto de Atacama cerca de la cierra de Chaco. Anal. Univers, d. Chile (Santiago) Bd. 25 , p. 289 - 3or. I864: D o m e y k o: Rapport sur deux Mémoires de M. J. Domeyko, relatifs, I'un à de grandes masses d'aérolithes trouvées dans le désert d'Atacama, près de Taltal; l'autre à plusieurs espèces minérales nouvelles du Chili, - I. Examen des aérolithes de Taltal. C. R. Bd. 58 , p. $55 \mathrm{I}-556$ (Analyse); s, auch Ann. des Mines (6) 5 (1864), p. 43I -450 ; Chemical News Bd. 9, p. 175; Journ. prakt. Chem. Bd. 95 (1865), p. 59-61 ; Liebig-Kopp, Jahresber. 1864, p. 903; Kenngott, Uebersicht I862|65, p. $449-450$.

1864: Joy: Analysis of a meteorite from Chili. Am. Journ. (2) 37, p. 243 -248 (Analyse); s. auch Bull. Soc. Chim, (2) 3, p. 426; Chem. Centr. 1864, p. 1055; Journ. prakt. Chem. Bd. 94, p. 167-169; Liebig-Kopp, Jahresber. 1864 , p. 902 ; Kenngott, Uebersicht 1862165 , p. $269,44^{8}-449$.

r864: H a i d in g e r : Eine grosskörnige Meteoreisen-Breccie von Copiapo. Sitzber. Wien. Akad. Bd. 49 II, p. 494496 (Mitteilung von v. Tschudi).

1864: Smith: A new Meteoric Iron from Wayne County, Ohio. - Some remarks on the recently described Meteorite from Atacama, Chili. - The new Atacama Meteorite. Am. Journ. (2) 38 , p. $386-387$; s. auch Original Researches 1884 , p. $432-433$; Journ. prakt. Chem. Bd. 95, p. 313-314; Kenngott, Uebersicht 1862165 , p. 450 .

1865: Buchner: Zweiter Nachtrag, Pogg. Ann. Bd. 124, p. $592-596,600$. 1867: D a u b r é e : Contribution à l'anatomie des météorites. C. R. Bd. 65 , p. $148-151$.

r869: B u chner: Vierter Nachtrag, Pogg. Ann. Bd. 136, p. 609. r869: M e unier: Recherches, Ann. Chim. Phys. (4) 17, p. $5^{2}$.

r870: Rammels berg : Meteoriten, p. $95-97$.

1872: $\mathrm{M}$ e unier: Analyse lithologique de la météorite de la Sierra de Chaco. Mode de formation de la logronite. C. R. Bd. 75 , p. $1547-1552$.

1875: Do me y k o: Note sur deux nouvelles météorites du désert d'Atacama et observations sur les météorites qui ont été découvertes jusqu'ici dans cette partie de l'Amérique méridionale. C. R. Bd. 81, p. 599-600 (Anmerkung Daubrées). Ref. Liebig-Kopp, Jahresber. 1875 , p. 1316.

1879: Do m e y k o: Mineralojia. 3. Aufl. (1879), p. 135-137; s, auch: Zweiter Nachtrag (1883), p. 39--4I (bezieht sich auf Vegas i Carrisalillo).

r88x : Brezina: Bericht III, Sitzber. Wien. Akad. Bd. 84 I, p. 278 .

1883: T s c h e r m a k: Beitrag, Sitzber. Wien. Akad. Bd. 88 I, p. 351,354 (schlägt den Namen Grahamit vor).

r884: Meunier: Météorites, p. 77 $-79,87,94,97,165,166-169,359$, $373,500-501$.

1884: W a ds w or th: Studies, p. 73. 1883/85: Tschermak: Photographien, T. 22, 23, p. 21, 22, 23, 24. 1885: Brezina: Wiener Sammlung, p. $19 \mathrm{I}-192,233$.

r889: F let c her: Atacama Meteorites, p. $224,225,231,233,234-243,252$ (Taltal ; Sierra de Chaco; Chili; Janacera Pass; Jarquera Pass; Mejillones). Ref. N. J. 1890 II, p. 230.

I889: v. Sandberger: Ein neuer Meteorit aus Chile. N. J. 1889 II, p. 175. 1893: B rez in a: Ueber neuere Meteoriten (Nürnberg), p. 162.

1893: Newton: Lines of structure in the Winnebago Co. Meteorites and in other Meteorites. Am. Journ. (3) 45, p. $152-153,355$. Ref. N. J. I894 I, p. $273-274$. 
1894: Cohen: Meteoritenkunde, an vielen Stellen.

r 894 : F l e t c h e r: Introduction, p. II,I4. 1895: Meunier: Revision des lithosidérites, p. $33,35-36,37-38$.

r895: Brezina: Wiener Sammlung, p. 262 .

Ursprüngliches Gewicht: Grosse Massen, deren ursprüngliches Gewicht sich nicht ermitteln lässt, weil vielfach Verwechslungen mit Imilac vorzuliegen scheinen. Zwei Zentner sollen in die Hände von Sammlern gelangt sein.

Nachweisbares Gewicht: 34060 gr.

\begin{tabular}{|c|c|c|c|}
\hline Bailey & $\left.57^{1}\right)$ & Hamburg & $\left.1612^{7}\right)$ \\
\hline Bement & $\left.5 \mathrm{II}^{2}\right)$ & Harvard, U. & 92 \\
\hline Berlin, U. & 453 & Howell & 6 \\
\hline Böhm & I 47 & Kopenhagen & 29 \\
\hline Bologna & 93 & London, B. M. & $\cdot 728_{3}$ \\
\hline v. Braun & 9 & London, P. G. & - 778 \\
\hline Brezina & $\left.1006^{3}\right)$ & de Mauroy & 9 \\
\hline Budapest & $35^{2}$ & Melion & 4 \\
\hline Calcutta & 9 & Moericke & 210 \\
\hline Cleveland & $\left.18^{4}\right)$ & Moskau & 10 \\
\hline Cohen & 536 & München & 247 \\
\hline Dorpat & 3 & New Haven & 39 \\
\hline Dresden, M. & $3^{8}$ & Newton & 1 \\
\hline Göttingen & $\left.1023^{5}\right)$ & Paris, E. & 2214 \\
\hline Gregory & $\left.211^{6}\right)$ & Paris, M. & $\left.205^{8}\right)$ \\
\hline
\end{tabular}

\begin{tabular}{l|l|l|} 
Pohl & 43 & Utrecht
\end{tabular}

Rom, U. $\quad 52$ Ward 32

v. Siemaschko $86^{\circ}$ ) Washington 449

Stockholm 14 Washingt., Sh. 28

Strassburg $\quad 6_{3}$ Wien, H. M. *)**)

Stürtz $\quad 54 \quad 2973^{10}$ )

$\begin{array}{llll}\text { Troyes } & 8 & \text { Wien, U. I. } & 16\end{array}$

Upsala 8

Das Museum in Manchester, Owens College, besitzt ein $1624 \mathrm{gr}$, schweres Eisen von $*$ Taltal, Atacama-Wüste, Chili๔. Herr B. Hobson teilte mir nit, dass dieses Stück kein Mesosiderit, sondern ein Meteoreisen sei. Es bedarf einer näheren Untersuchung, um dieses Eisen einreihen zu können. Klausenburg führt unter Mesosideriten Atacama Bolivia $185^{8} \approx 98 \mathrm{gr}$. auf, die vorläufig hier angeschlossen werden mögen; Name und Jahreszahl würden auf Joël-Eisen deuten.

Vago (Verona) bei Verona, Italien.

Stein, Ci (Bestimmung Brezina's nach dem grössten Pariser Stück von $7 \mathrm{gr}$.), gefallen 21 . Juni 1668 .

r671: Francesco Carli: Valisnieri Opere Bd. 2, p. 66 und Galeria di Minerva Bd. 6, p. 206; s, auch Ephemer. Akad. Nat. curios. Cent, 6, Obs. I3 (nach v, Ende).

I672: Conversations tirées de l'Académie

1) Davon 33 gr. »Taltal, Chili 1872 . - 2) Davon 12 gr. sTaltal, Chili . -3) Vaca Muerta (Atacama) 108 gr.; Vaca Muerta (Cachinal) 153 gr.; Vaca Muerta (Cerro la Bomba) 547 gr.; Vaca Muerta (Sierra de Chaco) 198 gr. - 4) Davon 15 gr. sTaltal 1872\%. - 5) Davon 12 gr. "Janacera *. - 6) Davon 185 gr. >Mejillones«; 24 gr. "Sierra de Chaco\&; 2 gr. Janacera Passe. - 7) Herr Dr. Gottsche teilte mir in Bezug auf dieses Stück noch mit: 1870 gekauft von Herrn C. W. Lüders, jetzt Vorsteher unseres Ethnogr. Museums. Herr Lüders, mit Philippi befreundet, brachte das Stück von drüben mit. Seine Originaletiquette lautet: $\gg 8600$ Fuss über dem Meer, 40 leguas von Cobija, 35 leguas von San Pedro de Atacama, I legua vom Flüsschen Imilac «. Auch Herr Prof. Brackebusch bezeichnet dies Stück bestimmt als von Imilac stammend; andererseits ist unser Stück nach Cohen und Brezina absolut identisch mit Tschermak's Grahamit von Sierra de Chaco. - 8) Davon 2 gr. Passe de Janacera, Atacama Bolivie; 7 gr. Pseudo-Mejillones. -- 9) Davon 16 gr. \$Sierra Mejillones 1867 M oder P «. - 10) Davon 1526 gr. Janacera-Pass u. $25^{8}$ gr. San Pedro de Atacama. Ausserdem nach 1. Juli 1893 erworben: 230 gr. Vaca muerta u. 217 gr. Chañaral. 
de M. de Bourdelot, contenant diverses recherches et observations physiques, par Legallois. Paris 1672 , p. 181 (s. auch Meunier 1884, p. $475-476$ ).

?: Opuscoli scelti da C.A moretti Bd.19,p.42. Die in Betracht kommenden Stellen der obigen Werke sind in Chladni's »Feuermeteore « übersetzt.

1749: Z ig a t a: Supplementi alla Cronica di Pier Zigata. Verona 1749, p. 108.

I802: Butenschön : >Moniteur « an XI. Nov. 2.

1803: $\mathrm{Ch} 1$ a d n i: Chronologisches Verzeichnis, Gilb. Ann. Bd. 15, p. 309, $314-315$.

1803: I z a rn: Lithologie, p. 329.

1804: Pötzsch: Kurze Darstellung, p. $106-107$.

1804: v. En de: Massen und Steine, p. $32-33$.

1806: L a ug i e r: Extrait d'un Mémoire sur l'existence du chrôme dans les pierres météoriques. Ann, Mus, d'hist. nat. Bd. 7 , p. $392-397$; Auszug in Gilb. Ann. Bd. 24 (18o6), p. $3^{80}, 3^{8} 3$. r808: de 1a Métherie: Journ. Phys. Bd. 66 .

1812: C h 1 a d ni: Verzeichnis, Schweigg. Journ. Bd. 4 Beil. I, p. Io.

1812: B igot de Morogues, p. (79), $83-84$

1814: Dr, Ch la d ni's vergebliche Bemühungen, verschiedene ältere Meteorsteine aufzufinden, nebst einigen ihn selbst betreffenden Nachrichten. Gilb. Ann. Bd, 47, p. 97, 99-100.

r815: $\mathrm{Chladni}$ : Neues Verzeichnis, Gilb. Ann, Bd, 5o, p. 244-245.

r8rg: $\mathrm{Ch}$ la d n i: Feuermeteore, p. 48, $57,66,68,71,73,233-236$ (!).

1823: v. $\mathrm{Z}$ a $\mathrm{c} \mathrm{h}$ : Ueber Feuerkugeln als Erdkometen. Schweigg. Journ. Bd. 38 , p. I9I.
1836: Kämtz: Meteorologie, p. 268 . 1843: P a r ts c h: Meteoriten, p. I4I.

1854: B a $1 \mathrm{c}$ e $11 \mathrm{~s}$ : Lithologia meteorica, p. 19.

1857: Arago: Astronomie populaire. Bd. 4, p. 192

1859: H a r r i s: Dissert. Gött., p. 58. r859: B u c h n e r : Feuermeteore, p. 39. 1863: B uc hner: Meteoriten, p. 4. 1884: M e unier: Météorites, p. 197, $475-476$

1885: Brez in a : Wiener Sammlung, p. 271 .

1887: Brezina: Reisebericht, Ann. Hof-Mus. Bd. 2 (Not.), p. 72.

1890: Brezin a : Reise, Ann. Hof-Mus. Bd. 4 (Not.), p. 1 9.

r894: Co hen: Meteoritenkunde, p. 8, 244, 245 .

1895: Brezina: Wiener Sammlung, p. 246,255 .

Ursprüngliches Gewicht: Mehrere sehr grosse Steine, sehr wenig erhalten.

Nachweisbares Gewicht: 9 gr.

Paris, M. $\quad 9^{1}$ )

\section{Valle de Allende}

\section{Huejuquilla-Gruppe \\ Valle de San Bartolomé \\ Huejuquilla-Gruppe}

Varas, Serrania de Varas, Atacama, Chile, Südamerika.

Eisen, Of, gefunden um 1875 , beschr. r889 (in Verzeichnissen schon früher erwähnt).

r885: Brezina: Wiener Sammlung, p. 268.

1889: F letcher: Atacama Meteorites, Mineral. Magaz. Bd. 8, p. 224, 230, 258-259 (Analyse).

1) ${ }^{2}$ Die Fragmente gehören einem Eukrit, einem $\mathrm{Ci}$ und einem $\mathrm{Cc}$ an, allerdings die grösseren Stïcke dem intermediären Chondrit, während die Ce Fragmente Albareto sein könnten (Brezina, Reise I890, p. I19). 
I892: v. H a uer: Ann. Hof.-Mus, Bd. 7 (Not.), p. 73.

r894: $\mathrm{F}$ le t c he r: Introduction, p. I4. 1895: Brez in a: Wiener Sammlung, p. 269 .

Ursprüngliches Gewicht: $1470 \mathrm{gr}$. Nachweisbares Gewicht: $1182 \mathrm{gr}$.

London, B. M. 1168 | Wien, H. M.

\section{Vaucluse}

Saurette

Vavilovka,Gouv.Cherson,Russland. Stein, Cwb, gefallen 19. Juni 1876 . 1877: Prendel: Description de la météorite de Vavilovka. Mém, de la Soc. nation. des Sc, nat. Cherbourg Bd, 2r, p. 205 (Analyse). Ref. N. J. 1878, p. 868; Liebig-Kopp, Jahresber. 1878 , p. 1319.

1882: Brezina: Bericht IV, Sitzber. Wien. Akad. Bd. 85 I, p. $33^{8}$ (Vergleich mit Mócs, s. dort).

1884: M e un i e r: Météorites, p. 257.

r885: Brezina: Wiener Sammlung, p. $181,232$.

1893: M elik off: Chemische Analyse des Meteoriten von Wawilowka. Ber. d. d. chem. Ges. 1893 II, Bd. 26, p. 1929-1932 (Analyse).

I894: C oh en: Meteoritenkunde, p, Ir2, 20I, 248.

Ursprüngliches Gewicht: ?

Nachweisbares Gewicht: $193^{2}$ gr.

Bologna

v. Braun

Budapest

Gregory

London, B. M.

\begin{tabular}{r|lr}
9 & Odessa & 1 395 \\
2 & Paris, M. & 8 \\
327 & v. Siemaschko & 126 \\
47 & Wien, H. M. & 16 \\
2 &
\end{tabular}

\section{Vegas i Carrisalillo Vaca Muerta}

\section{Venagas}

Descubridora

Veramin(Karand), Teheran,Persien. Mesosiderit, M, gefallen Febr.1880. ${ }^{1}$ ) 188I : D i z s c h: Geologisches, Bergund Hüttenmännisches aus Persien. -

Meteorsteinfall. Berg- u. Hüttenm.Zeitg. Bd. 40 , p. 100 .

I88I : B r ez in a : Bericht III. - 8)Veramin, Teheran, Persien, gefallen April 1880. Sitzber. Wien. Akad. Bd. 84 I, p. 277-279. Ref. N. J. 1883 I, p. 382 . r884: L in d ström : Förteckning öfver Riksmusei Meteoritsamling. Ofversigt af Kongl. Vetenskaps-A kademiens Förh. Stockholm (1884), Nr. 9. Ref. N. J. I886 II, p. 39 .

1884: Da u brée: Météorite tombée récemment en Perse, à Veramine, dans le district de Zerind, d'après une communication de M. Tholozan. C. R. Bd. 98, p. $1465-1466$ (Analyse ohne Zahlenangabe). Ref. N. J. I885 II, p. 269; Liebig-Kopp, Jahresber, $188_{4}$, p. 2040-204I.

1884: Me unier : Météorites, p. 165,174. 1885: Brezina: Wiener Sammlung, p. 156, 191, 233.

1887: F 1 ig h t: Meteorites, p. 214-215. r894: C o h e n : Meteoritenkunde, p. 289. r894: F l e t c her: Introduction, p. 14. r895: Me unier: Revision des lithosidérites, p. $33-37$.

Ursprüngliches Gewicht: Ein Stein von $45-54 \mathrm{Kgr}$.

Nachweisbares Gewicht: 573 gr.

Bailey

Budapest

Edinburg

Gregory

Harvard, U.

3 Paris, M.

62 Rom, U.

6 v. Siemaschko

I Stockholm

II Upsala 8

Kristiania $\quad 4 \mathrm{I}$ Wien, H. M. I6

London, B. M. $\quad 54$

Die grösste Masse soll sich im Besitz des Schah von Persien befinden.

Verezegyhaza

Verkhne

Vernon Co., Wisconsin, U. S. A.

Stein, Cka (früher als $\mathrm{Ck}$ aufge-

I) Nach Tholozan's Bericht an Daubrée Februar 1879. In der ersten Hälfte Mai r88o sah Diezsch ein Stück beim Schah von Persien. 
fasst), gefallen 25. März 1865 . Smith giebt in seiner Originalarbeit dieses Datum an, andererseits wird fast immer der 26. März als Falltag bezeichnet.

Hierher auch sThe Claywater-Meteorite «.

1875: S m it h: Am. Journ. (3) ro, p. 314 (Notiz, dass Smith in der Amer. Assoc. for the advanc. of Sc. diesen Meteoriten erwähnt habe).

r876: S mith: An account of a New Meteoric Stone that fell on the $25^{\text {th }}$ of March, 1865, in Wisconsin, identical with the Meno-Meteorite. Am. Journ.

(3) 12, p. 207-209 (Analyse); s. auch C. R. Bd. $83(1876)$, p. $161-163$; Original Researches 1884 , p. 519-522; N. J. 1877 , p. $4 \mathrm{IO}-4 \mathrm{II}$; Cohen, Campo de Pucará, N. J. 1887 II, p. 52 ; Liebig-Kopp, Jahresber. 1876, p. 1316 -1317 .

1879: R a m mels berg: Meteoriten, p. 14,25 .

1884: Meunier: Météorites, p. $18_{3}$, $184-185,335$.

r885: Brezina: Wiener Sammlung, p. 19I, 233 .

1887: F lig ht: Meteorites, p. I44.

r889: F l e t c her: Atacama Meteorites,

Mineral. Magaz. Bd, 8, p. 252.

r890: Eastma n: Met. Astron., p.316.

Ursprüngliches Gewicht: Ein Stück von $700 \mathrm{gr}$. Es wurde noch ein Stück von etwa 800 gr. gefunden, welches aber verloren ging. Nachweisbares Gewicht: 435 gr.

\begin{tabular}{lr|lr|} 
Bailey & 7 & Kopenhagen & 18 \\
Bement & 2 & London, B. M. & 52 \\
Budapest & 11 & Paris, M. & 69 \\
Gregory & 22 & Stockholm & 13 \\
Harvard, U. & 200 & Wien, H. M. & 29 \\
Heidelberg & 3 & Washington, Sh. & 9
\end{tabular}

Verona

Vago

Viasma

Kikino
Victoria 1854 (186r) Cranbourne Victoria $187 \mathrm{I} \quad$ Victoria

Victoria, Saskatchewan River, Iron Creek, Battle River, British Nordamerika.

Eisen, Om, gefd. 1871 , beschr. 1872 . r872: But 1 er: The Great Lone Land. London bei Sampson Low. 1872, p 304. 1887: Proc. and Trans. Royal. Soc.

Canada Bd. 4, Sektion 3, p. 97. I887: Flight: Meteorites, p. 53-54. r889: v. H a u e r: Ann. Hof-Mus, Bd. 4

(Not.), p. 64 ; s, auch Bd. 6 (I89I) (Not.), p. 54 .

1890: E a s t m a n : Met. Astron., p. 322. r 890: F l e t c her: Mexican Meteorites,

Mineral. Magaz. Bd 9, p. 103.

r894: Co he n: Meteoritenkunde, p. 73. r895: Brezina: Wiener Sammlung, p. 279 .

Ursprüngliches Gewicht: $175 \mathrm{Kgr}$. (386 lbs. nach Fletcher).

Nachweisbares Gewicht: 472 gr.

Bailey 12 Paris, M. IO

$\begin{array}{llll}\text { Gregory } & 28 & \text { Ward } & 88\end{array}$

Howell 13 Washington, Sh.125

London, B. M. 79 Wien, H. M. 117

Wo befindet sich die grosse Masse?

Victoria West, Cap-Kolonie, Südafrika.

Eisen, Ofv, gefunden (gefallen?) 1862 , beschrieben 1868 .

1868: Gregory: Meteoric Iron from South Africa, Geol. Magaz. Bd. 5, p. 532. r871: Tschermak: Meteoreisen von Victoria West. M. M. (1871), p. 109. 1873: Smith: A description of the Victoria Meteoric Iron, seen to fall in South Africa in 1862 , with some notes on Chladnite or Enstatite. Am. Journ. (3) 5, p. 107-ro (Analyse und Abbildung eines Schliffs); s. auch C. R. Bd. 76 (1873), p. 294-297; Original Researches 1884 , p. $469-470$; $>M i-$ 
neralogy and Chemistry a, p. 374; Liebig-Kopp, Jahresber. 1873, p. 1251 . 1874: S m it h: On a Mass of Meteoric Iron of Howard Co., Ind, ; with some remarks on the molecular structure of meteoric iron, and a notice concerning the presence of solid protochloride of iron in Meteorites. Am. Journ. (3) 7 , p. 394 .

I880: Brez in a: Reichenbach'scheLamellen, Denkschr.Wien. Akad, Bd. 43, p. 14 .

1884: M eunier: Météorites, p. 56 (Abb.), 116, 124.

1885: Brezina: Wiener Sammlung, p. $208,233$.

1887: Brezina u. Cohen: Photographien, T. 12, 13, 14.

r887: F lig ht: Meteorites, p. 137.

1893: Brez in a: Ueber neuere Meteoriten (Nürnberg), p. 164.

r893: M e unier: Revision des fers météoriques, p. 40, 41.

1894: Cohen: Meteoritenkunde, p. 193. 196.

1894: F le t $\mathrm{ch}$ er: Introduction,p.33,34. r895: Brezina: Wiener Sammlung, p. 267 .

Ursprüngliches Gewicht: 2944 gr. (6 lbs., 8 oz.).

Nachweisbares Gewicht: I175 gr.

Budapest

Calcutta

4 Paris, M. $\quad 98$

Gregory

221 v. Siemaschko 2

17 Stockholm 83

London, B. M. I58 Wien, H. M*) I72

London, P. G. $\quad 227$

Ist die Hälfte der Masse noch in dem South African Museum, wie Smith im Jahre 1873 sagt?

Villa Lujan

Lujan

Villa Nova

Canellas

Villa Nueva Huejuquilla-Gruppe

Ville franche

Salles

Virba

Wirba
Visa

Mócs

Vivionnère

La Vivionnère

Vouillé bei Poitiers, Dep. Vienne, Frankreich.

Stein, Cia, gefallen 13 . Mai $188_{31}$. 183I: : M. le ministre du Commerce adresse un Rapport du maire de Vouillé sur une chute d'aérolithe qui a eu lieu dans cette commune le 13 mai dernier. Ann. Chim. Phys. Bd. 47, p. 442.

r83r: Des rozi e rs: Bull. Soc. Agric. Belles-Lettr, et Sc. de Poitiers ( $183 \mathrm{I})$, p. 226 (Analyse).

1835 : v. H of $f$ : Neunter Nachtrag,Pogg. Ann. Bd. 34 , p. $34 \mathrm{I}-342$ (schöpft aus Biblioteca Italiana Bd. 64,1831 , p. 401$)$.

1836: K ä $\mathrm{m} \mathrm{tz}$ : Meteorologie, p. 299. 1843: Parts ch: Meteoriten, p. 60.

1857: Arago: Astronomie populaire. Bd. 4, p. 202.

1859: H a r r is: Dissert.Gött.,p. $82-83$. I863: B u c hner: Meteoriten, p. $54-55$. I863: Rose: Meteoriten, p. 155.

1864: D a u bréce: Note sur deux aérolithes, l'un tombé à Vouillé (Vienne), le 13 mai $188_{3}$, et offert au Muséum d'Histoire naturelle par la ville de Poitiers; l'autre tombé à Mascombes, département de la Corrèze, le $3^{1}$ janvier 1836 , et dont la chute était restée sans publicite. C. R. Bd. 58, p. 226 -228 .

1864: Buchner: Erster Nachtrag, Pogg. Ann. Bd. 122, p. 328-329.

1859/65: v. Re ic hen b a c h: IX I6I, 168, 178. XIII 360. XXV 321, 322. 1869: Buchner: Vierter Nachtrag, Pogg. Ann. Bd. 136, p. 439.

1875: vom R a th: Meteoriten, Verh. naturh. Ver. Bonn Bd. 32, p. 367.

1875: G î $\mathrm{m}$ b e l: Ueber die Beschaffenheit des Steinmeteoriten vom Fall am 12. Februar 1875 in der Grafschaft Iowa N. A. Sitzber. München. Akad. Bd. 5, p. $3 \mathbf{1 4}$. 
r884: M e un i e r: Météorites, p. 94, 95, 197, 198, 459.

1885: Sh e p ard: Am. Journ. (3) 30, p. 106 (Krystall von Nickeleisen).

1885: Brezina: Wiener Sammlung, p. 182,232 .

r894: C o hen: Meteoritenkunde,p.267.

Ursprüngliches Gewicht : Ein Stein von etwa $20 \mathrm{Kgr}$. (1864 gelangten nach Paris 15700 gr.).

Nachweisbares Gewicht: I6 6I9 gr.

\begin{tabular}{lr|lr} 
Bailey & 6 & London, P. G. & 54 \\
Bement & 2 & Madrid & I5 \\
Berlin, U. & 72 & Minneapolis & Spl. \\
Bonn & 2 & Moskau & I \\
Budapest & 38 & New Haven & 5 \\
Calcutta & 91 & Paris, M. & 15912 \\
Cambridge & 3 & Rom, U. & 6 \\
Cleveland & 5 & v. Siemaschko & 4 \\
Dorpat & 17 & Stockholm & 3 \\
Göttingen & 32 & Troyes & 8 \\
Gregory & 25 & Tübingen & Spl. \\
Hamburg & 3 & Upsala & 16 \\
Harvard, U. & I I 2 & Ward & 3 \\
Kunz & 20 & Washington,Sh. & 15 \\
London, B. M. & 61 & Wien, H. M.*) & 88
\end{tabular}

Waconda, Mitchel Co., Kansas, U. S. A.

Stein, Ccb, gefd. 1874 , beschr. 1876 . 1876: Shepard: Notice of the Meteoric Stone of Waconda,MitchelCounty, Kansas. Am. Journ. (3) II, p. $473-$ 474. Ref.Liebig-Kopp, Jahresber, 1876 , p. 1318 .

1876: vom R ath: Erwähnung eines seitens des Herrn C. U. Shepard dem Museum dargebrachten Geschenks eines Fragments ( 14 gr.) des Meteoriten von Waconda, Mitchel Co. Kansas, Am. 1872. Verh, naturh. Ver. Bonn Bd. 33 (Sitzber.), p. 103.

1877: $\mathrm{S} \mathrm{mith}$ : Examination of the Waconda Meteoric Stone, Bates County Meteoric Iron and Rockingham County Meteoric Iron. - Waconda Meteorite.
Am. Journ. (3) 13, p. 2II-213 (Analyse); s. auch Original Researches 1884 , p. 523-525; Brezina, Meteoritenstudien II, Denkschr. Wien. Akad. Bd, 44 (1881), p. 133; Liebig-Kopp, Jahresber. 1877, p. 1396.

r879: R a mmels berg : Meteoriten, p. $12,24,25$.

1882: W i e c hmann: Fusion-Structures in Meteorites. Ann. N. Y. Acad. Sc. Bd, 2, p. 293.

1884: Meunier: Météorites, p. 209, $224-225$.

1884: W a d s w or th: Studies,p.93-94. r885: Brezina: Wiener Sammlung, p. $168,177,232$.

r887: F light: Meteorites, p. 75-76. r889: v. H a u e r: Ann. Hof-Mus. Bd. 4 (Not.), p. 64.

1890: Eas t m a n: Met. Astron., p. 320. 1893: Brezin a: Ueber neuere Meteoriten (Nürnberg), p. I61.

1894: C o h e n: Meteoritenkunde, p.202, 206, 283 .

1895: Brezina: Wiener Sammlung, p. $24 \mathrm{I}, 25^{8}$.

Ursprüngliches Gewicht: Ein Stein, von welchem etwa die Hälfte, $26 \mathrm{Kgr}$. (58 lbs.), erhalten blieb.

Nachweisbares Gewicht: I 5786 gr.

\begin{tabular}{lr|lr} 
Bailey & 61 & Dresden, M. & 19 \\
Bayet & 2 & Dublin, M. & 138 \\
Bement & 138 & Göttingen & 12 \\
Berlin, G. & 17 & Gregory & 235 \\
Berlin, U. & 14 & Greifswald & 15 \\
Blatz & 106 & Harvard, U. & 3257 \\
Böhm & 42 & Howell & 44 \\
Bologna & 9 & Kopenhagen & 284 \\
Bonn & 22 & Krantz & 10 \\
v. Braun & 459 & Kunz & 5 \\
Brezina & 35 & London, B. M. & 467 \\
Brüssel & 90 & de Mauroy & 15 \\
Budapest & 182 & Minneapolis & 144 \\
Cambridge & 13 & Neumann & 2 \\
Cleveland & 7 I & New Haven & 104 \\
Dorpat & 507 & Newton & 38
\end{tabular}


Paris, E.

Paris, M.

Pohl

Prag, M.

Rom, U.

v. Siemaschko

Stockholm

Strassburg

\section{Wadee Banee Khaled}

Nejed

Wairarapa, Wairarapa Valley, Wellington, Neu-Seeland.

Stein, gefallen (?) 4. Dezember 1864 . Hierher vorläufig auch: Turakina (Turanaki), Wellington, Neu-Seeland, gefallen 4. Dez. 1864 .

1865: J u r o r s Report of the New Zealand Exhibition (1865). Appendix A, p. 410 ,

1865: Ha iding er: Der Meteorit von Turakina, Wellington, Neuseeland. Sitzber. Wien. Akad. Bd. 52 II, p. 15I153. Ref. Kenngott, Uebersicht 1862165 , p. 433.

1869: B u chner: Vierter Nachtrag, Pogg. Ann. Bd. 136, p. 455.

1887: F 1 ig ht: Meteorites, p. 140.

Ursprüngliches Gewicht: $13.6 \mathrm{Kgr}$. (480 oz.).

Nachweisbares Gewicht : Flight sagt, dass der Stein im Colonial-Museum in Wellington aufbewahrt werde.

\section{Waldau}

Waldo Co.

L'Aigle

Waldron Ridge, Claiborne Co., Tennessee, U. S. A.

Eisen, Og, gefd. und beschr. 1887 .

(Nach Kunz gehört dieses Eisen mit Cosby's Creek, Greenbrier Co. und Jennys Creek zu ein er Lokalität).

1887: $\mathrm{K}$ unz: On some American Me- teorites. - 4. On a mass of Meteoric iron from Waldron Ridge, Claiborne Co., Tenn. Am. Journ. (3) 34, p. 475 -476 . Ref. N. J. 1889 I, p. 62.

1889: L e d o ux: The Pipe-Creek Meteorite. Trans. of the New York Acad. of Sc. Bd. 8, p. 187 (Analyse). Ref. N. J. 1891 I, p. 50 ; s. auch Oesterr. Zeitschr. f. Berg- u. Hüttenw. Bd. 39 (1891), p. 344 .

1890 : E a s tm a n: Met. Astron., p. 320. r89r: v Ha u e r: Ann. Hof-Mus. Bd. 6

(Not.), p. 54 .

1893: Meunier: Revision des fers météoriques, p. 29, 32.

1894: H untington: The Smithville Meteoric Iron. Proc. Amer. Acad. Arts and Sc. Bd. 29, p. 259 (Situationsplan von Tennessee bis West Virginia).

1894: Co hen: Meteoritenkunde, p. 124. 1895: Brezina: Wiener Sammlung, p. 287.

UrsprünglichesGewicht: Etwa 8Kgr. (I 8 lbs., das grösste Stück 15 lbs.). Nachweisbares Gewicht: 8477 gr. Bement 371 Paris, M. 67 Gregory $\quad 3254$ v. Siemaschko 5 Harvard, U. $\quad 12$ Ward 825 London, B. M. $\quad 70$ Wien, H. M. 3873

Walker Co. Lime Creek-Walker Co. Walker Township Grand Rapids Warrenton, Warren Co., Missouri, U. S. A.

Stein, Cco, gefallen 3. Januar 1877 . 1877: $\mathrm{Smith}$ : Note of the recent fall of three Meteoric Stones, in Indiana, Missouri, and Kentucky. - No. 2. On January third, 1877 , at sunrise, in Warren County, Missouri. Am. Journ. (3) 13, p. 243 ; s. auch C. R. Bd. 84 (1877), p. 399; N. J. 1877, p. 735-736. 1877: S mit h: A description of the Rochester, Warrenton, and Cynthiana Meteoric Stones, which fell respectively

I) Ausserdem 319 gr. nach I. Juli 1893 erworben. 
Decembre 21 st, 1876 , January 3 d, 1877 , and January $23 \mathrm{~d}, 1877$, with some remarks on the previous falls of Meteorites in the same regions. - 2. Warrenton (Missouri) Meteorite. Am. Journ. (3) 14, p. 222-224 (Analyse), 227229 ; s. auch Original Researches 1884, p. $532-534$; C. R. Bd. 85 (1877), p. $679-680$; N. J. 1878 , p. $78-79$; Zeitschr, f. Kryst. Bd, 2 ( 1878$)$, p. IIo Liebig-Kopp, Jahresber. 1877, p. 1394 -1395.

1877: K ir k wood: On eight Meteoric Fireballs seen in the United States from July $x 876$ to February 1877 . $\mathrm{V}$ and VI. The Meteors of January 3 and January 20, 1877. Amer. Philos. Soc. March 16, 1877 , p. 595 ; s. auch Am. Journ. (3) 14 (1877), p. 75.

1879: R a mmels berg: Meteoriten,p.6r. r880: K l e in : Vermehrung, Gött. Gel.

Anz., p. 567 .

r884: Meuni er: Météorites, p. 280, 283 (Abb.), $284-285,494,523-524$. r885: Brezina: Wiener Sammlung,

p. $190-191,233$.

1887: F lig h t: Meteorites, p.I99-200. 1890: E a s tm a n: Met. Astron., p. 316. r895: Brezina: Wiener Sammlung, p. 259.

Ursprüngliches Gewicht: Von einem

Stein, der roo lbs. gewogen haben mochte, blieben nur 4-7 Kgr. (ro-I5 lbs.) erhalten.

Nachweisbares Gewicht: 16r4 gr.

\begin{tabular}{lr|lr} 
Bailey & IO & Minneapolis & 27 \\
Bement & 5 & New Haven & 260 \\
Bologna & 2 & Paris, M. & I 84 \\
v. Braun & 6 & v. Siemaschko & 5 \\
Breslau & 3 & Stockholm & 52 \\
Budapest & 79 & Troyes & I \\
Cohen & Spl. & Ward & 65 \\
Göttingen & 32 & Washington, & II \\
Gregory & 45 & Washington, Sh. 27 \\
Harvard, U. & 571 & Wien, H. M. & I47 \\
London, B, M & 82 & &
\end{tabular}

Warschau

Pultusk

Washington Co. 1858 Trenton

Washington Co. 1890 Farmington Waterloo

Wawilowka

Wayne Co. 1858

Seneca Falls

Vavilovka

Wooster

Wayne Co. $\mathrm{I}^{8} 83$ Jenny's Creek

Weichsel

Schwetz

Welland, Ontario, Canada.

Eisen, Om, gefd. r888, beschr. r8go.

r8go: Howe 11: Description of New Meteorites. - The Welland Meteorite. Proc. Rochester Acad. of Sc. Bd. I, p. $86-87$ (Analyse von Davison und Abbildung); auch abgedruckt in The Ward Collection of Meteorites Rochester, N. Y. 1892 bei R. W. Lace, p. $61-62$. Ref. N. J. 1892 II, p. 34 .

r8go: Ea s tm a n : Met. Astron., p. 320. r89r: Davis on: Analyses of Kamacite, Taenite and Plessite from the Welland Meteoric Iron. Am. Journ. (3) 42 , p. 64-66 (Read before the Rochester Acad. of Sc. and published in the Proc. for 1891 , where it is accompanied by a plate, not reproduced here). Ref. N. J. 1892 I, p. 269.

r892: v. Ha uer: Ann. Hof-Mus. Bd. 7 (Not.), p. 72.

r892: Cohen: Meteoreisen-Studien. Ann. Hof-Mus. Bd. 7, p. 159, 160, I6r. 1893: Brezina: Ueber neuere Meteoriten (Nürnberg), p. I64.

1894: Cohen: Meteoritenkunde, p. $64,83,96,102,104,106,108,115,24$ I. r895: Brezina: Wiener Sammlung, p. 284 .

r895: Co h en: Meteoreisen-Studien IV. Ann. Hof-Mus, Bd. 10, p. 82,87 .

Ursprüngliches Gewicht: $8 \mathrm{Kgr}$. Nachweisbares Gewicht: 5rI4 gr.

Bailey

Bement

4I Böhm

Berlin, U. 


\begin{tabular}{lr|lr} 
Budapest & 54 & Paris, E. & 54 \\
Dorpat & 516 & Prag, M. & 136 \\
Gregory & 202 & Rom, U. & 41 \\
Greifswald & 35 & v. Siemaschko & 75 \\
Harvard, U. & 138 & Stürtz & 75 \\
Howell & 38 & Ward & 773 \\
Kopenhagen & 35 & Washington & 36 \\
London, B. M. & 466 & Wien, H. M. & 1521 \\
de Mauroy & 25 & &
\end{tabular}

Wellington Eisen

Rowton

Wellington Stein

Wairarapa

WerchneDnieprowsk am Dniepr,

Gouv. Ekaterinoslaw, Russland.

Eisen, Off, gefunden 1876 , erwähnt I 885 , beschr. 1887 .

1885: Brezina: Wiener Sammlung, p. 208,233 .

1887: Brezina u. Cohen: Photographien, T. 12 ,

1893: Meunier: Revision des fers météoriques, p. 75 .

I894: F l et cher: Introduction, p. I4. r895: Brezina: Wiener Sammlung, p. 267 .

Worauf die Angabe der in manchen Verzeichnissen mitgeteilten Fundzeit (1876) sich stützt, habe ich nicht ermitteln können. Ist darüber eine russische Notiz publiziert ?

Ursprüngliches Gewicht: ?

Nachweisbares Gewicht: 465 gr.

Budapest

Calcutta

$8 \mid$ Paris, M.

3

Dorpat

33 Petersburg, B. 44

v. Leuchtenberg $72^{\prime}$

London, B. M. 25 Wien, H. M. 8

Werchne Tschirskaja, am Don im Gebiet der Don'schen Kosaken, Russland.

Stein, Cca, gefallen I2. Nov. 1843 . r847: Borissiak: Sur l'Aérolithe tombé près de Verkhne-Tschirskaia Stanitsa. Bull. Petersburg. Akad. Bd. 5,

1) Oxydiertes Eisen. p. 196-198; s. auch Pogg. Ann. Ergz.Bd. 2 (1848), p. $366-367$; LiebigKopp, Jahresber. $1847 / 1848$, p. 1299 -1300; Kenngott, Uebersicht 1844/49, p. 286; "L'Institute Bd. I5 (1847) Nr. 699, p. 174 .

r847: Eic hwald: Verzeichnis, Erman's Archiv f. wissensch, Kunde Russlands Bd. 5 , p. $\mathbf{I} 8 \mathrm{r}$.

r859: Bu chner: Feuermeteore, p. 97 -98 .

1863: Buchner: Meteoriten, p. 74 . 1884: Me uni er: Météorites, p. 75.

r885: Brezina: Wiener Sammlung, p. $190,233$.

1887: B rezin a : Neue Meteoriten III, Ann. Hof-Mus. Bd. 2 (Not.), p. 115.

Ursprüngliches Gewicht: Ein Stein von $8.30 \mathrm{Kgr}$. (8030 gr. ?)

Nachweisbares Gewicht: 177 gr.

Paris, M.

I 5 Wien, H. M.**) 94

Stockholm 68

Befindet sich der Stein noch an der Universität Charkow?

Werchne Udinsk am Fluss Niro, Seitenfluss des Witim, Ostsibirien, Russland.

Eisen, Om, gefd. 1854 , beschr. $186_{3}$. 1863: Ro se: Meteoriten, p. 65, 153.

1864: R o se legte als neue Erwerbungen des mineralogischen Museums der Universität Berlin Probẹn von zwei neuen Meteoriten vor: einem Meteoreisen [gefunden imWerchne-Udinskischen Kreise] und einem andern Meteoriten, der wahrscheinlich ein Mesosiderit ist [Breitenbach]. Zeitschr. d. d. geol. Ges. Bd. 16, p. $355-356$ (Analyse von v. Kotschubei). Ref. Kenngott, Uebersicht I862165, p. 269.

1865: B u chner: Zweiter Nachtrag, Pogg. Ann. Bd. 124, p. 599.

1865: Krantz sprach über das Meteor-Eisen von Werchneudinik; erhielt 
$6 \frac{1}{2} \mathrm{Kgr}$, in Abschnitten. Verh. naturh. Ver. Bonn (Sitzber.) Bd. 22, p. 19-20. 1867: Buchner: Dritter Nachtrag, Pogg. Ann. Bd, 132, p. 319.

r869: $\mathrm{B}$ u c h n e r : Die Aetzfiguren des Meteoreisens, Ber. Oberhess, Ver. f. Natur- u. Heilk. Giessen (1869), p. 108 - 109 .

1869: Buchner: Vierter Nachtrag, Pogg. Ann. Bd. 136, p. 600.

1875: vom Rath: Meteoriten, Verh. naturh. Ver. Bonn Bd. 32 , p. $363-364$. 1884: Meunier: Météorites, p. 127.

1885: Brezina: Wiener Sammlung, p. $152,210,233$.

1893: M e u n i e r: Revision des fers météoriques, p. 46.

1895: Las peyres u. Ka is er: Mittheilungen aus dem mineralogischen Museum der Universität Bonn VI. Theil. - 37. Chemische Zusammensetzung des Meteoreisens von Werchne Udinsk in Sibirien. Zeitschr. f. Kryst. Bd. 24, P. 493- 494 (neue Analyse).

r895: Co he n: Meteoreisen-Studien IV. Ann. Hof-Mus. Bd, 10, p. $83-84,90$.

Ursprüngliches Gewicht: $18 \frac{1}{2} \mathrm{Kgr}$. Nachweisbares Gewicht : $10859 \mathrm{gr}$.

\begin{tabular}{lr|lr|} 
Bailey & 70 & London, P. G & 189 \\
Bement & 46 & Madrid & 91 \\
Berlin, U. & 569 & Moskau & 411 \\
Bologna & 26 & München & 55 \\
Bonn & 577 & Neumann & 61 \\
v. Braun & 174 & Paris, M. & 278 \\
Budapest & 553 & Petersburg, A. & 537 \\
Calcutta & 460 & Pohl & 324 \\
Dorpat & 115 & Rom, U. & IIO \\
Dresden, M. & 54 & v. Siemaschko & 288 \\
Edinburg & 46 & Stockholm & 1588 \\
Göttingen & 15 & Strassburg & 8 \\
Gregory & 100 & Stürtz & 50 \\
Greifswald & 31 & Szamosujvar & 44 \\
Kopenhagen & 127 & Troyes & 75 \\
Kristiania & 44 & Tübingen & 133 \\
v. Leuchtenberg 247 & Washington, Sh. 36 \\
London, B. M, 2904 & Wien, H. M.*) & 423
\end{tabular}

Herr v. Kotschubei in Kasan soll nach Herrn Melnikoff's Mitteilung ebenfalls Stücke von diesem Eisen besitzen.

Wessely(Znorow),HradischerKreis, Mähren, Oesterreich.

Stein, Cga, gefallen 9. Sept. 1831 . 1832: v. Schreibers: Ueber den Meteorstein-Niederfall auf der Herrschaft Wessely in Mähren, am 9. Sept. 1831. Baumgartner's Zeitschr. f. Phys. u. verw. Wiss. Bd. I (1832), p. 193-239. 1832: v. Holger: Analyse des bei Wessely gefallenen Meteorsteines.Baumgartner's Zeitschr. etc. Bd. I (1832), p. $240-248$.

1832: v. S c hreibers: Bemerkungen über die Resultate der vorstehenden chemischen Analyse. Baumgartner's Zeitschr, etc. Bd. I (1832), p. 248-252; s. auch Froriep's Notizen aus der Naturund Heilkunde Nr. 70r, p. 298 (Bd. $32 ; 19)$.

1833: Oken's Isis $\approx$ Heft4-6, p. 479. r835: v. H off: Neunter Nachtrag, Pogg.

Ann. Bd. 34 , p. 342 .

1836: Kämtz: Meteorologie, p. 298. 1843: P a rts c h: Meteoriten, p.66-67. r854: v. Boguslawski: Zehnter

Nachtrag, Pogg. Ann. Ergz.-Bd. 4, p. I4. 1855: Giebel u. Heintz: Zeitschr.

f. d. ges. Naturw. Bd. 6 , p. 66,414 . 1857: Arago: Astronomie populaire. Bd. 4 , p. 202.

1859: H arris: Dissert. Gött., p. 83 . r863: B u c hner: Meteoriten, p. 55 . r863: Ro s e: Meteoriten, p. 92, 154. r857/65: v. Reichen ba ch: III 624. V 476,480 . VI 441 . IX 162, 171, 180. X $359,360,361$. XI 294, 300, 301, 302. XII 454. XIII 358. XV I0I, 121. XXIV 226. XXV 429 .

r885: Brezina: Wiener Sammlung, p. $182,233$.

1886: Melion: Die Meteorsteinfälle in Mähren. Notizbl. d. mähr.-schles. Ges. z. Beförd. d. Ackerbaus etc. (1886) 
Nr. 5 u. 6 , p. $4,7,9$, IO, II.

r89o: v. Niess l: Periheldistanzen,

Verh. naturf. Ver. Brünn Bd. 29, p. 189, $195,196,214,258$.

1894: F let c her: Introduction, p. 14.

Ursprüngliches Gewicht: Ein Stein von $3780 \mathrm{gr}$. (Als der Stein nach Wien kam, wog er $6 \mathrm{Pfd} .2 \mathrm{I}^{1 / 2}$ Lot; $2-3$ Lot mochten vorher abgeschlagen worden sein).

Nachweisbares Gewicht: 3720 gr.

Bement

Berlin, U.

Calcutta

London, B. M.

\section{$7 \mid$ Melion}

3 Neumann

4 Tübingen

London, P. G.

Western Port Distr. Cranbourne West Liberty Homestead

Weston, Fairfield Co., Connecticut, U. S. A.

Stein, Ccb, gefallen 14. Dez. I807. 1807: Medic. Reposit (1807), p. 202 (nach Buchner's Quellenverzeichnis).

1808: Gilbert: Steinregen im Dezember 1807 , in Massachusets in NordAmerika. Gilb. Ann. Bd. 29, p 2II, 352 (Aus französischen Zeitungen); s. auch ,Encyclopédie « Bd. 5 (1808) p. 58659I, 593, 602-606.

1808: C h la d n i : Beiträge, Gilb. Ann. Bd. 29, p. 375 .

1808: Gilbert: Nachträge zu der Nachricht von den Meteorsteinen, welche am 14. December 1807 zu Weston in Connecticut herabgefallen sind. Gilb. Ann. Bd. 3o, p. $421-423,427-430$. r808: Silliman u. K ingsley: An Account of a Phenomenon. Description d'un météore vu dans la province de Connecticut, aux Etats-Unis, et suivi de la chute d'un nombre d'aërolithes, tirée du papier Américain, intitulé, Connecticut Herald; et détails ultérieurs sur ces pierres, communiqués à l'In-

stitut de France, par M. A. Pictet, l'un de ses Correspondants, dans les séances des 4 et II avril. Bibl. Brit. Nr. 296 (April r808), p. $258-278$; s. auch Journ. Phys. Bd, 66 (1808), p. $379-380$ und ferner die hiernach erfolgte freie Bearbeitung Gilbert's in seinen Ann. Bd. 29 (1808), p. 353-370; Journ. Mines Bd. 23 (1808), p. 127, 142; v.Moll, Neues Jahrb. der Berg u. Hüttenkunde Bd. I (1809), p. 120-125.

1808: De Drée-Pictet: Description comparative de onze pierres météoriques, de la collection de Mr. De Drée, présentée à l'Institut de France, dans la séance du II avril. Bibl. Brit. Nr. 296 (April r8o8), p. 285-286, 287, 288. r8og: Silliman u. Kingsley: Memoir on the origin and composition of the meteoric stones which fell from the Atmosphere, in the County of Fairfield, and State of Connecticut, on the $14^{\text {th }}$ of December 1807 ; in a Letter, dated February 18 th 1808 , from Benjamin Silliman, Professor of Chemistry in Yale College, Connecticut, and $\mathrm{Mr}$. James L. Kingsley, to Mr.John Vaughan, Librarian of the American Philosophical Society. Read March $4^{\text {th }} 1808$. Trans. Amer. Philos. Soc. Philadelphia Bd. 6 (1809), p. $323-325,335-345$ : Chemical examination of the stones which fell at Weston (Connecticut) December $14^{\text {th }}, 1807$, by Benjamin Silliman, Professor of Chemistry, in Yale College.

18ro: Silliman: Note sur la chute d'un aérolithe, à Weston en Amérique. Ann. Chim. Bd. 73, p. 290-292.

18ro: Warden: Description et analyse d'une pierre météorique tombée à Weston dans l'Amérique septentrionale, le 4 décembre 1807 . Ann. Chim. Bd. 73 , p. 293-299; übersetzt in Gilb. Ann. Bd. 42 (1812), p. $210-$ 214 ; s. auch Journ. Phys. Bd. 70 (18Io), p. 424-426; auch in einer 
englischen (?) Zeitschrift: Description and Analysis of the Meteoric Stone, which fell at Weston in North America, the $4^{\text {th }}$ December, 1807 . Communicated to David Hosack M. D. etc. by David BailieWarden, Esquire Consul General of the United States at Paris, p. $4 \mathrm{I} 3-4 \mathrm{I} 6$. 1812: Bigot de Morogues, p. 21 r -222, 309, 333 .

r8r2: Ch 1 a d n i: Verzeichnis,Schweigg. Journ. Bd. 4 Beil. I, p. 16.

1815: Chladni: Neues Verzeichnis, Gilb. Ann. Bd. 5o, p. 254.

1815: B ow ditsch: An estimate of the height, direction, velocity and magnitude of the Meteor, that exploded over Weston in Connecticut, December 14, 1807. With methods of calculating observations made on such bodies. Mem. Amer. Acad. of Arts and Sc. (Cambridge) Bd. 3 Teil II, p. 213-236. 1816: Zeitschr. f. Astron. Januar u. Februar 1816, p. 137.

1816: Chladni: Erste Fortsetzung, Gilb. Ann. Bd. 53, p. $386-387$.

18r9: $\mathrm{C}$ h 1 a d n i: Feuermeteore, p. 48, 50, 58, 66, 69, 7I, 74, 9I, I48-I49, $253,267,268,271,282-284$ (1), 294, 429.

1836: K ä m tz: Meteorologie, p.258, 281 -282 .

r839: Herrick: Account of a Meteor seen in Connecticut, December 14, 1837; with some considerations on the Meteorite which exploded near Weston, Dec. 14, 1807. Am. Journ. (I) 37, p. $132-135$.

1843: P a rt s c h: Meteoriten, p. $4 \mathrm{I}-42$. r846: S hep a rd: Reporton Meteorites.

Am. Journ. (2) 2, p. 380, 392.

1848: $\mathrm{S}$ h e pard: Reporton Meteorites. Am. Journ. (2) 6, p. $403,410$.

1854: B a $1 \mathrm{c}$ e 11 s : Lithologia meteorica, p. 22.

1854: v.B o g u s 1 a w s k i: ZehnterNachtrag, Pogg. Ann. Ergz.-Bd. 4, p. 12-13. 1857: Arago: Astronomie populaire. Wü I fing, Meteoriten.
Bd. 4, p. $196,253$.

r859: B u chner: Feuermeteore, p. 77 -78 .

1859: Ha rris: Dissert. Gött., p. 67. r862: Kenngott u. Wiser: Zür-

cher Sammlung, p. I54.

1863: B u chner: Meteoriten, p. 22.

1863: R os e: Meteoriten, p. 27, 84, 154.

r858/65: v. Reichen b a ch: VI 44 I, 454, 456. IX 161, 170, 171, 179. X 359. XI 296, 297, 304. XIII 355 , $357,361,362,365$. XXIV 226. XXV 428,435 .

1867: Go e bel: Kritische Uebersicht, Mélanges phys. chim. Bd. 7 , p. 325 . r869: Silliman and Kingsley:

An account of the Meteor which burst over Weston in Connecticut, in December 1807 , and of the falling of stones on that occasion. Am. Journ. (2) 47, p. I-8 (Aus den Mem. of the Connecticut Acad. of Arts and Sc.). Ref. Liebig-Kopp, Jahresber. I869, p. 1303 -1304 .

1875: Ts chermak: Vulkanismus, Sitzber. Wien. Akad. Bd. 71 II, p. 663. 1875: vom R a th: Meteoriten, Verh. naturb. Ver. Bonn Bd. 32 , p. 367.

1876: Wright: On the Gases contained in Meteorites. Am. Journ. (3) II, p. $259-262$ u. (3) 12 (1876), p. 167,169 . r882: W i e c h m a n n: Fusion-Structures in Meteorites. Ann. N. Y. Acad, Sc. Bd, 2, p. $293,294-295$ (Taf. I9 u. 20), 306 .

I884: W a d s wort h: Studies, p. I05. 1884: v. $\mathrm{N}$ iess 1: Ueber die astronomischen Verhältnisse bei dem Meteoritenfalle von Mócs in Siebenbürgen am 3. Febr, 1882. Sitzber. Wien. Akad. Bd. 89 II , p. 291 (Hemmungshöhe).

Ref. N. J. 1886 I, p. 224.

1884: M e unier: Météorites, p. 227, 228, 444, 446, 459 .

r885: Brezina: Wiener Sammlung, p. $190,233$.

1887: Flight: Meteorites, p. 98. 
1888: N ew to n: Orbits, Am. Journ.

(3) 36, p. 4

r889: F l e t c h er: Atacama Meteorites, Mineral. Magaz. Bd. 8, p. 226 (Streufeld 10 miles).

I89o: v. $\mathrm{N}$ i e s $\mathrm{s} 1$ : Periheldistanzen,Verh. naturf. Ver. Brünn, Bd. 29, p. $\mathbf{1} 89$, I95, 214, 257 .

189o: E as t m a n : Met. Astron., p. 316. r893: N ew t on: Lines of structure in the Winnebago Co. Meteorites and in other Meteorites. Am. Journ. (3) 45, p. 153,355 .

r894: C o hen: Meteoritenkunde, p. 6I, I73, 174, 237.

I894: F le t c her: Introduction, p. 39.

Ursprüngliches Gewicht: Steinschauer. Der grösste Stein soll 225 lbs. gewogen haben; viel verschleppt.

Nachweisbares Gewicht: I8 267 gr.

Bailey

Belgrad

Bement

Berlin, U.

Blatz

Bologna

Bonn

v. Braun

Breslau

Budapest

Calcutta

Cambridge

Cleveland

Dorpat

Dresden, M.

Dublin, M.

- Freiberg, i. S.

Göttingen

Gregory

Harvard, U.

Heidelberg

Klausenburg

Kopenhagen

Kunz

London, B.M.

London, P. G. $\quad 154$

\section{Whitfield}

Dalton

Wichita Co., Brazos River, Texas, U. S. A.

Eisen, Og,bekannt 1836 , beschr. 1860 . 1860: $\mathrm{S}$ h u mard: Notice of Meteoric Iron from Texas. Trans. Acad. of Sc. St. Louis (1856/60) Bd. I, p. 622623 (Analyse von Riddell).

1860: $\mathrm{H}$ a id inger: Einige neuere Nachrichten über Meteoriten, namentlich die von Bokkeveld, New-Concord, Trenzano, die Meteoreisen von Nebraska, vom Brazos, von Oregon. Sitzber. Wien. Akad. Bd. 4I, p. $571-$ 572 ; s. auch Liebig-Kopp, Jahresber. 1860 , p. $850-851$; Kenngott, Uebersicht 1861, p. 107 .

1858/62: v. Reichen ba ch: VI 448. IX 174, 181. X 359, 365. XV nо, 124, 126. XVI 26I, 262. XVII 266, 272. XVIII 487. XIX 149, I54. XX 622. 1863: B u chner: Meteoriten, p. 16r. 1863: R o s e: Meteoriten, p. 152.

1876: B u c k l e y: Second Annual Rep. Geol, Surv. Texas (Houston 1876).

1884: M a 11 et: On a Mass of Meteoric Iron from Wichita County, Texas. Am. Journ. (3) 28, p. 285-288 (neue Analyse). Ref. N. J. 1886 I, p. 32; LiebigKopp, Jahresber. 1884, p. 2041.

1885: Brezina: Wiener Sammlung, p. 155, 207, 215-216, 234 (T. II u. III). r886: $\mathrm{H}$ u n tin g t o $\mathrm{n}$ : Crystalline Structure, Am. Journ. (3) 32 , p. 295.

r888: v. H a u e r: Ann. Hof-Mus. Bd. 3 (Not.), p. 42.

1889: v. H a u e r: Ann. Hof-Mus. Bd. 4 (Not.), p. 64.

1889: $\mathrm{B}$ r e z i n a : Cliftonit aus dem Meteoreisen von Magura, Arvaer Comitat. Ann. Hof-Mus. Bd. 4, p. 105.

1890: F l e t c h er: Mexican Meteorites, Mineral. Magaz. Bd. 9, p. 102.

r891: Cohen u, Weinschenk: Meteoreisen-Studien. Ann. Hof-Mus. Bd. 6, p. 131, 152-155 (Analyse), 161, $162,164,165$. 
1892: C o hen: Meteoreisen-Studien. Ann. Hof-Mus. Bd. 7 , p. $154-155$, $158(\mathrm{Cu}), 159,160,16 \mathrm{I}$.

1893: Brezina: Ueber neuere Meteoriten (Nürnberg), p. 165.

1893: Meunier: Revision des fers météoriques, p. 28-30 (Abbildung einer geätzten Platte).

1894: C o h en: Meteoritenkunde, p. 53, 57 , IOI, 102, 104, II5, I16, II7, г50, 152, 194.

1895: Brezina : Wiener Sammlung, p. 285 .

r895: Co h e n: Meteoreisen-Studien IV. Ann. Hof-Mus. Bd.ro, p. $83,84,85,86,91$.

Ursprüngliches Gewicht: I45 Kgr. I 860 befand sich in Austin, Texas, eine Masse von 320 lbs., von welcher etwa $3-4$ lbs. abgesägt waren.

Nachweisbares Gewicht: 29 172 gr.

\begin{tabular}{|c|c|c|}
\hline Bailey & I2 954 & München \\
\hline Bement & 1424 & New Haven \\
\hline Berlin, U. & Io & Paris, M. \\
\hline Böhm & 27 & Prag, M. \\
\hline v. Braun & $4^{1} 3$ & Riga \\
\hline Brezina & 1478 & Rom, U. \\
\hline Budapest & 949 & Sevilla \\
\hline Calcutta & 12 & v. Siemaschko \\
\hline Darmstadt, M & $3^{2}$ & Tübingen \\
\hline Dorpat & 399 & Turin, U. \\
\hline Gregory & 169 & Ward \\
\hline Hamburg & 91 & Washington \\
\hline Harvard, U. & 1797 & Washington,Sh. \\
\hline Howell & I 55 & Wien,H.M.*) 49 \\
\hline London, B. M & 1395 & Wien, U. II. \\
\hline de Mauroy & & \\
\hline
\end{tabular}

\section{Widdin}

Williams Port

Wilna

Wilson Co. Fisen

Wirba Wilson Co. Stein

\section{Windorah}

Winnebago Co.

Wirba, Widdin, Türkei.

Stein, Cwa, gefallen 20. Mai 1874 . Hierher auch: Belgradjek sgefallen 2. Juni $1883 \ll$.

1874: D a u b r é e: Note sur une météorite tombée le 20 mai 1874 , en Turquie, à Virba près Vidin. C. R. Bd. 79, p. 276-277; s. auch Am. Journ. (3) 8 (1874), p. 399; Wochenschr. f. Astr., Met. u. Geogr. 1874, p. 312, 354 . r884: M e u n i er: Météorites, p.209,225. 1885: Brezina: Wiener Sammlung, p. 272 ,

1887: F lig ht: Meteorites, p. 73 .

r887: v. H a u e r: Ann. Hof-Mus, Bd, 2 (Not.), p. $3^{8 .}$

1893: M eunier: Sur deux météorites turques récemment parvenues au Muséum d'Histoire naturelle. C. R. Bd, Ir7, p. 257-258 (Urba, Belgrade Djik). Ref. N. J. 1895 I, p. 276.

1895: Brezin a : Wiener Sammlung, p. 244 .

Ursprüngliches Gewicht: $3600 \mathrm{gr}$. Nachweisbares Gewicht: 3200 gr.

\begin{tabular}{lr|lr} 
v. Braun & 96 & Paris, M. & 16 \\
Eger & $\left.3040^{2}\right)$ & v. Siemaschko & 2 \\
London, B. M, & 38 & Wien, H. M. & 8
\end{tabular}
London, B. M. $\quad 38$ Wien, H. M. 8

Wisconsin Meteorite $185^{8}$ Trenton Wisconsin 1865 Vernon Co. Wisconsin $\mathrm{r} 884$ Hammond Withe Sulfur Springs

Witim

Greenbrier Co.

Wittmess

Wjasemsk

Werchne Udinsk

Eichstädt Kikino

Wöhler Eisen Campo del Cielo Wold Cottage, Yorkshire, England.

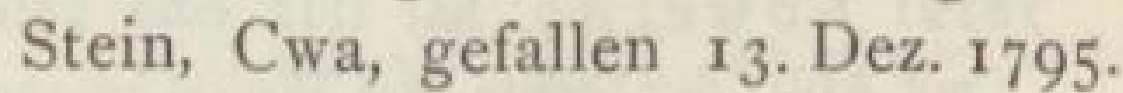

I) Ausserdem 68 gr. nach I. Juli 1893 erworben.

2) 1895 erworben. 
1796: Topham: Gentlemans magazine vom 8. Februar 1796; s. auch Gilb. Ann. Bd, I5 (1803), p. 318-319. 1796: $\mathrm{K}$ in g: Remarks, p. 21-22, 25, 33 .

I802: How a r d : Experiments and Observations on certain stony and metalline Substances, which at different Times are said to have fallen on the Earth ; also on various kinds of native Iron. Phil. Trans. (1802), p. 174-175, 183-184, 197 (Analyse). Auszug in Gilb. Ann. Bd. 13, p. 297-298, 305, 3II-312 ; s, auch Fourcroy's Uebersetzung in „Encyclopédie « Bd. 5 (1808), p. $546,548,549,554,559$; Klaproth, Abh. Berlin. Akad. 1803 , p. $32-33$. r803: C h 1 a d n i : Chronologisches Verzeichnis, Gilb. Ann. Bd. 15, p. 310, 318-319 (Anmerkung von Gilbert); s. auch v. Moll's Ann. Berg- u.Hüttenk. Bd, $2\left(18 \mathrm{o}_{3}\right)$, p. $319-32 \mathrm{I}$.

1803: de Dré e: Recherches, Journ. Phys. Bd. 56, p. $383-384,411,413$, $417,419,420$.

1803: I z a rn: Lithologie, p. 100, ror, 108, I7I, 175, 187-188, 209, 21 4, 231, $232,257,318,325,343,344,349$.

1804: Pötzsch: Kurze Darstellung, p. $78-79,92$.

1804: v. Ende: Massen und Steine, p. $54-55$.

1806: L a u g i e r : Chromium, Gilb. Ann. Bd. 24, p. 379; s. auch „Encyclopédie « Bd. 5 (1808), p. 583,592 .

1808: de Dré e-Pi c te t : Description comparative etc. (s. bei Weston). Bibl. Brit. No. 296 (April 1808), p. 28I, $287,288$.

1812: Bigot de Morogues, p. 150 $-\mathrm{I} 53,335$.

1812: Chla dni: Verzeichnis, Schweigg. Journ. Bd. 4, Beil. I, p. 14.

1812: Sowerby hat 1812 eine Tafel mit Abb. der Steine von Wold Cottage, Mooresfort und High Possil anfertigen lassen; s. auch Chladni, Feuermeteore, p. 293 (Ein Exemplar im k. k. Hofmuseum).

1815: Chladni: Neues Verzeichnis, Gilb. Ann. Bd. 50, p. 252.

r8rg: $\mathrm{Chladni:}$ Feuermeteore, p. 6, 9 , 10, 57, 66, 69, 71, 73, 75, 91, 258, $263-264(1), 293,428$.

r834: B e r z e li u s: Ueber Meteorsteine.

Pogg. Ann. Bd. 33, p. 1.

1836: Kä m tz: Meteorologie, p. 277.

r839: B e n z e n b er $\mathrm{g}$ : Sternschnuppen, p. $45-46$.

I843: Partsch: Meteoriten, p. 58. 1854: B a $1 \mathrm{c}$ e $11 \mathrm{~s}$ : Lithologia meteorica, p. 22.

1857: A rago: Astronomie populaire. Bd. 4, p. 195.

1859: Harris: Dissert. Gött., p. 63. 1859: B u c hn er: Feuermeteore, p. 56 -57 .

I863: B u c h n e r : Meteoriten, p.12-13. r863: Rose: Meteoriten, p. 155.

1858/65: v. Reichen b a ch: V 477 , 480,48 I. VI 454 . VII 552 . IX 155 , 161, 167, 168, 178. X 359. XI 294, 297. XIII 373 (Fig.), 383. XX 623 . XXV 318, 319, 320, 324, 432, 438. r884: M e un ier : Météorites, p. 95,98 , 208, 210, 459, 468 .

1885: S h ep a r d: Am. Journ. (3) 30, p. 106 (Krystall von Nickeleisen).

1885: Brezina: Wiener Sammlung, p. $180,232$.

1893: N ew to n: Lines of structure in the Winnebago Co. Meteorites and in other Meteorites. Am. Journ. (3) 45, p. 152,355 . Ref. N. J. 1894 I, p. 273 -274 .

r894: Cohen: Meteoritenkunde, p. 7, $23,6 \mathrm{I}, 185$.

1894: F let cher: Introduction, p. 22 $-23,24$.

r895: Brezina: Wiener Sammlung, p. 243 .

Ursprüngliches Gewicht: Ein Stein von $25^{1 / 2} \mathrm{Kgr}$. (56 lbs.). 


\begin{tabular}{lr|lr} 
Nachweisbares Gewicht: 2 I I 75 & gr. \\
Bailey & 8 & Klausenburg & 24 \\
Belgrad & $4 \mathrm{I}$ & London, B. M, 20 III \\
Bement & 42 & London, P. G. & 99 \\
Berlin, U. & 3 & Moskau & 33 \\
v. Braun & 4 & Newton & I \\
Budapest & 7 I & Oxford & 6 \\
Calcutta & 36 & Paris, M. & II5 \\
Cambridge & 15 & Pohl \\
Cleveland & I & v. Siemaschko \\
Dorpat & 14 & Stockholm \\
Dresden, M. & I3 & Troyes \\
Göttingen & I29 & Tübingen \\
Gregory & 53 & Ward \\
Greifswald & 27 & Washington, Sh. \\
Harvard, U. & 66 & Wien, H. M.*) \\
IO2 \\
Heidelberg & 3 &
\end{tabular}

Kristiania besitzt noch ein Stück von 27 gr. mit der Bezeichnung Yorkshire, welches ca. 1830 von Herrn Prof. Esmark geschenkt wurde und vielleicht hierher gehört.

\section{Wolfsegg}

s. Anhang

Wooster, Wayne Co., Ohio, U.S.A.

Eisen, Om, gefd, $185^{8}$, beschr. 1864 .

r864: S mith: A new Meteorite from Wayne County, O. Remarks on Meteorite from Atacama, Chili. Am. Journ. (2) 38 , p. $385-386$ (Analyse); s, auch Original Researches 1884, p. 431; Journ. prakt. Chem. Bd. 95 (1865), p. 313; Kenngott, Uebersicht $1862 / 65$, p. 269 ; Liebig-Kopp, Jahresber. I864, p. 904. 1865: B u chner: Zweiter Nachtrag, Pogg. Ann. Bd. 124, p. 599-600. r884: M e unier: Météorites, p. r16,r24. r885: Brezina: Wiener Sammlung, p. 211,234 .

1893: M e un i e r: Revision des fers météoriques, p. 50.

1894: C o h e n : Meteoritenkunde, p. 57.

Ursprüngliches Gewicht: $22^{1} / 2 \mathrm{Kgr}$. (50 lbs.).

Nachweisbares Gewicht: 49 gr.

\begin{tabular}{lr|lr} 
Bailey & J4 & London, B. M. & 5 \\
Berlin, U. & I & London, P. G. & 4 \\
Calcutta & 2 & Paris, M. & 5 \\
Göttingen & I & Stockholm & 3 \\
Gregory & 5 & Washington, Sh. & 3 \\
Harvard, U. & 6 & Wien, H. M.*) Spl. \\
Wo befindet sich die grosse Masse?
\end{tabular}

Worowa

Wyoming

Xiquipilco

s. Anhang Angara

Silver Crown

Toluca

Yanhuitlan

Misteca

Yardea Station, Gawler Range, Südaustralien.

Eisen?, gefunden 1875 , noch nicht beschrieben.

1893: Briefliche Mitteilung des Herrn Cleland, Hon. Curator, Mineralogical Collection, Adelaide Museum: ,The only specimen which has been found, or at any rate scientifically made known, up to the present time is in the form of a mass of meteoric iron obtained in the Gawler Range in November, 1875. The form is bounded by a series of more or less concave and irregularly-shaped planes. The surface is, for the most part, coated with a somewhat shining and dark-brown oxide of iron. This meteorite consists of metallic iron, and contains a small proportion of nickel. It weighs 3268.7 grams. As originally found it was a trifle heavier, a small piece having been broken off by the finder. The locality and circumstances attending the discovery of the meteorite are thus described by Mr. James Martlew: I found the stone on the flat in a mallee scrub about half a mile from the northern foot of the range, being distant four miles south of Yardea Station. It was about 15 inches under the surface, and was surrounded for about three feet by limestone broken into small pieces. All round this there was from 
four to eight inches of soil covering the limestone $\propto$.

Ursprüngliches Gewicht: 3269 gr. Nachweisbares Gewicht: 3269 gr.

Public Library Museum, and Art Gallery of South Australia

3269

Yarra-Yarra

Cranbourne

Yatoor (Nellore) bei Nellore, Madras, Ostindien.

Stein, Cc, gefallen 23. Januar 1852 . I86r: $\mathrm{H}$ a i dinge $\mathrm{r}$ : Der Meteorit von Yatoor bei Nellore in Hindostan. Sitzber. Wien. Akad. Bd. 44 II, p. $73-$ 74; s. auch Am. Journ. (2) 34 (1862), p. 152 ; N. J. 1862 , p. 597 ; Kenngott, Uebersicht $1862 / 65$, p. 435 ; Liebig-Kopp, Jahresber. 186r, p. I120 ; Zeitschr. f. d, ges. Naturw. Bd. I9, p. III ; L'Institute Bd. 29 (186r), Nr. 1446, p. 320.

1863: B u chner: Meteoriten, p. 82. 1863: Ha i d ing e r: Parnallee. Dritter Bericht. Sitzber. Wien. Akad. Bd. 47 II, p. $425-426$.

1863: Maskelyne und v. Lang: Mineralogical Notes. - Notices of Aërolites von Maskelyne. - 13. Nellore. Philos. Magaz. Bd. 25, p. $443-446$; s, auch Rep. Brit. Assoc, 1862 (Not. and $A b s t r)$, p. 190; Kenngott, Uebersicht $1862 / 65$, p. 442 .

I863: Ros e: Meteoriten, p. 154. 1865: Buchner: Zweiter Nachtrag, Pogg. Ann. Bd. 124, p. $5^{81}-5^{82}$.

1883: W i ik: Mikroskopisk undersökning af naagra paa universitetets mineralkabinett befindliga meteoriter. Ofvers. Finska Vetensk. Soc. Förh. Bd, 24 . Ref. N. J. 1883 I, p. 384 .

r884: Meunier: Météorites, p. I9I (Abb.), 192.

r885: Brezina: Wiener Sammlung, p. $185,233$.

1894: F let c h er: Introduction, p. Io, 12, 36 . r895: Brezina: Wiener Sammlung, p. 255 .

Ursprüngliches Gewicht: Ein Stein von über $I_{3} \mathrm{Kgr}$. Nach London gelangten 30 lbs.

Nachweisbares Gewicht: I 1987 gr.

\begin{tabular}{lr|lr} 
Bement & 2 & London, P. G. & 35 \\
Berlin, U. & 92 & New Haven & 47 \\
Budapest & 4 & Paris, M. & 62 \\
Calcutta & 30 & v. Siemaschko & 27 \\
Dorpat & I0 & Stockholm & 43 \\
Göttingen & 36 & Strassburg & 3 \\
Gregory & 3 & Ward & I \\
Harvard, U. & 99 & Washington, Sh. & 3 \\
Heidelberg & I & Wien, H. M.*) 202 \\
London, B.M. II 287 &
\end{tabular}

Yodzé, auch Jodzie, Russland.

Stein,Hob,gefallen I 7. (5. ?) Juni 1877 . 1892: v. Ha uer: Ann. Hof-Mus. Bd. 7 (Not.), p. 73 .

1893: B r e z in a : Ueber neuere Meteoriten (Nürnberg), p. 159.

r895: Brezina: Wiener Sammlung, p. $24 \mathrm{I}$.

Ursprüngliches Gewicht: ?

Nachweisbares Gewicht: 43 gr.

London, B. M. 2 Wien, H. M. Paris, M. 40

\section{Yokahima}

Yorkshire 1795

Yorkshire 1881 Middlesbrough

Yorktown Tomhannock Creek

Youndegin (Penkarring Rock), SubDistrictYoundegin; 7 o engl.Meilen östl. von York, Westaustralien,

Eisen, Og, gefd, 1884 , beschr. 1887 .

r887: Flet cher: On a meteoric iron found in 1884 in the sub-district of Youndegin, Western Australia, and containing cliftonite, a cubic form of graphitic carbon. Mineral. Magaz. Bd. 7, p. 121-130 (Analyse). Ref. N. J. 1888 
II, p. 225-226; s, auch Weinschenk, Ann. Hof-Mus. Bd. 4 (1889), p. 99. 1887: Fletcher: Cubic crystals of graphitic Carbon. *Nature Bd. 36 , p. 304-305.

r889: Brez in a: Cliftonit aus dem Meteoreisen von Magura, Arvaer Comitat. Ann. Hof-Mus. Bd. 4, p. $102,103$. I892: v. Ha uer: Ann. Hof-Mus, Bd. 7 (Not.), p. 73.

1893: B rez in a : Ueber neuere Meteoriten (Nürnberg), p. 165.

1893: v. H a uer: Ann. Hof-Mus. Bd. 7 (Not.), p. 49.

r893: Am. Journ. (3) 46, p. 76 ; s. auch Oesterr. Zeitschr. f. Berg- u. Hüttenw. Bd. 41 (1893), p. 453 (kurze Angabe, dass der jetzt im Wien. Hof-Mus. befindliche Meteorit von nahezu einer Tonne Gewicht bei Gregory in London eingetroffen sei).

1893: Gre gor y : Some Press notices of Mr.J. R. Gregory's large Meteorite, found at Youndegin, West Australia.Anzeige aus , Illustrated London News « vom Ir. März 1893, aus sIron * vom 17. März 1893, aus »Weekly Dispatch * vom 26. März 1893.

1893: Meunier: Revision des fers météoriques, p. 29, 33-34.

1894: Co he n: Meteoritenkunde, p. 68, II5, $125,140,145,146$.

1894: F let c her: Introduction, p. 14. r895: Brezina: Wiener Sammlung, p. $286-287$.

r895: C o h e n: Meteoreisen-Studien IV. Ann. Hof-Mus. Bd. 10, p. 82 .

Ursprüngliches Gewicht: $933 \mathrm{Kgr}$. Fünf Stücke: $900 ;$ 11.7; 10.9; 8 ; $2.7 \mathrm{Kgr}$. Ausserdem $7 \frac{3 / 4}{\mathrm{Kgr}}$. Verwitterungskruste aus Magnetit bestehend.

Nachweisbares Gewicht: $9273^{8} 5$ gr.

\begin{tabular}{lr|lr} 
Bement & 1805 & Brezina & I49 \\
Böhm & 78 & Calcutta & 85 \\
v. Braun & 116 & Dresden, M. & 23
\end{tabular}

\begin{tabular}{lr|l|r|} 
Gregory & I4 & Prag, M. & I48 \\
Greifswald & 20 & Rom, U. & IO3 \\
Kunz & 20 & Stockholm & II \\
London,B.M. & 13 235 & Strassburg & 38 \\
de Mauroy & 27 & Utrecht & 38 \\
Paris, E. & 72 & Ward & 1087 \\
Paris, M. & 406 & Wien,H.M. 909 910
\end{tabular}

Zaborzika, Volhynien, Russland.

Stein, Cw, gefallen ro. April r818. Hierher auch: Czartorya, Gouv. Volhynien, Russland. Stein, Cw, zuerst erwähnt 1859 .

1823: La ugi er: Bulletin des Sc. de la Soc. Philom. Juin 1823 (Analyse); übersetzt in Gilb. Ann. Bd. 75 (1823), p. 264-266 unter dem Titel: Vorläufige Nachricht von der chemischen Analyse zweier in Polen gefundenen Aërolithen und zweier Meteor-Eisen, mit Bemerkungen von Gilbert; s. auch Ann. Chim. Phys. Bd. 25 (1824), p. 219-220; Schweigg. Journ. Bd. 43 (1825), p. 27.

r823: Chladni: Dritter Nachtrag, Gilb. Ann. Bd. 75 , p. 230.

1824: Chla dni: Vierter Nachtrag, Pogg. Ann. Bd. 2, p. 153 .

1836: K ämtz: Meteorologie, p. 287. r837: R o s e : Reise nach dem Ural. Bd.I, p. 77 .

1843: Parts ch: Meteoriten, p. 53.

r847: Eichwald: Verzeichnis, Erman's Arch. f. wissensch. Kunde v. Russl. Bd. 5, p. 178 .

r848: B 1 o e d e: Tabelle, Bull. Petersburg. Akad. Bd. 6, p. Io.

1857: A rago: Astronomie populaire. Bd. 4, p. 199.

1859: H a r r i s: Dissert. Gött., p. 73. 1863: Buchner: Meteoriten, p. 37 $-38,{ }_{15}$ (Czartorya).

1863: Ros e: Meteoriten, p. 88, 155. 1858/65: v. Reichenbach: (Czartorya) V 476. VI 456. IX 161, 168, 178. XI 294, 297, 301. XIII 360, 372 (Fig.), $380,383$. XX 626. XXV $324,438,607$. 
r859/65: v. Re i chen ba c h: (Zaborzika) IX 161, 168, 178. X 359, 363. XIII 372 (Fig.). XX 623, 626. XXV $438,607,615$.

1867: Go e b e 1: Kritische Uebersicht, Mélanges phys. chim. Bd. 7, p. 338 . 1867: Buchner: Dritter Nachtrag, Pogg. Ann. Bd, 132, p. 318.

1884: M e unier: Météorites, p. 208, $212-213$.

r885: Brezina : Wiener Sammlung, p. $177,232,255$ (Czartorya).

Ursprüngliches Gewicht: Etwa 4 Kgr. Eichwald giebt 1847 an, dass in Kiew 9 Pfund aufbewahrt werden.

Nachweisbares Gewicht: $3867 \mathrm{gr}$.

\begin{tabular}{lr|lr} 
Bement & 3 & London, P. G. & 2 \\
Berlin, U. & 53 & Moskau & 2 \\
v. Braun & I4I & Neumann & 2 \\
Budapest & 39 & Paris, M. & 46 \\
Calcutta & I & Petersburg, A. & 431 \\
Dorpat & I9 & v. Siemaschko & 2 \\
Gregory & 3 & Tübingen & 16 $3^{1}$ ) \\
Kiew & 2834 & Washington & 4 \\
London, B. M. & 9 & Wien, H. M.*) & II3
\end{tabular}

Zabrodje, Gouv. Wilna, Russland. Stein, Cia, gefallen 22. Sept, 1893 . r89: Melikoff und $P$ issarjewski: Chemische Analyse des Meteoriten von Zabrodje. Ber, d, d. chem. Ges. Bd, 27, p. 1235-1238.

r895: Brezina: Wiener Sammlung, p. 248.

Ursprüngliches Gewicht : 3155 gr. Nachweisbares Gewicht: 300 gr.

Odessa 300 gr. Inzwischen erhielt Wien, H. M. 5 gr.

Wo befindet sich die grösste Masse des Steins?

I) Davon $\gg 105$ gr. Czartorya ; das einzige Stück, welches seit 1859 unter diesem offenbar irrtümlich für Zaborzika geschriebenen Namen vorkommt. Neuerdings giebt Herr Prof. Loewinson-Lessing für das Stück der Dorpater Sammlung an sZaboritza, Czartorya, Volhynien, Russland, gefallen II. April I8I8๕.

\section{Zacatecas, Mexico.}

Eisen, $\mathrm{Obz}$, zuerst erwähnt $\mathbf{r}_{79^{2}}$. 1792: Gazeta de Mexico. Tomo V Nr. 7 del Martes 3 de Abril de 1792, p. 58 -60 (ein Exemplar befindet sich im k. k. Hof-Mus. in Wien); s, auch N. J. 1856, p. $289-290$.

r804: S onnes c h mid: Beschreibung der vorzüglichsten Bergwerks-Reviere in Mexico oder Neuspanien, p. I92. Als Manuskript gedruckt (ein Exemplar befindet sich im k. k. Hof-Mus, in Wien, ein anderes nach Chladni in Freiberg). 1804: D e 1 Rio: Tablas mineralogicas, p. 56.

I $8 \mathrm{I}$ : v. $\mathrm{H}$ u m b o $1 \mathrm{~d} \mathrm{t}$ : Essai politique sur le royaume de la nouvelle Espagne Bd. I, p. 293 u. Bd. 2, p. 582 .

1812: Bigot de Morogues, p. 300. 1815: Chla d n i: Bemerkungen, Gilb. Ann. Bd. 50, p. 269.

r8rg: Chladni: Feuermeteore, p. 336338 (1), 434; s. auch Denkschr. München. Akad. 1812, p. 109-110.

1826: See beck: Ueber die magnetische Polarisation der Metalle und Erze durch Temperatur-Differenz. Pogg. Ann. Bd. 6 , p. 144 .

1836: B u r k a rt: Aufenthalt und Reisen in Mexico in den Jahren $\mathbf{1 8 2 5 - 1 8 3 4}$ Bd. I, p. 389 (Stuttgart 1836 ).

1843: P a rts c h: Meteoriten, p. 122125.

1849: B ergeman n: Ueber das Meteoreisen von Zacatecas. Pogg. Ann. Bd. 78 , p. $406-413$ (Analyse). Ref. Liebig-Kopp, Jahresber. 1849 , p. 827 -828; Kenngott, Uebersicht 1844/49, p. 226 ; Pharm. Centr. 1850 , p. 246 ; N. J. 1850 , p. 446.

1852 : Cla r k: Dissert. Gött., p. 2830 ; s. auch Am. Journ. (2) 15, p. II; 
Wöhler, Gött. Gel. Anz. (Nachr.), 26. Januar $185^{2}$.

r853: Nögger a th: Meteoreisen-Massen mit Widmanstädt'schen Figuren. N. J. 1853 , p. 174 (soll aus den Verh. d. Niederrh. Ges. Bonn vom 16. Dez. I852 sein, ist aber dort nicht zu finden); s. auch Kenngott, Uebersicht 1853 , p. 116.

1854: v. Boguslawski: Zehnter Nachtrag, Pogg. Ann. Ergz, - Bd, 4, p. $4 \mathrm{ro}-4 \mathrm{II}$.

1855: Ha i d ing e r: Bemerkungen über die zuweilen im geschmeidigen Eisen entstandene krystallinische Structur, verglichen mit jener des Meteoreisens. Sitzber. Wien. Akad. Bd. ${ }_{5}$ I, p. 357.

r856: Burkart: Fundorte I, N. J. $(1856)$, p. $283,284,285,288-297$, 299 ; s. auch Briefl. Mitt. N. J. 1857 , p. 54 .

r857: B e r g e m a n n : Untersuchungen von Meteoreisen. - Meteoreisen von Zacatecas. Pogg. Ann. Bd. 100, p. 255 -256 (neue Analyse); s, auch Chem. Centr. 1857, p. 746; Journ. prakt. Chem. Bd. $7 \mathrm{I}$ (1857), p. 59; Liebig-Kopp, Jahresber. 1857, p. 734 ; Kenngott, Uebersicht $1856 / 57$, p. 153 u. 1859, p. 106 ; N. J. 1859 , p. 737. 1857: Giebel u. Heintz: Zeitschr. f. d. ges. Naturw. Bd. 9, p. $5^{10}$ u. Bd. Io, p. 189.

1858: B urk art: Fundorte II, N. J. (1858), p. $772,773,774,775$.

1859: M üller: On a Meteoric iron from Zacatecas in Mexico. Quart. Journ. Chem. Soc. Bd, II, p. 236-240; s. auch Burkart in Verh. Niederrh. Ges. Bonn (Sitzber.) 1850, p. 84-90; Journ. prakt. Chem. Bd. 79 (1860), p. 23-26 (Analyse; Abbildung einer geätzten Platte); >L'Institut « Bd. 27 (1859), Nr. 1334 , p. $242-243$; Liebig-Kopp , Jahresber. $185^{8}$, p. 8II -812 ; Kenngott, Uebersicht I859, p. $105-106 ;$ N. J. 1859 , p. 736-737.
1859: B u c hn e r : Feuermeteore, p.146. 1859: H a r r is: Dissert. Gött., p. 105.

r860: $\mathrm{R}$ a m mel s ber $\mathrm{g}$ : Mineralchemie, p. 910, 947, 948, 1000.

r863: B u chner : Meteoriten, p. I44 -146 .

1863: Ros e: Meteoriten, p. 66-67, T. II, 153,159 .

r864: C a varoz: Ossements fossiles découverts en diverses parties du Mexique. -- Corps d'origine météorique. C. R. Bd. 59, p. $1099-\mathbf{1 1 0 0 .}$

I858/65: v. Re i chen b a ch: VI 443. VII 551,557 . VIII 488 . IX 162, 175 , 176, 182. X 359. XI 291. XII 456. XIII 364. XIV 390. XV I00, 125. XVII 273. XVIII $480,484,487,489$. XIX 155 . XX 622, 627,629. XXI 587 . XXV 437.

1865: B u chner: Zweiter Nachtrag, Pogg. Ann. Bd. 124, p. 596.

r869: Bu chner: Die Aetzfiguren des Meteoreisens. Ber. d. Oberhess. Ver. f.

Natur-u.Heilk. Giessen 1869, p.II2-II5. 1869: Meunier: Recherches, Ann, Chim. Phys. (4) 17, p. 30, 68, 69, 72. 1870: Ram melsberg: Meteoriten, p. 79 .

r870: Burkart: Fundorte IV, N. J. 1870, p. $686-688,692$.

1875: vom R a th: Meteoriten, Verh. naturh. Ver. Bonn Bd. 32, p. 356-357. 1875: Mohr: Entstehungsart, Ann.

Chem. Pharm. Bd. 179, p. 259, 268. r876: B a r cen a : On certain Mexican Meteorites. Proc. A cad, nat. hist, Philad. 1876, p. 123 .

I884: M e un i e r: Météorites, p. 26, 39, $44,45,48,94,96,98,99,116,117$. 1884: $\mathrm{H}$ ï p k e: Beiträge, Abh, naturh. Ver. Bremen Bd. 8, p. 520 .

1883/85: Tschermak: Photographien, p. 3 .

r885: Brezina: Wiener Sammlung, p. $200,217,234$.

1887: Brezin a: Neue Meteoriten III, Ann. Hof-Mus. Bd. 2 (Not.), p. 115. 
r889: C a sti110: Catalogue, p. 4. r889: C o h en: São Julião, N. J. r889 I, p. $223,224,225$.

r89o: Brezin a: Reise, Ann. Hof-Mus.

Bd. 4 (Not.), p. II7.

I890: F let cher: Mexican Meteorites, Mineral. Magaz. Bd. 9, p. 99, 104, $162-164,474$.

I890: Ea stma n: Met. Astron., p. 3 I8.

I892: Eastman: The Mexican Meteorites. Bull. Philos. Soc. Washington Bd. 12, p. 46.

1893: M e unier: Revision des fers météoriques, p. 52,56 .

1894: Cohen: Meteoritenkunde, an vielen Stellen.

1894: Flet cher: Introduction, p. 33. 1895: Brezina: Wiener Sammlung, p. 289 .

r895: C o h e n: Meteoreisen-Studien IV. Ann. Hof-Mus. Bd. io (Not.), p. $8_{3}$, 84,85 .

UrsprünglichesGewicht: Eine grosse Masse von etwa $1000 \mathrm{Kgr}$.

Nachweisbares Gewicht: 24424 gr.

\begin{tabular}{lr|lr} 
Bailey & 7 I & Hamburg & 38 \\
Belgrad & 4 & Harvard, U. & 28 I \\
Bement & 27 & Heidelberg & 1434 \\
Berlin, U. & 1410 & Klausenburg & 43 \\
Bologna & 166 & Kopenhagen & 9 \\
Bonn & 3436 & v. Leuchtenberg & I2I \\
v. Braun & $36 \mathrm{I}$ & Lissabon & 45 \\
Bremen & 69 & London, B. M. & 3847 \\
Budapest & 337 & London, P. G. & 140 \\
Calcutta & 766 & Manchester & 2 I \\
Cleveland & 16 & Mannheim & II9 \\
Darmstadt, M. & 134 & de Mauroy & 23 \\
Dorpat & 52 & Melion & 7 \\
Dresden, M. & 86 & Minneapolis & 4 \\
Edinburg & 91 & Moskau & 56 \\
Göttingen & 53 & München & 5 I \\
Graz, J. & 34 & Neumann & 84 \\
Gregory & 86 & Paris, M. & 2353 \\
Greifswald & 35 & Parma & 10 \\
Halle & I95 & Pohl & 42
\end{tabular}

\begin{tabular}{lr|lr} 
Prag, M. & 59 & Tübingen & $5128^{1}$ ) \\
Rom, U. & 123 & Upsala & 98 \\
v. Siemaschko & 98 & Ward & 56 \\
Stockholm & 34 & Washington & 15 \\
Strassburg & 237 & Washington, Sh. 175 \\
Stïrtz & 59 & Wien, H. M.* ${ }^{*}$ ) & 2014 \\
Stuttgart & 6 & Wien, U. I. & I50 \\
Troyes & 15 & &
\end{tabular}

Die Universität Basel und Herr Dr. Ulex in Hamburg besitzen ebenfalls ein Stück Zacatecas.

Zebrak (Praskoles), auch Hoíowic (Horzowitz), Kreis Beraun, Böhmen, Oesterreich.

Stein, Cc, gefallen 14. Okt. 1824 .

1825: v. Martius: Fernere Nachrichten von denen in vorigen Herbst in Böhmen gefallenen Meteorsteine. Kastner's Archiv f. d. gesammte Naturlehre Bd. 5, p. 417-4I9 (einem der Münchener Akademie mitgeteilten Bericht entnommen); s, auch Ann. Chim. Phys. Bd. 30 (1825), p. $42 \mathrm{I}-422$. 1826: Chla dni: Fünfter Nachtrag, Pogg. Ann. Bd. 6, p. $28-30,167$. 1836: K ä mtz: Meteorologie, p. 293. 1843: Partsch: Meteoriten, p. 80. 1854: v.B og u s l a w s k i: ZehnterNachtrag, Pogg. Ann. Ergz,-Bd. 4, p. 439. 1859: Harris: Dissert. Gött., p. 78. r859: v. R ei c hen b a c h: IX I6I, I69, 179. XI 294, 295.

r863: B u c hn er: Meteoriten, p.47-48. 1884: Meunier: Météorites, p. 72, 208, 214.

1885: Brezina: Wiener Sammlung, p. $185,233$.

1895: Brezina: Wiener Sammlung, p. 255 .

U rsprüngliches Gewicht: Ein Stein, von welchem die zwei grössten Bruchstücke zusammen 1873 gr. (ro7 Lot Wiener Gewicht) wogen. Nachweisbares Gewicht: 1271 gr.

I) Ausserdem $9250 \mathrm{gr}$. als Zacatecas bezeichnet, welche pseudometeorisch sein dürften. 


\begin{tabular}{lr|lr|} 
Brezina & II & Paris, M. & I \\
Budapest & $\mathbf{7}$ & Prag, M. & 870 \\
Calcutta & I & Stuttgart & 3 \\
London, B.M. & 8 & Tübingen & 3 \\
Neumann & I 4 & Wien, H. M.*) & $353^{1}$ )
\end{tabular}

\section{Zipaquira}

Rasgata

Zmenj bei Stolin, Gouv. Minsk, Russland.

Stein, Ho, gefallen August $185^{8}$, beschr. 1892 .

r892: Prende1: Meteorit >Zmenjк, gefallen im August 1858. - Odessa 1892 (Russisch); s, auch Revue des Sciences naturelles 1892 No. 9 (1893), p. 323-326. Ref. N. J. 1895 I, p. 33; Bibl, géol, de la Russie Bd. 8, p. 125. r894: v. Ha u e r: Ann. Hof-Mus. Bd. 9 (Not.), p. 3 I.

r895: Brezina: Wiener Sammlung, p. $240-241$.

Ursprüngliches Gewicht: $246 \mathrm{gr}$. Nachweisbares Gewicht: 155 gr.

\begin{tabular}{lr|l|l|} 
Brezina & 4 & Wien, H.M. & 116 \\
Odessa & 35 &
\end{tabular}

\section{Znorow Wessely}

Zsadany, Temeser Komitat, Ungarn.

Stein, Cc, gefallen 31 . März 1875 .

1875: Egyetértés és Magyar Ujság, 23. April u. 16. Juni 1875 .

r875: Wartha u. Krenner: Természettu dományi 1875 , p. 200.

r878: C o hen: Ueber den Meteoriten von Zsadány, Temesvar Comitat, Banat.
Verh. naturh.-med. Ver. Heidelberg Bd. 2 Heft 2, p. I-Io (Sep.) (Analyse). Ref. N. J. 1878 , p. $747-748$; Liebig-Kopp, Jahresber. 1878 , p. 1317 . 1878: Wartha: Vorläufige Anzeige bezüglich der Analyse des Zsadányer Meteoriten. Fresenius, Zeitschr. f. analyt.

Chem. Bd. 17, p. $43^{1-434}$. Ref. Liebig-Kopp,Jahresber. 1878. p I317-1318. 1879: R a m mels berg: Meteoriten, p. 24,25 .

1879: $\mathrm{P}$ ill it z: Analyse des Zsadányer Meteoriten. Fresenius, Zeitschr. f. analyt. Chem. Bd. 18, p. 58-68. Ref. LiebigKopp, Jahresber. 1879, p.1276-1277. 1879: K l e in : Göttinger Sammlung, Gött. Gel. Anz. 1879, p. 92.

r884: Me u n i e r: Météorites, p. 79, 209. r884: W a d s w or t h: Studies,p.96-97. 1885: Brezina: Wiener Sammlung, p. 185,233 .

I887: F lig ht: Meteorites, p.188-I9o. 1894: Co he n : Meteoritenkunde, p. 156, $177,205,248,249,266,277,283$.

1894: F le t c h e r: Introduction,p.r3,27. Ursprüngliches Gewicht: $55^{2} \mathrm{gr}$. Zusammen 16 Steine, der grösste $152 \mathrm{gr}$.

Nachweisbares Gewicht: 4I 4 gr.

\begin{tabular}{lr|lr} 
Böhm & 8 & Gregory & 5 \\
Budapest & 223 & London, B. M. & 25 \\
Cohen & 13 & Paris, M. & 12 \\
Debreczin & 69 & v. Siemaschko & 14 \\
Göttingen & Spl. & Wien, H. M.**) & 45
\end{tabular}

Zweibrücken Krähenberg

I) Nach r. Juli 1893 noch 96 gr. erworben. 


\section{Anhang.}

Dieser Anhang enthält in alphabetischer Reihenfolge: Meteoriten, welche wegen fehlender Literatur oder wegen ungenügender Angaben sich nicht einordnen liessen, sowie einige unzweifelhafte Pseudometeoriten, die auch in manchen der neuesten Verzeichnisse noch als echte Meteoriten aufgeführt werden; ferner Mitteilungen über drei neuere Meteoriten (Lesves, Madrid u. Nagy-Borove) und schliesslich eine Ergänzung zu dem Eisen von Forsyth County und eine Berichtigung zum Stein von Schellin.

\section{Alexander County, Nord-Carolina,} U. S. A.

Eisen, gefunden 1860 .

Bailey

Bement

193 Cleveland

12

Amates, Rancho de los Amates, Staat Morelos, Mexico.

Eisen, erwähnt 1889 .

1889: Casti11o: Catalogue, p. 3.

r89o: F1 et cher: Mexican Meteorites,

Mineral, Magaz. Bd. 9, p. 168-169.

1895: Brezina: Wiener Sammlung,

p. 274 .

Das Stück befand sich 1889 im Besitz Castillo's und wird in seinem Katalog ohne Gewichtsangabe aufgeführt.

Ameca-Ameca, Staat Mexico, Mex. Eisen, erwähnt 1889 .

1889: Casti110: Catalogue, p. 3.

1890: F le tc her: Mexican Meteorites,

Mineral. Magaz. Bd. 9, p. 168-169.

r895: Brezina: Wiener Sammlung, p. 274.

Das Stück befand sich 1889 im NationalMuseum in Mexico; Castillo führt es in seinem Katalog ohne Gewichtsangabe auf.

\section{Amerika.}

Eisen, ohne Fundzeit.

Kiew 9I gr.; Petersburg, B. 308 gr. ; v. Siemaschko $3 \mathrm{gr}$. >Amérique, localité inconnue (Websky) .

Amerika.

Stein, ohne Fundzeit.

Petersburg, B. 35 gr.

Angara, Gouvernement Jenisseisk, Ost-Sibirien.

Hierunter vorläufig vereinigt:

I) Grosse Muroshna, Nebenfluss der Angara. Eisen, in Goldseifen gefunden 1885 .

Petersburg, A, 116 gr.

2) Fluss Uderei, Eisen, in Goldseifen gefunden 1885 .

Petersburg, A. $16 \mathrm{gr}$.

3) Worowa, Nebenfluss der grossen Muroshna. Eisen in Goldseifen gefunden.

Petersburg, A. $68 \mathrm{gr}$.

Ausserdem siehe Ssyromolotow, p. 336 , auf welches zu verweisen bei Angara p. 8 versäumt worden ist. 
Arve.

»Aérolithe trouvée dans l'Arve, semblable à celle de Barbotan«. Paris, U. $\quad 82 \mathrm{gr}$.

Asien.

Bruchstück eines Meteoriten, einseitig berindet, angeblich aus Asien.

$$
\text { Krüger } 2 \mathrm{gr} \text {. }
$$

\section{Atacama.}

Eisen. Edinburg $\quad$ II6 gr.

Eisen. Würzburg $5 \mathrm{gr}$. Eisen, r86o. Cleveland $5 \mathrm{gr}$. Eisen, r864. Harvard, U. $15 \mathrm{gr}$. Eisen $>$ Holosiderit, geätzte (also wohl oktaëdrisch)

Dublin, R, C. 192 gr.

Eisen. „Dünne Eisenplatte, eine Seite poliert u. geätzt, gute Figuren

$$
\text { Hohenheim } 126 \text { gr. }
$$

\section{Australien.}

Eisen.

$$
\text { Bement } 20 \mathrm{gr} \text {. }
$$

\section{Australien.}

Pallasit, gefunden r880.

r888: H unting ton: Catalogue, Proc. Amer. Acad. Boston Bd. 23, p. 99.

Harvard, U. $90 \mathrm{gr}$.

Belmont (Simonod), Dep. Ain, Frankreich.

Meteor, beobachtet am 13 . November $r 8_{35}$.

Die Literaturangaben deuten auf einen kohligen Meteorstein. Die kleine Menge des erhaltenen Materials ist höchstwahrscheinlich pseudometeorisch.

1835: A r a go teilt einen Brief Millet d'Aubenton's mit. sL'Institute. Bd. 3, Nr. 134, p. 386 .

1835: Unter, Vermischte Notizen *: 4)
Sternschnuppen. Pogg. Ann. Bd. 36, p. $562-563$.

r836: Mille t schickt einige Stücke an die Akademie. sL'Institut $\propto$ Bd. 4, Nr. I4I, p. 17.

1836: Unter $>$ Vermischte Notizen $\propto$ : 7) Meteorstein im Departement Ain. Pogg. Ann. Bd. 37 , p. $460-46 \mathrm{r}$.

1839: B enzen berg: Sternschnuppen, p. 236.

1843: P a r t s c h: Meteoriten, p. I4-I5 r850: v. Humboldt: $\gg$ Kosmos Bd.III, p. 608 .

I854: v. Boguslawski: Zehnter Nachtrag, Pogg. Ann. Ergz.-Bd.4, p. 8o, 354 .

1859: v. Reichen bach: IX 163 (zweifelt an der Echtheit des Steins; verweist auf Dumas ${ }^{2}$ 'Institut Bd. 4 , Nr. 14I, p. 17, der die Untersuchung übernommen, aber nicht veröffentlicht habe).

r859: B u chner: Feuermeteore, p. 92 -93 .

1863: Ros e: Meteoriten, p. 25.

1865: B u chn er: Zweiter Nachtrag, Pogg. Ann. Bd. 124, p. 572.

1867: $\mathrm{H}$ a i d ing e r: Der Meteorit von Simonod. Sitzber. Wien. Akad. Bd. 55 II, p. $127-130$.

r867: Geinitz: N. J. 1867, p. 724 . r884: M e un ier: Météorites, p. 462. r885: Brezina : Wiener Sammlung, p. $184,233$.

Ursprüngliches Gewicht: ?

Nachweisbares Gewicht: Spl.

Calcutta Spl. Tübingen Spl.

London, P. G. Spl. Wien, H. M. *) Spl.

Bohême, sHolosidère de Bohême

$$
\text { Lausanne } 3 \mathrm{gr} \text {. }
$$

Botetourt, Virginia, U. S. A.

Eisen, gefunden um 1850 , beschr. I 866 .

r866: $S$ he p a rd: Brief Notices of serveral localities of Meteoric Iron. - 
2. Botetourt County, Virginia. Am. Journ. (2) 42, p. 250.

1869: Buchner: Vierter Nachtrag, Pogg. Ann. Bd. 136, p. 603 .

r885: Brezina: Wiener Sammlung, p. 221,234 .

I890: E as t m a n: Met. Astron., p. 318.

Ursprüngliches Gewicht: Eine grössere Masse, welche sich nicht gut zu Pferde transportieren liess.

Nachweisbares Gewicht: Die kleine für eine Analyse zu unbedeutende Masse, welche Shepard nach der oben angegebenen Notiz besass, ist in seiner in Washington befindlichen Sammlung jetzt nicht mehr aufgeführt. Herr Manross hat an einer Probe im Wöhler'schen Lab. mehr als $20 \%$ Nickel gefunden. Es ist sehr fraglich, ob diese von Shepard beschriebene Masse, die verloren gegangen zu sein scheint, als meteorisch anzusehen ist.

Calcutta Spl. Wien, H. M.*) Spl, Gehört hierher auch das Göttinger Stück $\$ 1866$ Virginien N. Am. (aus einer Petroleumquelle)\& ?

»Brésil, loc. inconnue .

Eisen, gefunden 1866.

1893: M e unier: Revision des fers météoriques, p. 75 .

Paris, M.

2 gr.

Brookville, Franklin Co., Indiana. Stein, gefunden $\mathrm{r} 866$.

Harvard, U. 7 gr.

\section{Bruce's Eisen.}

Eisen, erwähnt 1859 .

I859/62: v. Reichen b a c h: IX 162, 174, 181. XV 110, 124, 128. XVI 261, 262. XVII 266, 273. XIX 149.

r863: B u chner: Meteoriten, p. 200. Tübingen Io gr.
Die meteorische Natur dieses Eisens von unbekannter Herkunft ist zweifelhaft.

Buenos Ayres, Brasilien.

Eisen, ohne Fundzeit.

1879: K 1 e i n: Gött. Gel. Anz. (Nachr.) 1879, p. 99.

$$
\text { Göttingen } \quad 18 \mathrm{gr} \text {. }
$$

Nicht Santa Catharina, da hiervon 140 gr. getrennt aufgeführt werden.

\section{California «?}

Eisen, ohne nähere Bezeichnung.

$$
\text { Berkeley } 230 \mathrm{gr} \text {. }
$$

Caparrosa (Rincon de Caparrosa), südwestlich von Chilpanzingo, Staat Guerrero, Mexico.

Eisen, bekannt $185^{8}$, erwähnt 1866 . 1866: B u r k a rt : Fundorte III, N. J. 1866, p. 402.

1870: Burkart: Fundorte IV, N. J. 1870 , p. 692.

1889: C a st i110: Catalogue, p. I-2. 189o: F 1 e t c he r: Mexican Meteorites, Mineral. Magaz. Bd. 9, p. 174.

1893: M e un i e r : Revision des fers météoriques, p. 29,34 .

1895: Brezina: Wiener Sammlung, p. 274 .

Das Stück von ursprünglich 341 gr. Gewicht soll beim Durchbrechen einer Stufe von Kupferkies gefunden worden sein. Brezina konstatierte neuerdings grosse Aehnlichkeit mit Toluca. Ausser der Hauptmasse, welche in Mexico sein dürfte, besitzt Paris, M. I6 gr. u. Wien, H. M. 20 gr.

\section{Ceylon.}

Eisen, gefunden 1869 .

Herr Direktor Rudler teilte mir über dieses Eisen mit: $\gg$ The two pieces of meteoric iron are in a box with an old label in these words: 
Ceylon. Found 1869 , in the hands of the natives, working into charms as "Lightning Iron «.

London, P. G. zwei Stücke von 23.4 und $21.4 \mathrm{gr}$.

In der älteren Literatur wird ein Meteor stein fall von Ceylon vom 13. April 1795 erwähnt; einige Angaben über denselben mögen hier angeschlossen werden.

1800: Le B ek: Bemerkungen über einige Ceilonische Fossilien und ihre Schleif-Methode, \$Der Naturforscher* 29. Heft, p. $242-252$ (Halle, b. Gebauer $\left.8^{\circ}\right)$; s, auch v. Moll's Annalen der Berg- u. Hüttenkunde Bd. 2 ( $\left.18 \mathrm{O}_{3}\right)$, p. $97-98$.

r8r6: $\mathrm{Chl}$ a d n i : Zweite Fortsetzung, Gilb. Ann. Bd. 54 , p. $351-352$.

r8rg: $\mathrm{C}$ h 1 a d n i : Feuermeteore, p. 66, $69,74,75,262-263$ (1).

1854: B a l c e $11 \mathrm{~s}$ : Lithologia meteorica, p. 22.

I854: v. Boguslawski: Zehnter Nachtrag, Pogg.Ann. Ergz.-Bd. 4, p.423. r859: Harris: Dissert. Gött., p. 63.

Cincinnati, U. S. A.

Eisen.

Herr Dr. Weinschenk hatte die Freundlichkeit, mir Folgendes mitzuteilen : sDas Eisen von Cincinnati wurde von Hosaeus dem hiesigen Institut geschenkt; er besass eine ziemlich grosse Platte und war im Zweifel, ob es ein Meteorit sei. Das Eisen hat einen ziemlich hohen Gehalt an Nickel, ist ziemlich dicht und zeigt kleine glänzende Stäbchen; doch ist mir seine meteorische Natur zweifelhaft. Es soll bei einem Hausbau in Cincinuati gefunden worden seins.

München $28 \mathrm{gr}$.
Colorado River, La Paz, NeuMexico.

Eisen, bekannt $\mathbf{1 8 6 2}$.

$$
\text { New Haven II gr. }
$$

Concord, New Hampshire, U. S. A. Pseudometeorit.

1847: Am. Journ. (2) 4, p. $353-356$. r848: Am. Journ. (2) 6, p. $403,416-$ 417 ; s. auch Liebig-Kopp, Jahresber. 1847/48, p. 1313; Rammelsberg, Handwörterbuch Suppl. V (1853), p. 33.

1854: v. Bog us laws ki: Zehnter Nachtrag, Pogg. Ann. Ergz.-Bd. 4, p. 376 -377 .

1860: R a m mels berg: Mineralchemie, p. $941-942$.

r863: B u c hner: Meteoriten, p. 65 .

Cuernavaca, Staat Morelos,Mexico. Eisen, erwähnt 1889 .

r889: C a st illo: Catalogue, p. 3.

r89o: F 1 e t $\mathrm{c}$ h er: Mexican Meteorites, Mineral. Magaz. Bd. 9, p. 168.

1895: Brezina: Wiener Sammlung, p. 274 .

Das Stück befand sich 1889 im NationalMuseum in Mexico.

Curvello, Minas Geraës, Brasilien, gefallen $x$. April 1833 .

r84I: C l a us s e n: Bull. Acad. Bruxelles Bd. 8, Nr. 5 .

1863: B u chner: Meteoriten, p. 56. 1888: D e r by: Meteoritos Brasileiros. Revista do Observatorio Rio de Janeiro, p. 16-18 (Sep.).

Wahrscheinlich ein Pseudometeorit, der vermutlich verloren gegangen ist.

Eisenberg, Sachsen-Altenburg, Deutschland.

Eisen, gefunden 1873 .

1874: Geinitz: >Isis (1874), p. 5 u. Purgolt: 1882 , p. 9 .

Die Analyse ergab einen grossen Gehalt an Kohlenstoff und kein Nickel, es liegt also höchst wahr- 
Anhang. Eisenberg - Kaaba.

scheinlich ein Pseudometeorit vor. Die Hauptmasse besitzt Dresden mit 1394 gr.

Ferguson, Haywood Co., NordCarolina, U. S. A.

Stein, gefallen 18 . Juli 1889 . 1890: K u n z: Am. Journ. (3) 40. p. 320. Ref. N. J. 1891 II, p. 52 ; 1892 I, p. 267.

Der etwa 226 gr. ( 8 Unzen) schwere Stein ging durch Zufall verloren.

Florac (?), Dep. de la Lozère, Frankreich.

Eisen (also nicht Aumières) »découvert par M. Henry«.

$$
\text { Parma } 122 \text { gr. }
$$

Forsyth County, Nord-Carolina (nicht Georgia). Eisen, s. o. p. 123 r8g6: de Schweinitz: Meteorite from Forsyth Co., North Carolina. Am. Journ. (4) I, p. 208.

Grodno (?), Gouvernement Grodno (?), Russland.

Eisen, Fundzeit unbekannt.

$$
\text { Kiew } 253 \text { gr. }
$$

Haywood County, Nord-Carolina, U. S. A. Eisen, beschr. I 854 .

1854: S h ep a rd: Am. Journ. (2) 17, p. 327 .

Shepard erhielt ein kleines Stück von etwa 3 gr., an welchem er reichen Nickelgehalt nachwies. Nur Calcutta und London, B. M. besitzen Spl.

\section{Heidelberg.}

Die von Wawnikiewicz (Ann. Chem. Bd. 123 (I862), p. 254) mitgeteilte Analyse, welche u. a. o. $1 \% \mathrm{Ni}$ ergab, lässt die meteorische $\mathrm{Na}$ tur dieses Eisens als höchst zweifelhaft erscheinen. Wie Herr
Direktor Fletcher mir brieflich mitteilte, betrachtet er das Eisen ebenfalls als pseudometeorisch.

Calcutta 24 gr., London, B. M. 15 gr.

Hommoney Creek, Nord-Carolina. Pseudometeorit.

\section{Igast.}

Pseudometeorit ; s. Lit. bei Ösel. Dorpat $2 I /$ v. Siemaschko 120 Helsingfors $\quad 20$ Stockholm $\quad 56$ Paris, M. 46

\section{Indien, Stein,}

Edinburg besitzt zwei indischeSteine von 125 gr. u. 94 gr. ohne nähere Bezeichnung (s. bei Charwallas p. 74).

Kaaba, Stein. Der schwarze Stein der Kaaba in Mecca (el Hadschar el aswad), lange vor Mohammed bekannt und von den Arabern als Heiligtum verehrt; erwähnt 1772 .

1772: Carsten Niebuhr: Beschreibung von Arabien. Kopenhagen 1772, p. 362 .

I776: d'H e r b e lot: Bibliothèque orientale.Artikel $\gg$ Hagiar Allasovad * (Maestricht 1776. Folio), p. 390.

1816: A li B e y: Travels of Ali Bey in Marocco, Tripoli, Cyprus, Egypt, Arabia, Syria and Turkey Bd. 2 (Taf. 55, Abbildung). London 1816. Auch Voyage d'Ali Bey el Abassi, en Afrique et en Asie Bd. 2, p. 347 u. Taf. 55, Paris 1814.

1816: Chladni: Erste Fortsetzung, Gilb. Ann, Bd. 53, p. 392.

r819: $\mathrm{C}$ h1 a d n i: Feuermeteore, p. 184 -185 .

1829: $\mathrm{B} \mathrm{urk} \mathrm{hardt:} \mathrm{Travels} \mathrm{in} \mathrm{Arabia,}$ Bd, I, p. 249. London 1829. Uebersetzung: Weimar 1830 . Ref. Leonhard's Jahrbuch Bd. 2 (1831), p. 362. 
1832: v. H off: Achter Nachtrag, Pogg. Ann. Bd. 24, p. 233.

I857: $\mathrm{P}$ a r $\mathrm{ts} \mathrm{c} \mathrm{h}$ : Ueber den schwarzen Stein in der Kaaba zu Mekka. Mitgeteilt aus den hinterlassenen Schriften. Denkschr. Wien. Akad. Bd. 13, p. 1 -5 ; s. auch Sitzber. Wien. Akad. Bd. 22 (1856), p. 393-394; "L'Institut \& Bd. 24 (1856) Nr. 1199, p. 449-450. r859: B u c h n e r: Feuermeteore, p. 3r. 1885: M ü 11 er: Der Islam im Morgenund Abendland. Aus Oncken's *Allgemeine Geschichte in Einzeldarstellung. Bd. I, p. 198-203 (Abbildung). 1888: S n o u ck H urg ron je: Mekka I. Die Stadt und ihre Herren. Haag 1888, p. 2-3 (spricht von zwei Steinen, dem >schwarzen $\ll$ in der östlichen Ecke und dem »südlichen in der südlichen Ecke).

1894: S a l e h S o ub h y: Pèlerinage à la Mecque et à Médine. Le Caire. Imprimerie nationale. 1894 , p. 7 I.

Ursprüngliches Gewicht: Ein Stein von etwa 7 Zoll Durchmesser.

Nachweisbares Gewicht: Zu Anfang dieses Jahrhunderts gelangten, als infolge eines Aufstandes der Wahhabiten der Stein zertrümmert wurde, kleine Bruchstücke desselben an Mehmed Ali, nachmaligen Vizekönig von Egypten, an den Sultan, den Schah und den Beherrscher von Kabul.

\section{Kamtschatka.}

Eisen, bekannt um $1803-1806$; höchst wahrscheinlich pseudometeorisch.

Hierunter vereinigt: RasoumowskiEisen (v. Siemaschko); Eisen aus dem portugiesischen Amerika (John).

182I: John: Sur la Nature de grandes masses de fer métallique d'origine problématique, et sur celle du fer des aérolithes attirables par l'aimant. Ann. Chim. Phys. Bd. 18, p. 20I-202; s. auch Schweigg. Journ. Bd. 32 (I82I), p. 262. John sagt, dass von dem Rasoumowski-Eisen auch ein von Lavater herstammendes Stück in der Züricher Sammlung sich befinde und dass dieses von Peterson (und Krusenstern) aus Kamtschatka mitgebracht worden sei. 1859/62: v. Reic hen b a c h: IX 162, 175, 176, 182. XI 29I. XII 458. XIII 356. XV 10o. XVII 268, 269, 273. XVIII 488. XXI 578 .

r862: K e n ng ot $\mathrm{t}$ u. W iser: Zürcher Sammlung, p. $145-146$.

I89r: v. Siemas chko verweist in seinem Katalog p. 20 auf Journ. Phys. Bd, 92, p. 267.

1895: M e unier: Revision des lithosidérites, p. $26-27$.

Ursprüngliches Gewicht: ?

Nachweisbares Gewicht: $63 \mathrm{gr}$.
Paris, M.
131) Tübingen
Pohl
4 Zürich
$\left.33^{2}\right)$
v. Siemaschko I2

Lesves bei Namur, Belgien.

Stein, Cc (?), gefallen 13 . April I 896 . Nach der Beschreibung Renard's scheint ein Kügelchenchondrit vorzuliegen, daher die Bezeichnung $\mathrm{Cc}$ (?).

1896: Renard: Notice préliminaire sur la météorite de Lesves. Bull. Acad. roy. Belgique (3) 3I, Nr. 6, p. 654663 (Analyse von Stöber; Abb.); s. auch >Naturea vom 30. April $\mathbf{1} 896$.

Ursprüngliches Gewicht: Ein Stein von nahezu $2 \mathrm{Kgr}$.

Nachweisbares Gewicht: Nach gütiger Mitteilung von Herrn Dr. C. Klement in Brüssel erwarb Herr Prof. Dewalque in Lüttich

1) Pallasit, welcher Aehnlichkeit mit Imilac zeigt. - 2) Wahrscheinlich Pseudometeorit. W ü $1 \mathrm{fing}$, Meteoriten. 
die Hauptmenge im Gewicht von I360 gr., welche zwischen den Museen von Brüssel, Lüttich u. Gent zur Verteilung gelangte; Brüssel, Musée d'Hist. Nat. de Belgique, besitzt 330 gr.; London B. M. besitzt nach dem neuesten Verzeichnis von r 896 einen Spl.

Locust Grove, Henry Co., Georgia, U. S. A.

Eisen, gefunden 1857 , erwähnt 1895 (Brezina, Wiener Sammlung 1895 , p. 302,353$)$.

Ob Henry County?

Long Creek, Jefferson Co., Tenn., U. S. A.

Eisen, gefunden 1853 , beschr. 1854 . 1854 : $\mathrm{S}$ h e p a r d: Meteoric Iron? from Long Creek, Jefferson Co., Tennessee. Am. Journ. (2) 17, p. 329-330 (Analyse : $3.3 \%$ C u. kein $\mathrm{Ni}$ ).

1859: B u c hn e r : Feuermeteore, p. 133 -134 .

1887: Riggs: A new Meteoric Iron and an Iron of doubtful nature. Am. Journ. (3) 34, P. 60 (Analyse : $0.76 \%$ $\mathrm{Ni}, 6.73 \% \mathrm{Mn}, 0.86 \%$ Graphit und $1.46 \%$ gebundener Kohlenstoff).

1890: E a s t m a n : Met. Astron. p. 318, 322.

UrsprünglichesGewicht: Etwa r Kgr. $\left(2^{1 / 2}\right.$ lbs. $)$.

Nachweisbares Gewicht: $5^{86}$ gr.

Tübingen 15 $\left.{ }^{1}\right) \mid$ Washington

571

Lorana (ohne nähere Bezeichnung). Stein, 1697 .

New Haven 8 gr.

Macquaire River, Neu Süd-Wales, Australien.

Mesosiderit (?) oder Pallasit (?), gefunden 1857 .
Gregory

Paris, M.

58 v. Siemaschko

3

$\mathrm{Ob}$ mit Cowra $z \mathrm{u}$ vereinigen?

Madrid, Spanien.

Stein, Cwa (reich an Maskelynit), gefallen ro. Februar 1896.

r896: Calderon: Le bolide de Madrid. "Le Naturaliste 1 er mars 1896, (2) 18, Nr. 216, p. $55-56$.

1896: C a l d e r o n: Explosion d'un bolide à Madrid. Bull. Soc. Géol. France (3) 24, p. $117-120$.

1896: Merino: El bólide del to de Febrero de 1896 . Gazeta de Madrid vom II. Febr. 1896 ; s. auch Astr. Nachr. Bd. 140 Nr. 3347 (1896), p. 170.

1896: M eunier: Examen sommaire de la météorite tombée à Madrid le Io février 1896 . C. R. Bd. 122, p. 640 $-64 \mathrm{r}$.

I896: Co he n: Ueber den Meteoritenfall bei Madrid. Mitt. Naturw. Ver. Neu-Vorpommern u. Rügen Bd, 28 (Sep.), 6 Seiten.

r896: Gredilla y Gauna: C. R. Bd. 122 Nr. 26 (29. Juni 1896).

Ursprüngliches Gewicht: Noch nicht mit Sicherheit bekannt geworden.

Nachweisbares Gewicht: Nach Cohen's Aufsatz sind als Besitzer zu nennen: Professor Solano 125 gr.; Prof. Cánovas del Castillo einige Stücke; Prof. Iñiguez ein ganzer Stein; Observatorium Madrid 44 gr.; Prof. Cohen 4 gr.; London B. M. Spl. Auch dürften Paris M. und Wien H. M. kleine Stücke besitzen.

Manzanares, HaciendaManzanares, 5 Leguas (20 Km.) von San Luis de la Paz, Mexico.

Eisen, gefallen (?) 3o. März r $89 \mathrm{I}$.

I) Dichtes Eisen, ohne Analyse nicht zu bestimmen. 
1893: Brezin a: Ueber neuere Meteoriten (Nürnberg), p. I64.

Mexico, ohne nähere Bezeichnung. Eisen.

$$
\text { Cambridge } \quad \text { I9 gr. }
$$

Missouri, U. S. A.

Undescribed Iron. Slab, 245 grammes. By exchange with G. F. Kunz. Obtained by Kunz from Professor Potter, of Saint Louis. Washington $245 \mathrm{gr}$.

Mohilew, Gouvernement Mohilew. Stein, Fundzeit unbekannt.

$$
\text { Kiew } 22 \text { gr. }
$$

Monte Alto, Prov. Bahia, Brasilien. Eisen, erwähnt 1888 .

1888: D e r b y : Meteoritos Brasileiros. Notas sobre Meteoritos Brasileiros. Revista do observatorio. Rio de Janeiro (1888), p. 20 (Sep.-Adr.).

Ursprüngliches Gewicht: Das Eisen soll so gross sein, wie das Eisen von Bemdego.

Nachweisbares Gewicht: ?

Nagy-Borove, Liptoer Comitat, Ungarn.

Stein, gefallen 9. Mai 1895 , erwähnt 1895 (Brezina, Wiener Sammlung 1895, p. 307,356 ).

Nauheim, Bad Nauheim in der Wetterau, Grossherzgt. Hessen, Deutschland.

Eisen, gefunden 1826 , beschr. 1828 . Die meteorische Natur dieses Eisens ist noch nicht erwiesen.

1828: W i 11 e: Geognostische Beschreibung der Gebirgsmassen zwischen dem Taunus und Vogelsgebirge von der Lahn nach dem Main, Rhein und der Nahe, nebst besonderer Beachtung der daselbst vorkommenden verschieden- artigen Mineralquellen, Mainz $\mathbf{1} 828$ (bei Florian Kupferberg). - Anmerkung auf p. 5i-54. Wille hat keine quantitative Analyse ausgeführt und einen Nickelgehalt nur aus der schweren Löslichkeit des Eisens vermutet. Die Stelle lautet p. 54: >Nach diesen angeführten Fund- als Körper-Eigentümlichkeiten der entdeckten Eisenmasse, die ihre Schwerauflöslichkeit etwa einem Nickelgehalte zuzuschreiben hat, was in der Folge eine (bis hierhin aber noch nicht ausgeführte) quantitative Untersuchung näher aufklären wird - glaube ich sie für eine gediegene Eisenmasse meteorischen Ursprungs ansehen zu können, " Die Untersuchung Wille's beschränkt sich auf Bestimmung des spez. Gew., Beschreibung der Rinde und Oberfläche, Schneideversuche und Auflösung in Salpetersäure ohne qualitative chemische Untersuchung.

1859: B u c h n e r: Feuermeteore, p. II7. r863: B u c h n e r: Meteoritische Notizen aus dem Vereinsgebiete. Ber. d. Oberhess. Ges, f. Natur- u. Heilk. Giessen 1863, p. 94 ; s, auch Bd. 8 (1860), p. 84 .

1885: Brezina: Wiener Sammlung, p. 221, 234.

Ursprüngliches Gewicht: 3 kleine Stücke, zusammen 26 Lot. Die ursprüngliche Masse, welche einige Pfund schwer gewesen sein soll, ist grösstenteils verloren gegangen.

Nachweisbares Gewicht: $56^{1 / 2}$ gr.

London, B. $\quad 3^{1 / 2} \mid$ Wien, H. M.*) 53

Nevada, U. S. A.

Eisen, von unbekannter Fundzeit. Göttingen $6 \mathrm{gr}$.

Nordamerika (?), ohne nähere Bezeichnung.

Stein.

Turin, U. $\quad 14 \mathrm{gr}$.

$26 *$ 
Oroville, Butte Co., Californien, U. S. A.

Eisen, bekannt u. erwähnt 1894 in Bement's als Manuskript gedruckter "Third Rough List of Meteorites; C. S. B. June $1894 \%$.

$$
\text { Bement } \quad 184 \text { gr. }
$$

Ovifak, Disco, Jakobshavn, Niakornak u. Upernavik sind tellurische Eisen; s. auch Sowallik.

Pilot Grove, Lee Co., Iowa, U.S. A. Stein.

$$
\text { Bailey } \quad 7 \mathrm{gr} \text {. }
$$

Die Literatur: Am. Journ. (2) 30, p. 208; Buchner, Meteoriten 1863, p. 96; Pogg. Ann. Bd. 122 (1864), p. 321 bezieht sich wahrscheinlich auf einen Pseudometeoriten $s$ Pilot Grove, Independence Co., Iowa \&.

Ponta Grossa, Provinz Paraná, Brasilien.

Stein, gefallen April 1846 .

1888: De r b y : Meteoritos Brasileiros. Notas sobre Meteoritos Brasileiros. Revista do Observatorio. Rio de Janeiro, p. 15-16 (Sep.).

Ursprüngliches Gewicht: $667 \mathrm{gr}$. ( $\mathrm{r}^{1 / 4}$ libras).

Nachweisbares Gewicht: Der Stein scheint verloren gegangen $z u$ sein oder war ein Pseudometeorit.

Princetown, Highland Co., Ohio, U. S. A.

Eisen, gefallen (?) I3. Februar 1893 , erwähnt 1895 (Brezina, Wiener Sammlung, p. 305,359 ).

\section{Putnam, Georgia, U. S. A.}

Stein, gefallen Februar 1854 (also nicht das 1839 gefundene und I854 beschriebene Eisen von Putnam County).

$$
\text { Bologna } 2 \mathrm{gr} \text {. }
$$

\section{Rügen.}

Pseudometeorit.

Das Märkische Provinzial-Museum in Berlin besitzt dieses Eisen von 90 gr., welches Cohen als Spiegeleisen erkannte.

Mitt. naturw. Ver. Neu-Vorpommern u. Rügen Bd. 26 (1894) (Sep.), 2 Seiten.

Ruschany, $3^{8}$ Werst von Slonim, 42 Werst von Deretschin, Gouvernement Grodno.

Nach den sBremer Nachrichtene vom 28. Dezember 1894 soll am 7. Dezember $\mathbf{1} 894$ bei Ruschany ein grosser Meteorit gefallen sein.

Sachsen (Afzelius).

Pallasit (?) aus der Sammlung Afzelius.

$$
\text { Upsala } \quad 65 \mathrm{gr} \text {. }
$$

$\mathrm{Ob}$ vielleicht Steinbach?

Sanarka, Troizk, Ural.

Pseudometeorit.

1882: Grew ing k: Arch. f. d. Naturk. Liv. Esth. u. Kurl. Bd, 9. Ref. N. J. 1884 I, p. $29-30$.

Im Ganzen $3^{1 / 2}$ gr.; jetzt in Dorpat unter Pseudometeoriten aufgeführt.

San Cristobal, Provinz Antofogasta, Chile, S. A.

Pallasit, P.

Herr Dr. Frenzel teilte mir 1896 (Juni) mit, dass er einen neuen Meteoriten von 82 gr. erhalten habe, welcher in dem Goldgrubendistrikt SanCristobal gefunden worden sei und mit Imilac Aehnlichkeit besitze.

Schellin (s. o. p. $3 \mathrm{II}-3 \mathrm{I} 2$ ).

Freifrau v. Bredow hatte die Güte, mir eine Probe des auf Wagenitz unter der Etiquette s Schellin aufbewahrten Materials zu über- 
senden; leider hat sich dasselbe als unzweifelhaft pseudometeorisch erwiesen. $\mathrm{Ob}$ in der dortigen Sammlung doch noch etwas von dem Stein aufbewahrt wird, bedarf weiterer Nachforschung.

Seassport, Missouri, U. S. A.

Stein, gefunden $18 \mathrm{r}$.

Petersburg, B. 13 gr.

Sowallik, Grönland.

Eisen, gefunden $\mathrm{r} 8 \mathrm{r} 8$, höchst wahrscheinlich pseudometeorisch.

r819: Ross: Journ. of Sc. and Arts.

London Bd, 6 (Nr. XII), p. 369.

(Brande will $3 \%$ Nickel nachgewiesen haben); s. auch Voyage of Discovery etc. by Captain John Ross. London 18I9.

r8rg: C hla d n i: Feuermeteore, p.344 -345 .

1843: P a r t s c h : Meteoriten,p.135-136. 1852: C 1 a r k: Dissert. Gött., p. 71.

r863: Buchner: Meteoriten, p. 154 -155 .

r866: S h epard: Brief Notices of several localities of Meteoric Iron. I. Savisavik, North Greenland. Am. Journ. (2) 42, p. 249.

r885: Brezina: Wiener Sammlung, p. $234,269$.

1893: F let $\mathrm{cher}$ : Introduction, p. $8 \mathrm{r}$. r895: Brez ina: Wiener Sammlung, p. $297,299$.

Wien, H. M.*) besitzt 3 gr. u. Washington 16 gr. mit der Bezeichnung: Nr. 47 Greenland. Not Ovifac. Weight $\mathbf{1 5}_{5} .65$ grammes. By exchange from S. C. H. Bailey.

Sparta, Tennessee, U. S. A. v. Siemaschko $5 \mathrm{gr}$.

Stinking Creek, Campbell Co., Tennessee, U. S. A.

Eisen, gefd. 1853 , beschr. 1855 . Die meteorische Natur dieses Eisens ist höchst zweifelhaft.
1855: Smith: Memoir on Meteorites. - A description of five new Meteoric Irons, with some theoretical considerations on the origin of Meteorites based on their Physical and Chemical characters. - 2. Meteoric Iron from Campbell County, Tenn. Am. Journ. (2) 19, p. 159 (Analyse); s. auch Original Researches 1884, p. 374; Rep. Smithsonian Inst. 1855, p. 154; Journ. prakt. Chem. Bd. 66 (I855), p. 425 ; Weinschenk, Ann. Hof-Mus. Bd. 4 (1889), p. 96; Kenngott, Uebersicht 1855, p. 95-96; Liebig-Kopp, Jahresber. 1855 , p. 1026.

1859: B u c hn er : Feuermeteore, p.r3i. r859: H a r r is: Dissert. Gött., p. I2O. 1860: R a mme 1s berg: Mineralchemie, p. 916.

r863: Bu chner: Meteoriten, p. I9I $-192$.

1869: M e unier: Recherches, Ann. Chim. Phys. (4) 17, p. 36, 71, 72.

r884: M e unier: Météorites, p. 53, I3I, 132.

1885: Brezina: Wiener Sammlung, p. 269 .

1886: Brezina u. Cohen: Photographien, T. $\mathbf{1}, 2$.

1888: Huntington: On the Crystalline Structure of Iron Meteorites. Am. Journ. (3) 32, p. 303 (aus den Proc. Amer. Acad. Arts and Sc. vom 12. Mai 1886).

1890: E a s tma n: Met. Astron., p. 318. r893: Meunier: Revision des fers météoriques, p. 73-74.

r894: C o hen: Meteoritenkunde, p. r18. Ursprüngliches Gewicht: 123 gr.

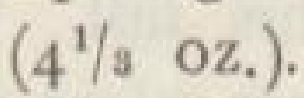

Nachweisbares Gewicht: roo gr. Calcutta $\quad 3 \mid$ Paris, M. 8 Harvard, U. $\quad 27 \mid$ Roebling $\quad 62$ Südamerika (Afzelius). Stein. Aus der Sammlung Afzelius. Upsala $\quad 279$ gr. 
$\mathrm{Ob}$ vielleicht Macao? da dies der einzige aus Südamerika stammende Stein älteren Datums ist.

Tamentit, Tuat, Afrika.

Eisen, bekannt I864, erwähnt 1865 . 1865: "Gerhard Roblf's Tagebuch seiner Reise durch Marokko nach Tuat, 1864. 3. Abschnitt: Reise von Karsas im Ued Sraura nach Ain Salah, 29. Juli bis I7. September, und: Allgemeine Beschreibung von Tuat $\alpha$. Petermann's Mitt. 1865 , p. 409 . Ref. Pogg. Ann. Bd. 129 (1 866), p. 176 ; Liebig-Kopp, Jahresber. 1866, p. 1007; Wochenschr. f. Astr., Met. u. Geogr. 1867, p. 40.

Nach der kurzen Beschreibung Rohlf's liegt wahrscheinlich ein Meteorit vor, auf welchen aufmerksam zu machen ich hierbei nicht unterlassen möchte.

Thunder Bay, Lake Superior, Ontario, Canada.

Eisen, gefunden 1826 , erwähnt 1895 . Brezina, Wiener Sammlung, p. 345 (unter Disco).

Bailey

75 Paris, M.

4

Thüringen, 1894 .

Pseudometeorit.

Nach Herrn Prof. Cohen's freundlicher Mitteilung Phosphoritknollen aus Podolien.

Tiree, Hebriden, Schottland.

Stein.

London, P. G. 4 gr.

Herr Direktor F. W. Rudler teilt mir hierüber mit: I can find no information about this fall. We have two small pieces of an undoubted meteoric stone in a glasstube, with an old label thus: 2Tyree, Scotland «?

Tocane, St. Apre, Dordogne, Frankreich.
Stein (?), gefallen 14. Februar 1861.

Wohl Pseudometeorit.

186I: »Cosmos $\alpha$ vom 26. April 1861.

r863: B u chner: Meteoriten, p. 106 -107.

1864: Pogg. Ann. Bd. 122 (1864), p. 329 -330 .

Es soll nur ein Stein von $7 \mathrm{gr}$. gefunden worden sein, der 1863 im Museum des Dep. Dordogne aufbewahrt wurde.

Transsylvania, Ungarn.

Eisen.

Calcutta IOI gr. nur mit der Bezeichnung: *From Transsylvania, from Mr. Nevill, I867.

Turon River, Neu Süd-Wales, Westaustralien.

Eisen, pseudometeorisch nach Brezina's Untersuchung des Stückes der Sammlung Siemaschko's.

$$
\begin{array}{lr|lr}
\text { Bailey } & 8 & \text { Paris, M. } & 6 \\
\text { Gregory } & 38 & \text { v. Siemaschko } & 6
\end{array}
$$

Unbekannte Fundorte von Eisen.

Calcutta 121 gr. One specimen, cut and polished on one face, the obverse shows natural surface.

Edinburg 123 gr.

Lissabon $35^{\circ}$ gr., Fisen, Og.

v. Siemaschko $15 \mathrm{gr}$. sLocalité inconnue (Paris). «*

\section{Unbekannte Fundorte vonSteinen.}

Calcutta 193 gr. One specimen cut and polished on one side showing metallic granules; a dull blac crust on the natural surface. $\&$

Edinburg 629 gr. (s. bei Charwallas, p. 74).

v. Leuchtenberg 6066 gr. (I4 Pfd. 78 Zolodnik). „Stein, vollständig umrindet, nur ein ganz kleines Stück abgeschlagen.\& 
Lissabon 270 gr. „Stein Cga? Tübingen 22 gr. + 9 gr. »Siena \&, welche aber zu einer andern Lokalität gehören.

Turin $7 \mathrm{gr}$.

\section{Wolfsegg.}

Eisen, pseudometeorisch.

1886: G u r 1t: Verh. naturh. Ver. Bonn

(Sitzber.) Bd. 43, p. $188 \rightarrow 189$; s. auch C. R. Bd. 103 (1886), p. 702 u. Dau- brée im gleichen Bd., p. 702-703; »Nature Bd. 35, p. 36.

1887: Brez in a : Ann. Hof-Mus. Bd. 2 (Not.), p. II3.

1888: Brezina: Allgemeiner Bergmannstag, Wien 1888 , p. $\mathrm{I}-6$ (Verlag des Komite's des Bergmannstages).

Yokahima, Hiokomo, Japan.

Pallasit.

v. Siemaschko $3 \mathrm{gr}$. (nach Brezina, Wiener Sammlung 1895, p. 265 ähnlich Imilac). 


\section{Verzeichnis der Sammlungen.}

Das folgende Verzeichnis zählt in alphabetischer Reihenfolge die Sammlungen auf, deren Meteoriten in den vorangegangenen Abschnitten berücksichtigt worden sind. Ausserdem haben hier noch Mitteilungen über die Sammlungen von Brüssel, Greifswald (Physikalisches Institut) und Stuttgart, (K. Realgymnasium), die erst nach teilweiser Drucklegung der vorangegangenen Abschnitte einliefen, Aufnahme gefunden,

Als Stichwort ist bei den öffentlichen Sammlungen der Name der Stadt, in welcher das betreffende Museum sich befindet, bei den Privatsammlungen der Name des Besitzers gewählt worden.

Mit einem * sind diejenigen Sammlungen versehen, deren Verzeichnisse nach ausdrücklicher Angabe der Herren Einsender sich auf den Bestand vom I. Juli 1893 beziehen. In gleicher Weise wurden auch solche Sammlungen bezeichnet, bei denen zwar dieser Termin nicht ausdrücklich hervorgehoben wurde, die Einsendung aber doch noch in den nächsten Monaten erfolgte oder bei denen man mit grosser Wahrscheinlichkeit annehmen durfte, dass seit dem 1 . Juli 1893 keine wesentlichen Veränderungen eingetreten waren.

Für jede Sammlung ist die Zahl ihrer Lokalitäten und ihr Gesamtgewicht mitgeteilt; das letztere wurde auf ganze Kilogramme abgerundet, wobei Gewichte unter $0.6 \mathrm{Kgr}$. nur durch einen Strich $(-)$ bezeichnet sind. Die hier mitgeteilten Zahlen weichen zuweilen von den früheren Angaben (Jahresh. d. Ver. f. vaterl. Naturk. Württemberg Bd. 5r (1 895$)$, p. 347$\left.35^{2}\right) \mathrm{ab}$; es findet dies seine Erklärung darin, dass ich auf spätere Anfragen hin über manche Einzelheiten der Kataloge jetzt besser unterrichtet bin. Ueber die Art der Zählung der Lokalitäten sind nähere Angaben in der Einleitung, sowie in den Erläuterungen zum Verzeichnis der Meteoriten zu finden.

Unter jeder Sammlung sind jene Meteoriten aufgeführt, von welchen die betreffende Sammlung die grösste Menge besitzt, und zwar ist das Ver. hältnis des in einer Hand befindlichen Maximalgewichts einer Lokalität zu dem gesamten nachweisbaren Material derselben durch Dezimalbrüche ausgedrückt. Beispielsweise soll die Angabe bei Bement \Bath 0.2; Kesen 0.3 ; Pacula 0.7 ; Plymouth 1.0 ; San Pedro Springs เ.0

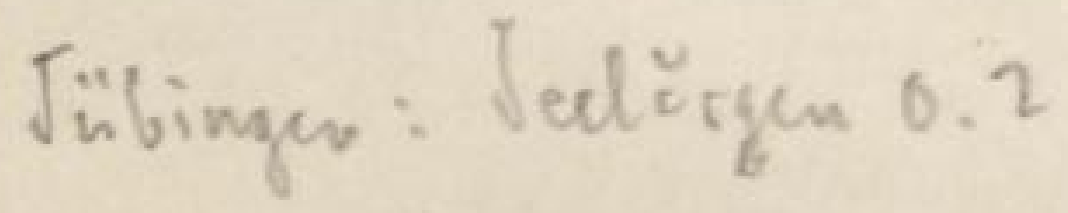


dieser Sammlung von Bath zwei Zehntel, von Kesen drei Zehntel, von Pacula sieben Zehntel und von den beiden letzten Fällen zehn Zehntel (oder wenigstens $96 \%$ ) des ganzen bis jetzt nachweisbaren Gewichtes dieser Lokalitäten vorhanden sind und dass davon keine andere Sammlung grössere Mengen besitzt. Diese Angaben dürften sich in jenen Fällen als zweckmässig erweisen, in denen der Tauschverkehr mit einer bestimmten Sammlung leichter auszuführen ist, als mit anderen Sammlungen und wo man gerne darüber unterrichtet sein will, welche Meteoriten in der betreffenden Sammlung besonders reichlich vertreten sind.

Die Literaturangaben beschränken sich in den meisten Fällen auf die Mitteilung der letzten vor 1894 im Druck erschienenen Verzeichnisse, welche mir mit den bis zum 1 . Juli 1893 reichenden handschriftlichen Nachträgen oder mit weiteren Angaben über später bekannt gewordene Meteoriten eingesandt wurden.

*Aachen: Mineralogisches Institut der K. Technischen Hochschule; mitgeteilt durch Herrn Prof. Dr. Arzruni

*A delaide: Public Library, Museum and Art Gallery of South Australia in Adelaide; mitgeteilt durch Herrn L. Cleland, Hon. Curator, Mineralogical Collection, Adelaide Museum

Yardea Station I.o.

Altenburg: Die Sammlungen der Naturforschenden Gesellschaft besitzen nach Herrn Kustos Dr. Koepert 5-6 Meteoriten

*Bailey: Privatsammlung des Herrn S. C. H. Bailey, Oscawana-on-Hudson, Westchester Co., N. Y., U. S. A.; mitgeteilt durch den Besitzer

Bethlehem 1.0; Deep Springs 0.8; Emmetsburg 0.4; Tomhannock Creek 0.9; Wichita County 0.4 ; Wooster 0.3 .

Base 1: Geologische und Mineralogische Sammlungen des Museum's. Vergl. Livret-Guide géologique dans le Jura et les Alpes de la Suisse, von Prof. Dr. C. Schmidt. Lausanne bei Briedel u. Cie. 1894 (Sep.), p. 4. (Kurze Aufführung der Fälle ohne Gewichtsangabe)

*B at avia: Museum des Hoofdbureau van het Mynwezen; mitgeteilt durch Herrn A. Renaud, Chef du service des mines aux Indes Orientales Hollandaises

Bandong 0.7; Djati-Pengilon t.o.

v. Ba um hauer: Die Privatsammlung von E. v. Baumhauer, Harlem, gelangte um r 885 grösstenteils an das NationalMuseum in Budapest.

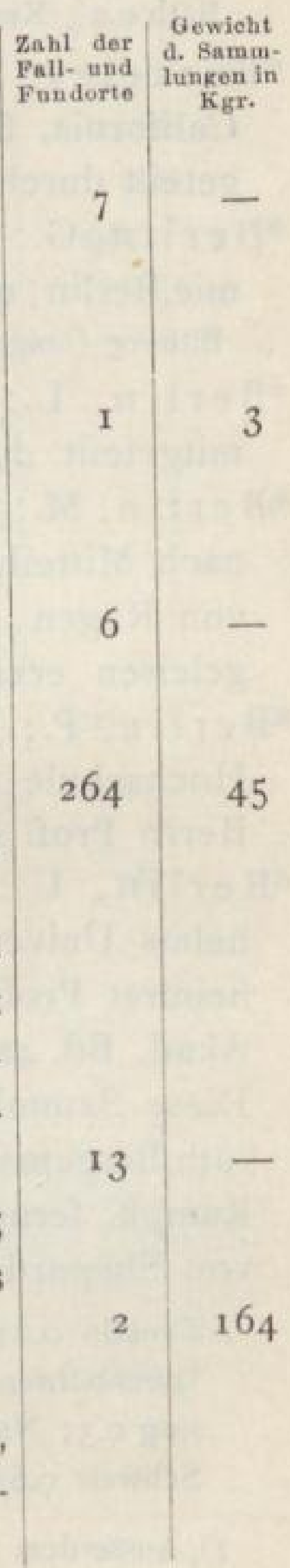


*B a yet: Privatsammlung des Herrn Ad. Bayet, Bruxelles, Nouveau Marche aux Grains 33 ; mitgeteilt durch denBesitzer *B elg rad: Museum; mitgeteilt durch Herrn Prof. Jovan Žujowiě, Vorstand des Museum's in Belgrad Guča 1.0; Jeliza-Gebirge o.8; Sokobanja o.6.

Bellucci: Privatsammlung des Herrn Prof. Joseph Bellucci, Perugia, Italien; mitgeteilt durch den Besitzer

*B ement: Privatsammlung des Herrn Clarence S. Bement, Philadelphia, Pa., 1804 Spring Garden Street, U. S. A.; mitgeteilt durch den Besitzer. S. auch Flugblatt: Second rough list of Meteorites. C.S. B. April 1893 , ferner Third rough list of Meteorites. C. S. B. June 1894

Bath 0.2 ; Kesen 0.3; Pacula o 7 ; Plymouth 1.0; San Pedro Springs 1.o.

*Berkeley: Berkeley bei San Francisco, University of California, Department of Geology and Mineralogy; mitgeteilt durch Herrn Professor Andrew C. Lawson, Ph. D.

*B e rlin, G.: K. Geologische Landesanstalt und Bergakademie,Berlin ; mitgeteilt durch Herrn Geh.Bergrat Hauchecorn Bitburg (umgeschmolzen) 0.7.

*Berlin, L.: K. Landwirtschaftliche Hochschule, Berlin; mitgeteilt durch Herrn Prof. Dr. H. Gruner

*B e r li n, M.: Märkisches Provinzial-Museum, Berlin; besitzt nach Mitteilung von Herrn Direktor E. Friedel ein Eisen von Rügen, welches aber von Cohen kürzlich als Spiegeleisen erkannt wurde; s. Anhang Rügen.

*Berlin, P.: Mineralogisches Institut der K. Technischen Hochschule zu Berlin-Charlottenburg; mitgeteilt durch Herrn Prof. Dr. Hirschwald

*Berlin, U.: Meteoritensammlung der K. Friedrich-Wilhelms Universität Berlin; mitgeteilt durch Herrn Geheimrat Prof. Dr. Carl Klein. S. auch Sitzber. Berlin. Akad. Bd. 4 I (1889), p. $843-864$. Ref. N. J. I89o I, p. 4 I3. Diese Sammlung besitzt die Meteoriten von Chladni, Klaproth,Bergemann, Rammelsberg, Tamnau, Erzherzog StephanRumpff, ferner eine grössere Anzahl aus den Sammlungen von Shepard u. Smith

Alfianello 0.2 ; Grüneberg o.8; Gütersloh o.8; Humboldt-Eisen 0.3; Ibbenbühren 1.0; Iquique 1.0; Klein-Wenden 0.9; Linum 1.0; Mässing 0.3; Nagaya 0.7; Petershurg 0.2; Sarepta 0.3; Schellin 0.7; Schwetz 0.6; Toke-uchi-mura 0.9.

I) Ausserdem $55 \mathrm{Kgr}$. umgeschmolzenes Bitburg.

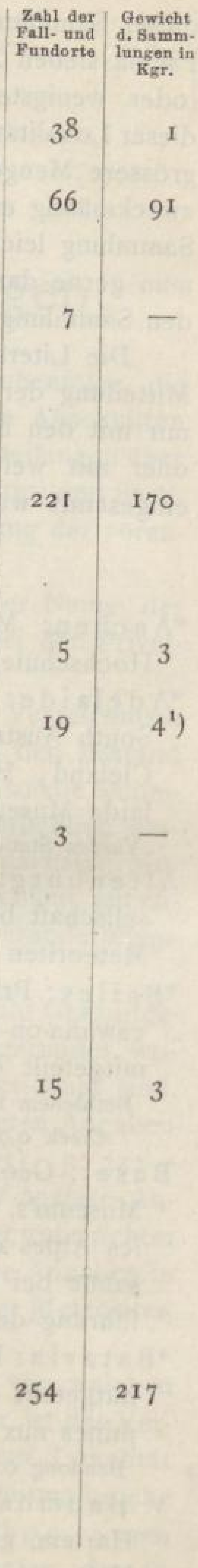


Bern, M.: Naturhistorisches Museum, Bern ; mitgeteilt durch den Direktor, Herrn Prof. Dr. Edm. Freiherrn v. Fellenberg

*Blatz: Privatsammlung des Herrn D. Blatz, MineralienComptoir, Heidelberg; mitgeteilt durch den Besitzer

*Böhm: Privatsammlung des Herrn Jul. Böhm, MineralienComptoir, Wien I, Maysedergasse 3 ; mitgeteilt durch den Besitzer

Bologna: Mineralogisches Kabinet der Universität; mitgeteilt durch Herrn Direktor Prof. Luigi Bombicci. S. auch Flugblatt von 1888 , Météorites du cabinet de minéralogie de la Royale Université Bologne. Imprimerie Fava et Garagnani

Renazzo 0.4; Trenzano 0.5.

B onn: Mineralogisches Institut der Universität; mitgeteilt durch Herrn Professor Dr. Laspeyres. S. auch Verh. naturh. Ver. Bonn Bd. 51 (1894), p. $83-156$ u. Bd. $5^{2}$ (1895), p. $14 \mathrm{I}-220$.

Bordeaux: Faculté des Sciences, Bordeaux; besitzt nach Mitteilung von Herrn Prof. Fallot ein Stück von Aussun Bordeaux: Museum in Bordeaux; besitzt nach Mitteilung von Herrn Prof. Fallot einige Bruchstücke von Meteoriten aus Amerika, Oesterreich und ein sehr kleines Stück von Orgueil

*v. Braun: Privatsammlung Sr. Exzellenz des Herrn Staatsrat Freiherrn von Braun in Wien, Hofstallstrasse; mitgeteilt durch den Besitzer

Braunau: Benediktinerstift, Braunau in Böhmen; im Auftrage Sr. Hochwürden des Herrn Abtes mitgeteilt durch Herrn Gymnasiallehrer V. Maiwald

Braunau 0.6.

v. Bredow: Sammlung von Bredow auf Wagenitz bei Paulinenau, Provinz Brandenburg; mitgeteilt durch Freifrau v. Bredow; s. Anhang Schellin.

*Bremen: Städtisches Museum für Natur-, Völker- und Handelskunde; mitgeteilt durch Herrn Dr. L. Häpke. S. auch Beiträge zur Kenntnis der Meteoriten, - 2. Die Meteoriten des Städtischen Museums zu Bremen. Abh. Naturw. Ver. Bremen Bd. 8 (1884), p. $5^{19}-5^{22}$

Bitburg (nicht umgeschmolzen) to $\mathrm{gr}$.

*Breslau: Mineralogisches Museum der K. Universität;

1) Ausserdem noch Stücke von 22 Lokalitäten, s. p. 45 Anm. 
im Auftrag von Herrn Prof. Dr. Hintze mitgeteilt durch Herrn Privatdozent Dr. L. Milch Gnadenfrei 0.7 .

Brezina: Unter dieser Bezeichnung sind Meteoriten aufgeführt, welche von Herrn Direktor Brezina angekauft wurden, um dieselben allmählich der Sammlung des K. K. Hof-Museum's in Wien einzuverleiben; mitgeteilt durch den Besitzer

Primitiva 0.9; São Julião de Moreira r.o.

*Brunner: Privatsammlung des Herrn J. Brunner, Magdeburg; mitgeteilt durch den Besitzer

*Brüss el: Musée Royal d'Histoire Naturelle de Belgique. Section de Minéralogie et de Lithologie; mitgeteilt durch Herrn Dr. C. Klement, Aide-Naturaliste au Musée d'Histoire Naturelle.

Die Sammlung besitzt folgende Meteoriten, von denen die drei letzten im obigenVerzeichnis derMeteoriten etc. noch eingereiht werden konnten. Alfianello 7 gr. ; Barbotan 12 gr.; Coahuila (Fort Duncan) 99 gr.; Crab Orchard 45 gr.; Eagle Station 46 gr.; Ensisheim I9I gr.; Forest City 22 gr.; Joe Wright Mountain 41 gr.; L'Aigle 95 gr.; Lenarto 218 gr.; Lesves 330 gr.; Mauerkirchen 336 gr.; Misteca 87 gr.; Mócs 97 gr.; Pultusk 792 gr.; Seeläsgen 7 gr.; Stannern 13 gr.; Tourinnes-la-Grosse 72 gr.; Trenton 9 gr.; Waconda 90 gr.

Buchner: Die Privatsammlung von Herrn Prof. Dr. O. Buchner gelangte vor etwa 20 Jahren durch Kauf an den Naturalienhändler Dr. Eger in Wien und von dort grösstenteils in den Besitz Sr. Exzellenz des Herrn Staatsrat Freiherrn von Braun.

*B u d a p e s t: Ungarisches National-Museum; mitgeteilt durch Herrn Dirig. Kustos Prof. J. A. Krenner. S. auch: Die Meteoritensammlung des Ungarischen National-Museums in Budapest. Systematisch zusammengestellt von Andor von Semsey. Hon. Obercustos des Ung. National-Museums. Budapest I886, Druck der Akt.-Ges. Athenaeum «. 15 Seiten. Das National-Museum enthält auch seit 1869 oder r870 die Meteoriten aus der Sammlung des Fürsten Lobkowitz (Mitteilung von Herrn Professor J. C. Hibsch), ferner den grössten Teil der v. Baumhauer'schen Sammlung

Assisi 0.2 ; Capeisen 0.9; Château-Renard 0.2; Gross-Divina 1.0; Indarch 0.3; Kikino 0.3; Lenarto 0.8; Pawlowka 0.2; Prambanan 0.4 ; Staunton 0.1; Tjabé 0.5 ; Utrecht 0.7 ; Zsadany 0.5 .

1) Worunter die Hauptmasse von São Julião im Gewicht von $162 \mathrm{Kgr}$. 
Calcutta: Indian Museum; Ergänzungen zum Verzeichnis von 1880 mitgeteilt durch Herrn T. H. Holland, Curator G. S. J.; s. auch Popular Guide to the Geological Collections in the Indian Museum, Calcutta. - 3. Meteorites, by F. Fedden. Government Central Printing Office, Calcutta, November 1880. 49 Seiten.

Bishunpur 0.8; Bori 0.9; Dandapur 0.5; Gopalpur 0.8; Iharaota 0.5 ; Judesegeri 0.7 ; Kakangarai o.6; Khairpur o.6 ; Khetree o.6; Lodran 0.8; Mainz 0.3; Manbhoom 0.8; Manegaon 0.7; Menow 0.4 ; Mooradabad 0.5; Motecka-nugla 0.7 ; Muddoor 0.8 ; Pirthalla 0.5 ; Pokra 0.7 ; Pulsora 0.7; Quenggouk 0.7; Segowlee 0.6 ; Shalka 0.5 ; Shergotty 0.9 ; Shytal 0.8 ; Sitathali 0.6 ; Umbala 0.5 .

C a mbridge: Mineralogisches Museum der Universität Cambridge,England; mitgeteilt durch Herrn Prof.W.J.Lewis

C a p p e 1: Privatsammlung des Herrn F. Cappel, Hamburg; mitgeteilt durch Herrn Kustos Dr. Gottsche

*Catania: Cabinetto di Mineralogia e Geologia della R. Universitá; mitgeteilt durch Herrn Prof. L. Bucca

$\mathrm{Ch}$ arkow: Herr Prof. A. Gurow hatte die Freundlichkeit mir mitzuteilen, dass im Geologischen Museum der Universität Charkow sich keine Meteoriten befänden, dass dagegen im Mineralogischen Kabinet (Prof. Briot) zwei russische Meteorsteine aufbewahrt würden. Ich vermute, dass hierunter die Steine von Bachmut und Jigalowka gemeint sind; auch soll in Charkow die Hauptmasse von Werchne Tschirskaja sein (Buchner r 863)

Chicago: Das Field Columbian Museum in Chicago erwarb nach 1 . Juli 1893 den grössten Teil der hier unter Kunz und Ward aufgeführten Meteoriten. S. auch Handbook and Catalogue of the Meteorite Collection, by Oliver C. Farrington, Ph. D., Curator, Department of Geology. Field Columbian Museum. Publication 3. Geological Series Bd. I, Nro. 1 , Chicago 1895, 66 Seiten u. 6 Tafeln.

$\mathrm{Clausthal:}$ Mineralogische Sammlung der K. Bergakademie; mitgeteilt durch Herrn Prof. Dr. Klockmann

*Cleveland: Collection of Adelbert College, Cleveland, Ohio, U. S. A., mitgeteilt durch Herrn S. C. H. Bailey ${ }^{*} \mathrm{C}$ linto $\mathrm{n}$ : Mineral Cabinet of Hamilton College, Clinton, N. Y., U. S. A. ; mitgeteilt durch Herrn S. C. H. Bailey 
*Cohen: Privatsammlung des Herrn Prof. Dr. E. Cohen, Greifswald; mitgeteilt durch den Besitzer

*C zernowitz: Mineralogisches Institut der Universität; mitgeteilt durch Herrn Prof. Dr. R. Scharizer

*Danzig: Westpreussisches Provinzial-Museum; mitgeteilt durch Herrn Direktor Professor Dr. H. Conwentz

*D a r m s t a d t, M. : Mineralienkabinet des Grossherzogl. Museums ; mitgeteilt durch Hrn. Geh. BergratProf.Dr.R.Lepsius

D e b r e c z in: Sammlung des Debrecziner Kollegium's ; mitgeteilt durch Herrn Kustos Prof. J. Kovács Kaba o.9.

*D e tmold: Museum; mitgeteilt durch Herrn Direktor Dr. O. Weerth Barntrup 0.6.

*D i j o n: Faculté des Sciences; nitgeteilt durch Herrn Prof. L Collot

*D o r p a t: Meteoritensammlung der Universität; mitgeteilt durch Herrn Prof. F. Loewinson-Lessing. S. auch Grewingh: Ueber ein nickelhaltiges Stück Eisen von Sanarka am Ural und Verzeichnis der Meteoriten-Sammlung der Universität Dorpat im Dezember 1888. Arch. f. d. Naturk. Liv. Esth. u. Kurl. Bd. 9. Ref. N. J. 1884 I, p. 29-30 Buschhof 0.4 ; Mordvinovka 0.3 ; Nerft 0.7 ; Ösel 0.3; Pillistfer o.8.

*Dos s: Herr Privatdozent Dr. Bruno Doss in Riga besitzt kleine Stücke von Misshof

*D res d e n, M.: K. Mineralogisches Museum in Dresden; mitgeteilt durch Herrn Geh. Hofrat Prof. Dr. H. B. Geinitz. S. auch: Die Meteoriten des K. Min. Mus. in Dresden, zusammengestellt auf Veranlassung des Direktors Dr. H. B. Geinitz, von Dr. J. V. Deichmüller. „Isis « 1886 , p. 92 -94. Ref. N. J. I888 I, p. 209

Nenntmannsdorf 1.0.

*D r e s d e n, P.: Mineralog.-geolog. Sammlung der K. Sächs. Technischen Hochschule Dresden; auf Veranlassung von Herrn Geh. Hofrat Prof. Dr. H. B. Geinitz mitgeteilt durch Herrn Dr. Hugo Francke

D u bl in, M.: Museum of Science and Art, Dublin; durch Vermittlung von Herrn Direktor A. Rambaut mitgeteilt durch Herrn Grenville A. J. Cole. S, auch Journ. Roy. Geol. Soc. Ireland Bd. VI (1886), p. ${ }_{15} 8$

Mooresfort 0.4 .

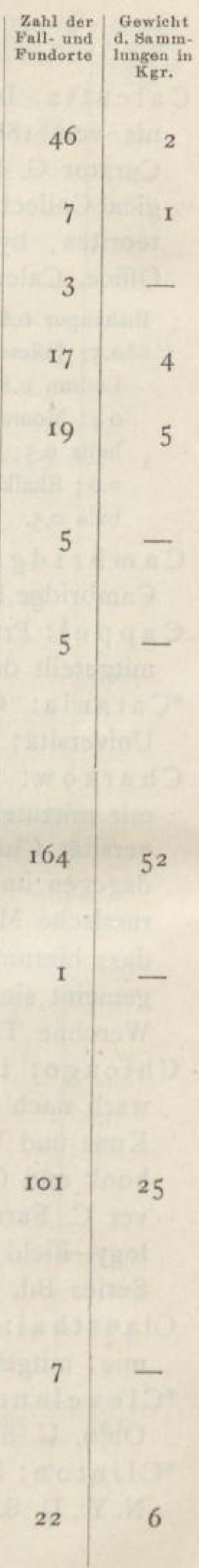


D u b lin, R. C. : Mineral-Collection of the Royal College of Science for Ireland, Dublin; mitgeteilt durch Herrn Grenville A. J. Cole

Edinburg: Natural History Collections in the Museum of Science and Art; im Auftrag von Herrn Prof. J. Geikie mitgeteilt durch Herrn Dr. R. H. Traquair, Keeper of the Nat. Hist. Department Mauritius 0.8 .

Eger: Privatsammlung des Herrn Dr. L. Eger, Naturalienund Lehrmittel-Comptoir, Wien I. Maximilianstr. Nr. II; mitgeteilt durch den Besitzer

Aleppo 0.9; Wirba 0.9.

Elbogen: Rathaus in Elbogen, Böhmen; mitgeteilt durch Herrn Bürgermeister Dörfler

En sis heim: Rathaus in Ensisheim, Elsass; mitgeteilt durch Herrn Bürgermeister Hermann

Ensisheim 0.8 .

*Erlangen: Mineralogisches Institut der Universität; im Auftrag des Herrn Prof. Dr. Oebbeke mitgeteilt durch Herrn Assistent Dr. Oels

*Frankfurt: Meteoritensammlung der SenckenbergischNaturforschenden Gesellschaft; mitgeteilt durch Herrn Dr. W. Schauf

*F r e i b e rg i. S.: K. Sächs. Bergakademie; mitgeteilt durch Herrn Geh. Bergrat Prof. Dr. A. Weisbach Steinbach (Rittersgrün) 0.7.

*Freiberg i. S. N.: Mineralienniederlage der K. Sächs. Bergakademie ; mitgeteilt durch Herrn Faktor H. Zinkeisen

Frenzel: Privatsammlung des Herrn Hüttenchemiker Dr. A. Frenzel, Freiberg i. S.; mitgeteilt durch den Besitzer Gent: Laboratoire de Minéralogie, Université de Gand; im Auftrag von Herrn Prof. A. E. Renard mitgeteilt durch den Herrn Präparator

Gera: Sammlung des Fürstlichen Gymnasium's; mitgeteilt durch Herrn Gymnasialoberlehrer Dr. Löscher Politz 0.3 .

Giessen: Mineralogisches Kabinet der Universität; mitgeteilt durch Herrn Geh. Bergrat Prof. Dr. A. Streng Hungen 0.5 .

*Goldschmidt: Privatsammlung des Herrn Prof. Dr. V. Goldschmidt, Heidelberg; mitgeteilt durch den Besitzer 
G o th a: Naturwissenschaftliche Sammlungen des Herzogl. Museums; mitgeteilt durch Herrn Kustos Dr. W. Pabst Göttingen: Mineralogisches Institut der Universität; mitgeteilt durch Herrn Prof. Dr. Th. Liebisch. S. auch: Klein: Die Meteoritensammlung der Universität Göttingen am 2. Januar 1879. Gött. Gel. Anz. (Nachr.) 1879 , p. $84-100$ und ferner: Ueber eine Vermehrung der Meteoritensammlung der Universität. Gött. Gel. Anz. (Nachr.) I 880, p. $565-569$

Avilez 0.6; Bremervörde 0.4; Erxleben 0.2; Heredia 0.6; Tabarz 0.4. *Graz, J.: Joanneum, Steiermärkisches Landesmuseum, Mineralogische Abteilung; mitgeteilt durch Herrn Kustos Dr. Hatle

Graz, U.: Mineralogisches Institut der K. K. Universität Graz; mitgeteilt durch Herrn Prof. Dr. C. Doelter

Gr e g o r y: Privatsammlung des Herrn J. R. Gregory, London, Nr. I, Kelso Place, Stanford Road, Kensington London W. Die Privatsammlung des Herrn J. R. Gregory hat in den letzten Jahren eine sehr wesentliche Bereicherung erfahren, so dass in dem neuesten Kataloge mit handschriftlichen Nachträgen 380 Lokalitäten nach Gregory's Zählung aufgeführt werden. Leider enthalten die verschiedenen sAddenda \& keine Mitteilung über die Zeit der Erwerbung, daher schien es nicht geboten, alle diese Nachträge aufzunehmen. Meine Auszüge wurden grösstenteils einem Kataloge des Wiener Hofmuseums entnommen, der mit den handschriftlichen Nachträgen um 1892 zum Abschluss gekommen sein dürfte

Eli Eluat 1.0 (?); Gilgoin 1.0; Pipe Creek 0.5; Thunda 0.5. .

*Greifswald: Mineralogisches Institut der Universität; mitgeteilt durch Herrn Prof. Dr. E. Cohen

Greifswald, Ph.: Physikalisches Institut der Universität; mitgeteilt durch Herrn Prof. Dr. E. Cohen.

Diese Sammlung besitzt folgende Meteoriten: Brenham $64 \mathrm{gr}$; Pultusk 86 gr.; Toluca 63 gr., von denen nur noch Toluca in dem obigen Verzeichnis aufgenommen werden konnte.

Grenoble: Mineralogische Sammlung der Faculté des Sciences; mitgeteilt durch Herrn Prof. W. Kilian

Ha arlem: Nach Mitteilung des Sekretärs Prof. Dr. J. Bosscha vom Dez. 1893 besitzt die Hollandsche Maatschappy der Wetenschappen keine Meteoriten. 
*Halle: Mineralogisches Institut der Universität; mitgeteilt durch Herrn Prof. Dr. Lüdecke

Nobleborough 0.8 .

$\mathrm{H}$ a m b u r g: Naturhistorisches Museum, Abteilung für Mineralogie; mitgeteilt durch Herrn Kustos Dr. C. Gottsche. Enthält die Fischer'sche und Zimmermann'sche Sammlung; s. Buchner $1863, \mathrm{p}$ X, XI.

Häp ke: Herr Dr. L. Häpke in Bremen besitzt ein Stück von Rancho de la Pila

H a r vard, U. : Harvard University Museum, Cambridge, Mass., U. S. A. Ausser der grossen Sammlung von Smith, über welche Huntington ein Verzeichnis in Proc. Amer. Acad. Arts and Sc., Boston Bd. 23 (1887/88), p. 37-iro veröffentlicht hat (Catalogue of all recorded Meteorites, with a description of the specimens in the Harvard College Collection, including the cabinet of the late J. Lawrence Smith), erwarb die Harvard-Universität noch ${ }_{5} 5$ Steine und 12 Eisen, über welche Herr Prof. J. E. Wolff mir Mitteilung machte

Butler 0.5; Casey County 0.4; Charlotte 0.7; Coopertown 0.3; Cranberry Plains 0.4; Cynthiana 0.8; Danville 0.5; Forest City 0.3 (Huntington); Frankfort (Eisen), 0.9; Harrison County 0.3 ; Kokomo 0.6 ; Lumpkin 0.5; Marshall County o.1; Mejillones o.6 ; New Concord 0.3; Oktibbeha 0.3; Putnam County 0.6; Rochester 0.4 ; Russel Gulch 0.4 ; Scriba 0.3 ; Smith's Mountain 0.3 ; Vernon County 0.5 ; Warrenton 0.3 .

*Heidelberg: Mineralogisches Institut der Universität; zusammengestellt durch den Verfasser

Darmstadt 0.6 .

*Helsingfors: Mineralogisches Institut der Universität; mitgeteilt durch Herrn Prof. Dr. F. J. Wiik Luotolaks 0.7.

Helsing for s: Die geologische Landesanstalt von Finnland besitzt nach Herrn Direktor Dr. J. J. Sederholm's Mitteilung keine Meteoriten.

Herzogenbusch: Provinciaal Genootschap van Kunsten en Wetenschappen in Noord-Brabant; mitgeteilt durch den Sekretär, Freiherrn Dr. Franz von Ryckevorsel Uden 1.o.

*H o h e nh e i m: Mineralien-Kabinet der K. Landwirtschaft- 
lichen Akademie ; mitgeteilt durch $\uparrow$ Herrn Prof. Dr. F. Nies *How e 11: Privatsammlung des Herrn Edwin E. Howell, National Science Establishment, Washington, D. C., I $7^{\text {th }}$ street 612, N. W.; mitgeteilt durch den Besitzer

Beaver Creek 0.9; Cañon Diablo 0.4 ; Cross Roads 1.o; El Capitan Range 1.0; Inca 0.4 .

*K a r ls ruhe, M.: Grossherzogl. Naturalien-Kabinet, Karlsruhe; mitgeteilt durch $\dagger$ Herrn Geh. Rat Prof. Dr. A. Knop

*Karlsruhe, P.: Mineralogische Sammlung der Technischen Hochschule, Karlsruhe; mitgeteilt durch den Vorigen

*K a s a n: Geologisches Kabinet der Universität; mitgeteilt durch Herrn Prof. Stuckenberg

Ochansk o.S.

K i e w : Mineralogisches Kabinet der Wladimir-Universität; durch gütige Vermittlung des Herrn Prof. Dr. Eichelmann mitgeteilt von Herrn Prof. Armaschewski

Bialystock 0.2; Bjelaja Zerkow 0.8; Brahin 0.9; Dolgowoli 0.7; Lixna 0.5 ; Zaborzika 0.6 .

K 1 a u s en burg: Siebenbürgisches Landes-Museum; mit geteilt durch Herrn Prof. Dr. A. Koch. S. auch Verzeichnis der Meteoritensammlung des Siebenbürgischen Landes-Museum's in Klausenburg von Prof. Dr. A. Koch. Klausenburg 1885 (ungarisch) Mócs 0.3.

*K ö ni g s b e r g, M. : Provinzial-Museum; mitgeteilt durch Herrn Prof. Dr. Jentzsch

K op en ha ge n: Mineralogisches Museum der Universität; mitgeteilt im Auftrag des Herrn Prof. Dr. N. V. Ussing durch Herrn cand. Aagaard Mern I.O.

*K ra ntz: Privatsammlung des Herrn Dr. F. Krantz, Rheinisches Mineralien-Contor, Bonn; mitgeteilt durch den Besitzer

Laborel 0.5; Pultusk 0.2.

*K ristiania: Mineralogisches Institut der K. FriedrichsUniversität; mitgeteilt durch Herrn Prof. W. C. Brögger Morradal r.o; Ski o.8; Tysnes o.9.

$\mathrm{K} r$ ü g e r: Privatsammlung des Herrn Stadtrat E. Krüger, Mitau, Kurland; mitgeteilt durch den Besitzer

K unz: Privatsammlung des Herrn George F.Kunz, New-York, 
I I-15 Union Square; mitgeteilt durch den Besitzer (jetzt grösstenteils in Chicago)

Brenham 0.7; Colfax 0.8; Fisher 1.0; Floyd Mountain 1.0; Forsyth

County 1.0 ; Henry County 0.5; La Charca 1.0 (?); Long Island I.o.

L a t $\mathrm{e} \mathrm{ux}$ : Ein Verzeichnis der Privatsammlung von Herrn Latteux von 1885 , welches die Bibliothek des Wiener HofMuseum's besitzt, führt 79 Lokalitäten im Gesamtgewicht von 2 Kgr. auf. Diese Sammlung wurde nicht in den obigen Index eingereiht, da dieselbe inzwischen vielleicht einer grösseren einverleibt worden ist und überdies meist sehr kleine Stücke ( 19 unter 1 gr.) enthält.

L a u s a n ne: Musée Géologique de l'Université; mitgeteilt durch Herrn Prof. E Renevier

*Leiden: 's Rijks Geologisch-Mineralogisch Museum; mitgeteilt durch Herrn Prof. K. Martin

Drake Creek 0.4; Nyawi o.9.

*Leipzig: Mineralogisches Museum der Universität; im Auftrag des Herrn Geh. Bergrat Prof. Dr. Zirkel mitgeteilt durch Herrn Prof. Dr. Lenk

*L e o be n: K. K. Bergakademie, Lehrkanzel für Mineralogie und Geologie; mitgeteilt durch Herrn Prof. H. Hoefer

*v. L e u c h t e n b e r $g$ : Sammlung des verstorbenen Herzogs Nicolai Maximilianowitsch von Leuchtenberg, aufbewahrt im Berginstitut zu St. Petersburg; auf Veranlassung von Herrn Prof. Karpinskij mitgeteilt durch Herrn Bergingenieur Michael Melnikoff

L is s a bo n: Museu Nacional de Lisboa, Secção Mineralogica; mitgeteilt durch Herrn J. P. Gomes

v. Lobkowitz: Die ehemalige Sammlung des Fürsten v. Lobkowitz befindet sich nach Mitteilung von Herrn Prof. Hibsch grösstenteils im National-Museum in Budapest.

*L o n d o n, B. M.: British Museum (Natural History), Mineral Department, London, S. W., Cromwell Road; mitgeteilt durch Herrn Direktor L. Fletcher. S. auch: An introduction to the study of Meteorites, with a list of the Meteorites represented in the Collection. 1893. Printed by order of the trustees. Die neueste Liste erschien November 1896 und verzeichnet 476 Lokalitäten

Akburpoor 0.9; Aldsworth 0.9; Assam 0.4; Aubres 0.9; Barranca

Blanca 1.0; Bherai 1.0; Bustee 0.9; Butsura 0.9; Cambria 0.4;

1) S. auch Anhang: Unbekannte Fundorte von Steinen. 
Campo del Cielo 1.o; Chail r.o; Chandpur 0.5; Charsonville 0.3; Charwallas 0.5 ; Chesterville 0.3 ; Coahuila o.I; Cold Bokkeveld 0.3; Cosby's Creek 0.5; Cowra 0.9; Cranbourne I.0; Cronstadt 0.9; Dakota 0.3; Daniels Kuil 0.5; Dhulia 0.5; Dhurmsala 0.4; Dundrum 0.7; Durala 1.0; Dyalpur o 9; Estherville 0.4 ; Futtehpore 0.4 ; Goalpora 0.5 ; Great Fish River 0.9 ; Greenbrier County 1.0; Gurram Konda 1.0; High Possil 0.6; Imilac o.8; Jackson County 0.4 ; Jamestown 0.4 ; Jamkheir 0.8 ; Jhung 0.6 ; Joël Iron 0.9; Kadonah 0.4; Kaee ı.o; Kheragur 0.6; Killeter 0.7; Kusiali 1.0; Lion River 0.2 ; Little Piney 0.2; Lutschaunig 0.7 ; Makariwa 1.0; Mantos Blancos 0.9; Mhow 0.5; Middlesbrough $0.9(2)$; Mornans 0.9 ; Murfreesboro 0.4 ; Nageria 0.9 ; Nammianthal 0.4; Nedagolla 0.9; Nejed r.o; Newstead 0.7; Obernkirchen 0.9; Oczeretna 0.9; Ogi 1.0; Parnallee o.8; Perth I.0; Pirgunje 1.0; Ploschkowitz 0.7; Quinçay 0.3; Rancho de la Pila 0.5; Rowton 1.0; San Francisco del Mezquital o 9; Smithland 0.5 ; Supuhee 0.9; Tarapaca 0.3; Udipi 0.9; Varas 1.0; Werchne Udinsk 0.3; Wold Cottage 0.9; Yatoor 0.9.

*London, P. G.: Museum of Practical Geology, Jermyn Street, London; mitgeteilt durch Herrn Kurator I. W. Rudler

Black Mountain 0.2; Lucky Hill 0.9; Madoc 0.2; Victoria West 0.2.

*L u n d, G.: Geologisches Museum der Universität Lund; mitgeteilt durch Herrn Prof. B. Lundgren

*L u n d, M. : Mineralogisches Museum der Universität Lund; mitgeteilt durch den Vorigen

L ï t t i c h: Mineralogische Sammlung der Universität; mitgeteilt durch Herrn Prof. G. Dewalque

de Luynes: Die Sammlung des Duc de Luynes soll sich nach Mitteilung vou Herrn Marquis de Mauroy auf dem Schloss Donpierre (Seine et Oise) befinden und 107 Stücke von 107 Fällen enthalten

* M a d i s o n: University of Wisconsin, Department of Geology and Mineralogy; mitgeteilt durch Herrn Wm. H. Hobbs Trenton 0.4 .

M a dras: Government Central-Museum; mitgeteilt durch Herrn H. Warth, D. Sc.

Madrid: Museo de Ciencias Naturales. Gabinete de Historia Natural. Nach dem Katalog, welchen Gredilla y $\mathrm{G}$ a una in seinen Estudio sobre los Meteoritos $189^{2}$ bei Escuela Tipográfica del Hispicio, Madrid, veröffent. licht hat 
Barea 1.0; Cabezzo de Mayo o.4; Canellas o.6; Cuba 1.0; Molina I.0; Monroe 0.4; Nulles 1.0; Oviedo 0.5; Sena 0.9; Sevilla I.o.

${ }^{*} \mathrm{M}$ a n c he st e r: Manchester Museum, Owens College; mitgeteilt durch Herrn Dozent Bernhard Hobson

* Ma n nheim: Grossherzogliches Naturhistorisches Museum; mitgeteilt durch Herrn Kustos G. Arnold

*M a r b u r g, Mineralogisches Institut der Universität; mitgeteilt durch Herrn Prof. Dr. R. Brauns

*de Mauroy: Privatsammlung des Herrn Marquis de Mauroy, Ingr. civil des mines, Wassy, Hte-Marne, Frankreich; mitgeteilt durch den Besitzer

Me li: Privatsammlung des Herrn Romolo Meli, Professor an der Scuola d'applicazione per gl'ingegneri, Rom; mitgeteilt durch den Besitzer

*Melion: Privatsammlung des Herrn Dr. Jos. Melion, Brünn, Kröna 52 ; mitgeteilt durch den Besitzer; s. auch: Die Meteoriten des Dr. Jos. Melion Brünn I889, im Selbstverlag d. Verf.

Minneapolis: S. Catalogue of the Meteorites in the University Collection, with references to literature describing them. I9th Annual Rep. of the Geological and Natural History Survey of Minnesota. Herausgegeben von N. H. Winchell, State Geologist, Minneapolis, I892, p. I $70-192$

Moden a: Mineralogisches und Geologisches Institut der Universität ; mitgeteilt durch Herrn Prof. Dante Pantanelli Albareto o.6.

M o e ri cke: Privatsammlung des Herrn Dr.W. Moericke, Stuttgart; mitgeteilt durch den Besitzer Ternera I.o.

*M o skau: Mineralogisches Kabinet der landwirtschaftlichen Akademie zu Petrowskoje-Rasumowskoje bei Moskau, Russland; mitgeteilt durch Herrn Assistent A. Kupffer

Rakowka 0.3 .

$\mathrm{M}$ ü $\mathrm{n}$ c h e n: Meteoritensammlung des K. Bair. Staates; mitgeteilt durch Herrn Privatdozent Dr. Weinschenk. S. auch das Verzeichnis, welches L. Frischmann im Auftrag von F. v. Kobell am r. März r 868 herausgegeben hat. $8^{\circ}{ }_{4}$ Seiten. Akad. Buchdruckerei F. Straub, München

Duruma 0.9; Eichstädt 0.5; Mauerkirchen 0.6; Schönenberg 1.o. 
*M ü nster: Mineralogisches Museum der K. Akademie; mitgeteilt durch Herrn Prof. Dr. O. Mügge

*N e a p e 1: Museo Mineralogico della R. Università di Napoli; mitgeteilt durch Herrn Prof. Eug. Scacchi

*N e umann: Privatsammlung des Herrn W. Max Neumann, K. K. Major a. D., Graz, Heinrichstrasse 65 ; mitgeteilt durch den Besitzer. S. auch: Verzeichnis der Meteoriten der Neumann'schen Sammlung, nach dem von J. G. Neumann verfassten Manuskript. Gedr. k. k. Universitäts-Druckerei Graz, 1886

*N e w - H a ven: Peabody Museum of Yale College, New Haven, Conn., U. S. A.; mitgeteilt durch Herrn Prof. E. S. Dana. S. auch Katalog in Am. Journ. (3) 32 (1886), Appendix. Ref. N. J. 1887 II, p. 44

Cape Girardeau 0.9; Castine 0.7 ; Cross Timbers 1.0; Forsyth 0.2 ; Frankfort (Stein) 0.5; Guilford County 0.3; Hammond 0.9; Homestead 0.3 ; Honolulu 0.2 ; Jewell Hill 0.5 ; Nanjemoy 0.3 ; Pittsburg 0.3; Richmond 0.3 ; Salt Lake City 0.9; Salt River 0.4 ; Sanct Augustine's Bay 0.3; Shingle Springs 0.6 ; Weston 0.8.

*N e w t o n: Privatsammlung des Herrn Prof. H. A. Newton, New Haven, Conn., U. S. A.; mitgeteilt durch den Besitzer

N ew-Y ork, M.: American Museum of Natural History, Geological Department, New York, U. S. A.; mitgeteilt durch Herrn E. O. Hovey. S. auch: Bulletin of the American Museum of Natural History Bd. VIII, p. 149-155, New York, Juli 3, r 896

N e w - Y ork: School of Mines Columbia College, Mineralogical Department, besitzt nach Herr Lea Mc. J. Luquer Io kleine und unbedeutende Stücke von Meteoriten

Odessa: Meteoriten-Sammlung der Neurussischen Universität; mitgeteilt durch Herrn Prof. R. Prendel

Grossliebenthal 0.9; Savtschénskoje 1.0; Vavilovka 0.7; Zabrodje I.o.

*O 1 d e n b u r g : Grossherzogl. Museum. Vergl.C.F.Wiepken : Notizen über die Meteoriten des Grossherzoglichen Museum's. Abh. naturw. Ver. Bremen Bd. 8 (1884), p. $5^{24}$ $-531$

O x f o r d: Magdalen College; mitgeteilt durch Herrn Prof. H. A. Miers

Chandakapoor 0.6 ; Limerick 0.8 . 
P a r is, E. : École des Mines, Paris ; mitgeteilt durch Herrn Präparator Ad. Richard

Bueste o 5 .

*Paris, M.: Muséum d'Histoire Naturelle au Jardin des Plantes; mitgeteilt durch Herrn Prof. Dr. Stanislas Meunier. S. auch: Guide dans la Collection de Météorites du Muséum d'Histoire Naturelle. Paris, bei G. Masson 1889 Adalia 1.0; Agen 0.9; Alais 0.6; Angers 0.5; Aumières 0.9; Aussun 0.2 ; Baratta 0.6 ; Berlanguillas 0.7 ; Bocas 0.6 ; Cachiyuyal 0.8 ; Charcas 1.0; Chassigny 0.5; Copiapo 0.6 ; Cosina 0.5; Deal 0.5; Deliesa o.8; Dellys 0.8; Epinal o.8; Favars 0.8; Feid Chair I.0; Galapian 0.8; Grazac 1.0; Haniet-el-Beguel 1.0; Hassi Jekna 1.0; Juncal 1.0; Juvinas 0.8; Kerilis 0.9; Kernouvé 0.5; La Bécasse 0.9; La Caille 1.0; L’Aigle 0.2; La Vivionnère 0.6; Le Pressoir 0.3; Les Ormes 0.8; Lonaconing 0.7; Mascombes 0.9: Mexico 0.7; Montlivault 0.9; Orgueil 0.8; Ornans 0.7; Phû Long 1.0; Pnompehn 1.0; Roda 0.7; Saint-Caprais 0.8; Saint-Mesmin 0.5; Salles 0.6; Santa Catharina 0.4 ; Sauguis 0.7; Saurette o.6; Senhadja I.0; Sewrukof 0.4; Tadjera 1.0; Toulouse 05 ; Tourinnesla-Grosse 0.4; Vaca Muerta 0.4; Vago 1.0; Vouillé 0.9; Yodzé 0.9.

Paris, U.: Laboratoire de Minéralogie, Faculté des Sciences à la Sorbonne; im Auftrage des Herrn Prof. Hautefeuille mitgeteilt durch Herrn Léopold Michel, répétiteur à la Faculté des Sciences de Paris

P a r ma: Università di Parma. Gabinetto di Geologia e Mineralogia; mitgeteilt durch Herrn Prof. V. Simonelli Borgo San Donino o.3.

P a u: Musée de Pau, Dep. Basses-Pyrénées, Frankreich; mitgeteilt durch Herrn Bibliothekar L. Soulice

Pavia: Mineralogisches Kabinet der Universität Pavia; im Auftrag des Herrn Prof. Sansoni mitgeteilt durch Herrn Privatdozent Dr. W. Salomon

P e c h: Privatsammlung des Herrn C. F. Pech, Mineralienhändler in Berlin N. W., Luisenstr. 19 ; mitgeteilt durch den Besitzer

Petersburg: Das Comité Géologique in Petersburg besitzt nach Mitteilung von Herrn Prof. A. Karpinsky keine Meteoriten.

*P e te rs burg, A.: Mineralogisches Museum der Kais. Akademie der Wissenschaften, Petersburg; im Auftrag Sr. Exzellenz des Herrn Direktor F. v. Schmidt mitgeteilt durch E. Freiherrn v. Toll 
Botschetschki 1.0; Grossnaja 0.6; Jigalowka o.6; Karakol ı o; Krasnojarsk 0.9; Krasnoj-Ugol I.o; Kuleschowka o.8 ; Simbirsk(Partsch) 1.0; Slobodka 0.9; Ssyromolotow 1.0; Stawropol o.8; Timoschin 1.0.

Petersburg, B.: Mineralogisches Museum des Berginstitut's (Gornyi Institut); mitgeteilt durch Herrn Bergingenieur Michael Melnikoff

Augustinowka 1.0; Bischtübe 0.9; Borodino 1.0; Nowo-Urei 0.8 ; Petropawlowsk 1.o.

Pierrotet: Privatsammlung des Herrn Paul Pierrotet, Comptoir Géologique, Paris, 53 rue Monsieur-le-Prince; mitgeteilt durch den Besitzer

Pl a g e m a n n: Privatsammlung des Herrn Dr. Plagemann in Hamburg; mitgeteilt durch Herrn Dr. Gottsche

Poh 1: Privatsammlung des Herrn Professor Dr. J. J. Pohl in Wien; mitgeteilt durch den Besitzer. S. auch: Nachtrag zum Kataloge der Meteoriten-Sammlung des Dr. J. J. Pohl in Wien, Ende 1878 ; ferner: Die neuen Erwerbungen für die Meteoriten-Sammlung des Dr. J. J. Pohl in Wien, Beginn 1879 bis Ende 1881. Selbstverlag des Verf. Druck bei F. Jasper, Wien.

Milena 0.5 .

Pr a g, B. U.: Geologische und Mineralogische Sammlungen der Böhmischen Universität, Prag; mitgeteilt durch Herrn Prof. Dr. K. Vrba

*P r a g, D. U. : Mineralogisches Institut der Deutschen Universität, Prag; mitgeteilt durch Herrn Prof. Dr. F. Becke

Prag, M.: Museum des Königreichs Böhmen, Prag; mitgeteilt durch Herrn Prof. Dr. K. Vrba Bohumilitz 0.8 .

Reva l, P. M. : Provinzial-Museum, Reval; mitgeteilt durch Rudolf Freiherrn von Schilling

*Rig a: Museum des Naturforschervereins, Riga; mitgeteilt durch Herrn Privatdozent Dr. Bruno Doss. S. auch: Korrespondenzblatt d. Naturf. Ver. Riga Bd, 35 (1892), p. $17-18$

Misshof 0.5 ; Werchne Dnieprowsk 0.5 .

Rig a, Ges.: Gesellschaft für Geschichte und Altertumskunde in Riga; mitgeteilt durch den Vorigen

Riga, P.: Mineralienkabinet des Polytechnikum's, Riga ; mitgeteilt durch den Vorigen

R i o: Museo Nacional, Rio de Janeiro; mitgeteilt durch 
Herrn Prof. O. A. Derby. S. auch: Notas sobre Meteoritos Brasileiros von Orville A. Derby in Revista do Observatorio. Rio de Janeiro $\mathbf{1} 888$.

Angra dos Reis 0.9; Bemdegó 1.0; Itapicuru-mirim 1.0; Macao 0.7; Minas Geraës 1.o; Santa Barbara I.o.

R o e b Jing: Privatsammlung des Herrn W. A. Roebling, Trenton, N. J., U. S. A.; mitgeteilt durch den Besitzer R o m, T.: Scuola d'applicazione per gl'ingegneri, Rom; mitgeteilt durch Herrn Prof. Romolo Meli

Ausser Colescipoli (13 gr.) besitzt die Sammlung noch Stücke von Cañon Diablo, Forest City, Pultusk und Toluca,

Ro m, S. Ap.: Naturhistorisches Museum des Collegium S. Apollinare in Rom; mitgeteilt durch Herrn Prof. Guiseppe Tuccimei

*R o m, U.: Mineralogisches Kabinet der Universität Rom; mitgeteilt durch Herrn Prof. G. Strüver

Girgenti 0.3 ; Monte Milone o.8; Orvinio 0.3.

* Schemnit $z$ : K. ungarische Berg- und Forstakademie; mitgeteilt durch Herrn Direktor Scholtz

*v. S c hilli n g: Privatsammlung des Freiherrn Rudolf von Schilling, Reval, Kentmannstr. 98 ; mitgeteilt durch den Besitzer

Tennasilm 0.5 .

Schulze: Die Privatsammlung des Herrn Dr. H. Schulze, Santiago, Südamerika, gelangte 1895 grösstenteils an das Wiener Hof-Museum.

Seligmann: Privatsammlung des Herrn Dr. Seligmann in Koblenz, Schlossrondel 18; mitgeteilt durch den Besitzer

Sevilla: Sammlung der Universität, Sevilla; mitgeteilt durch Herrn Prof. Dr. S. Calderón

Cangas de Onis 0.5 .

Sidney s. Sydney.

v. Si e mas ch ko: Privatsammlung des $\dagger$ Herrn Staatsrat Julien de Siemaschko, Exzellenz in St. Petersburg, Wass. Ostr., I3 Ligne, 2. S. Catalogue de la Collection de Météorites de Julien de Siemaschko. Petersburg 1891 Jamyschewa 0.5 ; Mighëi 0.5 .

1) Ausser diesen in dem Katalog von 1891 verzeichneten 347 Lokalitäten wurden im Verzeichnis der Meteoriten etc, noch einige weitere Fälle aufgenommen, welche sich als handschriftliche Nachträge in einem im Besitz des Herrn Direktor Dr. Brezina befindlichen Exemplar befanden. 
Siena: Accademia dei Fisiocritici di Siena; mitgeteilt durch Herrn Prof. Pietro Gucci

Siena 0.2.

*S p e y e r: Städtisches Museum; mitgeteilt durch den Herrn Konservator der naturhistorischen Abteilung Krähenberg r.o.

*Sto ckholm: Riksmusei Mineralogiska Afdelning; mitgeteilt durch Herrn Prof. G. Lindström. S. auch: Öfversigt af Kongl. Vetenskaps-Akademiens Förhandlingar I 884, Nr. 9, p. 209-222. Ref. N. J. 1886 II, p. 38-39

Hessle 0.3 ; Lundsgird 0.9 ; Ställdalen 0.7 ; Veramin 0.6 .

*Strass burg: Mineralogisches und petrographisches Institut der Universität; mitgeteilt durch Herrn Prof. Dr. H. Bücking (die Geologische Sammlung besitzt nach Mitteilung von Herrn Prof. Dr. Benecke keine Meteoriten)

Strüver: Privatsammlung des Herrn Prof. G. Strüver, Rom; mitgeteilt durch den Besitzer

*S t u e r: Privatsammlung des Herrn Alexander Stuer, Comptoir Géologique et Minéralogique, Paris, 40 Rue des Mathurins; mitgeteilt durch den Besitzer

*S t ü r t z: Privatsammlung und Meteoritenvorrat (am 1 . Juli 1893) des Herrn B. Stürtz, Mineralogisches und Palaeontologisches Comptoir, Bonn, Riesstrasse 2; mitgeteilt durch den Besitzer

*S t u t t g a r t: K. Naturalienkabinet, Stuttgart; mitgeteilt durch Herrn Prof. Dr. E. Fraas

Stuttgart, P.: Technische Hochschule, Stuttgart; besitzt nach Mitteilung des Herrn Prof. Dr. v. Eck ebenfalls einige Meteoriten.

Stuttgart, R.: K. Real-Gymnasium, Stuttgart; mitgeteilt durch Herrn Prof. Dr. Konrad Miller

Die Sammlung besitzt folgende Meteoriten: Alfianello $14 \mathrm{gr}$; Cold Bokkeveld 14 gr.; Stannern Spl.; Toluca 66 gr.

*Sydney: Australian Museum, Sydney; mitgeteilt durch Herrn Kurator Robert Etheridge

*S z a mosujvar: Armenisch-Katholisches Gymnasium; mitgeteilt durch Herrn Dr. L. Martonfi

*T h a r a n d: Mineralogisches Institut der K. Forstakademie; mitgeteilt durch Herrn Prof. Dr. H. Vater

T if lis: Musée d'Histoire Naturelle Tiflis, Kaukasien ; besitzt nach Mitteilung von Herrn Dr. Radde keine Meteoriten.

*Troyes: Musée de Troyes, Aube, Frankreich; mitgeteilt 

durch Herrn Marquis de Mauroy, Ingr. civil des Mines,
Wassy, Hte-Marne

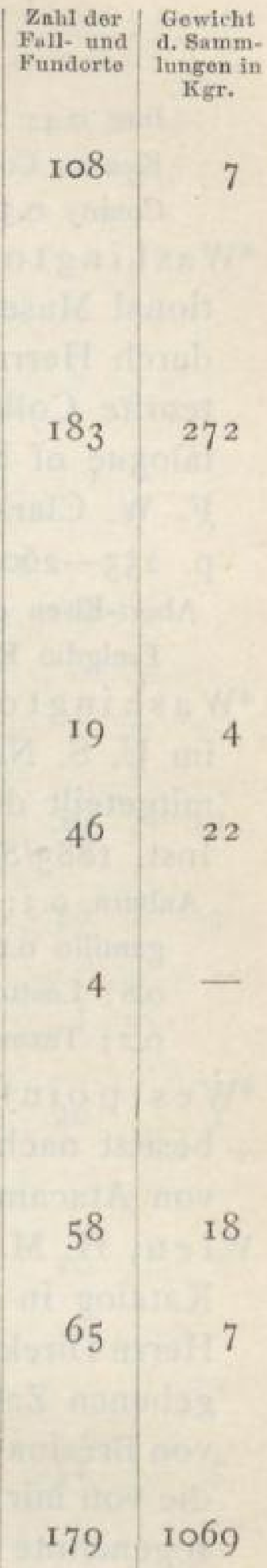

Turi n, J.: R. Scuola d'applicazione per gl'ingegneri, Turin; mitgeteilt durch Herrn Direktor A. Cossa

*Tu rin, U.: Museum der Universität, Turin; mitgeteilt durch Herrn Assistent Mylius

Alessandria 0.3 ; Cereseto 0.8 ; Motta di Conti o.8.

Ulex: Privatsammlung des Herrn Dr. Ulex in Hamburg; mitgeteilt durch Herrn Dr. Gottsche

*Upsala: Sammlung der Universität; mitgeteilt durch Herrn Prof. Dr. H. Sjögren. S. auch: Verzeichnis von G. Holm in Ofversigt af Kongl. Vetenskaps-Akademiens Förhandlingar 1885, Nr. 2, p. 23-28. Ref. N. J. 1886 II, p. 212

*Utrecht: Mineralogisch-Geologisches Institut der Universität; mitgeteilt durch Herrn Prof. Dr. Wichmann

*W a rd: Privatsammlung des Herrn Henry A. Ward, Natural Science Establishment, Rochester, N. Y., U. S. A.; gedruckter Katalog von Juni 1893 , übersandt vom Besitzer (jetzt grösstenteils in Chicago)

Bear Creek 0.2; Carlton-Hamilton 0.6; Crab Orchard 0.5; Doña

lung der Universität. Ein Verzeichnis der Sammlung wurde von Quenstedt im August $187 \mathrm{I}$ als Flugblatt herausgegeben. Kurze Erwähnung N. J. 1871, p. 940; Verh. geol. ReichsBarbotan 0.2; Blansko 0.4; Borkut 0.8; Carthago 0.5; Doroninsk

0.4; Hainholz o.6; Lime Creek 0.6; Lissa 0.3; Magura 0.3; Orange River (Eisen) 0.2 ; Rasgata 0.6 ; Seeläsgen 0.2 ; Slobodka (Partsch) 0.8 ; Smithville 0.6 ; Zacatecas 0.2 .

1) Ueler die Tübinger Sammlung ist kürzlich ein Verzeichnis herausgegeben worden (Ann. Hof-Mus. Bd, 10, p. 328 - 337). Dieses Verzeichnis wurde ohne Zustimmung des früheren und des jetzigen Vorstands der Sammlung publiziert und enthält ausserordentlich zahlreiche Ungenauigkeiten und Fehler. In einem Nachtrag auf p. 370 sagt der Verfasser, dass ich ihn sauf mehrfache Unrichtigkeiten, hauptsächlich in den Gewichtsangaben «, aufmerksam gemacht hätte. Es ist dies insofern nicht ganz richtig, als ich dem Verfasser vielmehr schrieb, >diese Ungenauigkeiten beschrïnken sich keineswegs auf die Art der Wiedergabe des Inhaltes der alten und neuen Etiquetten «. Bei Herausgabe des Verzeichnisses der Tübinger Sammlung werde ich auf diese Angelegenheit näher eingehen müssen; hier möge nur darauf hingewiesen werden, dass jenes Verzeichnis mit grösster Vorsicht $z u$ benutzen ist. 
428 Verzeichnis der Sammlungen. Washington - Wien, H. M.

Inez 0.4 ; Farmington 0.6; Fort Pierre 0.3; Grand Rapids 0.5 ; Kenton County 0.2 ; Merceditas 0.3 ; Puquios 0.3; Saint François County 0.3 ; Toluca o.I.

*Washington: Department of Mineralogy im U. S. National Museum in Washington, D. C., U. S. A.: mitgeteilt durch Herrn Kurator F. W. Clarke. S, auch: The Meteorite Collection in the U. S. National Museum. A Catalogue of Meteorites represented November I, 1886. By F. W. Clarke. Rep. Smithsonian Inst. $1885 / 86$, Part II, p. $255-260$. Ref. N. J. I 89 I I, p. 49

Abert-Eisen 0.6; Chihuahua 1.0; Ivanpah 0.2; Morristown 1.0; San

Emigdio Range 0.7; Travis County 1.0; Tucson 1.0.

*W a sh ing t on, Sh.: Frühere Shepard'sche Sammlung, jetzt im U. S. National Museum, Washington D. C., U. S. A.; mitgeteilt durch den Vorigen. S. auch: Rep. Smithsonian Inst. $1885 / 86$, Part II, p. $261-265$

Auburn 0.3; Bishopville 0.3 ; Burlington 0.3 ; Dalton 0.9; Gargantillo 0.7 ; Hartford 0.2; La Grange 0.3; Lexington County 0.8 ; Losttown 0.3; Ranchito 0.6; Ruffs Mountain 0.5; Searsmont 0.2 ; Tazewell 0.2 ; Union County 0.2 ; Victoria 0.3 .

*W e s t p o in $\mathrm{t}$ : Military Academy of Westpoint, N.Y., U.S.A.; besitzt nach Mitteilung von Herrn S. C. H. Bailey Stücke von Atacama und Homestead

Wi en, H. M.: K. K. Naturhistorisches Hof-Museum, Wien; Katalog in Ann. Hof-Mus. Bd. 10 (1895), p. 298-307 von Herrn Direktor Dr. A. Brezina. Die unter Wien, H. M. angegebenen Zahlen stimmen nicht immer mit denjenigen des von Brezina herausgegebenen Verzeichnisses überein, weil die von mir in diesem Index aufgeführten Zahlen noch eine sogenannte Format-Sammlung umfassen. Diese FormatSammlung befand sich bereits 1893 im Hof-Museum, wurde aber erst in allerjüngster Zeit erworben. Ueber die Bedeutung $\left.\operatorname{der}^{*}\right){ }^{* *}$ ) s. bei dem Verzeichnis der Abkürzungen unter Tschermak 1872 u. 1877

Asco 0.4; Babb's Mill 1.0; Bachmut 0.5; Baird's Farm 0.3; Bella

Roca 0.5 ; Benares 0.3; Bingera r.o; Bjelokrynitschie 0.4 ; Bluff 0.2 ; Bridgewater 0.9; Cabin Creek I.0; Calderilla 1.0; Castalia 0.9 ; Chantonnay 0.3 ; Chulafinnee 0.8 ; Cleveland 0.4 ; Collescipoli 0.2; Costilla 1.0; De Cewsville 1.0; Denton County 0.3; Descubridora 1.0; Eagle Station 0.7; Elbogen 0.8; Esnandes o.6; Fukutomi o.6; Glorieta 0.4 ; Hex River o.8; Hollands Store 0.5; Hraschina 1.0; Huejuquilla-Gruppe 0.5; Ilimaë 1.o; Jenny's Creek 
0.5 ; Joe Wright Mountain 0.9; Jonesboro 1.0; Jonzac 0.7; Kakowa Zahl der | Gewicht Fall- und d. Samm0.6 ; Kalumbio.8; Kendall County 0.7; Knyahinya 0.7; Kokstatu Lancé 1.0; Lick Creèk o.8; Linnville 0.5; Lucé o.6; Lujan I.o; Luponnas 0.3 ; Mac Kinney 0.6 ; Maêmê 0.4 ; Marmande 0.4 ; Mazapil 0.9; Mezö-Madaras 0.5; Miney 0.7; Misteca 0.3; Moonbi 1.0; Mount Joy 1.0; Mühlau 1.0; Nagy.Vazsony o.8; Narraburra Creek I.o; Nelson County 0.6; Netschaëvo 0.2; Ohaba 1.0; Okniny 0.3; Orange River (Stein) 1.0; Piquetberg 1.0; Port Orford 1.0; Prairie Dog Creek 0.7; Saint-Denis-Westrem o.6; Scottsville 0.2; Seneca Falls 0.2; Senegal 0.3; Seres 0.9; Silver Crown 0.8; Slavetic 0.9; Stannern 0.4; Summit 0.8; Tabor o.6; Tajgha 0.5 ; Tieschitz 0.9 ; Tounkin I.0; Waconda 0.3 ; Waldron Ridge 0.4 ; Welland 0.3 ; Werchne Tschirskaja 0.5 ; Wessely r.o; Youndegin 1.0 ; Zebrak 0.7 ; Zmenj 0.7 .

*W ien, U. I: Mineralogisch-Petrographisches Institut der Universität Wien; mitgeteilt durch Herrn Hofrat Tschermak

*Wi e n, U. II : Mineralogisches Institut der Universität Wien; mitgeteilt durch Herrn Hofrat Schrauf

Worlé e: Privatsammlung des Herrn F. Worlée, Hamburg; mitgeteilt durch Herrn Dr. Gottsche

Wrany: Privatsammlung des Herrn Dr. A. Wrany, Prag; mitgeteilt durch Herrn Prof. Dr. K. Vrba

*W ü r z burg: Mineralogisch-Geologisches Institut der Universität; mitgeteilt durch Herrn Geh. Bergrat Dr. von Sandberger

Carcote o.6.

$\mathrm{Z}$ ür i h: Mineralogische Sammlung der Universität und des Polytechnikum's ; mitgeteilt durch Herrn Prof. Dr. Grubenmann 


\section{Versuch einer Bestimmung des Tauschwertes der Meteoriten.}

In der Einleitung habe ich erwähnt, dass das Interesse, welches den Meteoriten gegenwärtig in weiteren Kreisen entgegengebracht wird, durch eine grössere Verbreitung des Materials erhöht werden könnte. Da ich nun diese weitere Verbreitung durch einen lebhaften Tauschverkehr für möglich halte und da ich ferner eine Erleichterung desselben in der wenn auch nur annähernden Bestimnung des gegenseitigen Wertverhältnisses der Meteoriten erblicke, will ich versuchen, den Tauschwert dieser Körper zu ermitteln.

Wenn die Tauschwerte allgemeine Gültigkeit erlangen sollen, so müssen die bei ihrer Berechnung in Betracht kommenden Faktoren möglichst allgemeinen Gesichtspunkten entnommen werden. Solcher Faktoren dürften im ganzen acht vorhanden sein, von denen die zuerst zu besprechenden drei, wie ich hier vorausschickend bemerke, sich bei einer formelmässigen Berechnung der Werte verwenden lassen, während die fünf übrigen dieses nicht gestatten, dafür aber auch von geringerer Bedeutung für die Bewertung sind.

I) Der Wert eines Meteoriten hängt an erster Stelle $a b$ von der Menge des erhaltenen Materials, so dass eine Lokalität als um so kostbarer betrachtet wird, je weniger davon gesammelt wurde. Nacb diesem Grundsatz werden die nachweisbaren Gewichte $(\mathrm{N})$ der in Sammlungen aufbewahrten Meteoriten eine grosse Rolle bei der Aufstellung einer Wertskala spielen; daher habe ich mich bemüht, alles in Sammlungen aufbewahrte Material so vollständig wie möglich zu ermitteln. Ich habe also versucht, die Differenz zwischen ursprünglichem und nachweisbarem Gewicht möglichst klein werden zu lassen. Trotz meiner Bemühungen zeigen diese beiden Grössen in sehr vielen Fällen noch erhebliche Differenzen, worauf ich bereits in der Einleitung näher eingegangen bin. In der systematischen Uebersicht (s. letztes Kapitel) wurden alle Lokalitäten mit unvollständigen Gewichtsangaben mit * versehen. Bei diesen liessen sich die Tauschwerte nicht mit Sicherheit ermitteln; es sind daher die Zahlen, welche ich dafür nach meiner weiter unten folgenden Formel berechne, mit Vorsicht aufzunehmen. 
Um indessen wenigstens annähernd die Grenzen, innerhalb welcher die Werte schwanken können, festzulegen, habe ich in den Rubriken unter W zuweilen zwei Werte aufgeführt; der eine ergiebt sich aus der Zahl N, der andere folgt unter Berücksichtigung eines grösseren Bruchteils des ursprünglichen Gewichtes.

Vielleicht erwartet man ein * auch bei vielen anderen Lokalitäten, wie $z$. B. bei Pultusk und Toluca; denn bei der ausserordentlich grossen Verteilung dieser Meteoriten habe ich sicherlich einen erheblichen Teil nicht registrieren können. Indessen dürften die in Schul- oder andern kleinen Sammlungen aufbewahrten kleinen Massen dieser so reichlich vertretenen Lokalitäten wegen der starken Abrundung der Gewichte kaum wesentlich den Wert verändern.

Nach einer Schätzung, über welche sich nähere Angaben in der Einleitung finden, darf man annehmen, dass meine Erhebungen dahin geführt haben, etwas mehr als $4 / 5$ des in Sammlungen aufbewahrten Materials zu verzeichnen, so dass sich wenigstens eine annähernde Wertbestimmnng auf Grund dieser nachweisbaren Gewichte durchführen lässt. Wenn es mir aber auch nur gelingen sollte, die Grenzen der Wertbestimmung soweit festzulegen, dass man wenigstens sagen kann, der Wert eines Meteoriten betrage nicht mehr als das Doppelte und nicht weniger als die Hälfte der mitgeteilten Zahl, so würde schon hiermit in Hinsicht auf die gegenwärtige grenzenlose Verwirrung in der Wertfrage ein Fortschritt zu verzeichnen sein. Bei den 5 Meteoriten der oktaëdrischen Eisen mit feinsten Lamellen (Gruppe 39 im letzten Kapitel) schwankt allerdings die Wertbestimmung in weiteren Grenzen wegen des Eisens von Ranchito; dasselbe gilt, wenn auch nicht in so hohem Grade, von den 2 Eisen der Zacatecas-Gruppe; bei den mit einem * versehenen Meteoriten lässt sich dagegen trotz der ungenügenden Kenntnis des nachweisbaren Gewichtes die Grenze bei sehr vielen enger ziehen.

2) Der Wert eines Meteoriten wird ferner durch die ihm eigentümlichen petrographischen Eigenschaften bestimmt, so dass sein Wert fällt, wenn Zahl oder Masse der mit ihm zur gleichen petrographischen Gruppe gehörenden Meteoriten steigt. Beispielsweise werden die Meteoriten von Angra dos Reis, Chassigny und Shergotty immer sehr viel höher geschätzt werden, als diejenigen von Le Pressoir, Salt Lake City und Monroe, obgleich von Angra dos Reis (397 gr.) und Le Pressoir (376 gr.), von Chassigny ( 854 gr.) und Salt Lake City ( 875 gr.), von Shergotty (4897 gr.) und Monroe ( $479^{8} \mathrm{gr}$.) je ungefähr gleiche Massen, nämlich die in () beigefügten Gewichte, nachweisbar sind. Die ersten drei Steine sind jeder für sich die einzigen Vertreter einer gut charakterisierten petrographischen Gruppe, wăhrend die letzteren zur Gruppe der Kügelchenchondrite (Cc) oder der grauen breccienähnlichen Chondrite $(\mathrm{Cgb})$ oder $\mathrm{zu}$ den geaderten grauen Chondriten (Cga) gezählt werden, welchen noch zahlreiche andere Lokalitäten angehören. Diese petrographische Eigentümlichkeit eines 
Meteoriten kann auf die Weise berücksichtigt werden, dass man annimmt, der Wert verhalte sich umgekehrt, wie die Zahl der Lokalitäten in einer Gruppe, oder umgekehrt, wie das Gesamtgewicht einer Gruppe; auch liessen sich vielleicht beide Grössen bei der Berechnung verwenden. Ich habe es vorgezogen, nur das Gruppengewicht $(\mathrm{G})$ in meine Formel aufzunehmen, weil die Zahl der Lokalitäten einer Gruppe schon durch den dritten gleich zu besprechenden Faktor wenigstens bis zu einem gewissen Grade zum Ausdruck kommt. Es zeigt sich nämlich, dass eine Gruppe wenigstens im allgemeinen eine um so grössere Verbreitung besitzt, je grösser die Zahl ihrer Lokalitäten und nicht je grösser ihr Gruppengewicht ist, was wiederum damit zusammenhängt, dass die einzelnen Lokalitäten sich grösstenteils in einer Hand befinden. Zur Illustration des zuletzt Gesagten können die in der systematisch angeordneten Uebersichts-Tabelle, wie sie in der Einleitung zu finden ist, unter $\mathrm{Z}, \mathrm{N}$ und $\mathrm{B}$ aufgeführten Zahlen dienen. Beispielsweise haben die weissen Chondrite $(\mathrm{Cw})$ und die intermediären breccienähnlichen Chondrite (Cib) ungefähr gleiches Gruppengewicht, nämlich 53 und $63 \mathrm{Kgr}$; in der ersteren Gruppe ergeben sich bei 24 Lokalitäten 321 , in der letzteren bei 7 Lokalitäten 176 Besitzer, wobei noch zu bedenken ist, dass die letztere Zahl durch den Fall von L'Aigle ungewöhnlich hoch ausfällt.

Dieser zweite Faktor hängt nun ganz wesentlich von der Art der Einteilung, also von dem System der Meteoriten ab. Ich habe meinen Berechnungen das Rose-Tschermak'sche System zu Grunde gelegt und noch jene Erweiterungen, welche Brezina in seiner letzten Arbeit beibehalten hat, wenigstens in der Hauptsache adoptiert. Es lassen sich gewiss manche Einwände gegen dieses System erheben; indessen muss man bei einer Kritik wohl bedenken, dass unsere Kenntnisse über die Gesamtheit des Materials ausserordentlich lückenhaft sind. Wenn man darauf Rücksicht nimmt, so wird man kaum leugnen können, dass diese Anordnung die Gesamtkenntnis über die petrographischen Eigenschaften der einzelnen Lokalitäten am besten zum Ausdruck bringt.

Als erstes Einteilungsprinzip ist die chemische Zusammensetzung, als zweites die Struktur, als drittes sind äussere Kennzeichen (Farbe, Adernbildung) gewählt worden. Ganz besonders wird man das letzte Prinzip zum Angriffspunkt gegen das System machen können, und ist es vor allem die Einteilung in weisse, intermediäre und graue Chondrite, welche von den einzelnen Forschern in verschiedener Weise durchgeführt werden dürfte. Die Unterschiede nach dem äussern Ansehen sind zuweilen bei den Uebergangsgliedern sehr gering, wie schon Tschermak (M. M. 1872, p. 166) hervorhebt. Ich bin wenigstens bei der Bestimmung der Tübinger Sammlung zuweilen zu Resultaten gekommen, die ein wenig von den Wiener Angaben abweichen; dennoch habe ich geglaubt, die letzteren adoptieren zu sollen, um einen Dualismus, der bei dem ganzen provisorischen 
Charakter dieser Bestimmungen nur Verwirrung anrichten würde, zu vermeiden. Nach diesem System lassen sich die Meteoriten in 5 Klassen und 50 Gruppen einteilen. Diese 50 Gruppen sind ausserordentlich ungleichartig; dennoch schien es mir zweckmässig, jede dieser Gruppen bei der Bewertung gleich hoch anzuschlagen d. h. mit andern Worten: Ich habe jeder Lokalität eine höhere individuelle Bedeutung beigelegt, als ihr nach diesem System zukommt. Die Meteoriten der 32. u. 50. Gruppe sind von der Bewertung natürlich ausgeschlossen, ebenso wie die im Anhang auf p. 396 und 407 angeführten Fälle mit Ausnahme von Lesves. Von Madrid fehlen noch zuverlässige Angaben über die gesammelten Mengen. Der Anhänger eines andern Systems wird auch andere Werte für die einzelnen Meteoriten berechnen. Wie weit diese Werte von den hier gegebenen abweichen, muss für jeden Fall besonders untersucht werden. Einige Lokalitäten wurden in dem Index nicht genügend charakterisiert, um sie hier leicht auffinden zu können, daher möchte ich bemerken, dass:

\begin{tabular}{|c|c|c|c|c|c|c|}
\hline Colfax & zur & Gruppe & 50 & Long Island & zur & Gruppe \\
\hline Cranberry Plains & , & $\rightarrow$ & 50 & Ngawi & , & $\rightarrow$ \\
\hline Hammond & s & 》 & $4 \mathrm{I}$ & Roda & $\Rightarrow$ & 》 \\
\hline Iharaota & $\gg$ & » & 9 & Victoria West & 3 & 》 \\
\hline Indarch & , & 》 & 28 & Yodzé & $\gg$ & " \\
\hline
\end{tabular}

gestellt wurde.

Es ist noch mit einigen Worten der A enderung zu gedenken, welche die Gruppengewichte dadurch erfahren können, dass die nachweisbaren Gewichte einiger Meteoriten noch nicht endgültig festgestellt werden konnten. Zum Verständnis muss ich vorausschicken, dass die Gewichte bei der Berechnung stark abgerundet wurden, worauf ich auf p. 438-439 noch zurückkomme. Ueberschreitet der grösstmögliche Einfluss, welchen die Aenderung des nachweisbaren Gewichtes auf das Gruppengewicht hervorbringt, nicht die Abrundungsgrenzen, so bleibt das Endresultat jetzt und später dasselbe. Wenn auch von Pultusk noch $100 \mathrm{Kgr}$. mehr erhalten sein sollten also anstatt $200 \mathrm{Kgr}$. - $300 \mathrm{Kgr}$. vorhanden wären, so würde das weder auf den Wert von Pultusk noch auf denjenigen der andern geaderten grauen Chondrite einen Einfluss haben, wie man aus den am Schluss auf p. $447 \mathrm{ff}$. folgenden Tabellen ersehen kann. Ist der Einfluss dieser wahrscheinlichen Aenderung aber so bedeutend, dass die Abrundungsgrenze überschritten wird, so sind die der Berechnung zu Grunde gelegten Gruppengewichte passend erhöht worden.

Aenderungen sind bei folgenden Gruppen vorgenommen. - Die Gründe hierfür sind im Index auf p. I-395 bei den einzelnen Lokalitäten zu finden. Gruppe I. Gruppengewichts-Grenze auf $0.5-1 \mathrm{Kgr}$. erhöht.

Gruppe 4. Gruppengewichts-Grenze hauptsächlich wegen Bialystok und

Petersburg auf 5-10 Kgr. erhöht.

W iilfing, Meteoriten. 
Gruppe 6. Gruppengewichts-Grenze auf $\mathrm{I}-2 \mathrm{Kgr}$. erhöht.

Gruppe I2. $\quad$, 20-50 * wegen Monte Milone und Pacula erhöht.

Gruppe I3. Gruppengewichts-Grenze wegen Alfianello auf $200-500 \mathrm{Kgr}$. erhöht.

Gruppe 14. Gruppengewichts-Grenze wegen Bori u. New Concord auf 200 $-500 \mathrm{Kgr}$. erhöht.

Gruppe r9. Gruppengewichts-Grenze wegen Baratta u. Sewrukof auf 200 $-500 \mathrm{Kgr}$. erhöht.

Gruppe 22. Gruppengewichts-Grenze wegen Werchne Tschirskaja auf 50 - $100 \mathrm{Kgr}$. erhöht.

Gruppe 26. Gruppengewichts-Grenze wegen PipeCreek auf 10--20K gr.erhöht.

Gruppe 27. Gruppengewichts-Grenze hauptsächlich wegen Bluff auf 200 $-500 \mathrm{Kgr}$. erhöht.

Gruppe 33. Gruppengewichts-Grenze wegen Inca u. Veramin auf 500I000 Kgr. erhöht.

Gruppe 39. Gruppengewichts-Grenze wegen Tazewell auf 50-100 Kgr. $\mathrm{zu}$ erhöhen, wegen Ranchito auf $2000-\infty$; indessen kann dies letztere vorläufig wohl noch nicht berücksichtigt werden. Die äussersten Wertgrenzen, die zu erwarten sind, wurden in Klammer () beigefügt.

Gruppe 40. Gruppengewichts-Grenze schon allein wegen Bear Creek, Grand Rapids u. Madoc auf 500-1000 Kgr. erhöht.

Gruppe 44. Gruppengewichts-Grenze wegen Zacatecas auf 500-1000 Kgr. erhöht.

Gruppe 48. Gruppengewichts-Grenze wegen Dehesa u. Shingle Springs auf $20-50 \mathrm{Kgr}$. erhöht.

Gruppe 49. Gruppengewichts-Grenze wegen Campo del Cielo, Rasgata u. Tucson auf $2000-\infty$ erhöht.

3) Den dritten Faktor für die Wertformel entnehme ich der Zahl der Besitzer (B) einer Lokalität. Hierüber habe ich mich in einem früheren Aufsatz ${ }^{1}$ ) folgendermassen ausgesprochen: „Wenn von einem Meteoriten sim Gewichte von $5000 \mathrm{gr}$. je $1000 \mathrm{gr}$. in fünf Sammlungen vorhanden sind, »so dürfte das Material leichter und billiger im Tausch zu erhalten sein, »als wenn es sich auf zwei Besitzer im Verhältnis von $4990 \mathrm{gr}$. und $10 \mathrm{gr}$. sverteilt. In ersterem Falle könn en mehrere Besitzer abgeben und man skann sich an mehrere wenden; im letzteren Fall kann füglich nur einer s abgeben und er wird daher höhere Forderungen stellen. Dies Moment >der Verbreitung wird aber um vieles vermindert werden, wenn man die "Voraussetzung macht, dass jede Sammlung gleiches Interesse daran hat, sunsere Kenntnis auf dem Gebiete der Meteoriten zu fördern, und wenn s die Ueberzeugung durchgedrungen ist, dass bei einem lebhaften Tausch-

I) Jahresh. d. vaterl. Naturk. Württembergs Bd. $5^{\text {I }}$ (1895), p. 343-344. 
»verkehr jede Sammlung, auch die grösste, eine Verbesserung erfährt.๔ Indessen bin ich nunmehr der Ansicht, dass - selbst wenn jene ideale Auffassung zur Geltung kommen sollte - die Zahl der Besitzer in beschränktem Masse zu berücksichtigen ist. Ein weit verbreiteter Meteorit muss nämlich aus dem Grunde geringer bewertet werden, weil er gerade wegen seiner grossen Verbreitung von einer kleineren Zahl von Sammlungen als wünschenswertes Tauschobjekt angesehen werden kann und er demnach unter dem Einfluss der geringeren Nachfrage zu leiden hat. Wenn ich also die Zahl der Besitzer in meine Formel einführe, so geschieht es hauptsächlich aus dem letzteren Grunde und nicht deswegen, weil ein in Händen weniger Besitzer befindlicher Meteorit als Spekulationsobjekt zuweilen missbraucht wird.

4) Als viertes Moment bei der Wertbestimmung wäre nach meinen früheren Darlegungen (1. c. p. 344) das in Zukunft fallende oder aufzufindende Material zu berücksichtigen. Freilich werden im allgemeinen die Meteoriten durch neues Material in ihrem Wert sinken. Von einschneidender Bedeutung ist das indessen nur bei jenen Gruppen, welche spärliche Vertreter aufweisen. Wäre Manbhoom der einzige Vertreter der Amphoterite geblieben, so würde sich sein Wert nach den weiter unten mitgeteilten Tabellen zu 176 Einheiten berechnen; nachdem nun aber der Fall von Jeliza uns weiteres, reichliches Material eines Amphoterits gebracht hat, sinkt der Wert von Manbhoom auf 82 Einheiten. In den weitaus überwiegenden Fällen, nämlich bei den Gruppen mit vielen Vertretern und reichlichem Material wird der Einfluss nicht so gross sein, bei manchen sogar ganz verschwinden; $z$. B. werden nach meiner Skala neu gefallene oder aufgefundene Massen von Eisen mit mittleren Lamellen (Om) oder mit groben Lamellen $(\mathrm{Og})$ niemals die Werte der andern beeinflussen. Und wenn man nach der Erfahrung der letzten roo Jahre annimmt, dass die Meteoriten, welche seltenen Gruppen angehören, auch in Zukunft selten fallen werden und dass man infolge dessen nicht oft eine Störung der Werte zu befürchten hat, so sollte man kein Bedenken tragen, dieses Moment ausser Betracht zu lassen. Verringern liesse sich sein Einfluss unzweifelhaft dadurch, dass man die neu aufzufindenden oder fallenden Meteoriten verstaatlicht. Durch eine solche Verstaatlichung würde nicht nur die irrtümliche Auffassung von dem hohen absoluten Geldwert der Meteoriten beseitigt werden - die doch nur durch die unkluge Konkurrenz einiger Sammler entstanden ist -, sondern es würde auch die Aufmerksamkeit der Behörden mehr wie bisher auf diesen Gegenstand gelenkt und dadurch viel Material, welches unter den jetzigen Verhältnissen verloren geht, der wissenschaftlichen Untersuchung zugeführt werden.

5) Als fünfter Faktor für die Berechnung des Wertes lassen sich die Gewinnungskosten anführen. Ich glaube, dass auch diese keine Rolle bei der Wertbestimmung spielen können, wobei ich nochmals hervorheben 
will, dass meineWertangaben nicht absolute Werte sondern nur Tauschwerte bedeuten sollen, welche beim Tausch von Meteoriten gegen Meteoriten geiten können. Wenn eine Sammlung einen Meteoriten billig erstanden hat, so wird sie den hieraus entspringenden Vorteil so lange geniessen, als dieser billig erstandene Meteorit sich als Tauschmaterial verwenden lässt und umgekehrt. Uebrigens sind die eigentlichen Gewinnungskosten bei den Meteoriten mit Einschluss des allerersten Kaufpreises verschwindend klein gegenüber den Preisen, welche nachher im Handel angesetzt zu werden pflegen. So schickte Krantz eine eigens zu dem Zweck ausgerüstete Expedition in das Toluca-Thal nach Mexiko, um die dortigen Meteoreisen zu sammeln; hiermit waren gewiss grosse Kosten verbunden und dennoch gehört das Meteoreisen von Toluca immer noch zu den billigst $z u$ erwerbenden.

6) Auch der Erhaltungszustand eines Meteoriten lässt sich natürlich nicht in einer Formel ausdrücken. Ueberdies hängt das Interesse, welches ein Meteorit für die Forschung hat, durchaus nicht mit seinem Erhaltungszustand zusammen, so dass selbst ein stark zersetzter Meteorit für das Studium eine viel wichtigere Rolle spielen kann, als ein gut erhaltener. In sehr vielen Fällen wird sich auch die Möglichkeit finden, gleich gutes Material gegen einander einzutauschen.

7) Ebensowenig kann man das historische Interesse, welches einige Meteoriten beanspruchen dürfen, quantitativ zum Ausdruck bringen. Ganz besonders kämen hier die Fälle von Blansko, Ensisheim, Hraschina, Krasnojarsk, L'Aigle und Siena in Betracht. Zum Glück aber sind diese Lokalitäten mit Ausnahme von Blansko (von dem überhaupt nicht viel gesammelt wurde) und Hraschina recht verbreitet, so dass die Nachfrage nach diesen weniger lebhaft sein wird, als nach vielen andern, und sie beim Tauschverkehr also keine grosse Rolle spielen werden. Die folgende kleine Tabelle giebt über die Verteilung näheren Aufschluss:

$\begin{array}{lcrr} & \text { Besitzer } & \text { Nachw. Gewicht } & \text { In einer Hand } \\ \text { Blansko } & \text { I3 } & 243 \mathrm{gr} . & 88 \mathrm{gr} . \\ \text { Ensisheim } & 65 & 70385 \mathrm{gr} . & 54800 \mathrm{gr} . \\ \text { Hraschina } & \text { r } 8 & 3967 \mathrm{gr} . & 39245 \mathrm{gr} . \\ \text { Krasnojarsk } & \text { 109 } & 567995 \mathrm{gr} . & 520000 \mathrm{gr} . \\ \text { L'Aigle } & 87 & 36843 \mathrm{gr} . & 8549 \mathrm{gr} . \\ \text { Siena } & 40 & 4 \mathrm{I} 63 \mathrm{gr} . & 1050 \mathrm{gr} .\end{array}$

8) Schliesslich wäre noch zu erwähnen, dass diejenigen Meteoriten, deren Fall beobachtet worden ist, vielfach höher geschätzt werden, als andere, welche zufällig im Boden aufgefunden wurden. Ganz besonders gilt dies von den Meteoreisen, von denen bis jetzt mit Sicherheit nur 7 (von 178): Braunau, Cabin Creek, Charlotte, Hraschina, Mazapil, Nedagolla und Rowton und mit grc̈sserer oder geringerer Wahrscheinlichkeit noch 2 andere: Nejed und Victoria West beim Falle beobachtet worden 
sind. $\mathrm{Ob}$ man bei diesen 7 oder 9 Eisen den aus meiner Formel sich ergebenden Wert verdoppeln oder noch weiter erhöhen will, mag unentschieden bleiben. Bei den Steinen, bei welchen umgekehrt die im Fall nicht beobachteten in der Minderzahl sind, scheint man im allgemeinen keinen grossen Unterschied zwischen der einen und der andern Art zu machen. Zur Orientierung mögen hier die ${ }_{2} 3$ Steine (von 335), deren Fallzeit nicht bekannt ist, aufgeführt werden:

\begin{tabular}{l|l|l|l} 
Assam & Goalpara & Mühlau & SanEmigdioRange \\
Bluff & Long Island & Oczeretna & San Pedro Springs \\
Carcote & Lutschaunig & Pipe Creek & Simbirsk-Partsch \\
Darmstadt & Mainz & Piquetberg & Slobodka-Partsch \\
Eli Eluat & Makariwa & Prairie Dog Creek & Waconda \\
Gilgoin Station & Morristown & Salt Lake City &
\end{tabular}

Ferner will ich noch anfügen, dass von den ro Pallasiten und vom Siderophyr bis jetzt mit Sicherheit noch keiner im Fall beobachtet worden ist, während dies bei den ro Mesosideriten für Barea, Estherville und Veramin und für den einen Lodranit der Fall ist.

Nach den Gesichtspunkten, welche unter 1), 2) und 3) erörtert sind, würde man etwa folgende Formel zur Berechnung des Tauschwertes (W) aus dem Gruppengewicht $(\mathrm{G})$, dem nachweisbaren Gewicht $(\mathrm{N})$ und der Zahl der Besitzer (B) aufstellen können

$$
\mathrm{W}=\frac{\mathrm{I}}{\mathrm{f}(\mathrm{GNB})}
$$

und es fragt sich nunmehr, welche Funktion man für $\mathrm{f}$ wählen soll. Ich habe für $f$ die $n^{\text {te }}$ Wurzel gesetzt, und es bleibt dann nur noch übrig, $n$ zu bestimmen oder, was dasselbe bedeutet, unter den folgenden Formeln diejenige zu wählen, welche als die passendste erscheint.

$$
\begin{aligned}
& W_{1}=\frac{1}{G N B} \\
& W_{2}=\frac{1}{\sqrt{G N B}} \\
& W_{3}=\frac{1}{\sqrt{G N B}} \\
& \vdots \\
& W_{n}=\frac{1}{\sqrt[n]{G N B}}
\end{aligned}
$$


Bevor ich nun an diese Wahl gehe, möchte ich einschalten, dass es zweckmässig ist, die Gewichte sowie die Zahl der Besitzer stark abzurunden, damit keine gar zu grosse $Z$ ahl von Werten, von denen sich viele nur wenig unterscheiden, auftritt.

Zunächst mögen alle Lokalitäten ausgeschlossen werden, von welchen sich nur $50 \mathrm{gr}$. oder weniger nachweisen lassen. Dahin gehören:

I) 18 Fälle, von denen noch weiteres Material erhalten sein dürfte, nämlich :

\begin{tabular}{l|l|l|l|} 
Bald Eagle & Feid Chair & Moonbi & Tonganoxie \\
Baratta & Fukutomi & Oktibbeha & Tounkin \\
Betlehem & Gilgoin & Rancho de laPila & Wairarapa \\
Blue Tier & La Charca & (Cacaria) & Wooster \\
Eli Eluat & Middlesbrough & Tajgha &
\end{tabular}

2) 37 Fälle, von denen höchstwahrscheinlich nur die im Index angegebenen kleinen Mengen erhalten blieben, nämlich:

\begin{tabular}{l|l|l|l} 
Adalia & Deal & Mooradabad & Quinçay \\
Asco & Dhulia & Mühlau & Sabetmahet \\
Barntrup & Grazac & Nageria & SaintChristophe- \\
Bherai & Gurram Konda & Orange River(Stein) & la-Chartreuse \\
Bitburg (nicht & Jamkheir & Oviedo & SanctAugustine's \\
\multicolumn{1}{r|}{ umgeschmolz.) } & Jonesboro & Perth & Bay \\
Bocas & Kusiali & Piquetberg & Schellin \\
Calderilla & Lujan & Ploschkowitz & Tabarz \\
Castine & Maêmê & Pnompehn & Vago \\
Chail & Manegaon & Port Orford & Yodzé
\end{tabular}

Alsdann sollen die Gewichte sowohl der Gruppen als der Lokalitäten auf $\mathrm{r}_{5}$ und die Zahl der Besitzer auf 4 Abteilungen beschränkt werden. Alle Gewichte

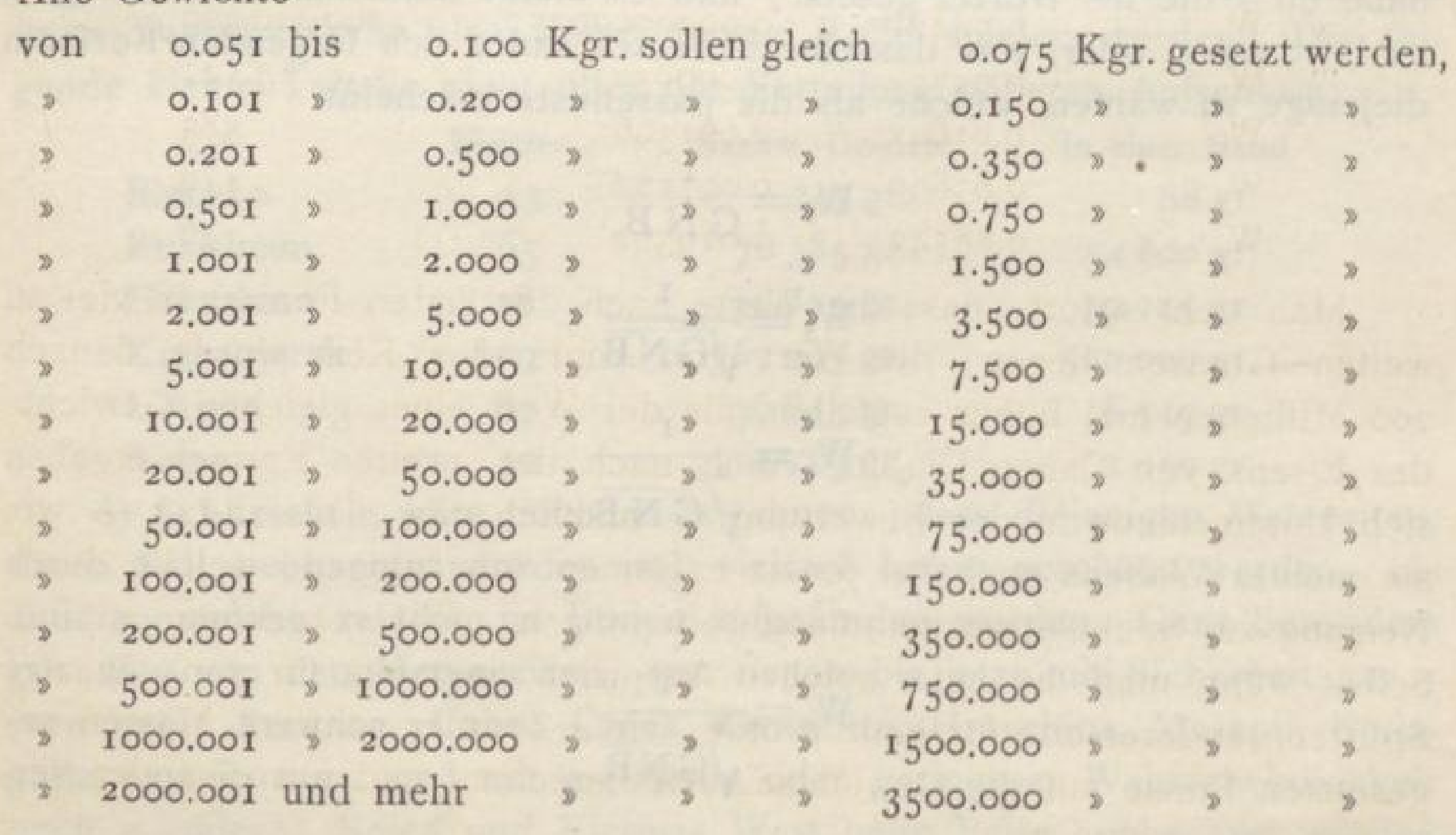


während die Zahl der Besitzer

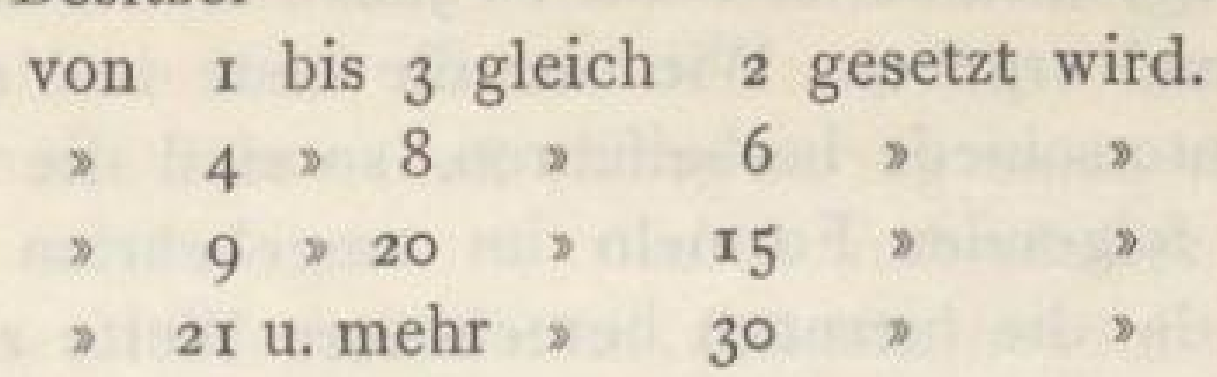

Die Besitzerzahlen sind besonders stark abgerundet, weil denselben I) überhaupt eine untergeordnete Bedeutung beigelegt wird und weil 2) die in der ersten Zeit des Tauschverkehrs sich fortwährend ändernden Werte um so eher konstant werden.

$\mathrm{Zu}$ einer Wahl unter den obigen Formeln kann man nun auf die Weise gelangen, dass man zu ermitteln versucht, innerhalb welcher Grenzen die Werte überhaupt auftreten können, dass man also $W_{1}, W_{2}, W_{3}, \ldots \ldots$, $W_{n}$ für einen der kostbarsten und für einen der geringsten Meteoriten ausrechnet. Es wird wohl kein Zweifel darüber sein, dass zu einem der kostbarsten Meteoriten der Stein von Angra dos Reis gehört, wie andererseits zu einem der am billigsten zu erwerbenden das Eisen von Cañon Diablo zu rechnen ist. Für diese beiden Lokalitäten erhält man als wirkliche und abgerundete Werte der Faktoren G, N und B folgende Zahlen:

\begin{tabular}{|c|c|c|c|}
\hline \multicolumn{2}{|c|}{ Angra dos Reis } & \multicolumn{2}{|c|}{ Cañon Diablo } \\
\hline $\begin{array}{l}\text { wirkliche } \\
\text { Werte }\end{array}$ & $\begin{array}{l}\text { abgerundete } \\
\text { Werte }\end{array}$ & $\begin{array}{l}\text { wirkliche } \\
\text { Werte }\end{array}$ & $\begin{array}{l}\text { abgerundete } \\
\text { Werte }\end{array}$ \\
\hline 397 gr. & $0.35^{\circ} \mathrm{Kgr}$. & 一 & $3500 \mathrm{Kgr}$. \\
\hline $397>$ & $0.350=$ & $4121734 \mathrm{gr}$. & $3500 \%$ \\
\hline II & 15 & 55 & 30 \\
\hline
\end{tabular}

Hieraus berechnet sich nach den obigen Formeln:

\begin{tabular}{lclr} 
& Angra dos Reis & \multicolumn{1}{c}{ Cañon Diablo } & \multicolumn{1}{c}{ W Angra } \\
W Cañon \\
$W_{1}$ & 0.54421 & 0.00000000272 & 200000000 \\
$W_{2}$ & 0.73772 & 0.00005216 & 14142 \\
$W_{3}$ & 0.81643 & 0.001396 & 585 \\
$W_{4}$ & $0.85^{8} 90$ & 0.007222 & 119 \\
$W_{5}$ & 0.88542 & 0.019362 & 46
\end{tabular}

Man sieht sofort, dass die Werte nach der ersten Formel in viel zu weiten Grenzen liegen; der Wert von Angra dos Reis würde danach 200 Millionen mal höher ausfallen, als der Wert eines gleichen Gewichts des Eisens von Cañon Diablo. Auch nach der zweiten Formel ergeben sich Unterschiede in der Bewertung - nämlich von $\mathrm{I}_{\text {bis }} \mathrm{I}_{4} \mathrm{I}_{4}{ }^{2}-$ wie sie meines Wissens auch bei jenen extremen Schätzungen, welche durch Neigung zur Spekulation veranlasst sein mögen, nicht vorgekommen sind. Selbst wenn man die unter solchen Auspizien einerseits für ganz winzige Splitter, andererseits für ganz grosse, viele Zentner schwere Massen angesetzten Preise auf die Gewichtseinheit berechnet und mit einander ver- 
gleicht, gelangt man noch nicht zu jenen Extremen, wie sie sich aus der zweiten Formel ergeben. Wie nun die erste und zweite Formel sicherlich zu grosse Unterschiede herbeiführen, so sind die fünfte, sechste und natürlich alle folgenden Formeln im umgekehrten Sinne nicht zweckentsprechend, da die hiernach berechneten Werte zu geringe Unterschiede aufweisen. Es bleibt dann nur die Wahl zwischen der dritten und vierten Formel übrig. Man könnte vielleicht glauben, dass Wertschwankungen innerhalb der Grenzen von 1 und $I_{1} 9$, wie sie sich nach $W_{4}$ berechnen, genügen; indessen überschreiten die im letzten Jahrzehnt für Meteoriten angesetzten Preise doch zu häufig diese Grenzen, während sie die aus der dritten Formel sich ergebenden erreichen oder auch wohl gelegentlich überschreiten. Mit Rücksicht auf diese praktischen Verhältnisse scheint es mir daher geboten, die dritte Formel zu wählen. Bei dieser Wahl unter den verschiedenen Formeln ist also jenes Moment, welches in den Formeln selbst nicht zum Ausdruck kommt, nämlich der Kaufpreis, nicht ohne Bedeutung gewesen. Diese dritte Formel liesse sich noch dadurch etwas umgestalten, dass man nicht alle drei Faktoren mit der Kubikwurzel belegt, sondern von einem Faktor die zweite oder vierte Wurzel nimmt. Eine derartige Abänderung erscheint mir indessen als eine Komplikation, welche der im Allgemeinen doch sehr unbestimmt gehaltenen Bewertung nicht entspricht. Ich beschränke mich deshalb darauf, die Formel

$$
W=\frac{I}{\sqrt[3]{G N B}}
$$

zur Tauschwert-Berechnung der Meteoriten vorzuschlagen.

Die folgenden 4 Tabellen enthalten alle Werte, welche nach dieser Formel mit Rücksicht auf die früher besprochenen Abrundungen möglich sind, so dass man daraus den Wert eines jeden Meteoriten unmittelbar finden kann. Die erste Tabelle bezieht sich auf die Meteoriten mit $\mathrm{I}-3$ Besitzern, die zweite auf $4-8$, die dritte auf $9-20$, die vierte auf 2 I und mehr Besitzer. In jeder Tabelle ist das Gewicht der Lọalität in horizontaler, das Gewicht der Gruppe in vertikaler Richtung angegeben. 
I bis 3 Besitzer.

Nachweisbares Gewicht $(\mathrm{N})$ einer Lokalität in $\mathrm{Kgr}$.

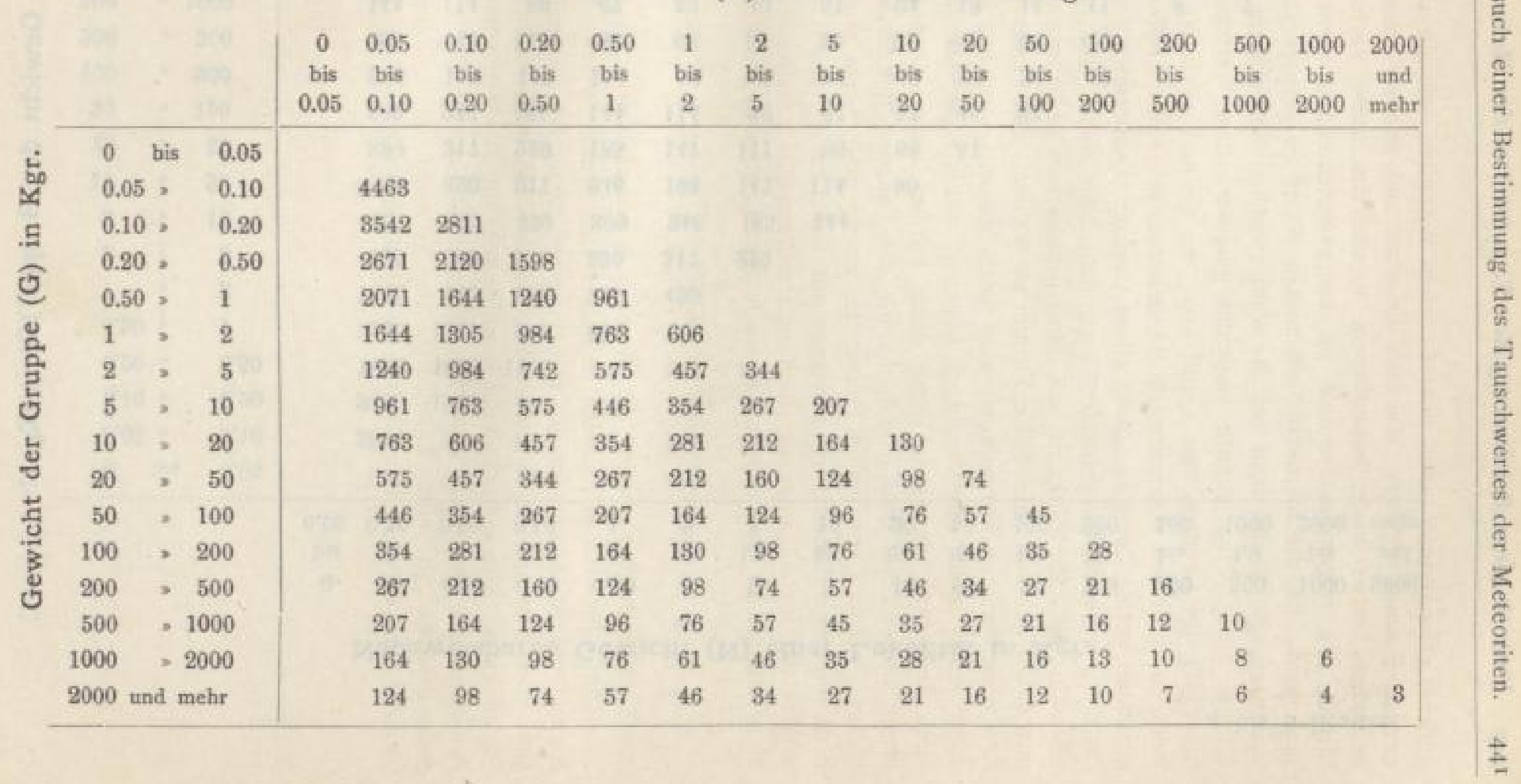




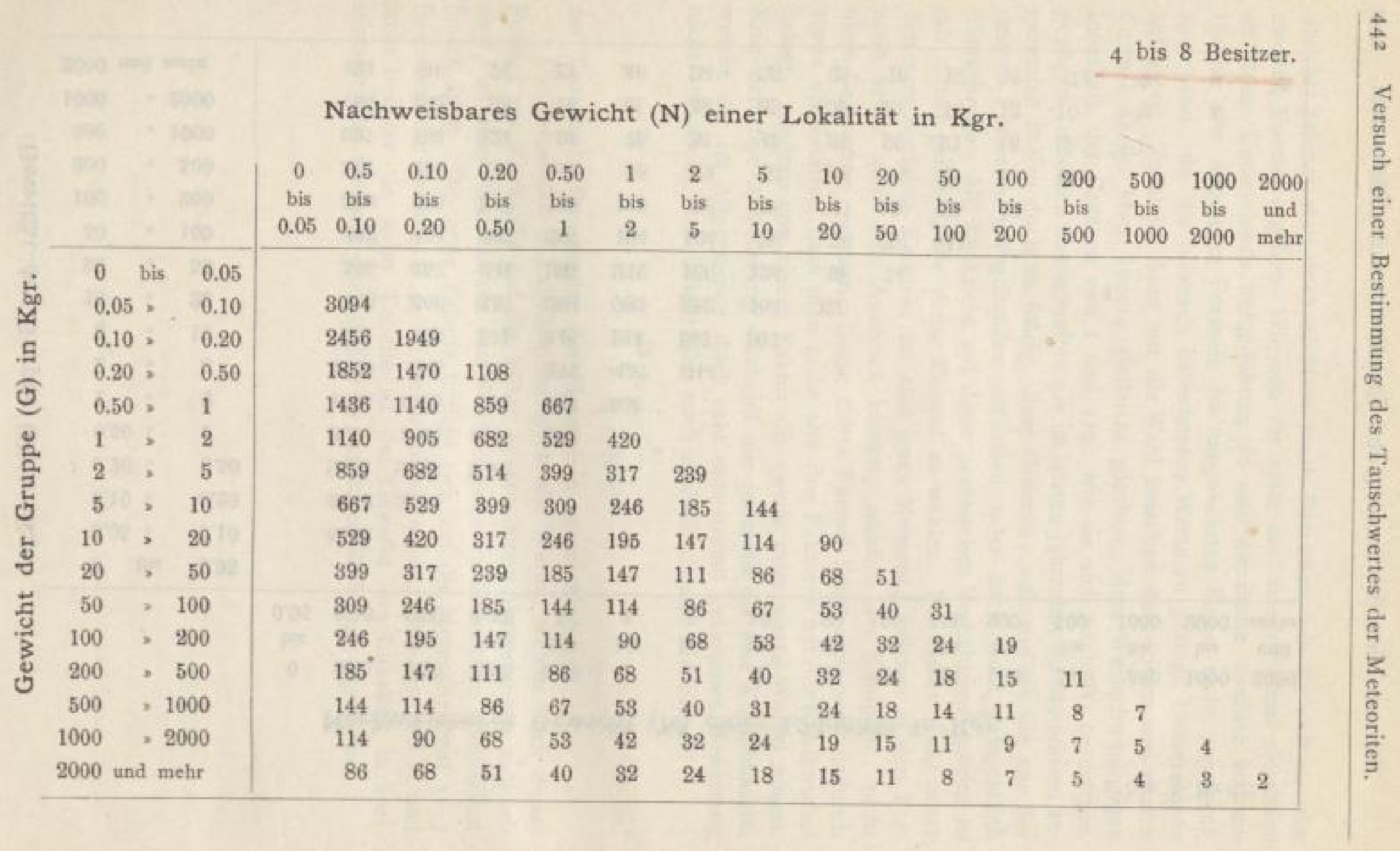

\section{SLUB}


9 bis 20 Besitzer.

Nachweisbares Gewicht $(\mathrm{N})$ einer Lokalität in $\mathrm{Kgr}$.

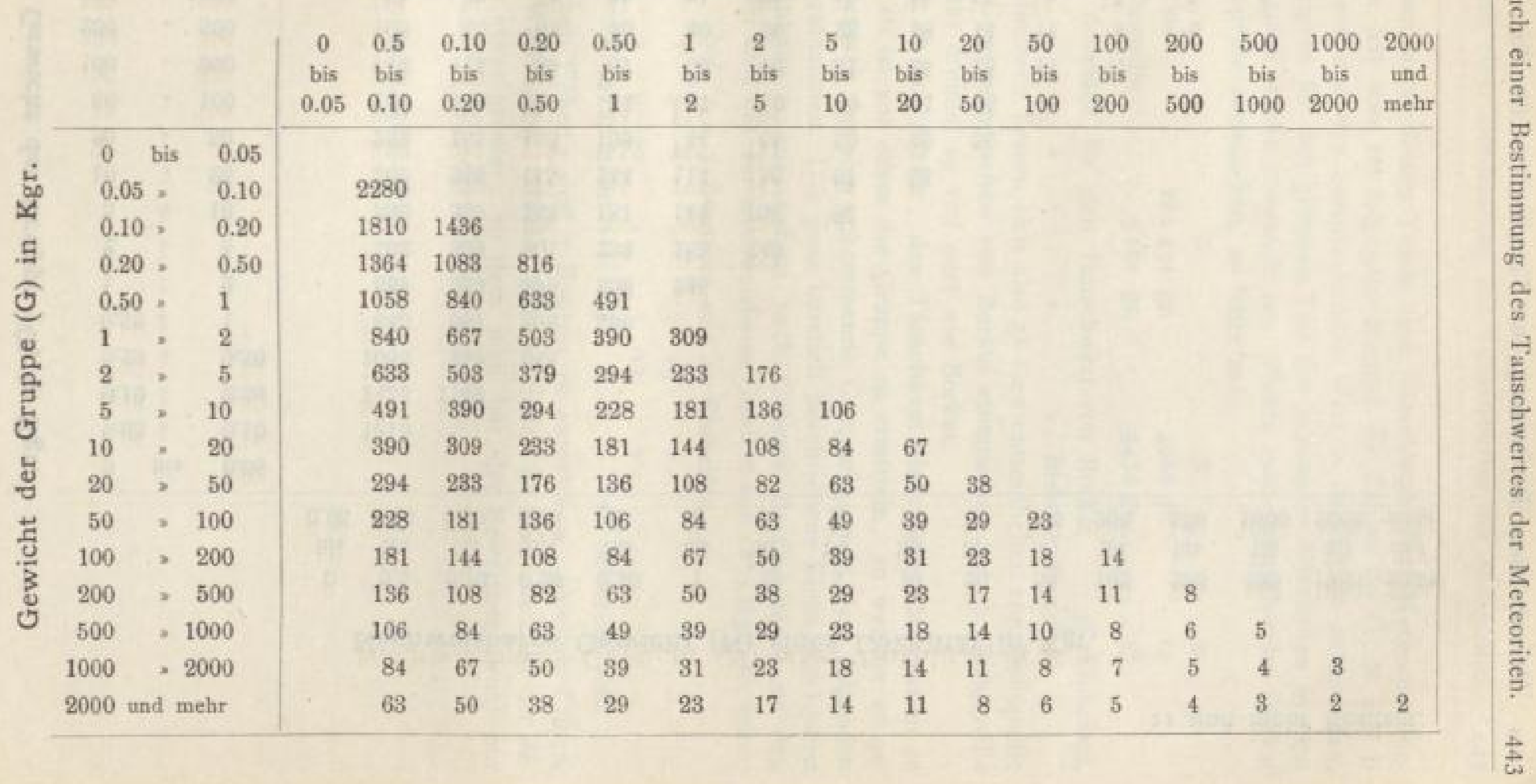

\section{ㄱ SLUB}

Wir führen Wissen. 


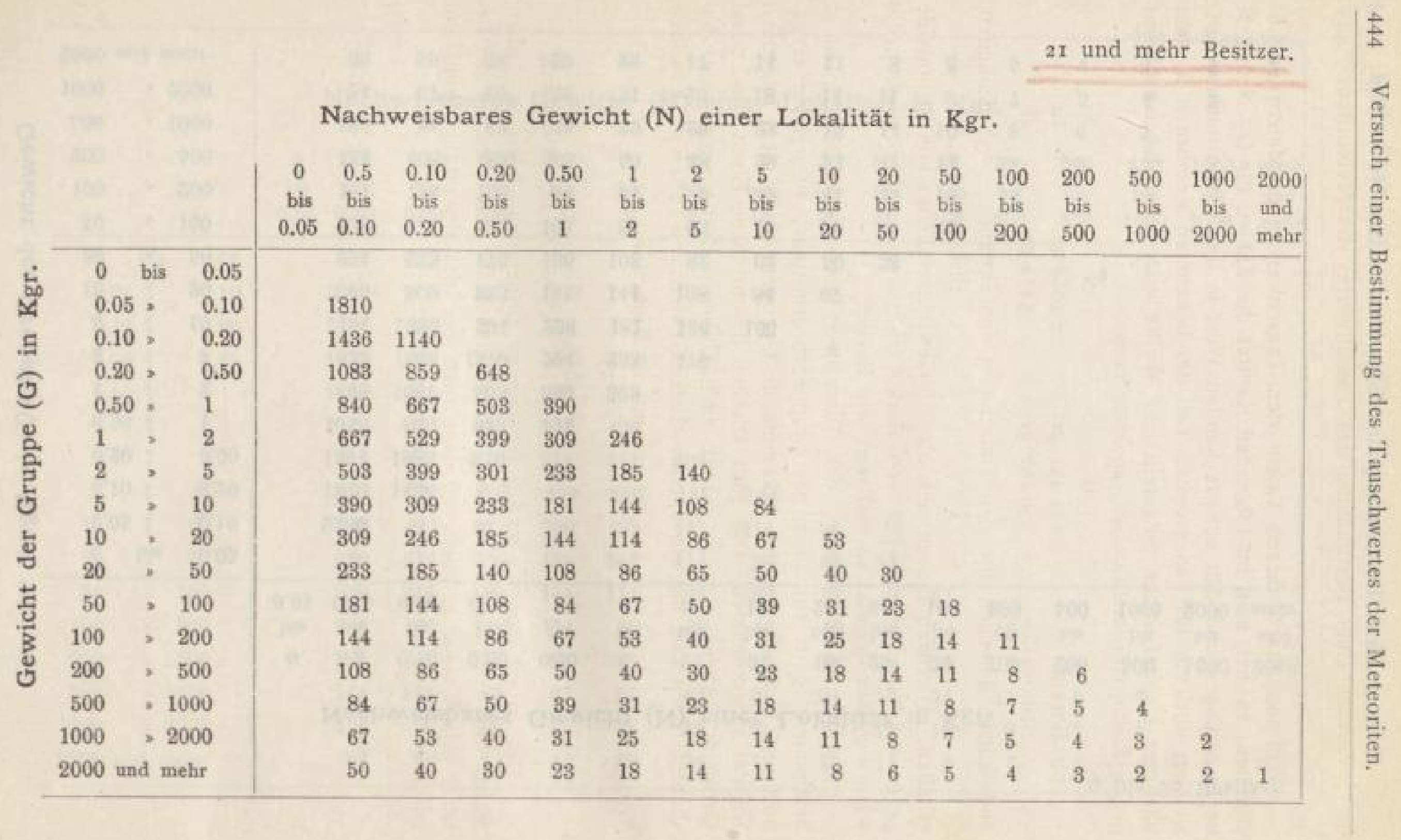


Will man nach diesen Tafeln den Tauschwert eines Meteoriten bestimmen, so hat man im folgenden Kapitel die Zahlen für $\mathrm{G}, \mathrm{N}$ und $\mathrm{B}$ nachzuschlagen und den entsprechenden Wert in den Tafeln aufzusuchen, welcher übrigens für den grössten Teil der bekannten Meteoriten in dem nächsten Kapitel schon mitgeteilt ist. Wollte man beispielsweise Borkut gegen Bishopville eintauschen, so hätte man

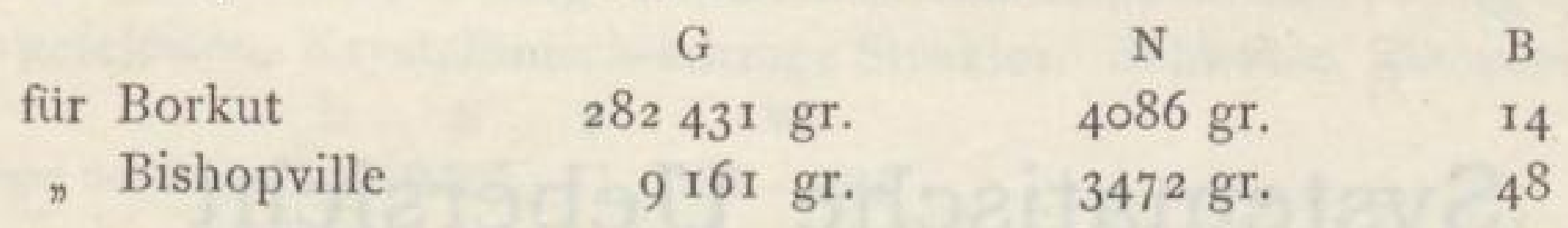

und würde demnach für den Tauschwert von Borkut ${ }_{3} 8$ Einheiten,

$$
\text { > " Bishopville ro8 " }
$$

erhalten. Hiernach liessen sich also $3^{8}$ Gewichtseinheiten von Bishopville gegen 108 Gewichtseinheiten von Borkut eintauschen, d. h. Bishopville wäre ungefähr dreimal so viel wert wie Borkut.

Handelt es sich darum, den Tauschwert eines neuen Meteoriten zu bestimmen, so ist vor allem die Gruppe zu ermitteln, zu welcher er gehört und hiernach das ihm zukommende Gruppengewicht dem folgenden Kapitel zu entnehmen. Das ganz kürzlich beschriebene Eisen von Locust Grove (s. Anhang p. 402) gehört nach Cohen's Bestimmung ${ }^{1}$ ) zu den dichten Eisen und hat ein nachweisbares Gewicht von $10^{1 / 3} \mathrm{Kgr}$. Es würde

$$
\begin{aligned}
& \text { bei } \mathrm{I}-3 \text { Besitzern mit } 2 \mathrm{I} \text { Einheiten } \\
& \text { 》 } 4-8 \text { \$ } \\
& \text { 》9-20 \# } \\
& \text { * } 2 \mathrm{Iu} \text { mehr } \text { 》 } 8 \text { " } 8 \mathrm{u} \text { bewerten sein. }
\end{aligned}
$$

Der Arbeit Cohen's sind 3 Besitzer zu entnehmen, nämlich Cohen, Stürtz und Washington. Höchst wahrscheinlich ist aber die Zahl der Besitzer schon etwas grösser, so dass man bei der Bewertung wohl nur

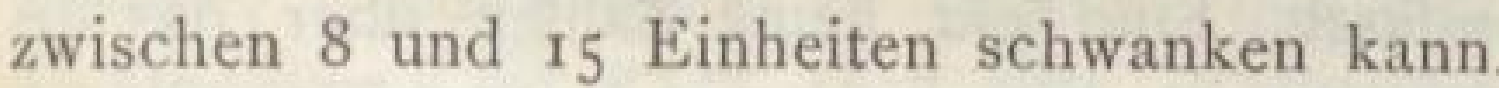

I) Sitzberr. Berlin. Akad, 1897, p. 76-8I. 


\section{Systematische Uebersicht der erhaltenen Meteoriten und ihrer Tauschwerte.}

Neben jeder Lokalität ist in der folgenden systematischen Uebersicht das ursprüngliche Gewicht $(\mathrm{U})$, das nachweisbare Gewicht $(\mathrm{N})$, die Zahl der Besitzer (B) und der Tauschwert (W) aufgezeichnet. Das ursprüngliche Gewicht habe ich nur in einer kurzen Zahl anzugeben versucht; in vielen Fällen ist das nicht möglich, weil die Angaben zu allgemein gehalten sind; so wird $\mathrm{z}$. B. bei manchen Lokalitäten von svielen Massen « gesprochen und nur das Gewicht des grössten und kleinsten Stücks mitgeteilt. Uebersteigt die Summe dieser beiden Gewichte das nachweisbare Gewicht, so ist dieselbe hier aufgeführt; übersteigt aber umgekehrt das nachweisbare Gewicht die Summe jener beiden Zahlen, so ist das ursprüngliche Gewicht nur durch einen Strich $(-)$ angedeutet. Hiermit soll die Vermutung - aber auch nicht mehr als eine Vermutung - ausgesprochen werden, dass die nachweisbare Menge den wesentlichen Teil des erhaltenen Materials umfasst. Sind gar keine Zahlen über das ursprüngliche Gewicht zu finden, so ist dies durch ein Fragezeichen (?) ausgedrückt. - Bei der Angabe der Werte sind in zweifelhaften Fällen, d. h. bei den hier mit einem * bezeichneten, die Grenzen angegeben, innerhalb welcher die Tauschwerte schwanken können. Durch die Einklammerung eines der Grenzwerte ist angedeutet, dass der andere wahrscheinlich der richtigere ist; z. B. ist bei Angra dos Reis der Wert (816-) 49r angegeben, womit ich eben die Vermutung ausdrücke, dass von Angra mehr als $1 / 2 \mathrm{Kgr}$. erhalten blieb und sich auch wahrscheinlich einstmals nachweisen lassen wird. Bei Cabezzo de Mayo $(\mathrm{Cw})$ in Gruppe ro ist der Wert (84-) 39 angegeben, d. h. das bis jetzt nachweisbare Gewicht von 1208 gr. ergäbe den Wert 84 ; da aber höchst wahrscheinlich zwischen 1o und $20 \mathrm{Kgr}$. erhalten sind, so wäre der richtigere Wert 39. Die Gründe für eine jede derartige unbestimmte Angabe sind im alphabetischen Verzeichnis der Meteoriten kurz mitgeteilt. 
A. Calcium- und aluminiumreiche Steine, welche frei oder nahezu frei von Nickeleisen sind und keine Chondren enthalten.

I. I. Angrit, A. $\mathrm{G}=0.397 \mathrm{Kgr}$. (0.5-I).

Schwarzbrauner, aussergewöhnlich calciumreicher Augit; wenig Olivin und Schwefeleisen. Krystallinisch-körnige Struktur. Schwarze, glänzende Rinde. *Angra dos Reis $\quad \begin{array}{cccc}1.7 & 0.397 & 11 & (816-) 491\end{array}$

2. II. Eukrite, Eu. $\mathrm{G}=90.6 \mathrm{I}$ o Kgr. (50- I00).

Augit und Anorthit; geringe Mengen von Schwefeleisen. Krystallinischkörnige Struktur, ähnlich manchen doleritischen Basalten. Schwarze, glänzende Rinde.

\begin{tabular}{lcccc|lcccc} 
& U & N & B & W & & U & N & B & W \\
Adalia & $?$ & 0.003 & 1 & - & Juvinas & - & 50.641 & 62 & 18 \\
*Jonzac & 5 & 1.552 & 22 & $67-50$ & Stannern & 52 & 38.414 & 95 & 23
\end{tabular}

3. III. Shergottit, Sh. $\mathrm{G}=4.897 \mathrm{Kgr}$. $(2-5)$.

Augit und Maskelynit, wenig Magnetit. Krystallinisch-körnige Struktur. Braune, glänzende Rinde.

$\begin{array}{ccccc} & \text { U } & \text { N } & \text { B } & \text { W } \\ \text { Shergotty } & 5 & 4.897 & 13 & 176\end{array}$

4. IV. Howardite, Ho u. Hob. $G=4.707$ Kgr. $(5-10)$.

Augit, Anorthit, Bronzit und Olivin. Tuffartige Grundmasse mit krystallinischen zum Teil eukritischen Ausscheidungen. Schwarze, glänzende Rinde.

\begin{tabular}{|c|c|c|c|c|c|c|c|c|c|}
\hline & $\mathrm{U}$ & $\mathrm{N}$ & B & W & & $\mathrm{U}$ & $\mathrm{N}$ & B & W \\
\hline *Bialystock & 2 & 0.627 & 18 & $228-181$ & Nobleborough & - & 0.078 & 11 & 491 \\
\hline Frank & 0.615 & 0.535 & 18 & 228 & Pawlowka & 2 & 1.123 & 19 & 181 \\
\hline La Vivionnère & 0.780 & 0.779 & 6 & 309 & *Petersburg & 1.764 & 0.399 & 29 & \\
\hline not & - & 0 . & 20 & 228 & Yodzé (Hol & & 0.043 & 3 & \\
\hline Iässing & 1.6 & 0.074 & 14 & 491 & Zmenj & 0.246 & 0.155 & & \\
\hline
\end{tabular}

\section{B. Magnesiumreiche Steine, welche frei oder nahezu frei von Nickeleisen sind und keine oder nahezu keine Chondren enthalten.}

5. I. Bustite, Bu. $G=1.650 \mathrm{Kgr}$. $(I-2)$.

Diopsid und Bronzit; in untergeordneter Menge Oldhamit, Plagioklas, Nickeleisen und Osbornit. Nahezu krystallinische Struktur. Sehr dünne, gelbbraune und matte Rinde.

\begin{tabular}{ccccc|crrrr} 
& $\mathrm{U}$ & $\mathrm{N}$ & $\mathrm{B}$ & $\mathrm{W}$ & & $\mathrm{U}$ & $\mathrm{N}$ & $\mathrm{B}$ & $\mathrm{W}$ \\
Aubres & 0.567 & 0.528 & 4 & 529 & Bustee & 1.3 & 1.122 & 16 & 309
\end{tabular} 
6.

II. Chassignit, Cha. $\mathrm{G}=0.854 \mathrm{Kgr} .(\mathrm{I}-2)$.

Eisenreicher Olivin und geringe Mengen von Chromit. Krystallinischkörnige Struktur. Dünne, schwarze und schwach glänzende Rinde.

$$
\begin{array}{ccccc} 
& \text { U } & \text { N } & \text { B } & \text { W } \\
* \text { Chassigny } & 4 & 0.854 & 33 & (390-) 246
\end{array}
$$

Hierher gehört vielleicht auch der Stein von Nowo-Urei, welcher vorläufig mit Dyalpur und Goalpara zur Gruppe der Ureilite vereinigt ist.

\section{III. Chladnite, Chl u. Chla. $G=9 \cdot 16$ I Kgr. $(5-10)$.}

Rhombischer Pyroxen. Krystallinisch-körnige Struktur. Die Rinde ist entweder weiss und glänzend und alsdann durch gelbliche bis blaue Partien und schwarze Adern marmoriert oder grauschwarz und matt. Im ersteren Fall besteht der Stein im wesentlichen aus Enstatit (Bishopville), im letzteren Falle aus Bronzit (Ibbenbühren, Manegaon und Shalka). Ibbenbühren soll auch ein Mineral mit schief auslöschenden Lamellen (Augit?) besitzen.

\begin{tabular}{llrrr|lrrrr} 
& $\mathrm{U}$ & $\mathrm{N}$ & $\mathrm{B}$ & $\mathrm{W}$ & & $\mathrm{U}$ & $\mathrm{N}$ & $\mathrm{B}$ & $\mathrm{W}$ \\
Bishopville (Chla) & 6 & 3.472 & 48 & 108 & Manegaon & - & 0.044 & 6 & \\
Ibbenbühren & 2.064 & 2.019 & 12 & 136 & Shalka & - & 3.626 & 29 & 108
\end{tabular}

\section{IV. Amphoterite, Am. G=39.949 Kgr. (20-50).}

Olivin und Bronzit; in untergeordneten Mengen Schwefeleisen und Nickeleisen. Struktur teils körnig, teils chondritisch. Bei Manbhoom sind die Chondren nur mikroskopisch klein entwickelt, bei Jeliza finden sich in einer krystallinischen Grundmasse grosse Ausscheidungen von polyedrischen Chondren. Schwarze und matte Rinde, an geflossenen Stellen glänzend. - Roda bedarf noch einer genaueren Untersuchung.

\begin{tabular}{lcrrr|rrrrr} 
& U & N & B & W & & U & N & B & W \\
Jeliza-Gebirge & - & 37.634 & 28 & 30 & Roda & 0.200 & 0.179 & 8 & 317 \\
Manbhoom & - & 2.136 & 12 & 82 & & & & &
\end{tabular}

\section{Magnesiumreiche Steine, welche im wesentlichen} aus Olivin, Bronzit, Nickeleisen und Schwefeleisen bestehen und mit Ausnahme von Nowo-Urei deutlich chondritische Struktur mit tuffartigem Charakter zeigen.

Die Rinde, welche in der Dicke stark schwankt, ist bei der ersten Abteilung der howarditischen Chondrite zum Teil glänzend, bei den übrigen Abteilungen gelblichbraun bis schwarzbraun und matt, bei den krystallinischen Chondriten sehr rauh und lose anhaftend, beim Tadjerit von der übrigen Masse nicht zu unterscheiden. Die als ge a dert bezeichneten Unterabteilungen weichen von den übrigen dadurch ab, dass die hierzu gerechneten Meteoriten von teils spärlich, teils reichlich auftretenden schwarzen oder 
auch metallischen Adern durchsetzt werden, die auf Bruchflächen häufig als Harnisch erscheinen. Die schwarzen Adern lassen sich in vielen Fällen als Rinden-Infiltrationen erklären. Der breccienartige Typus beruht auf einem Zusammenvorkommen der verschiedenfarbigen Massen, insbesondere auf einem Gemisch der weissen und intermediären chondritischen Massen einerseits und denjenigen der grauen Chondrite andererseits; infolgedessen finden sich viele breccienähnliche, graue Chondrite (23) gegenüber wenigen breccienähnlichen, weissen (6) und intermediären Chondriten (7).

9. I. Howarditische Chondrite, Cho u. Choa. $\mathrm{G}=108.3 \mathrm{O} 2 \mathrm{Kgr}$. $(100-200)$.

Diese Gruppe umfasst die Uebergangsglieder von den Howarditen zu den Chondriten.

\begin{tabular}{lcccc|lrrrr} 
& U & N & B & W & & U & N & B & W \\
BorgoSan Donino & - & 1.516 & 23 & 53 & Santa Barbara & 93 & 85.167 & 4 & 24 \\
*Harrison County & 0.700 & 0.300 & 21 & $86-67$ & Sevilla & $?$ & 0.095 & 4 & 246 \\
Iharaota (Choa) & 0.372 & 0.314 & 5 & 147 & Siena & - & 4.163 & 40 & 40 \\
Krähenberg & 16.5 & 15.091 & 17 & 31 & Sitathali & 1.413 & 1.413 & 10 & 67 \\
Mauritius & - & 0.243 & 5 & 147 & & & &
\end{tabular}

\section{Weisse Chondrite.}

Gelblich-weisse, tuffartige Massen mit meistens gleichfarbigen Chondren.

10.

a. Weisse Chondrite, Cw. $\mathrm{G}=53.995 \mathrm{Kgr}$. (50-100).

\begin{tabular}{|c|c|c|c|c|c|c|c|c|c|}
\hline & $\mathrm{U}$ & $\mathrm{N}$ & B & W & & & $\mathrm{N}$ & B & W \\
\hline Bachmut & 16 & 2.531 & $24:$ & $50(-31)$ & Mauerkirchen & 19 & 12.558 & 40 & 31 \\
\hline ocas & & 0.014 & 6 & & *Middlesbrougl & h 1.594 & 0.028 & 4 & (114) \\
\hline Cabezzo de & & & & & *Milena & 5.5 & 1.946 & 26 & $(67-) 50$ \\
\hline Mayo & 25 & 1.208 & 17( & $(84-) 39$ & Montlivault & - & 0.539 & 6 & 144 \\
\hline De Cewsville & .340 & 0.340 & 1 & 267 & & 0.070 & 0.049 & 8 & \\
\hline Dolgowol & 1.6 & 1.209 & 11 & 8 & *Mordvinovka & 33 & 3.481 & 32 & $50-31$ \\
\hline High P & - & 0.152 & 14 & 181 & $*$ Ogi & 10.2 & 4.283 & 6 & $86-67$ \\
\hline Karakol & 3 & 2.788 & 7 & 86 & Öst & 6 & 1.500 & 33 & $67-50$ \\
\hline Kusi: & ? & 0.004 & 3 & & & ? & 0.030 & 2 & \\
\hline $\mathrm{La} \mathrm{B}$ & 2.8 & 2.785 & 8 & 86 & Pnompehn & 1 & 0.041 & 2 & \\
\hline Les Ormes & 0.125 & 0.113 & 7 & 246 & *Tourinnes-la- & & & & \\
\hline Lundsgård & & & 7 & 58 & Grosse & 14.5 & 3.440 & 32 & $50-39$ \\
\hline Iascombe & & 0.566 & 7 & 144 & Zaborzika & 4 & 3.867 & 18 & 63 \\
\hline
\end{tabular}

II. b. Geaderte weisse Chondrite, Cwa, $\mathrm{G}=263.670 \mathrm{Kgr}$. $(200-500)$.

\begin{tabular}{|c|c|c|c|c|c|c|c|c|c|}
\hline & U & $\mathrm{N}$ & B & W & & $\mathrm{U}$ & $\mathrm{N}$ & B & W \\
\hline Angers & 1 & 0.149 & 9 & 108 & Chandpur & 1.201 & 0.974 & 6 & 86 \\
\hline Asco & - & 0.042 & 6 & & Dhulia & ? & 0.019 & 3 & \\
\hline Aumière & - & 1.556 & 12 & 50 & Drake Creek & - & 5.501 & 25 & 23 \\
\hline Buschhof & 5 & 3.521 & 30 & 30 & *Forsyth & 16 & 0.741 & 24 & $(50-) 23$ \\
\hline Castine & 0.042 & 0.022 & 13 & & Futtehpore & - & 3.300 & 21 & 30 \\
\hline
\end{tabular}


$45^{\circ}$ Systemat. Uebersicht der erhaltenen Meteoriten u. ihrer Tauschwerte.

\begin{tabular}{lcccc|lcrrr} 
& U & N & B & W & & U & N & B & W \\
Galapian & $?$ & 0.056 & 3 & 267 & *Maêmê & 0.328 & 0.027 & 3 & \\
Girgenti & 3.5 & 3.389 & 29 & 30 & Minas Geraës & 1.224 & 1.212 & 5 & 68 \\
Grossliebenthal 8 & 8.048 & 17 & 29 & Mócs & 174 & 155.632 & 103 & 8 \\
*Hartford & 21 & 9.177 & 47 & $23-18$ & Pirgunje & $?$ & 0.743 & 2 & 124 \\
Honolulu & - & 2.395 & 26 & 30 & Politz & 3.5 & 2.757 & 25 & 30 \\
Jigalowka & $?$ & 1.552 & 17 & 50 & Sauguis & 3 & 0.208 & 10 & $82(-50)$ \\
*Kalumbi & 4.6 & 0.192 & 2 & $(212-74)$ & Schönenberg & 8.015 & 7.846 & 11 & 29 \\
Kikino & $?$ & 0.193 & 8 & 147 & Senhadja & - & 10.334 & 16 & 23 \\
Killeter & - & 0.140 & 8 & 147 & Ski & 0.850 & 0.750 & 13 & 63 \\
Kuleschowka & 6 & 5.219 & 24 & 23 & Slobodka-Partsch ? & 0.940 & 3 & 124 \\
Lissa & - & 12.397 & 29 & 18 & Wirba & 3.600 & 3.200 & 6 & 51 \\
Lucé & 3 & 0.263 & 14 & $82(-50)$ & WoldCottage 25.5 & 21.175 & 31 & 14
\end{tabular}

12. c. Breccienähnliche weisse Chondrite, Cwb. $G=19.655 \mathrm{Kgr}$. (20-50).

\begin{tabular}{lcrrr|lrrrr} 
& U & N & B & W & & U & N & B & W \\
Aleppo & 3 & 2.225 & 5 & 111 & *Pacula & 3.361 & 1.191 & 7 & $147-111$ \\
Bandong & 11.25 & 11.110 & 26 & 40 & Uden & 0.720 & 0.708 & 9 & 136 \\
*Monte Milone & 5 & 2489 & 14 & 82 & Vavilovka & $?$ & 1.932 & 9 & 108
\end{tabular}

\section{Intermediäre Chondrite.}

Uebergangsglieder von den weissen zu den grauen Chondriten.

I3. a. Intermediäre Chondrite, Ci. G $=112.743 \mathrm{Kgr} .(200-500)$.

\begin{tabular}{lrrrc|lrrrr} 
& U & N & B & W & & U & N & B & W \\
*Alfianello & 200 & 54.866 & 75 & $11-8$ & Fisher & 0.500 & 0.500 & 2 & 124 \\
Butsura & 22 & 20.448 & 23 & 14 & Laborel & 2.257 & 2.047 & 9 & 38 \\
Canellas & 1 & 0.805 & 10 & 63 & Mhow & 0.354 & 0.333 & 6 & 111 \\
Charwallas & 3 & 0.071 & $9(136-) 63$ & Rakowka & - & 2.642 & 12 & 38 \\
Deal & - & 0.010 & 9 & & Saint Caprais & 0.282 & 0.202 & 3 & 160 \\
Dhurmsala & $(145)$ & 30.245 & 55 & 14 & Vago &.- & 0.009 & 1 & \\
*Favars & 1.5 & 0.555 & 13 & 63 & & & &
\end{tabular}

I4. Geaderte intermediäre Chondrite, Cia. $\mathrm{G}=180.199 \mathrm{Kgr} .(200-500)$.

\begin{tabular}{|c|c|c|c|c|c|c|c|c|c|}
\hline & U & $\mathrm{N}$ & B & W & & U & $\mathrm{N}$ & B & w \\
\hline Agen & 30 & 5.274 & 28 & 23 & Nerft & 10.349 & 9.972 & 23 & 23 \\
\hline Barntrup & 0.017 & 0.016 & 2 & & *NewConc. & 209 & 97.811 & 56 & $(11-) 8$ \\
\hline Berlanguillas & 2.5 & 1.376 & 17 & 50 & Orange River & ? & 0.008 & 1 & \\
\hline *Bori & 18.617 & 5.287 & 3 & $(57-) 32$ & Salles & 10 & 2.900 & 22 & 30 \\
\hline Château-Ren. & 20 & 17.922 & 52 & 18 & Schellin & - & 0.007 & 4 & \\
\hline Dandapur & - & 6.047 & 9 & 29 & Toulouse & - & 0.420 & 18 & 82 \\
\hline Durala & - & 12.763 & 10 & 23 & Vouillé & 20 & 16.619 & 30 & 18 \\
\hline Duruma & 0.577 & 0.575 & 9 & 63 & *Zabrodje & 3.155 & 0.300 & & $160-7$ \\
\hline Macao & - & 2.902 & 18 & 38 & & & & & \\
\hline
\end{tabular}


Systemat. Uebersicht der erhaltenen Meteoriten u. ihrer Tauschwerte. $45^{\mathrm{I}}$

15. c. Breccienähnliche intermediäre Chondrite, Cib. $\mathrm{G}=63.071 \mathrm{Kgr} .(50-100)$.

\begin{tabular}{lrrrr|llrrr} 
& U & N & B & W & & U & N & B & W \\
Bjelokrynitschie ? & 1.662 & 18 & 84 & Pulsora & - & 0.755 & 6 & 144 \\
Chandakapoor & - & 10.745 & 28 & 31 & Saint-Mesmin & - & 9.601 & 18 & 49 \\
L'Aigle & - & 36.843 & 87 & 23 & Shytal & - & -18.188 & 10 & 63 \\
Luponnas & 16 & 0.277 & 9 & $136(-63)$ & & & & &
\end{tabular}

\section{Graue Chondrite.}

Gelblich- bis bläulichgraue, tuffartige Massen mit verschiedenfarbigen Chondren, welche mit der Grundmasse fest verbunden sind.

\begin{tabular}{|c|c|c|c|c|c|c|c|c|c|}
\hline 6. & a. Graue & Chond & rite, & $\mathrm{Cg}$. & $\mathrm{G}=436.114 \mathrm{~K}$ & $\mathrm{Kgr}$ & $-500)$ & & \\
\hline & U & N & B & W & & U & $\mathrm{N}$ & B & w \\
\hline otschetschki & 0.614 & 0.550 & 6 & 86 & Knyahinya & 476 & 423.120 & 97 & \\
\hline ads & 0.161 & 0.159 & 2 & 212 & Lutschaunig & ? & 0.156 & 3 & 212 \\
\hline & 6 & 4.772 & 14 & 38 & Seres & 8.4 & 7.287 & 19 & 29 \\
\hline & : & 0.070 & 4 & 185 & Tounkin & 2 & Spl. & 1 & \\
\hline
\end{tabular}

17. b. Geaderte graue Chondrite, Cga. $G=345.728 \mathrm{Kgr}$. $(200-500)$.

\begin{tabular}{|c|c|c|c|c|c|c|c|c|c|}
\hline & U & $N$ & B & W & & U & $\mathrm{N}$ & B & W \\
\hline Aldsworth & 1 & 0.572 & 8 & 86 & *Limerick & 48 & 10.878 & 241 & $18-14$ \\
\hline Alessandria & 1.3 & 0.960 & 20 & 63 & Lixna & 20 & 5.213 & 30 & 28 \\
\hline Barbotan & - & 5.911 & 44 & 23 & Monroe & 8.8 & 4.798 & $42:$ & $30-88$ \\
\hline Blansko & $\left.0.350^{\prime}\right)$ & 0.243 & 13 & 82 & Mooresfort & 3.5 & 2.974 & 30 & 30 \\
\hline *Charsonville & & 6.903 & 40 & 23 & Mornans & 1.300 & 1.187 & 4 & 68 \\
\hline Cronstadt & - & 1285 & 6 & 68 & Oczeretna & ? & 0.124 & 3 & 212 \\
\hline Danville & 2.038 & 0.230 & 13 & $82-63$ & Ohaba & 16.25 & 16.022 & 13 & 23 \\
\hline Darmstadt & - & 0.102 & 9 & 108 & Parnallee & 74 & 72.237 & 44 & 11 \\
\hline *Fukutomi & 7.680 & 0.034 & 3 & $(267-) 40$ & Pultusk & - & 200932 & 129 & 6 \\
\hline Grüneberg & 1 & 0.971 & 15 & 63 & Saurette & 3 & 1.560 & 15 & 50 \\
\hline Hungen & 0.112 & 0.111 & 8 & 147 & Udipi & ? & 3.673 & 10 & 38 \\
\hline Kadonah & ? & 0.089 & 8 & 185 & Umbala & 0.100 & 0.068 & 8 & 185 \\
\hline Kakowa & 0.577 & 0.522 & 11 & 63 & Wessely & 3.780 & 3.720 & 9 & 38 \\
\hline Kerilis & 5 & 4.409 & 10 & 38 & & & & & \\
\hline
\end{tabular}

18. c. Breccienähnliche graue Chondrite, Cgb. $\mathrm{G}=363.571 \mathrm{Kgr} .(200-500)$.

\begin{tabular}{lcrrr|llrll} 
& $\mathrm{U}$ & $\mathrm{N}$ & $\mathrm{B}$ & $\mathrm{W}$ & & $\mathrm{U}$ & $\mathrm{N}$ & $\mathrm{B}$ & $\mathrm{W}$ \\
Akburpoor & 1.8 & 1.663 & 10 & 50 & Molina & - & 114.298 & 15 & 11 \\
Assam & $(1.163)$ & 1.251 & 9 & 50 & Nulles & - & 8116 & 11 & 29 \\
Borodino & $?$ & 0.325 & 4 & 111 & Okniny & 12 & 0.309 & 9 & $82-$ - \\
Bueste & 1.82 & 1.588 & 11 & 50 & Quinçay & $?$ & 0.031 & 4 & \\
Cangas de & - & 6.880 & 19 & 29 & Salt Lake City & 0.875 & 0.875 & 6 & 86 \\
Castalia & 7.800 & 6.317 & 21 & 23 & Sena & 4.5 & 2.045 & 9 & 38 \\
Chantonnay & 35 & 9.942 & 61 & $23-18$ & Slavetic & 1.708 & 1.420 & 8 & 68 \\
*Doroninsk & 3.8 & 0.571 & 11 & $63-50$ & Stalldalen & 34 & 31.926 & 29 & 14 \\
Homestead & 300 & 124.492 & 62 & $8-6$ & Supuhee & $?$ & 4.496 & 12 & 38 \\
Khetree & - & 0.078 & 6 & 185 & Tomhannock & & & & \\
Mexico & - & 0.169 & 6 & 147 & Creek & 1.5 & 1.496 & 19 & 50 \\
MezöMadaras & - & 24.393 & 47 & 14 & Tysnes & 21.7 & 20.894 & 19 & 17
\end{tabular}

I) Ausserdem noch ein Stein von 8 Lot, welcher 1866 aufgefunden wurde. 
$45^{2}$ Systemat. Uebersicht der erhaltenen Meteoriten u. ihrer Tauschwerte.

19. V. Schwarze Chondrite, Cs. $G=I 38.827$ Kgr. $(200-500)$.

Dunkelgraue bis schwarze, fest gefügte, chondritische Massen, deren Färbung teils auf fein-verteilte Kohle, teils auf Magnetkies zurückgeführt wird. Die aus der Grundmasse hervortretenden Chondren sind meist von heller Farbe.

\begin{tabular}{lcccc|lrrrc} 
& U & N & B & W & & U & N & B & W \\
*Baratta & 137 & 0.025 & 2 & $(?-21)$ & Mac Kinney & 100 & 79.613 & 3 & 27 \\
*Bishunpur & 1.038 & 0.570 & 2 & 124 & *Renazzo & 5 & 1.083 & 25 & $40-30$ \\
Farmington & 84 & 53.487 & 38 & 11 & *Sewrukof & 98 & 0.792 & 13 & $(63-) 14$ \\
Grossnaja & 3.980 & 3.257 & 22 & 30 & & & &
\end{tabular}

VI. Kügelchenchondrite.

Zahlreiche, feste und gut ausgebildete Chondren. Bei der ersten Abteilung (Cco u. Ccn) ist die tuffartige Grundmasse sehr spärlich entwickelt, so dass die Steine fast nur aus Chondren bestehen. Bei den folgenden 3 Abteilungen (Cc, Cca u. $\mathrm{Ccb}$ ) ist sie reichlicher vorhanden, und von lockerer Beschaffenheit, so dass die Chondren sich leicht loslösen lassen. Bei der letzten Abteilung (Cck) wird die Grundmasse etwas krystallinisch und fest, so dass beim Zerkleinern der Stücke die Chondren zum Teil mit entzwei brechen.

20. a. Ornansite und Ngawit, Cco u. Ccn. $\mathrm{G}=8.0 \mathrm{I}_{3} \mathrm{Kgr} .(5-10)$.

$\begin{array}{lcrrr} & \mathrm{U} & \mathrm{N} & \mathrm{B} & \mathrm{W} \\ \text { Ngawi (Ccn) } & 1.393 & 1.340 & 9 & 181 \\ \text { Ornans } & 6.050 & 5.059 & 18 & 106\end{array}$

2I. b. Kügelchenchondrite, Cc. $G=282.431 \mathrm{Kgr} .(200-500)$.

\begin{tabular}{|c|c|c|c|c|c|c|c|c|c|}
\hline & $\mathrm{U}$ & $\mathrm{N}$ & B & W & & $\mathrm{U}$ & $\mathrm{N}$ & B & W \\
\hline Albareto & - & 1.159 & 16 & 50 & Jhung & $?$ & 3.414 & 15 & 38 \\
\hline Assisi & 1.795 & 1.551 & 18 & 50 & Judesegeri & 0.716 & 0.531 & 4 & 86 \\
\hline Assun & 50 & 9.527 & 51 & $23-18$ & Kaee & - & 0.214 & 4 & 111 \\
\hline Avilez & $?$ & 0.236 & 4 & 111 & Kheragur & ? & 9.545 & 5 & 86 \\
\hline Benares & - & 2.416 & 26 & 30 & Krasnoj-Ugol & - & 2.530 & 6 & 51 \\
\hline Bjelaja Zerkow & - & 1.826 & 13 & 50 & Lancé & 51.75 & 48.883 & 14 & 17 \\
\hline Borkut & 6.7 & 4.086 & 14 & 38 & *Le Pressoir & 1.133 & 0.376 & 15 & $(82-) 63$ \\
\hline Cape Girardeau & $u-$ & 2.358 & 7 & 51 & Lesves & 2 & 1.360 & 4 & 68 \\
\hline Collescipoli & 3.430 & 1.802 & 21 & $40-30$ & *Little Piney & $20(?)$ & 0.409 & 25 & $(65-?)$ \\
\hline Eichstädt & 3.185 & 1.168 & 18 & 50 & Marmande & - & 0.063 & 6 & 185 \\
\hline Epinal & - & 0.301 & 10 & 82 & Misshof & 4991 & 4.182 & 18 & 38 \\
\hline Gargantillo & 1 & 0.789 & 13 & 63 & Motta di Conti & i 8.620 & 8.185 & 8 & 40 \\
\hline Gnadenfrei & 1.75 & 1.059 & 14 & 50 & Muddoor & - & 2.475 & 9 & 38 \\
\hline Gopalpur & 1.634 & 1.582 & 5 & 68 & Mühlau & 0.005 & 0.005 & 1 & \\
\hline Gross-Divina & - & 10.715 & 9 & 23 & Nanjemoy & 7 & 2.525 & 22 & 30 \\
\hline Hessle & - & 22.895 & 53 & 14 & Quenggouk & 6.044 & 5.541 & 21 & 23 \\
\hline Itapicuru-mirim & $\mathrm{m} 2.024$ & 2.024 & 6 & 51 & Rochester & 0.340 & 0.195 & 18 & $108-82$ \\
\hline
\end{tabular}


Systemat. Uebersicht der erhaltenen Meteoriten u. ihrer Tauschwerte. 453

\begin{tabular}{|c|c|c|c|c|c|c|c|c|c|}
\hline \multirow{2}{*}{\multicolumn{2}{|c|}{ SanEmigdioRange - }} & $\mathrm{N}$ & B & w & \multicolumn{2}{|r|}{ U } & $\mathrm{N}$ & B & w \\
\hline & & 0.180 & 10 & 108 & Timoschin & 65.5 & 50.099 & 29 & 11 \\
\hline *Searsmont & 0900 & 0.345 & 27( & $65-\lcm{50}$ & Yatoor & 13 & 11.987 & 19 & 23 \\
\hline Slobodka & 2.75 & 2.696 & 12 & 38 & Zebrak & 1.873 & 1.271 & 10 & 50 \\
\hline Sokobanja & 48 & 40.329 & 57 & 14 & Zsadany & 0.552 & 0.414 & 10 & 82 \\
\hline ieschitz & - & 28.233 & 19 & 17 & & & & & \\
\hline
\end{tabular}

22. c. Geaderte Kügelchenchondrite, Cca. G=48.498 Kgr. $(50-100)$.

\begin{tabular}{lcrrr|lrrrr} 
& $\mathrm{U}$ & $\mathrm{N}$ & $\mathrm{B}$ & $\mathrm{W}$ & & $\mathrm{U}$ & $\mathrm{N}$ & $\mathrm{B}$ & $\mathrm{W}$ \\
Nammianthal & 4.519 & 4.244 & 8 & 86 & Trenzano & - & -10.515 & 37 & 31 \\
Phû Long & $?$ & 0.365 & 1 & 267 & Utrecht & - & 9.870 & 30 & 39 \\
Piquetberg & $?$ & 0.038 & 1 & & *Werche Tschirs- & & & \\
Saint-Denis-Westrem & 0.700 & 0.575 & 11 & 106 & kaja & 8.30 & 0.177 & 3 & $(354-) 96$ \\
Tennasilm & 28.5 & 22.714 & 16 & 29 & & & &
\end{tabular}

23. d. Breccienähnliche Kügelchenchondrite, $\mathrm{Ccb} . \mathrm{G}=367.391 \mathrm{Kgr} .(200-500)$.

\begin{tabular}{lcrrr|llrrr} 
& $\mathrm{U}$ & $\mathrm{N}$ & $\mathrm{B}$ & $\mathrm{W}$ & & $\mathrm{U}$ & $\mathrm{N}$ & $\mathrm{B}$ & W \\
Bath & 21.2 & 14.648 & 14 & 23 & *Kesen & $120(?)$ & 7.088 & 18 & $29-$ ? \\
Bremervörde & 7.25 & 6.424 & 34 & 23 & Ochansk & - & 169.203 & 40 & 8 \\
Cereseto & - & 4.361 & 17 & 38 & Pirthalla & 1.160 & 1.041 & 4 & 68 \\
*Feid Chair & 0.380 & 0.025 & 1 & & Ploschkowitz & - & 0.037 & 4 & \\
Forest City & - & 122.037 & 42 & 8 & Tabor & - & 6.606 & 27 & 23 \\
Gütersloh & 1.5 & 1.161 & 13 & 50 & Waconda & 26 & 15.786 & 47 & 18 \\
Heredia & - & 0.707 & 10 & 63 & Weston & - & 18.267 & 51 & 18
\end{tabular}

24. e. Krystallinische Kügelchenchondrite, Cck. $G=24.634 \mathrm{Kgr} .(20-50)$.

\begin{tabular}{lcccc|lcccc} 
& $\mathrm{U}$ & $\mathrm{N}$ & $\mathrm{B}$ & $\mathrm{W}$ & & $\mathrm{U}$ & $\mathrm{N}$ & $\mathrm{B}$ & $\mathrm{W}$ \\
Beaver Creek & 14. & 11.864 & 10 & 50 & Prairie Dog Creek 2.9 & 2.900 & 3 & 160 \\
*Bethlehem & $0.03(?)$ & 0.003 & 3 & & Richmond & 1.8 & 0.849 & 22 & $108-86$ \\
Lumpkin & 0.357 & 0.128 & 12 & $233-176$ & Savtschénskoje & 2.565 & 2.565 & 2 & 160 \\
Menow & 10.5 & 6.325 & 26 & 50 & & & &
\end{tabular}

\section{Krystallinische Chondrite.}

Krystallinische Grundmassen mit harten Chondren fest verwachsen.

25. a. Krystallinische Chondrite, Ck. G=835.880 Kgr. (500- I000).

\begin{tabular}{|c|c|c|c|c|c|c|c|c|c|}
\hline & U & $\mathrm{N}$ & B & w & & U & N & B & W \\
\hline Carcote & - & 0.325 & 5 & 86 & Long Island & 558 & 558 & 1 & 10 \\
\hline${ }^{*}$ Cosina & 1.2 & 0.284 & 91 & $(63-) 49$ & $*_{\text {Mainz }}$ & 1.75 & 0.685 & 194 & $49(-39)$ \\
\hline Daniels Kuil & 1.048 & 0.897 & 20 & 49 & Motecka-Nu & gla ? & 2.295 & 12 & 29 \\
\hline Djati Pen- & & & & & Pillistfer & 25.5 & 23.561 & 30 & 11 \\
\hline gilon & 166.4 & 160.994 & 26 & 7 & Pokra & - & 0.342 & 4 & 86 \\
\hline *Dundrum & 2.225 & 0.340 & & $(86-) 53$ & Segowlee & - & 7.099 & 19 & 23 \\
\hline Erxleben & 2.25 & 1.166 & 32 & 31 & Simbirsk & $?$ & 1.517 & 3 & 76 \\
\hline Guareña & 32 & 32.000 & 5 & 18 & Stawropol & 1.632 & 1.527 & 13 & 39 \\
\hline Kernouvé & 40 & 31.142 & 22 & 11 & *Tjabé & 20 & 1.112 & 24( & $(31-14$ \\
\hline Khairpur & 13 & 9.809 & 17 & $23-13$ & Toke-uchi-n & ura 0.066 & 0.066 & 3 & 207 \\
\hline Kleinwenden & 3.25 & 2.719 & 11 & 29 & & & & & \\
\hline
\end{tabular}


26. b. Geaderte krystallinische Chondrite, Cka. G=7.849 Kgr. ( $10-20$ ).

\begin{tabular}{ccccc|lcccc} 
& U & N & B & W & & U & N & B & W \\
Pipe Creek & 13.5 & 7.414 & 18 & 84 & Vernon County & 0.700 & 0.435 & 12 & 233
\end{tabular}

27. c. Breccienähnliche krystallinische Chondrite, Ckb. G= $144.785 \mathrm{Kgr}$. $(200-500)$.

\begin{tabular}{ccccc|lcrrr} 
& U & N & B & W & & U & N & B & W \\
Bluff & 146 & 74.400 & 41 & $11-8$ & Ensisheim & 127 & 70.385 & 65 & 11
\end{tabular}

28. VIII. Kohlige Chondrite, $\mathrm{K}$ u. Kc. $\mathrm{G}=29.660 \mathrm{Kgr} .(20-50)$.

Durch einige Prozent Kohlenstoff mattschwarz gefärbte chondritische Massen, welche wenig oder kein Eisen enthalten und niedriges spezifisches Gewicht zeigen.

\begin{tabular}{|c|c|c|c|c|c|c|c|}
\hline & $\mathrm{N}$ & B & W & & U & $\mathrm{N}$ & B \\
\hline Alais & $\begin{array}{ll}6 & 0.266\end{array}$ & 32 & $140(-108)$ & Kaba & 4 & 2.316 & 22 \\
\hline *Cold Bokke- & & & & Mighëi & - & 7.948 & 27 \\
\hline veld $\quad(100$ ? & 4.108 & 49 & $65-50$ & Nagaya & - & 2.528 & 22 \\
\hline Grazac & 0.022 & 1 & & Orgueil & - & 11.523 & 50 \\
\hline Indarch & 0.949 & 11 & $(136-) 50$ & & & & \\
\hline
\end{tabular}

29.

IX. Orvinit, Co. $\mathrm{G}=2.324 \mathrm{Kgr} .(2-5)$.

Hellere Bruchstücke eines typischen Chondrit liegen in einer schwärzlichen Bindemasse, welch' letztere deutliche Fluidalstruktur zeigt.

$\begin{array}{ccccc} & \text { U } & \text { N } & \text { B } & \text { W } \\ \text { *Orvinio } & 3.396 & 2.324 & 26 & 140\end{array}$

30.

$$
\text { X. Tadjerit, Ct. } \mathrm{G}=8.843 \mathrm{Kgr} \text {. }(5-\mathrm{IO}) \text {. }
$$

Dunkler, grösstenteils aus halbglasiger Grundmasse bestehender Chondrit ohne erkennbare Rinde.

$\begin{array}{ccccc} & \text { U } & \text { N } & \text { B } & \text { W } \\ \text { Tadjera } & 8.843 & 8.843 & 13 & 106\end{array}$

31 .

XI. Ureilit, Cu. $\mathrm{G}=4.528 \mathrm{Kgr}$. $(2-5)$.

Schwarze, teils chondritische (Dyalpur, Goalpara), teils körnige und alsdann grösstenteils aus Olivin bestehende Massen, welche kontinuierliche Eisenadern (nach Brezina) enthalten und demnach als Uebergänge zur Klasse D aufgefasst werden können. Nowo-Urei zeichnet sich durch seinen Gehalt an Diamanten aus.

\begin{tabular}{lcccc|lcccc} 
& $\mathrm{U}$ & $\mathrm{N}$ & $\mathrm{B}$ & $\mathrm{W}$ & & $\mathrm{U}$ & $\mathrm{N}$ & $\mathrm{B}$ & $\mathrm{W}$ \\
Dyalpur & $?$ & 0.304 & 5 & 514 & Nowo-Urei & - & 2.058 & 16 & 176 \\
Goalpara & 2.636 & 2.166 & 9 & 176 & & & & &
\end{tabular}


32. XII. Meteorsteine, welche wegen mangelnder Bestimmung noch keine Stellung im System gefunden haben. $G=12.468 \mathrm{Kgr}$.

\begin{tabular}{|c|c|c|c|c|c|c|c|}
\hline & $\mathrm{U}$ & $\mathrm{N}$ & B W & & U & $\mathrm{N}$ & B \\
\hline *Bherai & ? & 0.017 & 1 & Mern & 4.1 & 3.790 & 1. \\
\hline Chail & - & Spl. & 1 & *Morristown & 16.3 & 1.820 & 1 \\
\hline *Eli Eluat & ? & 0.002 & 1 & Nageria & 0.018 & 0.014 & 2 \\
\hline *Gilgoin & 64 & 0.008 & 1 & *Nagy-Borove & ? & $?$ & ? \\
\hline Guễa & - & 1.915 & 1 & Perth & - & 0.001 & 2 \\
\hline Gurram Konda & ? & 0.010 & 1 & *Sabetmahet & 1.297 & 0.003 & 1 \\
\hline Jamkheir & ? & 0.024 & 2 & *Saint Christophe & & & \\
\hline Kakangarai & 0.347 & 0.342 & 3 & la-Chartreuse & 5.5 & - & - \\
\hline *La Charca & 0.399 & Spl. & 1 & San Pedro Springs & s - & 0.072 & 1 \\
\hline Linum. & 1.863 & 1.730 & 2 & Travis County & - & 2.657 & 2 \\
\hline *Makariwa & 2 & 0.063 & 1 & *Wairarapa & 13.6 & - & - \\
\hline
\end{tabular}

\section{Nickeleisen mit Silikaten.}

33. I. Lodranit, Lo. $\mathrm{G}=0.970 \mathrm{Kgr}$. $(0.5-\mathrm{I})$.

Krystallinisch-körniges Gemenge von Olivin und Bronzit in einem feinen, zusammenhängenden Netz von Nickeleisen. Auf polierten Schnittflächen erscheint dieses Eisennetz ohne Zusammenhang.

$\begin{array}{ccccc} & \text { U } & \text { N } & \text { B } & \text { W } \\ \text { Lodran } & \text { ? } & 0.970 & 11 & 491\end{array}$

34. II. Mesosiderite (Grahamite), M. G=482.925 Kgr. (500-1000).

Olivin, Bronzit, Plagioklas und Augit von teils chondritischer, teils krystallinischer Struktur in einem Netz von Nickeleisen. Auf polierten Schnittflächen erscheint dieses Eisennetz ohne Zusammenhang.

\begin{tabular}{lcrrr|lcccc} 
& $\mathrm{U}$ & $\mathrm{N}$ & $\mathrm{B}$ & $\mathrm{W}$ & & $\mathrm{U}$ & $\mathrm{N}$ & $\mathrm{B}$ & W \\
Barea & 3 & 2.306 & 4 & 40 & *Inca & 12.25 & 3.145 & 20 & $(29-) 23$ \\
Crab Orchard & 43 & 34.067 & 29 & 11 & Lujan & 0.009 & 0.008 & 1 & \\
Doña Inez & 7.25 & 5.686 & 22 & 18 & Miney & 90 & 57.022 & 34 & 8 \\
Estherville & 337 & 334.714 & 72 & 5 & Vaca Muerta ? & 34060 & 44 & 11 \\
Hainholz & 16.5 & 11.344 & 45 & 14 & *Veramin & 50 & 0.573 & 13 & $49-$ ?
\end{tabular}

\section{III. Siderophyr, S. $\mathrm{G}=8 \mathrm{I} .786 \mathrm{Kgr}$. $(50-\mathrm{IO0})$.}

Bronzit, Nickeleisen und accessorisch Asmanit (Tridymit). Das oktaëdrisch aufgebaute Eisen ist von einheitlicher Orientierung und zeigt deutliche Widmanstätten'sche Figuren. Auf Schnittflächen tritt es nur teilweise zusammenhängend auf.

$\begin{array}{ccccc} & \text { U } & \text { N } & \text { B } & \text { W } \\ \text { Steinbach } & - & 81.786 & 73 & 18\end{array}$


$45^{6}$ Systemat. Uebersicht der erhaltenen Meteoriten u. ihrer Tauschwerte.

36. IV. Pallasite, P. $G=I 742.519 \mathrm{Kgr}$. $(1000-2000)$.

Nickeleisen und Olivin. Auf Schnittflächen tritt das erstere in $\mathrm{zu}$ sammenhängendem Netz auf.

\begin{tabular}{|c|c|c|c|c|c|c|c|c|c|}
\hline $\begin{array}{r}\text { Bitburg/ } \\
\text { umgesc }\end{array}$ & U & 0.034 & B & W & $\begin{array}{l}\text { Eagle Station } \\
\text { Imilac }\end{array}$ & $\begin{array}{c}\mathrm{U} \\
36.5 \\
-\end{array}$ & $\begin{array}{c}\mathrm{N} \\
26.120 \\
282.317\end{array}$ & $\begin{array}{c}\text { B } \\
34 \\
93\end{array}$ & $\begin{array}{r}W \\
8 \\
4\end{array}$ \\
\hline Brahin & 100 & 92.886 & 24 & 7 & Jamyschewa & 6 & 4.036 & 19 & 23 \\
\hline Brenham & 900 & $\left.769.108^{1}\right)$ & 68 & 3 & Krasnojarsk & 687 & 567.995 & 109 & 3 \\
\hline Calderilla & 0.021 & 0.019 & 1 & & Port Orford & - & 0.004 & 4 & \\
\hline
\end{tabular}

37. V. Meteoreisenbreccie von Copiapo, Obc. $\mathrm{G}=22.939 \mathrm{Kgr}$. $(20-50)$.

Breccienähnliches, oktaëdrisches Nickeleisen mit krystallinischem Chondrit. $\begin{array}{ccccc} & \text { U } & \text { N } & \text { B } & \text { W } \\ \text { Copiapo } & ? & 22.939 & 13 & 38\end{array}$

38. VI. Meteoreisen von Netschaëvo, Omn. $G=5.858 \mathrm{Kgr}$. $(5-\mathrm{IO})$. Oktaëdrisches Nickeleisen mit krystallinischem Chondrit.

$\begin{array}{ccccc} & \text { U } & \text { N } & \text { B } & \text { W } \\ \text { Netschaëvo } & ? & 5.858 & 27 & 84\end{array}$

\section{E. Meteoreisen.}

Nickeleisen mit untergeordneten Mengen von Schwefeleisen oder Graphit oder Phosphornickeleisen (Schreibersit, Rhabdit) oder Cohenit oder mehreren dieser Verbindungen.

\section{Oktaëdrische Meteoreisen.}

Nickeleisen nach den Flächen eines Oktaëders schalig aufgebaut, so dass nickelreichere und nickelärmere Lamellen auf einander folgen. Beim Aetzen von polierten Flächen entstehen Widmanstätten'sche Figuren.

39. a. Oktaëdrische Eisen mit feinsten Lamellen (Lamellenbreite etwa o.r mm), Off. $\mathrm{G}=36.054 \mathrm{Kgr} .(50-100 ;$ ev. $2000-\infty)$.

\begin{tabular}{lcccc|lcccc} 
& $\mathrm{U}$ & $\mathrm{N}$ & $\mathrm{B}$ & $\mathrm{W}$ & & $\mathrm{U}$ & $\mathrm{N}$ & $\mathrm{B}$ & $\mathrm{W}$ \\
Butler & 36 & 27.550 & 35 & {$[23(-6)]$} & *Tazewell & 27 & 7.510 & 39 & {$[31(-11)]$} \\
Cowra & $?$ & 0.223 & 2 & {$[267(-74)]$} & Werchne Dnie- & & & & \\
*Ranchito & $(50000)$ & 0.306 & 9 & {$[136(-2)]$} & prowsk & - & 0.465 & 10 & {$[136(-38)]$}
\end{tabular}

1) Davon 272 gr. Brenham (Anderson). 
40. b. Oktaëdrische Eisen mit feinen Lamellen (Lamellenbreite $0.1_{5}-0.4 \mathrm{~mm}$ ), Of; auch VictoriaWest-Eisen, Ofv. $\mathrm{G}=224.263 \mathrm{Kgr}$. (500-1000).

\begin{tabular}{|c|c|c|c|c|c|c|c|c|}
\hline & U & $\mathrm{N}$ & W & & $\mathrm{U}$ & $\mathrm{N}$ & B & W \\
\hline *Bear Creek & 200 & 2.389 & $26(23-) 7$ & *Lion River & 80.5 & 1.913 & 22 & $(31-) 8$ \\
\hline Bella Roca & 33.0 & 26.387 & $26 \quad 11$ & *Madoc & 167.5 & 1.734 & 25 & $(31-) 7$ \\
\hline Bridgewater & 13.63 & 9.513 & $31-24$ & Mantos Blancos & s 10.3 & 9.436 & 4 & 31 \\
\hline Cambria & 16.3 & 12.018 & 30 & *Moonbi & 13 & 0.002 & 1 & $(?-)^{35}$ \\
\hline Carlton Hamilt & t. 81.5 & 65.681 & 31 & Obernkirchen & 41 & 37.674 & 24 & 11 \\
\hline Charlotte & 4 & 3.156 & $20 \quad 29$ & *Prambanan & $?$ & 0.252 & 9 & $63(-3)$ \\
\hline *Grand Rapids & s 51.5 & 16.834 & $24(14-) 11$ & *PutnamCounty & y32.5 & 4.101 & 35 & $(23-) 14$ \\
\hline Hassi Jekna & 1.250 & 1.250 & $1 \quad 76$ & *Russel Gulch & 13 & 4.278 & 21 & $(23-) 18$ \\
\hline Jamestown & 4.015 & 3.578 & 40 & Smith'sMountair & in 5 & 2.271 & 14 & 29 \\
\hline Jewell Hill & 13.5 & 11.117 & 26 & Varas & 1.470 & 1.182 & 2 & 76 \\
\hline Jonesboro & 0.030 & 0.028 & 1 & *Victoria West & & & & \\
\hline $\begin{array}{l}\text { *La Grange } \\
\text { Laurens County }\end{array}$ & $\begin{array}{l}51 \\
\text { y } 2.220\end{array}$ & $\begin{array}{l}6.544 \\
1.750\end{array}$ & $\begin{array}{c}27(18-) 11 \\
653\end{array}$ & $(\mathrm{Ofv})$ & 2.944 & 1.175 & 11 & $(39-) 29$ \\
\hline
\end{tabular}

4I. c. Oktaëdrische Eisen mit mittleren Lamellen (Lamellenbreite $0.5-1 \mathrm{~mm}$ ), Om; auch Hammond-Eisen, Oh. $\mathrm{G}=4694.865 \mathrm{Kgr} .(2000-\infty)$.

\begin{tabular}{|c|c|c|c|c|c|c|c|}
\hline $\mathrm{U}$ & $\mathrm{N}$ & B & W & $\mathrm{U}$ & $\mathrm{N}$ & B & W \\
\hline Abert-Eisen 0.456 & 0.324 & 7 & 51 & Haniet-el- & & & \\
\hline Baird's Farm - & 0.837 & 23 & 23 & Beguel & 2.000 & 1 & 46 \\
\hline Burlington 6 & 4.867 & 22 & 14 & Hraschina $\quad 48.75$ & 39.671 & 18 & 8 \\
\hline Cabin Creek 47.4 & 47.409 & 4 & 16 & *Huejuquilla- & & & \\
\hline *Cachiyuyal 2.550 & 0.434 & 3 & $(74-) 46$ & Gruppe (39 000) & 2.709 & 14 & {$[(17-)$} \\
\hline Carthago 127 & 123.596 & 42 & 4 & Humboldt- & & & \\
\hline Charcas - & 783.649 & 20 & 3 & Eisen & 2.842 & 16 & 17 \\
\hline Chulafinnee 14.750 & 14.020 & 18 & 11 & Ilimaë & 51.198 & 2 & 12 \\
\hline${ }^{*}$ Cleveland 115.5 & 2.751 & 13 & $(17-) 5$ & *Ivanpah 50 & 0.348 & 20 & $(38-) 8$ \\
\hline Coopertown 17 & 7.239 & 36 & $(11-) 8$ & Jackson Co. 0.425 & 0.209 & 6 & 51 \\
\hline *Costilla $\quad 35$ & 1.580 & 1 & $(46-) 12$ & Joël Iron $\quad 1.300$ & 1.296 & 9 & 23 \\
\hline Cross Timbers - & 748.662 & 34 & 2 & Joe Wright & & & \\
\hline Dalton $\quad 57.5$ & 55.288 & 20 & 6 & Mountain 42.5 & 37.497 & 31 & 6 \\
\hline Dellys ～- & 0.091 & 4 & 63 & Juncal $\quad-$ & 105.604 & 14 & 5 \\
\hline *Denton Co. 5.750 & 0.675 & 20 & $(29-) 17$ & *KentonCo.163 & 39.724 & 14 & $(8-) 5$ \\
\hline *Descubri- & & & & Kokstad $\quad 42.6$ & 40.812 & 1 & 16 \\
\hline dora $\quad 617.5$ & 42.207 & 9 & $(8-) 4$ & La Caille - & 626.896 & 23 & 2 \\
\hline Elbogen 107 & 103.613 & 51 & 4 & Lenarto $\quad 108.6$ & 91.595 & 57 & $5-4$ \\
\hline El Capitan & & & & *Losttown $\quad 6.8$ & 0.235 & 12 & $(38-\cdot) 17$ \\
\hline Range $\quad 27.5$ & 27.500 & 3 & 16 & Lucky Hill ? & 3.406 & 6 & 24 \\
\hline Emmetsburg ? & 0.177 & 10 & 50 & Marshall Co. 6.8 & 1.388 & 17 & $23-17$ \\
\hline Fort Pierre 13 & 7.379 & 20 & $14-11$ & Mazapil $\quad 3.950$ & 3.780 & 6 & 24 \\
\hline Frankfort 11 & 8.319 & 12 & 14 & Merceditas 43.4 & 30.749 & 25 & 6 \\
\hline Glorieta $\quad 159$ & 145.101 & 35 & 4 & *Misteca 421 & 6.263 & 28 & $11-3$ \\
\hline Co. 0.200 & 0.071 & 11 & 50 & Murfreesboro 8.5 & 6.848 & 19 & 14 \\
\hline $\begin{array}{l}\text { Hammond } \\
\text { (Oh) } 24\end{array}$ & & & & Nagy-Vazsony 1.980 & 1.750 & 6 & 32 \\
\hline$(\mathrm{Oh})$ & 28.910 & 8 & & Nejed $\quad-$ & 59.800 & 11 & 6 \\
\hline
\end{tabular}


$45^{8}$ Systemat. Uebersicht der erhaltenen Meteoriten u. ihrer Tauschwerte.

\begin{tabular}{|c|c|c|c|c|c|c|c|c|c|}
\hline & U & $\mathrm{N}$ & B & W & & $\mathrm{U}$ & $\mathrm{N}$ & B & W \\
\hline *Orange & & & & & *Staunton & 113.964 & 51.627 & 57 & 5 \\
\hline River & 148.5 & 1.063 & 17 & $(23-) 5$ & *Tajgha & 0.350 & 0.021 & 2 & \\
\hline Petropawlo & wsk - & 7.200 & 7 & 18 & *Thunda & 50 & 9.287 & 19 & $(14-) 8$ \\
\hline Plymouth & - & 3.676 & 2 & 34 & Toluca & 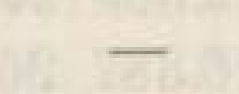 & 972.407 & 127 & 2 \\
\hline Puquios & 6.5 & 5.339 & 25 & 11 & *Tongano & & & & \\
\hline *Rancho de & & & & & xie & 11.5 & - & - & \\
\hline la Pila & 87.8 & 47.513 & 11 & $(8-) 6$ & *'Trenton & 65 & 24.615 & 39 & 6 \\
\hline Rowton & 3.5 & 3.228 & 9 & 17 & *Victoria & 175 & 0.472 & 8 & $(51-) 7$ \\
\hline${ }^{*}$ Ruff's Mou & & & & & Welland & 8 & 5.114 & 23 & 11 \\
\hline tain & 53 & 11.300 & 41 & $(8-) 6$ & Werchne & & & & \\
\hline Schwetz & 21.637 & 15.923 & 28 & 6 & Udinsk & 18.5 & 10.859 & 36 & 8 \\
\hline SenecaFalls & s 4 & 2.067 & 18 & 17 & *Wooster & 22.5 & 0.049 & 12 & \\
\hline Hition- & & & & & & & & & \\
\hline
\end{tabular}

42. d. Oktaëdrische Eisen mit groben Lamellen (Lamellenbreite $1.5-2 \mathrm{~mm}$ ), Og. $\mathrm{G}=14535.060 \mathrm{Kgr} .(2000-\infty)$.

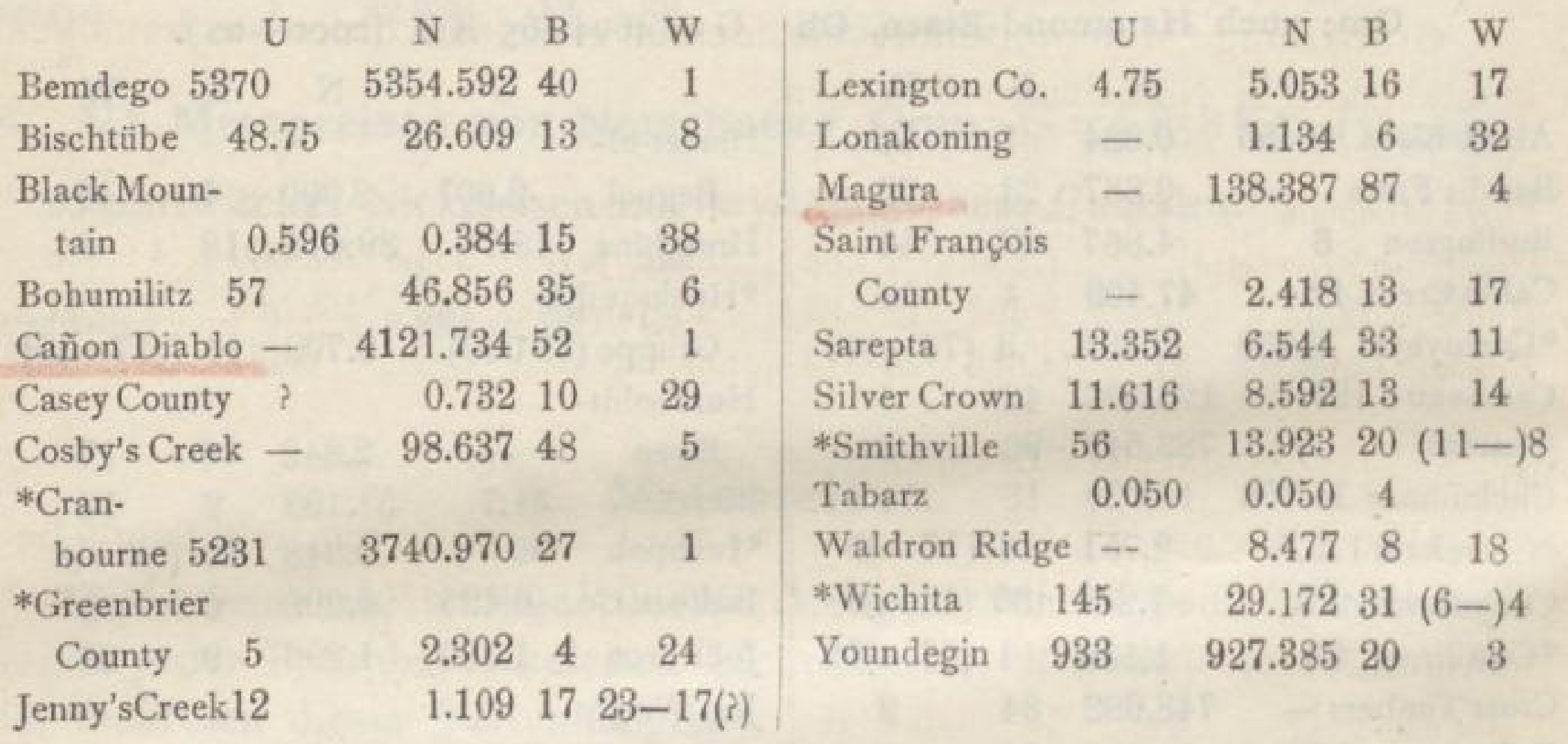

43. e. Oktaëdrische Eisen mit gröbsten Lamellen (Lamellenbreite über $2.5 \mathrm{~mm}$ ), Ogg. $\mathrm{G}=107.362 \mathrm{Kgr}$. $(100-200)$.

\begin{tabular}{|c|c|c|c|c|c|c|c|}
\hline & $\mathrm{U}$ & N & B & & U & $\stackrel{\mathrm{N}}{\mathrm{N}}$ & $\begin{array}{l}\text { W } \\
14\end{array}$ \\
\hline Creek & 32 & 0.052 & $1(354-) 46$ & *Union County & 6.8 & 0.711 & $22(67-) 40$ \\
\hline Nelson Cou & 73 & 38.703 & $41 \quad(18-) 14$ & & & & \\
\hline
\end{tabular}

44. f. Breccienähnliche oktaëdrische Eisen (Zacatecas-Gruppe), Obz.

$$
\mathrm{G}=36.657 \mathrm{Kgr} \text {. }(20-50 \text { ev. } 500-1000) \text {. }
$$

Grosskörnige Meteoreisenbreccie, deren einzelne haselnuss- bis wallnussgrosse Körner aus oktaëdrischem Nickeleisen bestehen.

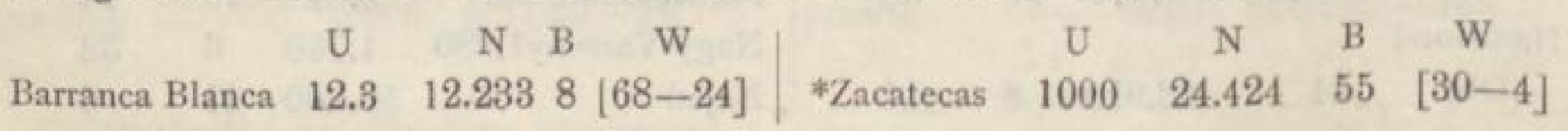




\section{Hexaëdrische Meteoreisen.}

Homogen krystallisiertes Nickeleisen mit deutlicher Spaltbarkeit nach dem Hexaëder und mit häufig eingelagerten Zwillingslamellen von Hexaëderkrystallen nach Oktaëderflächen, wodurch beim Aetzen Neumann'sche Linien entstehen.

45.

\begin{tabular}{lcrrc|lcrrr} 
& $\mathrm{U}$ & $\mathrm{N}$ & $\mathrm{B}$ & $\mathrm{W}$ & & $\mathrm{U}$ & $\mathrm{N}$ & $\mathrm{B}$ & W \\
*Auburn & 3.5 & 0.791 & 25 & $(31-)) 25$ & Lick Creek & 1.24 & 1.230 & 11 & 31 \\
Braunau & 40.710 & 29.367 & 56 & 8 & Lime Creek & 74.5 & 66.626 & 27 & 7 \\
Coahuila & - & 1751.178 & 72 & 2 & Nenntmannsdorf & 12.500 & 12.002 & 13 & 14 \\
*Dakota & 4.800 & 0.790 & 13 & $(39-) 23$ & Pittsburg & - & 0.592 & 13 & 39 \\
Hex River & $\left.61^{\prime}\right)$ & 45.644 & 19 & 11 & Scottsville & 10 & 6.771 & 22 & 14
\end{tabular}

46. b. Breccienähnliche hexaëdrische Eisen, Hb. G=581,409 Kgr. $(500--1000)$ Grobkörnige Meteoreisenbreccie, dessen einzelne Körner aus homogen krystallisiertem Nickeleisen bestehen.

\begin{tabular}{lrrrr|lrrrr} 
& U & N & B & W & & U & N & B & W \\
Floyd Mountain & 14.2 & 14.086 & 3 & 35 & Mejillones & $?$ & 1.185 & 10 & 39 \\
Great Fish River (Hb?) ? & 1.530 & 7 & 53 & Mount Joy & 383.5 & 383.500 & 4 & 8 \\
Hollands Store & 5 & 3.402 & 12 & 29 & São Julião ca. 162 & 162.569 & 8 & 11 \\
Kendall County & 21 & 14.666 & 26 & 14 & Summit & 1 & 0.471 & 4 & $86-67$
\end{tabular}

47.

c. Capeisen-Gruppe, Hca. G=83.135 Kgr. (50-100).

Hexaëdrisches (?) Nickeleisen, welches auf polierten, mit Säure behandelten Flächen eigentümliche Aetzbänder aufweist, die noch einer näheren Untersuchung bedürfen.

\begin{tabular}{llccc|lcccc} 
& $\mathrm{U}$ & $\mathrm{N}$ & $\mathrm{B}$ & $\mathrm{W}$ & & $\mathrm{U}$ & $\mathrm{N}$ & $\mathrm{B}$ & $\mathrm{W}$ \\
Capeisen & 85 & 71.789 & 37 & 18 & *Kokomo & 5.850 & 0.655 & 10 & $(106-) 63$ \\
Iquique & 12.5 & 10.691 & 2 & 76 & & & &
\end{tabular}

48.

d. Chesterville-Gruppe, Hch. G=19.402 Kgr. $(20-50)$.

Hexaëdrisches (?) Nickeleisen mit zahlreichen, durch die ganze Masse hindurch gleich orientierten Krystallen von Phosphornickeleisen (Schreibersit oder Rhabdit).

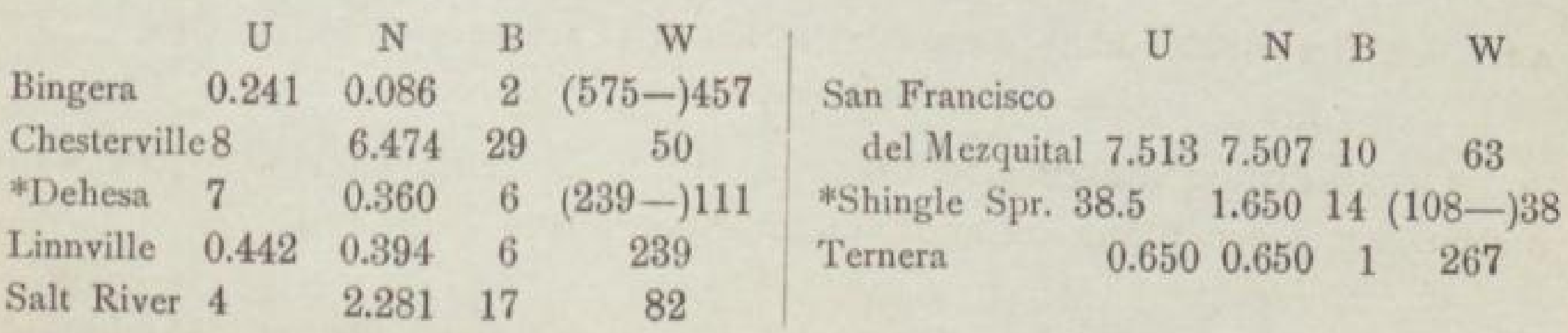

ז) Wie ich nachträglich bei Brezina, Wiener Sammlung 1895, p. 291 finde. 
460 Systemat. Uebersicht der erhaltenen Meteoriten u. ihrer Tauschwerte.

49. III. Dichte Meteoreisen, Db, Dn, Dp, Ds, Dt. G=I596.406 Kgr.

$$
(2000-\infty) \text {. }
$$

Amorphes Nickeleisen, welches also weder hexaëdrische Spaltbarkeit, noch Neumann'sche Linien, noch Widmanstätten'sche Figuren, noch irgend eine orientierte Anordnung erkennen lässt. Brezina teilt diese dichten Eisen in 5 Gruppen [Babb's Mill-Gruppe Db, Nedagolla-Gruppe Dn, Primitiva-Gruppe Dp, Senegal(Siratik)-Gruppe Ds, Tucson-Gruppe Dt] ein, welche ich hier zusammenfasse.

\begin{tabular}{|c|c|c|c|c|c|c|c|}
\hline & & \multicolumn{3}{|c|}{\begin{tabular}{lllll|llll} 
& U & N & B & W & U & N & B & W
\end{tabular}} \\
\hline & & & & & & & \\
\hline \multicolumn{4}{|c|}{ 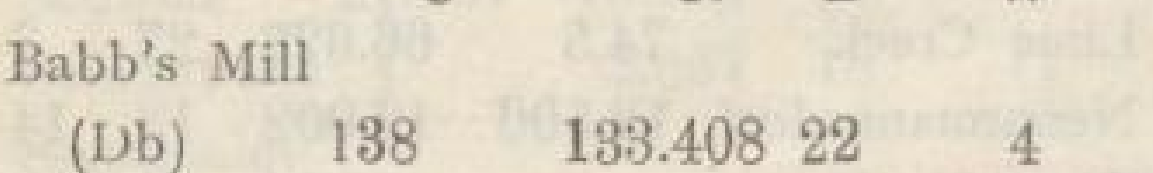 } & Bay (Dn) - & 0.020 & 5 & \\
\hline \multicolumn{4}{|l|}{ *Campo del Cielo } & $\begin{array}{l}\text { Santa Catha- } \\
\text { rina (Dn) }\end{array}$ & 137.453 & 46 & 4 \\
\hline Morradal (Db) 2.750 & 2.750 & 2 & 34 & Scriba (Dn) & 1.685 & 23 & $18(-14)$ \\
\hline Nedagolla (Dn) - & 4.701 & 9 & 17 & Senegal (Ds) & 1.710 & 15 & 23 \\
\hline Newstead(Dn) 14.830 & 11.458 & 20 & 11 & Smithland $(\mathrm{Db})$ - & 4.969 & 12 & 17 \\
\hline *Oktibbeha(Db) 0.155 & 50015 & 9 & & Tarapaca $(\mathrm{Db}) 7.5$ & 5.368 & 25 & 11 \\
\hline *Primitiva(DP) 3 & 1.348 & 2 & $(46-) 34$ & *Tucson(Dt) 1000 & 637.224 & 30 & 2 \\
\hline *Rasgata(Ds) 750 & 9.443 & 30 & $(11-)^{2}$ & & & & \\
\hline
\end{tabular}

50. IV. Meteoreisen, welche wegen mangelnder Bestimmung keine Stellung im System gefunden haben. $G=2229.995 \mathrm{Kgr}$.

\begin{tabular}{|c|c|c|c|c|c|c|c|}
\hline & $\mathrm{U}$ & $\mathrm{N}$ & W & & $\mathrm{U}$ & $\mathrm{N}$ & B \\
\hline Augustinowka 40 & & 400 & 3 & Cuba & - & 1.329 & 1 \\
\hline *Bald Eagle (O) & 3.3 & ? & ? & *Deep Springs & 11.5 & 2.259 & 5 \\
\hline *Blue Tier & 1.3 & ? & ? & Forsyth County & 20.943 & 20.943 & 1 \\
\hline Chihuahua & - & 1800.000 & 1 & *Henry County & 1.92 & 0.152 & 7 \\
\hline Colfax $(O)$ & 2400 & 1.954 & 3 & Yardea Station & 3.269 & 3.269 & 1 \\
\hline CranberryPlains( & O) - & 0.089 & 6 & & & & \\
\hline
\end{tabular}




\section{Berichtigungen.}

p. 4 ist bei $\gg$ Alais « noch zu verweisen auf: Wöhler, Gött. Gel. Anz. (Nachr.) 1864, p. 279.

p. 7 Z. 21 v. u. lies $\gg$ Graz, J. 76\& statt Graz 76\%.

p. 9 ist bei $>$ Asco \& Tübingen und London, B. M. mit Spl. hinzuzufügen.

p. IO ist bei $*$ Assame Tübingen mit 4 gr. hinzuzufügen.

p. 18 Z. 2 v. u. lies: sauch $\approx$ statt salso $<$.

p. 18 ist bei "Bairds Farm" noch Bonn mit $\mathbf{I}$ gr. hinzuzufügen

p. 28 ist bei "Bethlehem * London, B. M. mit Spl. hinzuzufügen.

p. 34 Z. 14 v. o. lies: s Washington « statt > Washington, M. «.

p. $3^{8}$ ist bei "Bohumilitz noch zu verweisen auf: Quenstedt >Klar und Wahr 1872 , p. $3^{13}$ (Abb. einer geätzten Platte).

p. 44 ist bei •Braunaus noch zu verweisen auf: Quenstedt "Klar und Wahr 1872 , p. 293 (Skizze eines Stïckes der Tübinger Sammlung).

p. 49 Z. 12 v. u. lies: „Meteoreisen-Studien \& statt sMeteoriten-Studien *

p. 55 ist einzuschalten: Cachinal Vaca Muerta.

p. 67 ist einzuschalten: $\mathrm{Chañal}$ Vaca Muerta,

p. 67 Z. 14 v. u. lies: "Chañaralino* statt *Chañarlino .

p. 145 Z. ro v, u. lies: $\$ 1875$ : Gümbel : « statt $₫ 1875$ : ,

p. 156 Z. 2 v. o. lies: »Washington, Sh. « statt „Washington *

p. 177 Z. 9 v. u. lies: $\gg 1848$ : Bloede: statt $\gg 1846$ : Bloede:

p. 192 ist bei $\$$ Krasnoj-Ugole London, B. M. mit Spl. hinzuzufügen.

p. 213 Z. 2 v. o. lies: $536 \mathrm{Kgr.<}$ statt $\$ 936 \mathrm{Kgr}$. .

p. 227 Z. 5 v. u, lies: $\gg 1836$ : Kämtz: statt $\gg 1830$ : Kämtz: ,

p. 251 Z. 20 v. u, lies: „Stein, Cca \&, statt »Stein Cc\&,

p. 253 Z. 7 v. o. lies: $>70$ lbs. 14 oz. \& statt $>70$ lbs. 40 oz.«.

p. 264 ist bei Ochansk " noch Newton mit 23 gr. hinzuzufügen.

p. 265 Z. I4 v. u. lies: >Oktibbeha Countye statt Oktibbeha e.

p. 336 Z. 8 v. o. lies: sSowallik $\pi$ statt iSwallike.

p. 367 Z. 2 v. o. lies: sArizona, U. S. A.\& statt >Arizona, Mex.e.

p. 432 Z. I4 v. o. lies: $>$ Z, G und $B \varepsilon$ statt $>Z, N$ und $B \varepsilon$. 

7 SLUB

Wir führen Wissen. http://digital.slub-dresden.de/id477902421/515
UNIVERSITÄTSBIBLIOTHEK 


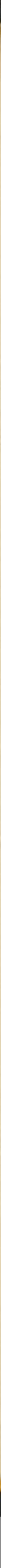


Büchere $i$

- Bergafademie. Freibera $i 5 a$

SLUB

Wir führen Wissen. 
